\title{
Py.hological
}

Applications

grid rêd:

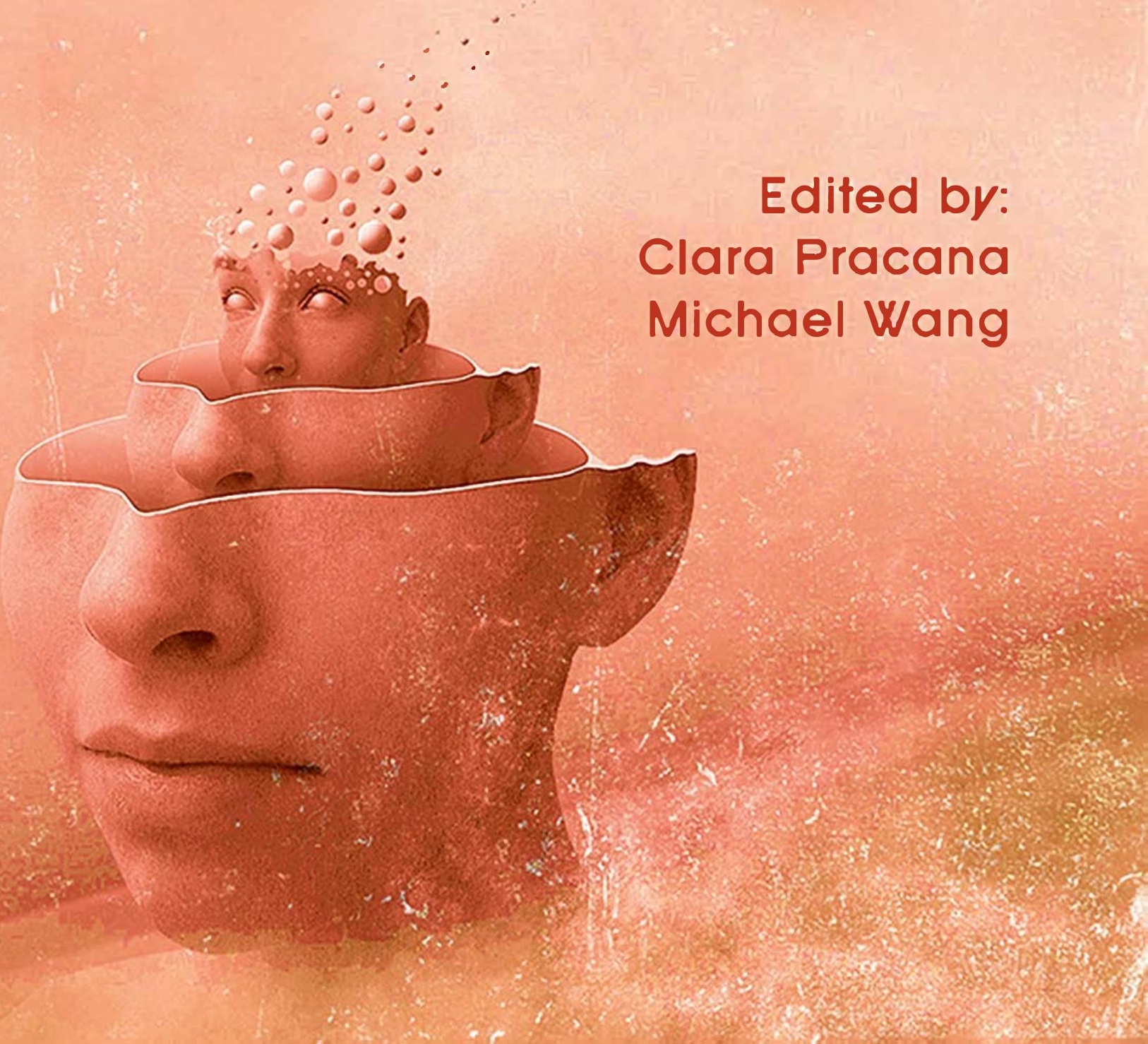




\section{Psychological Applications and Trends 2019}

Edited by:

Clara Pracana

\&

Michael Wang 


\section{Edited by:}

- Prof. Clara Pracana, Full and Training Member of the Portuguese Association of Psychoanalysis and Psychoanalytic Psychotherapy, Portugal,

- Prof. Michael Wang, Emeritus Professor of Clinical Psychology, University of Leicester, United Kingdom

Published by InScience Press, Rua Tomás Ribeiro, 45, $1^{\circ}$ D, 1050-225 Lisboa, Portugal

\section{Copyright @ 2019 InScience Press}

All rights are reserved. Permission is granted for personal and educational use only.

Commercial copying, hiring and lending is prohibited. The whole or part of this publication material cannot be reproduced, reprinted, translated, stored or transmitted, in any form or means, without the written permission of the publisher. The publisher and authors have taken care that the information and recommendations contained herein are accurate and compatible with the generally accepted standards at the time of publication.

The individual essays remain the intellectual properties of the contributors.

ISSN (electronic version): 2184-3414

ISSN (printed version): 2184-2205

ISBN: 978-989-54312-2-9

Legal Deposit: 440723/18

Printed in Lisbon, Portugal, by GIMA - Gestão de Imagem Empresarial, Lda. 
Keynote Lecture

Special Talks 



\section{FOREWORD}

This book contains a compilation of papers presented at the International Psychological Applications Conference and Trends (InPACT) 2019, organized by the World Institute for Advanced Research and Science (W.I.A.R.S.).

Modern psychology offers a large range of scientific fields where it can be applied. The goal of understanding individuals and groups (mental functions and behavioral standpoints), from this academic and practical scientific discipline, aims ultimately to benefit society. The International Conference seeks to provide some answers and explore the several areas within the Psychology field, new developments in studies and proposals for future scientific projects. The goal is to offer a worldwide connection between psychologists, researchers and lecturers, from a wide range of academic fields, interested in exploring and giving their contribution in psychological issues. We take pride in having been able to connect and bring together academics, scholars, practitioners and others interested in a field that is fertile in new perspectives, ideas and knowledge.

We counted on an extensive variety of contributors and presenters, which can supplement the view of the human essence and behavior, showing the impact of their different personal, academic and cultural experiences. This is, certainly, one of the reasons there are nationalities and cultures represented, inspiring multi-disciplinary collaborative links, fomenting intellectual encounter and development.

InPACT 2019 received 322 submissions, from more than 35 different countries from all over the world, reviewed by a double-blind process. Submissions were prepared to take form of Oral Presentations, Posters, Virtual Presentations and Workshops. 107 submissions (overall, 33\% acceptance rate) were accepted for presentation in the conference.

The conference also included:

- One keynote presentations by Prof. Dr. Glenys Parry (PhD, CPsychol, FBPsS, Emeritus Professor, School of Health \& Related Research, University of Sheffield, United Kingdom).

- Two Special Talks, one by Prof. Dr. Michael Wang (Emeritus Professor of Clinical Psychology, University of Leicester, United Kingdom), and the other by Prof. Dr. Luís Delgado (Applied Psychology Research Center Capabilities and Inclusion (APPsyCI) - University Institute, Portugal).

We would like to express our gratitude to all our invitees.

The Conference addresses different categories inside Applied Psychology area and papers fit broadly into one of the named themes and sub-themes. The conference program includes six main broad-ranging categories that cover diversified interest areas:

- CLINICAL PSYCHOLOGY: Emotions and related psychological processes; Assessment; Psychotherapy and counseling; Addictive behaviors; Eating disorders; Personality disorders; Quality of life and mental health; Communication within relationships; Services of mental health; and Psychopathology.

- EDUCATIONAL PSYCHOLOGY: Language and cognitive processes; School environment and childhood disorders; Parenting and parenting related processes; Learning and technology; Psychology in schools; Intelligence and creativity; Motivation in classroom; Perspectives on teaching; Assessment and evaluation; and Individual differences in learning.

- SOCIAL PSYCHOLOGY: Cross-cultural dimensions of mental disorders; Employment issues and training; Organizational psychology; Psychology in politics and international issues; Social factors in adolescence and its development; Social anxiety and self-esteem; Immigration and social policy; Self-efficacy and identity development; Parenting and social support; Addiction and stigmatization; and Psychological and social impact of virtual networks. 
- LEGAL PSYCHOLOGY: Violence and trauma; Mass-media and aggression; Intra-familial violence; Juvenile delinquency; Aggressive behavior in childhood; Internet offending; Working with crime perpetrators; Forensic psychology; Violent risk assessment; and Law enforcement and stress.

- COGNITIVE AND EXPERIMENTAL PSYChOlOGY: Perception, memory and attention; Decision making and problem-solving; Concept formation, reasoning and judgment; Language processing; Learning skills and education; Cognitive Neuroscience; Computer analogies and information processing (Artificial Intelligence and computer simulations); Social and cultural factors in the cognitive approach; Experimental methods, research and statistics; and Biopsychology.

- PSYCHOANALYSIS AND PSYCHOANALYTICAL PSYCHOTHERAPY: Psychoanalysis and psychology; The unconscious; The Oedipus complex; Psychoanalysis of children; Pathological mourning; Addictive personalities; Borderline organizations; Narcissistic personalities; Anxiety and phobias; Psychosis; Neuropsychoanalysis.

This book contains the results of the different researches conducted by authors who focused on what they are passionate about: to study and develop research in areas related to Psychology and its applications. It includes an extensive variety of contributors and presenters that are hereby sharing with us their different personal, academic and cultural experiences.

We would like to thank all the authors and participants, the members of the academic scientific committee, and of course, to the organizing and administration team for making and putting this conference together.

Looking forward to continuing our collaboration in the future,

Respectfully,

Prof. Clara Pracana

Full and Training Member of the Portuguese Association of Psychoanalysis and Psychoanalytic Psychotherapy, Portugal

Conference and Program Co-Chair

Prof. Michael Wang

Emeritus Professor of Clinical Psychology, University of Leicester, United Kingdom

Conference and Program Co-Chair

Zagreb, Croatia, 4-6 May, 2019 


\title{
SCIENTIFIC COMMITTEE
}

\section{Conference and Program Co-Chairs}

\author{
Clara Pracana
}

Full and Training Member of the Portuguese Association of Psychoanalysis and Psychoanalytic Psychotherapy, Portugal

Michael Wang

Emeritus Professor of Clinical Psychology, University of Leicester, United Kingdom

\section{International Scientific Committee}

A. Nuray Karanci, Middle East Technical University, Turkey

Acácia Santos, São Francisco University, Brazil

Adi Fahrudin, Universiti Malaysia Sabah, Malaysia

Adilia Silva, Independent Researcher/Clinician, South Africa

Agata Wawrzyniak, University of Szczecin, Poland

Alessio Avenanti, Università di Bologna, Italy

Ali Kemal Tekin, Sultan Qaboos University, Oman

Alois Ghergut, University Alexandru Ioan Cuza from Iasi, Romania

Ana Paula Couceiro Figueira, University of Coimbra, Portugal

Anastasia Alevriadou, University of Western Macedonia, Greece

Andreja Bubic, University of Split, Croatia

Anna Alexandrova-Karamanova, Institute for Population and Human Studies - BAS, Bulgaria

Anna Janovská, Pavol Jozef Šafárik University in Košice, Slovakia

Anne-Marie Émond, Université de Montréal, Canada

Anton James, The British Psychological Society; The Irish Psychological Society, United Kingdom / Ireland

Antonio Aiello, University of Pisa, Italy

Art Blume, Washington State University, USA

Assunta Marano, Themis Research Centre /

"Sapienza", University of Rome, Italy

Atmane Ikhlef, Independent Consultant/ Researcher, Algeria
Auksė Endriulaitienė, Vytautas Magnus University, Lithuania

Ayse Esra Aslan, Istanbul University, Turkey

Beata Gajdošová, Pavol Jozef Šafárik University in Košice, Slovakia

Beatriz Delgado Domenech, University of Alicante, Spain

Bernard Gangloff, University of Rouen, France

Binnur Yesilyaprak, Ankara University, Turkey

Carmen Berce Popa, University of Oradea, Romania

Chris McVittie, Queen Margaret University, United Kingdom

Claudio Sica, University of Firenze, Italy

Colette Dufresne-Tassé, Université de Montréal, Canada

Daniel Süss, Zurich University of Applied Sciences \& University of Zurich, Switzerland

Danijela S. Petrović, University of Belgrade, Faculty of Philosophy, Serbia

Daragh T. Mcdermott, Anglia Ruskin University, United Kingdom

David Aparisi, Universidad de Alicante, Spain

Deborah Wooldridge, Bowling Green State University, USA

Dweep Chand Singh, AIBHAS, Amity University Uttar Pradesh, NOIDA, India

Eda Kargi, East Mediterranean University, Cyprus

Ederaldo J. Lopes, Federal University of Uberlândia, Brazil

Edwin Herazo, Human Behavioral Research Institute, Colombia 
Ekaterina Usmanova, Saint Petersburg State University, Russia

Elena Levchenko, Perm State University, Russia

Eleni Petkari, European University of Cyprus, Cyprus

Elenita M. Tiamzon, World Citi Colleges, Philippines

Emel Kuruoglu, Dokuz Eylul University, Turkey

Emerson Rasera, Federal University of Uberlândia, Brazil

Fotios Anagnostopoulos, Panteion University of Social \& Political Sciences, Greece

Gail Tan-Ilagan, Ateneo de Davao University, Philippines

Gandharva Joshi, Saurashtra University, India

Gordana Đigić, University of Niš, Serbia

Hayder Aljouranj, Helsinki Deaconess Institute, Finland

Henry Grubb, University of Dubuque, USA

Igor Menezes, University of Lincoln, United Kingdom

Isabella Corradini, Themis Research Centre, Italy

Isabella Mcmurray, University of

Bedfordshire, United Kingdom

Jakob Pietschnig, University of Vienna, Austria

Jelisaveta Todorović, University of Niš, Serbia

Juliet Dinkha, American University of Kuwait, Kuwait

Kamna Chhibber, Fortis Healthcare, India

Katherine Makarec, William Paterson University, USA

Konstantinos Kafetsios, University of Crete, Greece

Kristi Kõiv, University of Tartu, Estonia

Lada Kaliská, Matej Bel University, Slovakia

Laura Furcsa, Eszterházy Károly University, Hungary

Laura Vilela e Souza, Universidade de São Paulo, Brazil

Liliana Mata, "Vasile Alecsandri" University of Bacau, Romania

Lilly Both, University of New Brunswick in Saint John, Canada
Lisa Best, University of New Brunswick, Canada

Ljiljana Gregov, University of Zadar, Croatia

Loreta Bukšnytė-Marmienė, Vytautas Magnus University, Lithuania

Luca Cerniglia, Università Telematica Internazionale UNINETTUNO, Italy

Lucia Hricová, Pavol Jozef Šafárik University in Košice, Slovakia

Luis Alberto Casado Aranda, University of Granada, Spain

Madelene Sta. Maria, De La Salle University Manila, Philippines

Maher Abu Hilal, Sultan Qaboos University, Oman

Marcelo F. Costa, University of São Paulo, Brazil

Marco Vassallo, Council for Agricultural Research and Economics (CREA), Italy

Margit Höfler, Danube University Krems, Austria

Maria Iakovleva, Saint Petersburg State University, Russia

Martin Eisemann, Tromso University, Norway Mary M. Chittooran, Saint Louis University, USA

Michael Zeiler, Medical University of Vienna, Austria

Miroslava Köverová, Pavol Jozef Šafárik University in Košice, Slovakia

Mojca Juriševič, University of Ljubljana, Faculty of Education, Slovenia

Nadia Mateeva, Bulgarian Academy of Sciences, Bulgaria

Neala Ambrosi-Randić, Juraj Dobrila University of Pula, Croatia

Nebojša Milićević, University of Niš, Serbia

Norihiro Kuroishi, International Christian University, Japan

Nuria Carriedo, UNED - Universidad Nacional de Educación a Distancia, Spain

Olga Deyneka, St. Petersburg State University, Russia

Olga Orosová, Pavol Jozef Šafárik University in Košice, Slovakia

Omar Rahman, University of South Florida, USA 
Otilia Clipa, Stefan cel Mare University, Romania

Páraic Scanlon, Birmingham City University, United Kingdom

Regina Fernández Morales, Universidad de Chile, Chile

Ronald Hambleton, University of Massachusetts, USA

Roxana-Maria Ghiațău, Alexandru Ioan Cuza University, Iasi, Romania

Sharo (Shahrokh) Shafaie, Southeast Missouri State University, USA

Shulamith Kreitler, Tel-Aviv University, Israel

Silvia Cimino, Sapienza University of Rome, Italy

Snežana Stojiljković, University of Niš, Serbia
Suppiah Nachiappan, Sultan Idris Education University, Malaysia

Suzie Savvidou, The University of Sheffield International Faculty, CITY College, Greece

Sylvia Kwok, City University of Hong Kong, China

Tali Heiman, The Open University of Israel, Israel

Veena Easvaradoss, Women's Christian College, Chennai, India

Victor Martinelli, University of Malta, Malta Vildan Mevsim, Dokuz Eylul University, Turkey

Werner Leitner, IB Hochschule, Germany

Yoriko Sano, Kanto Gakuin University, Japan

Zvjezdan Penezić, University of Zadar, Croatia 


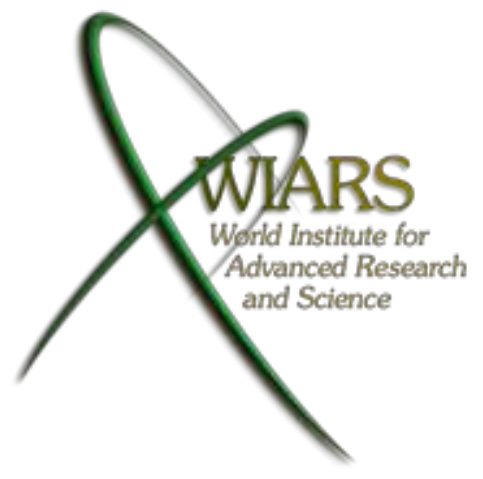

http://www.wiars.org 


\title{
HOW DO PSYCHOLOGICAL THERAPIES CAUSE HARM - AND WHAT SHOULD WE DO ABOUT IT?
}

\author{
Prof. Dr. Glenys Parry \\ PhD, CPsychol, FBPsS \\ Emeritus Professor, School of Health \& Related Research, University of Sheffield (United Kingdom)
}

\begin{abstract}
There is no doubt that psychological therapies are generally effective in helping people with a range of mental health difficulties find relief from distress and improve their quality of life. Both from practice-based evidence and well-controlled clinical trials, we see that those who engage in a bona fide therapy with a qualified practitioner are, on average, better off than those who do not. The effect sizes are, compared with many medical interventions, quite impressive.

Yet that phrase 'on average' hides a genuine problem; some people who undertake therapy have a bad experience, feel worse afterwards than when they started, and may experience lasting bad effects. Even a cursory internet search will reveal many blogs, websites, books and articles from people who say they have been harmed. Psychotherapy research has a history of neglecting the subject, but is now starting to take this problem seriously. Recent developments include psychometric measures of negative effects of treatment, qualitative and survey investigations and meta-analysis of negative outcomes. Understanding therapeutic harm is vital in taking steps to prevent it, and I shall give an overview of some key issues in appraising the evidence of whether therapy is harmful, what makes it harmful and what can be done to reduce the risk of harm.

We know a good deal about how successful therapy works, in terms of therapist competence, therapist characteristics, client characteristics, trajectories of change, therapeutic alliance rupture and repair, specific techniques and 'non-specific' factors. We know less about bad therapy or harmful therapy. For a start, these are not necessarily the same things. There is an important distinction between process and outcome; bad experience and poor outcome. Bad therapy, therapy which does not meet minimum standards of competent practice, is not necessarily harmful, and well-conducted therapy can have unwanted negative effects.

Unwanted negative effects are sometimes termed 'side' effects, although I reject this terminology and shall explain why. Much psychological research in therapy uses a medical framework, characterised by terms such as matching treatment to diagnosis, dose-response, side effects, and treatment compliance and so on. Whilst this metaphor has utility, to address the potential for harm, I advocate a dialogic understanding of the co-constructed therapeutic frame; two subjectivities meeting in the consulting room, each with their own histories and patterns of managing self in relation to others. I find a cognitive analytic approach the most useful in this regard.

Most therapists are neither excellent nor harmful, but have a mix of outcomes. Only a minority are psychotoxic, with fake qualifications, lack of training, narcissism, psychopathy, sexual abuse, financial abuse, emotional abuse, rigidity, lack of emotional intelligence or mentalisation capacity, and gross boundary violations.

However, I shall argue that the toxic few are not the main issue. I draw on evidence about clients' common experiences of harmful therapy, differences between therapists, therapist errors, inappropriate therapy choice, misdiagnosis, unresolved alliance ruptures, and what is known about the people most vulnerable to the risk of harm from therapy. From the therapist's perspective, I shall argue that there are clear limitations of therapist training and skill, and errors are inevitable. It is a natural defence to minimise and avoid discussion of our errors rather than accept them as a normal part of professional practice. A risk-averse, punitive system is not going to help, indeed it encourages defensive, secretive practice. Understanding the common causes of harm, creating a compassionate narrative and putting systems in place to mitigate risk is more useful. Therapy is undertaken within a systemic context a private practice, a professional ethics framework, a public sector mental health service. These systems are powerful influences on the extent to which potentially harmful practice can be detected and prevented. I make five recommendations for safer practice at the individual, professional and organisational levels.
\end{abstract}




\section{Biography}

Glenys Parry is a clinical psychologist and Emeritus Professor at the University of Sheffield. She practises cognitive analytic therapy in a not-for-profit group practice in Sheffield.

She worked in the NHS from 1974 to 2011, where her roles included Consultant Psychologist and Psychotherapist, Director of Psychology Services, and Director of R\&D. She has also been responsible for psychology and psychotherapy policy at the Department of Health (1992-1996) and has contributed to clinical guideline development at NICE. Professionally she is a Fellow of the British Psychological Society, MB Shapiro award holder, founder member of the Association for Cognitive Analytic Therapy and past Vice-President of the British Association for Counselling and Psychotherapy. As Professor of Psychological Therapies at the University of Sheffield (School of Health \& Related Research 2002-2016), she conducted randomised trials, systematic reviews, qualitative research and service evaluations in a range of topics including the impact of life events and social support on depression, cognitive behaviour therapy for panic fear in asthma, health status of Gypsies and Travellers, therapist competence in cognitive analytic therapy, IAPT services, computerised CBT, care pathways in persistent depression and borderline personality disorder. Her project 'Understanding and Preventing Adverse Effects of Psychological Therapies' was funded by the UK NIHR Research for Patient Benefit programme. 


\title{
BION AND THE POPES OF HORROR
}

\author{
Prof. Dr. Luís Delgado \\ Applied Psychology Research Center Capabilities and Inclusion (APPsyCI) - \\ ISPA - University Institute (Portugal)
}

\begin{abstract}
In this communication of psychoanalysis (extra-therapeutic) applied to artistic productions the author focuses on the (in)ability to think of horror through two of the, roughly, forty interpretations made by the painter Francis Bacon - The Screaming Pope (1953) and Figure with Meat (1954) - of the portrait of Pope Innocent X (1650) by Diego Velázquez, which can be understood in the light of Wilfred Bion`s Theory of Transformations (1965), in which each interpretation/transformation leads the painter to the intensification of an unnamed terror, viscerally felt, without any capacity for mentalization, for naming, for a dream and the diffusion of the feeling of identity. For this, he created a technique capable of reproducing the deep psychic reality instead of the appearance of people. His aim was not to paint the horror that gave rise to "scream", but to paint the very "scream" of terror, so as to make the inaudible audible.
\end{abstract}

Keywords: Velázquez, Bacon, Bion, Horror, Transformations theory.

Figure 1. Pope Innocent X (Velázquez, 1650).

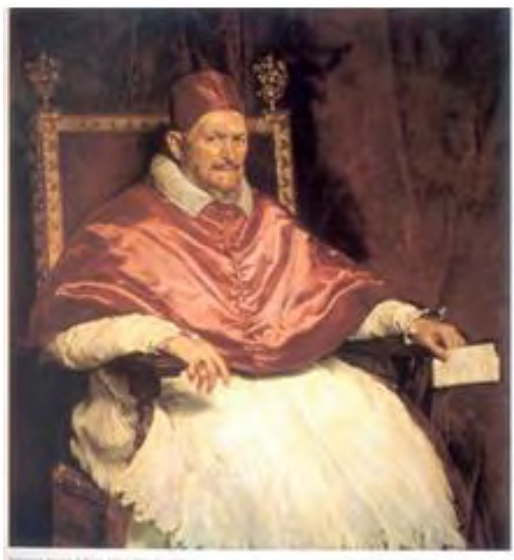

Diego Velázquez's painting, Pope Innocent X (1650), can be found in Rome, in the Doria Pamphilj Gallery. It is a realistic painting depicting a Pope who is brilliant, radiant, magnificent, and secure. In short, a Pope symbolizing the robustness of one of the fundamental pillars of western culture and civilization. When the Pope saw Vélazquez's painting for the first time, he exclaimed "Troppo vero!". Three hundred years later, Francis Bacon saw a reproduction of the painting and assembled a vast collection of reproductions, through which he came to study and know the painting deeply. Between 1951 and 1965, Bacon painted 45 studies, variations, reactions to Velázquez's painting, always feeling a strong obsession for it. 
Figure 2. Francis Bacon (1909-1992).

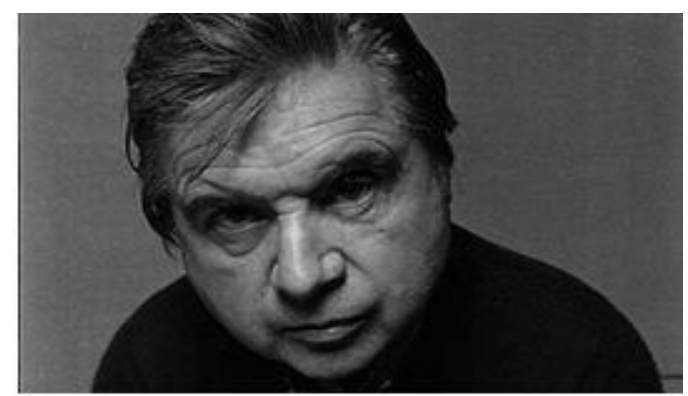

Before we go on to describe Bacon's first study of the painting, I propose that we first listen to the painter himself about the meaning and technique of his painting in general, as well as the historical and cultural circumstances of the time:

. "Art is a method of opening up areas of sensitivity, rather than the mere illustration of an object" (Bacon cited by Sinclair, 1995, p. 232).

. "I paint forces, not figures" (interview with Sylvester, 2007).

. "A big part of a painting is always a convention, the appearance, and that's what I try to eliminate from my paintings. I look for the essential, that the painting takes on, in the most direct way possible, the material identity of what it is I'm representing. My way of distorting images brings me far closer to human beings than if I just sat down and did their portrait (...) I tried to find a technique capable of reproducing the profound reality and not the appearance of people" (interview with Ramón Chao, 1982).

In short, what Bacon attempts to capture is the emotional and sensitive state of a subject in any given moment, his objective is to capture an identity.

Like many other post-World War II artists, he tried to reproduce the climate of absolute terror and disbelief in the culture, expressing what happened to humanity after the War and the Nazi Holocaust. Bacon never paints the figuration of horror, there is never any narration or illustration of a horror scene. What he paints is the scream as a capture of an invisible force. His objective is to express the torturous reality of the contemporary man, traumatized by the impossibility to eradicate Evil, worse yet, by the complete disbelief in civilizational and cultural progress, by the maddening paradox of the association between progress and barbarism. What Bacon intends to metaphorize is the afflictive and rabid helplessness of the disaster in culture, the disenchantment of the world. I remember the lucid words of Primo Levi in, and the title is accurate, If This Is A Man: "The offense made to the human being (...) the insurmountable nature of the offense that spreads like a disease, which is an inexhaustible source of evil" (The Truce, 1963/2010, p. 9). What Bacon lets us see is a metaphor of the internal feeling towards the total bankruptcy of culture, as Freud conceived in the beginning of his essay, The Future of an Illusion (1927/1981). Why choose the figure of the Pope? Precisely because he represents one of the major foundations of our western civilization.

Figure 3. The Screaming Pope (Bacon, 1953).

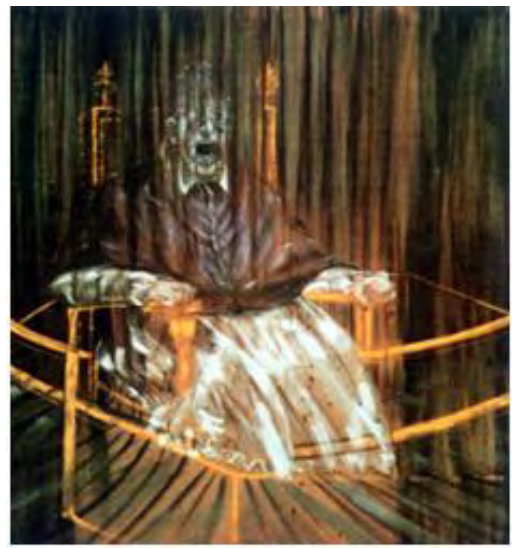

Francis Bacon's painting, Study After Velázquez's Portrait of Pope Innocent X (1953), better known as The Screaming Pope, presents us with a vociferous and desperate papal figure in free fall, enclosed in a chair and a cube, with his upper body blurred between the curtains in the background and a 
tassel on top of the place that should be occupied by the brain. Beyond the feeling of a downward movement of the figure, when Bacon paints the pope who screams, there is nothing that provokes horror, and the curtain before him is not only a way to isolate him - to subtract him from view - it is also to show that he sees nothing, he screams before the invisible.

In addition to the fierce criticism of "organized religion" in the form of screams and sneers of a king of the Catholic Church and monarch of the world, Bacon put his deepest fears and angers on the canvas, expelled them, and attained a new intensity. Let us not forget that Bacon brings with him a childhood marked by death (of two sisters), a youth disturbed by the extreme incomprehension of his father (he expelled him from home for his homosexual manifestations), the suicide of two of his lovers, as of all the horror of Ireland's civil war, both World Wars, and the beginning of the Cold War. The figure vertiginously falling and screaming, solitary and caged, without any possibility of escape, is an endless mask of suffering, anger, anguish, of helplessness and terror, of decrepitude and death.

Despite all the realism of his painting, Bacon believed that there were "inner realities", even admitting that Freud's works had modified his own sense of realism, "because we became more aware of how realism can be fed by the subconscious. (...) We live almost all the time covered by veils... It is a veiled existence." Indeed, Bacon's picture is a lifting of the veil of the appearances of Velázquez's representation, confronting himself and the spectator with "the brutality of the facts", with the spasmodic and convulsive cry, in order to directly and violently hit the nervous system. First sit down and then think. According to Paul Klee's famous formula, "instead of giving the visible, make it visible", in art it is not a matter of reproducing or inventing forms, but of grasping forces (Klee, cited by Deleuze, 1984). Bacon told a journalist that his studies of Velázquez were "an intention to turn a certain type of sensation into something visible".

Wilfred Bion states in his book Transformations (1965/1982), now a classic: "The theory of transformations and its development do not belong to the central corpus of psychoanalytic theory, but to the practice of psychoanalytic observation. Psychoanalytic theories, as well as the statements of the patient or the analyst, are representations of an emotional experience. Understanding the process of representation will help us to understand representation and what is represented "(pp. 43-44). The painter, through his artistic talent, managed to transform a person, a landscape ("the realization") into a painting ("representation"), thanks to the invariants. "I will call 'invariant' the elements that account for the unchanged aspect of transformation" (p.7). And, further on: "The analysis, in its pre-catastrophic state, is distinguished from the post-catastrophic state by the following characteristics: it is not emotional, theoretical, and devoid of any noticeable exterior modification... In the post-catastrophic state, on the contrary, violence is obvious, but its ideological content, hitherto evident, seems to be lacking. The emotion is evident and reaches the analyst" (p.15) - in our case it reaches the viewer. Bion proposes that the psychic transformations are processed by three different modalities, that he denominates "transformations of rigid movement", "projective transformations" and "transformations in hallucinosis".

In the present study, and according to Bion's theory, we conceive Velázquez's painting as a representation of Bacon's pre-catastrophic (the mind's neurotic part) state performing a rigid motion transformation, in which it minimally distorts the original fact - Innocent $X$ - and allows the viewer to find the invariant element very easily ("Troppo vero!"). The apparent characteristics of the pope are visible: power, serenity, infallibility, protection, trust in the life of the beyond, belief in love...

The Screaming Pope, however, represents the projection of the mind in a post-catastrophic state, in a projective-type transformation, distorting the original fact more intensely due to intense emotions, distorting the notions of space/time and papal posture without, however, completely preventing the viewer from recognizing the invariants that make recognition possible.

Figure 4. Figure with Meat (Bacon, 1954).

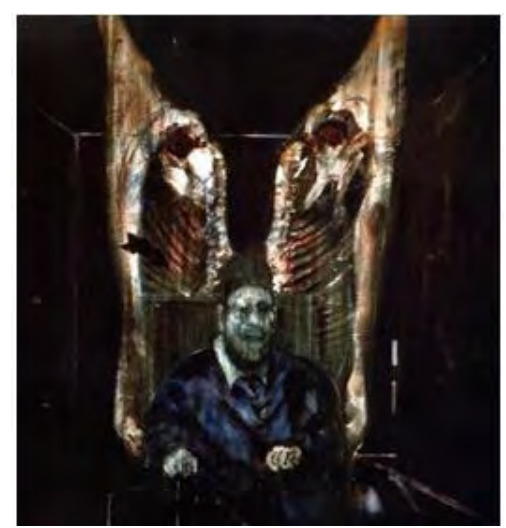


In another study of Pope Innocent X, titled Figure with Meat (1954), we see, in lugubrious hues, a body that vanishes and decomposes, escaping through a screaming mouth, behind which two huge carcasses of animals appear hanging. Here we no longer distinguish the man from the animal, the dead from the living. This totally disfigured and mutilated body, at the border of disappearance and confused with the flesh, suggests the loss of basic identity and the presence of death. "I am always aware of my mortal condition. And I hate this condition: I never want to die." Bacon feels touched by the smell of death and by the violence of life, in which "each one lives to eat the other" (Bacon cited by Sinclair, 1993/1995, 47). Bacon exposes in his work something of death and real incompleteness when he states: "There is always a sense of death in people when they see my paintings... I may carry this feeling of death all the time... I always surprise myself when I wake up in the morning "(op. cit., 78). In this artwork, the distortion is of such magnitude that it borders on the transformation into hallucinosis, related to a primitive catastrophe due to an anxiety of primordial annihilation, the subject finding no internal continent capacity, reintroduced in the form of an 'unnamed terror'. This canvas is clearly the representation of a catastrophic emotional experience: terror and pain in its pure state.

\section{References}

Bion, W.R. (1965/1982). Transformations - Passage de l'Apprentissage à la Croissance. Paris: PUF.

Deleuze, G. (1984). Francis Bacon, Logique de la Sensation. Paris: Édit. de la Différence.

Delgado, L. (2012). Psicanálise e Criatividade: estudo psicodinâmico dos processos criativos artísticos. Lisboa: ISPA.

Freud, S. (1927/1981). O Futuro de uma Ilusão. Sigmund Freud, Obras Completas (Tomo III, 2962-2992). Madrid: Biblioteca Nueva.

Levi, P. (1958/2010). Se Isto É um Homem. Lisboa: Teorema.

Levi, P. (1963/2010). A Trégua. Lisboa: Teorema.

Sinclair, A. (1995). Francis Bacon. Su Vida en una Época de Violência. Barcelona: S.A.

\section{Biography}

Luís Delgado studied clinical psychology at the Institute Henry Piéron - Université René Descartes, Paris $\mathrm{V}$ and is a professor at Instituto Superior de Psicologia Aplicada - University Institute - Lisboa, where he teaches methodology and projective techniques in clinical and teaches master and doctoral seminars. His greatest interest lies in the study of the psychodynamics of creativity and in psychoanalysis applied to artistic and literary objects. He has published two books on this subject and several articles in national and international journals. Luís Delgado is psychoanalyst and psychotherapist and, in addition to clinical practice, gives training to the students of his association of psychoanalysis - Associação Portuguesa de Psicanálise e Psicoterapia Psicanalítica - and promotes clinical cases supervision groups in a dynamic perspective. He is full member of Applied Psychology Research Center Capabilities \& Inclusion - ISPA - University Institute, which provides investigation of psychological and social problems. 


\title{
WHAT IS A CLINICAL PSYCHOLOGIST? INTERNATIONAL PERSPECTIVES
}

\author{
Prof. Dr. Michael Wang \\ Emeritus Professor of Clinical Psychology, University of Leicester (United Kingdom)
}

\begin{abstract}
There are significant variations in the characteristics, role, function, activity and training of the clinical psychologist across the globe. This talk will review these in the context of international cultural and historical factors. Professional clinical psychology in China, Russia, Australia, the UK and the USA will be described and discussed within the context of national healthcare systems, culture and politics. What should be our frame of reference and criteria when comparing these professional profiles? An attempt will be made to summarise commonalities in roles and definitions across nations and cultures and to evaluate whether certain characteristics are more helpful than others.
\end{abstract}

Keywords: Clinical psychology, international, profession, healthcare.

\section{Biography}

Michael Wang is Emeritus Professor of Clinical Psychology and former DClinPsy Course Director at the University of Leicester. Although he takes a broad bio psychosocial approach to clinical problems, he has particular training and expertise in cognitive behavioural therapy and is a recognised consultant clinical neuropsychologist. He is a former Chair of the Division of Clinical Psychology of the British Psychological Society and has broad knowledge and experience of the profession and of training at national level. He is Chair of the Association of Clinical Psychologists UK.

He holds Visiting Professorships at the Universities of Madras (India), Anamalai (India), Xi'an (China), Ryazan (Russia) and is a Fellow of Madras Medical College.

He has a research interest in psychological aspects of anaesthesia and surgery and in 2015 was awarded the Humphry Davy Medal by the Royal College of Anaesthetists in recognition of this work. 



\section{ORAL PRESENTATIONS}

\section{Clinical Psychology}

Quality of life in patients with spine tumors

Olga Y. Shchelkova, Aslan K. Valiev, Ekaterina B. Usmanova, \& Evgeny A. Sushentsov

among American and polish young adults

Chris Segrin, Katarzyna Adamczyk, Corey A. Pavlich, Małgorzata Osowiecka,

Radostaw Trepanowski, \& Agata Celejewska

Potentials of psychological prediction of patients' therapeutic behavior after myocardial revascularization

Maria Iakovleva, Nadezhda Kruglova, Olga Shchelkova, Ekaterina Lubinskaya

\& Olga Nikolaeva

The role of cognitive functioning in the success of social rehabilitation of cardiac patients

Daria Eremina

Features of the people rehabilitation potential with different characteristics of learned helplessness and human life-world stability

Olga Petriaeva, Irina Loginova, \& Olesya Volkova

The effects of civic participation on Japanese older adults

Keiko Katagiri

The effect of locus of control in responsibility among participants with traumatic event history

Merve Topcu, Deniz Köroğlu, Yusuf Coşku İnceler, \& Faruk Gençöz

Why are some people optimistic while others are not?

Lilly E. Both

E-mail counseling for the ultra-orthodox community

Osnat Rubin

"I think having Asperger's helped me with my transgender identity": The lived experiences of youth with gender diverse identities and autism spectrum disorders Wallace Wong, Jaime Semchuk, \& Veronique Nguy

Acting with awareness predicts negative but not positive affect in a sample of addicts

Tânia Caetano, Eduardo Ramadas, \& Jessica Lopes

Relation between subjective and physical well-being and mindfulness

Lisa A. Best, Cecile J. Proctor, Tracy A. Freeze, Derek J. Gaudet, Ryley Russell,

\& Rory McPhee

Pilot trial of stepped care CBT for pediatric OCD 
The thought of living with spinal cord injury: How an imagined disability affects life satisfaction

Derek J. Gaudet, Lisa A. Best, \& Najmedden Attabib

Association between perception of acceptance and rejection, and drawings of "person picking an apple from a tree" among primary school children

Or Shalev, Andriani Papadaki, Elias Kourkoutas, \& Michal Bat Or

Links between cognitive distortions and cognitive emotion regulation strategies in non-clinical young adulthood

Romain Deperrois, \& Nicolas Combalbert

Cognitive distortions and emotion regulation among post traumatic stress disorder victims

Nawal Ouhmad, Nicolas Combalbert, \& Wissam El-Hage

Acute polymorphous psychotic disorder. Evolutionary possibilities

Simona Trifu, Alexandra Popescu, \& Eduard George Carp

\section{Educational Psychology}

Development and psychometric properties of NEGORI (Negative Orientation

László Kasik, \& Zita Gál

Innovative method for introduction of written language: Experience in Mexico

Yulia Solovieva, \& Luis Quintanar Rojas

Social problem-solving in the classroom

Zita Gál, \& László Kasik

Tuning in to kids: A piloting study targeted to support Turkish parents' emotion socialization

Ayca Ulker Erdem, Mubeccel Gonen, \& Sophie Havighurst

Turkish validity and reliability of Watson-Glaser critical thinking appraisal test's short form

Ayse Esra Aslan, Faruk Bozdă̆, \& Burcu Aykaç

Mathematics teachers' professional development - Gender difference

Danijela S. Petrovic

Adult visitors' intellectual functioning exploring contemporary art: Experiences that generate self-discovery

Anne-Marie Émond

Adult reading and meaning making in a museum exhibition about ancient China

Colette Dufresne-Tassé

The teaching and integration of psychology into Saudi Arabian curriculum

Kyle A Msall

Understanding and defining instructional leadership: School principals' perspective 
The impact of adjustment, engagement and help-seeking behaviour towards academic well-being of international students from developed countries who study in developing countries

Erna Zulaeni Wiles, Tina Afiatin, \& Neila Ramdhani

Meaning of foreigners among Estonian, Latvian, Lithuanian and Russian university students

Kristi Kõiv, Svetlana Gurieva, Olga Deyneka, Vaiva Zuzeviciute, Anna Liduma,

\& Sandra Rone

The role of trait emotional intelligence in the process of career decision-making

Eva Sollarova, \& Lada Kaliska

Estonian and Finnish students' experiences about international career guidance e-learning course

Kristi Kõiv, Ari Jussila, Outi Rantanen, \& Triin Peitel

The relationship between learning style and anxiety among Japanese undergraduates Michiko Toyama, \& Yoshitaka Yamazaki

Cross-cultural research on discipline techniques, values, and academic expectations of Turkish and Finnish mothers

Melisa Kütük, \& Meral Atıcı

Mindfulness, self-esteem, and burnout in counselors and psychologists working at schools

Gökçe Bulgan

Supporting emotion socialization skills of preschool teacher candidates

Aysel Esen Coban, Ayca Ulker Erdem, \& Tulay Ilhan Iyi

\section{Social Psychology}

The organizational valorization of employees who are satisfied with their live Bernard Gangloff, \& Neila Malleh

An implicit model of assessment of attitude to health of specialists in an organization Elena Rodionova, Vladislav Dominiak, German Nikiforov, \& Zoya Dudchenko

Decision trees: Comparing reported intentions and theoretically expected intentions in urban context

Jimmy Bordarie

The HEXACO personality measure as a predictor of job performance and job satisfaction

Gaye Saltukoğlu, Arkun Tatar, \& Hüdanur Özdemir

Typology of insurance behavior of Russian citizens

Olga Medyanik

Student's ideas about the prerequisites and measures to combat corruptions

Olga Deyneka

A descriptive approach to mobbing: The role of self esteem and body image at work Nevin Kılıç, Berra Bekiroğlu, Hüdanur Özdemir, \& Arkun Tatar 
Personality traits and reasons to leave country in Serbian university students

Jelisaveta Todorovic, Snezana Stojiljkovic, \& Tamara Ciric

An investigation of the relation between personality, job performance, and job

satisfaction via profile analysis

Arkun Tatar, Gaye Saltukoğlu, Hüdanur Özdemir

Acculturation strategies and perception of a North African woman in a judicial context

Chloé Dougez, Anne Taillandier-Schmitt, \& Nicolas Combalbert

Holidays in cultural memory: Social media mining across generations

Tatiana Folomeeva, \& Fedor Vinokurov

Parent and children's characteristics by custody type: A high conflict sample

Marta Herrero, Ana Martínez-Pampliega, Irati Alvarez, Mireia Sanz-Vázquez,

Susana Cormenzana, \& Susana Corral

The health-promoting behaviors for married couples

Elçin Sakmar-Balkan, \& Hakan Kuru

Psychological determinants of youth participation in the life of the country

Tatiana Anisimova, Karina Bakuleva, \& Irina Samuylova

Asylum interview as a fork on the road - From asylum seeker to irregular migrant

Katri Johanna Gadd, \& Laura Sofia Lehtikunnas

Qualitative psychological effects of political information to high school students Irina Samuylova

\section{$\underline{\text { Legal Psychology }}$}

Self-esteem and conformism of female students as predictors of experienced violence by partner

Ivana Jankovic, Jelisaveta Todorovic, \& Snezana Stojiljkovic

\section{Cognitive and Experimental Psychology}

Malleability of working memory through chess training in school children

Ebenezer Joseph, Veena Easvaradoss, T. Edwin Prabhakaran, \& Sweta Jain

Developmental patterns of sex differences in verbal and visuospatial abilities

Efrat Barel, \& Orna Tzischinsky

Emotion reactions to economic predictions and its effects on reasoning and logical thinking

Jerônimo Soro, Mário B. Ferreira, Karen Gouveia, \& Joana Reis

Susceptibility of perception of vertical to blood glucose fluctuations in healthy young adults

Rima L. Abdul Razzak 


\section{Clinical Psychology}

A longitudinal study of school satisfaction - Mental health and social skills upon college admission-

Yuko Ikui

Relationship between mindfulness, self-compassion and difficulties in emotion regulation in addicts

Tânia Caetano, Eduardo Ramadas, \& Jessica Lopes

Re-connecting oneself to the world of peers via the contemplation of an artwork

Laurence Bertron-Gatier, \& Célia Vaz-Cerniglia

Motivation and emotions in effective eating habits change. Multi-method exploratory study

Grażyna Wąsowicz, Magdalena Poraj-Weder, \& Christophe Matthys

Combination of attachment patterns in romantic relationship and its quality

Dariusz Kuncewicz, \& Dorota Kuncewicz

\section{Educational Psychology}

Family functioning characteristics involved in adolescent depressive symptoms

Antonio Caño, \& Carmen Rodríguez-Naranjo

Adjustment of graduate students with attention deficit hyperactivity disorder (ADHD) Julien Dalpé, Georgette Goupil, France Landry, \& Rachel Paquette

Factorial validity of the descriptive/injunctive norm preference scale

Norihiro Kuroishi, \& Yoriko Sano

The rencontres focus: outcomes of a learning and study skills based program for undergraduate students with attention deficit hyperactivity disorder (ADHD) Rachel Paquette, France Landry, Georgette Goupil, \& Julien Dalpé

Developing academic skills in digital environments: Media effects on performance and metacognitive judgments

Gal Ben-Yehudah, \& Ronen Kasperski 
A structural model of the test for creative thinking-drawing production (TCT-DP) in adults- revised

Sara Ibérico Nogueira, Leonor Almeida, \& Tiago Souza Lima

Positive emotional atmosphere or technology-based training: Teachers' priorities depending on their personality Kristina Kovalcikiene, \& Sigitas Daukilas

Normative values for the test for creative thinking-drawing production (TCT-DP), over the school years in Portuguese private schools

Leonor Almeida, Sara Ibérico Nogueira, Tiago Souza Lima, \& Maria Manuel Sousa

Empathy and classroom climate as predictors of student's well-being: An international pilot study

Manja Veldin, Ana Kozina, Tina Vršnik Perše, Maša Vidmar, Ivana Jugović,

\& Magnus Oskarsson

EDU-CAR: Qualitative analysis of an intervention for the development of socio-emotional and career competences

Mara Leal, \& Lucy Melo-Silva

\section{Social Psychology}

Content of father and mother stereotypes in japan, compared to overall gender

Mizuka Ohtaka

The effects of psychological games on nonverbal communication skills of the youth

Koshi Makino

Content validity of the descriptive/injunctive norm preference scale

Yoriko Sano, \& Norihiro Kuroishi

Road less traveled: Motivations and pathways of Filipino lesbians and gays identifying as ex-gays

Nel Jayson Santos, Aaron Vichard Ang, Sigrid Joyce Dela Paz, \& Daniel Jan Duque

University teachers' professional identity: A role of self-determination theory

Kristina Kovalcikiene, Aurelija Stelmokiene, Loreta Gustainiene,

\& Giedre Geneviciute-Janone

Self-concept and anxiety of potential Slovak leaders

Lenka Ďuricová, \& Beata Žitniaková Gurgová

Perception of (the) psychological motives of tattooing of all ages

Anna Winiarek, \& Ludwika Wojciechowska

A case study on the process of interpersonal relationship formation between Muslims and Japanese hosts

Sachiko Nakano

Stigmatization of people with alcohol dependence compared to people with schizophrenia

Jana Serebriakova, \& Maria Hildegard Walter 


\section{VIRTUAL PRESENTATIONS}

\section{Clinical Psychology}

Parental support, mentoring relationship and 30-day prevalence of alcohol use among high school boys and girls

Marianna Berinšterová, Monika Magdová, \& Miroslava Bozogáňová

The addictive personality: Myth or cornerstone of prevention and treatment?

Donna L. Roberts

Spirituality, quality of life, depression and dementia

Océane Agli

Brief therapy approaches in the treatment of substance abuse and addiction

Donna L. Roberts

\section{Educational Psychology}

Gender-based linear correlation of emotional quotient with study habits and academic success of junior college students

Mary Ann I. Diaz, Elenita M. Tiamzon, M. ${ }^{a}$ Cristina J. Estioco, \& John Mark S. Borbon

Resting state EEG power analysis in Filipino children with dyslexia

Katherine Ko, Roann Ramos, \& Rosalito De Guzman

Positive self-esteem, satisfaction and the unplugged program among Slovak schoolchildren

Olga Orosová, Maria Bacikova-Sleskova, \& Beata Gajdosova

Psychological literacy of teachers in the role of youth mentors

Marianna Berinšterová, Katarína Fuchsová, \& Monika Magdová 


\section{Social Psychology}

Dynamics of socio-cultural and linguistic identity in the process of socialization in a multicultural society

Tatiana Martsinkovskaya, Vasilisa Orestova, Ekaterina Kiseleva, Maria Soboleva,

\& Evgenia Kriger

Violence against teachers in the school environment: An explorative study

Isabella Corradini, \& Assunta Marano

Attitudes of citizens to the policy of homelessness in St. Petersburg

Andrey Zaytsev, \& Olga Medyanik

Organizational support and work engagement of deaf and hard of hearing employees:

Diversity management challenges

Biljana Maleš Mutić, Svetlana Čizmić, Milica Vukelić, \& Ivana B. Petrović

Alcohol use in association with unorganized social activities in free time and accessibility of alcohol among early adolescents

Lenka Abrinkova, Olga Orosová, Maria Bacikova-Sleskova, \& Marcela Štefañáková

Increasing capacity in work with war victims and migrants: Pragmatic empowerment training (PET)

Charles David Tauber, \& Sandra Marić

Migration factors related to emigration intentions among university students in

Slovakia

Bohuš Hajduch, Olga Orosová, \& Marcela Štefaňáková

Workplace relationships as a symptom of organizational culture and a lever for culture transformation

Olga Tararukhina

Cyberbullying vs. Traditional bullying - Do victims react differently?

Meyran Boniel-Nissim

Effective measure of organizational culture

Olga Tararukhina

\section{Cognitive and Experimental Psychology}

The meaning-based approach to assessing the images of young and elderly women and men

Shulamith Kreitler

The role of training or hints relating to "thinking in opposites" in insight problem solving

Erika Branchini, Roberto Burro, Elena Capitani, Ugo Savardi, \& Ivana Bianchi

Knowledge of prospectively relevant information improves search performance 
Adolescent frontal lobe brain development: Effects of social and economic deprivation and implications for intellectual assessment, academic remediation, and court case disposition

Michael Lindsey

Comprehensive treatment for TIC disorders

Omar Rahman, \& Adam Lewin 



\section{* ORAL PRESENTATIONS *}

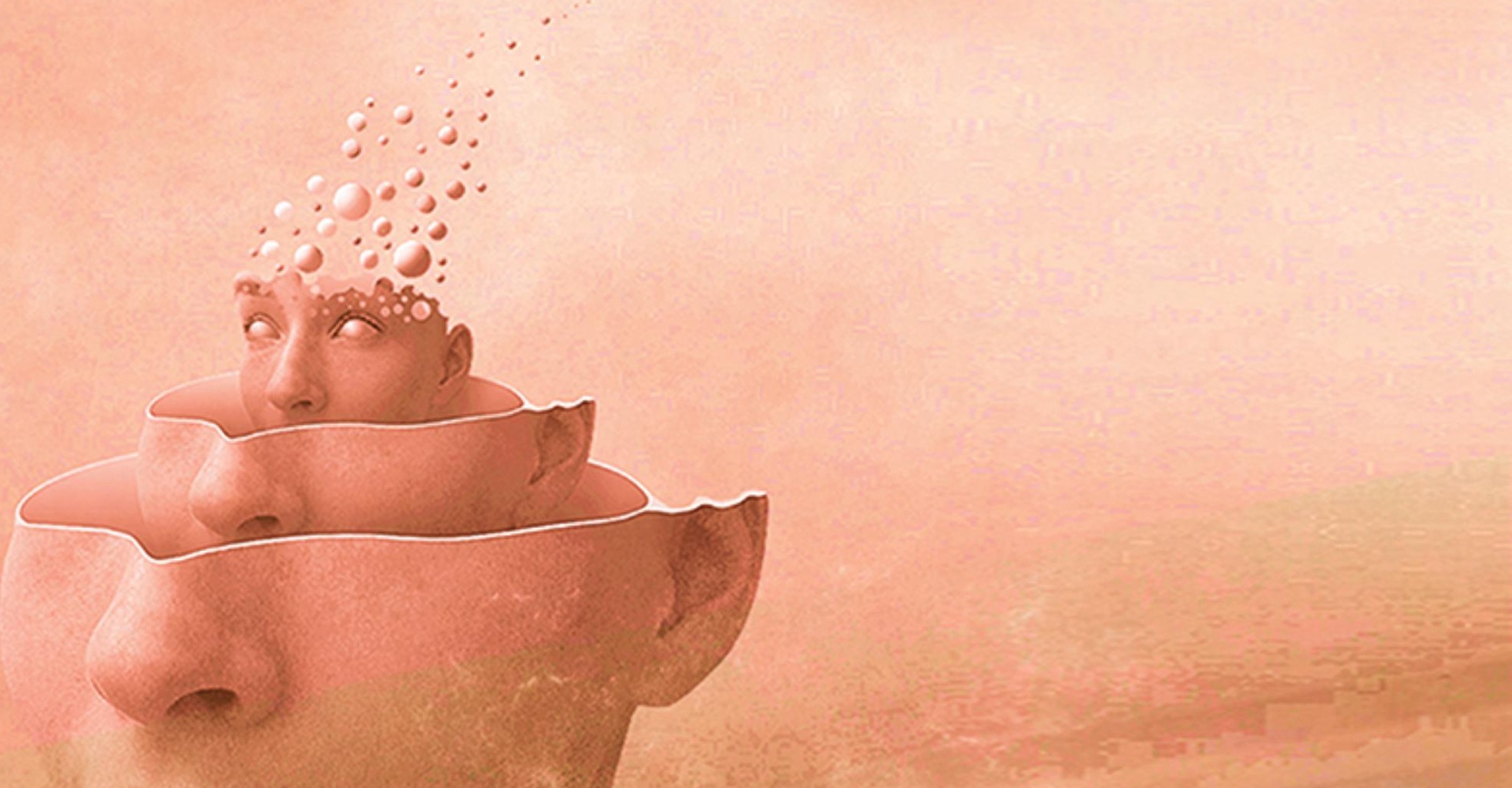





\title{
QUALITY OF LIFE IN PATIENTS WITH SPINE TUMORS
}

\author{
Olga Y. Shchelkova ${ }^{1}$, Aslan K. Valiev ${ }^{2}$, Ekaterina B. Usmanova ${ }^{3}$, \& Evgeny A. Sushentsov ${ }^{2}$ \\ ${ }^{1}$ St. Petersburg State University, Saint Petersburg, (Russia) \\ ${ }^{2}$ N.N. Blokhin Russian Cancer Research Center, Moscow (Russia) \\ ${ }^{3}$ International Preschool, Moscow (Russia)
}

\begin{abstract}
Introduction: Quality of life (QoL) in chronic somatic diseases is seen as integral characteristic of physical, psychological, emotional and social functioning of patient. QoL in oncological patients is studied intensively. Patients with spine tumors previously had poor prognosis. Modern surgical treatment in patients with spine tumors allows to increase survival rate. Consequently the issue of QoL in patients with spine tumors becomes especially actual.

The aim of the study was to reveal basic parameters of QoL in patients with spine tumors before and after surgical treatment.

Results: Study participants were 25 patients with spine tumors. The diagnosis was spine metastases from different primary sources. The methods were: VAS and Watkins scales, «SF- 36 Health Status Survey», Quality of Life Questionnaire-Core 30 of European Organization for Research and Treatment Cancer, Spine Oncology Study Group Outcomes Questionnaire. All patients had pain syndrome before treatment. Pain assessment using VAS and Watkins scales before and after treatment revealed pain reduction as a result of surgery. Patients with spine tumors assess their QoL in general after surgery significantly higher than before treatment. As well patients report increasing QoL after surgery on the parameters of neurological status and pain intensity which corresponds to VAS and Watkins scales assessment. Besides the results revealed improving of QoL in patients with spine tumors after treatment on the parameter of emotional functioning. The diagnosis of patients with spine metastases refers to last stages of the disease. So treatment in these patients in most cases initially aimed at palliative care helps not only reduce pain intensity but also increase their QoL and in this way allow patients have emotionally rich life.

Conclusion: Nowadays patients with spine tumors undergo effective surgical treatment which increases their survival rate and significantly improve their QoL.

The further research is needed to study psychological factors of QoL in patients with spine tumors in order to make relevant programs of functional, social and psychological rehabilitation for these patients.
\end{abstract}

Keywords: Quality of life, spine metastases, pain syndrome.

\section{Introduction}

The relevance of treatment in patients with spine metastases increased simultaneously with increasing in number of these patients in clinical practice. The issue of choosing the optimal method of treatment for patients with spine metastases is one of the most difficult and opened at the moment. The main criteria for choosing the method of treatment are patients' survival rate and quality of life (QoL). For patients with poor prognosis the only criterion for choosing treatment is QoL.

QoL in chronic somatic diseases is seen as integral characteristic of physical, psychological, emotional and social functioning of patient. QoL concept pays attention to emotional experience of disease in patients, particularly to personal sense of possibility to satisfy actual needs and possibility of full social functioning in spite of disease limitations.

\section{Patients characteristics}

Study participants since 2016 till 2018 (N=25) were inpatients of N.N. Blokhin Russian Cancer Research Center. The diagnosis was spine metastases from different primary sources.

All patients had surgical treatment. 


\section{Methods}

The methods were:

1. VAS and Watkins scales. VAS scale (Visual analog scale) was used for subjective assessment of pain by a patient. Watkins scale was used for an objective assessment of pain by a doctor.

2. SF- 36 Health Status Survey (Ware et al., 1993; Wasserman et al., 2011)

3. Quality of Life Questionnaire - Core 30 of European Organization for Research and Treatment Cancer (Aaronson et al., 1993; Fayers et al., 1995);

4. Spine Oncology Study Group Outcomes Questionnaire (SOSG-OQ) (Janssen SJ. et al., 2017).

\section{Results}

Study revealed that all patients with spine tumors had pain syndrome before treatment. Pain assessment using VAS and Watkins scales before and after treatment revealed pain reduction as a result of surgery.

Besides the results revealed significant differences between QoL parameters in patients before and after surgical treatment. According data of SF-36 and SOSG-OQ patients with spine tumors assess their QoL in general after surgery significantly higher than before treatment, as well as patients assess their emotional functioning after surgical treatment significantly better than before surgery.

The results of studying general QoL and emotional functioning in patients with spine tumors after surgery compared with QoL before surgery are presented on Figure 1.

Figure 1. General QoL and emotional functioning in patients with spine tumors before and after surgery.

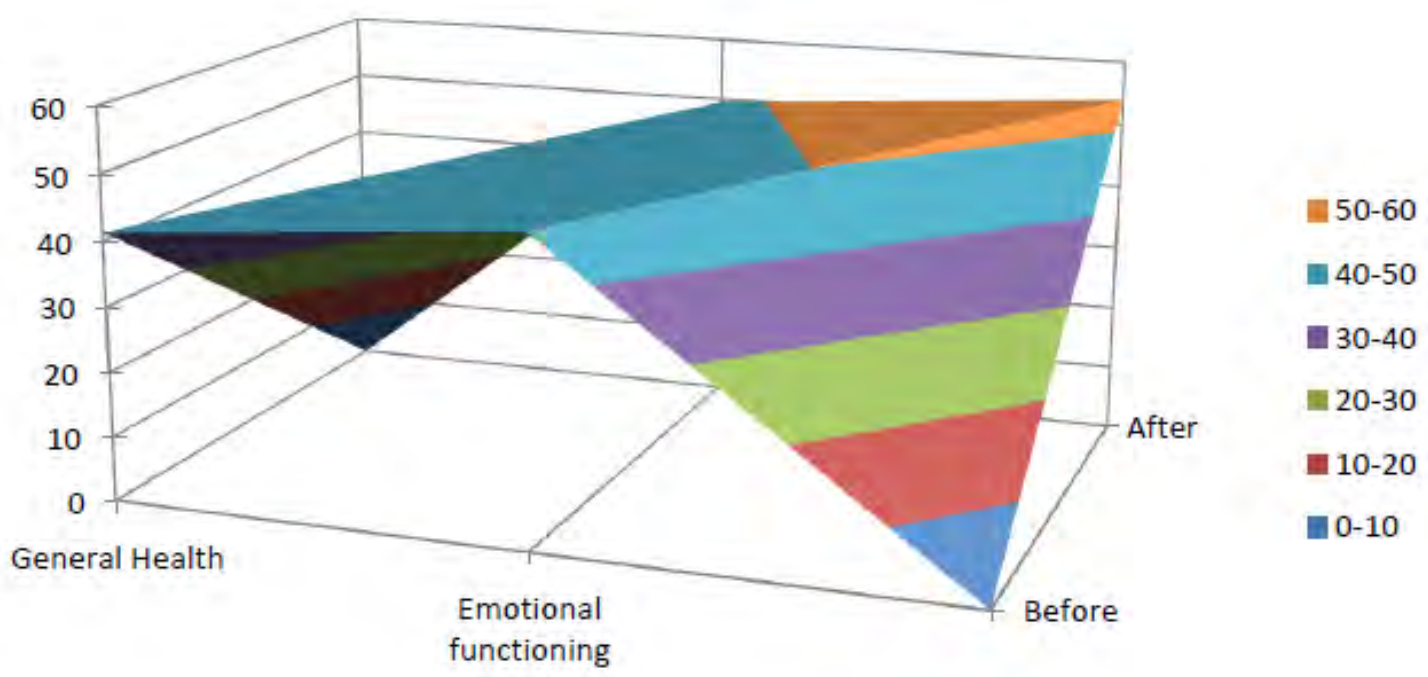

As well patients report increasing QoL after surgery on the parameters of neurological status and pain intensity according the results of SOSG-OQ and QLQ C-30.

The results of study neurological status and pain intensity in patients with spine tumors are presented on Figure 2. 
Figure 2. Neurological status and pain intensity in patients with spine tumors.

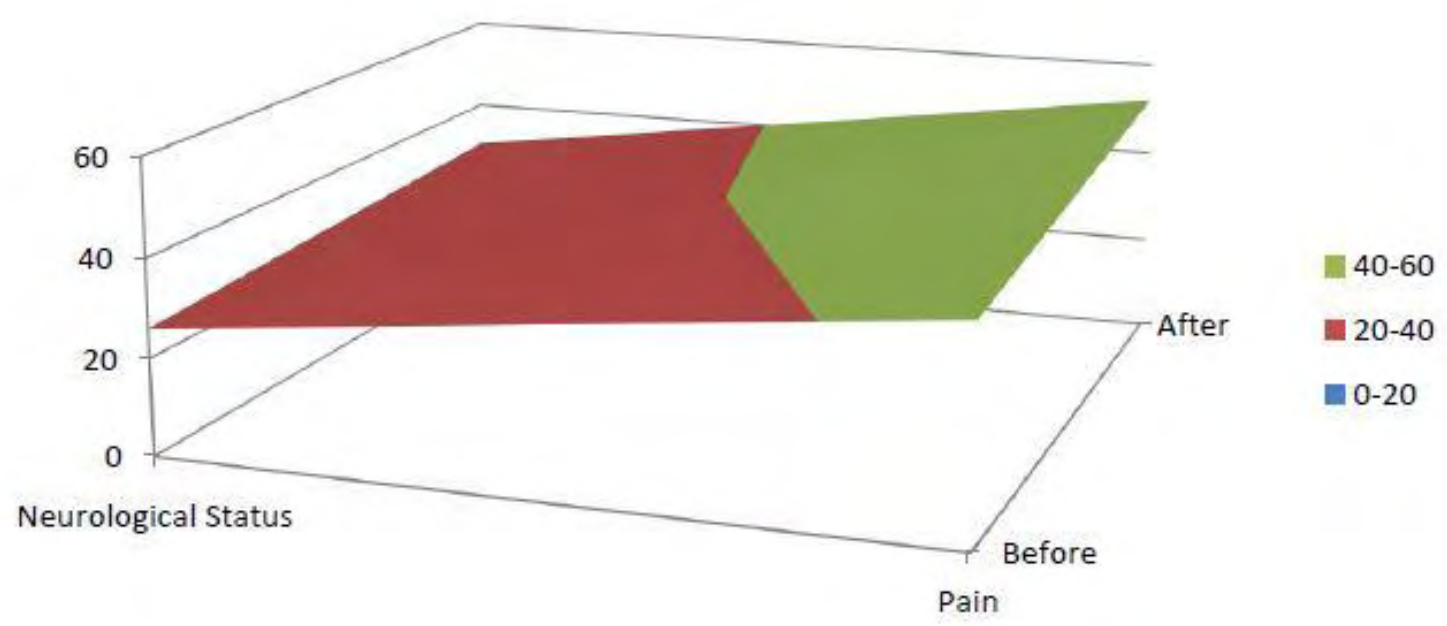

These results correspond to VAS and Watkins scales assessment.

In conclusion, patients with spine tumors assess their QoL on the basic parameters as general health, emotional functioning, neurological status and pain after surgery significantly higher than before treatment. Patients with spine tumors have an opportunity to undergo effective surgical treatment which increases their survival rate and significantly improve their QoL.

The further research is needed to study psychological factors of QoL in patients with spine tumors in order to make relevant programs of functional, social and psychological rehabilitation for these patients.

\section{Acknowledgements}

The research is supported with RFFI grant № 19-013-00154 A.

\section{References}

Aaronson, N. K., Ahmedzai, S., \& Bergman, B. (1993). The European Organisation for Research and Treatment of Cancer QLQ-C30: A quality of life instrument for use in international clinical trials in oncology. Journal of the National Cancer Institute, 85, 365-375.

Fayers, P., Aarson, N., Bjordal, K., \& Sullivan, M. (1995). QLQ C-30 Scoring Manual. Brussels: EORTC Study Group on Quality of Life.

Janssen SJ., Teunis T., van Dijk E. Validation of the Spine Oncology Study Group-Outcomes Questionnaire to assess quality of life in patients with metastatic spine disease // Spine J. - 2017. V. 17(6): 768-776.

Ware, J. E., Snow, K. K., Kosinski, M., \& Gandek, B. (1993). SF-36 Health Survey Manual and Interpretation Guide. Boston, MA: New England Medical Center, The Health Institute.

Wasserman, L. I., Trifonova, E. A., \& Shelkova A. Yu. (2011). Psychological diagnostics and intervention in somatic clinic. Saint Petersburg: Rech. 


\title{
DOES LONELINESS HAVE NATIONALITY? CULTURAL AND INDIVIDUAL DIFFERENCES IN LONELINESS AMONG AMERICAN AND POLISH YOUNG ADULTS
}

\author{
Chris Segrin ${ }^{1}$, Katarzyna Adamczyk ${ }^{2}$, Corey A. Pavlich ${ }^{3}$, Malgorzata Osowiecka ${ }^{4}$, \\ Radosław Trepanowski ${ }^{2}, \&$ Agata Celejewska ${ }^{2}$ \\ ${ }^{1}$ Department of Communication, University of Arizona (USA) \\ ${ }^{2}$ Adam Mickiewicz University in Poznan (Poland) \\ ${ }^{3}$ School of Communication, Cleveland State University (USA) \\ ${ }^{4}$ University of Social Sciences and Humanities in Warsaw (Poland)
}

\begin{abstract}
The current study was intended to determine the possible differences in social and emotional (i.e., family and romantic) loneliness a function of nationality and relationship status. Although the social construction of romantic relationships in Poland and the US has changed over recent years, the Polish culture with its stronger traditionalist value system may still place a higher premium on having a romantic or marital partner compared to the US. The family unit is also a more central and core element of one's social network in Poland compared to the US. Associations between loneliness and romantic partner status were tested in 431 US and 395 Polish participants (mean age $=22.92$ ) who completed the Social and Emotional Loneliness Scale for Adults - Short Form (SELSA-S; DiTommaso, Brannen, \& Best, 2004). The analyses demonstrated that Polish respondents reported higher loneliness than US respondents, Wilks' $\lambda=0.89, F(3,769)=30.78$, $p<.001$ and single individuals reported higher loneliness than partnered individuals, Wilks' $\lambda=0.60$, $F(3,769)=169.26, p<.001$. There were also significant nationality and relationship status interactions in the domain of family loneliness, $F(1,771)=6.47, p<.01$, and in the domain of romantic loneliness, $F(1,771)=71.82, p<.001$. More specifically, single Polish participants reported higher family loneliness than Polish partnered participants. American participants reported the exact same level of family loneliness, regardless of their relationship status. In turn, the lack of a romantic partner was more strongly associated with increased romantic loneliness for Polish than for American participants. Although all participants reported increased loneliness when they were without a partner, this effect was particularly exaggerated among Polish participants. At the same time, Polish participants seem to benefit more (in terms of decreased romantic loneliness) than Americans do as a function of having a romantic partner.
\end{abstract}

Keywords: Loneliness, relationship status, young adults, Poland, USA.

\section{Introduction}

In the research literature loneliness is conceptualized as a multifaceted or domain-specific phenomenon. The first researcher who described loneliness as a multidimensional experience was Weiss (1973) who proposed a distinction between social loneliness as a result of an inadequate access to social networks and emotional loneliness perceived as a lack of close or intimate relationships which are characteristic of ties with a romantic partner, parent, or child. Emotional loneliness is primarily related to "the absence of a partner, that is, with the absence of an exclusive, close, and intimate tie" (Dykstra \& Fokkema, 2007, p. 9). In turn, social loneliness is related to a perceived deficiency in social networks, or a lack of social relations or social activities (Russell, Cutrona, Rose, \& Yurko 1984; Weiss 1973). Furthermore, on the basis of Weiss' (1973) distinction between the experience of social isolation (social loneliness) and emotional isolation (emotional loneliness), DiTommaso and Spinner $(1993,1997)$ noted that emotional loneliness appeared to be comprised of two domains, that is, family emotional loneliness and romantic emotional loneliness.

Prior studies showed that the lack of a meaningful relationship with a partner or a spouse in emerging and young adulthood may be an important factor for the experience of loneliness (e.g., Russell, Cutrona, McRae, \& Gomez, 2012). At the same time, it is important to emphasize that in most prior studies loneliness was conceptualized as a unitary state. This unidimensional approach to loneliness recognizes 
loneliness as basically having the same form in different social contexts (e.g. Russell, Peplau, \& Cutrona, 1980). Research by DiTommaso and Spinner (1993) however indicated that involvement in a romantic relationship is associated with lower romantic loneliness, but to a weak degree with family and social loneliness. Furthermore, in a Polish study single individuals scored higher on romantic and family loneliness than did partnered individuals, and no differences emerged between single and partnered individuals in the domain of social loneliness (Adamczyk, 2016).

Alongside the discussion concerning the link between relationship status and loneliness it is also important to consider the fact that various cultures and societies may hold diverse attitudes and beliefs about marriage and possessing a partner (Gubernskaya, 2010). For instance, Poland has unique qualities derived from a traditional notion of life paths in adulthood, which includes heterosexual marriage and having children, relatively low rate of divorces, and less approval for alternative family and marital forms. In contrast, the American society is characterized by a higher level of divorces, and seems to be more approving towards alternative forms of marital and family life such as singlehood, marital non-cohabitation, and cohabitation without marriage. As a result, singlehood in Poland might be related to lower life satisfaction outcomes in comparison to singlehood in the United States of America, where singlehood might not be so detrimental for these outcomes.

\section{Objectives}

The primary aim of the present investigation was to determine the possible differences in social and emotional (i.e., family and romantic) loneliness a function of nationality and relationship status. In addition, we were also interested to determine if there is an interaction between nationality and relationship status such that differences between single and partnered people in terms of loneliness across the two nationalities. In regard to the linkage between relationship status and social and emotional (i.e., family and romantic) loneliness we expected that:

H1. Single individuals in Poland and in the United States would report higher romantic loneliness than partnered individuals.

H2. There will be an interaction between nationality and relationship status that Polish single individuals would report higher romantic loneliness than American single individuals.

\section{Method}

\subsection{Participants and procedure}

The study was conducted on a sample of 431 US and 395 Polish participants $(M=22.92$, $S D=4.24$ ) Women represented $67 \%$ of the sample and men represented $30 \%$, with $3 \%$ missing this information. There were 461 participants (56\%) who reported being in a romantic relationship at the time of the assessment, while 335 participants (41\%) were not, and 30 (4\%) were missing this information.

To recruit Polish and American participants, the authors distributed questionnaires in the form of online survey using a computer program 'Qualtrics' available at many American universities. Qualtrics allows users to view and complete the survey at their own pace on their computer, while storing all of the responses in single data file.

\subsection{Materials}

The questionnaire presented to participants was comprised of the following instruments:

Demographic Questionnaire. A series of demographic questions was asked to obtain general descriptive information about participants' background such as their age, sex, education level, and current relationship status.

The Social and Emotional Loneliness Scale for Adults - Short Form (SELSA-S; DiTommaso et al., 2004) (Polish adaptation - Adamczyk \& DiTommaso 2014). The SELSA-S is a multidimensional measure of loneliness that consists of 15 items rated on a 7-point Likert-type scale, ranging from 1 (strongly disagree) to 7 (strongly agree). It was designed to measure emotional (romantic and family) and social loneliness. Each subscale consists of five statements about feelings of loneliness within the past year. The family loneliness subscale assesses feelings toward family relationships. The social loneliness subscale measures feelings toward being part of a social group. The romantic loneliness subscale measures the degree to which participants feel they have significant others in their lives. Mean scores are calculated for each subscale, and higher SELSA-S scores indicate higher levels of loneliness in the particular domain. The SELSA-S's three subscales have high internal reliability, with Cronbach's alpha coefficients ranging from .87 to .90, and have been shown to be a valid measure of loneliness (DiTommaso et al., 2004). 


\section{Results}

The analyses demonstrated that Polish respondents reported higher loneliness than US respondents, Wilks' $\lambda=0.89, F(3,769)=30.78, p<.001$ and single individuals reported higher loneliness than partnered individuals, Wilks' $\lambda=0.60, F(3,769)=169.26, p<.001$. There were also significant nationality and relationship status interactions in the domain of family loneliness, $F(1,771)=6.47, p<.01$, and in the domain of romantic loneliness, $F(1,771)=71.82, p<.001$. More specifically, single Polish participants reported higher family loneliness than Polish partnered participants. American participants reported the exact same level of family loneliness, regardless of their relationship status. In turn, the lack of a romantic partner was more strongly associated with increased romantic loneliness for Polish than for American participants.

\section{Discussion}

The objective of the present study was to investigate whether potential differences in social and emotional (i.e., family and romantic) loneliness exists as a function of Polish and American nationality and relationship status among young adults. The findings revealed that Polish individuals experienced higher loneliness in comparison to their American counterparts and that single individuals reported higher loneliness than partnered individuals in both countries (H1). The analyses also revealed a significant interaction between nationality and relationship status in the domain of family and romantic loneliness. To be precise, single individuals in Poland reported higher family loneliness than Polish partnered participants, whereas American participants reported the same level of family loneliness, regardless of their relationship status. Moreover, we determined that single status was related to increased romantic loneliness in Poland in comparison to American participants and this pattern of results confirmed H2.

The observed interaction between nationality and relationship status in the domain of romantic loneliness as a result of the cultural and social specificity of Poland and the United States of America suggests that the differentiation in importance and evaluation of marriage and family [termed the Ideology of Marriage and Family by DePaulo and Morris (2005)] translate into the experience of increased loneliness associated with singlehood. Therefore, we may conclude that in cultures and societies where possessing a partner/spouse and marriage are highly valued (Poland) life satisfaction outcomes (i.e., loneliness) are lower among single people compared to their peers in cultures and societies that place a lower premium on possessing a partner or marriage (USA).

There are several limitations of this study. First, the correlational nature of the study precludes any causal inferences concerning the observed associations. Second, the age of the sample, even though representing a unique developmental state, precludes any generalizations to individuals in middle and late adulthood. For example, the consequences of singlehood and urgency for finding a life partner would presumably be very different among middle aged adults. Despite these limitations, the present findings underscore the importance of understanding how cultural values and norms may influence people's experience of possessing or not a partner and the possible consequences of relationship status.

\section{Acknowledgments}

The presented project proposal is financed by a grant from the Polish Scientific National Centre as part of the research project "A Longitudinal investigation of mental and physical health of Polish and American young adults” (UMO-2014/13/B/HS6/01382).

\section{References}

Adamczyk, K. (2016). An investigation of loneliness and perceived social support among single and partnered young adults. Current Psychology, 35(4), 674-689. doi: 10.1007/s12144-015-9337-7

Adamczyk, K., \& DiTommaso, E. (2014). Psychometric properties of the Polish version of the Social and Emotional Loneliness Scale for Adults (SELSA-S). Psychological Topics, 23(3), 327-341. UDK - 159.923.33.072-057.875(438)

DePaulo, B., Morris, W. (2005). Singles in society and science. Psychological Inquiry, 16(2), 57-83. doi: 10.1207/s15327965pli162\&3_01 
DiTommaso, E., Brannen, C., \& Best, L. A. (2004). Measurement and validity characteristics of the short version of the Social and Emotional Loneliness Scale for Adults. Educational and Psychological Measurement, 64, 99-119. doi: 10.1177/0013164403258450

DiTommaso, E., \& Spinner, B. (1993). The development and initial validation of the Social and Emotional Loneliness Scale for Adults (SELSA). Personality and Individual Differences, 14, 127-134. doi: 10.1016/0191-8869(93)90182-3

DiTommaso, E., \& Spinner, B. (1997). Social and emotional loneliness: A re-examination of Weiss' typology of loneliness. Personality and Individual Differences, 22, 417-427. doi: 10.1016/S0191-8869(96)00204-8

Dykstra, P.A., \& Fokkema, T. (2007). Social and emotional loneliness among divorced and married men and women: comparing the deficit and cognitive perspectives. Basic and Applied Social Psychology, 29(1), 1-12. doi: 10.1080/01973530701330843

Gubernskaya, Z. (2010). Changing attitudes toward marriage and children in six countries. Sociological Perspectives, 53(2), 179-200. doi: 10.1525/sop.2010.53.2.179

Russell, D. W., Cutrona, C., McRae, C., \& Gomez, M. (2012). Is loneliness the same as being alone? The Journal of Psychology, 146(1-2), 7-22. doi: 10.1080/00223980.2011.589414

Russell, D., Cutrona, C.E., Rose, J., \& Yurko, K. (1984). Social and emotional loneliness: An exploration of Weiss's typology of loneliness. Journal of Personality and Social Psychology, 46(6), 1313-1321. doi: 10.1037/0022-3514.46.6.1313

Russell, D., Peplau, L. A., \& Cutrona, C. E. (1980). The revised UCLA Loneliness Scale: Concurrent and discriminant validity evidence. Journal of Personality and Social Psychology, 39(3), 472-480.

Weiss, S. R. (1973). Loneliness: The experience of emotional and social isolations. London, England: The MIT Press. 


\title{
POTENTIALS OF PSYCHOLOGICAL PREDICTION OF PATIENTS' THERAPEUTIC BEHAVIOR AFTER MYOCARDIAL REVASCULARIZATION
}

\author{
Maria Iakovleva $^{1}$, Nadezhda Kruglova ${ }^{2}$, Olga Shchelkova ${ }^{1}$, Ekaterina Lubinskaya ${ }^{3}$, \\ \& Olga Nikolaeva ${ }^{3}$ \\ ${ }^{I}$ Department of Medical Psychology and Psychophysiology, Saint Petersburg State University (Russia) \\ ${ }^{2}$ Institute of Psychology, The Herzen State Pedagogical University of Russia (Russia) \\ ${ }^{3}$ Almazov National Medical Research Centre (Russia)
}

\begin{abstract}
Introduction. The prognostication of high-tech heart surgery outcomes is a relevant aspect in medicine. However, in this field a prognosis of psychological nature is also in evidence, such as predicting the patients' return to work after myocardial revascularization when there are no medical contraindications for it, as well as predicting patients' adherence to supportive treatment after revascularization.

Aim: to evaluate the predictive potentials of the patients' psychological characteristics in terms of their returning to work and adherence to treatment after bypass grafting.

Methods. An interview for patients performed at different stages of treatment, which included questions on patient's labor activities and their adherence to treatment; psychometric techniques, the Big Five Questionnaire, the SF-36 Questionnaire, the Integrative Anxiety Test (IAT), the Lazarus and Folkman Ways of Coping Questionnaire (WCQ), the Technique for Diagnosing the Types of Attitude toward the Disease ("TOBOL") among them. 159 patients were studied in 2 stages: 1) 63 employable patients were examined regarding their return to labor activity after surgery; 2) 96 patients - regarding their adherence to medical recommendations after surgery.

Results. The discriminant analysis allowed to obtain the following data. The higher the scores on the "Extroversion" scale (BIG V), on the "Social Activity" scale (SF-36) and on the "Social Protection" scale (IAT), the higher the probability of the patient returning to work (Lambda $=0.76, \chi^{2}=16.67 ; p<0.001$ ). The lower the value on the "anosognosic type" ("TOBOL") and the higher the value on the "sensitive type" ("TOBOL") in combination with high values of the "search for social support" scale (WCQ), the higher the likelihood of the patient being adherent to treatment (Lambda $=0.62, \chi^{2}=10.85 ; p<0.01$ ).

Conclusion. The findings show a high significance of psychological characteristics for predicting the behavior of patients after myocardial revascularization. Further detailed study of these correlations is required, as well as consideration of implementing in the psychological support of patients at the cardiac surgery departments the results obtained.
\end{abstract}

Acknowledgement. This research was supported by the Russian Foundation for Basic Research (RFBR) (Grant No 18-013-00689 A).

Keywords: Cardiology, health outcomes, prognosis, psychological characteristics.

\section{Introduction}

Myocardial revascularization (coronary bypass surgery or grafting) is a proven and effective method of treatment for the coronary heart disease (Bockeria, Alshibaya, Bendeliani, Nikonov, \& Krymov, 2012; Velazquez, 2004). The medical prognosis for patients who have undergone such operations is favorable - this is evidenced by satisfactory health status and high rates of quality of life after postsurgical rehabilitation; patients get an almost complete cure. However, there is a whole range of problematic aspects following high-tech surgical treatment. Patients do not return to work, despite the absence of medical contraindications, new manifestations of the disease appear that are not directly related to the effectiveness of the surgery (Fonager, Lundbye-Christensen, Andreasen, Futtrup, Christensen, Ahmad, \& Nørgaard, 2014; Sousa, Serruys, \& Costa, 2003). 
Thus, the psychological aspect of the treatment and rehabilitation processes (Glazer, Emery, Frind, et al., 2002; Vasserman, Trifonova, \& Shchelkova, 2011) comes to the fore, the role of the psychologist in following-up the patient at all stages of therapy becomes apparent - preparation for surgery, rehabilitation in a hospital, and then restoring a habitual way of the patient's life, taking into account the new possible limitations imposed by health.

At the same time, it is necessary to take into account which characteristics of patients' personality, their emotional state, coping strategies and attitude towards the disease affect rehabilitation, its effectiveness, patients' return to active life and work (in case of absence of contraindications), as well as adherence to supporting therapy, which helps to avoid relapses of the disease and prolongs the beneficial effect of the operation. Summarizing all the aforesaid, there is a need in considering a "psychological prognosis" in relation to patients undergoing surgical treatment of the coronary heart disease.

Within this context the aim of the research was set up, which was to evaluate the predictive potentials of the patients' psychological characteristics in terms of their returning to work and adherence to treatment after bypass grafting.

\section{Material}

159 patients of the Almazov National Medical Research Centre were studied in 2 stages.

1) 63 employable patients were examined regarding their return to labor activity after surgery. The mean age in this group was 57,7 years, with the following gender distribution: $87,3 \%$ men and $12,7 \%$ women.

This stage of the study included patients of working age who were able to and actually worked before the surgical treatment.

2) 96 patients - regarding their adherence to medical recommendations after surgery. The mean age in this group was 60,1 years, with the following gender distribution: 82,5\% men and 17,5\% women.

Patients from both groups suffered from coronary heart disease and underwent surgical treatment - coronary bypass grafting. The duration of the CHD at the time of surgery was different, from 1 year to 25 years. Most of the patients had secondary diagnosis such as hypertensia, gastric ulcer, obesity, arthrosis, colitis and some others.

Those patients who suffered mental disorders or postoperative complications were excluded from the study.

\section{Methods}

The methods used were the following. An interview for patients; an interview for doctors and several psychometric techniques. The patients' interview was performed at different stages of treatment, depending on the questions. Some of the measurements were made before the surgery and some of them - after it. It included questions on patient's socio-demographic characteristics, their family, labor activities, stress situations, habits and their therapeutic behavior. The doctors' interview included questions on patient's health status, surgery outcomes and their adherence to treatment, if they followed the recommendations and were willing to go on with the treatment. A wide range of psychometric techniques were also used, among them:

- The Big Five Questionnaire, to study patients' personality features,

- the SF-36 Questionnaire ("The Short Form (36) Health Survey"), to assess patients' health-related quality of life,

- the Integrative Anxiety Test (IAT), a modification of the Charles Spielberger's "State-Trait Anxiety Inventory", aimed to a differentiated assessment of anxiety as an emotional state and anxiety as a stable personality trait,

- the Lazarus and Folkman Ways of Coping Questionnaire (WCQ), to study patients' most frequently used coping strategies,

- the Technique for Diagnosing the Types of Attitude toward the Disease ("TOBOL"), which aims to diagnose various types of personal reaction to the disease (i.e., attitude toward the disease) in patients with somatic diseases, mainly chronic. 


\section{Results}

The characteristics that make an important contribution in determining whether the patient returns to labor after surgical intervention were selected from a wide range of data on the psychological characteristics of patients obtained in the study by means of mathematical-statistical analysis (discriminant analysis).

The formula of the function presented below is obtained from the results of the analysis and allows to evaluate the contribution of various variables to the definition of a patient, it allows to predict that each new coronary heart disease patient waiting for surgical treatment will return or not return to work after the surgery.

where the variables are:

$$
F 1=-0,113 \times A+0,235 \times B+0,038 \times C+0,512,
$$

A - the "Extraversion" scale (BIG V),

B - the "Social Protection" scale (IAT),

C - the "Social Role Functioning" scale (SF-36).

Thus, the above formula implies the following.

The higher the scores on the "Extroversion" scale (BIG V), on the "Social Role Functioning" scale (SF-36) and on the "Social Protection" scale (IAT), the higher the probability of the patient returning to work (Lambda $=0.76, \chi^{2}=16.67 ; p<0.001$ ).

Therefore, psychological features reflecting the characteristics of patients' social contacts and their quality have a prognostic value in relation to patients' return to work.

The data obtained in the study of the psychological characteristics of patients with varying degrees of adherence to treatment after coronary bypass grafting, were subjected to several types of mathematical-statistical analysis, including discriminant and multiple regression.

The formula of the function obtained from the results of the analysis is given below. It allows to evaluate the contribution of various variables to the classification of a patient in the group of those who are adherent or not adherent to treatment after surgical treatment.

where the variables are:

$$
F 2=-0,545 \times D+0,505 \times E+0,339 \times J
$$

$\mathrm{D}$ - the "Anosognosic type" of attitude toward the disease ("TOBOL"),

E - the "Search for social support" scale (WCQ),

$\mathrm{J}$ - the "Sensitive type" of attitude toward the disease ("TOBOL").

Thus, the above formula means as follows. The lower the value on the "anosognosic type" ("TOBOL") and the higher the value on the "sensitive type" of attitude toward the disease ("TOBOL") in combination with high values of the "search for social support" scale (WCQ), the higher the likelihood of the patient being adherent to treatment (Lambda $=0.62, \chi^{2}=10.85 ; p<0.01$ ).

\section{Discussion}

The patient's therapeutic behavior, in particular after coronary bypass grafting, is associated with a large number of factors. We cannot doubt the influence of the social environment, the economic status of the patient, factors of the health care system, etc. on the therapeutic behavior. All these aspects must be taken into account, but we must not forget the importance of patient's personality, his/her psychological characteristics. The way the patient understands and perceives the disease, how he/she perceives the treatment, what support he/she receives from the doctor and family, as well as his/her cognitive attitudes, personality traits and coping strategies directly influence the type of behavior that the patient will show after surgical treatment (Iakovleva, 2016). The role of the psychologist is to identify and adjust these factors with the aim of facilitating favorable patient's behavior in the therapy process and increasing the effectiveness of treatment.

\section{Conclusion}

The findings show a high significance of psychological characteristics for predicting the behavior of patients after myocardial revascularization. Further detailed study of these correlations is required, as well as consideration of implementing the results obtained in the psychological support of patients at the cardiac surgery departments. 


\section{Acknowledgements}

This research was supported by the Russian Foundation for Basic Research (RFBR) (Grant No 18-013-00689 A).

\section{References}

Bockeria, L. A., Alshibaya, M. M., Bendeliani, N. G., Nikonov, S. F., \& Krymov, K. V. (2012). Individual structured therapeutic training and intensive outpatient monitoring of patients after direct myocardial revascularization (results of a 12-month randomized clinical trial). The Bulletin of A.N. Bakoulev Scientific Center for Cardiovascular Surgery of Russian Academy of Medical Sciences "Cardiovascular Diseases", 13(1), 38-47.

Fonager, K., Lundbye-Christensen, S., Andreasen, J.J., Futtrup, M., Christensen, A.L., Ahmad, K., \& Nørgaard, M.A. (2014). Work Status and Return to the Workforce after Coronary Artery Bypass Grafting and/or Heart Valve Surgery: A One-Year-Follow Up Study. Rehabilitation Research and Practice, 2014, Article ID 631842, https://doi.org/10.1155/2014/631842.

Glazer, K.M., Emery, C.F., Frind D.J., et al. (2002). Psychological predictors of adherence and outcomes among patients in cardiac rehabilitation. J. Cardiopulm Rehabilitation, 22(1), 40-46.

Iakovleva, M. V. (2016). Adherence to treatment after coronary bypass surgery: psychological aspects. Revista Iberoamericana de Psicología y Salud, 7(1), 9-14. doi: 10.1016/j.rips.2015.10.003

Sousa, J.E., Serruys, P.W., \& Costa, M.A. (2003). New frontiers in cardiology: drug-eluting stents: Part I. Circulation, 107(17), 2274-2279.

Vasserman, L.I., Trifonova, E.A., \& Shchelkova, O.Y. (2011). Psychological diagnosis and correction in the somatic clinic. Saint Petersburg: Rech.

Velazquez, E.J. (2004). Heart failure and heart disease. Duke Clinical Research Institute, Duke University Medical Centre. Retrieved 12.15, 2018, from URL: https://medicine.duke.edu/sites/medicine.duke.edu/files/field/attachments/Velazquez\%20\%20HF\%20\%26\%20IHD.pdf 


\title{
THE ROLE OF COGNITIVE FUNCTIONING IN THE SUCCESS OF SOCIAL REHABILITATION OF CARDIAC PATIENTS
}

\author{
Daria Eremina \\ Saint-Petersburg State University (Russia)
}

\begin{abstract}
The paper presents the results of an investigation of clinical characteristics and indicators of cognitive functioning (indicators of active attention and mental capacity, memory, verbal-logical and spatial thinking, as well as neuropsychological indicators of visual recognition of fragmented images), which are potentially significant for the resumption of work in the late postoperative period of patients with coronary heart disease (CHD), undergoing coronary artery bypass grafting (CABG). An attempt has been made to extract from the whole mass of the data obtained the most prognostically significant, which will allow to differentiate patients at the stage of preparation for CABG to those who will be able to continue working after the operation, and those who are more likely not able to return to work.

The present study enrolled 118 patients (of average age $59.71 \pm 7.32$ years) who underwent coronary artery bypass grafting with standard cardiopulmonary bypass technique. The examination using a neuropsychological test battery (including WAIS, TMT-test, Stroop test, TAS, Benton test, etc.) was performed in three stages: two days before CABG, 12-14 days and three months after the surgery.

Patients with CHD experience significant postoperative cognitive decline mostly in verbal memory and attention, while positive dynamics was observed in the visual and logical memory, as well as in spatial and verbal-logical thinking. Using factor analysis we identified 4 factors of cognitive functioning, which were called verbal memory, thinking, cognitive control and visual recognition. As a result of the study of indicators of cognitive functioning, which are potentially significant for the resumption of work in the late postoperative period, we constructed a model, according to which the accuracy of prediction of belonging to the "working" group and to the group of "nonworkers" in the remote postoperative period is $83.9 \%$ (the significance of the canonical function obtained is $p=0.000066$ ). It was shown that the return to work in the long-term postoperative period is more typical for younger people who have lower indicators of the factors "Verbal memory" and "Thinking" before the operation and higher indicators of the factors "Verbal memory" and "Cognitive control" in the second and third stages of the study.
\end{abstract}

Keywords: Cognitive functioning, coronary artery bypass grafting, coronary heart disease, rehabilitation, labor forecast.

\section{Introduction}

Approximately 16.7 million people in the world die every year from cardiovascular diseases, including coronary heart disease (CHD). CHD is the leading disease in incidence and mortality in the general population (Leal, Luengo-Fernández, Gray, Petersen, \& Rayner, 2006; World Health Statistics 2006). One of the most important treatments for coronary heart disease is myocardial revascularization. It is carried out with the use of either coronary artery bypass surgery (CABG) or transluminal coronary angioplasty balloon. However, coronary bypass surgery has been the most commonly performed surgical procedure for more than 30 years.

However, despite the increase in the volume of aid and an improvement of health of the majority of operated patients, working capacity of patients is frequently reduced. For example, several studies observed a significant improvement in physical health after CABG in $90 \%$ of patients, but only $40-60 \%$ (Pinna Pintor et al., 1992; McCrone, Lenz, Tarzian, \& Perkins, 2001) of patients returned to their work without reducing the level of working capacity and preoperative qualification. Moreover, the level of social and professional functioning after cardiac surgery is largely determined by the emotional state, cognitive and personality characteristics of the patients, as well as by adherence to treatment during rehabilitation after cardiac surgery. 
According to the results of our previous studies, $73.7 \%$ of patients did not have objective (according to the cardiologist) contraindications regarding returning to work three months after the surgery. Moreover, it turned out that patients themselves assess the state of their physical health significantly better three months after surgery than before surgery $(\mathrm{p}<0.05)$ and in the early postoperative period ( $\mathrm{p}<0.001$ ). However, only $8.8 \%$ of the patients successfully returned to work three months after CABG, and only $35.1 \%$ were planning to return to work soon.

Thus, the present research aims at an investigation of clinical characteristics and indicators of cognitive functioning (indicators of active attention and mental capacity, memory, verbal-logical and spatial thinking, as well as neuropsychological indicators of visual recognition of fragmented images), which are potentially significant for the resumption of work in the late postoperative period of patients with CHD, undergoing CABG, in order to develop a model, which will allow to differentiate patients at the stage of preparation for CABG to those who will be able to continue working after the operation, and those who are more likely not able to return to work.

\section{Materials and methods}

118 patients undergoing coronary artery bypass grafting with standard cardiopulmonary bypass technique in Federal Almazov Medical Research Centre (Saint-Petersburg, Russia) were studied. Cardiopulmonary bypass (CPB) is a technique that temporary takes over the function of the heart. CPB is commonly used in heart surgery because of the difficulty of operating on the beating heart and it is well known to contribute to cognitive decline. The informed consent was obtained from all patients. Among them there were $58(82.9 \%)$ men and $12(17.1 \%)$ women; the average age of the patients was $59.71 \pm 7.32$ years. $48.6 \%$ of the patients were employed before the operation, $54.3 \%$ of patients were planning to return to their work after the operation. According to clinicians, the majority of the patients had no contradictions to come back to work three months after the surgery. But in fact, only $20.5 \%$ returned.

The examination was performed in three stages: two days before CABG, immediately before discharge from a hospital (12-14 days after CABG), and three months after CABG.

The methods used in the current study were selected with regard to the bio-psycho-social approach in modern clinical psychology and in accordance with the «Statement of Consensus on Assessment of Neurobehavioral Outcomes after Cardiac Surgery» (Zuyeva I.B. et al., 2011).

The study of cognitive functions of patients with CHD undergoing CABG was performed with the use of the following methods.

(1) Verbal learning test «10 words» was used in studying short- and long-term verbal memory.

(2) The method «Remembering stories» was used in studying logical memory.

(3) «The Benton Visual Retention Test» was used in studying visual perception and visual memory.

(4) The subtest «Similarities» of the Wechsler Adult Intelligent Scale (WAIS) was used in studying abstract verbal reasoning.

(5) The «Simple analogy» method was used in studying verbal-logical thinking.

(6) The subtest «Block Design» of the Wechsler Adult Intelligent Scale (WAIS) was used in studying spatial thinking.

(7) The Trail Making Test (TMT Parts A and B) was used in studying psychomotor speed, attention switching and mental flexibility.

(8) The Stroop Color-Word Test (SCWT) was used in studying two indicators: processing speed, as well as selective attention and resistance to cognitive interference.

(9) Computer technique «Visual acuity» was used to measure visual acuity.

(10) «Gollin Incomplete Figures Test» was used in studying the ability of visual recognition of the fragmented images.

\section{Results}

At the first stage of the research the whole set of studied psychometric and neuropsychological indicators of cognitive functioning was analysed using factor analysis. As a result, 4 factors of cognitive functioning, explaining $62 \%$ of the total dispersion of signs, were identified:

1. Thinking (this factor includes the following cognitive indicators: abstract verbal reasoning, verbal-logical thinking, spatial thinking, visual memory, logical memory); 
2. Visual recognition (visual recognition of the fragmented images);

3. Verbal memory (short-term and long-term verbal memory);

4. Cognitive control (psychomotor speed, attention switching and mental flexibility, processing speed, selective attention and resistance to cognitive interference).

At the next stage of the research discriminant analysis was used in order to identify clinical characteristics and indicators of cognitive functioning, which are potentially significant for the success of social and labour rehabilitation of patients (resumption of work in the late postoperative period). Using results of the discriminant analysis, we develop a model, which allows to differentiate patients at the stage of preparation for CABG to those who will be able to continue working after the operation, and those who are more likely not able to return to work.

The constructed model allows to predict correctly belonging to the group of "workers" and to the group "non-working" on the basis of discriminant variables in $83.9 \%$ of cases $(84.2 \%$ in the case of patients who returned to work, and $83.7 \%$ in the case of patients not returned to work).

The model includes such discriminant variables as age, the rate of compliance (measured by the Morisky-Green test), the number of heart attacks, the duration of illness, the number of hospitalizations in the past year, body mass index, the duration of the surgery, aortic cross-clamping and of the use of cardiopulmonary bypass during surgery, the number of diseased arteries, as well as 12 variables that reflect the level of cognitive functioning at different (three) stages of the investigation.

Table 1 presents the standardized coefficients of the canonical function, reflecting the most prognostically informative indicators regarding the division into groups of "working" and "non-working" and allowing to determine the ratio of contribution of each variable.

Table 1. Standardized coefficients of the canonical function for the most informative indicators.

\begin{tabular}{|l|l|}
\hline Psychodiagnostic indicators & Coefficient value \\
\hline Age & 0,805001 \\
\hline Verbal memory (1th stage) & 0,665799 \\
\hline Thinking (1th stage) & 0,295777 \\
\hline Verbal memory (2th stage) & $-0,575515$ \\
\hline Cognitive control (2th stage) & $-0,397905$ \\
\hline Cognitive control (3th stage) & $-0,532787$ \\
\hline Dynamics of visual recognition & 0,507482 \\
\hline
\end{tabular}

According to the data presented in Table 1, the most significant contribution to the fact that the patient will be classified as "working" or "non-working" three months after CABG has the variable "age": the older is the patient, the less likely he is to return to work after the operation. It also turned out that the higher were the indicators "Verbal memory" and "Thinking" before the operation, the less likely the patient is to continue his professional activity. This may be explained by the fact that the "Verbal memory" and "Thinking" factors were reduced in the preoperative period due to the underlying coronary disease, which indicates the possibility of a potential improvement of these factors after the surgery.

However, the higher were the indicators "Verbal memory" and "Cognitive control" at the second stage of the research and the higher was the indicator "Cognitive control" at the third stage of the research, the more likely patients are to return to work activity. In addition, the greater was the dynamics of "Visual recognition", the less likely the patients continue to work after surgery.

It should be emphasized that clinical indicators were not included in the constructed model, which indicates the high significance of cognitive functioning indicators in the social-labor prognosis of patients with CHD, undergoing CABG. It is also important to note that since the Age variable is included in the canonical function described above, the phenomenon of age-related changes in cognitive functions can be excluded from consideration.

\section{Acknowledgements}

This work was supported by the Russian Foundation for Basic Research (RFBR), project № 18-013-00689 A. 


\section{References}

Leal J, Luengo-Fernández R, Gray A, Petersen S, Rayner M. Economic burden of cardiovascular diseases in the enlarged European Union. Eur Heart J 2006; 27:1610-1619.

McCrone S, Lenz E, Tarzian A, Perkins S. Anxiety and depression: incidence and patterns in patients after coronary artery bypass graft surgery. Appl. Nurs. Res. 2001;14 (3):155-164.

Pinna Pintor, P., Torta, R., Bartolozzi, S., Borio, R., Caruzzo, E., Cicolin, A., Ravizza, L. (1992). Clinical outcome and emotional-behavioral status after bypass graft surgery. Quality of Life Research, 1(3), $177-185$.

World Health Organization. World Health Statistics 2006. Geneva, 2006.

Zuyeva IB, Vanaeva KI, Sanets EL, Piotrovskaya VR, Genihovich EL, Kirillova VI, Shlyakhto EV. The relationship of factors of cardiovascular risk with cognitive function in middle-aged patients. Hypertension 2011; 17(5):432-441. 


\title{
FEATURES OF THE PEOPLE REHABILITATION POTENTIAL WITH DIFFERENT CHARACTERISTICS OF LEARNED HELPLESSNESS AND HUMAN LIFE-WORLD STABILITY
}

\author{
Olga Petriaeva $^{1,2}$, Irina Loginova ${ }^{1}, \&$ Olesya Volkova ${ }^{1}$ \\ ${ }^{I}$ Chair of Clinical Psychology \& Psychotherapy, Krasnoyarsk State Medical University (Russia) \\ ${ }^{2}$ Chair of Nervous Diseases with a course of Medical Rehabilitation, Krasnoyarsk State Medical \\ University (Russia)
}

\begin{abstract}
Rehabilitation potential is considered as the ability of a person to activate his biological and socio-psychological mechanisms for restoring destroyed health, employability, personal status and estate. It can be realized under the certain conditions and the consolidated assistance of rehabilitation services and society. The efforts of specialists (ergotherapists, social workers, clinical psychologists) and the closest social environment can be effective in specific conditions. The major participant of the rehabilitation activity is initially the person who needs rehabilitation. It is suggested that the problem of rehabilitation potential correlates with problem of learned helplessness of the person and problem of human life-world stability (constructive, unconstructive, stagnant). These two phenomena are similar in their definition and revealing the level of responsibility of the person for his life (Deci \& Ryan, 2000). The learned helplessness as form of human life organization is the catalyst of psychological instability and somatic illness, it determines decrease in resilience of personality to harmful influences of the external environment, contributes to the development and exacerbation of psychological damages and somatic diseases of various etiologies. The helplessness is formed and "taught" gradually, under the influence of a factor of social response to failures in life events or features of somatic health rather than level of stress factor or disease nature and their objective influence on personality (Volkova, 2016). It is very important for a person to have successful experience in overcoming difficulties. Then rehabilitation activities can rely on this positive experience and provide more confident progress of a person towards the restoration of lost functions. The human life-world stability is considered in psychology as an essential indicator of the success of the life self-fulfillment and transference of abilities inherent in him (human) into reality (Loginova, 2012). The important indicator of rehabilitation success is patients' ability to use the newly obtained functions in everyday life.
\end{abstract}

Keywords: Rehabilitation potential, learned helplessness, human life-world stability.

\section{Introduction}

In the last decade rehabilitation as the direction of medicine is constantly developing, modifying, obtaining the new features and aspects. At the same time, violation of health acts as an important condition of this concept applicability, which in this connection allows to appear various aspects of rehabilitation ("recovery treatment", "medical rehabilitation", "recovery medicine", etc.). Rehabilitation in medicine is understood as restoration of organismic functions to adequate (normal) values after any organic injury (stroke).

Nevertheless, nowadays the idea that different people are restored differently becomes obvious: with different speed, with different staging of groups of muscles inclusion, with different opportunities of result maintaining after hospital treatment. For the description of the above listed parameters of rehabilitation course the term "rehabilitation potential" was endenizened into a scientific lexicon. Rehabilitation potential includes possibilities of an organism to restoration, motivation of the patient, possibility of patient's adaptation to the world around, his socialization process, etc. The idea of rehabilitation potential is being formed according to ICF (International Classification of Functioning, restrictions of activity and health), NIHHS (National Institutes of Health Stroke Scale), Renkin scale, ICD-10 (International Statistical Classification of Diseases and Related Health Problems) and other important for ergotherapy parameters. These scales widely clasp all spheres of the patient, but can mean absolutely different defeats at the same level: for example, sedentary in a carriage and able to operate it 
perfectly patient is as well socialized, as patient walking with a unilateral support; or the patient with aphasia is as deeply struck, as the patient confined to the bed.

The analysis of scientific definition of the term "rehabilitation potential" allowed us to reveal several essential positions:

1) The concept of "the rehabilitation potential" is inexact, insufficiently clear and is affected by the influence of not clinical context (Enderby, Pandyan, Bowen, et al., 2017).

2) Rehabilitation potential is considered as the ability of a person to activate his biological and socio-psychological mechanisms for restoring destroyed health, employability, personal status and estate, but not medical stuff activity (Sivukha, Enikeeva, 1997).

3) Representation and specification of patient rehabilitation potential is necessary not only for the correct rehabilitation program development, but also for forecasting the potential efficiency of the held rehabilitation activity (Haselbach, Renggli, Carda, et al., 2014), for estimation of the possible restoration level of a broken function and, therefore, the rehabilitation forecast.

4) The described in the contemporary studies clinical cases indicate the possibility of gradual change concerning the rehabilitation potential level: initially all patients by default have high rehabilitation potential and take a usual rehabilitation course, but during the subsequent hospitalization at the same medical institution doctors can estimate the remote results and change the level of rehabilitation potential. However, patients with various psychological characteristics can have problems with restoration of functions already at the first stage of rehabilitation that demands inclusion of the clinical psychologist in multidisciplinary team on a constant basis (Petryaeva, Shnayder, Artyukhov, Sapronova, Loginova, 2017).

We suggested that the problem of rehabilitation potential correlates with problem of learned helplessness of the person and problem of human life-world stability (constructive, unconstructive, stagnant). These two phenomena are similar in their definition and revealing the level of responsibility of the person for his life (Deci \& Ryan, 2000).

The learned helplessness as form of human life organization is the catalyst of psychological instability and somatic illness, it determines decrease in resilience of personality to harmful influences of the external environment, contributes to the development and exacerbation of psychological damages and somatic diseases of various etiologies. The helplessness is formed and "taught" gradually, under the influence of a factor of social response to failures in life events or features of somatic health rather than level of stress factor or disease nature and their objective influence on personality (Volkova, 2017).

It is very important for a person to have successful experience in overcoming difficulties. Then rehabilitation activities can rely on this positive experience and provide more confident progress of a person towards the restoration of lost functions.

The human life-world stability is considered in psychology as an essential indicator of the success of the life self-fulfillment and transference of abilities inherent in him (human) into reality (Loginova, 2012).

\section{Design}

The study was of comparative type and was organized in the two samples to reveal the degree of difference manifestation. All respondents subscribed voluntary informed consent to participate in the study in accordance with the norms of the Local Ethics Committee of Krasnoyarsk State Medical University. The study was conducted from 2017 until 2018.

Criteria of inclusion in the research:

- patients with a trauma of cervical section of the spine as one of the most severe injuries on consequences for the human;

- agreement to participate in the study subscribed in the informed consent;

- duration of a disease is no more than two years;

- status of a graduate (to provide high cognitive inclusiveness);

- age up to 50 years (to avoid cases of dementia processes influence on the research data);

- existence of close social environment for providing psychological support in rehabilitation process;

- the number of hospitalizations is more than one previous (that indicates adaptability to rehabilitation process);

- no more than one serious associated disease in anamnesis.

40 people were involved in the study.

The general number of patients was divided into two groups according to the level of rehabilitation potential.

- The first group - patients with low level of rehabilitation potential.

- The second group - patients with high level of rehabilitation potential. 


\section{Objectives}

The aim of the research is to study the features of the patients' rehabilitation potential with different characteristics of learned helplessness and human life-world stability:

The main objectives of the research are:

1) To explore how the state of the learned helplessness influences the features of rehabilitation potential?

2) To reveal how the specifics of human life-world stability influence the features of rehabilitation potential?

\section{Methods}

At the preparatory investigation stage, the assessment of rehabilitation potential was carried out by means of the following methods:

- neurologic (Renkin Scale, Bartel Index, OSS (Orgogozo Stroke Scale), ECOG (Karnovsky Index), RMI (Rivermead Mobility Index)),

- psychological (SF36, Riff scale, EPQ, E.B. Fantalova's questionnaire, BAI (Beck Anxiety Inventory), Cattel Sixteen Personality Factor Questionnaire, Dembo-Rubenstein test),

- neuropsychological techniques (MMSE, MOCA)

with deciphering of their result.

These scales are validated and have clear evidential base. The assessment of rehabilitation potential (high, average or low) was provided on the basis of the obtained data.

The open-type questionnaire "Learned Helplessness Genesis Value Judgment" (Volkova, 2016) was applied to study the unique ways of learned helplessness development and identification of its place in life of a specific person.

The method "Investigation of the Human Life-World Stability" (Loginova, 2012) was used as the research tool, which allows to study the features of the human life-world stability in the process of real-life activity. This method is aimed at studying the features of the human life organization and allows revealing the manifestations of the life stability (constructive, unconstructive, and stagnant).

Mathematical processing of the obtained results was carried out with use of the SPSS Statistics 21 software packaging.

\section{Discussion}

All data (responses and quotations) obtained in the two groups of respondents by means of the open-type questionnaire "Learned Helplessness Genesis Value Judgment" (Volkova, 2016) were systematized in accordance with the instruction and are presented in Table 1.

Table 1. Results of the questionnaire "Learned Helplessness Genesis Value Judgment".

\begin{tabular}{|c|c|c|c|c|}
\hline \multirow{2}{*}{$\begin{array}{l}\text { Learned } \\
\text { helplessness } \\
\text { criteria } \\
\end{array}$} & \multicolumn{2}{|c|}{$1^{\text {st }}$ group } & \multicolumn{2}{|c|}{$2^{\text {nd }}$ group } \\
\hline & Before disease & During disease & Before disease & During disease \\
\hline $\begin{array}{l}\text { Estimation of } \\
\text { the health state } \\
\text { (somatic status) }\end{array}$ & $\begin{array}{l}\text { I was diagnosed } \\
\text { with prematurity at } \\
\text { my birth } \\
\text { Frequent diseases of } \\
\text { various genesis } \\
\text { Asthenia } \\
\end{array}$ & $\begin{array}{l}\text { I feel deeply sick } \\
\text { I do not feel } \\
\text { healthy } \\
\text { I'm not alive, there } \\
\text { is no life in me }\end{array}$ & $\begin{array}{l}\text { Sometimes I suffered from } \\
\text { viral diseases } \\
\text { Something rarely } \\
\text { happened } \\
\text { I always considered } \\
\text { myself as healthy }\end{array}$ & $\begin{array}{l}\text { I'm overcoming the } \\
\text { disease } \\
\text { I do not define } \\
\text { myself as a patient } \\
\text { I'm restoring }\end{array}$ \\
\hline $\begin{array}{l}\text { Estimation of } \\
\text { the emotional } \\
\text { state }\end{array}$ & $\begin{array}{l}\text { I was morally } \\
\text { humiliated in my } \\
\text { family } \\
\text { I suffered from } \\
\text { experiencing } \\
\text { difficult life periods } \\
\text { Life has broken me, } \\
\text { I'm broken }\end{array}$ & $\begin{array}{l}\text { The trauma cracked } \\
\text { me even more } \\
\text { I am emotionally } \\
\text { devastated } \\
\text { I have no energy } \\
\text { for emotion }\end{array}$ & $\begin{array}{l}\text { Positive experiences of joy } \\
\text { from openings, } \\
\text { communication, } \\
\text { achievements }\end{array}$ & $\begin{array}{l}\text { Experiences of } \\
\text { impossibility to be } \\
\text { active, other } \\
\text { emotional reactions } \\
\text { remained the same }\end{array}$ \\
\hline $\begin{array}{l}\text { Estimation of } \\
\text { the motivation } \\
\text { level }\end{array}$ & $\begin{array}{l}\text { I always wanted to } \\
\text { be active, but it was } \\
\text { impossible }\end{array}$ & $\begin{array}{l}\text { There is no desire } \\
\text { to be active } \\
\text { I accepted this } \\
\text { situation (disease), } \\
\text { there is no } \\
\text { motivation on } \\
\text { changing it }\end{array}$ & $\begin{array}{l}\text { I was active, I clearly } \\
\text { understood what I want } \\
\text { I was strongly motivated } \\
\text { on achievements } \\
\text { I always competed with } \\
\text { others and myself }\end{array}$ & $\begin{array}{l}\text { I am ready to fight } \\
\text { for health } \\
\text { I have experience of } \\
\text { achievements, I use } \\
\text { it for achieving } \\
\text { results }\end{array}$ \\
\hline
\end{tabular}




\begin{tabular}{|c|c|c|c|c|}
\hline $\begin{array}{l}\text { Estimation of } \\
\text { the will-power } \\
\text { level }\end{array}$ & $\begin{array}{l}\text { Everything came } \\
\text { easily for me, I } \\
\text { never trained my } \\
\text { will-power } \\
\text { The will was weak - } \\
\text { I could achieve } \\
\text { nothing through } \\
\text { efforts }\end{array}$ & $\begin{array}{l}\text { If someone who } \\
\text { would direct me is } \\
\text { absent, I do nothing }\end{array}$ & $\begin{array}{l}\text { I was always a strong- } \\
\text { willed person } \\
\text { I was always on friendly } \\
\text { terms with my will-power: } \\
\text { I can do everything } \\
\text { overcoming my thought "I } \\
\text { do not want" to complete } \\
\text { anything I've started doing }\end{array}$ & $\begin{array}{l}\text { Now I became } \\
\text { weaker, wanted to } \\
\text { give up smoking, but } \\
\text { didn't succeed in it } \\
\text { I try to overcome the } \\
\text { situation: it's } \\
\text { impossible to reach } \\
\text { anything without } \\
\text { efforts }\end{array}$ \\
\hline $\begin{array}{l}\text { Locus of control } \\
\text { estimation }\end{array}$ & $\begin{array}{l}\text { While parents were } \\
\text { alive, they } \\
\text { controlled me. } \\
\text { When they died - it } \\
\text { was necessary to } \\
\text { become independent } \\
\text { Support of people } \\
\text { around me was } \\
\text { always necessary }\end{array}$ & $\begin{array}{l}\text { Now external } \\
\text { control is very } \\
\text { important (the } \\
\text { doctor, the nurse) } \\
\text { Without someone } \\
\text { who "adjusts" I } \\
\text { cannot do anything }\end{array}$ & $\begin{array}{l}\text { Generally, is was 50/50 } \\
\text { It differs from situation to } \\
\text { situation, but I tried to } \\
\text { control everything myself } \\
\text { I was accustomed to rely } \\
\text { on opinion of only the } \\
\text { closest - that's the way I } \\
\text { lived }\end{array}$ & $\begin{array}{l}\text { Now in those aspects } \\
\text { where I am not an } \\
\text { expert (concerning } \\
\text { treatment), I do not } \\
\text { interfere - I trust } \\
\text { experts } \\
\text { Situationally, about } \\
50-70 \% \text { is of my } \\
\text { control }\end{array}$ \\
\hline $\begin{array}{l}\text { Estimation of } \\
\text { cognitive sphere }\end{array}$ & $\begin{array}{l}\text { Though I was ill in } \\
\text { the childhood, I was } \\
\text { smart } \\
\text { I studied well at } \\
\text { school } \\
\text { I was a middling }\end{array}$ & $\begin{array}{l}\text { I try to maintain } \\
\text { my wits working } \\
\text { I puzzle crosswords }\end{array}$ & $\begin{array}{l}\text { My development was } \\
\text { normal } \\
\text { I never had problems with } \\
\text { study } \\
\text { I had excellent memory }\end{array}$ & $\begin{array}{l}\text { I do not notice any } \\
\text { deterioration so far } \\
\text { Everything is } \\
\text { interesting, I read a } \\
\text { lot }\end{array}$ \\
\hline $\begin{array}{l}\text { General } \\
\text { atmosphere in } \\
\text { child-parental } \\
\text { relations }\end{array}$ & $\begin{array}{l}\text { In my childhood I } \\
\text { was beaten by } \\
\text { parents } \\
\text { I was afraid of } \\
\text { parents } \\
\text { I did everything my } \\
\text { parents wanted only } \\
\text { because of their } \\
\text { rigid style of } \\
\text { education }\end{array}$ & $\begin{array}{l}\text { I do not want to be } \\
\text { a burden to my } \\
\text { children } \\
\text { I have difficult } \\
\text { relations with my } \\
\text { relatives }\end{array}$ & $\begin{array}{l}\text { We had a great family, I } \\
\text { have built my own family } \\
\text { by this example } \\
\text { Parents always understood } \\
\text { me } \\
\text { I was never shouted at, } \\
\text { parents explained me } \\
\text { everything }\end{array}$ & $\begin{array}{l}\text { My relations with } \\
\text { family remained } \\
\text { wonderful } \\
\text { I feel support } \\
\text { All relatives are } \\
\text { ready to help me }\end{array}$ \\
\hline $\begin{array}{l}\text { General } \\
\text { characteristic of } \\
\text { relations with } \\
\text { social } \\
\text { environment }\end{array}$ & $\begin{array}{l}\text { I had no friends } \\
\text { I was a soul of any } \\
\text { company } \\
\text { It was pleasant to } \\
\text { communicate }\end{array}$ & $\begin{array}{l}\text { I limited } \\
\text { communication } \\
\text { because of the } \\
\text { defect } \\
\text { I am ashamed of } \\
\text { friends as I am a } \\
\text { disabled person } \\
\text { I do not } \\
\text { communicate with } \\
\text { anybody, except } \\
\text { relatives }\end{array}$ & $\begin{array}{l}\text { Communication brought } \\
\text { me pleasure } \\
\text { I always was in the center } \\
\text { of events, possessed all the } \\
\text { information } \\
\text { I trusted to my social } \\
\text { environment }\end{array}$ & $\begin{array}{l}\text { My friends and } \\
\text { acquaintances often } \\
\text { visit me } \\
\text { I communicate } \\
\text { actively in social } \\
\text { networks } \\
\text { People often ask me } \\
\text { for advice }\end{array}$ \\
\hline
\end{tabular}

All results of the two groups of respondents obtained by means of the method "Investigation of the Human Life-World Stability" were systematized in accordance with the instruction and are presented in Table 2.

Table 2. Results of method "Investigation of the Human Life-World Stability"

\begin{tabular}{|l|l|l|}
\hline \multicolumn{1}{|c|}{ Options } & \multicolumn{1}{|c|}{$1^{\text {st } \text { group }}$} & \multicolumn{1}{c|}{$2^{\text {nd }}$ group } \\
\hline Temporarity of events tendency & $\begin{array}{l}\text { The present } 30 \% \\
\text { The past } 40 \% \\
\text { The past - the present 30\% }\end{array}$ & $\begin{array}{l}\text { The present - the future } 70 \% \\
\text { The past - the present - the future } 30 \%\end{array}$ \\
\hline The ratio of verbs & $\begin{array}{l}\text { The present } 40 \% \\
\text { The past } 40 \% \\
\text { The past - the present 20\% }\end{array}$ & $\begin{array}{l}\text { The present - the future } 70 \% \\
\text { The past - the present - the future } 30 \%\end{array}$ \\
\hline $\begin{array}{l}\text { Criterion for the described } \\
\text { events content selecting }\end{array}$ & $\begin{array}{l}\text { Chronotopic } 10 \% \\
\text { Topological 30\% } \\
\text { Biographical } 60 \%\end{array}$ & $\begin{array}{l}\text { Chronotopic } 40 \% \\
\text { Topological } 50 \% \\
\text { Biographical } 10 \%\end{array}$ \\
\hline $\begin{array}{l}\text { General emotional background } \\
\text { of events }\end{array}$ & $\begin{array}{l}\text { Positive } 10 \% \\
\text { Neutral } 40 \% \\
\text { Negative } 50 \%\end{array}$ & $\begin{array}{l}\text { Positive } 70 \% \\
\text { Neutral } 20 \% \\
\text { Negative } 10 \%\end{array}$ \\
\hline
\end{tabular}




\begin{tabular}{|c|c|c|}
\hline $\begin{array}{l}\text { The meaning of the described } \\
\text { life events }\end{array}$ & $\begin{array}{l}\text { The overall direction of the } \\
\text { development line is conserved 20\% } \\
\text { General orientation is not withheld } \\
20 \% \\
\text { Center of the development line } 20 \% \\
\text { Completion of the development line } \\
40 \%\end{array}$ & $\begin{array}{l}\text { The overall direction of the } \\
\text { development line is conserved } 60 \% \\
\text { General orientation is not withheld } \\
30 \% \\
\text { The beginning of the development line } \\
10 \%\end{array}$ \\
\hline Attitude to events & $\begin{array}{l}\text { Valuable } 10 \% \\
\text { Responsible } 10 \% \\
\text { Rational } 80 \% \\
\end{array}$ & $\begin{array}{l}\text { Valuable } 70 \% \\
\text { Responsible } 20 \% \\
\text { Rational } 10 \% \\
\end{array}$ \\
\hline Continuity of personal story & $\begin{array}{l}\text { Retained } 10 \% \\
\text { Situational } 30 \% \\
\text { Missing } 60 \% \\
\end{array}$ & $\begin{array}{l}\text { Retained } 70 \% \\
\text { Situational } 20 \% \\
\text { Missing } 10 \% \\
\end{array}$ \\
\hline Creative reflexive position & $\begin{array}{l}\text { Holistic reflexive position } 10 \% \\
\text { Situational reflexive attitude } 30 \% \\
\text { Absence of a reflexive relation } 60 \%\end{array}$ & $\begin{array}{l}\text { Holistic reflexive position } 70 \% \\
\text { Situational reflexive attitude } 20 \% \\
\text { Absence of a reflexive relation } 10 \%\end{array}$ \\
\hline
\end{tabular}

\section{Conclusions}

It was revealed that people with low rehabilitation potential had problems during the childhood or youth period which promoted formation of the learned helplessness syndrome. The difficulties in the course of personality development significantly reduce possibilities of present recovery period. It is hard for such patients to cope with difficulties independently, as an active person, from the subjective position. They narrow life space because of defect, experience mainly negative feelings and emotions. Besides, their life has fragmentary character: it is broken into two parts "before the disease" and "during the disease". They feel fear thinking about their future. They are not focused on the reflexive attitude to life and themselves. Their critical thinking is reduced.

As for patients with high rehabilitation potential they have resource for restoration during disease: they are optimistic, capable to control their own life, keep social contacts, try to be useful to others. Their life is represented as more complete, complex, filled with sense. They are capable to learn experience from current situation and to give support to people around.

The important indicator of rehabilitation success is patients' ability to use the newly obtained functions in everyday life. However, only $60 \%$ of patients with low rehabilitation potential demonstrate the ability to transfer the mastered skills to everyday life. We suppose that there are two major obstacles to it: the learned helplessness manifestations and not constructive or stagnant human life-world stability.

\section{References}

Deci, E.L., Ryan, R.M. (2000). The "What" and "Why" of goal pursuits: human needs and the self-determination of behavior. Psychological Inquiry, Vol. 11, 4, 227-268.

Enderby, P., Pandyan, A., Bowen, A., Hearnden, D., Ashburn, A., Conroy, P., Logan, P., Thompson, C., Winter, J. (2017). Accessing Rehabilitation after Stroke - A Guessing Game? Disabilitation Rehabilitation, 39 (7), 709-713. doi: 10.3109/09638288.2016.1160448.

Haselbach, D., Renggli, A., Carda, S., Croquelois, A. (2014). Determinants of neurological functional recovery potential after stroke in young adults. Cerebrovasc Dis Extra, 4, 77-83. doi: 10.1159/000360218.

Loginova, I.O. (2012). Research of stability of the vital world of the person: technique and psychometric characteristics. Psychological Science and Education, 3, 18-28.

Petryaeva, O.V., Shnayder, N.A., Artyukhov, I.P., Sapronova, M.R., Loginova, I.O. (2017). The role of orthotic service in modern rehabilitation of patients with Charcot-Marie-Tooth disease. Journal of Biosciences and Medicines, 6, 23-34.

Sivukha, T.A., Enikeeva, A.A. (1997). Methodical approaches to determining the rehabilitation of the brain vascular pathology. Medico-social examination and rehabilitation of disabled people, 21, 63-69.

Volkova, O.V. (2016). Prospects of a complex research model application in designing the program aimed to diagnostics, correction and prevention of the learned helplessness in ontogenesis. Siberian psychological journal, 61, 47-63. 


\title{
THE EFFECTS OF CIVIC PARTICIPATION ON JAPANESE OLDER ADULTS
}

\author{
Keiko Katagiri \\ Graduate School of Human Development / Kobe Active Aging Research Hub, Kobe University (Japan)
}

\begin{abstract}
The growing elderly population in Japan has invited many social problems, one such problem being a shortage of productive population members to support the large elderly dependent population. Another is the seniors themselves; normative roles for seniors after retirement do not exist. In 2013, the Japanese government amended the Elderly Persons Employment Stabilization Law, which requires companies to provide jobs to employees until the age of 65 . However, most Japanese companies have a mandatory retirement age of 60 , and once senior workers reach this age, the companies prepare new labour contracts under which they work at lower rates and no designation. Following this amendment, an increased number of workers in their 60s have joined the workforce. Work is one means for seniors to engage in society; however, it cannot be a source of self-esteem, as this changes after the age of 60, when retirement become mandatory. Katagiri proposed a model of civic participation for seniors; this model delineates three options: social participation, productive activity, and civic engagement. The purpose of this study was to explore the effect of working condition and social participation on health. We used data from a nationally representative sample of the 2012 Japanese General Social Survey. The responses of participants aged 60 to 74 years were analysed. Of these, $18.1 \%$ of respondents were full-time workers and $17.5 \%$ were part-time workers, while $66.4 \%$ did not work. We found that $32.5 \%$ people did not participate in any activities. About half the full-time and part-time workers did not engage in any non-work-related activities. We conducted an ANCOVA to compare mental health among individuals working under different conditions and engaging in non-work-related activities, controlling for subjective health and education. The results showed a main effect of participation in non-work-related activities for the male respondents. The male respondents engaging in social or civic activities showed better mental health than those not engaging in any activities, regardless of work status. For the female respondents, in addition to the same positive effect of participation, the respondents working part-time showed better mental health than those working full-time or not working. The effect of participation was similar to that seen in the male respondents. These results suggest that the meaning of work may differ for older workers. Work cannot be a source of self-esteem, and workers need meaningful activities to find meaning in life.
\end{abstract}

Keywords: Older workers, social participation, work life balance, civic engagement, meaning in life.

\section{Introduction}

\subsection{Increase of older workers in Japan}

The aging rate of the Japanese population has been increasing; it reached $27.7 \%$ in 2018 and is estimated to reach $33.3 \%$ in 2036 and $38.4 \%$ in 2065 . In tandem, the productive population has been decreasing. The implication of this for the younger generation is heavy burden in terms of social security costs. In the worst-case scenario, a ratio of one young person supporting one old person has been estimated. Such a state of affairs cannot be deemed sustainable.

The Japanese government is struggling to solve this problem by encouraging the elderly to remain a part of the work force as long as possible and to this effect revised the "Law Concerning Stabilization of Employment of Older Persons." Until recently, the mandatory retirement age in Japan was 60; however, the revision of the law obligated companies to retain their employees until the age of 65 . Note that this does not mean that the retirement age has been extended from 60 to 65. Most companies continue to set the retirement age as 60 , which means that the existing labour contract ends once the employee has turned 60 , and a new contract begins. This new contract is usually completely different; this is a significant reduction in salary, sometimes one third of the original. In addition, the employee serves as a member of staff, with no designation. The work hours remain the same: Monday to Friday, from 9 a.m. to 5 p.m. This means that seniors have to work under rather devalued conditions. Despite this, most choose to continue working for 
financial reasons. The annuity sum is estimated to decrease with the increase in the older population. At present, they have to support not only their older parents but also sometimes their adult children, who are still dependent on them after graduation from university. Furthermore, the extended life expectancy make it impossible for them to estimate how much they will need to save for retirement.

To add a different perspective, the devalued working conditions after the retirement age of 60 cannot serve as a source of self-esteem. Katagiri (2012) investigated the effect of work status and social participation on seniors in their 60s. Social participation was found to have a positive effect on self-esteem. Working men who engaged in a social activity were found to have higher self-esteem than those who did not engage in any social activity. She interpreted these results in light of the devalued working conditions for older workers.

One more aspect to be considered is work hours and days. Most older workers would rather not work the same hours as they did before; they prefer fewer hours a day or fewer days a week. A few studies have investigated differences in working conditions based on work hours. Corna (2016) compared changes in work and health status by gender using longitudinal data from Americans ranging from 52 to 69 years. Working condition was divided into full-time, part-time, and retired. Men were found to be in the best of health when they switched from full-time to part-time job in their mid-sixties, whereas for women, the same was true for those who continued part-time work from the ages of 52 to 69. This shows that working condition affects health status. However, to date no studies considering work status have been conducted in Japan.

\subsection{Purpose}

Katagiri (2017) proposed a model of civic participation for seniors; this model delineates three options for seniors: social participation, productive activity, and civic engagement. Social participation includes group activity, hobbies, sports, and lifelong learning etc. Productive activity includes paid or unpaid work. Civic engagement means engaging in civic or political activities. The first purpose of this study was to explore the effect of work (productive activity) and social activity (social or civic activity) on health; the second was to observe gender differences, if any.

\section{Method}

\subsection{Data}

We conducted a secondary analysis using data from the Japanese General Social Survey 2012, a nationally representative sample of Japan. A sub sample comprised participants in the age group of 60 to 74 years $(\mathrm{N}=1,929)$.

\subsection{Independent variables}

We created three kinds of working conditions - full-time workers, part-time workers, and not working - according to work hours. Full-time workers worked more than 40 hours a day, part-time workers less than 40 hours, and participants with 0 work hours were considered as not working.

We created a dummy variable for social or civic activities other than work. The basis was whether the participants engaged in at least one of the following group activities: political associations, residential/neighbourhood associations, and social service clubs (volunteer group/NPO9, citizens' movement/ consumers' cooperative groups, religious groups, alumni associations, recreational associations (hobbies and sports), labour unions, occupational/professional association/trade unions, etc.).

\subsection{Dependent variables}

Three items measuring mental health were taken from SF 36: "Have you felt calm and peaceful?" "Did you have a lot of energy?" and "Have you felt downhearted and blue? (reversed)?". Answers ranged from "1: None of the time," "2: Little of the time," "3: Some of the time," "4: Most of the time," and "5: All of the time," to organized, as higher scores indicated better mental health. As Cronbach's $\alpha$ was .64, we summed up the scores to determine each participant's mental health score.

\section{Results}

\subsection{Description of the participants}

Table 1 and Table 2 list the characteristics of the participants, who ranged in age from 60 to 89 and were on average healthy and from middle class backgrounds. 
Table 1. Descriptive statistics of participants.

\begin{tabular}{lrr}
\hline & $\mathrm{N}=1,929$ \\
& Mean & $S D$ \\
\hline Age & 70.1 & 7.27 \\
Subjective health (1: Very bad - 5: Very good) & 3.6 & 1.15 \\
Mental health (3-15) & 10.58 & 2.24 \\
Household income (1: Far below average - 5: Far above average) & 2.5 & 0.90 \\
Level of urbanization (1: rural - 5: urban) & 3.2 & 0.92 \\
\hline
\end{tabular}

Table 2. Distribution by demographic characteristics.

\begin{tabular}{llrr}
\hline & & $N$ & $\%$ \\
\hline \multirow{2}{*}{ Gender } & Male & 888 & 0.459865 \\
& Female & 1043 & 0.540135 \\
\hline \multirow{2}{*}{ Education } & Less than high school & 601 & 0.313 \\
& High school & 915 & 0.476 \\
& $1 \mathrm{z}$ & 405 & 0.211 \\
\hline \multirow{2}{*}{ Marital status } & Married & 1427 & 0.74 \\
& Not married & 502 & 0.26 \\
\hline \multirow{2}{*}{ Work status } & Full-time & 289 & 0.154 \\
& Part-time & 351 & 0.187 \\
& Not working & 1238 & 0.659 \\
\hline
\end{tabular}

\subsection{Mental health status by gender, work status, and age group}

Table 3 shows the mental health scores by gender, work status, and age group. First, we observed no statistical differences between men and women. Second, mental health differed based on work status. People working part-time showed better scores than the other two groups. Third, though we observed no differences among age groups, male respondents showed their peak of good mental health in their early 70s, while female respondents showed their peak in their late 60s.

Table 3. Mental health by gender, work status, and age group.

\begin{tabular}{clcc}
\hline & & $M$ & $S D$ \\
\hline \multirow{2}{*}{ Gender } & Men & 10.64 & 2.23 \\
& Women & 10.54 & 2.26 \\
\hline \multirow{3}{*}{ Work status } & Full-time & 10.61 & 2.32 \\
& Part-time & 10.90 & 2.18 \\
& Not working & 10.48 & 2.25 \\
\hline \multirow{3}{*}{ Age group } & $60-64$ & 10.52 & 2.15 \\
& $65-69$ & 10.67 & 2.11 \\
& $70-74$ & 10.79 & 2.30 \\
& $75-$ & 10.44 & 2.39 \\
\hline
\end{tabular}

\subsection{Results of the ANCOVA}

We conducted an ANCOVA to compare mental health by gender among individuals in different working conditions and engaging in activities other than work, controlling for subjective health and education.

Subjective health, educational status, and active engagement in social activity showed significant main effects for both men and women. We observed no statistical differences in work status among men. 
On the contrary, for women, subjective health, educational status, active engagement in social activity, and work status showed statistical differences (Figure 1). Women working part-time showed better mental health than those working full-time or not working.

Figure 1. Results of the ANCOVA.
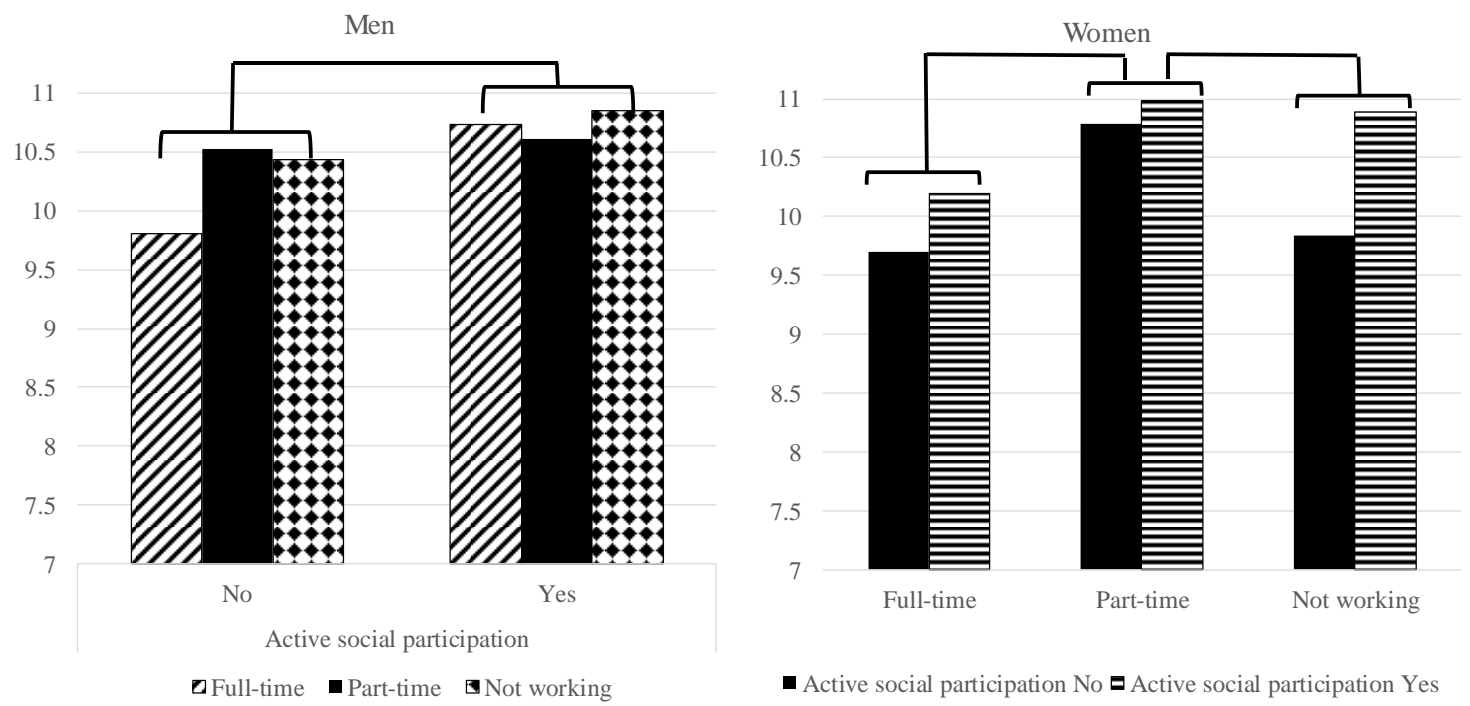

\section{Discussion}

The results show the robust positive effects of active social participation on mental health in both men and women; however, the same was not seen with working conditions. This suggests that men cannot find meaning in work after retirement. Thus, we observed no differences in mental health scores among the male respondents with regard to working conditions. Instead, men were found to have better mental health when they could find a role, network, or passion when engaging in social or civic activities. Conversely, men who could not find a substitute for work tended to feel depressed.

The working conditions of the female respondents were found to affect mental health. Women with part-time jobs were found to have the best mental health. When working full-time, women might have to play multiple roles: wife, mother, caregiver to old parents, and caregiver to grandchildren and this multiple role strain might cause them a great amount of stress (Marks, 1977). However, fewer such roles do not necessarily indicate less stress for middle-aged and old-aged women. Wives with retired husbands sometimes suffer from "homebound husband stress syndrome" and get depressed on account of their husbands staying home all day and not participating in domestic chores (Kurokawa, 2009). If such women are also not working, their retired husbands might be a source of stress.

This study also showed the robust strong positive effects of active social or civic activities. In Japan, the social engagement rate needs to be considered carefully as activeness ranges from merely having a membership in a group activity to active engagement in said activity (Katagiri \& Kim, 2018). Just having a membership alone does not contribute to better mental health; active engagement is the key.

There seem to be two levels of hurdles for Japanese seniors. The first is finding a good group in which to participate. The second is active engagement. Most men spent workaholic lives, devoting very little time to community activities and hobbies. It is therefore difficult for them find even one activity that interests them, and even when they do find one, it is not easy to adapt to a group activity. Sometimes, it may be not easy to get along with other group members, and sometimes they may not find the activity interesting or meaningful enough.

The Japanese government has been promoting the prolonged work life policy, one of the reasons being that work is assumed to have positive effects on health. While some studies do report the positive effects of work on health among seniors (e.g. Ilmarinen, 2001), the results of this study do not support this claim. Regardless of the results, we cannot conclude that working conditions do not matter for older adults, as the working conditions and health status of older workers are dramatically changing. Further investigation of both working conditions and health is needed.

At the same time, the results indicate the importance of work-life balance for older adults. Measures to improve working conditions and encourage active engagement in social or civic activities other than work must be considered. 


\section{References}

Corna, L. (2016). Later-life employment trajectories and health: Evidence from the US. Paper presented at the 45th Annual Conference of the British Society of Gerontology, Stirling, Scotland, P.121.

Ilmarinen, J. E. (2001). Aging workers. Occupational and Environmental medicine, 58(8), 546-546.

Katagiri, K. (2012). Japanese retirees and social participation: A challenge to the third age. Tokyo: University of Tokyo Press. (in Japanese)

Katagiri, K. (2017). Development among third age in super aging society. Tokyo: University of Tokyo Press. (in Japanese).

Katagiri, K., \& Kim, J. H. (2018). Factors determining the social participation of older adults: a comparison between Japan and Korea using EASS 2012. PloS One, 13(4), e0194703.

Marks, S. R. (1977). Multiple roles and role strain: Some notes on human energy, time and commitment. American Sociological Review, 42(6), 921-936.

Nobuo Kurokawa (2009). Homebound husband stress syndrome. Japanese Society of Psychosomatic Medicine, 49(2), 99. 


\title{
THE EFFECT OF LOCUS OF CONTROL IN RESPONSIBILITY AMONG PARTICIPANTS WITH TRAUMATIC EVENT HISTORY*
}

\author{
Merve Topcu ${ }^{1}$, Deniz Köroğlu ${ }^{1}$, Yusuf Coşku İnceler ${ }^{1}$, \& Faruk Gençöz ${ }^{2}$ \\ ${ }^{1}$ Department of Psychology, Çankaya University (Turkey) \\ ${ }^{2}$ Department of Psychology, Middle East Technical University (Turkey)
}

\begin{abstract}
Individuals' causal attributions between their behavior and a reward determine their future behaviors (LoC). When individual perceive limited control over an action, then the behavior appraised as a result of luck, and fate, as under the control of powerful others, or as unpredictable. It is called external LoC. When the event is perceived as contingent upon his/her behavior, it is internal LoC. Traumatic events are defined as disruptive to the daily routine, develop suddenly and unexpectedly, create horror, anxiety and panic, and disturb the process of making sense of the world. However, after traumatic event exposure it is possible to show psychological improvement, which is entitled as posttraumatic growth. Positive correlation between posttraumatic growth and responsibility has been found in the literature. Responsibility defined as individual's fulfilment of antecedents and consequences of their emotions, cognitions, and behaviors. It was thought that understanding the responsibility could be essential on the way through posttraumatic growth. The role of LoC in responsibility among people with traumatic event history was examined. A cross-sectional paper-pencil format study conducted with 139 participants who reported at least one traumatic event exposure included in the analysis. Informed consent, socio-demographic form, the Positive (PA) and Negative Affect (NA) Scale, the LoC Scale, the Social Desirability Inventory, and the Responsibility Scale were given to the participants. Correlational analysis shows that responsibility was positively correlated with PA for last six months and social desirability. The responsibility was negatively correlated with NA for last six months and belief in an unjust world. 2-stepped hierarchical regression analysis run to examine the role of $\mathrm{LoC}$ in the prediction of responsibility. Social desirability, PA and NA were entered in the first step. Significantly correlated belief in an unjust world dimension of LoC with responsibility entered in the second step. Results demonstrated that belief in an unjust world significantly predicted responsibility when controlled for social desirability and PA and NA. That is, those who believe in that world is an unjust place may think, feel or act responsibly more. And also, thinking, feeling or acting responsibly may be appraised as protective against feelings of failure, guilt or shame, fear of punishment, uncertainty and being victim of an uncontrolled event. Eventually this protection may be rewarding. On the way through posttraumatic growth, belief in an unjust world and responsibility may be influential factors especially on therapeutic process with individuals with traumatic event history.
\end{abstract}

Keywords: Trauma, growth, locus of control, responsibility.

\section{Introduction}

Individuals' causal attributions to the relationship between their behavior and a reward determine their future behaviors. This is called the locus of control (LoC). When individuals perceive limited control over an action, then the behavior appraised as a result of luck, and fate, as under the control of powerful others, or as unpredictable, called external LoC. When the event is perceived as contingent upon his/her behavior, it is internal LoC (Rotter, 1966). Traumatic events are defined as disruptive to the daily routine, develop suddenly and unexpectedly, create horror, anxiety and panic, and disturb the process of making sense of the world (APA, 2013). However, after traumatic event exposure, it is possible to show psychological improvement, which is entitled as posttraumatic growth (Tedeschi \& Calhoun, 1995; 2004). Positive correlation between posttraumatic growth and responsibility has been found (Topcu, 2016). Responsibility is defined as an individual's fulfillment of antecedents and consequences of their emotions, cognitions, and behaviors (Topcu, Öge, \& Gençöz, 2018; Topcu, 2016). It was thought that understanding

*Additional information: The study presented in this manuscript is part of the doctorate thesis of the first author. 
the responsibility could be essential on the way through posttraumatic growth. The role of the LoC in responsibility among people with traumatic event history was examined.

The aim of the current paper is to assess the role of the LoC in the prediction of responsibility while controlling the effects of social desirability and positive (PA) and negative (NA) affect for last six months in a sample of individuals with at least one traumatic event history. It was hypothesized that when social desirability and PA and NA for last six months were controlled, significant association between the LoC and responsibility are still expected.

\section{Methods}

\subsection{Participants}

139 participants who reported at least one traumatic event history included in the study to examine the relationship of responsibility and dimensions of the LoC. Age of the participants varied between 18 to 52 years $(\mathrm{M}=32.86, \mathrm{SD}=12.55) .25 .2 \%(n=35)$ of the participants are male, $74.8 \%(n=104)$ of them are female. SD, PA and NA for last 6 months and the LoC group mean scores compared across gender via Mann-Whitney U test. Though the gender ratio was in favor of females, Bonferroni correction was considered as well. All of them was insignificant across gender ( $\mathrm{p} \geq .05$ ).

\subsection{Measures}

2.2.1. Socio-demographic form. The sociodemographic form includes age, educational status, and gender variables. The definition of the trauma which have defined based on DSM V (APA, 2013) was given to participants. Then asked the participants whether they had any prior experience which fits with the definition.

2.2.2. The Positive and Negative Affect Scale (PANAS). The PANAS which was developed by Watson, Clark, and Telegen (1988) to measure general tendencies toward positive affect and negative affect. Different timeframes can supply different scores. In this study, timeframe was their affectivity for 'last 6 months'. Indicating how they feel on general on a 5 point Likert-type scale in the form of from 1 ("very slightly") to 5 ("extremely") were asked from the participants for all 20 items. Turkish adaptation of PANAS was done by Gençöz (2000).

2.2.3. The Social desirability inventory (SDI). The SDI which was originally developed by Stöber (2001) measures the extent that individuals' respond in a favorable manner. Participants were asked to evaluate social desirability by rating 16 dichotomous questions ("true" / "false"). Higher socially desirable responding is shown by higher scores. Scores for social desirability ranged between 0-20 totally. The Turkish version of social desirability inventory which was adapted by Kozan (1983) includes a list of 20 items.

2.2.4. The Responsibility Scale (RS). The Responsibility Scale (RS) which was developed by Topcu (2016; 2018) assess individuals' perceptions about responsibility. Participants were asked to evaluate responsibility by rating on a 5-point Likert-type scale ranging from 0 ("never") to 4 ("always") on 24 items. Inventory has 3 dimensions, namely, emotional, behavioral and cognitive responsibility. Only total score of the scale was used in this study.

2.2.5. The Internal - External Locus of Control Scale (LoC). The LoC was originally developed by Rotter (1966) in order to evaluate the individual's attributions to their consequences of their behaviors. It consists of 29 items and is scaled as dichotomous. Total score ranges from 0 to 23. Personal control, belief in chance, meaningless of the effortfullness, belief in fate and belief in an unjust world are the subscales of the LoC. Participants were asked to evaluate their behaviors by rated on a 5-point Likert-type scale ranging from 1 ("strongly disagree") to 5 ("strongly agree"). The Turkish form of the scale was adapted by Dağ (2002).

\subsection{Procedure}

A part of the first author's doctorate thesis is presented in this study (Topcu, 2016). Additionally, part of the data was presented in one of the previous Inpact conference series (Topcu, Köroğlu, \& Gençöz, 2018). A cross-sectional paper-pencil format study was conducted with conveniently sampled 139 participants. For eliminating the sequencing effect instruments in the study were presented in random order. The informed consent and a brief explanation of the study were placed on the first page. The study was approved by the Middle East Technical University Ethical Committee. All statistical analyses were done with the use of IBM SPSS 21. 


\section{Results}

Pearson correlation coefficients were calculated. Results revealed that the Responsibility scale total score was only negatively and significantly correlated with belief in an unjust world ( $r=-.24, p<.01)$. Relationship among variables were summarized in Table 1.

To assess the role of LoC in the prediction of responsibility while controlling the effects of social desirability and positive and negative affect for last six months in a sample of individuals with past traumatic event history, 2-stepped hierarchical regression analysis run. To learn the impact of LoC in the prediction of responsibility, social desirability and positive and negative affect for last six months were entered in the first step; and significantly correlated EMSs with responsibility entered in the second step of the Linear regression analyses (Enter), respectively. In the prediction of responsibility, the first model was significant $(F(3,135)=22.403, p<0.001)$. Only social desirability $(t=6.674 .082, p<.001)$ and PA scores for last six months $(t=3.488, p<.001)$ significantly contributed to the regression. Adjusted $R^{2}$ revealed that both responsibility and PA scores accounted for the variance of responsibility with the proportion of $32 \%$. $\beta$ coefficients showed that exploratory proportions of SD and PA on responsibility were $48 \%$ and $25 \%$, respectively. In the second step of the analyses, only significantly and negatively correlated dimension of LoC, belief in an unjust world, were added. Results revealed that in the prediction of responsibility, the second model was significant $(F(4,134)=18.322, p<0.001)$.SD $(t=6.60, p<.001)$, PA for last six months $(t=3.28, p<.01)$ and belief in an unjust world subscale of $\operatorname{LoC}(t=-2.10, p<.05)$ score significantly contributed to the regression. Adjusted $R^{2}$ revealed that SD, PA for last six months, and belief in an unjust world scores accounted for the variance of responsibility with the proportion of $33 \%$. $\beta$ coefficients showed that exploratory proportions of SD, PA for last six months, and belief in an unjust world on the responsibility were $21 \%, 5 \%$ and $2 \%$, respectively (Table 2 ).

\section{Discussion}

Results revealed that responsibility was only negatively and significantly correlated with belief in an unjust world, which is an external LoC subscale. Also, SD, PA for the last six months and belief in an unjust world scores significantly contributed to the responsibility. In a world where individuals often get what they do not deserve or do not get what they do deserve, responsibility may serve as rationalization and/or coping mechanism (Lench, \& Chang, 2007). Traumatic events are defined as disruptive to the daily routine, develop suddenly and unexpectedly, create horror, anxiety and panic, and disturb the process of making sense of the world (APA, 2013). Individuals with higher anxiety tend to remember more negative events. Easy access to negative events in the memory is related to higher beliefs in an unjust world (Koster, De Raedt, Goeleven, Franck, \& Crombez, 2005). Higher belief in an unjust world is related to greater anxiety, depressive symptoms, neuroticism, and defensiveness (Lench, \& Chang, 2007). Since the sample represents individuals with at least one traumatic event history, it can be concluded that those who believe in less that world is an unjust place may think, feel or act responsibly more which are especially in a socially desirable manner. Thinking, feeling or acting responsibly may be appraised as protective against feelings of failure, guilt or shame, fear of punishment, uncertainty and being victim of an uncontrolled event; ease the escape from the punishment, and prospective and cumulative traumatic effects, and protect themselves against punishment and feelings of guilt, shame, and anger resulted from 'failing' responsibility (Topcu, Köroğlu, \& Gençöz, 2018). Eventually protection and escape may be rewarding. Being rewarding may be related to higher PA for the last six months. On the way through posttraumatic growth, the relationship between belief in an unjust world and responsibility may be influential especially on therapeutic process with individuals with traumatic event history.

Although posttraumatic growth mentioned in this study, it was not assessed any measurement or statistically. It can be considered as a limitation. However, previous literature findings determine the way through posttraumatic growth (Tedeschi \& Calhoun, 1995; 2004). Belief in an unjust world may inhibit individuals from psychological growth (Topcu, Köroğlu, \& Gençöz, 2018) by less likely to cope successfully with their trauma (Lench, \& Chang, 2007) rather than manifesting self-reflective examination, evaluating their behaviors, accepting their emotions, feeling relieved from frightening images of facing emotions, finding peace, contentment, and control over the circumstances, and taking a social role and responsibility (Chandler, 1975; cited in Dawes, Montada, Filipp \& Lerner, 1992).

Limitations were stated previously (Topcu, Köroğlu \& Gençöz, 2018; Topcu, 2016). In this study, only the total score of responsibility was used. However, examining the dimensions of responsibility may lead detailed information about the relationship between responsibility and the LoC. In addition, the assumption which belief in an unjust world could be an inhibitor factor for PTG. This assumption could be tested statistically, too. Further studies can consider these limitations of the current study as well. 
Table 1. Correlations between scale scores $(N=139)$.

\begin{tabular}{|c|c|c|c|c|c|c|c|c|c|c|}
\hline & \multicolumn{2}{|c|}{$\begin{array}{c}\text { Personal } \\
\text { control }\end{array}$} & \multicolumn{2}{|c|}{$\begin{array}{c}\text { Belief in } \\
\text { chance }\end{array}$} & \multicolumn{2}{|c|}{$\begin{array}{c}\text { Meaningless of the } \\
\text { effortfullness }\end{array}$} & \multicolumn{2}{|c|}{$\begin{array}{c}\text { Belief in } \\
\text { fate }\end{array}$} & \multicolumn{2}{|c|}{$\begin{array}{l}\text { Belief in an unjust } \\
\text { world }\end{array}$} \\
\hline & $\boldsymbol{R}$ & $p$ & $r$ & $p$ & $r$ & $p$ & $r$ & $p$ & $r$ & $p$ \\
\hline RS & -.072 & .397 & .038 & .659 & -.132 & .121 & .067 & .434 & -.243 & $.004 *$ \\
\hline SD & -.157 & .065 & -.082 & .337 & .018 & .829 & .175 & $.039 * *$ & -.102 & .234 \\
\hline PA & -.174 & $.041^{* *}$ & -.121 & .155 & -.016 & .854 & .052 & .544 & -.122 & .154 \\
\hline NA & -.170 & $.045^{* *}$ & .166 & .051 & .259 & $.002 *$ & .090 & .291 & .213 & $.012^{* *}$ \\
\hline
\end{tabular}

Table 2. Linear regression analyses (Enter) with responsibility total score as dependent variable and SD, PA, NA and Belief in an unjust world subscale score of LoC as independent variables.

\begin{tabular}{|c|c|c|c|c|c|c|}
\hline \multirow{2}{*}{\multicolumn{2}{|c|}{ Model }} & \multicolumn{2}{|c|}{$\begin{array}{c}\text { Unstandardized } \\
\text { Coefficients }\end{array}$} & \multirow{2}{*}{$\begin{array}{c}\begin{array}{c}\text { Standardized } \\
\text { Coefficients }\end{array} \\
\beta \\
\end{array}$} & \multirow[b]{2}{*}{$t$} & \multirow[b]{2}{*}{$p$} \\
\hline & & B & SE & & & \\
\hline \multirow[t]{3}{*}{ Step 1} & SD & 1.156 & .173 & .475 & 6.674 & .000 \\
\hline & PA & .160 & .046 & .246 & 3.488 & .001 \\
\hline & NA & -.063 & .040 & -.110 & -1.545 & .125 \\
\hline \multirow[t]{4}{*}{ Step 2} & SD & 1.132 & .172 & .465 & 6.601 & .000 \\
\hline & PA & .149 & .046 & .230 & 3.275 & .001 \\
\hline & NA & -.045 & .041 & -.080 & -1.114 & .267 \\
\hline & $\begin{array}{l}\text { Belief in an Unjust } \\
\text { World }\end{array}$ & -.094 & .045 & -.150 & -2.096 & .038 \\
\hline
\end{tabular}

\section{References}

American Psychiatric Association. (2013). Diagnostic and statistical manual of mental disorders (5th ed.). Arlington, VA: American Psychiatric Publishing.

Dağ, İ. (2002). Kontrol Odağı Ölçeği (KOÖ): Ölçek geliştirme, güvenirlik ve geçerlik çalışması. Türk Psikoloji Dergisi, 17(49), 77-90.

Dawes, R. M., Montada, L., Filipp, S. H., \& Lerner, M. J. (1992). Life crises and experiences of loss in adulthood.

Gençöz, T. (2000). Pozitif ve negative duygu ölçeği: Geçerlik ve güvenirlik çalışması. [Positive and Negative Affect Schedule: A study of validity and reliability] Turkish Journal of Psychology, 15(46), 19-26.

Koster, E. H. W., De Raedt, R., Goeleven, E., Franck, E., \& Crombez, G. (2005). Mood congruent attentional bias in dysphoria: Maintained attention to and impaired disengagement from negativeinformation. Emotion, 5, 446-455.

Kozan, K. (1983). Davranış bilimleri araştırmalarında sosyal beğenirlik boyutu ve Türkiye için bir sosyal beğenirlik ölçeği. ODTÜ Gelişme Dergisi, 10(3), 447-478.

Lazarus, R. S., \& Folkman, S. (1984). Stress, appraisal, and coping. New York: Springer Publishing Co.

Lench, H. C., \& Chang, E. S. (2007). Belief in an unjust world: When beliefs in a just world fail. Journal of Personality Assessment, 89(2), 126-135.

Rotter, J. B. (1966). Generalized expectancies for internal versus external control of reinforcement. Psychological monographs: General and applied, 80(1), 1.

Stöber, J. (2001). The Social Desirability Scale-17 (SDS-17): Convergent validity, discriminant validity, and relationship with age. European Journal of Psychological Assessment, 17(3), 222.

Tedeschi, R. G., \& Calhoun, L. G. (1995). Trauma \& transformation: Growing in the aftermath of suffering. Thousand Oaks, CA: Sage Publications.

Tedeschi, R. G., \& Calhoun, L. (2004). Posttraumatic growth: Conceptual foundations and empirical evidence. Psychological Inquiry, 15, 1-18. 
Topcu, M. (2016). The Concept of Gratitude and its Relationship with Posttraumatic Growth: Roles of Early Maladaptive Schemata and Schema Coping Styles, Locus of Control and Responsibility (Unpublished Doctoral dissertation). Retrieved from http://etd.lib.metu.edu.tr/upload/12619691/index.pdf

Topcu, M., Köroğlu, D. \& Gençöz, F. (2018) The Role of Schemata in Responsibility among People with Traumatic Event History. In Inpact: Psychological Applications and Trends, (p. 68-72), Porto, Portugal.

Topcu, M., Öge, B. \& Gençöz, F. (2018). Psychometric properties of the Responsibility Scale, Ankara University the Journal of the Faculty of Languages and History-Geography, 58(1), 1056-1078.

Watson, D., Clark, L. A., \& Tellegen, A. (1988). Development and validation of brief measures of positive and negative affect: the PANAS scales. Journal of personality and social psychology, 54(6), 1063. 


\title{
WHY ARE SOME PEOPLE OPTIMISTIC WHILE OTHERS ARE NOT?
}

\author{
Lilly E. Both \\ Department of Psychology, University of New Brunswick (Canada)
}

\begin{abstract}
The purpose of this study was to examine factors that predict optimism and pessimism. Optimism, or positive bias, is the belief that undesirable events are more likely to happen to other people, than to oneself. Pessimism, on the other hand, is the belief that negative life events are more likely to happen to oneself. Although pessimism and optimism are inversely related, they are not opposite ends of the same continuum and should be measured separately. In this study, both dispositional traits (personality) and situational influences (coping styles) were examined in relation to optimism and pessimism. The sample consisted of 178 individuals $(\mathrm{M}$ age $=23.00 ; \mathrm{SD}=6.27$; range $=19-50$ years; $79 \%$ women $)$ who completed an online survey. Participants completed the BFI-2 to assess personality, the Ways of Coping Scale to determine coping styles, and the Future Events Scales to measure optimism and pessimism. The results found a moderate negative correlation between optimism and pessimism, suggesting that although these constructs are related, they are still distinct. A hierarchical multiple regression analysis was conducted using optimism as the criterion variable. The overall model was statistically significant and accounted for $42 \%$ of the variance in optimism scores. Significant predictors were lower scores on negative emotionality (neuroticism), and higher scores on extraversion, agreeableness and conscientiousness. As well, problem-focused coping made a unique contribution. Thus, optimists are emotionally stable individuals who are outgoing and sociable, easy to get along with, and responsible. They also are more likely to cope with a stressor by dealing directly with it. A second hierarchical multiple regression analysis was conducted using pessimism as the criterion variable, and again, the overall model was statistically significant, with $36 \%$ of the variance accounted for. However, a different pattern emerged with respect to the predictors. In this case, pessimism was predicted by age (being older), gender (being female), and higher negative emotionality (neuroticism) scores. Also, higher scores on emotion-focused coping contributed to the model. Pessimists, therefore, tend to be older and have more life experiences under their belts. They also tend to be women who are more anxious and depressed, and tend to put off dealing with stressors, which may not diffuse the situation. Taken together, these results suggest that our perceptions - whether we have a positive or negative bias - are influenced by both dispositional factors (like personality) and situation influences (like coping).
\end{abstract}

Keywords: Personality, coping, optimism, pessimism, positive bias.

\section{Introduction}

The optimistic bias occurs when an individual believes an undesirable event is more likely to happen to someone else than to oneself (Shepperd, Waters, Weinstein, \& Klein, 2015). Past research has focused on documenting the events for which the optimistic bias occurs, such as health risks like a fatal heart attack (Radcliffe \& Klein, 2002), or addiction to cigarette smoking and alcohol (Masiero, Riva, Oliveri, Fioretti \& Pravettoni, 2018), as well as environmental disasters such as hurricanes (Trumbo, Meyer, Marlatt, Peek \& Morrisey, 2014), and even chance events (Weinstein, 1980). Other studies have focused on the cognitive and motivational reasons for the distortion (Weinstein, 1980), as well as the consequences (both harmful and beneficial) of having a positive bias (Shepperd, Pogge, \& Howell, 2017).

Optimists tend to be more resilient (Davis \& Asliturk, 2011) and report using active coping in stressful situations (Carver et al., 1993). Pessimists, who believe negative life events are more likely to happen to themselves than to others, report using more escape strategies (Carver et al., 1993). Thus coping mechanisms play a role in the perceived risk of positive and negative life events. These strategies are considered a situational influence because they are learned and are amenable to change. 
Despite the large database on the pervasiveness of the optimistic bias, few studies to date have focused on dispositional influences such as personality. In one study, Borkenau and Mauer (2006) found personality influenced risk estimates. However, the authors only examined neuroticism and extraversion in their model of positive and negative emotionality. Personality is generally examined using the five factor model, namely the traits of neuroticism, extraversion, openness, conscientiousness and agreeableness (Costa \& McCrae, 1992). This study extended the literature by examining all five personality factors and coping styles in relation to the optimistic and pessimistic bias.

\subsection{Purpose of the present study}

The purpose of this study was to examine factors that predict optimism and pessimism. Both dispositional factors (personality) and situational influences (coping styles) were assessed.

\section{Method}

\subsection{Participants}

The sample consisted of 178 individuals ( $M$ age $=23.00 ; S D=6.27$; range $=19-50$ years; $79 \%$ women) who completed an online survey. Although the survey was open to members from the general public, the vast majority of them were university students who were informed of the study through SONA, an online recruiting tool. The majority of participants were single (85\% single; $12 \%$ married or common law; 3\% divorced) and Caucasian (86\% White or Caucasian, 7\% Asian, 2\% Black or African American; 5\% Other). University students could earn one bonus point towards their final grade for participating in this research. As well, all participants had the opportunity to be entered into a draw for a \$50 Amazon gift card (i.e., they sent an email at the completion of the study that was separate, and not linked to their data).

\subsection{Measures}

Demographic Questionnaire. This brief measure asked participants to report their age, gender, marital status, race/ethnicity, and education level.

The Big Five Inventory - 2 (BFI-2; Soto \& John, 2017). This measure consists of 60 items (some reverse coded) that assess personality factors commonly known as the Big Five - extraversion, agreeableness, conscientiousness, negative emotionality (neuroticism), and open-mindedness. Participants indicate the extent to which they agree or disagree with each statement on a 5 point scale where $1=$ disagree strongly and 5 = agree strongly. This inventory is used widely in personality research, due to its established reliability and validity (Soto and John, 2017). In the present study, the subscale or factor scores had excellent reliability (Cronbach's a = .87 extraversion; .80 agreeableness; .79 conscientiousness; .91 negative emotionality; and .76 open-mindedness).

The Ways of Coping Checklist (Vitaliano, Russo, Carr, Maiuro, \& Becker, 1985). This scale is a 42 item self-report measure that asks participants to assess their coping strategies in stressful situations. Participants rate the degree (from $0=$ not used to $3=$ used a great deal) to which they used certain strategies such as "blamed yourself" or "talked to someone who could do something about the problem." Three subscale scores are computed that assess problem-focused coping (15 items), emotion-focused coping (21 items), and seeking support (6 items). In the present study, only the problem- and emotion-focused subscales were utilized. This scale has good reliability and validity scores (see Vitaliano et al., 1985 for details). In the present study, Cronbach's a = .87 for problem-focused coping, and .90 for emotion-focused coping.

The Future Events Scale (Wichman, Reich, \& Weary, 2006). This scale consists of 23 items (the original scale had 26 items but new factor structure has dropped 3 items; see Wichman et al., 2006). Participants indicate the likelihood (on a scale from -5 extremely unlikely to +5 extremely likely) that certain events (such as "to have a loved one die in the next year") will happen to them. Two subscale scores were computed - one for optimism (Cronbach's $a=.88$ ) and one for pessimism (Cronbach's a = .81).

\subsection{Procedure}

All participants were directed to Qualtrics, an online survey platform. Participants read a consent form describing the nature of the study, and indicated whether they wished to participate by either clicking on the consent button or exiting the survey. Once inside the survey, the demographic measure was always presented first, followed by the remaining measures in random order. The survey took approximately 20 minutes to complete. 


\section{Results}

\subsection{Gender differences}

Independent samples $t$-tests were conducted to determine if there were any gender differences. Women scored higher than men on negative emotionality (neuroticism; $M$ women $=3.25, S D=.87$; $M$ men $=2.41, S D=.60 ; t=-6.56, p<.001)$, on emotion-focused coping $(M$ women $=2.50, S D=.59$; $M$ men $=2.21, S D=.40 ; t=-3.32, p=.001)$ and on pessimism $(M$ women $=5.55, S D=1.65$; $M$ men $=4.77, S D=1.62 ; t=-2.46, p=.015)$.

\subsection{Correlations}

The bivariate correlations are presented in Table 1. Age was correlated with open-mindedness, agreeableness, and pessimism (older adults scored higher on these measures). Negative emotionality (neuroticism) was correlated positively with emotion-focused coping and pessimism, and was negatively correlated with problem-focused coping and optimism. The remaining personality factors, by and large, showed the opposite pattern in that they correlated positively with problem-focused coping and optimism, and correlated negatively with emotion-focused coping and pessimism. There was no statistically significant correlation between problem-focused and emotion-focused coping. However, problem-focused coping was correlated positively with optimism, whereas emotion-focused coping correlated negatively with optimism and positively with pessimism. Finally optimism and pessimism were inversely and only moderately correlated.

Table 1. Bivariate Correlations with Age, Personality Factors, Coping and Optimism.

\begin{tabular}{|c|c|c|c|c|c|c|c|c|c|}
\hline & $\mathrm{N}$ & $\mathrm{E}$ & $\mathrm{O}$ & A & $\mathrm{C}$ & PFC & EFC & OPT & PES \\
\hline Age & -.04 & .04 & $.19 *$ & $.18 *$ & .05 & .11 & -.10 & -.04 & $.15^{*}$ \\
\hline $\mathrm{N}$ & & $-.42 * * *$ & -.01 & $-.33 * * *$ & $-.26 * * *$ & $-.27 * * *$ & $.52 * * *$ & $-.40 * * *$ & $.50 * * *$ \\
\hline $\mathrm{E}$ & & & $.22 * *$ & .15 & $.24 * *$ & $.22 * *$ & $-.27 * * *$ & $.47 * * *$ & $-.32 * * *$ \\
\hline $\mathrm{O}$ & & & & $.21 * *$ & $.20 * *$ & $.22 * *$ & $-.16 *$ & $.21 * *$ & -.07 \\
\hline A & & & & & $.43 * * *$ & $.19 *$ & $-.30 * * *$ & $.33 * * *$ & $-.26 * * *$ \\
\hline $\mathrm{C}$ & & & & & & $.35 * * *$ & $-.30 * * *$ & $.38 * * *$ & $-.23 * *$ \\
\hline PFC & & & & & & & -.04 & $.43 * * *$ & -.11 \\
\hline EFC & & & & & & & & $-.28 * * *$ & $.44 * * *$ \\
\hline OPT & & & & & & & & & $-.40 * * *$ \\
\hline
\end{tabular}

$\mathrm{N}$ is Negative Emotionality, E is Extraversion, $\mathrm{O}$ is Open-Mindedness, A is Agreeableness, C is Conscientiousness, PFC is problem-focused coping, EFC is emotion-focused coping, OPT is optimism, PES is pessimism

\subsection{Hierarchical regression analyses}

A hierarchical regression analysis was conducted to determine whether personality and coping strategies predicted optimism. Age and gender were entered on the first step to control for their effects. On the second step, the five personality factors were added. Finally, on the third step, the two coping strategies were added. Tolerance and VIF (variance inflation factor) were all within acceptable levels for the analysis. The overall model was statistically significant and accounted for $42 \%$ of the variance $(F(9,165)=13.34, p<.001$, multiple $R=.65)$. Age and gender were not statistically significant predictors $\left(F(2,172)=1.47, p=.23, R^{2}=.02\right)$. The five personality factors were entered on the second step and produced a statistically significant change in the model $\left(R^{2}\right.$ change $\left.=.34, F_{\text {inc }}(5,167)=17.48, p<.001\right)$. Significant predictors were Negative Emotionality (neuroticism) $\left(\beta=-.17, t=-2.17, p=.03, s r^{2}=.02\right)$, Extraversion $\left(\beta=.31, t=4.29, p<.001, s r^{2}=.07\right)$, Agreeableness $\left(\beta=.15, t=2.08, p=.04, s r^{2}=.02\right)$, and Conscientiousness $\left(\beta=.19, t=2.61, p=.01, s r^{2}=.03\right)$. Finally, the coping strategies were entered on the last step and produced a statistically significant change in the model $\left(R^{2}\right.$ change $=.07$, $\left.F_{\text {inc }}(2,165)=9.50, p<.001\right)$. The only significant predictor at this step was problem-focused coping $\left(\beta=.29, t=4.36, p<.001, s r^{2}=.07\right)$. The adjusted $R^{2}$ value of .39 in the overall model indicates that more than a third of the variability in optimism scores was predicted by personality traits and coping, namely lower scores on negative emotionality (neuroticism), and higher scores on extraversion, agreeableness, conscientiousness, and problem-focused coping.

A second hierarchical regression analysis was conducted using pessimism as the criterion variable. Again, tolerance and VIF were within acceptable limits for the analysis. The overall model was statistically significant and accounted for $36 \%$ of the variance $(F(9,165)=10.23, p<.001$, multiple 
$R=.60)$. The first step of the model was statistically significant $\left(F(2,172)=7.42, p=.001, R^{2}=.08\right)$. Significant predictors were age $\left(\beta=.17, t=2.31, p=.02, s r^{2}=.03\right)$ and gender $(\beta=.24, t=3.25$, $\left.p=.001, s r^{2}=.06\right)$. The five personality factors were entered on the second step and produced a statistically significant change in the model $\left(R^{2}\right.$ change $\left.=.24, F_{\text {inc }}(5,167)=11.80, p<.001\right)$. The significant predictor at this stage was Negative Emotionality (neuroticism) $(\beta=.37, t=4.59$, $\left.p<.001, s r^{2}=.09\right)$. Finally, the coping strategies were entered on the last step and produced a statistically significant change in the model $\left(R^{2}\right.$ change $\left.=.04, F_{\text {inc }}(2,165)=4.93, p=.008\right)$. The only significant predictor at this step was emotion-focused coping $\left(\beta=.24, t=2.97, p=.003, s r^{2}=.04\right)$. The adjusted $R^{2}$ value of .32 in the overall model indicates that a third of the variability in pessimism scores was predicted by being older, being female, having higher negative emotionality (neuroticism) scores, and using emotion-focused coping strategies.

\section{Discussion}

Why are some people optimistic while others are not? This study examined dispositional factors (personality traits) and situational influences (coping styles) to examine this question.

In this study, optimism was predicted by personality traits and coping, namely lower scores on negative emotionality (neuroticism), and higher scores on extraversion, agreeableness, conscientiousness, and problem-focused coping. However, the largest proportion of variance was explained by the personality factors, with extraversion contributing the most unique variance. Extraverts are sociable, assertive and have high energy levels (Soto \& John, 2017). Indeed, extraverts have been described as optimistic (Costa \& McCrae, 1992).

Pessimism was predicted by age (being older), gender (being female), having higher negative emotionality (neuroticism) scores, and using emotion-focused coping. Again, the largest proportion of variance was explained by personality factors, namely negative emotionality. Negative emotionality is comprised of three subscales: anxiety, depression and emotional volatility (Soto \& John, 2017). These characteristics are associated with maladjustment and individuals high on negative emotionality tend to experience more negative affective states and do not cope well in the face of adversity (Costa \& McCrae, 1992). As well, women tend to score higher than men on neuroticism (Costa \& McCrae, 1988; Fowler \& Both, 2017) and on the use of emotion-focused coping strategies (Eaton \& Bradley, 2008). However, in this study, the gender difference should be interpreted with caution given the majority of participants were women.

Optimism and pessimism were measured separately in this study and were inversely but only moderately correlated. However, for both the optimistic and pessimistic bias, personality factors accounted for the lion's share of the variance. These results underscore the importance of assessing personality. Personality is considered a dispositional trait that is relatively stable over the adult years (Costa \& McCrae, 1988). One's personality can be viewed as a lens through which one perceives and interprets the world. Individuals who are extraverts, agreeable and conscientious experience their world differently than those who live their lives with high anxiety and depression.

Both problem-focused and emotion-focused coping strategies were assessed in this study. Interestingly, the two coping styles were not correlated, but differentially predicted the outcome variables. Problem-focused coping - dealing directly with a stressor by problem-solving solutions - predicted optimism, whereas pessimism was predicted by emotion-focused coping, such as blaming oneself or wishful thinking. Coping styles have been targeted in interventions (Powell, Wegmann, \& Shin, 2019) and respond well to therapy. Thus, in order to address the pessimistic bias, therapists should focus on influences that are amenable to change.

\section{Conclusion}

Who are optimists? They are emotionally stable individuals who are cheerful and friendly, easy to get along with, and reliable. They are also more likely to cope with a stressor by facing it directly. Pessimists, on the other hand, tend to be older individuals who, by definition, have more life experiences under their belts. Perhaps they are disillusioned by the cumulative effect of long-term, everyday frustrations. They tend to be women and are more anxious, depressed, and emotionally volatile. Pessimists also tend to put off dealing with stressors, which may not diffuse the situation. Wishing something will go away does not make it happen. The bottom line is that our perceptions - whether we have a positive or negative bias - are influenced by both dispositional factors (like personality) and situational influences (like coping). 


\section{References}

Borkenau, P., \& Mauer, N. (2006). Personality, emotionality and risk perception. Journal of Individual Differences, 27(3), 127-135.

Carver, C.S., Pozo, C., Harris, S.D., Noriega, V., Scheier, M.F., Robinson, D.S., ... Clark, K.C. (1993). How coping mediates the effect of optimism on distress: A study of women with early stage breast cancer. Journal of Personality and Social Psychology, 65(2), 375-390.

Costa, P.T., \& McCrae, R.R. (1988). Personality in adulthood: A six-year longitudinal study of self-reports and spouse ratings on the NEO Personality Inventory. Journal of Personality and Social Psychology, 54(5), 853-863.

Costa, P.T., \& McCrae, R.R. (1992). Revised NEO Personality Inventory (NEO-PI-R) and NEO Five Factor Inventory (NEO-FFI) professional manual. Odessa, FL: Psychological Assessment Resources.

Davis, C.G., \& Asliturk, E. (2011). Toward a positive psychology of coping with anticipated events. Canadian Psychology, 52(2), 101-110.

Eaton, R.J., \& Bradley, G. (2008). The role of gender and negative affectivity in stressor appraisal and coping selection. International Journal of Stress Management, 15(1), 94-115.

Fowler, S. A., \& Both, L. E. (2017). The influence of personality and copying styles on forgiveness. In C. Pracana \& M. Wang (Eds.), Psychology application \& developments III (pp. 109-120). Lisbon: inScience Press.

Masiero, M., Riva, S., Oliveri, S., Fioretti, C., \& Pravettoni, G. (2018). Optimistic bias in young adults for cancer, cardiovascular and respiratory diseases: A pilot study on smokers and drinkers. Journal of Health Psychology, 23(5), 645-656.

Powell, T. M., Wegmann, K. M., \& Shin, O. J. (2019, February 7). Stress and coping in social service providers after superstorm Sandy: An examination of a postdisaster psychoeducational intervention. Traumatology. Advance online publication. http://dx.doi.org/10.1037/trm0000189

Radcliffe, N.M., \& Klein, W.M.P. (2002). Dispositional, unrealistic, and comparative optimism: Differential relations with the knowledge and processing of risk information and beliefs about personal risk. $P S P B, 28(6), 836-846$.

Shepperd, J. A., Pogge, G., Howell, J.L. (2017). Assessing the consequences of unrealistic optimism: Challenges and recommendations. Consciousness and Cognition, 50, 69-78.

Shepperd, J.A., Waters, E.A., Weinstein, N.D., \& Klein W.M.P. (2015). A primer on unrealistic optimism. Current Directions in Psychological Science, 24(3), 232-237.

Soto, C.J., \& John, O.P. (2017). The next Big Five Inventory (BFI-2): Developing and assessing a hierarchical model with 15 facets to enhance bandwidth, fidelity, and predictive power. Journal of Personality and Social Psychology, 113(1), 117-143.

Trumbo, C., Meyer, M.A., Marlatt, H., Peek, L., \& Morrisey, B. (2014). An assessment of change in risk perception and optimistic bias for hurricanes among gulf coast residents. Risk Analysis, 34(6), 1013-1024.

Vitaliano, P.P., Russo, J., Carr, J.E., Maiuro, R.D., \& Becker, J. (1985). The Ways of Coping Checklist: Revision and psychometric properties. Multivariate Behavioral Research, 20(1), 3-26.

Weinstein, N.D. (1980). Unrealistic optimism about future life events. Journal of Personality and Social Psychology, 39(5), 806-820.

Wichman, A.L., Reich, D.A., \& Weary, G. (2006). Perceived likelihood as a measure of optimism and pessimism: Support for the Future Events Scale. Psychological Assessment, 18, 215-219. doi: 10.1037/1040-3590.18.2.215 


\title{
E-MAIL COUNSELING FOR THE ULTRA-ORTHODOX COMMUNITY
}

\author{
Osnat Rubin \\ Counseling and Human Development, University of Haifa (Israel)
}

\begin{abstract}
This study examines a unique project of its kind among the ultra-Orthodox community, in which ultra-Orthodox M.A. students consult via e-mail to members of the community. The procedure is anonymous: the client's personal email details are stored in the system, students are given the content of the request without the identifying information; and they write a reply, which is transmitted through the system to the client, within a week at the most. Counseling model includes one answer via e-mail, with no further interaction.

The advantages of using e-mail as a medium for counseling include: therapeutic aspects of writing, the ability to maintain anonymity on the part of the client, asynchronous that allows the consultant to plan, design, correct, re-examine and save the text, as well as the independence of time and place for consultation process.

Psychotherapy or mental health care in the ultra-Orthodox sector involves shame, reluctance and resistance. An ultra-Orthodox applying psychological help within this enclave community, risks a negative label, both regarding himself and his family. Thus, the anonymity that characterizes the online counseling may be a solution and may have an additional value as first-aid for those who avoid turning to face-to-face help.

The present study analyzed a total of 60 cases of e-consultation within this project. Five stages were identified in the process, as well as unique aspects of e-counseling in the ultra-Orthodox sector. These findings will be discussed in light of counseling literature and methods of discourse analysis.
\end{abstract}

Keywords: E-counseling, ultra-orthodox, credibility, faith.

\section{References}

Rubin, O. (2017). "Good advice": e-mail consultation to the ultra-Orthodox sector. Efshar: Social Work Journal, 28, 39-42. [Hebrew]. 


\title{
“I THINK HAVING ASPERGER'S HELPED ME WITH MY TRANSGENDER IDENTITY": THE LIVED EXPERIENCES OF YOUTH WITH GENDER DIVERSE IDENTITIES AND AUTISM SPECTRUM DISORDERS
}

\author{
Wallace Wong ${ }^{1}$, Jaime Semchuk ${ }^{2}$, \& Veronique Nguy $^{2}$ \\ ${ }^{1}$ Diversity Emotional Wellness Centre (Canada) \\ ${ }^{2}$ University of British Columbia (Canada)
}

\begin{abstract}
With a growing body of research suggesting the co-occurrence of autism spectrum disorders (ASD) and gender variance (GV), only a handful of published studies have investigated the perspectives and experiences of GV youth with ASD. Current clinical care guidelines for this population have generally been obtained through expert knowledge and fail to consider the contribution and perspectives of key stakeholders with an insider perspective such as youth and their caregivers. As such, a semi-structured focus-group and interview were conducted to explore the experiences and perspectives of four GV youth with ASD. Interviews were transcribed and analyzed for themes. The key themes identified were: 1 ) a clear understanding of their gender identity and related needs 2) significant perceived stigma related to their ASD diagnosis, 3) recognition of how ASD identity and gender identity intersect and impact one another, 4) complexities of medical systems can be challenging to navigate for youth with ASD, so a comprehensive team approach to services is important. This study seeks to increase our understanding of this population and contribute to improving the quality of clinical services for this transgender youth with ASD.
\end{abstract}

Keywords: Gender variance, autism spectrum disorders, gender identity, access to services, youth perspectives.

\section{Introduction}

Accumulating research indicates that children and adolescents with co-occurring autism spectrum disorders (ASD) and gender variance (GV) are identified at higher rates than would be expected by chance (de Vries, Noens, Cohen-Kettenis, van Berckelaer-Onnes, \& Dorelihers, 2010; Skagerberg, Di Ceglie, \& Carmichael, 2015; Janssen, Huang, \& Duncan, 2016; Shumer, Reisner, Edwards-Leeper, \& Tishelman, 2016; van der Miesen, Hurley, Bal, \& de Vries, 2018). Current available studies looking at rates of clinical ASD diagnosis in gender-referred youth suggest a co-occurence rate of 6.3 to 13.3\% (de Vries et al., 2010; Holt, Skagerberg, \& Dunsford, 2016; Nahata, Quinn, Caltabellotta, \& Tischelman, 2017; Shumer et al., 2016).

The clinical assessment and treatment of gender-variant children and youth with autism can prove complex, due to the developmental aspects of ASD (de Vries et al., 2010; Strang, et al., 2018). Recently, clinical care guidelines for this population were developed by obtaining consensus among professionals with expertise in the field (Strang, et al., 2018). However, these guidelines lacked the contribution of the expertise afforded by those with lived experience. To our knowledge, there are only a handful of studies to date that addresses the clinical needs, perspectives, and experiences of gender variant-youth with autism (Strang et al., 2018; Strang et al., 2018). In order to better understand and provide effective clinical services for this population, it is important to learn about the perspectives of key stakeholders with an insider perspective (Strang et al., 2018; Strang et al., 2018). The present study seeks to contribute to this body of research by asking the following question: What are the experiences of GV youth with ASD related to understanding their identities and accessing clinical services? 


\section{Participants and methods}

Table 1. Participant Demographics.

$\begin{array}{cccc}\begin{array}{c}\text { Participan } \\ \text { t }\end{array} & \text { Age } & \begin{array}{c}\text { Gender } \\ \text { Identity }\end{array} & \text { Diagnosis } \\ \begin{array}{c}\text { Participant } \\ 1\end{array} & 13 & \begin{array}{c}\text { Transgender } \\ \text { Female }\end{array} & \begin{array}{c}\text { Pervasive Developmental Disorder-Not } \\ \text { Otherwise Specified }\end{array} \\ \begin{array}{c}\text { Participant } \\ 2\end{array} & 16 & \begin{array}{c}\text { Transgender } \\ \text { Male }\end{array} & \text { Autism Spectrum Disorder } \\ \begin{array}{c}\text { Participant } \\ 3\end{array} & 17 & \begin{array}{c}\text { Transgender } \\ \text { Female }\end{array} & \text { Asperger's Syndrome } \\ \text { Participant } & 19 & \text { Transgender } & \\ 4 & & \text { Male } & \text { Asperger's Syndrome }\end{array}$

Four participants were recruited from a community-based mental health services clinic in the Lower Mainland of British Columbia, Canada. All participants identified as gender variant and had a diagnosed Autism Spectrum Disorder. Informed consent was obtained from each participant and their legal guardian to take part in the study. This study was conducted in March 2018 using a qualitative semi-structured interview format. An initial focus group was conducted with three of the participants, and a separate interview was conducted individually with another youth who also wished to participate, but was unavailable during the time of the initial focus group. All interviews were audio recorded, transcribed, and analyzed for emergent themes using qualitative thematic analysis (Braun \& Clarke, 2006).

\section{Results}

Figure 1. Organization of Themes.

\begin{tabular}{|c|c|c|c|c|}
\hline Broad Themes & $\begin{array}{c}\text { Understanding Gender } \\
\text { Identity (GI) }\end{array}$ & $\begin{array}{c}\text { Understanding } \\
\text { Autism Spectrum } \\
\text { Disorders (ASD) }\end{array}$ & $\begin{array}{c}\text { Intersection of } \\
\text { ASD and GI }\end{array}$ & $\begin{array}{c}\text { Navigating } \\
\text { Systems }\end{array}$ \\
\hline Sub-themes & Self-Awareness & \begin{tabular}{c} 
ASD Stigma and \\
Stereotypes \\
\cline { 2 - 5 }
\end{tabular} & $\begin{array}{c}\text { Invalidation of GI } \\
\text { due to ASD } \\
\text { Diagnosis }\end{array}$ & $\begin{array}{c}\text { Additional } \\
\text { Challenges }\end{array}$ \\
\hline
\end{tabular}

\subsection{Understanding gender identity}

Participants discussed their self-awareness of their gender identity and indicated their transgender identity was something that felt innate to them. For instance, one participant stated, "It is something I'm born with. It wasn't something I chose to be...I can't force myself not to be transgender. Either you are or you're not. That's the best I can describe it." Participants also identified challenges describing their gender identity to others. The fluid nature of gender made it difficult for participants to communicate with others about their transgender identity. As one participant described, "Because I take things so literal - gender can sometimes be very fluid and vague, the definition, so it's kind of hard to tell people how you feel."

\subsection{Understanding autism spectrum disorder}

During interviews, participants highlighted the perceived stigma and stereotypes others attribute to ASD. One participant shared, "People think I'm just great with anything with science and math. And anything to do with socializing, I'm bad at. Like two extremes." Relatedly, another participant stated, "Living with autism, it sucks. To be honest, just because of the fact you're different from other people, you 
receive doubts from other people because they think you're not mentally capable of comprehending things." Due to perceived negative stereotypes, participants reported they were unlikely to disclose their ASD diagnosis to others. According to one participant, "No, I just mostly ignore it. I don't talk about being autistic at all."

\subsection{Intersection of ASD and gender identity}

Participants reported experiencing invalidation of their transgender identity due to their ASD diagnosis, including having others disregard their gender identity, or expressing disbelief. A participant shared, "Because people always assume because someone has a disorder like autism or something, they don't understand anything...just people trying to tell you how you are feeling, just assuming." Participants also identified the benefits of having both ASD and a transgender identity. For instance, participants perceived that living in their affirmed gender helped facilitate an increase in social interactions, addressing a deficit associated with ASD. Further, another participant indicated that symptoms of ASD helped them when facing mental health challenges related to their transgender identity, as illustrated in the following excerpt, "I think having Asperger's helped me with my transgender identity, especially during times you get depressed...I know having Asperger's made me stick with my schedule...it helped keep me going."

\subsection{Navigating systems}

Finally, participants identified additional challenges navigating systems when accessing clinical services related to their ASD and transgender identity. Specifically, communicating with professionals to access services, relying on others for help, and misunderstanding information all posed challenges for participants. A participant shared, "To be transgender you have to make a lot of phone calls with people you've never heard of... and having autism can be difficult. You have to rely on other people to help." However, participants also experienced helpful supports when accessing services. These supports included having parents who validate their child's gender identity and are willing to attend appointments with their children. One participant highlighted, "My mom was always with me at appointments because sometimes I wouldn't understand the doctor. She's known me a lot longer and she would know how to explain it to me." Other helpful supports included accessing services from professionals who were willing to simplify information and provide extra time during appointments. A participant suggested, "Definitely try to outline steps more clearly...try talking more slowly."

\section{Discussion}

In this study, all participants were aware of their gender identity, but found it challenging to explain their identity to others. Youth with ASD commonly experience challenges with social communication (American Psychiatric Association, 2013). As a result, some caregivers or professionals may underestimate their ability to understand their gender identity. Additionally, others may question the authenticity of a transgender identity for a youth with ASD. Due to perceived negative stereotypes, some trans-youth with ASD reported that they were unlikely to disclose their ASD diagnosis to others, fearing that other people may not believe or challenge the authenticity of their gender identity. However, when a transgender youth with ASD chooses to withhold information, at times, it may limit services and impede the opportunity for them to receive specialized services and appropriate care. Finally, participants found it challenging to navigate our medical system and access related clinical services. They found comfort in having a supportive adult by their side during appointments. Medical and mental health professionals may also lack training and understanding of how to work with this specific population, which has the potential to affect the quality of services.

\subsection{Limitations}

In this study, participants consisted of four transgender youth with ASD accessing publicly funded mental health and medical services. They all had a basic level of family support and were under the age of twenty. Thus, the generalizability of our results to other transgender youth who are not accessing healthcare services, supported by family or older than nineteen years old is unknown. The sample size of this study is relatively small. A larger sample size may have produced different results. Lastly, having the psychologist as a focus group facilitator may have biased the responses of some participants who were also under his care.

\subsection{Conclusion}

In our study, participants had a clear understanding of their gender identity and related needs. Participants' greatest challenges were caregivers', professionals', and the public's' lack of understanding of their intersecting identities related to their transgender identity and ASD diagnosis, as well as difficulties 
associated with navigating and accessing related services. There is very limited research focused on this population and professionals may lack appropriate training and guidelines when providing services. Although this study has its limitations, we believe that the results from this study can inform healthcare and clinical professionals of specific considerations when providing services for transgender youth with ASD. For a transgender youth diagnosed with ASD, learning how to navigate the medical and mental health systems is not easy. For this reason, increasing public awareness and providing a wraparound, step-by-step approach to services may prove helpful for this population. The findings from this study may provide some direction for future research.

\section{References}

American Psychiatric Association, American Psychiatric Association. DSM-5 Task Force, \& PsychiatryOnline

Premium Package. (2013). Diagnostic and statistical manual of mental disorders: DSM-5 (5th ed.). Washington, D.C: American Psychiatric Association.

Braun, V., \& Clarke, V. (2006). Using thematic analysis in psychology. Qualitative research in psychology, 3(2), 77-101.

De Vries, A. L., Noens, I. L., Cohen-Kettenis, P. T., van Berckelaer-Onnes, I. A., \& Doreleijers, T. A. (2010).

Autism spectrum disorders in gender dysphoric children and adolescents. Journal of autism and developmental disorders, 40(8), 930-936.

Holt, V., Skagerberg, E., \& Dunsford, M. (2016). Young people with features of gender dysphoria: Demographics and associated difficulties. Clinical Child Psychology and Psychiatry, 21(1), 108-118.

Janssen, A., Huang, H., \& Duncan, C. (2016). Gender variance among youth with autism spectrum disorders: A retrospective chart review. Transgender Health, 1(1), 63-68.

Nahata, L., Quinn, G. P., Caltabellotta, N. M., \& Tischelman, A. C. (2017). Mental health concerns and insurance denials among transgender adolescents. LGBT Health, 4(3), 188- 193

Shumer, D. E., Reisner, S. L., Edwards-Leeper, L., \& Tishelman, A. (2016). Evaluation of Asperger syndrome in youth presenting to a gender dysphoria clinic. LGBT Health, 3(5), 387-390.

Skagerberg, E., Di Ceglie, D., \& Carmichael, P. (2015). Brief report: Autistic features in children and adolescents with gender dysphoria. Journal of autism and developmental disorders, 45(8), 2628-2632.

Strang, J. F., Meagher, H., Kenworthy, L., de Vries, A. L. C., Menvielle, E., Leibowitz, S., ... Anthony, L. G. (2018). Initial clinical guidelines for co-occurring autism spectrum disorder and gender dysphoria or incongruence in adolescents. Journal of Clinical Child and Adolescent Psychology, 47(1), 105-115.

Strang, J. F., Powers, M. D., Knauss, M., Sibarium, E., Leibowitz, S. F., Kenworthy, L., ... \& Pervez, N. (2018). "They thought it was an obsession": Trajectories and Perspectives of Autistic Transgender and Gender-Diverse Adolescents. Journal of autism and developmental disorders, 48(12), 4039-4055.

van der Miesen, A. I., Hurley, H., Bal, A. M., \& de Vries, A. L. (2018). Prevalence of the Wish to be of the Opposite Gender in Adolescents and Adults with Autism Spectrum Disorder. Archives of sexual behavior, 47(8), 2307-2317. 


\title{
ACTING WITH AWARENESS PREDICTS NEGATIVE BUT NOT POSITIVE AFFECT IN A SAMPLE OF ADDICTS
}

\author{
Tânia Caetano, Eduardo Ramadas, \& Jessica Lopes \\ Department or Research and Development, VillaRamadas International Treatment Centre (Portugal)
}

\begin{abstract}
Objective: Mindfulness has been associated with not only lower levels of psychopathology but also higher levels of positive psychological characteristics. Research has found that its impact is especially relevant in disorders related to emotion dysregulation, such as addiction. Mindfulness can be a valuable resource in addiction treatment and it is important to understand how the different dimensions may influence relevant therapeutic variables.

Our objective for this exploratory study was to not only explore the impact of the different mindfulness dimensions on symptoms of depressive, anxiety and stress, but also on the prevalence of positive and negative affect on the patients. Our hypothesis was that various dimensions of mindfulness would be significant predictors of both psychopathological symptoms, but also positive affect in treatment.

Method: The present study has a cross-sectional design, and was conducted as an exploratory analysis of the first results of a wider quasi-experimental study. Current results relate to 17 patients receiving treatment in VillaRamadas treatment center for addiction. The evaluation protocol was administrated before any mindfulness training was given. From the original and more extensive evaluation protocol, the relevant psychological measures for this study were the Kentucky Inventory of Mindfulness Skills (KIMS), the Depression, Anxiety and Stress Scale, 21 items (DASS-21), and the Positive Affect and Negative Affect Scale (PANAS).

Results: From 17 participants, 13 were male. Age varied from 19 to 64 years $(M=33, S D=10.13)$.

Age presented moderate negative associations with stress and negative affect. Years of education had a moderate positive association with the describing dimension of mindfulness. Acting with awareness presented strong negative associations with depression, anxiety and negative affect, and a very strong negative association with stress. Depression, anxiety and stress showed strong positive associations with negative affect, but only depression presented a strong negative association with positive affect.

The mindfulness dimension of acting with awareness, was a significant predictor of depression (35\%), anxiety (42.9\%), stress (65.7\%) and PANAS' negative affect (52.1\%). It was not a significant predictor of positive affect.

Discussion: The only dimension of mindfulness significantly associated with the measures of psychopathology and negative affect, was acting with awareness. Contrary to our original hypothesis, even this dimension did not present a significant association with positive affect. Being an exploratory study, more research needs to be conducted to validate and better understand these results.
\end{abstract}

Keywords: Mindfulness, acting with awareness, addiction, negative affect, positive affect.

\section{Introduction}

The interest in mindfulness has grown among psychology researchers and practitioners alike during the past two decades. Although a hard concept to describe due to its complex and rather foreigner cultural background, mindfulness or being mindful has been described as paying attention to the present moment in a purposeful and nonjudgmental way, with the goal of developing greater awareness, clarity and acceptance of the present reality (Kabat-Zinn, 1994).

Although some authors attempted to operationalize mindfulness as a single faceted construct, mainly through the development of unidimensional psychometric instruments (Buchheld, Grossman, \& Walach, 2001; Brown \& Ryan, 2003; Kumar, Feldman, \& Hayes, 2008), conceptualizing it as a both a multifaceted trait and set of different but related skills, allows for a more nuanced study of the construct and its relationship with other relevant psychological concepts such as affect. For the purpose of this study, a multifaceted operationalization was used with the Kentucky Inventory of Mindfulness Skills 
(KIMS; Baer, Smith, \& Allen, 2004), that measures four dimensions hat can also be conceptualized as skills to be trained. The considered dimensions are Observing (observing, noticing various stimuli such as internal or external phenomena), Describing (describing by applying words in a nonjudgmental way), Acting with awareness (being fully engaged in each activity), and Accepting without judgment (accept reality as it is without judging, avoiding or escaping it).

It has been an accepted reality that subjective well-being is not a unitary construct, but instead composed of two independent dimensions: positive affect and negative affect (Bradburn, 1969; Diener \& Emmons, 1985).

Positive affect has been shown to promote a range of psychological and physical health outcomes and some studies have found it more predictive of resilience, physical health and life satisfaction than the absence of negative affect (e.g. Cohen, Alper, Doyle, Treanor, \& Turner, 2006; Cohn, Fredrickson, Brown, Mikels, \& Conway, 2009). Because of their independence, the reduction of negative affect does not lead directly to the promotion of positive affect (Watson \& Clark, 1997), underlining the importance of understanding how different psychological constructs and subsequent therapies influence both the reduction of negative affect and the promotion of positive affect.

Mindfulness has been positively associated with various positive psychological constructs such as life satisfaction (Brown \& Ryan, 2003), self-esteem (Brown \& Ryan, 2003; Rasmussen \& Pidgeon, 2011) and positive affect (Brown \& Ryan, 2003), and negatively associated with negative affect such as depression (Cash \& Whittingham, 2010), neuroticism (Giluk, 2009) and general psychopathological symptoms (Baer et al., 2006).

However, the independence of positive and negative affect has been of some note in previous studies looking into the impact of mindfulness meditation and mindfulness-based interventions. A meta-analysis conducted by Sedlmeier et al. (2012), found that for nonclinical samples, meditation had particularly strong effect on negative affect. Another more recent study from Menezes and Bizarro (2015), a pilot study investigating the effect of brief training of focused meditation on a nonclinical sample, found significant changes regarding negative affect and trait-anxiety, but reported no change on positive affect.

Although it is difficult to generalize these few results, specially taking into account the different conceptualizations of mindfulness, it at least raises the question of how mindfulness impacts positive affect and negative affect differentially, especially taking into consideration non-clinical and clinical samples.

In this exploratory study, we proposed ourselves to study the relationship between the four dimensions of mindfulness suggested by Baer, Smith, \& Allen (2004), and both negative emotional states such as depression, anxiety and stress, and negative and positive affect, in a clinical sample of addicts.

\section{Methods}

\subsection{Participants and procedure}

The sample of the present exploratory study is a small sample composed of 17 Portuguese patients, all receiving treatment for an addiction disorder, at VillaRamadas International Treatment Centre throughout December of 2018 and January of 2019.

From the 17 patients, 13 were male and 4 were female, and age varied between 19 and 64 years $(M=33, S D=10.13)$. All the patients had been diagnosed at admission into treatment, either with a substance use disorder or with an impulsive disorder (behavioral addiction).

The relevant data for this study was collected as part of an experimental study looking at the impact of a 30-day program of mindfulness on top of treatment as usual in the treatment center. As part of this study, patients participated in a group session design to present the study and its goals and to allow informed consent, which was given in written from.

The evaluation protocol was administered by licensed clinical psychologists, members of the multidisciplinary staff.

\subsection{Measurements}

Kenucky Inventory of Mindfulness Skills (KIMS; Baer, Smith, \& Allen, 2004; Portuguese translation and adaptation by Carvalho, 2011), is a self-report instrument composed of 39 items scored on a 5-point Likert scale from 1 ("Never/Almost Never") to 5 ("Always"). Originally created to assess the efficacy of Dialectical Behavior Therapy, it measures the following four subscales: Observing (12 items), Describing (8 items), Acting with Awareness (10 items) and Accept without Judgement (9 items). Higher scores represent a greater report of the corresponding dimension. 
Depression, Anxiety and Stress Scale-21 (DASS-21; Lovibond \& Lovibond, 1995; Portuguese version by Pais-Ribeiro, Honrado, \& Leal, 2004), is a set of three self-report scales, each with 7 items scored on a 4-point Likert scale from 0 ("Did not apply to me at all") to 3 ("Applied to me very much or most of the time"), measuring depression, anxiety and stress. The total score of each scale can vary from 0 to 21 , with higher scores indicating higher levels of the emotional state. It is based on a dimensional conception of psychopathology, created on the assumption that differences in depression, anxiety and stress between normal and clinical population are differences of degree.

Positive Affect and Negative Affect Scale (PANAS; Watson \& Clark, 1988; Portuguese version by Galinha \& Pais-Ribeiro, 2005), is a set of two self-report scales, each one with 10 items scored on a 5-point Likert scale from 1 ("Not at all") to 5 ("Very much"), one measuring positive and another measuring negative affect. Each item consists of an emotion, positive or negative, and the participant was asked to consider the frequency with which he felt each emotion during the week prior. The total score of each scale can vary from 10 to 50, with higher scores indicating a greater report of the affect.

\subsection{Data analysis}

The IBM SPSS software package, version 23, was used to perform the necessary data analysis.

Descriptive statistics were used to explore the data and describe the sample.

Routine assumption testing was performed to better choose the appropriate statistical tests. Considering the small sample size, non-parametric alternatives were preferred.

Spearman correlation values we used as a non-parametric alternative to Pearson's correlation, with the intent of studying the relationships between the variables in study, more specifically, between the four dimensions of mindfulness considered in the present study and positive and negative affect.

Simple linear regression was used to test the possible predictive value of acting with awareness regarding the dependent variables.

\section{Results}

\subsection{Descriptive statistics}

Years of education ranged from 10 to 16 years, with a mean of 13.47 years $(S D=1.84)$.

The four mindfulness scales Observing, Describing, Acting with Awareness and Accept without Judgement, presented mean results of $37.50(S D=9.93), 27.87(S D=5.55), 32.13(S D=5.98), 26.44$ $(S D=4.95)$, respectively.

Depression showed a mean result of $6.82(S D=6.17)$, anxiety of $2.65(S D=2.87)$, and stress of $8.06(S D=5.70)$. $(S D=10.95)$.

Finally, positive affect had a mean result of $29.18(S D=11.49)$, and negative affect of 21.82

\subsection{Correlation values between the studied variables (Spearman's correlation)}

Age presented a moderate positive association with years of education, and moderate negative associations with stress and negative affect. Years of education had a moderate positive association with the describing dimension of mindfulness.

Acting with awareness was the only mindfulness dimension that showed significant correlation values with variables related to emotional states and affect, presenting strong negative associations with depression, anxiety and negative affect, and a very strong negative association with stress.

Depression, anxiety and stress showed strong positive associations with negative affect, but only depression presented a strong negative association with positive affect.

The complete results are presented in Table 1. 
Table 1. Correlation values between the studied variables.

\begin{tabular}{|c|c|c|c|c|c|c|c|c|c|c|c|}
\hline & 1 & 2 & 3 & 4 & 5 & 6 & 7 & 8 & 9 & 10 & 11 \\
\hline 1. Age & - & & & & & & & & & & \\
\hline $\begin{array}{l}\text { 2. Years of } \\
\text { Education } \\
\text { KIMS }\end{array}$ & $.510^{*}$ & - & & & & & & & & & \\
\hline 3. Observing & -.136 & 322 & - & & & & & & & & \\
\hline 4. Describing & .291 & $.539^{*}$ & $.499^{*}$ & - & & & & & & & \\
\hline $\begin{array}{l}\text { 5. Act with } \\
\text { Awareness }\end{array}$ & .475 & .099 & -.141 & .159 & - & & & & & & \\
\hline $\begin{array}{l}\text { 6. Accept } \\
\text { without } \\
\text { Judgement } \\
\text { DASS-21 }\end{array}$ & .064 & -.119 & .300 & -.194 & -.026 & - & & & & & \\
\hline 7. Depression & -.264 & -.012 & -.251 & -.124 & $-.636^{* *}$ & -.403 & - & & & & \\
\hline 8. Anxiety & -.415 & -.057 & .237 & .091 & $-.681^{* *}$ & -.078 & $.744^{* *}$ & - & & & \\
\hline $\begin{array}{l}\text { 9. Stress } \\
\text { PANAS }\end{array}$ & $-.517^{*}$ & -.274 & .001 & -.170 & $-.834^{* *}$ & -.210 & $.852 * *$ & $.767^{* *}$ & - & & \\
\hline $\begin{array}{l}\text { 10. Positive } \\
\text { Affect }\end{array}$ & .291 & .123 & .445 & .321 & .223 & .470 & $-.616^{* *}$ & -.339 & -.438 & - & \\
\hline $\begin{array}{l}\text { 11. Negative } \\
\text { Affect }\end{array}$ & $-.589^{*}$ & -.215 & .071 & -.264 & $-.750^{* *}$ & -.042 & $.710^{* *}$ & $.764^{* *}$ & $.788^{* *}$ & -.387 & - \\
\hline
\end{tabular}

\subsection{Simple linear regression}

Five simple linear regression analysis were conducted, to study the predictive value of the mindfulness subscale Acting with Awareness regarding the five dependent variables (DASS-2: depression, anxiety and stress; PANAS: positive affect and negative affect).

Acting with Awareness was a significant predictor of depression $(\mathrm{F}(1,14)=9.063, \mathrm{p}=.009)$, with an adjusted $R^{2}$ of .350 , anxiety $(\mathrm{F}(1,14)=12.275, \mathrm{p}=.004)$, with an adjusted $R^{2}$ of .429 , stress $(\mathrm{F}(1,14)=29.726, \mathrm{p}<.000)$, with an adjusted $R^{2}$ of .657 , and negative affect $(\mathrm{F}(1,14)=17.306, \mathrm{p}=.001)$, with an adjusted $R^{2}$ of .521 .

Lastly, Acting with Awareness was not a significant predictor of positive affect.

\section{Discussion}

The goal of the present study was to evaluate the possible differential impact of mindfulness, taking into consideration four dimensions, on negative affect (and more specifically depression, anxiety and stress) and positive affect in a clinical sample of addicts receiving treatment.

Interestingly, from the four dimensions of mindfulness considered (observing, describing, acting with awareness and accept without judgement), only acting with awareness presented moderate to strong significant associations with the relevant variables and only with those on the negative side of the emotional spectrum. Acting with awareness did not shown any association with positive affect in our sample. The only significant association with positive affect was a moderate negative association with depression, which was an expected result considering that lack of pleasure in previously pleasurable activities is one of the main criteria for a depression diagnosis.

From the theoretical conceptualization of each of the four dimensions of mindfulness considered, it would have been expected that instead of one of the frequently dubbed "what" dimensions (observing, describing and acting with awareness), the "how" dimension (accept without judgement) would have a particularly relevant role in emotion regulation and, therefore, the decrease of negative affect. In fact, studies looking into the impact of mindfulness in patients with auditory hallucinations of distressing voices (the perfect case study of high vulnerability to negative affect) found that acceptance significantly negatively associated with depression, anxiety, stress and negative affect (PANAS-negative; e.g. Brockman, Kiernan, \& Murrell, 2014).

Acting with awareness showed itself to be a significant predictor of depression, anxiety, stress and negative affect in general in our sample.

It is crucial that these results are interpreted taking into account the vast limitations of what is only an exploratory study. In addition to its cross-sectional design, that does not allow us to establish any causal relation, the sample size, which was very small, could be affecting the results, explaining the lack of association between the other mindfulness dimensions and the other studied variables. 
Nonetheless, the results raise interesting research questions that may be better explored in future studies with a more adequate sample and the possibility to consider the moderation value of other variables such as sex.

\section{References}

Baer, R. A., Smith, G. T., Allen, K. B. (2004). Assessment of mindfulness by self-report: the Kentucky inventory of mindfulness skills. Assessment, 11(3), 191-206.

Baer, R. A., Smith, G. T., Hopkins, J., Krietemeyer, J., \& Toney, L. (2006). Using self-report assessment methods to explore facets of mindfulness. Assessment, 13(1), 27-45.

Bradburn. N. M. (1969). The structure of psychological well-being. Chicago: Aldine.

Brockman, R., Kiernan, M., \& Murrell, E. (2014). Psychometric properties of two brief versions of the voices acceptance and action scale (VAAS): implications for the second-wave and third-wave behavioural and cognitive approaches to auditory hallucinations. Clin. Psychol. Psychother. doi: 10.1002/cpp.1916.

Brown, K. W., \& Ryan, R. M. (2003). The benefits of being present: Mindfulness and its role in psychological well.being. Journal of Personality and Social Psychology, 84, 822-848.

Buchheld, N., Grossman, P., \& Walach, H. (2001). Measuring mindfulness in insight meditation (Vipassana) and meditation based psychotherapy: The development of the Freiburg Mindfulness Inventory (FMI). Journal for Meditation and Meditative Research, 1, 11-34.

Carvalho, M.P.S. (2011). Validação dos Conceitos de Atenção Plena e Evitamento Experiencial segundo a Terapia da Aceitação e Compromisso (ACT) na Explicação do Comportamento Alimentar- Indicações para o Tratamento da Obesidade. Master's Degree Dissertation, Universidade Católica Portuguesa, Braga, Portugal.

Cash, M., \& Whittingham, K. (2010). What facets of mindfulness contribute to psychological well-being and depressive, anxious, and stress-related symptomatology? Mindfulness, 1, 177-182.

Cohen, S., Alper, C. M., Doyle, W. J., Treanor, J. J., \& Turner, R. B. (2006). Positive Emotional Style Predicts Resistance to Illness After Experimental Exposure to Rhinovirus or Influenza a Virus. Psychosomatic Medicine, 68(6), 809-815.

Cohn, M. A., Fredrickson, B. L., Brown, S. L., Mikels, J. A., \& Conway, A. M. (2009). Happiness Unpacked: Positive Emotions Increase Life Satisfaction by Building Resilience. Emotion, 9(3), 361-368.

Diener, E., \& Emmons, R. A. (1985). The Independence of Positive and Negative Affect. Journal of Personality and Social Psychology, 47(5), 1105-1117.

Galinha, I. O., \& Pais-Ribeiro, J. (2005). Contribuição para o estudo da versão portuguesa da Positive and Negative Affect Schedule (PANAS): II - Estudo psicométrico. Análise Psicológica, 2(23), 219-227.

Giluk, T. L. (2009). Mindfulness, big five personality, and affect: A meta-analysis. Personality and Individual Differences, 47, 805-811.

Kabat-Zinn, J. (1994). Wherever you go, there you are: mindfulness meditation in everyday life. New York: Hyperion.

Kumar, S. M., Feldman, G. C., \& Hayes, S. C. (2008). Changes in mindfulness end emotion regulation in an exposure based cognitive therapy dor depression. Cognitive Therapy \& Research, 32, 734-744.

Lovibond, S. H., \& Lovibond, P. F. (1995). Manual for the Depression Anxiety \& Stress Scales (second edition). Psychology Foundation.

Pais-Ribeiro, J., Honrado, A., \& Leal, I. (2004). Contribuição para o estudo da adaptação portuguesa das escalas de ansiedade, depressão e stress (eads) de 21 itens de lovibond e lovibond. Psicologia, Saúde \& Doenças, 5(2), 229-239.

Rasmussen, M. K., \& Pidgeon, A. M. (2011). The direct and indirect benefits of dispositional mindfulness on self-esteem and social anxiety. Anxiety, Stress \& Coping, 24, 227-233.

Sedlmeier, P., Eberth, J., Schwarz, M., Zimmermann, D., Haarig, F., Jaeger, S., \& Kunze, S. (2012). The psychological effects of meditation: A meta-analysis. Psychological Bulletin, 138(6), 1139-1171. doi:10.1037/a0028168

Watson, D., \& Clark, L. A. (1988). Development and Validation of Brief Measures of Positive and Negative Affect: The PANAS Scales. Journal of Personality and Social Psychology, 54(6), 1063-1070.

Watson, D., \& Clark, L. A. (1997). Measurement and Mismeasurement of Mood: Recurrent and Emergent issues. Journal of Personality Assessment, 68(2), 267-296. 


\title{
RELATION BETWEEN SUBJECTIVE AND PHYSICAL WELL-BEING AND MINDFULNESS
}

\author{
Lisa A. Best, Cecile J. Proctor, Tracy A. Freeze, Derek J. Gaudet, Ryley Russell, \\ \& Rory McPhee \\ Department of Psychology, University of New Brunswick (Canada)
}

\begin{abstract}
An individual's sense of well-being involves the complex interaction of psychological and health-related quality of life. Satisfaction with Life is reported subjectively and encompasses cognitive (assessment of life circumstances) and emotional (assessment of negative emotions) factors (Tay, Kuykendall, \& Diener, 2015). Physical health is a more objective measure of overall physical and emotional functioning, social engagement, emotional well-being, energy levels, fatigue, pain, and general health perceptions (Hays \& Morales, 2001). There is increasing evidence that mindfulness is associated with psychological and physical outcomes (Ludwig \& Kabat-Zinn, 2008). Our purpose was to examine how different aspects of mindfulness (observing, describing, acting with awareness, non-judging of inner experience, and non-reactivity to inner experience) were related to physical and psychological well-being. In total, 513 non-clinical undergraduate participants completed questionnaires to measure life satisfaction, physical and psychological wellness and mindfulness. The current results highlight how personality and mindfulness affect both physical and psychological wellness. Specifically, emotional stability, extraversion, conscientiousness, and agreeableness were associated with better health outcomes and increased mindfulness. Some aspects of mindfulness (awareness and non-judging) were associated with both physical and psychological health. Given these results, we would suggest that individuals interested in improving their physical and psychological health might attend to an increased and non-judgemental focus on acting in the present moment.
\end{abstract}

Keywords: Five factor mindfulness, personality, psychological wellness, physical wellness.

\section{Introduction}

General well-being involves the complex interaction of psychological and health-related quality of life (Diener, Emmons, Larsen, \& Griffin, 1985). Physical health is an objective measure of overall physical and emotional functioning, social engagement, emotional well-being, energy levels, fatigue, pain, and general health perceptions (Hays \& Morales, 2001). In health-related research, it is common to focus on the measurement of specific mental health problems (i.e., anxiety, depression) in order to determine if psychological symptomology is co-morbid with specific medical conditions and their associated symptoms (i.e., limited mobility, pain). Satisfaction with life is reported subjectively and encompasses cognitive (assessment of life circumstances) and emotional (assessment of negative emotions) factors (Tay, Kuykendall, \& Diener, 2015). Measures of life satisfaction focus on the overall perception of one's life, with specific measures focused on the subjective experience of "happiness".

\subsection{Mindfulness}

Mindfulness is been shown to ameliorate work stress (Bostock, Crosswell, Pratha, \& Steptoe, 2018), and depression (Li \& Bressington, 2019). Marzabadi, Mills, and Valikhani (2018) reported significant differences in the levels of depression, anxiety, stress, physical and psychological health among those with low, medium, and high levels mindfulness. Further, there are significant associations between symptoms of psychological disorders (Obesssive Compulsive, Major Depression, Borderline Personality Disorder) and facets of mindfulness, indicating that those suffering from psychological disorders practice lower levels of mindfulness compared with healthy controls. Overall, research suggests that the ability to be present in the moment and aware of personal emotions without reaction and judgement has impacts across physical and psychological domains of well-being.

Research on mindfulness has suggested that there are five factors that contribute to being mindful: Observing; Describing; Acting with awareness; Non-judging of inner experience; and, Non-reactivity to inner experience (Baer et al., 2008). Observing involves attending to or taking notice of internal and external bodily experiences, including sensations, emotions, sounds, and smells. Observing involves the conscious awareness of both personal emotions (positive and negative) and environmental 
sensations (i.e., noticing how the rain sounds vs. an awareness of the fact that it is raining). Describing is being able to translate internal experiences to words (e.g., being able to differentiate between anger, frustration, and jealousy). The ability to be present in the moment is the defining quality of acting with awareness. Simply stated, acting with awareness is paying attention to a task without allowing the mind to wander. The non-judgement of inner experiences involves the ability to observe inner thoughts and feelings without placing positive or negative value onto them. The lack of judgement means that one is able to allow thoughts to come and go, without assigning a valence to them. When one avoids value labels, the avoidance of negative thoughts is unnecessary. Non-reactivity to inner experience is the ability to experience positive and negative emotion without acting in accordance with them (Baer, Smith, Hopkins, Krietemeyer, \& Toney, 2006; Baer et al., 2008).

\subsection{Personality}

The most common model of personality is the Five Factor Model, in which personality is defined by Extroversion, Agreeableness, Openness to Experience, Conscientiousness and Neuroticism (or Emotional Stability). These factors are traits that can explain and predict behavior (Costa \& McCrae, 1992). Neuroticism involves increased levels of psychological distress and unpleasant feelings and emotions. Extraversion, or sociability, is associated with higher levels of friendliness, activity, and the experience of positive emotions. Openness to experience involves intellectual curiosity, flexibility in thoughts and behaviours, and a readiness to adjust in different situations. Agreeableness involves feelings of sympathy, cooperation, and trustworthiness, with lower scores indicating distrust and pessimism. Conscientiousness is associated with an increased propensity for both organization and diligence. In adults, personality is relatively stable even during major life changes or events and affects individual coping styles (Allemand, Steiger, \& Hill, 2013). Personality factors are commonly studied in relation to well-being (Hayes \& Joseph, 2003); individuals with lower neuroticism and higher extroversion generally have higher subjective well-being (Schimmack, Radhakrishnan, Oishi, Dzokoto, \& Ahadi, 2002). Additionally, individuals who suffer from depression report higher levels of emotional instability and lower mindfulness indicating possible crossover between these factors and the relation to psychological health.

\section{Purpose of the current study}

Our overall goal was to examine how mindfulness (observing, describing, acting with awareness, non-judging of inner experience, and non-reactivity to inner experience) was related to psychological and physical health. Further, we were interested in examining if personality factors (emotional stability, extraversion, conscientiousness, agreeableness, openness to experience) were related to specific aspects of mindfulness and overall wellness.

\section{Methods}

In total, 513 undergraduate student participants volunteered to complete an online questionnaire package and received course credit for participation. Participants ranged in age from 17 to 52 years $(M$ age $=20.55, S D=5.13)$. Approximately half of the participants reported being in a romantic relationship; 65\% of these participants reported that their relationship had lasted more than a year. The questionnaire package included measures of personality (BFI-2; Soto \& John, 2017), psychological well-being (Satisfaction with Life Scale, SWLS; Diener et al., 1985), general health (RAND-36 it Health Survey; Hays \& Morales, 2001), common life stressors (Social Readjustment Rating Scale, SRRS; Holmes \& Rahe, 1967), and mindfulness (Five Factor Mindfulness Questionnaire; Baer et. al., 2006).

\section{Results}

The RAND-36 subscales range from 0 (worst possible health) to 100 (best possible health). Descriptive statistics are presented in Table 1. To test specific differences between our sample and published norms, one sample t-tests were conducted. Overall, current participants had higher scores on scales associated with overall physical health and lower scores on scales associated with emotional and social well-being. Further, participants in this sample reported a variable amount of stress, with some reporting stress at levels that could impact their physical health (Holmes \& Rahe, 1967). Although the stress reported was high, it is important to note that the SRRS includes items that indicate stress associated with positive (i.e., getting married) and negative (i.e., death of a family member) events. Given that the majority of participants likely faced many changes associated with entering adulthood, these high and variable scores were expected. To highlight further that not all stress leads to decreases in life satisfaction, it is important to note that the mean SWLS indicated average/above average life satisfaction, which are typical of individuals who live in economically developed nations. More than half of the current participants had SWLS scores that indicated high satisfaction, suggesting that they have an 
enjoyable life, in which their needs are being fulfilled (Diener, n.d.). Overall, these results are not surprising. The young adults who participated in this study reported excellent physical health but are experiencing some emotional and social struggles in response to the increased responsibilities associated with young adulthood.

Table 1. Average Physical and Psychological Well-Being of Participants.

\begin{tabular}{|c|c|c|c|c|c|}
\hline \multirow[b]{2}{*}{ Rand-36 Subscale } & \multicolumn{2}{|c|}{$\begin{array}{l}\text { Current Sample } \\
(\mathrm{N}=405)\end{array}$} & \multicolumn{2}{|c|}{$\begin{array}{c}\text { Published Norms } \\
(\mathrm{N}=2471)\end{array}$} & \multirow[b]{2}{*}{ t-score } \\
\hline & Mean & SD & Mean & SD & \\
\hline Physical Functioning & 89.92 & 17.77 & 70.61 & 27.42 & $21.84 * * *$ \\
\hline Energy/Fatigue & 42.65 & 19.96 & 52.15 & 22.39 & $-9.58 * * *$ \\
\hline Emotional Well-Being & 58.13 & 20.87 & 70.38 & 21.97 & $-11.10 * * *$ \\
\hline Social Functioning & 74.51 & 24.03 & 78.77 & 25.43 & $-3.57^{*}$ \\
\hline Pain & 79.56 & 19.11 & 70.77 & 25.46 & $9.26^{* * *}$ \\
\hline Physical Limitations & 82.22 & 29.73 & 52.97 & 40.78 & $19.80 * * *$ \\
\hline Emotional Limitations & 59.78 & 41.81 & 65.78 & 40.71 & $-2.88 *$ \\
\hline General Health & 64.55 & 21.19 & 56.99 & 21.11 & $7.18^{* *}$ \\
\hline SRRS Total Score & 235.75 & 125.95 & & & \\
\hline SRRS Total Stressors & 9.04 & 4.49 & & & \\
\hline Satisfaction with Life & 23.65 & 6.73 & & & \\
\hline
\end{tabular}

Correlational analyses indicated statistically significant positive associations between the RAND Health Scores and the mindfulness factors (see Table 2). Overall, the Describing, Awareness, and Non-judging factors were consistently associated with physical and psychological wellness. As would be expected, the magnitude of the correlations between mindfulness and psychological health were larger than those between mindfulness and physical health.

Table 2. Relation between Mindfulness Factors and Indicators of Physical and Psychological Wellness.

\begin{tabular}{|c|c|c|c|c|c|}
\hline & \multicolumn{5}{|c|}{ Mindfulness Factors } \\
\hline & Observing & Describing & Awareness & Non- Judging & Non-reactive \\
\hline Physical Limitations & -.022 & $.134^{*}$ & $.162 * *$ & $.158 * *$ & .060 \\
\hline Emotional Limitations & -.076 & $.183 * * *$ & $.367 * * *$ & $.318 * * *$ & $.144^{*}$ \\
\hline Physical Functioning & .061 & $.141^{*}$ & $.127^{*}$ & .089 & .085 \\
\hline Energy & .013 & $.278 * * *$ & $.431 * * *$ & $.383 * * *$ & $.226 * * *$ \\
\hline Emotional Well-Being & -.003 & $.340 * * *$ & $.492 * * *$ & $.456 * * *$ & $.339 * * *$ \\
\hline Social Functioning & -.033 & $.205^{* * *}$ & $.338 * * *$ & $.342 * * *$ & $.198^{* *}$ \\
\hline Pain & $.183^{* *}$ & $.264 * * *$ & $.209 * * *$ & $.126 * * *$ & $.387 * * *$ \\
\hline General Health & $.195 * *$ & $.320 * * *$ & $.211^{* *}$ & $.168 * *$ & $.336 * * *$ \\
\hline SRRS (\# Stressors) & $.177 * * *$ & .011 & -.099 & $-.176^{* * *}$ & .074 \\
\hline Satisfaction with Life & $.262 * *$ & $.329 * *$ & $.359 * *$ & $.276^{* *}$ & $.233^{* *}$ \\
\hline
\end{tabular}

There were consistent low to moderate correlations between mindfulness and personality (see Table 3). In general, having high levels of factor and facet Extraversion, Agreeableness, Conscientiousness and low factor and facet Emotional Instability were associated with higher Mindfulness Describing, Awareness, and Non-Judging. The Observing factor of Mindfulness was associated with higher Agreeableness, Conscientious, and Openness. It is interesting to note that individuals who reported higher levels of Neuroticism had lower scores on Describing, Awareness, Non-Judging and Non-Reacting Mindfulness. This suggests that the inability to regulate one's emotions is associated with the inability to consider thoughtfully changing environmental circumstances.

\subsection{Does increased mindfulness predict physical and psychological health}

Two hierarchical regressions allowed us to examine if specific aspects of mindfulness predicts better physical and psychological well-being. In both regressions, age, relationship status, and unscaled SRRS were entered in Block 1 and the mindfulness factors were entered in Block 2. The first regression predicting RAND General Health was statistically significant, $\mathrm{F}(8,234)=5.10, p<.0001$, with $15.3 \%$ of the variability in physical health accounted for by the predictor variables. Block 1 demographic variables were not statistically significant. Block 2 was statistically significant $\left(\mathrm{R}^{2}\right.$ change $\left.=.13\right)$, with Mindfulness-Observing ( $t=-1.71 ; \beta=-.11)$, Mindfulness-Awareness $(t=3.73 ; \beta=.27)$, and Mindfulness-Non Reacting $(t=2.09 ; \beta=.14)$ predicting RAND General Health. Thus, higher levels of 
Awareness and Non-Reacting coupled with lower levels of Observing was associated with better physical health. In the second regression, SWLS was the criterion variable, $\mathrm{F}(8,240)=10.33, p<.0001$, $\mathrm{R}^{2}=26.3 \%$. Block 1 was statistically significant and accounted for $6 \%$ of the variability in SWLS. Specifically, Relationship status $(t=-2.57 ; \beta=-.16)$ and Number of Stressors $(t=-2.72 ; \beta=-.17)$ predicted SWLS. Block 2 was also statistically significant $\left(\mathrm{R}^{2}\right.$ change $\left.=.20\right)$, with Mindfulness-Awareness $(t=2.36 ; \beta=.16)$, and Mindfulness-Non Judging $(t=3.68 ; \beta=.24)$, and Mindfulness-Non Reacting ( $t=2.78 ; \beta=.17)$ contributing significantly to the model. Thus, being in a romantic relationship, having fewer life stressors, and higher scores on the Awareness, Non-Judging, and Non-Reacting factors of mindfulness contributed positively to life satisfaction.

Table 3. Relation between Mindfulness Factors and BFI-2 Factors and Facets.

\begin{tabular}{|c|c|c|c|c|c|}
\hline & \multicolumn{5}{|c|}{ Mindfulness Factors } \\
\hline & Observing & Describing & Awareness & Non-Judging & Non-Reacting \\
\hline Extraversion (E) & .041 & $.424 * * *$ & $.302 * * *$ & $.373 * * *$ & $.171 *$ \\
\hline E1 Sociability & .008 & $.271 * * *$ & $.188 * *$ & $.267 * * *$ & .094 \\
\hline E2 Assertiveness & .029 & $.414^{* * *}$ & $.211 * * *$ & $.290 * * *$ & $.149 * *$ \\
\hline E3 Energy Level & .068 & $.353 * * *$ & $.347 * * *$ & $.359 * * *$ & $.178 * *$ \\
\hline Agreeableness (A) & $.171 *$ & $.270 * * * *$ & $.250 * * *$ & $.245 * * *$ & $.158 *$ \\
\hline A1 compassion & .088 & $.218 * * *$ & $.113^{*}$ & $.164^{* *}$ & .028 \\
\hline A2 Respectfulness & $.173 * *$ & $.175 * *$ & $.279 * * *$ & $.176^{* *}$ & $.161^{*}$ \\
\hline A3 Trust & $.151^{* *}$ & $.255^{* * *}$ & $.214 * * *$ & $.249 * * *$ & $.192 * * *$ \\
\hline Conscientiousness (C) & $.165 * *$ & $.312 * * * *$ & $.485 * * *$ & $.291 * * *$ & $.198 * * *$ \\
\hline C1 Organization & $.135 *$ & $.133^{*}$ & $.341 * * *$ & $.188^{*}$ & $.120 *$ \\
\hline C2 Productiveness & $.163 * *$ & $.383 * * *$ & $.466 * * *$ & $.290 * *$ & $.199 * *$ \\
\hline C3 Responsibility & .099 & $.268 * * *$ & $.385 * * *$ & $.242 * * *$ & $.173 * *$ \\
\hline Emotional Instability (N) & -.017 & $-.355 * * *$ & $-.487 * * *$ & $-.435 * * *$ & $-.473 * * *$ \\
\hline N1 Anxiety & -.002 & $-.291 * * *$ & $-.373 * * *$ & $-.312 * * *$ & $-.410 * * *$ \\
\hline N2 Depression & .012 & $-.353 * * *$ & $-.497 * * *$ & $-.485 * * *$ & $-.376 * * *$ \\
\hline N3 Emotional Volatility & -.051 & $-.292 * * *$ & $-.409 * * *$ & $-.346 * * *$ & $-.457 * * *$ \\
\hline Openness $(\mathrm{O})$ & $.266 * * *$ & $.357 * * *$ & .102 & .050 & .054 \\
\hline O1 Intellectual Curiosity & $.291 * * *$ & $.344 * * *$ & .068 & .038 & .042 \\
\hline O2 Aesthetic Sensitivity & $.159 * *$ & $.181^{* *}$ & -.027 & -.055 & -.045 \\
\hline O3 Creative Imagination & $.208 * * *$ & $.359 * * *$ & $.222 * * *$ & $.153^{* *}$ & $.149 * *$ \\
\hline
\end{tabular}

\section{Discussion and conclusions}

The comparisons between the current study and published norms elucidate potential stressors associated with young adulthood. As would be expected of young university students, participants in the current study were in good physical health. Scores on subscales measuring physical functioning, pain, physical limitations, and general physical health were higher than published norms. Conversely, the current participants had scores indicative of lower emotional well-being; scores on RAND scales measuring emotional well-being, social functioning, limitations on emotional functioning, and energy/fatigue were lower than published norms. In addition, participants reported that they had encountered a large number of stressors over the past year and, given that the first years of university represent a transitional period between childhood and adulthood, the increase in stressors is not surprising (Denovan \& Macaskill, 2017). In spite of the lower emotional and social functioning, participants in the current sample had above average satisfaction with life, which was indicative of Canadian undergraduate students (Fowler, Davis, Both \& Best, 2018).

Overall, our results highlight that specific aspects of mindfulness are associated with both physical and psychological health. Both correlational and regression analyses suggested that higher levels of Awareness and Non-Reacting were associated with physical and psychological wellness. The current research confirms that higher levels of mindfulness is associated with increased emotional regulation, decreased rumination, and non-attachment with psychological distress (Baer, 2007; Coffey, Hartman, \& Fredrickson, 2010). Being able to act intentionally while avoiding the impulse to assign negative value judgements to current circumstances leads to positive health outcomes. The current results also highlight how other aspects of mindfulness differentially affects physical and psychological wellness. In this study, higher levels of mindful observing were associated with lower levels of perceived health. Further, the absolute number of stressors reported was positively related to Mindful Observing and negatively associated with Mindful Non-judging. Thus, it appears that an increased focus on one's bodily sensations may actually lead to an increased focus on negative sensations and stressors. The non-judging aspect of mindfulness was predictive of psychological well-being. Taking a non-judgmental approach to one's inner experiences would involve dealing with internal thoughts and feelings in a neutral manner, which allows one to avoid excessive rumination and a detachment from emotional distress (Coffey et al., 2010). 
To conclude, increased mindfulness has been associated with positive health outcomes. The current results expand upon previous research and highlights the interplay between personality and mindfulness. These results have important clinical applications. Extraversion, Conscientiousness, and Agreeableness were associated with higher scores on the factors of mindfulness while higher Emotional Instability was related to lower levels of mindfulness. Although personality factors are considered stable, mindfulness is amenable to change. Thus, regardless of personality, practicing mindfulness may lead to positive health changes. It is also interesting to note the complex associations between mindfulness, reported stress, and physical health; in this study, Mindful-Observing was associated with greater pain and life stressors, indicating it may bring undue attention to these ailments. Future research should examine mediation models to determine how personality and mindfulness influence physical and psychological well-being.

\section{References}

Allemand, M., Steiger, A. E., \& Hill, P. L. (2013). Stability of personality traits in adulthood: Mechanisms and implications. Geropsych: The Journal of Gerontopsychology and Geriatric Psychiatry, 26(1), 5-13. doi:10.1024/1662-9647/a000080

Baer, R. A. Mindfulness, assessment, and transdiagnostic processes. Psychological Inquiry, 18, 238-242.

Baer, R. A., Smith, G. T., Lykins, E., Button, D., Krietemeyer, J., Sauer, S., \& Williams, J. M. G. (2008). Construct Validity of the Five Facet Mindfulness Questionnaire in Meditating and Nonmeditating Samples. Assessment, 15(3), 329-342. https://doi.org/10.1177/1073191107313003

Baer, R. A., Smith, G. T., Hopkins, J., Krietemeyer, J., \& Toney, L. (2006). Using self-report assessment methods to explore facets of mindfulness. Assessment, 13(1), 27-45.

Bostock, S., Crosswell, A. D., Prather, A. A., \& Steptoe, A. (2019). Mindfulness on-the-go: Effects of a mindfulness meditation app on work stress and well-being. Journal of Occupational Health Psychology, 24(1), 127-138. https://doi.org/10.1037/ocp0000118

Coffey, K. A., Hartman, M., \& Fredrickson, B. L. (2010). Deconstructing mindfulness and constructing mental health: understanding mindfulness and its mechanisms of action. Mindfulness, 1(4), 235-253.

Costa, P. T., Jr., \& McCrae, R. R. (1992). Revised NEO Personality Inventory (NEO-PI-R) and the NEO Five-Factor Inventory (NEO-FFI) Professional Manual. Odessa, FL: Psychological Assessment Resources.

Costa, P. T., Jr., \& McCrae, R. R. (2010). Revised NEO Personality Inventory (NEO-PI-R) and the NEO Five-Factor Inventory (NEO-FFI) Professional Manual. Odessa, FL: Psychological Assessment Resources.

Denovan, A., \& Macaskill, A. (2017). Stress and subjective well-being among first year UK undergraduate students. Journal of Happiness Studies, 18(2), 505-525.

Diener, E. (n.d.). Understanding Satisfaction with Life Scores. Retrieved from: http://labs.psychology.illinois.edu/ ediener/SWLS.html (2 April 2019).

Diener, E. D., Emmons, R. A., Larsen, R. J., \& Griffin, S. (1985). The satisfaction with life scale. Journal of Personality Assessment, 49(1), 71-75.

Fowler, S. A., Davis, L. L., Both, L. E., \& Best, L. A. (2018). Personality and perfectionism as predictors of life satisfaction: The unique contribution of having high standards for others. Facets, 3(1), 227-241.

Hayes, N., \& Joseph, S. (2003). Big 5 correlates of three measures of subjective well-being. Personality and Individual Differences, 34, 723-727. DOI: 10.1016/S0191-8869(02)00057-0

Hays, R. D., \& Morales, L. S. (2001). The RAND-36 measure of health-related quality of life. Annals of medicine, 33(5), 350-357.

Holmes, T. H., \& Rahe, R. H. (1967). The Social Readjustment Rating Scale. Journal of psychosomatic research, 11(2), 213-218.

Li, S. Y. H., \& Bressington, D. (2019). The effects of mindfulness-based stress reduction on depression, anxiety, and stress in older adults: A systematic review and meta-analysis. International Journal of Mental Health Nursing. https://doi.org/10.1111/inm.1256

Marzabadi, E., Mills, P. J., \& Valikhani, A. (2018). Positive personality: Relationships among mindful and grateful personality traits with quality of life and health outcomes. Current Psychology: A Journal for Diverse Perspectives on Diverse Psychological Issues. https://doi.org/10.1007/s12144-018-0080-8

RAND Health Care (n.d.). 36-item short form survey (SF-36) scoring instructions. Retrieved from https://www.rand.org/health-care/surveys_tools/mos/36-item-short-form/scoring.htm

Soto, C. J., \& John, O. P. (2017). The next Big Five Inventory (BFI-2): Developing and assessing a hierarchical model with 15 facets to enhance bandwidth, fidelity, and predictive power. Journal of Personality and Social Psychology, 113(1), 117-143. 


\title{
PILOT TRIAL OF STEPPED CARE CBT FOR PEDIATRIC OCD
}

\author{
Adam Lewin, \& Omar Rahman \\ University of South Florida (USA)
}

\begin{abstract}
Introduction: Obsessive Compulsive Disorder (OCD) affects 1-2\% of youth, with $75 \%$ of those having a comorbid disorder. OCD is typically impairing and disruptive to social, academic, and family functioning. Impairment related to OCD usually compounds over time and leads to significant distress, impairment, and dysfunction.

Evidence-based treatment for OCD includes cognitive behavioral therapy with exposures and response prevention (CBT-ERP) and selective seratonin reuptake inhibitors (SSRIs). These are often used in combination. However, for pediatric OCD, CBT-ERP is the preferred first line of treatment, with SSRI recommended for severe cases, non-responders to CBT, and for those where CBT is not available or acceptable.

Despite this, CBT-ERP has limited accessibility and dissemination for several reasons: relatively few trained experts, long waitlists, distance and travel time, requirement of 12-14 visits, and expenses (treatment, missed work, etc.). As a result, many youth do not receive the recommended treatment. Bridging this gap between research knowledge and implementation of good treatment is a primary concern.

We investigated an alternate treatment approach, Stepped Care CBT (SSCBT), to address the above concern. SSCBT has the advantage of allowing for larger caseloads for therapists, shorter wait times for patients, decreased life disruption, and decreased cost. The primary question was this: Can a parent-delivered, therapist-assisted CBT mitigate the need for full course, therapist-directed treatment?

Method: Forty-five youth were screened for the study. Thirty-four youth with OCD were randomized at a 2:1 ratio into SSCBT $(n=22)$ or standard therapy $(n=12)$. SSCBT consisted of Step One: a low therapist-intensity treatment, consisting of 3 clinic sessions with therapist, six weekly phone contacts, and 11 parent-child meetings guided by a workbook; and Step Two: for non-responders in Step One, nine sessions of therapist-directed standard in-clinic CBT-ERP. Standard care consisted of 12 sessions of in-clinic, therapist directed CBT-ERP.

Results: In the standard group, the treatment response rate was 83\%. In SSCBT, the response rate was 79\% after Step One and 93\% after Step Two.

Discussion: The results provide support for the stepped care model as applied to CBT for OCD in youth. This has the potential to make effective treatment available to more youth, and at a significantly reduced cost and disruption. We also discuss secondary findings from the study, as well as strength, limitations, and suggestion for future research.
\end{abstract}

Keywords: Obsessive compulsive disorder, cognitive behavioral therapy, exposure and response prevention, stepped care, therapy. 


\title{
THE THOUGHT OF LIVING WITH SPINAL CORD INJURY: HOW AN IMAGINED DISABILITY AFFECTS LIFE SATISFACTION
}

\author{
Derek J. Gaudet ${ }^{1}$, Lisa A. Best ${ }^{2}$, \& Najmedden Attabib ${ }^{3}$ \\ ${ }^{I}$ M.A., Department or Psychology, University of New Brunswick Saint John (Canada) \\ ${ }^{2}$ Ph.D., Department or Psychology, University of New Brunswick Saint John (Canada) \\ ${ }^{3}$ M.D., Department of Neurosurgery, Saint John Regional Hospital (Canada)
}

\begin{abstract}
Paralysis resulting from a spinal cord injury is a traumatic, life changing event having both acute and prolonged effects on an individual's life satisfaction (LS). Ethical and methodological considerations make research on the initial impact of paralysis on LS using a patient population difficult. As spinal cord injury and other debilitating injuries can affect anyone, understanding how the average person thinks about living with paralysis caused by spinal cord injury offers important insight to how individuals might the injury during the initial days. Using an online-survey methodology we examined how the average person believes two levels of paralysis, paraplegia and quadriplegia, could affect their overall LS. Four-hundred and eighteen participants completed a demographics questionnaire, the Life Satisfaction Questionnaire-11, and the Big Five Inventory-2. Additionally, the participants indicated the degree to which they thought that their life was worth living. After completing these measures, they were asked to imagine themselves living with paraplegia. While doing this, they re-completed the LiSAT-11 and rated the degree to which they thought life would be worth living. They completed these measures one additional time, after imagining themselves living with quadriplegia. Analyses revealed a significant main effect of tSCI scenario on LiSAT-11 scores. LiSAT-11 scores were considerably lower than the participant's actual LS in both imagined paralysis conditions, being lowest in the quadriplegia condition. The degree to which they believed that their life would be worth living followed the same trend. Although participants believed that their lives were currently worth living, they believed life would be substantially less worth living if they were either paraplegic or quadriplegic. Several personality domains were also found to be associated with these factors. Discussion: The results of the present study provide insight into how traumatic spinal cord injury might affect LS and self-worth in, at least, the initial days of injury. The findings could also have implications for early psychological intervention.
\end{abstract}

Keywords: Life satisfaction, spinal cord injury, disability, paralysis.

\section{Introduction}

Life satisfaction refers to an important component of subjective well-being and consist of a global evaluation of one's life as a whole (Diener et al.,1985). Traumatic spinal cord injuries (tSCI) are life changing events that can have a huge impact on life satisfaction. Depending on the severity and location of the injury, the effects of a tSCI can range from minor numbness to complete paralysis of the limbs. In Canada, there are an estimated 43,974 individuals living with a tSCI, with 43\% classified as having some level of paraplegia (disability in the lower limbs) and 56\% with some degree of quadriplegia (reduced function and sensation from the neck down). Traumatic spinal cord injury most commonly occurs between 15 to 29 years of age (Noonan et al., 2012). This is of particular importance as this age range also corresponds to the years for which an individual is striving to establish their independence and autonomy. These factors are associated with life satisfaction (Cavazos Arroyo, 2013). As severe tSCI removes some degree of a sense of autonomy and independence, at least initially, it is likely that tSCI will have a considerable impact on an individual's life satisfaction when it occurs during those years.

For obvious methodological constraints, most studies examine life satisfaction recovery of individuals living with severe tSCI injuries after discharge. That research has demonstrated that some recovery of life satisfaction occurs following the first two years after discharge (van Leeuwen et al., 2012), but little is understood about the effect that tSCI has on an individual's pre-injury life satisfaction. Obtaining a reliable measure of pre-injury satisfaction is almost impossible, as it would be certainly be influences by their present circumstances. One way to examine the potential effects of tSCI on life satisfaction is to ask a healthy population to compare current aspects of their lives to imagined scenarios in which they are living with a severe tSCI and then compared those results with a sample of individuals living with the condition. 
Gerhart et al. (1993) reported that many people strongly believe that their lives would change for the worse and that a satisfactory life would be impossible after a spinal injury. Further, it is interesting to note that the participants in this study were emergency care professionals who deal with traumatic situations on a regular basis. In spite of their personal experience with injury, participants still held negative beliefs about the realities of living with severe disability. When asked to imagine themselves living with a severe tSCI, only $18 \%$ of participants could imagine that they would be glad to be alive. In fact, $92 \%$ of those actually living with a severe tSCI reported that they were happy to be alive. These results suggest that people hold largely negative and inaccurate beliefs about the well-being and life satisfaction of those living with severe disability. This is important to consider, as these inaccurate beliefs likely affect how individuals process a tSCI event immediately after injury.

Regardless of what we think we "know" about living with severe tSCI, there are numerous famous cases that seemingly contradict our largely negative beliefs. Rick Hansen, Christopher Reeves, and Stephen Hawking are all famous cases of individuals who appear(ed) perfectly satisfied with their lives, even after acquiring a severe disability. In addition to examining the extent to which individuals believe that their overall life satisfaction would decrease as a result of severe tSCI, we were also interested in the possibility that certain personality traits might act as "protective factors" that might allow an individual to imagine a life with severe tSCI, while also maintaining a certain degree of life satisfaction. Here, we consider how basic personality domains (i.e., Extraversion, Agreeableness, Conscientiousness, Neuroticism, and Openness to Experience) are related to how individuals think about living with paraplegia and quadriplegia.

In the present study, we asked people to imagine how satisfied they be with different aspects of their lies if they were living with 1) paraplegia and 2) Quadriplegia. We compared measures of their actual life satisfaction to measures of life satisfaction in each of these scenarios. We hypothesized that a decrease in life satisfaction would occur, and that people would believe that their lives would be less worth living. We also suspected that personality, specifically negative emotionality, would be negatively correlated with perceived life satisfaction, whereas agreeableness, extraversion, conscientiousness, and open-mindedness would be positively correlated with perceived life satisfaction.

\section{Method}

\subsection{Measures}

Participants were required to complete a series of survey's and questionnaires including a demographics section, the Big Five Inventory-2 (BFI-2), the Life Satisfaction Questionnaire-11 (Li-SAT-11). In addition to this, participants were asked to respond on a seven-point Likert scale, ranging from not at all (1) to completely (7), the extent to which they believed life is worth living. The demographics questionnaire asked participants for their age, gender, as well as for information on several other aspects of their life, such as education and vocation, not considered in the present analysis. The BFI-2 measures five domains of personality (Extraversion, Agreeableness, Conscientiousness, Negative Emotionality, and Open Mindedness), as well as three facets of each domain. The BFI-2 has been demonstrated to have excellent psychometric properties (Soto \& John, 2017). The Life Satisfaction Questionnaire - 11, asks individuals to indicate the extent to which they are satisfied with 11 areas of their lives on a six-point Likert scale ranging from very dissatisfying (1) to very satisfying (6). The LiSAT-11 has been shown to have good psychometric properties (Post et al. 2012). Participants also responded to a single Likert scale item asking them to rate the degree from not at all (1) to completely (7) that they believed that their life was worth living.

\subsection{Procedure}

Study participants were directed to the study by one of two methods. In the first method, the study was circulated via social media relying on a snowball sampling approach. In the second method, participants were recruited from psychology courses at the University of New Brunswick. Responses remained entirely anonymous in both situations. All participants were provided with an internet link that they could follow to the study. University students followed a link through a campus research participant recruitment site which automatically granted them a bonus point upon completion of the study. All participants provided informed consent. The study was a repeated measures design divided into 3 phases. In the first phase, they responded to a series of demographics questions which were followed by the BFI-2, LiSAT-11, and a single Likert scale item asking them to rate the extent to which they felt that their life was worth living. This phase acted as a means to collect "baseline information" and always came first. Personality and life satisfaction measures were presented in a randomized order. After completion of the first phase, the experimental manipulation phase took place. Participants were told to imagine a scenario that they were living with a tSCI that resulted in paraplegia. While imagining this, they were then asked to complete the LiSAT-11 in a way that reflected how they would feel about their life satisfaction. After completing the LiSAT-11, they were also asked to rate the extent to which they believed that their life would be worth living. They followed this procedure one final time, while imagining that they were living with Quadriplegia. The order of Paraplegia and Quadriplegia tSCI scenarios was counterbalanced to control for order effects. 


\section{Results}

Four hundred and fifty-six respondents provided informed consent and completed the online study. The final sample was $81 \%$ female, with a mean age of 20.41 years $(S D=4.73)$. The online study took approximately 30 minutes to complete.

A mixed design 3x2 (tSCI scenario by gender) two-way repeated measures ANOVA was conducted to determine whether people believed that their life satisfaction would decrease following tSCI, as measured by LiSAT-11 scores. The results of this analysis indicate that the assumption of sphericity was not met, therefore the Greenhouse-Geisser correction was used. The Analysis revealed a statistically significant main effect of tSCI scenario on LiSAT scores, $F(1.83,759.42)=628.71, p<.001, \eta^{2}=.602$. Gender was not found to have had an effect on LiSAT-11 scores, nor was the interaction effect between tSCI scenario and gender found to be statistically significant. Pairwise comparisons indicate that life satisfaction decreased with severity of imagined impairment. LiSAT-11 scores were found to decrease as a result of imagining themselves living with tSCI resulting in paraplegia $(p<.001)$, and then again when imagining themselves living with quadriplegia $(p<.001)$. The decrease from the paraplegia scenario to the quadriplegia scenario was also found to be statistically significant $(p<.001)$. Results of the analysis are displayed in figure 1.

Figure 1. Life satisfaction scores by tSCI scenario.

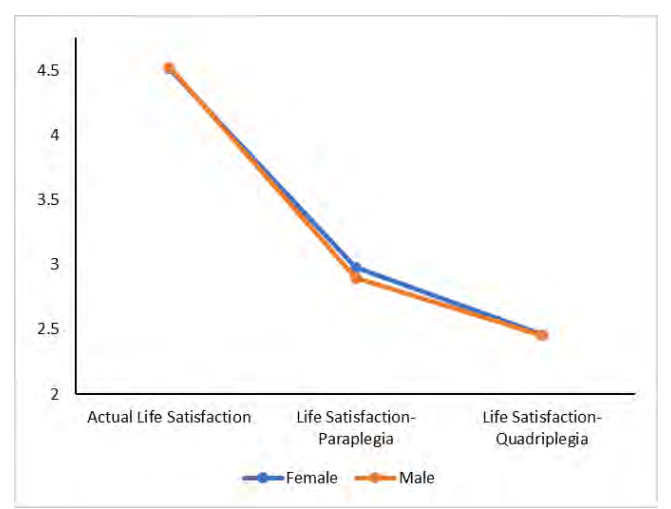

A mixed design 3x2 (tSCI scenario by gender) two-way repeated measures ANOVA was conducted to determine whether people believed that their life would be worth living following tSCI, as measured by responses to a single Likert scale question. Again, the assumption of sphericity was found to be violated, so a Greenhouse-Geisser correction was used. The analysis revealed a statistically significant effect of tSCI scenario on whether participants believed that their life would be worth living, $F(1.61,710.40)=522.43, p<.001, \eta^{2}=.542$. The main effect of gender was not found to be statistically significant. The interaction effect between gender and tSCI scenario was found to be statistically significant, $F(1.61,710.40)=7.639, p=.001, \eta^{2}=.017$. Specifically, feeling that life would be worth living dropped when participants imagined themselves having to live with a tSCI resulting in paraplegia $(p<.001)$ or Quadriplegia $(p<.001)$. This decrease was also statistically significant between tSCI paraplegia and tSCI Quadriplegia scenarios. Post hoc analysis of the interaction effect revealed a statistically significant difference between genders in feeling that life would be worth living for paraplegia, but no difference in ratings for the No tSCI or tSCI quadriplegia conditions. The results of this analysis are displayed below (figure 2).

Figure 2. Feeling that life is worth living Likert responses by $t$ SCI scenario.

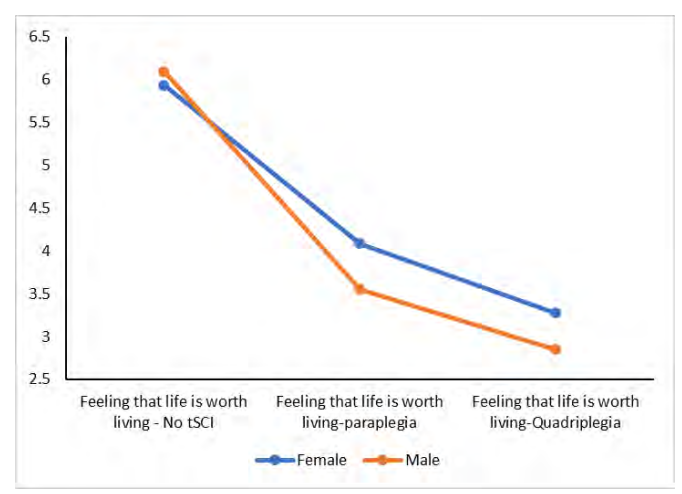


To determine the relationship between personality domains and LiSAT-11 scores, as well as with the life worth living variable, we examined the correlations between each of the BFI domain scale factors and LiSAT-11 scores in each of the tSCI scenarios. Life Satisfaction in the tSCI condition was found to be positively correlated with Agreeableness, suggesting that when imagining oneself living with paraplegia, higher levels of agreeableness are associated with higher levels of predicted life satisfaction. Correlations are presented in the table below. Negative emotionality was the only other personality trait associated with LiSAT scores in the tSCI scenarios. It was found to be negatively correlated with LiSAT scores in both paraplegia and quadriplegia scenarios. Next, we examined the correlations between LiSAT scores in the tSCI scenarios and each personality trait. Many more of the correlations emerged as statistically significant in this analysis, although some of the effect sizes are quite small.

Table 1. Correlations between LiSAT scores / life worth living scores and BFI-2 personality domains.

\begin{tabular}{|c|c|c|c|c|c|}
\hline & Extraversion & Agreeableness & Conscientiousness & $\begin{array}{c}\text { Negative } \\
\text { Emotionality }\end{array}$ & $\begin{array}{c}\text { Open- } \\
\text { Mindedness }\end{array}$ \\
\hline $\begin{array}{l}\text { LiSAT } \\
\text { No tSCI }\end{array}$ & $.327 * * *$ & $.377 * * *$ & $.297 * * *$ & $-.479 * * *$ & .003 \\
\hline $\begin{array}{c}\text { LiSAT } \\
\text { tSCI Paraplegia }\end{array}$ & .043 & $.118 *$ & .077 & $-.138 * *$ & .043 \\
\hline $\begin{array}{c}\text { LiSAT } \\
\text { tSCI Quadriplegia }\end{array}$ & .048 & .057 & .032 & $-.130 * *$ & .037 \\
\hline $\begin{array}{c}\text { Life Worth Living - } \\
\text { No tSCI }\end{array}$ & $.291 * * *$ & $.321 * * *$ & $.297 * * *$ & $-.493 * * *$ & .051 \\
\hline $\begin{array}{c}\text { Life worth living - } \\
\text { Paraplegia }\end{array}$ & $.093 *$ & $.200 * * *$ & $.099 *$ & $-.132 * *$ & $.119 *$ \\
\hline $\begin{array}{c}\text { Life worth living - } \\
\text { Quadriplegia }\end{array}$ & .014 & $.145 * *$ & $.107 *$ & $-.142 * *$ & .086 \\
\hline
\end{tabular}

As we can see, in some cases personality variables are related to LiSAT-11 scores and feeling that life is worth living across all three conditions in a similar way. To determine whether there is a statistically significant difference in the effect size of these correlations, Fisher's r-to-z transformation was used. The results indicated statistically significant differences between some of the correlation effect sizes. In all cases except openness, the effect size of the correlation between personality domains and LiSAT-11 scores was larger for the No tSCI condition, then in the other two scenarios. The difference between the effect size of the correaltion between LiSAT-11 scores and personality domains in the No tSCI condition and the same variables in the two tSCI scenarios (where both correlations are statistically significant) were found to be statisitically significant. The same pattern was found for the life worth living variable.

\section{Conclusions}

The present study examined the extent to which individuals believed that their life satisfaction would be impacted by severe disability caused by tSCI. Here, we found that when participants imagined themselves living with paraplegia or quadriplegia, they believed that their overall life satisfaction would decrease. In addition to this, it was shown that the more severe the disability, the greater the decrease in life satisfaction. The drops in life satisfaction were not only statistically significant, but also substantial enough to be quite meaningful. Overall, present life satisfaction $(M=4.52, S D=.741)$ dropped from "rather satisfying" to "dissatisfying", when participants were imagining themselves living with paraplegia $(M=2.95, S D=.985)$, and almost to "very dissatisfying" when imaging themselves living with quadriplegia $(M=2.457, \mathrm{SD}=.998)$. Participants also indicated that feeling as though their lives were worth living also depended on whether they were living with a severe disability. On a seven-point Likert scale, from not at all (1) to completely (7), participants indicated a strong belief that their life, at present, was worth living $(M=5.97, \mathrm{SD}=1.344)$. When asked to imagine themselves living with paraplegia, there was a considerable drop to just below the theoretical neutral point of " 4 " $(M=3.96, S D=1.786)$. There was another considerable decrease when imagining themselves living with quadriplegia $(M=3.18, S D=1.828)$. The interesting finding here is not necessarily that people believe that life satisfaction and feeling that life is worth living would decrease under these conditions, but rather, the degree to which they believe that these aspects of their lives would decrease. In feeling that life was worth living, there was also an interesting interaction effect for gender and tSCI scenario. Males and females both felt to the same degree that their lives were worth living without tSCI., but when imagining themselves living with paraplegia, they differed in the extent to which they believed they would still feel that way. Females fell above the theoretical neutral point $(M=4.09, S D=1.756)$, but males fell below it by a considerable amount $(M=3.55, S D=1.776)$, this difference was statistically significant. This difference between genders disappeared when males and female imagined themselves living with quadriplegia, suggesting that such a disability is perceived as 
simply too much to handle. This suggest that females believe that they would still be able to manage their lives with paraplegia, but males would be more heavily burdened by the disability. The reason for such a difference might be understood when considering traditional gender roles that could play a role in subconscious processing. Traditional gender stereotypes suggest that males need to be perceived as strong and powerful to first to attract a mate, and then be able to protect and provide for their families. Any form of paralysis would hurt that perception. Although the social climate on traditional gender roles has been changing for some time, there is still evidence of their persistence in society. It's likely that multiple factors play a role in this particular interaction effect, but it will require additional research to determine the cause.

We also examined whether or not there was evidence that how participants perceived that their life satisfaction in tSCI scenarios. When examining the correlations between these variables and the personality domains of the BFI-2, Extraversion, Agreeableness, Conscientiousness, were positively correlated with life satisfaction and Negative Emotionality was negatively correlated with Life satisfaction. Openness was not significantly correlated with life satisfaction. This exact pattern has commonly been found in the literature (Steel et al., 2008). When participants imagined themselves living with either paraplegia or quadriplegia, most of these relationships, except Negative Emotionality and Agreeableness in the paraplegia scenario, disappeared. In terms of agreeableness, this suggests that the more Agreeable and individual is, the better their life satisfaction. This relationship carries over to some extent, although with a statistically significant reduction in effect size, when asked to imagine what their life satisfaction would be if they were living with paraplegia. The relationship, however, disappears in the quadriplegia scenario. In terms of Negative Emotionality, the higher levels of Negative Emotionality are associated with lower life satisfaction in all three scenarios. Although Negative Emotionality maintains a negative correlation with perceived life satisfaction in the two tSCI scenarios, the correlations again show a statistically significant decrease in effect size. This again suggests that personality plays less of a role in perceiving your life satisfaction in the two tSCI scenarios than it does in determining your actual life satisfaction.

In the present study, we examined the extent to which healthy people believed that a tSCI resulting in paraplegia and then quadriplegia would affect their life satisfaction, as well as whether they still believed that their lives would be worth living in those scenarios. This was done as a means to examine the potential effect that a real tSCI injury has on an individual's life satisfaction. A limitation to this method is that none of these participants actually had a tSCI, and many never will. Although this may be true, it is possible for anyone to sustain a tSCI. It is likely safe to assume that the individuals in this sample are not much different from those who actually sustain a tSCI. A better understanding of how the general population thinks about living with tSCI informs us of how those same individuals might process a tSCI injury during the initial days of recovery. This study is a smaller part of a much larger project. The next step will involve comparing these perceptions of life satisfaction to the life satisfaction of individuals who are actually living with paraplegia and quadriplegia caused by a tSCI. In addition to this, the LiSAT-11 also focuses on 11 specific areas of life satisfaction. A closer examination of these areas should reveal which areas people believe will be most impacted by paraplegia and quadriplegia and could inform early intervention strategies to address those concerns.

\section{References}

Cavazos Arroyo, J. (2013). Personality factors, affect, and autonomy support as predictors of life satisfaction. Universitas Psychologica, 12(1), 41-53.

Diener, E. D., Emmons, R. A., Larsen, R. J., \& Griffin, S. (1985). The satisfaction with life scale. Journal of personality assessment, 49(1), 71-75.

van Leeuwen, C. M., Post, M. W., van Asbeck, F. W., Bongers-Janssen, H. M., van der Woude, L. H., de Groot, S., \& Lindeman, E. (2012). Life satisfaction in people with spinal cord injury during the first five years after discharge from inpatient rehabilitation. Disability and rehabilitation, 34(1), 76-83.

Noonan, V. K., Fingas, M., Farry, A., Baxter, D., Singh, A., Fehlings, M. G., \& Dvorak, M. F. (2012). Incidence and prevalence of spinal cord injury in Canada: a national perspective. Neuroepidemiology, 38(4), 219-226.

Post, M. W., van Leeuwen, C. M., van Koppenhagen, C. F., \& de Groot, S. (2012). Validity of the Life Satisfaction questions, the Life Satisfaction Questionnaire, and the Satisfaction with Life Scale in persons with spinal cord injury. Archives of physical medicine and rehabilitation, 93(10), 1832-1837.

Soto, C. J., \& John, O. P. (2017). The next Big Five Inventory (BFI-2): Developing and assessing a hierarchical model with 15 facets to enhance bandwidth, fidelity, and predictive power. Journal of personality and social psychology, 113(1), 117.

Steel, P., Schmidt, J., \& Shultz, J. (2008). Refining the relationship between personality and subjective well-being. Psychological bulletin, 134(1), 138. 


\title{
ASSOCIATION BETWEEN PERCEPTION OF ACCEPTANCE AND REJECTION, AND DRAWINGS OF "PERSON PICKING AN APPLE FROM A TREE" AMONG PRIMARY SCHOOL CHILDREN
}

\author{
Or Shalev ${ }^{1}$, Andriani Papadaki ${ }^{2}$, Elias Kourkoutas ${ }^{2}$, \& Michal Bat Or ${ }^{1}$ \\ ${ }^{\prime}$ The Graduate School of Creative Arts Therapies, University of Haifa (Israel) \\ ${ }^{2}$ Special Education and Psychology, University of Crete (Greece)
}

\begin{abstract}
The present study examined and compared associations between perceptions of parental acceptance/rejection in 644 Greek school age children (323 boys and 321 girls, age range 10-12), and their "Person Picking an Apple from a Tree" (PPAT) drawings. Perception of parental behavior was measured by the "Parental Acceptance-Rejection Questionnaire" (Rohner and Khaleque, 2005). Drawing content was analyzed quantitatively according to a reliable rating system called the Symbolic Content in PPAT drawings (SC-PPAT: Bat Or et al., 2014, 2017). We employed k-means cluster analysis and obtained three relatively discrete PPAT scripts. Drawing scripts were found to be associated with children's perceptions of parental behavior; these associations were found mainly among boys, especially to highly aggressive parents. These results demonstrate how empirical inquiry into PPAT content contributes to identifying implicit relational representations; furthermore, they reinforce the need to examine drawings from a holistic perspective, while focusing on the relational experience of children as expressed through their pictorial PPAT narratives.
\end{abstract}

Keywords: Parental acceptance-rejection, children, PPAT drawings, gender difference.

\section{Introduction}

The parent-child relationship is critical to child development, and considered to be the origins of later relational attributes and personal qualities in an adult (Clarke \& Scharff, 2014). This refers not only to "real" parent-child relationships, but also to internal mental representations of the relationships with others (Flanagan, 2016). The Interpersonal Acceptance-Rejection Theory (IPARTheory; Rohner, 2016) is an evidence-based theory pertaining to socialization and life span development of children and adults, initially focused mostly on the effects of perceived parental acceptance-rejection in childhood, and was formed mainly through the use of verbal tools such as interviews, and self-report questionnaires (Rohner, 2015). Parental acceptance-rejection refers to a bipolar dimension of parental warmth, with parental acceptance at the positive end of the continuum, and parental rejection at the negative end. Acceptance refers to parents' love, affection, care, comfort, support, or nurturance of children. Rejection refers to the absence or withdrawal of parental warmth, love, or affection from their children (Khaleque, 2015). According to Rohner (1980, 2004), children and adults organize their perceptions of parental acceptance-rejection around four universal categories: a) warmth/affection - the quality of the affectionate relationship between the parents and their children, and the physical, verbal, and symbolic behaviors parents use (or are perceived to use) to express these feelings and behaviors; b) hostility/aggression - either physical, verbal, active and/or passive, and problems with the management of hostility and aggression; c) indifference/neglect - a lack of parental concern or interest in the child; and d) undifferentiated rejection - the individuals' belief that his/ her parent/s do not really care about him or her, without necessarily being able to prove this based on their behavior. Two meta-analyses found that children who perceived themselves as accepted by their parents tended to have socially accepted behaviors and positive personality characteristics (Khaleque, 2013; Khaleque \& Rohner, 2012). At the same time, empirical studies worldwide have shown a correlation between parental rejection and children's psychological maladjustment; behavioral problems (including conduct disorder, externalizing behaviors, and delinquency); psychological disorders; and decreased school performance (Dwairy, 2010; Groh, Roisman, IJzendoorn, Bakermans-Kranenburg, \& Fearon, 2012; Miles \& Harold, 2003; Putnick et al., 2015). Some gender differences were found in children's perception of parental acceptance-rejection in recent years. In a sample of 168 Greek children (aged 7-12), both boys and girls tended to perceive their parents as accepting, but girls perceived significantly more maternal acceptance than boys did. 
There was significant correlation between psychological adjustment of boys and perceived paternal, but not maternal, acceptance (Giotsa \& Touloumakos, 2014).

Projective Drawings (PDs) have been proposed for testing and evaluating mental development and intelligence levels in children, and later expanded to provide additional information about the individual, such as personality traits and emotional and cognitive development. A large body of evidence suggests that there are differences between the drawings of boys and those of girls, which can manifest in the pictorial content (Turgeon, 2008; Wright \& Black, 2013). The "Person Picking an Apple from a Tree" (PPAT) drawing task (Gantt, 1990) is an art-therapy assessment that has been found to be valuable in revealing associations between emotional-behavioral problems and cognitive (executive functions) aspects in preschool children (Bat Or, Ishai, \& Levi, 2014) and highly aggressive school-age children (Bat Or, Kourkoutas, Smyrnaky, \& Potchebutzky, in press). Significant associations between PPAT drawings and relationship representations has only been found among adults (Bat Or \& Ishai, 2016), whereas there is still a need to study these possible associations among children. The present study examined whether the content of children's PPAT drawing task (Gantt, 1990) was associated with their perceived parental attitude (acceptance and rejection) as measured by the "Parental Acceptance-Rejection Questionnaire" (Child PARQ; Rohner \& Khaleque, 2005).

Our research hypotheses were: (a) Associations will be found between perceived parental acceptance-rejection and the pictorial contents of PPAT drawings. Specifically, parental acceptance will correlate with the positive aspects of PPAT drawings (for example, active drawn person, successful picking, tree with apples); and, parental rejection will correlate with negative aspects in the PPAT (for instance, weak tree, passive drawn person, tree inclining away from the person, etc.). (b) Associations between PARQ and PPAT pictorial content will differ substantially between genders.

\section{Method}

\subsection{Participants}

The sample of 644 Greek school age children (age range 10-12) were randomly selected from public schools in three prefectures of the island of Crete (Heraklion, Chania, and Rethymnon). 277 children were from the fifth grade, and 367 from the sixth grade, $51 \%$ of the participants were boys and $49 \%$ were girls. Participant distribution was $86 \%$ urban residents and $14 \%$ semi urban residents.

\subsection{Instruments}

2.2.1. Parental Acceptance-Rejection Questionnaire (Child PARQ). Parental Acceptance-Rejection Questionnaire (Child PARQ) (Rohner, 1990; Adaptation in Greek in Giovazolias et al., 2010). The current study used the short form of the Parental Acceptance-Rejection Questionnaire: Child version (Child PARQ: Mother version, Child PARQ: Father version; Rohner and Khaleque, 2005). The Child PARQ short version encompasses 24 items and asks children to interpret their caregiver's behavior through their own personal experiences. Participants were asked to evaluate each statement on a four-point Likert scale ranging from 1 (almost never true) to 4 (almost always true). The scales were summed and keyed in the direction of perceived rejection. Mother and Father Child PARQ questionnaires are identical. The Warmth/Affection Scale is composed of eight items, for example, "My father/mother says nice things about me." Scores were inverted, thus high scores indicate lack of parental Warmth/Affection. The Hostility/Aggression Scale is composed of six statements, for example, "My father/mother hits me, even when I do not deserve it." The Indifference/Neglect Scale has six items, including statements such as "My father/mother pays no attention to me." Finally, the Undifferentiated Rejection Scale incorporates four statements such as "My father/mother seems to dislike me." The Greek Child PARQ was found to be a reliable and valid instrument (Artemis and Touloumakos, 2016). In the current study, the internal consistency of the total PARQ scores of mothers and fathers in each sub-scale were good (Cronbach's alphas were 0.853 and 0.851 , respectively, $\mathrm{N}=644$ ).

2.2.2. "Person Picking an Apple from a Tree" Drawing Task (PPAT). "Person Picking an Apple from a Tree" drawing task (Gantt, 1990, Unpublished). We followed the instructions proposed by Gantt and Tabone (1998) for administration of the PPAT process. Accordingly, participants were given white sheets of paper (21by $29.5 \mathrm{~cm}$ ) and markers in 12 colors (red, orange, blue, turquoise, green, dark green, hot pink, gray, purple, brown, yellow, and black), and were asked to draw "a person picking an apple from a tree" (Gantt and Tabone, 1998). Due to the slightly different composition of colors in the 12 pack markers sold in Greece, the gray colored marker replaced the magenta color noted in the original Gantt and Tabone (1998) instructions. The 'Symbolic Contents in "Person Picking an Apple from a Tree" for school-age children' (SC-PPAT/c2 Bat Or et al., 2017), comprises nine Likert-scales that range between 0 (the rated feature is absent) and 5 or 6 (the rated feature at its maximum). The scales measure three central aspects of the PPAT drawing: characteristics of the tree (for example the number of apples on the tree); characteristics of the person (for instance, the degree in which a person is active/passive in 
the apple picking process); and characteristics of the tree-person relationship (for example, the position of the tree truck in relation to the person's location). The drawings $(\mathrm{N}=644)$ were rated according to the SC-PPAT/c2 rating system; two trained raters coded 10\% of each of the drawings, until they achieved substantial agreement. The inter-rater reliabilities were calculated by the Intra-Class Correlation coefficient, which ranged between good and excellent.

\subsection{Procedure}

Researchers initially secured approval from the Educational Institute of the Ministry of Education as well as the ethics committee of the University of Crete. Furthermore, meetings were held with the parents of the participants to inform them of the purposes of this research. Parents were asked to sign consent forms. The research was conducted in the schools, and researchers entered the class accompanied by the class teacher. On the first day, the researchers introduced themselves and administered the Child PARQ-mother/father questionnaires, and on the second day they administered the PPAT drawing task. Participants were individually asked to draw a person picking an apple from a tree; no time limitation was set. Researchers assured the children that there were no right or wrong answers, and no drawing would be considered an ugly drawing. They informed the children that the questionnaires and the drawings would be collected by the researchers.

\section{Results}

\subsection{SC-PPAT Factor Analysis and Cluster Analysis}

Three main factors were obtained by Confirmatory Factor Analysis. Each factor consists of two scales. Person Agency pertains to the drawn person's activity, including the degree in which the person is active/passive in the apple picking, and if there is contact between person and tree. Tree Accessibility pertains to the tree's orientation toward the drawn person, including the position of the tree trunk in relation to the person, and placement of branches in relation to the person. Finally, Tree Potency pertains to the characteristics of the tree, including the number of apples it bears and its strength vs. weakness. These factors yield a total of $68 \%$ of the explained variance. Inter-factor associations were also measured. The main finding is a medium positive association between Person Agency and the Tree Accessibility. In specific, as much as the person is more agent, the tree is more accessible for the person. K-means cluster analysis was conducted for identifying groups of PPAT drawings with different combinations of PPAT's main factors values. One-way ANOVA showed significant differences between the three main factors in each cluster. As can be seen in Table 1, three clusters were identified in PPAT drawings. Figure 1 illustrates drawings in cluster A: a potent tree, a low agent person, and a neutral-to-less-accessible tree $(n=295)$; Figure 2 illustrates drawings in cluster B: a non-potent tree, a person with medium agency, and a fairly accessible tree $(n=153)$; Finally, Figure 3 illustrates PPAT drawings in cluster C: a potent tree, a person with agency, and an accessible tree $(n=307)$. In terms of the PPAT narrative, cluster $\mathrm{C}$ describes the best scrip, in terms of coherency and reciprocity, while the two other clusters reveal gaps between personal agency and tree potency. While cluster B describes a narrative with a weak though accessible tree and medium personal agency, cluster A reflects a potent though less accessible tree and low personal agency. For the whole sample, children who drew cluster B PPAT tended to perceive their mothers as more rejecting, in comparison to children who drew cluster C PPAT.

Table 1. Final Cluster Centers.

\begin{tabular}{lccc}
\hline & \multicolumn{3}{c}{ Cluster } \\
\cline { 2 - 4 } PPAT's main factors & $\mathrm{A}$ & $\mathrm{B}$ & $\mathrm{C}$ \\
\hline Potency of tree & 4.80 & 2.77 & 4.87 \\
Agency of person & 2.06 & 2.75 & 3.35 \\
Accessibility of tree & 2.69 & 2.86 & 2.99 \\
Number of PPAT & 295 & 153 & 307 \\
\hline
\end{tabular}

3.1.1. Gender differences. Pearson's correlation-coefficients were calculated between PARQ categories and SC-PPAT/c2 factors for both boys and girls. Person Agency was the only main factor associated with PARQ categories. Person Agency was negatively associated with the mother's Hostility/Aggression, $r=-.207, \mathrm{p}<0.01$; in other words, the more the boy perceived his mother as hostile and aggressive towards him, his drawn person's agency was lower, and vice versa: the less hostile and aggressive the boy perceived his mother to be, the more agent person was drawn. In order to examine the differences in correlations that were found between the boys' group and the girls' group, z-tests for differences between Pearson's correlations - coefficients were conducted. Negative significant differences between boys and girls were found, correlated in the boys group $(r=-.207, \mathrm{p}<.001)$ but not in the girls 
group $(\mathrm{r}=-.017, \mathrm{~ns}), \mathrm{z}=2.33, \mathrm{p}<0.05$. In addition to these analyses, a one-way ANOVA analysis was conducted in each gender group between the three clusters and validity variables. Among boys, two significant differences relating to parental hostility levels were found. Specifically, a significant difference between clusters $A$ and $C$ in terms of their scores of father's hostility $F(2,347)=3.42, p=.034$; and a significant difference between clusters A and C in terms of their scores of mother's hostility $F(2,337)=3.72, p=.025$ were detected. In other words, only among boys was a significant difference found between cluster A and C (between PPAT with a potent tree, a low agent person, and a neutral to less-accessible tree and PPAT with coherent and reciprocal relations between the person and the tree. i.e., they are both potent and the tree is also accessible) in relation to reports about parental hostility levels. By the same token, boys who reported higher parental hostility tended to draw cluster A PPAT (with a mixed script: a potent tree, a person with low agency, and a neutral to a less-accessible tree) in comparison to boys that reported lower parental hostility levels and tended to draw cluster C PPAT (with a coherent script, in which all the three factors -a potent and accessible tree, and a person with agency_-are positive).

\section{Figure 1. Cluster A PPAT with a potent and neutral tree in terms of accessibility, and a person with low agency.}

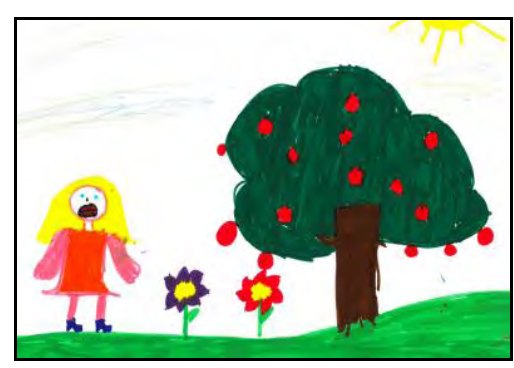

Figure 2. Cluster B PPAT with a non-potent and accessible tree, and a person with medium agency.

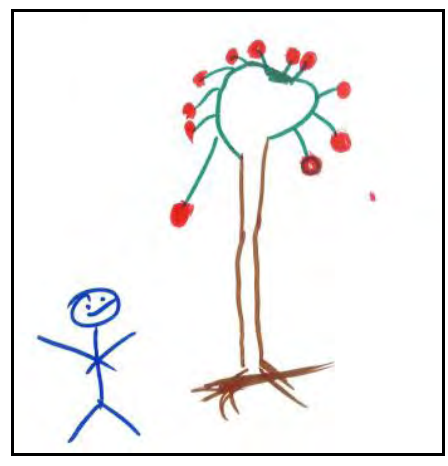

Figure 3. Cluster C PPAT with a potent and accessible tree, and $a$ person with agency.

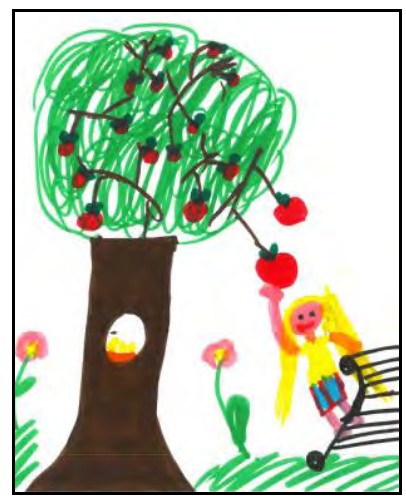

\section{Discussion and conclusion}

The present study contributed to the validity of the SC-PPAT rating system among school age children with a self-report relational measure (perception of parental acceptance-rejection). The preliminary analysis showed that the PPAT drawings of school age children, like those as adults comprise three main content factors: the tree potency, the tree accessibility, and the drawn person's agency. This supports the claim about the efficiency of the holistic approach in analysing projective drawings.(McGrath \& Carroll, 1992). Results demonstrated that certain PPAT narratives were associated with maternal hostility: specifically, children that reported higher levels of maternal rejection tended to draw a less potent tree and a person with neutral to low agency. These drawn objects might be representative of the children's internal working models, and thus influenced by their relational expectations, i.e. their hope to receive assistance from other people, how cooperatively they interact, their self-worth, and their ability to achieve goals (Grossmann, Grossmann, \& Waters, 2006). As regards to gender diferences, associations between parental hostility and a non-coherent PPAT script were found only among boys. The results indicate that the pictorial space of children's drawings, communicated through various symbolization and a thematic script may reveal their subjective experience (Malchiodi, 1998). This research establishes that a broader observation of drawing narratives or script is required to understand the child's subjective relational experience, especially when observing normative middle-childhood children, as was the case in this study. This is similar to clinical work with clients, where clinicians attempt to gain access to the client's relational scripts through personal narratives (McLeod, 1997).

\section{References}

Artemis, G., \& Touloumakos, A. K. (2016). “They Accept Me, They Accept Me Not” Psychometric Properties of the Greek Version of the Child Parental Acceptance Rejection Questionnaire-Short Form. Journal of Family Issues, 37(9), 1226-1243.

Bat Or, M., \& Ishai, R. (2016). Form and content in adults 'Person Picking an Apple from a Tree' drawings as related to their attachment. International Journal of Art Therapy, 21(1), 2-14. 
Bat Or, M., Ishai, R., \& Levi, N. (2014). Validating PPAT's symbolic meanings of emotional and cognitive functioning among children. The Arts in Psychotherapy, 41(4), 309-319.

Bat Or, M., Ishai, R., \& Levi, N. (2017). Symbolic Contents in PPAT drawings (SC-PPAT/c): Children (10-12 years). A Manual for rating PPAT drawings. University of Haifa.

Clarke, G. S., \& Scharff, D. E. (2014). Fairbairn and the object relations tradition. London: Karnac Books.

Dwairy, M. (2010). Parental acceptance-rejection: a fourth cross-cultural research on parenting and psychological adjustment of children. Journal of Child and Family Studies, 19(1), 30-35.

Flanagan, L. M. (2016). Object relations theory. In J. Berzoff, L. M. Flanagan, P. Hertz (Eds). Inside out and outside in: Psychodynamic clinical theory and psychopathology in contemporary multicultural contexts ( $4^{\text {th }}$ ed, pp. 123-165). Rowman \& Littlefield.

Gantt, L. (1990). A validity study of the Formal Elements Art Therapy Scale (FEATS) for diagnostic information in patients' drawings (Unpublished doctoral dissertation). University of Pittsburgh, Pittsburgh, PA.

Gantt, L. (2004). The Case for Formal Art Therapy Assessments. Art Therapy: Journal of the American Art Therapy Association, 21(1), 18-29.

Gantt, L., \& Tabone, C. (1998). The Formal Elements Art Therapy Scale: A rating manual. Morgantown, WV: Gargoyle Press.

Giotsa, A., \& Touloumakos, A. K. (2014). Perceived Parental Acceptance and Psychological Adjustment The Moderating Role of Parental Power and Prestige Among Greek Pre-Adolescents. Cross-Cultural Research, 48(3), 250-258.

Giovazolias, T., Kothali, P., Louvrou, B., \& Mitsopoulou, E. (2010). The Role of Father Involvement in Children's Bullying Behavior: Empirical Findings from a Greek Sample. 3rd Congress on Interpersonal Acceptance-Rejection. Padua, Italy: 28-31 July.

Groh, A.M., Roisman, G.I., van IJzendoorn, M.H., Bakermans-Kranenburg, M.J., \& Fearon, R.P. (2012). The significance of insecure and disorganized attachment for children's internalizing symptoms: A meta-analytic study. Child Development, 83, 591-610.

Grossmann, K. E., Grossmann, K., \& Waters, E. (2006). Attachment from infancy to adulthood: The major longitudinal studies. New York, NY: Guilford Press.

Khaleque, A. (2013). Perceived parental warmth, and children's psychological adjustment, and personality dispositions: A meta-analysis. Journal of child and Family studies, 22(2), 297-306.

Khaleque, A. (2015). Parental acceptance and children's psychological adjustment. In Promoting Psychological Well-Being in Children and Families (pp. 226-243). UK: Palgrave Macmillan.

Khaleque, A., \& Rohner, R. P. (2012). Pancultural associations between perceived parental acceptance and psychological adjustment of children and adults: A meta-analytic review of worldwide research. Journal of cross-cultural Psychology, 43(5), 784-800.

Malchiodi, C. (1998). Understanding children's drawings. New York: Guilford Press.

McLeod, J. (1997). Narrative and psychotherapy. London: Sage.

Miles, C. J., \& Harold, G. T. (2003). Parents warmth and hostility, children's depression and aggression: A cross-lagged, longitudinal analysis. Paper presented at the meeting of the Society for Research in Child Development, Tampa, FL.

Putnick, D. L., Bornstein, M. H., Lansford, J. E., Malone, P. S., Pastorelli, C., Skinner, A. T., \& Alampay, L. P. (2015). Perceived mother and father acceptance-rejection predict four unique aspects of child adjustment across nine countries. Journal of Child Psychology and Psychiatry, 56(8), 923-932.

Rohner, R. P. (1980). Worldwide tests of parental acceptance-rejection theory: An overview. Cross-Cultural Research, 15(1), 1-21.

Rohner, R. P. (1990). Handbook for the study of parental acceptance and rejection ( $3^{\text {rd }}$ ed.). Storrs, CT: Rohner Research Publications

Rohner, R. P. (2015). Itroduction to interpersonal acceptance-rejection theory (IPARTheory), methods, evidence, and implications. Retrieved from http://scholarworks.gvsu.edu/orpc/vol6/iss1/4/

Rohner, R. P. (2016). Introduction to Interpersonal Acceptance-Rejection Theory (IPARTheory) and Evidence. Online Readings in Psychology and Culture, 6(1), 4.

Rohner, R. P., \& Khaleque, A. (2005). Parental acceptance-rejection questionnaire (PARQ): Test manual. Handbook for the study of parental acceptance and rejection, 4, 43-106.

Teglasi, H. (2013). The Scientific Status of Projective Techniques as Performance Measures. In D. H. Saklofske, C. R. Reynolds, \& V. L. Schwean (Eds.). The Oxford handbook of child psychological assessment. (pp. 113-128). New York: Oxford University Press.

Wright, L., \& Black, F. (2013). Monochrome males and colorful females: do gender and age influence the color and content of drawings?. SAGE Open (4)1-9. 


\title{
LINKS BETWEEN COGNITIVE DISTORTIONS AND COGNITIVE EMOTION REGULATION STRATEGIES IN NON-CLINICAL YOUNG ADULTHOOD
}

\author{
Romain Deperrois, \& Nicolas Combalbert \\ EA.2114, PAVeA Laboratory, University of Tours (France)
}

\begin{abstract}
The cognitive content-specificity hypothesis, based on Beck's cognitive theory (1976), suggests that automatic thoughts and emotional status are positively related. Cognitive distortions, which involve erroneous information processing, and cognitive emotion regulation strategies, which are strategies implemented at the cognitive level to modulate the emotional response, could be linked. The objective of this study was to study these relationships between cognitive distortions (positive/negative) and cognitive emotion regulation strategies (adaptive/non-adaptive) in a non-clinical young adult population. 96 participants (age 18-39 years; 48 men; 48 women) completed the French version of the Cognitive Emotion Regulation Questionnaire and the French Cognitive Distortions Scale for adults, a recent tool to quantify the behavioural expression of the presence of cognitive distortions in individuals' usual reasoning, using a dichotomy between positive/negative poles, according to the Franceschi's model (2007, 2008). Negative distortions were positively correlated with the use of non-adaptive strategies of cognitive emotion regulation, while positive distortions were negatively correlated with the use of adaptive strategies. The main distortion linked to the use of non-adaptive strategies was selective abstraction, while those linked to the use of adaptive strategies were positive dichotomous reasoning, positive neutral omission, and minimization. The results obtained make it possible to consider the possibility that there may be interaction profiles between the presence of cognitive distortions in individuals' usual reasoning and the use of cognitive emotion regulation strategies. Cognitive distortions interfere with adaptive and non-adaptive strategies in a non-clinical young adult population. Mental health cannot therefore be characterized by the absence of cognitive distortions and the use of only adaptive strategies of cognitive emotion regulation.
\end{abstract}

Keywords: Cognitive emotion regulation, cognitive distortions, information processing, young adulthood, cognitive content.

\section{Introduction}

Cognitive distortions, introduced by Beck (1964) in his work on depression, refer to attitudes, thoughts and beliefs, false or erroneous, that lead the individual to perceive events inappropriately and attribute negative meanings to their experiences (Kendall, 1991). Varying numbers and types of cognitive distortion have been identified and recognized by different authors (Kramer \& Drapeau, 2011). However, Franceschi (2007, 2008) proposed a model based on the classic classification of the cognitive distortions identified by Beck, specifying the relationships between them. This conceptual model is based on three notions: the reference class, which consists of a set of events, phenomena, objects, or stimuli in general; duality, which makes it possible to characterize an event of the reference class according to a dichotomy between 2 poles (positive/negative, internal/external, collective/individual, etc.); and the taxon system, which refers to an individual-specific classification of elements of the reference class in relation to each other according to a given duality. Franceschi (2007) also defined cognitive distortions as general (dichotomous reasoning, maximization, minimization, arbitrary focus, omission of the neutral and requalification in the opposite pole) or specific, regarded as instances of general cognitive distortions (disqualification of the positive, selective abstraction, catastrophism). Even if the presence of cognitive distortions in normal reasoning is related to the development of mood disorders (Beck, 1995), behavioural disorders, such as pathological gambling (Barrault \& Varescon, 2012), or personality disorders (Del Pozo, Harbeck, Zahn, Kliem \& Kröger, 2018), they are not specific to a clinical population, and any individual is prone to produce reasoning bias (Pennequin \& Combalbert, 2017).

It has frequently been shown that emotions and cognitive activity are linked, in both pathological and non-pathological populations (Krimbel, Nelson-Gray \& Mitchell, 2012). The hypothesis of specific cognitive content, based on Beck's cognitive theory (1976), postulates that an individual's mood can be 
characterized by a specific cognitive content. According to this hypothesis, the system of thought and emotional status would therefore be positively linked.

Introduced by studies in clinical psychology, emotion regulation refers to the set of extrinsic and intrinsic strategies implemented to reduce, maintain or increase emotion or emotional responses, and in particular their intensive and temporal characteristics (Gross, 2001). Concerning the conscious cognitive component of emotion regulation in Gross's model (1998), otherwise known as cognitive emotion regulation, a number of strategies have been observed, divided into two categories: adaptive strategies (i.e. those that allow a beneficial mode of functioning over the long term), and non-adaptive strategies (Garnefski, Kraaij \& Spinhoven, 2001).

The relationship between cognitive distortions and cognitive emotion regulation has been emphasized by the study of the attention process. This process has been shown to be involved in information processing, and therefore in the presence of cognitive distortions (Franceschi, 2008), as well as in the cognitive emotion regulation process (Van der Linden, Rochat \& Billieux, 2006). The relationship between cognitive distortions and cognitive emotion regulation has also been highlighted by studies of emotional disorders. Depression is characterized by a disruption of the emotion regulation process underpinned by excessive use of cognitive distortions (Joormann \& Stanton, 2016).

To date, few studies have looked at the links between the presence of cognitive distortions in normal reasoning and the use of cognitive emotion regulation strategies in individuals in the general population (Robert, Combalbert \& Pennequin, 2017). For this reason, this study proposes to explore the links between these different types of cognitive information processing in a non-clinical young adult population, using Franceschi's model to draw the profile of cognitive distortions, and to identify distortions likely to be most associated with the use of cognitive emotion regulation strategies. Based on previous results obtained from clinical populations, we made the general hypothesis that the type and frequency of cognitive distortions would influence the type of cognitive emotion regulation used by a non-clinical young adult population.

\section{Method}

\subsection{Participants and procedure}

The sample consisted of 96 participants from the general population, including 48 men $(M=25.17 \pm 5.48$ years $)$ and 48 women $(M=24.79 \pm 5.73$ years $)$, aged 18 to $39(M=24.98 \pm 5.58$ years). Average years of formal education was 13.93 (SD = 2.23; $13.92 \pm 2.21$ for men, $13.94 \pm 2.26$ for women), which corresponds to a two year degree after the baccalaureate. None of the participants were receiving professional medical or psychological care. Each participant completed the Cognitive Emotion Regulation Questionnaire and the Cognitive Distortions Scale for adults, alone and in a quiet environment. Participants were informed that their responses would be anonymous. A single experimenter collected the data at the participants' homes. The order in which the tools were presented was counterbalanced. An Internet version of the form was also made available online, using the Google Forms application. Participants were instructed to find a quiet place to answer the questions; at the end of the form they were asked whether they had done so. No significant differences were found between the results of the paper and the Internet versions of the form (T-test carried out for each variable with $\mathrm{p}<.01$ ). The results were therefore aggregated. Time to complete the questionnaire was 15-20 minutes.

\subsection{Measures}

1- To assess the presence of cognitive distortions in the normal reasoning of individuals, we used the French Cognitive Distortions Scale for adults developed by Robert et al. (Robert, Combalbert, Pennequin, Deperrois \& Ouhmad). This is a self-report questionnaire composed of 42 scenarii related to everyday life events, with suggestions about what individuals might think in a similar situation. For each scenario, participants assign a score to the corresponding proposal, ranging from (0) doesn't correspond at all to what I think to (10) corresponds absolutely to what I think. The cognitive distortions thus evaluated are: dichotomous reasoning (i.e. seeing events in black or white, without shades of gray, and hence perceiving a reference class only in relation to the extreme taxon of each pole), omission of the neutral (i.e. disregarding neutral aspects of events and seeing them only as positive or negative), disqualification of one pole (i.e. giving arbitrary preference to one pole to characterize the events of the reference class), requalification in the opposite pole (i.e. characterizing as negative an event that should objectively be seen as positive and vice versa), arbitrary focus (i.e. focusing on one event in the reference class while ignoring all others), minimization (i.e. assigning less significance to an event in the reference class than its objective importance), and maximization (i.e. assigning greater significance to an event in the reference class than its objective importance). Each cognitive distortion is targeted by 6 scenario/proposal pairs, 3 suggesting a positive distortion (i.e. carried out on the positive side of the spectrum), and the other 3 suggesting a negative distortion (i.e. carried out on the negative side of the spectrum). 
The Cognitive Distortions Scale for adults has good internal consistency for each subscale, with an alpha coefficient of 0.67 to 0.87 , and excellent internal consistency for the whole scale, with an alpha coefficient of 0.94 (Robert et al.).

2- The Cognitive Emotion Regulation Questionnaire, developed by Garnefski, Kraaij and Spinhoven (2001), is a 36-item self-report questionnaire designed to assess individuals' cognitive emotion regulation capacity. The French version was developed and validated by Jermann et al. (Jermann, Van der Linden, d'Acremont \& Zermatten, 2006). This tool makes it possible to measure the preferential use of nine distinct cognitive emotion regulation strategies, in relation to what individuals think (and not what they do) following a negative and stressful life event. The strategies are divided into two categories: (1) adaptive strategies, including acceptance, positive refocusing, refocusing on planning, positive reappraisal (i.e. giving positive meaning to the event and drawing positive conclusions from it in terms of personal growth, for example), and putting into perspective, and (2) non-adaptive strategies, which include self-blame, blaming others, rumination, and catastrophizing. Participants rate the items on a Likert scale, ranging from (1) almost never to (5) almost always, according to what they think when confronted by a negative or unpleasant event. Each strategy is targeted by 4 items.

Each subscale of the French version of the Cognitive Emotion Regulation Questionnaire has good internal consistency, with an alpha coefficient of 0.68 to 0.87 (Jermann et al., 2006).

\section{Results}

There was no significant effect of age on any of the cognitive distortions and the cognitive emotion regulation strategies. We found a positive correlation between level of education and distortions: disqualification of one pole $+(\mathrm{r}=0.35, \mathrm{p}<.05)$, requalification in the opposite pole $+(\mathrm{r}=0.22, \mathrm{p}<.05)$, and arbitrary focus $+(\mathrm{r}=0.34, \mathrm{p}<.05)$. T-tests also revealed an effect of sex on requalification in the opposite pole $-(\mathrm{t}(94)=2.72, \mathrm{p}<.01)$ and on maximization $+(\mathrm{t}(94)=10.27, \mathrm{p}<.05)$. Both types of distortion were found more among men than women. We found a positive correlation between level of education and acceptance $(r=0.22, \mathrm{p}<.05)$, and between level of education and catastrophizing $(\mathrm{r}=0.23, \mathrm{p}<.05)$. We also observed an effect of sex on acceptance $(\mathrm{t}(94)=2.20, \mathrm{p}<.05)$ and blaming others $(\mathrm{t}(94)=3.04, \mathrm{p}<.05)$; men used both these strategies more than women.

We observed a positive correlation between negative distortions and the use of non-adaptive cognitive emotion regulation strategies $(r=0.33, \mathrm{p}<.001)$, and a negative correlation between positive distortions and the use of adaptive strategies $(r=-0.23, p<.05)$. We also analyzed the effect of cognitive distortions on the use of cognitive emotion regulation strategies using T-Tests. This enabled us to divide participants into 2 separate groups based on the overall score for the Cognitive Distortion Scale according to the standard defined by the tool $(\mathrm{M}=144.21 \pm 36.94)$ : group $1(\mathrm{~N}=51, \mathrm{M}=117.59 \pm 18.46)$ consisted of participants with scores below the standard, and group $2(\mathrm{~N}=45, \mathrm{M}=165.89 \pm 14.39)$ consisted of participants with scores above the standard. The data show that participants in group 1 used adaptive cognitive emotion regulation strategies more than those in group $2(\mathrm{t}(94)=2.49, \mathrm{p}<.05)$, and that there was no significant difference in the use of non-adaptive strategies $(\mathrm{t}(94)=-1.85$, NS).

In order to identify the cognitive distortions most associated with adaptive and non-adaptive cognitive emotion regulation strategies, we conducted two forward stepwise regression analyses (Table 1 \& 2).

Table 1. Steps of the forward stepwise regression concerning adaptive cognitive emotion regulation strategies for each cognitive distortion.

\begin{tabular}{lllll}
\hline Step & Variables & $\boldsymbol{\beta}$ & $\mathbf{R}^{2}$ & F Change \\
\hline Step 1 & Dichotomous reasoning + & $0.3464 *$ & 0.1200 & $12.816 *$ \\
Step 2 & Dichotomous reasoning + & $0.3102 *$ & 0.1200 & $12.816 *$ \\
& Minimization + & $-0.2387 *$ & 0.1757 & $9.909 *$ \\
Step 3 & Dichotomous reasoning + & $0.2445 *$ & 0.1200 & $12.816 *$ \\
& Minimization + & $-0.2533 *$ & 0.1757 & $9.909 *$ \\
& Neutral omission + & $-0.2337 *$ & 0.2260 & $8.957 *$ \\
Step 4 & Dichotomous reasoning + & $0.2896 *$ & 0.1200 & $12.816 *$ \\
& Minimization + & $-0.2914 *$ & 0.1757 & $9.909 *$ \\
& Neutral omission + & $-0.2276 *$ & 0.2260 & $8.957 *$ \\
& Minimization - & $0.2010 *$ & 0.2625 & $8.098 *$ \\
\hline
\end{tabular}

NS: Non-Significant; * $\mathrm{p}<.05$ 
Concerning the use of adaptive cognitive emotion regulation strategies, the best model corresponds to step 4, which explained $26.25 \%$ of the score variance, and included dichotomous reasoning $+(12 \%$ of the variance), minimization $+(5.57 \%)$, neutral omission $+(5.03 \%)$, and minimization $-(3.65 \%)$.

Table 2. Steps of the forward stepwise regression concerning non-adaptive cognitive emotion regulation strategies for each cognitive distortion.

\begin{tabular}{lllll}
\hline Step & Variables & $\boldsymbol{\beta}$ & $\mathbf{R}^{2}$ & F Change \\
\hline Step 1 & Arbitrary focus - & $0.3255 *$ & 0.1059 & $11.139 *$ \\
Step 2 & Arbitrary focus - & $0.2486 *$ & 0.1059 & $11.139 *$ \\
& Maximization - & 0.1825 NS & 0.1333 & $7.155 *$ \\
\hline NS: Non-Significant; * $<-05$ & & &
\end{tabular}

Concerning the use of non-adaptive strategies, the best explanatory model is the one presented in step 1, explaining $10.59 \%$ of the score variance, composed of arbitrary focus.

\section{Discussion}

The purpose of this research was to show that the type and frequency of cognitive distortions affect the type of cognitive emotion regulation used by non-clinical young adults in the general population. A further aim was to study which of the distortions identified by Franceschi (2007) are most associated with the use of adaptive and non-adaptive cognitive emotion regulation strategies.

According to our results with non-clinical adults, greater use of non-adaptive cognitive emotion regulation strategies is related to negative distortions in normal reasoning. By contrast, less use of adaptive cognitive emotion regulation strategies is related to positive distortions. More cognitive distortions (positive and negative) led to greater recourse to non-adaptive cognitive emotion regulation strategies, at the expense of adaptive strategies. These data are comparable to the results of studies of clinical populations with emotional disorders (Joormann \& Stanton, 2016). Accordingly, our research hypothesis is validated. However, it would appear that the propensity to use non-adaptive strategies depends only on the presence of cognitive distortions in normal reasoning, whereas the use of adaptive strategies is modulated by the number and intensity of cognitive distortions. These observations show that the ability to consciously regulate emotional status is affected, along with mood, by the presence of errors of reasoning in cognitive information processing. The cognitive component of emotion regulation would therefore depend on the specific cognitive content of each individual (Kendal \& Ingram, 1989).

Our results show that the cognitive distortions most associated with the use of adaptive cognitive emotion regulation strategies are positive dichotomous reasoning, minimization and positive neutral omission. Dichotomous reasoning is polarized reasoning, devoid of nuances. This is a cognitive distortion that leads the individual to perceive a reference class only according to the extreme taxa of each pole. Thus, preference is attributed to the extreme taxon of the corresponding pole. For example, a positively oriented person who was not hired following a job interview would think that they were too good for the job. Neutral omission leads the individual to ignore the neutral taxon of the reference class. This absence of neutrality leads the person to consider all possible neutral events as either positive or negative. In its positive orientation, the neutral omission is biased towards the corresponding pole and neutral events are thus seen as positive. The common point between these 2 cognitive distortions is that they direct the individuals' reasoning only to positive elements. The subjective interpretation of the situation on which the reasoning is based is erroneous because negative elements are not considered. Minimization is a cognitive distortion whereby an element of the reference class is assigned a lower taxon based on duality. Thus, negative events are considered less negative and positive events less positive. Reasoning is based on elements of the situation, whose emotional and intensity valence is subjectively attenuated. The presence of these different distortions in normal reasoning tends to explain the observed decrease in the use of adaptive cognitive emotion regulation strategies, which require objective consideration of all aspects (positive and negative) of an event, based on a well-functioning cognitive process of emotion regulation.

The cognitive distortion most associated with the use of non-adaptive cognitive emotion regulation strategies is negative arbitrary focus. Arbitrary focus leads to giving precedence to one of the taxa in the reference class by ignoring the others. In its negative orientation, negative arbitrary focus (or selective abstraction) leads the individual to consider the reference set only in terms of an event with a negative connotation. The individual's reasoning is then biased by that experience. For example, a person who received a fine in the past will expect to relive this negatively charged event every time they receive a letter. This reduction of the multiple elements of a situation to a single element with negative emotional 
connotation leads the individual to construct an erroneous interpretation of the situation, and underlies the use of non-adaptive cognitive emotion regulation strategies. This type of reasoning centered on the negative aspects of a situation is found more commonly in depressive symptomatology (Ehring \& Watkins, 2008).

Our results show that the cognitive process of emotion regulation is dependent on the individual's own cognitive processing. Our predictive models suggest that the preferred use of adaptive or non-adaptive cognitive emotion regulation strategies by a non-clinical young adult population is affected by the ability, underpinned by automatic thoughts, to focus reasoning on positive events and to minimize or even ignore negative events, while the use of non-adaptive strategies is linked to the tendency to make a negative and pessimistic judgment of events, underpinned by personal experience and cognitive patterns (Beck, 1964).

\section{References}

Barrault, S., \& Varescon, I. (2012). Cognitive distorsions and gambling behaviours: State of the question. Psychologie Française, 57, pp. 17-29.

Beck, A.T. (1964). Thinking and depression: II. Theory and therapy. Archives of General Psychiatry, 10 (6), pp. 561-571.

Beck, A.T. (1976). Cognitive therapy and the emotional disorders. New York: New American Library.

Beck J.S. (1995). Cognitive therapy: basics and beyond. New York: Guilford.

Del Pozo, M.A., Harbeck, S., Zahn, S., Kliem, S., \& Kröger, C. (2018). Cognitive distortions in anorexia nervosa and borderline personality disorder. Psychiatry Research, 260, pp. 164-172.

Ehring, T. \& Watkins, E.R. (2008). Repetitive negative thinking as a transdiagnostic process. International Journal of Cognitive Therapy, 1, pp. 192-205.

Franceschi, P. (2007). Compléments pour une théorie des distorsions cognitives. Journal de Thérapie Comportementale et Cognitive, 17 (2), pp. 84-88.

Franceschi, P. (2008). Théorie des distorsions cognitives: application à l'anxiété généralisé. Journal de Thérapie Comportementale et Cognitive, 18, pp 127-131.

Garnefski, N., Kraaij, V. \& Spinhoven, P. (2001). Negative life events, cognitive emotion regulation and emotional problems. Personality and Individuals Differences, 30, pp. 1311-1327.

Gross, J.J. (1998). The emerging field of emotion regulation: An integrative review. Review of General Psychology, 2, pp. 271-299.

Gross, J.J. (2001). Emotion regulation in adulthood: Timing is everything. Current Directions in Psychological Science, 10, pp 214-219.

Jermann, F., Van der Linden, M., D'Acremont, M. \& Zermatten, A. (2006). Cognitive Emotion Regulation Questionnaire (CERQ): Confirmatory factor analysis and psychometric properties of the french translation. European Journal of Psychological Assesment, 22, pp. 126-131.

Joormann, J. \& Stanton, C.H. (2016). Examining emotion regulation in depression: A review and future directions. Behaviour Research and Therapy, 86, pp. 35-49.

Kendall, P.C. (1991). Guide theory for therapy with children and adolescents. In: Child and Adolescent Therapy: Cognitive-Behavior Procedures (pp. 3-24). New York: Guilford.

Kramer, U. \& Drapeau, M. (2011). Etude de validation de la version française des échelles de codage du coping et des erreurs cognitives (CE-CAP) sur une population non clinique. Annales Médico-Psychologiques, 169, pp. 523-527.

Kendall, P.C. \& Ingram, R.E. (1989). Cognitive-behavioral perspectives: theory and research on depression and anxiety. In: Kendall PC, Watson D, editors. Anxiety and Depression: Distinctive and Overlapping Features (pp. 27-53). San Diego: Academic Press.

Krimbel, N.A., Nelson-Gray, R.O. \& Mitchell, J.T. (2012). BIS, BAS, and bias: the role of personality and cognitive bias in social anxiety. Personality and Individuals Differences, 52, pp. 395-400.

Pennequin, V., \& Combalbert, N. (2017). L'influence des biais cognitifs sur l'anxiété chez des adultes non cliniques. Annales Médico-Psychologiques, 175, pp. 103-107.

Robert, A., Combalbert, N. \& Pennequin, V. (2018). Etude des profils de distorsions cognitives en fonction des états anxieux et dépressifs chez des adultes tout-venants. Annales Médico-Psychologiques, 176 (3), pp. 225-230.

Van der Linden, M., Rochat, L. \& Billieux, J. (2006). Troubles du comportement socio-émotionnel et impulsivité: une approche cognitive et neuropsychologique. In: P. Azouvi, J-M. Mazaux \& P. Pradat-Diehl (Eds), Comportement et lésions cérébrales. Paris: Frison-Roche. 


\title{
COGNITIVE DISTORTIONS AND EMOTION REGULATION AMONG POST TRAUMATIC STRESS DISORDER VICTIMS
}

\author{
Nawal Ouhmad ${ }^{1}$, Nicolas Combalbert ${ }^{1}$, \& Wissam El-Hage ${ }^{2}$ \\ ${ }^{1}$ Laboratory PAVEA, University of Tours (France) \\ ${ }^{2}$ Psychiatric Clinic, Saint Cyr sur Loire (France)
}

\begin{abstract}
Many authors have studied the links between cognition and emotions, including the links between cognitive distortions and emotional cognitive regulation strategies. Nevertheless, the explorations of these two instances in a clinical population presenting a posttraumatic stress disorder have very little been explored. The literature on this subject suggests that a specific profile could emerge in these subjects compared to a different clinical population and / or non-clinical. 184 subjects divided into three groups (60 subjects exposed to a traumatic event with PTSD, 61 subjects exposed to a traumatic event without PTSD, 63 control subjects) passed the CERQ, PCL-5 and a cognitive distortions scale.

The main results, which are preliminary results. highlight the presence of a profile distinguishing subjects with PTSD. They have difficulties in managing their emotions, as well as increased use of cognitive distortions, but they do not use them the most. The results lead us to question the cognitive functioning of people with PTSD but also the importance and consequences of therapeutic and drug monitoring of these patients.
\end{abstract}

Keywords: Cognitive distortions, cognitive emotional regulation, psychic trauma, posttraumatic stress disorder.

\section{Introduction}

Post-Traumatic stress disorder (PTSD) is a psychopathological disorder that is characterized by "the development of specific symptoms following exposure to a traumatic event in the context of death, death threats, serious injury or sexual assault "(WHO, 2016). Since 2013, PTSD has been classified as a "trauma and stress disorder" in the Diagnostic and Statistical Manual of Mental Disorders, Fifth Edition (DSM-5). PTSD is characterized by the appearance of psychiatric symptoms after exposure to one or more traumatic events. Generally, the diagnosis of PTSD is made after the month following the traumatic event (American Psychiatric Association, DSM 5, 2013). When an individual experience or witnesses a traumatic event, he may experience intense fear, helplessness or horror. Following this, a series of symptoms may appear, like cognitive distortions or difficulties to regular our emotions.

Beck $(1963,1964)$ defines cognitive distortions as fallacious reasoning that plays a determining role in the emergence of a number of mental disorders. Franceschi (2007) clarified the conceptual framework of cognitive distortions, distinguishing three key notions. The reference class consists of a set of phenomena or objects, for example, the reference class is composed of the character traits of the patient. The notion of duality, which corresponds to a pair of concepts such as positive / negative, internal / external, collective / individual, etc. In concrete terms, this refers to a criterion from which the elements of the reference class can be evaluated. We can note by A / $\bar{A}$ a given duality or A and $\bar{A}$ for dual concepts. And the taxon system. The taxonomy is a series of elements forming lists which concerns a domain. This taxonomy allows the patient to evaluate and classify the reference class according to criteria corresponds to a given duality A / $\overline{\mathrm{A}}$.

Regarding regulation of emotions, James Gross (1998) reintroduces the concept of emotional regulation into the field of cognitive science. The regulation of emotions refers to "the modulation of current emotional responses through a feedback loop on these responses" (Oschsner \& Gross, 2005). This refers to the set of strategies used by an individual to reduce, maintain or increase emotion or emotional responses. Garnefski et al. (2001) developed a scale to measure the preferred use of nine cognitive regulation strategies for emotions. 
Adaptive strategies group together several domains. Acceptance, when the individual expresses thoughts of acceptance and resignation in the face of the lived event. Positive concentration, focusing on more joyous and pleasant thoughts to deal with the lived event while concentration on the action is the concentration on the steps to follow to effectively manage the lived event. The positive reassessment will be when the individual will determine a positive sense of the event lived and will draw positive conclusions, finally, putting into perspective that is the fact of minimizing the seriousness of the event lived and highlighting its relative relativity. to other events considered more serious. Non-adaptive strategies take up the blame of oneself, which is the fact of taking responsibility for the lived event, contrary to the blame of others, where the responsibility will be attributed to the other. The ruminating of focusing on the feelings and thoughts associated with the lived event and dramatization, when the individual explicitly emphasizes the negative aspects of the lived event.

Studies have highlighted the relationship between emotion regulation and cognitive distortions. Gratz and Roemer (2004) showed that people suffering from PTSD had difficulty regulating their own emotions during an experience of emotional distress. Bardeen's (2013) study suggests an association between difficulties related to cognitive regulation of emotions and symptoms of PTSD. Other studies show that emotional regulation strategies in subjects with PTSD are generally unsuitable (Foa, 2007, Fairholme et al., 2013). However, few studies have investigated the link between cognitive distortions and cognitive regulation of emotions (for review Joorman \& Stanton, 2016). However, it seems that individuals who have been exposed to a traumatic event and suffer from PTSD have an alteration of the processes of regulation of emotions and produce cognitive distortions. The interest of this study is therefore to establish the link between cognitive distortions and cognitive regulation of emotions within a pathological population.

To our knowledge, few studies have been completed with possible interactions between cognitive regulation of emotions and cognitive distortion in a population reported by PTSD (Lau, Pettit \& Creswell, 2013). This study has several main objectives, like to link the production of cognitive distortions with emotion regulation strategies. The second is to determine that there is a profile of ESPT subjects regarding these two dimensions. The goal of this research is spreading beyond the positive impact of the specific profile of cognitive distortions and cognitive regulation of emotions. A profile that would not be present in exposed subjects without PTSD.

\section{Method \& protocol}

One hundred and eighty-three subjects divided into three groups participated in our study. A group with PTSD following exposure to a traumatic event $(n=60)$, a group of subjects exposed to a traumatic event but who did not develop PTSD $(n=63)$ and a control group $(n=63)=61)$ to identify possible differences between the 3 groups.

The PCL-5 (Weathers, 2013) is a 20-item self-administered questionnaire that assesses the 20 symptoms of PTSD according to DSM 5. The PCL-5 has several goals, including assessing changes in symptoms before and after treatment, screening of individuals with PTSD, and the provisional diagnosis of PTSD. Responses are rated from 0 (not at all) to 4 (extremely).

The assessment scale of adult cognitive distortions of Robert, Combalbert, Pennequin, Deperrois \& Ouhmad (2018) was validated on a population of 916 French subjects (765 women and 151 men) aged 18 to $85(\mathrm{M}=33.9, \mathrm{SD}=14.8)$. This scale includes 42 mini-scenarios on everyday life based on cognitive distortions presented in Franceschi's (2007) model. Each scenario is accompanied by a proposal of what one might think in a similar situation. The response proposals correspond to cognitive distortions. The distortions are divided into 7 subscales (dichotomous reasoning, omission of the neutral, disqualification of one of the poles, arbitrary focus, maximization, minimization, requalification in the other pole) each comprising 6 scenarios. Three suggest "positive" valence distortion, 3 suggesting "negative" valence distortion. The subjects must assign a score ranging from 0 (does not correspond at all to what I think) to 10 (corresponds quite to what I think). A high score signifying the subject's adherence to distortion.

The aim of the Cognitive Emotion Regulation Questionnaire, developed by Garnefski, Kraaij and Spinhoven (2001), is to evaluate the cognitive and emotional regulation capacity of individuals. It contains 36 items and was developed and validated in its French version (Jermann et al., 2006). This tool measures the preferential production of emotional regulation strategies about what people think and not what they do. Each of the strategies listed below is targeted by 4 different items.

This research was conducted in compliance with the Code of Ethics (1996 updated in February 2012). Participation was free, entirely voluntary and anonymous. The results have been submitted to statistical studies, respecting the confidentiality of each one. The participants were informed that they could stop their participation in the study and that they had access to give them, they also received no bonuses and they signed a free and informed consent form. 
All three groups were split according to the PCL-5 score. The data was analyzed using the Statistica software (Version 13). One-way ANOVAs (Analysis of Variance) were conducted to compare our three groups. We also have correlation analyzes between the two variables emotional cognitive regulation and cognitive distortions.

\section{Results}

Figure 1. Differences in cognitive distortions profiles (positive and negative) between the three groups.

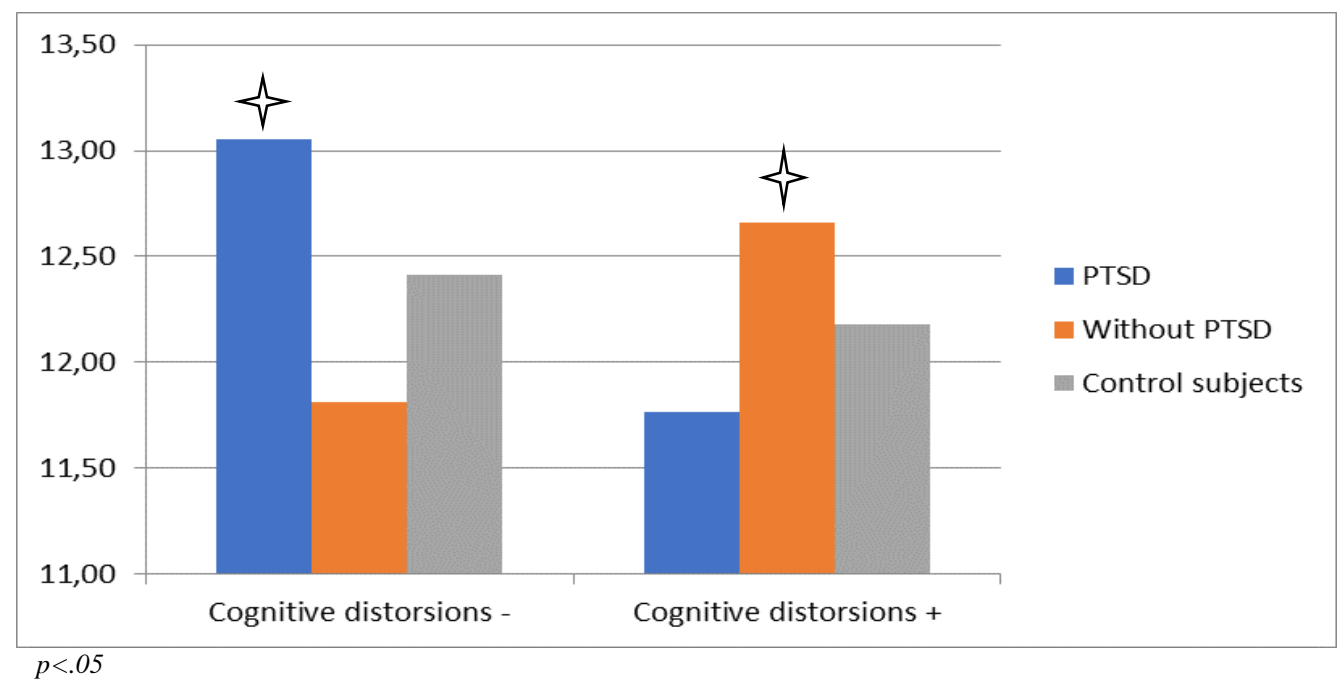

The results show that the group "exposed without PTSD" uses significantly more CD- than CD + . There is also a significant difference between the groups concerning positive cognitive distortions $(\mathrm{F}(2,181)=8,727, \mathrm{p}<.01)$. This is not the case for the negative cognitive distortions $(\mathrm{F}(2,181)=1,985$, $\mathrm{p}>1)$.

Table 1. Differences in non-adaptative emotional regulation between the three groups.

\begin{tabular}{|c|c|c|c|}
\hline & PTSD & Whhout PTSD & $\begin{array}{l}\text { Control } \\
\text { Subjects }\end{array}$ \\
\hline Blâme de soĩ & $10,92(4,85)$ & $8,84(4,45)$ & $10,48(4,44)$ \\
\hline Rumination & $13,45(4,07)^{*}$ & $10,79(4,34)$ & $12,64(4,40)$ \\
\hline Dramatisation & $10,75(4,42)^{*}$ & $8,18(4,01)$ & $7,77(3,55)$ \\
\hline Blâme d'autruĩ & $9,20(4,79)^{*}$ & $9,10(5,28)$ & $7,03(3,35)$ \\
\hline NAR Total & $44,32(12,54)^{*}$ & $36,90(13,16)$ & $37,92(11,75)$ \\
\hline
\end{tabular}

In terms of emotional cognitive regulation, the results show that all groups have significantly higher scores on non-adaptive regulation. Significant results are noted notably for rumination ( $\mathrm{f} 2,181=5,919)$ at $\mathrm{p}<.05)$, dramatization $(\mathrm{F} \mathrm{2,181=8,52)}$ at $\mathrm{p}<.05)$, and blame of others $(\mathrm{F} 2,181=4,67)$ at $\mathrm{p}<.05)$.

\section{Discussion}

The main objective of the study was to verify the presence of specific profile in people with PTSD. The results do suggest the existence of this specific profile concerning cognitive distortions and emotional cognitive regulation in individuals with post-traumatic stress disorder. 
One the one hand, we hypothesized that PTSD would use more negative cognitive distortions because we thought they would be more likely to blame themselves and feel responsible. According to Chung et al., (2014), the characteristics of a trauma can influence the management techniques of the traumatic event as well as its consequences. It is at this point that individuals will negatively evaluate events and thus develop erroneous thought patterns. The results of our study seem to invalidate this postulate. Although individuals with PTSD have a particular profile of cognitive distortions compared to other groups, they seem to be moving in the opposite direction. Indeed, the individuals ESPT seem to use more positive distortions and less negative distortions than the other two groups. These results can be explained by the fact that ESPT subjects benefit from a therapeutic follow-up (psychologist / psychiatrist and / or drug treatment) directly related to developed PTSD.

One the other hand, and consistent with previous studies, we hypothesized that PTSD would use more non-adaptive emotional regulation strategies than the other two groups. The results confirm our hypothesis. Indeed, PTSD is positively associated with strategies of emotional avoidance and control (Roemer et al., 2001, Bardeen et al., 2015) but also with regulatory strategies considered appropriate (Aldao et al. 2010, Webb et al., 2012). In addition, the results show that ESPT individuals preferentially use non-adaptive strategies such as rumination, dramatization, and blaming others. This suggests that PTSD individuals are more likely to blame themselves than others. Our results therefore suggest that people exposed to trauma who developed PTSD would have a particular profile of cognitive distortion production. Indeed, it seems that they produce more positive valence cognitive distortions. In addition, it appears that ESPT subjects use cognitive regulation strategies for inappropriate emotions.

Despite the observations made, it is pertinent to consider the practical and methodological limits of this research. Concerning the protocol: first of all, subjects do not have a conscious access to all the strategies they use, be they cognitive distortions or strategies of regulation of emotions. These are not strategies that subjects voluntarily put in place. Second, the form of the handover (in-line or face-to-face) may be biased. Indeed, only the subjects ESPT were received by the experimenter in order to pass the protocol. For the sake of social desirability, they have been able to minimize or manage some of their emotions. On the other hand, the control and exposed groups without PTSD responded to the online protocol; again, biases such as social desirability may occur. All of these biases are difficult to control. Concerning the population: ESPT subjects are followed by a professional (psychologist or psychiatrist) and can have a treatment directly related to the trauma. Moreover, follow-ups and treatments can significantly reduce the symptomatology of the trauma.

In order to refine this study, it seems interesting to control as much as possible the impact of a possible therapeutic follow-up and a drug treatment on the individuals. By comparing for example, the effects of therapies: type of follow-up, drug treatment and the combination of both on the regulation of emotions and / or cognitive biases. One could see, for example, what types of bias these methods of care take first.

\section{References}

Aldao, A., Nolen Hoeksema, S \& Schweizer, S. (2010). Emotion-regulation strategies across psychopathology: A meta-analytic review. Clinical Psychology Review, 20, 217-237.

American Psychiatric Association. (2013). Diagnostic and statistical manual of mental disorders (5th edition text revision). Washington, DC: American Psychiatric Publishing.

Bardeen, J. R., Tull, M. T., Stevens, E. N., \& Gratz, K. L. (2015). Further investigation of the association between anxiety sensitivity and posttraumatic stress disorder: Examining the influence of emotional avoidance. Journal of Contextual Behavioral Science, 4, 163-169.

Chung MC, Jalal S, Khan NU. Posttraumatic stress disorder and psychiatric comorbidity following the 2010 flood in Pakistan: Exposure characteristics, cognitive distortions, and emotional suppression. Psychiatry 2014; 77:289-304.

Fairholme CP, Nosen EL, Nillni YI, Schumacher JA, Tull MT, Coffey SF. Sleep disturbance and emotion dysregulation as transdiagnostic processes in a comorbid sample. Behav Res Ther. 2013;51: 540-546.

Franceschi, P. (2007). Compléments pour une théorie des distorsions cognitives. Journal de Thérapie Comportementale et Cognitive, pp. 84-88.

Franceschi, P. (2008). Théorie des distorsions cognitives: application à l'anxiété généralisé. (Elsevier, Ed.) Journal de Thérapie Comportementale et Cognitive, 18, pp 127-131.

Foa EB, Hembree EA, Rothbaum BO. Prolonged Exposure Therapy for PTSD: Emotional Processing of Traumatic Experiences - Therapist Guide. Oxford, England: Oxford University Press, 2007. 
Garnefski, N., Kraaij, V., \& Spinhoven, P. (2001). Negative life events, cognitive emotion regulation and emotional problems. Personality and Individuals Differences, 30, pp. 13111327.

Gratz, K. L., \& Roemer, L. (2004). Multidimensional assessment of emotion regulation and dysregulation: Development, factor structure, and initial validation of the Difficulties in Emotion Regulation Scale. Journal of Psychopathology and Behavioral Assessment, 36, 41-54.

Gross, J.J. (2001). Emotion regulation in adulthood: Timing is everything. Current Directions in Psychological Science, 10, pp 214-219.

James, W. (1890). The principles of Psychology. New York : Henry Holt.

Jermann, F., Van der Linden, M., D'Acremont, M. \& Zermatten, A. (2006). Cognitive Emotion Regulation Questionnaire (CERQ): Confirmatory factor analysis and psychometric properties of the french translation. European Journal of Psychological Assesment, 22, pp. 126-131.

Joormann, J. \& Stanton, C.H. (2016). Examining emotion regulation in depression: A review and future directions. Behav Res Ther, 86, pp. 35-49.

Lau, J.Y.F., Pettit, E. \& Creswell, C. (2013). Reducing children's social anxiety symptoms: exploring a novel parent-administered cognitive bias modification training intervention. Behav Res Ther, 51, pp. 333-337.

Ochsner, K.N. and Gross, J.J. (2005) The cognitive control of emotion. Trends in Cognitive Sciences, 9, 242-249.

Webb, T. L., Miles, E., \& Sheeran, P. (2012). Dealing with feeling: A meta-analysis of the effectiveness of strategies derived from the process model of emotion regulation. Psychological Bulletin, 138, 775-808 


\title{
ACUTE POLYMORPHOUS PSYCHOTIC DISORDER. EVOLUTIONARY POSSIBILITIES
}

\author{
Simona Trifu ${ }^{1}$, Alexandra Popescu ${ }^{2}, \&$ Eduard George Carp ${ }^{3}$ \\ ${ }^{1}$ University of Medicine and Pharmacy "Carol Davila”, Bucharest (Romania) \\ ${ }^{2}$ Hospital for Psychiatry “Alex. Obregia”, Bucharest (Romania) \\ ${ }^{3}$ Hospital for Psychiatry Sapunari (Romania)
}

\begin{abstract}
Motivation: psychotic confusion is often a psychiatric emergency encountered in several mental disorders, making it difficult to distinguish between a major depressive episode that overlaps perceptual distortions and delirious ideation, and an acute psychotic episode that risks developing into schizophrenia. A meticulous psychiatric interview performed after the remission of the psychotic phenomenology, bearing in mind the chronology of events and the fine analysis of the dispositional changes dictate the evolution and prognosis.

Objectives: we aim to highlight the functioning mechanisms and the therapeutic response of a 27-year-old female patient, admitted for an acute polymorphic psychotic episode, who presented schizophrenic symptoms, developed on a paranoid-narcissistic personality structure. The existence of rejection sensitivity prior to this episode calls for a differential diagnosis with a depressive episode of psychotic intensity, which gives rise to the opportunity of treatment with antidepressants, with careful consideration of the potential effect on the current psychotic episode.

Methods: emergency psychiatric hospitalization, medical surveillance, injectable antipsychotic administration due to negative symptoms and food refusal, daily psychiatric monitoring, EEG, cerebral MRI, psychological evaluation, psychodynamic interview, supportive psychotherapy for self-help.

Results: in the construction of the patient's diathesis, we identify early abandonment and change of attachment figure, personality traits from the narcissistic registry, fragility of the ego, sensitivity. Premorbid, uses defense mechanisms such as denial, repulsion, and vice versa. At the beginning of a young adult's life, the patient encounters a few failures in the workplace as well as in private life, which has changed his mood in the depressed sense over the past 6 months, the patient refusing SSRI type anti-depressives and psychotherapy. Later on, hallucinations and auditory pseudo-hallucinations, delusional preoccupation concerns centered on body function, and the idea of maintaining body image, the somatic delirium sketch, with bizarre behavioral changes, stereotypes and postures, in the last days before admission overwhelming food negativity and psychotic confusion. The rapid cutback of the episode is done with the classic injectable neuroleptic with subsequent passage on Aripirazole and the re-evaluation of the depressive component for the choice of the dual antidepressant SSRI.

Conclusions: the paranoid-narcissistic personality structure and the prevalence of denial mechanisms dictated the diagnosis of polymorphic psychotic disorder, wherein the central concerns are of a somatic nature, necessitating antidepressants and being post psychotic. There was an unfavorable response to the SSRI (Sertraline) which led to the psychotic distress becoming more intense. The treatment with dual AD (Mirtazapine) ultimately gave a positive result.
\end{abstract}

Keywords: Polymorphous psychotic disorder, somatic delirium, psychotic denial, paranoid-narcissistic personality structure.

\section{Patient history}

Patient D., a 27-year-old girl, graduate of the Law School, at a leading university, has passed the entrance examination at the Bar of Law after the fourth attempt. She is currently working at a law firm, where, she says, she is overwhelmed by the amount of work and unsatisfied with the lack of immediate rewards. His parents were preoccupied of their personal careers, working in diplomacy and being attached to various embassies for long periods of time, being distant for the patient both physically and emotionally. The patient grew up with her grandparents until the age of eight, and at 17, her parents 
re-established themselves in Belgium, where D. was unable to integrate socially, returning alone to the country.

About his current social relationships, the patient claims he only has superficial friendships ("My colleagues have always been jealous of me, they always wanted to sabotage me through the advice they gave me."). She had sex with a faculty colleague, of whom she fell in love, a unidirectional feeling, present today even though she had no contact for three years.

\section{Disease history}

Depressive symptomatology started three months prior to admission, the patient had two ambulatory visits during this period. By not complying with the recommended medical treatment, his mental condition worsens, reaching a psychotic rank.

\section{Examination of psychic state}

\subsection{Observations}

The patient, in relatively unkempt condition, a state of inner disorder found in clothing, intrapsychic tension, temporo-spatial disorientation and personal life history, confusion, psychomotor unrest; rises in the cabinet several times, looks in the mirror, with a gesture in which he tries to reposition his jaw, a blank look, a facies flattened, tics present in the form of convulsions in the limbs and the body, grimaces incongruent with mood and concord with the world indoor. (Kernberg 1995)

\subsection{Perception}

She has hallucinations and pseudo-hallucinations, predominantly proprioceptive but also auditory, the presence of voices being suggested by bizarre psychotic behavioral changes of perplexity and emphatic mutant changes. Relate later how the content of the voices is an encouragement; possibly in the past, the presence of positive auditory hallucinations with negative reference to the patient's inability and incapacity (Cassidy \& Shaver, 2016). Body pattern disruptions, mainly at the face, punctuated by small ophthalmic defects, as well as oral-maxillo-facial corrections by previous interventions and presently fixation points, in which the patient presents cenesthopathies and subsequently qualitative perception disorders. (Cournut, 1991)

\subsection{Attention}

Attention Deficit Disability, which generates perplexity, as well as increased latency in responses. Psychotic confusion due to difficulty switching in the concentrated focus field. (Scheffer, 2004)

\subsection{Memory}

She presents hypomnesia, with hypermnesia for some delusional integrated pseudomonas.

\subsection{Thinking}

Hypochondriac delusional ideation, mainly centered on altering the body's scheme, on minimal defects and persistent beliefs in the need to modify them; functioning on the projection mechanism, psychotic denial of the undesirable aspects of reality, reversal of the effect with the cause, easy skating between the concrete and the metaphorical plane, need of narcissistic mirroring, mentality deficit, magical belief according to which the external reality imprints the internal.

\subsection{Affectivity}

It refers to a major depressive mood several months ago, lived for a short duration, then denied. (Kohut, 2009) The patient clears from the depressed side by itself, experiencing the emphatic self-sufficiency delusion, with a later hypochondriacal turn, with a return to primary narcissism and the importance of body needs. Affective inversion towards parents.

\subsection{Activity}

Activity is characterized by the presence of attitudes and postures, grimaces and tics, in which the symbolism of delusional ideas is represented in the act. Verbal, nutritional and physiological negative at admission. Regression to early behaviors. (Freud, 2014) 


\title{
3.8. Personality
}

Psychotic anguish of destruction of her Ego.

\subsection{The consciousness of the disease}

\author{
Absent.
}

\section{Patient evolution}

During the meetings, D. presents encounters in which he behaves neurotic, in a psychotic continuum. We identify the early traumas of the separation and the defense mechanisms in which the house and the country become shields of resistance ("I wanted to be closer to what I felt at home. My parents ... I did not represent them as my home... "). The second shield of resistance becomes work, along with narcissistic mechanisms to strengthen the Ego ("I wanted to be able to progress professionally, to be the best in everything. I always told myself I could do more!"). Her labor of habituation with loneliness is narcissistic, the excessive use of suppression causing the formation of a repressed, which in adult life turns aggressively towards itself in the form of the delusional hypochondriac idea of the major depressive episode. (Roussillon, 2011)

Manifestly, the trigger for the current episode is isolation and intense intellectual effort for the Bar entrance examination, held for the fourth time ("I isolated myself to learn, I refused to go out, I slept, I ate, and I learned"). Latently, the trigger is a failing pseudo-relationship with a narcissistic invisible man, with whom D. had sex occasionally three times and admires him with the conviction that he should know the feelings she carries. In relation to this man, the patient slides into a trap: "Let me cover myself to show that I can. I could not tell him how I felt. " The girlfriends refuse to be "relatives" between her and this man and retreat feeling too much pressure on D.

The current episode starts right after entering Law Bar and starting the job as a trainee lawyer, the patient trying to deny his sad disposition, despair, insecurity and self-esteem (Krumwiede, 2014): "I was sad since I start working, I was coming home crying that I was not well seen by the rest of the world. I felt the need for help, I wanted to talk to someone, to say that I'm not afraid of being kicked out because I do not know everything". As an elaboration attempt, her mother is calling for a meeting. In the tumult of the afflictions revealed in the blast of crying, the mother puts funny pictures on the phone, refuses any negative thought coming from $\mathrm{D}$. and the only statement he can make is: "If you continue like this, you will not get well."

Concretely, D.'s parents return to the country, which the patient interprets as a violation of the borders as a moment of intrusion, breaking his protective barriers: "My mother settled in the living room and invaded my space. Before that, I could not say that something bothers me, and now I came to write them to tell them that I want to remain alone".

After the change of mood in depressive way, the hypochondria concerns appears, the delusional beliefs related to the perception of the body's scheme, along with the hallucinations and pseudo-hallucinations of the hearing, meant to cancel the depression (Gide, 2001) appear: "The voice I was hearing was my internal intellect, what I thought was well to say, I have no problem and I will go over. " The delusional idea develops on pseudo-reminiscences related to anterior oral-maxillo-facial interventions with a corrective role, as well as ophthalmic correction of dioptric vision, D. being an inclined beard to sustain its image mainly on the physical side. Thus, any attack on his bodily health is profoundly psychologically disturbing (Adler, 2010). The delusional ideas are based on anxiety of non-self-indulgence and non-recognition of feelings, along with the easy glide between the metaphorical plane and the concrete one: "I made myself a certain image in the faculty, and now I was looking in the mirror, I was more admired. I feared myself because I could not figure out what I was looking at. "

At the admission, D. presents quasi-continuous psychotic-altered behaviors in which he looked in the mirror, repositioned his jaw, adopted bizarre physiognomies and positions, which he later described as having a role to reduce psychotic anxiety: "I was looking in the mirror to confirm that I look like the one in my mind". Mirroring is a primitive narcissism-paranoiac mechanism in which the external reality is positioned as true before the internal reality, imposing it (Gabbard, 2014): "I was not able to make my own personality. I took part of the good things of men"," Can I hear voices in my ears? I wanted to hear their voices! It was a dark nightmare - shadows. The voices told me to shut up".

The peculiarity of this case is represented by proprioceptive hallucinations on which the hypochondrial delusion originates in the fragility of self-image (Williams \& Gabbard, 2007). In another, we note the perpetual effort to maintain the narcissistic boundaries of the Ego in the absence of the parental mirror mechanism ("I was trying to repress myself, to make them proud"). Transgression depression - delirium arises from inability mother containment suffering daughter when, metaphorically speaking, it did not break the soul, but jaw (Winnicott, 2005): "When I complained, the effort that a lot, 
my jaw went down. Then my face took the form of crying. That's why I looked sad in the hospital. I was depressed for what I was looking at. I felt like I needed someone in a way that I had not felt before", " I behaved like this because I refused to think I was in the hospital. I did not want to know I was there; I did not want to know I was so bad. "

Self-defense systems developed by the patient in recent months have registered in the psychotic area, with behavior change in a bizarre way and regression to baby's age (refuses to go to the toilet, unable to function excretory than the tub full of water: "It happened to me because they came over me!"). Tics and grimaces are interpreted as symbolic moments of solitude with himself: "I was in a crisis and I was walking alone in the house, thinking what to do. There were moments of focus, not tics. It was my way of struggling with being in the hospital". The mechanism of denial of reality functioned in a cleaved manner until the patient was in love: "I cannot deny the reality of others, I can only deny mine". Love is a land on which D. does not step, choosing unconsciously for the hypochondriac delirium and not the erotic one. (Klein, 2011)

\section{Treatment}

Throughout hospitalization, the patient was treated with a combination of antipsychotic (Aripiprazole $30 \mathrm{mg}$ ) and two antidepressants (Mirzaten $30 \mathrm{mg}+$ Sertraline $100 \mathrm{mg}$ ) along with benzodiazepines. Under this medication, reduced psychotic dimension was observed, with the cancellation of denial and the promise of continuing outpatient treatment. The treatment schedule was also recommended at home, with monthly psychiatric monitoring and psychotherapy sessions. Once discharged, the patient accuses multiple adverse reactions to psychiatric treatment and threatens replacement of drugs with a natural approach, it is found the reinstatement of somatic delirium. For the risk of non-compliance, Flupentixol $20 \mathrm{mg}$, twice intramuscular injection is recommended. Also, it is chosen to remove the SSRI (Sertraline) from the treatment scheme, as it is observed that hypochondriac phenomenology is under this drug.

\section{References}

Adler, A (2010). Understanding Human Nature. Eastford: Martino Fine Books; Tra edition. Cassidy, J. \& Shaver, P. (2016). Handbook of Attachment, Third Edition: Theory, Research, and Clinical Applications. New York: The Guilford Press.

Cournut, J (1991). L'ordinaire de la passion: Névroses du trop, névroses du vide (Le Fil rouge) (French Edition). Paris: Presses universitaires de France.

Freud, S (2014). Sigmund Freud 7 book premium collection: General Introduction to Psychoanalysis; Totem and Taboo: Interpretation of Dreams; Dream Psychology; and many more (Timeless Wisdom Collection 626) Kindle Edition. Business and Leadership Publishing.

Gabbard, G (2014). Tratat de psihiatrie psihodinamica. Bucuresti : Editura Trei.

Gide, A (2001). If it dies ... an autobiography. New York: Vintage.

Kernberg, O (1995). Borderline Conditions and Pathological Narcissism (The Master Work Series). Lanham: Jason Aronson, Inc.

Klein, M (2011). Love, Guilt and Reparation Kindle Edition. New York: Vintage.

Krumwiede, A (2014). Attachment Theory According to John Bowlby and Mary Ainsworth. Munchen: GRIN Publishing.

Kohut, H (2009). The Analysis of the Self: A Systematic Approach to the Psychoanalytic Treatment of Narcissistic Personality Disorders. Chicago: The University of Chicago Press.

Roussillon, R (2011). Primitive Agony and Symbolization (IPA: Psychoanalytic Ideas and Applications). London: Karnac Books ltd.

Scheffer, M (2004). Keys to the Soul: A Workbook for Self-Diagnosis Using the Bach Flowers. Ashingdon: C.W. Daniel Company ltd.

Williams, P \& Gabbard, G (2007). Key Papers in Literature and Psychoanalysis (The IJPA Key Papers Series). New York: Karnac Books.

Winnicott, D.W. (2005). Spaima de prabusire - Explorari psihanlitice. Bucuresti: Editura Fundatiei Generatia. 


\title{
DEVELOPMENT AND PSYCHOMETRIC PROPERTIES OF NEGORI (NEGATIVE ORIENTATION QUESTIONNAIRE)
}

\author{
László Kasik ${ }^{1}$, \& Zita Gál ${ }^{2}$ \\ ${ }^{1}$ University of Szeged, Institute of Education / Social Competence Research Group (Hungary) \\ ${ }^{2}$ University of Szeged, Institute of Psychology / Social Competence Research Group (Hungary)
}

\begin{abstract}
There are several available questionnaires, internationally known and accepted, which measure negative problem orientation with one factor (e.g., Social Problem-Solving Inventory-Revised, SPSI-R, D'Zurilla et al., 2002; Social Problem-Solving Inventory-Adolescents, SPSI-A, Frauenknecht \& Black, 2002). Our aim was to create such a multi-factor questionnaire that enables a more detailed and reliable analysis of interpersonal problems and one's negative problem orientation towards their solutions in adolescence. We carried out two data collections during the development of the questionnaire $\left(\mathrm{N}_{2016}=952, \mathrm{~N}_{2017}=835\right)$ among 12-, 15- and 18-year-olds in Hungary. The distribution of mothers' highest educational qualification in the three age groups was similar. The tested statements were chosen based on the category system of an earlier pilot research as well as students' and professionals' statements which were then organised into a factor structure. To analyse convergent and discriminative validity, SPSI-R (D'Zurilla et al., 2002) was used. The exploratory factor analysis and confirmatory factor analysis greatly support the theoretical factor structure in all age groups, and the path analysis also confirmed what had been thought about the system of connections of the variables. The result of the questionnaire development is the 21-item, 6-factor NEGORI (Negative Orientation Questionnaire) which bears quite good reliability indexes in all age groups and which can measure the following aspects within negative problem orientation: negative self-efficacy, the bad feeling caused by negative consequences, not dealing with the problem as being positive, problem avoidance as well as habits pointing to negative orientation and waiting with the solution. With the multi-factor questionnaire, we gained different and more detailed information as compared to previous data related to age and gender, which enables taking a lot more personal characteristics into consideration in addition to problem-solving styles when creating and using development programmes at schools that focus on social problem-solving and thus better concentrate on orientation which profoundly defines problem-solving.
\end{abstract}

Keywords: Social problem-solving, negative problem orientation, questionnaire development, NEGORI, adolescents.

\section{Introduction}

The success of social problem-solving largely depends on one's problem orientation in general and the given problem, problem-solving as a process and how effective problem-solvers we regard ourselves (e.g., D’Zurilla et al., 2002; Frauenknecht \& Black, 2010; Strough \& Keener, 2013). National and international research (e.g., Eskin, 2013; Kasik, 2015) both show that if one's orientation is negative, for example he/she believes that the given problem is unsolvable and he/she thinks that nothing can be done for a solution and the likelihood of unsuccessful problem-solving increases. Our problem-orientation is primarily influenced by family socialisation and negative orientation is mostly defined by one's mother's (or tender's) negative orientation and problem-solving behaviour (e.g., Nezu et al., 2004). It is also proven that one's negative orientation during childhood and adolescence may bear long-term effects on academic-professional success and the quality of one's social connections, and may play a significant role in the development of the feeling of hopelessness, stress and depression (e.g., Eskin, 2013).

\section{Aims}

In 2016, a questionnaire (NEGORI) was developed based on the theoretical models, the items expressing negative orientation from the presented and partly used questionnaires, and the system of categories of the reasons of negative orientation in order to measure negative orientation. With the data 
collected in 2016, an exploratory factor analysis (EFA) was carried out and the connection between the factors was examined along with age and gender related differences. With the data collected in 2017, a confirmatory factor analysis (CFA) was carried out as well as structural equation model (SEM) to show the causal dependences between the (endogenous and exogenous) dimensions. The aim of the current study was - with the EFA and CFA - to develop a multi-factor questionnaire which enables a more detailed examination of interpersonal problems and negative orientation towards their solution. Furthermore, age and gender differences were revealed in this sample as well and the results of the also completed SPSI-R (D'Zurilla et al., 2002) were used to examine the convergent and discriminative validity of the NEGORI (Kasik et al., 2018).

\section{Methods and measurements}

The first data collection took place in 2016, the second in 2017 (with a repeated data collection). The first data collection was carried out in autumn 2016 among 12-, 15- and 18-year-old $\left(\mathrm{M}_{12}=12.13\right.$ $\left.\mathrm{SD}_{12}=.78 ; \mathrm{M}_{15}=15.07 \mathrm{SD}_{15}=.45 ; \mathrm{M}_{18}=18.11 \mathrm{SD}_{18}=.51\right)$ primary and secondary school students. 952 students were involved in the research $\left(\mathrm{N}_{12}=310, \mathrm{~N}_{15}=301, \mathrm{~N}_{18}=341 ; \mathrm{N}_{\text {girls: } 12,15,18}=56,54,58 \%\right)$. Based on the recorded background variables, the distribution of mothers' highest educational qualification (Hungarian system: 8 years of primary school, vocational school certificate, technical college certificate, grammar school certificate, college/university degree) in the three age groups was similar $\left(\chi^{2}=21.19 \mathrm{p}=.25\right)$. The other data collection was carried out on a sample of 835 students $\left(\mathrm{N}_{12}=290, \mathrm{~N}_{15}=270, \mathrm{~N}_{18}=275\right)$ in spring $2017\left(\mathrm{M}_{12}=12.05 \mathrm{SD}_{12}=.76 ; \mathrm{M}_{15}=15.12 \mathrm{SD}_{15}=.41\right.$; $\left.\mathrm{M}_{18}=17.98 \mathrm{SD}_{18}=.62 ; \mathrm{N}_{\text {girls: }} 12,15,18=55,56,57 \%\right)$. In the case of this one, another data collection was carried out after two weeks. Based on mothers' highest educational qualification the three subsamples do not differ significantly from one another either $\left(\chi^{2}=20.23 p=.29\right)$. The data collection was carried with the headmasters' and parents' consent in all cases, who were informed about its content and aim. The data collection took the time of one lesson in all cases (Kasik et al., 2018).

The convergent and discriminative examination of the NEGORI was done with SPSI-R (D'Zurilla et al., 2002). The questionnaire consists of 25 items and the statements are grouped into five factors: Positive orientation, Negative orientation, Rationality, Impulsivity, Avoidance (for their contents, see Introduction). The statements have to be evaluated on a five-fold scale ( 1 = Absolutely not true for me -5 = Absolutely true for me). Based on national research, the factor structure of the questionnaire is completely the same as the original English version's factor structure and measures well among 11-18-year-olds. Its reliability index (Cronbach- $\alpha$ ) is above .76 at all ages (for more detail about the national cross-sectional and longitudinal studies see: Kasik, 2014; Kasik et al., 2016).

\section{Results}

An EFA was carried with the data from the 2016 data collection. Based on the analysis, the same six factors were distinguished at all three ages: Fending off the problem (4 items), Negative consequences (4 items), Negative self-efficacy (3 items), Positive consequences (3 items), Habit, pattern (3 items), Waiting (3 items). The validity and reliability indexes of the factors and the NEGORI (in the case of the whole sample and the age groups) are summarised in Table 1.

Table 1. The results of the EFA and reliability.

\begin{tabular}{|c|c|c|c|c|c|}
\hline \multirow[b]{2}{*}{ Factor/NEGORI } & \multirow{2}{*}{$\begin{array}{l}\text { Number of } \\
\text { items }\end{array}$} & \multicolumn{4}{|c|}{ Cronbach- $\alpha$} \\
\hline & & $\begin{array}{l}\text { Whole } \\
\text { sample }\end{array}$ & 12-year-olds & 15-year-olds & 18-year-olds \\
\hline Fending off the problem & 4 & .85 & .80 & .84 & .89 \\
\hline Negative consequences & 4 & .86 & .84 & .88 & .85 \\
\hline Negative self-efficacy & 4 & .84 & .87 & .85 & .78 \\
\hline Positive consequences & 3 & .68 & .69 & .70 & .71 \\
\hline Habit, pattern & 3 & .69 & .66 & .69 & .70 \\
\hline Waiting & 3 & .77 & .71 & .79 & .78 \\
\hline NEGORI & 21 & .78 & .88 & .90 & .88 \\
\hline $\mathrm{KMO}$ & & .90 & .88 & .89 & .87 \\
\hline Bartlett & & 7026.86 & 2015.28 & 2615.01 & 2683.53 \\
\hline $\mathrm{df}$ & & 210 & 210 & 210 & 210 \\
\hline $\mathrm{p}$ & & .00 & .00 & .00 & .00 \\
\hline Variance $(\%)$ & & 57.66 & 68.11 & 70.22 & 65.84 \\
\hline
\end{tabular}


The factor structure of NEGORI was tested on the data collected in 2017 with CFA. The fitness of the model is satisfactory; the CFA carried out with the six factors separated during the 2016 EFA also show satisfactory fitness indexes: whole sample: $\chi^{2}=386.4 \mathrm{p}<.001 \mathrm{df}=174 \chi^{2 / \mathrm{df}}=2.22 \mathrm{CFI}=.96 \mathrm{TLI}$ $=.95 \mathrm{RMSEA}=.04(\mathrm{p}=1.00) \mathrm{SRMR}=.04 ; 12: \chi^{2}=289.98 \mathrm{p}<.001 \mathrm{df}=174 \chi^{2 /} \mathrm{df}=1.67 \mathrm{CFI}=.92 \mathrm{TLI}$ $=.90$ RMSEA $=.05(\mathrm{p}=.25)$ SRMR $=.06 ; 15: \chi^{2}=237.12 \mathrm{p}<.001 \mathrm{df}=174 \chi^{2 /} \mathrm{df}=1.36 \mathrm{CFI}=.97 \mathrm{TLI}$ $=.96$ RMSEA $=.04(\mathrm{p}=.98)$ SRMR $=.05 ; 18: \chi^{2}=285.65 \mathrm{p}<.001 \mathrm{df}=174 \chi^{2 /} \mathrm{df}=1.64 \mathrm{CFI}=.95 \mathrm{TLI}$ $=.94$ RMSEA $=.04(\mathrm{p}=.88)$ SRMR $=.05$. SEM was used to describe the system of connections between the factors and theoretical model of the effects the variables have on one another (Figure 1) with the data from the second collection. Based on the research that has been carried out so far and the inter-correlational coefficients revealed in this research, it was hypothesised that the central latent variable of the model was Negative self-efficacy, which directly effects all factors, and that Fending off the problem, Negative consequence and Positive consequence directly affect the Habit, pattern and Waiting variables.

Figure 1. The theoretical model of the connection of the variables.

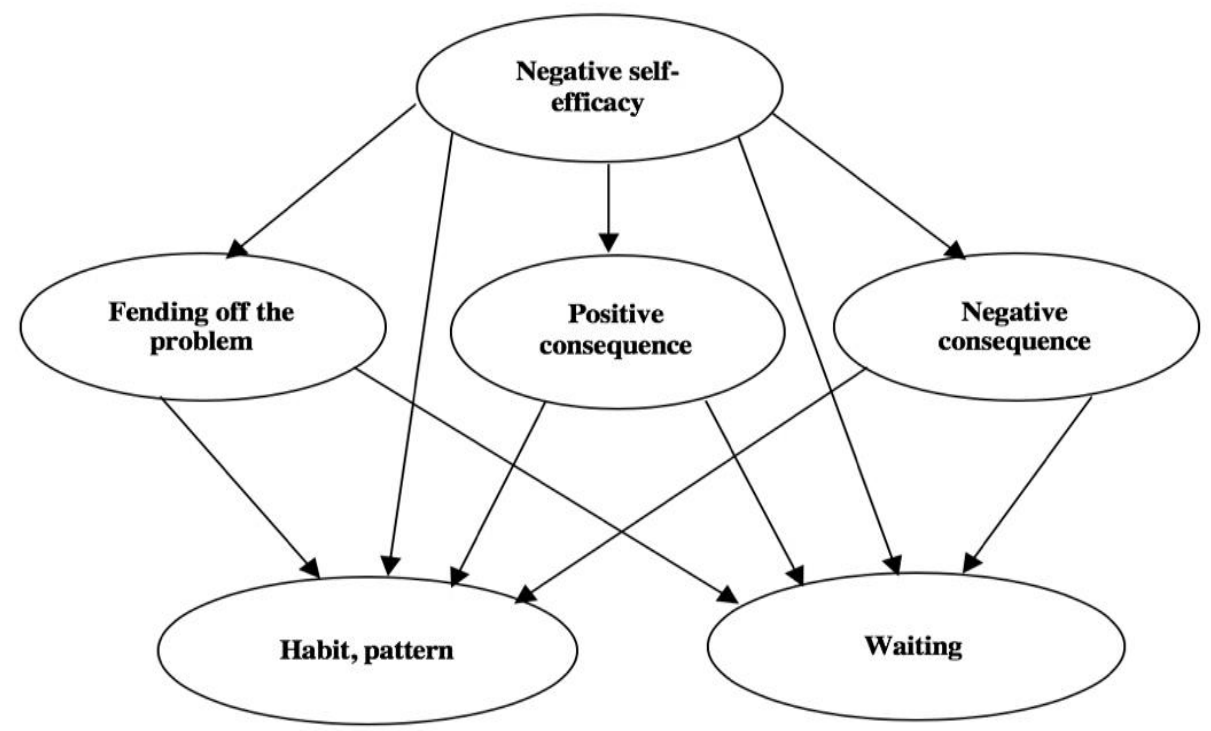

The fitness of the theoretical model is satisfactory: whole sample: $\chi^{2}=466.06 \mathrm{p}<.001 \mathrm{df}=174$ $\chi^{2 / \mathrm{df}}=2.68 \mathrm{CFI}=.94 \mathrm{TLI}=.93 \mathrm{RMSEA}=.04(\mathrm{p}=.98) \mathrm{SRMR}=.06 ; 12: \chi^{2}=319.49 \mathrm{p}<.001 \mathrm{df}=176$ $\chi^{2 /} \mathrm{df}=1.81 \mathrm{CFI}=.90 \mathrm{TLI}=.88 \mathrm{RMSEA}=.06(\mathrm{p}=.67) \mathrm{SRMR}=.08 ; 15: \chi^{2}=266.44 \mathrm{p}<.001 \mathrm{df}=176$ $\chi^{2 / \mathrm{df}}=1.51 \mathrm{CFI}=.95 \mathrm{TLI}=.94 \mathrm{RMSEA}=.04(\mathrm{p}=.87) \mathrm{SRMR}=.07 ; 18: \chi^{2}=325.71 \mathrm{p}<.001 \mathrm{df}=176$

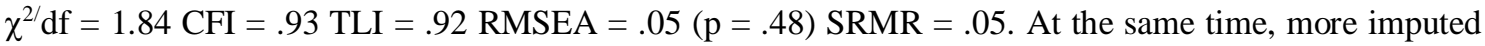
relationships were not significant; therefore, those were deleted. The fitness thus received is satisfactory, it is consistent with the theoretical model in whole sample and in all age subsamples: whole sample: $\chi^{2}=466.06 \mathrm{p}<.001 \mathrm{df}=176 \chi^{2 /} \mathrm{df}=2.64 \mathrm{CFI}=.94 \mathrm{TLI}=.93 \mathrm{RMSEA}=.04(\mathrm{p}=.98) \mathrm{SRMR}=.06$; 12: $\chi^{2}=272.54 \mathrm{p}<.001 \mathrm{df}=132 \chi^{2 /} \mathrm{df}=2.06 \mathrm{CFI}=.92 \mathrm{TLI}=.90 \mathrm{RMSEA}=.06(\mathrm{p}=.14) \mathrm{SRMR}=.08$; 15: $\chi^{2}=266.44 \mathrm{p}<.001 \mathrm{df}=176 \chi^{2 /} \mathrm{df}=1.51 \mathrm{CFI}=.95 \mathrm{TLI}=.94 \mathrm{RMSEA}=.04(\mathrm{p}=.87) \mathrm{SRMR}=.07$; 18: $\chi^{2}=327.80 \mathrm{p}<.001 \mathrm{df}=177 \chi^{2 /} \mathrm{df}=1.85 \mathrm{CFI}=.93 \mathrm{TLI}=.92 \mathrm{RMSEA}=.05(\mathrm{p}=.48) \mathrm{SRMR}=.05$.

The analysis of the convergent and discriminative validity of NEGORI was carried out on the data from the second collection (2017) for which we used SPSI-R Based on the correlations, the Positive orientation is a negative significant connection with Negative self-efficacy and Waiting in all three age groups (Table 2). Negative orientation is in a positive connection with all NEGORI factors and in almost all age groups; of these, the values in connection with the factors of Fending off the problem and Negative self-efficacy are the highest among 12- and 15-year-olds. The factor of Avoidance bears no significant connection with any NEGORI factor among 12-year-olds; however, it does with 15- and 18-year-olds. The connection between the factors of Avoidance, Waiting and Negative self-efficacy are the strongest among 15-year-olds. Rationality and Impulsivity is a significant connection with only the factors of Habit, pattern and Waiting (the former is negative, the latter is positive) among 15- and 18-year-olds. 
Table 2. The connection between the factors of NEGORI and SPSI-R (Pearson $r ; p<.01)$.

\begin{tabular}{|c|c|c|c|c|c|c|}
\hline Factor & $\begin{array}{c}\text { Sub- } \\
\text { sample }\end{array}$ & $\mathrm{PO}$ & NO & $\mathrm{R}$ & I & A \\
\hline \multirow{3}{*}{ Fending off the problem } & 12 & n.s. & .35 & n.s. & n.s. & n.s. \\
\hline & 15 & n.s. & .48 & n.s. & n.s. & .31 \\
\hline & 18 & n.s. & .29 & n.s. & n.s. & .17 \\
\hline \multirow{3}{*}{ Negative consequence } & 12 & n.s. & n.s. & n.s. & n.s. & n.s. \\
\hline & 15 & n.s. & .20 & n.s. & n.s. & .16 \\
\hline & 18 & n.s. & .16 & n.s. & n.s. & .17 \\
\hline \multirow{3}{*}{ Negative self-efficacy } & 12 & -.13 & .35 & n.s. & n.s. & n.s. \\
\hline & 15 & -.22 & .55 & n.s. & n.s. & .43 \\
\hline & 18 & -.15 & .28 & n.s. & n.s. & .22 \\
\hline \multirow{3}{*}{ Positive consequence } & 12 & n.s. & .21 & n.s. & n.s. & n.s. \\
\hline & 15 & n.s. & .33 & n.s. & n.s. & .31 \\
\hline & 18 & n.s. & .20 & n.s. & n.s. & .23 \\
\hline \multirow{3}{*}{ Habit, pattern } & 12 & n.s. & n.s. & n.s. & n.s. & n.s. \\
\hline & 15 & n.s. & .26 & -.16 & .19 & .29 \\
\hline & 18 & n.s. & .25 & -.18 & .21 & .22 \\
\hline \multirow{3}{*}{ Waiting } & 12 & -.18 & .29 & n.s. & n.s. & n.s. \\
\hline & 15 & -.19 & .25 & -.15 & .21 & .52 \\
\hline & 18 & -.13 & .18 & -.19 & .18 & .31 \\
\hline
\end{tabular}

Note. $\mathrm{PO}=$ Positive orientation, $\mathrm{NO}=$ Negative orientation, $\mathrm{R}=$ Rationality, $\mathrm{I}=$ Impulsivity, $\mathrm{A}=$ Avoidance.

\section{Discussion}

The results of the research unequivocally supported the assumption that in order to understand negative problem orientation, fields other than the ones covered by the questionnaires should also be taken into consideration. The categories of reasons revealed in the pilot largely contributed to the development of the NEGORI. Based on the analysis of the factor structure of the original, 40-statement questionnaire, the final, 21-item NEGORI has good reliability indexes in all three age groups. The six factors partially cover the fields that measure negative orientation in PSI, SPSI-R and SPSI-A, and most categories of the pilot measurement. The results of the CFA confirmed the six-factor model in all three ages. The 'I do not want to solve my problem because...' statement beginnings most likely account for the fact that the connection between the NEGORI factors are positive in all age groups. Based on the analysis of the convergent and discriminative validity, the Negative orientation factor of SPSI-R is in a positive connection with all NEGORI factors at almost all ages, which was confirmed by other tools that measure negative orientation as well (e.g., Robichaud \& Dugas, 2005). One of the reasons for this is the content overlap of the items of Negative orientation and the NEGORI factors.

\section{Limitation}

Even though the NEGORI can be considered to be apt to shed light on fending off the problem, negative and positive consequence, negative self-efficacy, habit and pattern, and waiting as reasons for negative orientation, several further studies are needed in order to refine the questionnaire, on the one hand and to analyse the data in more detail, on the other hand. Upon completion, the students could think of problems, problematic situations related to anyone; therefore, it will be useful to create the person-specific version of the NEGORI in the future (as in the case of SPSI-R, Kasik, 2015) which requires the changing of the statements in the Habit, pattern factor because those contain family, peer and teacher model people. It will also be important to shed light on the connection between the NEGORI and other fields, such as anxiety, general self-efficacy, coping, self-evaluation and the aforementioned responsibility-taking. The results of these correlation analyses will also contribute to making school development programmes for children and adolescents that focus on problem-solving even better and to be able to concentrate on the solution-defining orientation as well by taking more personal characteristics into consideration in addition to problem-solving styles. 


\section{References}

D’Zurilla, T. J., Nezu, A., \& Maydeu-Olivares, A. (2002). Social Problem-Solving Inventory-Revised (SPSI-R): Technical Manual. North Tonawanda, New York: Multi-Health Systems.

Eskin, M. (2013). Social Problem Solving Therapy in the Clinical Practice. Elsevier. Turkey.

Frauenknecht, M. \& Black, D. R. (2010). Is it social problem solving or decision making? Implications for health education. American Journal of Health Education, 41(2), 112-123.

Kasik, L. (2014). Development of social problem solving - A longitudinal study (2009-2011) in a Hungarian context. European Journal of Developmental Psychology, 12(2), 142-158.

Kasik, L., Gál, Z., \& Tóth, E. (2018). Development and Psychometric Properties of NEGORI (Negative Orientation Questionnaire). Journal of Relationships Research, 9(8), 1-13.

Kasik, L., Gáspár, CS., Guti, K., \& Zsolnai, A. (2016). Relationship between Social Problem Solving, Anxiety and Empathy among Adolescents in Hungarian Context. In K. Newton (Ed.), Problem-Solving: Strategies, Challenges and Outcomes (pp. 177-196). New York: NOVA Science Publishers, Inc.

Nezu, A. M., Wilkins, V. M., \& Nezu, C. M. (2004). Social problem solving, stress, and negative affect. In E. C. Chang, T. J. D'Zurilla, \& L. J. Sanna (Eds.), Social problem solving (pp. 49-65). Washington, DC: American Psychological Association.

Robichaud, M. \& Dugas, J. (2005). Negative problem orientation (Part I): Psychometric properties of a new measure. Behaviour Research and Therapy, 43(3), 391-401.

Strough, J. \& Keener, E. (2013). Interpersonal problem solving across the life span. In P. Verhaeghen, P. \& C. Hertzog (Eds.), The Oxford handbook of emotion, social cognition, and everyday problem solving during adulthood. Oxford University Press, The Oxford Library of Psychology Series. 


\title{
INNOVATIVE METHOD FOR INTRODUCTION OF WRITTEN LANGUAGE: EXPERIENCE IN MEXICO
}

\author{
Yulia Solovieva, \& Luis Quintanar Rojas \\ Master Program in Neuropsychological Diagnosis and Rehabilitation, \\ Faculty of Psychology, Puebla Autonomous University (Mexico)
}

\begin{abstract}
Introduction of written language at primary school represents one of the important aspects of school learning. In Mexico, as in many other countries, common methods of teaching are repetition, memorization and reproduction of given information: letters or syllables. Our presentation shows an example of implementation of a new method for introduction of written language based on activity theory and concept of guided orientation and comparison of the results with traditional methods. Written language is analyzed as symbolic codification and de-codification of oral words according to phonological and phonetic system of language. The Program for Training of Written language implies fulfilment of joint actions of children guided by teacher. Collective dialogue is the main type of interaction and children are free to move from place to place in the classroom. Children learn how to codify and represent oral words of Spanish language with the help of external materialized and perceptive schemes. The results of application of the method show correct pronunciation of all known and unknown words, usage of correct space between words in writing, reduced number of orthographic mistakes. After working with the method for one year children commit less mistakes in reading and writing in comparison with the pupils who learn according to traditional methods. Our proposal is a new alternative method for initial education and especially for introduction of reading and writing in primary school. The main challenges of activity theory are preparation of specific orientation within conception of the zone of proximate development.
\end{abstract}

Keywords: Innovative education, methods of teaching, written language, primary education, orientation in teaching.

\section{Introduction}

The process of teaching of reading and writing at primary school represents one of the most important aspects of child's life during this period. Learning to read and write is the point of attraction for psychologists and teachers. However, there are no real intents for searching of new ways of psychological understanding of the child's actions during introduction of reading and writing at school. In Mexico, as in many other countries, the process of reading and writing at school traditionally is based on isolated training of this cognitive ability. Common methods of teaching of reading and writing are repetition, memorization and reproduction of given information: letters or syllables. In public schools in Mexico, children never read entire books. Only pages or paragraphs of reduced and simple texts appear in official programs for primary school. Even in private schools reading acquisition is a mechanic process, which starts from the first grade of pre-school age and continues up to the sixth grade of primary school (Quintanar, \& Cols., 2011). Cognitive tests measure the quantity of words, which the child can read loudly in a minute. Understanding of the meaning and sense of texts is never taken into account. Situation with reading and understanding at all education levels in Latin America is a serious problem. Such countries as Mexico and Colombia normally occupy last places within OGDA tests. Alternative methods within active school and global reading can't solve this problem. These methods never take into account orientation, analysis of content of actions and reflexive participation of the children in their own school actions (SEP, 2010). Alternative methods are always based on the theory of constructivism or conceptions of competences.

Activity theory is a new option for proposal of new alternative methods for initial education and especially for introduction of reading and writing in primary school. The main challenges of activity theory are preparation of specific orientation for each part of knowledge. Our presentation shows an 
example of usage of orientation for initial introduction of reading and writing process in primary school. The method of orientation is based on previous methodological conceptions of Elkonin $(1980,1995)$, Galperin $(1979,1992)$, Talizina $(1988,2009)$ as main representatives of activity theory and cultural psychology. Reading and writing process is understood as a reflective and voluntary process of symbolic codification and de-codification of oral words by a subject (Luria, 1980).

\section{Method}

The authors of the method present detailed analysis of phonological and phonetic system of Spanish language as the base for introduction of symbolic codification and graphic representation of kinds of sounds of Spanish language. The Program for Training of Written language implies fulfilment of joint actions of children guided by teacher (Solovieva \& Quintanar, 2016). On each stage of the work with the method, the teacher shows children what and how should they do. The work in classroom is always collective and interactive process with no kind of individual tasks. The teacher helps children always in cases of difficulties. Children are encouraged to ask and answer questions collectively, to help each other to correct mistakes or to put examples of words and sounds. All kinds of initiative and interest of children are taken into account by teacher (Oboukhova, 2006). All tasks are shared in the group and are fulfilled collectively together with the teacher (Solovieva \& Cols., 2017). The teacher takes part as another mate of the children and as a guided of intellectual activity. Collective dialogue is the main type of interaction and children are free to move from place to place in the classroom. All procedures of the method are based on the concept of the zone of proximate development introduced by Vigotsky (1996). Children learn how to codify and represent oral words of Spanish language with the help of external materialized schemes. Collective dialogue and joint creative tasks are the main type of interaction and children are free to move from place to place in the classroom. On perceptive stage, children draw these schemes in notebooks together with examples of drawings corresponding to the words they choose. The method includes introduction of actions of codification at materialized, perceptual and verbal levels (Solovieva, 2014). Gradually, children pass to the level of reading and writing of words and sentences in Spanish. Different creative tasks and work in groups are used during the work with the method. Children use reflective orientation of all kinds of types of correspondence between the sounds and letters in Spanish language.

Stages of the Innovative Method for introduction of reading and writing:

1. Oral phonetic analysis of sounds in words. On this stage children say loudly different words they like. The teacher helps children to identify orally the sounds in each pronounced word.

2. Phonetic analysis with the help of external materialized action. On this stage, the teacher shows the scheme for words, in which the quantity of squares correspondents to quantity of sounds in the word. Children choses correspondent scheme according to the quantity of sounds and fill the schemes with the circles in correspondent places.

3. Introduction of general phonological differentiation of vowels and consonants. The vowels are symbolized as red circles, while the consonant sounds as green circles in external schemes.

4. Introduction of letters with the help of materialized external action:

- Introduction of letters which correspond to vowel sounds;

- Introduction of letters which correspond to consonant sounds (according to the order of rules for correlation between setter and sound of Spanish language);

5. Introduction of the action of writing and reading of letters:

- Writing and reading of letters which determine vowels of Spanish language;

- Writing and reading of letters which determine consonants of Spanish language;

6. Independent reading and writing of words and sentences.

\section{Results}

Our program has been applied in private primary school "Kepler" in the city of Puebla (Mexico) for six years (Solovieva \& Quintanar, 2016). The results of application of the method show correct pronunciation of all known and unknown words, usage of correct space between words in writing, reduced number of orthographic mistakes. The teachers who work now using our Method expressed notable differences between possibilities of this group and other children who were learning by traditional (analytic or global) training of reading. The children showed no difficulties in final psychological assessment. They were able to pass naturally to reading of complex narrative and artistic texts at the end of the first year of primary school. High cognitive motivation permitted introduction of profound analysis of meaning and sense of stories and short novel for children with good understanding. Children were eager to read books independently, which rarely happen in traditional Mexican schools. 


\section{Discussion}

Among strong advantages of the method is correct pronunciation of all known and unknown words in Spanish, usage of correct space between words in writing, reduced number of orthographic mistakes. The method guarantees better understanding of all regularities and exceptions of correspondence between phonological level of words and its representation with graphic symbols (letters). After working with the method for one year children commit less mistakes in reading and writing in comparison with the pupils who learn according to traditional methods (Solovieva, Torrado \& Quintanar, 2018). Another important achievement of the method is the platform for gradual introduction of theoretical thinking instead of empirical thinking predominant in pre-school age (Talizina, 1988, 2009; Davidov, 1988). We are sure that the main starting point of our Method is usage of specific orientation for oral and written analysis of words. Such orientation, introduced from external level by joint collective actions between teacher and children, permitted correct fulfillment of intellectual verbal actions of analysis and production of words and sentences. We would like to stress that the introduction of new methods elaborated according to theoretical and methodological proposals of cultural-historic psychology and activity theory could be very useful and productive. Specific orientations might be created for correction in cases of learning disabilities and prevent mistakes of confusion, inversion, omission, wrong separation and anticipation in reading and writing. Intellectual reflexive analysis of words and separation of level of sounds and letter might be used as the basis for but also as a method for acquisition of foreign language (Solovieva, 2015), which is especially important in modern global world of communication.

\section{Conclusion}

The authors are convinced that high interest and constant motivation for learning depend on orientation of each action of a pupil introduced by guidance of the teacher. Such kind of pedagogical proposal is completely based on the principles of activity theory applied to teaching process. Our proposal might be a new alternative method for initial education and especially for introduction of reading and writing in primary school. The main challenges of activity theory are preparation of specific orientation within conception of the zone of proximate development.

\section{References}

Davidov, V. V. (1988). La enseñanza escolar y el desarrollo psíquico. Moscú: Progreso.

Elkonin, D.B. (1980). Psicología del Juego. Madrid. Pablo del Rio.

Elkonin, D.B. (1995). Desarrollo psicológico en las edades infantiles. Moscú: Academia de Ciencias Pedagógicas y Sociales.

Galperin, P. Ya. (1979). Introducción a la psicología. Un enfoque dialéctico. Madrid: Pablo del Río.

Galperin, P. (1992). Stage-by-stage formation as a method of psychological investigation. Journal of Russian and East European Psychology, 30, (4), 60-80.

Luria, A. R. (1980). Fundamentos de neurolingüística. Barcelona: Toray-Mass.

Obukhova, L.F. (2006). Psicología del desarrollo. Moscú: Educación superior.

Quintanar, L., Solovieva, Yu., Lázaro, E., Bonilla, R., Mejía, L. \& Eslava, J. (2011). Dificultades en el proceso lectoescritor. México: Trillas.

SEP (2010). Manual de procedimientos para el fomento y la valoración de la competencia lectora en el aula. México: SEP.

Solovieva, Yu. (2014). Desarrollo de la actividad intelectual desde paradigma histórico-cultural. México: CEIDE.

Solovieva, Yu. (2015). Estrategias introductorias de la lectoescritura en el idioma inglés. México: Plaza y Valdés.

Solovieva, Y., \& Quintanar, L. (2016). Enseñanza de la lectura. Un método práctico. México: Trillas.

Solovieva, Yu., Baltazar Ramos, A.M., Escotto, A. \& Quintanar, L. (2017). Training of Teachers: Difficulties and Proposals in Mexico. Journal of Studies in Education. 7 (4): 78-91.

Solovieva, Yu., Torrado, O. \& Quintanar, L. (2018). Orientation for Initial Introduction of Written Speeach in primary School. Journal of Education, Society and Behavioural Science. 24 (4): 1-18.

Talizina, N. F. (1988). Psychology of teaching. Moscow: Progress.

Talizina, N. F. (2009). La teoría de la actividad aplicada a la enseñanza. México: BUAP.

Vigotsky, L. S. (1995). Obras escogidas Tomo IV. Madrid: Visor. 


\title{
SOCIAL PROBLEM-SOLVING IN THE CLASSROOM
}

\author{
Zita Gál ${ }^{1}$, \& László Kasik ${ }^{2}$ \\ ${ }^{1}$ University of Szeged, Institute of Psychology / Social Competence Research Group (Hungary) \\ ${ }^{2}$ University of Szeged, Institute of Education / Social Competence Research Group (Hungary)
}

\begin{abstract}
In Hungary, the examinations of social problem-solving and their factors mainly carried out by self-reported questionnaires both among adolescents and adults. On the other hand, it is necessary to apply other research methods in order to identify and understand interpersonal problems and solution processes. In our current research, we used observation to identify student-student and teacher-student problems during a lesson. The study occurred in 2017, when 34 lessons were observed (in all grades except 3 and 4). Among these, 8 took place in lower primary schools (6-10-year-olds), 12 in upper primary schools (10-14-year-olds), 14 in high schools (14-18-year-olds). We carried out the observations using a set of criterias established by our research group based on Chang et al. (2004), Eskin (2012) and Strough \& Kenner (2012): the source of the problem, the subject of the problem, time perspective, frequency, organization, attitude, feasibility, problem solving styles and strategies, relationship between the members, behaviour of the teachers. The identified problems (based on age and gender) more or less are the same as we found during previous researches carried out by questionnaires: in lower primary school it is the mockery, and later the physical and verbal aggression which appears to be the main problems of the students. In the case of mockery and verbal aggression it is clear that they want to settle their differences as soon as possible. We experienced both in the questionnaire using researches and this observation, that negative orientation manifests in the verbal expression of inaccessibility. Teachers, except teachers in lower primary school, also have negative orientation towards a problem, and they think of a problem as a distractor, so their aim was the fast, behaviour-focused elimination of the situation. The problem-solving in upper primary and high schools were clearly teacher controlled, they immediately gave instructions to the students how to solve it based on their own consideration. We did not experience any kind of discussions, rethinking or evaluation of the situation among teachers. What we saw pays the attention to effect of the problems to classroom work, and interpersonal problems can influence students subject attitude and learning motivation. In the future, we are going to carry out other observations as well, and also planning to use a questionnaire to analyze as many aspects of problems solving as possible. This way we could get more and more precise data in order to develop a program for students and teachers.
\end{abstract}

Keywords: Social problem solving; classroom observation; behavior of teachers and students.

\section{Introduction}

Following observations at schools, Singleton and Asher (1977) categorised students' interpersonal problems. The most common of them among children and adolescents were insulting, decrying, looking down and physical abuse. In both cases of research, students stated problems which other people do not regard as problems but positive occurrences (e.g., receiving a gift), which reinforced earlier conclusions of the relative nature of problems and drew attention to the dependence of problems on social environments (only disadvantaged children gave such an example). Similar problem groups were revealed among primary and secondary school (7-18-year-old) students (Kasik \& Gál, 2015; Kasik, 2015): the most frequent problems claimed among lower year students were mockery, telling on each other and exclusion; among upper year students they were exclusion, physical abuse by peers and favouritism by teachers; and among secondary school students they were teasing and verbal abuse by peers. Parent-related problems appeared in a significant proportion on the problem list even among lower year students and this only grew among adolescents (physical and verbal abuse by parents, parents' problems related to alcohol, and family feuds and fights also in relation to those). 
Based on the work done by Bedell et al. (1980), Chang et al. (2004), D'Zurilla and Goldfried (1971), Eskin (2012), Hunt (1965), Singleton and Asher (1977) and Strough and Kenner (2012), we summarised the aspects used to interpret social problems in Table 1.

Table 1. Aspects of interpreting social problems.

\begin{tabular}{|c|c|}
\hline Main aspects & Sub-aspects \\
\hline Origin & $\begin{array}{ll}- & \text { Own (goal, need, interest, attribute) } \\
\text { - } & \text { Another person, others (goal, need, interest, attribute) } \\
\text { - } & \text { Environment, situation, social task } \\
\text { - } & \text { Group and social process }\end{array}$ \\
\hline Definition & $\begin{array}{ll} & \text { Well-defined } \\
- & \text { Less well-defined or not well-defined }\end{array}$ \\
\hline Manifestation & $\begin{array}{ll} & \text { In behaviour } \\
\text { - } & \text { In the expression of thoughts, feelings }\end{array}$ \\
\hline Chronology & $\begin{array}{ll}\text { - } & \text { Happening in the present or past } \\
\text { - } & \text { Happening in the future (possibly or certainly) }\end{array}$ \\
\hline Frequency & $\begin{array}{ll}- & \text { Occurs once or rarely } \\
\text { - } & \text { Occurs multiple times (repeats frequently) } \\
\text { - } & \text { Nearly constant } \\
\end{array}$ \\
\hline Consequence & $\begin{array}{ll}\text { - } & \text { Small degree } \\
\text { - } & \text { Considerable impact on how one leads his/her life }\end{array}$ \\
\hline Relation & $\begin{array}{ll}\text { - } & \text { Negative } \\
\text { - } & \text { Positive } \\
\end{array}$ \\
\hline Organisation & $\begin{array}{ll}\text { - } & \text { No connection with another (isolated) } \\
\text { - } & \text { Few causal relations } \\
\text { - } & \text { Many, forming a hierarchic system, causal relation }\end{array}$ \\
\hline Connection of members & $\begin{array}{ll}\text { - } & \text { Interpreted in a submissive relation } \\
\text { - } & \text { Interpreted in a coordinative relation }\end{array}$ \\
\hline Solvability & $\begin{array}{ll} & \text { Solvable (necessary, possible) } \\
\text { - } & \text { Unsolvable (not needed, not possible) } \\
\end{array}$ \\
\hline
\end{tabular}

The mode of problem solving is influenced by a considerable number of factors. Of these, family background emerges during childhood and adulthood as well, especially with regard to parents' problem solving and the examples they provide. With the advancement of age, peers' influence (in terms of their behaviour, suppositions and expectations) grows exponentially and teachers also play an influential role with their suppositions, expectations, the way they disciple and the examples they provide (Webster-Stratton, 2011). According to Webster-Stratton (2011), the effectiveness of problem-solving in the classroom is influenced by the system of values and norms mediated by the teacher; their problem-solving style; students' personal traits (e.g., communication and problem-solving ability, family examples for problem-solving); the network of power, emotions and communication in the group; and the emotional and confidential relation between the teacher and the student.

Based on Goldstein's research (1999), teachers teaching 7-12-year-olds dedicate more time to understanding and dealing with a problem, they use positive reinforcement, talk about proper behaviour and problem-solving strategies more often than those who teach adolescents (12-18-year-olds) as they aim to end problematic situations in order to maximise the time dedicated to mediating the material to be learnt. These teachers approach problems as factors of interference; however, this does not appear to be an effective strategy in terms of students' social development. In fact, it often serves as a negative example and can thus reinforce non-adequate forms of avoidance (e.g., negligence) as well as a negative approach to problems, the parts of which are insolvability or the feeling of pointlessness of making an effort to solve the problem.

\section{Aims and methods}

The aim of classroom observations carried out during spring 2017 was to identify interpersonal problems, their manifestations, styles and modes of solution between students and in student-teacher relationships. We received directorial authorisation in all schools (in four Hungarian counties) to carry out our observations. The lessons were chosen randomly, and the teachers were told that our aim was to see teaching methodologies and students' reactions to their impact. 
All the lessons were observed by two teacher trainees who were trained by the researchers and who positioned themselves in the back of the classrooms. They saw 34 lessons altogether (all years, except 3 and 4): 8 of these were at lower primary school (ethics, mathematics, geography, foreign language); 12 were at upper primary school (biology, physics, Hungarian grammar, mathematics, geography, foreign language); and 14 were at secondary school (Hungarian grammar and literature, mathematics, physics, geography, foreign language). Of the 14 secondary school lessons, 10 took place at a (four-year) grammar school and 4 took place at a technical grammar school (formerly: technical college). Of the 34 teachers, 7 were male and 27 were female, all of them between the ages of 35 and 45 , and with 10 to 20 years of teaching experience.

The observations were carried out by the observers based on a system of aspects (Table 2) as developed by us which was based on models and research methods presented in the theoretical part.

Table 2. Aspects of observation.

\begin{tabular}{|c|c|}
\hline Main aspects & Sub-aspects \\
\hline Characteristics of the class & $\begin{array}{l}\text { - Type of school (primary school, secondary school) } \\
\text { - } \quad \text { Year } \\
\text { - Number of students (present) } \\
\text { - } \quad \text { Desk arrangement (e.g., in rows, U-shape, circular, in groups) } \\
\text { - Place of teacher's desk (e.g., in the forefront, on the side, in the middle) }\end{array}$ \\
\hline Source of the problem & $\begin{array}{l}\text { - Among who did the problem emerge: student-student, student-teacher } \\
\text { - How many are involved } \\
\text { - Distribution of sexes }\end{array}$ \\
\hline Subject & $\begin{array}{l}\text { - What is the problem based on what has been said (e.g., interest, value, } \\
\text { communication style, attribute) }\end{array}$ \\
\hline Chronology & $\begin{array}{l}\text { - Happened during the lesson } \\
\text { - Happened in the past and mentioned during the lesson } \\
\text { - Will happen in the future (possibly or certainly) }\end{array}$ \\
\hline Frequency & $\begin{array}{l}\text { - } \text { Expressed once } \\
\text { - Recurring during the lesson } \\
\text { - Permeates the entirety of the lesson }\end{array}$ \\
\hline Organisation & $\begin{array}{l}\text { - No connection with another (isolated) } \\
\text { - Few causal relations } \\
\text { - Many, forming a hierarchic system, causal relation }\end{array}$ \\
\hline Relation & $\begin{array}{ll}\text { - } & \text { Negative } \\
\text { - } & \text { Positive } \\
\end{array}$ \\
\hline Statements about solvability & $\begin{array}{l}\text { - Solvable (necessary, possible) } \\
\text { - Unsolvable (not needed, not possible) }\end{array}$ \\
\hline $\begin{array}{r}\text { Styles and strategies of } \\
\text { solution }\end{array}$ & $\begin{array}{l}\text { - Styles: rational, impulsive, avoiding } \\
\text { - Strategies: talking it through, aspiring to talk it through, making a } \\
\text { compromise, 'defeating' the other one, verbal/physical aggression }\end{array}$ \\
\hline $\begin{array}{r}\text { Connection between } \\
\text { members }\end{array}$ & $\begin{array}{ll}- & \text { Submission } \\
\text { - } & \text { Coordination } \\
\end{array}$ \\
\hline Teacher's behaviour & $\begin{array}{l}\text { - How does (s)he approaches the problem situation, how does (s)he reacts } \\
\text { - What does (s)he says during the solution }\end{array}$ \\
\hline
\end{tabular}

The observers were asked to make a report of all the situations that they deemed problematic; however, only those situations were analysed which manifested in direct, open communication. Therefore, the 45 instances of being on the phone, six instances of sending messages under the table, two instances of doing crosswords and four instances of reading magazines or books are not included in the analysis - none of these became a problem source (or the teacher did not want to make it one; we do not have insight into this). Based on the system of aspects, all the observers wrote detailed records and another evaluator, who was not present during the lesson, also analysed them. Following this, what was seen was analysed and talked through in smaller groups. No significant discrepancy was noticed between those who observed the same classes; it was only with regard to the reason behind certain problems and their consequences that different opinions emerged. 


\section{Results}

The interpersonal problems identified during the observation are in line with the formerly discovered ones, even in terms of age and sex (Kasik, 2015; Kasik \& Gál, 2014, 2016): in lower years, mockery emerges as the main problem while physical and verbal abuse emerges in upper years. Favouritism by the teacher, one of the most frequent student-teacher problems identified, was not seen by the observers during these lessons. Of all the problem situations among students, it was apparent from communication between them on two occasions (mockery, verbal absuse) that they wanted to solve the disagreement as soon as possible. It was seen among secondary school students that negative orientation is closely connected to the thought of insolvability (e.g. in the case of verbal abuse: 'Leave me alone, I do not want to deal with this. - I do not even care about you, you do not even understand. '). This supports the data from the questionnaire: negative orientation towards the problem and its solution becomes more and more prominent in adolescence.

Teachers' ability to recognise the situation, their intention for the problem situation, their communication technique and their relationship with the children defines how students interpret and deal with the problem and could counterpoint the often non-adequate social problem-solving style and mode acquired as part of the family socialisation (Webster-Stratton, 2011). Based on the observations, all teachers except lower primary school teachers regarded the problems that emerge between students and students and teachers as trouble, uncomfortable and as an obstacle; their aim was to quickly put an end to the situation. It was also with the exception of lower year teachers that their orientation towards the problem situation was significantly negative. This is in accordance with Goldstein's conclusion (1999) that pre-adolescent and adolescent students' problems are dealt with in a different way by teachers. In the case of lower year teachers, this can be accounted for by the number of hours spent with children (higher for lower year teachers), which enables them to get to know them better in terms of, among many things, their problems and the reasons behind them as well as their problem-solving styles and behaviour. Age-related differences in social attributes can be an important accounting factor too (e.g. emotion control, communication with a person of authority).

Problem-solving among upper primary school and secondary school teachers (both in terms of student-student and student-teacher problems) was largely dominated by them; they state what the good solution is, and they order its execution right away. It could not be even seen in the case of problems between students that they let them deal with it; they formed a suggestion for the solution. In the case of verbal and physical abuse as well as upon instances of taking liberties and scoffing, teachers do not really deal with what could be in the background of these based on their reactions. For example, what the reason behind scoffing could be (e.g. the suppression of some sort of past event), why an argument resulted in the beating of the other one, what they said to each other, why they said that. In addition to the behavioural approach, the acknowledgement of emotions and thoughts fails even though this is important both in terms of dealing with the problems and what there is behind them, and this would be of significant importance in early and later adolescence as well.

Though to varying degrees, but all teachers drew students' attention to their place in the hierarchy and their own position (e.g. in the case of taking liberties: 'My child, you cannot lecture me.'; 'Know who you are speaking to.'). Their communication was based on authority and they showed offence. Burnout can be hypothesised in the case of those teachers who utilised shaming, which typically manifests as loss of control in a tense situation (Petróczi, 2007). This is supported by the fact that this happened in the presence of an observer as well as running out of the classroom. The question arises: what does the teacher do when (s)he is alone with the children?

Examples were also seen where the problems that manifest themselves during the lesson have serious precursors; the events that took place outside of the lesson continue into it, emerge as even more complex problems (physical abuse) and they continue unless the situation is dealt with. Thinking and talking things through, evaluating the problem situation after it was dealt with, were not observed either. According to Webster-Stratton (2011), one of the foundations of the emergence of social problems and, most importantly, dealing with them is that the teacher knows what kind of emotional state the students arrive to a lesson, and this is expected from teachers. However, this requires time and it can hardly become a part of the culture of teaching in light of the fact that the observed teachers want to solve the problems as quickly as possible, without shedding light on the underlying reasons in most cases. 


\section{Discussion and limitation}

What was observed draws attention to the effect problems have on the performance (of dealing with the learning material) in the lesson because its course apparently breaks, especially after verbal and physical abuse; in the case of five lessons, teachers indicated that they could not do the tasks designated (in three cases, they were given as homework). Social problems in lessons can also influence students' attitude towards subjects and they can impact their motivation as well. Further observations are planned for the future and, in order not to only compare what was seen with data from earlier questionnaires, we are planning to have questionnaires completed in the observed classes which enables the analysis of several aspects of problem-solving. Such a questionnaire is NEGORI (Kasik et al., 2018), which measures negative orientation, or the Avoidance questionnaire (Kasik et al., 2016). The combined data collection ensures more precise and additional information in order to develop the most complex developmental programme for children and adolescents - and their teachers.

\section{References}

Bedell, J. R., Archer, R. P., \& Marlowe, H. A. Jr. (1980). A description and evaluation of a problem solving skills training programs. In: D. Upper, \& S. M. Ross (Eds.), Behavioral group therapy: An annual review (pp. 3-35). Champaign: Research Press.

Chang, E. E., D'Zurilla, T. J., \& Sanna, L. J. (2004) Social problem solving. Theory, research, and training. Washington, DC: American Psychological Association.

Eskin, M. (2012). Social Problem Solving Therapy in the Clinical Practice. Turkey, Elsevier.

Goldstein, A. P. (1999). The PREPARE curriculum: Teaching prosocial competencies. Champaign, IL: Research Press.

Hunt, J. McV. (1963). Motivation inherent in information processing and action. In O. J. Harvey (Ed.), Motivation and social interaction (pp. 35-94). New York: Ronald Press.

Kasik, L. (2015). Személyközi problémák és megoldásuk. Budapest: Gondolat Kiadó.

Kasik, L., \& Gál, Z. (2014). Mit tekintenek 7, 10 és 14 éves diákok személyközi problémának? Iskolakultúra, 24(9), 3-25.

Kasik, L., \& Gál, Z. (2015). A helyzet- és személyspecifikus problémamegoldás kapcsolata a tudatelméleti müködéssel 4-6 éves korban. Magyar Pedagógia, 115(4), 297-318.

Kasik, L., \& Gál, Z. (2016). Parents' and teachers' opinions of preschool children's social problem-solving and behavioural problems. Early Child Development and Care, 118(10), 1632-1648.

Kasik, L., Gál, Z., \& Tóth, E. (2018). Development and Psychometric Properties of NEGORI (Negative Orientation Questionnaire). Journal of Relationships Research, 9(8), 1-13.

Kasik, L., Guti, K., Tóth, E., \& Fejes, J. B. (2016). Az elkerülés mint folyamat - az Elkerülés kérdőív bemérése 15 és 18 évesek körében. Magyar Pedagógia, 116(2), 219-253.

Petróczi, E. (2007). Kiégés - elkerülhetetlen? Budapest: Eötvös József Könyvkiadó.

Singleton, L. C., \& Asher, S. R. (1977). Peer preferences and social interaction among third-grade children in an integrated school district. Journal of Educational Psychology, 69, 330-336.

Strough, J., \& Keener, E. (2013). Interpersonal problem solving across the life span. In P. Verhaeghen \& C. Hertzog (Eds.), The Oxford handbook of emotion, social cognition, and everyday problem solving during adulthood (pp. 134-152). Oxford University Press: The Oxford Library of Psychology Series.

Webster-Stratton, C. (2011). The Incredible Years. Parents, Teachers, and Children's Training Series. Program Contetnt, Methods, Research and Dissemination 1980-2011. file:///The-IncredibleYears-Parent-Teacher-Childrens-Training-Series-1980-2011p.pdf 


\title{
TUNING IN TO KIDS: A PILOTING STUDY TARGETED TO SUPPORT TURKISH PARENTS' EMOTION SOCIALIZATION*
}

\author{
Ayca Ulker Erdem ${ }^{1}$, Mubeccel Gonen ${ }^{1}$, \& Sophie Havighurst ${ }^{2}$ \\ ${ }^{1}$ Hacettepe University, Department of Early Childhood Education (Turkey) \\ ${ }^{2}$ University of Melbourne Department of Psychiatry (Australia)
}

\begin{abstract}
This study examined the contribution of Tuning in to Kids (TIK) Program on emotion socialization practices of Turkish parents as well as reducing preschoolers' emotional and behavioral problems. TIK is an evidence-based parenting program particularly teaches parents emotion coaching skills, which is to recognize, understand and respond to children's emotions in a reassuring way. Randomized controlled trials of TIK have found increases in parent emotion coaching and child emotion knowledge, and reductions in difficult child behaviors. On this basis, TIK helps parents to develop appropriate emotion socialization skills. When emotion socialization studies conducted with parents in Turkey are examined, significant numerous research on parents' emotion socialization has been documented; however, an intervention targeted to support emotion socialization practices of parents has never studied in this context. In this regard, the effectiveness of TIK Program among Turkey sample was examined in the current research. As a first stage TIK was adapted to Turkish under the guidance of field experts and a pilot implementation was performed. The content of program was organized in terms of clearance, comprehensibleness and applicability. In the second stage, a quasi experimental design was used to consider program impact on certain parent and child outcomes. Ethical permits to conduct this research was obtained by the Ethics Committee of the researcher's university. With the approval of ethics committee, researchers obtained the required informed assents of all parents. 60 parents, who has at least one child between three to six years old, were recruited via preschools and were assigned in experiment and wait list control groups according to their demographics. Parents filled a battery of assessment in order to assess their emotion socialization practices and to rate their children's emotional and behavioral problems. Parents in experiment condition participated in the 6 session TIK program between pre and posttests. They learnt emotion coaching skills and how to regulate their own emotions throughout the sessions. Follow up assessments were employed to examine retention effects. Data analysis was performed using a mixed model ANOVA. Preliminary results showed that program completion rates were high. Most of the participants reported high satisfaction with the program. There are statistically significant improvements in parents' non-supportive emotion socialization practices between pretests and posttests $(F(1,55)=20.464, p<0.01)$. However, there is no significant improvement was seen in children's parent-reported outcomes. This study which explores the potential use of TIK in Turkey sample is thought to make a significant contribution to the field. All the findings would be evaluated to use Tuning into Kids Program for early childhood education settings in Turkey.
\end{abstract}

Keywords: Emotion socialization, parenting program, early childhood, early intervention, tuning in to kids.

*This research covers a part of the first author's doctoral dissertation under the supervision of the second and the third author and supported by The Scientific and Technological Research Council of Turkey- TUBITAK. 


\title{
TURKISH VALIDITY AND RELIABILITY OF WATSON-GLASER CRITICAL THINKING APPRAISAL TEST'S SHORT FORM*
}

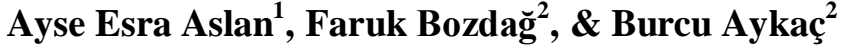 \\ ${ }^{I}$ Faculty of Education, Istanbul University-Cerrahpasa/Prof. Dr. (Turkey) \\ ${ }^{2}$ Faculty of Education, Istanbul University-Cerrahpasa/Research Assistant (Turkey)
}

\begin{abstract}
Problem: Critical thinking has been the focus of attention for both philosophers and psychology experts since ancient times. Criticism is expressed etymologically in Greek as "critic" or "kritike", and in Latin as "criticus". Ennis, with his essay "Critical Thinking Concept" in Harvard Educational Review (1962), is the pioneer of contemporary critical thinking in the United States. In this article, Ennis tries to clarify the concept of critical thinking and proposes a taxonomy that includes twelve critical thinking structures.

Critical thinking is addressed in the field of education when Dewey started to use the concept of reflective thinking. According to Dewey (1909), reflective thinking is an active, permanent and careful evaluation of any belief or knowledge in the light of the ground that supports it.

According to the Delphi Report prepared by experts in the field of critical thinking (Facione, 1990), critical thinking is the explanation of purposeful, conceptual, methodological, critical or contextual ideas on which the decision is based, self-regulated judgment, resulting in interpretation, analysis, evaluation and inference The main aim of this study is to develop the Turkish version of the critical thinking scale which is an important concept in the evaluation of individual differences.

Method: Psychological test development and adaptation steps will be followed.

Instruments: Watson-Glaser Critical Thinking Appraisal Form S (Short Form) consists of 16 scenarios, 40 items, and 5 sub-tests. The sub-tests are Making Inferences, Recognition of Assumptions, Deduction, Interpretation, Evaluation of Arguments. Parallel forms of the test are available.

Data analysis: In data analysis, factor analysis will be carried out according to the structural equation model after obtaining an available Turkish scale form for linguistic equivalence study.
\end{abstract}

Keywords: Critical thinking, Watson-Glaser critical thinking scale-form S, university students critical thinking ability.

\section{Introduction}

Critical thinking, one of the favorite subjects of education and cognitive psychology, is both a product of intelligence and a thinking skill that develops intelligence. According to the Delphi Report, critical thinking is defined as a purposeful, self - regulatory judgment resulting in interpretation, analysis, assessment and inference, as well as explanation of the evidence, conceptual, methodological, criteriological or contextual considerations on which that judgment is based (Facione, 1990). Socrates developed the method of Socratic Questioning for critical thinking (Schreglmann, 2011), while Dewey (1909, cited in O'Hare, 2004) said that critical thinking under reflective thinking should be considered within the educational objectives. Bloom (Ennis, 1993) includes critical thinking within the group of higher achievements in the taxonomy of educational objectives. Although it is important to develop critical thinking skills within the Turkish National Education objectives, it is observed that this cannot be developed through the implemented curriculum and practice (Ersoy \& Baser, 2011; Bulut, Ertem \& Sevil, 2009). The teacher is evaluated in terms of attitudes and behaviors, teaching methods and creating educational materials in developing critical thinking skill (Alkın-Şahin \& Gözütok, 2013). However, there is a need for a special program and training material prepared by the expert to develop critical thinking (Aybek, 2006; Aybek, 2010; Gürkaynak, Üstel, \& Gülgöz, 2008; Tok \& Sevinç, 2010). The critical intellectual characteristics of the critical thinkers are listed. These are; 1) disciplined features, 2) intellectual integrity, 3) intellectual modesty, 4) intellectual justice, 5) intellectual determination, 6) intellectual objectivity, 7) intellectual confidence in reasoning, 8) intellectual courage, 9) intellectual empathy ( Paul \& Elder, 2013).

*This research was carried out with the research scholarship within the scope of project no 36913 in the scientific research projects of Istanbul University. 


\section{Objectives}

The main aim of this study is to develop the Turkish version of the Watson-Glaser Critical Thinking Appraisal Test-Short Form. The long form of the test has been adapted to Turkish previously, however, the validity and the reliability of the test were problematic. In this context, it is aimed to analyze the reliability and the construct validity of the Turkish version of the Watson-Glaser Critical Thinking Appraisal Test- Short Form.

\section{Methods}

In the research, psychometric scale development steps were followed. The sample of the study was selected from the prospective teachers, aged between 18-24, that are enrolled in the education faculty of Istanbul University. Because it is a state university, the students have enrolled in the faculty with a placement test and came from various regions of Turkey. $\mathrm{N}=773$ people from 13 departments within the faculty were reached. Analyzes were performed according to classical test theory and results at least $\mathrm{p}<.05$ confidence interval were evaluated.

The linguistic equivalence studies were analyzed with the data collected from a group of $\mathrm{N}=57$ people studying in English language teaching department and have a good mastery of both languages. Firstly, the English form was applied to the study group and after a 15-day interval, the Turkish form was applied. Pearson Product Moment Correlation (r) and related group t-test were used between these two groups of scores.

For reliability applications, Cronbach Alpha analysis was performed. The construct validity was tested within the scope of validity analyzes. Item total, item remainder, and item analysis were performed. For item total and item remainder analysis correlation was used and unrelated group t-test was used for item analysis.

\subsection{Data collection tool}

Watson-Glaser Critical Thinking Appraisal Test-Short Form consists of five subtests and 40 questions. A high level of competency in critical thinking, as measured by the Watson-Glaser, may be operationally defined as the ability to correctly perform the domain of tasks represented by the five tests (Glaser, 2008).

1. Inference. Discriminating among degrees of truth or falsity of inferences drawn from given data.

2. Recognition of Assumptions. Recognizing unstated assumptions or presuppositions in given statements or assertions.

3. Deduction. Determining whether certain conclusions necessarily follow from information in given statements or premises.

4. Interpretation. Weighing the evidence and deciding if generalizations or conclusions based on the given data are warranted.

5. Evaluation of Arguments. Distinguishing between arguments that are strong and relevant and those that are weak or irrelevant to a particular issue.

For each subtest, a reading text and related premises were given. The selected texts are similar to the daily newspaper or journal articles. Test takers are asked to give a judgment/comment to premises depending on the text. For the inference subtest, 5-point Likert scale was used and the other four tests were answered in $1 / 0$ form.

\section{Conclusion and discussion}

The research data were collected from a total number of 219 people $(\mathrm{N}=773$, Nfemale $=554$, $\mathrm{N}_{\text {male }}=219$ ) from 13 departments of Istanbul University Faculty of Education that educates teacher candidates in various fields. The relationship between the data obtained with English and Turkish test forms that were applied to assess linguistic equivalence was calculated by Pearson Product Moment Correlation Coefficient and statistically significant relationship was determined for all of the subtests as follws; Inference $(\mathrm{r}=.984$, sig. $=.000, \mathrm{t}=-1,694$, sig. $=.000, \mathrm{p}>\mid .05)$, Recognition of Assumptions $(\mathrm{r}=.995$, sig. $=.000$, $\mathrm{t}=1$,427, sig. $=.000, \mathrm{p}>\mid .05$ ), Deduction. ( $\mathrm{r}=.955$, sig.=.000, $\mathrm{t}=-6,051$, sig. $=.000, \mathrm{p}<.05$ ), Interpretation $(\mathrm{r}=.961$, sig. $=.000, \mathrm{t}=-1,000-$, sig. $=.322, \mathrm{p}>\mid .05)$, Evaluation of Arguments $(\mathrm{r}=.981$, sig. $=.000, \mathrm{t}=1,351$, sig. $=.182, \mathrm{p}>\mid .05)$ and for total $(\mathrm{r}=.988$, sig. $=.000, \mathrm{t}=-5,519$, sig. $=.000, \mathrm{p}<.001)$. In addition, meaningless results were obtained except for the deduction subtest in the related group t-test analysis that was performed between the mean values of the two groups. Significant relationships for these analyses are expected to be meaningless t-test. In the reliability analysis, the following Cronbach's Alpha values were obtained for the 
subtests: Inference $(\alpha=.595)$, Recognition of Assumptions $(\alpha=.705)$, Deduction $(\alpha=.705)$, Interpretation $(\alpha=.660)$, Evaluation of Arguments $(\alpha=.695)$. For all subtests, validity analyses were performed because the degree of reliability was considered to be within acceptable limits.

Factor analysis was done for the construct validity of the scale. Factor analysis is a multivariate statistical method that aims to find a less significant number of new variables conceptually by combining a number of interrelated variables (Çokluk, Şekercioğlu \& Büyüköztürk, 2012). For the validity analysis, all sub-tests were accepted as a factor and item total and item remainder analyzes were performed. For these five subtests, a significant result was obtained at $\mathrm{p}<.01$ level. This result was interpreted as a pattern between the subtests of the test and the items in terms of measuring critical thinking and the discriminant analysis of the subtests were done. When the same analyses were repeated on the basis of the items covered by the sub-tests, the relationship between the total subtest and the whole test for the validity of the test was accepted, although meaningless results were obtained from the item total and item remainder analysis for some items. Considering the limit values that can be taken into consideration in interpreting the item-total correlation, in general items with a total correlation of 0.30 or higher are known to differentiate the individuals well (Büyüköztürk, 2006, Ennis, 1996).

The use of construct validity of a test provides the internal consistency of the items within the subtests and the subtests within the whole. The correlation coefficient of Person Product Moments is used to determine the correlation. Pearson Product Moment Correlation coefficient is used to find and interpret the relationship between two variables, while regression analysis is used to determine how independent variables explain the changes observed in the dependent variable, whether they predict the dependent variable in a meaningful way and their relative significance on the dependent variable (Büyüköztürk, 2007). In a subsequent study, with confirmatory factor analysis, it can be tested whether subtests predict the five subfactor structures as in the original. Confirmatory factor analysis is a fairly large statistical technique used at a higher level in the testing of related theories and latent variables (Tabachnick and Fidell, 2014). In the confirmatory factor analysis, the researcher specifies in the hypothesis or model that s/he established before the analysis that which variables are related to which dimensions and which dimensions are related to each other (Stevens, 2002). It is possible to see studies, which develop tools to measure the elements that will directly or indirectly affect the critical thinking for different age levels, that apply the structural equation model as another analysis model in the construct validity and factor analysis (Gülveren, 2007; Kayagil \& Erdoğan, 2011; Vural \& Kutlu, 2004). Structural equation model is a statistical technique used for testing models where causal and correlational relationships coexist between observed variables and latent variables. This method is a multivariate method which is formed by combining analysis such as variance, covariance analysis, factor analysis and multiple regression to predict dependency relationships (Tüfekçi \& Tüfekçi, 2006). It is mainly a combination of factor analysis and regression analysis. This analysis tests the compliance of the observed covariance matrix with the covariance matrix according to the theoretical model (Hox \& Bechger, 1995, p. 356). The two basic characteristics of structural equation modeling are as follows (Hair et al., 1998, p. 584); firstly, multiple and interdependent relationships are tested in a single analysis.; secondly, it is assumed that the indicator variables cannot be measured perfectly and error variances of the indicators are included in the calculations.

\section{References}

Alkın-Şahin, S. \& Gözütok, F. D. (2013). Eleştirel düşünmeyi destekleyen öğretmen davranışları envanteri (EDDÖDE): Geliştirilmesi ve uygulanması [Critical thinking supportive teacher behaviors inventory (CTSTBI): Its development and application]. Eğitim Bilimleri Araştırmaları Dergisi - Journal of Educational Sciences Research, 3(2), 223-254. http://ebad-jesr.com/

Aybek, B. (2006). Konu ve beceri temelli eleştirel düşünme öğretiminin öğretmen adaylarının eleştirel düşünme eğilimi ve düzeyine etkisi [The effect of subject-based and skill-based critical thinking teaching on the critical thinking disposition and level of teacher candidates.]. (Unpublished doctoral dissertation). Çukurova University Institute of Social Sciences Department of Educational Sciences.

Aybek, B. (2010). Örneklerle düşünme ve eleştirel düşünme [Thinking and critical thinking with examples]. Ankara: Nobel Publishing.

Bulut, S., Ertem, G. \& Sevil, Ü. (2009). Hemşirelik öğrencilerinin eleştirel düşünme düzeylerinin incelenmesi [Examining the critical thinking levels of nursing students]. Dokuz Eylül Üniversitesi Hemşirelik Yüksekokulu Elektronik Dergisi- Dokuz Eylül University Electronic Journal of School of Nursing, 2(2), 27-38.

Büyüköztürk, Ş. (2007). Veri analizi el kitabı [Handbook of data analysis]. Ankara: PegemA Publishing.

Çokluk, Ö., Şekercioğlu, G., \& Büyüköztürk, Ş. (2012). Sosyal bilimler için çok değişkenli istatistik spss ve lisrel uygulamalarl [Multivariate statistics spss and lisrel applications for social sciences]. Ankara: PegemA Publishing. 
Doğan, N. (2013). Eleştirel düşünmenin ölçülmesi [Measurement of critical thinking]. Cito Eğitim: Kuram ve Uygulama- Cito Education: Theory and Practice, 22, 29-42.

Dutoğlu, G. \& Tuncel, M. (2008). Aday öğretmenlerin eleştirel düşünme eğilimleri ile duygusal zeka düzeyleri arasındaki ilişki [The relationship between the teacher candidates' critical thinking dispositions and the levels of emotional intelligence]. Abant İzzet Baysal Üniversitesi Ĕgitim Fakültesi Dergisi- Abant Izzet Baysal university journal of Education Faculty, 8(1);11-32.

Ennis, R. H. (1993). Critical thinking assessment. Theory into practice, 32 (3), 179-186.

Ersoy, E. \& Başer, N. (2011). İlköğretim ikinci kademede eleştirel düşünmenin yeri [The place of critical thinking in the secondary stage of primary education]. Adnan Menderes Üniversitesi Ĕgitim Fakültesi Eğitim Bilimleri Dergisi- Adnan Menderes University Journal of Educational Sciences, 2(1), 1-10. http://dergi.adu.edu.tr/egitimbilimleri/

Facione, P. (1990). Critical thinking: A statement of expert consensus for purposes of educational assessment and instruction (The Delphi Report).

Gülveren, H. (2007). Ë̆itim fakültesi öğrencilerinin eleştirel düşünme becerileri ve bu becerileri etkileyen eleștirel düșünme faktörleri [Critical thinking skills of the faculty of education and critical thinking factors affecting these skills]. (Unpublished doctoral dissertation). Dokuz Eylül University Graduate School of Educational Sciences, İzmir.

Gürkaynak, İ, Üstel, F., \& Gülgöz, S. (2008). Eleştirel Düşünme, [Critical thinking]. $3^{\text {rd }}$ Edition. Education Reform Initiative (ERI) reports.

Hair, J.F., R.E. Anderson, R.L. Tahtam \& W. C. Black (1998). Multivariate data analysis. Prentice Hall International Inc., New Jersey.

Hox, J.J. ve T.M. Bechger (1995). An introduction to structural equation modeling. Family Science Review, $11,354-373$.

Kartal, T. (2012). İlköğretim fen Bilgisi öğretmen adaylarının eleştirel düşünme eğilimlerinin incelenmesi [An investigation of the critical thinking disposition of elementary science teacher candidates]. Ahi Evran Üniversitesi Kırşehir Eğitim Fakültesi Dergisi- Ahi Evran University Kırşehir Educational Faculty Magazine (KEFAD), 13(2); 279-297.

Kayagül, S. \& Erdoğan, A. (2011). Bazı değişkenlerin ilköğretim yedinci sınıf öğrencilerinin eleştirel düşünme becerilerini yordama gücü [The prediction level of some variables in elementary school seventh grade students' critical thinking skills]. Selçuk Üniversitesi Ahmet Keleşoğlu Ĕgitim Fakültesi Dergisi- Selçuk University Ahmet Keleşoğlu Journal of Education Faculty, 31, 21-334.

O'Hare, L. C. (2005). Measuring critical thinking skills and dispositions in undergraduate students. (Unpublished doctoral dissertation). Queen's University Belfast Faculty of Science and Agriculture, Northern Ireland.

Paul, R. \& Elder, L. (2013). Kritik düşünce: Yaşamınızın ve öğrenmenizin sorumluluğunu üstlenmek için araçlar [Critical thinking: Tools for taking charge of your learning and your life]. $3^{\text {rd }}$ Edition. İstanbul: Nobel Akademi.

Schreglmann, S. (2011). Konu temelli eleştirel düşünme ögrretiminin öğretmen adaylarının akademik başarl, eleştirel düşünme eğilimine ve düzeyine olan etkisi [The effect of subject-based critical thinking teaching on the academic achievement, critical thinking disposition level of teacher candidates]. (Unpublished Master Thesis). Çukurova University Institute of Social Sciences, Adana.

Stevens, J. P. (2002). Applied multivariate statistics for the social sciences. New Jersey: Lawrance Erlbaum Association, Inc

Tabachnick, B. G. \& Fidel, L. S. (2014). Using multivariate statistics. USA: Pearson Education Limited.

Tok, E. \& Sevinç, M. (2010). Düşünme Becerileri Eğitiminin Eleştirel Düşünme ve Problem Çözme Becerilerine Etkisi [Effects of Thinking Skills Training on Critical Thinking and Problem Solving Skills]. Pamukkale Üniversitesi Eğitim Fakültesi Dergisi- Pamukkale University Faculty of Education Journal, 27, 67-82.

Tüfekçi, N. \& Tüfekçi, Ö. K. (2006). Bankacılık sektöründe farklı olma üstünlüğünün ve müşteri sadakatinin yarattığı değer: Isparta ilinde bir uygulama [The advantage of being different in the banking sector and customer loyalty: An application in the province of Isparta]. Süleyman Demirel Üniversitesi Sosyal Bilimler Enstitüsü Dergisi- Journal of Süleyman Demirel University Institute of Social Sciences, 2(4), 170-183.

Vural, R. A. \& Kutlu O. (2004). Eleştirel düşünme: Ölçme araçlarının incelenmesi ve bir güvenirlik çalışması [Critical thinking: Examination of measurement tools and a reliability study]. Sosyal Bilimler Enstitüsü Dergisi- Journal of Social Sciences Institute, 13(2), 189-200.

Watson, G., \& Glaser, E. M. (2008), Watson-Glaser Critical Thinking Appraisal Short Form, USA: Pearson Education, Inc.

Yıldırım, H. İ. \& Şensoy, Ö. (2011). İlköğretim 7. Sınıf öğrencilerinin eleştirel düşünme eğilimi üzerine eleştirel düşünme becerilerini temel alan fen öğretiminin etkisi [The effect of science teaching based on critical thinking skills on 7th grade students' critical thinking disposition]. Kastamonu Eğitim Dergisi- Kastamonu Education Journal, 19(2); 523-540. 


\title{
MATHEMATICS TEACHERS' PROFESSIONAL DEVELOPMENT - GENDER DIFFERENCE
}

\author{
Danijela S. Petrovic \\ Department of psychology, Faculty of Philosophy, University of Belgrade (Serbia)
}

\begin{abstract}
As professionals, teachers need to invest into their professional development on a long-term basis in order to fulfil the job demands across their entire career. Additional insight into this issue can be obtained by exploring how teachers perceive their professional development. Therefore, the aim of this paper is to examine gender differences related to participation in various professional development activities, perceived impact of professional development, support for and obstacles to professional development, as well as, the need for professional development. Data was collected within the 2013 Teaching and Learning International Survey (TALIS 2013) on a sample of 440 Serbian mathematics teachers (ISCED 2) from 191 schools (65.7\% female and 34.3\% male teachers). A standardized TALIS questionnaire for teachers was used for data collection. The results show that more female teachers participated in at least one professional development activity during the previous 12 -month period $\left(93.1 \%\right.$ vs. $86.2 \%, \chi^{2}(1,444)$ $=5.559, \mathrm{p}<.05)$ than their male colleagues. A similar number of female and male teachers attended various types of professional development activities (such as workshops, observation visits to other schools, in-service training courses, participation in teachers' networks, collaborative research, peer observation, etc.), except education conferences which were attended by more female teachers (63.4\% vs. $\left.48.1 \%, \chi^{2}(1,444)=9.795, \mathrm{p}<.01\right)$. Additionally, female teachers spend more days in professional development activities in general (27 vs. 17 days). Furthermore, female teachers expressed a higher need for professional development than their male colleagues, especially in teaching students with special needs $\left(68.1 \%\right.$ vs. $\left.58 \%, \chi^{2}(1,435)=4.358, \mathrm{p}<.01\right)$, new technologies in the workplace $(54.9 \%$ vs. $45.6 \%$, $\left.\chi^{2}(1,437)=3.346, \mathrm{p}<.05\right)$, approaches to individualised learning $\left(48.8 \%\right.$ vs. $39.7 \%, \chi^{2}(1,438)=3.263$, $\mathrm{p}<.05)$, teaching cross-curricular skills $\left(46.7 \%\right.$ vs. $\left.32.1 \%, \chi^{2}(1,437)=7.959, \mathrm{p}<.01\right)$ and student behaviour and classroom management $\left(45.8 \%\right.$ vs. $\left.31.3 \%, \chi^{2}(1,438)=8.581, \mathrm{p}<.01\right)$. Male teachers more often highlighted a lack of employer support as a barrier to participation in professional development $\left(38.3 \%\right.$ vs. $\left.28.6 \%, \chi^{2}(1,439)=4.206, \mathrm{p}<.05\right)$. There is no deference between female and male teachers regarding their perception of professional development activities' impact and costs, as well as, the support obtained by the school. Based on the results it can be concluded that female mathematics teachers invest more in their professional development and they are more sensitive to development of competences that directly support teaching and learning.
\end{abstract}

Keywords: Teachers, mathematics, professional development, gender difference, TALIS 2013.

\section{Introduction}

TALIS - the OECD Teaching and Learning International Survey examines both the conditions under which learning takes place and under which teachers work. TALIS examines the ways in which teachers' work is recognised, appraised and rewarded and assesses the degree to which teachers perceive that their professional development needs are being met. The study provides insights into the beliefs and attitudes about teaching that teachers bring to the classroom and the pedagogical practices that they adopt. Additionally, TALIS examines the extent to which certain factors relate to teachers' reports of job satisfaction and self-efficacy. Finally, recognising the important role of school leadership, TALIS examines the roles of school leaders and the support that they give their teachers (OECD, 2009, 2014).

The TALIS survey focuses on the following policy issues: (1) The appraisal of teachers' work in schools and the form and nature of the feedback they receive, as well as the use of outcomes from these processes to reward and develop teachers; (2) The amount and type of professional development available to teachers and their needs and barriers for accessing training; (3) The impact that school-level policies and practices, including those of the school leadership, shape the learning environment in schools and 
impact the work of teachers; (4) The creation and support of effective school leadership in an era of accountability and devolution of educational authority; (5) The extent to which recent trends in school leadership and management have an impact on teachers; (6) The profiles of countries with regard to teaching practices, activities, professional development needs, beliefs and attitudes, and variation in these according to teachers' background characteristics (OECD, 2009, 2013).

Research conducted over the past two decades (e.g., Darling-Hammond, 1999; Hargreaves, 2002; Hattie, 2009; Johnson, Kraft \& Papay, 2012; OECD, 2009, 2014), unambiguously shows that professional development of teachers is one of the key factors both for improving the educational achievements of students and for greater teachers' work commitment.

Most scholars, regardless of their theoretical orientation (e.g., Desimone, 2009; Guskey, 2002 and 2009; Fessler, 1995; Tickle, 2000), when talking about the professional development of teachers, point to three phases - the phase of initial education (pre-service training), the introduction to work (induction phase) and the phase of continuous professional development (in-service training). The initial teacher education represents a formal preparation of teachers during which basic teaching competences are acquired. The induction phase is a stage when, with the support of mentors, real first independent steps into the role of teachers are made, i.e. when, for the first time, teaching is carried out independently. It is also a phase in which a novice teacher faces the professional reality. The phase of continuous professional development is the phase in which a teacher overcomes initial challenges and continues to work on improving their competences.

Different definitions of professional development can be found in the relevant literature. According to the definition used in TALIS, professional development of teachers includes activities that develop individual skills, knowledge, expertise and other characteristics of teachers in order to improve their teaching practice (OECD, 2013).

\section{Objectives}

As professionals, teachers need to invest into their professional development on a long-term basis in order to fulfil the job demands across their entire career. Additional insight into this issue can be obtained by exploring how teachers perceive their professional development. Therefore, the aim of this paper is to examine mathematics teachers' gender differences related to continuous professional development. The following elements were investigated: participation in various professional development activities, perceived impact of attended professional development activities, need for further professional development, as well as, support for and obstacles to professional development (OECD, 2014).

Mathematics teachers were selected for several reasons. Firstly, mathematics is not a favourite school subject for Serbian students. Only $15.6 \%$ of elementary school students like mathematics and $38.4 \%$ think that mathematics is useful subject (Petrović, 2000). In general, mathematics has been shown to be related with less interest and enjoyment than other domains, eliciting most anxiety among students (OECD, 2010; Radišić, Videnović \& Baucal, 2015). Secondly, Serbian students underachieve in mathematics. For example, data collected in PISA 2012 survey show that students from Serbia on average have significantly lower performance in mathematics literacy (449 points, which is about 45 points lower average performance) compared to OECD countries. This difference corresponds roughly to the effects of 1 year of schooling (Pavlović, Babić \& Baucal, 2013). Finally, Serbian mathematics teacher expresses a traditional set of beliefs about teaching and learning to a higher extent than teachers form other domains (e.g., Serbian language teachers) (Radišić \& Baucal, 2012).

\section{Methods}

Data was collected within the Teaching and Learning International Survey (TALIS 2013) on a sample of 440 Serbian mathematics teachers (ISCED 2) from 191 schools (65.7\% female and 34.3\% male teachers).

The conducted research is non-experimental, survey type. A standardized TALIS questionnaire for teachers, developed by an international expert group, was used for data collection (OECD, 2013). The team of domestic experts in the field of education adapted the questions in the questionnaire (to the extent that it was possible) to the Serbian education system, and some issues that are of particular importance to Serbian educational context have been added. Questions in the questionnaire (there are 48 in total) are closed type, with two or more offered answers (e.g. Likert scales). Prior to use in the main research, the questionnaire was standardized and psychometrically validated in a pilot study involving 332 teachers from Serbia. Descriptive statistics were used for data processing, and one-factor analysis of the variance and the hi-square test was used to test differences between categorical and numerical variables. 


\section{Results}

The results show that more female teachers participated in at least one professional development activity during the previous 12 -month period $\left(93.1 \%\right.$ vs. $\left.86.2 \%, \chi^{2}(1,444)=5.559, p<.05\right)$. As shown in Table 1, a similar number of female and male teachers attended various types of professional development activities (such as workshops, observation visits to other schools, in-service training courses, participation in teachers' networks, collaborative research, peer observation, etc.), except education conferences which were attended by more female teachers $\left(63.4 \%\right.$ vs. $\left.48.1 \%, \chi^{2}(1,444)=9.795, p<.01\right)$. There is no significant difference between female and male teachers regarding the duration of particular professional activities. However, female teachers in general spend more days in professional development activities (27 vs. 17 days) than their male colleagues (see Table 1).

Table 1. Teachers ' professional development activities during the previous 12 month period.

\begin{tabular}{|c|c|c|c|c|c|c|}
\hline \multirow{2}{*}{$\begin{array}{l}\text { Type of professional development } \\
\text { activities }\end{array}$} & \multicolumn{3}{|c|}{ Participation } & \multicolumn{3}{|c|}{ Duration in days } \\
\hline & Female & Male & $\Sigma$ & Female & Male & $\Sigma$ \\
\hline Courses/workshops & $71 \%$ & $63 \%$ & $67 \%$ & 7 & 5 & 6 \\
\hline Education conferences or seminars & $63.8 \% * *$ & $48.1 \% * *$ & $55.9 \%$ & 7 & 5 & 6 \\
\hline Observation visits to other schools & $10.3 \%$ & $13.6 \%$ & $11.9 \%$ & 6 & 2 & 4 \\
\hline $\begin{array}{l}\text { Observation visits to business } \\
\text { premises, public organisations, NGOs }\end{array}$ & $3.4 \%$ & $6.5 \%$ & $4.9 \%$ & 5 & 2 & 3.5 \\
\hline $\begin{array}{l}\text { In-service training courses in business } \\
\text { premises, public organisations, NGOs }\end{array}$ & $7.9 \%$ & $7.8 \%$ & $7.9 \%$ & 2 & 3 & 2.5 \\
\hline Qualification programme & $28 \%$ & $31.2 \%$ & $29.6 \%$ & / & / & / \\
\hline Participation in a network of teacher & $28 \%$ & $31.2 \%$ & $29.6 \%$ & / & / & l \\
\hline $\begin{array}{l}\text { Individual or collaborative research on } \\
\text { a topic of interest to you professionally }\end{array}$ & $24.6 \%$ & $28.1 \%$ & $26.4 \%$ & / & / & 1 \\
\hline $\begin{array}{l}\text { Mentoring and/or peer observation and } \\
\text { coaching }\end{array}$ & $23.9 \%$ & $29.2 \%$ & $26.5 \%$ & I & / & 1 \\
\hline
\end{tabular}

Analyses of chosen areas of professional development (see Table 2) show that majority of teachers attained professional development activities related to student evaluation and assessment practice (78.2\%) or to their discipline, such as, knowledge and understanding of a subject field (77.1\%) and pedagogical competencies in teaching a subject field (67.9\%). The lowest interest teachers show for school management and administration (10.1\%), approaches to developing cross-occupational competencies for future work or future studies (8.5\%) and teaching in a multicultural or multilingual setting (6.1\%). Differences in a chosen area of professional development, among male and female teachers (in favour of male teachers), are obtained for knowledge of the curriculum (38.5\% vs. 27.6\%, $\left.\chi^{2}(1,319)=3.971, \mathrm{p}<.05\right)$, ICT skills for teaching $\left(57.8 \%\right.$ vs. $\left.40.0 \%, \chi^{2}(1,318)=6.344, \mathrm{p}<.01\right)$ and new technologies in the workplace (38.9\% vs. $\left.25.2 \%, \chi^{2}(1,319)=9.148, \mathrm{p}<.01\right)$ )

Mathematics teachers expressed the highest need for professional development in the following areas (see Table 3): teaching students with special needs (63\%), new technologies in the workplace (50\%), approaches to individualised learning (44.3\%) and ICT skills for teaching (44\%). Regarding gender differnces, female teachers expressed a higher need for professional development than their male colleagues, especially in teaching students with special needs $\left(68.1 \%\right.$ vs. $58 \%, \chi^{2}(1,435)=4.358$, $\mathrm{p}<.01)$, new technologies in the workplace $\left(54.9 \%\right.$ vs. $\left.45.6 \%, \chi^{2}(1,437)=3.346, p<.05\right)$, approaches to individualised learning $\left(48.8 \%\right.$ vs. $\left.39.7 \%, \chi^{2}(1,438)=3.263, \mathrm{p}<.05\right)$, teaching cross-curricular skills $\left(46.7 \%\right.$ vs. $\left.32.1 \%, \chi^{2}(1,437)=7.959, \mathrm{p}<.01\right)$ and student behaviour and classroom management $(45.8 \%$ vs. $\left.31.3 \%, \chi^{2}(1,438)=8.581, \mathrm{p}<.01\right)$. The lowest need for professional development teachers have for school management and administration (27.3\%) and knowledge and understanding of subject (19.3\%).

Regarding barriers to participation in professional development, the only significant gender difference is that female teachers perceive lack of employer support as a more important problem (38.3\% vs. $\left.28.6 \%, \chi^{2}(1,439)=4.206, p<.05\right)$. For both female and male teachers, the main obstacles for professional development are expensiveness of professional development activities and a lack of incentives for participating in such activities (see Figure 1). 
Table 2. Chosen areas of professional development during the previous 12 month period.

\begin{tabular}{|l|l|l|l|}
\hline The areas of professional development & $\begin{array}{l}\text { Female } \\
\text { teachers }\end{array}$ & $\begin{array}{l}\text { Male } \\
\text { teachers }\end{array}$ & $\Sigma$ \\
\hline Student evaluation and assessment practices & $74.8 \%$ & $81.7 \%$ & $78.2 \%$ \\
\hline Knowledge and understanding of my subject field(s) & $75.2 \%$ & $78.9 \%$ & $77.1 \%$ \\
\hline Pedagogical competencies in teaching my subject field(s) & $65.2 \%$ & $70.6 \%$ & $67.9 \%$ \\
\hline ICT (information and communication technology) skills for teaching & $\mathbf{4 0 . 0 \%} * *$ & $\mathbf{5 7 . 8 \%} * *$ & $48.9 \%$ \\
\hline Approaches to individualised learning & $46.2 \%$ & $50.0 \%$ & $48.1 \%$ \\
\hline Student behaviour and classroom management & $43.8 \%$ & $48.1 \%$ & $46 \%$ \\
\hline Teaching students with special needs & $32.5 \%$ & $35.2 \%$ & $33.9 \%$ \\
\hline Teaching cross-curricular skills (e.g. problem solving) & $32.9 \%$ & $34.3 \%$ & $33.6 \%$ \\
\hline Knowledge of the curriculum & $\mathbf{2 7 . 6 \%} *$ & $\mathbf{3 8 . 5 \%}$ & $33.1 \%$ \\
\hline New technologies in the workplace & $\mathbf{2 5 . 2 \%} * *$ & $\mathbf{3 8 . 9 \%} * *$ & $32 \%$ \\
\hline Student career guidance and counselling & $24.3 \%$ & $27.8 \%$ & $26.1 \%$ \\
\hline School management and administration & $10.0 \%$ & $10.2 \%$ & $10.1 \%$ \\
\hline Approaches to developing cross-occupational competencies & $7.7 \%$ & $9.3 \%$ & $8.5 \%$ \\
\hline Teaching in a multicultural or multilingual setting & $6.7 \%$ & $5.6 \%$ & $6.1 \%$ \\
\hline
\end{tabular}

Note. ${ }^{* *} p<.01, * p<.05$

Table 3. High level of need for professional development.

\begin{tabular}{|l|l|l|l|}
\hline The areas of professional development & $\begin{array}{l}\text { Female } \\
\text { teachers }\end{array}$ & $\begin{array}{l}\text { Male } \\
\text { teachers }\end{array}$ & $\Sigma$ \\
\hline Teaching students with special needs & $\mathbf{6 8 . 1} \%^{*}$ & $\mathbf{5 8 . 0} \%^{*}$ & $63 \%$ \\
\hline New technologies in the workplace & $\mathbf{5 4 . 9} \%^{*}$ & $\mathbf{4 5 . 6 \%}$ & $50 \%$ \\
\hline Approaches to individualised learning & $\mathbf{4 8 . 8} \%^{*}$ & $\mathbf{3 9 . 7} \%^{*}$ & $44.3 \%$ \\
\hline ICT (information and communication technology) skills for teaching & $47.9 \%$ & $40.0 \%$ & $44 \%$ \\
\hline Approaches to developing cross-occupational competencies & $45.9 \%$ & $37.1 \%$ & $41.5 \%$ \\
\hline Teaching cross-curricular skills (e.g. problem solving) & $\mathbf{4 6 . 7 \%}$ & $\mathbf{3 2 . 1 \%}$ & $39.4 \%$ \\
\hline Student behaviour and classroom management & $\mathbf{4 5 . 8} \% * *$ & $\mathbf{3 1 . 3} \%^{* *}$ & $38.5 \%$ \\
\hline Student evaluation and assessment practice & $36.3 \%$ & $34.0 \%$ & $35.1 \%$ \\
\hline Knowledge of the curriculum & $32.9 \%$ & $32.0 \%$ & $32.5 \%$ \\
\hline Pedagogical competencies in teaching my subject field(s) & $31.5 \%$ & $33.1 \%$ & $31.7 \%$ \\
\hline Teaching in a multicultural or multilingual setting & $29.1 \%$ & $32.0 \%$ & $30.5 \%$ \\
\hline School management and administration & $27.7 \%$ & $26.8 \%$ & $27.3 \%$ \\
\hline Knowledge and understanding of my subject field(s) & $19.4 \%$ & $19.1 \%$ & $19.3 \%$ \\
\hline
\end{tabular}

Note. $* * p<.01, * p<.05$

Figure 1. Barriers to participation in professional development (\%).

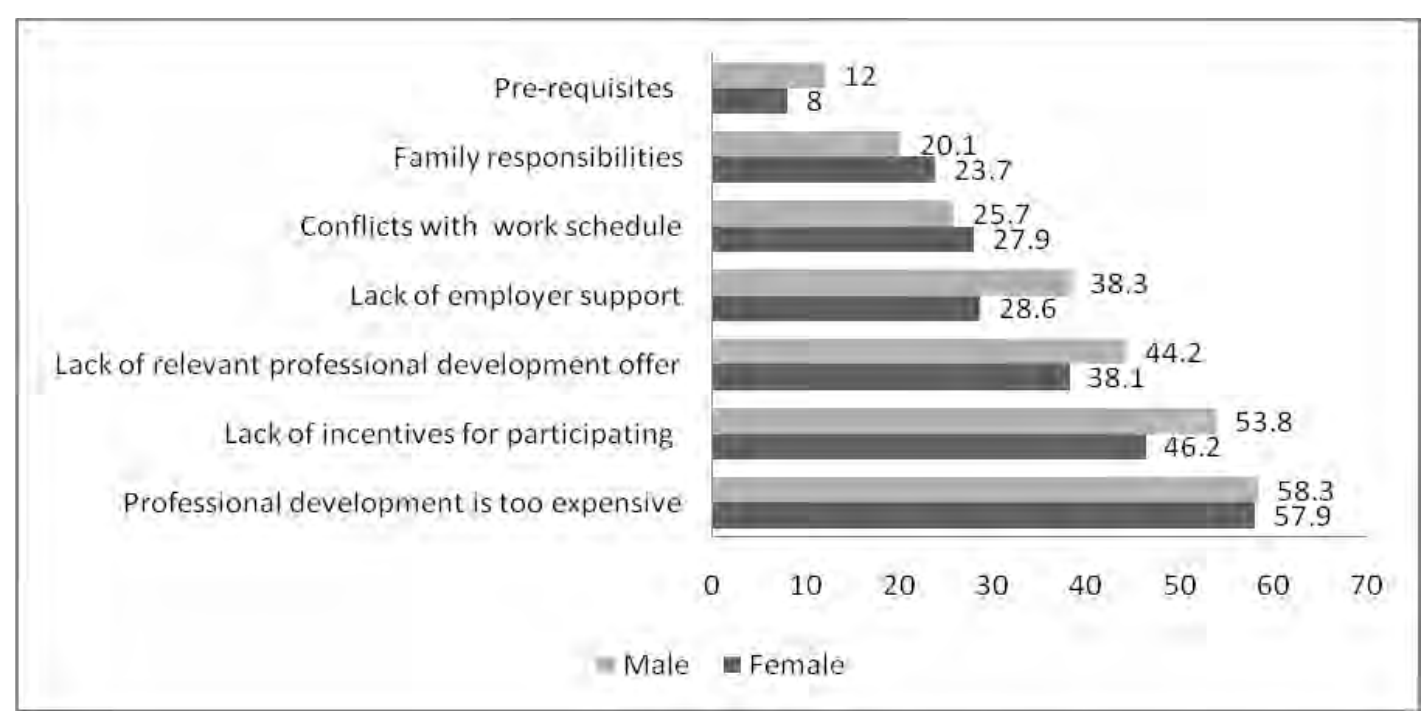




\section{Discussion and conclusion}

Compared to other Serbian teachers (see Petrović, Kuzmanović, Jošić, Jovanović, 2015), mathematics teachers attended more a qualification programme (e.g. a degree programme) and chose, to a lager extent, areas of professional development closely related to their subject field, such as knowledge of a subject field and pedagogical competencies in teaching a subject field. On the other hand, female mathematics teachers expressed a higher need for professional development related to teaching students with special needs, approaches to individualised learning and new technologies in the workplace than male teachers, and in that way, female mathematics teachers are more similar to other Serbian teachers. Based on the results, it can be concluded that female mathematics teachers invest more in their professional development and they are more sensitive to development of competences that directly support teaching and learning. Furthermore, the results obtained in this survey show that the priority of educational policy in Serbia, in terms of professional development of teachers, should be directed towards providing conditions for acquiring competencies for inclusive education and for the application of ICT and modern teaching technologies. Additionally, in order to provide greater coverage and greater participation of teachers from Serbia in continuous professional development it is necessary to increase the offer of professional development activities which are less expensive or free of charge, and to use additional incentives to stimulate teachers to participate in professional development activities (e.g. adapting the school schedule, gaining free days for professional development at the expense of additional engagement in teaching and extracurricular activities, increase of teachers' salaries, etc.).

\section{Acknowledgements}

This work was supported by the Ministry of Education, Science and Technological Development, Republic of Serbia (project number 179018).

\section{References}

Fessler, R. (1995). Dynamics of Teacher Career Stages. In T. Guskey and M. Huberman (eds.), Professional Development in Education, New Paradigms and Practices (171-192). New York: Teachers College, Columbia University.

Guskey, T. R. (2003). Professional development that works: What makes professional development effective?. Phi delta kappan, 84(10), 748.

Guskey, T. R. (2009). Closing the knowledge gap on effective professional development. Educational horizons, 224-233.

OECD (2009). Creating Effective Teaching and Learning Environments: First results from TALIS. Paris: OECD Publishing.

OECD (2010). Mathematics teaching and learning strategies in PISA. Paris: OECD Publishing.

OECD (2013). Teaching and Learning International Survey TALIS 2013: Conceptual Framework. Paris: OECD Publishing.

OECD (2014). TALIS 2013 Results: An International Perspective on Teaching and Learning. Paris: OECD Publishing.

Pavlović-Babić, D., \& Baucal, A. (2013). Podrži me, Inspiriši me, PISA 2012 u Srbiji: Prvi rezultati. Beograd: Institut za psihologiju.

Petrović, D.S. (2000). Stavovi učenika prema školi [Students’ Attitudes towards School]. magistarska teza., Beograd: Filozofski fakultet, Univerzitet u Beogradu.

Petrović, D.S., Kuzmanović, D., Jošić, S. \& Jovanović, V. (2015). Obuhvat, trajanje i dostupnost stručnog usavršavanja nastavnicima u Srbiji [The scope, duration and availability of professional development activities for teachers in Serbia] u J. Radišić i N. Buđevac (ured.) Sekundarne analize istraživačkih nalaza u svetlu novih politika u obrazovanju (str. 9-27), Beograd: Ministarstvo prosvete, nauke i tehnološkog razvoja i Društvo istraživača u obrazovanju u Srbiji.

Radišić, J., Videnović, M., \& Baucal, A. (2015). Math anxiety-contributing school and individual level factors. European Journal of Psychology of Education, 30(1), 1-20.

Radišić, J., \& Baucal, A. (2012). Understanding practice of mathematics and language teachers from their own perspective, paper presented at the 25th International Congress for School Effectiveness and $\begin{array}{lllll}\text { Improvement, } & \text { Malmo 5-8 January 2012. Retrieved from }\end{array}$ http://www.icsei.net/fileadmin/ICSEI/icsei_2012/papers/1791918_ABS.pdf

Scheerens, J. (Ed.) (2009). Teachers' professional development: Europe in international comparison. A secondary analysis based on the TALIS dataset. The Netherlands: University of Twente.

Wei, R. C., Darling-Hammond, L., and Adamson, F. (2010). Professional development in the United States: Trends and challenges. Dallas, TX. National Staff Development Council. 


\title{
ADULT VISITORS' INTELLECTUAL FUNCTIONING EXPLORING CONTEMPORARY ART: EXPERIENCES THAT GENERATE SELF-DISCOVERY ${ }^{1}$
}

\author{
Anne-Marie Émond \\ Département de didactique, Université de Montréal (Canada)
}

\begin{abstract}
Objectives: The aim of our research centres around accessing and understanding the intellectual functioning of adults' art experience encountering contemporary art in a museum context.

Methods: In order to access a visitor's aesthetic experience, the Thinking Aloud protocol (Dufresne-Tassé et al., 1998) was used to collect adult visitors' talk during their exploration of artworks at the Montreal Museum of Fine Arts. This method consists of recording what an individual visitor says, during his visit, that is, thoughts, ideas, reflections that might emerge during the exploration of artworks. The verbal comments of 67 visitors, aged 18 to 80 years old that possess different levels of education and frequent museums more than 3 times per year were recorded. The recorded comments were transcribed and analyzed using different instruments created in previous research projects (Emond, 2017).

Results: Our initial results are set in relation to Pelowski and Akiba's (2011) psychological model of art experience. Specifically, we present how visitors' contemporary art appreciation, that goes beyond the meta-cognitive stage, is the result of an art exploration that encourages imagining different selves and self-discovery (Minissale, 2013). Conclusion: This is to say that for a transformative aesthetic experience, an individual has to consider the pleasures of ambiguity featured by contemporary art. Our results reflect on the exploration of contemporary art as a form of creativity as proposed by Minnisale (2013), where visitors learn to valorize the process of adjusting their self-image through new experiences brought about by artworks.
\end{abstract}

Keywords: Adult visitors, intellectual functioning, art museum, contemporary art, aesthetic experience.

\section{Rationale of the research}

Psychological aesthetics research was often conducted in the context of a laboratory, one that is very different from an actual gallery space in a museum. Our previous research showed the importance of the artwork materiality, the museography and all the information provided by the museum on the quality of visitors' aesthetic experiences (Émond, 2016a,b) and in doing so, identifying the necessity to favour more research in such a context. In this study, we pursue our investigation on museum visitors aesthetic experience regarding contemporary art. More specifically, we are interested in documenting what actually happens to visitors during an encounter with contemporary art. We want to understand their art appreciation practices: what are their thoughts, how they deal with moments of harmony or conflict. By visitors' aesthetic responses, we refer to all the verbal comments made by visitors during their museum visit which gives us access to the different mental operations they might use to make sense of the artworks such as identifying, judging and predicting.

The purpose of the study is to identify the components of a transformative aesthetic experience in the context of a museum visit. How can a visitor, who experiences moments of conflict (dissonance) or of harmony (consonance), while viewing contemporary art in a museum, attain a transformative aesthetic experience?

\footnotetext{
${ }^{1}$ This research has been funded by the Social Sciences and Humanities Research Council of Canada (SSHRC).
} 


\section{The choice of participants}

Our 67 participants are frequent museum visitors, that is, they visit museums more than three times per year. They are all members of the Montreal Museum of Fine Arts where the research was conducted. Aged 18 to 80 years old they possess different levels of education ranging from less than twelve years of education to post-secondary education. It is important, in the context of our research, to have participants that are familiar with museums and feel at ease exploring works of art in order to maximize the potential of identifying the components of a transformative aesthetic experience.

\section{Procedure for collecting data}

In the 1990s, a number of researchers used the thinking aloud protocol to study problem resolution (Ericsson \& Simon, 1993; Winburg, 1991), text comprehension (Kukan \& Beck, 1997; Pressley \& Afflerbach, 1995), and decision-making (Rosman, Lubatkin, \& O’Neill, 1994). In 1995, Dufresne-Tassé and the Université de Montréal Groupe de recherche sur les musées et l'éducation des adultes - GRMEA (Research group for museums and adult education) adapted this method with a view to collecting adult visitors' comments in the instant where they take shape in the museum exhibition galleries, in order to study visitors' psychological functioning (Dufresne-Tassé \& Lefebvre, 1995). This method consists in recording what visitors say at the time of their visit. They don't have to make any effort to explain or justify their remarks, they are simply asked to express out loud what comes to their minds throughout their visit. They are invited to verbalize their experience as it unfolds.

As mentioned by Dufresne-Tassé and Lefebvre in their book Psychologie du visiteur de musée: contribution à l'éducation des adultes en milieu muséal [Psychology of museum visitors: a contribution to adult education in the museum context] (1995), there are conditions to be met in order to successfully obtain visitors' reactions during their exploration of artworks. Firstly, it is very important that individuals be willing to visit the museum. Secondly, they must be aware of the fact that they are going there to participate in research in which their role is essential. Thirdly, they must understand that they will be expressing their thoughts out loud throughout the visit. Consequently, the research situation created is one where individuals who accept to become visitor-participants wish to cooperate with the researcher and this encourages them to verbalize their thoughts as they come. The participants are unrestricted in their choice of contemporary artworks to be viewed in the permanent collection of international contemporary art at the Montreal Museum of Fine Arts in Quebec, Canada. During their museum visit, one visitor at a time, is being accompanied by the researcher who is attentive to the visitors' comments that are recorded for the purpose of the study. Depending on each visitor, the duration of the visit varies between one and three hours. The data collected during this time is later transcribed and consist of what we call a 'discourse'. This discourse is then used to analyse each visitor's aesthetic experience.

\section{Treatment of data}

Through a visitor's discourse, an analysis is made using instruments created in previous research. One consists of a typology of consonances and dissonances to identify moments of harmony and/or of conflict, and another, regroups eight mental operations that could be used by the visitor during the art exploration (see Émond, 2017 for details). Once all the discourses are coded, we are able to put visitors' consonant and dissonant moments and the different mental operations used in relation to Pelowski and Akiba's (2011) psychological model of art experience. This model presents five different stages of the aesthetic experience: Stage 1: Pre-expectations and self-image; Stage 2: Cognitive mastery and introduction of discrepancy; Stage 3: Secondary control and escape; Stage 4: Meta-cognitive re-assessment; Stage 5: Aesthetic outcome and new mastery, with a total of three possible Outcomes to the aesthetic experience.

\section{Results and discussion}

\subsection{Meta-cognitive re-assessment and aesthetic outcome and new mastery}

In Pelowski and Akiba's (2011) psychological model of art experience, as presented by Émond (2018), all stages have a particular role to play in the quality of visitors' museum experience. Nonetheless, it is important in the context of this study to concentrate our analyses on what we consider a crucial stage in the aesthetic experience of contemporary art, that is, Stage 4: Meta-cognitive re-assessment. As reported by Émond (2018): 
When a visitor finds himself prisoner in a situation where he has no way of escaping or easily resolving the dissonance, he/she is experiencing while being engaged with a contemporary work of art, he can decide to enter in "a period of active experiential and expectational re-assessment" (p. 89). If the visitor decides to continue with an experiential re-evaluation, "this event is often accompanied or preceded by acute self-focused attention" (Steele et al., 1993, as cited by Pelowski \& Akiba, 2011, p. 89), which seems to have an impact on dissonant situations (Pelowski \& Akiba, 2011). Becoming self-focused, "leads [the visitor] to the feeling of "increased submissiveness" and "individuation", causes the individual to "focus attention on personal limitations and to increase perceived uncontrollability" (Rothbaum et al., 1982, p. 23), increases negative affect and anxiety (Mor \& Winquis, 2002); and induces expectational re-assessment (Ingram, 1990)" (Pelowski \& Akiba, 2011, p. 89). The visitor, then gives up total control and revises his expectations acknowledging the presence of the dissonance or conflict and switches to a meta-cognitive approach in his art appreciation. The visitor who can reach this stage is gearing towards a transformative aesthetic experience. There is therefore a metacognitive re-assessment. If successful, the visitor will modify his self-image and achieve self-transformation (Rothbaum et al., 1982, as cited by Pelowski \& Akiba, 2011).

When the visitor completes Stage 4 and enters into Stage 5: aesthetic outcome and new mastery, he is getting closer to an Outcome 3, that is, a transformative aesthetic experience. To complete Stage 5, the visitor must change his pre-expectations and self-image. We could say that the visitor is receptive to new experiences.

Being in a creative pursuit, allows the visitor to explore his identity, forging new knowledge and self-insight. It is as if the contact with contemporary art, almost conceived to create dissonances on the part of the visitor, challenges the way he appreciates art and ferment change. As mentioned by Radford (2004) "[it] may be that some works of conceptual art are simply centered on challenging boundaries, at setting up dissonances" (p. 56). The role of cognitive dissonances is unavoidable in the exploration of contemporary art, it contains "[the] notion of tension and reconciliation, and of the creative act as speaking to something "within us" and revealing to us something about ourselves..." (Radford, 2004, p. 56).

The visitor that overcomes the challenges inherent to the exploration of contemporary artworks, seems to put into action his creativity. In these circumstances, the visitor appears to tolerate ambiguity and is willing to go beyond his comfort zone in his art appreciation. In doing so, the visitor withholds his aesthetic judgement and, as Gomez (2007) mentions, "enables an idea to survive long enough to generate other ideas" (p. 35) and prolonging the aesthetic experience. This type of aesthetic experience generates, on the part of the visitor a certain fascination with the unusual. When this occurs, we can find evidence of metacognitive knowledge, experiences and skills on the part of the visitor.

Identifying metacognitive knowledge in a visitor's discourse "refers to a person's conceptions of cognitive processes, such as thoughts about mental images, beliefs, and insights" (Gutwill \& Dancstep, 2017, p. 73). To illustrate this, the visitor could say "I'm a person that looks for beautiful works of art" (MBAM AA). As for, metacognitive experiences we find in a visitor's discourse "awareness of thoughts or feeling, such as frustration or excitement" (Gutwill \& Dancstep, 2017, p. 73). An example would be, "I am angry when I see found objects in an artwork" (MBAM AA). Finally, concerning metacognitive skills, a visitor might say "When I am not sure about the meaning of the artwork, I read the label to find out what is happening" (MBAM AA) as it involves "strategies aimed at monitoring cognition, such as orientation, planning and evaluation” (Gutwill \& Dancstep, 2017, p. 73).

Art becomes a vehicle to construct and understand the self (Brinck, 2007). This entails, on the part of a museum visitor, to create meaning from his exploration of an artwork, and this, through time. The exploration of an artwork becomes a work in progress where every encounter contributes to self-discovery.

When the visitor is capable of understanding the divergent or dissonant elements of his aesthetic experience and integrates them as new self-knowledge, this will influence his future art exploration. Kokkos (2010) refers to Mezirow (2000) in saying that:

[...] transformative learning occurs when there is a transformation in our set of specific beliefs, feelings and attitudes (points of view) or in our broader orienting predisposition (habit of mind). Transformation in a habit of mind may be sudden (epochal) or slower through incremental changes in various points of view. The entry point to a transformative process is the disorienting dilemma - a kind of learning disability felt by a person... (pp. 173-174). 
At this point, just has Minissale (2013) pointed out, contemporary art appreciation requires that a visitor takes pleasure being in a state of ambiguity. On the part of the visitor, it does not require definitive conclusion of his art appreciation, just the recognition of multiple possibilities of interpretations that reinforces the pleasure of ambiguity. As an example, our visitor MBAM BR said: "In the past, I passed right off the things I did not like... because I did not like it, it's not beautiful, it doesn't tell me anything. But now I'm going to spend a little more time looking..." This visitor is now capable of understanding the divergent or dissonant elements of his aesthetic experience and integrate this new self-knowledge for his future encounters with artworks and in doing so, modifies his preconceptions about artworks, thus his self-image.

When this happens, the museum visitor may experience a sense of "fitness" with the artwork and the artist. A sense of "true self" (Csikszentmihalyi, 1990) occurs and the visitor might lose track of time. At this point, we might find moments akin to flow experiences that entails, amongst others, the loss of self-consciousness. The museum visitor might experience a sense of enlightenment, epiphany and pleasure that calls for a repeat museum experience in the exploration of contemporary works of art.

\section{Conclusion}

Our results indicate that to help visitors achieve the transformative aesthetic experience, educators need to develop educational programs accompanied by mediation that would help visitors question their preconceptions about art and accompany them in their exploration of contemporary art, in a spirit of openness. As Minissale (2013) claims:

In this way, art can be seen as some kind of exchange between different selves, helping to structure self-knowledge....The experience of the work of art is akin to enjoying the exploration of the self as something unresolved, which may or may not dissipate when one has left the gallery space" (p. 5318, p. 5322).

Change occurred in our visitor MBAM AA while he was exploring contemporary artworks. What he experienced may have an incidence on his future museum visits. In his words:

I think that art must be beautiful. It must be fun. But it makes me fall into a trap of the artist in fact, I finally see...It's a revelation...I admit that...that's it, it's exactly what I had to experience looking at the work of art...because that was it. I will have learned a lot of things today, it's fascinating... But that's it, I feel assaulted...Oh, it's very funny. We do not give the artist enough credit. For us it's a reaction, then we do not go further...to say is that really what he wanted us to do? But then I realize...he does something like that to make us react. It makes sense, it's very funny.

Detailing the exact experiences that an artwork might provoke on a museum visitor is very difficult to accomplish, since it seems that there are as many ways to do so, as there are visitors. Although, let's not forget that the aesthetic experience involves elements that are shared by all human beings and can be identified. We can find both positive such as pleasure or negative emotions such as disgust and some self-consciousness emotions such as embarrassment and some cognitive emotions such as confusion while exploring artworks (Silvia, 2009). One thing that became evident for some of our participants, like MBAM AA, is that exploring contemporary artworks demands an active approach, one that asks for a co-creation of the work of art.

With these words, we must now continue to grasp the components and dynamics of an awareness of self, experienced by visitors during an aesthetic experience, to better informed our actions as museum educators.

\section{References}

Brinck, I. (2007). Situated cognition, dynamic systems, and art: on artistic creativity and aesthetic experience. Janus Head, 9(2), 407-431. Retrieved December 1, 2018, from: http://www.janushead.org/9-2/brinck.pdf

Csikszentmihalyi, M. (1990). Flow; the psychology of optimal experience. New York: Harper Perennial.

Dufresne-Tassé, C. \& Lefebvre, A. (1995). Psychologie du visiteur de musée : Contribution à l'éducation des adultes en milieu muséal. Montréal, QC : Éditions Hurtubise HMH.

Dufresne-Tassé, C. Sauvé, M., Weltzl-Fairchild, A., Banna, N., Lepage, Y. \& Dassa, C. (1998). Pour des expositions muséales plus éducatives, accéder à l'expérience du visiteur adulte. Développement d'une approche. Canadian Journal of Education/Revue canadienne de l'éducation, 23(3), 302-315. 
Ericsson, K. A., \& Simon, H. A. (1993). Protocol analysis. Cambridge, MA: MIT Press.

Émond, A.M. (2016a). La pertinence pour les musées de développer des outils de médiations écrites pour l'appréciation de l'art contemporain. In A. Savoie, A.M. Émond, F. Gagnon-Bourget \& P. Gosselin (Eds.), Actes du Colloque sur la recherche en enseignement des arts visuels (pp. 26-32). Montréal, QC : CRÉA.

Émond, A.M. (2016b). Connaître les opérations mentales utilisées par les étudiants à la formation des maîtres pour traiter les œuvres d'art contemporain. In M. Théberge \& F. Chaîné (Eds.), Réfléchir à la formation artistique (48-76). Québec, QC : Éditions FRÉA.

Émond, A.M. (2017). La voix des visiteurs en contexte muséal : matière première dans l'élaboration d'instruments permettant d'analyser et de cartographier le fonctionnement intellectuel d'adultes lors de leur traitement d'œuvres d'art contemporain. In C. Dufresne-Tassé \& A.M. Laraignée (Eds.), Numéro spécial sur la recherche, Volume 2 ICOM Éducation 27 (13-44). Retrieved from https://drive.google.com/file/d/1H4RsNSMKOtiqhGSz2rf9wVBF3CRAeC4W/view

Émond, A.M. (2018). The intellectual functioning of art museums' visitors: Identifying components of a transformative aesthetic experience. In C. Pracana \& M. Wang (Eds.), Psychological applications and trends 2018 (156-160). Retrieved from http://inpact-psychologyconference.org/wpcontent/uploads/2018/05/Psychological-Applications-and-Trends-2018.pdf

Gomez, J. G. (2007). What do we know about creativity? The Journal of Effective Teaching, 7(1), 31-43. Retrieved December 1, 2018, from https://pdfs.semanticscholar.org/3ad5/30c1138e10eb674e456c3ec4ed52cb0a209c.pdf

Gutwill, J.P. \& Dancstep, T. (2017). Boosting metacognition in science museums: Simple exhibit label designs to enhance learning. Visitor Studies, 20(1), 72-88.

Kokkos, A. (2010). Transformative learning through aesthetic experience: Towards a comprehensive method. Journal of Transformative Education, 8(3), 155-177. Retrieved December 1, 2018, from: https://pdfs.semanticscholar.org/5934/7b9e3b38154e84884162439feac8e2f21147.pdf

Kukan, L. \& Beck, I. L. (1997). Thinking aloud and reading comprehension research: Inquiry, instruction, and social interaction. Review of Educational Research, 67(3), 271-299.

Minissale, G. (2013). The psychology of contemporary art. New York, Cambridge University Press.

Pelowski, M., \& Akiba, F. (2011). A model of art perception, evaluation and emotion in transformative aesthetic experience. New Ideas Psychology. 29, 80-97. doi: 10.1016/j.newideapsych.2010.04.001

Pressley, M. \& Afflerbach, P. (1995). Verbal Protocols of reading: The nature of constructively responsive reading. Hillsdale, NJ: Lawrence Erlbaum.

Radford, M. (2004). Emotion and creativity. The Journal of Aesthetic Education, 38(1), 53-61. Retrieved December 1, 2018, from https://www.jstor.org/stable/3527362?seq=1\#metadata_info_tab_contents

Rossman, A., Lubatkin, M. \& O'Neill, H. (1994). Rigidity in decision behaviors: A within-subject test of information acquisition using strategic and financial cues. Academy of Management Journal, 37(4), 1017-1033.

Silvia, P. J. (2009). Looking past pleasure: Anger confusion disgust pride surprise and other unusual aesthetic emotions. Psychology of Aesthetics, Creativity, and the Arts, 3, 48-51. Retrieved December 1, 2018, from: http://psycnet.apa.org/fulltext/2009-01867-011.html

Winburg, S. S. (1991). Historical problem solving. A study of the cognitive processes used in the evaluation of documentary and pictorial evidence. Journal of educational psychology, 83(1), 73,87. 


\title{
ADULT READING AND MEANING MAKING IN A MUSEUM EXHIBITION ABOUT ANCIENT CHINA
}

\author{
Colette Dufresne-Tassé ${ }^{1}$ \\ Maîtrise en muséologie, Université de Montréal (Canada)
}

\begin{abstract}
Adults of the general public type who visit an exhibition whose subject is almost unknown to them attach great importance to reading the major texts (panels) of that exhibition. These texts provide them with the conceptual structure of the exhibition. They allow them to discover and appropriate the subject of the exhibition with very few mistakes. But they do not support its enrichment through personal contributions, for example in the form implications derived from reasoning. Why? This is the simple, but unexplored question in the museum context, that is studied here. ${ }^{2}$
\end{abstract}

Keywords: Adult visitors, reading, exhibition, meaning making.

\section{Introduction}

In a thematic temporary exhibition ${ }^{3}$, reading the texts that accompany the objects is a major activity. It is the main way to discover the meaning of each object and to understand the phenomenon presented, for example, an historical fact, the way of life of a cultural group, a natural phenomenon or the work of an artist (Hooper Greenhill, 1994 ; Merleau-Ponty and Ezrati, 2005; Poli, 2002; Ravelli, 2006; Serrel, $1996{ }^{* 4}$ ). In other words, the production of meaning corresponds to an appropriation, i.e. a recreation by the visitor of the content of the exhibition, the abundance and characteristics of the production of meaning determining the breath of the visitor's recreation (Dufresne-Tassé, O'Neill, Sauvé and Marin, 2014).

Regarding the characteristics of the production of meaning, M.C. O'Neill of the École du Louvre and a group of her students ${ }^{5}$ observed several times six different orientations of this production, only one corresponding exactly to the recreation of the exhibition content. The orientations identified are as follows:

- Meaning is in line with what the curator is saying in the exhibition; it fits perfectly its content;

- It is beyond the content of the exhibition; it complements and enriches it;

- It constitutes a clear opposition to what the curator says in the exhibition;

- It is incomplete in the sense that it takes the form of a question or hypothesis;

- It is simply not true, because it contradicts generally accepted knowledge;

- It is completely foreign to the content of the exhibition.

\section{Context of data collection}

The present research was carried out in a large temporary thematic exhibition on ancient China offered in Quebec City by the Musée de la civilisation. This exhibition was chosen because its subject was quasi unknown to the Quebec public and so, we expected, would lead to ample reading and an opportunity to study it.

\footnotetext{
${ }^{1}$ Have also contributed to this research: Marie-Clarté O'Neill from the École du Louvre, and Anne-Marie Émond from the Université de Montréal.

${ }^{2}$ This research has been funded by the Social Sciences and Humanities Research Council of Canada (SSHRC), by the Fonds pour la formation de chercheurs et l'aide à la recherche, as well as by the Fonds Québécois de la recherche sur la société et la culture (FQRSC) of the Province of Quebec Government. It also received some logistic support from the Université de Montréal.

${ }^{3}$ Temporary exhibitions are usually thematic, i.e. they present their content according to a general theme divided in a certain number of sub-themes.

${ }^{4}$ As the subject matter has been treated in many other publications, references marked with an $\left({ }^{*}\right)$ are only examples.

5 See: Adjadj, 2004 ; Baudon, 2002 ; Cabille, 2001 ; Hely, 2001 ; Jeannoutot, 2003 ; Krummenaker, 2001; Letellier, 2003 ; Pralus, 2004 ; Quantin, 2002 ; Rey-Jouvin, C. 2002 ; Rondet, 2001 ; Vicedo, 2003
} 


\section{Sample}

A group of 30 visitors, from 28 to 64 years of age (mean 34.8), including the same proportion of men and women of three levels of schooling (baccalaureate, less than a baccalaureate, and more than a baccalaureate), and low visiting habits (once or twice a year); in other words, a sample of "the general public" type.

\section{Data on the reading of the 30 visitors of the sample}

The group of 30 visitors read 4,532 times, with an average per visitor of 161, and a standard deviation of 68.29. The main texts of the exhibition, namely its panels (introductory texts and texts presenting each of its parts) and the labels attached to each object, were read as follows:

- Panels: Average readings: 20.52 per visitor, with a standard deviation of 11.95; this represents the reading of $81.58 \%$ of all the panels;

- Labels: Average readings: 39.76 per visitor, with a standard deviation of 24.60; this represents the reading of $41.67 \%$ of all the labels.

According to what is known about reading museum texts (Black, G. 2005; Daignault, 2011; Serell, 1996*), one could consider that the reading rate of panels is particularly high, while that of labels is rather low. It can therefore be assumed that the novelty of the exhibition's subject matter captured the visitors' attention more than the exoticism of the objects presented, and that visitors preferred to discover the exhibition's content based on the general information it presented rather than on the information attached to each object.

\section{Means of collecting information on meaning production}

As it was not possible to use a standardized questionnaire (there was no relevant one), and because a post-visit interview would have led to invalid data (due to many factors like memory overload), information from each visitor was obtained through the Thinking Aloud Technique (Ericcson and Simon, 1993). A version that we adapted and validated for the museum situation was used. It consists in asking a visitor who is arriving at the museum to do her/his visit as she/he wishes, saying aloud what comes to her/his mind without bothering remembering or justifying it (Dufresne-Tassé and al., 1998a, 1998b).

The visitor voicing her/his experience as it unfolds (Hirtle, 2013), she/he reveals what she/he is thinking, imagining or feeling, i.e. her/his production of meaning while observing or reading. We called this visitor's oral production "a discourse". We tape registered and then computerized it, so it could be studied in its written form.

\section{Hypotheses}

The visitor's reading behaviour described above, combined with the six outcomes of the meaning production identified by O'Neill and her students suggested the following hypotheses.

Correspondence of meaning production with exhibition content (70\%)

1. The majority (50\% or more) of the production of meaning is in line with what the curator is saying in the exhibition, and represents a recreation for the visitor;

2. The creation of meaning that enriches the content of the exhibition is relatively frequent (about $20 \%$ ) because by reading the panels, the visitor has a representation that is both precise and articulated and can thus use her/his imagination or her/his ability to reason and produce implications.

Uncertainty of meaning production in relation to exhibition content $(15 \%)$

3. Given the precise and structured representation that the visitor can develop of the exhibition's content, its production of meaning in the form of questions or hypotheses should be very limited. However, its relatively low consultation (less than one label out of two) is likely to generate uncertainties and doubts that would lead to questions or hypotheses.

Divergence of meaning production from exhibition content (10\%)

4. The production of meaning that is in direct opposition to the curator's thought should be relatively rare (about 5\%), because it is difficult for a visitor to oppose an articulated discourse that deals with a subject with which she/he is very little or not at all familiar.

5. As for the creation of meaning that leads to notorious falsehoods, it should be relatively rare (5\%), because, as we have seen, the texts of the panels are precise and articulated, so they safely guide the visitor's production of meaning. 
Escape of meaning production from the content of the exhibition (5\%)

6. Finally, for similar reasons, a production of meaning foreign to the content of the exhibition should be very rare (5\% or less). Indeed, guided by a precise and articulated discourse about a subject matter that she/he has chosen to discover, the visitor should not be tempted to think of something else.

\section{Results}

Correspondence of the production of meaning coming from the visitors who participated in this research with the exhibition content is less strong (see Table 1$)$ than what was expected $(48.11 \%+6.49 \%$, or $54.60 \%$ versus $70 \%$ - see Table 1 ). This is due to the fact that the enrichments of this content were much less numerous than expected (6.49\% versus $20 \%)$.

The same applies to the uncertainty of the production of meaning in relation to the content of the exhibition. It is lower than expected (9.33\% versus 15\%).

But the expectations regarding the divergence of the production of meaning from the exhibition content (10\%) are correct. Indeed, direct oppositions (7.43\%) and falsehoods (2.86\%) total 10.29\%.

Finally, the escapes from the subject of the exhibition are much more frequent than expected. Indeed, they are five times more numerous (25. $78 \%$ versus $5 \%$ ).

\section{Discussion}

\subsection{Orientation of visitors' reading}

We have seen that the visitors of this research read only a small portion of the labels, but a large proportion of the panels. How can these data be explained? It is known that the general public visitors come to the museum mainly to see objects and discover their meaning (Dufresne-Tassé and Lefebvre (1996); Dufresne-Tassé, O'Neill, Sauvé and Marin, 2014; Hooper-Greenhill, 1994*). The texts of the labels that closely accompany them physically and intellectually should therefore be of great interest to them, and read in a very high proportion. But in some exhibitions, such as the one chosen in this research, the texts of the labels are usually limited to identifying the objects they accompany, as well as some of their characteristics, such as the materials from which they are made and the time they were created or used. So they present details that remain isolated from each other, i.e. disparate elements located throughout the vast semiological space of an ancient Chinese culture. Such labels therefore have only sporadic and limited interest for a visitor who does not have the knowledge necessary to insert their contents into an organised whole that would make them attractive and give them a real value.

On the other hand, the texts of the panels first present a general description of the subject of the exhibition, then an articulated description of each of the aspects that the exhibition explores. So, for a visitor who knows little about this subject, panels are a much more advantageous source of information than the labels. Therefore, it is not surprising that the visitors of this research read them more than the labels.

However, according to Grassin (2006), the label texts could gain in interest if they dealt with some characteristics of the objects that visitors can observe, and if, at the same time, they systematically detailed the content of a panel devoted to a particular aspect of the exhibition.

\subsection{Visitors' production of meaning}

We just saw that the visitors of this research read panels rather than labels. Because of the articulation between them of the panels, their careful reading allowed the visitors, although they had little or no knowledge of the subject of the exhibition, to develop a structured schematic representation of it. This succinct representation also seems to have led the visitors to deal with the content of the exhibition with very few errors. In addition, it was sufficient for them to associate many elements foreign to the exhibition, elements of course drawn from their personal universe. This association has an important function. It links the content of the exhibition to various elements, memories or ideas of the visitor, and facilitates their integration, retention and subsequent use (Dufresne-Tassé, 2001).

However, the succinct representation acquired by the visitors does not seem to have enabled them to enrich the very content of the exhibition. This is probably because their personal universe did not contain any directly relevant knowledge or experience, so their only appropriate resource was their ability to reason and derive implications from what they observed or read.

Why insisting on the enrichment of the exhibition content? It seems important for two reasons. Firstly, because it establishes a strong link between what the visitor discovers and her/his personal universe. Secondly and probably more importantly, because it contributes to the visitor's immersion in the subject of the exhibition (Dufresne-Tassé, 2014, 2016) and to the appearance of an intellectually and emotionally 
intense but also pleasant experience, which, as stressed by Parker (2008, 2014) or Steptoe, Deaton and Stone (2013) contributes greatly to the visitor's psychological and physical well-being.

The accuracy of the interpretation just presented of the reading and meaning production of adults visiting a thematic temporary exhibition will have to be verified during investigations realized in several exhibitions. The huge variation in the characteristics of these exhibitions, but also in the level of knowledge that visitors bring in, should also make it possible to deepen and broaden the interpretation offered here.

\section{References}

Adjadj, J. (2004). Étude du fonctionnement psychologique du visiteur adulte inactif de l'exposition "Edouard Vuillard 1868-1940)". Mémoire de muséologie. Paris: École du Louvre.

Black, G. (2005). The Engaging Museum. Abingdon: Routledge.

Baudon, A. (2002). Étude du public actif de l'exposition "L'Or des Rois Scythes". Mémoire de muséologie. Paris: École du Louvre.

Cabille, E. (2001). Étude sur le fonctionnement psychologique d'un visiteur d'exposition. Monographie de muséologie. Paris: École du Louvre.

Daignault, L. (2011). L'évaluation muséale. Savoirs et Savoir-Faire. Québec, QC: Presses de l'Université du Québec.

Dufresne-Tassé, C. et Lefebvre, A. (1996). Psychologie du visiteur de musée. Contribution à l'éducation des adultes en milieu muséal. Montréal, QC: Éditions Hurtubise HMH.

Dufresne-Tassé, C. (2001). Y si supierammos lo que pensan, imaginan y sentan los visitantes frente a los objetos museales. En S. Singer y H. Ribero-Borel (org.), La pedagogía en el museo: Corrientes actuales (p. 12-32). Mexico: Museo Franz Mayer.

Dufresne-Tassé, C. (2014). Experiencia intensa e experiencia de imersão: Relatório de observações diretas. Museion, Revista do Museu e Arquivo Históerico La Sallem, 19, 27-42.

Dufresne-Tassé, C. (2016). Distinguer une expérience intense courte à propos d'un objet d'art d'une expérience rudimentaire de même longueur. Revue Canadienne de recherches et enjeux en éducation artistique / Canadian Review of Art Education: Research and Issues, 43, 1, 7-16.

Dufresne-Tassé, C., O' Neill, M.C., Sauvé, M. et Marin, D. (2014). Un outil pour connaître de minute en minute l'expérience d'un visiteur adulte. Revista Musologia \& Interdisciplinaridade, 3, 6, 187-204.

Dufresne-Tassé, C., Sauvé, M., Weltzl-Fairchild, A., Banna, N., Lepage, Y. et Dassa, C. (1998 a). Pour des expositions muséales plus éducatives, accéder à l'expérience du visiteur adulte. Développement d'une approche. Canadian Journal of Education, 23, 3, 302-316.

Dufresne-Tassé, C., Sauvé, M., Weltzl-Fairchild, A., Banna, N., Lepage, Y. et Dassa, C. (1998 b). Pour des expositions muséales plus éducatives, accéder à l'expérience du visiteur adulte. Élaboration d'un instrument d'analyse. Canadian Journal of Education, 23, 4, 421-438.

Ericcson, K.A. and Simon, H.A. (1993). Protocol Analysis. Verbal Reports as Data. Cambridge, MA: The MIT Press.

Grassin, A.S. (2006). Observation des objets et lecture des cartels. Le "jonglage" comme stratégie de visite. Le cas de l'exposition "Xi'an, capitale éternelle. Dans C. Dufresne-Tassé (dir.), Familles, écoliers et personnes âgées au musée: Recherche et perspectives / Families, Schoolchildren and Seniors at the Museum: Research and Trends / Familias, escolares y personas de edad en el museo: Investigaciones y perspectivas (p. 177-205). Paris, Conseil international des musées.

Hely, K. (2001). Le fonctionnement psychologique du visiteur en salle d'exposition. Public actif, deuxième partie de l'exposition. Monographie de muséologie. Paris: École du Louvre.

Hirtle, W. (2013). Making Sense out of Meaning. Montréal, QC and Kingston, ON: McGill-Queen's University Press.

Hooper-Greenhill, E. (1994). Museums and their Visitors. London: Routledge.

Jeannoutot, M. (2003). Le fonctionnement psychologique du visiteur inactif pendant la visite de l'exposition "Matisse-Picasso". Mémoire de de muséologie. Paris: École du Louvre.

Krummenaker, C. (2001). "L'exposition Visions du Futur" aux Galeries nationales du Grand Palais,

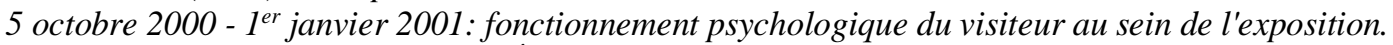
Monographie de muséologie. Paris: École du Louvre.

Letellier, E. (2003). Étude du fonctionnement psychologique du visiteur adulte appliquée à l'exposition "Matisse-Picasso". Mémoire de Muséologie. Paris: École du Louvre.

Merleau-Ponty, C. et Ezrati, J.J. (2005). L'exposition, théorie et pratique. Paris: L'Harmattan.

Packer, J. (2008). Beyond Learning: Exploring Visitors' Perceptions of the Value and Benefits of Museum Experiences. Curator: The Museum Journal, 51, 1, 33-55. 
Packer, J. (2014). Visitors' Restorative Experiences in Museums and Botanic Gardens Environments. In S. Filep and P. Pearce (Eds.), Touristic Experience and Fulfilment: Insights from Positive Psychology (p. 202-222). London: Routledge.

Poli, M.S. (2002). Le texte au musée: Une approche sémiotique. Paris: L'Harmattan.

Pralus, S.A. (2004). Le fonctionnement psychologique des visiteurs inactifs dans l'exposition "Edouard Vuillard (1968-1940)". Mémoire de muséologie. Paris: École du Louvre.

Quantin, B. (2002). Étude du public actif de l'exposition "L'Or des Rois Scythes". Mémoire de muséologie. Paris: École du Louvre.

Ravelli, L.J. (2006). Museum Texts. Abingdon: Routledge.

Rey-Jouvin, C. (2002). "Le fonctionnement psychologique des visiteurs adultes inactifs. Exposition L'Or des Rois Scythes". Mémoire de muséologie. Paris: École du Louvre.

Rondet, A. (2001). Un public de l'exposition "Visions du Futur": Une approche du fonctionnement psychologique. Monographie de muséologie. Paris : École du Louvre.

Serrell, B. (1996). Exhibit Labels. An Interpretive Approach. Walnut Creek, CA: AltaMira Press.

Steptoe, A., Deaton, A. and Stone A. (2015). Subjective Wellbeing, Health, and Ageing. The Lancet 385, No. 9968, 640-648.

Vicedo, M. (2003). Approche du fonctionnement psychologique du visiteur d'exposition. L'étude du public actif pendant l'exposition "Matisse-Picasso". Mémoire de muséologie. Paris: École du Louvre.

\section{Appendix}

Table 1. Meaning production of 30 adult visitors according to six orientations.

\begin{tabular}{|c|c|c|c|c|c|}
\hline \multicolumn{2}{|c|}{$\begin{array}{l}\text { Meaning corresponds to } \\
\text { content of exhibition } \\
\text { (expected } 70 \% \text { ) }\end{array}$} & $\begin{array}{l}\text { Meaning appears as } \\
\text { uncertain } \\
\text { (expected 15\%) }\end{array}$ & \multicolumn{2}{|c|}{$\begin{array}{l}\text { Meaning diverges from } \\
\text { content of exhibition } \\
\text { (expected } 10 \%) .\end{array}$} & $\begin{array}{l}\text { Meaning escapes content } \\
\text { of exhibition } \\
\text { (expected } 5 \%\end{array}$ \\
\hline $\begin{array}{l}\text { In line with } \\
\text { curator } \\
\text { discourse }\end{array}$ & $\begin{array}{l}\text { Enriches } \\
\text { curator } \\
\text { discourse }\end{array}$ & $\begin{array}{l}\text { Expressed as } \\
\text { question or } \\
\text { hypothesis }\end{array}$ & $\begin{array}{l}\text { Opposes } \\
\text { curator } \\
\text { discourse }\end{array}$ & $\begin{array}{l}\text { Is } \\
\text { notoriously } \\
\text { false }\end{array}$ & $\begin{array}{l}\text { Foreign to } \\
\text { curator } \\
\text { discourse }\end{array}$ \\
\hline Total 4532 & 611 & 879 & 700 & 269 & 2479 \\
\hline Mean 156.28 & 21.07 & 30.31 & 24.14 & 9.28 & 85.48 \\
\hline 131.06 & 22.60 & 24.84 & 23.49 & 8.40 & 83.66 \\
\hline 48.11 & 6.49 & 9.33 & 7.43 & 2.86 & 25.78 \\
\hline
\end{tabular}




\title{
THE TEACHING AND INTEGRATION OF PSYCHOLOGY INTO SAUDI ARABIAN CURRICULUM
}

\author{
Kyle A Msall \\ PhD King Fahd Security College (Saudi Arabia)
}

\begin{abstract}
The current state of psychology is examined in Saudi Arabia which includes stigma, professional outlook, and underserved populations. These have an effect on instructors and professors that are teaching psychology within the region and/or to this population. Teaching psychology in the Gulf Cooperation Council (GCC) countries has several challenges that one must take into consideration while developing and delivering the course. In Saudi Arabia, there are several aspects of psychology that can be incorporated and must be dismissed due to the conservative Islamic society. Psychology courses must be adapted to the cultural context. Courses that are well adapted to the cultural context in undergraduate education throughout the Middle East have the possibility to bring about acceptance of psychology and reduce stigma overall. At some universities in Saudi Arabia, the integration of psychology into the curriculum is meant to support specific programs such as criminal justice. Not only does it support criminal justice, but students are learning basic and more advanced psychological concepts through different means than most university settings. This forces psychology to become a more interdisciplinary field at an earlier stage of learning. A review of the literature on integrating non-western cultures into psychology courses is discussed to incorporate psychology into other programs.
\end{abstract}

Keywords: Teaching psychology, Middle East, interdisciplinary psychology, non-western cultures.

\section{Introduction}

The Kingdom of Saudi Arabia is the largest country in the Gulf Cooperation Council (referred to as GCC henceforth). It includes the two holy sites of Islam and is therefore subject to strict Sharia law that all citizens, residents, and visitors must abide by. Although Saudi Arabia has been viewed as very strict in this regard in the past, there is a sense of change toward a more progressive society, albeit slowly. In the past year, females have begun to drive, different economic policies have been promoted, and several major sporting and music events have taken place in the Kingdom for the first time ever. This is great for the people living there but it also has an impact on education within Saudi Arabia.

Public education in Saudi Arabia in the past has lacked the necessary resources to become a serious contender on the world education stage. However, since 1992, the literacy rate among adults in Saudi Arabia has increased from $70 \%$ to $95 \%$ (Knoema). Although literacy rates have increased, the overall effectiveness of the education system and the students' ability to apply what they have learned has been in question for quite some time. The notion of Academic Entitlement is usually placed upon Western students and suggests that these students believe they are entitled to an education and is often associated with problematic behaviors and attitudes, which could include incivility in the classroom and the endorsement and normalcy of cheating among students (Blincoe and Garris, 2017). However, the study conducted by Blinco and Garris (2017) revealed that the rates of Academic Entitlement were much higher in Saudi Arabia when compared to rates among the US. This Academic Entitlement, it seems, is a major interruption within the classroom and can be noticed throughout the semester by the students.

Currently in the GCC, there are minimal laws and regulations for practicing psychologists or therapists. It is very easy for someone to claim they are a psychologist and provide "therapy" for individuals which could lead to more harm than good. As with other regions in the world, stigma is a major issue throughout the Middle East (McCrae, Shari, and Norman, 2019). It is often thought of as demeaning to go to therapy or the individual is automatically labelled as "crazy" which is similar to stigma across the world. However, a unique aspect of the Middle East, and especially Saudi Arabia, is the family name and tribe (AlAteeq, et al., 2018). The gravity that the family name plays in the future of the 
individual, and even the extended family, is immense. For this reason, many individuals do not seek out mental health services resulting in many mental health issues left untreated.

The high rates of Academic Entitlement and the stigma of psychology do become issues when teaching psychology within Saudi Arabia and one must integrate many different aspects in to the psychology curriculum in order to satisfy the cultural norms. Psychology as a field was founded by western philosophers and academics and has developed over time within these western contexts. It is often exported around the globe without any additional cultural relevance research or cultural modification done to the curriculum. If this is not enough, many non-western clinicians are trained in western countries and then go back to their home countries to practice western psychology within non-western contexts (Badri, 2016). This not only shows the ethnocentrism of the west but could lead to difficulties in therapy and cultural faux paus in the classroom. This is especially the case in Saudi Arabia where Sharia law rules. The current paper examines literature that offer ways for psychology instructors to modify their courses to a more culturally relevant course. This could do two things for the field of psychology: the first being, reducing stigma in societies that have historically not been able to openly address mental health issues; the second being, to spread psychology to non-western cultures in order to make the field more culturally relevant for specific cultural contexts.

\section{Method}

The current study's aim is to identify relevant strategies for modifying psychology courses to fit non-western cultures and in this particular study, the Islamic culture within Saudi Arabia. A literature review was carried out with the criteria of the literature set at adopting western psychology to non-western cultures. There is a limited amount of research published on this subject related to Islamic societies so best practices from other cultures were also sought after. The articles had to be peer-reviewed and published on or after 2015 until present to make sure the articles were appropriate and relatable.

\section{Results and discussion}

Although there are official divisions within the American Psychological Association focusing on cultural, international, and teaching psychology, the number of articles published since 2015 which related to modifying psychology courses to non-western courses was minimal. Of the nine published articles that were read focusing on this subject, only two were directly related to modifying a psychology course to an Islamic culture. This is an interesting find since there seems to be a consensus in the literature that psychology has failed to incorporate minority and diverse populations' experiences and worldviews into the curriculum (Chitindingu \& Mkhize, 2016; Long, 2013; Blokland-Eskell, 2005; Pillay \& Kramers, 2003). In fact, Chitindingu and Mkhize (2016) found that none of their participants reported that any of the curriculum had any coverage of indigenous knowledge systems of their particular African culture. An additional find was that psychology courses were tailored to the English language which non-English language natives found difficult to understand some of the psychological concepts. Although it may not be possible for instructors to translate some of these concepts to the language and culture they are teaching in, there is the possibility that instructors could use examples from the local culture to emphasis the concept.

An example of this specifically for Islam is for the instructor to make an example of Freud's Id, Ego, and Superego. The Quran describes each of these through the Islamic perspective of Nafs. The word nafs lexically refers to our soul, psyche, and heart or mind (Samah, 2018). By bringing in examples from the Quran and drawing comparisons between those examples and psychological concepts, students may be able to identity and understand these concepts better because it becomes more relevant to their own experiences and culture. With the Saudi Vision 2030, one of the goals is to improve education while ensuring that the curriculum and courses are consistent with Islamic beliefs (Mitchell \& Alfuraih, 2018). This practice of bringing in local cultural and traditional examples would support Saudi Vision 2030.

One of the major reasons instructors who teach psychology in non-western cultures have difficulty in modifying their psychological courses to a more culturally relevant course is due to the lack of research conducted with non-western populations. According to Rad, Martingano, and Ginges (2018), not a single study in the issues of Psychological Science in 2017 included samples of Africa or the Middle East while over $70 \%$ of the studies included samples from western countries in North American, western Europe, and Australia. While this may be due to the resources available for psychological research within these countries, the researchers and academics that carry out studies could include non-western samples as well. It seems that although it is agreed that psychology has a diversity problem, no action has been taken to improve this issue. Research that includes different cultures, ethnicities, and religions is essential 
to the development of a more inclusive psychology and the development of more culturally relevant psychology courses for non-western cultures.

A recommendation for the universities and instructors in non-western countries for teaching psychology is to include psychology in curriculum of other majors. An example of this is having psychology in a criminology program at a college in Riyadh. Although the course is entitled introduction to psychology, the content can be modified not only toward being more culturally relevant but also to include aspects of psychology that is pertinent to the students' major. The course may not be a traditional introduction to psychology course but would fit the program better. One aspect that could be focused on more in this course could be the subject of memory in which you could include eye witness testimony. This would enforce the need of psychology for the program and give a reason for the students to study this particular subject. Including these courses in other programs in non-western colleges and universities could also introduce mental health into a society where mental health is not confronted or a society where stigma is prominent.

\section{References}

AlAteeq, D., AlDaoud, A., AlHadi, A., AlKhalaf, H., \& Milev, R. (2018). The experience and impact of stigma in Saudi people with a mood disorder. Annals of general psychiatry, 17(1), 51.

Badri, M. (2016). Cultural and Islamic adaptation of psychology: A book of collected papers (Vol. 1). Human Behaviour Academy.

Blincoe, S., \& Garris, C. P. (2017). Challenging the assumption of a Western phenomenon: Academic entitlement in Saudi Arabia. The Journal of Experimental Education, 85(2), 278-290.

Blokland-Eskell, L.M. 2005. Voice to the Silent: An Ecology of Local Knowledge in Psychology. Unpublished Doctoral Thesis. Pretoria, South Africa: University of Pretoria.

Chitindingu, E., \& Mkhize, N. (2016). Listening to black African psychologists' experiences of social and academic inclusion: Incorporating indigenous knowledge systems into the curriculum. Alternation Special Edition, 18, 72-98.

Long, W. 2013. 'Market Relevance', 'Social Relevance', and Psychology in South Africa. South African Journal of Psychology 43, 4: 434-444.

Mitchell, B., \& Alfuraih, A. (2018). The Kingdom of Saudi Arabia: Achieving the Aspirations of the National Transformation Program 2020 and Saudi Vision 2030 Through Education. Journal of Education and Development, 2(3), 36.

McCrae, N., Sharif, L., \& Norman, I. (2019). Media portrayals of mental disorder in Saudi Arabia: a review of popular newspapers. Transcultural Psychiatry, 1363461518819117.

Pillay A.L. \& A.L. Kramers 2003. South African Clinical Psychology, Employment (In)Equity and the Brain Drain. South African Journal of Psychology 33,1: 52-60.

Rad, M. S., Martingano, A. J., \& Ginges, J. (2018). Toward a psychology of Homo sapiens: Making psychological science more representative of the human population. Proceedings of the National Academy of Sciences, 115(45), 11401-11405.

Samah, F. (2018, June). The Qur'an and mental health. In The Psychologist.

Saudi Arabia - Adult literacy rate. (n.d.). In Knoema. 


\title{
UNDERSTANDING AND DEFINING INSTRUCTIONAL LEADERSHIP: SCHOOL PRINCIPALS' PERSPECTIVE
}

\author{
Danijela S. Petrovic, \& Selena Vracar \\ Department of psychology, Faculty of Philosophy, University of Belgrade (Serbia)
}

\begin{abstract}
School principal's activities related to the positive effect on the quality of education are manifested in the domain of teaching and learning, i.e. instructional leadership. In the Republic of Serbia, school principals were unjustifiably unattended for a long period of time, and therefore the goal of this exploratory research was to investigate understanding of instructional leadership concept from school principals' perspective. Research sample consisted of 20 elementary and high schools principals and semi-structured interview was conducted. Inductive thematic analysis was used as a method of qualitative data analysis. The following topics were identified in participants' responds: Specifics, Priority and Variability of the instructional leadership. Theme Specifics implies that instructional leadership is defined through activities that constitute it. The narrow comprehension of Specifics is related with the understanding of instructional leadership as a process of improving quality of education through direct work and influence on teachers, while broad comprehension encompasses providing resources so that teachers work could be improved. Priority implies that instructional leadership is defined in the context of other principals' activities (such as finance and administration). Variability is different from other themes because it takes into consideration the change of instructional leadership practice over time and practice by different leaders. Brought together, the three themes mentioned above give a picture of all relevant aspects of instructional leadership. Direct work with teachers that relates with narrow comprehension of instructional leadership versus broad comprehension, represents the dichotomy of work activities presented in the relevant literature. Moreover, when discussing positioning instructional leadership in relation to other principal activities, it is noted that principals recognize its significance, but exert doubt about its priority. It is reasonable that the solution to this dilemma needs to be taken at the national level, making the necessary specifications in the legislation. Interesting questions that these findings raise are related with presence and universality of the mentioned transformation of instructional leadership in the school practice. Finally, it can be concluded that the defining concept of instructional leadership is wide, but within the frames of existent models. Investigating comprehensions of instructional leadership is important for improving the work of school principals. Thanks to the obtained data, it is possible to plan strategies for principals' training in various instructional leadership practices, as well as initiating changes in the legislative framework that regulates the work of principals.
\end{abstract}

Keywords: School leadership, instructional leadership, qualitative study, conceptions, effective schools.

\section{Introduction}

The interest for principals as important actors in the process of improving student achievement dates back to the 70s of the last century (Scheerens, 2012). At that time, a large base of research aimed at discovering the characteristics of effective schools (see, for example, Hall \& Hord, 1987; Edmonds, 1979; Rutter et al., 1979; Leithwood \& Montgomery, 1982; Weber, 1971; Brookover \& Lezotte, 1979; Purkey \& Smith, 1983). The conclusion of the aforementioned research was that principals, as instructional leaders, were one of the key features of effective schools (Leithwood, Begley, \& Cousins, 1990).

In the literature, various models of understanding the effects of instructional leadership are cited (see, for example, Pitner, 1988; Hallinger \& Heck, 1998). A simple, but deliberate division gives Shepard (1996) on instructional leadership in the narrow and broad sense. Broadly speaking, instructional leadership implies dealing with organizational issues and issues of school culture, while in a narrower sense it is confined to behavior that focuses on teaching practices. The goal of instructional leadership (whether it is understood in a narrower or broader sense) is to improve student achievement.

Of all the models of instructional leadership that have been developed, two models predominate over others. These are models developed by Bossert and associates (Bossert et al., 1982), and Hallinger and Murphy (Hallinger \& Murphy, 1985). Bossert and associates use the term "instructional 
management" that conceptualize as actions and strategies of the principals directed at the school's instructional organization and the development of a learning culture aimed at improving educational outcomes. In his work, Bossert et al. (1982) chose the term instructional management because they concluded that the role of director is reduced to managerial tasks oriented to the coordination and control of curriculum and teaching. Another dominant model that provides a clear definition of instructional leadership is, as mentioned above, presented in the work of Hallinger and Murphy (Hallinger \& Murphy, 1985). The conceptual framework of this model consists of three dimensions: defining a school mission, managing the curriculum and developing a learning culture. Each of the three dimensions is further described by the functions of instructional leadership.

In the Republic of Serbia school principals are in a very challenging position in view of the disproportion of set requirements (regulated by legal acts), and the support provided to them (in the form of training programs or seminars intended for professional development). Data from the TALIS 2013 survey (OECD, 2014) indicate, on one hand, the high representation of instructional leadership practice in schools in Serbia: the cumulative frequency (for answers to all three items related to instructional leadership $^{1}$ ) for Serbia is 249.3, while TALIS average is 208.2. Therefore, the percentage of principals who say that "often" or "very often" engage in these activities is over $80 \%$. On the other hand, the data indicates that the principals are undertrained (in the sense of not attending relevant training) either during initial education, or during later professional development. In Serbia, training from instructional leadership has never been attended by as many as $53.4 \%$ of principals (TALIS average is $22.2 \%$ ). There are several actors, from whom they can seek support, starting from the resources in the school itself, through the Society of School Principals of Serbia, to institutions and organizations of a national and international character. Common to all mentioned, potential, sources of assistance is that their inclusion depends, and it is left to the initiative of the director himself. Taking into account the importance they have when it comes to ensuring the quality of the work of the institution, it is clear that their professional development should not be left to their initiative, and that they must be systematically addressed, in the same way as the Law on the Foundations of the Education prescribes (Official Gazette RS, No. 72/2009). Considering the importance of instructional leadership, and in contrast, a modest database on the same in a domestic context, the goal of this exploratory research is to investigate the understanding of instructional leadership from the perspective of the school principals themselves.

\section{Methodology}

\subsection{Qualitative paradigm}

A qualitative paradigm is set up as an adequate research response to the study of instructional leadership for several reasons. Firstly, the instructional leadership in Serbia was not at all the subject of researchers' interest, and it is therefore impossible to formulate clear and precise hypotheses for quantitative research. Secondly, it is suitable for the purpose of deeper understanding and clarification of data related to the instructional leadership gathered in the TALIS 2013 survey (OECD, 2014). The designed study thus provides an opportunity for research participants to share with the researcher their personal experience of the investigated phenomenon.

Qualitative research is a constant reminder that the views and activities of other people are an inexhaustible source of knowledge and can never be fully covered. In this respect, the data collected by the qualitative study not only represent a complement to quantitative indicators but potentially can produce some relevant findings that could be obtained exclusively through this method, thanks to a particular type of sensitivity of qualitative studies (Viling, 2016).

\subsection{Sample}

Four principals were selected by the recommendation of the expert in educational leadership, while the rest of the sample was collected by the snowball method - based on the recommendations given by the initial sample of four principals. Besides to the recommendations of expert and colleagues, two additional indicators for the selection of principals to enter the sample were: (1) training in the field of instructional leadership, and (2) evaluation scores from the external evaluation of schools (for two domains: the School Ethos and the School Management and Leadership). The reason for choosing the School Ethos as an additional criterion is based on data from TALIS 2013 for Serbia - namely, the connection between practicing instructive leadership and indicators: the school climate - respect for each other is 0.26 (OECD, 2014). In total 20 school principals participated in the research (see Table 1).

\footnotetext{
${ }^{1}$ Principals were asked to indicate how frequently they engaged in the following activities in their school during the preceding 12 months: I took actions to support co-operation among teachers to develop new teaching practices; I took actions to ensure that teachers take responsibility for improving their teaching skills; I took actions to ensure that teachers feel responsible for their students' learning outcomes (OECD, 2014).
} 
Table 1. Characteristics of the sample.

\begin{tabular}{|c|c|c|c|c|c|}
\hline \multicolumn{2}{|c|}{ Gender distribution } & \multicolumn{2}{|c|}{ Type of the school } & \multicolumn{2}{c|}{ City } \\
\hline Male & Female & Elementary school & Secondary school & Belgrade & Other \\
\hline $45 \%$ & $55 \%$ & $55 \%$ & $45 \%$ & $80 \%$ & $20 \%$ \\
\hline
\end{tabular}

The principals of the sample have on average 17.3 years of teaching experience (which is 3.4 years more than the average for the Republic of Serbia in the TALIS 2013 study (OECD, 2014)). Most principals previously worked as subject teachers (i.e. they were teachers from 5th to 8th grade in elementary school, or in secondary school), two principals had previously been in the position of a professional associate - pedagogue, and one director previously worked as a class teacher (i.e. teaching from 1th to 4th grade in elementary school). On average, principals have 9.2 years of experience in a given position (which is 1.8 years more than the average for the Republic of Serbia in the TALIS 2013 study (OECD, 2014)). When it comes to external evaluation data for the assessment of the ethos and organization of school work and management, only good and fully-matched standards were represented in the sample, with the majority of the sample (70\%) being schools where these areas were rated at the highest level, the level 4. For the three schools from the sample (15\%), there are no data on the listed areas, because the external evaluations were not yet conducted in the given schools. Participation in training from instructional leadership is characterized for respondents who are on the master program "Leadership in Education" (25\% of the sample).

\subsection{Data collection and analysis}

A semi-structured interview was selected as a data collection technique. Some examples of questions from the interview guide are: "How do you understand the concept of instructional leadership?", "How do you see its significance?", with questions to encourage conversations such as: "Why do you think that?", "Can you explain a bit what did you say?", etc. Principals were invited to express their personal opinion, to speak from their own perspective. In the transcription of the interview, a denaturalized approach was used (Elo \& Kyngäs, 2008), which represents a complete and faithful form of transcription, but without the requirement to indicate idiosyncratic speech elements.

Inductive thematic analysis was used as a method of qualitative data analysis in this study. In general, the thematic analysis represents a method for identifying and organizing patterns in the content and meaning of qualitative data (Viling, 2016). An inductive approach functions from the bottom up - researchers access the data without a theoretically framed encoding frame, the topics are based on data (Boyatzis, 1998 according to Wing, 2016).

\section{Results} in Table 2.

In the director's narratives, three themes have been identified. The tabular display of data is given

Table 2. How school principals define instructional leadership.

\begin{tabular}{|c|l|c|c|}
\hline & \multicolumn{3}{|c|}{ Instructional leadership } \\
\hline Themes & \multicolumn{1}{|c|}{ Specifics } & Priority & Variability \\
\hline Categories & The narrow & Extremely important & \\
& comprehension & Relatively important & \\
& The broad comprehension & & \\
\hline
\end{tabular}

A. Specifics. Theme Specifics implies that instructional leadership is defined through activities that constitute it. Within the theme "Specifics" the following categories have been identified:

A1. The narrow comprehension. This category includes the answers of principals who see instructional leadership as a process of improving the quality of education through direct work with teachers. The principal follows the work of teachers, gives suggestions for their improvement, takes care of maintaining teacher motivation, and is present during the whole process of adaptation to working conditions. The principal who does so has the characteristics of an instructional leader. Also, this category includes a response group that emphasizes the word "leader". Namely, the principal is seen as someone who leads the introduction of innovations in educational practice or encourages teachers to do this, if he/she is "not innovative" in this regard. The reason why these responses also fall into this category is reflected in the fact that there is still direct influence and work between principals and teachers, with the specification of the operation present here. 
Example: The school principal as an instructional leader must seriously address the teacher motivation (female, elementary school).

A2. The broad comprehension. Principals whose answers fall into this category do not limit instructional leadership solely to the process of direct influence on teachers. Namely, they treat as an equally important aspect of instructional leadership the procurement of equipment and resources so that teachers can work efficiently. In other cases, the only possible direction of the activity of instructional leadership is seen in the case of procurement of the mentioned funds. Thus, this category includes answers through which instructional leadership is seen as a type of leadership which primarily has indirect consequences for improving the quality of teaching. Principals represent a secondary link in this process, in the sense that they operate through the creation of adequate working conditions or the formation of teams that will directly work with teachers.

Example: Instructional leadership implies the creation of an incentive environment in school, both in terms of material resources, the acquisition of other resources ... (female, elementary school).

$B$. Priority. The concept is defined by the significance it has, in terms of how it is positioned in the context of all the work that the director is doing. Within the theme "Priority" the following categories have been identified:

B1. Extremely important. For principals whose answers fall into this category, instructional leadership is the most important aspect of the principals' work. The education and quality of education acquired in school is seen as the primary function of a school, and principals perceive their role as bearers in achieving the set goal of quality education. Furthermore, it is important to note that some principals fail to put instructional leadership as a priority of their work, but regardless of the fact that they do not come to deal with it, they see it as extremely important as something that should give priority to their work.

Example: The basic mission of each school is quality teaching (male, secondary school).

B2. Relatively important. Everything is important and everyone is important. Instructional leadership is one aspect of the work of the principal whose importance is not denied, but also does not stand out in relation to others. Administrative tasks, dealing with finances and the like are also the responsibilities of principal which must not be ignored. Therefore, the principal should take actions so that everything functions well, perform all the tasks prescribed to him/her, and no job has, nor should have, a priority over another.

Example: The principal must be a lawyer, an electrician and a plumber and he must supervise everything that happens in school... (female, primary school).

C. Variability. This category of responses differs in relation to others by adding the time component to the concept of instructional leadership. Namely, principals with longer working years, find their role changed. The beginning is seen as a period of intensive work and "putting things in order", while work in the later years refers to the maintenance of a defined structure and established processes. In addition, the variability of performing the role itself can be seen in the way that principals use different approaches for organizing it.

Example: Like every beginner, you have the need to organize everything, to be in control of everything. It is much easier now, now I only coordinate the work of the teams... (female, elementary school).

\section{Discussion and conclusion}

As an expression of the efforts of the principal to determine the instructional leadership, three tendencies were identified: defining over the tasks from which it consists, defining in the context of other tasks and finally, defining the changes in the operation of which it consists. When it comes to defining instructional leadership through the jobs it consists of, it can be recognized dichotomy given by Sheppard (1996) to the instructional leadership in narrow and instructional leadership in the broad sense. So, we can notice that principals have different conceptions about the breadth of their field of activity as instructional leaders. Considering that the same disagreement exists in scientific circles, the registered situation isn't a surprise. Furthermore, when it comes to the second tendency of determining through positioning in relation to other jobs, we testify to the situation of recognized importance, but also the uncertainty about allocating it in relation to other tasks. It seems that the principals are guided by the responsibilities as defined by the legislation and that they want to stay within the given framework and perform all their duties equally, without any special allocation that the law does not do. Therefore, it is very clear that the educational law influences the formation of practice (Official Gazette RS, No. 72/2009). The law does not give priority to one area of work in relation to the other, and neither principal in his/her practices do so. Finally, it is interesting to note the presence of observations concerning the transformation of instructional 
leadership during the course of time. The transformation itself is moving from direct engagement and high presence to more mediocre and delegated tasks. Mentioned observations provoke interesting questions about presence and universality of this transformation of instructional leadership in practice.

On the basis of data acquired, the following recommendations have been formulated:

- Revisions to the legislation regarding the allocation of instructional leadership as a priority of the principal work;

- Expert support should be designed to follow the jobs that fall into the narrow and broad understanding of instructional leadership;

- Organize seminars that will be directed toward developing the strategies for determining work priorities;

- To establish a network of principals from different schools in order to provide mutual support and horizontal learning.

\section{Acknowledgements}

This work was supported by the Ministry of Education, Science and Technological Development, Republic of Serbia (project number 179018).

\section{References}

Bossert, S., Dwyer, D., Rowan, B., \& Lee, G. (1982). The instructional management role of the principal. Educational Administration Quarterly, 18(3), 34-64. doi: 10.1177/0013161x82018003004.

Brookover, W. B., \& Lezotte, L. W. (1979). Changes in school characteristics coincident with changes in student achievement. East Lansing, MI: Institute for Research on Teaching, Michigan State University.

Edmonds, R. (1979). Effective schools for the urban poor. Educational Leadership, 37(1), 15-24. Elo, S., \& Kyngäs, H. (2008). The qualitative content analysis process. Journal of Advanced Nursing, 62(1), 107-115. doi: 10.1111/j.1365-2648.2007.04569.x.

Elo, S. \& Kyngäs, H. (2008). The qualitative content analysis process. Journal of Advanced Nursing, Vol. 62, No. 1, 107-115.

Hall, G. E., \& Hord, S. M. (1987). Change in schools: facilitating the process. Albany: State University of New York Press.

Hallinger, P., \& Heck, R. H. (1998). Exploring the principal's contribution to school effectiveness: 1980-1995. School Effectiveness and School Improvement, 9(2), 157-191. doi: 10.1080/0924345980090203.

Hallinger, P., \& Murphy, J. (1985). Assessing the instructional management behavior of principals. Elementary School Journal, 86(2), 217-248. doi: 10.1086/461445.

Leithwood, K. A., Begley, P. T., \& Cousins, J. B. (1990). The Nature, Causes and Consequences of Principals' Practices: An Agenda for Future Research.Journal of Educational Administration, 28(4). doi:10.1108/09578239010001014.

Leithwood, K., \& Montgomery, D. (1982). The role of the elementary principal in program improvement. Review of Educational Research, 52(3), 309-339. doi: 10.3102/00346543052003309.

OECD (2014). TALIS 2013 Results: An International Perspective on Teaching and Learning. Paris: OECD.

Law on the Foundations of the Education System', Official Gazette RS, No. 72/2009, 52/2011 and $55 / 2013$

Pitner, N. (1988). The study of administrator effects and effectiveness. In N. Boyan (Ed.), Handbook of research in educational administration (106-132). New York: Longman.

Purkey, S., \& Smith, M. (1983). Effective schools: A review. The Elementary School Journal, 83 (4), 427-452. doi: 10.1086/461325.

Rutter, M., Maughan, B., Mortimore, P., Ouston, J., \& Smith, A. (1979). Fifteen Thousand Hours: Secondary schools and their effects on children. Cambridge: Harvard University Press.

Scheerens, J. (2012). School leadership effects revisited review and meta-analysis of empirical studies. Dordrecht: Springer.

Sheppard, B. (1996). Exploring the transformational nature of instructional leadership. The Alberta Journal of Educational Research, 42(4), 325-344.

Viling, K. (2016). Kvalitativna istraživanja u psihologiji. Beograd: CLIO.

Weber, G. (1971). Inner-city children can be taught to read: four successful schools. Washington: Council for Basic Education. 


\title{
THE IMPACT OF ADJUSTMENT, ENGAGEMENT AND HELP-SEEKING BEHAVIOUR TOWARDS ACADEMIC WELL-BEING OF INTERNATIONAL STUDENTS FROM DEVELOPED COUNTRIES WHO STUDY IN DEVELOPING COUNTRIES
}

\author{
Erna Zulaeni Wiles ${ }^{1}$, Tina Afiatin ${ }^{2}, \&$ Neila Ramdhani ${ }^{2}$ \\ ${ }^{1}$ Doctoral student of The Faculty of Psychology, Gadjah Mada University (Indonesia) \\ ${ }^{2}$ Faculty of Psychology, Gadjah Mada University (Indonesia)
}

\begin{abstract}
To seek evidence of the correlation between college adjustment (CA), academic engagement (AE), help-seeking behaviour (HSB) and students' academic well-being (AW), quantitative research was conducted at STIE Triatma Mulya Stenden, Bali, Indonesia, involving 235 grand tour students from NHL Stenden University of Applied Sciences of Dutch and German nationalities who took minors for ten weeks. Analysis with Structural Equation Model shows that the model provided acceptable fit with CFI $(0.992>0.9)$ and RMSEA $(0.031<0.06)$. All parameter estimates were at 0.05 significant level $(\mathrm{z}>2.58)$. With the note "minimum was achieved" and the value of $d f 14$ (positive two digit numbers) the chi-square value is acceptable. Detailed findings are 1) with the regression coefficient value $(\beta)=0.286, p$-value $=0.001$ and $c r$-value of less than 3.321, CA has a significant effect on AE; 2$)$ with $\beta=-0.436, p=0.031$ and $c r<-2.152$, AE has a significant effect on HSB; 3 ) with $\beta=0.205, p=0.125$ and $c r<-1.533$, CA has no significant effect on HSB; 4) with $\beta=0.020, p=0.728$ and $c r<3.372$, HSB does not have a significant effect on AW; 5) with $\beta=0.379, p=* * *$ and $c r<3.372$, AE has a significant effect on AW; 6) with $\beta=0.508, p=0.001$ and CR $<5879$, CA has a significant effect on AW. To conclude, students' short stay, international education standard, the locals' hospitality and English language competency enable students to adapt and become academically engaged. If issues persist, they are mostly related to their inability to resist invitations to socialize during school days. Regrettably, the short stay discourages the students from seeking help when facing problems.
\end{abstract}

Keywords: College adjustment, academic engagement, help-seeking behaviour, academic well-being.

\section{Introduction}

Internationalization at the national/sector/institutional levels is defined as "the process of integrating an international, intercultural or global dimension into the purpose, functions or delivery of higher education at the institutional and national levels" (Knight, 2008). The Organization for Economic Co-operation and Development (OECD) reported that in 2016, within its members, 35 countries from North and South America to Europe and Asia Pacific, 3.5 million are international students (OECD, 2018), and 55\% enrolled in bachelor's degree. Out of that number 1.9 (55\%) are students from Asia studying mostly in three developed countries i.e. Australia (15\%), the UK (11\%) and the USA (38\%). On the contrary, only 845.000 European students are recorded studying abroad mostly in other European countries and only 24\% studies in OECD countries. consequently, most research was carried out to this population and little was done to students from developed countries who study in developing countries.

The question is raised as to whether students from developed countries who study in developing countries encounter the same challenges. As mentioned by Yeoh, Le, and Doan (2013), students from developing countries face academic issues due to language barriers and different educational system. These students also are confronted with non-academic issues i.e. psychological, social and financial. The first and foremost psychological difficulty is culture shock - "a negative state generated by being in an unfamiliar cultural environment" (Ward, Bochner, \& Furnham, 2001). Physical issues can be caused by fatigue, lack of or imbalanced nutritious meals, lack of exercises, and other health-related risk-taking behaviour (Rajapaksa \& Dundes, 2003). Social issues, rooted from the lack of language proficiency, cultural differences and heavy load studies, create students' reluctance to socialize (Wiles, 2017). Financial issue persists because of high tuition fees, exchange rates discrepancies (Banjong, 2015), fundamental welfare issues due to limited work rights (Alghamdi \& Otte, 2016), and inaccessibility to health care and support services. This study is conducted to seek the answer to the afore-mentioned question. 


\section{Grand tour students and their issues}

NHL Stenden University of Applied Sciences (NHL SUAS) with the headquarter in Leeuwarden, the Netherlands and its branches in Thailand, Indonesia, Qatar, South Africa, and China is one of the largest institutions of higher education in the Netherlands. 2.900 out of over 24,000 enrolees are international students from 90 different citizenships (Stenden University, 2017). With that, the institution is committed to keep its education International. The primary focus is the Grant Tour (GT) program. Almost 100 minor programmes are offered all over campus sites including STIE Triatma Mulya Stenden (STMS) Bali, Indonesia - the venue of this research, which offers namely Entrepreneurship in Action, Event Management, Marketing and Management in Asia, Spa Business Strategy, Media Tourism Innovation and Mindfulness Leadership. STMS receives 100 - 120 GT students per module (10 weeks).

As most GT students have never travelled in developing countries before, two sessions of cultural orientations are conducted prior to the departure and right after the arrival to ensure the students adjust well. In her research, Wiles (2017) recorded that in every module there are always incidents related to various health issues, (traffic) accidents, thefts and street robberies, social issues like the accessibility of Bali night lives and the incapability to resist friends' invitation to go out during the school days. Psychological issues range from homesickness, loneliness, stress, depression, frustration, anger, and many other. Financial issues are not detected until in the later phase, where money has been spent for nightlife and travels. This results in the decrease of the students' engagement in the educational activities. Subsequently, this also leads to the students' low academic well-being and conversely to the achievement. Academic issues are related to module assignments which require students to work in groups. The dynamic of the groups normally dwindling around mid-module. Nothing recorded as an indication of academic issues due to the lack of English competency and the difference between the educational system implemented in NHL Stenden and STMS. Unfortunately, it is recorded that the number of the students failing the units in the module is $10-20 \%$; big enough to draw a conclusion that there is something not right with the students' well-being and that some measures need to be done in order to reduce the number. Focus is directed towards students' ability to adjust the new (academic) environment, students' engagement and initiative to seek help when facing challenges.

\section{Well-being, adjustment to college, academic engagement, and help-seeking behaviour}

From qualitative research employing N-Vivo data analysis, Wiles (2017) constructed a definition of academic well-being i.e. "the balance between sources owned and students' academic achievement, and when throughout their time in the institution, students are healthy physically and psychologically, have good social relationships with the academic staff, friends and the people where they live, and have future visions when there is assistance and support from the staff'. Engagement is defined as an activity that results in an increase or growth carried out by an individual by actively allocating his attention in his environment (Csikszentmihalyi, 2008) and the initiation of the efforts, actions and persistence of students in school assignments and their emotional state during the learning process (Skinner, Kinderman, \& Furrer, 2009). Adjustment includes the organization of personalities capable of directing students, towards stability, which in this case is the active adjustment of an individual with his social and physical environment (Skinner, 1936). Help-seeking behaviour is "The process of actively searching for relevant resources, carried out by a student who considers himself in need of personal, psychological, affective, health or social services, both informal and formal services, in a positive way with the purpose of meeting their needs and solving the problems they carry" (Spendelow \& Jose, 2010). The assumption is, should students find it easy to adjust with the (academic) environment, they will be engaged and should issues related to the adjustment and engagement arise, and students seek help, students will be academically wellbeing. To ensure students' academic well-being SUB provides study career advice and counselling, unfortunately, it is recorded that on average only 2 to 5 students seek psychological help.

\section{Methodology}

To find the solutions, quantitative research to test a theoretical model implying that student college adjustment, academic engagement mediated by help-seeking behaviour determine the students' academic well-being fits with the empirical data, was conducted, involving 235 GT students. Two nationalities, Dutch and Germans, were involved, representing developing countries. Four scales are employed, which are 1) Academic Wellbeing Scale (Borgonovi \& Pál, 2016), 2) General Help-Seeking Questionnaire (Wilson, Deane, Ciarrochi, \& Rickwood, 2005), 3) The Student Adjustment to College Questionnaire (Baker \& Siryk, 1999), and Academic Engagement (Maroco, Maroco, Campos, \& Fredricks, 2016) were replicated and modified. Upon tested by a panel of 15 experts from psychological and educational backgrounds, the reliability and validity of all instrument are proven to be above .3 and .7 respectively. The data was analysed by using Structural Equation Model. 


\section{Results}

The results show that all evidence supports the claim.

Table 1. Goodness of Fit.

\begin{tabular}{ccrc}
\hline Goodness of Fit Measure & Cut of value & Value Generated & Evaluation \\
\hline Chi Square $\left(\mathrm{X}^{2}\right)$ & Expected to be small & 17,175 & Good \\
Degree of freedom & Two digits & 14 & Good \\
CMIN/DF & $\leq 2.00$ & 1,227 & Good \\
GFI & $\geq 0,9$ & 0,982 & Good \\
AGFI & $\geq 0,9$ & 0,954 & Good \\
RMR & $\leq 0,084$ & 0,028 & Good \\
RMSEA & $\leq 0,080$ & 0,031 & Good \\
NFI & $.>0,90$ & 0,961 & Good \\
IFI & $\leq 1$ & 0,993 & Good \\
TLI & $.>0,95$ & 0,985 & Good \\
CFI & $.>0,95$ & 0,992 & Good \\
HOELTER & $\geq 124$ & 320 & Good \\
\hline
\end{tabular}

The absolute Fit Indices, Incremental Fit Indices, Parsimony Fit Indices tests, conducted and analyzed by employing SEM - Amos generated CMIN/DF value of 1,227, GFI value of 0.982, AGFI value of 0.954 , RMR value of 0.028 , RMSEA value of 0.031 , NFI value of 0.961 IFI value of 0.993 , TLI value of 0.985 , CFI value of 0.992 and the HOELTER value of 323.

Figure 1. The model.

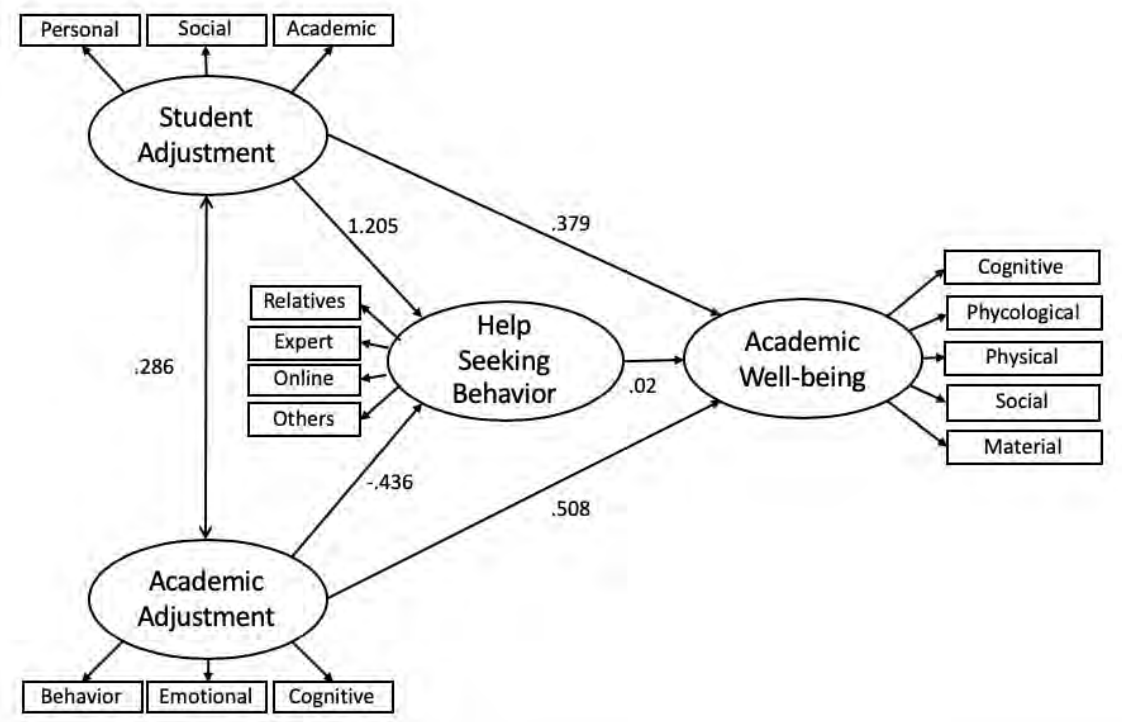

Results of the hypothesis testing using the standardised regression weight with the significant level $p$ of .05, however, show that two of the six assumptions are rejected.

Table 2. Standardized regression weight.

\begin{tabular}{cccccccc}
\hline & & & Estimate & S.E. & C.R. & P & Results \\
\hline Academic_Engagement & $<---$ & Student_Adjustment & .286 & .086 & 3.321 & $* * *$ & Accepted \\
Help-Seeking Behaviour & $<---$ & Academic Engagement & -.436 & .203 & -2.152 & .031 & Accepted \\
Help-Seeking Behaviour & $<---$ & Student Adjustment & -.205 & .134 & -1.533 & .125 & Rejected \\
Academic Well-being & $<---$ & Help-Seeking Behaviour & .020 & .057 & .347 & .728 & Rejected \\
Academic Well-being & $<---$ & Academic_Engagement & .379 & .112 & 3.372 & $* * *$ & Accepted \\
Academic Well-being & $<---$ & Student_Adjustment & .508 & .086 & 5.879 & $* * *$ & Accepted \\
\hline
\end{tabular}

Table 2 shows that the student adjustment variable has a significant effect on the academic engagement variable, the academic engagement variable has a significant effect on the help-seeking behaviour variable, the student adjustment variable has no significant effect on the help-seeking behaviour variable, the help-seeking behaviour does not have a significant effect on the academic wellbeing variable, the academic engagement variable has a significant effect on the academic wellbeing variable, and the student adjustment variable has a significant effect on the academic wellbeing variable. 


\section{Discussion}

The fitness of the model shows that student adjustment and academic engagement, when mediated by help-seeking behaviour will determine the students' academic well-being. Thus, it is crucial for STMS to provide assistance for their GT students during their adjustment period to ensure that the students engagement is preserved throughout the module. GT students are also exposed to factors which may influence their adjustment to their new environment in Bali such as culture and language differences, however this does not affect their ability to adapt to the new educational and social environment. The adoption the Netherlands' policy on teaching learning activities does not contribute to issues, except that students feel STMS has been too rigid and strict in the implementation. The use of English as the classroom language, the staff high level of English competency, the Balinese' level of English has also contributed to the speed of the adjustment and intensified the degree of the social interaction, which according to Church (1982) may also determine the adjustment. The 10-week duration of the stay in Bali may also contribute to the adjustment, forces the students to adapt to the new environment as fast as possible. All in all, this condition confirms the statement of Zhou, et al, (2008) that the least issues faced by international students the faster the speed of the acculturation process. In relation to research findings by Iglesias-Benavides, et.al., (2017), in which adjustment is related to students' depression and academic problems, only a few incidents are recorded. Despite the fact that on average up to $20 \%$ of the students fail the units, students' academic achievement overall is still above the par indicated by high level of grades. That is why statistically it is not proven that student adjustment significantly correlates with their academic wellbeing.

Confirming statements by Finn and Zimmer, (2012), academic engagement categories, behavioural, emotional and cognitive are proven to be influencing the students' academic well-being. It is recorded, there is a unique pattern in this case. Due to their accessibility, affordability, and the temptation, students mostly spend their night life outside of their accommodation and their weekends in or outside of the island. This has affected their academic well-being. Tutors often express their concern that students do not do the weekly assignments during the CBL or EMBL as expected, such as late completion of homework, the presence of disturbing behaviour, low level of attentiveness, interest and contributions to class discussions. On week six, however, students start showing a high level of engagement. Confirming research findings by Maroco, et al. (2016), the students' engagement can be used as an antidote to low academic achievement, school burnout, insecurity, and dissatisfaction. This new-found engagement has improved the quality of the relationship with friends, teachers and staff; health and physical and psychological well-being (Wang \& Eccles, 2012); and ultimately improved the willingness to learn, generating satisfying school achievement as stated by (Reschly \& Christenson, 2012).

The management of STMS has provided instrumental support - transportation and accommodation, health related-assistance to health-related information, hospitalization and insurance organization as well as emotional support via the study advisor and even educational support. Out of possible health and physical issues a few are recorded, mostly related to Bali belly and injuries, whereas mental health problems are related to anxiety, depressive and stress symptoms, and interpersonal problems, which mostly started when students were still in their country. The impact can be severe as all module assignments are carried out in groups. One student's problem may turn into a group problem.

Out of 100 students in a module, recorded only two to five students contact the student advisor for psychological/mental assistance. This might be the reasons why statistical analysis does not generate a significant correlation between help-seeking behaviour and academic wellbeing. Data shows that most students would rather go to roommates, friends and family, some would rather convince themselves that focusing on the assignments and exam will distract themselves from the issue. Increasing international students' awareness on the significance in seeking help when facing either personal, physical, and psychological problem to increase the students' well-being is big homework for an educational institution. Short time stay in the university should not be a hindrance since any type of support will be, if not solving, easing the issues. With that in my optimum academic well-being can be reached.

\section{Conclusion and recommendations}

Despite the fact that the number of international students is small, and that their stay in the developing country is quite short, their well-being should also be prioritised. First of all, the institution should ensure that students are able to adjust to the new life, the education and to the society where they live. Secondly, even though student enjoy the new life with its temptations, students need to increase their awareness on the necessity to focus on the education. The inability to decline invitations to have fun and enjoy the new life, will reduce the students' academic engagement. Low level of engagement will definitely affect their academic achievement. The institution should also be more active encouraging the students to seek a proper help should they face any issue or challenges. 
For future research, it is recommended that to see the generality, a replication is conducted for international students who 1) study in other university outside of Bali, Indonesia or Asia, 2) originate from other English-speaking developed countries like the UK, Sweden, Australia or the USA, 3) non-English speaking countries like France, 4) take a degree which means staying longer than merely 10 weeks. It will be not that surprizing it generates different results.

\section{References}

Alghamdi, H., \& Otte, S. (2016). Challenges and Benefits of Study Abroad. International Journal of Humanities and Social Science, 6(5), 16-22. ISSN 2220-8488.

Csikszentmihalyi, M. (1997). Finding Flow: The Psychology of Engagement with Everyday Life. New York, NY, The USA: Basic Books. ISBN: 0465024114.

Finn, J., \& Zimmer, K. (2012). Student engagement: What is it? Why does it matter? In S. Christenson, A. Reschly, C. Wylie, \& S. Christ, Handbook of research on student engagement (pp. 97-131). New York, NY, The USA: Springer Science \& Business Media. ISBN-13: 987-1461467915.

Iglesias-Benavides, J., Blum-Valenzuela, E., López-Tovar, A., Espinosa-Galindo, A., \& Rivas-Estilla, A. (2017). The College Adjustment Scales (CAS) test and recent students' school performance upon entry into a medical school. Medicina Universitaria, 18(73), $201 \quad$ - 214. DOI: 10.1016/j.rmu.2016.10.005.

Knight, J. (2008). Higher Education in Turmoil: The Changing World of Internalization (Vol. 13). Rotterdam, The Netherlands: Sense Publisher. ISBN: 978-90-8790-520-0 (paperback).

Maroco, J., Maroco, A., Campos, J., \& Fredricks, J. (2016). University student's engagement: development of the University Student Engagement Inventory (USEI). Psicologia: Reflexão e Crítica, 29(1), 1-12. DOI 10.1186/s41155-016-0042-8.

OECD. (2018). Education at a Glance 2018: OECD Indicators. Organisation for Economic Co-operation and Development, The Secretary General of the OECD. Paris: OECD Publishing. http://dx.doi.ord/10.1787/eag-2018-en.

Paltridge, T., Mayson, S., \& Schapper, J. (2012). Covering the Gap: Social Inclussion, International Students and the Role of Local Government. Australian Universities' Review, 54(2), 29-39. ISSN: 0818-8068. http://dx.doi.org/1.

Rajapaksa, S., \& Dundes, L. (2003). It's a Long Eay Home: International Student Adjustment to Living in the United States. Journal of College Student Retention, 4(1), 15-28. ISSN: ISSN-1521-0251.

Skinner, E., Kinderman, T., \& Furrer, C. (2009). A motivational perspective on engagement and disaffection: conceptualization and assessment of Children's behavioral and emotional participation in academic activities in the classroom. Educational and Psychological Measurement, 69(3), 493-525. DOI: 10.1177/0013164408323233

Spendelow, J., \& Jose, P. (2010). Does the optimism bias affect help-seeking intentions for depressive symptoms in young people? The Journal of General Psychology, 137(2), 190 - 209. DOI: 10.1080/00221301003645277.

Stenden University. (2017). Stenden University of Applied Science. Retrieved from https://stenden.com/exchangetostenden/

Wang, M., \& Eccles, L. (2012). Social support matters: longitudinal effects of social support on three dimensions of school engagement from middle to high school. Child Development, 83, 877-95. DOI: 10.1111/j.1467-8624.2012.01745.x.

Ward, C., Bochner, S., \& Furnham, A. (2001). The Psychology of Culture Shock. East Sussex, The UK: Routledge. ISBN: 9780415162357.

Wiles, E. (2017). Psycho-social Determinants of Academic Well-being for International Students from Developed Countries Who Study in Developing Countries. Stenden University Bali. Denpasar, Indonesia: Stenden University Bali. https://doi.org/10.2991/acpch-17.2018.43.

Yeoh, J., Le, T., \& Doan, T. (2013). Student Studying in an Australia University Context. In T. Le, \& Q. Le, Conducting Research in a Changing and Challenging World (pp. 303-309). Hauppauge, NY, The USA: Nova Science Publishers Inc. ISBN 978-1-62618-651-4.

Zhou, Y., Jindal-Snape, D., Topping, K., \& Todman, J. (2008). Theoretical models of culture shock and adjustment in international students in higher education. Studies in Higher Education, 33(1), 63-75. http://dx.doi.org/10.1080/03075070701794833. 


\title{
MEANING OF FOREIGNERS AMONG ESTONIAN, LATVIAN, LITHUANIAN AND RUSSIAN UNIVERSITY STUDENTS
}

\author{
Kristi Kõiv ${ }^{1}$, Svetlana Gurieva ${ }^{2}$, Olga Deyneka ${ }^{3}$, Vaiva Zuzeviciute ${ }^{4}$, Anna Liduma ${ }^{5}$, \\ \& Sandra Rone ${ }^{5}$ \\ ${ }^{I}$ Institute of Education, University of Tartu (Estonia) \\ ${ }^{2}$ Department of Social Psychology, Saint Petersburg State University (Russia) \\ ${ }^{3}$ Political Psychology Department, Saint Petersburg State University (Russia) \\ ${ }^{4}$ Faculty of Public Security, Mykolas Romeris University (Lithuania) \\ ${ }^{5}$ Faculty of Education, University of Latvia (Latvia)
}

\begin{abstract}
The purpose of this pilot study was to explore how Estonia, Latvian, Lithuanian and Russian university students conceptualize the meaning of foreigners. The present study examines similarities and differences between Estonian $(\mathrm{N}=118)$, Latvian $(\mathrm{N}=101)$, Lithuanian $(\mathrm{N}=101)$, and Russian $(\mathrm{N}=92)$ university students' understandings about foreigners by self-reported open-ended questionnaire. The applied categorical quantitative analysis of the data was the basis for statistical analysis. Results revealed quite distinctive meanings associated with foreigners when comparing four samples' conceptualizations. The meaning of foreigners among university students was generally conceptualized in society level as an exclusion of people connected with different nationality and language, whereby Russian respondents empathized more differences in citizenship and three Baltic states respondents in attitudes and values. Overall acceptance or unacceptance of foreigners tended to depend on the level - foreigners were more accepted in personal level and unaccepted in society level. Additionally, all university students were generally open and tolerant toward foreigners in the area of individual differences evoked from cultural enrichment, but Latvian, Lithuanian and Russian students were more prone to accept foreigners when there was a mutual respect with common values, and Estonians expressed more the attitude about equality of people. University students in four study groups were generally agree, that foreigners have influenced them more positive than negative way, but reasons were different: Lithuanians empathize more sympathy and helping behavior; Estonian and Latvian more enlargement of knowledge's with increase of tolerance; and Russian students' opinions were more connected with undirect influence by means of media, art and literature.
\end{abstract}

Keywords: Concept of foreigners, attitudes towards foreigners, university students.

\section{Introduction}

In the last century, immigrants have entered Europe in large numbers, leading to a drastic change in demographic build-up (McLaren, 2003) and the population of foreigners in the countries of the European Union has risen sharply in recent years playing a dominant role in population growth in some countries (Organisation for Economic Co-operation and Development, 2012). The growth of immigration in Europe has been associated with an increase of anti-foreigner attitudes in a variety of European countries (Gang, Rivera-Batiz, \& Yun, 2013). Immigrants (or foreigners) are perceived not only as outsiders in their new societies but also as a threat to the social, political and economic order as well as a threat to the cultural homogeneity and the national identity of the state (e.g. Scheepers, Gijberts, \& Coenders, 2002). The growing body of research on attitudes toward out-group populations in general and foreigners in European countries reveal that most people express negative attitudes toward foreigners (Gorodzeisky \& Semyonov, 2009). Also, it was reported more prejudice toward foreigners perceived as culturally dissimilar as toward more similar foreigners (Asbrock et al., 2014).

Previous researches (e.g. Gang et al., 2013; Ostapczuk, Musch, \& Moshagen, 2009) had showed that attitude toward foreigners were influenced by age and education: the more highly-educated and younger citizens tend to be more positive towards foreigners. Potential main reasons underlying the education effect include a different number of positive contacts with foreign people (Wagner, van Dick, Pettigrew, \& Christ, 2003) and an increased commitment to democratic norms of equality possibly associated with a higher formal education (Condran, 1979). Research had indicated that higher education is the key for decreasing attitudes towards minorities, but few studies (e.g. Kim, 2004; Sakai \& Koike, 
2011) have taken university students opinions into consideration. Due to this, university students were respondents of the present study in order to get their perspective on this matter.

In the era of globalization that accelerates personal and cultural exchanges across countries, understanding and respecting other cultures has become more important. This is true for the three Baltic countries (Kovalenko, 2010; Mensah, 2010; Leončikas \& Žibas, 20109 and Russia (Bisson, 2016) as these countries had experienced new migration views. For example, intolerance towards foreigners is a problem in three Baltic countries young people - surveys (e.g., Torney-Purta et al. 2001) suggest that in Estonia, Latvia and Lithuania adolescents hold relatively negative views towards foreigners, whereby in most of the European countries' respondents had positive attitudes about immigrants. When we look at previous studies among adults in three Baltic countries (Paas \& Halapuu, 2012) then we can identify differences in attitudes toward foreigners - Latvians and Estonians were less tolerant towards immigrants and Lithuanians were more tolerant. This rise a new research question: Is there similarities and differences between Estonian, Latvian, Lithuanian and Russian university students' conceptualization of the meaning of foreigners? The research is important because previous research (e.g. Hjerm, 2001; Ostapczik et al., 2009) had indicated that higher education is the key for influencing attitudes towards minorities.

The aim of the study was to explore how Estonia, Latvian, Lithuanian and Russian university students conceptualize the meaning of foreigners.

\section{Method}

\subsection{Samples}

Four samples of university students participated in the study: 118 Estonian (189 of them were women and 29 men), 101 Latvian (96 of them were women and 5 men), 101 Lithuanian (67 of them were women and 34 men), and 92 respondents (women 54 and 28 men). Totally, there were 412 respondents.

\subsection{Instrument}

Questionnaire consists of four open-ended questions expressing subjective feelings of acceptance and un-acceptance of foreigners in five areas: Meaning of foreigners (What do you understand by definition „, foreigners"?); reasons for acceptance of foreigners (Are you ready to accept foreigners or otherwise minded people? Why?); reasons for non-acceptance of foreigners (What would you never accept concerning with foreigners or otherwise minded people? Why?); and influence of foreigners (Have you been influenced by foreigners or otherwise minded people? How?).

Quantitative content analysis was used to code and analyze the open-ended questions answers categories. Chi-square was used to test for the differences between the three study groups responses categories calculated by percentages.

\section{Results}

The quantitative content analysis technique was used to categorize answers of open-ended question: What do you understand by definition of foreigners "? Table 1 shows findings among three samples of Baltic state university students as calculated as frequencies of key categories and between-group differences of categories analyzed by the $\chi^{2}$-test, whereby all differences were significant at $\mathrm{p}<.01$ level.

Table 1. Frequencies of key categories of descriptions of meaning of foreigners among four samples of students.

\begin{tabular}{|c|c|c|c|c|c|c|c|c|c|c|}
\hline Category & $\begin{array}{l}\text { Estonia } \\
\text { (A) }\end{array}$ & $\begin{array}{l}\text { Latvia } \\
\text { (B) }\end{array}$ & $\begin{array}{l}\text { Lithuania } \\
\text { (C) }\end{array}$ & $\begin{array}{l}\text { Russia } \\
\text { (D) }\end{array}$ & $\begin{array}{l}\chi^{2} \\
\text { (A vs.B) }\end{array}$ & $\begin{array}{l}\chi^{2} \\
\text { (A vs.C) }\end{array}$ & $\begin{array}{l}\chi^{2} \\
(\mathrm{~A} \text { vs.D) }\end{array}$ & $\begin{array}{l}\chi^{2} \\
\text { B vs.C) } \\
\end{array}$ & $\begin{array}{l}\chi^{2} \\
\text { (B vs.D) }\end{array}$ & $\begin{array}{l}\chi^{2} \\
\text { (C vs.D) }\end{array}$ \\
\hline $\begin{array}{l}\text { Different nationality } \\
\text { and language }\end{array}$ & $19 \%$ & $24 \%$ & $15 \%$ & $51 \%$ & ns & ns & 45.12 & ns & 15.29 & 28.11 \\
\hline $\begin{array}{l}\text { Different } \\
\text { citizenship or no } \\
\text { citizenship }\end{array}$ & $2 \%$ & $6 \%$ & $4 \%$ & $28 \%$ & ns & ns & 78.82 & ns & 54.78 & 56.76 \\
\hline $\begin{array}{l}\text { Poor economic } \\
\text { situation and } \\
\text { physical state }\end{array}$ & $6 \%$ & $7 \%$ & $30 \%$ & $4 \%$ & ns & 20.32 & ns & 15.03 & ns & 23.21 \\
\hline $\begin{array}{l}\text { Different attitudes } \\
\text { and values }\end{array}$ & $30 \%$ & $39 \%$ & $39 \%$ & $5 \%$ & ns & ns & 22.88 & ns & 32.98 & 33.74 \\
\hline $\begin{array}{l}\text { Marginalization of } \\
\text { people: Different } \\
\text { life style }\end{array}$ & $12 \%$ & $14 \%$ & $11 \%$ & $11 \%$ & ns & ns & ns & ns & ns & ns \\
\hline $\begin{array}{l}\text { Individualistic } \\
\text { viewpoint }\end{array}$ & $30 \%$ & $11 \%$ & $2 \%$ & $1 \%$ & 13.35 & 27.87 & 23.53 & ns & ns & ns \\
\hline
\end{tabular}


Research results showed that four samples of students conceptualize foreigners mainly in society level: (1) differences in nationality and language, and (2) marginalization, whereby Russian students empathized more differences in nationality and language, and also differences in citizenship; and three Baltic counties students evaluated more differences in attitudes and values. Lithuanian university students conceptualize foreigners more often in terms of individual physical differences between people and Estonians expressed their own individualistic personal viewpoint by separating self from other groups of people.

An analyze of reasons for acceptance of foreigners among university students reveal overwhelming positive attitude toward foreigners with regards to emplacing several cultures are enriching people (Table 2). The reasons why young people in three Baltic countries accepted foreigners were different in personal level: (1) Estonian students were more prone to accept foreigners and otherwise minded people by expressing more often the attitude that all people are equal; (2) Latvian, Lithuanian and Russian students expressed more often the opinion to accept foreigners when there is a mutual respect, common values, moral and understandings between people.

Table 2. Frequencies of key categories of descriptions of reasons of acceptance of foreigners among four samples.

\begin{tabular}{|c|c|c|c|c|c|c|c|c|c|c|}
\hline Category & $\begin{array}{l}\text { Estonia } \\
\text { (A) }\end{array}$ & $\begin{array}{l}\text { Latvia } \\
\text { (B) }\end{array}$ & $\begin{array}{l}\text { Lithuania } \\
\text { (C) }\end{array}$ & $\begin{array}{l}\text { Russia } \\
\text { (D) }\end{array}$ & $\begin{array}{l}\chi^{2} \\
\text { (A vs.B) }\end{array}$ & $\begin{array}{l}\chi^{2} \\
\text { (A vs.C) }\end{array}$ & $\begin{array}{l}\chi^{2} \\
\text { (A vs.D) }\end{array}$ & $\begin{array}{l}\chi^{2} \\
\text { B vs.C) }\end{array}$ & $\begin{array}{l}\chi^{2} \\
\text { (B vs.D) }\end{array}$ & $\begin{array}{l}\chi^{2} \\
\text { (C vs.D) }\end{array}$ \\
\hline $\begin{array}{l}\text { Acceptance: Differences } \\
\text { enrich and are } \\
\text { interesting culturally }\end{array}$ & $31 \%$ & $28 \%$ & $30 \%$ & $31 \%$ & ns & ns & ns & ns & ns & ns \\
\hline $\begin{array}{l}\text { Acceptance: When there } \\
\text { is mutual respect, } \\
\text { common values } \\
\text { understanding }\end{array}$ & $14 \%$ & $32 \%$ & $36 \%$ & $36 \%$ & 8.05 & 4.05 & 4.08 & ns & ns & ns \\
\hline $\begin{array}{l}\text { Acceptance: When there } \\
\text { is no dangerous and } \\
\text { violent behaviour }\end{array}$ & $14 \%$ & $12 \%$ & $13 \%$ & $14 \%$ & ns & ns & ns & ns & ns & ns \\
\hline $\begin{array}{l}\text { Attitude: All people are } \\
\text { equal }\end{array}$ & $47 \%$ & $20 \%$ & $21 \%$ & $19 \%$ & 26.44 & 23.29 & 32.37 & ns & ns & ns \\
\hline
\end{tabular}

The third question in questionnaire was: What would you never accept concerning with foreigners or otherwise minded people? Results of analyze of reasons for non-acceptance of foreigners among three study samples is presents in the table 3. It was revealed that foreigners were less accepted on society level being not tolerant against aggression and violence, whereby three Baltic countries respondents tolerate less discrimination and religious extremism, and Russian compliance of cultural traditions and rules.

Table 3. Frequencies of key categories of descriptions of un-acceptance of foreigners among four samples.

\begin{tabular}{|c|c|c|c|c|c|c|c|c|c|c|}
\hline Category & $\begin{array}{l}\text { Estonia } \\
\text { (A) }\end{array}$ & $\begin{array}{l}\text { Latvia } \\
\text { (B) }\end{array}$ & $\begin{array}{l}\text { Lithuania } \\
\text { (C) }\end{array}$ & $\begin{array}{l}\text { Russia } \\
\text { (D) }\end{array}$ & $\begin{array}{l}\chi^{2} \\
\text { (A vs.B) }\end{array}$ & $\begin{array}{l}\chi^{2} \\
\text { (A vs.C) }\end{array}$ & $\begin{array}{l}\chi^{2} \\
(\mathrm{~A} \text { vs.D) }\end{array}$ & $\begin{array}{l}\chi^{2} \\
\text { B vs.C) }\end{array}$ & $\begin{array}{l}\chi^{2} \\
\text { (B vs.D) }\end{array}$ & $\begin{array}{l}\chi^{2} \\
\text { (C vs.D) }\end{array}$ \\
\hline $\begin{array}{l}\text { Society level: } \\
\text { Aggression and } \\
\text { violence }\end{array}$ & $28 \%$ & $22 \%$ & $24 \%$ & $30 \%$ & ns & ns & ns & ns & ns & ns \\
\hline $\begin{array}{l}\text { Society level: } \\
\text { Discrimination and } \\
\text { extremism }\end{array}$ & $49 \%$ & $56 \%$ & $50 \%$ & $17 \%$ & ns & ns & 18.63 & ns & 6.96 & 19.39 \\
\hline $\begin{array}{l}\text { Society level: People } \\
\text { do not follow cultural } \\
\text { traditions and rules } \\
\text { Group level: Sexual } \\
\text { minorities and } \\
\text { disabled }\end{array}$ & $17 \%$ & $20 \%$ & $23 \%$ & $35 \%$ & ns & ns & 31.92 & ns & 36.73 & 27.85 \\
\hline
\end{tabular}

The last question in the questionnaire related to personal influence of foreigners: Have you been influenced by foreigners or otherwise minded people? Research result indicated that university students in four study groups had generally more positive than negative views toward foreigners connected with the influence on them, whereby negative feelings related to personal experiences about insecurity and unpredictable behavior and customs; and at the other side - foreigners as positive models as strong people who can survive and adapt in society. Reasons why foreigners can positively influence personally students were different: (1) Lithuanians emphasized more sympathy and helping behavior, (2) Estonians and Latvians more enlargement of their knowledges with an increase of tolerance towards foreigners as positive models, and (3) and Russian students' opinions were more connected with undirect positive influence by means of media, art and literature (Table 4). 
Table 4. Frequencies of key categories of personal descriptions of influence of foreigners among four samples.

\begin{tabular}{|c|c|c|c|c|c|c|c|c|c|c|}
\hline Category & $\begin{array}{l}\text { Estonia } \\
\text { (A) }\end{array}$ & $\begin{array}{l}\text { Latvia } \\
\text { (B) }\end{array}$ & $\begin{array}{l}\text { Lithuania } \\
\text { (C) }\end{array}$ & $\begin{array}{l}\text { Russia } \\
\text { (D) }\end{array}$ & $\begin{array}{l}\chi^{2} \\
\text { (A vs.B) }\end{array}$ & $\begin{array}{l}\chi^{2} \\
\text { (A vs.C) }\end{array}$ & $\begin{array}{l}\chi^{2} \\
\text { (A vs.D) }\end{array}$ & $\begin{array}{l}\chi^{2} \\
\text { B vs.C) }\end{array}$ & $\begin{array}{l}\chi^{2} \\
\text { (B vs.D) }\end{array}$ & $\begin{array}{l}\chi^{2} \\
\text { (C vs.D) }\end{array}$ \\
\hline $\begin{array}{l}\text { Positive influence: } \\
\text { Sympathy and } \\
\text { helping behaviour }\end{array}$ & $5 \%$ & $8 \%$ & $40 \%$ & $4 \%$ & ns & 16.13 & ns & 10.66 & ns & 14.82 \\
\hline $\begin{array}{l}\text { Positive model of } \\
\text { strong people }\end{array}$ & $28 \%$ & $27 \%$ & $11 \%$ & $5 \%$ & ns & ns & 9.54 & ns & 8.70 & 9.73 \\
\hline $\begin{array}{l}\text { Positive influence: } \\
\text { Experiences with } \\
\text { increase of tolerance }\end{array}$ & $31 \%$ & $30 \%$ & $10 \%$ & $10 \%$ & ns & 9.94 & 9.14 & ns & 7.51 & ns \\
\hline $\begin{array}{l}\text { Negative influence: } \\
\text { Insecurity about } \\
\text { peoples unpredictable } \\
\text { behavior and customs }\end{array}$ & $26 \%$ & $25 \%$ & $28 \%$ & $25 \%$ & ns & ns & ns & ns & ns & ns \\
\hline $\begin{array}{l}\text { Undirect positive } \\
\text { influence by music, } \\
\text { literature, movies etc }\end{array}$ & $10 \%$ & $10 \%$ & $11 \%$ & $56 \%$ & ns & ns & 37.98 & ns & 36.62 & 43.22 \\
\hline
\end{tabular}

\section{Conclusions}

This study follows a key aspect of sociocultural approaches - the focus on the situated nature of behavior and how it is socially constituted in the specific area of conceptualization of meaning of foreigners among university students. The meaning of foreigners among Estonian, Latvian, Lithuanian and Russian university students was generally conceptualized in society level as an exclusion of people with different nationality and language and marginalization of people. Braun, Behr and Kaczmirek (2013) analyzed the cross-national equivalence of the meaning of the term immigrants and found that the perception of immigrants was determined by a general representation of immigrants as well as a representation by the most dominant ethnic minority group, which differed from country to country. Also, Asbrock et al. (2014) found that the meaning of foreigners was dominantly connected with largest groups of people with migration background in the country. Our results indicated that university students conceptualized foreigners broader than ethnic minority group in society - excluded and marginalized people. Following sociocultural perspectives present study reveal that university students' understandings and interpretations of foreigners as socially contracted societal constructs differed somewhat - Russian respondents empathized more differences in citizenship and three Baltic states respondents in attitudes and values. Additionally, it was revealed that the meaning of foreigners for Estonian students was conceptualized more from individualistic, than collectivistic, perspective, supporting tendencies among young peoples' citizenship behavior (Krzywosz-Rynkiewicz et al., 2018).

All four countries university students were generally open and tolerant toward foreigners in individual differences evoked from cultural enrichment, but their attitudes showed some variation: Estonian students expressed more the attitude that people are equal; and Latvian, Lithuanian and Russian respondents were more prone to express positive attitude towards foreigners when there is mutual respect and common values. Thus, acceptance of foreigners of university students depends on the attitudes in the individual level, the unacceptance of the foreigners reflects the quality of opinions in the society level. Namely, negative attitudes toward foreigners among four groups of university students tended to be more pronounced against aggression, violence, discrimination and extremism in society, whereby the dominant reason for non-tolerance tended to be different - discrimination and extremism for three Baltic countries students, and not following of cultural traditions and rules for Russian students, showing an overall acceptances in the personal level, but un-acceptance in society level in terms of threatens of equality and security. Also, Sakai \& Koike (2015) found that university students' attitudes towards foreigners were confounding, but dominantly positive.

Although, university students tended to have tolerant attitudes towards foreigners, but ambivalent experiences concerning with influence of foreigners. Specifically, the influence of foreigners in terms of personal positive experiences for three Baltic counties students was direct and to Russian university students tended to be indirect; and at one side - negative personal experiences for most of the respondents related to insecurity evoked from peoples' unpredictable behavior and customs. Also, Kim (2004) found that college students' attitudes toward minorities were more influenced by subjective factors than by demographic characteristics and family backgrounds. Even though we analyzed data among four country university students' samples, we argue that our pilot study results are important for other countries as well, especially planning surveys for attitudes toward foreigners or immigrants, there is a challenge for specification of the meaning of main concept for the specific target group. 


\section{References}

Asbrock, F., Lemmer, G., Becker, J. C., Koller, J., \& Wagner, U. (2014). „Who are these foreigners anyway?" The content of the term foreigner and its impact on prejudice. SAGE Open, April-June, $1-8$.

Bisson, L. (2016). Russia's immigration policy: new challenges and tools. Russie.Nei.Visions, No.91. Paris: Ifri.

Braun, M., Behr, D., \& Kaczmirek, L. (2013). Assessing cross-national equivalence of measures of xenophobia: Evidence from probing in web surveys. International Journal of Public Opinion Research, 25, 383-395.

Condran, J. G. (1979). Changes in white attitudes toward blacks: 1963-1977. Public Opinion Quarterly, 43, 463-476.

Gang, I. N., Rivera-Batiz, F. L. \& Yun, M. S. (2013). Economic strain, education and attitudes towards foreigners in the European Union. Review of International Economics, 21(2), 177-190.

Gorodzeisky, A., \& Semyonov, M. (2009). Terms of exclusion: Public views toward admission and allocation of rights to immigrants in European countries. Ethnic and Racial Studies, 32, 401-423.

Hjerm, M. (2001). Education, xenophobia and nationalism: A comparative analysis. Journal of Ethnic and Migration Studies, 27(1), 37-60.

Kim, S. H. (2004). College students attitudes toward and social distance feelings toward minorities. Social Research, 1, 169-206.

Kovalenko, J. (2010). Situation of new immigrants in Estonia. In New immigrants in Estonia, Latvia and Lithuania (pp. 7-24). Tallinn, Estonia: Legal Information Centre for Human Rights.

Krzywosz-Rynkiewicz, B., Zalewska, A. M., Kõiv, K., Zuzeviciute, V., \& Vidnere, M. (2018). Young people citizenship activity in post-soviet states - comparison across countries. In: B. Krzywosz-Rynkiewicz, A. M. Zalewska, \&amp; K. J. Kennedy (Eds.). Young People and Active Citizenship in Post-Soviet Times: A Challenge for Citizenship Education (pp. 41-51). London and New York: Routledge, Taylor \& Francis Group.

Leončikas, T., \& Žibas, K. (2010). Situation of new immigrants in Lithuania. In New immigrants in Estonia, Latvia and Lithuania (pp. 41-73). Tallinn, Estonia: Legal Information Centre for Human Rights.

McLaren, L.M. (2003). Anti-immigrant prejudice in Europe: Contact, threat perception, and preferences for the exclusion of immigrants. Social Forces, 81(3), 909-36.

Mensah, P. (2010). Situation of new immigrants in Latvia. In New immigrants in Estonia, Latvia and Lithuania (pp. 25-40). Tallinn, Estonia: Legal Information Centre for Human Rights.

Organisation for Economic Co-operation and Development (OECD) (2015). International Migration Outlook, Paris: OECD Publishing.

Ostapczuk, M., Much, J., \& Moshagen, M. (2009). A randomized-response investigation of the education effect in attitudes towards foreigners. European Journal of Social Psychology, 39, 920-931.

Paas, T. \& Halapuu, V. (2012). Attitudes towards immigrants and the integration of ethnically diverse societies. Discussion Paper No. 2012-23. Tartu: NORFACE Migration.

Sakai, H., \& Koike, H. (2011). Implicitly and explicitly measured attitudes towards foreigners: A dual-process model perspective. JABAET Journal, 14/15, 39-58.

Scheepers, P., Gijberts, M., \& Coenders, M. (2002). Ethnic exclusionism in European countries: Public oppositions to civil rights for legal migrants as a response to perceived threat, European Sociological Review 18, 17-34.

Semyonov, M., Raijman, R., Gorodzeisky, A. (2008). Foreigners' impact on European societies: Public views and perceptions in a cross-national comparative perspective. International Journal of Comparative Sociology, 49, 5-29.

Torney-Purta, J., Lehmann, R., Oswald, H., \& Schulz, W. (2001). Citizenship and education in twenty-eight countries: Civic knowledge and engagement at age fourteen. Amsterdam: IEA.

Wagner, U., van Dick, R., Pettigrew, T. F., \& Christ, O. (2003). Ethnic prejudice in East and West Germany: The explanatory power of intergroup contact. Group Processes and Intergroup Relations, 6, 22-36. 


\title{
THE ROLE OF TRAIT EMOTIONAL INTELLIGENCE IN THE PROCESS OF CAREER DECISION-MAKING ${ }^{1}$
}

\author{
Eva Sollarova, \& Lada Kaliska \\ Department of Psychology, Faculty of Education, Matej Bel University in Banska Bystrica (Slovakia)
}

\begin{abstract}
The process of career decision-making is an important experience in the adolescent's period often manifested by decision-making difficulties. The study analyzes the influence of trait emotional intelligence (TEI) in the process of career decision-making, especially on career decision-making difficulties, career decidedness and career decision self-efficacy (CDSE) assessed in a sample of high school students during their $2^{\text {nd }}$ career choice orientation testing. TEI as defined by Petrides (2009) and CDSE as defined by Betz et al. (1996), representing more stable personality-related variables, were studied in the context of career decision-making covering both more stable variables (emotional and personality-related aspects of career decision-making difficulties as specified by Saka, Gati \& Kelly, 2008) and more developmental variables (career decidedness as defined by Lounsbury \& Gibson, 2011). The study aims at the trait EI prediction level (as a personality trait) assessed by Trait Emotional Intelligence Questionnaire - Short Form (TEIQue-SF) created by Petrides (2009) adapted to the Slovak conditions by Kaliská, Nábělková and Salbot (2015) of career decidedness and career decision self-efficacy above the career decision-making difficulties by correlation and regression analysis. Career decision-making difficulties were assessed by the Emotional and Personality Career Decision-Making Difficulties Scale (EPCD; Saka, Gati \& Kelly, 2008), career decidedness was assessed by Lounsbury and Gibson's Career Decidedness Scale (CDS, 2011), career decision self-efficacy by the Career Decision Self-Efficacy Scale-Short Form (CDSES-SF, Betz et al., 1996). The research sample consisted of 322 high school students (average age: 17.7 /SD=.46/; 58.4\% of females). Results proved that high trait EI is significantly positively correlated to decidedness, to career decision self-efficacy, and significantly negatively to all the factors and global level of career decision-making difficulties. Trait EI significantly predicts career difficulties over and above career decidedness and career decision self-efficacy. The results empirically support the relevance of both trait EI and career decision self-efficacy in the career decision process, with practical implications for diagnostics and intervention within career counseling, as a means to strengthen the effectiveness of the process of career decision making in career choice and in career development.
\end{abstract}

Keywords: Trait emotional intelligence, career indecision, career decidedness, career decision-making difficulties, career decision self-efficacy.

\section{Introduction}

Career decision-making is typically a stressful experience, often manifested by decision-making difficulties. It is especially important at the end of adolescence period when high school students face challenges to make a choice regarding their future studies or a work profession. Career decision-making combined with personality variables is a well-researched empirical area. However, there is a place for further exploration by incorporating the emotional intelligence (EI) construct in relation to career decision-making constructs. The relationships between EI and career decision-making is important to be explored, as the resulting information can contribute to both research-based knowledge for vocational psychology and to career guidance practice.

Career indecision is defined as difficulties encountered by individuals while making career-related decisions and refers to all problems and challenges that need to be addressed prior to, during, or after the decision-making process (Saka, Gati, \& Kelly, 2008, 403). It can be differentiated between temporary, developmental indecision on one side and more pervasive, chronic indecisiveness derived predominantly from personality and emotional factors. Saka, Gati and Kelly (2008) developed a theoretical framework for analysing the emotional and personality-related aspects of career decision-making difficulties. They proposed a hierarchical taxonomy with three major clusters of difficulties - pessimistic views, anxiety, and self-concept and identity - that are then subdivided into 11

${ }^{1}$ The research was supported by Scientific Grant Agency in Slovakia, grant project VEGA No. 1/0654/17. 
specific categories based on finer distinctions. The first major cluster - pessimistic views - refers to negative cognitive biases and perceptions. The second major cluster is defined as anxiety refering to the possible effects of anxiety on specific aspects of decision-making process. The third major cluster is labelled as self-concept and identity, which refers to developmental personality aspects of the individual. Based on the proposed model, the authors developed the Emotional and Personality Career Difficulties Scale $(E P C D)$ and empirically verified the above-mentioned model. It has been verified within the Slovak context as well (Sollárová, 2016; Sollárová \& Kaliská, 2018).

The literature reveals a growing interest in studying individual variables associated with the career decision-making process. In the search for variables that potentially affect the career decision-making process, the importance of self-perceptions, or how the individual views himself or herself, has emerged as a recent focus of research. Bandura's concept of self-efficacy was integrated into the career decision-making process by Hacket and Betz (1981) and defined "career decision self-efficacy" as an individual's belief that s/he is capable of successfully completing tasks and specific behaviors required in career decision-making (Taylor \& Betz, 1983). A meta-analysis by Choi et al. (2012) investigated the relationship between career decision-making self-efficacy and a selection of related variables including gender, age, race, self-esteem, vocational identity, career barriers, peer support, vocational outcome expectations, and career indecision. Career indecision was found to have a strong negative correlation with career decision self-efficacy.

Apart from personality traits, the specific role of career decision-making process is generally recognized and agreed upon among researchers (Martincin \& Stead, 2015), emotional intelligence (EI) represents an additional potentially critical variable in the career decision-making process (Di Fabio \& Palazzeschi, 2009), yet being rarely studied. In studying the role of EI in career decision-making difficulties, Mayer-Salovey's ability-based model and Bar On's model as the so-called mixed model linking EI with personality and abilities, have been mostly investigated (Di Fabio, \& Palazzeschi, 2009; Di Fabio, Palazzeschi, \& Bar-On, 2012). The studies indicate that EI is inversely associated with decision-making difficulties and that EI also explains a significant percentage of the incremental variance when compared with personality traits in explaining the impact on the career decision-making difficulties involved in Gati's model (Di Fabio, \& Palazzeschi, 2009). Research exploring the relations between EI and career difficulties is still limited, especially the relations between trait EI models (e.g., Schutte or Petrides' model). Thus, investigating a role of trait EI as another EI model offers a new research opportunity. Trait EI, investigated in this study, is explained by its author, Petrides (2009), as a constellation of emotion-related self-perceptions and dispositions located at the lower levels of personality hierarchies. The model consists of 15 facets (13 of them forming 4 factors: emotionality, sociability, well-being and self-control and 2 independent facets stand by themselves) forming the global level of trait EI (more detailed characteristics of the factors in Petrides, 2009; Kaliská \& Nábělková, 2015). Petrides also created questionnaires to measure trait EI (Trait Emotional Intelligence Questionnaire - TEIQue) for three developmental stages (children, adolescents, and adults) of two forms (short and long form), adapted in Slovakia by Kaliská, Nábělková, \& Salbot (2015).

There have been conducted very few investigations on EI in relation to career indecision, specifically analyzing the relationship between trait EI based on Petrides' model and emotional and personality-related aspects of decision-making difficulties based on Saka, Gati, and Kelly's model (2008). The first attempt of the authors (Sollárová, \& Kaliská, 2018) showed significant positive, though weak, relationships between global trait EI level and career decidedness, however significant negative moderate to strong relationship between global trait EI level and global career decision-making difficulties as well as its three factors (especially factor of self-concept and identity) in the sample of 156 Slovak high school students. Trait EI predicted a significant almost $7 \%$ of unique variance in career decision-making difficulties after controlling for decidedness level with remaining significant negative moderate correlation.

\section{Objectives}

The present study aims to analyse the relationship between emotional intelligence, career decidedness, career decision self-efficacy and career decision-making difficulties. More succinctly, the purpose of the study is to verify whether EI can explain a significant percentage of incremental variance compared with career decidedness and career decision self-efficacy in explaining career decision-making difficulties. We questioned the following:

First, what is the relationship between trait emotional intelligence, career decidedness, career decision self-efficacy and career decision-making difficulties? Based on our reading of the literature we hypothesized that

(H1) individuals with higher trait emotional intelligence scores would display higher levels of career decidedness.

(H2) individuals with higher trait emotional intelligence scores would display higher levels of career decision-making self-efficacy. 
(H3) individuals with higher trait emotional intelligence scores would display lower levels of career decision-making difficulties.

Second, what clusters of career decision-making difficulties as defined by Saka, Gati, and Kelly's model (2008) of emotional and personality-related decision-making difficulties are most associated with total trait emotional intelligence as defined by Petrides?

Third, can trait emotional intelligence predict a significant percentage of incremental variance above career decidedness and career decision self-efficacy in explaining career decision-making difficulties? We hypothesized that (H4) trait emotional intelligence will explain a significant percentage of incremental variance compared with career decidedness and career decision-making self-efficacy in predicting the career decision-making difficulties.

\section{Methods}

Research sample. The research sample consisted of 322 high school students (average age: 17.7 /SD=.46/; 58.4\% of females) from three high schools of the central Slovak region. The research sample was obtained by targeted and occasional sampling as a part of professional orientation testing. Either the parental or individual (18-year-old ones do not need parental approval) informed consent was signed voluntarily two weeks before testing.

Research methods. Trait EI was assessed by the short Slovak version of the Trait Emotional Intelligence Questionnaire-Short Form (TEIQue-SF, Kaliská, Nábělková, \& Salbot, 2015) created by Petrides (2009). The instrument consists of 30 items answered by a seven-point Likert scale (1 - completely disagree to 7 - completely agree), a higher rating indicate a higher level of TEI; Cronbach a was .83.

To evaluate career decision-making difficulties, participants responded to the Slovak version of the Emotional and Personality Career Difficulties Scale (EPCD, Saka, Gati, \& Kelly, 2008). The scale consists of 53 items, each item representing one of the 11 difficulty categories, answered on a 9-point scale (1 - does not describe me to 9 - describes me well), a higher rating indicates a higher level of difficulty. The total score and the sum from the three subscales were calculated. Cronbach $\alpha$ was 0.945 .

To evaluate career in/decidedness, the Career Decidedness Scale (CDS; Lounsbury, \& Gibson, 2011) was used. It is a 5-item one-dimenzional scale with the score range from 6 to 30, a higher score indicates a higher decidedness. Cronbach $\alpha$ was .949.

Career decision self-efficacy was assessed by The Career Decision Self-Efficacy Scale - Short Form (CDSE-SF; Betz, Klein, \& Taylor, 1996). The scale includes 25 items divided by five scales, namely, accurate self-appraisal, gathering occupational information, goal selection, making plans for the future, and problem solving. The answers were obtained using a scale with five alternatives, ranging from $1=$ not al all confident to $5=$ totally confident. Higher scores indicated higher levels of career decision self-efficacy. Cronbach $\alpha$ was .907.

Data analysis. Descriptive statistics, Pearson $\mathrm{r}$ correlations, and a hierarchical regression analysis were performed.

\section{Results}

The basic descriptive indicators for global trait EI assessed by TEIQue-SF questionnaire, for three main factors and global level of career difficulties assessed by EPCD, the level of decidedness by CDS and career decision self-efficacy by CDSE-SF of our research sample are presented in Table 1.

Table 1. Descriptive indicators of all variables in a sample of the Slovak adolescents $(N=322)$.

\begin{tabular}{|c|c|c|c|c|c|c|c|c|}
\hline & & Min & Max & $\mathrm{AM}$ & SD & Skewness & Kurtosis & $\alpha$ \\
\hline TEIQue-SF & Global Trait EI & 2.47 & 6.63 & 4.83 & .72 & -.473 & .345 & .853 \\
\hline \multirow{4}{*}{ EPCD } & Pessimistic views & 1.50 & 7.33 & 4.89 & 1.01 & -.253 & .117 & \multirow{4}{*}{.944} \\
\hline & Anxiety & 1.19 & 9.00 & 5.32 & 1.87 & -.338 & -.673 & \\
\hline & Self-concept and Identity & 1.00 & 7.94 & 4.19 & 1.34 & .119 & -.357 & \\
\hline & $\begin{array}{l}\text { Global Career } \\
\underline{\text { Difficulties }}\end{array}$ & 1.79 & 7.89 & 4.89 & 1.22 & -.280 & -.379 & \\
\hline CDS & Decidedness & 1.00 & 5.00 & 2.68 & 1.12 & .420 & -.878 & .940 \\
\hline CDSE & $\begin{array}{l}\text { Career Decision Making } \\
\text { Self-efficacy }\end{array}$ & 1.84 & 4.88 & 3.47 & .59 & -.075 & -.308 & .907 \\
\hline
\end{tabular}

We can also conclude all of the observed inner consistencies of the instruments used estimated by Cronbach's alpha coefficients reach acceptable values. Statistical analysis of skewness and kurtosis of the variables proves the normal distribution of the analyzed variables therefore the relation estimate was 
carried out using parametric correlation analysis. Pearson's correlation coefficient (r) enables to determine the direction and strength of relations between variables presented in Table 2.

Table 2. Correlation analysis of the variables $(N=322)$.

\begin{tabular}{|c|c|c|c|c|c|c|c|}
\hline & & 2 & 3 & 4 & 5 & 6 & 7 \\
\hline TEIQue-SF & 1 Global Trait EI & $-.307^{* * * *}$ & $-.343^{* * *}$ & $-.608^{* * *}$ & $-.476^{* * *}$ & $.247^{* * *}$ & $.552^{\text {***** }}$ \\
\hline \multirow{4}{*}{ EPCD } & 2 Pesimistic views & 1.00 & $.547^{* * *}$ & $.472^{* * *}$ & $.682^{* * *}$ & $-.426^{* * *}$ & $-.343^{* * *}$ \\
\hline & 3 Anxiety & & 1.00 & $.683^{* * *}$ & $.946^{* * *}$ & $-.766^{* * *}$ & $-.536^{* * *}$ \\
\hline & $\begin{array}{l}4 \text { Self-concept and } \\
\text { Identity }\end{array}$ & & & 1.00 & $.853^{* * *}$ & $-.559^{* * *}$ & $-.598^{* * * *}$ \\
\hline & $\begin{array}{l}5 \text { Global Career } \\
\underline{\text { Difficulties }}\end{array}$ & & & & 1.00 & $-.738^{* * *}$ & $-.595^{* * *}$ \\
\hline CDS & 6 Decidedness & & & & & 1.00 & $.526^{* * *}$ \\
\hline CDSE & $\begin{array}{l}7 \text { Career Decision Making } \\
\text { Self-efficacy }\end{array}$ & & & & & & 1.00 \\
\hline
\end{tabular}

Referring to the correlation analysis it can be stated that global level of trait EI was negatively correlated to all the scales and global level of career difficulties (supporting H3). The strongest negative and significant correlation was between global level of trait EI and EPCD cluster of Self-Concept and Identity within career decision-making difficulties (Q2). The global trait EI is in (positive) weak significant relation to the level of decidedness as hypothesized in $\mathrm{H} 1$, and in positive significant strong relation to career decision-making self-efficacy supporting the hypothesis $\mathrm{H} 2$.

Further on there was a hierarchical two-step regression analysis conducted to determine if global level of the career difficulties as dependent variable could be predicted by the decidedness, career decision-making self-efficacy level and global trait EI level to support also the incremental validity of trait EI. The results are presented in Table 3.

Table 3. Hierarchical regression analysis.

\begin{tabular}{|c|c|c|c|}
\hline & \multicolumn{3}{|c|}{ Career Difficulties } \\
\hline & $\begin{array}{l}\text { Step } 1 \\
\text { Step } 2 \\
\text { Step } 3\end{array}$ & $\begin{array}{r}\mathrm{F} \\
\mathrm{F}_{\text {change }}(2,3 \\
\mathrm{F}_{\text {change }}(3,3\end{array}$ & $\begin{array}{l}29^{* * *}, \mathrm{R}^{2} \text { adj. }=.545 \\
\mathrm{R}^{2} \text { adj. }=.606, \mathrm{R}^{2} \text { change }=.062 \\
\mathbf{R}^{\mathbf{2}} \text { adj. }=\mathbf{= . 6 4 9}, \mathbf{R}_{\text {change }}=\mathbf{. 0 4 4}\end{array}$ \\
\hline & Beta & $\mathrm{t}$ & Partial correlations \\
\hline $\begin{array}{l}\text { Decidedness } \\
\text { (Step 1) }\end{array}$ & -.739 & $-19.176^{* * *}$ & -.739 \\
\hline $\begin{array}{l}\text { Decidedness } \\
\text { Career Decision Making }\end{array}$ & -.586 & $-13.887^{* * *}$ & -.623 \\
\hline $\begin{array}{l}\text { Self-efficacy } \\
\text { (Step 2) }\end{array}$ & -.292 & $-6.915^{* * *}$ & -.369 \\
\hline Decidedness & -.604 & $-15.126^{* * * *}$ & -.656 \\
\hline $\begin{array}{l}\text { Career Decision Making } \\
\text { Self-efficacy }\end{array}$ & -.142 & $-3.051^{* *}$ & -.173 \\
\hline $\begin{array}{l}\text { Trait EI } \\
\text { (Step 3) }\end{array}$ & -.253 & $-6.211^{* * *}$ & -.336 \\
\hline
\end{tabular}

A three-step hierarchical regression was performed to investigate the prediction potential of trait EI of career decision-making difficulties level and at the same time to prove the incremental influence of trait EI over and above the decidedness and career decision-making self-efficacy level. The decidedness was entered at step 1, decidedness and career decision-making self-efficacy at step 2 and trait EI was added on its own at step 3. At step 1, the model was statistically significant and decidedness level predicted almost $55 \%$ of the variance in career difficulties level. Then at step 2, the career decision-making self-efficacy was added, and the model remained statistically significant predicting 61\% of the variance in career difficulties level. And as the last step, the trait EI was entered on its own. The model was again found to be significant, and trait EI remained a significant negative predictor of career decision-making difficulties, over and above decidedness and career decision-making self-efficacy level (H3). Trait EI predicted a significant almost $4 \%$ of unique variance in career decision-making difficulties after controlling for decidedness and career decision-making self-efficacy level supporting incremental validity of trait EI with remaining partial medium strength correlation of $r=-.336$. 


\section{Discussion}

Relationships between global trait emotional intelligence (TEI) as measured by the Petrides' TEIQue-SF (2009), career decidedness as measured by Lounsbury and Gibson's CDS (2011), career decision self-efficacy by Betz et al.'s $C D S E S$-SF and career decision-making difficulties as measured by Saka, Gati and Kelly's EPDS scale were investigated in a population of the Slovak high school students.

As expected ( $\mathrm{H} 1$ and $\mathrm{H} 2$ ), individuals who showed higher global trait emotional intelligence displayed higher career decidedness and higher career decision-making self-efficacy level. The result supports our previous findings (Sollárová, 2016; Sollárová \& Kaliská, 2018) where the level of emotional and personality-related aspects of career decision-making difficulties was differentiated by the level of undecidedness of high school and university students where students with higher level of undecidedness (related to their study and profession choice) showed higher level of career decision-making difficulties.

As expected (H3), individuals who showed higher global trait emotional intelligence level displayed less career decision-making difficultes, both in overall difficulties and in all three areas of difficulties as defined in the model by Saka, Gati, and Kelly (2008). The strongest negative and significant correlation was found between global level of trait EI and the third major area of difficulties defined as "self-concept and identity" (Q2).

Prediction of the emotional and personality-related career decision-making difficulties by the decidedness and career decision-making self-efficacy level and over and above by the global trait EI level (H4) was investigated and examined by means of hierarchical regression analysis. Our research supported that global trait EI significantly predicted higher level of career decidedness and career decision self-efficacy and lower level of emotional and personality-related career-decision-making difficulties, over and above the decidedness and career decision self-efficacy level. The findings support the role of trait EI as a predictor of also more stable, pervasive emotional and personality-related aspects of career decision-making difficulties (as defined in the model by Saka, Gati, \& Kelly, 2008), above and over studied career-related construct of self-efficacy. The results also empirically support the relevance of both trait EI and self-efficacy (generalized and career decision) in the career decision process, with practical implications for diagnostics and intervention within career counseling, as a means to strengthen the effectiveness of the process of career decision making in career choice and in career development.

\section{References}

Betz, N. E., Klein, K. L., \& Taylor, K. M. (1996). Evaluation of a short-form of the Career Decision-Making Self-Efficay Scale. Journal of Career Assessment, 4, 413-428.

Choi, B. Y., Park, H., Yang, E., Lee, S. K., Lee, Y., \& Lee, S. M. (2012). Understanding career decision self-efficacy. A meta-analytic approach. Journal of Career Development, 39, 443-460.

Di Fabio, A., \& Palazzeschi, L. (2009). Emotional intelligence, personality traits and career decision difficulties. International Journal for Educational and Vocational Guidance (9), 135-146.

Di Fabio, A., Palazzeschi, L., \& Bar-On, R. (2012). The role of personality traits, core self-evaluation, and emotional intelligence in career decision-making difficulties. Journal of employment counseling (49), 118- 129.

Hacket, G., \& Betz, N. E. (1981). A self-efficay approach to the career development of women. Journal of Vocational Behavior, 18, 326-339.

Kaliská, L., \& Nábělková, E. (2015). Psychometrické vlastnosti a slovenské normy Dotazníkov črtovej emocionálnej inteligencie pre deti, adolescentov a dospelých. Banská Bystrica: Belianum.

Kaliská, L., Nábělková, E., \& Salbot, V. (2015). Dotazníky črtovej emocionálnej inteligencie TEIQue-SF/TEIQue-CSF: manuál k skráteným formám. Banská Bystrica: Belianum.

Lounsbury, J. W., \& Gibson, L. W. (2011). Career Decidedness Scale. Knoxville, TN USA, Resource Associates, Inc.

Martincin, K. M., \& Stead, G. B. (2015). Five-Factor Model and Difficulties in Career Decision Making: A Meta-Analysis. Journal of Career Assessment, 23(1), 3-18.

Petrides, K.V. (2009). Trait Emotional Intelligence Questionnaire (TEIQue). Technical Manual. London: London Psychometric Laboratory.

Saka, N., Gati, I., \& Kelly, K. R. (2008). Emotional and Personality-Related Aspects of Career-Decision-Making Difficulties. Journal of Career Assessment 16(4), 403-424.

Sollárová, E. (2016). Ťažkosti v kariérovom rozhodovaní pred a po druhej smerovej vol'be. Rozhodovanie $v$ kontexte kognície, osobnosti a emócií V., Nitra, UKF, 20-37.

Sollárová, E. \& Kaliská, L. (2018). Trait emotional intelligence as a predictor of career indecision. Psychological applications and trends 2018: InPACT 2018. Lisbon: InScience Press, 102-106.

Taylor, K.M., \& Betz, N.E. (1983). Applications of self-efficacy theory to the understanding and treatment of career indecisiion. Journal of Vocational Behavior, 22, 63-81. 


\title{
ESTONIAN AND FINNISH STUDENTS' EXPERIENCES ABOUT INTERNATIONAL CAREER GUIDANCE E-LEARNING COURSE
}

\author{
Kristi Kõiv ${ }^{1}$, Ari Jussila ${ }^{2}$, Outi Rantanen ${ }^{2}$, \& Triin Peitel $^{1}$ \\ ${ }^{1}$ University of Tartu, Institute of Education (Estonia) \\ ${ }^{2}$ Tampere University of Applied Sciences, School of Professional Techer Education, (Finland)
}

\begin{abstract}
The present study consists of a need for assessment of Estonian and Finnish university students' experiences of developed common career guidance international course as a part of teacher training programs in two higher education institutions - University of Tartu and Tampere University of Applied Sciences. The research was based on a survey developed on small sample of Estonian $(\mathrm{N}=19)$ and Finnish $(\mathrm{N}=9)$ students who participated in integrated e-learning platform lasting one academic semester. At the end of the course the collected data had been both quantitative and quantitative. The self-reported questionnaire for students consists of 13 questions about cognitive and learning skills concerning with participating in career guidance course; and for the qualitative approach as the concept mapping about the course as a base of data of quantitative content analysis. Results showed that the top learning experiences used during course by participants were collaborative work skills in international learning context and cognitive skills in the area of critical thinking as analyzing and evaluation of knowledges' in career guidance. Empirical findings provide evidence that course was suitable at enhancing students' academic skills and experiences.
\end{abstract}

Keywords: Career guidance, university students, one-group posttest research design.

\section{Introduction}

Career courses and other career interventions have an important role in preparing students for transitions throughout lifelong learning (Reardon, Lenz, Sampson, \& Peterson, 2017). Previous research had documented the efficacy of a career course as an intervention for university students. For example, reduction of participants negative traits (Oliver \& Spokane, 1988) and negative career thoughts (Osborn, Howard, \& Leierer, 2007; Reed, Reardon, Lenz, \& Leierer, 2001); increasement of positive traits (Peng, 2001) and self-concept (Carver \& Smart, 1985); and development of career development processes and career maturity (Clark \& Wayment, 2017; Freeman, Lenz, \& Reardon, 2017).

Understanding how university students learn about various aspects of career guidance course will also be important to analyze and consider how best to infuse curricula with information about career development. In the current study, the development and implementation of new common international career guidance course and the assessment of university students experiences about the targeted course took place after fulfillment this course. A research question was evoked: Do common international career guidance e-learning course is beneficial for Estonian and Finnish students' cognitive and learning skills?

The aim of this work was twofold: (1) to develop and implement a common international study course in the area of career guidance at two universities in Estonia and Finland; and (2) to describe participants' self-reported learning experiences after the end of this integrated e-learning course in two areas: learning experiences and academic (cognitive and learning) skills.

\section{Method}

\subsection{International career guidance e-learning course}

The developed common international e-learning course in the area of career guidance was a regular part of both participating countries' universities teacher training curriculum. The two credit point course addresses to (1) encourage and build understanding of the international context of career guidance, 
(2) identify cultural concepts and presumptions in the field of career guidance, and (3) familiarize learners to how and on what basis education and career guidance systems process in two participating countries. During 11-week semester the course exposes the target audience masters' degree students to a logical progression of topics intended to build knowledge's, skills and attitudes in the area career guidance systems in two targeted countries as a basis of international contexts. Each week's module requires students to study a topic including self-directed research, develop of personal reflections, engage with peers in online discussion boards, prepare groupwork and video-presentations.

At the end of the course, students were able to describe and compare the main aspects of their own and neighboring country's career system from international perspective, whereby the competence in understanding and applying concepts were measured through tests and completed homework assignments.

\subsection{Participants}

The research was conducted on a sample of all 28 participants of international career guidance course, with ages ranging between 21-33 years $(\mathrm{M}=25.3, \mathrm{SD}=1.2)$. All participants were teacher training students at University of Tartu $(\mathrm{N}=19)$ and at Tampere University of Applied Sciences $(\mathrm{N}=9)$. Participation in the investigation was voluntary and anonymous, data confidentiality and other ethical aspects were assured.

\subsection{Study design and instruments}

A one group post-test pilot study design was used, and Estonian and Finnish university students completed post-questionnaires in the 11th weeks of their respective course. The self-reported questionnaire consists of 13 questions as short descriptions of two areas of academic skills. The investigation themes include: Cognitive skills (based on the typology: analyzing, evaluation, applying, understanding, remembering, and creating: Anderson \& Krathwohl, 2000) and learning skills (active learning, enjoyment of learning, group work, meaningful learning, intrinsic motivation, performance, and problem solving). This process resulted in a list with 13 single-answer items with a three-point scale, where students had to rate their level of agreement: Completely (3) Partly (2) and Not at all (1) with answering the question: What kind of skills I had during my studies in the area of the international course?

For the qualitative approach the concept mapping methodology was used in this study to answer the question: What kind of experiences I had during my studies in the area of the international course? with use of computer drawing program or free-hand drawings. Concept mapping as a research method was used to assess participants' experiences of a topic and data were analyzed using a quantitative content analysis.

\section{Results}

Students self-reported data of the pilot study showed that international career guidance courses had developed their cognitive skills connected with analyzing and evaluation of information as two aspects of critical thinking, whereby the same tendency was revealed analyzing qualitative data of the study (Figure 1, Table 1). In addition, significant difference using by t-tests were found at the level of 0.05 between the mean scores of students self-reported cognitive skills comparing analyzing and evaluation with remembering and creating (accordingly: $t=1.75 ; t=2.24 ; t=1.72 ; t=2.44$ ), showing that students evaluated their cognitive skills in the area of analyzing and evaluation higher than remembering and creating.

Also, group work and active learning skills were more highly evaluated by university students than self-assessment skills and intrinsic motivation (accordingly: $t=2.83, \mathrm{p}<.01 ; t=1.83, \mathrm{p}<.05 ; t=2.80$, $\mathrm{p}<.01 ; t=1.72, \mathrm{p}<.05$ ), whereby other learning skills - meaningful learning, enjoyment of learning, problem solving and performance skills, were moderately evaluated abilities (Figure 1). 
Figure 1. Course participants self-reported learning and cognitive skills (means).

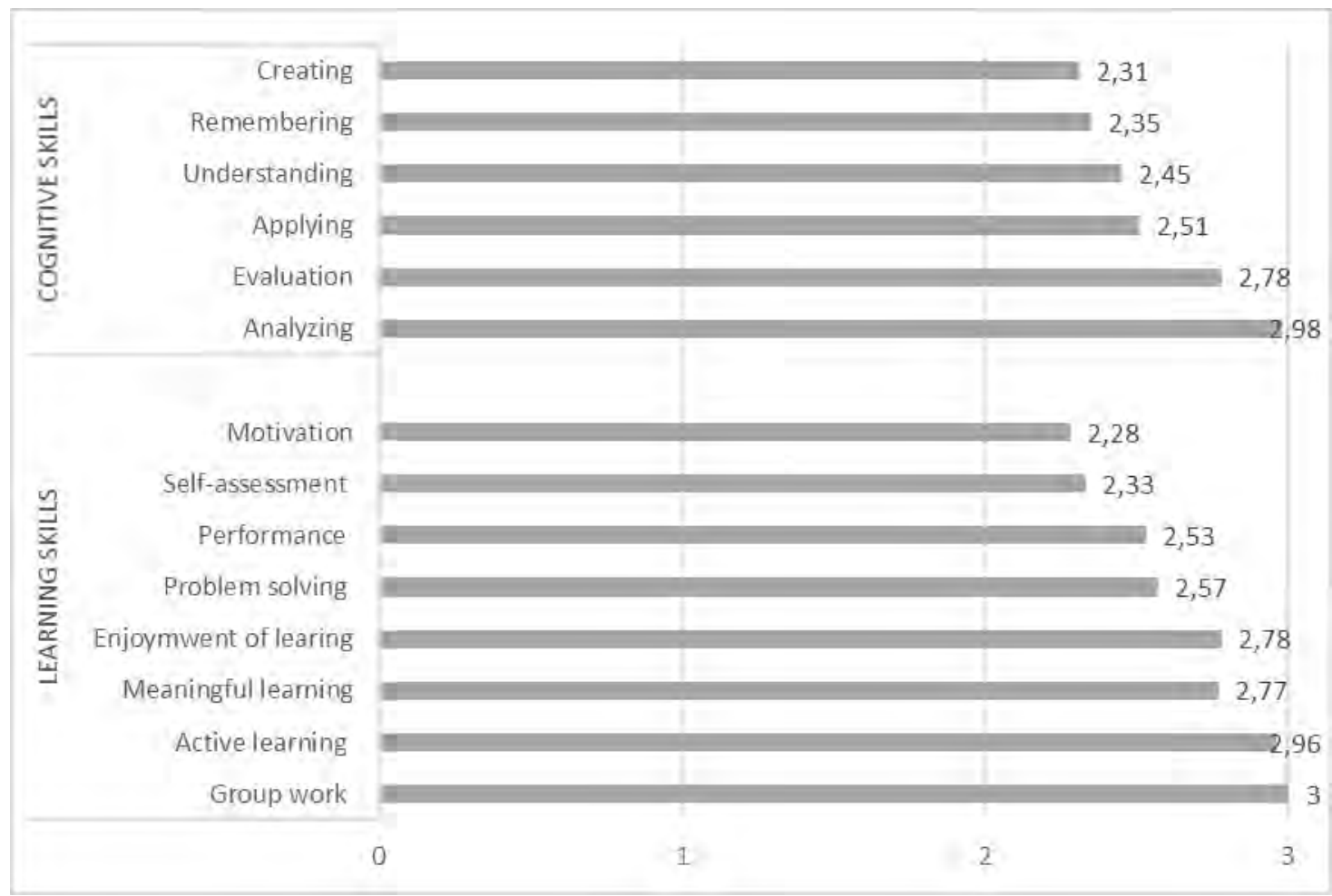

Table 1. Categories and subcategories of participants experiences about international common career guidance course.

\begin{tabular}{lll}
\hline Category & Subcategory & $\begin{array}{c}\text { Frequency } \\
\mathbf{\%}\end{array}$ \\
\hline Cognitive competence & & $21 \%$ \\
& Analyzing & $6 \%$ \\
& Evaluation and synthesis & $5 \%$ \\
& Applying & $4 \%$ \\
& Understanding & $3 \%$ \\
& Remembering & $2 \%$ \\
& Creation & $1 \%$ \\
Learning skills & & $17 \%$ \\
& Active learning & $13 \%$ \\
& Passive learning & $4 \%$ \\
Professional and identity & & $28 \%$ \\
& & $15 \%$ \\
& Development of collaborative learning & $8 \%$ \\
& Accept of different viewpoints and common values & $5 \%$ \\
& Valuing of learning experiences & $9 \%$ \\
& & $6 \%$ \\
& & $3 \%$ \\
& Development of professional identity & $25 \%$ \\
& Self-assessment and reflection & $19 \%$ \\
& & $4 \%$ \\
& Rich/good international learning experience & $1 \%$ \\
& Common learning goals & $1 \%$ \\
\hline
\end{tabular}

Results from post-course qualitative data gathered by concept mapping showed that course participants' experiences were frequently connected with development of their social and emotional learning skills, especially with collaborative learning and active learning experiences with possibilities for value education - internalizing values. Also created good and rich international learning environment with common study aims was empathized by participants, but relatively lesser were expressed skills connected with development of professional identity and self-reflection/assessment (Table 1). 


\section{Conclusions}

The most relevant results of the study show that content of the course was highly evaluated as: (1) collaborative and active learning context with perceived possibilities for development of professional identity development; (2) high level academic cognitive skills of analyzing and evaluating information as components of critical thinking; and (3) possibilities for development of social and emotional learning skills (e.g. internalizing values). In contrast, intrinsic learning motivation and course flexibility in the area of practical orientation with possibilities for deeper self-assessment and reflection was perceived as critical factors for future development of the course.

In conclusion, findings of the pilot study imply preliminary results that the developed and applied international career guidance e-learning course as a part of regular teacher training curriculum at the higher education was suitable learning context for enriching participants' multi-dimensional academic skills and experiences in the area of career guidance.

\section{References}

Anderson, L. W., \& Krathwohl, D. R., et al (2000) A Taxonomy for Learning, Teaching, and Assessing: A Revision of Bloom's Taxonomy of Educational Objectives. New York: Allyn \& Bacon.

Carver, D. S., \& Smart, D. W. (1985). The effects of a career and self-exploration course for undecided freshmen. Journal of College Student Personnel, 26, 37-43.

Clark, T. S. C., \& Wayment, H. A. (2017). Integrating online career-development skills in a management course: Findings of increased career confidence and adult development. Journal of the Academy of Business Education, 18, 68-81.

Freeman, V. F., Lenz, J. G., \& Reardon, R. (2017). Career Course Impact on College Students' Career Decision and Affective States. VISTAS Online, 1-13.

Osborn, D. S., Howard, D. K., \& Leierer, S. J. (2007). The effect of a career development course on the dysfunctional career thoughts of racially and ethnically diverse college freshmen. The Career Development Quarterly, 55, 365-377.

Oliver, L. W., \& Spokane, A. R. (1988). Career-intervention outcome: What contributes to client gain? Journal of Counseling Psychology, 35, 447-462.

Peng, H. (2001). Comparing the effectiveness of two different career education courses on career decidedness for college freshmen: An exploratory study. Journal of Career Development, 28, 29-41.

Reardon, R. C., Lenz, J. G., Sampson, J. P., Jr., \& Peterson, G. W. (2017). Career development and planning: A comprehensive approach. Dubuque, IA: Kendall Hunt.

Reed, C. A., Reardon, R. C, Lenz, J. G., \& Leierer, S. J. (2001). A cognitive career course: From theory to practice. The Career Development Quarterly, 50, 158-167. 


\title{
THE RELATIONSHIP BETWEEN LEARNING STYLE AND ANXIETY AMONG JAPANESE UNDERGRADUATES
}

\author{
Michiko Toyama, \& Yoshitaka Yamazaki \\ Department of Business Administration, Bunkyo University (Japan)
}

\begin{abstract}
This study examined the relationship between learning style and anxiety among university freshmen, controlling for gender. Although many studies on learning style have been conducted since the 1960s, relatively little research has highlighted an association between learning style and anxiety. Particularly, there is limited information on how learning style relates to anxiety at a time when students experience the important transition from high school to university. This study sought to fill this gap.

Participants of the study consisted of 194 freshmen of a Japanese university located near Tokyo. The third version of Kolb's Learning Style Inventory was used to identify students' learning style, while the State-Trait Anxiety Inventory (STAI) developed by Spielberger was employed to examine the level of state and trait anxiety. Data were collected in a required course and analyzed using two-way analysis of variance (i.e., learning style and gender). In our sample, 71 students had an Accommodating learning style; 69, Diverging; 41, Assimilating; and 13, Converging. Thus, as a whole, Japanese freshmen were more inclined towards a feeling than a thinking learning orientation. The study included 143 men and 51 women.

Analysis of variance results revealed that the four learning styles significantly differed in both state and trait anxiety variables. However, there was an insignificant difference in both anxiety types between male and female students. Additionally, there was no interaction effect of learning style and gender in terms of both types of anxiety. The Tukey post hoc test showed that the Diverging learning style had a significantly higher level of state anxiety than the Accommodating style and Converging style. Also, the Diverging style had a significantly higher level of trait anxiety than the Accommodating style and a marginally higher level than the Converging style. These results suggest that regardless of gender, freshmen with a Diverging learning style, compared with other learning styles, tend to have the highest level of state and trait anxiety during this important academic transition.
\end{abstract}

Keywords: Learning style, state anxiety, trait anxiety, Japanese undergraduate students.

\section{Introduction}

Freshmen encounter psychological challenges when beginning their university life (Basco \& Olea, 2013; Clinciu, 2013; Pancer, Hunsberger, Pratt, \& Alisat, 2000). One such challenge, student anxiety as a negative emotional experience derived from the unfamiliar university environment, leads to adverse consequences (Von Ah, Ebert, Ngamvitroj, Park, \& Kang, 2004) such as poor academic adjustment and performance (Levitz \& Noel, 1989; Saklofske, Austin, Mastoras, Beaton, \& Osborne, 2012), mental and physical illness (Boery et al., 2017), and even dropout (Clinciu, 2013). Research on efforts to alleviate freshman anxiety has reported the effectiveness of social support (Compas, Wagner, Slavin, \& Vannatta, 1986; Sato et al., 2017), which includes institutional aids and school counselors. Although the effect of freshman anxiety as well as methods to reduce it have been investigated, little research has examined the relationship between freshman anxiety and individual differences - especially learning style. Using a sample of university students in Jordan, Kadiem and Hamzah (2004) documented the association of gender, personality, and trait-anxiety with learning styles, but their study did not highlight the context of transition to university. Based on studies performed by Spielberger (1972) and colleagues (Spielberger, Gorsuch, Lushene, Vagg, \& Jacobs, 1983; Vagg, Spielberger, \& O’Hearns, 1980), state anxiety was also identified as a crucial construct that hinges more on environmental conditions. As a consequence of the limited research, it is still unknown whether there is a relationship between learning style and state as well as trait anxiety of university freshmen. Accordingly, the aim of this study was to examine how learning style relates to state and trait anxiety in this group. 


\section{Literature review}

\subsection{Learning style}

Over several decades, learning style has been of interest to scholars and practitioners in multiple fields (Honigsfeld \& Schiering, 2004). A large number of learning style studies have indicated that people have a distinctive way of learning (Dunn \& Dunn, 1978; Kolb, 1984; Kolb \& Kolb, 2017; Peterson, Rayner, \& Armstrong, 2009). The term "learning style" refers to a person's preferred way of responding to tasks, assignments, or problems in a learning situation (Peterson et al., 2009). This study chose Kolb's (1984) learning model because it is based on individuals' experiences as a source of learning, and in this study the learning context involved freshmen experiencing an academic transition.

According to Kolb's (1984) model, learning has four modes: concrete experience (CE), reflective observation (RO), abstract conceptualization (AC), and active experimentation (AE). The CE learning mode is dialectically opposed to the AC mode in the grasping experience dimension, while the $\mathrm{RO}$ learning mode is dialectically contrasted with the $\mathrm{AE}$ mode in the transforming experience dimension. A combination of two learning modes from each learning dimension creates four basic learning styles. The Diverging learning style consists of the $\mathrm{CE}$ and RO modes and is characterized by the competencies of understanding people, having strong interpersonal relationships, and being imaginative and patient. The Assimilating learning style, made up of the AC and RO modes, relates to the characteristics of making ideal plans, building theories and models, and viewing things from various perspectives. The Converging learning style is composed of the AC and AE modes; it has the features of solving problems practically, making decisions, and establishing pragmatic goals. Finally, the Accommodating learning style, with the CE and AE modes, is characterized by a trial-and-error approach, motivating and leading people, and making things happen.

\subsection{State and trait anxiety}

Anxiety refers to an unpleasant emotion such as apprehension and worry (Kazdin, 2000). Spielberger (2013) indicated that the term "anxiety" has been used to describe not only certain types of emotions but also diverse cognitive actions or processes. He argued that anxiety is exhibited in two dimensions: state and trait anxiety (Spielberger, 2013). State anxiety involves a transitory excitement in a short-term condition based on a specific situation; thus, it is thought that the change of situation affects its occurrence. Trait anxiety is characterized as a relatively stable and acquisitive attitude; thus, it is recognized as a personality trait. It tends to make people see a broad scope of safe conditions as dangerous (Nazerian, Zamani, \& Soltani, 2011).

\section{Methods}

This study was part of a project that explored multiple features of freshmen in a Japanese university located near Tokyo. To collect data for the study, one of the authors asked four instructors who taught a course required for freshmen in the management department to distribute paper-based surveys in their classes at the beginning of the semester. There were 194 participants, 143 men and 51 women. To examine state and trait anxiety, this study employed the State-Trait Anxiety Inventory (STAI) designed by Spielberger. STAI questions have a 4-point Likert-type scale, ranging from 1, not at all, to 4, very much so. For this sample, the Cronbach's alpha was 0.86 for the STAI state scale and 0.84 for the STAI trait scale. The third version of Kolb's Learning Style Inventory (Kolb, 1999) was applied to investigate freshmen's learning style. Research has shown that the third version has better reliability than previous versions (Kayes, 2005). In this sample, 71 students had an Accommodating learning style (37\%); 69, Diverging (36\%); 41, Assimilating (21\%); and 13, Converging (8\%). Accordingly, as a whole, Japanese freshmen exhibited a preference for a feeling (CE) rather than a thinking (AC) learning orientation. Most Japanese freshmen had an Accommodating or Diverging learning style, which is consistent with past learning style study results (Toyama \& Yamazaki, 2018; Yamazaki, Toyama, \& Attrapreyangkul, 2018). To analyze how freshmen's learning style related to their state and trait anxiety, this study used two-way analysis of variance (i.e., learning style and gender) by controlling gender.

\section{Results}

Results of two-way analysis of variance illustrated significant differences among the four learning styles in terms of state anxiety $(\mathrm{F}=3.38, p<0.05)$ and trait anxiety $(\mathrm{F}=4.07, p<0.01)$; however, gender did not have a significant relationship with the two anxiety variables (state: $\mathrm{F}=0.22$, $p>0.05$; trait: $\mathrm{F}=1.38, p>0.05$ ). Additionally, there was no interaction effect of learning style and gender in terms of anxiety. Based on the Tukey post hoc test, the Diverging learning style had a 
significantly higher level of state anxiety than the Accommodating style $(p<0.01)$ and Converging style $(p<0.05)$. Also, the Diverging style had a significantly higher level of trait anxiety than the Accommodating style $(p<0.01)$ and a marginally higher level than the Converging style $(p<0.10)$. These results indicated that regardless of gender, freshmen with a Diverging learning style, compared with other learning styles, were likely to have the highest level of state and trait anxiety during their academic transition. Furthermore, freshmen who preferred to learn through active experimentation (AE), which represents the common learning mode of the Converging and Accommodating styles, tended to exhibit a lower level of state and trait anxiety when beginning their university studies. Table 1 summarizes results of two-way analysis of variance and the Tukey post hoc test, and Figure 1 shows state and trait anxiety levels according to learning style and gender.

Table 1. Results of two-way analysis of variance with the Tukey test.

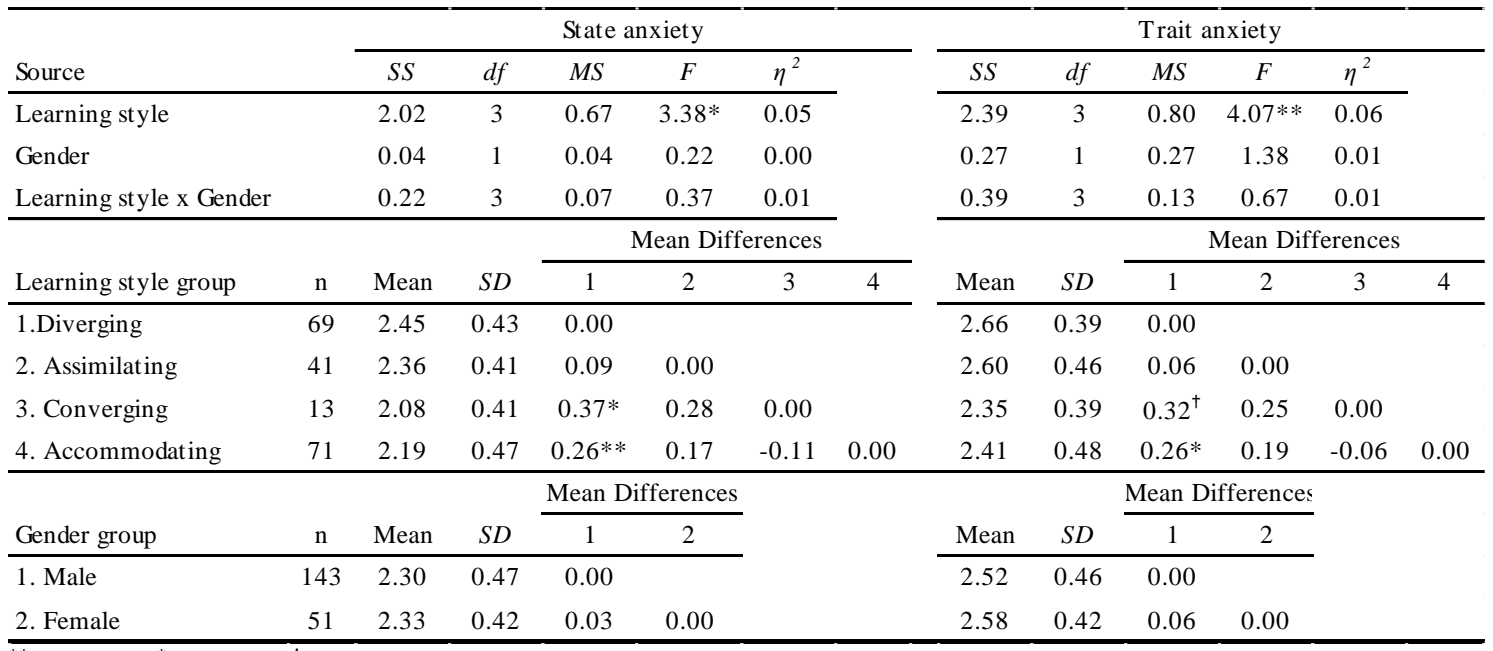

${ }^{* *} p<0.01,{ }^{*} p<0.05,{ }^{\dagger} p<0.10$.

Figure 1. State and trait anxiety levels according to learning style and gender.
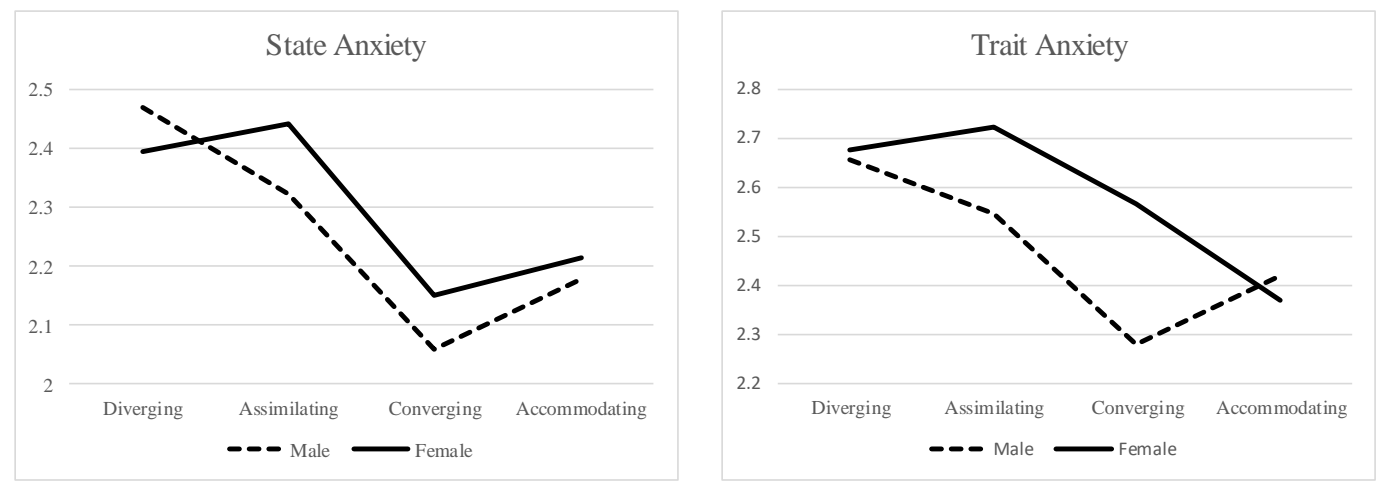

\section{Discussion}

This study showed a strong relationship between learning style and state-trait anxiety among university freshmen. Although several previous studies on learning style did not focus on freshmen (Ayalp \& Özdemir, 2016; Kadiem \& Hamzah, 2004; Yazici, 2017), their results are partly congruent with our results. For example, Ayalp and Özdemir (2016) reported a significant association between learning style and test anxiety using a sample from Turkish universities. More specifically, Turkish students with a Diverging learning style had a higher level of test anxiety than those with a Converging learning style. The other two studies applied different measures for learning style, which do not allow for direct comparisons. However, it should be noted that they showed that learning style was relevant to trait anxiety (Kadiem \& Hamzah, 2004) and text anxiety (Yazici, 2017). 
An interesting question is raised in terms of why the Diverging learning style relates to a higher level of state and trait anxiety. As those who learn through a Diverging learning style use the two modes of concrete experience (CE) and reflective observation (RO), they are sensitive to their internal and external environments. If they face a situational, unfamiliar problem, they may find it challenging to quickly resolve the problem or make decisions to cope with it. These activities require action rather than reflection. Thus, characteristics of learning style seem to be related to the anxiety. Furthermore, the learning tendencies of the Diverging learning style may hinder self-confidence development, which is thought to reduce anxiety. A recent study by Yamazaki, Toyama, and Ubed (2018) using a sample of Indonesian managers documented that abstract conceptualization (AC) over concrete experience (CE), and active experimentation (AE) over reflective observation (R), were associated with self-efficacy beliefs. In other words, people with a Diverging learning style have a lower level of self-efficacy than those with a Converging learning style. In the area of sport study, Nazerian et al. (2011) found a relationship between state and trait anxiety and self-confidence; thus, a future study should explore how learning style, anxiety, and self-confidence are interrelated.

As a practical implication, educational institutions and university teachers may want to pay special attention to freshmen with a Diverging learning style, who tend to have a higher level of state and trait anxiety. Since there is matching between the Diverging learning style and the Facilitator educator role (Kolb \& Kolb, 2017), if university teachers employ a warm, friendly, and interpersonal approach to Diverging-style freshmen, their anxiety level may be decreased. These actions may also increase students' motivation to learn (Toyama \& Yamazaki, 2019).

\section{References}

Ayalp, G. G., \& Özdemir, N. (2016). Relationship between test anxiety and learning styles of architecture undergraduates. Creative Education, 7(2), 364-375. doi:10.4236/ce.2016.72036

Basco, R. E., \& Olea, M. T. (2013). Correlation between anxiety level and academic performance of BS biology freshmen students. International Journal of Educational Research and Technology, 4(1), 97-103.

Boery, E. N., Casotti, C. A., Freire, I. V., Pereira, R., de Oliveira, B. G., \& Ribeiro, Í. J. S. (2017). Stress and quality of life among university students: A systematic literature review. Health Professions Education, 4(2), 70-77. doi:10.1016/j.hpe.2017.03.002

Clinciu, A. I. (2013). Adaptation and stress for the first year university students. Procedia - Social and Behavioral Sciences, 78, 718-722. doi:10.1016/j.sbspro.2013.04.382

Compas, B. E., Wagner, B. M., Slavin, L. A., \& Vannatta, K. (1986). A prospective study of life events, social support, and psychological symptomology during the transition from high school to college. American Journal of Community Psychology, 14, 241-257.

Dunn, R., \& Dunn, K. (1978). Teaching students though their individual learning styles: A practical approach. Upper Saddle River, NJ: Prentice-Hall.

Honigsfeld, A., \& Schiering, M. (2004). Diverse approaches to the diversity of learning styles in teacher education. Educational Psychology, 24(4), 447-507.

Kadiem, S., \& Hamzah, M. D. (2004). The influence of sex, personality types and trait-anxiety of learning styles among university students in Jordan. Journal Pendidik dan Pendidikan, 19, 97-106.

Kayes, D. C. (2005). Internal validity and reliability of Kolb's Learning Style Inventory version 3 (1999). Journal of Business and Psychology, 20(2), 249-257.

Kazdin, A. E. (2000). Encyclopedia of psychology, Vol. 1, 8th ed. Washington, DC: American Psychological Association.

Kolb, A. Y., \& Kolb, D. A. (2017). The experiential educator: Principles and practices of experiential learning. Kaunakakai, HI: EBLS Press.

Kolb, D. A. (1984). Experiential learning: Experience as a source of learning and development. Englewood Cliffs, NJ: Prentice-Hall.

Kolb, D. A. (1999). Learning Style Inventory, Version 3. Boston, MA: TRG Hay/McBer.

Levitz, R., \& Noel, L. (1989). Connecting students to institutions: Keys to retention and success. In M. L. Upcraft, J. N. Gardner, \& Associates (Eds.), The freshman year experience: Helping students survive and succeed in college (pp. 65-81). San Francisco, CA: Jossey-Bass.

Nazerian, I., Zamani, A., \& Soltani, A. (2011). The comparison of trait anxiety, state anxiety, and self-confidence among male athletes of team sports and individual sports in the country. Journal of Physical Education and Sport Science, 3, 3. Retrieved from https://pdfs.semanticscholar.org/078e/ 4ef93269b8d6f8aaaa23e2b1e339396b06a0.pdf 
Pancer, S. M., Hunsberger, B., Pratt, M. W., \& Alisat, S. (2000). Cognitive complexity of expectations and adjustment to university in the first year. Journal of Adolescent Research, 15(1), 38-57. doi:10.1177/0743558400151003

Peterson, E., Rayner, S. G., \& Armstrong, S. J. (2009). Researching the psychology of cognitive style and learning style: Is there really a future? Learning and Individual Differences, 19(4), 518-523.

Saklofske, D. H., Austin, E. J., Mastoras, S. M., Beaton, L., \& Osborne, S. E. (2012). Relationships of personality, affect, emotional intelligence and coping with student stress and academic success: Different patterns of association for stress and success. Learning and Individual Differences, 22(2), 251-257. doi:10.1016/j.lindif.2011.02.010

Sato, S., Suzuki, T., Kawashiri, T., Yamaguchi, M., Murata, T., \& Unemoto, S. (2017). Characteristics in mental health and stress coping behaviors of the university freshmen who are unwilling to join the university and who feel anxiety about academic learning. KIT Progress, 25, 47-56.

Spielberger, C. D. (1972). Anxiety as an emotional state. In C. D. Sprielberger (Ed.), Anxiety: Current trends in theory and research (pp. 23-49). New York, NY: Academic Press.

Spielberger, C. D. (2013). Theory and research on anxiety. In C. D. Spielberger (Ed.), Anxiety and behavior (pp. 3-20). New York, NY: Academic Press.

Spielberger, C. D., Gorsuch, R. L., Lushene, R. E., Vagg, P. R., \& Jacobs, G. A. (1983). Manual for the State-Trait Anxiety Inventory. Palo Alto, CA: Counseling Psychologist Press.

Toyama, M., \& Yamazaki, Y. (2018). A cross-cultural comparison of learning style and self-efficacy between employees in Japan and Indonesia. International Journal of Education, Psychology and Counselling, 3(13), 44-56.

Toyama, M., \& Yamazaki, Y. (2019). Are there effects of a match between learning style and teaching style in an EFL classroom? Innovation in Language Learning and Teaching (online version), 1-16. doi:10.1080/17501229.2019.1575386

Vagg, P. R., Spielberger, C. D., \& O’Hearns, T. P. (1980). Is the State-Trait Anxiety Inventory multidimensional? Personality and Individual Differences, 1, 207-214.

Von Ah, D., Ebert, S., Ngamvitroj, A., Park, N., \& Kang, D. (2004). Predictors of health behaviours in college students. Journal of Advanced Nursing, 48(5), 463-474.

Yamazaki, Y., Toyama, M., \& Attrapreyangkul, T. (2018). Cross-cultural differences in learning style and learning skills: A comparison of Japan, Thailand, and the USA. In C. Maheshkar \& V. Sharma (Eds.), The handbook of research on cross-cultural business education (pp. 160-182). New York, NY: IGI Global.

Yamazaki, Y., Toyama, M., \& Ubed, R. S. (2018). Exploring how learning style relates to general and career management self-efficacy beliefs in a managerial context. Organization Management Journal, 15(4), 201-213. doi:10.1080/15416518.2018.1528859

Yazıc1, K. (2017). The relationship between learning style, test anxiety and academic achievement. Universal Journal of Educational Research, 5(1), 61-71. doi:10.13189/ujer.2017.050108 


\title{
CROSS-CULTURAL RESEARCH ON DISCIPLINE TECHNIQUES, VALUES, AND ACADEMIC EXPECTATIONS OF TURKISH AND FINNISH MOTHERS
}

\author{
Melisa Kütük, \& Meral Atıcı \\ Department of Education Sciences, Psychological Counseling and Guidance Division, \\ Cukurova University (Turkey)
}

\begin{abstract}
The modernization and economic growth of Turkey has had an impact on parenting practices, values, and expectations of Turkish families. Similarly, a change towards further individualism and independence can be noticed in child rearing practices and values in Finland. This study aimed to investigate child rearing and discipline approaches, values transferred to children, academic and other expectations and views of Turkish and Finnish mothers. Qualitative data was collected by conducting interviews with 15 Turkish and 15 Finnish mothers, 30 mothers in total, who had adolescent children. The data was analyzed using content analysis. Findings indicated that Turkish and Finnish mothers' views differed, but similarities were also present. Both groups valued family lives and benevolence, Turkish mothers reported setting more rules regarding the child, positive punishments were the most used disciplinary techniques, and they also had high academic expectations. Finnish mothers, on the other hand, were seen to have lower academic expectations, more rules regarding the family and the home, and the most used disciplinary methods were negative punishments. Since there are no studies comparing Turkish and Finnish mothers in this field, this study is regarded to be valuable in the field of cross-cultural research.
\end{abstract}

Keywords: Child rearing, disciplinary practices, values, parental expectations, culture. 


\title{
MINDFULNESS, SELF-ESTEEM, AND BURNOUT IN COUNSELORS AND PSYCHOLOGISTS WORKING AT SCHOOLS
}

\author{
Gökçe Bulgan \\ Department of Psychological Counseling and Guidance, MEF University (Turkey)
}

\begin{abstract}
Burnout is a struggle of today's workforce and is regarded as a risk factor for mental health professionals working with countless social, emotional, and career related issues. Understanding the role of resources would help with its prevention and early intervention. The aim of this study is to investigate the role of mindfulness and self-esteem as possible resources in relation to burnout in counselors and psychologists working at schools. Data were collected from 338 (229 females and 109 males) counselors and psychologists working at public schools in Turkey, among them 184 (54.4\%) were counselors and 154 (45.6\%) were psychologists. Participants' age ranged between 21 and 51 with a mean of 31.41 $(S D=8.09)$. Data were collected using a Demographic Information Form, and Turkish versions of the Mindful Awareness Attention Scale (Brown \& Ryan, 2003; Özyeşil, Arslan, Kesici, \& Deniz, 2011), the Self-Liking Subscale of the Two Dimensional Self-Esteem: Self-Liking and Self-Competence Scale (Doğan, 2011; Tafarodi \& Swann, 2001), and the Burnout Measure Short Version (Pines, 2005; Tümkaya, Çam, \& Çavuşoğlu, 2009). The internal consistencies of the scales in the current study were $.87, .85$, and .90 respectively. The results indicated no significant differences between the genders of the participants on variables of mindfulness, self-esteem, and burnout. There were significant negative correlations between mental health professionals' mindfulness and burnout levels $(r=-.34 ; \mathrm{p}<.001)$ and their self-esteem and burnout levels $(r=-.53$; $\mathrm{p}<.001)$. In addition, there were positive relationships between mental health professionals' mindfulness and self-esteem levels $(r=.39 ; \mathrm{p}<.001)$. Results of multiple regression analyses indicated that mindfulness and self-esteem accounted for $30.2 \%$ of variance in mental health professionals' burnout levels. In addition, mindfulness $(\beta=-.15, \mathrm{p}<.01)$ and self-esteem ( $\beta=-.47, p<.001$ ) both uniquely contributed to burnout. Considering that mindfulness and self-esteem had significant unique contributions to burnout, teaching simple yet effective interventions and strategies for increasing mindfulness and self-esteem during their undergraduate program could be useful in preventing counselor and psychologist burnout in later years. As school counselors and psychologists feel more resourceful in preventing and dealing with burnout, they would be more effective in their work with students. Based on the results, the counseling and psychology curriculums could be reconstructed by including courses that focus on mindfulness based practices and those that strengthen one's relationship with himself/herself. Including courses such as positive psychology or increasing happiness and wellbeing could be valuable goals to focus on within the future directions of the counseling field.
\end{abstract}

Keywords: Mindfulness, self-esteem, burnout, mental health, school psychology.

\section{References}

Brown, K. W., \& Ryan, R. M. (2003). The benefits of being present: Mindfulness and its role in psychological well-being. Journal of Personality and Social Psychology, 84, 822-848.

Doğan, T. (2011). İki boyutlu benlik saygısı: Kendini sevme / Öz-yeterlik Ölçeği’nin Türkçe’ye uyarlaması, geçerlik ve güvenirlik çalışması. Eğitim ve Bilim, 36(162), 126-137.

Özyeşil, Z., Arslan, C., Kesici, Ş., \& Deniz, M. E. (2011). Bilinçli Farkındalık Ölçeği’ni Türkçe’ye Uyarlama Çalışması, Eğitim ve Bilim, 36(160), 224-235.

Pines, A. M. (2005). The Burnout Measure Short Version (BMS). International Journal of Stress Management, 12(1), 78-88.

Tarafodi, R. W., \& Swan, W. B. (2001). Two-dimesional self-esteem: Theory and measurement. Personality and Individual Differences, 31, 653-673.

Tümkaya, S., Çam, S., \& Çavuşoğlu, İ. (2009). Tükenmişlik Ölçeği Kısa Versiyonu'nun Türkçe'ye uyarlama, geçerlik ve güvenirlik çalışması. Ç. Ü. Sosyal Bilimler Enstitüsü Dergisi, 18(1), 387-398. 


\title{
SUPPORTING EMOTION SOCIALIZATION SKILLS OF PRESCHOOL TEACHER CANDIDATES
}

\author{
Aysel Esen Coban, Ayca Ulker Erdem, \& Tulay Ilhan Iyi \\ Department or Early Childhood Education, Hacettepe University, Turkey
}

\begin{abstract}
This research aims to investigate early childhood teacher candidates' emotion socialization skills and to provide support to improve those skills. As related to the basic purpose of the study, qualitative research method was used for sample selection, data collection and data analysis process. Participants were 22 early childhood teacher candidates. A semi structured interview, a focus group interview and reflective journals were used for data gathering. Data was analyzed by using content analysis. Findings showed that training support was effective for improving their emotion socialization skills. While teacher candidates' socialization practices of positive emotions enriched, they claimed to use more supportive socialization practices of negative emotions after the training. This study is believed to have direct impacts on preservice and in-service training plans and indirect effects on early support for children's emotional development.
\end{abstract}

Keywords: Emotion socialization, preschool teaching, teacher candidates, teacher training.

\section{Introduction}

Children express their positive and negative feelings in a variety of ways in their daily lives and receive reactions from their immediate surroundings to these feelings. This process, which is called emotion socialization, refers to parents' teaching about emotions by responding to children's emotions (Eisenberg, Cumberland \& Spinrad, 1998) and the way that the parents express their feelings and become a model for children (Morris, Silk, Steinberg, Myers \& Robinson, 2007). In this period, guiding the positive and negative emotional experiences of the child and giving supportive reactions to their feelings will positively affect the emotional and social development of the child. Studies conducted with parents show that positive and rewarding responses of parents to their children's feelings increased their positive social behaviors (Denham \& Kochanoff, 2002) while the children of mothers who did not accept the feelings of their children and ignored their emotions were less able to regulate their emotions and accordingly they showed more aggressive behavior (Ramsden ve Hubbard, 2002).

While this process is limited by the parents in early years, by the start of the preschool period, peers and teachers are included in the emotional interactions of the child. Therefore, teachers are also considered as important emotion socialization agents for children. Although there are many studies on the parents' emotion socialization strategies, little work has been found with preschool teachers who have an important role on the development of children in early ages. Researchers propose that preschool teachers have a great deal of emotion socialization opportunities for children during daily interactions (Denham, Bassett, \& Zinsser, 2012) and highlighted that they assume to provide an emotion-focused care (Ahn, 2005; Ahn \& Stifter, 2006; Hyson, 2002). However, although the importance of teacher-child interaction in terms of learning environments has been revealed; teacher training programs have limited content (such as Incredible Years, PATHS, I Can Problem Solve) on promoting emotional development of children through interactions.

Similarly, early childhood teacher candidates' emotion socialization practices were less focused which is a significant gap in the national and international literature. In this regard, it is thought that developing training programs to increase teacher candidates' awareness towards emotion socialization and to enable them to gain appropriate emotion socialization skills will contribute to the field. Considering that teachers' emotion socialization practices are important models for children's emotional experiences it is important to draw attention to these skills at the stage of gaining their professional identity and recommended to gain awareness and skills through practical courses. On this basis literature offers certain methods such as reflective supervision (Emde, 2009), mindfulness, emotional intelligent training, stress reduction and teaching new skills (Jennings \& Greenberg, 2009). Within this context, this study firstly aimed to investigate teacher candidates' emotion socialization strategies and to provide support for improving their emotion socialization skills with a 10-session training which is based on the techniques defined in the literature. 


\section{Method}

As related to the basic purpose of the study, qualitative research method was used for sample selection, data collection and data analysis process in this study. A case study approach was conducted to deeply examine participants' emotion socialization practices and the effectiveness of the proposed support. Creswell (2007) states that case study is an approach in which the researcher examines one or more cases in depth, over a limited time, using multiple data collection tools and defines themes related to the cases.

\subsection{Procedure}

The research process for this study began with the sample selection. As a part of a larger research study which aims to develop social emotional learning/ teaching practices of early childhood teacher candidates, an interaction group was announced and a purposeful sample was identified. The sample for this study comprised 22 early childhood teacher candidates studying at a government based university in Ankara, Turkey. They were all studying preschool teaching at different class levels in spring term of 2018.

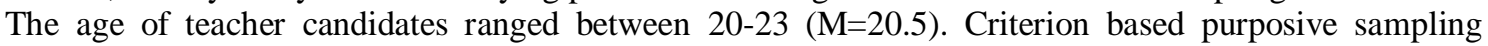
technique was used to select the participants. Depending on the aim of the study, all participants were considered to have an ongoing teaching experience at a public kindergarten with 3-6 year old children. Oral consents and the informed assents of all participants were obtained. Additionally, a group protocol was signed in order to protect participant rights in the group process with an emphasis on confidentiality. Then semi-structured interview forms were presented to the participants to assess their baseline emotion socialization skills and practices. The form compiled open ended questions in order to gain information about teacher candidates' emotion socialization skills. The type of questions asked to participants during the research were, but not limited, as follows: (1) children's challenging emotions faced in their practices, (2) their own responses to children's negative emotions, (3) their own responses to children's positive emotions. The aim of the initial interviews was to define participants' current emotion socialization levels.

As a next step, participants attended to a 10-session training which was formed and piloted prior to the current research. The first author directed the group with two co-authors as co-facilitators. This training included activities to teach new skills for responding children's emotions through psychoeducation and experiential techniques such as play, drama and art. This training was considered to improve professional development and socio-emotional learning/teaching practices. Sessions covered certain themes which are important for gaining supportive emotion socialization skills. The content of the first five sessions is as follows respectively; an intro session with an emphasis on the participants' desire to be an early childhood teacher; a psychoeducation session about negative emotions and theoretical base of emotion socialization, a creative drama session about the basic emotion socialization strategies and an experiential session about individual emotional awareness. Fifth and sixth sessions covered some exercises for highlighting the importance of meta-emotion philosophy and sampling how to develop empathy. After that, sessions continued with a reflective supervision session in which participants brought their own examples from the field practice; an emotion coaching approach to anger and anxiety; a prompter game to make participants notice different emotional experiences with lower and higher intensity and finally a focus group session to explore participants' metacognitions about their emotion socialization skills. This was an evaluation session and after group interactions were completed semi structured interviews were implemented as posttests to investigate the change in participants' emotion socialization levels. Additionally, participants were asked about their observations, experiences and practices on emotion socialization throughout individual reflective journals during the research process.

\subsection{Data analysis}

The findings of this study were derived from interviews and reflective journals to ensure the triangulation. Content analysis was used for analyzing the interview data. Participants' reflections in reflective journals were used as supportive evidence to show the change in their emotion socialization skills and representative quotations from the interview and reflections are presented in the findings.

Data analysis process involved the researchers working on the themes and the data constantly until an inclusive set of major themes are established (Creswell, 2007). The fundamental process in content analysis was to bring together similar data in the context of certain themes and categories to interpret them in a way that the reader can understand (Yıldırım \& Şimşek, 2011). In the second stage; reduced data was used and it was tried to reveal patterns between the data. In this regard the whole interview data was read by two researchers and analyzed under the codes and the themes generated from the theoretical background of socializing positive and negative emotions and previous research (Ahn \& Stifter, 2006; Kılıç, 2015). In order to ensure the reliability an independent researcher was asked to analyze $15 \%$ of data. Reliability was measured by the technique suggested by Miles and Huberman (1994) as .92 which is an agreeable level for social research. 


\section{Findings}

According to the information obtained from the interview, it is seen that the most compelling emotion experienced by teacher candidates is anger $(f=11)$, which is followed by sadness $(f=6)$, curiosity $(f=2)$ and anxiety $(f=1)$. The themes and codes related to the emotion socialization reactions adopted by the teacher candidates towards those challenging emotions and also children's positive emotions before the training support are presented in Table 1.

Table 1. Themes and codes about emotion socialization skills of participants before the training.

\begin{tabular}{|c|c|c|c|}
\hline & Pre-test & & \\
\hline Theme & Sub-theme & Code & Frequency \\
\hline \multirow{2}{*}{$\begin{array}{l}\text { Socializing } \\
\text { positive } \\
\text { emotions }\end{array}$} & Sharing & $\begin{array}{l}\text { - sharing emotion } \\
\text { - asking for sharing the emotion with friends }\end{array}$ & 18 \\
\hline & Emotion focused response & $\begin{array}{l}\text { - find out the reason of the emotion } \\
\text { - talking about the emotion }\end{array}$ & 9 \\
\hline \multirow[t]{5}{*}{$\begin{array}{l}\text { Socializing } \\
\text { negative } \\
\text { emotions }\end{array}$} & Solution focused response & $\begin{array}{l}\text { - trying to solve the problem } \\
\text { - look for a solution } \\
\text { - solving problem for the child }\end{array}$ & 8 \\
\hline & Distraction & $\begin{array}{l}\text { - eliminating the reason } \\
\text { - focusing on the solution by ignoring emotion }\end{array}$ & 6 \\
\hline & Minimizing & $\begin{array}{l}\text { - using behavior change techniques } \\
\text { - find out the reason of the emotion and make } \\
\text { suggestions } \\
\text { - using I language } \\
\text { - encouraging children to show empathy to peers }\end{array}$ & 4 \\
\hline & Emotion focused response & $\begin{array}{l}\text { - appreciating the emotion } \\
\text { - giving opportunity to talk about emotion } \\
\text { - expressing the emotion its normal } \\
\text { - showing empathy } \\
\text { - considering the emotion }\end{array}$ & 4 \\
\hline & Physical affection and comfort & - comforting, calming down, physical contact & 2 \\
\hline
\end{tabular}

Table 1 shows that sharing and emotion focused response was used for the socialization of positive emotions before the training. However, prior to the support, teacher candidates' strategies for socializing negative emotions were mainly solution focused response, distraction and minimizing. Positive emotion socialization responses such as emotion focused response and physical affection and comfort were less used. The participants' own statements about these responses are as follows:

"If there is a negative external factor, I try to eliminate it. If the problem is not related to the environment, I find the problem and apply behavior management methods."(TC2, interview) interview)

"I try to solve, eliminate or differentiate the event that causes children to feel that way." (TC5,

"I noticed a few mistakes I made during my practice, I was very uncomfortable. I realized that I didn't give the children any opportunity, and I showed them directly the solution." (TC3, reflective journal).

When Table 1 and 2 are assessed together, it is observed that the training was effective for improving teacher candidates' emotion socialization skills. Table 2 shows that strategies adopted by teacher candidates towards positive emotions was enriched after the support. On the other hand, it is seen that the quality of the reactions they adopt in socializing negative emotions has changed. Teacher candidates interiorize more supportive emotion socialization strategies such as physical affection and comfort, helping to regulate negative emotion, problem focused response, emotion focused response, labeling, positive interaction and modeling after the training. Sample quotations showing these changes are as follows:

"[Previously] I was more focused on problem solving. After having knowledge about socialization of emotion (...) I started to give place to what I learned in my practice. I guided children by using the emotion coaching method. (TC1: reflective journal)"

"During my practice, I applied the steps of labeling the emotion, defining the situation, asking questions that would lead to a solution, and becoming a model. Realizing that the child is getting calm and these steps work has shown that I must continue to follow these steps in all my teaching experiences. "(TC2, reflective journal)

"I was getting into a lather when the kids got angry before. I could not reach them. I learned what to say to the child when I recognized the concept of emotion socialization (...) when the child feels sorry, 
instead of understanding his sorrow, giving a reward to him and passing it off, causes the child not to define his sense." (TC17, reflective jorunal)

"I was repeating a very frequent mistake and trying to make children forget their negative feelings and distract them. I was exaggerating their positive feelings; much more enthusiastic way I was transferring them. Now, I tell children that the emotions they experience are actually very natural and give them time to feel that emotion." (TC20, reflective journal)

Table 2. Themes and codes about emotion socialization skills of participants after the training.

\begin{tabular}{|c|c|c|c|}
\hline & Post-test & & \\
\hline Theme & Sub-theme & Code & Frequency \\
\hline \multirow[t]{2}{*}{$\begin{array}{l}\text { Socializing } \\
\text { positive } \\
\text { emotions }\end{array}$} & Sharing & $\begin{array}{l}\text { - sharing the emotion } \\
\text { - asking for sharing the emotion with friends } \\
\text { - projecting the emotion but not magnifying }\end{array}$ & 22 \\
\hline & Emotion focused response & $\begin{array}{l}\text { - find out the reason of the emotion } \\
\text { - considering the emotion } \\
\text { - appreciating the emotion } \\
\text { - talking about the emotion } \\
\text { - projecting the emotion physically } \\
\text { - asking for accompanying emotions } \\
\text { - enabling the child to understand the emotion } \\
\text { - permitting the child to express emotion }\end{array}$ & 13 \\
\hline \multirow[t]{8}{*}{$\begin{array}{l}\text { Socializing } \\
\text { negative } \\
\text { emotions }\end{array}$} & $\begin{array}{l}\text { Physical affection and } \\
\text { comfort }\end{array}$ & $\begin{array}{l}\text { - giving time to calm down } \\
\text { - comforting, calming down, physical contact } \\
\text { - giving trust }\end{array}$ & 14 \\
\hline & $\begin{array}{l}\text { Helping to regulate } \\
\text { negative emotion }\end{array}$ & $\begin{array}{l}\text { - finding the solution together } \\
\text { - permitting the child to experience the emotion } \\
\text { - searching for a solution } \\
\text { - enabling child to cope with the emotion } \\
\text { - expressing the emotion its normal } \\
\text { - enabling the child to understand the emotion }\end{array}$ & 12 \\
\hline & Problem focused response & $\begin{array}{l}\text { - observing } \\
\text { - finding out reason of the emotion } \\
\text { - finding out the problem } \\
\text { - asking questions }\end{array}$ & 9 \\
\hline & $\begin{array}{l}\text { Showing empathy to } \\
\text { negative emotion }\end{array}$ & $\begin{array}{l}\text { - appreciating the emotion } \\
\text { - projecting the emotion } \\
\text { - showing empathy }\end{array}$ & 5 \\
\hline & Emotion focused response & $\begin{array}{l}\text {-talking about emotion (physical projections, } \\
\text { accompanying other emotions) } \\
\text {-considering the emotion, appreciating the emotion }\end{array}$ & 4 \\
\hline & Labeling & $\begin{array}{l}\text { - asking to label the emotion } \\
\text { - naming the emotion }\end{array}$ & 2 \\
\hline & Positive interaction & - positive communication & 2 \\
\hline & Modeling & $\begin{array}{l}\text { - modelling } \\
\text { - guiding }\end{array}$ & 2 \\
\hline
\end{tabular}

\section{Discussion}

The aim of this study was to investigate and support emotion socialization strategies of early childhood teacher candidates. The findings showed that their response repertoires were enriched. Moreover, teacher candidates were found to interiorize more supportive socialization strategies such as physical affection and comfort, emotion focused response, labeling, helping to regulate negative emotion, problem focused response, positive interaction, modelling and showing empathy to negative emotions.At the same time, the teacher candidates stated that they felt more competent in coping with children's negative emotions and started to use these supporting strategies in their teaching practices. As a result of the relational culture structure of Turkey, different from the Western literature, pre-service teachers seemed to socialize positive emotions through suggesting to share with others and making conscious efforts in order to maintain children's positive mood. In addition to this, prior to the training, teacher candidates reported that they often focused on the event which elicit negative emotion and used solution focused response so that they could eliminate the cause of the negative emotion. Solution focused response is different from the non-supportive emotion socialization responses defined in the literature and this could be explained by the didactic and behavior-focused early childhood learning environment which is commonly observed in 
Turkey. This approach, which is used as a way of preventing misbehaviors in learning environments, provides an idea that teacher candidates focus on behavior by ignoring emotion. However, it is known that children express and control their feelings much easier when teachers comfort their negative emotions (Gottman, Katz \& Hooven, 1997). According to Furman (1995) children might not know emotion words and might not be able to verbally express their feelings. Therefore, teachers' definitions and labels of their emotions is highly important in terms of children's recognition of their own emotions. At this point, the current study's findings revealed that after the support, teacher candidates adopted problem-focused responses and helping to regulate negative emotion strategies rather than searching for solutions to help children coping with their emotions. Similarly, in Ahn's study (2005) it was concluded that when teachers help children for coping with their emotions and offer concrete suggestions, their pro-social development has improved. Considering the fact that the teacher candidates use non-supportive emotion socialization strategies before the training such as distraction and minimizing, training provided in this research was found to be effective in supporting teacher candidates' emotion socialization skills. Teachers play a very important role in terms of creating an environment that supports children's emotion regulation skills and ability to respond appropriately to the feelings of others (Hyson, 1994). Consistently, the current study showed that the support provided to the teacher candidates has increased their use of positive emotion socialization strategies. Thus, it is thought that this training will enable teacher candidates to create an environment that supports children's emotion regulation skills. With all these contributions, this study is believed to have direct impacts on pre-service and in-service trainings and indirect effects on early support for children's emotional development.

\section{References}

Ahn, H. J. (2005). Child care teachers' strategies in children's socialization of emotion. Early Childhood Development and Care, 175(1), 49-61.

Ahn, H. J. \& Stifter, C. (2006). Child care teachers' response to children's emotional expression. Early Education and Development,17(2), 253-270.

Creswell, J. W. (2007). Qualitative inquiry and research design: Choosing among five approaches (2nd ed.). Thousand Oaks, CA, US: Sage Publications, Inc.

Denham, S. A., Bassett, H. H., \& Zinsser, K. (2012). Early childhood teachers as socializers of young children's emotional competence. Early Childhood Education Journal, 40(3), 137-143.

Denham, S.A. \& Kochanoff, A.T. (2002). Parental contributions to preschoolers' understanding of emotion. Marriage \& Family Review, 34(3-4), 311-343. doi: 10.1300/J002v34n03_06

Eisenberg, N., Cumberland, A., Spinrad, T.L. (1998). Parental socialization of emotion. Psychological Inquiry, 9(4), 241-273.

Emde, R. N. (2009). Facilitating reflective supervision in an early child development center. Infant Mental Health Journal, 30(6), 664-672.

Furman, R. A. (1995). Helping children cope with stress and deal with feelings. Young Children, 50(2), 33-41.

Gottman, J.M., Katz, L.F. \& Hooven, C. (1997). Meta-emotion: How families communicate emotionally. Mahwah, NJ: Lawrence Erlbaum Associates.

Hastings, P.D. \& De, I. (2008). Parasympathetic regulation and parental socialization of emotion: Bio-psychosocial processes of adjustment in preschoolers. Social Development, 17 (2). 211-237.

Hyson, M. C. (1994). The emotional development of young children: Building an emotion-centered curriculum. New York: Teachers College.

Hyson, M. (2002). Emotional development and school readiness. Professional Development, 57(6),76-78.

Jennings, P.A. \& Greenberg, M.T. (2009). The prosocial classroom: Teacher social and emotional competence in relation to student and classroom outcomes. Review of Educational Research, 79(1), 491-525.

Kılıç, Ş. (2015). Preschool teachers' emotional socialization responses to 4-6 year old Turkish preschoolers' emotional expressions. EJRE, 3(1). 53-63.

Miles, M, B., \& Huberman, A. M. (1994). Qualitative data analysis: An expanded sourcebook. (2nd ed). Thousand Oaks, CA: Sage

Morris, A. S., Silk, J. S., Myers, S.S., Steinberg, L.\& Robinson, L. R. (2007). Role of family context in the development of emotion regulation. Social Development, 16(2), 361-388. doi:10.1111/j.14679507.2007.00389.x

Ramsden, S. R. \& Hubbard, J.A. (2002). Family expressiveness and parental emotional coaching: their role in children's emotion regulation and aggression. Journal of Abnormal Child Psychology, 30(6), 657-667.

Yıldırım, A., \& Şimşek, H. (2011). Qualitative research methods in Social Sciences (8th. ed). Ankara: Seçkin Publishing. 


\title{
THE ORGANIZATIONAL VALORIZATION OF EMPLOYEES WHO ARE SATISFIED WITH THEIR LIVE
}

\author{
Bernard Gangloff ${ }^{1}, \&$ Neila Malleh $^{2}$ \\ ${ }^{1}$ Laboratoire Parisien de Psychologie Sociale, Université Paris 10 (France) \\ ${ }^{2}$ Institut Supérieur de l'Animation pour la Jeunesse et la Culture, Université de Tunis (Tunisia)
}

\begin{abstract}
Satisfaction with life seems to have many positive aspects. Our general hypothesis is that employees who feel satisfaction with life will be positively valued by their superiors, and that this positive value, which evokes the concept of social norms, allows us to assign to satisfaction with life a normative status. Three populations (128 line managers, 176 students destined to become human resource managers, and 307 employees) responded to a satisfaction with life questionnaire. Managers had to indicate, for each item, if they appreciated (or not) an employee feeling the satisfaction presented in the item; students had to indicate which items an employee should check if s/he wants to be positively (versus negatively) appreciated by his/her superior; and employees had to indicate if each item corresponded to the satisfaction they used to feel. Three hypotheses are developed and confirmed: managers positively value employees feeling satisfaction with life; students are aware of this positivity; spontaneously employees show satisfaction with life, which means, knowing that spontaneous self-presentation is often produced by social desirability, that employees are aware of the satisfaction positivity. These data are discussed under a conceptual and applied angle.
\end{abstract}

Keywords: Satisfaction with life, organizational normativity.

\section{Introduction}

Numerous theories on satisfaction have been formulated so far (Diener et al., 1999). Bouffard (1997) has proposed a classification that differentiates the general theories from the so-called specific ones. The former can be divided into two groups. The ascending theories (including the works of Diener, Sandvik, Pavot \& Gallagher, 1991; Okun, Olding \& Cohn, 1990; Cohen, Towbes \& Flocco, 1988), suggest that satisfaction comes from the aggregation of all micro-satisfactions resulted from various areas of life (such as familial, professional, conjugal, friendly). The descending theories (Lykken \& Tellegen, 1996; McCrae \& Costa, 1991; Seligman, 1991) propose a reversed order: the existence of a personality structure that predisposes to satisfaction or dissatisfaction and influences the interpretation of life events in terms of satisfaction or dissatisfaction. In what regards the specific theories, meant to complement the general theories, they particularly include the cognitive theories (which examine the manner in which the cognitive operations transform the objective information into subjective data : e.g. Lent et al., 2005; Ryff \& Essex, 1992; Weiner, 1992); the theory of standards (according to which the satisfaction in life comes from evaluating the distance that separates us from a standard, the latter being generally other people: Buunk, Collins, Taylor, Van Yperen \& Dakof, 1990; Easterlin, 1995; Emmons \& Diener, 1985; Frieswijk, Buunk, Steverink \& Slaets, 2004; Pelham et Wachsmuth, 1995); or the theory of goals, which is based on intrapersonal comparisons that relate aspirations and their achievement (such as Oishi et al., 1999; Ryan \& Deci, 2000; Spence, Oades \& Caputi, 2004).

However, these different points of view do not hinder a consensual definition of satisfaction. Andrews \& Withey (1976) point out that life satisfaction corresponds to one of three components of subjective wellbeing characterised, alongside two emotional components (positive and negative emotions: see Bradburn, 1969), by a cognitive and evaluative component. The feeling of satisfaction, according to Mercier \& Filion (1987, p. 137), entails in fact a judgement, an evaluation. Life satisfaction is thus defined as «a global evaluation of a person's life quality, according to their own criteria" (Shin \& Johnson, 1978, p.478). 
In the professional field, numerous studies have highlighted the fact that work satisfaction has a positive effect on several areas: performance (Aziri, 2011; Javed, Balouch \& Hassan, 2014; Lai Wan, 2007; Nanda \& Browne (1977)), organisational commitment and loyalty (Chen, 2006; Fletcher \& Williams, 1996; Javed et al. 2014; Martensen \& Gronholdt, 2001; Vanderberg \& Lance, 1992), attachment to the company and desire to remain in it (Chen, 2006; Muchinsky \& Morrow, 1980), etc. Certain authors considered that such positive consequences could also be derived from the individuals' evaluation of their life as a whole (and not only of their satisfaction within the professional area), leading to the company managers valuing the employees who evaluate their life as a whole in a positive manner. Such is the case of the study conducted by Gangloff and Malleh (2016). But these authors have used a multidimensional scale (the Psychological Wellbeing Manifestation Scale of Massé et al., 1998), which refers to different strata of life (need of self-esteem, of sociability, self-control and events, etc.). Due to the fact that the appreciation criteria for life quality vary according to individuals, Diener, Emmons, Larsen \& Griffin (1985, p. 71) find it more pertinent to examine people's global satisfaction than to interrogate them regarding their satisfaction in one field or another, or in one stratum or another and then sum it up. Therefore, we wanted to resume the idea of Gangloff and Malleh, but by applying a global scale. More specifically, we wanted to test the hypothesis according to which satisfaction meets certain characteristics of a social norm.

The term "norm" refers to «that which seems desirable, suitable within a specific society or group" (Maisonneuve, 1973, p.59). Dubois also writes (1994, p.28) that «a norm defines a set of events judged as being good or desirable by the collective from which they emerge, as opposed to another set of events regarded as being less good or less desirable, even frankly bad». Dubois (1994, p.28-29) states that all norms result from social learning (which means that the propagation and integration of norms vary according to social groups) and are based on an informal value allocation (this value being related to the utility and/or the desirability attributed to the norm within the framework of social functioning: Dubois, 2005). This article aims to show that life satisfaction meets certain aspects of this definition and could therefore be attributed the first constituent elements of a normative status. Therefore, we formulate the following operational hypotheses:

- executives value employees who report a high level of satisfaction (H1),

- non-executives are aware of this valuation and they usually have a high level of satisfaction (H2),

- non-executives are aware of this valuation and, in order to give a good image of themselves, they declare to have a high level of satisfaction (H3).

\section{Method}

Gosselin (2005) has classified the measurement tools for investigating life satisfaction into two categories: global instruments (which allow the measuring of satisfaction within an integrative spirit, e.g. the scale of Diener et al., 1985), and the instruments measuring various facets of satisfaction corresponding to different areas of life (work, family, personal relations, leisure) or to different strata of life. We have emphasized the criticism brought by Diener et al. (1985) against faceted scales. Moreover, Diener (1984) remarks that the global satisfaction scales often contain only one item, or still yet, are meant for specific populations (particularly elderly people), or even relate to aspects not inherent to satisfaction. Excluding such instruments, we have used ten items (five of satisfaction and five of dissatisfaction ${ }^{1}$ ), two of which were extracted directly from the SWLS by Diener et al. (1985), seven were developed starting from that same scale, and a tenth were derived from Huebner's students' life satisfaction scale (SLSS) (1991). We asked three populations (two populations of employees - executives and non-executives, and one population of students) to respond individually and voluntarily to that scale, and we obtained a satisfactory Cronbach alpha (.738).

The population of executives ( $\mathrm{N}=128$ men) was subjected to the legislator's paradigm (Gangloff, 2008). Those executives were thus instructed to indicate, for each item, if they would appreciate (or not) if an employee adopted the behaviour presented in the item. The respondents thus checked each item according to a dichotomous principle: appreciated response versus rejected response. The non-executive employees ( $\mathrm{N}=307$ men) were confronted with the self-presentation paradigm with neutral instructions, meaning they had to indicate, in all sincerity, on a 4-point scale (true, rather true, rather false, false), the degree to which each sentence corresponded to a conduct they normally adopted. Finally, the students ( $\mathrm{N}=176$ : 134 women and 42 men) were confronted to a new paradigm, created for this occasion: the

\footnotetext{
1 1) So far, I've obtained the important things that I wanted from life (extracted from the SWLS scale), 2) So far, I've not obtained much of what I wanted in my life, 3) I often envy the lives of others, 4) I have a life that is more satisfying than that of many others, 5) When I am not satisfied with something, all the other areas of my life are affected , 6) It suffices for everything to go well in one area, for me to feel satisfied, 7) So far, I am satisfied with the choices I've made in my life, 8) If I could, I would change many of the decisions I've made during my life, 9) I am satisfied with my life (extracted from the SWLS scale), 10) I would love to change many things in my life (extracted from SLSS).
} 
paradigm of «courtesan self-presentation»: the participants were confronted with the following instructions: "You were asked to respond to the following questionnaire by imagining the answers that an employee should check in order to be appreciated by their superior, and which should they check if they wanted to be badly regarded by their superior». The respondents thus checked each item according to a dichotomous principle: in order to be well regarded versus in order to be badly regarded².

\section{Results}

\subsection{For executives $(\mathbf{N}=128)$}

We notice (Table 1 ) that the positive answers are significantly more frequent than the negative ones $\left(\chi^{2}=455,88 ; p \approx .00\right)$.

Table 1. Distribution of executive employees' answers in raw data (and \% between parenthese.) NB: due to 3 non-answers, the total is less than 1280 (128 participants $x 10$ items).

\begin{tabular}{cc}
\hline Positive answers & $1020(79.87 \%)$ \\
\hline Negative answers & $257(20.13 \%)$ \\
\hline Total & $1277(100 \%)$ \\
\hline
\end{tabular}

\subsection{For non-executives $(\mathbf{N}=307)$}

Here once more (Table 2), the positive answers are more frequent than the negative ones $\left(\chi^{2}=504,10 ; p \approx .00\right)$. It should however be noted that the executives provided more positive answers than the non-executives $\left(\chi^{2}=42,07 ; p \approx .00\right)$.

Table 2. Distribution of non-executive employees' answers in raw data (and \% between parentheses). NB: due to 10 non-answers, the total is less than 3070 (307 participants $x 10$ items).

\begin{tabular}{cc}
\hline Positive answers & $2151(70.29 \%)$ \\
\hline Negative answers & $909(29.71 \%)$ \\
\hline Total & $3060(100 \%)$ \\
\hline
\end{tabular}

\subsection{For students ( $\mathrm{N}=176$ : 134 women and 42 men)}

Taken globally (i.e. for both men and women), the positive answers are more frequent than the negative answers (Table $3 ; \chi^{2}=966.75 ; p \approx .00$ ), which is also observed for women (Table 3: $\chi^{2}=682.33$; $p \approx .00$ ) and for men (Table $3 ; \chi^{2}=288.62 ; p \approx .00$ ). However, men provide more positive answers than women $\left(\chi^{2}=9.65 ; p=.001\right)$.

Moreover, we notice that the students systematically provide more positive answers than the executives, both men and women together $\left(\chi^{2}=32,35 ; p \approx .00\right)$ and men $\left(\chi^{2}=31,79 ; p \approx .00\right)$ or women $\left(\chi^{2}=18,00 ; p \approx .00\right)$ taken individually. Similarly, the students' answers are more frequently positive than those of the non-executive employees, both for men and women together $\left(\chi^{2}=181,59 ; p \approx .00\right)$ and for men $\left(\chi^{2}=85,74 ; p \approx .00\right)$ or women $\left(\chi^{2}=122,70 ; p \approx .00\right)$ taken individually.

Table 3. Distribution of the students' answers, in raw data (and \% between parentheses). NB: For men and women combined, due to 44 non-responses, the total is below 1760 (176 participants $x 10$ items). For women, due to 34 non-responses, the total is below 1340 (134 participants $x 10$ items). For men, due to 10 non-responses, the total is below 420 (42 participants $x 10$ items).

\begin{tabular}{cccc}
\hline & Men + Women & Men & Women \\
\hline Positive answers & $1502(87.53 \%)$ & $1125(86.14 \%)$ & $377(91.95 \%)$ \\
\hline Negative answers & $214(12.47 \%)$ & $181(13.86 \%)$ & $33(8.05 \%)$ \\
\hline Total & $1716(100 \%)$ & $1306(100 \%)$ & $410(100 \%)$ \\
\hline
\end{tabular}

\footnotetext{
2 The self-presentation paradigm is usually of three types ( $a c c$. to Jellison \& Green, 1981): 1) self-presentation with neutral or standard instructions (answer the questionnaire as sincerely as possible); 2) with self-valuation instructions (answer trying to render the best self-image possible); 3) with self-devaluation instructions (answer trying to render the worst self-image possible). Usually, the self-valuation and self-devaluation instructions are used together: the difference in answers obtained between the two sets of instructions is considered to emphasize the perception of respondents in what regards the difference in social value awarded to one or the other type of answers. But this combined usage seems methodologically problematical, whether carried out intra-subjectively (each respondent answers successively according to the set of instructions received, which leads to a potential halo effect which can only be compensated by controlling the order effect) or inter-subjectively (in this case, the inter-subjective differences could bias the results). The method we have chosen (the courtesan self-presentation paradigm) allows us to avoid each of those two pitfalls.
} 


\section{Discussion-Conclusion}

We hypothesized that satisfaction would meet the main criteria characterising a social norm. Our results indicate that this is indeed the case: the executives claim to value the employees who report high satisfaction (hypothesis 1). In addition, we observe that declaring such an elevated feeling is effective for non-executive employees (hypothesis 2), which could mean, if we take into consideration the fact that the self-presentation paradigm with neutral instructions frequently produces answers impregnated with social desirability, a clairvoyance of the employees in terms of such valuation: the latter would declare themselves to be satisfied because they would have integrated the fact that such statement of satisfaction is valued. Finally, this clairvoyance is more directly confirmed on our third population by the fact that the future employees, in this case, the students, are indeed aware of this valuation and report a higher satisfaction in order to demonstrate their worth and, a contrario, they display a weaker satisfaction in order to render a bad image of themselves (hypothesis 3). Finally, we observe that the executives provide more positive answers than the non-executives, and that the students provide more positive answers than executive employees and non-executive employees.

One of the limitations of this study is having considered the status of our three populations to be their sole descriptive criterion (employees or students). However, the results obtained, far from being inconsistent, respond well to the hypotheses formulated. The mutations faced by organisations, particularly as a result of globalisation and the competition it induces, leads them to extend their exigencies in terms of individual performance and flexibility. The competences sought are indeed more and more frequently related to adaptability. The studies conducted by Pulakos (e.g. Pulakos, Schmitt, Dorsey, Hedge \& Borman, 2002) on adaptive performance (which translate the individuals' capacity to adapt to the new exigencies of a job), underline the fact that such performance is supported by an adaptive competence consisting of eight factors (interpersonal adaptability - being capable of working with new colleagues, customers, providers; cultural adaptability - being capable of working within different cultural contexts; physical adaptability being capable of working within various and difficult environments; solving of uncertain and unpredictable work situations; etc.). Confronted with these new exigencies, it is obvious that the employees have to be optimistic in what regards their capacity to respond to them, and it is logical that superiors, by renewing their criteria for appreciating the personnel and by integrating the new economic data, would value the subordinates who experience (or state to experience) such a feeling. Our results follow this logic. This being the case, instead of studying the normativity of an object and thus align with a socio-cognitive approach, we could now continue this study by examining the proven level of life satisfaction of the employees, i.e. independently of all research on desirability.

\section{References}

Andrews, F.M., \& Withey, S.B. (1976). Social indicators of well-being: American's perception of life quality. New-York: Plenum. DOI:10.1007/978-1-4684-2253-5

Aziri, B. (2011). Job satisfaction: a literature review. Management Research and Practice, 3 (4), 77-86.

Bouffard, L. (1997). Variations sur le thème du bonheur. Revue Québécoise de Psychologie. 18.3-12.

Buunk, B.P., Collins, R.L., Taylor, S.E., Van Yperen, N.W., \& Dakof, G.A. (1990). The affective consequences of social comparison: Either direction has its ups and downs. Journal of Personality and Social Psychology. 59.1238-1249.

Bradburn, N.M. (1969). The structure of psychological well-being. Chicago: Aldine

Chen, C. (2006). Job satisfaction, organizational commitment and flight attendants turnover intentions: a note. Journal of Air Transport Management, 12 (5), 274-276. doi:10.1016/j.jairtraman.2006.05.001

Cohen, L.H., Towbes, L.C., \& Flocco, R. (1988). Effect of induced mood on self-reported life events and perceived and received social support. Journal of Personality and Social Psychology. 55.669-674.

Diener, E. (1984). Subjective well-being. Psychological Bulletin, 95, 542-575.

Diener, E., Emmons, R. A., Larsen, R. J., \& Griffin, S. (1985). The Satisfaction with Life Scale. Journal of Personality Assessment, 49, 71-75.

Diener, E., Sandvik, W., Pavot, W., \& Gallagher, D. (1991). Response artifacts in the measurement of subjective well-being. Social Indicators Research. 24.35-56.

Diener, E., Suh, E.M., Lucas, R.E., \& Smith, H.L. (1999). Subjective well-being: Three decades of progress. Psychological Bulletin. 125.276-302.

Dubois, N. (1994). La norme d'internalité et le libéralisme. Grenoble : PUG.

Dubois, N. (2005). Normes sociales de valeur et jugement : ancrage sur l'utilité et ancrage sur la désirabilité. Revue Internationale de Psychologie Sociale, 3, 43-80.

Easterlin, R.A. (1995). Will raising the incomes of all increase the happiness of all ? Journal of Economic Behavior and Organization. 27.35-47. 
Fletcher, C., \& Williams, R. (1996). Performance management, job satisfaction and organizational $\begin{array}{llllll}\text { commitment. British Journal of } & \text { Management, } 7 \text { (2), }\end{array}$ doi: 10.1111/j.1467-8551.1996.tb00112.x

Frieswijk, N., Buunk, B.P., Steverink, N., \& Slaets, J.P.J. (2004). The effect of social comparison information on the life satisfaction of frail older persons. Psychology and Aging. 19.183-190.

Gangloff, B. (2008). Normativity of the acceptance of professional injustices via the legislator's paradigm: a study on recruiters. Actes de la $5^{\text {th }}$ International Conference of Applied Psychology (Timisoara, Roumanie, 2007). Timisoara (Roumanie) : Editura Eurobit, 219-228.

Gangloff, B., \& Malleh, N. (2016). French managers and their subordinates'well-being. World Academy of Science, Engineering and Technology. International Journal of Social, Education, Economy, Business and Industrial Engineering, 10(12), 3416-3419.

Gosselin, E. (2005). Contribution à une synthèse des connaissances sur la satisfaction dans la vie: regard psychologique sur une réalité plurielle. Revue de l'Université de Moncton, 36 (2), 131-169. Doi: 10.7202/014502ar

Huebner, E. S. (1991). Initial development of the Student's Life Satisfaction Scale. School Psychology International, Vol 12(3), 231-240.

Javed, M., Balouch, R., \& Hassan, F. (2014). Determinants of job satisfaction and its impact on employee performance and turnover intentions. International Journal of Learning and Development, 4 (2), 120-140. doi: 10.5296/ ijld.v4i2.6094

Jellison, J.M., \& Green, J. (1981). A self-presentation approach to the fundamental attribution error: the norm of internality. Journal of Personality and Social Psychology, 40(4), 643-649.

Lai Wan, H. (2007). Human capital development policies: enhancing employee's satisfaction. Journal of European Industrial Training, 31 (4), 297-322. doi: org/10.1108/03090590710746450

Lent, R.W., Singley, D., Sheu, H.-B., Gainor, K.A., Brenner, B.R., Treistman, D., \& Ades, L. (2005). Social cognitive predictors of domain and life satisfaction: Exploring the theoretical precursors of subjective well-being. Journal of Counseling Psychology. 52.429-442.

Lykken, D., \& Tellegen, A. (1996). Happiness is a stochastic phenomenon. Psychological Science. 7.186-189.

McCrae, R.R., \& Costa, P.T. (1991). Adding liebe und arbeit The full five-factor model and well-being. Personality and Social Psychology Bulletin. 17. 227-232. DOI:10.1177/014616729101700217

Maisonneuve, J. (1973). Introduction à la psychosociologie. Paris: PUF.

Martensen, A. \& Gronholdt, L. (2001). Using employee satisfaction measurement to improve people management: an adaptation of Kano's quality types. Total Quality Management, 12 (7/8), 949-57. doi: 10.1080/09544120100000020

Massé, R. Poulin, C., Dassa, C., Lambert, J., Bélair, S., \& Battaglini, M.A. (1998). Elaboration et validation d'un outil de mesure du bien-être psychologique: l'ÉMMBEP. Revue Canadienne de Santé Publique, 89 (5), 352-357. Doi: http://dx.doi.org/10.17269/cjph.89.1014

Mercier, C., \& Filion, J. (1987). La qualité de vie: perspectives théoriques et empiriques. Santé Mentale au Québec, 12(1), 135-143.

Muchinsky, P. M., \& Morrow, P. C. (1980). A multidisciplinary model of voluntary employee turnover. Journal of Vocational Behavior, 17 (3), 263-290. doi: 10.1016/0001-8791(80)90022-6

Nanda, R., \& Browne, J. J. (1977). Hours of work, job satisfaction and productivity. Public Productivity Review, 2 (3), 46-56. doi: 10.2307/3380223

Oishi, S., Diener, E., Suh, E., \& Lucas, R.E. (1999). Value as a moderator in subjective well-being. Journal of Personality. 67.157-184.

Okun, M.A., Olding, R.W., \& Cohn, C.M.G. (1990). A meta-analysis of subjective well-being interventions among elders. Psychological Bulletin. 108.257-266.

Pelham, B.W., \& Wachsmuth, J.D. (1995). The waxing and waning of the social self: Assimilation and contrast in social comparison. Journal of Personality and Social Psychology. 57.825-838.

Pulakos, E.D., Schmitt, N., Dorsey, D.W., Hedge, J.W., \& Borman, W.C. (2002). Predicting adaptive performance: Further tests of a model of adaptability, Human Performance, 15, 299-323.

Ryan, R.M., \& Deci, E.L. (2000). Self-determination theory and the facilitation of intrinsic motivation, social development, and well-being. American Psychologist. 55.68-78.

Ryff, C., \& Essex, M.J. (1992). The interpretation of life experience and well-being: The sample case of relocation. Psychology and Aging. 7.507-517.

Seligman, M.E.P. (1991). Learned optimism. New York: Random House.

Shin, D.C., \& Johnson, D.M. (1978). Avowed happiness as an overall assessment of the quality of life. Social Indicators Research. 5, 475-492.

Spence, G., Oades, L.G., \& Caputi, P. (2004). Trait emotional intelligence and goal self-integration: Important predictors of emotional well-being. Personality and Individual Differences. 37.449-461.

Vanderberg, R. J., \& Lance, Ch. E. (1992). Examining the causal order of job satisfaction and organizational commitment. Journal of Management, 18 (1), 153-167. doi: 10.1177/014920639201800110

Weiner, B. (1992). Human motivation: Metaphors, theories and research. Newbury Park, California: Sage. 


\title{
AN IMPLICIT MODEL OF ASSESSMENT OF ATTITUDE TO HEALTH OF SPECIALISTS IN AN ORGANIZATION
}

\author{
Elena Rodionova ${ }^{1}$, Vladislav Dominiak ${ }^{2}$, German Nikiforov $^{1}$, \& Zoya Dudchenko ${ }^{1}$ \\ ${ }^{I}$ Department of Psychology, St. Petersburg State University (Russia) \\ ${ }^{2}$ Graduate School of Management, St. Petersburg State University (Russia)
}

\begin{abstract}
Attitude to health can be considered as one of the most important factors of efficiency and professional success of employees today, as a regulator of human behavior in a challenging and controversial professional situation. Studies of psychologists (starting with R. La Pierre's phenomenon, 1934) often fix the discrepancy between the declared attitude to health and true attitude and behavior. The imperfection of methods of diagnostics of attitude to health may be one of the reasons for such discrepancy. The authors suggest studying the attitude to health of specialists in an organization not only by traditional survey methods (for example, R.A. Berezovskaya's attitude-to-health questionnaire, a questionnaire on studying the barriers of health seeking behavior by Nikiforova G.S. and Dudchenko Z.F.), but also by means of an implicit method (based on the priming effect, implicit associative test). The article presents the results of the study conducted by using the implicit methodology for studying the attitude to health, which is based on a model of polar values.
\end{abstract}

Keywords: Implicit method, attitude to health, occupational health, dual model.

\section{Introduction}

The relevance of the study of attitude to health with the purpose of predicting an employee's professional conduct in situations related to a high level of stress, hazardous production and the importance of a clear and rapid response to a professional situation is acute in organizational psychology, where there is a likelihood (and sometimes it is very high) of life- and health-threatening situations (in the profession of oil refining industry specialists, specialists responsible for the life and health of other people, pilots, engine-drivers, doctors, etc.). For some professions a stress factor is concurrent, it is associated with experiencing mental tension due to the highest responsibility in making decisions. Under current conditions, health gains an economic value and there is no doubt about the relevance of maintaining occupational health. Prediction of an employee's behavior in such situation is one of the key tasks that can be resolved by psychological methods.

Attitude to health is understood as "a system of individual selective links of a person with various phenomena of the surrounding reality, contributing to or, conversely, threatening human health, as well as determining personal assessment of physical and mental state" [Zhuravleva I.V.].

A number of authors (G. Allport, 1935; Haber and Fried, 1975; Rokic, 1960) think that attitude leads to certain behavior, i.e. attitude determines behavior.

A concept of "implicit personality theory" was proposed by J. Bruner and R. Tagiuri in 1954 and is still used to denote the unconscious hierarchical system of ideas about the mental structure of other people. Two main approaches are distinguished in the study of implicit personality theories - traditional and alternative (psycho-semantic) ones. The traditional direction is represented by the works of J. Bruner and R. Tagiuri, common sense psychology by L. Ross, a causal attribution theory by G. Kelly, D. Shader, etc. An alternative approach, which was called like that by its ancestor J. Kelly, originated in line with a personal construct theory and was developed as a psycho-semantic direction (P. Vernon, V.F. Petrenko, A.G. Shmelev, and others). Representatives of the latter approach, in addition to highlighting the substantial components of the implicit personality theory, conduct a factor analysis, which allows evaluating and combining the qualities and links between separate components into a personal semantic space.

In recent years, when psychological studies of social attitudes were conducted, interest in the use of a special subgroup of indirect measuring methods (implicit methods) has grown significantly 
(Greenwald, McGhee \& Schwartz, 1998; Nosek \& Banaji, 2001; De Houwer, 2003; Fazio \& Olson, 2003, T.G. Stefanenko, 2008). The active use of these methods in this area of research was due to the fact that they allow to study "true" and not only declared socially desirable attitudes, as well as to avoid the effect of insufficient introspection, the "Rosenberg effect" and other distorting effects. The authors have suggested that such implicit methods may also be applicable to the attitude to health; however the authors do not know such works.

\section{Methods}

In the framework of this project the authors aim to explore the possibility of predicting human behavior in tense and dangerous professional situations based on an employee's "attitude to health" profile. For this purpose, the following tasks have been formulated: 1) to analyze existing methods of study of the attitude to health (identifying rather a declared attitude to health) and to develop an implicit method that would allow to study the deepest level of attitude to health, 2) to empirically test a dual model of attitude to health, 3) to analyze the results of the implicit diagnostics of attitude to health and distinguish types of employees in relation to real (objective) data on health groups.

In the study the authors used an implicit method, which has recently become widely used in the study of attitudes. This method is based on the priming effect, measuring the time of reaction of test subjects (Greenwald, McGhee \& Schwartz, 1998; Nosek \& Banaji, 2001; De Houwer, 2003; Fazio \& Olson, 2003; Stefanenko, 2008). Allowing excluding a factor of social desirability, this method reveals the inner, hidden attitudes of a person. In this study an implicit method is used to study the attitude to health, which is a relatively new approach.

One hundred and fifty-two people engaged in professional activities took part in testing the model. The average age of respondents was 26 years old. At this stage of the study the authors did not take into account the scope of activities, it was important for us that respondents worked in organizations. Besides, such indicators as self-assessment on a health scale, a health group and quality-of-life assessment were an important criterion for assessing the results. The study contains the data on internal/external goals (Desi and Ryan's concept).

\section{Findings and discussion}

Implicit characteristics of attitude to health and illness are studied in a dual model. A model is understood as an object that reflects the most important features of the studied phenomenon, object or process. In our case, these are attitudes to such concepts as "health" and "illness". The authors assume that there may be several variations of models of one and the same object and several objects can be described by a single model. In our study, the authors propose a dual model, which is shown in Figure 1.

Figure 1. A dual model of attitude to health

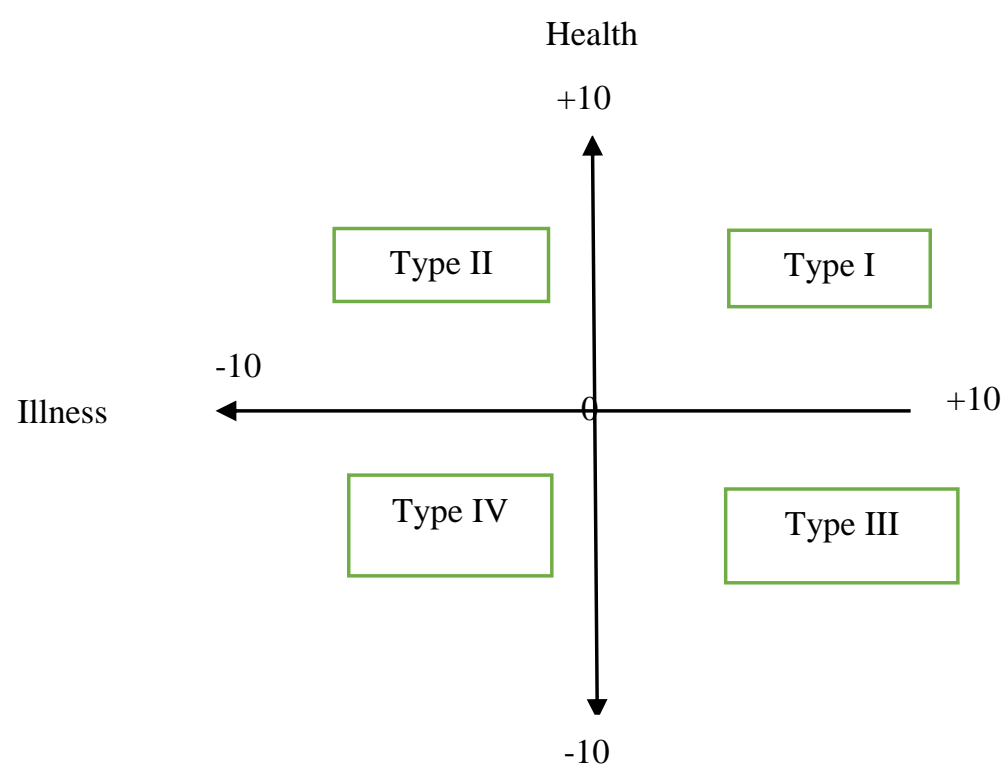


Hypothetically, four types of employees are distinguished in this model; they have qualitative characteristics and belong to risk groups, where Type I is the lowest risk group, Type IV - the highest risk group, which includes employees, who are most at risk of losing health and prone to occupational injuries. It is dangerous to engage such employees in the work on challenging and stressful work sites.

Implicit assessments of the attitude to health and illness were confirmed in health groups of the respondents: Health Group 1 (employees who get sick very rarely) included Type I and Type II respondents (positive attitude to health), Health Groups 2 and 3 included Type III respondents and Health Group 4 (employees who get sick very often) included Type IV employees. Thus, an implicit attitude to health and illness is directly related to the respondents' quality of life and the number of illnesses (health group). At the same time, the self-assessment of one's state of health is not related to the implicit attitude. The authors got a direct correlation of health self-assessment with a positive attitude to illness, i.e. employees really assess an illness as a positive state and highly assess their state of health.

Interesting indicators were the data on the ratio of types of employees with behavioral characteristics that contribute to maintaining a healthy lifestyle (sporting activities, active recreation, the ratio of work and rest, cold water treatment, balanced diet, etc.) or, on the contrary, causing the adherence to destructive lifestyles (alcohol consumption, eating junk food, etc.). At the same time, Type I and Type II employees adhered to behavioral characteristics of a healthy lifestyle, unlike Type III and Type IV employees. The largest distribution of destructive behavioral norms was observed in the group of Type IV employees. A qualitatively significant difference in rational habits of maintaining a healthy lifestyle was observed in Type I and Type II respondents. Type I respondents actively kept a healthy lifestyle through a balanced diet, an active lifestyle, but their average duration of sleep and rest was statically significantly lower than that of Type II employees.

Thus, the implicit attitude to health was confirmed by behavioral manifestations of the respondents and the objective data on health indicators (health group). Besides, the authors have not revealed a connection between the self-assessment of the attitude to health and implicit evaluations.

\section{Conclusion(s) and recommendations/future directions}

The study of the attitude to health in the context of professional activity is clearly of interest, since it reveals not only the possibilities of preventing the specialist's health problems, but also gaining theoretical and practical knowledge that allows creating conditions for preservation and development of regulatory properties of the organism, its physical, mental and social well-being. In turn, this ensures high reliability of professional activity, career longevity and maximum life expectancy. In this case, it is important to develop models of a professionally healthy employee and methods of diagnostics of the attitude to health.

The authors assume that not a single model can fully replace the studied object, since it displays only some of its properties. But sometimes, when solving certain tasks, in our case - the identification of types of employees that can be successful in a tense and dangerous professional activity, the dual model of attitude to health can be interesting and practically significant.

The study of the ratio of the true profound attitude to health and professional behavior also remains relevant. There is a need for a theoretical model of "occupational health" based on the identification and classification of implicit and explicit factors that shape the attitude to health.

The authors suppose that the study of a person's attitude to health, to the issues of maintaining occupational health can fully reveal the nature and structure of a healthy personality, solve theoretical-methodological and methodical issues in developing programs of psychological support for specialists in maintaining occupational health.

\section{Acknowledgements}

The article was prepared with the support of the RFBR grant, project number 18-013-00568. 


\section{References}

Dominyak, V.I. Implicit diagnostics of personal values (article). - Scientific-Technical Bulletin of Saint-Petersburg State Polytechnical University. Series "Humanities", no. 1(143), 2012, pp.137-143.

Nikiforov, G.S., Rodionova, E.A., Vodopyanova, N.E., Dudchenko, Z.F Psychological factors of professional burnout// International scientific-practical congress of pedagogues, psychologists and medical workers "Grow up", the 30th of November, 2016, Geneva (Switzerland)/ Publishing Center of the European Association of pedagogues and psychologists "Science", Geneva, 2016, pp.74-79.

Management Psychology. Theory and Practice / edited by Nikiforov G.S. - Saint-Petersburg, Rech Publ., 2010. 816 p.

Rodionova, E.A., Dominyak, V.I. Socio-psychological characteristics of occupational health of specialists (article). Ananyevskie Readings - 2013. Psychology in Health Care: Materials of the scientific conference, October 22-24, 2013/Editor-in-chief O.Yu. Shchelkova. - Saint-Petersburg: Skufia-Print, 2013, pp. 362-364. 


\title{
DECISION TREES: COMPARING REPORTED INTENTIONS AND THEORETICALLY EXPECTED INTENTIONS IN URBAN CONTEXT
}

\author{
Jimmy Bordarie \\ Departement Techniques of Commercialization, EE 1901 QualiPsy, University of Tours (France)
}

\begin{abstract}
The research concerns the validation of an extended version of the theory of planned behaviour (TPB). This model argues that the prediction of intentions depends on three determinants: attitude, perceived social norms and behavioural control. Our model integrates cognitive aspects (such as beliefs, importance or perceived effect) and past behaviours (based on habits such as frequency or duration of behaviour).

The second objective is to provide a differential perspective. The construction of a decision tree will make it possible to distinguish and to compare reported intention and the expected intention that participants should have reported based on the prediction model.

In order to test our model, we are interested in a new public policy in Angers (France, Pays de la Loire). The latter concerns traffic calming with the generalised reduction of the speed limit from $50 \mathrm{~km} / \mathrm{h}$ to $30 \mathrm{~km} / \mathrm{h}$. We are studying the intention of young drivers to respect this $30 \mathrm{~km} / \mathrm{h}$ speed limit.

Participants are drivers $(n=129$, Mean Age $=22.4$ y.o., $S D=3.8$ ). They completed a questionnaire based on the extended version of the TPB model related to the $30 \mathrm{~km} / \mathrm{h}$ speed limit. Bayesian analyses are used to do the linear regression and build the decision tree.

The results confirmed the relevance of the model which is able to explain $53 \%$ of the behavioural intention variance. Linear regression confirmed previous results from the literature: attitude and behavioural control are the two most significant determinants of intention prediction according to TPB. In addition, perceived importance and frequency are two significant dimensions in this study. The decision tree is doubly relevant. On the one hand, it determines the most significant questions for predicting intent in nearly $60 \%$ of cases, and it is much better than a random prediction $(\mathrm{p}=.01)$. On the other hand, it identifies drivers who report an intention that matches their answers to other questions and drivers who report a dissonant intention in relation to their answers to significant questions in the model.

The study provides support to politicians, researchers and communicators who are charging the implementation of this type of measures. Awareness campaigns can have an impact on representational aspects in order to develop a sense of perceived importance. Urban planning in conjunction with public policies to reduce speed limit can also help to increase the frequency of behaviour related to compliance with the $30 \mathrm{~km} / \mathrm{h}$ and to perceive this speed as legitimate.
\end{abstract}

Keywords: Theory of planned behavior, past behaviors, decision trees, public policy, speed limit.

\section{Theoretical framework}

\subsection{Decision trees}

Decision trees can help to predict certain behaviours (Quinlan, 1993). They are easy to interpret and understand to predict situations and/or determine outcome preferences in different contexts. In this study, the decision tree compares drivers' self-reported intentions and their intentions predicted by their answers to a questionnaire. Ordinary trees consist of a root, branches, nodes and leaves. In statistics, decision trees are composed of circles (nodes) and segments (branches). The first node is the root. Two or several branches may grow from it. The last node of the chain is a leaf and no branches grow from it. Each node represents a variable, and branches give a set of values, which can be predicted from observations of individuals, social groups, or specific characteristics. Decision trees have already been used in medicine (Li, Gluer, Eastell, Felsenberg, Reid, Rox and Lu, 2012; Podgorelec, Kokol, Stiglic and Rozman, 2002), and notably to identify significant predictors of child abuse in Canada (Fallon, Ma, Allan, Pillhofer, Trocmé and Jud, 2013). 


\subsection{Theory of planned behaviour}

This research is based on the Theory of Planned Behaviour (TPB) (Ajzen, 2011; Ajzen and Albarracin, 2007). According to this model, intention to enact a behaviour can be predicted by taking into account three factors: attitude, subjective norms and perceived behavioural control (PBC). Attitude refers to the individual's positive or negative evaluation of a specific behaviour. Subjective norms are based on whether individuals think that people of importance to them (e.g. parents, friends) approve or disapprove of the behaviour. Finally, PBC refers to individuals' perceptions of their capacity to achieve the behaviour. These three factors are also impacted by beliefs (Emin, 2003) and past behaviors (Ajzen, 1991). The latter are the best predictor of future behaviour (Conner and Armitage, 1998; Ouellette and Wood, 1998). In this study, we especially focus on the influence of social-cognitive factors on the intention to comply with the specific speed limit of 30km/h. Research has shown the relevance of the TPB for mobility, risk-taking and traffic issues by studying the consumption of alcohol (McGhie, Lewis and Hyde, 2012; Moan and Rise, 2011), the use of mobile phones (Przepiorka, Błachnio and Sullman, 2018; Waddell and Wiener, 2014), the behaviour of pedestrians (Xu, Li and Zhang, 2013) and motorcyclists (Özkan, Dogruyol, Lajunen, Yıldırım and Çoymak, 2012). The TPB has also been applied to the compliance with speed limits, as well for motorists as motorcyclists (Chorlton, Conner and Jamson, 2012; Elliott, 2010; Eyssartier, 2012; Leandro, 2012; Wallén Warner and Åberg, 2008).

\subsection{Self-report habit index}

According to the contribution of past behaviours in the prediction of future behaviours, studies have tried to combine the TPB model with the Self-Report Habit Index (SRHI) (Cestact, 2009). The SRHI (Verplanken and Orbell, 2003) measures several dimensions of habit: automatic activation, perceived ease, behavioural frequency, relevance to self-identity and history of enactment. It has been used in various fields, and notably in the field of transportation and travel mode choices (Aarts, Verplanken and von Knippenberg, 1997; Verplanken, Myrbakk and Rudi, 2005). But it has never been used to understand drivers' habits with regard to speed or compliance with speed limits. However, drivers often object to speed limit reduction measures because compliance with speed limits usually depends on two major factors. Environmental conditions (curved or straight sections, visibility, reliability of road signs and posted speed limits) are one of the most impact factor influencing drivers' speed (Fitzpatrick, Carlson, Brewer and Wooldridge, 2001). This point refers to drivers' habits to comply with speed limit according to environmental circumstances. Secondly, drivers generally drive faster than the limit that they themselves regard as being safe (Goldenbeld and van Schagen, 2007). This point refers to drivers' beliefs in circumstances in which they can comply or not speed limits.

\subsection{Aims of the current study}

The purpose of the current study is to understand drivers' intentions by comparing drivers' self-reported intentions and the theoretical intentions expected from the drivers' answers given to different variables. From a theoretical perspective, this research aims to provide information on socio-cognitive determinants of drivers' decisions. From an applied perspective, the study aims to facilitate the implementation of speed limit reduction measures.

\section{Method}

\subsection{Participants}

Participants (n=129, Mean Age=22.4 y.0., SD=3.8, range: 18-30) are drivers and students at the University of Angers. There are $78 \%$ females and $22 \%$ males. They anonymously answer an online questionnaire in French. They were informed that their participation was voluntary and unpaid.

\subsection{Instrument}

The questionnaire comprised fifteen items, based on the TPB and the SRHI. There were five items related to the TPB (attitude, subjective norms, PBC). Three of them were related to subjective norms according to different social referents (parents, friends, motorists). Five further items were related to beliefs and identification. According to the SRHI, four items were related to habits such as frequency, automatic activation history of enactment and ease. Questions were presented randomly. Answers were given on a 6-point scale from 1 (completely disagree) to 6 (totally agree). 


\subsection{Analysis}

Cronbach's alpha $(\alpha=.92)$ indicates very good reliability (Nunnaly, 1978). Analyses were conducted using R software and Bayesian analysis. Regarding our methodology with R software, we used the BayesFactor package and the regressionBF function. The calculation method is based on the JZS (Rouder, Speckman, Sun, Morey and Iverson, 2009). Here, the dependent variable is the intention and the 14 other questions are used as independent variables. The determinants explained $53 \%$ of the variance for intention to comply with the $30 \mathrm{~km} / \mathrm{h}$ speed limit $[\mathrm{F}(14,376)=30.25, \mathrm{p}<.0001, \mathrm{r} 2=.53]$. This is a very high value with regards to the average explanation of the TPB around 39\% (Armitage and Conner, 2001).

\section{Results}

\subsection{Construction of the decision tree}

The construction of the decision tree is based on the CART algorithm (Breitman, Friedman, Olshen and Stone 1984). Each node divides the tree into two branches that lead to variables. The first node (top of the tree) reveals the most significant variable splitting the sample into two groups. Each branch concerns the part of the sample answering upper than the modal value or answering lower than the modal value. At the end of the tree (bottom), the last leaf indicates the theoretical intention to comply with the $30 \mathrm{~km} / \mathrm{h}$ speed limit. This intention is represented by a number between 1 and 6 ; the higher the number, the greater the theoretical intention. This theoretical intention is resulting from drivers' answers to the different questions selected for the model.

Figure 1. Decision tree contributing to predict the intention of driving at $30 \mathrm{~km} / \mathrm{h}$.

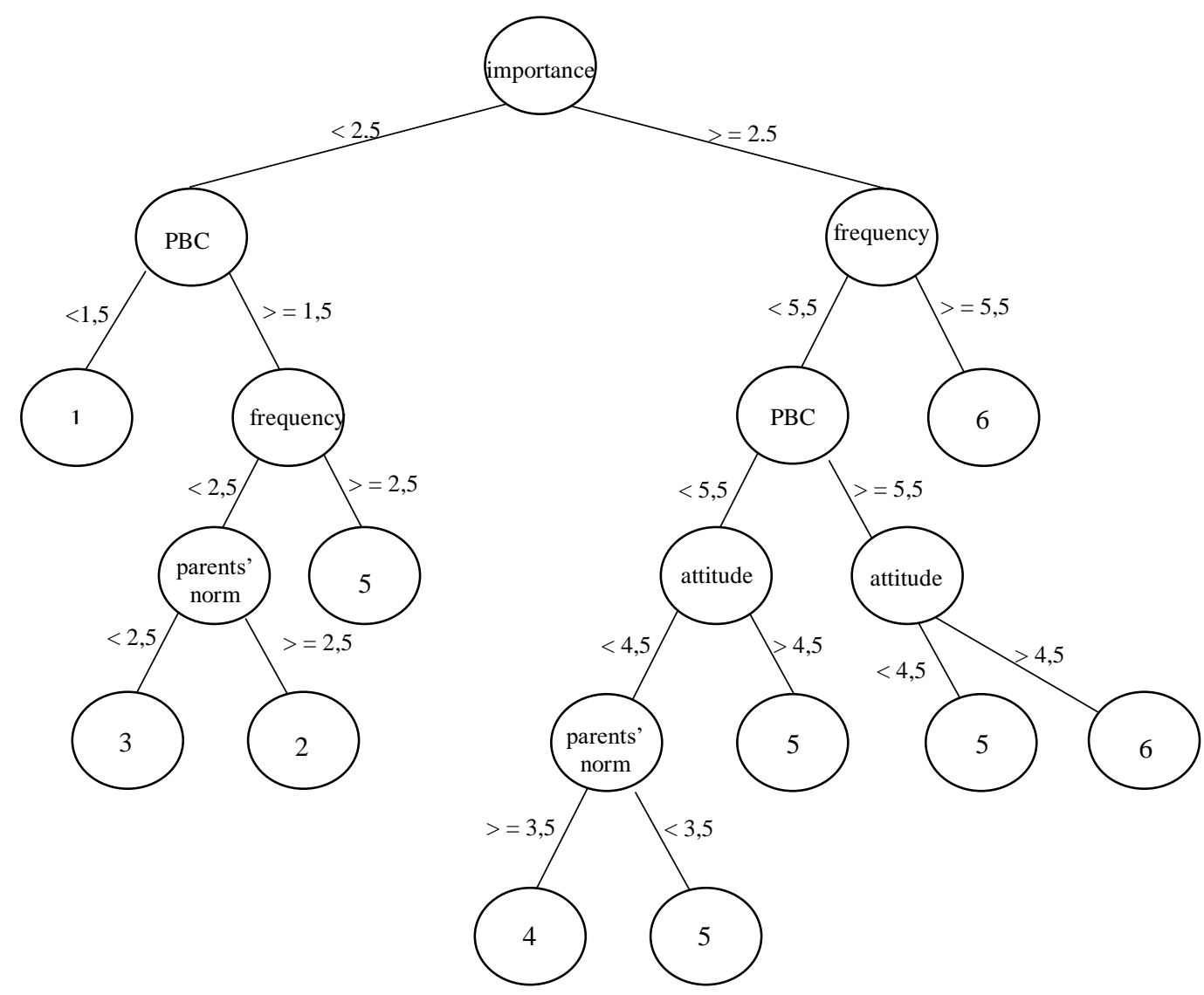

The model that we can use to predict participants' intentions to comply with the $30 \mathrm{~km} / \mathrm{h}$ speed limit is illustrated on Figure 1 and it refers to the variable perceived importance, frequency, PBC, attitude and eventually perceived parental norm. The number at the end of the tree corresponds to the theoretically expected intention of the participant. We can then compare this theoretical answer (prediction) with the participant's self-reported intention (intention) (Table 1). Numbers in bold correspond to the number of participants for whom the predicted and self-reported intention values were the same. 
The decision tree in our model has a significant predictive value of drivers' intention to comply with the $30 \mathrm{~km} / \mathrm{h}$ speed limit. It gives an exact prediction for $58.1 \%$ of the participants (i.e. 75 participants) when random predictions would obtain an exact prediction for $16.67 \%$. The difference between exact predictions and random predictions is significant $\left(\chi^{2}\right.$ Yates Correction $(Y C)=14.47, \mathrm{df}=5, \mathrm{p}<.013$ ). The same can be calculated for acceptable predictions (equal to or within one point of difference with the participant's answer). This calculation gives an acceptable prediction for $84.5 \%$ of the participants (i.e. 109 participants) when the random predictions would be acceptable for $44.4 \%$. The difference between acceptable predictions and random predictions is significant $(\chi 2 \mathrm{YC}=22.84, \mathrm{df}=5, \mathrm{p}<.001)$.

Table 1. Data of the first group for constructing the decision tree.

\begin{tabular}{|c|c|c|c|c|c|c|c|}
\hline \multicolumn{8}{|c|}{ Prediction } \\
\hline \multirow{7}{*}{ Intention } & & 1 & 2 & 3 & 4 & 5 & 6 \\
\hline & 1 & 5 & 0 & 1 & 0 & 0 & 0 \\
\hline & 2 & 1 & 7 & 3 & 2 & 3 & 0 \\
\hline & 3 & 0 & 3 & 6 & 3 & 8 & 0 \\
\hline & 4 & 0 & 2 & 0 & 19 & 12 & 2 \\
\hline & 5 & 1 & 0 & 0 & 4 & 23 & 3 \\
\hline & 6 & 0 & 0 & 0 & 1 & 5 & 15 \\
\hline
\end{tabular}

\section{Discussion}

The results confirmed the relevance of combining the TPB and SRHI variables for predicting drivers' intention to comply with the $30 \mathrm{~km} / \mathrm{h}$ speed limit. The study confirms that habits such as the frequency of doing the behaviour influences intentions. Results also confirm the role of beliefs, such as the perceived importance of the speed limit, confirming the relationship between legitimacy and compliance with speed limits (Goldenbeld and van Schagen 2007).

Using a decision tree to predict intention is more effective than traditional predictions like linear regression because it makes possible the comparison between self-reported intentions and theoretical intentions expected from participants' answers about the other variables. When the two values differ, it would be interesting to examine in more depth the reasons for the discrepancy and rationalization process (Bordarie, 2015, 2017).

This study has some limitations starting with the sample which has the concern of not-being representative of the general population as it was composed of a majority of female students. Moreover, there is an issue with regards to the self-reported data that can imply social desirability related to the compliance with laws. However, guaranteeing anonymity and confidentiality should have minimized this effect. Finally, we should remind that intentions and behaviours are different.

\section{References}

Aarts, H., Verplanken, B., \& Knippenberg, A. van (1997). Habit and information use in travel mode choices. Acta Psychologica, 96, 1-14. https://doi.org/10.1016/S0001-6918(97)00008-5

Ajzen, I. (1991). The theory of planned behavior. Organizational Behavior and Human Decision Processes, 50, 179-211. https://doi.org/10.1016/0749-5978(91)90020-T

Ajzen, I. (2011). The theory of planned behaviour: Reactions and reflections. Psychology and Health, 26, 1113-1127. https://doi.org/10.1080/08870446.2011.613995

Ajzen, I., and Albarracin, D. (2007). Predicting and changing behavior: A reasoned action approach. In I. Ajzen, D. Albarracin, and R. Hornik (Eds.), Prediction and Change of Health Behavior: Applying the reasoned action approach (pp. 3-21). Mahwah, NJ: Lawrence Erlbaum Associates Publishers.

Armitage, C. J., and Conner, M. (2001). Efficacy of the theory of planned behaviour: A meta-analytic review. British journal of social psychology, 40(4), 471-499. https://doi.org/10.1348/014466601164939

Bordarie, J. (2015). Représentations sociales et mobilité urbaine durable. Thèse de Doctorat, Université d'Angers.

Bordarie, J. (2017). Rationalization processes between social representations and semantic blocks theory. In D. Compagno (Dir.), Quantitative Semiotic Analysis. Springer.

Breitman, L., Friedman, J.H., Olshen, R.A., and Stone, C.J. (1984). Classification and Regression Trees. Wadsworth, Belmont, California.

Cestac, J. (2009). Habitude et principe de compatibilité dans le modèle du comportement planifié: décisions pro-environnementales et automobile. Thèse de Doctorat, Université de Nanterre-Paris X. 
Chorlton, K., Conner, M., and Jamson, S. (2012). Identifying the psychological determinants of risky riding: An application of an extended Theory of Planned Behaviour. Accident Analysis and Prevention, 49, 142-153. https://doi.org/10.1016/j.aap.2011.07.003

Conner, M., and Armitage, C. J. (1998). Extending the theory of planned behavior: A review and avenues for further research. Journal of Applied Social Psychology, 28, 1429-1464. https://doi.org/10.1111/j.1559-1816.1998.tb01685

Elliott, M.A. (2010). Predicting motorcyclists' intention to speed: Effects of selected cognitions from the theory of planned behaviour, self-identity and social identity. Accident Analysis and Prevention, 42, 718-725. https://doi.org/10.1016/j.aap.2009.10.021

Emin, S. (2003). L'intention de créer une entreprise des chercheurs publics: le cas français. Thèse de doctorat, Université Pierre Mendès France, Grenoble, France.

Eyssartier, C. (2012). Dépassement de la limitation de vitesse sur route limitée à $90 \mathrm{~km} / \mathrm{h}$ : étude sur les motards peu expérimentés du département de la Sarthe. In S. Gaymard and A. Egido (Eds.), Sécurité et facteurs humains dans les transports: une approche multidisciplinaire (pp. 253-266). Paris: L'Harmattan.

Fallon, B., Ma, J., Allan, K., Pillhofer, M., Trocmé, N., and Jud, A. (2013). Opportunities for prevention and intervention with young children: lessons from the Canadian incidence study of reported child abuse and neglect. Child and adolescent psychiatry and mental health, 7(1), 4. https://doi.org/10.1186/1753-2000-7-4

Fitzpatrick, K., Carlson, P., Brewer, M., and Wooldridge, M. (2001). Design factors that affect driver speed on suburban streets. Transportation Research Record: Journal of Transportation Research Board, 1751, 18-25. https://doi.org/10.3141/1751-03

Goldenbeld, C., and Schagen, I. van (2007). The credibility of speed limits on $80 \mathrm{~km} / \mathrm{h}$ rural roads: the effects of road and person(ality) characteristics. Accident Analyses and Prevention, 39 (6), 1121-1130. https://doi.org/10.1016/j.aap.2007.02.012

Leandro, M. (2012). Young drivers and speed selection: A model guided by the Theory of Planned Behavior. Transportation Research Part F: Traffic Psychology and Behaviour, 15(3), 219-232. https://doi.org/10.1016/j.trf.2011.12.011

Li, C., Gluer, C.C., Eastell, R., Felsenberg, D., Reid, D.M., Rox, D.M., and Lu, Y. (2012). Tree-structured subgroup analysis of receiver operating characteristic curves for diagnostic tests. Academic Radiology, 19(12), 1529-1536. https://doi.org/10.1016/j.acra.2012.09.007

McGhie, A., Lewis, I., and Hyde, M.K. (2012). The influence of conformity and group identity on drink walking intentions: comparing intentions to drink walk across risky pedestrian crossing scenarios. Accident Analysis and Prevention, 45(2), 639-645. https://doi.org/10.1016/j.aap.2011.09.035

Moan, I., and Rise, J. (2011). Predicting intentions not to "drink and drive" using an extended version of the theory of planned behavior. Accident Analysis and Prevention, 43(4), 1378-1384. https://doi.org/10.1016/j.aap.2011.02.012

Nunnaly, J. (1978). Psychometric theory. New York: McGraw-Hill.

Ouellette, J. A., and Wood, W. (1998). Habit and intention in everyday life: The multiple processes by which past behavior predicts future behavior. Psychological Bulletin, 124, 54-74. https://doi.org/10.1037/0033-2909.124.1.54

Podgorelec, V., Kokol, P., Stiglic, B., and Rozman, I. (2002). Decision trees: an overview and their use in medicine. Journal of medical systems, 26(5), 445-463. https://doi.org/10.1023/A:1016409317640

Przepiorka, A. M., Błachnio, A. P., and Sullman, M. J. (2018). Factors influencing intentions to text while driving among Polish drivers. Transportation Research Part F: Traffic Psychology and Behaviour, 55, 306-313. https://doi.org/10.1016/j.trf.2018.03.015

Quinlan, J.R. (1993). C4.5: Programs for Machine Learning, Morgan Kaufmann, San Francisco.

Rouder, J.N., Speckman, P.L., Sun, D., Morey, R.D., and Iverson, G. (2009). Bayesian t-tests for accepting and rejecting the null hypothesis. Psychonomic Bulletin and Review, 16, 225-237. https://doi.org/10.3758/PBR.16.2.225

Verplanken, B., and Orbell, S. (2003). Reflections on Past Behavior: A Self-Report Index of Habit Strength1. Journal of Applied Social Psychology, 33(6), 1313-1330. https://doi.org/10.1111/j.15591816.2003.tb01951.x

Waddell, L. P., and Wiener, K. K. K. (2014). What's driving illegal mobile phone use? Psychosocial influences on drivers' intentions to use handheld mobile phones. Transportation Research Part F: Traffic Psychology and Behaviour, 22, 1-11. https://doi.org/10.1016/j.trf.2013.10.008

Wallén Warner, H., and Åberg, L. (2008). Drivers' beliefs about exceeding the speed limits. Transportation Research Part F: Traffic Psychology and Behaviour, 11, 376-389. https://doi.org/10.1016/j.trf.2008.03.002

Xu, Y.S., Li, Y.J., and Zhang, F. (2013). Pedestrians' intention to jaywalk: Automatic or planned? A study based on a dual-process model in China. Accident Analysis and Prevention, 50, 811-819. https://doi.org/10.1016/j.aap.2012.07.007 


\title{
THE HEXACO PERSONALITY MEASURE AS A PREDICTOR OF JOB PERFORMANCE AND JOB SATISFACTION
}

\author{
Gaye Saltukoğlu ${ }^{1}$, Arkun Tatar ${ }^{1}, \&$ Hüdanur Özdemir ${ }^{2}$ \\ ${ }^{I}$ Department of Psychology, FSM Vaklf University / Associate Professor (Turkey) \\ ${ }^{2}$ Psychologist (Turkey)
}

\begin{abstract}
The study aimed to examine the relationship between personality traits according to the Six Factor Personality Inventory (HEXACO), and job performance and job satisfaction. A total of 852 people (433 women and 419 men) from 154 different professions participated in the study. The age range of the participants was between 20 and 74, and they were working at a job for a period between 1-40 years. Participants answered the HEXACO Personality Inventory long form and evaluated their job performance and job satisfaction on a scale between 1 and 5 . To predict job performance and job satisfaction, the data was analyzed by multiple linear regression. The sub-dimensions of Modesty, Fairness, Social Self-Esteem, Liveliness, Perfectionism, Diligence and the factors of Extraversion, Conscientiousness predicted job performance, and sub-dimensions of Modesty, Liveliness, Social Boldness, Forgiveness, Patience, Diligence and the factors of Extraversion, Agreeableness, and Conscientiousness predicted job satisfaction. Afterwards, job performance and job satisfaction levels of the participants were grouped as low, moderate, high. Total HEXACO scores of these groups were compared by MANOVA for sub-dimensions and factors. Job performance groups differed in the factors of Honesty-Humility, Extraversion, Conscientiousness, Openness to Experience, and job satisfaction groups differed in the factors of Extraversion, Agreeableness, and Conscientiousness. As for the sub-dimensions, groups that were high in job performance and job satisfaction differed from the groups that were low in many personality traits.
\end{abstract}

Keywords: Job performance, job satisfaction, HEXACO, personality.

\section{Introduction}

Various factor analytic studies have revealed three, five, six, seven and eight factor theories to assess personality (Almagor, Tellegen and Waller, 1995; Barrett, Petrides, Eysenck and Eysenck, 1998; Caprara, Barbaranelli and Comrey, 1992; Eysenck, Eysenck and Barrett, 1985; Hogan and Hogan, 2007; Goldberg, 1990; McCrae and Costa, 1987; Saucier, 2002; Zuckerman, Kuhlman, Joireman, Teta and Kraft, 1993). Even though the Five Factor Model has been widely used in personality research, there is no consensus about which model better explained personality traits (Block, 1995; Piedmont and Aycock, 2007; Simms, Williams and Nus, 2017). On the other hand, the HEXACO Personality Model has been revealed relatively recently and its validity has been tested in many languages. For this reason, in recent studies this model excels (Ashton and Lee, 2002; 2007; Ashton, Lee and Goldberg, 2004; Lee and Ashton, 2008; 2018). The psycho-lexical approach to personality structure rests on the assumption that personality variation would be represented by adjectives in all languages, and would helps to differentiate most important personality traits. The main difference that distinguishes the HEXACO Model from the Five Factor Model is the sixth factor that has emerged in the psycho-lexical studies and included in the model. This factor is called Honesty-Humility (Allport and Odbert, 1936; Ashton and Lee, 2005; 2008a; 2008b; Goldberg, 1993; Lee, Ogunfowora and Ashton, 2005; Lee and Ashton, 2014). The inclusion of the sixth factor into the model of personality taxonomy, made the HEXACO Model more compressive and powerful than the Five Factor Model (Ashton, Lee, de Vries, Perugini, Gnisci and Sergi, 2006; Ashton, Lee, Perugini, Szarota, de Vries, di Blas, Boies and de Raad, 2004). The HEXACO model consists of Humility-Honesty, Emotionality, Extraversion, Agreeableness, Conscientiousness and Openness to Experience factors (Ashton and Lee, 2009a; Lee and Ashton, 2004).

The relationship between personality, and job performance and job satisfaction is an often discussed topic in the related studies (Abraham and Morrison, 2003; Rothmann and Coetzer, 2003; Saltukoğlu and Tatar, 2018). It is also seen that the Five Factor Model of personality is the preferred model (Barrick and Mount, 1991; 1993; Barrick, Stewart and Piotrowski, 2002; Mount, Barrick, and Stewart, 1998; Salgado, 1997; Saltukoğlu and Tatar, 2018). The number of studies for business life seems to be on the rise with the widespread acceptance of the Five Factor Model and the development of valid 
measuring instruments based on this model. Thus, more systematic and comprehensive personality studies about job performance and job satisfaction were conducted (Alessandri and Vecchione, 2012; Barrick and Mount, 1991; 1993; Barrick, Mount and Judge, 2001; Judge and Zapata, 2015; Rothstein and Goffin, 2006; Salgado, 1997; Zhao and Seibert, 2006). On the other hand, selecting the proper individuals for the job is important. Selecting individuals with appropriate personality characteristics for the job is important in terms of job satisfaction and job performance (Guion and Gottier, 1965; Rothstein and Goffin, 2006).

Although there are many studies that associate the Five Factor model is associated with job satisfaction and job performance, similar studies conducted with the relatively new HEXACO Model are rare. For this reason, in this study it is intended to examine job performance and job satisfaction in the context of the HEXACO Personality Model. Thus, the aim of the study is to compare the personality profiles of the groups created according to the evaluations they made about their job performance and job satisfaction levels.

\section{Methods}

\subsection{Participants}

A total of 852 people working at a job for a year or longer and working in 154 different professions, 433 women (50.8\%) and 419 men (49.2\%) participated in the study. Their age range was between 20 and 74 (mean $=37.36$, sd = 10.73), and they worked at a job for a period between 1 and 40 years $($ mean $=13.30$, sd $=10.19)$. Participants consisted of 35 elementary school graduates $(4.1 \%), 47$ middle school graduates (5.5\%), 244 high school graduates (28.6\%) and 526 university graduates (61.7\%). Economic status of the participants was as follows: 42 (4.9\%) low income, 557 (65.4\%) middle income and $248(29.1 \%)$ high income. 5 people did not specify their income $(0.6 \%)$. Of all the participants 359 (42.1\%) were single, 448 (52.6\%) were married and 45 (5.3\%) were widowed or divorced.

\subsection{Instruments}

A socio-demographic questionnaire along with the Personality Inventory long form was used in the study. Participants evaluated their job performances and job satisfactions on a 1-5 point scale. The HEXACO Personality Inventory consists of six factors and 24 sub-dimensions located under these factors (Ashton, Lee and de Vries, 2014; Lee and Ashton, 2006). The inventory was translated into Turkish and its psychometric properties were tested (Tatar, 2018). The inventory has two forms, long form with 100 items and short form with 60 items (Ashton and Lee, 2009b; Lee and Ashton, 2004; 2006; 2018). Long form of the Turkish adaptation showed internal consistency coefficients ranging from 0.72 to 0.78 for six factors and from 0.29 to 0.77 for 24 sub-dimensions (Tatar, 2018).

\subsection{Application}

The study was conducted in Istanbul with those who voluntarily accepted to participate. It took 20-30 minutes for a person to complete the form. All applications were completed in nearly one year.

\subsection{Results}

First, internal consistence coefficients of the factors and the sub-dimensions were calculated and were found between 0.32-0.76 for the sub-dimensions and between 0.71-0.77 for the factors. Subsequently, to predict job performance and job satisfaction, the data was analyzed by multiple linear regressions. Social Self-Esteem, Liveliness, Perfectionism, Diligence sub-dimensions were found effective in predicting job performance and Modesty, Liveliness, Social Boldness, Forgiveness, Patience, Diligence sub-dimensions were found effective in predicting job satisfaction. Models generated to predict job performance $\left(\mathrm{R}^{2}=0.140, \mathrm{~F}_{24,851}=5.588, \mathrm{p}<0.001\right)$ and job satisfaction $\left(\mathrm{R}^{2}=0.100, \mathrm{~F}_{24,851}=3.826\right.$, $\mathrm{p}<0.001$ ) for sub-dimensions were statistically significant.

Table 1. To predict job performance and job satisfaction with factors of HEXACO Personality Inventory.

\begin{tabular}{|c|c|c|c|c|c|}
\hline \multirow{2}{*}{ Factors } & \multirow{2}{*}{ Alpha } & \multicolumn{2}{|c|}{ Job Performance } & \multicolumn{2}{|c|}{ Job Satisfaction } \\
\hline & & $\beta$ & $\mathbf{t}$ & $\boldsymbol{\beta}$ & $\mathbf{t}$ \\
\hline Constant & & & $7.825 * * *$ & & $3.818 * * *$ \\
\hline Honesty-Humility & 0.74 & -0.060 & -1.716 & 0.008 & 0.218 \\
\hline Emotionality & 0.71 & -0.052 & -1.544 & 0.003 & 0.081 \\
\hline Extraversion & 0.77 & 0.132 & $3.721 * * *$ & 0.142 & $3.902 * * *$ \\
\hline Agreeableness & 0.74 & 0.045 & 1.320 & 0.146 & $4.239 * * *$ \\
\hline Conscientiousness & 0.75 & 0.224 & $6.495 * * *$ & 0.088 & $2.497 *$ \\
\hline Openness to Experience & 0.74 & 0.035 & 1.001 & 0.011 & 0.318 \\
\hline
\end{tabular}


For the factors, Extraversion and Conscientiousness were found effective in predicting job performance, and Extraversion, Agreeableness, and Conscientiousness factors were effective in predicting job satisfaction (Table 1). Models generated for the prediction of job performance $\left(\mathrm{R}^{2}=0,093\right.$, $\left.\mathrm{F}_{6,851}=14,403, \mathrm{p}<0.001\right)$ and job satisfaction $\left(\mathrm{R}^{2}=0,057, \mathrm{~F}_{6,851}=8,461, \mathrm{p}<0.001\right)$ for the factors were statistically significant.

Afterwards, job performance and job satisfaction levels of the participants were grouped as low, moderate, high. The total factor scores of these groups were compared by MANOVA. Main effect was statistically significant in the comparison of total factor scores of job performance (Wilk's $\lambda=0.895, F_{12}$, $1688=8.008, \mathrm{p}<0.001$, partial $\eta^{2}=0.054$ ) and job satisfaction (Wilk's $\lambda=0.940, \mathrm{~F}_{12,1688}=4.412$, $\mathrm{p}<0.001$, partial $\eta^{2}=0.030$ ) groups. Factors when examined individually, job performance groups differed in the factors of Honesty-Humility $\left(F_{2}, 849=5.122, p<0.01\right.$, partial $\left.\eta^{2}=0.012\right)$, Extraversion $\left(\mathrm{F}_{2,849}=18.406, \mathrm{p}<0.001\right.$, partial $\left.\eta^{2}=0.042\right)$, Conscientiousness $\left(\mathrm{F}_{2,849}=27.924, \mathrm{p}<0.001\right.$, partial $\left.\eta^{2}=0.062\right)$, Openness to Experience $\left(F_{2,849}=6.898, p<0.001\right.$, partial $\left.\eta^{2}=0.016\right)$; as for job satisfaction groups, they differed in the factors of Extraversion $\left(F_{2}, 849=11.530, p<0.001\right.$, partial $\left.\eta^{2}=0.026\right)$, Agreeableness $\left(F_{2,849}=7.889, p<0.001\right.$, partial $\left.\eta^{2}=0.018\right)$, Conscientiousness $\left(F_{2,849}=9.083\right.$, $\mathrm{p}<0.001$, partial $\eta^{2}=0.021$ ) (Table 2).

Table 2. Comparison of total factor scores of job performance and job satisfaction groups by MANOVA.

\begin{tabular}{|l|r|r|r|r|}
\hline Factors & \multicolumn{2}{|c|}{ Job Performance } & \multicolumn{2}{c|}{ Job Satisfaction } \\
\hline & \multicolumn{1}{|c|}{$\mathbf{F}$} & Partial $\boldsymbol{\eta}^{2}$ & $\mathbf{F}$ & Partial $\boldsymbol{\eta}^{\mathbf{2}}$ \\
\hline Honesty-Humility & $5.122^{* *}$ & 0.012 & 1.299 & 0.003 \\
\hline Emotionality & 2.136 & 0.005 & .498 & 0.001 \\
\hline Extraversion & $18.406^{* * *}$ & 0.042 & $11.530^{* * *}$ & 0.026 \\
\hline Agreeableness & 0.990 & 0.002 & $7.889^{* * *}$ & 0.018 \\
\hline Conscientiousness & $27.924^{* * *}$ & 0.062 & $9.083^{* * *}$ & 0.021 \\
\hline Openness to Experience & $6.898^{* * *}$ & 0.016 & 2.116 & 0.005 \\
\hline
\end{tabular}

\section{Discussion}

This study aims to detect the personality traits predicting job performance and job satisfaction by the HEXACO model. To this end, first it has to be determined which personality traits in the HEXACO model predicts job performance and job satisfaction. Afterwards, it has been indented to determine whether employees with different job performance and job satisfaction levels differ in terms of personality traits. The originality of the study is the combination of employees from many different professions. The presence of employees from many different professions, at the same time created the difficulty of the study.

Results of both statistical methods are taken together, has shown that only the emotionality factor is clearly ineffective in terms of job performance and job satisfaction. The Emotionality factor consists of Fearfulness, Anxiety, Dependence and Sentimentality sub-dimensions. This factor corresponds to the Emotional Stability Factor in the Five Factor Model however, does not cover characteristics such as hostility, rage and anger (Ashton and Lee, 2005; 2007; 2008a; 2008b; Ashton, Lee and de Vries, 2014; Ashton, Lee, Perugini, Szarota, de Vries, di Blas, Boies and de Raad, 2004).

In terms of other factors, in terms of both job performance and job satisfaction and also in regression analysis and comparison of groups, it is observed that more or less there is an influential factor or sub-dimension. Effective factors in terms of job performance and job satisfaction in the HEXACO model have the following properties.

The Humility-Honesty factor of the HEXACO Personality model consists of Sincerity, Fairness, Greed Avoidance and Modesty sub-dimensions. This factor includes characteristics such as sincere, honest, faithful/loyal, modest/unassuming, fair-minded versus sly, greedy, pretentious, hypocritical, boastful, pompous (Ashton and Lee, 2005; 2007; 2008a; 2008b). The Extraversion factor consists of Social Self-Esteem, Social Boldness, Sociability and Liveliness sub-dimensions. This factor includes characteristics such as lively, extraverted, sociable, talkative, cheerful, and active versus shy, passive, withdrawn, introverted, quiet, and reserved. On the other hand, this factor does not include characteristics such as bravery and durability as in the Five Factor Model (Ashton and Lee, 2007; 2009a; Lee and Ashton, 2004; 2018). The Agreeableness factor consists of Forgiveness, Gentleness, Flexibility and Patience sub-dimensions. This factor includes characteristics such as patient, tolerant, peaceful, mild, agreeable, lenient, gentle versus ill-tempered, quarrelsome, stubborn, choleric. It does not include emotionality in the Five Factor Model (Ashton and Lee, 2007; 2009a; Ashton, Lee and de Vries, 2014; Lee and Ashton, 2004; 2018). The Conscientiousness factor consists of Organization, Diligence, Perfectionism and Prudence sub-dimensions. The characteristics of this factor on the one end are organized, disciplined, diligent, careful, thorough, precise and on the other end are sloppy, negligent, reckless, lazy, irresponsible, absent-minded (Ashton and Lee, 2007; 2009a; Ashton, Lee and de Vries, 
2014; Lee and Ashton, 2004; 2018). The Openness to Experience factor consists of Aesthetic Appreciation, Inquisitiveness, Creativity and Unconventionality sub-dimensions. This factor includes characteristics such as intellectual, creative, unconventional, innovative, ironic (Ashton and Lee, 2007; 2009a; Lee and Ashton, 2004; 2018).

The results obtained are especially important for the selection of personnel, especially in terms of job performance and job satisfaction. On the other hand personality traits have a relationship in the similar direction with both job performance and job satisfaction (Barrick, Mount and Judge, 2001; Rothmann and Coetzer, 2003). Besides, these two facts are correlated with each other and job satisfaction is accepted as the predictor of job performance (Wright and Cropanzano, 2000). This study addressed the relationship of personality traits, job performance and job satisfaction, a topic with many examples in the literature, in terms of a relatively new HEXACO model.

\section{References}

Abraham, J. D., \& Morrison, J. D. (2003). Relationships between the Performance Perspectives Inventory's Selling scale and job performance of real estate agents. Applied Human Resource Management Research, 8(1), 45-48.

Alessandri, G., \& Vecchione, M. (2012). The higher-order factors of the Big Five as predictors of job performance. Personality and Individual Differences, 53(6), 779-784.

Allport, G. W., \& Odbert, H. S. (1936). Trait-names: a psycho-lexical study. Psychological Monographs, 47(1), 1-171.

Almagor, M., Tellegen, A., \& Waller, N. G. (1995). The Big Seven model: A cross-cultural replication and further exploration of the basic dimensions of natural language trait descriptors. Journal of Personality and Social Psychology, 69(2), 300-307.

Ashton, M. C., \& Lee, K. (2002). Six independent factors of personality variation: a response to Saucier. European Journal of Personality, 16(1), 63-75.

Ashton, M. C., \& Lee, K. (2005). Honesty-humility, the Big Five, and the Five-Factor Model. Journal of Personality, 73(5), 1321-1354.

Ashton, M. C., \& Lee, K. (2007). Empirical, theoretical, and practical advantages of the HEXACO model of personality structure. Personality and Social Psychology Review, 11(2), 150-166.

Ashton, M. C., \& Lee, K. (2008a). The HEXACO model of personality structure and the importance of the H factor. Social and Personality Psychology Compass, 2(5), 1952-1962.

Ashton, M. C., \& Lee, K. (2008b). The prediction of Honesty-Humility-related criteria by the HEXACO and Five-Factor Models of personality. Journal of Research in Personality, 42(5), 1216-1228.

Ashton, M. C., \& Lee, K. (2009a). An investigation of personality types within the HEXACO personality framework. Journal of Individual Differences, 30(4), 181-187.

Ashton, M. C., \& Lee, K. (2009b). The HEXACO-60: A short measure of the major dimensions of personality. Journal of Personality Assessment, 91(4), 340-345.

Ashton, M. C., Lee, K., \& de Vries, R. E. (2014). The HEXACO Honesty-Humility, Agreeableness, and Emotionality factors: a review of research and theory. Personality and Social Psychology Review, 18(2), 139-152.

Ashton, M. C., Lee, K., \& Goldberg, L. R. (2004). A hierarchical analysis of 1,710 English personality-descriptive adjectives. Journal of Personality and Social Psychology, 87(5), 707-721.

Ashton, M. C., Lee, K., de Vries, R. E., Perugini, M., Gnisci, A., \& Sergi, I. (2006). The HEXACO model of personality structure and indigenous lexical personality dimensions in Italian, Dutch, and English. Journal of Research in Personality, 40(6), 851-875.

Ashton, M. C., Lee, K., Perugini, M., Szarota, P., de Vries, R. E., di Blas, L., Boies, K., \& de Raad, B. (2004). A six-factor structure of personality-descriptive adjectives: solutions from psycholexical studies in seven languages. Journal of Personality and Social Psychology, 86(2), 356-366.

Barrett, P. T., Petrides, K., Eysenck, S. B., \& Eysenck, H. J. (1998). The Eysenck Personality Questionnaire: An examination of the factorial similarity of P, E, N, and L across 34 countries. Personality and Individual Differences, 25(5), 805-819.

Barrick, M. R., \& Mount, M. K. (1991). The Big Five personality dimensions and job performance: A meta-analysis. Personnel Psychology, 44(1), 1-26.

Barrick, M. R., \& Mount, M. K. (1993). Autonomy as a moderator of the relationships between the Big Five personality dimensions and job performance. Journal of Applied Psychology, 78(1), 111-118.

Barrick, M. R., Mount, M. K., \& Judge, T. A. (2001). Personality and performance at the beginning of the new millennium: What do we know and where do we go next? International Journal of Selection and Assessment, 9(1-2), 9-30.

Barrick, M. R., Stewart, G. L., \& Piotrowski, M. (2002). Personality and job performance: Test of the mediating effects of motivation among sales representatives. Journal of Applied Psychology, 87(1), 43-51. 
Block, J. (1995). A contrarian view of the Five-Factor Approach to personality description. Psychological Bulletin, 117(2), 187-215.

Caprara, G. V., Barbaranelli, C., \& Comrey, A. L. (1992). Validation of the Comrey Personality Scales on an Italian sample. Journal of Research in Personality, 26(1), 21-31.

Eysenck, S. B., Eysenck, H. J., \& Barrett, P. (1985). A revised version of the psychoticism scale. Personality and Individual Differences, 6(1), 21-29.

Goldberg, L. R. (1990). An alternative" description of personality": the big-five factor structure. Journal of Personality and Social Psychology, 59(6), 1216-1229.

Goldberg, L. R. (1993). The structure of phenotypic personality traits. American Psychologist, 48(1), 26-34.

Guion, R. M., \& Gottier, R. F. (1965). Validity of personality measures in personnel selection. Personnel Psychology, 18(2), 135-164.

Hogan, R., \& Hogan, J. (2007). The Hogan Personality Inventory Manual. Tulsa, OK: Hogan Assessment Systems.

Judge, T. A., \& Zapata, C. P. (2015). The person-situation debate revisited: Effect of situation strength and trait activation on the validity of the Big Five personality traits in predicting job performance. Academy of Management Journal, 58(4), 1149-1179.

Lee, K., \& Ashton, M. C. (2004). Psychometric properties of the HEXACO Personality Inventory. Multivariate Behavioral Research, 39(2), 329-358.

Lee, K., \& Ashton, M. C. (2006). Further assessment of the HEXACO Personality Inventory: two new facet scales and an observer report form. Psychological Assessment, 18(2), 182-191.

Lee, K., \& Ashton, M. C. (2008). The HEXACO Personality Factors in the Indigenous Personality Lexicons of English and 11 Other Languages. Journal of Personality, 76(5), 1001-1053.

Lee, K., \& Ashton, M. C. (2014). The Dark Triad, the Big Five, and the HEXACO model. Personality and Individual Differences, 67, 2-5.

Lee, K., \& Ashton, M. C. (2018). Psychometric properties of the HEXACO-100. Assessment, 25(5), 543-556.

Lee, K., Ogunfowora, B., \& Ashton, M. C. (2005). Personality traits beyond the big five: Are they within the HEXACO space? Journal of Personality, 73(5), 1437-1463.

McCrae, R. R., \& Costa, P. T. (1987). Validation of the Five-Factor Model of personality across instruments and observers. Journal of Personality and Social Psychology, 52(1), 81-90.

Mount, M. K., Barrick, M. R., \& Stewart, G. L. (1998). Five-Factor Model of personality and performance in jobs involving interpersonal interactions. Human Performance, 11(2-3), 145-165.

Piedmont, R. L., \& Aycock, W. (2007). An historical analysis of the lexical emergence of the Big Five personality adjective descriptors. Personality and Individual Differences, 42(6), 1059-1068.

Rothmann, S., \& Coetzer, E. P. (2003). The Big Five personality dimensions and job performance. $S A$ Journal of Industrial Psychology, 29(1), 68-74.

Rothstein, M. G., \& Goffin, R. D. (2006). The use of personality measures in personnel selection: What does current research support? Human Resource Management Review, 16(2), 155-180.

Salgado, J. F. (1997). The Five Factor Model of personality and job performance in the European community. Journal of Applied Psychology, 82(1), 30-43.

Saltukoğlu, G., \& Tatar, A. (2018). İş performansının öngörülmesinde kişilik ölçümünün rolü: Öğretmen örneği. Journal of Human Sciences, 15(1), 619-634.

Saucier, G. (2002). Gone too far-or not far enough? Comments on the article by Ashton and Lee (2001). European Journal of Personality, 16(1), 55-62.

Simms, L. J., Williams, T. F., \& Nus, E. (2017). Assessment of the Five Factor Model. In T. A. Widiger (Ed.), The Oxford handbook of the Five Factor Model (pp. 353-380). New York: Oxford University Press.

Tatar, A. (2018). Gözden Geçirilmiş Uzun ve Kısa Form Altı Faktörlü Kişilik Envanteri (HEXACO-PIR) Türkçe formunun psikometrik özelliklerinin incelenmesi. Anadolu Psikiyatri Dergisi, 19(1), 5-13.

Wright, T. A. ve Cropanzano, R. (2000). Psychological well-being and job satisfaction as predictors of job performance. Journal of Occupational Health Psychology, 5(1), 84-94.

Zhao, H., \& Seibert, S. E. (2006). The Big Five personality dimensions and entrepreneurial status: A meta-analytical review. Journal of Applied Psychology, 91(2), 259-271.

Zuckerman, M., Kuhlman, D. M., Joireman, J., Teta, P., \& Kraft, M. (1993). A comparison of three structural models for personality: The Big Three, the Big Five, and the Alternative Five. Journal of Personality and Social Psychology, 65(4), 757-768. 


\title{
TYPOLOGY OF INSURANCE BEHAVIOR OF RUSSIAN CITIZENS
}

\author{
Olga Medyanik \\ Department of Political Psychology, St. Petersburg state University (Russia)
}

\begin{abstract}
Ensuring the economic security of the personality is to create a mechanism of protection against internal and external threats in modern Russia. In this connection, the role of the policy of insurance of citizens as the most effective institution of risk management and security of health, personal property of citizens. The article presents the results of an empirical study of the attitude of Russian citizens to insurance policy of insurance and investment. The study consisted of three stages: at the preparatory stage - a longitudinal study of Russian clients of insurance companies was conducted, at the pilot study - a survey of policyholders on two developed scale multifactorial questionnaires investigating insurance preferences and financial anxiety. In the main study, which was presented by a heterogeneous sample, an Arsenal of techniques was used, which included a basic survey, a TAS questionnaire (in Soldatova's approbation), a questionnaire of economic attitudes (Deineka, Zabelin) and a Dinner satisfaction scale. A total of 1409 people took part in the study.

The article presents the results of theoretical understanding of the factors of economic security of the personality, which affect the insurance behavior of citizens. The questions of scientific development of insurance research in politics and Economics, the problem of psychology of personal security in the economy, the phenomenon of insurance in the economic life of the citizen and the phenomenon of trust as an indicator of political and economic reality. The problem of insurance culture and financial planning as a measure to reduce economic risks at the level of the state and the individual is raised. The theoretical model of insurance behavior is developed.

The Institute of insurance in our work is considered in the framework of psychological science as a category of risk avoidance, danger, threat, overcoming fear, as a system of economic security, creating a mechanism to protect the individual from internal and external fears and threats.
\end{abstract}

Keywords: Insurance behavior, insurance risks, economic security of the person, the typology of the insured, financial anxiety.

\section{Introduction}

In nowadays conditions, insurance provides the opportunity for market system of economic management to work in any state. To provide economic security of the person it is needed to create a mechanism of protection against internal and external threats in modern Russia. That's why the role of citizens insurance policy is increasing as the most effective risk management institute and achieving safety of health and private property of citizens

The goal of this research is to study Russian citizens' attitudes towards insurance policy and investment using the conception of personal economic security.

Theoretical analysis of insurance policy psychological problems has formed the basis for searching the case of pretty low preparedness of citizens to use the range of insurance service to advance personal economic security. The low level of confidence in private companies and banks, and the lack of financial literacy may be seen as the main reasons of restrained insurance behavior.

\section{Methods}

The preparatory study stage was focused on theoretical understanding of economic security problems taking insurance field as an example. Empirical research consisted of three stages. At the preparatory stage, the research was conducted of Russian insurance companies clients using longitudinal observation and interviews.

At the stage of pilot study insurance preferences and financial anxiety of citizens were measured using official Multiphase scale Inventories. 
At the stage of the main research characteristics of the attitude of citizens to the economic policy of the Russian Federation and, in particular, to the policy of insurance and investment was being studied. On the third and the main stage the characteristics of citizens' relations to economic of Russian federation and mainly to insurance and investment policy were studied using authorial method "CRPII" and economic attitudes (Deyneka's and Zabelina's inventory). Such personality characteristics as tolerance/intolerance to ambiguity were measured additionally using Bunder's TAS inventory in Soldatova's adaptation, life satisfaction using E.Diners method in Leontieva's and Osina's adaptation, economic attitudes (Dejneka's and Zabelina's iventory).

There were 1409 people taking part in this research.

\section{Results and discussion}

Basing on a theoretical understanding of the factors of personal economic security which are affecting the insurance behavior of citizens, on analysis of problems of confidence in Russian economics, insurance culture and financial planning as economic risk reduction remedy for the whole country and for the personality we worked out a theoretical model of insurance behavior.

Longitudinal observation of insurant behavior incombination with an unstructured interview during eight years (the first stage) allowed to work out a typology of insurant behavior based on psychology. Four types of insurants are marked out: sensible, confiding, anxious and risky.

A pilot study was made using the inventory of citizens' preferences in insurance (Deyneka's inventory algorithm) and the inventory of financial (second stage). It allowed us to transform the typology supplementing its foundations with the attitude characteristics to the economic policy of the Russia.

Exploratory factor analysis of the data received from inventory of citizens' preferences in insurance policy allowed to mark out two orthogonal factors: voluntaristic/fatal investment policy factor and paternalistic/market-oriented policy factor, which lay the foundation to correct typology that we got in the result of first stage of the research.

Also, two more factors were marked out, such as: seeking-oriented factor of insurants activity and reason factor, which holds back insurance policy. There were marked out three factors based on factor analysis inventory of financial anxiety. Such as financial anxiety factor, lack of money experience factor, value of insurance security factor.

Basing on received data it is advisable to consider the insurance institute not only as an economic functional, but also as a psychological and political institution that helps to reduce the experience of fear, danger, risk, as a system of economic security which creates a mechanism of personality protection from internal and external threats.

At the third stage of the research was worked out an inventory which made it possible to study the attitude of citizens to insurance and investment policies in the context of attitudes toward domestic economic policy in general. The study carried out with its help gave grounds to confirm the typology of citizens on the basis of their attitude to insurance and investment policies: paternalists, marketers, fatalists, voluntarists.

Additional methods of assessment of person's tolerance (Soldatova) and satisfaction with the quality of life (Diner) allowed me to carry out correlational analysis of psychological indicators and relation to economical policy of the government. Correlation analysis showed that the higher the tolerance to novelty, the less the need to insure the property and expand insurance through non-state and voluntary insurance services.

Relationships of life satisfaction with attitudes towards economic policy were revealed. The higher the satisfaction with life, the more positive is the attitude towards the results of economic policy in Russia (the welfare of citizens, the fairness of the tax system, satisfaction with consumption, the balance of effort and income, expectations of an increase in well-being).

The correlation of the data received from the inventory of attitudes towards insurance and investment policies and data from the inventory of economic policy confirmed the reliability of the research results. For example, the radical attitudes of those who represent the economic institutions of insurance and investment are associated with the condemnation of entrepreneurship and dissatisfaction with their consumer possibilities.

\section{Conclusions}

1. The program of the research of relation is developed and approved of citizens to the insurance policy, which included the design of the longitudinal research using the method of observation and semi-structured individual interviews with insurance clients companies and more scale multifactor questionnaire of citizens' preferences in insurance policy, financial anxiety, attitudes citizens to the 
policy of insurance and investment (the questionnaire CRPII) in combination with tested questionnaires of other authors-methods of economic attitudes (Deyneka, Zabelina), the scale of tolerance-intolerance identity uncertainty (Budner, adaptation of Soldatova), scale life satisfaction (Diner) for both topical and potential policyholders.

2. The results of the study of the attitude of Russian citizens to politics insurance and investment showed a contradictory attitude of citizens to insurance policy and lack of psychological readiness to shift economic risks to insurance companies.

3. The use of the term "economic security" is justified personality" as a long-term state of personal financial stability provisions for the manifestation of external and internal threats to the personal financial sovereignty, independence, capacity for economic development that provides the opportunity to meet and expand economic need.

4. Longitudinal monitoring of the behavior of insurance consumers services allowed to develop a typology of policyholders, which included four types: rational, anxious, trusting, risky. Describe there are also four types of strategies of insurance behavior: rational and alarming, rational-risky, anxious-trusting, risky-trusting.

5. Factor structure of preferences in insurance policy, obtained on the sample of actual policyholders, contains the factor market installation, the paternalistic factor installation factor tentatively-search activity, the factor of financial literacy, factor of the reasons constraining insurance policy. Cluster analysis of empirical data confirmed the presence of three types of policyholders: "marketers", "paternalists" and "neutral". "Paternalists" link the problems of the insurance industry and distrust of insurance companies with poor state control of activities insurance companies and other financial institutions, as well as the lack of fair distribution of income of the population. For such citizens the need to adopt fair economic policies is important laws'. "Marketers" distance themselves from the request for state control. The third category - "neutral" - are those insurers who are clearly not trust market institutions, but also to the role of the state are without special hope.

6 . The factor structure of the financial anxiety questionnaire included factors affecting situational behavior that is associated with the loss of money, experiences of their deficit, awareness of investment risks, needs insurance coverage, financial optimism. Financial anxiety and uncertainty triggers consumer interest in insurance and investment products that can give him confidence in tomorrow and get rid of the feelings of ruin or loss of property.

7. Analysis of gender differences in attitudes towards economic policy showed that women have higher social internality than men. They are more likely to attribute blame for the problems in the country's economy citizens and more trust the policy pursued state. Women, unlike men, advocate for targeted assistance in preferential security policy. They believe that benefits should be received only families in need. Women are more prone to stability and conservatism, trusting and "socially naive", men-more are prone to risk and rational strategies in economic behavior.

8. Empirical research has shown that tolerant (positive) the attitude of citizens to insurance is a factor of increase economic security and tolerance to the economic situation uncertainty: more tolerant to uncertainty citizens will find themselves more satisfied with economic policy of their country. As there is a relationship between satisfaction life and attitude to economic policy: the higher satisfaction with life, the more positive attitude to the results economic policy in Russia.

9. The results of the study conducted on a sample of relevant and potential policyholders, confirmed the typological differences, obtained in the study of current policyholders.

The typology dividing citizens into "paternalists" is confirmed", "marketers" and "neutral" in relation to different aspects economic policy, including insurance was made.

10. On the basis of the theoretical analysis the generalizing the structure of the concepts of insurance behavior of a citizen in modern market economy, where the basis of perception of financial well-being is the economic security of the individual. A conceptual framework is proposed model of studying insurance behavior as a factor of economic security of person. The author's vision of the key elements is proposed effective insurance policy, taking into account the psychological aspects of modern Russia.

11. On the basis of the generalized structure of the concepts of insurance behavior and the empirical study of the attitude to politics insurance designed tiered structure that favors formation of insurance trust of citizens, including the block of financial anxiety, the unit value of insurance coverage, block financial literacy, insurance culture, economic security unit persons (in the context of insurance trust). 


\section{References}

Bazanov A. N. etc. (total 19 people) Insurance and risk management (textbook) for students / under the editorship of G. V. Chernova. - 2nd ed., - M.: yurait publishing House, 2014.

Belozerov S. A. The influence of insurance on the choice of the savings strategy of the household (Theses; RSCI) // V SB.: international economic Symposium - 2015: Proceedings of the II international scientific and practical conference dedicated to the 75th anniversary of the faculty of Economics of St. Petersburg state University. 22-25 April 2015-St. Petersburg.: Scythia print publishing house, 2015. - P. 402-407.

Belozerov S. A. trends in the development of insurance in the digital economy // Article from the collection of works "Insurance in The digital economy: problems and prospects: proceedings of the XIX international.scientific. - prakt.Conf."(Yoshkar-Ola, 5-7 June 2018) in 2T. T. 1 / Rosgosstrakh: Mari state UN-t: edited by E. V. Zlobin, T. V. Sarycheva. - Yoshkar-Ola: publishing House of Mari state University, 2018. -300 p.

Deyneka O. Dysfunctional Economic Behaviour: Victims of Financial Debt (The Case of Microfinance Organisation Clients) // Psychology and Law in Europe: When West Meets East / Ed. By Pär-Anders Granhag, Ray Bull, Alla Shaboltas, Elena Dozortseva. CRC Press, 2017 P. 125-135

Deyneka O. S. Methodology of economic and psychological research at the Department of political psychology of St. Petersburg state UNIVERSITY. Theory and practice of Russian political psychology // Proceedings of the scientific conference devoted to the Department of political psychology. Edited by Prof. Yuriev A. I.-St. Petersburg: St. Petersburg state University, 2008. - P. 174.

Deyneka O. S. Psychology of economic policy // anthology to the textbook on political psychology. SPb: "Kolo", 2012. - P. 129 -175.

Deyneka O. S. Social regulators as factors of realization of economic power and policy // Psychology of power - 2008: Proceedings of the Second international scientific conference / edited by Yuriev A. I. - SPb., 2008. - Pp. 37-41.

Deyneka O. S., Zabelina E. V. Results of the development of a multiple-factor questionnaire for rapid diagnosis of economic attitudes. Psychological research, 2018, 11(58), 9. 


\title{
STUDENT'S IDEAS ABOUT THE PREREQUISITES AND MEASURES TO COMBAT CORRUPTIONS
}

\author{
Olga Deyneka \\ Saint Petersburg State University (Russia)
}

\begin{abstract}
In the context of "creeping" everyday corruption, it is extremely important to monitor attitudes towards corruption, especially among young people. The objective of the study was to identify the problems in attitude to corruption in Russia among students. The total sample included 181 students: 106 university students from St. Petersburg, Moscow and Chelyabinsk, and 81 students from St. Petersburg colleges. The study showed that the students associated responsibility for the level of corruption primarily with the institutions of power, accusing legislative branch and sharing the view of the weak interest of the government in eliminating corruption. According to students, combating corruption is also difficult due to the fact that corruption is not condemned in society. Correlation analysis of the data showed that those students who know the articles of the Criminal code of the Russian Federation related to corruption, higher assess the effectiveness of Russian legislation in combating corruption. The result confirms the importance of improving the legal culture of students, as in the protest movement corruption is often the cause of destructive protests. As the most effective measures to combat corruption, students identify anti-corruption civic education and interaction with international organizations, and the least effective measure is considered fines. The results can be used in the development of educational programs.
\end{abstract}

Keywords: Attitudes, corruption, students, countermeasures, prerequisites.

\section{Introduction}

In everyday consciousness, corruption is most often reflected as a bribe. However, corruption is a broader concept. Corruption refers to deviations from a binding legal norm, arbitrariness in the exercise of authority and the illegal use of public resources. Indeed, no one is surprised at the offers to buy any product or service, provided that part or full payment passes without going through the cashier's office (for example, this happens with theater tickets, technical inspections of cars).

Corruption acts include bribery of officials and public and political figures, bribery for legal and illegal representation of benefits and advantages, protectionism. Against the background of the promotion of enterprise among public servants while reducing the control of their activities in the early 1990s (and in the absence of legislative reinforcement for many transactions between administrations and businesses) moral deformations have intensified (Nestik, 2002), corruption has become a way of life and a certain social norm (Simoniya, 2000).

Recently, Russia has been actively, but to a certain degree, working to combat corruption. It should be noted public exposures of corrupt officials from the authorities. Prevention of corruption is carried out through the introduction of special disciplines and online courses in educational programs in universities. In this regard, it is important to monitor attitudes towards corruption, especially among young people.

\section{Background}

The consequences of acts of corruption can be economic, political and psychological. As the socio-psychological mechanisms of corruption are compelled conformal behavior (Nestik, 2002), "corporate" morality (Deyneka, 2013), lack of social capital (Coleman, 1988; Corruption and..., 2007), Eastern tradition's archetypes (Boronoev \& Smirnov, 2000; Reshetnikov, 2008; Kitova, Zhuravlev, Sosnin, Yurevich, 2017). Psycho-political prerequisites of corruption are paternalism, hierarchy and reliance on informal relations with the authorities, as well as periods of modernization (Huntington, 1996) in the absence of a clear concept and ignoring the socio-cultural traditions of society, low legal culture of citizens, combined with religious and national heterogeneity of the population. 
Despite their relevance, empirical work on the economics and psychology of corruption has long been presented poorly (Braun M., Di Tella R., 2004). Currently, the interest of scientists to the problem of corruption has intensified.

To substantiate the relevance of the new research on attitudes towards corruption, we analyzed the dynamics of the reflection of corruption as an acute problem of society among student youth in the period from 2010 to 2018 using the Citizen Questionnaire. In the block of problems of modern society, young people consistently put the problem of corruption in the first place, along with a large stratification of society. Less important were such issues as raising the retirement age, unemployment and migration. Table 1 shows descriptive statistics of student research data in different years.

Table 1. Assessment of the severity of the problem of corruption in different years among students.

\begin{tabular}{|c|c|c|c|c|c|}
\hline \multicolumn{6}{|c|}{ The degree of students' consensus that corruption is the main problem of Russian society } \\
\hline 2010 & 2013 & 2014 & 2015 & 2016 & 2017 \\
$(\mathrm{n}=94)$ & $(\mathrm{n}=208)$ & $(\mathrm{n}=227)$ & $(\mathrm{n}=227)$ & $(\mathrm{n}=231)$ & $(\mathrm{n}=211)$ \\
\hline $\mathrm{M}=6,56 ;$ & $\mathrm{M}=5,69 ;$ & $\mathrm{M}=5,19 ;$ & $\mathrm{M}=5.64 ;$ & $\mathrm{M}=5.65 ;$ & $\mathrm{M}=6,05 ;$ \\
$\sigma=0,07$ & $\sigma=1,36$ & $\sigma=1,33$ & $\sigma=1,69$ & $\sigma=1,46$ & $\sigma=1,16$ \\
\hline
\end{tabular}

Over the past years, the problem of corruption in the minds of young people has retained the status of the most acute problem and, as can be seen from table 1, does not lose relevance.

The objective of this study was to identify problems in attitude to corruption in Russia among students, to study students' ideas about the prerequisites and measures to counter corruption.

\section{Method}

The method of the research was the short version of the questionnaire "Attitudes towards corruption", consisting of 18 claims with a 7-point scale responses. The questionnaire includes the following blocks: attitudes to anti-corruption measures; pretensions to power, critical attitude toward authority; assessment of the current state of corruption in government institutions in the country.

Two groups of students took part in this study. The total sample included 181 students: 106 university students from St. Petersburg, Moscow and Chelyabinsk (the sample consisted of 75\% female and 25\% male students; the average age was 22.9), and 81 students from St. Petersburg colleges (78\% female and 22\% male students; the average age was 18.0).

\section{Results}

The results showed that the students associated responsibility for the level of corruption primarily with the institutions of power, accusing legislative branch and sharing the view of the weak interest of the government in eliminating corruption $(\mathrm{M}=5.26, \mathrm{SD}=1.74)$. According to students, combating corruption is also difficult due to the fact that corruption is not condemned in society $(\mathrm{M}=4.42, \mathrm{SD}=1.71)$. At the same time, respondents do not consider the use of bribes as the norm of modern business $(\mathrm{M}=2.98, \mathrm{SD}=1.73)$.

As the most effective measures to combat corruption, students identify anti-corruption civic education and interaction with international organizations, and the least effective measure is considered fines (table 2).

Table 2. Evaluating the effectiveness of anti-corruption measures.

\begin{tabular}{|c|c|c|c|}
\hline $\begin{array}{l}\text { Countermeasures and Prevention of } \\
\text { Corruption }\end{array}$ & Rank & $\mathrm{M}$ & SD \\
\hline $\begin{array}{l}\text { Anti-corruption civic upbringing and } \\
\text { education }\end{array}$ & I & 5.74 & 1.38 \\
\hline $\begin{array}{l}\text { Interaction with international } \\
\text { organizations }\end{array}$ & II & 5.01 & 1.53 \\
\hline Citizens' Anti-Corruption Education & III & 4.43 & 1.72 \\
\hline $\begin{array}{l}\text { Public condemnation of corruption in the } \\
\text { media and the Internet }\end{array}$ & IV & 4.02 & 1.90 \\
\hline $\begin{array}{l}\text { Ethical standards and commissions for } \\
\text { civil servants }\end{array}$ & V & 3.51 & 1.55 \\
\hline Fines & VI & 3.45 & 1.80 \\
\hline
\end{tabular}


"Anti-corruption education" is considered as one of the directions of the general prevention of crimes and represents the education of human values (Nikolaev, 2011). The result obtained is an indirect evidence of the approval among students of the introduction of special programs in universities and colleges. Such programs could increase the "anti-corruption resistance" (Vanovskaya,2013).

Estimates of the causes (preconditions) of corruption in modern Russian society are given in Table 3 in descending order of their importance.

Table 3. Estimates of the preconditions of corruption.

\begin{tabular}{llll}
\hline \multicolumn{1}{c}{$\begin{array}{c}\text { Countermeasures and Prevention of } \\
\text { Corruption }\end{array}$} & Rank & M & SD \\
\hline $\begin{array}{l}\text { Low effectiveness of anti-corruption } \\
\text { legislation }\end{array}$ & I & 5.91 & 1.38 \\
$\begin{array}{l}\text { Low salaries of employees } \\
\text { No Condemnation of Corruption in }\end{array}$ & II & 4.84 & 1.73 \\
$\begin{array}{l}\text { Society } \\
\text { Gaps in the laws }\end{array}$ & IV & 3.41 & 1.71 \\
\hline
\end{tabular}

It should be noted that the assessment of the effectiveness of anti-corruption laws in Russia was closely related to economic optimism (I think that the economic situation in Russia will improve in the next 5 years) among university students $(r=0.49)$ and even more among college students $(r=0.56)$.

Four factors were identified as a result of factor analysis of the questionnaire data. As can be seen from table 3, the data of the questionnaire formed four well-interpretable factors: the factor of the effectiveness of the fight against corruption; the anti-corruption measures factor; the corruption prerequisites factor; the factor of social norms due to corruption.

Table 3. The factor structure of the questionnaire "Attitudes towards corruption" data.

\begin{tabular}{|c|c|}
\hline Statements & $\begin{array}{l}\text { Factor } \\
\text { loading }\end{array}$ \\
\hline \multicolumn{2}{|l|}{ The Factor of the Effectiveness of the Fight against Corruption (9.6) } \\
\hline Existing Russian legislation is effective in the fight against corruption. & -0.750 \\
\hline I think that the economic situation in Russia will improve in the next 5 years. & -0.731 \\
\hline I am convinced that our government is not interested in eliminating corruption. & 0.718 \\
\hline $\begin{array}{l}\text { I believe in the principle of good faith in relation to competitions and tenders in the Russian } \\
\text { Federation. }\end{array}$ & -0.486 \\
\hline I am convinced that corruption undermines the economy and national security & 0.415 \\
\hline \multicolumn{2}{|l|}{ The Anti-Corruption Measures Factor (7.2) } \\
\hline $\begin{array}{l}\text { Public condemnation of corruption in the media and the Internet is the most effective } \\
\text { measure to eliminate corruption. }\end{array}$ & 0.788 \\
\hline Citizens' anti-corruption education is the best measure to fight corruption. & 0.722 \\
\hline I am convinced that anti-corruption civic education and upbringing are necessary. & 0.615 \\
\hline \multicolumn{2}{|l|}{ The Corruption Prerequisites Factor (6.4) } \\
\hline $\begin{array}{l}\text { The lure of corruption is especially great against the background of low wages of } \\
\text { employees. }\end{array}$ & 0.585 \\
\hline $\begin{array}{l}\text { The most dangerous type of corruption for modern Russia is corruption in law } \\
\text { enforcement. }\end{array}$ & 0.570 \\
\hline Gaps and vagueness of interpretations in laws are the main cause of corruption. & 0.500 \\
\hline I know about articles of the Criminal Code related to corruption. & -0.495 \\
\hline $\begin{array}{l}\text { Active interaction with international organizations in the fight against corruption can have } \\
\text { positive consequences for Russia. }\end{array}$ & 0.430 \\
\hline \multicolumn{2}{|l|}{ The Factor of Social Norms due to Corruption (5.2) } \\
\hline I consider the use of bribes the norm of modern bu & 0.725 \\
\hline I am convinced that corruption undermines the economy and national security. & -0.506 \\
\hline Anti-corruption is difficult because it is not condemned in society. & 0.448 \\
\hline
\end{tabular}

Correlation analysis of the data showed that those students who know the articles of the Criminal Code of the Russian Federation related to corruption, higher assess the effectiveness of Russian legislation in combating corruption $(r=0.28, p<0.01)$. The result confirms the importance of improving the legal culture of students, as in the protest movement corruption is often the cause of destructive protests. 
The role of interaction with international organizations in the fight against corruption was considered significant by those students who advocate anti-corruption education $(\mathrm{r}=0.30, \mathrm{p}<0.01)$, and those who are more concerned about corruption in law enforcement $(r=0.29, p<0.01)$. The result obtained is not unfounded. Corruption in law enforcement agencies, designed to satisfy the motive of citizens' safety, is still present. As shown by our comparative studies of attitudes towards the corruption of citizens of Russia and Italy (Deyneka, 2015), among Russians there were more of those who had claims to law enforcement agencies than among Italians.

\section{Conclusions}

The triad of representation of the prerequisites of corruption in the minds of students includes problems related to power and laws, economics, and public opinion.

The students identify anti-corruption civic education, interaction with international organizations, and citizens' anti-corruption education as the most effective anti-corruption measures. They consider fines to be the least effective measure.

Thus, it can be said that in the public opinion of young people there is a willingness to educate as part of special educational programs to improve anti-corruption sustainability and legal culture.

\section{References}

Boronoev A.O., Smirnov P.I. (2000). Russian mentality and reform. (Ed. V. Bolshakov). Society and Politics: Current research, search concepts. St. Petersburg: St. Petersburg State University. 274 -303. (in Russia).

Braun M., Di Tella R. Inflation, Inflation Variability, and Corruption. Economics and Politics. 2004. 16(1), 77-100.

Coleman, J. S. (1988). Social capital in the creation of human capital. American Journal of Sociology. 94, 95-120.

Deyneka O.S. (2013). Political psychology and crime prevention in economics and business. Saarbruecken: Palmarium Academic Publishing (in Russia).

Deyneka O. (2015). Attitude toward corruption: two comparative studies. InPACT 2015. Ljbljna, Slovenia. 2-4 May. Proceedings. Ed. by Clara Pracana. Lisbon: W.I.A.R.S. 156 -159. http://inpactpsychologyconference.org/wp-content/uploads/2014/04/InPACT-2015_Proceedings.pdf

Corruption and the Secret of Law: A Legal Anthropological Perspective. (2007). Ed. Nuijten M, Anders G. London: Routledge.

Huntington S. F. (1996). Clash of Civilizations and the Remarking of Word Order. New-York: Simon \& Schuster.

Kitova D.A., Zhuravlev A.L., Sosnin V.A., Yurevich A.V. (2017). Corruption as an object of socio-psychological research: status and prospects. Institute of Psychology of the Russian Academy of Sciences. Social and economic psychology. V. 2. № 3 (7). 6-38. http://soc-econompsychology.ru/engine/documents/document370.pdf

Nestik T.A. (2002). Corruption and Culture. The economic theory of crime and punishment. 4 (2). http://corruption.rsuh.ru/magazine/4-2/n4-05.html

Nikolaev S.M. The concept and essence of anti-corruption education. Historical, philosophical, political and legal sciences, cultural studies and art history. Questions of theory and practice. Tambov: Diploma, 2011. 7 (2), 159-162. http://www.gramota.net/materials/3/2011/7-2/42.html

Reshetnikov M.M. (2008). Psychology of Corruption: utopia and dystopia. St.-Petersburg: East European Institute of Psychoanalysis.

Simonia N.A. On corruption and the state. Person and the world. Moscow. Russian Academy of Natural Sciences. Institute of Federalism and Civil Society. 2000. 7.7-15.

Vanovskaya O.V. (2013). Psychology of corrupt behavior of civil servants. St. Petersburg: LLC "Book House". (in Russia). 


\title{
A DESCRIPTIVE APPROACH TO MOBBING: THE ROLE OF SELF ESTEEM AND BODY IMAGE AT WORK
}

\author{
Nevin Kılıç ${ }^{1}$, Berra Bekiroğlu², Hüdanur Özdemir ${ }^{2}, \&$ Arkun Tatar ${ }^{1}$ \\ ${ }^{1}$ Department of Psychology, FSM Vakif University / Assistant Professor (Turkey) \\ ${ }^{2}$ Psychologist (Turkey)
}

\begin{abstract}
The present study is aimed to investigate perceived mobbing, self-esteem, and body image together. In this direction, relations between these constructs were examined, and mobbing scores of different socio-demographic groups were compared. Participants of the study were 223 female and 219 male, a total of 442 people, who have been working in public (165 people) or private sector (277 people) more than a year. Data were collected through the A Mobbing Scale, the Two Dimensional Body Image Scale, and the Coopersmith Self-Esteem Inventory. In order to explain perceived mobbing, different regression models and in order to demonstrate the relations between variables, different path models were tested. Results suggest that, self-esteem is the most important predictor of mobbing. Respectively, body image affects perceived mobbing indirectly through self-esteem. According to the results of covariance analysis that was performed for comparing socio-demographic groups, number of working years, and age has no significant effect on mobbing scores. In addition, results reveal that especially poor body image, low self-esteem, and bad economic conditions may be prominent risk factors for exposure to mobbing.
\end{abstract}

Keywords: Mobbing, self-esteem, body image.

\section{Introduction}

Body image, which has become an increasingly popular research topic within various fields such as social and health sciences, is a notion that is often used interchangeably with physical appearance (Cash, 2004; Furnham, Badmin \& Sneade, 2002). Although body image is frequently equated with physical appearance, it refers to a much broader concept than the latter, and it is conceptualized as a multidimensional construct that encompasses individuals' cognitive, emotional and behavioral evaluations of their physical appearances. It is stated that body image consists of two main components, namely, perceptions and attitudes. The perceptual component contains individuals' personal evaluations of their physical appearances whereas the attitudinal component contains individuals' feelings or thoughts about their own bodies (Chase, 2001; Hayes, D’Anci \& Kanarek, 2011; Stokes \& Frederick-Recascino, 2003; Wenninger, Weiss, Wahn \& Staab, 2003).

Although body image is conceptualized as a subjective construct, it is demonstrated that individuals' perceptions of and attitudes towards their bodies are influenced by socio-cultural ideals and expectations that are presented in media through various messages (Cash \& Labarge, 1996; Lee, Lee, Choi, Kim \& Han, 2014; Sainsbury, 2009; Veldhuis, Konijn \& Seidell, 2012). Internalization of these ideal body portrayals has an important impact on individuals' body image (Bessenoff, 2006; Chase, 2001). The perceived difference between ideal norms and the personal evaluations of one's own physical appearance may lead to dissatisfaction with body and cause body image distortion. Negative evaluation or distorted perspective towards body is linked to psychological problems such as depression, anxiety, eating disorders and so on (Friederich et al., 2007; Lee et al., 2014; Swami, Hadji-Michael \& Furnham, 2008; Thompson, Dinnel \& Dill, 2003; Weawer \& Byers, 2006)

In previous studies, body image has been found to be closely related to self-esteem which also encompasses overall perception of self (Latha, Hegde, Bhat, Sharma \& Rai, 2006; Sainsbury, 2009). In this direction, some researchers suggest that body image is an essential component of self-esteem, whereas others hold the view that body image and self-esteem are related but different constructs (Kostanski \& Gullone, 1998; Sainsbury, 2009). Despite the different approaches on the relation between these two constructs, it has commonly been assumed that body image distortion may have negative affects on self-esteem. In other words, it is considered that negative body image is reflective of low 
self-esteem (Latha et al., 2006). On the other hand, it is widely believed that people who look physically attractive are more likely to be successful, happy, intelligent, sociable, and emotionally stable (Chase, 2001). Besides, physically attractive and successful people are judged more favorably, treated better, appreciated greatly by society (Sainsbury, 2009). However, in the job context, individuals' physical appearances comprise risk factors for becoming target of regularly repeated, undesirable, destructive, hostile, and inappropriate behaviors such as ostracizing, insulting, humiliating and so on (De Pedro, Sánchez, Navarro \& Izquierdo, 2008; Einarsen, 1999; Leymann, 1990; Tomi?, 2012; Turaç \& Şahin, 2014; Tutar \& Akbolat, 2012). Whatever the reason, exposure to mobbing may lead to severe stress related outcomes such as psychosomatic symptoms, depression, anxiety, PTSD (Post-Traumatic Stress Disorder), suicidal thoughts and behaviors (Szigety, 2012; Notelaers, Vermunt, Baillien, Einarsen \& De Witte, 2011; Leymann \& Gustafsson, 1996). Low self-esteem is considered both a consequence of mobbing and a vulnerability factor for it (Einarsen, Raknes \& Matthiesen, 1994; Einarsen, 2000; Şenol et al., 2015; Szigety, 2012).

As discussed above, although several divergent accounts on the relation between physical appearance and exposure to mobbing have been proposed, there is a notable paucity of research on body image and mobbing relation in job context. In this direction, the present study is aimed to examine this relation.

\section{Methods}

\subsection{Participants}

Primary exclusion criteria for participation in the study were working at non-revenue generating jobs, working less than a year, and working in a job that there are less than five employees. Participants were 223 female (50.5\%) and 219 male (49.5\%), a total of 442 people, ranging in age between 18 and 64 (Mean $=29.50, \mathrm{SD}=7.79$ years). $165(37.3 \%)$ of the participants have been working in public sector and $277(62.7 \%)$ in private sector. Working years of the participants ranged between 1 and 48 (Mean $=6.32$, $\mathrm{SD}=7.38$ years).

\subsection{Materials}

Data were collected through the A Mobbing Scale, the Two Dimensional Body Image Scale, and the Coopersmith Self-Esteem Inventory.

The A Mobbing Scale, consists of 30 items $(\alpha=0.96)$ rated on a 5-point Likert-type scale (on which 1 = Very Inaccurate and 5 = Totally Accurate). High scores indicate higher level of perceived mobbing (Tatar, Namlı et al., 2017).

Two Dimensional Body Image Scale, is a self-report questionnaire, consisting of 63 items scored on a 5-point Likert scale ranging from 1 (Dislike Extremely) to 5 (Like Extremely). Besides the overall score $(\alpha=0.93)$, the scale also provides scores for the two sub-dimensions called Functions $(\alpha=0.83)$ and Appearance $(\alpha=0.92)$. Higher scores indicate satisfaction with body in general, physical appearance, and functions of body parts (Tatar, Saltukoğlu et al., 2017).

Short Form of the Coopersmith Self-Esteem Inventory for Adults, consists of 25 items $(\alpha=0.81)$ scaled dichotomously (Yes $=4$, No $=0$ ) with higher scores indicating higher level of self-esteem (Eriş \& İkiz, 2013).

\subsection{Procedure and data analysis}

Data were collected through an online survey within a 1-month period. Convenient sampling method was used for sharing the web link to the survey with people who met the inclusion criteria and accepted to participate in the study voluntarily. In the first place, internal consistency reliability coefficients for the scales were calculated. Afterwards, for determining the relationships between the variables, Pearson correlation analyses; for predicting the perceived mobbing scores, simple and multiple linear regression analyses; and for examining the direct and indirect effects of the variables on each other, path analyses were performed. Finally, the mean scores of the groups were subjected to the one-way Analysis of Covariance (ANCOVA) in terms of the perceived mobbing scores.

\section{Results and discussion}

In this study, results of the different regression and path analyses were reported together designedly with the aim of demonstrating the differences between the models and the changes in results with the transition from one model to the other. Another advantage of evaluating variables both together and separately is that it provides comparable results that lead to a better understanding of their relation to mobbing. 
Results of the simple and multiple linear regression analyses showed that in predicting the perceived mobbing scores, self-esteem is the most important predictor. The increase in the $\mathrm{R}^{2}$ values (0.055-0.152) obtained in the five different regression models indicates that body image has a significant complementary role in self-esteem while predicting the mobbing scores. Although regression analyses resulted in the expected direction, for investigating the relations between body image, self-esteem, and mobbing further, nine different models were tested through the path analysis in order to demonstrate the differences between models and determine the better fitting model. Besides the direct relationship between body image and mobbing, results of the last model, in which the overall total score of the Two-Dimensional Body Image Scale and its sub-dimensions were correlated with mobbing both directly and through self-esteem, reveals that body image has an impact on mobbing and self-esteem may play a mediating role between body image and perceived mobbing. Although the significantly improved fit indices and compatible results with the findings of regression analyses in general, there was a difference between the two methods that sub-dimensions of the Two Dimensional Body Image Scale did not contribute much in explaining the variance related to mobbing in regression analysis whereas specification of these sub-dimensions improved the model fit significantly in path analysis.

In order to support the results obtained from previous analyses and to explain the difference observed between these two different methods, mobbing scores were compared in terms of body image and self-esteem via covariance analysis. In this direction, perceived mobbing levels of different groups that were formed based on the socio-demographic characteristics of the participants were compared. It has been thought that especially age (in terms of body image) and number of the working years of participants (in terms of interpersonal relationships at workplace) may have a common effect on perceived mobbing scores, therefore these variables were included and controlled as covariates. However, contrary to expectations, findings suggest that these variables did not affect the mobbing scores as covariates. ANCOVA results support the results obtained from both regression and path analyses. While there is no difference found between the body image groups in terms of the mobbing scores, there is a significant difference found between the self-esteem groups. On the other hand, it was hypothesized that that there may be differences in mobbing perceptions of body image groups relying on the previous studies which have suggested that both being physically unattractive and having an extremely attractive physical appearance are risk factors for exposure to mobbing (Tomic, 2012; Turaç \& Şahin, 2014). However, results of the present study do not support the previous findings as there was no difference found in this direction, additionally no interaction was observed between the self-esteem and body image groups. On one hand, this result indicates that there is no interaction between these constructs. On the other hand, this contradictory result may be due to unmeasured variables (such as body image, which could account for some aspects of the results) or other uncontrolled error sources in previous studies. Either way, it is concluded that relations between these constructs need to be reconsidered with more sophisticated and complex designs. When results for the socio-demographic variables are evaluated as a whole, it is understood that "weak" people seem to be more vulnerable to mobbing in general. In this study, it is seen that especially distorted body image, low self-esteem, and bad economic conditions are prominent risk factors for exposure to mobbing.

\section{References}

Bessenoff, G. R. (2006). Can the media affect us? Social comparison, self-discrepancy, and the thin ideal. Psychology of Women Quarterly, 30(3), 239-251.

Cash, T. F. (2004). Body image: past, present, and future. Body Image, 1(1), 1-5.

Cash, T. F., \& Labarge, A. S. (1996). Development of the Appearance Schemas Inventory: a new cognitive body-image assessment. Cognitive Therapy and Research, 20(1), 37-50.

Chase, M. E. (2001). Identity development and body image dissatisfaction in college females. (MD thesis), The Graduate Collage University of Wisconsin, Menomonie, WI.

De Pedro, M. M., Sánchez, M. I. S., Navarro, M. C. S., \& Izquierdo, M. G. (2008). Workplace mobbing and effects on workers' health. The Spanish Journal of Psychology, 11(1), 219-227.

Einarsen, S. (1999). The nature and causes of bullying at work. International Journal of Manpower, 20(1/2), 16-27.

Einarsen, S. (2000). Harassment and bullying at work: a review of the Scandinavian approach. Aggression and Violent Behavior, 5(4), 379-401.

Einarsen, S., Raknes, B. R. I., \& Matthiesen, S. B. (1994). Bullying and harassment at work and their relationships to work environment quality: an exploratory study. European Journal of Work and Organizational Psychology, 4(4), 381-401. 
Eriş, Y., \& İkiz, F. E. (2013). Ergenlerin benlik saygısı ve sosyal kaygı düzeyleri arasındaki ilişki ve kişisel değişkenlerin etkileri. Turkish Studies, 8(6), 179-193.

Friederich, H. C., Uher, R., Brooks, S., Giampietro, V., Brammer, M., Williams, S. C., Herzog, W., Treasure, J., \& Campbell, I. C. (2007). I'm not as slim as that girl: neural bases of body shape self-comparison to media images. Neuroimage, 37(2), 674-681.

Furnham, A., Badmin, N., \& Sneade, I. (2002). Body image dissatisfaction: gender differences in eating attitudes, self-esteem, and reasons for exercise. The Journal of Psychology, 136(6), 581-596.

Hayes, J. F., D’Anci, K. E., \& Kanarek, R. B. (2011). Foods that are perceived as healthy or unhealthy differentially alter young women's state body image. Appetite, 57(2), 384-387.

Kostanski, M., \& Gullone, E. (1998). Adolescent body image dissatisfaction: relationships with self-esteem, anxiety, and depression controlling for body mass. The Journal of Child Psychology and Psychiatry and Allied Disciplines, 39(2), 255-262.

Latha, K. S., Hegde, S., Bhat, S. M., Sharma, P. S. V. N., \& Rai, P. (2006). Body image, self-esteem and depression in female adolescent college students. Journal of Indian Association for Child and Adolescent Mental Health, 2(3), 78-84.

Lee, H. R., Lee, H. E., Choi, J., Kim, J. H., \& Han, H. L. (2014). Social media use, body image, and psychological well-being: a cross-cultural comparison of Korea and the United States. Journal of Health Communication, 19(12), 1343-1358.

Leymann, H. (1990). Mobbing and psychological terror at workplaces. Violence and Victims, 5(2), 119-126.

Leymann, H., \& Gustafsson, A. (1996). Mobbing at work and the development of post-traumatic stress disorders. European Journal of Work and Organizational Psychology, 5(2), 251-275.

Notelaers, G., Vermunt, J. K., Baillien, E., Einarsen, S., \& De Witte, H. (2011). Exploring risk groups workplace bullying with categorical data. Industrial Health, 49(1), 73-88.

Sainsbury, D. C. (2009). Body image and facial burns. Advances in Skin and Wound Care, 22(1), 39-44.

Senol, V., Avsar, E., Akca, P. R., Argun, M., Avsarogullari, L. \& Kelestimur, F. (2015). Assessment of mobbing behaviors exposed by the academic personnel working in a university. Turkey. Journal of Psychiatry, 18(212), 2-10.

Stokes, R., \& Frederick-Recascino, C. (2003). Women's perceived body image: relations with personal happiness. Journal of Women and Aging, 15(1), 17-29.

Swami, V., Hadji-Michael, M., \& Furnham, A. (2008). Personality and individual difference correlates of positive body image. Body Image, 5(3), 322-325.

Szigety, T. (2012). Early predictors of workplace mobbing. Procedia-Social and Behavioral Sciences, 33, 418-422.

Tatar A., Namlı M., Özüdoğru, M.T., Uysal, A.R., Yeşilkanat G., Bahadır, E., Kalender, B., \& Aydın, S. (2017). A Psikolojik Yıldırma Ölçeği'nin geliştirilmesi ve psikometrik özelliklerinin incelenmesi. Journal of Behavior at Work, 2(2), 79-88.

Tatar, A., Saltukoğlu, G., Aksu, F., Haşlak, D. F., Zekioğlu, A., Kılıç, N., \& Bekiroğlu, B. (2017). İki Boyutlu Beden İmgesi Ölçeği'nin genel örnekleme uyarlanması, psikometrik özelliklerinin madde cevap kuramı ile incelenmesi ve cinsiyet farklılıklarının karşıllaştırılması. Researcher: Social Science Studies, 5(4), 21-40.

Thompson, T., Dinnel, D. L., \& Dill, N. J. (2003). Development and validation of a body image guilt and shame scale. Personality and Individual Differences, 34(1), 59-75.

Tomic, M. (2012). Mobbing: the incidence of mobbing activities and differences regarding workplace and gender. Megatrend Review, 9(1), 243-252.

Turaç, I. S., \& Şahin, B. (2014). Mobbinge maruz kalma durumunun belirlenmesi: hemşireler üzerine bir çalışma. Eskişehir Osmangazi Üniversitesi Sosyal Bilimler Dergisi, 15(1), 87-112.

Tutar, H., \& Akbolat, M. (2012). Sağlık çalışanlarının yönetici cinsiyetleri bakımından mobbing algıları. Selçuk Üniversitesi Sosyal Bilimler Enstitüsü Dergisi, 28, 19-29.

Veldhuis, J., Konijn, E. A., \& Seidell, J. C. (2012). Weight information labels on media models reduce body dissatisfaction in adolescent girls. Journal of Adolescent Health, 50(6), 600-606.

Weaver, A. D., \& Byers, E. S. (2006). The relationships among body image, body mass index, exercise, and sexual functioning in heterosexual women. Psychology of Women Quarterly, 30(4), 333-339.

Wenninger, K., Weiss, C., Wahn, U., \& Staab, D. (2003). Body image in cystic fibrosis-development of a brief diagnostic scale. Journal of Behavioral Medicine, 26(1), 81-94. 


\title{
PERSONALITY TRAITS AND REASONS TO LEAVE COUNTRY IN SERBIAN UNIVERSITY STUDENTS
}

\author{
Jelisaveta Todorovic ${ }^{1}$, Snezana Stojiljkovic ${ }^{1}, \&$ Tamara Ciric $^{2}$ \\ ${ }^{1}$ Department of Psychology, University of Nis (Serbia) \\ ${ }^{2}$ City of Nis government (Serbia)
}

\begin{abstract}
This paper is referring to the problem of brain drain, used to describe 'the situation in which large numbers of educated and very skilled people leave their own country to live and work in another one where pay and conditions are better'. It is why this phenomenon has often been connected to the depletion of the intellectual and professional resources of a country or a region. Serbia has suffered a huge brain drain in recent years. The goal of this research is to examine what are the main reasons of university students to leave Serbia and whether it is possible to find out some personal dispositions for that. In addition, it is important to determine what percentage of students has a wish to go abroad and what are their plans and expectations. The research sample is consisted of 355 students of University of Nis, Serbia. About $62 \%$ of respondents sample was female $(\mathrm{N}=219)$. A questionnaire about students' interests and reasons for going abroad and leave Serbia was prepared by authors for research purposes. Personality traits are defined according to the Big Five model and assessed by The Big Five Inventory - BFI (John \& Srivastava, 1999), intending to measure: extroversion, neuroticism, openness to experience, agreeableness, and conscientiousness. The main results are as following. About $36.2 \%$ of students planned to leave country, $49.2 \%$ of students said that they didn't sure yet, while only $14.6 \%$ didn't show willingness to go abroad and live in foreign country. The most common reasons for going abroad is searching for a work (46\%) and the second one is a wish to continue education (19.7\%). The greatest percentage of students plan to go by the help of scholarship (24.5\%) or by the help of relatives (21.4\%). Students of different faculties have different levels of priorities when it comes to economic reasons or independence as a reason for going abroad. Some of them are ready to get jobs they would not accept to do in Serbia. Concerning the dimensions of personality, the results showed that primarily high openness to experience and high enough extroversion could be connected to students' willingness to leave their own country. Obtained data are useful for better explanation of strong interest and tendency to go abroad in students' population in Serbia. The findings are discussed in the light of up-to-date literature and studies.
\end{abstract}

Keywords: Brain drain, students, personality traits, reasons for leaving country, Serbia.

\section{Introduction}

This paper is referring to the problem of brain drain, used to describe 'the situation in which large numbers of educated and very skilled people leave their own country to live and work in another one where pay and conditions are better'. During the 1960s and 1970s, this term was used to describe the migration of experts and scientists from underdeveloped regions into developed countries. The term is also used for a widespread phenomenon that students from poor countries go to studies and remain permanently working and living in developed countries. In the region of Southeastern Europe and in Serbia, a high percentage of qualified professionals had left their homeland and employed abroad for a long time, sometimes during the whole working life, and returning after receiving the pension. Several studies have shown that mass migration from Eastern Europe during the 1990s was in fact brain waste, because very few people from those countries found employment that meet their qualifications. It is why this phenomenon has often been connected to the depletion of the intellectual and professional resources of a country or a region.

According to the data collected by The Institute for Sociological Research of the Faculty of Philosophy in Belgrade, during the 1990s about 5\% of the total population of the former Yugoslavia was displaced, which was more than in the previous decades (1971 - 2.5\% 1981 - 3.6\%, 1991 - 3.9\%). 
Moreover, the majority (91\%) of people were under 40 years, mainly students and graduated people, with more than 13 years of schooling (Grečić, 2010; Grupa 484, 2010). As a consequence of such mass migrations, the vitality of the labor force had been significantly reduced, the inflow of money from foreign exchange was not so great, and the migrants mainly returned as elderly and not healthy enough people. Such a huge migration represents a real devastation in developing countries while developed countries got benefits on the basis of the influx of cheap and qualified workforce.

Going abroad of young educated personnel in order to continue their education and professional development is not only the result of social and historical circumstances in the country. Family and personal parameters can be closely related to emigration. Recent studies discuss whether there is a certain type of personality who is more inclined than others to leave homeland and live abroad.

\section{Research problem and objectives}

The scope and actuality of migration of the Serbian population is basically our motivation to deal with this problem. The goal of this research is to examine what are the main reasons of university students to leave Serbia and whether it is possible to find out some personal dispositions for that.

In addition, our research is aimed to determine what percentage of students show an intention to emigrate, and what are their plans and expectations. We also want to examine how well they are informed about the possibilities and conditions of emigration and living in foreign countries.

\section{Method}

\subsection{Participants}

The sample consisted of 355 students of University of Nis, Serbia, aged 19 to 27 years. About $62 \%$ of respondents sample was female $(\mathrm{N}=219)$ and $38 \%$ was males $(\mathrm{N}=136)$. Respondents are studying the following faculties: Faculty of Electronic Engineering (47), Faculty of Science (47), Faculty of Medicine (33), Faculty of Civil Engineering and Architecture (61), Faculty of Economics (58), Faculty of Philosophy (57), Faculty of Arts (51). Further data will be given to describe the sample of the subjects.

Participants live primarily with both parents in complete families (64\%), and $9.6 \%$ of them live in a single-parent family, due to divorce or death of a father or mother. About $15 \%$ of students live alone or with a roommate, mainly because studying outside their place of residence. $52.4 \%$ of respondents have changed their place of living, whether due to studying or because of migration to larger cities for better living conditions. Respondents are living mainly in town or city (77.5\%).

The education of the parents of the respondents is predominantly at the secondary level, while a third of them have university education, which is significantly higher than the average educational level of the population in Serbia. More precisely, 50.4\% of fathers finished secondary school, $7.3 \%$ of fathers finished college and $32 \%$ graduated at university; education of mothers is at a similar level: secondary school 54.1\%, higher school 5.9\% and university level 29.6\%.

Regarding the socio-economic status (SES) of the origin-families, respondents' estimations are as the following: very bad $-2.8 \%$, unsatisfactory $-25.1 \%$, satisfactory $-53.1 \%$, quite well $-17.2 \%$. As can be seen from the data, about $70 \%$ of the respondents consider the socioeconomic situation in the family to be satisfactory or completely good, which indirectly indicates that the unfavorable economic situation is not the only reason they want to go abroad for a shorter or longer period of time. Only $6 \%$ of respondents have a permanent job, and about $14 \%$ of them work occasionally part-time job.

When asked if they have traveled abroad so far, the respondents gave the following answers: $37.7 \%$ rarely travel abroad, occasionally $33.8 \%$, often $11 \%$ and $4.9 \%$ have not traveled yet.

\subsection{Variables and instruments}

A questionnaire about students' interests and reasons for going abroad and emigrate from Serbia was made by authors for research purposes. The questions were designed to examine what are the most frequent reasons for going abroad, for a shorter or longer time: economic reasons, for example, job search, higher incomes and better paid jobs, the possibility of career development and promotion; continuing education and professional development; a quest for adventure and a desire to get to know other cultures; the need for independence and opportunity for personal development. The process of developing the questionnaire is based on the review of the available literature in this field. In terms of content, the questionnaire can be partly compared with aspects of migration from the MBM-Migration Belief Model (Groenewold, Bruijn, \& Bilsborrow, 2012; Hoppe, \& Fujishiro, 2015). Precisely, MBM assumes that emigration intensions and plans are determined by perceived economic threat in the domain of living conditions, perceived emigration benefits, perceived emigration barriers (obstacles in emigration process) and emigration self-efficacy (confidence in one's ability to effectively perform in a foreign country). 
Personality traits are defined according to the Big Five model and assessed by The Big Five Inventory - BFI (John \& Srivastava, 1999), 44-item inventory, intending to measure: extroversion, neuroticism, openness to experience, agreeableness, and conscientiousness. In this conception, personality traits are considered to be time-stable inner dispositions of a person that are able to initiate and direct person's thinking, emotions and behavior. Extroversion vs. introversion (E) refers to person's sociability, activity and excitement-seeking, assertiveness, and positive emotions as warmth and cordiality. Neuroticism vs. emotional stability (N) refers to the negative effects, such as anxiety, hostility, sadness, depression and vulnerability too. Openness vs. closedness to experience $(\mathrm{O})$ refers to person's intellectual curiosity, open-mindedness and novelty seeking, wide interests and imagination. Agreeableness (A) refers to tendency to be trusting, not demanding, altruistic, tender-minded and benevolent in relations with people. Conscientiousness (C) refers to person's dutifulness, achievement striving, tendency to be selfdisciplined, persistent, organized, with strong sense of responsibility to commitments.

\subsection{Procedure}

The survey was conducted in above mentioned faculties, situated in Nis, Serbia. The questionnaires were administered in groups during regular classes, in the period April-June 2016. Testing was anonymous and it took about 40 minutes. Students were informed about the research purposes and their participation was voluntary.

\section{Results and discussion}

\subsection{Reasons, intensions, and emigration plans in university students in Serbia}

For the purposes of this paper, only the most important results of the research will be presented. According to data, 36.2\% of students are planning long-term stay abroad, i.e. to emigrate from Serbia, $49.2 \%$ of students said that they didn't sure yet about that, while only $14.6 \%$ didn't show willingness to go abroad and live in foreign country. The most common reasons for leaving the country are economic: finding jobs, better paid jobs, whether it is in line with professional education of students or less attractive jobs, higher income that enables a better life and the founding of one's own family. It should be emphasized that dissatisfaction with the political situation and the perspective offered to young people for life in the country is also an important motivating factor for the emigration of young people from Serbia.

If we take into consideration both students planning long-term and short-term stay abroad, the goals that students want to achieve by going abroad are: a search for a job of $45.9 \%$; continuing education 19.7\%; professional development 9,9\%; the desire to get to know other cultures $3.4 \%$; the desire to separate from the parents $1.4 \%$. Students of different faculties have somewhat different priorities when it comes to the goals for going abroad. Besides, some of them are ready to get jobs they would not accept to do in Serbia.

The largest migration of students and professionals is registered in the field of medicine, electronics, and technical sciences which is talked about a lot but there are no precise data; migration is considered to be greater than that recorded in statistical data (Grupa 484,2010; Santric-Milicevic et al., 2014).

Regarding the way students would go abroad, the following results were obtained: expectation that they will receive scholarships - $24.5 \%$, with the help of their relatives - $21.4 \%$, by friends' help - 11\%, at own risk - 18\%; by a partner's help - 2.8\%, getting a job - $3.4 \%$, working on the pool, babysitting etc. - 2,8\%.

As can be seen from the above mentioned data, about $70 \%$ of the respondents consider the socioeconomic situation in the family to be satisfactory or completely good, which indirectly indicates that the unfavorable economic situation is not the only reason they want to go abroad for a shorter or longer period of time.

When it comes to informing the students of the University of Niš about going abroad, the data showed the following: only $8.5 \%$ of students are very well informed; $23.4 \%$ of those informed; they know something - 44.8\%; very little information has $19.2 \%$ of students, and $4.2 \%$ are not informed at all.

Bearing in mind the situation in Serbia, characterized by high youth unemployment and unsatisfactory economic situation, it is clear why such a large percentage of youth wants to emigrate and believes that much more opportunities are offered by foreign economically developed countries, in Europe or USA. Although the economic situation in Slovakia is better than in Serbia, recent findings indicate that $23.7 \%$ of Slovak university students planned to emigrate long-term, while $12.3 \%$ of them planned short-time stay abroad, primarily for economic reasons and secondly due to their confidence in one's ability to effectively perform the emigration behavior, called 'emigration self-efficacy' (Dobrowolska-Kulanova, \& Orosova, 2018; Hajduch, Orosova, \& Dobrowolska-Kulanova, 2018). 


\subsection{The role of personality dispositions in the context of migration}

Concerning the dimensions of personality, the results showed that primarily high openness to experience and high enough extroversion could be connected to students' willingness to leave their homeland. Comparing to the university students without emigration intensions, students with emigration plans were scored higher on dimension of openness $(p<0.01)$ and dimension of extroversion $(p<0.05)$. Openness to experience relates to intellectual curiosity, a wide range of interests, and openness to change and novelty in general. Accordingly, it's understandable that students of these personality traits show more inclination and readiness to leave their homeland and go to another country. As is well-known, Extroversion refers to person's high involvement in the outside world, the ability to keep interpersonal relations, a tendency to experience positive emotions and an assertive attitude to the social environment. The aforementioned personality dispositions represent the precondition for the person to fit in well in the new environment, to meet the expectations of others and to adapt good enough, and thus show that he/she is able to function adequately in new circumstances.

The finding of an important role of openness in the context of population migration studies is in line with numerous studies conducted around the world as well as in Serbia. Some researchers believe it's possible to talk about a particular type of personality that is prone to migration, so-called "migrant personality" (Boneva, \& Frieze, 2001; Li, Olson, \& Frieze, 2013). That term means that an individual has a personal predisposition for migration and that this decision is not largely conditioned by situational factors, and can manifest in migration from one place to another, but also in the desire to travel for leisure or entertainment and excitement. On the sample of Croatian students, Boneva and Frieze (2001) found that high openness and low "place attachment" are associated with the desire to emigrate. There are also somewhat different views of the essence of migration. "Existential migration" is the term introduced by Madison (2009, according to Luca \& Filipopoulos, 2014), in order to describe a person who has a psychological need to leave home and "explore the world".

Researching the tendencies towards migration to Singapore, on a sample of 1915 young people aged 16 to 26, Chan-Hoong (2008) found that 21\% of young people want to emigrate because of their dissatisfaction with the prospect in their homeland, and they are characterized by openness, high motive of achievement and experiencing self-efficacy. On the other hand, about $23 \%$ of youth didn't want to go abroad because of high conservatism and strong attachment to family and nation, and that could be seen as cultural resilience. In a survey conducted in America on a sample of 2,772 students abroad (Salisbury at all, 2008), it was found that the decision to go to study outside the country is influenced by social, cultural, financial and personal factors that are accumulating since high school. Factors that contribute to the decision of young people to leave are: higher socioeconomic status of the family, higher educational level of parents, school success, as well as openness to differences, for new ideas and experiences.

The Office for cooperation with the Diaspora of the City of Nis (South East Serbia) conducted a survey in February 2014 on the sample of students of the upper secondary school, aiming to examine how many young people tend to study and work outside Serbia, what are the most important reasons for going to abroad, what are their personality traits and what kind of families they live in (Ćirić, Sinđić and Matković, 2014). The main results are as following: students who wish to study abroad are open-minded persons, with wide interest to get know new culture and people, they are successful in school, not satisfied with socioeconomic status of families living in.

\section{Implications and future research direction}

Findings indicate that high percentage (more than 30\%) of university students from the sample show emigration intentions and plan long-term staying abroad. The economic and political situation in state and lack of possibilities to get an adequate and well paid job are the most frequent reasons for emigration among students. The second motive to go abroad is related to professional development and educating purposes. Openness and wish to get to know some other cultures is the third reason for leaving Serbia, but it's not frequent. Concerning personality dimensions, students' high openness to experience and high enough extroversion could be connected to students' willingness to emigrate and leave their homeland. Accordingly, that finding may be seen partly in line with concept of 'migrant personality' but more profound research is needed in the future. Furthermore, broader coverage of personality variables are desirable; thus, the level of aspiration, motive of achievement, expectations of living abroad, and life philosophy of the person should be included.

It's worthy to make some concluding remarks in the context of research problem in general. Many academics and successful professionals, mainly young adults, apply for a job abroad and sometimes decide to live as emigrants in foreign countries with no intention to return to homeland. Having in mind that university students, graduated persons and professionals represent the most potent 
part of general population (they are young, skillful and high educated persons), there are a lot of reasons to worry about the future in Serbia.

According to the data, about $68 \%$ of respondents have been poorly informed about the way of going abroad and living conditions in foreign countries. It is therefore important to improve information through providing accurate and reliable information on opportunities and benefits, but also on the risks of emigration. This would not directly prevent the migration of young people and professionals, but it could contribute to making the decision based on reliable and verified information.

Migration for employment and schooling is nowadays a very widespread process in the world, but this is a much bigger problem for a small and economically underdeveloped country such as Serbia. It is crucial to stimulate the return of young people to their country or to develop good business cooperation with successful professionals, and in this way, at least partially reduce the adverse effects of mass migrations.

\section{Acknowledgments}

This paper was created as a part of the research project No. 179002, supported by the Ministry of Education, Science and Technological Development, Republic of Serbia, and within the scope of project supported by Faculty of Philosophy, University of Nis, Serbia, No. 183/1-16-9-01

\section{References}

Boneva, B. S., \& Frieze, I. H. (2001). Toward a concept of migrant personality. Journal of Social Issues, 57, 477-491.

Chan-Hoong, L. (2008). Attitudes on emigration among young Singaporeans. National University of Singapore: Working Paper No. 19.

Ćirić, T., Sinđić, M., Matković, J. (2014). Osobine ličnosti i porodični odnosi maturanata koji imaju tendenciju da studiraju ili rade van Republike Srbije. 62. Sabor psihologa Srbije, Knjiga rezimea /Book of abstracts, 27-28. Beograd: CPP.

Dobrowolska-Kulanova, M., \& Orosova, O. (2018). Students' emigration intensions and emigration plans in the context of the Migration Belief Model. In C. Pracana \& M. Wang (Eds.) Psychology Applications and Developments IV (178-187). Lisboa, Portugal: InScience Press.

Grečić, V. (2010). Srpska naučna dijaspora. Beograd: Institut za međinarodnu politiku i privredu.

Groenewold, G., Bruijn, B., \& Bilsborrow, R. (2012). Psychosocial factors of migration: Adaptation and application of the health belief model. International Migration, 50 (6), 211-231.

Grupa 484 (2010). Odliv mozgova iz Srbije - problemi i moguća rešenja /Brain drain in Serbia problems and possible solutions/ www.researchgate.net/publictopics.PublicPostFileLoader.html

Hajduch, B., Orosova, O., \& Dobrowolska-Kulanova, M. (2018). Factors related to life satisfaction, cultural resilience and emigration intensions among Slovak university students. In C. Pracana \& M. Wang (Eds.) Psychology Applications and Developments IV (87-95). Lisboa, Portugal: InScience Press.

Hoppe, A., \& Fujishiro, K. (2015). Anticipated job benefits, career aspiration, and generalize self-efficacy as predictors for Migration decision-making. International Journal of Intercultural Relations, 47, 13-27.

John, O. P, \& Srivastava, S. (1999). The Big-Five trait taxonomy: History, measurement, and theoretical perspectives. In L.A. Pervin \& O.P. John (Eds.), Handbook of personality: Theory and research (Vol.2, pp. 102-138). New York: Guilford Press.

Li, M., Olson, J., \& Frieze, I. (2013). Students' Study Abroad Plans: The Influence of Motivational and Personality Factors. Frontiers: The Interdisciplinary Journal of Study Abroad, 23, 73-87.

Luca, M. \& Filipoppoulos, P. (2014). Motivational and adaptation experiences of returnees and migrants to Cyprus: A grounded theory study with Counselling Psychology application and practice implications in Europe. The European Journal of Counselling Psychology, Vol. 3(1), 20-41.

Salisbury, M., Umbach, P., Paulsen, M., Pascarella, E. (2008). Going Global: Understanding the Choice Process of the Intent to Study Abroad. Research in Higher Education Journal. DOI 10.1007/s11162-008-9111-X.

Santric-Milicevic, M. M., Terzic-Supic, Z. J, Matejic, B. R., Vasic, V., Ricketts III, T. C. (2014). First- and fifth-year medical students' intentions for emigration and practice abroad: A case study of Serbia. Health Policy, 118(2), 173-183. 


\title{
AN INVESTIGATION OF THE RELATION BETWEEN PERSONALITY, JOB PERFORMANCE, AND JOB SATISFACTION VIA PROFILE ANALYSIS
}

\author{
Arkun Tatar ${ }^{1}$, Gaye Saltukoğlu ${ }^{1}$ \& Hüdanur Özdemir ${ }^{2}$ \\ ${ }^{1}$ Department of Psychology, FSM Vaklf University / Associate Professor (Turkey) \\ ${ }^{2}$ Psychologist (Turkey)
}

\begin{abstract}
The widespread use of personality tests in the process of personnel recruitment to evaluate the suitability of applicants for a particular job and to select employees who display personality traits that are informative in terms of high performance and satisfaction, demonstrates the need for further investigation of the relation between personality, job performance and job satisfaction. In this context, the present study aimed to determine the personality traits that predict job performance and job satisfaction. For this purpose, personality profiles of the participants were compared in terms of their levels of job performance and job satisfaction, depending on the Five Factor Model. From 278 different occupational groups, 4975 people (2833 men and 2142 women) between the ages of 19 and 85 participated in the study. The primary criterion for inclusion was working at a job for more than one year. Personality profiles were gathered through the long form of the Five Factor Personality Inventory. In order to assess perceived job performance and job satisfaction levels, participants were asked to rate how well they find themselves successful in their work and how satisfied they are with their work on linear scales ranging from 1 to 5 with half-units of increase. In the first place, multiple linear regression analyses were conducted in order to determine effects of personality traits in predicting job performance and job satisfaction. Results showed that all of the factors are significant predictors of job satisfaction. However, Openness to Experience factor found to be ineffective in predicting job performance evaluations. In the second place, based on their job performance and job satisfaction levels subjects were categorized into four groups and personality profiles of the groups were compared with MANOVA. Results indicate that the group who has high job performance and job satisfaction differed in many personality dimensions.
\end{abstract}

Keywords: Job performance, job satisfaction, personality, personality profile, Five-Factor Model of Personality.

\section{Introduction}

The relation of personality to job performance, and job satisfaction has been the subject of much systematic investigation within the field of industrial and organizational psychology over the past century (Rothmann and Coetzer, 2003; Saltukoğlu and Tatar, 2018; Tatar, Şahintürk, Saltukoğlu and Telvi, 2013). Questions have been raised about the utility of personality measures in predicting job performance and satisfaction, especially for personnel selection purposes (Rothstein and Goffin, 2006). Early studies on the subject were conducted when there was no commonly accepted taxonomy of personality traits (Guion and Gottier, 1965; Schmitt, Gooding, Noe and Kirsch, 1984). In addition, the focus was on the validity of personality measures in occupational groups / settings, rather than investigating the effects of personality on job performance and job satisfaction in detail (Barrick and Mount, 1991). Consequently, this theoretical issue has led the conclusion that personality is not a powerful predictor of job performance and its validity is low (Guion and Gottier, 1965; Schmitt et al., 1984). However, this concept has been challenged by the emergence of the Five Factor Model of personality, which has provided more comprehensive framework (Rothmann and Coetzer, 2003; Salgado, 1997; Saltukoğlu and Tatar, 2018). The vast majority of studies, which were conducted based on the Five Factor Model, have been demonstrated that especially Conscientiousness and Emotional Stability are central factors influencing performance and satisfaction (Barrick and Mount, 1991; Barrick, Mount and Judge, 2001; Judge, Heler and Mount, 2002). 
Job performance, which is a multidimensional construct, reflects the time, energy, and resources that employees spend on tasks while fulfilling their responsibilities and it is stated that it could be affected by situational (e.g. job characteristics, structure of the organization, nature of the co-workers' interpersonal relationships) and dispositional (e.g. locus of control, self-esteem, motives, needs, achievement motivation, skills, and personality characteristics) factors (Rothmann and Coetzer, 2003). It has been reported that Conscientiousness is the strongest predictor of job performance compared to other factors (Alessandri and Vecchione, 2012; Zhao and Seibert, 2006). This finding is not surprising considering that the high end of this factor indicates being responsible, purposeful, self-disciplined, task-oriented, attentive, success oriented, determined, and trustworthy (Bhatti, Battour, Ismail, and Sundram, 2014; Somer, Korkmaz and Tatar, 2004). The second most important factor for job performance is Emotional Stability (Barrick and Mount, 1991; Zhao and Seibert, 2006). Low scores on this factor are indicative of being worried, anxious, hesitant, irritable, and unstable (Somer et al., 2004). It is stated that people who are on the low pole of this factor are vulnerable to negative life events, and when they face various problems at work their performance gets poorer, considering that they have the tendency to act accordingly (Rothmann and Coetzer, 2003).

However, research findings into Agreeableness, Extraversion and Openness to Experience have been inconsistent. This inconsistency can be explained by the fact that different jobs have and require different characteristics (Blickle et al., 2008; Costa, McCrae and Holland, 1984; Judge and Zapata, 2015; Ozer and Benet-Martinez, 2006; Saltukoğlu and Tatar, 2018; Tatar, Saltukoğlu and Teoman, 2017; Zhao and Seibert, 2006). However, it is generally reported that these factors are positively related to job performance in various occupational groups (Bhatti et al., 2014; Zhao and Seibert, 2006). Respectively, it is stated that employees who underperform are more resistant to new ideas, more conventional, less emphatic, and less inquisitive regarding the relationship between Openness to Experience and job performance. Additionally, it is suggested that employees who are at the high pole of this factor can perform better in educational settings and jobs like training (Barrick and Mount, 1991; Rothmann and Coetzer, 2003; Zhao and Seibert, 2006). Agreeableness is indicative of being open to criticisms, mild, cooperative, easygoing, helpful, and understanding and people who are at the high pole of this factor perform better in customer services or in jobs that include teamwork (Bhatti et al., 2014; Costa et al., 1984; Rothmann and Coetzer, 2003; Somer et al., 2004). Lastly, extravert people perform better in areas that include interaction with other employees or teamwork such as sales and marketing, or in managerial positions, considering that the high pole of Extraversion is reflective of positive experiences and emotions besides sociability, assertiveness, and talkativeness (Barrick, Stewart and Piotrowski, 2002; Blickle et al., 2015; Mount et al., 1998). Previous research findings into the relation between job performance and job satisfaction have been contradictory. However, it is observed that both constructs are affected by personality traits respectively and there is a strong relationship between them (Christen, Iyer and Soberman, 2006; Judge, Weiss, Kammeyer-Mueller and Hulin, 2017; Karim, 2017; Tatar, Saltukoğlu, Dal and Atay, 2013; Wright and Cropanzano, 2000). It is stated that the most important predictor of job satisfaction is Emotional Stability instead of Conscientiousness (Barrick and ark., 2001; Judge et al., 2002; Rothmann and Coetzer, 2003). Additionally, Extraversion plays an important role in predicting job satisfaction (Avery, Smillie and Fife-Schaw, 2015; Judge et al., 2002).

Regarding the inconsistency in findings, the overall aim of this study is to determine which personality traits are affective in predicting job performance and job satisfaction. In this direction, subjects from various occupational groups were compared in terms of their job performance and satisfaction levels.

\section{Method}

\subsection{Participants}

The subjects were selected on the basis of working at revenue generating jobs for more than one year (housewives, retirees, students, and people who work at charities voluntarily were excluded). Participants were 5040 people from 278 different occupations. However, the data obtained from 65 people were excluded because they did not answer questions properly. 2142 (43.1\%) of the participants were female and 2833 (56.9\%) were male, ranging in age between 19 and $85(\mathrm{M}=35.95$, SD = 12.85). Working years of the participants ranged from 1 to 63 years $(M=11.82$, SD =11.07).

\subsection{Materials}

Data were collected through long form of the Five Factor Personality Inventory (Somer, Korkmaz, and Tatar, 2002; 2004) and a questionnaire to determine socio-demographic characteristics of the subjects. Long form of the Five Factor Personality Inventory is a 5-point Likert type scale (on which $1=$ Totally Accurate, 5 = Very Inaccurate) consisting of 220 items and 17 personality dimensions. 
Employees were asked to rate how well they find themselves successful in their work and how satisfied they are with their work on a linear scale, ranged from 1 to 5, with half-units of increase.

\subsection{Procedure and data analysis}

Data were obtained from people whom accepted to participate in the study voluntarily, using convenient sampling method in Istanbul. Data collection process lasted for about four years, and an application for one individual lasted around 30-60 minutes depending on subject's reading speed.

In the first place, the internal consistency coefficients of the Five Factor Personality Inventory's factors and dimensions were calculated for the whole group, female, and male groups separately. Afterwards, the mean scores of gender groups and the groups that were formed based on job performance and job satisfaction levels were subjected to Multivariate Analysis of Variance (MANOVA) in terms of the five factors and 17 dimensions.

\subsection{Results}

The reliability coefficients for the factors in the whole group were $0.837-0.928$, in the female group were $0.815-0.932$, and in the male group were $0.829-0.921$. The reliability coefficients for the dimensions in the whole group were 0.667-0.856, in the female group were $0.644-0.868$, and in the male group were $0.665-0.837$. The correlation coefficients between employee's job performance and the dimensions of the inventory ranged from -0.004 to -0.271 , and between factors from 0.086 to -0.214 . The correlation coefficients between employee's job satisfaction and the dimensions of the inventory ranged from -0.030 to -0.243 , and between factors from 0.086 to -0.214 . The correlation coefficient between job performance and job satisfaction was calculated 0.403

Secondly, multiple linear regression analyses were conducted in order to determine effects of personality traits in predicting job performance and job satisfaction. Results showed that, Assertiveness, Tolerance, Responsibility / Deliberateness, Self Assuredness, Sensitivity, and Openness to Newness dimensions and Extraversion, Agreeableness, Conscientiousness, and Emotional Instability factors significantly predict the job performance evaluations. However, Liveliness, Interaction, Calmness, Agreement, Soft Heartedness / Altruism, Orderliness, Compliance with the Rules, Excitement Seeking, Emotional Lability, Proneness to Anxiety, and Analytical Thinking dimensions and Openness to Experience factor found to be ineffective in predicting job performance evaluations.

The results for job satisfaction indicate that Liveliness, Interaction, Calmness, Orderliness, Compliance with the Rules, Responsibility / Deliberateness, Self Assuredness, and Sensitivity dimensions and all of the factors is significant predictors. However, Assertiveness, Tolerance, Agreement, Soft Heartedness / Altruism, Excitement Seeking, Emotional Lability, Proneness to Anxiety, Analytical Thinking, and Openness to Newness dimensions found to be ineffective in predicting job satisfaction.

Afterwards, based on their job performance $(\mathrm{M}=4.07, \mathrm{SD}=0.77)$ and job satisfaction levels $(\mathrm{M}=3.87, \mathrm{SD}=1.02)$, subjects were categorized into four groups: Low Performance - Low Satisfaction (55.4\%), Low Performance - High Satisfaction (13.0\%), High Performance - Low Satisfaction (12.6\%), and High Performance - High Satisfaction (19.0\%). Gender and job performance-job satisfaction groups ( 2 x 4) were compared in terms of 5 factors and 17 dimensions separately with MANOVA.

When gender differences are compared in terms of personality dimensions, females' mean scores found to be significantly higher than males' on the Liveliness, Assertiveness, Tolerance, Soft Heartedness / Altruism, Orderliness, Responsibility / Deliberateness, Emotional Lability, Proneness to Anxiety, Self Assuredness, Analytical Thinking, Sensitivity, and Openness to Newness dimensions, while males scored significantly higher than females only on the Calmness and Agreement dimensions. In addition, females scored higher on the Extraversion, Conscientiousness, Emotional Instability, and Openness to Experience factors. There were no significant differences found on the Agreeableness factor between gender groups.

\section{Conclusion}

The aim of the present study was to determine which personality traits are affective in predicting job performance and job satisfaction. The mean scores of the subjects were subjected to regression analysis and MANOVA, and presented separately for the factors and 17 dimensions. It was observed that results obtained from sub-dimensions have provided more detailed information compared to factors. Together these findings allow comparisons across studies that based on other theoretical models apart from the Five Factor Model of Personality. Respectively, results presented here for factors allow comparisons between findings obtained from different measures of personality across studies, and establish a cultural framework. Another conclusion can be drawn from the present study is that different statistical techniques may offer contradictory findings on the same subject. Although regression and variance analyses indicate similar results, there are differences between the two methods in detail. 


\section{References}

Alessandri, G., \& Vecchione, M. (2012). The higher-order factors of the Big Five as predictors of job performance. Personality and Individual Differences, 53(6), 779-784.

Avery, R. E., Smillie, L. D., \& Fife-Schaw, C. R. (2015). Employee achievement orientations and personality as predictors of job satisfaction facets. Personality and Individual Differences, 76, 56-61.

Barrick, M. R., \& Mount, M. K. (1991). The Big Five personality dimensions and job performance: A meta-analysis. Personnel Psychology, 44(1), 1-26.

Barrick, M. R., Mount, M. K., \& Judge, T. A. (2001). Personality and performance at the beginning of the new millennium: What do we know and where do we go next? International Journal of Selection and Assessment, 9(1-2), 9-30.

Barrick, M. R., Stewart, G. L., \& Piotrowski, M. (2002). Personality and job performance: Test of the mediating effects of motivation among sales representatives. Journal of Applied Psychology, 87(1), 43-51.

Bhatti, A. M., Mohamed Battour, M., Rageh Ismail, A., \& Pandiyan Sundram, V. (2014). Effects of personality traits (Big Five) on expatriates adjustment and job performance. Equality, Diversity and Inclusion: An International Journal, 33(1), 73-96.

Blickle, G., Meurs, J. A., Wihler, A., Ewen, C., Merkl, R., \& Missfeld, T. (2015). Extraversion and job performance: How context relevance and bandwidth specificity create a non-linear, positive, and asymptotic relationship. Journal of Vocational Behavior, 87, 80-88.

Blickle, G., Meurs, J. A., Zettler, I., Solga, J., Noethen, D., Kramer, J., \& Ferris, G. R. (2008). Personality, political skill, and job performance. Journal of Vocational Behavior, 72(3), 377-387.

Christen, M., Iyer, G., \& Soberman, D. (2006). Job satisfaction, job performance, and effort: A reexamination using agency theory. Journal of Marketing, 70(1), 137-150.

Costa, P. T., McCrae, R. R., \& Holland, J. L. (1984). Personality and vocational interests in an adult sample. Journal of Applied Psychology, 69(3), 390-400.

Guion, R. M., \& Gottier, R. F. (1965). Validity of personality measures in personnel selection. Personnel Psychology, 18(2), 135-164.

Judge, T. A., \& Zapata, C. P. (2015). The person-situation debate revisited: Effect of situation strength and trait activation on the validity of the Big Five personality traits in predicting job performance. Academy of Management Journal, 58(4), 1149-1179.

Judge, T. A., Heller, D., \& Mount, M. K. (2002). Five-Factor Model of personality and job satisfaction: A meta-analysis. Journal of Applied Psychology, 87(3), 530-541.

Judge, T. A., Weiss, H. M., Kammeyer-Mueller, J. D., \& Hulin, C. L. (2017). Job attitudes, job satisfaction, and job affect: A century of continuity and of change. Journal of Applied Psychology, 102(3), 356-374.

Karim, N. H. A. (2017). Investigating the correlates and predictors of job satisfaction among Malaysian academic librarians. Malaysian Journal of Library and Information Science, 13(2), 69-88.

Mount, M. K., Barrick, M. R., \& Stewart, G. L. (1998). Five-Factor Model of personality and performance in jobs involving interpersonal interactions. Human Performance, 11(2-3), 145-165.

Ozer, D. J., \& Benet-Martinez, V. (2006). Personality and the prediction of consequential outcomes. Annual Review of Psychology, 57, 401-421.

Rothmann, S., \& Coetzer, E. P. (2003). The Big Five personality dimensions and job performance. SA Journal of Industrial Psychology, 29(1), 68-74.

Rothstein, M. G., \& Goffin, R. D. (2006). The use of personality measures in personnel selection: What does current research support? Human Resource Management Review, 16(2), 155-180.

Salgado, J. F. (1997). The Five Factor Model of personality and job performance in the European community. Journal of Applied Psychology, 82(1), 30-43.

Saltukoğlu, G., \& Tatar, A. (2018). İş performansının öngörülmesinde kişilik ölçümünün rolü: Öğretmen örneği. Journal of Human Sciences, 15(1), 619-634.

Schmitt, N., Gooding, R. Z., Noe, R. A., \& Kirsch, M. (1984). Metaanalyses of validity studies published between 1964 and 1982 and the investigation of study characteristics. Personnel Psychology, 37(3), 407-422.

Somer, O., Korkmaz, M., \& Tatar, A. (2002). Beş Faktör Kişilik Envanterinin geliştirilmesi-I: Ölçek ve alt ölçeklerin oluşturulması. Türk Psikoloji Dergisi, 17(49), 21-37.

Somer, O., Korkmaz, M., \& Tatar, A. (2004). Kuramdan Uygulamaya Beş Faktör Kişilik Modeli ve Beş Faktör Kişilik Envanteri (5FKE). Ege Üniversitesi Basımevi, İzmir. 
Tatar, A., Şahintürk, H., Saltukoğlu, G., \& Telvi, S. (2013). Tiyatro oyuncularının Beş Faktör Modeli çerçevesinden kişilik özelliklerinin incelenmesi ve meslek grubu profillerinin oluşturulması. Türk Psikoloji Dergisi, 28(72), 1-16.

Tatar, A., Saltukoğlu, G., \& Teoman, D. D. (2017). Çalışan genç kadınların kişilik profilleri ve bireycilik-toplulukçuluk özellikleri. $\dot{I}_{S ̧}^{\prime}$ te Davranış Dergisi, 2(1), 34-45.

Tatar, A., Saltukoğlu, G., Dal, G., \& Atay, B. (2013). Eczacıların Beş Faktör Modeli çerçevesinden kişilik özelliklerinin incelenmesi ve meslek grubu profillerinin oluşturulması. FSM İlmî Araştırmalar Insan ve Toplum Bilimleri Dergisi, 2, 323-341.

Wright, T. A., \& Cropanzano, R. (2000). Psychological well-being and job satisfaction as predictors of job performance. Journal of Occupational Health Psychology, 5(1), 84-94.

Zhao, H., \& Seibert, S. E. (2006). The Big Five personality dimensions and entrepreneurial status: A meta-analytical review. Journal of Applied Psychology, 91(2), 259-271. 


\title{
ACCULTURATION STRATEGIES AND PERCEPTION OF A NORTH AFRICAN WOMAN IN A JUDICIAL CONTEXT
}

\author{
Chloé Dougez ${ }^{1}$, Anne Taillandier-Schmitt ${ }^{1,2}, \&$ Nicolas Combalbert ${ }^{1}$ \\ ${ }^{1}$ Université de TOURS (France) \\ ${ }^{2}$ Université Rennes 2 (France)
}

\begin{abstract}
This study examines some extrajudicial factors that may influence the judgment of a woman from North African immigration. The perception of women with North African origin in our society is a poorly researched subject in psychology whereas they are the target of many stereotypes. In this study, we have been interested in the judgments of men and women on a North African woman who committed an assault on another woman (with a French-sounding name). We particularly studied the effects of acculturation strategies adopted by the perpetrator (adoption of the culture of the host country/retention of the original culture) and the profile of the participant (gender and social dominance orientation, SDO. Two hundred and eighty-six students read a scenario describing an assault committed by a North African woman who was then described according to one of the four acculturation strategies (assimilation, integration, separation, and marginalization) in many areas of daily life (work, family, social life...). The consequences of the assault for the victim were also manipulated (serious or minimal). After reading the scenario, participants judged the act and its perpetrator in four dimensions: judgment of the act, behavior explanation, and judgment of the perpetrator, perception of her masculine and feminine traits. Our results suggested that when the perpetrator did not adopt French culture and/or she maintained her original culture, participants judged her more negatively than in the other conditions and her behaviour was explained more by internal factors than external factors. These results were particularly observed for participants with high level of SDO. Otherwise, depending on the conditions of the experimentation, the judgments of women and men were different. The results of this study were discussed in view of stereotypical norms and threat feeling.
\end{abstract}

Keywords: Acculturation strategies, North African origin, gender, judgements, women delinquency.

\section{Introduction}

This study examines three extrajudicial factors that may influence the judgment of a woman from North African immigration. The perception of women with North African origin in our society is a poorly researched subject in psychology whereas they are the target of many stereotypes (Boukhobza, 2005; Beski 2011). Some studies highlight that people with an immigrant background are judged by the host population differently depending on the way they fit in the culture of the host country (Maisonneuve \& Testé, 2007; Maisonneuve, Testé, Taillandier-Schmitt, \& Dambrun, 2014). Furthermore, two studies have shown that a man or a woman with North African origin is judged more negatively and more severely if they do not adopt the French culture or they maintain their original culture, in a judicial context (Dougez, Taillandier-Schmitt, 2018; Taillandier-Schmitt and Combalbert, 2017). In this study, we are interested in the difference of judgments depending on the gender of the participant at the look of the status of the North African woman. Indeed, women of North African origin have long been absent from the social scene and are portrayed in the media in controversial contexts, such as wearing headscarves or burkinis (Gourdeau, 2015). These polemical issues confirm the stereotypes of women from North Africa, who are often considered as oppressed and subject to male authority. In this case, a North African woman who commits an assault transgresses several stereotypes. As described above, the transgression of stereotyped norms gives rise to harsh judgments (Delacolette, Dardenne, \& Dumond, 2010), especially if the person is likely to undermine a dominant position (for instance, for men when a woman has masculine characteristics). In this study, the perpetrator is described according one of the four acculturation strategies described by Berry (2005) on the bi-dimensional model, which may influence the perception of her and its behavior. Our questions are focused on the influence and interactions of the effects of gender and acculturation strategies on the judgement on a North African woman. We have included a measure of 
level of SDO (Pratto, Sidanius, Stallworth, \& Malle, 1994) because it may influence the judgment in judicial context (Fosterlee, Horowitz, Fosterlee, King \& Ronlund, 1999) and related to acculturation strategies (Levin \& al., 2012).

\section{Objectives}

The purpose of this study was to examine the effects and the interaction of the acculturation strategy adopted by a North African woman who committed an assault with the profile of participants who judged her. Women with North African origin are the target of some gender stereotypes so we decided to observe the gender variable and participants' level of SDO.

\section{Hypotheses}

We hypothesized that the target will be judged less negatively by participants when she adopts the French culture and if she does not maintain exclusively her original culture. We also hypothesized that men will judge more negatively the target depending on the condition of the experimentation (serious consequences and no retention of the original culture).

\section{Method}

Two hundred and eighty-six students (140 men and 146 women) aged 18 to 39 ( $M=20.962$, $S D=2.756$ ) participated in the study. They had different academic backgrounds. They read a scenario describing an assault committed by a North African woman (named "Jamila") against another woman (with a French-sounding name, "Valérie"). The consequences of the assault for the victim were also manipulated: either the victim managed to get away (minimal consequences) or she was knocked unconscious (serious consequences). A description then identifies whether or not she adopts the French culture and/or whether or not she maintains her original culture; depending on the bi-dimensional model of Berry (2005) : 1) she has adopted French culture and rejected her original culture (assimilation), 2) she has adopted French culture and maintained her original culture (integration), 3) she has rejected French culture and maintained her original culture (separation), 4) she has not adopted French culture or conserved her original culture (marginalization). After reading the scenario and the description, participants judged the act and its perpetrator in four dimensions: judgment of the act, behavior explanation, and judgement of the perpetrator, perception of her masculine and feminine traits. The level of SDO was also examined (Pratto, Sidanius, Stallworth, \& Malle, 1994).

\section{Results}

Our results may partly answer to our hypotheses in that we have observed the effects of the adoption of the culture of the country and retention of the original culture, and differences in judgments between women and men, related to the acculturation strategy of the perpetrator and participant's level of SDO. We observe an influence of acculturation strategies on three variables: aggravating circumstances, risk of recidivism and masculine traits (Table 1). Then the results show a main effect of the gender on the measures of level of SDO, custodial sentence, mitigating circumstances and threatening (Figure 1). And we found an effect of participant's level of SDO on the measures of custodial sentence, general impression, and risk of recidivism and attribution of feminine traits (Figure 2). Depending on the seriousness of the consequences for the victim, the adoption of the French culture and the retention of the original culture have influenced participants' judgment : our analyses revealed that when the consequences of the act were serious, the participants attributed the act more to the situation when the perpetrator adopted an integration strategy (adoption and retention) $(M=-.351, S D=2.176)$ than when she adopted a separation strategy (no adoption and retention) $(M=1.211, S D=2.622, p=.008$ ). By contrast, the participants attributed the behavior more to personal factors when the consequences were minimal and the perpetrator adopted either an integration strategy (adoption and retention) $(M=1.108$, $S D=2.283, p=.01$ ) or an assimilation strategy (adoption and no retention) $(M=.811, S D=2.196$, $p=.04)$. Then, men considered generally the North African woman as more threatening than students women $\left(F(1,254)=4.730, p=.03, \eta^{2}=.02\right)$, even more so if the consequences for the victim are serious $\left(F(1,254)=6.257, p=.01, \eta^{2}=.02\right)$. If the perpetrator does not adopt the French culture, men attributed more aggravating circumstances to her than women $\left(F(1,254)=5.301, p=.02, \eta^{2}=.02\right)$. Furthermore, the higher level of SDO predicted a more negative judgment of the perpetrator, and the judgment of women seems to be more depending on their level of SDO than men although that of men is generally higher than that of women. Finally, we observed effects of gender, acculturation strategy and level of SDO of the attribution of masculine and feminine traits to the perpetrator. 


\section{Discussion}

We discuss these results in view of stereotypical norms (Delacolette, Dardenne, \& Dumond, 2010) and threat feeling. In this study, the perpetrator transgresses several stereotypes and the participants judgments may be influenced by their perception of the level of the transgression, which is different depending on the gender, the level of SDO or the acculturation strategy of the perpetrator. Furthermore, depending on the condition of this experimentation and the profile of the participant, the threatening perception of the perpetrator may influence judgements. Men may have felt threatened by the perpetrator because in our study she does not correspond to the female stereotype and adopts male characteristics (violence). We can also interpret the results of this study in terms of adherence to stereotypes, which is consistent with the idea that men have a higher level of SDO than women: the more a person adheres to the stereotypes, the more he will judge negatively a perpetrator who transgresses stereotypes.

\section{Conclusion}

The study of the perception of North African women is a little explored subject in psychology, whereas they are the target of several stereotypes and they can experiment it, especially in the field of employment (Meurs \& Pailhé, 2010). This study is part of a desire to understand social dynamics related to the interaction between gender stereotypes and acculturation phenomena. In the judicial context, it is particularly important to focus on it as perceptions of women and migrants are discussed in the social scene.

Table 1. Means and standard deviations of measures related to retention of the original culture and adoption of French culture.

\begin{tabular}{ccccc} 
& \multicolumn{2}{c}{ Adoption } & \multicolumn{2}{c}{ No adoption } \\
& $\begin{array}{c}\text { Retention } \\
\text { (integration) }\end{array}$ & $\begin{array}{c}\text { No retention } \\
\text { (assimilation) }\end{array}$ & $\begin{array}{c}\text { Retention } \\
\text { (separation) }\end{array}$ & $\begin{array}{c}\text { No retention } \\
\text { (marginalization) }\end{array}$ \\
\hline $\begin{array}{c}\text { Aggravating } \\
\text { circumstances }\end{array}$ & $2.635(1.61)$ & $3.200(1.682)$ & $3.147(1.95)$ & $2.597(1.86)$ \\
Risk of recidivism & $3.649(1.557)$ & $3.486(1.759)$ & $4.587(1.779)$ & $3.97(1.696)$ \\
Masculine traits & $5.088(1.077)$ & $5.021(1.352)$ & $5.607(1.098)$ & $4.754(1.369)$
\end{tabular}

Figure 1. Main effect of the gender on the measures of level of SDO, custodial sentence, mitigating circumstances and threatening.

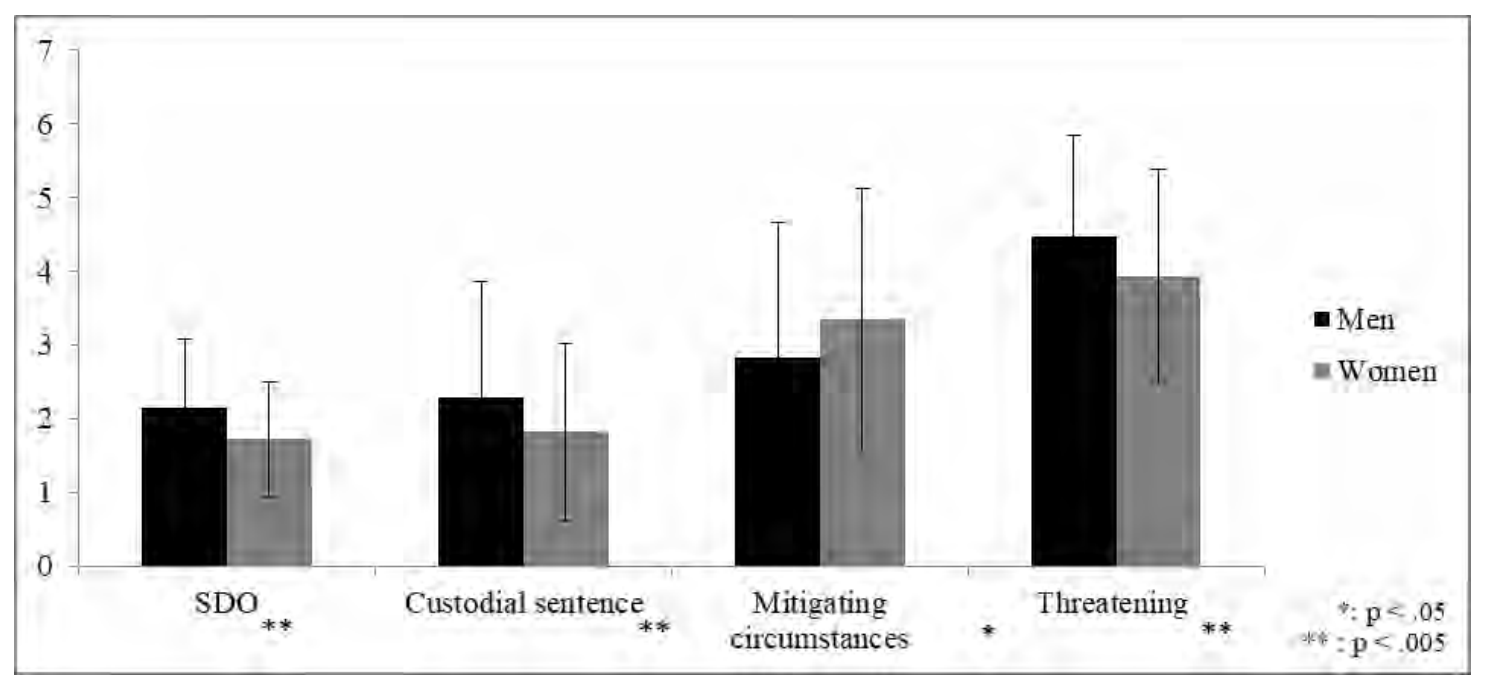


Figure 2. Effect of participant's level of SDO on the measures of custodial sentence, general impression, risk of recidivism and attribution of feminine traits.

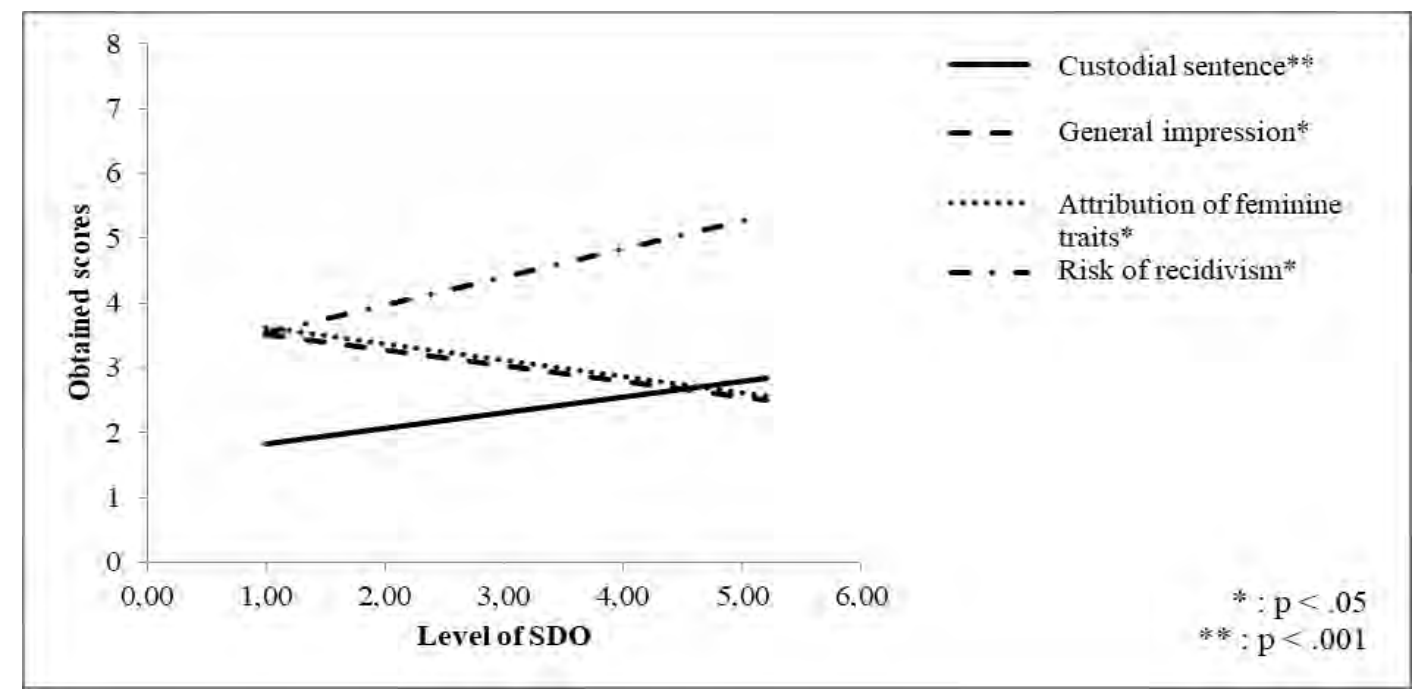

\section{References}

Berry, J. W. (2005). Acculturation: Living successfully in two cultures. International Journal of Intercultural Relations, 29(6), 679-712. doi: 10.1016/j.ijintrel.2005.07.013

Beski, C. (2011). Femmes issues de l'immigration: L'urgence d'une approche complexe. Ville école intégration, 13, 37-41. Retrived from https://www.adric.eu/index.php/nos-publications/66publications/152-chahka-beski-femmes-issues-des-immigrations-lurgence-dune-approchecomplexe

Boukhobza, N. (2005). Les filles naissent après les garçons. Revue Européenne des Migrations Internationales, 21(1), 227-242. Retrieved from http://remi.revues.org/2333

Delacolette, N., Dardenne, B. \& Dumont, M. (2010). Stéréotypes prescriptifs et avantages des groupes dominants. L'année psychologique, 110, 127-156.doi: 10.4074/S0003503310001053

Dougez, C., Taillandier-Schmitt, A., and Combalbert, N. (2018). Influence of acculturation strategies on the judgement of violent act committed by a North African woman. International review of social psychology, 31(1). doi: 10.5334/irps.106

Fosterlee, L., Horowitz, I. A., Fosterlee, R., King, K., \& Ronlund L. (1999). Death penalty attitudes and juror decisions in Australia. Australian Psychologist, 24, 64-69. doi.: 10.1080/00050069908257427

Gourdeau, C. (2015). Une politique d'intégration au service des femmes étrangères? Hommes et Migrations, 1311, 23-29. Retrieved from https://www.cairn.info/revue-hommes-et-migrations2015-3-page-23.htm

Levin, S., Matthews, M., Guimond, S., Sidanius, J., Pratto, F., Kteily, N., Pitpitan, E.V., \& Dover, T. (2012). Assimilation, multiculturalim, and colorblindness: Mediated and moderated relationschips between social dominance orientation and prejudice. Journal of Experimental Psychology, 48(1), 207-212. doi: 10.1016/j.jesp.2011.06.019

Maisonneuve, C., \& Testé, B. (2007). Acculturation preferences of host community: The effect of immigrants acculturation strategies on evaluation and impression formation. International Journal of Intercultural Relation, 31, 669-688. doi: 10.1016/j.ijintrel.2007.06.001

Maisonneuve, C., Testé, B., Taillandier-Schmitt, A., \& Dambrun, M. (2014). Host populations' perceptions toward migrants: The effect of private/public settings and the moderating role of threat. International Journal of Intercultural Relations, 41, 45-56. doi: 10.1016/j.ijintrel.2014.05.006

Meurs, D., \& Pailhé, A. (2010). Position sur le marché du travail des descendants directs d'immigrés en France: les femmes doublement désavantagées? Economie et Statistique, 431-432, 129-151. doi: 10.3406/estat.2010.8078

Pratto, F., Sidanius, J., Stallworth, L. M., \& Malle, B. F. (1994). Social dominance orientation: A personality variable predicting social and political attitudes. Journal of Personality and Social Psychology, 67, 741-763. doi: 10.1037/0022-3514.67.4.741

Taillandier-Schmitt, A., and Combalbert, N. (2017). Influence of acculturation strategies on the judgement and punishment of an offender of North African descent. Psychology, Crime \& Low, 23(9). doi: 10.1080/1068316X.2017.1335731 


\title{
HOLIDAYS IN CULTURAL MEMORY: SOCIAL MEDIA MINING ACROSS GENERATIONS
}

\author{
Tatiana Folomeeva, \& Fedor Vinokurov \\ Faculty of Psychology, Lomonosov Moscow State University (Russia)
}

\begin{abstract}
We present the first results of an ongoing study of the construction and transmission of cultural memories about national holidays in Russia. Due to a widespread adoption of social media among multiple generations as a first- or second-choice way of communication and social interaction, we were able to retrieve a relatively large volume of public data for a representative sample of urban population of Russia older than 14 years. A sample of 2200 public profiles and 13800 of their publications was downloaded from Vk.com - the most popular social media website in Russia (43\% of urban population older than 12 years use it daily according to Kantar TNS MediaScope). Research sample was constructed using a public Vk.com API and Web Census method. Our analysis involved only posts (not reposts) with textual content that were published in the interval of two days before and two days after the date of a national holiday in 2010-2018: January $1^{\text {st }}$ (The New Year), February $23^{\text {rd }}$ (Army Day or Men's Day), March $8^{\text {th }}$ (Women's Day) and May $9^{\text {th }}$ (Victory Day). Content analysis involved standard text-mining procedures of data cleansing, stemming and spam filtering (most frequent terms were reviewed by experts to create a list of stop-words). An explorative topic modelling with LDA algorithm for four separate text corpuses has shown that the publications were mostly connected with a consumer behavior on holidays, but one topic was found to be specific for authors older than 39 years - greeting cards. Greeting cards were the most accessible, affordable and familiar element of a festive ritual in Russia during $20^{\text {th }}$ century. A demographic group also known as "Generation X", those who have matured before public Internet and social media, are now transmitting their cultural memories digitally but with the most familiar and natural form of a pre-Internet illustrated message - a greeting card. Our research illustrates the advantages and limitations of automated analysis of big textual data in social psychology. We propose a novel procedure for automated longitudinal studies of cultural memory and social representations.
\end{abstract}

Keywords: Cultural memory, holidays, social media, data mining.

\section{Introduction}

Cultural memory research is a modern trend in social science, but the definition of "cultural memory" is still a point of discussion. The term is used as often as "collective memory" but the underlying theory is still under development (Wertsch, Roediger, 2008). Even those who are concerned with scientific usefulness of this term do not doubt the social determinants of memory and its dynamics. Employing Vygotsky's sociocultural theory we can describe human memories as result of communication, social rituals, signs and events involving others. The representations of the past are volatile and transform constantly while embedded in social change. Our research suggests that while social media stays the first- or second-choice way of communication and social interaction for the majority of country's population, researchers could monitor public posts and extract the contents of cultural memory, which becomes a great source of insights about social structure and dynamics.

We discuss the construction and transmission of cultural memories about national holidays in Russia. Holidays play a major role in different cultures and are a well-researched phenomenon in anthropology, psychology, social and cultural studies. A. Comte describe holiday as a way of social control, E. Durkheim and M. Mead write about its symbolic meaning, J. Taylor and S. Turner discuss holiday myths and rituals, P. Berger and T. Luckmann view holiday as an opposite of everyday behavior. Based on the previous research of holiday rituals (Karpova, 2008), we propose that festive communication of multiple generations on social media would reflect differences in their cultural memory. 


\section{Objectives and methods}

The goal of our research was to examine differences in social media communication on four national holidays among various demographics.

To gather a representative sample of festive communication about national holidays in Russia we applied social media mining techniques specific for the most popular social media platform in Russia VK.com (also known as "VKontakte" or "In Contact"). According to TNS Gallup Mediascope - a leading media-measuring agency - there were 73\% of monthly average internet users in Russia at the beginning of 2018 (Mediascope, 2018). A bit less than a half (43\%) of population older than 12 years used VK.com website or mobile application daily. VK.com is more popular than Facebook, Instagram or any other social media among Russian population. Since it provides all the same features and content types as Facebook with a similar user interface, there are mostly technical differences between VK.com and Facebook as sources of social data.

At first, we have gathered a representative sample of Russian urban population consisting of 2200 public profiles (last active in March of 2018) using Web Census method (Chekmyishev, Yashunskiy, 2014) which was proposed specifically for accomplishing this type of tasks with VK.com public API.

The second step of social media mining involved gathering public communication on national holidays: January $1^{\text {st }}$ (The New Year), February $23^{\text {rd }}$ (Army Day or Men's Day), March $8^{\text {th }}$ (Women's Day) and May $9^{\text {th }}$ (Victory Day). Publications by these 2200 authors from January 2010 till March 2018 were downloaded for dates in the interval between two days before and two days after the date of a national holiday. A single publication in VK.com includes some textual data and optional media attachments (mostly images or videos). Each record in the resulting dataset of 13800 publications was augmented with the information from author's public profile: age, gender, city of residence, etc.

Profiles and publications data was sampled and retrieved with a public VK.com API. Text data preparation involved standard text-mining procedures of data cleansing, stemming, filtering of common words and spam messages. Experts (students in social psychology) reviewed most frequent terms to create a list of stop-words for a spam filter. Spam publications had low term variability, so we applied simple filtering rules based on keyword occurrence.

At the end of a data collection step, we had four separate text corpuses for each holiday prepared for explorative topic modelling with latent Dirichlet allocation (LDA) algorithm. LDA is a machine-learning model well suited for topic discovery tasks with "bag of words" approach (Nikolenko et al., 2015). LDA generates and continuously refines multiple hypothesis about implicit topics in a corpus of documents. A "topic" is a sample of words that frequently co-occur in different documents. In text classification tasks LDA calculates a probability that a document belongs to each of the previously learned topics. We performed both topic discovery and document classification for the current research.

The technological stack consisted of a Python notebook running on a Google Colab service, a PyMorphy2 package for word lemmatization, pandas and scikit-learn packages for data analysis and modelling.

\section{Results and discussion}

For each of four holidays we have constructed a separate model of nine topics. The final number of modelled topics is a result of manual calibration for an optimal balance of topic interpretability and quantity.

Five to six topics in each model were linked to consumer behavior on holidays: buying flowers, pastries, various beauty services. One topic in all models was found to be specific for authors older than 39 years - greeting cards. This topic has slight variation in core terms depending on the holiday. These are top keywords sorted by weight (or importance): "The New Year" - greeting card, new year, picture, beautiful, greeting, here, watch, winter, hello, horse, happiness, rooster, goat, luck, dog, sheep, wish, success; "Victory Day" - victory, may, picture, postcard, day, beautiful, friend, great, sky, peaceful, head, answer, holiday; "Women's Day" - postcard, march, picture, beautiful, women day, huge, holiday, sunshine, joy, colors, blossom; "Army Day or Men's Day" - picture, postcard, February, beautiful, defender, fatherland, holiday, male.

Each of the discovered "greeting card" topics contains the word "picture" or "postcard" or both. The majority of posts classified with this topic also contain media attachments. A quick manual sampling of these images showed that these are indeed digital postcards - thematic images with holiday greetings. This topic is specific for publications authored by mature and senior demographic groups (39 years or older). Publications like these are almost non-existent among younger authors. 
These results could illustrate a changing role of a greeting card as an element of festive rituals in Russian culture. From the beginning of mass production of paper postcards in the post-war USSR in 1945 till nowadays greeting cards are still the most affordable holiday gifts available to just about anyone. The evolution of telecommunication and digital services have transformed the look and feel of the modern greeting card but its importance for older generations preserved.

Our research shows that social media mining and topic modelling are suitable and versatile techniques to study large groups in social psychology. Explorative analysis could be performed on historical data (87 months in our case) or almost in real-time with online monitoring and online algorithms for machine learning. Unsupervised topic modelling with a raw social media data allowed us to grasp the festive traditions among different generations in Russia. These are only first steps in making big data analysis and text mining algorithms widely available for psychologists.

\section{References}

Karpova, G. (2008). Prazdnik v kontekste sotsialnyih izmeneniy: tradicii $i$ vlast [Holiday in context of social change: traditions and authority]. Saratov: Nauchnaya kniga.

Chekmyishev, O.A., Yashunskiy, A.D. (2014) Izvlechenie i ispolzovanie dannyih iz elektronnyih sotsialnyih setey [Extracting and using data from online social networks]. Working Paper IPM im. M.V. Keldysha [Keldysh Institute of Applied Mathematics preprints], no 62. Retrieved March 18, 2019, from: http://library.keldysh.ru/preprint.asp?id=2014-62

Nikolenko, S., Koltcov, S., Koltsova, O. (2015) Topic modelling for qualitative studies. Journal of Information Science 2015. Vol 43, Issue 1. Pages 88-102.

Wertsch, J.V., Roediger, H.L. (2008) Collective Memory: Conceptual foundation and theoretical approach. Memory, 16 (3). Pages 318-326.

Mediascope (July 2018) Mediascope Web Index. Retrieved April 3, 2018, from URL: http://mediascope.net/services/media/media-audience/internet/information. 


\title{
PARENT AND CHILDREN'S CHARACTERISTICS BY CUSTODY TYPE: A HIGH CONFLICT SAMPLE
}

\author{
Marta Herrero $^{1}$, Ana Martínez-Pampliega ${ }^{1}$, Irati Alvarez ${ }^{1}$, Mireia Sanz-Vázquez ${ }^{2}$, \\ Susana Cormenzana ${ }^{1}$, \& Susana Corral ${ }^{1}$ \\ ${ }^{1}$ Department of Psychology and Education, Deusto University (Spain) \\ ${ }^{2}$ Department of Psychopedagogy, Begoñako Andra Mari (Spain)
}

\begin{abstract}
Divorced parents face challenges that might lead to detrimental psychological difficulties to their own and their children. These challenges are even higher in high conflict interparental conditions. In this context, there are differential positions about the adequacy of shared or exclusive custody based on their influence on parents' divorce adaptation. The goal of this study is to examine the psychological characteristics of parents and children in families with high interparental conflict by custody type. On this goal, a cross-sectional study was developed in Spanish public centers that support divorced parents with high interparental conflict. The study sample was 254 parents (39\% fathers, $61 \%$ mothers) who ranged between 23 to 63 years old. Parents who participated had mainly exclusive custody (80\%). We developed mean comparisons to test the differences between parents who had shared or exclusive custody. Results indicated that children of both groups had similar levels of somatization, anxiety/depression and aggressive symptoms. As well, parents who had shared or exclusive custody had similar parenting styles and general levels of psychological symptoms. Nevertheless, differences were encountered regarding co-parenting and general adjustment to divorce. Concretely, parents who had shared custody were those who indicated higher levels of coparenting and divorce adjustment. These results indicate that both types of custody might be linked to comparable psychological functioning for both children and parents. Complementary, these results pinpoint that custody assignments could be related to differential divorce adjustment that could influence parents and children long-term adaptation.
\end{abstract}

Keywords: Custody, parenting style, psychological symptoms, divorce, co-parenting.

\section{Introduction}

The number of divorces increased in the 60s-70s linked to their legalization and, nowadays, data show a similar reality (OECD Family Database, 2018). Besides, more than $60 \%$ of these couples have children (OECD Family Database, 2018). This noteworthy prevalence is very important since divorce circumstances imply demanding stressful life changes that can negatively affect children and parents (v.g. Amato, 2000; Haimi \& Lerner, 2016; Kiecolt-Glaser, 2018; Sbarra, 2015). In this context, societies have evolved and adapted their legal procedures to give solutions focused on setting the best conditions for children. In fact, current research keeps on the need of further evidence about the implications of custody arrangements (v.g. Nielsen, 2017).

The most frequent custody arrangements are exclusive and shared (Utrera, 2009). Exclusive custody implies that children cohabitate with one parent while the other parent has limited fixed contact/visiting periods. Besides, shared custody implies a more balanced cohabitation for both parents and more flexibility on the contact/periods. Therefore, shared custody can have multiple shapes depending on the cohabitation period with each parent (Utrera, 2009).

The existing literature about the benefits of each custody type supports both types of arrangements and there are no clear recommendations (Braver \& Votruba, 2018). Even so, previous research seems to pinpoint towards specific conditions that may explain the differential benefit of shared or exclusive custody. Concretely, the presence of parental conflict and co-parenting are key aspects (Nielsen, 2017). At this regard, children would not benefit from shared custody if their parents have high conflict levels or parents show low cooperation on their parenting responsibilities (Fabricius, Aaron, Akins, Assini, \& McElroy, 2018; Fernández-Rasines, 2017). This can be even more important in high conflict families (Zumbach, 2016). Moreover, authors as Nielsen (2017) indicate that co-parenting can be especially important in high interparental conflict conditions since it would allow to preserve parent-children relationships with both parents. 


\section{Objective}

Based on the exposed, the objective of this study is to examine the characteristics of children and parents who go through divorce depending on the custody type. Concretely, we aimed to test de differences on children psychological symptoms (i.e. somatization, anxiety/depression, and aggressiveness), parents' psychological symptoms, parenting styles (i.e. inductive, indulgent and strict parenting styles), adaptation to divorce and co-parenting by custody type (i.e. exclusive and shared custody).

\section{Method}

A cross-sectional study was developed in Spain directed to divorced parents who attended to public centers that specially give support in high interparental conflict situations.

\subsection{Sample}

The sample was 254 parents (39\% fathers, 61\% mothers) who ranged between 23 to 63 years old. Participants had been married for 10.57 years on average (SD $=6.36)$ and more than three $(52 \%)$, two $(13 \%)$ or one years $(19 \%)$ had been passed from divorce. They were mostly parents of one or two children (90\%).

The great majority of parents had the exclusive custody of their children (80\%). In fact, court cases rule predominantly on exclusive custody (60\%). In fact, exclusive custody arrangements were solved mainly after legal dispute (64\% of the exclusive custody cases) while shared custody arrangements were achieved mainly by mutual agreement (61\% of the shared custody cases).

\subsection{Scales}

Children somatization, anxiety/depression and aggressive symptoms. Children symptomatology was informed by the parents through the Spanish version of the Child Behavior Checklist (CBCL; Achenbach, 1991). This measure assesses the prevalence of specific symptoms in children. Specifically, we asked participants about children somatization, anxiety/depression and aggressiveness. Answers were recruited on a 3-point Likert scale ( $0=$ Not true - 2 = Usually true).

Parents' psychological symptoms. We measured parents' symptoms by the Spanish adaptation of the Symptoms Checklist (SCL-90; González de Rivera, De las Cuevas, Rodríguez, \& Rodríguez, 2002). Parents were asked how often they felt signs of somatization, interpersonal sensitivity, depression, and anxiety. Answers were recruited in 5-point Likert scales ( $0=$ Nothing at all $-5=\mathrm{Alot})$.

Parenting style. We measured the parenting styles using the Rules-Demands Scale (Bersabé, Fuentes, \& Motrico, 2001). This scale evaluates three parenting styles (i.e. inductive, strict and indulgent styles) concerning the way obedience to rules is established and commanded. Answers were requested on a 5-point Likert scale ( 1 = never -5 = always).

Adjustment to divorce. This variable was measure through the CAD-S (Yárnoz-Yaben \& Comino, 2010). This instrument measures both, parents' adaptation to their new parental commitments versus the general maladjustment described as psychological difficulties of parents to assume the break up, interparental conflict and the socioeconomic impact of divorce. This measure was answered on a

Co-parenting. This variable was measure through the eight items $(1=$ Totally disagree 5 = totally agree) of the CARE questionnaire (Yárnoz-Yaben, 2010). This scale assesses the perception that divorced parents have regarding their ex-partner commitment with their parental responsibilities.

\section{Results}

We developed t-test comparisons in order to examine the differences between the custody groups (i.e. shared and exclusive custody). Results are displayed in Table 1. Regarding children symptoms, t-test indicated that both custody groups reported similar levels of children's somatization, anxiety/depression and aggressive symptoms. Regarding parents' psychological symptoms, results showed that there were no significant differences between both study groups. Likewise, both groups reported similar parenting styles (i.e. inductive, indulgent and strict parenting styles).

Oppositely, both groups differed on adjustment to divorce and co-parenting. Concretely, the shared custody group reported higher levels of both, divorce adjustment to divorce and co-parenting, than the exclusive custody group. 
Table 1. Means, Standard Deviations and Comparisons between Exclusive and Shared Custody Groups.

\begin{tabular}{lccccccc}
\hline & \multicolumn{2}{c}{$\begin{array}{c}\text { Total sample } \\
(\mathrm{n}=254)\end{array}$} & \multicolumn{2}{c}{$\begin{array}{c}\text { Shared } \\
\text { custody } \\
\text { On= }\end{array}$} & \multicolumn{2}{c}{$\begin{array}{c}\text { Exclusive } \\
\text { custody } \\
(\mathrm{n}=202)\end{array}$} & \\
\cline { 2 - 8 } \multicolumn{1}{c}{ Outcome variable } & $\mathrm{M}$ & $\mathrm{SD}$ & $\mathrm{M}$ & $\mathrm{SD}$ & $\mathrm{M}$ & $\mathrm{SD}$ & t-test \\
\hline Children somatization & 2.73 & 3.17 & 2.33 & 2.68 & 2.83 & 3.29 & 1.02 \\
Children anxiety/depression & 4.66 & 4.15 & 3.73 & 3.32 & 4.90 & 4.32 & 1.82 \\
Children aggressiveness & 8.99 & 6.92 & 7.71 & 6.57 & 9.32 & 6.98 & 1.50 \\
Parents' psychological symptoms & 38.16 & 32.86 & 38.32 & 35.18 & 38.12 & 32.33 & -0.04 \\
Inductive parenting style & 43.83 & 5.41 & 43.58 & 5.73 & 43.90 & 5.34 & 0.38 \\
Indulgent parenting style & 13.77 & 4.16 & 13.27 & 3.98 & 13.90 & 4.21 & 0.97 \\
Strict parenting style & 23.48 & 6.68 & 22.73 & 5.91 & 23.67 & 6.87 & 0.90 \\
Adjustment to divorce & -0.23 & 1.22 & 0.18 & 1.45 & -0.33 & 1.13 & $-2.74^{* *}$ \\
Co-parenting & 2.18 & 0.96 & 2.63 & 1.20 & 2.07 & 0.86 & $-3.85^{* * *}$ \\
\hline
\end{tabular}

Note. t-test compared exclusive custody vs. shared custody.

${ }^{* *} p<.01,{ }^{* * *} p<.001$

\section{Discussion}

The present research approach the study of parent and children's characteristics after divorce by custody type. Our results indicate that high conflict divorces seem to influence similarly parents' and children's psychological symptoms irrespectively of the custody arrangements. Moreover, parents showed similar parenting styles irrespectively of the custody type. However, exclusive and shared custody arrangements differed on the prevalence of adjustment to divorce and co-parenting. Concretely, shared custody was related to higher adjustment and coparenting after divorce.

These results have two main implications. First, the similar values on parenting and parents and children's psychological symptoms may indicate that both custody types, exclusive and shared, could lead towards positive outcomes. This would explain that both custody types found previous empirical support (vg. Nielsen, 2017). Moreover, this imply that custody arrangements cannot determine the family members' health and that parents can equally exert their parenting capabilities.

Second, shared and exclusive custody groups differed on adaptation to divorce and co-parenting. Thus, parents on the shared custody group had better adjustment to the divorce demands and greater commitment to their responsibilities. This highlights that, to have the same benefits on psychological health, families with shared custody need a better divorce adjustment and higher involvement of both parents on children care. These results is in line with previous concerns about setting shared custody arrangements despite taking into account parents equal involvement on children's care (Fernández-Rasines, 2017) and family adjustment (Parkinson, 2018).

The main limitations of this research are its correlation nature and that only parents participate. Future studies may direct their efforts towards longitudinal with parent and children participants. Moreover, future research could deepen on the understanding of divorce profiles by custody type. Concretely, what happens when parents who have shared custody arrangements are not adjusted to divorce and have low co-parenting levels?

\section{Conclusions}

Custody arrangements define family structures and influence the relationships between the family members. We observed that exclusive and shared custody might lead to similar psychological symptoms of parents and children, but on different conditions. Specifically, shared custody leads to similar psychological adjustment but from higher adaptation to divorce and higher co-parenting than exclusive custody. This fact highlights the need of being cautious and considering family situations before suggesting shared custody in high conflict families to promote parents and children's health after divorce.

\section{References}

Achenbach, T. M. (1991). Manual for the child behavior checklist (4-18) and 1991 profile. Burlington, VT: University of Vermont, Department of Psychiatry.

Amato, P. R. (2000). The consequences of divorce for adults and children. Journal of Marriage and Family, 62(4), 1269-1287. https://doi.org/10.1111/j.1741-3737.2000.01269.x 
Bersabé, R., Fuentes, M. J., \& Motrico, E. (2001). Análisis psicométrico de dos escalas para evaluar estilos educativos parentales. Psicothema, 13(4), 678-684. Retrieved from https://dialnet.unirioja.es/servlet/articulo?codigo=2007083\&info=resumen\&idioma=SPA

Braver, S. L., \& Votruba, A. M. (2018). Does joint physical custody “cause” children's better outcomes? Journal of Divorce and Remarriage, 59(5), 452-468. https://doi.org/10.1080/10502556.2018.1454203

Fabricius, W. V., Aaron, M., Akins, F. R., Assini, J. J., \& McElroy, T. (2018). What happens when there is presumptive 50/50 parenting time? An evaluation of arizona's new child custody statute. Journal of Divorce and Remarriage, 59(5), 414-428. https://doi.org/10.1080/10502556.2018.1454196

Fernández-Rasines, P. (2017). Sharing child custody: Co-parenting after divorce in Spain. Oñati Socio-Legal Series [Online], 7(6), 1229-1246.

González de Rivera, J. L., De las Cuevas, C., Rodríguez, M., \& Rodríguez, F. (2002). Cuestionario de 90 síntomas SCL-90-R de Derogatis, Adaptación española. Madrid: TEA Ediciones.

Haimi, M., \& Lerner, A. (2016). The impact of parental separation and divorce on the health status of children, and the ways to improve it. Journal of Clinical \& Medical Genomics, 4(1), 1-7. https://doi.org/10.4172/2472-128X.1000137

Kiecolt-Glaser, J. K. (2018). Marriage, divorce, and the immune system. American Psychologist, 73(9), 1098-1108. https://doi.org/10.1037/amp0000388

Nielsen, L. (2017). Re-examining the research on parental conflict, coparenting, and custody arrangements. Psychology, Public Policy, and Law, 23(2), 211-231. https://doi.org/10.1037/law0000109

OECD Family Database. (2018). SF3.1: Marriage and divorce rates. Retrieved from http://www.oecd.org/els/family/database.htm

Parkinson, P. (2018). Shared physical custody: What can we learn from Australian law reform? Journal of Divorce and Remarriage, 59(5), 401-413. https://doi.org/10.1080/10502556.2018.1454197

Sbarra, D. A. (2015). Divorce and health. Psychosomatic Medicine, 77(3), 227-236. https://doi.org/10.1097/PSY.0000000000000168

Utrera, J. L. (2009). Guía básica para un buen divorcio. Barcelona: Ediciones B, S. A.

Yárnoz-Yaben, S. (2010). Cuestionario de Apoyo Recibido de la Ex pareja (CARE): Un instrumento breve paraevaluar la co-parentalidad post divorcio. Revista de Psicopatología y Psicología Clínica, 15(2), 133-142. https://doi.org/10.5944/rppc.vol.15.num.2.2010.4092

Yárnoz-Yaben, S., \& Comino, P. (2010). El CAD-S, un instrumento para la evaluación de la adaptación al divorcio-separación. Psicothema, 22(1), 157-162.

Zumbach, J. (2016). Mental disorders in children and parents in family law proceedings: Cases on child protection matters versus child custody and visitation issues. Journal of Child and Family Studies, 25(10), 3097-3108. https://doi.org/10.1007/s10826-016-0476-8 


\title{
THE HEALTH-PROMOTING BEHAVIORS FOR MARRIED COUPLES
}

\author{
Elçin Sakmar-Balkan ${ }^{1}$, \& Hakan Kuru ${ }^{2}$ \\ ${ }^{I}$ Department of Psychology, Nuh Naci Yazgan University, Kayseri (Turkey) \\ ${ }^{2}$ Department of Physical Education and Sports, Middle East Technical University, Ankara (Turkey)
}

\begin{abstract}
The central aim of the current research is to understand the association between adult attachment style (i.e., attachment avoidance and attachment anxiety) and health-promoting behaviors of married couples in the Turkish sample. The data was gathered using demographic information form (including height and weight), Experiences in Close Relationships-Revised, and Healthy Life Style Behavior Scale II from 269 married couples. The actor-partner interdependence model (APIM) was performed to realize both intrapersonal and interpersonal effects simultaneously. Attachment avoidance and attachment anxiety were used as independent variables, health-promoting behaviors and body mass index (BMI) were used as dependent variables, and the duration of the marriage, the number of children, and the education levels of individuals were used as control variables. The saturated model demonstrated that some associations among variables were insignificant. Therefore, these insignificant relationships were reduced from the model one by one. Eventually, the final model fit the data very well $[\chi 2(13, N=269)=9.131, p=.763$, GFI $=.994, \mathrm{AGFI}=.966, \mathrm{CFI}=1, \mathrm{RMSEA}=.00]$. Dyadic analyses demonstrated that the attachment anxiety of wives was not associated with any dependent variables. The results also showed that the attachment avoidance of both husbands and wives were significantly related to their own health-promoting behaviors. In other words, both husbands and wives who had higher levels of attachment avoidant were less likely to occupy in health-promoting behaviors. Moreover, the attachment anxiety of husbands was related to the BMI of wives. In the literature, it was indicated that individuals who have attachment avoidance reported unwilling to search for medical help about their complaints because of having problems in trusting health care professionals, the worse perception of general health, and fewer exercise behaviors. Additionally, the association between husbands' high attachment anxiety and their wives low BMI may be related to the critical and often coercive style of spouses who have attachment anxiety may influence their wives' weight perception and control.
\end{abstract}

Keywords: Adult attachment, health-promoting behaviors, BMI, married couples.

\section{Introduction}

Couples start to influence each other in different perspectives during the marriage. It is known that relational factors that influence overall health (Kiecolt-Glaser \& Newton, 2001). Besides, a few reports recommend that the changes in lifestyles alters couples lifestyle behaviors and cause a direct and indirect impact on each other's wellness such as weight gain, increase or decrease in physical activity levels (Craig \& Truswell, 1988; Kahn \& Williamson, 1990). Although the effect of health-promoting behaviors on individuals' health is noticeable, the influence of marital factors needs more attention.

Many lifestyle behaviors are becoming symphonious across couples (Meyler, Stimpson, \& Peek, 2007), including nutrition (Macario \& Sorensen, 1998) and smoking (Graham \& Braun, 1999). This is partly due to assortative mating; in other words, couples with similar characteristics are more likely to marry and may also reflect the influence spouses have on each other's health behaviors (Wilson, 2002). Couple concordance may explain risk factors for the disease at the household level (Wilson, 2002). For example, spouses of patients are at increased risk of conditions including hypertension and diabetes (Hippisley-Cox \& Pringle, 1998). Also, health behavior change tends to be accordant. For instance, in a study about couples participating a family health check-up, smoking behavior, blood pressure, blood glucose, and cholesterol level were correlated across couples one year after a cardiovascular lifestyle intervention program (Pyke, Wood, Kinmonth, \& Thompson, 1997). Further, when one partner adopts a healthier behavior, the other is more likely to make a positive health behavior change (Jackson, Steptoe, \& Wardle, 2015). 
For the reason that the features of individuals have a crucial role in health-promoting behavior, several psychological variables were researched. The adult attachment styles of individuals are one of the central psychological variable associated with health-promoting behaviors. The adult attachment styles generally are classified as secure and insecure attachment; and insecure attachment includes attachment avoidance and attachment anxiety (Mikulincer \& Shaver, 2007). Resignation from closeness and dependency is mentioned as attachment avoidance, while the feelings of rejection and desertion and the attempts at excessive intimacy is stated as attachment anxiety (Harma \& Sümer, 2016). Secure attachment is revealed with low attachment avoidance and low attachment anxiety (Pietromonaco, Uchino, \& Schetter, 2013). It was indicated that securely attached individuals occupy in health-promoting behaviors and talk about their symptoms (Huntsinger \& Luecken, 2004; Scharfe \& Eldredge, 2001). On the other hand, insecurely attached individuals, who have attachment avoidance or attachment anxiety, show more risky behaviors and lower health-promoting behaviors (Huntsinger \& Luecken, 2004; Savada, Busseri, Molnar, Perrier, \& DeCourville, 2009; Scharfe \& Eldredge, 2001). In other words, individuals who show insecure attachment style (i.e., anxious and avoidant attachment) tend to show riskier and fewer healthy behaviors rather than individuals who show secure attachment style.

Since marriage supplies the critical resource of attachment for many individuals (Sandberg, Busby, Johnson, \& Yoshida, 2012), it may significantly impact health behaviors (Sandberg et al., 2013). However, the majority of the research related to attachment and health-promoting behaviors examining the individual level of the relationship, not dyadic level. Moreover, the most of the studies were associated with the investigation of marriage and health indicators, such as cardiovascular risk, heart rate, and stress responses (Kiecolt-Glaser \& Newton, 2001), not health-promoting behaviors. Therefore, the main aim of the current study was to understand the association between adult attachment style (i.e., attachment avoidance and attachment anxiety) and health-promoting behaviors of married couples. Generally, it was hypothesized that attachment style of husbands and wives would predict health-promoting behaviors of couples. Specifically, the first hypothesis of the study was a spouse's attachment style would predict his or her health-promoting behaviors. The second hypothesis of the study was a spouse's attachment style would predict his or her partner's health-promoting behaviors.

\section{Method}

\subsection{Participants}

The current research included 269 heterosexual Turkish married couples (269 husbands and 269 wives). All couples had only been married once at the time of the study and had been living in big cities such as İstanbul, Ankara, and Kayseri. The length of marriages of couples ranged from 1 to 56 years with the average of 19.39 years $(S D=11.89)$. When the number of children was considered, $16.4 \%$ of couples had no child, $12.3 \%$ of couples had one child, $34.2 \%$ of couples had two children, and $37.2 \%$ of couples had three or more children. With respect to education level, $20.4 \%$ of husbands and $29.8 \%$ of wives had primary-secondary school education, $24.5 \%$ of husbands and $29.4 \%$ of wives had high school education, $47.2 \%$ of husbands and $35.3 \%$ of wives had applied schools or university degree, and $7.8 \%$ of husbands and $5.6 \%$ of wives had graduate/doctorate degree. The age of husbands ranged between 23 and 85 with the mean of 45.05 ( $s d=11.57$ ), and the age of wives ranged between 19 and 75 with the mean of 41.41 $(s d=10.80)$.

\subsection{Instruments} scales;

The instruments of the study consisted of the demographic information form and following two

Healthy Lifestyle Behavior Scale-II (HLBS-II): The HLBS-II estimates six-way of life practices under six subscales. It is a 52-item survey examines health responsibility, physical activity, nutritional habits, stress management, mental development, and interpersonal relations (Walker \& Hill-Polerecky, 1996). The Turkish version of HLBS-II has the reliability coefficients as .77 for health responsibility, .79 for physical activity, .68 for nutrition, .79 for mental development, .80 for interpersonal relationships and .64 for stress management (Bahar, Beşer, Gördes, Ersin, \& Kıssal, 2008).

Experiences in Close Relationships-Revised (ECR-R): ECR-R assesses the adult attachment styles as avoidance and anxiety (Fraley, Waller, \& Brennan, 2000). The scale contains 36 items, in which 18 items measure the attachment anxiety, whereas the other 18 items measure the attachment avoidance. The Turkish version of ECR-R has the reliability coefficients as .90 for attachment avoidance and .86 for the attachment anxiety (Selçuk, Günaydın, Sümer, \& Uysal, 2005). Regard as test-retest reliability, .82 for the attachment anxiety and .81 for the attachment avoidance were shown. 


\subsection{Data analysis}

Obtained data were analyzed using several techniques. Descriptive statistics and bivariate analysis were conducted by using the IBM SPSS 20 program. The model among adult attachment style, health-promoting behaviors, and BMI was formed by controlling the length of the marriage, the number of children, and education levels of spouses. In order to test the model, APIM (Ledermann, Macho, \& Kenny, 2011), which is to comprehend both intrapersonal and interpersonal effects simultaneously, were conducted by using IBM AMOS.

\section{Results}

\subsection{Descriptive statistics and bivariate analysis}

Regarding the main variables of the current research, the mean scores were calculated for wives and husbands separately. The mean score of the attachment avoidance for husbands was $2.03(s d=0.81)$ and for wives was $2.13(s d=0.97)$. The mean score of the attachment anxiety for husbands was 2.77 $(s d=0.86)$ and for wives was $2.89(s d=0.92)$. The mean score of the health-promoting behaviors (HPB) for husbands was $131.53(s d=20.27)$ and for wives was $133.78(s d=17.37)$. The mean score of the BMI for husbands was $27.07(s d=3.28)$ and for wives was $25.91(s d=4.30)$.

Both intrapersonal and interpersonal correlations were calculated for the main variables and are shown in Table 1.

Table 1. Bivariate correlations of main variables of the study.

\begin{tabular}{lllllllll}
\hline & $\mathbf{1}$ & $\mathbf{2}$ & $\mathbf{3}$ & $\mathbf{4}$ & $\mathbf{5}$ & $\mathbf{6}$ & $\mathbf{7}$ & $\mathbf{8}$ \\
\hline 1. Wives' avoidance & 1 & & & & & & & \\
2. Husbands' avoidance & $.51^{* *}$ & 1 & & & & & & \\
3. Wives' anxiety & $.45^{* *}$ & $.40^{* *}$ & 1 & & & & & \\
4. Husbands' anxiety & $.28^{* *}$ & $.56^{* *}$ & $.45^{* *}$ & 1 & & & & \\
5. Wives' HPB & $-.29^{* *}$ & $-.20^{* *}$ & $-.13^{*}$ & $-.13^{*}$ & 1 & & & \\
6. Husbands' HPB & $-.19^{* *}$ & $-.26^{* *}$ & $-.16^{* *}$ & $-.21^{* *}$ & $.30^{* *}$ & 1 & & \\
7. Wives' BMI & $.14^{*}$ & .02 & -.04 & -.08 & -.08 & -.02 & 1 \\
8. Husbands' BMI & .03 & .05 & -.08 & .01 & -.01 & .01 & $.31^{* *}$ & 1 \\
\hline
\end{tabular}

Note. ${ }^{*} p<.05 ;{ }^{* *} p<.001$

\subsection{Testing the model}

The conceptual model was committed to as the attachment avoidance and anxiety would have direct effects on health-promoting behaviors and BMI by controlling the length of the marriage, the number of children, and the education levels of spouses. Hereby, the model was saturated indicating observed and implied covariance matrices fitted exactly. The saturated model comprised insignificant links, so they were dropped from the model. Eventually, the final model fit the data very well $[\chi 2(13, N=269)=9.131, p=.763, \mathrm{GFI}=.994, \mathrm{AGFI}=.966, \mathrm{CFI}=1, \mathrm{RMSEA}=.00]$.

Figure 1. Actor and partner effects in predicting health-promoting behaviors and BMI.

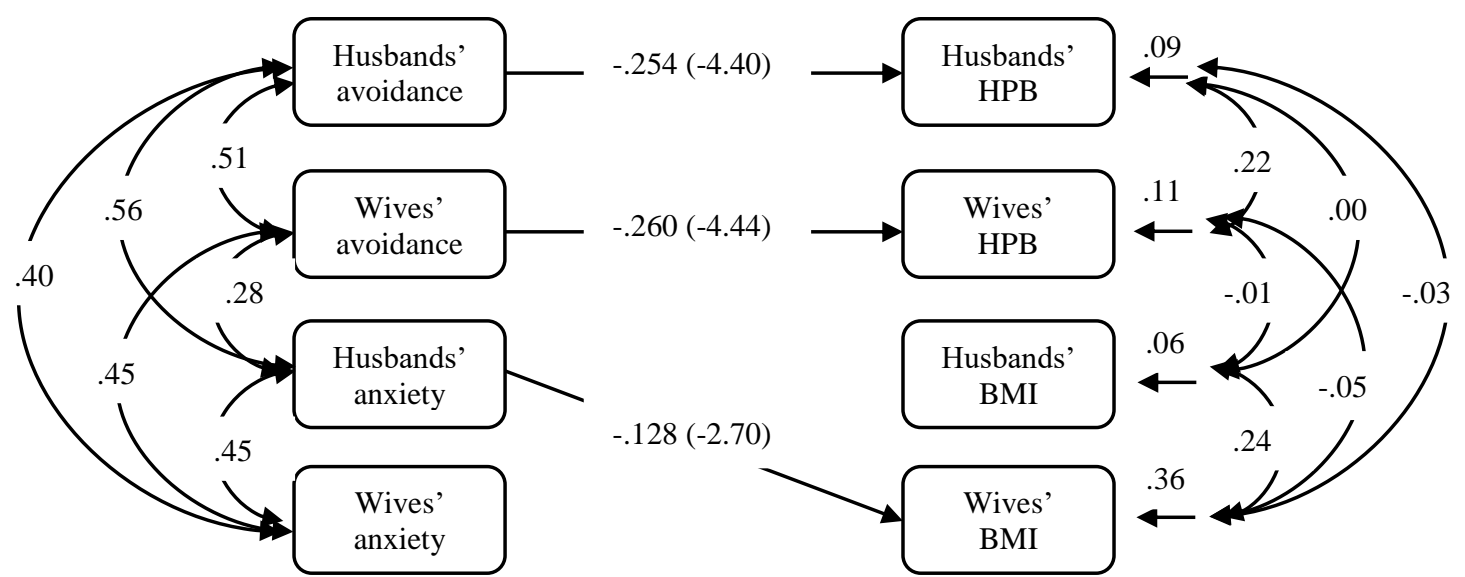

Note. Error terms represent the percent of the unexplained variance. For ease of interpretation, control variables, and correlation among IVs and control variables are not shown. $t$ values are presented in the parentheses next to standardized regression weights. 
The APIM analysis disclosed significant relationships for both actor and partner effects. Specifically, both husbands and wives high in the attachment avoidance reported low health-promoting behaviors $(\beta=-0.254, p<.001 ; \beta=-0.260, p<.001$, respectively). In other words, both husbands and wives who had higher levels of attachment avoidant were less likely to occupy in health-promoting behaviors. Moreover, husbands high in the attachment anxiety lead to low BMI of their wives $(\beta=-0.128$, $p<.007)$, indicating that husbands reported high attachment anxiety predicted low wives' BMI. Overall, adult attachment style explained $11.3 \%, 9.2 \%$, and $36.5 \%$ of the total variances in wives' and husbands' health-promoting behaviors and wives' BMI, respectively. Results showed that the paths for husbands and wives were comparable and there was no gender difference in the association between own attachment avoidance and own health-promoting behaviors.

\section{Discussion}

The study aimed to identify the relationship between adult attachment style and health-promoting behaviors. As expected, the current research found that both husbands and wives who showed higher attachment avoidance tended to behave less health-promoting. In the literature, it was indicated that attachment avoidance of individuals reported lower health-promoting behaviors such as unwilling to search for medical help, having problems in trusting health care professionals (Feeney \& Ryan, 1994), the worse perception of general health, and the physical inactivity (Kafescioglu, Thomas, \& Shields, 2010). On the other hand, attachment anxiety of individuals was not related to their health-promoting behaviors in the current study. Feeney (1995) reported that attachment anxiety is negatively associated with reporting the level of exercise and positively associated with reporting need to lose weight. Therefore, to examine health-promoting behaviors specifically may be more helpful to understand the relation.

Inconsistent with our expectations, the paths from adult attachment style to health-promoting behaviors are much more of an intrapersonal than an interpersonal phenomenon. In other words, both attachment avoidance and anxiety of individuals was not associated with their partners' health-promoting behaviors; partner effects were not shown for health-promoting behaviors. Individuals who have high attachment avoidance do not feel comfortable with providing and receiving care and support in their relationships (Kafescioglu, et al., 2010). They may see this kind of behaviors as restraining their independence. So, they may avoid affecting their spouse's health-promoting behaviors. Further, direct or indirect attempts of any behavioral change to spouse have a risk to be understood as the dissatisfaction of the relationship and/or to cause the dissatisfaction of their spouses. Hence, because of the fear of abandonment and rejection in a relationship is one of the main characteristics of individuals with high attachment anxiety (Mikulincer \& Shaver, 2007) who are sensitive to cues of possible rejection (see, Campbell, Simpson, Boldry, \& Kashy, 2005), they may abstain from any conscious or unconscious interfere to their spouse's health-promoting behaviors.

Considering the BMI of individuals, the findings, which showed no relationship between own attachment style and BMI, are consistent with the literature such as Koskina and Giovazolias' study (2010). The attachment anxiety of husbands, but not wives, was related to the BMI of their spouses in the current research. Being married has a more significant influence on men's health than relationship quality (Umberson, 1992). So, the characteristics of wives may be not so important for husbands' BMI. On the other hand, husbands reported high attachment anxiety lead to low wives' BMI in the current study. It is known that women may be sensitive to social pressure towards thinness. Individuals whose spouses have higher attachment anxiety reported their interactions as dissatisfying, negative in tone, low in amount, and low in the intimacy of disclosure (Bradford, Feeney, \& Campbell, 2002). The critical and often coercive style of these spouses may influence their wives' weight perception and control.

\section{References}

Bahar, Z., Beşer, A., Gördes, N., Ersin, F., \& Kıssal, A. (2008). Sağlıklı yaşam biçimi davranışları ölçeği II'nin geçerlik ve güvenirlik çalısması [Healthy life style behavior scale II: A reliability and validity study]. Cumhuriyet Üniversitesi Hemşirelik Yüksekokulu Dergisi, 12(1), 1-13.

Bradford, S. A., Feeney, J. A., \& Campbell, L. (2002). Links between attachment orientations and dispositional and diary-based measures of disclosure in dating couples: A study of actor and partner effects. Personal Relationships, 9, 491-506.

Campbell, L., Simpson, J. A., Boldry, J. G., \& Kashy, D. A. (2005). Perceptions of conflict and support in romantic relationships: The role of attachment anxiety. Journal of Personality and Social Psychology, 88, 510-531. 
Craig, P. L., \& Truswell, A. S. (1988). Dynamics of food habits of newly married couples: food-related activities and attitudes towards food. Journal of Human Nutrition and Dietetics, 1(6), 409-419.

Feeney, J. A. (1995). Adult attachment, coping style and health locus of control as predictors of health behavior. Australian Journal of Psychology, 47(3), 171-177.

Feeney, J. A., \& Ryan, S. M. (1994). Attachment style and affect regulation: Relationship with health behavior and family experiences of illness in a student sample. Health Psychology, 13(4), 334-345.

Fraley, R. C., Waller, N. G., \& Brennan, K. A. (2000). An item-response theory analysis of self-report measures of adult attachment. Journal of Personality and Social Psychology, 78, 350-365.

Graham, K., \& Braun, K. (1999). Concordance of use of alcohol and other substances among older adult couples. Addictive Behaviors, 24(6), 839-856.

Harma, M., \& Sümer, N. (2016) Are avoidant wives and anxious husbands unhappy in a collectivist context? Dyadic associations in established marriages. Journal of Family Studies, 22, 63-79.

Hippisley-Cox, J., \& Pringle, M. (1998). Are spouses of patients with hypertension at increased risk of having hypertension? A population-based case-control study. British Journal of General Practice, 48(434), 1580-1583.

Huntsinger, E. T., \& Luecken, L. J. (2004). Attachment relationships and health behavior: The mediational role of self-esteem. Psychology and Health, 19(4), 515-526.

Jackson, S. E., Steptoe, A., \& Wardle, J. (2015). The influence of partner's behavior on health behavior change: the English Longitudinal Study of Ageing. JAMA Internal Medicine, 175(3), 385-392.

Kafescioglu, N., Thomas, V., \& Shields, C. G. (2010). Dyadic and mediation analyses of coping with cardiovascular disease. Procedia Social and Behavioral Sciences, 5, 216-220.

Kahn, H. S., \& Williamson, D. F. (1990). The contributions of income, education and changing marital status to weight change among US men. International Journal of Obesity, 14(12), 1057-1068.

Kiecolt-Glaser, J. K., \& Newton, T. L. (2001). Marriage and health: His and hers. Psychological Bulletin, 127(4), 472-503.

Koskina, N., \& Giovazolias, T. (2010). The effect of attachment insecurity in the development of eating disturbances across gender: The role of body dissatisfaction. The Journal of Psychology, 144(5), 449-471.

Ledermann, T., Macho, S., \& Kenny, D. A. (2011). Assessing mediation in dyadic data using the actor-partner interdependence model. Structural Equation Modeling, 18, 595-612.

Macario, E., \& Sorensen, G. (1998). Spousal similarities in fruit and vegetable consumption. American Journal of Health Promotion, 12(6), 369-377.

Meyler, D., Stimpson, J. P., \& Peek, M. K. (2007). Health concordance within couples: a systematic review. Social Science \& Medicine, 64(11), 2297-2310.

Mikulincer, M., \& Shaver, P. R. (2007). Attachment in adulthood: Structure, dynamics, and change. New York: Guilford.

Pyke, S. D., Wood, D. A., Ann-Louise, K., \& Thompson, S. G. (1997). Change in coronary risk and coronary risk factor levels in couples following lifestyle intervention: the British Family Heart Study. Archives of Family Medicine, 6(4), 354.

Pietromonaco, P. R., Uchino, B., \& Schetter, C. D. (2013). Close relationship processes and health: Implications of attachment theory for health and disease. Health Psychology, 32(5), 499-513.

Sandberg, J. G., Busby, D. M., Johnson, S. M., \& Yoshida, K. (2012). The brief accessibility, responsiveness, and engagement (BARE) scale: A tool for measuring attachment behavior in couple relationships. Family Process, 51(4), 512-526.

Sandberg, J. G., Harper, J. M., Hill, E. J., Miller, R. B., Yorgason, J. B., \& Day, R. D. (2013) "What happnes at home does not necessarily stay at home": The relationship of observed negative couple interaction with physical health, mental health, and work satisfaction. Journal of Marriage and Family, 75(4), 808-821.

Savada, S. W., Busseri, M. A., Molnar, D. S., Perrier, C. P. K., \& DeCourville, N. (2009). Investigating a four-pathway model of adult attachment orientation and health. Journal of Social and Personal Relationships, 26(5), 604-633.

Scharfe, E., \& Eldredge, D. (2001). Associations between attachment representations and health behaviors in late adolescence. Journal of Health Psychology, 6(3), 295-307.

Selçuk, E., Günaydın, G., Sümer, N., \& Uysal, A. (2005). A new measure for adult attachment styles: The psychometric evaluation of experiences in close relationships-revised (ECR-R) on a Turkish sample. Turkish Psychological Articles, 8, 1-11.

Umberson, D. (1992). Gender, marital status, and the social control of health behavior. Social Science \& Medicine, 34, 907-917.

Walker, S. N., Hill-Polerecky, D. M. (1996). Psychometric evaluation of the Health Promoting Lifestyle Profile II. Unpublished manuscript, University of Nebraska Medical Center.

Wilson, S. E. (2002). The health capital of families: an investigation of the inter-spousal correlation in health status. Social Science \& Medicine, 55(7), 1157-1172. 


\title{
PSYCHOLOGICAL DETERMINANTS OF YOUTH PARTICIPATION IN THE LIFE OF THE COUNTRY
}

\author{
Tatiana Anisimova ${ }^{1}$, Karina Bakuleva ${ }^{2}, \&$ Irina Samuylova ${ }^{2}$ \\ ${ }^{I}$ Department of Human Psychology, Institute of Psychology, The Herzen University (Russian Federation) \\ ${ }^{2}$ Department of Political Psychology, Faculty of Psychology, Saint-Petersburg State University
}

(Russian Federation)

\begin{abstract}
Today's youth as a socio-demographic group is one of the main capital of society, who directly affects on the innovative, creative and labor participation inside each country. A young age is a period when new rights and obligations for direct participation in the political life of society appear, and as a result - the transition from youth to civil maturity, social and legal responsibility.

In November-December 2018, a socio-psychological study was conducted, where the specificity of youth participation in the life of the country were analyzed. 1,000 people aged 18 to 30 years, $65 \%$ - women, residents of 5 countries: Azerbaijan, Armenia, Kyrgyzstan, Moldova and Russia. Methods: questionnaire, method of unfinished sentences, content-analysis. Data processing: primary descriptive statistics, correlation, factor and variance analysis.

The data on the level of knowledge of respondents about the political structure and characteristics of the electoral system of the state were received. The attitude of young people to the electoral process, as well as the priority forms and motives of their participation in political governance were revealed. The analysis of cognitive, emotional and behavioral component of young people's ideas about political elections is carried out.

In general, young people are well informed about their civil rights and responsibilities. Opportunities for participation in political governance are assessed as significant, associated with concern for the surrounding space and positioning of their interests. Elections are seen as an important democratic resource for change within the state, requiring respect for the principles of law and justice.

Youth largely has a degree of mobility, awareness and physical health, which allow it to react quickly and to change behavior, therefore, the identification of universal and specific ways of interaction between young people from different countries with the political system and the priority forms of participation in the life of his country, is particularly relevant.
\end{abstract}

Keywords: Youth, civic activity, participation, election, country.

\section{Introduction}

The youth is a special social-age group, distinguished by the age framework and its status in society: the transition from childhood and adolescence to social responsibility. As a real social group, young people have three main signs of collective subjectivity: interconnectedness, joint activity and group self-reflexivity.

The psychology of youth received the most complete coverage in the age psychology of adolescence. Theoretical models of adolescence are presented in the approaches of leading Soviet psychologists and social educators (L.S. Vygotsky, S.L. Rubinstein, D.B. Elkonin, L.I. Bozovic, I.S. Kon, A.V. Mudrik, I.M. Ilyinsky et al.) and many other scientists (E. Sprangler, S. Buhler, S. Hall, K. Levin, R. Benedict, M. Mead, J. Piaget, E. Erickson, and others) (Volkov, 2006).

The characteristics of young people are determined by their intermediate social position, psychological characteristics of a given age period, the type of social structure, culture and socialization patterns peculiar to a given society, the discrepancy between various types of maturity - physical, mental, sexual, civil and labor (Emmons, 2004).

Political participation in the modern world is understood as actions aimed at exercising the influence of groups and individuals on the political system. Most often, political participation is seen as: the involvement of members of society in political and power relations, in the process of management and 
political decision-making; as the actions of people or groups taken in order to express their interests and influence the content of decisions made at various levels of state power implementation; as an element of political culture and a phenomenon due to the level of economic development and state (stability, transition, crisis) of society (Kirichek, 2011).

Studying the characteristics of political participation and behavior of representatives of various groups, many authors consider it necessary to pay attention to the following psychological components: the needs of the individual and the group participating in the activity; the motives that guide the subject; attitudes, values, orientations, beliefs and goals of the subject; personal characteristics of the role, decision-making style; interpersonal style; cognitive style; proper actions and deeds; feedback between the behavior and the conditions that formed it.

Political participation of young people is activities by which young people can individually or within any political or social movements, organizations, etc., influence the functioning, transformation or development of the political system, thereby defending their own subjectivity in youth policy (Chirun, 2010).

In recent years, the use of mass media to get information about politics, knowledge of current events, participation in political processes have declined among young people in almost all countries with established democratic institutions (Wattenberg, 2006). These changes reflect a decline in both opportunities for participation and civic interest from young people.

The study of the characteristics of the participation of young people in the life of the country is important because the experience of civic activity in youth forms the personality and has a lasting impact on the degree and nature of political participation throughout life (World Development Report 2007..., 2006).

\section{Objectives and design}

The main objective of the research is to study the specificity of the political participation of young people from different countries and psychological determinants of their involvement in politics.

Subject of research: motives, attitudes, cognitions (level of knowledge), political behavior, political participation.

The study was conducted in November-December, 2018, using written and Internet form of special questionnaire.

\section{Methods}

\subsection{Participants}

Young people aged 18 to 30 years from 5 countries: Russia $(n=200)$, Armenia $(n=200)$, Kyrgyzstan ( $n=200)$, Moldova $(n=200)$, Azerbaijan $(n=200)$. The total sample size: 1000 respondents. By gender, $35 \%$ - males, $65 \%$ - females.

Age: from 18 to 30 years - acquiring a new status and the formation of basic values. Respondents have sufficient knowledge to interpret social and political factors and civil rights to participate in political elections and other political events.

\subsection{Instruments}

The main research method was a questionnaire method - a method that uses a specially designed questionnaire question list as a means of collecting information from a respondent. The survey was conducted in writing, as well as through a special Internet form (a special link was given to the respondents).

In addition to the socio-demographic characteristics of sample (gender, age, year and direction of education), the questionnaire included:

- questions to identify the level of knowledge of young people about the political system of their country (respondents chose one of three response options: a parliamentary republic, a parliamentary-presidential republic or a presidential republic), electoral system (respondents chose one of three response options: majority, representative or proportional), passive and active electoral law (age to vote and to be elected);

- questions about preferred sources of political information (12 sources at total, measure scale $-\min =1$, $\max =5$ );

- questionnaire to identify the motives (8 items) and forms of political participation (7 items) (based on theoretical approaches of D.Olshansky (2001) and E.Shestopal (2001)), and the most effective forms of citizen participation in political governance ( 11 items), measure scale $-\min =1$, $\max =5$; 
- a modified version of the method of «Unfinished Proposals», revealing attitudes towards political elections (Samuylova, 2013) (4 items). For high-quality processing of the obtained data, the method of content analysis was used.

Data processing: 1) descriptive statistics methods (means, standard deviation, etc.); 2) the correlation analysis (Pearson), for detecting the presence of connections between the metric variables under study; 3) factor and variance analysis (reduction of dimension). The processing of initial data, calculation of results and statistical analysis were performed using the methods of mathematical statistics included in Microsoft Excel software and IBM SPSS Statistics 23.0.0.

\section{Results and discussion}

Analysis of the data showed that the main sources of information about politics for modern youth are the Internet $(M=4, \sigma=1.1)$, social networks $(M=3.73, \sigma=1.23)$, the mass media (television, newspapers, radio) $(M=3.37, \sigma=1.28)$ and family $(M=3.23, \sigma=1.26)$. The least significant are political parties $(M=2.58$, $\sigma=1.32)$, political institutes $(M=2.39, \sigma=1.23)$, public organizations $(M=2.38, \sigma=1.16)$, law enforcement agencies $(\mathrm{M}=2.00, \sigma=1.19)$, religious associations, the church $(\mathrm{M}=1.49, \mathrm{\sigma}=0.96)$.

It is said that political parties, political advertising and political institutes create significant electoral information space. At least, they significantly fill the real space, occupy public communication and consume financial resources. The study proved that for young people from different countries information about policies originating from the sources mentioned above is not much important. Thus, it can be assumed that Internet communication becomes the most important player in the electoral field of many countries.

The level of knowledge of young people about the electoral system and the political structure of their country turned out to be quite high (more than $60 \%$ of the correct answers).

Young people quite correctly indicate the age when they can participate in the voting (more than $50 \%$ of the correct answers). This age has already come for them, and they exercised their right, that is, personal experience is well preserved in memory.

Assessing their ability to stand for political elections, the opinions of young people differed more significantly (less than $30 \%$ of correct answers). Also a high percentage of those who find it difficult to answer were received. The revealed lack of knowledge clearly testifies to the absence of formed attitudes to participate in the political life of the country as subjects of politics and a desire to assume leadership functions.

The most manifested among the active forms of youth political participation are electoral behavior $(\mathrm{M}=3.47, \mathrm{\sigma}=1.54)$ and the simplest reactions to signals emanating from the political system (the behavior of an observer, a spectator) $(\mathrm{M}=3.07, \sigma=139)$. Coming out with comrades at a public meeting or building barricades is not the leading setting for political participation of young people $(\mathrm{M}=1.59, \sigma=1.08)$. In case of disillusionment with political institutions and rejection of the system, it is more likely that the reaction of switching off from political relations is demonstrated $(M=2.82, \sigma=1.44)$. Among the passive forms of political participation, respondents particularly noted the estrangement from politics due to excessive bureaucratization and disruption of the effective feedback between the political system and civil society as a whole $(\mathrm{M}=2.67, \mathrm{\sigma}=1.37)$.

Young people consider the most effective and most likely forms of participation in political management: environmental improvement measures (11.9\%), personal appeals to local governments (11.5\%), collection of signatures, petitions (10.3\%), discussion in the Internet of draft decisions of the authorities (9.9\%), work in public associations (9.4\%), participation in municipal elections (8.1\%). Such an experience of concrete «small deeds» that give a direct visible result can become a strategy for the formation of civic maturity and an active position of the young.

Describing their ideas and understanding «what is elections?», young people pointed out that elections are democracy and the opportunity to choose the future and the best candidate, emphasized the unifying power of the elections themselves for citizens.

Among the reasons for participating in elections, the most common were the answers: «because it is a civic duty», «a way to show one's activity», «to choose a worthy future», «to resist falsification». In their expectations of the election, young people rely on honesty, openness, respect for the legal rules, and the absence of corruption.

On an emotional level, the participation in politics should be meaningful and influential. The perception of elections as predictable, meaningless, not taking into account their interests and desires, push away young people from participating in politics. 
The results of the content analysis of the method of unfinished sentences (attitudes towards elections) were confirmed by quantitative data on the method of measuring motives for political participation. The basic motives for the political participation of young people from different states are the motive of interest $(M=3.04, \sigma=1.64)$, cognition $(M=2.79, \sigma=1.41)$ and world transformations $(M=2.69$, $\sigma=1.47)$. Low rates were obtained about motive of power (participation in politics for the getting power) $(\mathrm{M}=1.95, \sigma=1.22)$ and mercantile motive (participation due to monetary reward) $(\mathrm{M}=1.64$, $\sigma=1.14$ ). Low rates in terms of the traditional motive of political participation («this is customary in their environment, culture») ( $M=1.85, \sigma=1.21)$ and ideological motive (participation due to agreement with political ideology) $(M=2.26, \sigma=1.32)$ indicate that at this age the formation of their own motivational and value system occurs. This process enhances a critical attitude to the external, «adult» model of values. Young people have enough knowledge and interest in the socio-political situation in the country. Awareness of the inconsistencies with the reality of life principles proclaimed by parents and the environment is manifested in their alienation, revision and idealization of the values of their generation.

Despite the high rates of the motive of world transformation, when answering the question about the possibility to influence oneself on solving the problems of a city or a district, the most common answer was «I don't know I haven't tried yet» (32.4\%). In the second place was the answer «I can, only for some problems» (29.1\%). In third place was the answer «I can't» (20.3\%).

The analysis of variance showed that there were no significant differences between respondents from different countries in the ranking of the most preferred sources of information, motives and forms of political participation.

Conducting a correlation analysis (Pearson) made it possible to establish the existence of correlations between electoral activity, using sources for obtaining information about politics, motives and forms of political participation.

So, electoral activity of youth is correlated at a high level of significance $(\mathrm{p}<0.001)$ with:

- obtaining information from the mass media $(\mathrm{r}=0.219)$, the Internet (blogs, forums, websites) $(r=0.142)$, the family $(r=0.180)$, political institutes $(r=0.161)$ and political parties $(r=0.171)$;

- forms of political participation: the behavior of the observer, the spectator $(r=0.336)$, participation in politics as an ordinary functionary $(r=0.161)$, alienation from politics due to the loss of trust in political institutions $(\mathrm{r}=0.230)$, the non-acceptance of the political system $(\mathrm{r}=0.219)$ and hostility towards it $(\mathrm{r}=0.231)$;

- motives of political participation: the motive of interest $(r=0.249)$, the cognitive motive $(r=0.283)$, the motive of the world transformation $(r=0.244)$, the motive of power $(r=0.124)$.

\section{Conclusions}

A study conducted on a sample of young people from 5 countries showed that respondents are ready to participate in the political life of the country not only as voters, but also as active citizens who is involved in the process of adopting and implementing policies at the local, regional and national levels.

Of great importance for young people is the perception of their participation as significant and potential, as well as the legality and fairness of the electoral process.

One of the possible obstacles for young people to involve into politics as an initiator and executor of changes may be an insufficient level of knowledge about their capabilities (participation in elections as a candidate, options and opportunities to participate in the life of their city or country). Filling this gap is repairable through the process of education and disseminating information about the possibilities of realizing not only passive but also active law, as well as attracting young people to various forms of political participation.

For young people from different countries, information about politics emanating from institutions of purposeful political socialization (government, political parties, political advertising, etc.) is not influential. The most important player in the electoral field of all countries today is Internet communication (including social networks) and the immediate environment of young people (family, friends, referent groups).

Getting young people involved in social and political life in order to gain personal experience of participation is important because it determines the beginning of their further civil development and possible inclusion in power relations, management and political decision-making processes not only to protect their interests, but also for real influence on it. 


\section{References}

Chirun, S. N. (2010). Politicheskaja aktivnost' i politicheskoe uchastie molodezhi: problemy i vozmozhnosti [Political activity and political participation of youth: problems and opportunities]. Vestnik Tomskogo gosudarstvennogo universiteta [Tomsk State University Bulletin], 332, 50-54 (in Russian).

Emmons, R. (2004) Psihologija vysshih ustremlenij: motivacija i duhovnost' lichnosti [Psychology of Higher Aspirations: Motivation and Personality Spirituality]. Ed. D.A.Leontiev. Moscow (in Russian).

Kirichek, A. I. (2011). K voprosu o differenciacii soderzhanija kategorij «politicheskaja aktivnost'», «politicheskoe povedenie», i «politicheskoe uchastie» [On the issue of differentiating the content of the categories «political activity», «political behavior» and «political participation»]. Obshhestvo: politika, jekonomika, pravo [Society: politics, economics, law], 3, 34-37 (in Russian).

Olshansky, D. V. (2001). Osnovy politicheskoj psihologii [Fundamentals of Political Psychology]. Ekaterinburg (in Russian).

Samuylova, I. A. (2013). Ustanovki studencheskoj molodezhi Sankt-Peterburga po otnosheniju $\mathrm{k}$ vyboram v Rossii kak faktor ih politicheskogo uchastija [Attitudes of young people living in Saint-Petersburg toward the elections in Russia as a factor of their political participation]. Peterburgskij psihologicheskij zhurnal [Petersburg Psychological Journal], 3 (in Russian). Retrieved 04.04.19 from: http://ppj.spbu.ru/index.php/psy/article/view/35/17

Shestopal, E. B. (2002). Politicheskaja psihologija [Political Psychology]. Moscow: INFRA-M (in Russian).

Volkov, B. S. (2006). Psikhologiya yunosti i molodosti: Uchebnoye posobiye [Psychology of youth and adolescence: Textbook]. Moscow: Akademicheskiy Proyekt: Triksta.

World Development Report 2007: Development and the Next Generation (2006). Washington, The International Bank for Reconstruction and Development / World Bank.

Wattenberg, Martin P. (2006). Is Voting for Young People? New York, NY: Longman. 


\title{
ASYLUM INTERVIEW AS A FORK ON THE ROAD - FROM ASYLUM SEEKER TO IRREGULAR MIGRANT
}

\author{
Katri Johanna Gadd ${ }^{1}, \&$ Laura Sofia Lehtikunnas ${ }^{2}$ \\ ${ }^{1}$ Department of Geography and Geology, University of Turku (Finland) \\ ${ }^{2}$ Department of Law, University of Turku (Finland)
}

\begin{abstract}
Many asylum seekers have encountered various types of violence in their lives including physical and verbal harm, but also emotional violence. This paper evaluates yet another type of violence, namely epistemic violence, which asylum seekers may encounter when they arrive to a country seeking asylum. Epistemic violence is a failure of hearers to understand and acknowledge the speech of speakers in linguistic exchanges causing a negative impact on the speaker. Thus, epistemic violence is indirect and non-physical, yet it might have extremely severe legal and psychological consequences, such as a negative decision on an asylum request and the trauma caused by the decision and the situation in which person encounters the fork on the road: "Should I stay in Finland, or should I go?".

In 2015, Finland received an eightfold number of asylum applications compared with the previous years. Finnish authorities were not well prepared for the increase and in 2016 laws and regulations regarding immigration and legal aid were amended. According to the amended law, the legal assistance in the asylum interview is in practice no longer possible unless there are particularly serious reasons.

In this paper we report findings from interviews with 70 former asylum seekers regarding their experiences of their asylum interviews. We illustrate that many former asylum seekers did not experience to have been able to tell their story in such a way that their realities would have been understood and now they are irregular migrants.

Irregular migration is a timely phenomenon. Furthermore, it is highly multidisciplinary phenomenon requiring a holistic evaluation and discussion gathering academics from various disciplines including (but not being limited to) psychology, geography, sociology and law.

Epistemic violence needs to be avoided as much as possible in European immigration and social policies as it might increase irregular migration. Moreover, most importantly, it increases unnecessary agony and psychological stress for individual asylum seekers. Epistemic violence, as any other violence, has significant psychological effects through the negotiation of individual's self-esteem on a moment in which many experience to have lost the direction of the life. Consequently, we stress that the decision makers ought to acknowledge the possible side effects of the chosen social policies as those side effects such as an increase in experienced epistemic violence, might have extensive impact on living conditions and the quality of life of people in the society.
\end{abstract}

Keywords: Irregular migrants, asylum seekers, asylum interview, epistemic violence, Finland.

\section{Introduction}

The whole asylum process is about a story. The story needs to be personal, coherent, well-founded, credible and detailed. This story needs to be told in one asylum interview. If a person fails to tell the story in a right way, s/he risks of receiving a negative decision on his/her asylum request and facing the question whether to stay in a country as irregular migrant or to leave the country (Lehtikunnas, 2018). Asylum seekers' personal qualities, the abilities to understand the asylum process and individual perseverance become emphasised in the processes. The asylum seeker needs to be capable to analyse how the interpreters have put the words and to insist on correcting the possible shortcomings or even mistakes. However, as the translation is to a language asylum seekers do not often know, assuring that the translation is correct is challenging. Moreover, it is very difficult to know how the individual story will be understood in the asylum process; how the interpreter translates it and how the interviewer then interprets it. For some asylum seekers trusting fully in authorities is counterintuitive and even impossible. Taking 
into account, moreover, the fact that the asylum seekers are sometimes traumatised, the possibilities for a coherent storytelling might be jeopardised.

In 2015, nearly 33000 people came to Finland to seek asylum (Migri 2018). Finnish authorities were not well prepared to this increase in the amount of asylum seekers (the normal annual amount of new asylum seekers in Finland varies from 3000 to 4000). At European level the absolute number of asylum seekers arriving in Finland in 2015 was not high. However, the relative increase in the asylum applications was, in fact, among the highest in Europe (Jauhiainen, 2017). If the conditions for asylum or subsidiary protection were not fulfilled but the applicant could not return to their home country due to a poor security situation or an environmental disaster, residence permits could be obtained on the basis of humanitarian protection (Aer, 2016, p. 253). In 2016 the conditions for obtaining asylum and other residence permits in Finland were tightened and this possibility was removed from the Finnish law. Moreover, in 2016 restrictions on legal assistance in asylum process were introduced. The legal assistance by private lawyers in the asylum process at the Finnish Immigration Service (Migri) is no longer possible unless the presence of the assistant is necessary for particularly serious reasons such as particularly weak mental or health situation or if the asylum seeker is under 18 years old and in the country without a guardian (Changes on Aliens Act 9§ and the Legal Aid Act). Relatively common depression and anxiety or trauma are not enough to fulfil this condition. Due to the tightening asylum policies of the European Union (EU) and Finland, more people become irregular migrants as not all who get a negative decision to their asylum requests want to leave or feel that they could leave (Düvell, 2011).

\section{Epistemic violence}

Post-colonial researcher and philosopher Gayatri Spivak (1988) raised up the discussion about epistemic violence by questioning if the subaltern, the lowest strata of the urban sub proletariat, has a voice. She used the term epistemic violence in a rather general sense whenever the other had been constructed (Dotson, 2011). Spivak (1988) questioned the possibilities of the marginalised groups to speak, express themselves and for example give a testimony. According to her, epistemic violence is practiced when damaging a given group's ability to speak and be heard (Spivak, 1988). Epistemic violence has been discussed also in psychology, especially in relation to the hermeneutic processes of interpretation of data (see Teo, 2008; 2010), the statements of others.

In the same vein we understand the epistemic violence as a failure of hearers to meet the vulnerabilities of speakers in linguistic exchanges and recognise the speaker as knower (see also Hornsby, 1995; Dotson, 2011; Berenstain, 2016). Here the word 'epistemic' suggests that interpretations in linguistic exchanges are framed as knowledge and 'violence' denotes that this 'knowledge' has potentially a negative impact on the other (Teo, 2010). Practicing epistemic violence (i.e. damaging other person's possibility to speak, express him-/herself and be heard) may be intentional as well as unintentional (Dotson, 2011) and thus, might be difficult to address (Fricker, 2007). Consequently, the violence is indirect and non-physical (Teo, 2010), yet it can potentially have very material consequences.

Possibility for epistemic violence can be seen to be created in dependence relation between the speakers and the audience. The audience needs to understand the speaker's words and understand what the speaker is doing with the words. Therefore, the communication is about the speaker being able not only to express meaningful thoughts but also to be heard leaving the communication ultimately depending on the audience (Hornsby, 1995). Hence, in linguistic communication between the speaker and audience there is a fundamental need for reciprocity. Without the reciprocity the possibilities for epistemic violence are increased.

Lack of knowledge has been presented as one factor creating possibilities for epistemic violence (see e.g. Berging, 2002; Townley, 2006; Dotson, 2011). Refusal or inability, intentional or unintentional, of an audience to communicatively reciprocate a linguistic exchange can follow from the lack of knowledge (Dotson, 2011). The lack of knowledge is not necessarily related to intelligence, but can also be prompted by social positioning causing epistemic difference and inadequate hermeneutical resources (Berenstain, 2016). Epistemic difference here can be understood as a gap between different worldviews caused by differing social situations (e.g. economic, sexual, cultural) producing different understanding of the world (Bergin, 2002, 198). However, lack of knowledge may also be useful if it is used as impetus for study (Townley, 2006). Then, the fact that audience did not understand or was unable to reciprocate in the communication can, at best, lead into a further investigation to increase the understanding.

Dotson (2011; 2014) has researched epistemology and epistemic violence especially through different practices of silencing testimony given by oppressed individuals and has highlighted practices that silence, de facto, speakers. One form of epistemic violence as silencing is microinvalidation in power laden communication. It is characterised by communications that exclude, negate, or nullify the psychological thoughts, feelings, or experiential reality of a person (Sue et al. 2007, 274). These communications are violently interpreted in a certain way even though an equally valid alternative interpretation would be possible (Teo, 2008). 


\section{Methods}

Material of this research consists of official documents of international law and the Finnish constitution as background material and in-debt interviews with seventy irregular migrants living in Finland (24 females and 46 males). The interviews were conducted individually or in small groups depending on the preference of the research participants. All the participants have experiences from at least one asylum interview. Moreover, all the research participants have gotten negative decision on their asylum requests. All the interviews were conducted during the years 2018 and 2019.

The empirical data and the background literature were analysed using theory oriented content analysis in order to detect possibilities for epistemic violence in the asylum process and especially in the asylum interview. The material from the interviews was analysed also using thematic content analysis concentrating on the elements of epistemic violence (e.g. mistakes in understanding or in being understood, power imbalance in the interview, nullifying to mention but a few). Those elements were colour coded in order to detect their prevalence in the research participants' experiences of the asylum interviews.

\section{4. "Should I stay or should I go?"}

Asylum seekers' dependence on the hearer, the interviewer, is evident in the asylum interview. Does the interviewer have a common goal with the asylum seeker to make the story as strong as possible? Does the interviewer intend to ask, based on his/her expertise, the right questions benefiting the individual asylum-seeker? Does s/he have hermeneutical resources to understand or does the story remain unintelligible? This relation of dependence is prone to epistemic violence. 42 of our 70 participants had experienced epistemic violence in their asylum interviews.

For the successful interpretations to be formed, there is a great need of reciprocity not only to understand the speaker's words but also in taking the words as they are meant to be taken (Dotson, 2011). Some of the participants of this study explicitly said that they were not happy with the translation made by the interpreter. They told to have corrected various expressions in the minutes. However, in some worst cases the flaws were not detected or the audience, the interviewer was unable to understand the details of the story and the speaker, the asylum seeker was unable to detect the failures in understanding. Failures were only detected when the asylum seeker got a negative decision to his/her asylum request based on misunderstanding in the communication. One participants described this: "Only when I got the first negative decision and read through the papers with my Finnish friend did I realise that no, this wasn't what I meant'. This highlights the difficulty of asylum seekers perceive epistemic violence before the violence actually is materialised as a negative decision. Furthermore, the failures in the linguistic exchange in the interview are extremely difficult to correct afterwards. Changing the wording or expression often leads into a situation in which the creditability of the story is decreased as one of the participants explained: "I made the corrections and appealed. I got second negative decision fast as they [Finnish Immigration Service] said that my story wasn't coherent anymore". Another participant continued: "In my papers there is a line saying that I cannot be granted asylum as I have not been threatened in my home country in 2014 or 2015. I tried to correct this by saying that of course not, because I had already escaped and I was already in a refugee camp. I showed a document from UNHCR. Still negative." In these cases it is evident that the testimony of these individuals have not been heard and there has been severe difficulties in the understanding.

In the negative decisions, it is often claimed that the applicant has been asked to tell everything and there is no legal grounds to give asylum. Nearly all the participants questioned how they would know what is relevant to be told. This problem grew after the amendments in the immigration law in Finland as the legal advice might nowadays be scarce. The lack of advice and knowledge increases the possibility for epistemic violence in the asylum process as it jeopardises the ability of asylum seekers to speak. The lack of knowledge, however, is twofold. On the one hand asylum seekers might not know what to reveal. On the other hand the interviewer might be (intentionally or unintentionally) unable to meet the difference of the other person and thus, understand the linguistic exchange. This ignorance (see Sue et al. 2007) might be caused by the economic, political, sexual, cultural differences between interviewer and the asylum seeker (and the interpreter) producing different understanding of the world (see Bergin, 2002).

In this sense it would be beneficial for the asylum seeker if the interpreter has the same cultural or religious background as the asylum-seeker. However, five participants told to have been afraid of being interfered or judged by the interpreter as the interpreter might have different perception of the situation back home. On the other hand, without having the understanding on the background, it is even more difficult to hear the meaningful aspects of the full story and understand it clearly. Besides these challenges, ten participants told to have used Google Translator in the official asylum interview. These 
aspects create a real danger of intentional as well as unintentional epistemic violence, and the possibility to hinder open communication. Even little misunderstandings and differences in the terminology used in the interview can create dearth in the coherency and credibility of the asylum seeker 's story. Nevertheless, as asylum-seekers' claims are often related to issues strongly based on cultural, political, traditional and religious aspects, the interviewer and interpreter should be able to act and listen objectively without making conclusions and/or interpretations founded on their own experiences and general assumptions.

In order to treat all asylum seekers equally there is "knowledge" called country information of different countries of origin, towards which the stories of individual asylum seekers are mirrored. However, in the cases where the country information is in contradiction with the story told by the asylum seeker, the communication gets characteristics of epistemic violence as it excludes, negates, and/or nullifies the psychological thoughts, feelings, or experiential reality of the asylum seeker (see Sue et al. 2007) as most often the decision on the asylum claim in these cases is negative. The individual asylum seekers becomes disqualified as knower about his/her own situation and the interpretations done about a specific country become epistemic truths overriding individual experiences having severe consequences on the self-esteem of the person.

As described, the question in epistemic violence is whether or not there is a real possibility for a person to speak, express him-/herself and be heard. In particular, it is important to take into account how previous experiences and trauma affect the ability to communicate and/or remember things and events, and above all to express these things in a consistent and open manner. Elements, such as challenging experiences and the causes of traumas, are likely to have significant impacts on the safety aspects in the country of origin despite the fact that the individual is not necessarily able to tell them in the right way during the process. Moreover, as one of the participants explained: "In my home country I needed to fear militia. So, I learned very young to talk without telling much. I learned that I cannot trust. When I arrived, I should have told everything directly but I was too afraid to do so. I got negative [decision]. I noticed I need to trust and tell everything and I appealed. They said my story is not coherent and that I should have told everything directly".

Individuals of this research experience the consequences of (possible) epistemic violence e.g. distress, trauma and the loss of the direction of the life. As in various European countries immigration policies are tightened, these individuals are further negated as they are physically present in the societies but socially and legally negated and absent, alone with the psychological consequences of the past and the current situation. One participant told about his feelings: "I keep wondering what is wrong with me. Doesn't anybody like me or accept me as I am? I have no home country to go to or any family anywhere. Would it be better if I was dead? Then I think that no, not yet. I still can help someone else. I consciously think that so I can carry on".

\section{Concluding remarks}

The structure of the asylum interview is such that it creates vulnerabilities, threat of failure and being returned to the home country. Based on our analysis of the experiences, asylum seekers do not always feel they have been understood nor even heard. Sometimes there are smaller and bigger errors left in the documents from the interviews, which are difficult to retrieve afterwards. In some cases, there is lack in both, hermeneutical resources and desire to hear and understand the stories presented.

Epistemic violence is often characterised as personal violence (vs. structural). Even in the case presented here, there is a psychological and legal contact between interviewer and the asylum seeker; a subject and an object. However, the fact that epistemic violence occurs in an official hearing, transforms the violence into both; personal and structural.

Irregular migration is highly multidisciplinary phenomenon requiring a holistic evaluation and discussion about social policies gathering academics from various disciplines including (but not being limited to) psychology, geography, sociology and law. Epistemic violence needs to be avoided as much as possible in European immigration and social policies as it might, in fact, increase irregular migration. Additionally, most importantly, epistemic violence increases unnecessary torment and psychological distress for individual asylum seekers. Epistemic violence, as any other violence, has significant psychological effects through the negotiation of individual's self-esteem on a moment in which many experience to have lost the direction of the life. Consequently, we stress that the decision makers ought to acknowledge the possible side effects of the chosen social policies as those side effects, such as an increase in experienced epistemic violence, might have extensive impact on living conditions and the quality of life of people in the society. 


\section{References}

Aer, J. (2016). 'Ulkomaalaisoikeuden perusteet', Helsinki: Alma Talent.

Berenstain, N. (2016). Epistemic exploitation. Ergo, an Open Access Journal of Philosophy 3(22), 569-590.

Bergin, L. (2002). Testimony, epistemic difference, and privilege. Social Epistemology 16(3), 197-213.

Dotson, K. (2011). Tracking epistemic violence, tracking practices of silencing. Hypatia 26(2), 236-257.

Düvell, F. (2011). Paths into irregularity. The legal and political construction of irregular migration. European Journal of Migration and Law 13, 275-295.

Fricker, M. (2007). Epistemic injustice: Power and the Ethics of Knowing. Oxford: Oxford University Press.

Hornsby, J. (1995). Illocution and its significance in foundations of speech act theory - Disempowered speech. Philosophical topics 23(2), 127-147.

Jauhiainen, J. (2017). Turvapaikka Suomesta? Vuoden 2015 turvapaikanhakijat ja turvapaikkaprosessit Suomessa. Turku: University of Turku

Lehtikunnas, L. (2018). Should I stay or should I go - Irregularly somewhere in the middle. Master's thesis in international law. Turku: University of Turku

Migri (2018). Maahanmuuttoviraston selvitys sisäministerille turvapaikkapäätöksentekoon ja -menettelyyn liittyen. Helsinki: Finnish Immigration Service

Spivak, G. (1988). Can the Subaltern Speak? In C. Nelson \& L. Grossberg (Eds). Marxism and the Interpretation of Culture (pp. 271-317). Illinois: University of Illinois Press.

Sue, D. W., Capodilupo, C., Torino, G., Bucceri, J., Holder, A., Nadal, K. \& Esquilin, M. (2007). Racial microaggressions in everyday life: Implications for clinical practice. American Psychologist 62(4), 271-286.

Teo, T. (2008). From speculation to epistemological violence in psychology - A critical-hermeneutic reconstruction. Theory \& Psychology 18(1), 47-67.

Teo, T. (2010). What is epistemic violence in the empirical social sciences? Social and Personality Psychology Compass 4(5), 295-303.

Townley, C. (2006). Towards revaluation of ignorance. Hypatia 21(3), 37-55. 


\title{
QUALITATIVE PSYCHOLOGICAL EFFECTS OF POLITICAL INFORMATION TO HIGH SCHOOL STUDENTS ${ }^{1}$
}

\author{
Irina Samuylova \\ Department of Political Psychology, Faculty of Psychology, Saint-Petersburg State University
}

(Russian Federation)

\begin{abstract}
The paper presents the results of an empirical study of high school students' assessment of the qualitative characteristics of political information and its relationship with the emergence of emotional (affective) and cognitive-behavioral reactions. Teenagers and young people are especially sensitive to media influence. Having a clear understanding of the nature of politics, its goals, objectives, means and other subtleties at this age is rare. This makes young people vulnerable to suggestion and manipulation. Violation of the principles of information and psychological security can lead to General neurotization, the formation of depressive States, increased anxiety, distortion of the picture of the world, the manifestation of other negative effects in the younger generation.

In 2016-2018, an empirical study was conducted, which tested hypotheses about the relationship between the assessment of high school students (senior pupils) of the qualitative characteristics of political information, the level of their media competence and self-assessment of their own mental states of recipients caused by the impact of political information.

As the object of study were selected high school students (10-11 grade) from St. Petersburg and Mirny, Republic Sakha, Yakutia, a total of 115 people (75 girls and 40 boys), aged 15 to 18 years (average age 16). Data was collected using questionnaires.

Analysis of the results of the study showed that the most important sources of political information for high school students are the Internet, the mass media and the family, the least important sources are religious associations. Assessing the political information, high school students especially noted such qualities as clarity, discrediting and disintegrating character, low level of disorganization.

In the process of interaction with political information, high school students actualized concealment as a mechanism of psychological protection (delay or rejection of reactions, concealment of information about yourself).

In general, the study identified and described the main mental areas of potential psychological and political effects in the interaction with political information. Specific psychological characteristics that can be subject to potential transformations, as well as factors of "motivation for political participation" and political "conformism-nonconformism" are highlighted. The quality of political information contributes to the emergence of conditionally "positive" psychological effects (in terms of the stability of the political system), like "the effect of involvement" and the development of political conformism. The qualities of falsifying, misinforming and disorienting political information contribute to the emergence of "negative" affective (hatred, antipathy, criticality) and cognitive-behavioral effects (rivalry, radicalism).
\end{abstract}

Keywords: Political information, psychological effects, information psychological safety, high school students.

\section{Introduction}

Despite the development of the topic of media influence in psychological science, the specificity of the impact of political information is not enough studied today. As a rule, researchers focus on the study of negative aspects in a specific area of information (advertising, propaganda, transfer of political experience and political culture), without taking into account the effects associated with the generalized perception of political information.

${ }^{1}$ With the financial support of RFFI: project «Media image of Russia in context of national security», № 19-013-00725 
Minors are particularly sensitive to the effects of the media, namely adolescents and young men who are on the verge of adulthood and the acquisition of new civil rights. The transition to the stage of active political participation is accompanied by the formation of a system of beliefs and personal values, as well as the life and political position of the young personality. National policy and social conditions of life, as well as political information, assimilated by a senior high school student, are of tremendous importance. The manifestation of various psychological effects can be expressed most vividly in this age group.

Studying the designated problem will help establish whether there is a relationship between how senior high school students assess political information and what ideas about politics they have? Of particular interest is the definition of the relationship between the qualities of political information and the willingness to assimilate and relay political attitudes and values, as well as mental states of young people that are caused by political messages.

\section{Design}

The organization of the study included three stages. At the first, preparatory, stage, in order to identify and systematize the various psychological effects resulting from interaction with political information, a preliminary analytical review of the literature on the features of the psychological impact of mass and political information was conducted. The specific signs of political information and psychological characteristics of a person, which may be affected by media impact, were analyzed.

At the main stage, an empirical study was conducted aimed at studying the psychological characteristics that are actualized during the interaction of high school students with political information. Data collection was carried out using a written questionnaire (the time of filling is not more than 45 minutes). The questionnaire was formed from specially selected methods (see Methods). The study tested the hypothesis that there is a correlation between assessments of political information, the level of media competence and mental states experienced by high school students as a result of its common perception. The relationship between the qualities of political information and the parameters of social subordination / social aggressiveness of the respondents was also investigated.

For qualitative and quantitative processing of the obtained data, the following methods were used: content analysis, correlation analysis, factor analysis (method of principal components).

The obtained quantitative results were subjected to further interpretation in order to establish the specific qualitative psychological effects of the influence of political information on high school students.

\section{Objectives}

The main objective of the research is to identify qualitative psychological effects arising from the interaction of high school students with political information. The variety of existing forms of political information, types of audience, channels of communication and impact objectives prompted us not to limit ourselves to studying the effects of specific stimulus, which can be established by conducting an experiment, but to analyze the overall result of the intellectual expansion.

The subject of the research are specific psychological characteristics affecting the emotional, cognitive and behavioral spheres of the psyche: mental states, level of media competence, socio-political beliefs, parameters of social aggressiveness / social subordination.

Senior high school students (grades 10-11) from city Saint-Petersburg (Russia) and city Mirny, Republic of Sakha (Yakutia, Russia) were chosen as the object of study. A total of 115 respondents (75 girls and 40 boys), aged 15 to 18 years old (mean age 16.7).

\section{Methods}

During the research we used the method of theoretical analysis of literature, questionnaire, method of content analysis.

Questionnaire method - a method that uses a specially designed questionnaire question list as a means of collecting information from a respondent. The questionnaire procedure involves a written survey using prepared forms.

1) Questionnaire for identifying representations about the qualities of political information (A.Shlionsky, I.Samuylova, 2007). The questionnaire consists of 22 statements, 5 gradations of the degree of agreement are given (from 1 to 5 points). As a result of filling in the questionnaire, the following are revealed: psychological defense mechanisms (care, blocking, control, hiding) [Grachev, 1998], actualized in the process of interaction with information; criteria of information and psychological security (completeness, clarity, trust, relevance); qualities of adequate (sufficiency, clarity, objectivity, 
consistency, organization, concreteness, practicality and necessity) and inadequate political information (falsifying, disorienting, discrediting, destabilizing, misinforming, disorganizing, demoralizing, disintegrating) [Yuriev, 1992].

2) Questionnaire to identify the level of political media competence [Bakuleva, 2015]. It is aimed at studying the degree of emotional involvement in the perception of political messages, the propensity to analyze and interpret information of a political nature, knowledge about the features of the media, as well as the frequency of contacts with the media.

3) Methods for assessing mental states resulting from interaction with political information (to obtain subjective, deferred assessments of conditions arising from respondents during interaction with political information) [Yuryev, 1995; Bianki, 2006].

4) Method of measuring levels of social aggressiveness and social subordination [Samuylova, 2015]. Developed on the basis of the scheme of the psychological and political stability of society proposed by A.I. Yuriev [Yuriev, 1992]. It consists of 8 statements that diagnose the characteristics of the social subordination of the respondent (indifference, conservatism, adaptability, dependency) and social aggressiveness (initiative, radicalism, rivalry, creativity). The respondent expressed the degree of his agreement with each of the proposed statements (a scale from 1 to 5 points).

5) The method of measuring the system of political beliefs (operational code). Developed on the basis of A. George's operational coding theory [George, 1969]. It consists of 10 statements that diagnose a person's ideas about politics: philosophical (about the nature of politics, predictability of the future, the role of chance, etc.) and instrumental (about means of achieving goals, their realism, risk, level of motivation, etc.) issues. The respondent expressed the degree of his agreement with each of the proposed statements (a scale from 1 to 5 points).

For high-quality processing of the obtained data, the method of content analysis was used. The methods of mathematical statistics were used for quantitative data analysis: 1) descriptive statistics methods (means, standard deviation, etc.); 2) the correlation analysis (Pearson), for detecting the presence of connections between the metric variables under study; 3) factor analysis (reduction of dimension). The processing of initial data, calculation of results and statistical analysis were performed using the methods of mathematical statistics included in Microsoft Excel software and IBM SPSS Statistics 23.0.0.

\section{Discussion}

The theoretical analysis showed that informational and psychological, including politico-informational, impact causes a person a number of psychological effects that affect perceptual (sensations, perception), affective (mental states, emotions, feelings), cognitive (ideas, attitudes, beliefs, knowledge) and behavioral (motives, will, activity) areas.

Perceptual sphere affects the perception of political information and subjects of communication. Depending on the nature of the message being transmitted, the transmission, the channel of transmission and the direction of influence, various psychological effects can manifest themselves. For example, the perception of a speech by an authoritative politician may occur with a distortion created by the halo effect.

The affective sphere correlates with the concept of actualization of the mental states of a person as a reaction to political incentives developed for political psychology [Yuriev, 1995].

The cognitive sphere includes mechanisms for collecting and processing information, political views and beliefs of the person. Changing and shaping ideas about the nature of politics, specific events or groups of people most often becomes the goal of political communication. Accordingly, its expected effects often affect this particular area.

The behavioral sphere includes the needs, motives and forms of political behavior. Control and inducements of the political activity of the population are one of the direct tasks of the political system. Consequently, this area is also necessarily affected in the process of political communication.

The effect of political information is positive when it contributes to political socialization, that is, human development as a member of a politicized society, a participant in the political process. In the case of inverse changes, the effect may be negative. Also differ: individual (intrapersonal) and group effects; short-term effects (changes that have arisen under the influence of information are not permanent and disappear after some time) and long-term effects (changes persist for a period much longer than the duration of the impact itself). The effect may manifest itself situational and not extend to a person's daily life, however, the opposite is also true - some effects may have a totality of manifestation (for example, a gap in thinking). According to the objectives of the impact can be distinguished: the expected or side effect. The last one can be both positive and negative. 
Empirically, is was tested that there is a relationship between assessments of political information, the level of media competence and mental states experienced by high school students as a result of its holistic perception. The relationship between the qualities of political information and the parameters of social subordination / social aggressiveness of the respondents was also under investigation.

The general level of media competence of senior high school students can be defined as medium, $57 \%$ of respondents $(M=21.67, \sigma=+8.991)$, low $-29 \%$, high $-21 \%$.

The content analysis of the definitions of the concept "political information" that were given by respondents (the answer to the open question "Political information is ...") allowed us to establish general tendencies of understanding of political information by senior high school students (H.Lasswell's communication model was used for the analysis).

What is said? $75.8 \%$ of answers: "information about politics", "about the sphere of politics" (27\%), "about the state of the country" (15\%), "about events, situations" (14\%), "about foreign countries" (13\%). The last of answers are not significant (no more than 5\%): about political activity, about current incidents, about politicians, military-political news, about the government, propaganda information, "false information" and "theory of politics" (1.8\%). Most of the answers affect the content side of the information, a large proportion of formal-logical answers.

What effect? $10.6 \%$ of answers: awareness, familiarization (7.9\%), agitation (1.8\%), inducement to action $(0.9 \%)$, misleading, misinformation $(0.9 \%)$.

Who is talking to? $6.2 \%$ of responses: society (3.5\%), citizens (1.8\% of people), population $(0.9 \%)$.

Who is talking? 2.6\% of answers: "parties and their members", "mass media", "top power". In most cases, the source of political information is not called.

Where (by what channel)? $1.8 \%$ answers: books, television, radio.

Thus, high school students, making a judgment on political information in general, do not concentrate on the addressee or addressee of messages, as well as specific technical means of their transmission. Political information, to a greater extent, is perceived from the point of view of the subject and consequences of perception by the audience.

The most important sources of political information credit for senior high school students (among 12 sources) are the Internet, the mass media and the family, the least important sources religious associations.

By its qualities, political information is assessed by young people as clear $(M=3.9, \sigma=0.9)$ and not disruptive $(\mathrm{M}=2.4, \sigma=1.2)$, but discrediting $(\mathrm{M}=3.9, \sigma=1)$ and disintegrating $(\mathrm{M}=3.6, \sigma=1.2)$. Thus, political information, despite its clarity, can, according to high school students, mislead people and divide society, divide it into opposing parts. The main mechanism of psychological protection of respondents is the hiding mechanism $(\mathrm{M}=2.5, \sigma=1.2)$, which suggests that high school students tend to delay or reject reactions to incoming information, possibly concealing feelings and expressions of emotions.

Political information causes negative states: disturbance $(\mathrm{M}=2.3, \sigma=0.8)$, hostility $(M=2.5$, $\sigma=0.9)$, tension $(M=2.3$, $\sigma=0.9)$, anxiety $(M=2.4, \sigma=1.1)$ and criticality $(M=2.3, \sigma=1.1)$. This suggests that the existing impact of political information is perceived by them as negative, causes discontent, mistrust, doubts about its reliability and can push away high school students from the political sphere. At the same time, a state of curiosity is manifested, that is, a need for information, there is interest in it, but apparently it is not being satisfied.

Analysis of the results of measuring social aggressiveness/social subordination showed the severity of parameters of radicalism $(M=2.2, \sigma=1.2)$, competition $(M=3.6, \sigma=1.2)$, indifference $(M=2.2, \sigma=1.2)$, dependency $(M=3.5, \sigma=1.3)$ and lack of initiative $(M=2.3, \sigma=1.2)$. High school students have an interest in a political system that seems to be unsatisfactory, requiring changes, but they are not yet ready to take the initiative and take responsibility for any actions.

The policy is presented to high school students as a hostile world $(M=3.8, \sigma=1.2)$, where it is necessary to take risks in order to achieve goals $(M=2.5, \sigma=1.3)$, the achievement of which is quite realistic $(M=2.1, \sigma=0.9)$. As a means of achievement in politics, the word $(M=3.6, \sigma=1.5)$ and teamwork $(\mathrm{M}=2, \sigma=1.3)$ prevail.

As a result of the correlation analysis, the hypothesis was confirmed that there is a relationship between the assessment of the quality of political information by high school students, their level of media competence, political beliefs, self-esteem of mental states and social subordination/social aggressiveness parameters.

The factor analysis allowed us to distinguish two main factors: the factor of incentives for political participation (factor weight=8.31) and the factor of conformism-non-conformism (factor weight=7.29). 
The variables included in the first factor cause the emergence of the "engagement effect", which can be defined as an increase in interest in politics in the process of updating the motives for political participation that occurs as a result of adequate intellectual expansion. Increasing the necessity and practicality of political information causes a mental state of curiosity (affect) in a high school student, "turning off" the mechanisms of psychological defenses, increasing the level of motivation (behavior). Thus, the "engagement effect" is caused by the necessity, practicality of political information and affects all three selected areas of psychological effects (affective, cognitive and behavioral).

The variables of the second factor are arranged in a dichotomy, each pole of which corresponds to the "contentment-dissatisfaction" of the political system, the "social subordination/social aggressiveness" of the senior pupil and cause manifestations of opposite effects. The objectivity and sufficiency of political information contributes to the emergence of positive mental states and social subordination. Conversely, falsifying, misinforming and misleading information causes negative affective (hate, antipathy, criticality) and cognitive-behavioral (radicalism, rivalry) effects.

\section{Conclusions}

The research allowed us to identify and describe the main mental areas of the potential occurrence of qualitative psychological effects as a result of interaction with political information: affective, perceptual, cognitive and behavioral.

It was confirmed that there is a relationship between the assessment of the quality of political information by senior high school students, their level of media competence, political beliefs, self-assessment of mental states and parameters of social subordination / social aggressiveness. The factors of incentives for political participation and the factor of conformism-non-conformism are highlighted. The "effect of engaging in politics" has also been identified and described.

When a nationwide system of political education is not so strong or crucial, the presence and nature of the psychological effects of the influence of political information can become the defining criteria for evaluating the effectiveness of the political socialization of young people. Thus, we have touched upon a topic that requires careful study by psychologists, which provides a great deal of scope for scientific research. As a promising direction, we see the study of the specific manifestations of the obtained psychological effects in different age and social groups of people, deepening the theoretical and methodological development of the problem, conducting experiments to study the perceptual sphere that we ignored and confirm the effects established in this study.

\section{References}

Bakuleva K.K. (2015) Psihologicheskie aspekty izuchenija politicheskoj mediakompetentnosti // Vestnik Sankt-Peterburgskogo universiteta. Serija 12: Psihologija. Sociologija. Pedagogika, 2014. - № 3. Pp. 121-127. Bakuleva K.K. (2005) Psychological aspects of the study of political media competence // Bulletin of St. Petersburg University. Series 12: Psychology. Sociology. Pedagogy, 2014. - № 3. - Pp. 121-127.

Bianki V.A. (2006) Psihologija formirovanija politicheskogo prostranstva Sankt-Peterburga: dis. na soisk. uchen. step. kand. psih. nauk (19.00.12) / Saint-Petersburg. Bianki V.A. (2006) Psychology of the formation of the political space of St. Petersburg. St. Petersburg.

George A. (1969) The "Operational Code": A Neglected Approach to the Study of Political Leaders and Decision-Making. International Studies Quarterly. Vol. 13.

Grachev G.V. (1998) Informacionno-psihologicheskaja bezopasnost' lichnosti: sostojanie i vozmozhnosti psihologicheskoj zashhity (1998). Moscow. Grachev G.V. (1998) Information and psychological security of the individual: state and possibilities of psychological protection. - M.: RGAS, 1998.

Yuriev A.I. (1992) Vvedenie v politicheskuju psihologiju. - SPb.: Izd. S.-Peterburgskogo un-ta, 1992. Yuriev A.I. (1992) Introduction to political psychology. - SPb.: Ed. St. Petersburg University, 1992. 


\title{
SELF-ESTEEM AND CONFORMISM OF FEMALE STUDENTS AS PREDICTORS OF EXPERIENCED VIOLENCE BY PARTNER
}

\author{
Ivana Jankovic, Jelisaveta Todorovic, \& Snezana Stojiljkovic \\ Department of Psychology, University of Nis (Serbia)
}

\begin{abstract}
Violence in partner relationship is a serious social problem. People in relationships could be victim to different forms of physical, psychological or sexual violence. There can be numerous consequences of such relationship, so it is crucial to thoroughly examine the problem and prevention measures. The goal of this research is to examine whether partner violence in female students can be predicted based on their self-esteem and conformism. Self-esteem is defined as evaluative part of self-image that is reflected throughout positive and negative feelings towards self and indicates the level of confidence, worth and value, whereas conformism is a personality trait characterized by passive acceptance of standards and values of relevant social group, without prior critical analysis.

The sample consisted of 180 female students from Faculty of Philosophy in Nis, who have been in a relationship within past six months. Questionnaire of experienced violent behavior in partner relationship (Ajdukovic, Löw and Susac, 2011) was used, that contains sub scales for assessment of physical, psychological and sexual violence; self-esteem scale (Rosenberg, 1965) and conformism scale (Pantic, 1981) were used also.

The applied linear regression analysis showed, that the examined indicative variables account for 7.7\% variance of experienced psychological violence $(\mathrm{R}=.296, \mathrm{R} 2=.077, \mathrm{p}<.000)$, and that both self-esteem $(\beta=-, 197 \mathrm{p}<.008)$ and conformism $(\beta=, 183 \mathrm{p}<.014)$ are statistically significant predictors. For experienced physical violence the examined variables account for $12.1 \%$ variance $(\mathrm{R}=.361, \mathrm{R} 2=.121$, $\mathrm{p}<.000)$ and again both self-esteem $(\beta=-, 261, \mathrm{p}<.000)$ and conformism $(\beta=, 200 \mathrm{p}<.006)$ are statistically significant predictors. The scale used on a sample for sexual abuse assessment turned out to be unreliable, and as such it was omitted from further analysis. Cronbach's alpha coefficients was .518.

Even though there was not high enough percentage of explained variance, the obtained results showed that experienced physical and psychological violence in female students' partner relationships can be predicted based on their self-esteem and conformism. The direction of Beta coefficients showed that contribution of these variables were different. The experienced partner violence in female students will be higher if they have lower self-esteem and higher conformism.
\end{abstract}

Keywords: Violence, partner relationship, self-esteem, conformism.

\section{Introduction}

Intimate partner violence (IPV) is the violence that happens in a close relationship often categorized by cohabitation, marriage, or dating and it represents widespread, but preventable, public health problem. Adolescence and early adulthood are particularly vulnerable times characterized by the exploration of romantic relationships, sexuality, identity, and values. Indeed, $71 \%$ of female and $58 \%$ of male IPV victims first experience IPV before age 25 years (Breiding, Smith, Basile, Walters, Chen \& Merrick, 2014). IPV encompasses physical, psychological, and sexual abuse. Physical violence involves forceful physical contact that may vary from light pushes and slaps to severe beatings and lethal violence. Sexual abuse includes coercive and physical behaviors varying from trying to persuade someone to perform a sexual act against their will, ignoring "no" responses, to physically forced sex acts (Teten, Hall, \& Capaldi, 2009; Tjaden \& Thoennes, 2000) Psychological violence is the dominant, most important and most common form of violence and is used to establish control (Babović, Ginić, Vuković, 2003; Ignjatović, 2011) The term psychological aggression (or emotional abuse) refers to acting in an offensive or degrading manner toward another, usually verbally, and may include threats, shouting, cursing, insulting, degrading, ignoring, forbidding visiting (O’Leary \& Maiuro, 2001). Some degree of psychological abuse, at least at a minor or occasional level, is very common (Shortt, Capaldi, Kim, Kerr, Owen, Feingold, 2011). Consequences of IPV victimization and perpetration can be severe and long-lasting and include depression, substance abuse, antisocial behavior, disordered eating, and suicidal ideation (Banyard \& Cross, 2008; Bonomi, Anderson, Nemeth, Rivara, \& Buettner, 2013). Given the 
substantial health consequences, identifying factors that put individuals at risk for involvement in IPV is essential to creating effective prevention strategies. Organized by levels of a dynamic developmental systems perspective, risk factors for intimate partner violece included (a) contextual characteristics of partners (demographic, neighborhood, community, and school factors), (b) developmental characteristics and behaviors of the partners (e.g., family, peer, psychological/behavioral, and cognitive factors), and (c) relationship influences and interactional patterns (Capaldi, Knoble, Shortt, Kim, 2012). In this paper we deal with some of the psychological factors that may be associated with partner violence. More precisely, we wanted to examine the relationship of self-esteem and conformity with the perception of partner violence in relationship.

Self-esteem is defined as a subjective experience of one's own value, expressed by attitudes about yourself; that is, the degree to which a person believes that he/she is competent, successful, significant and valuable (Copersmith,1967). According to Coopersmith, self-esteem has four constructs: (a) success, comprised of power, significance, virtue, and competence, internally defined by one's perception of accomplishment; (b) values or importance one places on a situation; (c) aspirations or personal and public goals an individual establishes for self; and (d) defenses or ability to resist devaluation of one's self-esteem. Block and Robins (1993), observing the importance of the link between the real and the desired image of themselves, suggest that self-esteem is defined as the degree to which a person sees himself as a closer to the ideal, that is, away from an unwanted image of himself. Considered a personality trait, it is referred to as general self-esteem and been shown to be a stable variable throughout adulthood (Neiss, Sedikides, \& Stevenson, 2002). Coopersmith (1967), based on a three-year longitudinal study, concludes that the general estimate of one's own value reaches in early adolescence and remains relatively stable and durable over a period of several years. It also states that sudden changes can reduce (or increase) self-esteem, but it returns to the normal level when the conditions get 'normal' and a typical level. Depending on whether our experiences, starting from early childhood, generally positive or negative, we will build our own view of ourselves. Low self-esteem shows that people have many negative beliefs about themselves (Newark, Elsässer, and Stieglitz, 2016). The longitudinal study of Blocka and Robins (1993), which monitored changes in the development of self-esteem from adolescence to adulthood, provides information on differences in the self-esteem of men and girls. Although the results pointed to the invariability of self-esteem over the years, there was a tendency for a young man to increase self-esteem and to decrease in girls. The authors this difference explains the greater cultural pressure on young men to be more active, more productive, while the role of women in the society is much more passive.

Conformism is defined as accepting the behavior of a group or majority because a group requires and expects such behavior or because it simply presents it as a way of behaving. Conformism, therefore, exists not only when there is a direct or indirect pressure of the group, but also when, the behavior of the majority is accepted without thinking and without assessing its justification (Rot, 1999). Conformity is usually quite adaptive overall, both for the individuals who conform and for the group as a whole. Conforming to the opinions of others can help us enhance and protect ourselves by providing us with important and accurate information and can help us better relate to others (Stangor, Jhangiani, Tarry, 2014). There are only small differences between men and women in the amount of conformity they exhibit, and these differences are influenced as much by the social situation in which the conformity occurs as by gender differences themselves (Stangor, Jhangiani, Tarry, 2014). In some studies, however, it has been confirmed that women are more conformable than men (Costanzo and Shaw, 1966, according to: Šuković, 1986). The observed gender differences in conformity have social explanations - namely that women are socialized to be more caring about the desires of others-but there are also evolutionary explanations. Men may be more likely to resist conformity to demonstrate to women that they are good mates (Stangor, Jhangiani, Tarry, 2014). Margaret Mead (Mead, 1949, according to Rot, 1999) considers that the greater conformity in women is an integral part of the gender roles, acquired and imposed since childhood. In almost all cultures, girls learn to listen, to obey and not to stand out independently.

\section{Design}

The research was conducted in 2018 at the Faculty of Philosophy in Nis. The sample consisted of 180 students, aged 19-25 (M=20.94, SD=1.44). In the last six months, all students had experience of a partner relationship before testing. This was a condition for completing the questionnaire on the experience of violence. Prior to testing, all respondents received a formal consent to participate in the research.

\section{Objectives}

The subject of this research is to investigate the relationship between self-esteem, conformism and experienced violence in partner relationship. The specific goals were: 
- Examine the expression of self-esteem and conformism of female students and their experience of partner violence-psychological, physical and sexual violence

- Examine the correlations between self-esteem, conformism and the experience of partner

- Examine whether partner violence in female students can be predicted based on their self-esteem and conformism.

\section{Instruments}

Rosenberg Self-Esteem Scale (Rosenberg, 1965). The scale consisted of 10 items. Participants were requested to indicate the extent of their agreement or disagreement with each of these 10 statements using a 5-point scale from 1-strongly disagrees to 5-strongly agree. Example items: "Generally speaking, I'm satisfied with myself". Cronbach's alpha coefficient was .769.

Conformism scale (Pantic, 1981). The scale consisted of 5 items. Participants were requested to indicate the extent of their agreement or disagreement with each of these 5 statements using a 5-point scale from 1-strongly disagree to 5 - strongly agree. Example item: „I try to be as less as possible different from the others “. Cronbach's alpha coefficient was .669.

Questionnaire of experienced violent behavior in partner relationship (Ajdukovic, Löw and Susac, 2011). The scale contains sub scales for assessment of psychological (19 items), physical (7 items) and sexual violence (4 items). Participants were asked to indicate how many times their partner behaved in these ways on a scale of 0 (not once) to 5 (very often, several times a week). Examples of the items are: "The young man insulted me (psychological violence)", "The young man twisted my arm and roughly pulled my hair (physical violence)", "The young man threatened me to coerce me into sexual intercourse (sexual violence". Cronbach's alpha coefficients were: a sub scale for assessing psychological violence .891; a sub scale for assessing physical violence .897; a sub scale for assessing sexual violence .518. Due to low reliability on the sample, the scale for assessing sexual violence is exempt from further analysis.

\section{Results}

The following tables show obtained the results.

Table 1. Descriptive statistic.

\begin{tabular}{llllll}
\hline & $\mathrm{N}$ & $\mathrm{MIN}$ & $\mathrm{MAX}$ & $\mathrm{M}$ & $\mathrm{SD}$ \\
\hline Self-esteem & 180 & 2,50 & 5.00 & 4.08 & 0.55 \\
$\begin{array}{l}\text { Conformism } \\
\begin{array}{l}\text { Experienced } \\
\text { violence }\end{array}\end{array}$ & 180 & 1.08 & 4.15 & 2.22 & 0.56 \\
$\begin{array}{l}\text { Experienced physical violence } \\
\text { psychological }\end{array}$ & 180 & 0.00 & 2.74 & 0.43 & 0.52 \\
\hline
\end{tabular}

The obtained data show that the mean value for self-esteem is shifted to higher values (AS $=4.08$ ) on the scale from 1 to 5 . The mean value for conformism is grouped around lower values, $($ AS $=2.22)$. The mean values for experienced psychological and experienced physical violence are very low and amount to 0.43 (experienced psychological violence) and 0.18 (experienced physical violence).

Table 2. Correlations between self-esteem, conformism and experienced psychological and physical violence.

\begin{tabular}{lllll}
\hline & & & $\begin{array}{l}\text { Experienced } \\
\text { psychological } \\
\text { violence }\end{array}$ & $\begin{array}{l}\text { Experienced } \\
\text { physical violence }\end{array}$ \\
\hline Self-esteem & & $\mathrm{r}$ & -.23 & -.30 \\
& $\mathrm{p}$ & & .001 & .000 \\
Conformism & & $\mathrm{r}$ & .22 & .25 \\
& $\mathrm{p}$ & & .001 & .000 \\
\hline
\end{tabular}

All examined correlations show statistical significance. A negative correlation between the height of self-esteem and experienced psychological and physical violence in partner relationships was identified. Positive correlation exists between conformism and experienced psychological and physical violence. All examined correlations are relatively low. 
Table 3. Self-esteem and conformism as predictors experienced psychological and physical violence.

\begin{tabular}{lllllll}
\hline & \multicolumn{2}{l}{$\begin{array}{l}\text { Experienced psychological } \\
\text { violence }\end{array}$} & Model & $\begin{array}{l}\text { Experienced } \\
\text { violence }\end{array}$ & physical & Model \\
& Beta & $\mathrm{p}$ & & Beta & $\mathrm{p}$ & \\
Self-esteem & -.197 & .008 & $\mathrm{R}=.296$ & -.261 & .000 & $\mathrm{R}=.361$ \\
Conformism & .183 & .014 & $\mathbf{R}^{\mathbf{2}=.077}$ & .200 & .006 & $\mathbf{R}^{\mathbf{2}=.121}$ \\
& & & $\mathrm{p}<.000$ & & & $\mathrm{p}<.000$ \\
\hline
\end{tabular}

The results of linear regression analysis show that both self-esteem and conformism are significant predictors of experienced psychological and physical violence. The direction of the Beta coefficient indicates that the lower self-esteem increases the probability of the experienced of psychological and physical violence. On the other hand, the greater conformity increases the probability of the experienced of psychological and physical violence. Examined predictors could explain $7.7 \%$ variance of experienced psychological violence and 12.1\% variance of experienced physical violence.

\section{Discussion and conclusion}

The main goal of this paper was to investigate the relationship between self-esteem, conformism and experienced psychological and physical violence female students in romantic relationship by partner. The main goal was broken down into several specific and narrower goals: first to determine the correlations of variables, and then to investigate whether partner violence in female students can be predicted based on their self-esteem and conformism.

The results of descriptive statistics are in favour of the high agreement with the items that are the manifestations of self-esteem. The level of agreement with the items that are the indicators of conformism indicates low conformism female students. The level of agreement with the items that are the indicators of experienced psychological and physical violence is extremely low. This means that the female students have little experience with these forms of violence in partner relationships. The results are in line with the results of the research on the Croatian sample of high school students using the same questionnaire for assessing partner violence (Ajdukovic, Low, Susac, 2011). The average frequency of experienced psychological and violence is found in the lower part of the possible results on the questionnaire.

After examining the correlations between self-esteem, conformism and partner violence, we noticed a relatively similar connection between self-esteem and conformism with experienced psychological violence, and self-esteem and conformism with experienced physical violence. The results show a negative correlation between of self-esteem and the experienced psychological and physical violence. In other words, female students with lower self-esteem experience more violence by partners. As for the association between low self-esteem and violence victimization, several examples are observed within literature (O’Moore \& Kirkham, 2001; Slee \& Rigby, 1993, Papadakaki, Tzamalouka, Chatzifotiou, Chliaoutakis, 2009). Conformism is in a positive relation with experienced psychological and physical violence. This would mean that people who are more submissive to the authorities and accept the opinion of the majority can more often experience violence by partner.

After reviewing the results of the linear regression analysis, it was determined that both self-esteem and conformism are statistically significant predictors of experienced psychological and physical violence by partner. Even though there was not high enough percentage of explained variance, the obtained results showed that the experienced partner violence in female students will be higher if they have lower self-esteem and higher conformism.

Based on the results of this research, it could be concluded that, in order to reduce the experience of partner violence, the programs for the prevention and suppression of violence in partner relationships should develop and strengthen self-esteem in girls and at the same time encourage them to increase autonomy and independence in thinking and acting.

\section{Acknowledgments}

This paper was created as part of the project 179002 financed by the Ministry of Education, Science and Technological Development of the Republic of Serbia and within the scope of project Role of Applied psychology in Quality of Life of an Individual in Society. Psychology department Faculty of Philosophy in Nis, Faculty of Philosophy's Education-Science Board decision No. 183/1 in Nis (from 16-9-01 to 28.06.2018). 


\section{References}

Ajduković, D., Löw, A., i Sušac, N. (2011). Rodne razlike i prediktori partnerskog nasilja u mladenačkim vezama. Ljetopis socijalnog rada, 18(3), 527-553.

Ajduković, D., Löw, A., i Sušac, N. (2011). Upitnik doživljenih nasilnih ponašanja u vezi. Zagreb: Društvo za psihološku pomoć.

Babović, M., Ginić, K. i Vuković, O. (2010). Mapiranje porodičnog nasilja prema ženama u Centralnoj Srbiji. Beograd: SeConS.

Banyard, V. L., \& Cross, C. (2008). Consequences of teen dating violence: Understanding intervening variables in ecological context. Violence Against Women, 14, 998-1013.

Block, J., \& Robins, R. (1993). A longitudinal study of consistency and change in self- esteem from early adolescence to early adulthood. Child Development, 64, 909-923.

Bonomi, A. E., Anderson, M. L., Nemeth, J., Rivara, F. P., \& Buettner, C. (2013). History of dating violence and the association with late adolescent health. BMC Public Health, 13, Article 821.

Breiding, M. J., Smith, S. G., Basile, K. C., Walters, M. L., Chen, J., \& Merrick, M. T. (2014). Prevalence and characteristics of sexual violence, stalking, and intimate partner violence victimizationNational intimate partner and sexual violence survey, United States, 2011. MMWR Surveillance Summaries, 63(8), 1-18. https://www.cdc.gov/mmwr/preview/mmwrhtml/ss6308a1.htm

Capaldi, D.M., Knoble, N.B., Shortt, J.W., \& Kim, H.K. (2012). A Systematic Review of Risk Factors for Intimate Partner Violence. New York: Springer Publishing Company.

Coopersmith, C. (1967). Anticendents of self- esteem. Palo Alto: Consulting Psychologists Press.

Ignjatović, T. (2011). Nasilje prema ženama u intimnom partnerskom odnosu: Model koordiniranog odgovora zajednice. Beograd: Rekonstrukcija Ženski Fond.

Neiss, M. B., Sedikides, C., \& Stevenson, J. (2002). Self-esteem: A behavioural genetic perspective. European Journal of Personality, 16, 1-17.

Newark,P.E., Elsässe1, M., \& Stieglitz, R-D. (2016). Self-Esteem, Self-Efficacy, and Resources in Adults with ADHD. Journal of Attention Disorders, Vol. 20(3) 279- 290.

O'Leary, K.D., \& Maiuro, R.D. (Eds.). (2001). Psychological abuse in violent domestic relations. NewYork: Springer Publishing.

O'Moore, M., \& Kirkham, C. (2001). Self-esteem and its relationship to bullying behavior. Aggressive Behavior, 27, 269-283.

Pantić, D. (1981). Vrednosne orjentacije mladih u Srbiji. Istraživačko - izdavački centar SSO Srbije.

Papadakaki, M., Tzamalouka, G.S., Chatzifotiou, S., \& Chliaoutakis, J. (2009). Seeking for Risk Factors of Intimate Partner Violence (IPV) in a Greek National Sample the Role of Self-Esteem. Journal of Interpersonal Violence, Vol. 24(5), 732-750.

Rosenberg, M. (1965). Society and the adolescent self-image. Princeton, NJ: Princeton University Press.

Rot, N. (1999). Psihologija grupa. Beograd: Zavod za udzbenike i nastavna sredstva.

Shortt, J. W., Capaldi, D. M., Kim, H. K., Kerr, D. C., Owen, L. D., \& Feingold, A. (2011). Stability of intimate partner violence by men across 12 years in young adulthood: Effects of relationship transitions. Prevention Science, Online. Retrived on: https://www.ncbi.nlm.nih.gov/pmc/articles/PMC3134584/

Slee, P. T., \& Rigby, K. (1993). The relationship of Eysenck's personality factors and self-esteem to bully-victim behavior in Australian schoolboys. Personality and Individual Differences, 14, 371-373.

Stangor, C., Jhangiani, R., Tarry, H. (2014) Principles of Social Psychology - 1st International Edition. BC Open Textbook Project \& British Columbia Ministry of Advanced Education.

Šuković, F. (1986). Organizacija, grupe i motivacija. Beograd: Jugoslovenski zavod za produktivnost rada.

Teten, A. L., Hall, G. C. N., \& Capaldi, D. M. (2009). Use of coercive sexual tactics across 10 years in at-risk young men: Developmental patterns and co-occurring problematic dating behaviors. Archives of Sexual Behavior, 38(4), 574-582.

Tjaden, P., \& Thoennes, N. (2000). Extent, nature, and consequences of intimate partner violence: Findings from the National Violence against Women Survey (Publication No. NCT 181867). Washington, DC: Department of Justice. 


\title{
MALLEABILITY OF WORKING MEMORY THROUGH CHESS TRAINING IN SCHOOL CHILDREN
}

\author{
Ebenezer Joseph $^{1}$, Veena Easvaradoss ${ }^{2}$, T. Edwin Prabhakaran ${ }^{3}, \&$ Sweta Jain ${ }^{4}$ \\ ${ }^{I}$ Cognitive Science Research Initiative, Government of India; P\&T Audit Office (India) \\ ${ }^{2}$ Department of Psychology, Women's Christian College (India) \\ ${ }^{3}$ Department of Statistics, Loyola College (India) \\ ${ }^{4}$ Department of Psychology, Ethiraj College for Women (India)
}

\begin{abstract}
Working memory refers to a cognitive processing space where information is received, managed, transformed, and briefly stored. It is an operational process of transforming information for the execution of cognitive tasks in different and novel ways. Many class room activities require children to remember information and mentally manipulate it. While the effect of chess training on intelligence and academic performance has been examined, its impact on working memory needs to be studied. This study, funded by the Cognitive Science Research Initiative, Department of Science and Technology, Government of India, analyzed the effect of one-year chess training on the working memory of children. A pretest-posttest with control group design was used. The sample consisted of 88 children in the experimental group and 90 children in the control group. It was selected from children of both the genders studying in school (grades 3 to 9). The experimental group underwent weekly chess training for one year, while the control group was involved in extracurricular activities offered by the school such as cricket, hockey, football etc. Working memory was measured by two subtests of WISC-IV INDIA. The Digit Span Subtest involves recalling a list of numbers of increasing length presented orally in forward and in reverse order, and the Letter-Number Sequencing Subtest involves rearranging jumbled alphabets and numbers presented orally following a given rule. Both tasks require the child to receive and temporarily store information, manipulate it, and present it in a changed format. The children were trained using Winning Moves curriculum, audio-visual learning method, hands-on chess training and recording the games using score sheets, analyze their mistakes, thereby increasing their Meta-Analytical abilities. They were also trained in Opening theory, Checkmating techniques, End-game theory and Tactical principles. Analysis of Covariance revealed that the experimental group had significant gains in working memory compared to the control group. The present study ascertains a link between chess training and working memory. The transfer of chess training to the improvement of working memory could be attributed to the fact that while playing chess, children evaluate positions, visualize new positions in their mind, evaluate the pros and cons of each move, and decide moves based on the information stored in their mind. If working-memory's capacity could be expanded or made to function more efficiently, it could result in the improvement of executive functions as well as the scholastic performance of the child.
\end{abstract}

Keywords: Chess training, cognitive development, executive functions, school children, working memory.

\section{Introduction}

Working memory refers to a mental workspace that is involved in controlling, regulating, and actively maintaining relevant information to accomplish complex cognitive tasks (Miyake \& Shah, 1999). It is the small amount of information that can be held in mind and used in the execution of cognitive tasks, in contrast to long-term memory, which is the enormous amount of information saved in one's life. It has often been related to intelligence, information processing, executive function, comprehension, problem solving, and learning in people ranging from infancy to old age (Cowan, 2014).

Research evidence has linked performance on working memory tasks to vocabulary acquisition (Engel de Abreu, Gathercole, \& Martin, 2011); early academic success in reading (Gathercole \& Alloway, 2008; Stevenson, Bergwerff, Heisera, \& Resinga, 2014); mathematics (Arndt, Sahr, Opferman, Leutner, \& Fritz, 2013); and comprehension (Seigneuric, Ehrlich, Oakhill, \& Yuill, 2000). Furthermore, working memory ability measured when the child begins formal education strongly predicted success in reading, spelling and mathematics compared to IQ scores (Alloway \& Alloway, 2010) and short-term memory ability (Engle, Tuholski, Laughlin, \& Conway, 1999). 
A study carried out by Joseph, Easvaradoss, Kennedy, and Kezia (2016) assessed the impact of chess intervention on the general intelligence of children and analyzed the cognitive functions that contributed to the IQ gain. Significant increases were observed in IQ, and regression analysis indicated that nonverbal reasoning, language, and memory significantly contributed to the dependent variable IQ. Similar findings have been reported by Grabner, Stern, and Neubauer (2007). Since working memory is a better predictor of academic performance than IQ, it is imperative that research assesses the impact of programs that develop working memory. Rajah and Sundaram (2011) established that brief computer-aided intervention for improving neuropsychological functions such as attention and working memory has had a positive impact on the cognitive and academic skills of children who were average in academic performance.

However, research on the impact of chess training on working memory is not available. Chess is a game which involves working memory. While playing chess, the child mentally manipulates an enormous quantum of information, drawing on information stored in the long term memory and the data at hand. For example, the child concurrently uses blindfolded thinking (i.e. visualizing the positions of the chess game without looking at the board or picturing moves which had not occurred at all on the board), recalls and evaluates case studies of many typical positions while playing a chess game, connecting it to the game at hand. The outcomes of these mental operations are utilized to evaluate the positions, weigh various options, assess each potential move and make the optimum choice with the best outcome.

In the present study, it is hypothesized that systematic chess training would significantly increase working memory in children. The objective of the study was to assess the impact of a one - year chess training program with weekly sessions on the working memory of school going children.

\section{Methodology}

The research design used for the study was pretest-posttest with control group design (Edwards, 1985). The independent variable was the Chess training program and the dependent variable was the Working Memory of children. The sample comprised 178 children, 88 in the experimental group and 90 in the control group. The experimental group comprised 38 girls and 50 boys and the control group had 33 girls and 57 boys. The criteria for selecting the sample was children of both genders who are studying in schools, falling in the age range between 6 and 14 years (grade 3 to grade 9).

\subsection{Tools}

Assessment of Working Memory was carried out using the WISC-IV (Wechsler Intelligence Scale for Children-Fourth Edition, 2012). The test provided subtest and composite scores which represented intellectual functioning in specific cognitive domains and a composite score which represented general intellectual ability. To ensure accurate assessment, an Indian edition of WISC IV was used. Scores on the Digit Span subtest and the Letter-Number Sequencing subtest yielded the Working Memory Index.

Digit Span is a core Working Memory subtest composed of two parts: Digit Span Forward and Digit Span Backward. Digit Span Forward requires the child to repeat numbers in the same order as read aloud by the examiner, and Digit Span Backward requires the child to repeat the numbers in the reverse order of that presented by the examiner. This subtest is designed as a measure of auditory short term-memory, sequencing skills, attention and concentration. The Digit Span Forward task involves rote learning and memory, attention, encoding and auditory processing. Digit Span Backward involves working memory, transformation of information, mental manipulation and visuo-spatial imaging. The shift from the Digit Span Forward task to the Digit Span Backward task requires cognitive flexibility and mental alertness.

Letter-Number Sequencing is another core Working Memory subtest. A jumbled sequence of numbers and letters are read out by the examiner and the child recalls the numbers in ascending order and the letters in alphabetical order. This subtest involves sequencing, mental manipulation, attention, shortterm auditory memory, visuo-spatial imaging and processing speed.

\subsection{Chess training methodology}

The training methodology comprised Winning Moves Chess Learning Program (Joseph, 2008) Episodes 1-22, lectures with the demonstration board, on-the-board playing and training, chess exercise through workbooks (Chess school 1A, Chess school 2, and tactics) and working with chess software. Further children's games were mapped using chess base software and the brain patterns of the child were understood. They were taught the ideas behind chess openings and exposure to classical games were also given. The children participated in mock as well as regular tournaments. 


\section{Procedure}

Schools were identified and permission was obtained. Contracts were signed with the school to carry out the study. The children were randomly selected based on the inclusion criteria. Informed consent was obtained from the parents and the children. Random sampling within each school was used to form the experimental and control groups. Assessment was done for all students. Based on the IQ scores, children with identical IQ scores were paired, taking into consideration gender and age.

Clustering technique was used to form the training groups of 6 to 8 children. The chess training consisted of once a week chess classes conducted for one hour during school hours at the end of the day over one year. The children were given a standardized Winning Moves Chess Learning Program (Joseph, 2008) and played at tournaments also.

\section{Results}

The analysis was carried out using SPSS. Analysis of Covariance (ANCOVA) was used to compare the experimental and control groups on the dependent variable and to test the significance of difference between the means following chess training

Table 1. Descriptive statistics for working memory.

\begin{tabular}{|l|l|c|c|}
\hline \multirow{2}{*}{ Variable } & Assessment & $\begin{array}{c}\text { Experimental Group } \\
(\mathrm{N}=88) \\
\text { Mean/SD }\end{array}$ & $\begin{array}{c}\text { Control Group } \\
(\mathrm{N}=90) \\
\text { Mean/SD }\end{array}$ \\
\hline Working & Pre & 90.92 & 90.1 \\
Memory & & $(15.201)$ & $(13.692)$ \\
\cline { 2 - 4 } & Post & 97.57 & 91.64 \\
& & $(14.585)$ & $(12.706)$ \\
\hline
\end{tabular}

Table 2. ANCOVA between intervention groups and control group on working memory at postintervention.

\begin{tabular}{|l|r|r|r|r|r|}
\hline \multicolumn{1}{|c|}{ Sources of variance } & \multicolumn{1}{c|}{$\begin{array}{c}\text { Sum of } \\
\text { Squares }\end{array}$} & \multicolumn{1}{c|}{ df } & \multicolumn{1}{c|}{$\begin{array}{c}\text { Mean } \\
\text { Square }\end{array}$} & \multicolumn{1}{c|}{ F } & \multicolumn{1}{c|}{ Sig. } \\
\hline Corrected Model & 14891.816 & 2 & 7445.908 & 66.673 & 0.000 \\
\hline Intercept & 7051.475 & 1 & 7051.475 & 63.141 & 0.000 \\
\hline Pre Working Memory & 13330.48 & 1 & 13330.48 & 119.365 & 0.000 \\
\hline Exp Con & 1310.777 & 1 & 1310.777 & 11.737 & $0.001^{* *}$ \\
\hline Error & 19543.74 & 175 & 111.678 & & \\
\hline Total & 1626478 & 178 & & & \\
\hline Corrected Total & 34435.55 & 177 & & & \\
\hline
\end{tabular}

Table 1 shows that the mean working memory increased in the experimental group from 90.92 to 97.57 following intervention, compared to the control group which showed an increase from 90.1 to 91.64. Table 2 indicates that there was a significant effect of intervention on Working Memory at postintervention $(\mathrm{p}<0.01)$.

Figure 1. Probability Density Function plot of pre- and postexperimental (left) and control (right) for working memory.
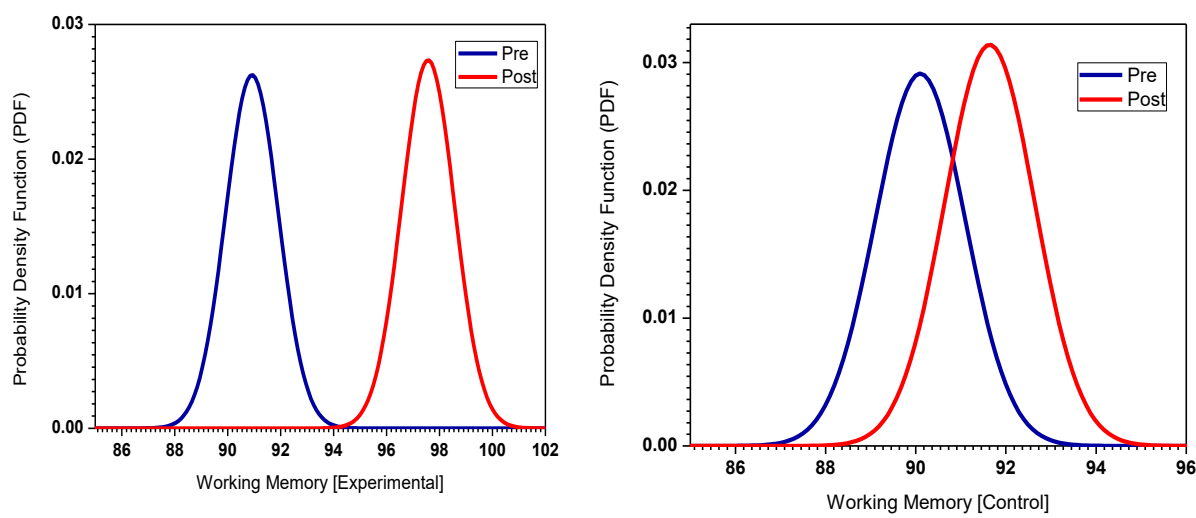
Figure 2. Cumulative Distribution Function plot of pre- and postexperimental (left) and control (right) for working memory.

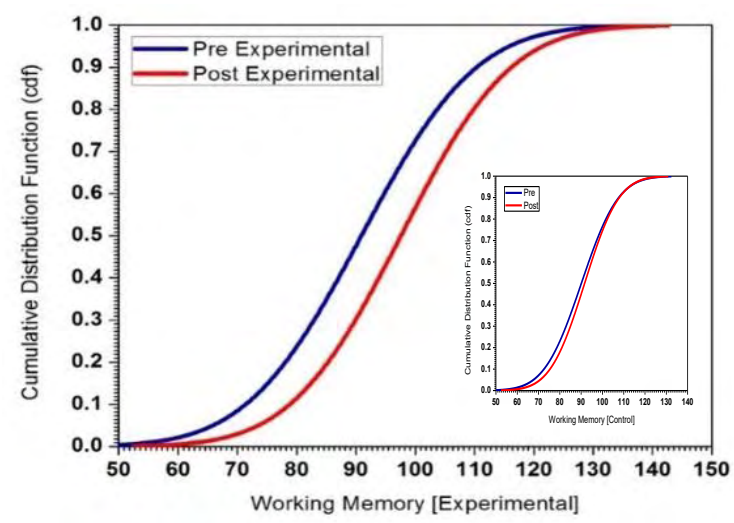

\subsection{Analysis of working memory-Gaussian normality curve}

The probability density function (PDF) plot in Figure 1 (left) clearly shows that pre- and postexperimental working memory distribution curves are distinctly separated and a higher mean is observed for postexperimental working memory. This indicates greater improvement in working memory in the children who underwent chess training (experimental group). However, the PDF plot in Figure 1 (right) clearly shows that pre- and postworking memory for control group are overlapped with each other, indicating not much improvement in working memory for control. The cumulative distribution function plots for pre- and postworking memory for experimental and control group are shown in Figure 2.

\section{Discussion}

The results clearly demonstrate that chess training has increased the working memory of the children who underwent the training when compared to the control group.

Children who received chess training appeared to have acquired the ability to juggle information while keeping in mind the already known information and the principles and rules of manipulation. In the present study, it was demonstrated that who children who had received chess training were able to transfer the ability to hold and manipulate information acquired during the training to tasks requiring similar abilities (namely the digit span and letter number sequencing tasks).

This effect has occurred after a year of chess intervention. It is probably the intensive and focused nature of the chess training which was individualized to the child's playing strength that resulted in the clear increase in scores. Further, a multisensory training approach was used ensuring that effective learning and development of chess playing skill was occurring.

Research has emphasized the encouraging effects resulting from working memory training. They have found a transfer effect on related tasks, such as tasks requiring following instructions, nonverbal reasoning and mathematical problem solving, and tasks involving attentional control (Brehmer, Westerberg, \& Backmann, 2012; Holmes, Gathercole, \& Dunning, 2009; Jaeggi, Buschkuehl, Jonides, \& Perrig, 2008).

Melby-Lervåg and Hulme (2013), on the other hand, reported contradictory findings that working memory training does not generalize to other reasoning tasks. However the training programs are of short duration with a mean training duration of 12 hours across all the studies reviewed by them. Clearly a working memory training program of longer duration is required for transfer to be observed.

Many studies have established a link between working memory and academic achievement (Gathercole \& Pickering, 2000). In one study, working memory independently predicted the children's achievements in reading and to a lesser extent in mathematics, thereby indicating that working memory was common to the domains of reading and mathematics (Gathercole, Alloway, Willis, \& Adams, 2006). Furthermore, working memory tests (digits backwards and sentence repetition) appeared to be the best predictors of mathematical test scores and may represent a major cognitive defect in children with specific defects in mathematics(Rosselli, Matute, Pinto, \& Ardila, 2006).

\section{Implications}

It is evident from the present study that there is a relationship between chess training and working memory. When systematic chess training with proper curriculum is offered, one could expect a significant gain in the working memory which can lead to educational implications for both normal children and special 
children. Strengthening working-memory through chess intervention could optimize its functioning; the positive outcomes would be seen not only in scholastic functioning but on other cognitive-behavioral attributes as well leading to holistic psychological well-being in the child. The potential educational gains of interventions using working memory training are valuable and therefore should be explored. Impacting working memory through chess training is thus a significant goal for psychologists, educationists, chess coaches and cognitive scientists.

\section{References}

Alloway, T. P., \& Alloway, R. G. (2010). Investigating the predictive roles of working memory and IQ in academic attainment. Journal of Experimental Child Psychology, 106(1), 20-29.

Arndt, D., Sahr, K., Opferman, M., Leutner, D., \& Fritz, A. (2013). Core knowledge and working memory as prerequisites of early school mathematics. South African Journal of Childhood Education, 3(1), $1-20$.

Brehmer, Y., Westerberg, H., \& Backmann, L. (2012). Working memory training in younger and older adults: Training gains, transfer and maintenance. Frontiers in Human Neuroscience, 6(63), 1-7.

Cowan, N. (2014). Working memory underpins cognitive development, learning and education. Educational Psychology Review, 26(2), 197-223.

Edwards, A. L. (1985). Experimental designs in psychological research. New York, NY: Harper \& Row.

Engel de Abreu, P. M. J., Gathercole, S. E., \& Martin, R. (2011). Disentangling the relationship between working memory and language: The roles of short-term storage and cognitive control. Learning and Individual Differences, 21(5), 569-574.

Engle, R. W., Tuholski, S. W., Laughlin, J. E., \& Conway, A. R. A. (1999). Working memory, short-term memory and general fluid intelligence: A latent-variable approach. Journal of Experimental Psychology, General, 128(3), 309-331.

Gathercole, S. E., \& Alloway, T. P. (2008). Working memory and learning: A practical guide for teachers. London: Sage.

Gathercole, S. E., Alloway, T. P., Willis, C., \& Adams, A. M. (2006). Working memory in children with reading disabilities. Journal of Experimental Child Psychology, 93, 265-281.

Gathercole, S. E., \& Pickering, S. J. (2000). Working memory deficits in children with low achievements in the national curriculum at 7 years. British Journal of Educational Psychology, 70, 177-194.

Grabner, R. H., Stern, E., \& Neubauer, A. C. (2007). Individual differences in chess expertise: A psychometric investigation. Acta Psychologica, 124, 398-420. Retrieved from http://dx.doi.org/10.1016/j.actpsy.2006.07.08

Holmes, J., Gathercole, S. E., \& Dunning, K. (2009). Adaptive training leads to sustained enhancement of poor working memory in children. Developmental Science, 12, F1-F7.

Jaeggi, S. M., Buschkuehl, M., Jonides, J., \& Perrig, W. J. (2008). Improving fluid intelligence with training on working memory. Proceedings of the National Academy of Sciences of the USA, 105(19), 6829-6833.

Joseph, E. (2008). India Patent No. L-32958/2009.

Joseph, E., Easvaradoss, V., Kennedy, A., \& Kezia, E. J. (2016). Chess training improves cognition in children. GSTF Journal of Psychology, 2(2), 1-6. doi:10.5176/2345-7872_2.2_33

Melby-Lervåg, M., \& Hulme, C. (2013). Is working memory training effective? A meta-analytic review. Developmental Psychology, 49, 270-291.

Miyake, A., \& Shah, P. (1999). Models of working memory: Mechanisms of active maintenance and executive control. Cambridge: Cambridge University Press.

Rajah, A., \& Sundaram, K. R. (2011). Changes in intellectual and academic performance of children following computer-based training: Preliminary results. Indian Journal of Psychiatry, 53(3), 249-252.

Rosselli, M., Matute, E., Pinto, N., \& Ardila, A. (2006). Memory abilities in children with subtypes of dyscalculia. Developmental Neuropsychology, 30(3), 801-818.

Seigneuric, A., Ehrlich, M. F., Oakhill, J. V., \& Yuill, N. M. (2000). Working memory resources and children's reading comprehension. Reading and Writing, 13(1/2), 81-103.

Stevenson, C. E., Bergwerff, C. E., Heisera, W. J., \& Resinga, W. C. M. (2014). Working memory and dynamic measures of analogical reasoning as predictors of children's math and reading achievement. Infant and Child Development, 23(1), 51-66. 


\title{
DEVELOPMENTAL PATTERNS OF SEX DIFFERENCES IN VERBAL AND VISUOSPATIAL ABILITIES
}

\author{
Efrat Barel, \& Orna Tzischinsky \\ Department of Behavioral Sciences, The Max Stern Academic College of Emek Yezreel (Israel)
}

\begin{abstract}
The present study explored the developmental patterns of sex differences in verbal and visuospatial abilities. Three hundred and twenty-six children and adults completed a battery of six cognitive tasks including two sets of abilities: The verbal cognitive battery included verbal fluency and short-term memory tasks. The visuospatial battery included mental rotation, localization, and form completion tasks. Results showed significant sex $\times$ age interaction on the mental rotation task, with men outperforming women in the 3-D task, but with no sex differences shown in childhood in the 2-D task. Sex differences in verbal fluency were found with girls and women outperforming boys and men in this task. Findings are discussed within an integrative approach of biological as well as environmental factors.
\end{abstract}

Keywords: Cognitive abilities, sex differences, developmental pattern.

\section{Introduction}

The present study aimed at investigating sex differences in various verbal and visuospatial abilities in childhood and adulthood. Sex differences in cognition received consistent support in adults. Men outperform women in visuospatial abilities, whereas women outperform men in verbal abilities (Halpern, 2012; Hines, 2004). Although research focusing on visuospatial abilities found that men outperform women on many tasks (e.g., navigation strategies and geographic orientation; Driscoll, Hamilton, Yeo, Brooks, \& Sutherland, 2005), the largest effect size has been found on mental rotation (e.g., Barel \& Tzischinsky, 2017; Burton \& Henninger, 2013; Hines et al., 2003; also see Linn \& Petersen, 1985; and Voyer, Voyer, \& Bryden, 1995, for meta-analyses). Women have been found to outperform men in verbal abilities, especially verbal memory (e.g., Bleecker, Bolla-Wilson, Agnew, \& Meyers, 1988; Kramer, Delis, \& Daniel, 1988) and verbal fluency (e.g., Burton \& Henninger, 2013; Weiss, Kemmler, Deisenhammer, Fleischhacker, \& Delazer, 2003). In other areas, such as vocabulary, verbal reasoning, and line orientation (Kimura, 2002), the findings are inconsistent.

In comparison with the consistency found in adults for sex differences in cognitive abilities, the age at which these differences emerge is unclear. Empirical evidence for sex differences in cognitive abilities prior to puberty did not produce unequivocal findings. Some studies have suggested that sex differences in verbal abilities appear early in life. For example, Lutchmaya, Baron-Cohen, and Raggatt (2002) demonstrated that girls show superiority to boys in vocabulary development, with 2-year-old girls using significantly more words than boys. Zambrana, Ystrom, and Pons (2012) documented sex differences in language comprehension at 18 and 36 months of age favoring girls at both time points.

Studies exploring individual differences and developmental aspects in visuospatial abilities used various tasks, including mental rotation, the Water Level Test, and block design. Studies of prepuberty children provided mixed results with regard to sex differences in visuospatial abilities. Some studies have demonstrated that the male advantage in mental rotation is apparent as early as infancy (Moore \& Johnson, 2008; Quinn \& Liben, 2008). Nevertheless, not all infant studies provided support for sex differences in mental rotation task in infancy (e.g., Hespos \& Rochat, 1997). In a similar vein, studies of preschool and school-aged children also provided mixed results. For example, Frick, Ferrrara, and Newcombe (2013) found sex differences in a mental rotation task. Palejwala and Goldenring Fine (2015) have examined, among others, sex differences in visual processing via block design and object assembly tasks, and found that sex differences at ages 2 to 3 were absent, whereas they emerged at ages 4 to 7 .

Sex differences in short-term memory in children usually do not generate significant results. For example, no sex differences were found in a picture memory task and in a location memory task in children aged 2 to 7 (Palejwala \& Goldenring Fine, 2015). In contrast, Keith, Reynolds, Roberts, Winter, 
and Austin (2011) reported sex differences in short-term memory with the use of a latent variable approach. Girls outperformed boys at ages 5 to 13, whereas boys outperformed girls at ages 14 to 17 .

The accumulated evidence regarding sex differences in cognitive abilities throughout the life span suggests that sex differences in cognition are magnified or become more common during adolescence (Herlitz, Reuterskiöld, Lovén, Thilers, \& Rehnman, 2013). The underlying mechanism for the developmental pattern of sex differences in cognitive abilities involves a complex interplay between biological and environmental variables. Among the environmental variables, play experiences (e.g., Caldera et al., 1999) and socio-cultural gender stereotypes (Quaiser-Pohl \& Lehmann, 2002) have been suggested as important factos related cognitive skills. Among the biological variables, endocrine factors such as sex hormones have been suggested as important factors (Halpern, 2012). Sex hormones, including androgens, estrogens, and progestins, can affect a wide range of organs, including the brain. Their greatest effect occurs during two sensitive periods in development: The first is during the prenatal and/or neonatal period, the second during the postnatal period (Collaer, Reimers, \& Manning, 2007; Halpern, 2012). It has been suggested that these sensitive periods in hormonal secretion are associated with sex differences in cognitive abilities (Hines, 2011; Kimura, 2002).

In sum, previous studies have suggested that sex differences in cognitive abilities are magnified and established during adolescence and adulthood, and are proposed to be mediated by neuroendocrine development in adolescence. The present study was designed to investigate developmental patterns in sex differences in cognitive abilities.

The hypotheses are as follows:

a. It is hypothesized that a Sex $\times$ Age interaction on visuospatial abilities is found in adults, with men outperforming women, and no sex differences in children.

b. It is hypothesized that sex differences in verbal abilities are found with female participants outperforming males, in both children and adults, with a magnified effect in adults.

\section{Methods}

\subsection{Participants}

Three hundred and twenty-six children and adults participated in the present study. One hundred and fifty-seven were undergraduate students from various departments: behavioral sciences (Psychology, Education), social sciences (Sociology and Anthropology, Information Systems, Economics, Accounting, and Management), and communication, at a college in the north of Israel. Eighty of the participants were female $\left(\mathrm{M}_{\text {age }}=26.04 \pm 3.45\right)$ and seventy-seven were male $\left(\mathrm{M}_{\text {age }}=26.18 \pm 2.78\right)$. One hundred and sixty-nine were children in grades 4 and 5 from 4 schools in the north of Israel (two schools from a medium-high socioeconomic status background, and two schools from a low socioeconomic status background). Eighty-seven of the participants were female $\left(\mathrm{M}_{\mathrm{age}}=10.34 \pm 0.61\right)$ and eighty-two were male $\left(\mathrm{M}_{\mathrm{age}}=10.45 \pm 0.61\right.$; see Figure 1$)$. All participants were right-handed according to their subjective reports.

Adult participants were recruited through advertisements at the college, and did not receive monetary compensation for their participation. Participants gave their informed consent. Children were recruited as part of their school assignment, following their parents providing their consent for their child's participation.

\subsection{Measures}

Cognitive test battery -Six types of tasks were presented to the participants: three verbal and three visuospatial cognitive tasks (Gordon \& Harness, 1977): Serial sounds; Serial digits; Verbal fluency; Mental rotation (2D and 3D stimuli); Localization; Form completion.

\section{Results}

\subsection{Hypothesis a: Sex $\times$ Age interaction on visuospatial abilities}

There was a statistically significant interaction between sex and age group on mental rotation $\left(\mathrm{F}(1,317)=8.78, \mathrm{p}<.01, \eta_{\mathrm{p}}{ }^{2}=.03\right)$. Adult males outperformed adult females $(\mathrm{p}<.001)$, whereas there were no sex differences in children $(\mathrm{p}>.05)$.

\subsection{Hypothesis b: Sex differences on verbal abilities}

For verbal fluency, the difference between males and females was significant, $\left(\mathrm{F}(1,322)=26.27, \mathrm{p}<.001, \eta_{\mathrm{p}}{ }^{2}=.08\right)$, with female participants outperforming males. 


\section{Discussion}

The present study assessed sex differences in visuospatial and verbal abilities in children and adults. The hypotheses were partially supported. Sex differences in verbal fluency were identified in childhood as well as in adulthood, with females outperforming males. This finding is in accord with previous results demonstrating the emergence of sex differences in verbal abilities in childhood, with no indication of these differences magnified throughout development (Herlitz et al., 2013). The findings suggest that sex differences in verbal abilities emerge before puberty and imply that the source of these differences should be sought early in development. Findings on other verbal tasks (short-term memory tasks) failed to produce significant results. This is line with the empirical evidence with regard to sex differences in memory tasks in children, usually implying no sex differences (e.g., Palejwala \& Goldenring Fine, 2015).

With regard to visuospatial tasks, empirical literature reported consistently that the largest effect size of sex differences was found for the mental rotation task (Levine, Foley, Lourenco, Ehrlich, \& Ratliff, 2016). The present study revealed that sex differences are not apparent in children. In adults, our findings supported previous studies demonstrating a large effect size $(d=0.84)$. Previous meta-analyses (Linn \& Peterson, 1985; Voyer et al., 1995) confirmed that there is substantial male advantage on mental rotation tasks, and pointed out that the tasks which involve 3D rotations (as opposed to rotating simpler shapes in the picture plane) produce the largest effect size, probably due to females' difficulty to mentally rotate objects in depth. In children, the present study used 2D stimuli, which have not produced significant sex differences. Several studies tested children's performance on various 2D stimuli using animal drawings (Kucian, von Aster, Loenneker, Dietrich, Mast, \& Martin, 2007) or other familiar objects (Ruthsatz, Neuburger, Jansen, \& Quaiser-Pohl, 2015), alphanumeric stimuli (Hoyek et al., 2012), or abstract characters (Hoyek et al., 2012; Kail, Pellegrino, \& Carter, 1980). They have demonstrated that various 2D stimuli differ in their difficulty level. Performances for familiar 2D stimuli (e.g., animal drawings or alphanumeric stimuli) were found to elicit higher accuracy scores and shorter response times as opposed to abstract characters (e.g., Kail et al., 1980). Kail et al. (1980) proposed that abstract characters are unfamiliar to both children and adults as opposed to familiar (e.g., alphanumeric) stimuli.

The underlying mechanism for the developmental pattern of sex differences' emergence in mental rotation received much attention from a biological perspective, especially the role of sex hormones and sex differences in brain structure and function. The most notable influence of sex hormones occurs during sensitive periods in development: prenatal and postnatal (Collaer et al., 2007; Halpern, 2012). Puberty has been suggested as a sensitive period of sex hormone-dependent brain organization, with increased levels of sex hormones secretion influencing changes in cognitive performance (Berenbaum \& Beltz, 2011). In a recent review, Levine et al. (2016) have postulated an integrative approach, including biological as well as environmental factors accounting for sex differences in mental rotation abilities. The suggested model could explain the differences in magnitude from childhood through adulthood in sex differences in mental rotation shown in the present study, as well as in former studies. One possible integrative attempt regarding Gene $\times$ Environment interaction influencing mental rotation performance suggests that the preliminary male advantage in these tasks led them to become more interested in these activities, which in turn led to a greater spatial advantage (Pezaris \& Casey, 1991). This notion is also supported by an evolutionary approach suggesting that differing evolutionary forces imposed on human males and females resulted in differences in anatomy followed by sexual differences in function (Lenroot \& Giedd, 2010). Males engaged in more spatially demanding tasks and, therefore, differential corresponding neuroendocrine infrastructures were needed to develop in human males and females.

To summarize, the present findings suggest that whereas females outperform males in verbal fluency throughout development, sex differences favoring males occur after puberty. Although the findings regarding verbal fluency are not consistent across studies, studies concerning the emergence and increase of sex differences in spatial abilities, and especially mental rotation ability, seem consistent (Berenbaum \& Beltz, 2011). Four decades ago, Waber (1976) postulated that sex differences in cognitive abilities could be explained by maturation rate. He suggested that through the mediation role of the development of hemispheric specialization, late maturers have better spatial abilities, whereas early maturers have better verbal abilities. The present findings provide support for this suggestion.

The present study has some limitations. First, the nature of the mental rotation task used for children and adults in the current study was different. Although the abstract 2D measure for mental rotation capability in children has been validated as capturing a high level of complexity of mental rotation ability, still, future studies are needed for developing 3D stimuli appropriate for children. Second, we have not controlled for the time of day or stage in the menstrual cycle of data collection. Since testosterone, estrogen, and progesterone vary across day time and the menstrual cycle, further studies 
should control for these factors. Third, although the adult sample consisted of participants from various departments (including social as well as formal sciences), future studies should still examine a wide range of study disciplines, especially from natural sciences and STEM fields in order to uncover the influence of field orientation and field expertise on the magnitude of sex differences in various cognitive abilities.

Table 1. Means (SD, Z-Scores, Raw Scores) and Cohen's D For Sex Differences in Verbal and Visuospatial Cognitive Abilities.

\begin{tabular}{|c|c|c|c|c|c|c|}
\hline & & Children & & & Adults & \\
\hline & Boys $(\mathrm{N}=82)$ & Girls (N=87) & $\mathrm{d}$ & Men $(\mathrm{N}=77)$ & $\begin{array}{l}\text { Women } \\
(\mathrm{N}=80)\end{array}$ & $\mathrm{d}$ \\
\hline Serial Sounds & 81.96 (37.66) & 91.98 (43.07) & 0.25 & 111.09 (55.34) & $114.68(42.21)$ & 0.08 \\
\hline Serial Digits & $7.72(2.57)$ & $7.95(2.56)$ & 0.32 & $9.58(1.06)$ & $9.88(1.58)$ & 0.23 \\
\hline Verbal Fluency & $17.80(6.47)$ & $21.93(6.65)$ & $0.63 * * *$ & 39.43 (10.69) & $44.88(10.82)$ & $0.50 * *$ \\
\hline Mental Rotation & $15.41(5.74)$ & $14.98(5.54)$ & 0.14 & $15.23(4.10)$ & 11.35 (4.57) & $0.84 * * *$ \\
\hline Localization & $1.93(0.36)$ & $2.03(0.45)$ & 0.21 & $0.98(0.74)$ & $1.06(0.72)$ & 0.14 \\
\hline Form Comletion & $13.43(7.15)$ & 13.67 (7.17) & 0.00 & $12.18(4.38)$ & 11.34 (4.33) & 0.15 \\
\hline
\end{tabular}

Note. The localization score is higher for poorer performance

$* * \mathrm{p}<.01 \quad * * * \mathrm{p}<.001$

\section{References}

Barel, E., \& Tzischinsky, O. (2017). The role of sex hormones and of 2D:4D ratio in individual differences in cognitive abilities. Journal of Cognitive Psychology, 29, 497-507.

Berenbaum, S. A., \& Beltz, A. M. (2011). Sexual differentiation of human behavior: Effects of prenatal organizational hormones. Frontiers in Neuroendocrinology, 32:10.

Bleecker, M. L., Bolla-Wilson, K., Agnew, J., \& Meyers, D. A. (1988). Age-related sex differences in verbal memory. Journal of Clinical Psychology, 44, 403-411.

Burton, L. A., \& Henninger, D. (2013). Sex differences in relationships between verbal fluency and personality. Current Psychology, 32, 168-174.

Caldera, Y. M., Mc Culp, A., O’Brien, M., Truglio, R. T., Alvarez, M., \& Huston, A. C. (1999). Children's play preferences, construction play with blocks, and visual-spatial skills: are they related? International Journal of Behavioral Development, 23, 855-872.

Collaer, M. L., Reimers, S., \& Manning, J. T. (2007). Visuospatial performance on an internet line judgment task and potential hormonal markers: Sex, sexual orientation, and 2d:4d. Archives of Sexual Behavior, 36, 177-192.

Collins, D. W., \& Kimura, D. (1997). A large sex difference on a two-dimensional mental rotation task. Behavioral Neuroscience, 111, 845-849.

Driscoll, I., Hamilton, D. A., Yeo, R. A., Brooks, W. M., \& Sutherland, R. J. (2005). Virtual navigation in humans: The impact of age, sex, and hormones on place learning. Hormones and Behavior, 47, 326-35.

Frick, A., Ferrara, K., \& Newcombe, N. S. (2013). Using a touch screen paradigm to assess the development of mental rotation between 3.5 and 5.5 years of age. Cognitive Processing, 14, 117-127.

Gordon, H. W. (1986). The cognitive laterality battery: Tests of specialized cognitive function. International Journal of Neuroscience, 29, 223-244.

Gordon, H. W., \& Harness, B. Z. (1977). A test battery for diagnosis and treatment of developmental dyslexia. DASH Speech and Hearing Disorders, 8, 1-7.

Gordon, H. W., \& Lee, P. A. (1993). No difference in cognitive performance between phases of the menstrual cycle. Psychoneuroendocrinology, 18, 521-531.

Halpern, D. F. (2012). Sex differences in cognitive abilities (4th ed.). New York, NY: Psychology Press.

Halpern, D. F., Benbow, C. P., Geary, D. C., Gur, R. C., Shilbey Hyde, J., \& Gernsbacher, M. A. (2007). The science of sex differences in science and mathematics. Psychological Science in the Public Interest, 8, 1-51. 
Herlitz, A., Reuterskiöld, L., Lovén, J., Thilers, P. P., \& Rehnman, J. (2013). Cognitive sex differences are not magnified as a function of age, sex hormones, or puberty development during early adolescence. Developmental Neuropsychology, 38, 167-179.

Hespos, S. J., \& Rochat, P. (1997). Dynamic mental representation in infancy. Cognition, 64, 153-188.

Hines, M. (2004). Brain gender. Oxford, England: Oxford University Press.

Hines, M. (2011). Gender development and the human brain. Annual Review of Neuroscience, 34, 69-88.

Hines, M., Fane, B. A., Pasterski, V. L., Mathews, G. A., Conway, G. S., \& Brook, C. (2003). Spatial abilities following prenatal androgen abnormality: Targeting and mental rotations performance in individuals with congenital adrenal hyperplasia. Psychoneuroendocrinology, 28, 1010-1026.

Hoyek, N., Collet, C., Fargier, P., \& Guillot, A. (2012). The use of the Vandenberg and Kuse Mental Rotation Test in Children. Journal of Individual Differences, 33, 62-67.

Kail, R., Pellegrino, J., \& Carter, P. (1980). Developmental changes in mental rotation. Journal of Experimental Child Psychology, 29, 102-116.

Keith, T. Z., Reynolds, M. R., Roberts, L. G., Winter, A. L., \& Austin, C. A. (2011). Sex differences in latent cognitive abilities ages 5 to 17: Evidence from the Differential Ability Scales (2nd ed). Intelligence, 39, 389-404.

Kimura, D. (2002). Sex hormones influence human cognitive pattern. Neuroendocrinology Letters, 23, 68-77.

Kramer, J. H., Delis, D. C., \& Daniel, M. (1988). Sex differences in verbal learning. Journal of Clinical Psychology, 44, 907-915.

Kucian, K., von Aster, M., Loenneker, T., Dietrich, T., Mast, F. W., \& Martin, E. (2007). Brain activation during mental rotation in school children and adults. Journal of Neural Transmission, 114, 675-686.

Lenroot, R. K., \& Giedd, J. N. (2010). Sex differences in the adolescent brain. Brain and Cognition, 72, 46-55.

Levine, S. C., Foley, A., Lourenco, S., Ehrlich, S., \& Ratliff, K. (2016). Sex differences in spatial cognition: Advancing the conversation. WIREs Cognitive Science, 7, 127-155.

Linn, M. C., \& Petersen, A. C. (1985). Emergence and characterization of sex differences in spatial ability: A meta-analysis. Child Development, 56, 1479-1498.

Lutchmaya, S., Baron-Cohen, S., \& Raggatt, P. (2002). Foetal testosterone and vocabulary size in 18 and 24 month old infants. Infant Behavior and Development, 24, 418-424.

Moore, D. S., \& Johnson, S. P. (2008). Mental rotation in human infants: A sex difference. Psychological Science, 19, 1063-1066.

Palejwala, M. H., \& Goldenring Fine, J. (2015). Gender differences in latent cognitive abilities in children aged 2 to 7 . Intelligence, 48, 96-108.

Pezaris, E., \& Casey, B. M. (1991). Girls who use "masculine" problem-solving strategies on a spatial task: Proposed genetic and environmental factors. Brain and Cognition, 17, 1-22.

Quaiser-Pohl, C., \& Lehmann, W. (2002). Girls' spatial abilities: Charting the contributions of experiences and attitudes in different academic groups. British Journal of Educational Psychology, 72, 245-260.

Quinn, P. C., \& Liben, L. S. (2008). A sex difference in mental rotation in young infants. Psychological Science, 19, 1067-1070.

Ruthsatz, V., Neuburger, S., Jansen, P., \& Quaiser-Pohl, C. (2015). Cars or dolls? Influence of the stereotyped nature of the items on children's mental-rotation performance. Learning and Individual Differences, 43, 75-82.

Voyer, D., Voyer, S., \& Bryden, M. P. (1995). Magnitude of sex differences in spatial abilities: A meta-analysis and consideration of critical variables. Psychological Bulletin, 117, 250-270.

Waber, D. P. (1976, May). Sex differences in cognition: A function of maturation rate? Science, 192(4239), 572-574.

Weiss, E. M., Kemmler, G., Deisenhammer, E. A., W. Fleischhacker, W. W., \& Delazer, M. (2003). Sex differences in cognitive functions. Personality and Individual Differences, 35, 863-875.

Zambrana, I. M., Ystrom, E., \& Pons, F. (2012). Impact of gender, maternal education, and birth order on the development of language comprehension: A longitudinal study from 18 to 36 months of age. Journal of Developmental and Behavioral Pediatrics, 33, 146-155. 


\title{
EMOTION REACTIONS TO ECONOMIC PREDICTIONS AND ITS EFFECTS ON REASONING AND LOGICAL THINKING
}

\author{
Jerônimo Soro, Mário B. Ferreira, Karen Gouveia, \& Joana Reis \\ Faculdade de Psicologia da Universidade de Lisboa (Portugal)
}

\begin{abstract}
The goal of the present work was to explore the possibility that emotions evoked by a threat of financial difficulties could be enough to affect logical reasoning particularly in problems related to financial issues. For this, we manipulated emotional priming via the presentation of excerpt texts from the Portuguese media news that favor either a pessimistic or an optimistic view about the country's economic future. Afterwards, participants judged the logical validity of several syllogisms that had neutral content ("All flowers need water; Roses are flowers; Roses need water"), negative financial ("All minimum wage workers have financial difficulties; Call-center workers are payed the minimum wage; Call-center workers have financial difficulties"), or negative non-financial content (All cancer treatments are painful; chemotherapy is a cancer treatment; chemotherapy is painful.). Furthermore, participants also expressed in a rating scale how confident they were in their responses to each syllogism. Our hypothesis was that negative emotions triggered by the pessimistic view about the future of the Portuguese economy would lead to more financial preoccupation and attention focus to financial issues, which would be translated in an advantage in logical reasoning in responding to syllogistic problems involving financial content. Preliminary results indicate that participants primed with pessimistic news about the economic future of Portugal (compared to participants primed with optimistic news) performed better in syllogistic problems of financial content but showed lower confidence in their responses. These results, particularly the dissociation between performance and subjective confidence, will be discussed in light of their contribution to the extant literature on the impact on judgment and reasoning of emotional distress caused by financial difficulties.
\end{abstract}

Keywords: Reasoning, emotion, syllogism, decision-making, economic crisis.

\section{Introduction}

As the result of the bailout loan in 2010, the Portuguese society was besieged by severe austerity (e.g., salary reduction and increased taxation) due to so-called punitive interest rates often justified as the consequence of years of collective overspending. We posit that the effects of such an intimidating social environment is that more and more people are mentally preoccupied with making ends meet, which triggers increased needs of self-regulation and snowballing adverse effects on subsequent self-control

More recently, the Portuguese economy has shown signs of recovery from this economic crisis (e.g., decrease in unemployment rates and loosening of the austerity measures). However, experts and opinion makers are divided as to how solid and stable this recovery really is. This divide is well captured by the media, with commentators presenting conflicting views concerning the country's economic future. There are those who convey the notion that the Portuguese economy is in the path of structural recovery, generating in consumers some relief and hope in the future; whereas other opinion makers find the current signs of recovery illusive (resulting mostly from European conjunctural factors) and argue that the Portuguese economy is on the verge of another crisis, potentially generating stress and preoccupation among consumers.

The present study was intended to explore how the consumer's emotions, reasoning and decision behavior are affected by these opposing views. Emotions were manipulated between-participants by priming participants with news from one of the two aforesaid current views about the future of Portuguese economy. As expected, emotional valence was positive after priming with encouraging news, and negative after priming with deleterious news about the state of the economy (a similar contrast was found when participants were primed with other positive and negative media news which content matter was unrelated to economical or financial issues). Participants then responded to several conditional 
reasoning problems (syllogisms) which content matter was neutral or negative (with financial or non-financial contents (see the Method section for examples).

Based on previous research showing that a) negative emotions lead to more systematic processing and positive emotions lead to more heuristic processing (Bless, Bohner, Schwarz \& Strack, 1990; Worth \& Mackie, 1987; and b) the elicitation of strong emotions that match the content of the reasoning problems enhance performance (Blanchette \& Campbell, 2012); we hypothesize that negative emotions that are relevant to the problem content would lead to better performance in that type of problem.

\section{Method}

\subsection{Participants}

The study had 118 subjects (66 women), with ages ranging between 18 and 52 years old $(\mathrm{M}=25.6 ; \mathrm{SD}=7.88)$. Data was collected via online questionnaire.

\subsection{Material}

Twelve syllogisms were created with different contents: four with neutral content, 4 with negative non-financial content (e.g., content mater involved diseases) and 4 with negative financial content (content mater involved debts and interest rates). The syllogisms validity and credibility were manipulated orthogonally so that there were 4 configurations corresponding to each of the 4 syllogisms from each content. Syllogisms in which credibility and validity did not match (credible but not logically valid or not credible but logically valid) were considered conflict problems (as they present a conflict between the logical deduction and the credibility of the conclusion) and the remaining were considered non-conflict problems (as the logical validity and credibility of the conclusion converge; see Table 1).

\subsection{Design}

The study had a $4 \times 3 \times 2 \times 2$ experimental design, with Emotional priming (emotional financial positive, $\mathrm{N}=26$; emotional financial negative, $\mathrm{N}=37$; emotional non-financial positive, $\mathrm{N}=28$; emotional non-financial negative, $\mathrm{N}=28$ ) as a between-participants factor and syllogism contents (neutral, non-financial, financial), syllogism credibility (credible, not credible), syllogism validity (valid, not valid) as within-participants factor.

\subsection{Procedure}

Participants were invited to participate in two separate studies. In the first study, they would be asked to respond to a pre-test of items to be included in a questionnaire concerning people's perception of the present state of the national economy (the emotional priming task). In the second study, they would be requested to respond to a series of conditional reasoning problems or syllogisms (the reasoning task).

The priming task was inspired in priming manipulations developed by Salancik (1974; Salancik \& Conway, 1975), and consisted in presenting to participants 6 pairs of paragraphs (each accompanied by a related image to reinforce the emotional response) describing the Portuguese economic context; or 6 pairs of paragraphs with brief news unrelated to economic or financial issues (e.g., sports). Additionally, the 6 pairs of descriptions could be either positive or negative in valence. For each pair, participants were asked to choose the one they considered more relevant and striking. After evaluating the six pairs of paragraphs, participants expressed the emotions induced by the priming task indicating in a seven points rating scale ("1-did not feel..." to "7-felt totally") how intensely did they feel each of 19 emotions.

Following the priming task, participants responded to the reasoning task where they were asked to indicate, for each presented syllogism, whether the conclusions followed logically from the premises or not and how confident they were in their answer in a scale from "1-not confident at all" to "7-completely confident"). After responding to the 12 syllogisms, participants were debriefed and thanked for their participation. 
Table 1. Examples of the 4 configurations of problems with neutral content

\begin{tabular}{|c|c|c|c|}
\hline & Content & Valid & Not valid \\
\hline \multirow{3}{*}{ Credible } & Neutral & $\begin{array}{c}\text { (Non-conflict) } \\
\text { All flowers need water. } \\
\text { All roses are flowers. } \\
\text { Hence, all roses need water. }\end{array}$ & $\begin{array}{c}\text { (Conflict) } \\
\text { All fruits have vitamins } \\
\text { Oranges have vitamins. } \\
\text { Hence, oranges are fruits. }\end{array}$ \\
\hline & $\begin{array}{l}\text { Negative } \\
\text { Non- } \\
\text { Financial }\end{array}$ & $\begin{array}{l}\text { All cancer treatments are painful. } \\
\text { Chemotherapy is a cancer treatment. } \\
\text { Hence, chemotherapy is a painful } \\
\text { treatment. }\end{array}$ & $\begin{array}{l}\text { All contagious diseases are serious. } \\
\text { AIDS is a serious disease. } \\
\text { Hence, AIDS is a contagious disease. }\end{array}$ \\
\hline & Financial & $\begin{array}{l}\text { All minimum wage workers have } \\
\text { financial difficulties. } \\
\text { Call-center workers are payed the } \\
\text { minimum wage. } \\
\text { Hence, call-center workers have } \\
\text { financial difficulties. }\end{array}$ & $\begin{array}{l}\text { All financial products have bank fees. } \\
\text { Credit cards have fees. } \\
\text { Hence, credit cards are financial } \\
\text { products. }\end{array}$ \\
\hline \multirow{3}{*}{$\begin{array}{c}\text { Not } \\
\text { credible }\end{array}$} & Neutral & $\begin{array}{l}\text { (Conflict) } \\
\text { All animals have eyes. } \\
\text { Viruses are animals. } \\
\text { Hence, viruses have eyes. }\end{array}$ & $\begin{array}{c}\text { (Non-conflict) } \\
\text { All birds have wings. } \\
\text { Dogs have wings. } \\
\text { Hence, all dogs are birds. }\end{array}$ \\
\hline & $\begin{array}{l}\text { Negative } \\
\text { Non- } \\
\text { Financial }\end{array}$ & $\begin{array}{l}\text { All criminals cause suffering to their } \\
\text { victims. } \\
\text { Psychotherapists are criminals. } \\
\text { Hence, psychotherapists cause suffering } \\
\text { to their victims. } \\
\end{array}$ & $\begin{array}{l}\text { All infectious diseases cause many } \\
\text { deaths. } \\
\text { Gastritis cause many deaths. } \\
\text { Hence, gastritis is an infectious disease. }\end{array}$ \\
\hline & Financial & $\begin{array}{l}\text { All government companies charge fees. } \\
\text { NGOs are government companies. } \\
\text { Hence, NGOs charge fees. }\end{array}$ & $\begin{array}{l}\text { All motor vehicles are taxed. } \\
\text { Bicycles pay taxes. } \\
\text { Hence, bicycles are motor vehicles. }\end{array}$ \\
\hline
\end{tabular}

\section{Results}

\subsection{Manipulation check}

Responses to the 19 emotions were aggregated into positive and negative emotions and included in a 2 X 2 X 2 ANOVA with Emotion Valence (positive, negative) as a within-participants factor, Priming Valence (positive, negative) and Content (financial, non-financial) as between-participants factors. There was only a significant interaction between Emotion Valence and Priming Valence $F(2,228)=104.54, p<.001, \eta_{p}{ }^{2}=.48$, showing the expected pattern of stronger negative emotions than positive emotions after negative valence priming when compared to positive valence priming. This indicates that the priming manipulation elicited the desired emotions in participants.

\subsection{Accuracy in syllogisms}

Accuracy was analyzed in a 2 X 2 X 3 X 2 ANOVA with Priming Valence (positive, negative) and Priming Content (financial, non-financial) as between-participants factors and Syllogism Content (neutral, non-financial, financial) and syllogism Type (conflict, non-conflict) as within-participants factors. There was only a significant main effect for syllogism Type, $F(1,115)=77.64, p<.001$, $\eta_{p}{ }^{2}=.40$, indicating, as expected, more correct responses for non-conflict problems than for conflict problems. There was an interaction between Syllogism Content and syllogism Type, $F(2,230)=4.07$, $p=.018, \eta_{p}^{2}=.03$, indicating that conflict problems with neutral content led to lower accuracy $(M=1,09$, $S E=0.08)$ than both non-financial $(M=1,24, S E=0.07)$ and financial content problems $(M=1,22$, $S E=0.07), F(1,115)=5.22, p=.024, \eta_{p}^{2}=.04$, while in non-conflict problems accuracy for neutral content $(M=1,69, S E=0.05)$ was not significantly different from accuracy for both non-financial $(M=1,66, S E=0.05)$ and financial content problems $(M=1,61, S E=0.05), F(1,115)=1.81, p=.181$, $\eta_{p}{ }^{2}=.01$. This interaction was qualified by a second order interaction between Syllogism Content, Syllogism Type and Priming Valence, $F(2,230)=3.58, p=.029, \eta_{p}{ }^{2}=.03$, showing that the previous pattern of lower accuracy for conflict neutral problems is observed only after positive valence priming and not after negative valence priming. This is confirmed by a significant difference in accuracy between problems with neutral content $(M=0.94, S E=0.12)$ and problems with both financial $(M=1.16$, $S E=.10)$ and non-financial content $(M=1.24, S E=.10), F(1,115)=8.74, p=.004, \eta_{p}{ }^{2}=.07$, after positive priming but not after negative priming, $F<1$ (Figure 1 ). 
Figure 1. Accuracy means for conflict and non-conflict syllogism problems with different contents under positive and negative priming valences.

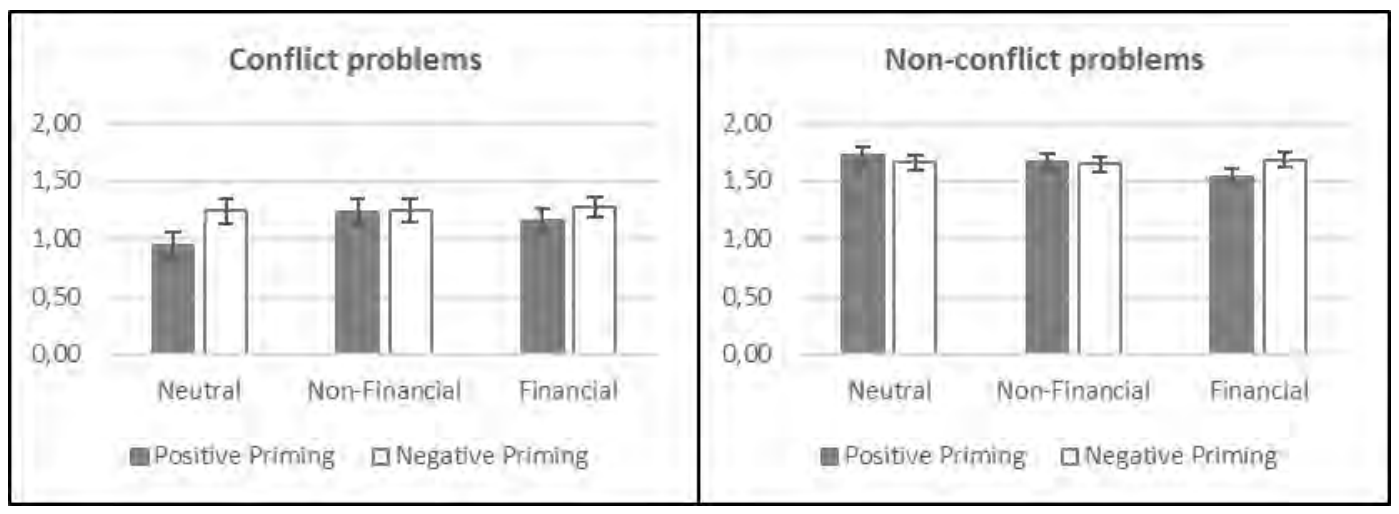

\subsection{Confidence in responses to syllogisms}

The same 2 X 2 X 3 X 2 ANOVA was performed on participants' confidence estimates. There was a significant main effect of syllogism Content, $F(2,230)=18.27, p<.001, \eta_{p}^{2}=.14$. Planned comparisons indicated that confidence for problems with neutral content $(M=5.99, S E=.11)$ was higher than for both financial and non-financial contents, $F(1,115)=28.15, p<.001, \eta_{p}{ }^{2}=.20$, while confidence for problems with non-financial content $(M=5.78, S E=.11)$ was higher than problems with financial content $(M=5.56, S E=.13), F(1,115)=9.22, p=.003, \eta_{p}{ }^{2}=.07$. There was also a syllogism Content $\mathrm{x}$ syllogism Type interaction, $F(2,230)=3.45, p=.033, \eta_{p}{ }^{2}=.03$. Confidence between problems with neutral content in a conflict version $(M=5.89, S E=.12)$ and non-conflict version $(M=6.10, S E=.11)$ were significantly different, $F(1,115)=5.78, p=.018, \eta_{p}{ }^{2}=.05$, as was confidence between problems with financial content in a conflict version $(M=5.46, S E=.14)$ and in a non-conflict version $(M=5.66$, $S E=.14), F(1,115)=3.72, p=.056, \eta_{p}{ }^{2}=.03$. However, confidence in problems with non-financial content showed no significant difference between conflict $(M=5.83, S E=.12)$ and non-conflict versions $(M=5.73, S E=.12), F(1,115)=1.30, p=.257, \eta_{p}^{2}=.01$ (Figure 2).

Figure 2. Confidence means for conflict and non-conflict syllogism problems with different contents.

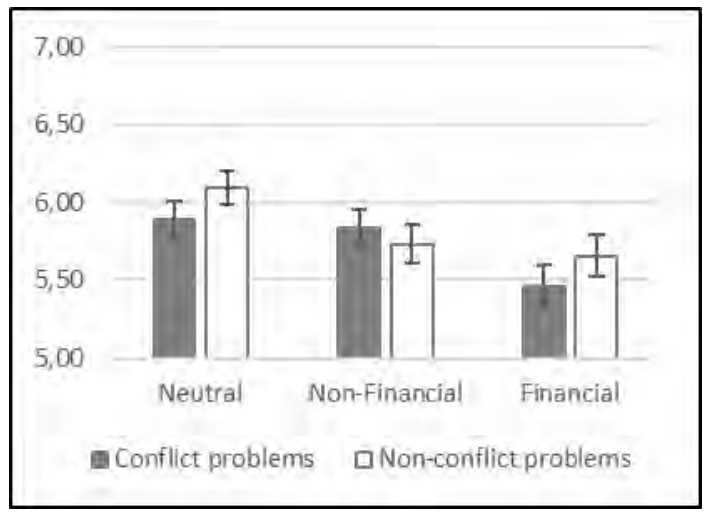

\section{Discussion}

Taken together these results suggest that positive priming leads to worse performance in neutral problems. In contrast, negative priming (financial or non-financial) and/or responding to negative valence problems (regardless of the financial or non-financial content of these problems) seems to be enough to enhance decision behavior in logic deductive tasks such as syllogism that present a conflict between logic validity and credibility. These results are in line with the argument that negative emotions lead to a more analytical processing whereas positive emotions lead to more heuristic processing (Worth \& Mackie, 1987). The effect of emotion appropriateness found in Blanchette and Campbell (2012) was not replicated. That is, negative financial priming (compared with positive financial priming) enhanced accuracy for all conflict problems and not specifically for problems with financial content.

Interestingly, syllogisms content seems to have had somewhat opposite effects on accuracy and confidence in the case of conflict problems. When compared to neutral content, negative (financial and non-financial) content improved performance but lead to a decrease in confidence (particularly in the case 
of financial content). Such pattern of results suggests that financial related negative content enhanced attention and recruited participants' cognitive resources to more carefully deliberate and respond to logical reasoning problems. However, such content may also have increased subjective uncertainty and hesitation translated into lower confidence levels on participants' own answers.

Such increase in deliberation and decrease in subjective confidence might signal the beginning of a response to a potentially emotionally stressful context of economic difficulties. Had this context been maintained and the mental (and physical) weariness brought about by long-term deliberation efforts and preoccupation could have increased levels of stress and deteriorate performance (Lund et al., 2010). In fact, recent research has shown that cognitive impairment can be the product of long-term financial preoccupations of making ends meet (e.g., Mani, Mullainathan, Shafir \& Zhao, 2013). In the short term, however, priming negative scenarios of economic difficulties seems to have boost logical decision-making. An interesting venue for future research would be to show both effects in the same task. In other words, to show that priming participants with scenarios of economic difficulties triggers a stress related response that improves logical reasoning in the short run but impairs it in the long run.

\section{References}

Blanchette, I., \& Richards, A. (2004). Reasoning about emotional and neutral materials: Is logic affected by emotion?. Psychological Science, 15, 745-752. doi:10.1111/j.0956-7976.2004.00751.x

Blanchette, I., Richards, A., Melnyk, L., \& Lavda, A. (2007). Reasoning about emotional contents following shocking terrorist attacks: A tale of three cities. Journal of Experimental Psychology: Applied, 13, 47. doi:10.1037/1076-898X.13.1.47

Blanchette, I., \& Campbell, M. (2012). Reasoning about highly emotional topics: Syllogistic reasoning in a group of war veterans. Journal of Cognitive Psychology, 24, 157-164. doi:10.1080/20445911.2011.603693

Bless, H., Bohner, G., Schwarz, N., \& Strack, F. (1990). Mood and persuasion: A cognitive response analysis. Personality and Social Psychology Bulletin, 16, 331-345. doi:10.1177/0146167290162013

Lund, C., Breen, A., Flisher, A. J., Kakuma, R., Corrigall, J., Joska, J. A., Swartz, L., Patel, V. (2010). Poverty and common mental disorders in low and middle income countries: A systematic review. Social Science \& Medicine, 71, 517-528. doi:10.1016/j.socscimed.2010.04.027

Mani, A., Mullainathan, S., Shafir, E., \& Zhao, J. (2013). Poverty impedes cognitive function. Science, 341, 976-980. doi:10.1126/science.1238041

Salancik, J. R. (1974). Inference of one's attitude from behavior recalled under linguistically manipulated cognitive sets. Journal of Experimental Social Psychology, 10(5), 415-427. doi: 10.1016/00221031(74)90010-9

Salancik, G. R., \& Conway, M. (1975). Attitude inferences from salient and relevant cognitive content about behavior. Journal of Personality and Social Psychology, 32, 829-840. doi: 10.1037/00223514.32.5.829

Worth, L. T., \& Mackie, D. M. (1987). Cognitive mediation of positive affect in persuasion. Social Cognition, 5, 76-94. doi:10.1521/soco.1987.5.1.76 


\title{
SUSCEPTIBILITY OF PERCEPTION OF VERTICAL TO BLOOD GLUCOSE FLUCTUATIONS IN HEALTHY YOUNG ADULTS
}

\author{
Rima L. Abdul Razzak \\ Department of Physiology, College of Medicine and Medical Sciences, \\ Arabian Gulf University (Kingdom of Bahrain)
}

\begin{abstract}
Most studies on the relationship between glucose levels and cognitive function have focused on investigating the effects of exogenous glucose administration on cognitive abilities, with results pointing to inconsistencies in glucose-related effects on cognitive tasks. Disadvantages of such an experimental manipulation include discrepancy among studies in the timing of administration of tests, namely the timing between food intake and cognitive assessment, ranging from 15 min to 4 hours from the glucose drink, and cognitive testing occurring mainly during the postprandial state, leaving the later postabsorptive and possibly fasting states void of cognitive evaluation. It is preferable to evaluate how cognitive tasks can be affected by natural variations in blood glucose levels throughout the day. Perception of subjective visual vertical (SVV), which is essential for spatial orientation and daily activities may be susceptible to glucose fluctuations since it is continuously constructed and updated by brain regions essential for human perception for verticality. SVV perception was assessed with a computerized rod and frame test (CRFT), a clinical exam that evaluates a subject's ability to align a rod to vertical position, with the recorded alignment errors providing information on the accuracy of spatial orientation. The test is carried out without a vertical reference in which the rod is displayed on a blank background and measures the subject's internal representation of vertical. Rod alignment deviation errors from gravitational vertical and time for alignment were compared between the postprandial (within 6 hours after last meal) and postabsorptive / fasting (more than 8 hours from last meal) states in 31 and 33 healthy young males respectively. The mean duration of food omission was shorter $(P<0.0001)$ and mean glucose level was significantly greater $(P=0.0008)$ in the postprandial phase. Significantly greater SVV deviation error was observed during the postprandial phase (Postprandial SVV: $0.88^{\circ} \pm 0.45^{\circ}$, Postabsorptive / Fasting SVV: $0.58^{\circ} \pm 0.35^{\circ} ; P=0.007$ ), while the mean time for rod alignment was significantly shorter (Postprandial SVV time: $7.44 \pm 2.65 \mathrm{~s}$, Postabsorptive / Fasting SVV time: $9.09 \pm 3.44 \mathrm{~s} ; P=0.04$ ).

Verticality perception is susceptible to blood glucose fluctuations throughout the day, with more accurate but slower response time during the postabsorptive/fasting phase. Activities that rely on verticality perception/spatial orientation may be performed more efficiently outside the postprandial phase.
\end{abstract}

Keywords: Verticality perception, CRFT, postprandial glucose, postabsorptivelfasting glucose.

\section{Introduction}

A large body of evidence suggests that cognitive functioning is susceptible to the level of blood glucose, its basic fuel. Most previous studies on the relationship between glucose levels and cognitive function have focused on investigating the effects of exogenous glucose administration on cognitive abilities. However, the beneficial effect attributed to exogenous glucose ingestion is not consistent. This may be as result of the many different factors that potentially affect cognitive abilities, such as the differential sensitivity of cognitive processes to glucose enrichment, the degree of cognitive demand for a certain task and variations in normal glucose regulation capacity.

Glucose enrichment usually involves experimental elevation of blood glucose concentrations levels in the euglycemic range (72-126 mg/dl) by a pure glucose drink before initiating the cognitive tasks after an overnight fast. Such an experimental manipulation has a few disadvantages, one of which is the inconsistency among studies in the timing of administration of tests, namely the timing between food intake and cognitive assessment, ranging from 15 min to 4 hours from the glucose drink. Because assimilation of a pure carbohydrate load is complete within 4 hours (McMahon et al, 1989), the postabsorptive and possibly fasting states are exempt of cognitive evaluation in this case, since with glucose enrichment, cognitive testing occurs mainly during the postprandial state. Moreover, as most of the cognitive testing occurs in the 
morning after an overnight fast, this does not necessarily reflect similar responses to glucose manipulations at lunch or dinner.

In healthy young adults, research on the effect of meals on cognitive function has yielded few conclusions about the effects of specific macronutrients on cognitive performance (Bellisle et al, 1998; Kenarek, 1997). More importantly, there are indications that blood glucose levels can affect cognitive function independent of any glucose enrichment, with higher level of baseline blood glucose being associated with better performance. This is especially true for memory (Parker and Benton, 1995), performance on a vigilance task (Benton et al, 1994) and on the Water Jars task (Donohoe \& Benton, 1999). If we accept that higher glucose levels facilitate cognitive function, then our hypothesis is that cognitive function may be enhanced during the postprandial state, in which there is an increase in blood glucose levels after food consumption, in comparison to performance in the postabsorptive state.

\section{Design}

To test our hypothesis, we selected the cognitive aspect of human vertical perception and orientation, known as subjective visual vertical (SVV). SVV is a psychophysical measure of the angle between perceptual vertical and gravitational vertical, and represents a fundamental spatial reference for maintaining an erect bipedal posture, for balance on Earth (Mazibrada et al, 2008; Van Pelt et al, 2005) and for judging visual orientations in space. The importance of investigating susceptibility of SVV glucose is that it is continuously constructed and updated (Barra, 2010) by brain regions, which play a pivotal role in human perception for verticality, and thus may be impacted due to natural variations in blood glucose levels throughout the day. SVV is typically assessed by the rod-and-frame task (RFT) (Asch \& Witkin, 1948; Baccini et al, 2014; Docherty \& Bagust, 2010), a clinical exam that evaluates a subject's ability to align a line to vertical position, with the recorded alignment errors providing information on the accuracy of spatial orientation. The test is carried out without a vertical reference in which the rod is displayed on a blank background and measures the subject's internal representation of vertical. The capacity to do so requires a complex integrative mechanism of visual, vestibular, and proprioceptive information relative to gravity (Vingerhoets et al, 2009). The design of the study included the independent variable of glucose level and the dependent variables were average rod alignment errors and alignment times.

\section{Objectives}

The aim of this study was to evaluate verticality perception through assessing subjective visual vertical (SVV) at different blood glucose levels that are more representative of real-life daily conditions, and not only falling within the postprandial state, but also within the longer postabsorptive state. Results may have implications to the adequacy of daily timing for performing some activities that heavily rely on spatial orientation.

\section{Methods}

Since restoration of the postabsorptive state takes at least 6 hours after a meal (McMahon et al, 1989), we evaluated SVV during the postprandial (within 6 hours after last meal) and postabsorptive/fasting (more than 8 hours from last meal) phases in 31 and 33 healthy young males respectively. Participants did not receive any incentives for their participation, and they all gave written informed consent.

\subsection{The computerized rod and frame test (CRFT)}

We have utilized a computerized version (Bagust, 2005) of the Rod and Frame Test (RFT) to assess the spatial measures of verticality perception. This test is a modified version of the CRFT (Dochety \& Bagust, 2010) in which the rod displayed in the center of the screen consisted of five white dots and had two starting positions, tilted 20 degrees in either a clockwise (CW) or counter clockwise (CCW) direction from vertical. The CRFT comprised 6 presentations and consisted of four replicates of SVV presentation (Figure 1a). A round black paper ring was stuck on the laptop screen to conceal its edges and reduce clues to verticality, while exposing the rod presentation in the center of the screen (Figure 1b). The test was performed in a dark room minimizing further any vertical cues within the room.

\subsection{Procedure}

Information on the times of the last meal and the blood collection just before cognitive testing were recorded., Prior to beginning the test, random blood glucose levels were measured with the ACCU-CHEK ACTIVE (Roche, Germany) glucometer. Participants were then shown two instructional presentations that allowed them to familiarize themselves with the process but were not included in the analysis. During testing, participants were seated in front of the laptop screen and asked keep their head in 
a fixed position without tilting or turning. They were instructed to rotate the dots using the right and left mouse buttons to a position perceived to be vertical. The dots rotated around their virtual midpoint in 0.5 degrees increments. When the participant was satisfied with the alignment of the dots, the program was advanced using the space bar of the computer keyboard. Positioning errors were recorded by the computer in degrees from gravitational vertical. The time for each rod alignment was also recorded.

Figure 1. a) Presentations of the rod during testing. The order of presentation was randomly assigned by the computer. b) Set up showing the concealment of the vertical edges of the laptop to obscure any cues of verticality from the subject.
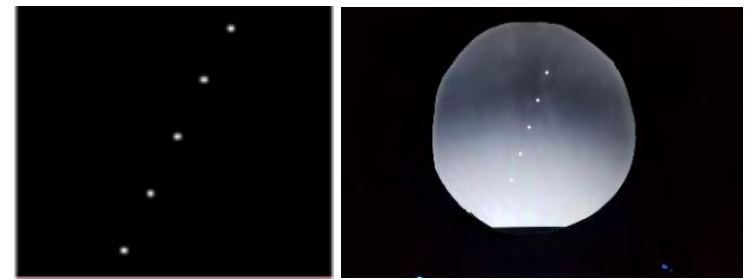

\subsection{Spatial error calculations \& statistical analysis}

Deviations from vertical were recorded in degrees as positive values if the deviation was in a CW direction, and negative values for CCW deviations. These values were used to calculate the mean signed errors and the mean absolute (unsigned) errors for the four SVV presentations for each participant. The reported alignment errors in this study are the absolute (unsigned) values. The SVV alignment errors of the two glucose criteria were then compared. All statistical analyses were carried out using INSTAT (Graph Pad Inc.). Data were tested for normality using the Kolmogorov-Smirnoff method. Differences between the two groups were determined by unpaired t-test. Data were reported as mean $\pm \mathrm{SD}$, and level of significance was set at $p<0.05$.

\section{Results}

All data passed the normality test. Figure 2 reveals negative correlation between food omission and glucose levels, but the association was significant only for the fasting state (Postprandial: $r=-0.27$, $\mathrm{P}=0.14$; Fasting: $\mathrm{r}=-0.46, \mathrm{P}=0.008$ ). Table 1 illustrates the differences in glycemic and verticality perception parameters between the postprandial and fasting states. Mean glucose level was significantly greater during the postprandial phase. Significantly greater SVV deviation error was observed during the postprandial phase, while the mean time for rod alignment was significantly shorter. In figure 3 , the mode of deviation errors for both groups was in the same error bin, however, the mode was greater for the fasting group. Almost all subjects falling within the larger error bins were in the postprandial phase.

Figure 2. Relationship between food omission and glucose levels during the postprandial and postabsorptive state.

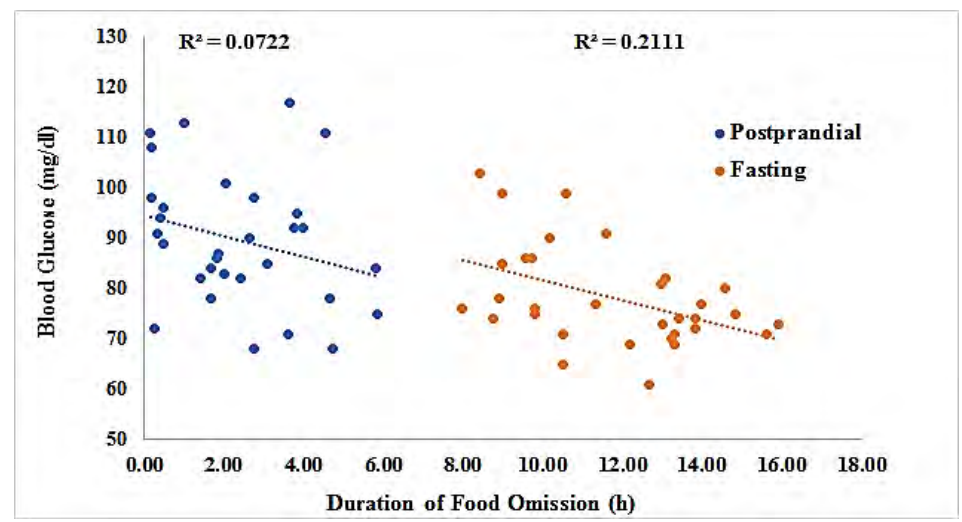

\section{Discussion}

This is the first study to examine the effects of glucose on verticality perception as measured by SVV. Our knowledge of SVV perception as a cognitive task that is mediated by complex integration of sensory inputs led us to hypothesize it may be cognitively demanding enough to influence brain glucose consumption. As it is constructed and updated continuously, it may be susceptible to actual changes in blood glucose concentrations throughout the day. We examined cognitive perceptual function without 
manipulating participants' levels of blood glucose. It is true that majority of the day is spent in the postprandial state because people usually eat at least three times a day, but this is not applicable to those who skip meals or those who are observing religious fasting.

Table 1. Glycemic and verticality perception parameters in the postprandial and postabsorptive states. Values represent mean $\pm S D$ and (range of values) for each parameter.

\begin{tabular}{lcccc}
\hline & $\begin{array}{c}\text { Duration of Food } \\
\text { Omission (h) }\end{array}$ & $\begin{array}{c}\text { Blood Glucose } \\
\text { level }(\mathrm{mg} / \mathrm{dl})\end{array}$ & $\begin{array}{c}\text { SVV Deviation } \\
\text { Error }^{\circ}\end{array}$ & $\begin{array}{c}\text { Time for SVV } \\
\text { Alignment (s) }^{\circ}\end{array}$ \\
\hline \multirow{2}{*}{ Postprandial } & $2.40 \pm 1.72$ & $89.7 \pm 13.2$ & $0.88 \pm 0.45$ & $7.44 \pm 2.65$ \\
& $(0.17-5.87)$ & $(68.0-117.0)$ & $(0.25-2.00)$ & $(4.32-15.38)$ \\
Postabsorptive / Fasting & $11.72 \pm 2.24$ & $78.6 \pm 9.9$ & $0.58 \pm 0.35$ & $9.09 \pm 3.44$ \\
& $(8.00-15.92)$ & $(61.0-103.0)$ & $(0.00-1.25)$ & $(3.85-15.83)$ \\
$\mathrm{t}(P$-vlaue $)$ & $18.55(<0.0001)$ & $3.89(0.0002)$ & Welch $\mathrm{t}=3.26(0.002)$ & $2.06(0.04)$ \\
\hline
\end{tabular}

Figure 3. Distribution of SVV alignment errors in 0.5 degree bins for subjects within postprandial or postabsorptive state.

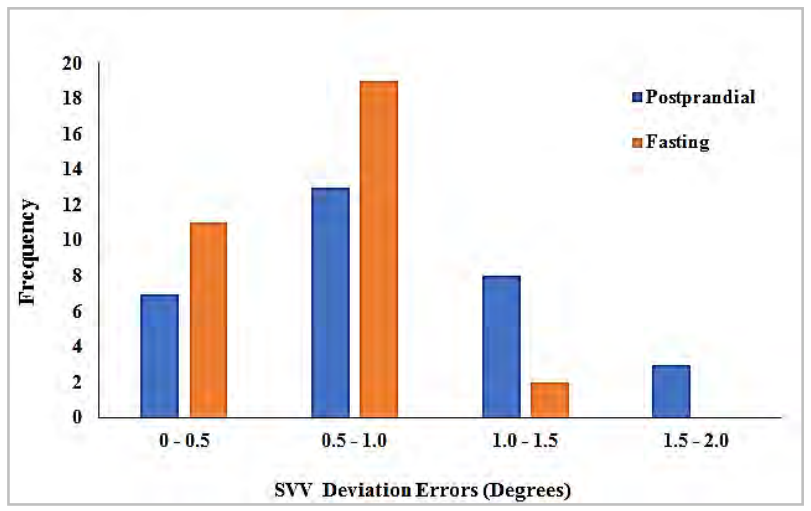

This is advantageous over glucose enrichment because the use of a single nutrient in the glucose drink is not representative of our usually mixed meals, and consuming a meal to increase blood levels may lead to different results from when the same amount of glucose is consumed in just a drink (Gold, 1986; Green et al., 2001). Because the assimilation of the constituents of a mixed meal and restoration of the postabsorptive state takes at least 6 hours, we carried our cognitive testing within 6 hours of meal ingestion, a relatively prolonged postprandial period.

Our results indicate to better verticality perception during the postabsorptive/fasting state as measured by smaller SVV mean alignment errors than during the postprandial phase. This may seem equivocal, since the glucose levels were higher in the postprandial phase in comparison the later glycemic phase. A possible explanation is that food omission for longer than 8 hours for our subjects in the postabsorptive states would induce a stable supply of glucose through glycogenolysis, while during the postprandial period, there will be post-meal glucose excursions as well. Such excursions, even if they are within the normal glucoregulation process, may negatively affect cognitive function. However, a stable supply of glucose as a result of glycogenolysis may be beneficial since stable blood glucose which avoids regular peaks and troughs in glucose is associated with better cognitive function (Sunram-Lea et al, 2016). A new hypothesis put forward by Wheeler et al (2017) states that on a daily basis there are dynamic interactions among hyperglycemia, hypoglycemia and cerebral blood flow, with brain exposure to circulating glucose excursions causing reduction in cerebral flow and early stage damage. If hyperglycemia time is long, this would result in adaptation to inhibit blood to brain glucose transport (Wheeler et al, 2017).

It is important to note the relatively small absolute values of the errors in the current study may seem within the acceptable normal range in comparison to that of the 3D mechanical RFT (Asch \& Witkin, 1948), in which most people deviate within $\pm 2^{\circ}$ from gravitational vertical. This may render the interpretation and functional significance of the present results inconclusive. However this is expected as the CRFT has been reported to produce smaller errors (Isableu et al; 2008) in comparison to the mechanical system.

The shorter alignment times during the postprandial phase are consistent with studies reporting that blood glucose levels influence the speed of cognitive processing, such that, enriched blood glucose 
levels are associated with faster cognitive processing and shorter response times (Owens \& Benton, 1994). The higher accuracy of rod alignment with low glucose levels might have been at the expense of response time which slowed with the need to concentrate.

\section{Conclusions}

Verticality perception over the course of the day may be impacted due to natural variations in blood glucose levels, with less accurate but shorter response time during the postprandial phase. Given the frequent use of sugary drinks or snacks in modern society, these results suggest a possible detriment of postprandial elevated glucose levels for verticality perception, and activities that rely on verticality perception/spatial orientation may be performed more efficiently outside the postprandial phase. For optimal verticality perception, it may be beneficial to adopt mechanisms that reduce blood glucose fluctuations throughout the day such as eating foods that cause a slow and steady rise in blood glucose.

\section{References}

Asch S E \& Witkin H A. (1948). Studies in space orientations: I. Perception of the upright with displaced visual fields. Journal of Experimental Psychology, 38, 325 - 337.

Baccini M, Paci M, Del Colletto M, Ravenni M \& Baldassi S. (2014). The assessment of subjective visual vertical: comparison of two psychophysical paradigms and age-related performance. Attention Perception Psychophysics, 76, 112-122.

Bagust J A. (2005). Computer based version of the rod and frame test-preliminary observations on the use of a VDU screen and video eyeglasses. Archives of Physical Medicine and Rehabilitation, 86, 1062-1064.

Barra J, Marquer A, Joassin R, Reymond C, Metge L, Chauvineau V., et al. (2010). Humans use internal models to construct and update a sense of verticality. Brain, 133(12), 3552-3563.

Bellisle F, Blundell JE, Dye L, et al. (1998). Functional food science and behaviour and psychological functions. British Journal of Nutrition, 80, S173-S193.

Benton D, Owens DS \& Parker PY. (1994). Blood glucose influences memory and attention in young adults. Neuropsychologia, 32, 595-607.

Docherty S \& Bagust J. (2010). From line to dots: an improved computerized rod and frame system for testing visual vertical and horizontal. BMC Research Notes, 3(1), 3-9.

Donohoe RT \& Benton D. (1999). Cognitive functioning is susceptible to the level of blood glucose. Psychopharmacology, 145, $378-385$.

Gold PE.(1986). Glucose modulation of memory storage processing. Behavioral and Neural Biology, 45, 342 -349.

Green MW, taylor MA, Elliman NA \& Rhodes O. (2001). Placebo expectancy effects in the relationship between glucose and gognition. British journal of Nutrition, 86, 173-179.

Isableu B, Gueguen M, Fourré B, Giraudet G \& Amorim M A. (2008). Assessment of visual field dependence: comparison between the mechanical 3D rod-and-frame test developed by Oltman in 1968 with a 2D computer-based version. Journal of Vestibular Research, 18(5-6), 239-247.

Kanarek R. (1997). Psychological effects of snacks and altered meal frequency. British Journal of Nutrition, 77, S105-120.

Mazibrada G, Tariq S, Pérennou D, Gresty M, Greenwood R \& Bronstein A M. (2008). The peripheral nervous system and the perception of verticality. Gait \& Posture, 27, 202-208.

McMahon M, Mash HM, \& Rizza RA. (1989). Effects of basal insulin supplementation on disposition of mixed meal in obese patients with NIDDM. Diabetes, 38, 291-303.

Mittelstaedt H. (1995). Somatic graviception. Biological Psychology, 42, 53 -74.

Owens D S \& Benton D. (1994). The impact of raising blood glucose on reaction time. Neuropsychobiology, 30, 106-113.

Parker PY \& Benton D. (1995). Blood glucose levels selectively influence memory for word lists dichotically presented to the right ear. Neuropsychologia, 33, 843-854.

Sünram-Lea SI, Foster JK, Durlach P, et al. (2001). Glucose facilitation of cognitive performance in healthy young adults: examination of the influence of fast-duration, time of day and pre-consumption on plasma glucose levels. Psychopharmacology, 157:46-54.

Van Pelt S, Van Gisbergen J A M \& Medendorp W P. (2005). Visuospatial memory computations during whole body rotations in roll. Journal of Neurophysiology, 94(2), 1432 - 1442.

Wheeler MJ, Dempsey PC, Grace MS, Ellis KA, Gardiner PA, Green DJ, Dunstan DW. (2017). Sedentary behavior as a risk factor for cognitive decline? A focus on the influence of glycemic control in brain health. Alzheimers Dement (N Y), 2;3(3):291-300. 


\title{
IN SEARCH OF EVIL: AFFECTIVE DIMENSIONS OF PRESIDENTIAL ELECTIONS 2018 IN GEORGIA
}

\author{
Marine Chitashvili, Davit Machavariani, \& Otar Sokhadze \\ Department of Psychology, Ivane Javakhishvili Tbilisi State University (Georgia)
}

\begin{abstract}
The paper discusses how employing theoretical elements and interpretive frames of psychoanalytic anthropology, particularly from Lacanian perspective, help to conceptualize and analyze the affective dimensions in elections by creation the image of enemy as evil. Psychoanalytical theoretical vocabulary became the main source to capture affective dimension of politics by analyzing the texts, images and discourse how various emotions are signified in political speech. Election as a conventional public activity involves different political parties declaring their political values and competing to convince the large groups of population to vote for and follow specific discourses in order to get the majority in elections and win by setting parameters through which desire is produced, regulated and channeled (Kolvraa, 2018). The common knowledge of building the electoral discourses involves construction of image of enemy, evil, that has to carry the guilt for the perpetual postponement of utopia (Zizek, 1990), i.e. fulfillment of the emotional pull of certain political ideas. By Lacan human subject is never integrated into the symbolic world of language and traced by feelings of something loss and driven by the desire to recapture the lost enjoyment. By Glynos (2010), Starvakakis (2007) and Zizek (2005) this framework was used to understand the affective dimensions of ideological discourses. "This means that ideological discourses must produce fantasmatic narratives or scenarios which promise a utopian future of full satisfaction" (Kolvraa, 2018). In narratives like fascism, radical nationalism, Stalinism loss of enjoyment is replaced as a "stolen" by the enemy than contributes to maintain expectation of impossible - "a return to the fullness of jouisance in a utopian society" (Kolvraa, 2018). However the question of enjoyment still remains open in terms of affective dimension of political discourse - how it is achieved by the discourses of regaining of lost or defeating the enemy. We are analyzing the concrete presidential elections in Georgia, former soviet and new democratic country as a multidimensional social phenomenon coming from Stalinist and nationalist authoritarian past straggling to establish democratic institutions, laws and regulations, implementing elections as a main source of democratic governance. The case of last presidential elections is discussed as ambiguous model where discourses of enemy is floating form lost to stolen enjoyment of utopian society
\end{abstract}

Keywords: Psychosocial, affective dimension, elections, enemy.

\section{Understanding psychosocial}

Psychoanalytical epistemology, as a new academic and therapeutic discourse, expands from psychology to other fields of social sciences from the very beginning of its existence. Freud used psychoanalysis as a methodology to explore the cultural and social phenomenon from the perspective of unconscious, drives, internalization, defense mechanisms, superego, id, etc. Erich Fromm in his book "Sigmund Freud's Mission: An Analysis of His Personality and Influence" (Fromm, 1978) argues, that Freud's personal interest was more in understanding of mankind, culture, world order and not particularly the patients' behavior. According to Fromm he (Freud) used the clinical material to write the macro theory of interplay of social and individual across the civilization.

Debating the core psychoanalytical concepts and its' applications led to establishing the new paradigms, concepts and hypothesis in anthropology, sociology, political science, law, gender studies, literary critic, cinema, etc. (Bocock, 1977).

Stephen Frosh states that the psychoanalysis contributed to the development of poststructuralist and critical social theories and it itself is a social theory (Frosh, 2012). Frosh as a founder of a new discipline of psychosocial studies looks at psychological, social and cultural applications of psychoanalytic theory. The mostly used concepts are repression (oppressive societies denying their 
subjects to access their desires), projection (explaining the mechanism of racism and war), identification (identity issues), internalization (concept of "ungrieved' due to the collective trauma), and castration (Lacanian idea of "lack", as a core principle of subjecthood, that could not be never achieved). Frosh himself uses the idea of haunting to explore how identities, beliefs, intimacies and hatreds are transmitted across generation and between people. Transmissions of cognitive schemas are widely explored in social psychology on IDPs and traumatized groups. (Hentschel et.al, 1996).

Robert Paul (Paul, 1989) rewiring the different aspects of psychoanalytical anthropology, points that those who use psychoanalysis in anthropology assume that human life is influenced by unconscious and is mirrored in culture and symbols people use and follow in every day life and in social actions. For Paul (1987) collective phenomena are the products of individual minds, thus psychoanalysis could be legitimately applied to interpret social phenomena. Aggression as a central impulse of the individual and society could be understood either in positive outcome of internalization (Freud, 1930) or as a projected outwards and directed toward enemies (scapegoating and splitting also enables group to deal with aggression). Paul (1987) defines applied psychoanalysis as a method to interpret cultural, collective, public bodies of symbolism. This approach has been widely used in recent years to understand the political behavior (Marcus, 2003, Brader, Marcus, 2013, Redlawsk, Lau, 2013, Kølvraa, 2018).

Christoffer Kølvraa (2018) points that Lacanian perspective help to conceptualize and analyze the affective dimensions of politics by analyzing texts, images and discourses - how various emotions are signified in political speech. As stated by Lacan, "discourse is "a necessary structure", that "subsists in certain fundamental relations". These "fundamental relations" are of several different orders: intrasubjective or psychological, intersubjective or social, and relations with the nonhuman world. Discourse, according to Lacan, plays formative and transformative roles in each of these orders.“ (Bracher, 1994, pp.117).

Election as a conventional public activity involves different political parties declaring their political views and competing to convince the large groups of population to vote for them by using specific discourses through which desire is produced, regulated and channeled. "This means that ideological discourses must produce fantasmatic narratives or scenarios which promise a utopian future of full satisfaction" (Kølvraa, 2018).

In this paper we analyze affective dimensions of transformative narrative of evil/enemy during 2018 presidential elections in Georgia.

\section{Background story and case}

Georgia is one of the newly independent states with the Soviet past gaining independence after dissolution of the Soviet Union in 1991. The country has a long history and is known as one of the oldest civilizations of the world (Reyfied, 2012). In the beginning of XX century (1918-21) Georgia became an independent republic, but soon after was occupied by Russia and became one of the republics of the Soviet Union. In 1990, after declaring independence and becoming the member of UN in 1992, Georgia starts a new era as a new democratic state oriented towards the EU integration (Jones, 2012). Since 2008, after the war with Russia, Georgia's 20\% has been occupied by Russia. In 2014 Georgia signed the association agreement with EU; in addition, it has been declared aspiration to join NATO.

Political development in last 25 years till 2016 encounter civil war, frozen conflicts and then occupation, forced change of governments and turmoil of unrest and civil protest, dramatical changes in social structure and growing social inequality. Georgia by the constitution (the last changes were introduced in 2018) is a parliamentary republic, with very limited power of the President as a representative of the country in foreign relations and supreme-commander-in-chief of defense forces (Constitution of Georgia, 2018).

The presidential elections 2018 (the fifth in raw) was the last direct elections. By the new constitution the next president will be elected by the electoral college, without debates and open ballot. Unexpectedly current elections became the special case for political parties to promote their candidates (even there is no any power assigned to the president other than representative). Intention to gain the office became the vital target.

2018 elections became the hot topic in reports of local and international observers highlighting the usage of hate speech, harsh rhetoric and discriminative statements in media (Benidze et.al, 2018, OSCE ODIHR, 2019).

Presidential elections were held in two rounds. During the first round 25 individuals were participating (CEC, 2018). Only three subjects were running very active pre-electoral campaign - Salome Zourabishvili, independent candidate (backed by the parliamentary constitutional majority party Georgian Dream, GD), Grigol Vashadze (supported by the parliamentary faction, political party - United National Movement, UNM) and David Bakradze (supported by the parliamentary opposition faction, 
political party - European Georgia, EG). These three became the main target of negative campaigning and harsh rhetoric from private and social media, most intensively toward S. Zourabishvili (ISFED, 2018).

The main argument provided by the candidates in their speeches was blaming the opponent as a Russian spy or associated with Russia either in past or present. Media and particularly social media ("Facebook" pages) were accusing two candidates S. Zourabishvili and G. Vashadze of pursuing Russian interests. A large portion of the pages that were active in the "Facebook" space, except for discrediting the election subjects, aimed to discredit the current political processes, civil activists and opinion leaders. Photo and video material published by social media pages show that uniform multimedia technologies are used, indicating that their source of creation may be one organized group (ISFED, 2018).

Two candidates, S.Zourabishvili and G.Vashadze, continued the run-off in second round. The use of negative, harsh and at times violent rhetoric significantly overshadowed the campaign. (OSCE ODIHR, 2019, p. 4-5). The ODIHR EOM noted a widespread use of aggressive and violent rhetoric on TV programs and social media, and during demonstrations as well as in individual statements by many high-ranking party members and public officials. Several statements, including on comparing the election to a civil war and calling for the destruction of their opponents, bordered on xenophobia and hate speech. The ODIHR EOM observed that UNM representatives periodically referred to Ms. Zourabishvili as a "traitor" and called the GD "an immoral armed gang"; during anti-UNM public demonstrations, the UNM was compared to "Nazis, terrorists or Satan" and the organizers called to "destroy or annihilate the party"; GD representatives periodically referred to UNM as a "bloody criminal regime" and their coveted victory as “civil war and revenge”(OSCE ODIHR, 2019, p. 28).

\section{Case Analysis}

The objective of case analysis is to identify affective mechanisms of the electoral campaign in whole. Freud understand social structures as formations to satisfy the individual's need for protection from the threat/enemy. Paul points that psychoanalytically oriented research "attempts to discover the fate of aggression, even-or especially-in societies in which its overt expression is normally inhibited .... Aggressive impulses suppressed within a group or community may be projected outward and directed toward "enemies"'” (Paul, 1989, p. 190-91).

During the presidential election social media (in particular Facebook) became one of the most influential channels to communicate with public. Overall only official 19 FB pages for candidates and supporting parties display 7. 224 posts covered 1,261,968 users who liked these pages. We are analyzing the secondary data of social media covering the both rounds of the elections 2018 concentrating only on messages with discredited character, where Russia is symbolized as an enemy and candidate is accused to serve the Russian interests. The evidence-based reports of International Society for Fair Elections and Democracy (ISFED, 2018) gives the full account on different types of misuse of facts during elections. We use only small part of that data, discrediting messages that vary on large scale. Quantitative data (Table 1) shows the breadth and intensity of that. Discrediting messages were grouped by the themes. Second round repeated statements from the first one and were enriched with new content. Data show that in second round discrediting messages against Grigol Vashadze were intensively used associating him with all the wrongdoings of the UNM government he was a part and mocking him as an alter-ego of UNM and former president Michael Saakashvili.

To show the dynamics of affective dimension we classified discrediting messages contents (DMC) in three groups. First one is the facts (DMC F), the second, half facts with deliberate targeted interpretation (DMC FI), third statements without any facts, pure interpretation (DMC I). In the first round DMC was targeted creating candidate as a symbolic image of real threat, Russia, and allowing opponents freely projecting aggression outwards. In the second round Georgian Dream who took the campaigning on behalf of SZ openly declared that this is "principal choice" against the GV, as UNM representative. The DMC content in second round against GV combined his symbolization as a Russia plus as a threat of past government and its violence, brutality, elective justice, insulting moral authority and values.

The common knowledge of building the electoral discourses involves construction of image of enemy, evil, that has to carry the guilt for the perpetual postponement of utopia (Zizek, 1990), i.e. fulfillment of the emotional pull of certain political ideas. Was there any political idea for the presidential elections? From the very beginning the ruling party Georgian Dream underlined the presidential elections to be less important, thus did not nominate its own candidate and supported independent one. However, unexpected activity from the opposition parties made ruling party to be engaged in full scale. The first round of the election campaign crystalized around the idea to have president not affiliated to any treats Georgia had and has nowadays, i.e. Russia as a major threat of independence of the country. Russia as 
symbol of loss of enjoyment being free country converted to symbolic representation by the candidates as unacceptable (DMC I round). The first-round results (SZ 39\% and GV 38\%) clearly show that none of them worked as acceptable and convincing symbols as enemies).

By Lacan human subject is never integrated into the symbolic world of language and traced by feelings of something loss and driven by the desire to recapture the lost enjoyment. By Glynos (2010), Starvakakis (2007) and Zizek (2005) this framework was used to understand the affective dimensions of ideological discourses.

Table 1. Data from social media targeted on discreditation of presidential candidates.

\begin{tabular}{|c|c|c|c|c|}
\hline candidate & \multicolumn{2}{|c|}{ Salome Zourabishvili (SZ) } & \multicolumn{2}{|l|}{ Grigol Vashadze (GV) } \\
\hline round & $I-01.06-18.10$ & $I I-29.10-02.12$ & $I-01.06-18.10$ & $I I-29.10-02.12$ \\
\hline \#FB pages & 26 & 43 & 27 & 72 \\
\hline \# Posts & 10538 & 8383 & 4766 & 3164 \\
\hline \# likes & 452572 & 721646 & 403417 & 712924 \\
\hline $\begin{array}{l}\text { Discrediting } \\
\text { Massages } \\
\text { Content (DMS) }\end{array}$ & $\begin{array}{l}\text { a) Putin ordered } \\
\text { Ivanishvili to } \\
\text { support SZ } \\
\text { b) No to Salome } \\
\text { betrayer } \\
\text { c) Don't vote for } \\
\text { betrayer }\end{array}$ & $\begin{array}{l}\text { d) SZ against Zviad } \\
\text { Gamsaxurdia ( } I^{\text {st }} \\
\text { president of } \\
\text { Georgia, symbol of } \\
\text { anti-Russian } \\
\text { nationalist) } \\
\text { e) Voting for SZ is } \\
\text { betraying the } \\
\text { homeland } \\
\text { (archbishop's } \\
\text { statement) } \\
\text { f) No to SZ- } \\
\text { Betrayer of } 2008 \\
\text { war } \\
\text { g) SZ - Resident, } \\
\text { Spy }\end{array}$ & $\begin{array}{l}\text { a) } 2008 \text { video on } \\
\text { positive attitude } \\
\text { toward Russia } \\
\text { b) UNM signed EU } \\
\text { Council resolutions } \\
\text { acknowledging the } \\
\text { war started after } \\
\text { bombing Tskhinvali } \\
\text { c)GV got education } \\
\text { in Russia and is } \\
\text { KGB associate } \\
\text { d) GV was Russian } \\
\text { citizen till } 2009 \text { and } \\
\text { at the same time } \\
\text { Georgian Minister } \\
\text { for foreign affairs } \\
\text { e) GV interview } \\
\text { saying the he is the } \\
\text { part of Russian } \\
\text { culture, i.e. } \\
\text { Russians' candidate }\end{array}$ & $\begin{array}{l}\text { f) visual material } \\
\text { mocking GV } \\
\text { transforming into } \\
\text { Micheil Saakashvili } \\
\text { ( } 3^{\text {rd }} \text { president of } \\
\text { Georgia, UNM } \\
\text { founder and leader, } \\
\text { symbol of injustice } \\
\text { and brutality of } \\
\text { 2007-12) } \\
\text { g) No to Misha (as a } \\
\text { symbol of violence) } \\
\text { h) visual material of } \\
\text { riot police during } \\
\text { the } 2007 \text { and } 2011 \\
\text { unrest against } \\
\text { government } \\
\text { i) GV misinterpret- } \\
\text { ting the Patriarch } \\
\text { message (the only } \\
\text { moral authority in } \\
\text { Georgia } \\
\text { j) GV presidential } \\
\text { PR lead by Russian, } \\
\text { i.e. Russian } \\
\text { involvement }\end{array}$ \\
\hline \#DMC Fact & & & 2 & 1 \\
\hline \#DMC half Fact & & & 3 & 3 \\
\hline \#DMC Interpreted & 3 & 4 & & 2 \\
\hline
\end{tabular}

Russian threat and perception of Russia as an enemy is not unequivocal for the political landscape of Georgia ("Alliance of patriots of Georgia" anti-western right-conservative party represented in parliament with faction), thus creating the need for clearer and more adequate enemy. In second-round DMC for GV are clear evidence of such type of mythology creation. In 2012, the first-time in Georgia, government was changed by the elections, UNM (GV) lost elections and GD (SZ supporter) got parliamentary majority seats. From 2016 elections GD has constitutional majority in parliament.

Political rivals run-off second round of presidential elections and GD openly supporting SZ started to appeal to nearest past and directly channeling threatening message of possibility power return of UNM (GV second round DMC). Russia as a threat was replaced by coming back of UNM (DMC for Vashadze), while his ties with Russia still remained to be part of the discourse.

The major shift of composing the image of enemy for the second round was re-exposing the traumatic experience of nearest past where GV as a representative UNM government was exposed as a threat. Actually, two traumas - the long standing and realistic, Russia, was challenged with the new and realistic, UNM. The object of enjoyment (freedom) in case of Russia is lost and in case of UNM, the same is twofold (Russia - lost, and personal security - stolen). The heroes of this myths transformed accordingly. SZ who is born as an emigrant from Georgia after the first occupation of Russia at beginning of XX century is returning to regain lost enjoyment and GV who is born in soviet times, enjoyed all privileges under soviets and UNM, both as a brutal regime, could not be trusted as a reliable personality. This statement (equilibrium of two traumas and dynamics of enjoyment) needs to be supported by the evidence. 


\section{References}

Benidze, M, Bartaia T, Rizhamadze N, Nizharadze E, Kinkladze T, Baramidze R. (2018). Final report of monitoring the 2018 presidential elections. Tbilisi: ISFED. Retrieved March 21, 2019 from https://www.scribd.com/document/402537696/ISFED-Final-Report-of-Monitoring-of-the-2018Presidential-Elections\#from_embed

Bocock R.J. (1977). Freud and the centrality of Instincts in Psychoanalytic Sociology. The British Journal of Sociology, Vol.28, No 4, pp.467-480.

Brader, T, Marcus G.E. (2013). Emotion and political psychology. In L Huddy, D.O. Sears, J.S Levy (Eds.), Oxford handbook of political psychology. (pp. 165-204). Oxford: Oxford University Press.

Bracher, M. (1994). On the psychological and social functions of language: Lacan's theory of the four discourses. In M. Bracher, M.W. Alcorn Jn., R.J. Corthell, F. Massadier-Kenny (Eds.), Lacanian theory of discourse: subject, structure and society. (pp. 107-128). New York and London: New York University Press.

CEC, (2018). October 28, 2018 Election of president of Georgia. Interim report. Tbilisi: Election administration of Georgia. Retrieved January 20, 2019, from http://cesko.ge/eng/elections/2018

Constitution of Georgia. Retrieved February 7, 2019, from https://matsne.gov.ge/en/document/view/30346?publication=35

Glynos, J. (2001). The grip of ideology: A Lacanian approach to the theory of ideology. Journal of political ideologies, Vol.6, No.2, pp.191-214.

Gugushvili, A., Kabachnik P. (2015). Stalin is dead, long live Stalin? Testing socialization, structural, ideological, nationalist, and gender hypotheses. Post-Soviet Affairs, Vol.35, issue.1, pp. 1-36.

ISFED, (2018). Interim report of the pre-election monitoring of the runoff - 2018 presidential elections. Tbilisi. Retrieved February 7, 2019, from http://www.isfed.ge/eng/angarishebi/Interim-Report-ofthe-Pre-Election-Monitoring-of-the-Runoff-2018-Presidential-Elections

ISFED, (2018). Second Interim report of the pre-election monitoring of the runoff - 2018 presidential elections. Tbilisi. Retrieved February 7, 2019, from http:/www.isfed.ge/eng/angarishebi/Numberof-Discrediting-Facebook-Pages-Tripled-for-the-Runoff-Second-Interim-Report-of-Social-MediaMonitoring

Hentschel U., Sumbadze N., Sadzaglishvili S, Mamulashvili M., Ulumberashvili S. (1996). Defensive and affective-emotional reactions to war: the Abkhazian war as reflected in people's subjective reactions. Psychological reports, Vol.78, issue 1, pp. 134-143.

Jones, S. (2012). Georgia: the political history since independence. London New York: I.B. Tauris.

Kølvraa, C. (2018). Psychoanalyzing Europe? Political Enjoyment and European Identity. Political Psychology, Vol. 39, No.6, pp. 1405-1418.

Marcus G.E. (2003). The psychology of emotion and politics. In D.O. Sears, L. Huddy. R. Jervis (Eds.), Oxford handbook of political psychology. (pp. 182-221). Oxford: Oxford University Press.

Freud, S. (1930). Civilization and its discontents. The standard edition of the complete psychological works of Sigmund Freud. Volume XX1. pp. 59-145. London: Hogarth

Fromm, E. (1978). Sigmund Freud's mission: an analysis of his personality and influence. Gloucester: Peter Smith.

Frosh, S. (2013). Hauntings: psychoanalysis and ghostly transmissions. London: Palgrave.

Frosh, S. (2012). A brief introduction to psychoanalytic theory. London: Palgrave.

OSCE ODIHR. (2019). Georgia presidential elections 28 October 28 November 2019. ODIHR Election observation final report. Warsaw: Office for Democratic Institution of Human Rights. Retrieved February 7, 2019, from https://www.osce.org/odihr/elections/georgia/412724?download=true

Paul, R. (1987). The question of applied psychoanalysis and the interpretations of cultural symbolism. Ethos Vol. 15, N. 1, pp. 82-103.

Paul, R. (1989). Psychoanalytic anthropology. Annual Review of Anthropology, Vol.18, pp.177-202.

Redlawsk, D.P., Lau R.R. (2013). Behavioral decision-making. In L Huddy, D.O. Sears, J.S Levy (Eds.), Oxford handbook of political psychology. (pp. 130-164). Oxford: Oxford University Press.

Reyfield, D. (2012). Edge of empires: a history of Georgia. London: Reaktion Books.

Stavrakakis, Y. (2007). The Lacanian left: Psychoanalysis, theory, politics. Edinburgh, Scotland: Edinburgh University Press.

Zizek, S. (2005). Interrogatin the real. London, United Kingdom: Continuum 

* POSTERS *

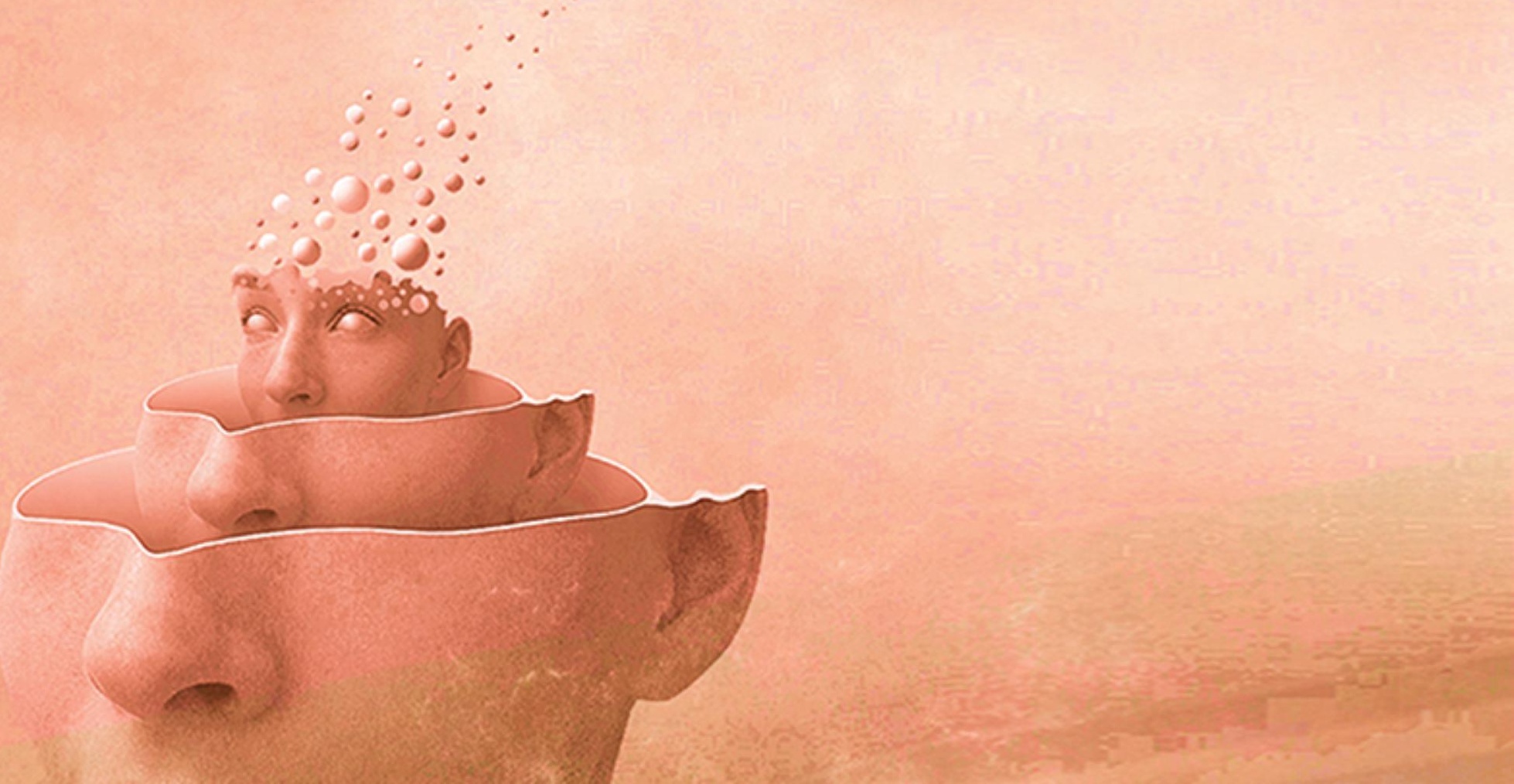





\title{
A LONGITUDINAL STUDY OF SCHOOL SATISFACTION -MENTAL HEALTH AND SOCIAL SKILLS UPON COLLEGE ADMISSION-
}

\author{
Yuko Ikui \\ Faculty of Humanities, Seisen Jogakuin College (Japan)
}

\begin{abstract}
The university entrance rate in Japan has been increasing over decades. Since 2005, it has finally exceeded fifty percent of high school graduates (Katase, 2007). As the entrance rate has gone up, the number of students who suffers from university maladjustment has also been increasing (Yamada, 2006). In this research, the author examined the possibility of using satisfaction with school life as an indicator to prevent such maladjustment, and conducted a two-year longitudinal study on female college students (four-year college and two-year college). The state of mental health and social skills was examined just after the commencement of the semester in April, and satisfaction with school life (motivation for school life; approval; maladjustment) was examined in the same students three months later in July, and a year later in July.

Based on the results of a $t$-test between 19 students who were in a poor mental health state upon admission and the remaining 162 students, significant statistical differences were found in satisfaction levels in the sophomore year between two groups (Motivation/ Approval $p<.01$; Maladjustment $p<.05$ ). Moreover, among the 19 students with poor mental health, there was no significant change in school satisfaction from freshman to sophomore, although 5 students with relatively higher social skills showed a lower score of Maladjustment $(\mathrm{p}<.05)$ than the other students. Even if students were at risk of poor mental health upon admission, it seems to be safe to assume that acquisition of social skills, possibly through institutional support, will be helpful to increase their resistance to anticipated maladjustment.
\end{abstract}

Keywords: College student, college adjustment, prevention of maladjustment, mental health, social skills.

\section{Introduction}

The university entrance rate in Japan has been increasing over decades. In 2005, the university entrance rate exceeded 50\% of high school graduates (Katase, 2007). As the entrance rate increased, the number of students entering university involuntarily and suffering from university maladjustment also increased (Yamada, 2006). To prevent university maladjustment or drop out, the University Personality Inventory (UPI) was developed in Japan (1966). The UPI is used to check the states of mental health of freshmen in select universities and colleges in Japan (Oka et al., 2015).

Musashi, et al (2013) investigated school satisfaction (Kawamura, 2010), an indicator of students' senses of adjustment to university and mental health. The study revealed a relationship between a low school satisfaction score and the tendency to suffer from depression and social anxiety. Ikui (2018) further investigated the influence of mental health and social skills on university and college admission and on school satisfaction. According to the results, the tendency to suffer from depression and the level of social skills affected the school satisfaction score and motivation to engage in school life after three months. Students whose state of mental health was at risk (i.e. UPI high group) demonstrated a tendency towards a lower motivation to build relationships with new friends and classmates than the other group.

\section{Objectives}

The purpose of this research is to further examine the influence of mental health states and social skills at admission on school adjustment by conducting a longitudinal study. The transition of students'school satisfaction and motivation for school life from freshman to sophomore is investigated through a sample from a college for female students. It will provide a proposal for the support system of a university to relieve maladjustment, especially for students with mental health problems.

\section{Methods}

\subsection{Survey period and participants}

As the first phase of the research, the author implemented the UPI questionnaire (Japan University Health Association, 1966) and KiSS-18 (Kikuchi, 1988/2004) to 239 freshmen during 
the orientation session in April 2017, and 220 students agreed to submit the informed consent form and participate in the research. The second phase of the research was conducted 3 months later, in July 2017, and 237 freshmen took Hyper-QU for university (Kawamura, 2014). After 1 year, the same students completed the second Hyper-QU in July 2018. The informed consent form was handed out to students again. Data from the 180 students who completed all the questionnaires and agreed to participate in the research were used for the analysis.

\subsection{Measurement}

UPI (University Personality Inventory, 1966): The questionnaire comprises 60 items and five lower factors: i) Subjective Symptoms (16 items), ii) Depression (20 items), iii) Social Anxiety (10 items), iv) Obsession (10 items) and v) Positive emotion (4 items). Choose (Yes or No). If the sum score of i) -iv) is greater than 30 , the state of mental health is assessed as high risk and should be given the appropriate support.

KiSS-18 (Kikuchi's Scale of Social skills, 1988/2004): Questionnaire with 18 items that measure the overall social skills for a smooth interpersonal relationship. 5-point Likert Scale.

Hyper-QU (2010: Standardized test comprising three scales-School Satisfaction, Motivation for School Life and Social Skills-measured on a 5-point Likert Scale. In this research, only School Satisfaction and Motivation scale were used for the analysis.

School Satisfaction: This scale comprises 30 items and two lower factors; Approval (how one's existence or behavior is approved by others; 15 items) and Maladjustment (how one experience the sense of maladjustment; 15 items).

Motivation for School Life: Scale comprises 30 items and six lower factors: Relationship with Friend (5 items), Desire to learn (5 items), Relationship with Faculty/Staff (5 items), Support System of College (5 items), Career Consciousness (5 items) and Relationship with Classmate (5 items). In this research, only the total score of this scale was used for the analysis.

\section{Results}

Table1 shows the means and standard deviations of UPI/KiSS-18, which was investigated in April 2017.

Table 1. Dean and SD of UPI (Total/UPI high/low Group) and KISS-18(Total).

\begin{tabular}{|c|c|c|c|c|c|c|c|c|c|}
\hline \multirow{2}{*}{ Scale } & \multirow{2}{*}{\multicolumn{3}{|c|}{ Factor }} & \multicolumn{2}{|c|}{ Total $(N=180)$} & \multicolumn{2}{|c|}{ UPI high G $(N=19)$} & \multicolumn{2}{|c|}{ UPI low $G \quad N=161)$} \\
\hline & & & & $M$ & $S D$ & $M$ & $S D$ & $M$ & $S D$ \\
\hline \multirow[t]{6}{*}{$\overline{\text { UPI }}$} & Total & Mental Health) & & 14.7 & 10.58 & 34.9 & 4.85 & 12.3 & 8.09 \\
\hline & & (1) Physical/Meı & bjective Symptoms & 3.6 & 3.06 & 8.5 & 2.54 & 3.1 & 2.59 \\
\hline & & & (2) Depression & 5.7 & 4.51 & 13.6 & 2.70 & 4.7 & 3.51 \\
\hline & & & (3) Social Anxiety & 3.1 & 2.61 & 7.3 & 1.72 & 2.7 & 2.16 \\
\hline & & & (4) Obsession & 2.6 & 2.41 & 6.4 & 1.80 & 2.1 & 2.05 \\
\hline & & & *(5) Positive Items & 1.7 & 1.28 & 0.9 & 1.00 & 1.8 & 1.28 \\
\hline KiSS-18 & Total & \$ocial Skill) & & 56.9 & 11.07 & & & & \\
\hline
\end{tabular}

The cut-off point of the total UPI score is 30. Table1 indicates that all scores in UPI high group are twice as high as that of the UPI low group. To compare school satisfaction scores and motivation for school life in 2017 and in 2018, a paired t test was conducted for the total $(N=180)$, UPI high group $(N=19)$ and UPI low group $(N=161)$. Table2 represents the findings.

Table 2. Mean, SD and t-test score of School Satisfaction Scale and Motivation for School Life Scale for two years.

\begin{tabular}{|c|c|c|c|c|c|c|c|c|c|c|}
\hline \multirow[b]{2}{*}{ Scale } & \multirow[b]{2}{*}{ Factor } & \multicolumn{3}{|c|}{ Total $(N=180)$} & \multicolumn{3}{|c|}{ UPI high G $(N=19)$} & \multicolumn{3}{|c|}{ UPI low $\mathrm{G}(N=161)$} \\
\hline & & $\begin{array}{c}2017 \\
M(S D)\end{array}$ & $\begin{array}{c}2018 \\
M(S D)\end{array}$ & $t$ & $\begin{array}{c}2017 \\
M(S D)\end{array}$ & $\begin{array}{c}2018 \\
M(S D)\end{array}$ & $t$ & $\begin{array}{c}2017 \\
M(S D)\end{array}$ & $\begin{array}{c}2018 \\
M(S D)\end{array}$ & $t$ \\
\hline \multirow{2}{*}{ School Satisfaction } & Approval & $\begin{array}{c}53.0 \\
(8.41)\end{array}$ & $\begin{array}{c}54.5 \\
(9.62)\end{array}$ & $-2.708 * *$ & $\begin{array}{c}48.3 \\
(11.14)\end{array}$ & $\begin{array}{c}53.6 \\
(8.09)\end{array}$ & 0.204 & $\begin{array}{c}53.4 \\
(8.19)\end{array}$ & $\begin{array}{c}55.3 \\
(8.99)\end{array}$ & $-3.411 * *$ \\
\hline & Maladaptation & $\begin{array}{c}25.4 \\
(8.17)\end{array}$ & $\begin{array}{c}25.1 \\
(8.35)\end{array}$ & 0.518 & $\begin{array}{c}31.3 \\
(9.01)\end{array}$ & $\begin{array}{c}24.5 \\
(7.32)\end{array}$ & 0.614 & $\begin{array}{c}24.9 \\
(7.84)\end{array}$ & $\begin{array}{c}24.6 \\
(8.06)\end{array}$ & 0.403 \\
\hline \multicolumn{2}{|c|}{ Motivation for School Life } & $\begin{array}{c}119.7 \\
(15.90)\end{array}$ & $\begin{array}{c}122.3 \\
(16.72)\end{array}$ & $-2.359 *$ & $\begin{array}{c}111.4 \\
(22.05)\end{array}$ & $\begin{array}{c}120.1 \\
(14.96)\end{array}$ & 0.470 & $\begin{array}{c}120.5 \\
(15.19)\end{array}$ & $\begin{array}{c}123.5 \\
(15.39)\end{array}$ & $-2.796 * *$ \\
\hline
\end{tabular}

The result of the $t$-test reveals significant differences between the scores of approval and motivation for school life in the total group and the UPI low group. This result means that a student who has no obvious mental problem eventually adjusts to school life better as a sophomore than a freshman. 
Among the UPI high group, however, no significant differences are observed in all three factors. Because of the small number of participants, the observed changes of adjustment seem to be the result of the individual differences.

Additionally, students were further divided into two groups - the high social skills group and low social skills group-according to the mean (56.9) of KiSS-18. An independent t test was conducted to compare the high social skill group and low social skill group among each of the UPI high and low groups. The compared scores were school satisfaction (approval and maladaptation) and motivation for school life in the second year. Table3 presents the representations.

Table 3. Mean, SD and T-test Score of Second Year School Satisfaction and Motivation for School Life by Groups.

\begin{tabular}{|c|c|c|c|c|c|c|c|}
\hline \multirow{3}{*}{ Scale } & \multirow{3}{*}{ Factor } & \multicolumn{3}{|c|}{ UPI high $\mathrm{G}(N=19)$} & \multicolumn{3}{|c|}{ UPI low $\mathrm{G}(N=161)$} \\
\hline & & $\begin{array}{c}\mathrm{K}-18 \text { high } \mathrm{G} \\
\quad(N=5)\end{array}$ & $\begin{array}{c}\mathrm{K}-18 \text { low } \mathrm{G} \\
(N=14)\end{array}$ & $t$ & $\begin{array}{l}\text { K-18 high } \mathrm{G} \\
\quad(N=89)\end{array}$ & $\begin{array}{c}\mathrm{K}-18 \text { low } \mathrm{G} \\
\quad(N=72)\end{array}$ & $t$ \\
\hline & & $M(S D)$ & $M(S D)$ & & $M(S D)$ & $M(S D)$ & \\
\hline \multirow{2}{*}{ School Satisfaction } & Approval & $52.2(19.01)$ & $46.1(9.44)$ & 0.939 & $57.8(7.91)$ & $52.1(9.29)$ & $4.188^{* *}$ \\
\hline & Maladaptation & $23.0(6.48)$ & $31.9(9.83)$ & $-1.872 \dagger$ & $22.9(7.17)$ & $26.7(8.62)$ & $-3.085 * *$ \\
\hline \multicolumn{2}{|c|}{ Motivation for School Life } & $118.4(34.22)$ & $108.9(18.57)$ & 0.789 & $126.1(14.91)$ & $119.7(15.90)$ & $2.612^{*}$ \\
\hline
\end{tabular}

In UPI low group, significant differences are observed between the high social skill group and low social skill group for approval, maladaptation and motivation for school life. Even among the healthy students, students with higher compared with lower social skills naturally adjust to school life after 1 year. In UPI high group, the only significant difference is at maladaptation. Using the skill to build the connection with their supporter (i.e. friends, classmates or teachers), the students who had mental health problems and high social skills at admission could prevent maladaptation.

\section{Conclusions}

The results of this study indicate that the students with mental health problems at admission showed low school satisfaction after 3 months. This tendency continued in the second year. Among this group, however, the maladaptation score of the students who have relatively high social skills decreased compared with the students with low social skills. Thus, a safe assumption is that having higher social skills naturally allows these students to, for example, connect with individuals, rely on others as necessary and share their weakness. Although these students may not be able to feel fully satisfied with or have a sufficient amount of motivation to engage in school life, they might at least be able to feel accepted into the school community.

The methodological limitation of this study is the insufficiency of participants, especially the at-risk freshmen who joined this longitudinal study. Additionally, because this research was conducted at a college for female students, the participants were naturally limited. To increase the number of participants, further research will be conducted widely among various colleges and universities.

\section{References}

Ikui, Y. (2018) The effect of mental health and social skills on satisfaction with school life. Proceedings of the International Psychological Applications Conference and Trends 2018.

Katase, K (2007) The sufferings of university professors in universal era: in the case of Tohoku Gakuin University. Tohoku Gakuin University Educational Research Institute Bulletin. 7, 5-40.

Kawamura, S. (2010) Hyper-QU for university. Toshobunka Company.

Kikuchi, A (1988/2004) Notes on the Researchers Using KiSS-18. Bulletin of the Faculty of Social Welfare, Iwate Prefectual University, 6(2), 41-51.

Japan University Mental Health Association (1966) The handbook of University Personality Inventory. Sozo Publishing.

Musashi, Y., Yamamoto, Y., Shinada, E., Kawamura, S. (2013) The relationship between the satisfaction with school life and the mental health of undergraduates. Japanese Journal of Counselling Science, 45(3), 165-174.

Oka, I., Yoshimura, M., Yamagishi, T. (2015) Forty three years of changes in mental health of Tsuda College freshmen: Results from University Personality Inventory. Tsuda College Departmental Bulletin, 47, 175-195.

Yamada, Y.(2006) Investigation of freshman's adjustment to college life. Journal of Nagoya Bunri University, 6, 29-36. 


\title{
RELATIONSHIP BETWEEN MINDFULNESS, SELF-COMPASSION AND DIFFICULTIES IN EMOTION REGULATION IN ADDICTS
}

\author{
Tânia Caetano, Eduardo Ramadas, \& Jessica Lopes \\ Department or Research and Development, VillaRamadas International Treatment Centre (Portugal)
}

\begin{abstract}
Difficulties in emotion regulation is one of the main characteristics of addictive disorders. Both mindfulness and self-compassion have been offered as possible alternative strategies of emotion regulation and have also been found to be connected.

Our objective was to understand how predisposition to be mindful (to be in the present moment), self-compassion and difficulties in emotion regulation relate to each other in a sample of addicts.

The present study was exploratory and has a cross-sectional design. Current results relate to 17 patients who were receiving treatment in VillaRamadas treatment center for addiction. The psychological measures used were: Mindful Attention and Awareness Scale (MAAS), Self-Compassion Scale (SCS) and Difficulties in Emotion Regulation Scale (DERS).

From the 17 participants, 13 were men. Age varied from 19 to 64 years $(M=33, S D=10.13)$. MAAS presented strong negative association with self-judgment and all its subscales, and a moderate negative association with the DERS total score. It did not show any significant association with self-compassion or any of its subscales.

The DERS total score showed strong positive associations with both the total score of self-judgment, and the subscales self-criticism and over-identification and a moderate association with isolation. It also presented a moderate negative association with self-compassion, and strong negative associations with the subscales self-kindness and mindfulness.

MAAS was a significant predictor of self-judgment, explaining $50.9 \%$ of its variability. Both self-compassion and self-judgment were significant predictors of DERS, explaining $27.4 \%$ and $55.3 \%$ of its variance, respectively.
\end{abstract}

Keywords: Mindfulness, self-judgment, self-compassion, emotion regulation, addiction.

\section{Introduction}

Mindfulness and self-compassion are constructs that frequently appear connected as important predictors of psychological health (e.g. Keng, Smoski, \& Robins, 2011; MacBeth, \& Gumley, 2012), and are growing in popularity as emerging strategies to be integrated in clinical practice. Both have been proposed as emotion regulation strategies (e.g. Tang, Hölzel, \& Posner, 2015; Berking, \& Whitley, 2014) that can be particularly useful for disorders characterized by a deficit related skills, such as addiction disorders.

Our objective with this small exploratory study was to explore the relationship between predisposition to mindfulness, self-compassion (and self-judgment) and difficulties in emotion regulation, in a clinical sample of addicts receiving treatment.

\section{Methods}

\subsection{Participants}

The sample was composed of 17 Portuguese patients, receiving treatment for an addiction disorder at VillaRamadas International Treatment Centre throughout December of 2018 and January of 2019.

From the 17 patients, 13 were male and 4 were female, and age varied between 19 and 64 years $(M=33, S D=10.13)$. All the patients had been diagnosed at admission into treatment, either with a substance use disorder or with an impulsive disorder (behavioral addiction).

\subsection{Measurements}

Mindfulness Attention and Awareness Scale (MAAS; Brown \& Ryan, 2003; Portuguese translation and adaptation by Pinto Gouveia, \& Gregório, 2013), is a 15-item self-report measure of a core 
characteristic of dispositional mindfulness, which is open awareness and attention of the present moment. The items are scored on a 6-point Likert scale from 1 ("almost always") to 6 ("almost never").

Self-Compassion Scale (SCS; Neff, 2003; Portuguese version by Castilho, Pinto-Gouveia, \& Duarte, 2015), is a set of two scales, self-compassion and self-judgment, which score is composed by the added scores of three subscales each. The subscales for the self-compassion scale are Self-Kindness, Common Humanity and Mindfulness; for the self-judgment scale are Self-Criticism, Isolation and Over-identification. All the items are scored on a 5-point Likert scale from 1 ("almost never") to 5 ("almost always").

Difficulties in Emotion Regulation Scale (DERS; Gratz, \& Roemer, 2004; Portuguese version by Veloso, Pinto-Gouveia, \& Dinis, 2011), is a self-report instrument that can calculate scores for six subscales and a total score. The 36 items that compose the scale are scored on a 5-point Likert scale from 1 ("almost never") to 5 ("almost always"). For the present study, only the total score was considered. Higher scores indicate greater emotion regulation difficulties.

\section{Results}

\subsection{Descriptive statistics}

Years of education ranged from 10 to 16 years, with a mean of 13.47 years $(S D=1.84)$.

The MAAS total score mean result was of 56.06, with $S D=11.09$.

From the SCS, the Self-Compassion total score presented a mean result of $35.12(S D=10.15)$, with a mean result of $12.76(S D=4.51)$ for Self-Kindness, of $11.47(S D=3.71)$ for Common Humanity, and of $10.88(S D=3.12)$ for Mindfulness. The Self-judgment total score presented a mean result of 39.88 $(S D=10.84)$, with a mean result of $15.88(\mathrm{SD}=4.58)$ for Self-Criticism, of $11.29(\mathrm{SD}=3.46)$ for Isolation, and of $12.71(\mathrm{SD}=3.72)$ for Over-identification.

Finally, the DERS total score had a mean result of 94.18, with a $S D=27.11$.

\subsection{Correlation values between the studied variables (Spearman's correlation)}

Age showed a moderate positive association with both years of education and the mindfulness subscale of self-compassion.

MAAS presented strong negative association with self-judgment and all its subscales, and a moderate negative association with the DERS total score. It did not show any significant association with self-compassion or any of its subscales.

The DERS total score showed strong positive associations with both the total score of self-judgment, and the subscales self-criticism and over-identification and a moderate association with isolation. It also presented a moderate negative association with self-compassion, and strong negative associations with the subscales self-kindness and mindfulness.

The complete results are presented in Table 1.

Table 1. Correlation values between the studied variables.

\begin{tabular}{|c|c|c|c|c|c|c|c|c|c|c|c|c|}
\hline & 1 & 2 & 3 & 4 & 5 & 6 & 7 & 8 & 9 & 10 & 11 & 12 \\
\hline 1. Age & - & & & & & & & & & & & \\
\hline $\begin{array}{l}\text { 2. Years of } \\
\text { Education }\end{array}$ & $.510^{*}$ & - & & & & & & & & & & \\
\hline $\begin{array}{l}\text { 3. MAAS } \\
\text { SCS }\end{array}$ & .438 & .112 & - & & & & & & & & & \\
\hline $\begin{array}{l}\text { 4. Self- } \\
\text { Kindness }\end{array}$ & .331 & .059 & .201 & - & & & & & & & & \\
\hline $\begin{array}{l}\text { 5. Common } \\
\text { Humanity }\end{array}$ & -.116 & -.032 & .158 & $.608^{* *}$ & - & & & & & & & \\
\hline $\begin{array}{l}6 . \\
\text { Mindfulness }\end{array}$ & $.483^{*}$ & .047 & .301 & $.743^{* *}$ & $.546^{*}$ & - & & & & & & \\
\hline $\begin{array}{l}\text { 7. Self- } \\
\text { Compassion } \\
\text { Total }\end{array}$ & .315 & .102 & .203 & $.930^{* *}$ & .750 ** & $.849^{* *}$ & - & & & & & \\
\hline $\begin{array}{l}\text { 8. Self- } \\
\text { Criticism }\end{array}$ & -.427 & .183 & $-.651^{* *}$ & $-.671^{* *}$ & -.347 & $-.644^{* *}$ & $-.600^{*}$ & - & & & & \\
\hline $\begin{array}{l}\text { 9. Isolation } \\
\text { 10. Over- }\end{array}$ & -.093 & .250 & $-.668^{* *}$ & -.342 & -.414 & -.304 & -.399 & $.610^{* *}$ & - & & & \\
\hline identificatio & -.265 & .235 & $-.662^{* *}$ & $-.637^{* *}$ & -.353 & $-.603^{*}$ & $-.557^{*}$ & $.954^{* *}$ & $.603^{*}$ & - & & \\
\hline $\begin{array}{l}\text { 11. Self- } \\
\text { judgment } \\
\text { Total }\end{array}$ & -.326 & .162 & $-.750^{* *}$ & $-.639^{* *}$ & -.422 & $-.583^{*}$ & $-.598^{*}$ & $.950^{* *}$ & $.797^{* *}$ & $.943^{* *}$ & - & \\
\hline 12. DERS & -.385 & .038 & $-.568^{*}$ & $-.648^{* * *}$ & -.210 & $-.603^{*}$ & $-.564^{*}$ & $.850^{* *}$ & $.491 *$ & $.803^{* *}$ & $.815^{* *}$ & - \\
\hline
\end{tabular}

Note. Values in bold indicate a statistically significant association between the respective variables. Significance level: $*=\mathrm{p}<.05 ;$ $* *=\mathrm{p}<.01 ; * * *=\mathrm{p}<.001$. 


\subsection{Linear regression}

Three simple regression analyses were conducted, the first one with MAAS as a possible predictor of self-judgment, and the second and third with self-judgment and self-compassion as predictors of difficulties in emotion regulation, respectively.

MAAS was a significant predictor of self-judgment $(\mathrm{F}(1,15)=17.617, p=.001)$ with an adjusted $R^{2}$ of .509 . Both self-compassion $(\mathrm{F}(1,15)=7.053, p=.018)$ and self-judgment $(\mathrm{F}(1,15)=20.783$, $p<.001)$ were significant predictors of DERS, with adjusted $R^{2}$ of .274 and .553 respectively.

\section{Discussion}

Our results indicate that predisposition to be mindful is strongly associated with and a significant predictor of self-judgment in addicts, but not with self-compassion. Given the strong association between self-judgment and difficulties in emotion regulation, and taking into consideration its importance in addiction, this result could partially explain the success of mindfulness-based programs for addiction.

Given the very small sample size, the results have to be interpreted with extreme caution. Although mindfulness was not a predictor of difficulties in emotion regulation in this study, future research could explore this potential relationship and, if found, the possible mediation effect of self-judgment.

\section{References}

Berking, M., \& Whitley, B. (2014). Affection regulation training: A practitioner's manual. New York: Busines Media. doi: 10.1007/978-1-4939-1022-9_ 1.

Brown, K.W. \& Ryan, R.M. (2003). The benefits of being present: Mindfulness and its role in psychological well-being. Journal of Personality and Social Psychology, 84, 822-848. doi: 10.1037/0022-3514.84.4.822

Castilho, P., Pinto-Gouveia, J., \& Duarte, J., (2015). Evaluating the Multifactor Structure of the Long and Short Versions of the Self-Compassion Scale in a Clinical Sample. Journal of Clinical Psychology, 71(9), 856-70. doi: 10.1002/jclp.22187

Gratz K. L., \& Roemer L. (2004). Multidimensional assessment of emotion regulation and dysregulation: development, factor structure, and initial validation of the difficulties in emotion regulation scale. J. Psychopathol. Behav. Assess. 26, 41-54. doi: 10.1007/s10862-008-9102-4

Gregório, S., \& Pinto-Gouveia, J. (2013). Mindful attention and awareness: relationships with psychopathology and emotion regulation. The Spanish Journal of Psychology, 16. doi: 10.1017/sjp.2013.79.

Keng, S. L., Smoski, M. J., \& Robins, C. J. (2011). Effects of mindfulness on psychological health: a review of empirical studies. Clinical Psychology Review, 31, 1041-1056. doi:10.1016/ j.cpr.2011.04.006.

MacBeth, A., \& Gumley, A. (2012). Exploring compassion: a metaanalysis of the association between self-compassion and psychopathology. Clinical Psychology Review, 32, 545-552. doi:10.1016/j.cpr.2012.06.003.

Neff, K. D. (2003). Development and validation of a scale to measure self-compassion. Self and Identity, 2, 223-250. doi: 10.1080/15298860390209035

Tang, Y., Hölzel, B. K., \& Posner, M. I. (2015). The neuroscience of mindfulness meditation. Nat. Rev. Neurosci. 16, 213-225. doi: 10.1038/nrn3916

Veloso, M., Pinto-Gouveia, J., \& Dinis, A. (2011). Estudos de validação com a versão portuguesa da Escala de Dificuldades na Regulação Emocional (EDRE). Psychologica, 54, 87-110. 


\title{
RE-CONNECTING ONESELF TO THE WORLD OF PEERS VIA THE CONTEMPLATION OF AN ARTWORK
}

\author{
Laurence Bertron-Gatier, \& Célia Vaz-Cerniglia \\ The Catholic University of Lyon (France)
}

\begin{abstract}
Some teenagers have no more confidence to exchange with each other and with adults through words. Not desire can enroll in the relation because it has become suspicious; or even defying due to psychic and emotional pain. This suffering is trying to be hidden because is even hardly accessible, intelligible to their conscience. They are in a vulnerable period and some of them are closing them in on themselves.

These teenagers, who lock themselves certainly to looking away, might catch their gaze in a pictorial scene. This scene included in a frame containing an art production which is given to see, that invites contemplation without risk of intrusion. It would provide, without doubt, a space to rush, or even to take refuge there. It would be a landscape that would allow these teenagers to flee in a space deeper and deeper, echoing the depth of their physical and emotional proven. Maybe in this place at once more and less distanced, these young teens might find a correct distance, more adjusted; they can develop more openness and further more deploy their senses.

By sense, we mean both the sensory modalities, the sense of direction and meaning.

Take the time to let yourself be surprised by an artist's painting which has been chosen, be able to choose it to contemplate in his own way, in a small room would promote an authentic encounter between the teenager and the artist via the painting. his intertwining of spaces and double projections and transfers build an environment sufficiently containing. It invites to daydreaming, poetry and a great sense of wonder offsetting an environment of the depression and destruction.

This moment of intimate and authentic meeting would promote the deployment of self-confidence and in each other. Effectively, the young teenager feels intimately known by this other who expresses through his artwork. Thus, the identification process is initiated.

Thus, the aim of this oral paper is to emphasize the importance of a device such as the Artothèque to accompany early adolescents who are in down development and have difficulty in communication and relationship with the other.

Some clinical cases will be presented to illustrate the authentic encounter between the young and the artwork at one point and thereafter.
\end{abstract}

Keywords: Artothèque, artwork, wonderment, the encounter with the other, senses.

\section{Problematic}

How can we render the meeting between an adolescent in a precarious relational situation with the work of an artist in an authentic and reciprocal manner? Convoked in new feelings, how can they transform their defensive acts into symbolised objects? We are going to attempt to capture the process of this psychic task, with a possible challenge concerning the restauration of the narcissism and a "re-connection" to the world.

\section{Hypothesis}

The contemplation of an artwork, a veritable landscape, allows certain adolescents in crisis points to find refuge. Without a fear of being seen or judged in this more isolated and deep space, they can preserve their sense of privacy, which also echoes the depth of their corporal and emotional feelings. 


\section{Framework}

Public: adolescents in relational crises concerning the familial, social and scholarly domains, in a space named the "Resource area" (day centres, childhood protection, and legal youth protection).

Art Library: Contemplating an ensemble of artworks in a public space and then choosing one that the adolescent will bring and present in the Resource Area.

\section{Results}

Certain adolescents are emotionally touched by the contemplation of an artwork in situ. The moving of the chosen artwork, when the adolescent actually puts the piece in his hands in front of everyone, is accompanied by a psychic movement that operates in reciprocity (the convergence between the psychic world of the artist and that of the adolescent), (Guillaumin, 1998).

A psychic and physical movement is initiated following a sudden emotional experience. Certain people begin to feel once again. Working from the artwork that seems to protect the adolescent almost as if being a new containing envelope, they begin to experiment more and more with their fixed and deeply buried experiences.

Their feelings are anchored in either the intention of the artist (painting by V. Velickovic, "Experience", 1974), or the artist themselves (H. Michaux and painting, no title, 1949), and sometimes in both. For the first, the teenager can, via the painting makes himself the experience of an intrusion like a dissection. For the second, he can feel and understand how cannabis enables him to hide and put out his depression.

\section{Discussion}

If there is a convergence between the adolescent and the artwork, this would invoke a psychic space that liberates itself from real time while driving a subjective appropriation of experiences and feelings, which are sometimes traumatic for certain people. This thereby participates in narcissistic restauration through the introjection of the beauty or strangeness perceived within the process of identification to an artist or his artwork. The adolescents are eventually able to find themselves, in part, in the artist's sublimated reality. This encounter offers the opportunity to distance oneself from the destructive movements (Winnicott, 1994) that animated them until that point, in order to open and re-connect themselves to a shared sensitivity. These meetings, surprising due to their unexpected nature, have a strong re-narcissising effect for certain people, because their sensitivity is then socially legitimised in their eyes (Chouvier, 2002).

\section{Conclusion}

The contemplation of an artwork that has captured their attention, an artwork that is both a pictorial representation of another's psychic space and as well as an opportune space for their own projections, these adolescents are then enabled to find the right distance between what the artwork invokes (intentionality of the artist) and what they themselves perceive and feel. The aesthetic implies that a process of transformation is at work, rather than displacement, concerning that which was until that point inexpressible. It initiates an inter and intra psychic movement within the adolescent that is ideal for the task of symbolization.

\section{References}

Chouvier, B. (2002). Les processus psychiques de la médiation. Paris: Dunod.

Guillaumin, J. (1998). Le jugement esthétique, un instrument logique étrange entre l'intime et l'universel. In B. Chouvier \& al. (Eds.), Symbolisation et processus de création: Sens de l'intime et travail de l'universel dans l'art et la psychanalyse (Inconscient et culture) (pp. 35-56). Paris: Dunod.

Winnicott, D.W., (1994). La tendance antisociale. In D.W. Winicott (Ed.), déprivation et délinquance (pp. 145-158). Paris: Payot (article original publié en 1956) 


\title{
MOTIVATION AND EMOTIONS IN EFFECTIVE EATING HABITS CHANGE. MULTI-METHOD EXPLORATORY STUDY
}

\author{
Grażyna Wąsowicz ${ }^{1}$, Magdalena Poraj-Weder ${ }^{1}, \&$ Christophe Matthys ${ }^{2,3}$ \\ ${ }^{I}$ Faculty of Psychology, University of Warsaw, Warsaw (Poland) \\ ${ }^{2}$ Nutrition \& Obesity, Clinical and Experimental Endocrinology, Department of Chronic Diseases, \\ Metabolism and Aging, KU Leuven (Belgium) \\ ${ }^{3}$ Clinical Nutrition, Department of Endocrinology, University Hospitals Leuven (Belgium)
}

\begin{abstract}
Many studies have shown that attempts to change one's behaviour often end in failure. Old habits reassert themselves and new attempts to alter them are necessary, leading to frustration and disappointment.

The aims of the study were to explore within one theoretical framework the importance of motivation and emotion in the process of changing eating habits, and to estimate the role of health professionals in the process. The research employed a multi-method approach, and quantitative and qualitative studies were conducted. The main goal of the quantitative study 1 . was to assess the role of health professionals in achieving effective change and to widen the knowledge of psychological (motivational and emotional) determinants of the effectiveness of eating habits change. The purpose of the quantitative study 2 . was to deepen the knowledge acquired in the first study. A total of 420 men and women undergoing the process of change were recruited to participate in the first study. Motivation was measured by Treatment Self-Regulation Questionnaire (Diet) (Ryan \& Connell, 1989; Levesque, Williams, Elliot, Pickering, Bodenhamer et al., 2007), emotions experienced in the process were assessed by Scale of Positive and Negative Experience (Diener \& Biswas-Diener, 2009) and emotional attitude towards a process of change - by Scale on Emotions Related to the Behaviour Change, created for the purpose of the study. Three measures of declared effectiveness of diet change were applied: objective (change in weight in kilograms) and subjective (evaluation of dietary change into more healthful food consumption and satisfaction with self-efficacy in the process). Study 2. was conducted using individual, semi-structured, in-depth interviews (IDIs) (Angrosino, 2010; Kvale, 2010). Two groups of respondents were examined: 6 individuals changing their eating habits and 7 health professionals. The thematic analysis (TA; Braun \& Clark, 2006; Clark et al., 2015) deductive method was used to analyze collected data. Results from both studies show that emotional attitudes toward dietary changes and emotional experiences during the process of change - neglected thus far in empirical studies - are crucial for the effectiveness of the process. Moreover, the emotional aspects modify the relationship between different types of motivation (as defined within the Self-Determination Theory) and the effectiveness of the process.
\end{abstract}

Keywords: Weight loss, motivation, emotions, health professionals, patients, self-determination theory. 


\title{
COMBINATION OF ATTACHMENT PATTERNS IN ROMANTIC RELATIONSHIP AND ITS QUALITY
}

\author{
Dariusz Kuncewicz ${ }^{1}$, \& Dorota Kuncewicz ${ }^{2}$ \\ ${ }^{1}$ Department of Clinical Psychology, University of Social Sciences and Humanities (Poland) \\ ${ }^{2}$ Department of Psychology, The John Paul II Catholic University of Lublin (Poland)
}

\begin{abstract}
The aim of this study was to explore the connection between a combination of attachment patterns in a couple and their relationship`s quality. The study involved 200 heterosexual couples $(N=400)$, aged 21 to 45 with the relationship's length from 0,5 to 25 years. We used Experiences in Close Relationships questionnaire and cluster analysis ( $k$-means procedure) to isolate three main attachment patterns: secure, anxious, avoidant. We also referred to Couple Satisfaction Index-32 and Partnership Questionnaire to measure different aspects of a relationship`s quality: satisfaction, quarreling, tenderness and mutual communication. The key results of the comparisons between couples, isolated on the basis of a combination of attachment patterns showed that: (1) a combination of two secure patterns was the most while a combination of anxious-avoidant and two avoidant patterns the least beneficial to a relationship s quality; (2) a combination of secure and insecure patterns turned out to be more favourable than a combination of two insecure ones; (3) a configuration of two anxious patterns appeared to be partly beneficial and partly detrimental; (4) a combination of a man with a secure and a woman with an insecure pattern would be more advantageous than a "reversed" combination of a man with an insecure pattern and a woman with a secure pattern.
\end{abstract}

Keywords: Romantic relationship, combination of attachment patterns, self-report measures.

\section{Introduction}

The aim of the study was to check the connection between a combination of attachment patterns in a relationship and its quality. The results of the previous research consistently indicate that a combination of secure attachment patterns favours getting more satisfaction from a relationship than a combination of insecure ones (e.g., Banse, 2004, MacLean, 2002). There is, however, a disagreement over a combination of secure and insecure patterns. The results of some research show that in such a configuration relationship satisfaction is on a level similar to two insecure patterns while other findings suggest the buffering effect of a secure pattern (for a review of the research - see. Mikulincer \& Shaver, 2007). So far the researchers have rarely differentiated one type of insecure pattern from another; nor have they tried a multifaceted approach to a relationship`s quality (they have mainly checked satisfaction in a relationship).

In our study we wanted to check the connection between a combination of attachment patterns in a relationship and its quality in a more comprehensive way. We considered various types of insecure patterns, gender and different dimensions of a relationship`s quality.

\section{Method}

The study involved 200 heterosexual couples $(N=400)$, aged 21 to $45(M=29,37 ; S D=5,35)$ with the relationship's length from 0,5 to 25 years $(M=6,23 ; S D=5,05)$. We used three questionnaires: (1) Experiences in Close Relationships (Brennan, Clark \& Shaver, 1998), including the Anxiety and Avoidance scales (Min = 1, Max = 7); (2) Couple Satisfaction Index-32 (Funk i Rogge, 2007), designed to measure the intensity of Satisfaction in a relationship (Min $=0 ;$ Max $=5$ ), and (3) Partnership Questionnaire (Hahlweg, 1996): including the Quarreling, Tenderness and Mutual communication scales $(\operatorname{Min}=0, \operatorname{Max}=3)$. 


\section{Results}

Cluster analysis ( $k$-means procedure) was performed using the two dimensions, Anxiety and Avoidance of the ECR questionnaire to isolate three main attachment patterns in a group of women and men. In both gender groups, low scores on the Anxiety $(M=3.11,2.57)$ and low on the Avoidance scale $(M=1.71,1.81)$ constituted a secure pattern (in 102 women and 91 men); high scores on the Anxiety $(M=4.83$; 4.33$)$ and low on the Avoidance scale $(M=1.83 ; 1.81)$ - an anxious pattern (in 74 women and 80 men); high on the Avoidance $(\mathrm{M}=3.58 ; 3.44)$ and average on the Anxiety scale $(M=3.88$; 3.02) - an avoidant pattern (in 24 women and 29 men).

Next, we isolated six types of couples, on the basis of a combination of attachment patterns in a relationship, and six subtypes, additionally including the partner's sex (Table 1).

Table 1. Types and subtypes of couples isolated on the basis of a combination of attachment patterns in a relationship.

\begin{tabular}{|c|c|c|c|}
\hline $\begin{array}{l}\text { Type and subtype } \\
\text { of a couple }\end{array}$ & $\begin{array}{l}\text { a combination of attachment patterns } \\
\text { in a relationship }\end{array}$ & $N$ & $\%$ \\
\hline I & secure-secure & 52 & 26 \\
\hline II & secure-anxious & 65 & 32,5 \\
\hline IIa & secure (female)-anxious (male) & 36 & 18 \\
\hline Ilb & anxious (female)-secure (male) & 29 & 14,5 \\
\hline III & secure-avoidant & 24 & 15,5 \\
\hline IIIa & secure (female)-avoidant (male) & 17 & 8,5 \\
\hline IIIb & avoidant (female)-secure (male) & 7 & 7 \\
\hline IV & anxious-anxious & 33 & 16,5 \\
\hline V & anxious-avoidant & 22 & 11 \\
\hline Va & anxious (female)-avoidant (male) & 11 & 5,5 \\
\hline $\mathrm{Vb}$ & avoidant (female)-anxious (male) & 11 & 5,5 \\
\hline VI & avoidant-avoidant & 4 & 2 \\
\hline
\end{tabular}

In order to check whether there is a connection between a type of a couple and different dimensions of their relationship`s quality, the one-way ANOVAs between subjects were conducted. To maintain the optimal size of compared groups, we excluded Type VI (avoidant-avoidant) from our calculations, and randomly selected 30 people from Type I (secure-secure) and Type II (secure-anxious).We found significant effects of a couple`s type in all four dimensions of their relationship`s quality (see Figure 1).

Figure 1. Average intensity of different dimensions of a relationship`s quality versus a type of couples isolated on the basis of a combination of attachment patterns (I, II, III, IV, V, VI).

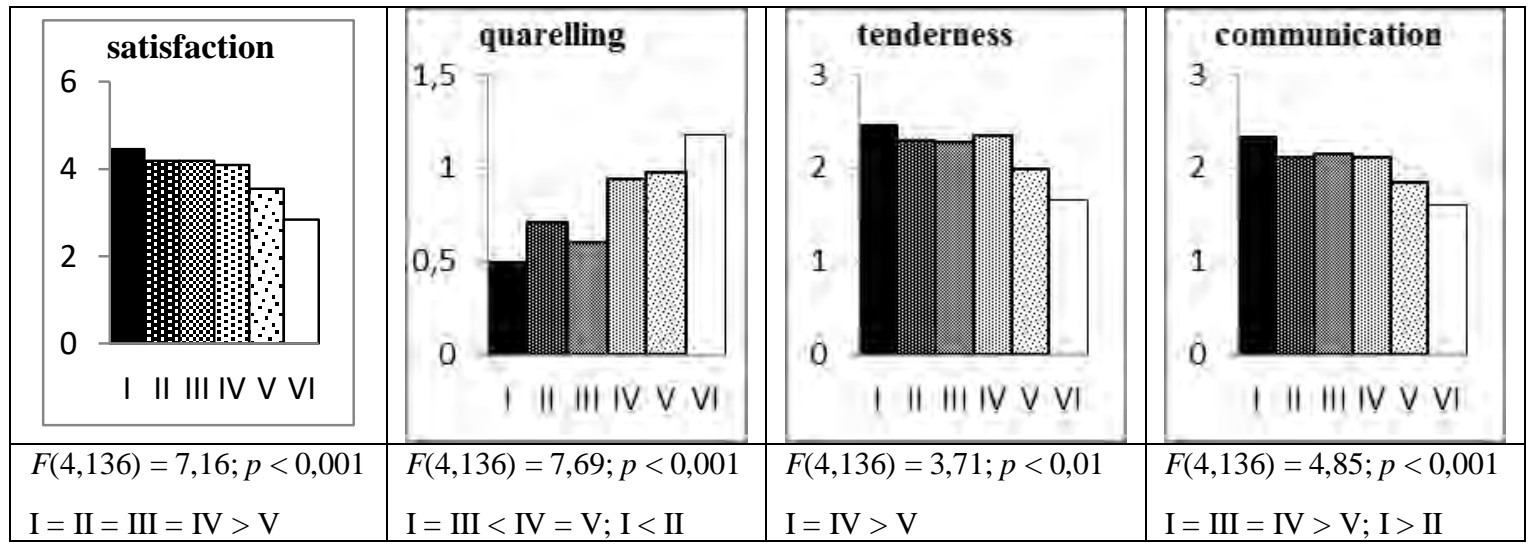

Post hoc comparison, using the Tukey HSD (the signs ">", "<" symbolize the significance and direction of differences between the average dimensions of a relationship`s quality at least on the $p<0.05$ level; the sign "=": lack of significant differences), indicated that a combination of secure-secure patterns (I) turned out to be the most beneficial for all dimensions of a relationship`s quality, and a combination of anxious-avoidant patterns (V) proved the least advantageous. The couples assigned to the avoidant-avoidant type (VI), excluded from post hoc pair comparison, scored the lowest average in all dimensions of a relationship`s quality. 
The buffering effect of a secure pattern took place. A combination of secure-avoidant patterns (III) coexisted with satisfaction in a relationship, negative behavior during quarrels, and mutual communication at a level similar to that which occurred in a combination of secure-secure patterns (I), but higher than the anxious-avoidant (V) [and the avoidant-avoidant type (VI)]. An analogous effect happened in a combination of secure-anxious patterns (II), but only in terms of relationship satisfaction.

A combination of two anxious patterns seems to be partly beneficial and partly detrimental to the quality of a relationship. The anxious-anxious combination (IV) was accompanied by relationship satisfaction, intimacy, and mutual communication at a level similar to that which occurred in the secure-secure combination, and higher than in the avoidant-anxious (V) [and the avoidant-avoidant type (VI)]. In turn, the anxious-anxious combination (IV) had a detrimental effect only in the case of negative behavior during quarrels. Its level was higher than in the secure-secure type and similar to that in the anxious-avoidant combination (V).

We observed that a person`s gender had an effect in the secure-avoidant (III) and secure-anxious (II) combinations. The arrangement in which the man presented a secure and the woman an avoidant pattern (IIIb) was characterized by less negative behaviour during quarrels and more intimacy than the reverse situation (IIIa), in which the woman displayed a secure and the man an avoidant pattern ( $U=25.50, p<0.05 ; U=24.00, p<0.05$ respectively). Similarly, the arrangement which involved a man with a secure pattern and a woman with an anxious one (IIb) was marked by less negative behaviour in quarrels than the opposite one (IIa), formed by a woman with a security and a man with an anxious pattern $[t(63)=1.97 ; p<0.05 ; d$ Cohen $=0.48]$. Gender effect did not occur in the anxious-avoidant combination.

\section{Discussion}

The research results which indicate that secure patterns are the most beneficial for the relationship while insecure and especially avoidant ones are the least - are consistent with previous findings (for a review of the research - see: Mikulincer and Shaver, 2007).

What is more puzzling is the specificity of a combination of two anxious patterns. As demonstrated by the obtained results, during quarrels anxious partners can hurt each other more deeply and frequently than those with secure patterns. The former, however, equal the latter in the overall assessment of satisfaction in their relationships; reciprocity in communicating their own thoughts and feelings, agreeing on plans, and discussing everyday matters (see Hahlweg, 1996); even in experiencing intimacy. By living in fear of being rejected, anxious couples may make life really difficult for each other (see Feeney, 2003), yet - at a more fundamental level - they share it and manifest how much they care about each other.

Gender effect can be accounted for by the unabated attraction of a traditional division of gender roles, according to which it is the man who provides stronger support for the woman than vice versa.

\section{References}

Banse, R. (2004). Adult attachment and marital satisfaction: Evidence for dyadic configuration effects. Journal of Social and Personal Relationships, 21, 273-282.

MacLean, A. P. (2002). Attachment in marriage: Predicting marital satisfaction from partner matching using a three-group typology of adult attachment style. Dissertation Abstracts International: Section B, 63(2-B) (UMI No. AAI3043755)

Brennan, K. A., Clark, C. L., \& Shaver, P. R. (1998). Self-report measurement of adult romantic attachment: An integrative overview. In J. A. Simpson \& W. S. Rholes (Eds.), Attachment theory and close relationships (pp. 46-76). New York: Guilford Press.

Funk, J. L., \& Rogge, R. D. (2007). Testing the Ruler with Item Response Theory: Increasing Precision of Measurement for Relationship Satisfaction with the Couples Satisfaction Index. Journal of Family Psychology, 21, 572-583.

Hahlweg, K. (1996). Fragebogen zur Partnerschaftsdiagnostik (FPD). Göttingen-Bern-Toronto-Seattle: Hogrefe, Verlag für Psychologie.

Mikulincer, M., \& Shaver, P. R. (2007). Attachment in adulthood: Structure, dynamics, and change. New York: Guilford Press.

Feeney, J. A. (2003). The systemic nature of couple relationships: An attachment perspective. In P. Erdman \& T. Caffery (Eds.), Attachment and family systems: Conceptual, empirical, and therapeutic relatedness (pp. 139-164). New York: Brunner-Routledge. 


\title{
FAMILY FUNCTIONING CHARACTERISTICS INVOLVED IN ADOLESCENT DEPRESSIVE SYMPTOMS
}

\author{
Antonio Caño ${ }^{1}, \&$ Carmen Rodríguez-Naranjo ${ }^{2}$ \\ ${ }^{l}$ Department or Personality, Assessment and Psychological Treatment, Faculty of Psychology, \\ University of Málaga (Spain) \\ ${ }^{2}$ Department of Basic Psychology, Faculty of Psychology, University of Málaga (Spain)
}

\begin{abstract}
Research has shown that family functioning contributes to depressive symptoms in adolescents, with a wide range of family functioning characteristics associated to adolescent depressive symptoms. However, these family attributes have been studied through different studies, methodologies and theoretical frameworks, and do not allow envisaging a single whole picture of the family attributes associated to adolescent depressive symptoms. The objective of this study was to overcome this deficit. We followed a systematic approach and used the Family Assessment Device (FAD), which comprehensively identify six family variables in which healthy and unhealthy families differ: Problem Solving (PS), Communication (CM), Roles (RL), Affective Responsiveness (AR), Affective Involvement (AI) and Behaviour Control (BC). Independent regression analyses conducted for each variable showed that all the FAD variables significantly predicted BDI scores. However, when the six variables were introduced simultaneously in the same equation to control for the shared explained variance, only AR and AI showed significant effects, with BC approaching significance. These results were confirmed through Pratt's measure, which showed that the non-overlapping effects of AR, AI and BC accounted for virtually the whole variance explained by the FAD dimensions. Conclusions at both methodological and applied levels emerge from these results. At a methodological level, these results prove the need for controlling the shared variance between family variables before deriving any conclusion about their role. At an applied level, they showed that the family affective aspects are the most important regarding adolescent depression, with only behaviour control playing a role within the non-affective variables.
\end{abstract}

Keywords: Family functioning, adolescent depression.

\section{Introduction and objectives}

Family functioning has demonstrated to be a strong risk factor for developing depressive symptoms during adolescence, which has been primarily defined by broadband variables like family cohesion, warmth, acceptance or support, which represent family as a whole (Guassi Moreira and Telzer, 2015). However, it is necessary to carry out a more fine-grained analysis of the specific family functioning variables that affect adolescent depressive symptoms and this is the main purpose of this study. With this purpose, the variables of the MacMaster Model of Family Functioning (MMFF; Miller, Ryan, Keitner, Bishop, and Epstein, 2000) were used in this study, which have been found useful in distinguishing healthy and unhealthy families in clinical and research settings. Research has shown that adolescents' global scores of the MMFF are related to their depressive symptoms (Millikan, Wamboldt, and Bihun, 2002). More recently, Rodriguez-Naranjo and Caño (2018) carried out a study to test the relationship between the variables included in the MMFF and depressive symptoms in adolescents, finding that both global scores of the MMFF and also each MMFF variable on its own predicted depressive symptoms. However, in that study it remains the possibility that the different MMFF variables predicted depressive symptoms due to its shared variance and the prediction was not a genuine effect of each single variable. To our knowledge no study has aimed to differentiate between the ability of the MMFF variables to predict depressive symptoms, once the shared variance between them have been controlled, and this is the main objective of this study. 


\section{Method}

Our sample consisted of 643 secondary-students between 12 and 17 years old. To assess the specific variables of family functioning, and its overall functioning, we used the Family Assessment Device (FAD; Epstein, Baldwin \& Bishop, 1983), including its six subscales: Problem Solving (PS), Communication (CM), Roles (RL), Affective Responsiveness (AR), Affective Involvement (AI) and Behaviour Control (BC). The Beck Depression Inventory (BDI; Beck, Ward, Mendelson, Mock, \& Erbaugh, 1961) was used to assess depressive symptoms in adolescents. We also measure socio-economic status (SES) through a six-item scale assessing the education and occupation of the parents, and housing conditions. All these scales showed good internal consistencies in our sample, with ranges of .65 to .89 .

\section{Design and results}

We conducted seven hierarchical regression equations to predict depressive symptoms. In all of them sex, age and SES were introduced in the first step for control purposes. In six of them one single MMFF variable was included in the second step, while in the seventh regression analyses all the MMFF variables were introduced jointly in the second step. When there were introduced in separate analyses, all the FAD variables significantly predicted depressive symptoms (all $p s<.001$ ). However, the results were at contrast when the six FAD variables together in the same equation, in order to control for the shared explained variance between FAD variables. Once controlled for the common variance, only AR and AI showed significant effects $(\beta=.21, p<.001$ for AR; $\beta=.16, p<.01)$, with BC approaching significance $(\beta=.09, p=.07)$.

Additionally, to this significance tests, Pratt's measures were calculated to identify the amount of unique variance accounted for each variable (Pratt, 1987). This is a measure aimed to study unique effects of predictors in a multiple regression analyses, and one of its important attributes is that their aggregation across the different predictors equals the overall explained variance $\left(\mathrm{R}^{2}\right)$, thus it distributes $\mathrm{R}^{2}$ between the different predictors avoiding any overlap between them. As a rule of thumb, predictors with Pratt's measures higher to the inverse of the number of predictors included in the regression equation are considered important (Wu, Zumbo \& Marshall, 2014). Results are presented in Table 1, showing that the non-overlapping variance accounted for $\mathrm{AR}$, $\mathrm{AI}$, and $\mathrm{BC}$ were above the criterion recommended to consider important a predictor (.11 for nine predictors). Actually, these three FAD dimensions accounted up to $95 \%$ of the total variance explained by the demographics and FAD dimensions.

Table 1. Unique effects of demographics and FAD dimensions for the prediction of BDI.

\begin{tabular}{lccc}
\hline & $\beta$ & $\begin{array}{c}\text { Cross product } \\
\beta^{*} \mathrm{r}\end{array}$ & $\begin{array}{c}\text { Pratt's measure } \\
\mathrm{d}=\left(\beta^{*} \mathrm{r}\right) / \mathrm{R} 2\end{array}$ \\
\hline Gender & .38 & .001 & .004 \\
Age & -.034 & -.001 & -.005 \\
SES & $-.096^{*}$ & .011 & .069 \\
PS & -.025 & -.006 & -.039 \\
CM & -.049 & -.012 & -.076 \\
RL & .052 & .016 & .098 \\
AR & $.213^{* * *}$ & .072 & .453 \\
AI & $.159^{* *}$ & .051 & .322 \\
BC & $.093+$ & .028 & .174 \\
\hline \multicolumn{4}{c}{$\sum \beta^{*} \mathrm{r}=$} \\
& \multicolumn{2}{c}{$\mathrm{R}^{2}=.016$} & $\sum \mathrm{d}=1$ \\
\hline$* p \leq .05 ; * *$ & $* .01 ; * * * p \leq .001 ;+p \leq .10$.
\end{tabular}

\section{Conclusions}

Conclusions at both methodological and applied levels emerge from these results. At a methodological level, these results prove the need for controlling the shared variance between predictors in regression analyses before reaching any conclusion about them. In our study the results were totally at odds depending on weather we regressed BDI scores on each FAD variable in different equations -thus without controlling its communality with other FAD variables- or we regressed BDS scores on the six variables at the same time -then controlling the shared explained variance between them. In the first case, all the FAD dimensions significantly predicted BDI scores, what did not allow revealing any 
discrimination between the different family functioning dimensions in order to predict BDI scores. However, more interesting results emerged once the shared variance between family functioning variables. Thus only two out of six (affective response, affective involvement) significantly predicted BDI scores, with behaviour control being very close to the signification. But the important point is that, in contrast to this variables, three family functioning variables (problem solving, communication and roles) were irrelevant in order to predict BDI scores in adolescents. Thus, an analytical approach that adequately attributes and distinguish unique versus shared effects between predictors is required to allow a fine-grained analysis of the specific family functioning variables that affect adolescent depressive symptoms.

At an applied level, our results show that the family affective aspects are the most important regarding adolescent depression, with only behaviour control playing a role within the non-affective variables. The importance of affective aspects within the family in order to prevent depressive symptoms are in line with other results that shown the importance of emotional bonding between family members to foster adolescents' emotional regulation and prevent depressive symptoms in adolescents (Yap, Allen, \& Sheeber, 2007). Of particular importance is the finding that adolescent perceptions of behavioural control also contributed to their depressive symptoms, which is consistent with other findings for parental monitoring (e.g., Kim \& Ge, 2000). Yap et al. (2007) argue that affective and behavioural impulses raise in adolescence simultaneously to a decrease of external monitoring, thus explaining heightened vulnerability to emotional problems at these ages. Our findings support this and suggest that both family affection and behavioural control are instrumental in promoting adolescents' emotional regulation in response to developmental challenges.

\section{References}

Miller, I. W., Ryan, C. E., Keitner, G. I., Bishop, D. S., \& Epstein, N. B. (2000). The McMaster Approach to Families: Theory, assessment, treatment and research. Journal of Family Therapy, 22(2), 168-189.

Millikan, E., Wamboldt, M. Z., and Bihun, J. T. (2002). Perceptions of the family, personality characteristics, and adolescent internalizing symptoms. Journal of the American Academy of Child \& Adolescent Psychiatry, 41, 1486-1494.

Rodríguez-Naranjo \& Caño, A. (2018). Family functioning, socio-economic status and adolescents' depressive symptoms: the mediating role of hopelessness. In C. Pracana and M. Wang (Eds). Psychological Applications and Trends (308-310). Lisbon: InSciencePress.

Epstein, N.B., Baldwin, L., \& Bishop, D.S. (1983). The McMaster Family Assessment Device. Journal of Marital and Family Therapy 9: 171-180.

Beck, A. T., Ward, C. H., Mendelson, M., Mock, J., \& Erbaugh, J. (1961). An inventory for measuring depression. Archives of General Psychiatry, 4, 561-571.

Pratt, J. W. (1987). Dividing the indivisible: Using simple symmetry to partition variance explained. In T. Pukilla \& S. Duntaneu (Eds.), Proceedings of Second Tampere Conference in Statistics (pp. 245-260). Tampere, Finland: University of Tampere.

Wu, A. D., Zumbo, B. D., \& Marshall, S. K. (2014). A method to aid in the interpretation of EFA results: An application of Pratt's measures. International Journal of Behavioral Development, 38 (1), 98-110.

Yap, M.B.H, Allen, N.B., and Sheeber, A.L. (2007). Using an emotion regulation framework to understand the role of temperament and family processes in risk for adolescent depressive disorders. Clin. Child Family Psychol. Rev., 10,180-196.

Kim, S. Y., \& Ge, X. (2000). Parenting and adolescent depressive symptoms in Chinese American Families. Journal of Family Psychology, 14, 420-435. 


\title{
ADJUSTMENT OF GRADUATE STUDENTS WITH ATTENTION DEFICIT HYPERACTIVITY DISORDER (ADHD)
}

\author{
Julien Dalpé, Georgette Goupil, France Landry, \& Rachel Paquette \\ Department of psychology, Faculty of Human Sciences, Université du Québec à Montréal (Canada)
}

\begin{abstract}
The difficulties of undergraduate students with ADHD are widely documented (Emmers, Jansen, Petry, van der Oord, \& Baeyens, 2017). This allows the development of support measures tailored to their needs. Many of these students complete their undergraduate studies and enter master's and doctoral programs. Graduate studies present additional challenges, particularly related to independent research and writing. However, few studies have explored the adjustment of students with ADHD at the graduate level, that is their ability to meet the demands of their study environment. This study aims to compare the adjustment of master's and doctoral students with ADHD $(n=16)$ and without ADHD. Participants completed a French translation of the Student Adaptation to College Questionnaire (Pariat, 2008, Baker \& Syrik, 1999). This scale measures four dimensions of adjustment: academic, social, personal-emotional and goal commitment/institutional attachment. Students with ADHD present average scores for the overall scale and the four subscales. Moreover, their scores do not differ significantly from those of the students without ADHD. These results indicate that graduate students with ADHD may respond as well to the demands of their study environment as their peers without ADHD, despite the additional challenges associated with their diagnosis. Further studies should explore the conditions and mechanisms facilitating their adaptation.
\end{abstract}

Keywords: ADHD, adjustment, university, graduate students.

\section{Introduction}

The prevalence of Attention deficit and hyperactivity disorder (ADHD) in higher education students is estimated between 3 and 7 \% (Emmers, Jansen, Petry, van der Oord, \& Baeyens, 2017). In Quebec (Canada), 6113 university students have requested institutional support regarding a diagnosis of ADHD, which makes ADHD the most frequent mental health diagnosis in Quebec universities (Association québécoise interuniversitaire des conseillers aux étudiants en situation de handicap, 2018). Compared to their peers without ADHD, students with ADHD tend to present lower grade point averages, less developed study skills and greater social and emotional difficulties. They are more likely than their peers to abandon their program or to be on academic probation (DuPaul, Weyandt, O'Dell, \& Varejao, 2009; Weyandt et al., 2013). To help these students to overcome their difficulties, universities offer support services tailored to their needs (Dupaul et al., 2009).

Many students with ADHD manage to complete their undergraduate studies and enter master's and doctoral programs. Graduate studies present new challenges, such as a greater focus on independent research and writing as well as a greater need for adequate time management and work organisation. Despite this, few studies have explored the ability of graduate students with ADHD to meet the demands of their study environment.

According to Baker and Syrik (1984, 1999), adjustment to university has four dimensions. Academic adjustment refers to the ability to meet educational and institutional demands. Social adjustment refers to the integration to the university community as well as the relationships with colleagues. Personal-emotional adjustment is the ability to maintain good physical and mental health despite stress. The last dimension is goal commitment and institutional attachment, that is the feelings toward the institution of attendance and toward university as a whole.

Previous studies found that students with ADHD had lower adjustment than their peers without ADHD (Jones, Rabinovitch, \& Hubbard, 2015; Shaw-Zirt, Popali-Lehane, Chaplin, \& Bergman, 2005). However, no study to our knowledge has investigated the adjustment of students with ADHD at the graduate level in comparison with their peers. This is the aim of the current study. 


\section{Method}

\subsection{Participants}

Participants were 46 French-speaking Canadian master's and doctoral students, including 16 with ADHD and 28 without ADHD (control group). Table 1 details the descriptive statistics for the participants.

Table 1. Descriptive statistics for the participants.

\begin{tabular}{lcc}
\hline & Students with ADHD $(n=16)$ & Control group $(n=28)$ \\
\hline Gender & & \\
Men & $3(19 \%)$ & $4(14 \%)$ \\
$\quad$ Women & $13(81 \%)$ & $24(86 \%)$ \\
Mean age & 29 years & 28,2 years \\
Educational level & & \\
$\quad$ Master & $7(44 \%)$ & $4(14 \%)$ \\
$\quad$ Doctorate & $9(56 \%)$ & $24(86 \%)$ \\
\hline
\end{tabular}

\subsection{Instrument and procedure}

Participants completed a French translation of the Student Adaptation to College Questionnaire (SACQ; Baker \& Syrik, 1999), either in paper form or online. The SACQ is a 67-item self-reported questionnaire that measures the four dimensions of student adjustment as well as a global adjustment index. Items are answered on a 9-point scale ranging from "does not apply to me at all "to "applies very closely to me ". Scores for the total scale and the four subscales can be converted into $T$ scores $(M=50$, $S D=10$ ), a higher score reflecting a higher level of adjustment. The normative sample was composed of 1424 American undergraduate students. For the French translation used in this study (Pariat, 2008), Cronbach's alpha based on the scores of 465 Canadian undergraduate students was .91 for the total scale, .87 for the academic subscale, .85 for the social subscale, .84 for the personal-emotional subscale and .84 for the institutional attachment subscale.

\subsection{Data analysis}

Data were analyzed using the SPSS software, version 25. First, scores for the total scale and each of the four subscales were compiled and converted into $T$ scores. Following the authors' instructions (Baker \& Syrik, 1999), missing data on specific items were replaced with the mean score of the subscale to which the item was associated. Then, the mean score for the total scale and each subscale was calculated for each of the two groups. Scores between 40 and 60 (one standard deviation from the mean) were considered average. Finally, $t$-tests were used to compare the scores of the students with ADHD to those of the control group. A significance level of .05 was used. Effect size was estimated using Cohen's $d$. A $d$ of .20 indicates a small effect, a $d$ of .50 indicates a medium effect and a $d$ of .80 indicates a large effect (Cohen, 1992).

\section{Results}

Table 2 shows the mean scores of the students with ADHD and the control group for the total scale and each of the subscales of the SACQ. Students from the two groups presented average mean scores for the total scale and each of the subscales. There were no significant differences between the two groups. There was a medium, but non-significant difference between the scores of the two groups for the personal-emotional subscale $(d=.49)$, with students from the control group presenting slightly lower scores than those with ADHD.

Table 2. SACQ scores of students with ADHD and control group.

\begin{tabular}{lccccccc}
\hline & \multicolumn{3}{c}{ ADHD } & \multicolumn{2}{c}{ Control } & & \\
\cline { 2 - 5 } & $M$ & $S D$ & $M$ & $S D$ & $t(42)$ & $p$ & Cohen's $d$ \\
\hline Academic & 46.00 & 11.06 & 47.79 & 12.20 & 0.48 & .63 \\
Social & 43.44 & 9.73 & 44.39 & 9.30 & 0.32 & .75 & .15 \\
Personal-emotional & 44.06 & 6.85 & 39.64 & 10.05 & -1.56 & .13 & .49 \\
Attachment & 47.63 & 10.36 & 47.29 & 8.46 & -0.12 & .91 \\
Total & 44.38 & 9.68 & 43.96 & 10.58 & -0.13 & .90 \\
\hline
\end{tabular}

\section{Discussion}

The goal of the present study was to measure the adjustment of graduate students with ADHD on the four dimensions defined by Baker and Syrik (1999) and to compare them with a control group of 
graduate students without ADHD. The results suggest that graduate students with ADHD are as well-adjusted as their peers without ADHD. Moreover, students with ADHD may be better adjusted than their peers on the personal-emotional dimension. However, as this difference is not statistically significant, it must be replicated with larger samples. Other studies on the adjustment of students at the undergraduate level showed that ADHD was related to lower adjustment (Jones et al., 2015; Shaw-Zirt et al., 2005). Thus, it is possible that students with ADHD who manage to enter master's and doctoral programs are better adjusted than the average student with ADHD.

As ADHD is the most frequent diagnosis in Quebec universities, the development of support services for graduate students with this condition should be a priority. Further studies should explore the conditions and coping mechanisms that allow these students to respond to the demands of their study environment, including the compensatory strategies developed during undergraduate studies. Studies should also investigate the potential beneficial effects of ADHD in graduate studies, such as creativity.

\section{Contributions, limitations and future directions}

The present study is one of the first to investigate the adaptation of graduate students with ADHD. The results suggest that these students are as well-adjusted as their peers in spite of the additional challenges associated with their diagnosis.

One limitation of the study is that the small size of the sample does not allow to take into account the great diversity of graduate study programs. Some programs require students to conduct a research project of a larger scope than other. The amount of autonomous work required of students can also vary from one program to the other. Thus, it is possible that the adjustment of graduate students, including those with ADHD, is partially dependant on the specific demands of their program. A second limitation is that the number of master's students is greater in the group with ADHD than in the control group. Thus, the two groups are not entirely comparable.

In light of those limitations, future studies should use larger samples that are comparable and representative of the various graduate programs offered in Quebec universities. Various instruments should also be used to investigate the adjustment of graduate students, including objective questionnaires to compare participants and open-ended interviews to better understand their specific experience. Such studies would help to understand the skills, challenges and needs of graduate students with ADHD.

\section{References}

Association québécoise interuniversitaire des conseillers aux étudiants en situation de handicap. (2015). Statistiques concernant les étudiants en situation de handicap dans les universités québécoises, 2014-2015 [Statistics regarding students with disabilities in Quebec universities]. Retrieved from http://aqicesh.ca/docs/AQICESH_stat2017-2018-Sans-univ.pdf

Baker, R. W., \& Syrik, B. (1984). Measuring adjustment to college. Journal of Counselling Psychology, $31,179-189$.

Baker, R. W., \& Syrik, B. (1999). Student Adaptation to College Questionnaire (Manual). Torrance, CA: Western Psychological Services.

Cohen, J. (1992). A power primer. Psychological Bulletin, 112, 155-159.

DuPaul, G. J., Weyandt, L. L., O’Dell, S. M., \& Varejao, M. (2009). College students with ADHD: Current status and future directions. Journal of Attention Disorders, 13, 234-250.

Emmers, E., Jansen, D., Petry, K., van der Oord, S., \& Baeyens, D. (2017). Functioning and participation of students with ADHD in higher education according to the ICF framework. Journal of Further and Higher Education, 41, 435-447.

Jones, H. A., Rabinovitch, A. E., \& Hubbard, R. R. (2015). ADHD symptoms and academic adjustment to college: The role of parenting style. Journal of Attention Disorders, 19, 251-259.

Pariat, L. (2008). Étude des liens entre le soutien social, l'ajustement universitaire et la persévérance en première année de baccalauréat [Study of the links between social support, adaptation to college and perseverance in first year of undergraduate studies] (Doctoral thesis, Université du Québec à Montréal, Montreal, Canada). Retrieved from https://archipel.uqam.ca/2214/1/D1797.pdf

Shaw-Zirt, B., Popali-Lehane, L., Chaplin, W., \& Bergman, A. (2005). Adjustment, social skills, and self-esteem in college students with symptoms of ADHD. Journal of Attention Disorders, 8, 109-120.

Weyandt, L. L., Dupaul, G., Verdi, G., Rossi, J. S., Swentosky, A. J., ... Carson, K. S. (2013). The performance of college students with and without ADHD: Neuropsychological, academic and psychosocial functioning. Journal of Psychopathology and Behavioral Assessment, 35, 421-435. 


\title{
FACTORIAL VALIDITY OF THE DESCRIPTIVE/INJUNCTIVE NORM PREFERENCE SCALE
}

\author{
Norihiro Kuroishi ${ }^{1}, \&$ Yoriko Sano ${ }^{2}$ \\ ${ }^{1}$ Institute for Educational Research and Service, International Christian University (Japan) \\ ${ }^{2}$ College of Interhuman Symbolic Studies, Kanto Gakuin University (Japan)
}

\begin{abstract}
This study was the second report of developing the Descriptive/Injunctive Norm Preference Scale. This scale intended to measure individual differences in personal attitudes against social norms. Cialdini et al. (1991) distinguished social norms into two types. Descriptive norm is decided by what behavior most people engage in a particular situation, which is reflected in perceived typicality. Injunctive norm, on the other hand, is defined by habits or moral rules, which represents what people approve/disapprove. This study was a part of the process to develop the scale that assess the tendency how people prefer to obeying/violating descriptive/injunctive norms. The pilot study created 90 items inquiring ones' attitudes (cognition, affection, and behavior) toward descriptive/injunctive norms.

Previous exploratory factor analysis (EFA) extracted 3 factors from the selected 56 items out of the pilot scale; F1: Apprehension of deviance from descriptive norms, F2: Regard for injunctive norms, and F3: Aversion to injunctive norms. This study tried to replicate the factor structure, and to examine the construct validity of the scale. Participants were recruited anew from a research company panel, and 400 hundred adults (200 females and 200 males) responded to online questionnaires. Confirmatory factor analysis (CFA) indicated the goodness of fit to be fair to the 3-factor model hypothesized from a priori analysis. Further verification in comparison with other related psychological constructs would be needed to examine its content validity.
\end{abstract}

Keywords: Scale development, factorial validity, injunctive and descriptive norms.

\section{Introduction}

Social norms influence an individual's behaviors. According to Cialdini et al. (1991), social norms can be distinguished into two types. Descriptive norm is decided by what most people do in a particular situation, which may bring about perceived typicality. On the other hand, injunctive norm is defined by moral rules, which reflects what people approve/disapprove. In many cases, these types of norms agree with each other. People recognize thieving as vice, and most people do not engage in such a misdeed. In some cases, however, descriptive norms can conflict with injunctive norms. Although people think they should not litter in public places, rubbish on the ground may indicate that many people litter habitually. When the two types of norms are disparate, the descriptive norms have greater effects on individuals' behaviors than the injunctive norms do.

Our previous research tried to reveal affective states when people obey/violate social norms, and showed that Japanese people feel calmer and have less negative affects when they follow descriptive norms. These tendencies seemed to be robust across demographic and cultural backgrounds. Exploration of genders, age-groups, and some other individual differences such as individualism-collectivism, need for uniqueness, and fear of success, indicated that these factors were irrelevant to the affective states. Only a within-subject examination revealed that rejection sensitivity moderate the affective reactions to social norms (Kuroishi \& Sano, 2017).

This study was the second step to develop a scale that directly assess the individual differences in how people prefer to obeying/violating descriptive/injunctive norms, and tried to examine the factorial validity of the scale by a confirmatory factor analysis (CFA). 


\section{Methods}

\subsection{Measure}

Fifty-five items were selected from the items used in the pilot study (Kuroishi \& Sano, 2018). The items were originally generated according to the original Cialdini's theory, and referred to cognition, emotion, or behavior when people obey or violate descriptive/injunctive norms.

Table 1. Standardized Factor Loadings Estimated by Confirmatory Factor Analysis (CFA).

\begin{tabular}{|c|c|c|c|}
\hline Item Details & F1 & F2 & F3 \\
\hline I want to do the same with everyone. & .789 & & \\
\hline It is embarrassing unless I do the same behavior as the surroundings. & .731 & & \\
\hline I am worried about whether I behave differently from the surrounding people. & .718 & & \\
\hline I'd like to do the same thing as everyone. & .727 & & \\
\hline I want to do the same by looking at the behavior of people around me. & .798 & & \\
\hline I am careful not to get out of what everyone is doing. & .724 & & \\
\hline I am worried that I am not doing the same thing as everyone else. & .734 & & \\
\hline I am relieved that I am doing the same as the surrounding people. & .753 & & \\
\hline I want to adopt what many people do. & .762 & & \\
\hline I am too embarrassed to behave in the different way from others. & .680 & & \\
\hline I am concerned about being out of touch with other people. & .774 & & \\
\hline I feel nervous when I am different from other people. & .715 & & \\
\hline I act like people around me before I know it. & .761 & & \\
\hline It is better to tailor to the behavior many people do. & .735 & & \\
\hline I am okay when I follow the things people do. & .736 & & \\
\hline I cannot stop looking at what everybody is doing. & .644 & & \\
\hline I feel quite safe when keeping the same behavior as other people. & .741 & & \\
\hline I often behave like the surrounding people at first. & .778 & & \\
\hline It is safe to obey the major opinion, even though it is different from my own opinion. & .671 & & \\
\hline It is better to do in concert with everyone. & .700 & & \\
\hline I am concerned about the state of the surrounding people. & .721 & & \\
\hline Apart from my opinions, it is better to behave in the same way as everyone. & .706 & & \\
\hline I look down on those who behave differently from everyone. & .540 & & \\
\hline I often slip my eyes attracted towards behaviors of the surrounding people. & .619 & & \\
\hline I don't want to act out of the surroundings. & .769 & & \\
\hline I try to keep rules and regulations. & & .756 & \\
\hline It is better to keep rules. & & .778 & \\
\hline Rules are important for everyone to live comfortably. & & .759 & \\
\hline I want to act socially appropreate. & & .784 & \\
\hline It is natural to observe rules. & & .781 & \\
\hline It is not good to break rules. & & .746 & \\
\hline I feel anger for those who do not follow rules. & & .677 & \\
\hline It is not good to ignore customs and customs. & & .513 & \\
\hline I want to make rules so that confusion will not occur. & & .617 & \\
\hline I feel guilty when in breaking rules and regulations. & & .662 & \\
\hline An old custom has some meaning. & & .527 & \\
\hline There are many customs which do not fit the present era. & & .406 & \\
\hline There are some worthless rules. & & .425 & \\
\hline I am relieved when under rules and regulations. & & .680 & \\
\hline I feel uneasy when I don't keep rules and regulations. & & .639 & \\
\hline Rules and regulations are not necessary for our lives. & & -.336 & \\
\hline I am sensitive to the rule and the regulation to protect. & & .572 & \\
\hline I do not want to obey customs and traditions. & & & .719 \\
\hline I am not concerned about customs and traditions. & & & .679 \\
\hline Traditions and customs are stuffy. & & & .659 \\
\hline I don't want to be tied down with customs. & & & .614 \\
\hline I don't want to be bound by rules. & & & .560 \\
\hline I don't like to behave in the same way as everyone else. & & & .491 \\
\hline I don't like customs or traditions. & & & .718 \\
\hline It does not matter whether I can follow customs and traditions. & & & .676 \\
\hline I don't mind even if I break customs and traditions. & & & .637 \\
\hline I don't care about customs and traditions. & & & .672 \\
\hline I really hate rules and regulations. & & & .542 \\
\hline I feel stressed in observing rules and regulations. & & & .509 \\
\hline $\begin{array}{l}\text { One should carry the opinion once he/she believe to be right, even though contrary to } \\
\text { the public convention. }\end{array}$ & & & .506 \\
\hline
\end{tabular}




\subsection{Data collection}

Web questionnaire survey was applied in this study. Four-hundred Japanese of the panel who have registered with Neo Marketing Inc. participated in our study. Respondents were planned to be obtained equally from 4 demographic groups; genders (males and females) x age-groups (aged 20-39 and 30-59). Data were collected successfully, and the sample consisted of 100 younger males (aged 33.35), 100 older males (aged 51.02), 100 younger females (aged 32.76), and older female (aged 48.85). Participants got access to the website and completed the questionnaire with agreement with providing their data for the study.

\subsection{Procedure}

Respondents were asked to read the statements about descriptive/injunctive norms carefully, and decide how they agree to each opinion on 5-point rating from "strongly disagree" (1) to "strongly agree" (5). The items were randomly displayed to each respondent.

\section{Results and Discussion}

Preliminarily, an exploratory factor analysis (EFA) by maximum likelihood extraction and with a promax rotation was conducted. Three-factor solution was adopted according to the pilot study. All the items loaded the most by the expected factor. The factor structure of the pilot data was replicated by the current data.

Mainly, a confirmatory factor analysis (CFA) indicated the goodness of fit to be fair to the goodness of fit to the 3-factor model which was hypothesized from the pilot study (RMR=.070, and RMSEA $=.068,90 \%$ CI $[.065, .070]$ ). Table 1 showed the standardized factor loadings. The factors were interpreted as the pilot study; F1 Apprehension of deviance from descriptive norms, F2 Regard for injunctive norms, and F3 Aversion to injunctive norms.

The inter-factor correlation coefficients were $r_{F 1-F 2}=.367, \quad r_{F 2-F 3}=-.142$, and $r_{F 3-F 1}=.117$, respectively. The significance of the coefficients suggested the only correlation between F1 and F2, and simple structures among other pairs of the factors.

\section{Conclusions}

These results manifested the factorial validity the Descriptive/Injunctive Norm Preference Scale (DINPS). This study validated the robustness and the goodness of fit to the hypothesized 3-factor structure of the items. It might indicate the feasibility to assess how people prefer to obeying/violating descriptive/injunctive norms, and predict the tangible behaviors people adopt to descriptive/injunctive norms.

There is, however, plenty of room for improvement. First, this scale is needed to verify its construct validity with comparing to the relevant psychological concepts: For examples, authoritarian personality (F-scale: Adorno et al., 1950) for a tendency to obey injunctive norms, and need for uniqueness (Snyder \& Fromkin, 1980) for a tendency to violate descriptive norms. Second, the original scale is in Japanese, and it is needed to develop some versions in other language such as English to examine its cross-validity. These attempts will enable international comparative studies across cultures.

\section{References}

Adorno, T. W., Frenkel-Brunswik, E., Levinson, D. J., \& Sanford, R. N. (1950). The Authoritarian Personality. New York: Harper and Row.

Cialdini, R. B., Kallgren, C. A., \& Reno, R. R. (1991). A focus theory of normative conduct. Advances in Experimental Social Psychology, 24, 201-234.

Kuroishi, N. \& Sano, Y. (2017). A within-subject study of affective reactions to social comparisons by considering individual differences in need for uniqueness and rejection sensitivity. Comprehensive Studies of Education: Journal of Japan Professional School of Education, 10, 63-79.

Kuroishi, N. \& Sano, Y. (2018.05) Development of a descriptive/injunctive norm preference scale. Poster Presentation at the International Psychological Applications Conference and Trends 2018.

Snyder, C. R., \& Fromkin, H. L. (1980). Uniqueness: The human pursuit of difference. New York: Plenum Press. 


\title{
THE RENCONTRES FOCUS: OUTCOMES OF A LEARNING AND STUDY SKILLS BASED PROGRAM FOR UNDERGRADUATE STUDENTS WITH ATTENTION DEFICIT HYPERACTIVITY DISORDER (ADHD)
}

\author{
Rachel Paquette, France Landry, Georgette Goupil, \& Julien Dalpé \\ Department of psychology, Université du Québec à Montréal (Canada)
}

\begin{abstract}
Undergraduate studies require good organizational, planning and time management skills. As several studies showed, students with Attention Deficit Hyperactivity Disorder (ADHD) have weaker learning and study strategies than students without ADHD (Gormley, DuPaul, Weyandt, \& Anastopoulos, 2016; Reaser, Prevatt, Petscher, \& Proctor, 2007). Some universities have therefore implemented support measures for those students. In Quebec (Canada), Landry (2012) developed the Rencontres FOCUS, which aim to enhance learning and study skills. This study evaluated the effects of the Rencontres FOCUS on the learning and study strategies of thirteen undergraduate students with ADHD (six men and seven women, $M_{\mathrm{age}}=29.2$ years). The French version of the Learning and Study Strategy Inventory (LASSI, Weinstein and Palmer, 2002) was used to evaluate participants' study skills. Dependent $t$-tests compared the pre and post-intervention scores. Participants significantly improve on Anxiety, Concentration, Selecting Main Ideas and Test Strategy subscales with a moderate to large size effect. However, results indicate that the participants remain below the $50^{\text {th }}$ percentile of the LASSI. This suggests that, despite their progress, those students have to continue to improve their study skills. The results also reveal that the Rencontres FOCUS seems relevant for the development of study strategies for students with ADHD.
\end{abstract}

Keywords: ADHD, university, study skills, group intervention.

\section{Introduction}

The prevalence of Attention deficit hyperactivity disorder (ADHD) in higher education students is estimated between 3 and 7 \% (Emmers, Jansen, Petry, van der Oord, \& Baeyens, 2017). ADHD is associated with long-term impairment in educational attainment, occupational status and social relationship (Barkley, Murphy, \& Fischer, 2008). Students with ADHD are more likely to present lower grade point average, to drop out of their program or to complete their program later than their peers without ADHD (Gormley, DuPaul, Weyandt, \& Anastopoulos, 2016). Barkley's theoretical framework (1997) places the self-regulatory deficits at the center of many problems students with ADHD tend to present, such as difficulties in concentration, information processing, organization, time management, motivation, etc.

Although several programs have been developed specifically for enhancing learning and study skills, none of them were adapted in French. To answer the increasing demands in Université du Québec à Montréal's student services, Landry (2012) developed the Rencontres FOCUS, a six weeks program aiming to enhance study skills of students with ADHD using cognitive and behavioral theories. Since 2012, seven cohorts participated in the program. In order to evaluate the students' appreciation of the program, Dalpé, Landry and Goupil (2019) conducted a qualitative data analysis and found that many participants mentioned they felt they improved their study skills. In continuation with their work, this study aims to empirically assess the effects of the Rencontres FOCUS program on learning and study skills of participants using a pre and post-intervention research design.

\section{Method}

\subsection{Participants}

Participants were 13 French-speaking Canadian undergraduate students with ADHD (six men and seven women, $M_{\text {age }}=29.2$ years). Data were collected on three consecutive cohorts of the Rencontres FOCUS between January 2017 and May 2018. 


\subsection{Instrument and Procedure}

Intervention. An educational psychologist and a doctoral student in psychology conducted the two-hour group sessions in a university setting. They presented the content mainly using a question-and-answer format to stimulate active student participation in the group discussion. Lecture format and explicit teaching were sometimes used when illustrating certain strategies. Table 1 details the session-by-session topics for the Rencontres FOCUS.

Table 1. Session-by-session topics of the Rencontres FOCUS.

\begin{tabular}{|c|c|c|c|c|c|c|}
\hline Sessions & $\begin{array}{l}\text { 1. ADHD and } \\
\text { educational } \\
\text { project }\end{array}$ & $\begin{array}{c}\text { 2. Time } \\
\text { management } \\
\text { and activity } \\
\text { planning }\end{array}$ & $\begin{array}{l}\text { 3. Attention and } \\
\text { material } \\
\text { organization }\end{array}$ & $\begin{array}{l}\text { 4. Reading and } \\
\text { writing }\end{array}$ & $\begin{array}{c}\text { 5. Stress } \\
\text { management }\end{array}$ & $\begin{array}{c}\text { 6. Motivation } \\
\text { and transition to } \\
\text { future } \\
\text { workplace }\end{array}$ \\
\hline
\end{tabular}

Pre- and post-intervention. Participants completed the French version of the Learning and Study Strategy Inventory (LASSI, Weinstein \& Palmer, 2002) at the first and last sessions. The 80-item test contains 10 eight-item scales. Students respond to items using a five-point Likert scale, ranging from "not at all typical of me" to "very much typical of me". For each subscale, the scores obtained are presented in the form of raw scores and percentiles.

\subsection{Data analysis}

Statistical analyzes used Excel to perform means, standard deviations, dependent $t$-tests between pre and post-intervention and Cohen's $d$. When significant differences on $t$-tests were found, the effect size was calculated by Cohen's $d$, where a $d$ of $.2, .5, .8$ respectively indicate a small, moderate, and large effect. Since the goal of this study was to see if there were any effects on the learning strategies, the analysis took into account percentile and raw data, so we could detect any variations between pre and post-intervention. The results of these analyzes are presented in Table 2.

\section{Results}

Results shown in Table 2 reveal improvement in four out of ten study strategies measured by the LASSI. Analyzes show a significant improvement in the raw scores of the following subscales: anxiety, $t(12)=-3.25, p=0.004$ and a moderate to large size effect. Analyzes also indicate a significant improvement in concentration $t(12)=-3.42, p=0.025$; main idea selection strategies $t(12)=-1.96$, $p=0.037$ and test strategies $t(12)=-1.80, p=0.049$ The effect size for the last three subscales shows a moderate range. Although we can observe that the percentile for all of the subscales improve, nine of the ten subscales remain below the $50^{\text {th }}$ percentile.

Table 2. LASSI pre and post-intervention raw and percentile scores of students with ADHD $(N=13)$.

\begin{tabular}{|c|c|c|c|c|c|c|c|c|}
\hline \multirow{2}{*}{ Subscales } & \multicolumn{2}{|c|}{ Raw Scores } & \multirow[b]{2}{*}{$t$} & \multirow[b]{2}{*}{$d$} & \multicolumn{2}{|c|}{ Percentile } & \multirow[b]{2}{*}{$t$} & \multirow[b]{2}{*}{$d$} \\
\hline & $\frac{\text { Pre-int. }}{M(S D)}$ & $\frac{\text { Post-int. }}{M(S D)}$ & & & $\frac{\text { Pre-int. }}{M(S D)}$ & $\frac{\text { Post-int. }}{M(S D)}$ & & \\
\hline Anxiety & $20.9(7.3)$ & $24.3(7.9)$ & $-3.25^{* *}$ & 0.66 & $29.8(29.0)$ & $44.8(32.2)$ & $-2.95 * *$ & 0.69 \\
\hline Attitude & $30.0(2.9)$ & $30.3(3.0)$ & -0.39 & & $19.7(17.6)$ & $23.2(19.0)$ & -0.72 & \\
\hline Concentration & $20.1(5.7)$ & $22.8(7.8)$ & $-3.42 * *$ & 0.40 & $18.8(17.3)$ & $32.9(29.7)$ & $-3.28 * *$ & 0.55 \\
\hline Self-testing & $19.9(3.9)$ & $20.9(6.2)$ & -0.90 & & $23.1(21.5)$ & $32.1(29.9)$ & -1.57 & \\
\hline Selecting Main Idea & $22.1(8.1)$ & $24.4(6.8)$ & $-1.96 *$ & 0.44 & $28.5(28.8)$ & $32.5(28.8)$ & -0.85 & \\
\hline Study Aids & $24.0(4.4)$ & $25.2(6.5)$ & -1.17 & & $41.2(26.8)$ & $49.9(30.7)$ & -1.65 & \\
\hline Time Management & $20.2(4.9)$ & $21.5(7.3)$ & -1.02 & & $20.9(20.4)$ & $28.2(30.8)$ & -1.55 & \\
\hline Test Strategies & $25.8(5.9)$ & 27.7 (5.9) & $-1.80 *$ & 0.48 & 33.7 (24.8) & $43.2(29.5)$ & -1.55 & \\
\hline
\end{tabular}




\section{Discussion}

The goal of this study was to measure and compare the learning and study skills of undergraduate students using the LASSI in a pre- and post-intervention research design. For the raw data comparison, the results suggest that undergraduate students with ADHD significantly improved their study skills on four out of ten subscales: Anxiety, Concentration, Selecting Main Idea and Test Strategies. Also, even if there were no significant differences, we can observe that participants improved on the six other subscales, showing a great potential for this novel and francophone program. Those results are consistent with research concerning group-based and peer-based intervention for students with ADHD (Anastopoulos \& King, 2015; Prevatt \& Yelland, 2013).

Although results showed that participants were able to enhance their study skills, we can notice that they remain under the $50^{\text {th }}$ percentile in most subscales. Thus, they remain in the "at risk" category, the last of the three LASSI ranking categories. This suggests that, despite their progress, additional initiatives are required to improve their study skills. Considering the prevalence of students with ADHD, support programs in universities could include individualized support from professionals, follow up sessions or peer-coaching.

\section{Limitations and future directions}

This study has some limitations. First, due to sample and institutional constraints, we were not able to assemble a control group to assess the program's direct effect on the participants. Second, the small sample size may have been insufficient to note significant differences between pre- and post-intervention. Future research should focus on exploring the psychosocial factors implicated in the success of the program such as the level of implication of individuals, the group cohesion, the role of the group counselors, etc. More studies on college and university initiatives like the Rencontres FOCUS could contribute to bring to light new and useful ways of helping students with ADHD.

\section{References}

Anastopoulos, A. D., \& King, K. A. (2015). A cognitive-behavior therapy and mentoring program for college students with ADHD. Cognitive and Behavioral Practice, 22(2), 141-151.

Barkley, R. A. (1997). Behavioral inhibition, sustained attention, and executive functions: Constructing a unifying theory of ADHD. Psychological Bulletin, 121(1), 65-94.

Barkley, R. A., Murphy, K. R., \& Fischer, M. (2008). ADHD in adults: What the science says. New York, NY: Guilford Press.

Dalpé, J., Landry, F., \& Goupil, G. (2019). Étude exploratoire sur la validité sociale des Rencontres FOCUS pour les étudiants universitaires ayant un TDAH [Exploratory study on social validity of the Rencontre FOCUS for university student with ADHD] (accepted for publication). Science et comportement.

Emmers, E., Jansen, D., Petry, K., van der Oord, S., \& Baeyens, D. (2017). Functioning and participation of students with ADHD in higher education according to the ICF framework. Journal of Further and Higher Education, 41, 435-447.

Gormley, M. J., DuPaul, G. J., Weyandt, L. L., \& Anastopoulos, A. D. (2016). First-year GPA and academic service use among college students with and without ADHD. Journal of Attention Disorders, 20(1), 1-14.

Landry, F. (2012). Programme de renforcement des stratégies d'étude pour les étudiants universitaires ayant un TDAH. [Study strategies enhancing program for university student with ADHD] (Unpublished doctoral dissertation). Université du Québec à Montréal: Montréal, Canada.

Prevatt, F., \& Yelland, S. (2015). An empirical evaluation of ADHD coaching in college students. Journal of attention disorders, 19(8), 666-677.

Reaser, A., Prevatt, F., Petscher, Y., \& Proctor, B. (2007). The learning and study strategies of college students with ADHD. Psychology in the Schools, 44(6), 627-638.

Weinstein, C. E., \& Palmer, D. R. (2002). LASSI user's manual: For those administering the Learning and Study Strategies Inventory. Clearwater, Floride: H \& H Publishing. 


\title{
DEVELOPING ACADEMIC SKILLS IN DIGITAL ENVIRONMENTS: MEDIA EFFECTS ON PERFORMANCE AND METACOGNITIVE JUDGMENTS
}

\author{
Gal Ben-Yehudah ${ }^{1}, \&$ Ronen Kasperski ${ }^{2,3}$ \\ ${ }^{1}$ Department of Education and Psychology, The Open University (Israel) \\ ${ }^{2}$ Department of Special Education, Gordon Teachers College (Israel) \\ ${ }^{3}$ Department of Special Education, Shaanan Teachers College (Israel)
}

\begin{abstract}
The prevalence of digital academic materials and their use is constantly growing. However, surveys report that most readers prefer to read long texts such as academic papers and textbooks in print rather than digital format. Moreover, accumulating evidence on in-depth processing of digital text points to the disturbing finding - understanding digital text is inferior to that of printed text. In an effort to overcome these contradictory trends, the current study investigated the effectiveness of an instructional intervention for preservice teachers, which focused on developing academic reading and writing skills in a digital environment. The instruction took place in a computer lab, during which the instructor demonstrated reading and writing strategies in a digital environment using frontal teaching methods. Then, the participants practiced these strategies in their scientific reading and writing. Multiple testing points were implemented in both media conditions: digital and paper. The mid-term testing point demonstrated that emphasizing in-depth processing diminished the 'media effect' (differences in performance between digital and paper conditions) reported in the literature for reading comprehension. Also there were no differences in academic writing between paper and digital groups. Confidence ratings for academic reading and writing were higher in the digital setting. Interestingly, differences in the evaluation (calibration analyses) of reading and writing were observed among media groups. While the digital group was more calibrated on their assessment of writing quality, the paper group was more calibrated in their assessment of reading comprehension. In this paper, we will discuss the importance of incorporating a digital module into the curriculum of preservice teachers, which should increase students' awareness to biases they may have about academic performance in digital environments.
\end{abstract}

Keywords: Academic writing, academic reading, preservice teachers, prediction of performance, judgement of learning.

\section{Introduction}

Digital materials are frequently used in higher education (Siemens, Gašević \& Dawson, 2015), despite evidence indicating inferior comprehension of digital text relative to printed text - 'digital text inferiority’ (e.g., Ben-Yehudah \& Eshet-Alkalai, 2018; Delgado, Vargas, Ackerman, \& Salmerón, 2018; Kong, Seo, \& Zhai, 2018; Singer \& Alexander, 2017a). The negative effects of reading on screen extend also to metacognitive evaluation of learning, namely, students are more overconfident in their success for reading on screen than on paper (Ackerman \& Goldschmidt, 2011; Singer \& Alexander, 2017b). One explanation for these effects is that shallow processing occurs more on screen than on paper (Chen, Cheng, Chang, et al., 2014; Wolf \& Barzillai, 2009), which leads to overconfidence and inferiority of digital text comprehension. This media effect can be reduced by "forcing" more in-depth processing on screen (Ackerman \& Lauterman, 2012). Complementing these findings are studies on reading preferences in higher-education students, which show that most students prefer to read long texts such as textbooks and academic papers in print rather than in digital format (Mizrachi, 2015). Today, 21st century skills include reading a large amount of scientific papers in digital format and writing with word processors, thus, it is necessary to develop these skills in future teachers.

The current study examined an instructional intervention for preservice teachers, which focused on developing academic reading and writing skills in digital environments. We investigated the effects of the media on academic performance (comprehension of academic papers and the quality of writing an 
integrative essay) and their corresponding confidence ratings before (prediction of performance, POP) and after (judgment of learning, JOL) each assessment. During the intervention participants received content instruction (understanding text and academic writing) together with ICT instruction over an entire semester (13 weeks, 52 hours). Here we report results for the mid-term assessment.

Our research questions (RQ) were: 1 . Does an instructional intervention diminish the "digital text inferiority" effect on the academic reading and writing of preservice teachers? 2. To what extent are confidence ratings influenced by the media (paper, digital) of reading and writing?

\section{Methods}

Participants were 126 preservice teachers who were required to take a prerequisite course to improve their academic skills. Instruction took place in a computer lab using frontal-teaching methods. During the instruction phase, the instructor demonstrated reading and writing strategies for processing digitally displayed academic papers. These included extracting main ideas and organizing them with a digital graphic organizer, and integrating the ideas into a digital summary. Next, students practiced these strategies while using a common word processing software. During the assessment phase, classes were quasi-randomly assigned to a medium condition: screen $(n=71)$ or paper $(n=55)$. The mid-term assessment was similar to previous practice sessions, in which the participants were required to integrate a text they had not seen previously (i.e., unseen) with texts that they had studied and then write an integrative essay. All the texts were available to them during the assessment.

We calculated two performance indices for the mid-term assessment, based on participants answer to an open-ended question. The indices were: 1. comprehension of academic texts (i.e., identifying main ideas, connecting prior knowledge to new knowledge); and 2. academic writing quality (i.e., writing coherence and connectivity). In addition, confidence ratings were collected for each academic subskill (academic reading and academic writing) just before (POP) and right after (JOL) the mid-term assessment. The four confidence indices were: 1) POP-reading ("...do you think you will be able to understand the article?"); 2) JOL-reading ("...do you think you were able to understand the article?"); 3) POP-writing ("...do you think you will be able to write an integrative answer?"); 4) JOL-writing ("...do you think you were able to write the integrative answer?"). We also calculated calibration measures for each type of confidence rating (i.e., POP-calibration and JOL-calibration). Calibration is the difference between the actual performance and the metacognitive judgement of performance.

\section{Results}

\subsection{Media effects on performance (RQ1)}

Two performance indices were derived from the mid-term assessment: academic text comprehension and writing quality (see methods). There were no significant effects of the media (digital, paper) on academic reading: digital (59.17\%) and paper (57.56\%), $t(121)=-0.42, p=.67, d=0.08$; nor on academic writing: digital $(66.4 \%)$ and paper $(70.4 \%), t(121)=0.82, p=.41, d=0.15)$. These results show that emphasizing in-depth processing through a cycle of instruction and practice sessions did indeed reduce the digital text inferiority effect.

\subsection{Metacognitive judgements (RQ2)}

Confidence ratings for each academic subskill (text comprehension and writing quality) were collected before (POP) and after (JOL) the mid-term assessment. Before participants began the assessment, their confidence ratings (POP) were significantly higher for the digital relative to print condition, for both academic reading and writing. As reflected by the significant media effect observed in both POP measures. For POP-reading, digital (68.68\%) was larger than paper (58.13\%), $t(117)=-2.65$, $p<.01, d=0.49$. For POP-writing, digital (71.75\%) was larger than paper (58.53\%), $t(121)=-3.73$, $p=.001, d=0.69$. Despite this overconfidence, analyses of POP-calibration indicated that the paper group (19.55\% bias) was significantly more calibrated than the digital group (26.55\% bias) in their evaluation of reading comprehension, $t(117)=-2.19, p=.03 ; d=0.44$. The groups did not differ in their POP-calibration for writing quality. In contrast, after the assessment, calibration analyses for JOL showed that the digital group (23.33\% bias) was significantly better at evaluating their writing quality than the paper group (33.25\% bias), $t(117)=2.72, p=.026 ; d=0.49$. 


\section{Discussion}

Our findings show that the instructional intervention was effective in reducing the media effect on academic performance, as the mid-term assessment showed no significant effect of the media (digital, paper) on academic reading or writing. This bares important educational implications, namely, to encourage preservice teachers to develop in-depth processing of digital text, which should help minimize the inferiority of digital text comprehension (Delgado et al., 2018; Kong et al., 2018; Singer \& Alexander, 2017a).

Consistent with the literature, higher levels of confidence in academic reading were observed in the digital as compared to paper setting (Ackerman \& Goldschmidt, 2011). A similar pattern of findings was observed for academic writing ability. To the best of our knowledge, confidence ratings have not been assessed for digital versus paper based writing. Thus, our finding in this area is novel and worth further investigation. Interestingly, after the mid-term assessment, the digital group's evaluation of academic writing (JOL-writing) was in fact less biased than the paper group's evaluation of their writing performance. In contrast, evaluation of reading comprehension (POP-reading) was more calibrated in the paper group than the digital group.

These encouraging findings suggest the importance of developing academic subskills (academic reading and writing) in digital environments, particularly in preservice teachers that are entrusted with educating our youth on 21st century skills. Given the prevalence of digital academic materials in higher education, it is important to increase students' awareness of the effect the media has one's own evaluation of reading and writing performance.

\section{References}

Ackerman, R., \& Goldsmith, M. (2011). Metacognitive Regulation of Text Learning: On Screen Versus on Paper. Journal of Experimental Psychology: Applied, 17(1), 18-32.

Ackerman, R., \& Lauterman, T. (2012). Taking reading comprehension exams on screen or on paper? A metacognitive analysis of learning texts under time pressure. Computers in Human Behavior, 28(5), 1816-1828.

Ben-Yehudah, G., \& Eshet-Alkalai, Y. (2018). The contribution of text-highlighting to comprehension: A comparison of print and digital reading. Journal of Educational Multimedia and Hypermedia, 27(2), 153-178.

Chen, G., Cheng, W., Chang, T., Zheng, X., \& Huang, R. (2014). A comparison of reading comprehension across paper, computer screens, and tablets: Does tablet familiarity matter? Journal of Computers in Education, 1, 213-225.

Delgado, P., Vargas, C., Ackerman, R., \& Salmerón, L. (2018). Don't throw away your printed books: A meta-analysis on the effects of reading media on reading comprehension. Educational Research Review.

Kong, Y., Seo, Y. S., \& Zhai, L. (2018). Comparison of reading performance on screen and on paper: A meta-analysis. Computers \& Education, 123, 138-149.

Mizrachi, D. (2015). Undergraduates' academic reading format preferences and behaviors. The Journal of Academic Librarianship, 41, 301-311.

Siemens, G., Gašević, D., \& Dawson, S. (2015). Preparing for the digital university: A review of the history and current state of distance, blended, and online learning. http://linkresearchlab.org/PreparingDigitalUniversity.pdf

Singer, L. M., \& Alexander, P. A. (2017a). Reading on paper and digitally: What the past decades of empirical research reveal. Review of Educational Research, 87(6), 1007-1041.

Singer, L. M., \& Alexander, P. A. (2017b). Reading Across Mediums: Effects of Reading Digital and Print Texts on Comprehension and Calibration. Journal of Experimental Education, 85(1), 155-172.

Wolf, M., Barzillai, M., (2009). The importance of deep reading. Educational Leadership, 66(6), 32-37. 


\title{
A STRUCTURAL MODEL OF THE TEST FOR CREATIVE THINKING-DRAWING PRODUCTION (TCT-DP) IN ADULTS- REVISED
}

\author{
Sara Ibérico Nogueira ${ }^{1}$, Leonor Almeida ${ }^{2}, \&$ Tiago Souza Lima ${ }^{3}$ \\ ${ }^{1}$ ULHT-Universidade Lusófona de Humanidades e Tecnologias / Associate Professor (Portugal) \\ ${ }^{2}$ ISG-Business \& Economics School / Associate Professor (Portugal) \\ ${ }^{3}$ UNIFOR-University of Fortaleza / Assistant Professor (Brasil)
}

\begin{abstract}
The Test for Creative Thinking-Drawing Production (TCT-DP, Urban \& Jellen, 1996) is one of the best instruments for the creative potential assessment, which has been considered a crucial dimension in the organizational context. Considering its factorial structure, most studies have been limited using an exploratory approach. This research aims to assess the factorial structure of the TCT-DP (Form A) for a Portuguese adult sample, with a total of 620 workers, revisiting the results obtained in a study of Almeida, Ibérico Nogueira and Lima (2018). Two studies were performed. In Study 1, an exploratory factor analysis (EFA) was performed to assess the factorial structure of the TCT-DP. Take part in this study three hundred two individuals from Portugal, most of them were women $(55,6 \%)$ and had an undergrad degree $(74,8 \%)$, with age range from 18 to 86 years $(M=41.1, S D=10.7)$. In Study 2, there were three hundred eighteen individuals from Portugal, most of them were women $(56,6 \%)$ and had an undergrad degree $(79,9 \%)$, with age range from 19 to 70 years $(M=40.6, S D=10.1)$. We perform a confirmatory factor analyses aiming to replicate the factorial solution identified in Study 1. The two-factor solution (F1- Adaptiveness; F2- Innovativeness) proposed in Study 1 had acceptable to marginal fit indices: $\chi^{2}=127,8, d f=34, \chi 2 / d f=3.76$, GFI $=.93$, CFI $=.83$, RMSEA $=.093$, CI $90 \%$ $[.076, .0111]$, ECVI $=0.54$. As in the study of 2018, two factors, Adaptiveness and Innovativeness, emerged and are composed by the same items, which led us, in the discussion, to a reflection about the parental practices features. Our model, already called TTT - Two Tracks of Thought, highlights the importance of both non-conventional and conventional thinking for the creative process.
\end{abstract}

Keywords: Creativity, TCT-DP, confirmatory factorial analysis.

\section{Introduction}

Creative thinking has been seen has a relevant dimension for those individuals and organizations that aim to reach their diversified goals, to solve their demanding problems and to be competitive and thus achieving a prolonged success (Hennessey \& Amabile, 2010). Despite the importance that has been given to creativity, organizations have not been able to promote it (Amabile, 1998). According to Halpern (2003), if we want to increase the creative potential, we must value it in the first place.

Jellen and Urban (1986) presented a comprehensive model of creativity that sustains the Test for Creative Thinking - Drawing Production (TCT-DP) that aims to evaluate several dimensions of the creative thinking, such as elaboration, composition, risk-taking, unconventional thinking and humor. The debate about the evaluation measures of creativity has unleashed one very important question related to the underlying construct of the creative assessment instruments. If some authors (e.g. Clapham, 1998) suggests its unidimensionality, Guilford (1956) and Torrance (1988) traditionally consider the multidimensionality of divergent thinking. Some other authors, namely Kim (2006), support the two-dimensionality of divergent thinking, defending the importance of both conventional and non-conventional way of thinking for the creative process. The dichotomy between divergent and convergent thinking was seen as a fake dichotomy, by Runco (2007), since developing and producing new ideas, by the divergent way of thinking, inevitably demands an evaluation and selection of the best and most appropriate ones, by the convergent way of thinking.

The study of Ibérico Nogueira, Almeida and Lima (2017) was the first to test the factor structure of the TCT-DP, through a confirmatory approach, with 969 Portuguese university students. A study of, Almeida, Ibérico Nogueira and Lima (2018) tested the factorial structure of TCT-DP for 883 Portuguese 
workers. A two-factor solution was obtained for the referred studies. The factors, called Adaptiveness and Innovativeness, seem to represent both conventional and non-conventional thinking. However, for the last study with Portuguese workers, some of the items that were part of the Adaptiveness factor, raised some questions about its pertinence. The present study aims to assess the factorial structure of the TCT-DP, through a confirmatory approach, for another Portuguese adult sample, of 620 participants in order to clarify the structure previously obtained with Portuguese workers.

\section{Method}

Testing the factor structure of the TCT-DP, a two-step approach was used. The sample originated two groups. In study 1 , the first group $(\mathrm{N}=302)$ was used for an exploratory approach aiming to investigate the factorial structure of the TCT-DP. In study 2, the second group $(\mathrm{N}=318)$ was used, aiming to replicate, through a confirmatory approach, the factor structure observed in Study 1.

The Instruments and Procedure were the same for both studies.

\subsection{Study 1}

2.1.1. Participants. In this study, participated 302 Portuguese workers, most of them women (55,6\%), with an undergraduate degree $(74,8 \%)$, with age range from 18 to 86 years $(M=41.1, S D=10.7)$.

2.1.2. Instruments. Urban and Jellen (1996) developed the Test for Creative Thinking-Drawing Production (TCT-DP) aiming to assess the creative potential of individuals from five years' age. It has a figurative pattern and fourteen criteria assessment. It has good psychometric qualities for the Portuguese population (e.g. Ibérico Nogueira, Almeida, \& Lima, 2017). A sociodemographic questionnaire was also applied.

2.1.3. Procedure. Between October of 2018 and February of 2019, the authors of the present study contacted, via telephone, e-mail or in person, the directors of companies of secondary and tertiary sectors, public and private, of small, medium and large dimension. After the permission to assess either lieders or employees, the authors explained the study objectives, instruments, the confidentiality and withdraw policy.

2.1.4. Results. To assess the factor structure of the scale, an exploratory factor analysis (EFA) was performed. A principal axis factoring (PAF) was used as the extraction method with a varimax rotation. To determine the number of factors to be extracted in the final solution we use Horn's parallel analysis and the interpretability of the solution. The cut-off used for factor loading was .30. A Kaiser-Meyer-Olkin $(\mathrm{KMO}=0.71)$ and Bartlett's Test of Sphericity, $\chi^{2}(45)=612, p<.001$, indicated the sampling adequacy for the analysis. Three components had eigenvalues greater than the Kaiser criterion of 1, which accounted for $55.3 \%$ of the variance. Additionally, a parallel analysis (100 datasets; IC 95\%) indicated the extraction of two factors. A second PAF was conducted, fixing the extraction of two factors. The two factors explained $44.6 \%$ of the variance. The first factor was referred to as Adaptiveness or Conventional thinking, which explained $29.3 \%$ of the variance and retained four items (Cn-Continuations, Cm-Completions, Bfd-Boundary-breaking being fragment dependent and Bfi-Boundary-breaking being fragment-independent), with factor loadings that ranged from .72 to .50 (Cronbach alpha $=0,72$ ). The second factor, referred to as Innovativeness or Non-conventional thinking, explained $15.3 \%$ of the variance and retained six items (Nee-New elements, Hu-Humor/emotionality, Cth-Connections to Theme, Uct-Unconventionality A, B, C and D, Pe-Perspective and Cl-Connections with lines) with factor loadings that ranged between .62 and .30 (Cronbach alpha $=0,63$ ).

\subsection{Study 2}

2.2.1. Participants. In the second study, participated 318 workers from Portugal, most of them women (56,6\%) and with an undergraduate degree $(79,9 \%)$, with age range from 19 to 70 years $(\mathrm{M}=40.6$, $\mathrm{SD}=10.1)$.

2.2.2. Results. The confirmatory factor analyses aim to replicate the factorial solution identified in Study 1. The estimation method used was the Maximum Likelihood Estimation (MLE) using the variance-covariance matrix, and the missing cases were replaced by the mean. The two-factor solution (F1, Conventional; F2, Non-conventional) proposed in Study 1 had acceptable to marginal fit indices: $\chi 2=127,8, d f=34, \chi 2 / d f=3.76$, GFI $=.93$, CFI $=.83$, RMSEA $=.093$, CI 90\% [.076, .0111], ECVI $=0.54$. All the factor loadings are statistically significant $(p<0,05)$. Both factors had acceptable values of Cronbach's alpha (F1, $\alpha=.69 ; \mathrm{F} 2, \alpha=.63)$. 


\section{Discussion and conclusion}

The aim of this study is to clarify the factorial structure of the TCT-DP for Portuguese workers. Using a confirmatory factor analysis, Study 2 replicates the factor structure of Study 1 . Two factors were obtained. The Innovativeness factor includes the items related to the Unconventional way of thinking (Uct), New elements (Nee), Humor (Hu), Perspective (Pe), Connections to theme (Cth) and Connections with lines $(\mathrm{Cl})$. The second factor, Adaptiveness, includes more conventional items such as Continuations (Cn) and Completions (Cm), as well the Bfd (Boundary-breaking being fragment dependent) and Bfi (Boundary-breaking being fragment-independent), traditionally considered as evidence of the willingness to take risks undeniably being among the most creative. A two-factor solution supports the two-factor solution of the previous study with a worker sample (Almeida, Ibérico Nogueira, \& Lima, 2018) in which there were found the exactly same items for each factor. In that previous study, the inclusion in Adaptiveness factor of those items dealing with the risk assumption was puzzling, in a certain way. The confirmation with the present study led us to rethink the pertinence of this structure and to raise the hypothesis that risk-taking may be a feature present in most individuals, regardless of their levels of creativity, given that possibly due to parental educational practices, individuals are less and less censored for their less-compliant behavior. The two-factor solution, Innovativeness and Adaptiveness, and the correlation between them, suggests that both forms of thinking seem inseparable, despite they can occur at different stages of the creative process, as Runco (2007) or Jaarsveld, Lachmann and Leeuwen (2012) highlighted. Since this study was carried out with working adults, it is not possible to generalize the results to the rest of the Portuguese population. In the future, further confirmatory factor analysis studies will be conducted with other specific population groups (e.g. private schools, different education areas).

\section{References}

Amabile, T. M. (1998). How to Kill Creativity. Harvard Business Review, 76(5), 76-87.

Almeida, L., Ibérico-Nogueira, S., \& Lima, T. (2018, July). In the path of Two Tracks of Thought: A Structural Model of the Test for Creative Thinking-Drawing Production (TCT-DP) in Portuguese workers. 16th ICIE conference 2018 on Excellence, Innovation, \& Creativity in Basic-Higher Education \& Psychology - University Paris Descartes, July 3-6, 2018.

Clapham, M. M. (1998). Structure of figural forms A and B of the Torrance Tests of Creative Thinking. Educational and Psychological Measurement, 58(2), 275-283. doi: 10.1177\%2f0013164498058002010

Finke, R. A., Ward, T. B., \& Smith, S. M. (1992). Creative Cognition: Theory, Research, and Applications. Cambridge, MA: MIT Press.

Guilford, J. P. (1956). The structure of intellect model. Psychological Bulletin, 53, 267-293.

Hennessey, B. A., \& Amabile, T. M. (2010). Creativity. Annual Review of Psychology, 61, 569-598.doi:10.1146/annurev.psych.093008.100416

Ibérico Nogueira, S., Almeida, L., \& Lima, T. S. (2017). TTT- Two Tracks of Thought: A structural model of the Test for Creative Thinking-Drawing Production (TCT-DP). Creative Research Journal, 29(2), 206-211. doi: 10.1080/10400419.2017.1303312

Jaarsveld, S., Lachmann, T., \& Leeuwen, C. V. (2012). Creative reasoning across developmental levels: Convergence and divergence in problem creation. Intelligence, 40, 172- 188. doi:10.1016/j.intell.2012.01.002

Jellen, H., \& Urban, K. K. (1986). The TCT-DP (Test for Creative Thinking - Drawing Production): An instrument that can be applied to most age and ability groups. Creative Child and Adult Quarterly, 11, 138-155.

Kim, K. H. (2006). Is creativity unidimensional or multidimensional? Analyses of the Torrance Tests of Creative Thinking. Creativity Research Journal, 18(3), 251-259. doi: 10.1207/s15326934crj1803_2

Runco, M. A. (2007). Creativity. Theories and themes: research, development, and practice. USA: Elsevier Academic Press.

Torrance, E. P. (1988). The nature of creativity as manifest in its testing. In R. J. Sternberg (Ed.), The nature of creativity: contemporary psychological perspectives (pp. 43-75). NY: Cambridge University Press.

Urban, K. K., \& Jellen, H. G. (1996). Manual of Test for Creative Thinking-Drawing Production (TCT-DP). Amsterdam: Harcourt Test Publishers. 


\title{
POSITIVE EMOTIONAL ATMOSPHERE OR TECHNOLOGY-BASED TRAINING: TEACHERS' PRIORITIES DEPENDING ON THEIR PERSONALITY
}

\author{
Kristina Kovalcikiene, \& Sigitas Daukilas \\ Department of Philosophy, Psychology and Vocational Education, Vytautas Magnus University \\ (Lithuania)
}

\begin{abstract}
Positive classroom atmosphere is one of the most powerful tools to enhance and promote students' learning. Moreover, a modern teacher is encouraged to keep up with technology and use it in the teaching process. From the perspective of professional roles multidimensionality approach, psychologists emphasize that we cannot perform all roles equally. Because each role involves different contexts, relationships, responsibilities and likely different personality characteristics. This study aimed to identify teacher's personality characteristics (personality traits and professional identity), which determines the teachers' priority in classroom. 436 vocational teachers from Lithuania filled up a self-administered questionnaire. It consisted of the Teachers' Professional Identity Questionnaire (Beijaard et al., 2000), Big Five Inventory (BFI; John et al., 2008) and two items to clarify VET teachers' priorities in the classroom. The results revealed that two completely different characteristics of personality are related to different preferences in the classroom. The personality trait of agreeableness is significant in predicting preference to favor a good emotional atmosphere $(\mathrm{p}<.01)$, and the didactical expert role predicts teachers' preference to favor technology-based training $(\mathrm{p}<.001)$.
\end{abstract}

Keywords: Vocational teachers, professional identity, personality traits, technology-based training, classroom atmosphere.

\section{Introduction}

Researchers emphasize the teacher's personality in the process of successful education. The activities of a modern teacher, innovativeness and creativity are an important incentive to motivate society to progress. There is enough evidence that students' learning achievements are related to teacher's experience, the style of teaching, the teaching tools used and the initiatives of a teacher (Blazar, 2016). With appearance of new technologies (photography, film, radio, television) and the whole social life has taken up by the media (Masschelein \& Simons, 2015), teachers have got greater opportunities for showing ideas in different ways, while presenting new materials and thereby motivating students to learn (Jokic, et al., 2009). On the other hand, "teaching cannot be reduced to a technical or instrumental action that results in learning gains with students" (Beijaard et al., 2000, p. 751). Social, emotional, and moral support for students, positive classroom climate, and student well-being are essential for teaching and learning. Creating a positive and engaging classroom atmosphere is one of the most powerful tools teachers can use to enhance, promote, and encourage students' learning and prevent problem behaviors (Wilson-Fleming \& Wilson-Younge, 2012).

Two of the most common characteristics of teachers for successful or effective education: personality traits and professional identity. Professional identity formation is a dynamic process, where teachers constantly organize their personal and professional "selves" based on their experiences (Cruess et al., 2015; Kavanoz \& Yüksel, 2017). Professional identity encompasses the teachers' perceptions of themselves as teachers (Canrinus, 2011). Why is professional identity so important? Clarity of professional identity leads to a sense of meaning in activity (Morgan, 2017). Also, professional identity is important for various work processes and outcomes, such as motivation, engagement, innovative behavior and creativity, relationships, work efficiency, etc. (Kavanoz \& Yüksel, 2017; Schutz et al., 2018). Teachers' professional identity determines teaching methods, attitudes towards professional development, preparation to meet the educational challenges in their teaching practice, the quality of education, the ability to promote change and foster innovation (Sardabi et al., 2018; Thomas \& Mockler, 2018). 
Personality traits are identified as important factors in various working situations, because they define stable personal characteristics and determine behavior (Mount et al., 2005). Personality traits, as well as professional identity, are one of the most important elements in the education, because the researchers emphasize personality traits as substantial to positive processes and results of any professional activity (Eryilmaz, 2014; Paleksić et al., 2017; Rusbadrol et al., 2015). Teachers' personality traits are related with their job satisfaction (Paleksić el al., 2017), work performance (Rusbadrol et al., 2015), subjective wellbeing and students' academic performance (Eryilmaz, 2014).

The current study attempted to reveal what psychological factors of a teacher (i.e., personality traits and professional identity) predict preferences of positive emotional classroom atmosphere or technology-based training. A more thorough understanding of the significance of teacher's psychological characteristics for working preferences in classroom is relevant, because different professional roles require different skill sets and attitudes. Thus, the analysis of the predictive value of the teachers' personality characteristics (personality traits and professional identity) for their preferences (for technology use or positive emotional atmosphere) were chosen in this article.

\section{Methods}

The data were collected in 2016 and 2017 as part of a large national survey of vocational teachers in Lithuania. 436 vocational teachers completed paper-based self-administered questionnaires and returned to researchers in sealed envelopes. Teachers' Professional Identity Questionnaire (Beijaard et al., 2000) was used to measure three professional roles of teachers: subject matter expert $(\alpha=.637)$, pedagogical expert (.692), and didactical expert (.700). Big Five Inventory (BFI; John et al., 2008) was used to assess vocational teachers' personality traits: extraversion $(\alpha=.671)$, agreeableness (.584), conscientiousness (.739), neuroticism (.676), and openness to experience (.558). Also, preferences for good emotional atmosphere or technology-based training were assessed by asking to answering two items: "I try to create a good emotional atmosphere in lectures" and "I try to use training based on information technology" in 5-point Likert scale (form $1=$ do not use at all to $5=$ often use).

\section{Results}

The analysis of descriptive statistics and correlations presented modest correlations among good emotional atmosphere and professional identity (.239; .264; .255, $\mathrm{p}<.01$ in all cases), as well as personality traits (.159; .246; .235; -.170; .188, $\mathrm{p}<.01$ in all cases). Modest correlations were also found between technology-based training and professional identity $(.171 ; .248 ; .111, \mathrm{p}<.01$ in all cases) and were not related with personality traits. Vocational teachers more often tend to favor a good emotional atmosphere in the classroom $(\mathrm{M}=4.47, \mathrm{SD}=.656)$ rather than using training-based information technology $(\mathrm{M}=3.83$, $\mathrm{SD}=.908)$.

Two regression equations were conducted to identify psychological factors that are important for the preferences to favor a good emotional atmosphere (Model 1) and technology-based training (Model 2) by vocational teachers in their lectures. Both regression models are statistically significant $(\mathrm{F}(8)=8.091$, $\mathrm{p}<.001 ; \mathrm{F}(8)=3.893, \mathrm{p}<.001)$. The results of the regression analysis revealed that the personality trait of agreeableness $(\beta=.140, \mathrm{p}<.01)$ is significant in predicting vocational teachers' preference to favor a good emotional atmosphere in classroom. Also, the analysis highlighted that didactical expert professional role of vocational teachers predicts their preference to favor technology-based training $(\beta=.267, \mathrm{p}<.001)$. The results showed that vocational teachers' altruism, inclination to help others, the ability to understand and sympathize with others are the psychological characteristics of teacher who creates a positive school atmosphere and emotionally healthy classroom environment; second, a didactical expert is a teacher who bases his or her work on knowledge and skills regarding the planning, execution, and evaluation of teaching and learning processes and this ensures the use of advanced and innovative technology-based teaching in lectures.

\section{Discussion and conclusions}

A teacher's work is strictly regulated and defined in written documents according to global and local standards from the one side. From the other side, a teacher is expected to be creative and able to think and work in an innovative manner (Duobiene, 2017), decreasing of studying durability, faster matter update, providing constant availability of data and information, individualization of course (Jokic et al., 2009). The results of this study revealed that two completely different personalities of teachers are related to their different preferences in the classroom. Whereas we can't do everything equally well because of 
our personality peculiarities, so we give priority to the activities, roles or functions we perform. Thus, managers of educational institutions should not expect teachers to be good in all areas. Therefore, it is recommended to form school personnel and to assign tasks according to the peculiarities of teachers' personality. Also, teamwork provides opportunities to learn from each other and achieve desired results, motivate society to progress, etc.

Summarizing the results of this study, it can be said that further research in this topic is desirable. It would be useful to explore other psychological characteristics of teachers that are related to their preferences for activities in classroom. In addition, it would be useful to include a learning achievement variable and see what is more effective - using modern technologies in the education process or maintaining a supportive classroom atmosphere that encourages students to feel comfortable in the teacher-student relationship.

\section{References}

Beijaard, D., Verloop, N., \& Vermunt, J. D. (2000). Teachers' perceptions of professional identity: an exploratory study from a personal knowledge perspective. Teaching and Teacher Education, 16, 749-764.

Blazar, D. (2016). Teacher and Teaching Effects on Students' Academic Performance, Attitudes, and Behaviors (Doctoral dissertation), Harvard Graduate School of Education.

Canrinus, E. T. (2011). Teachers' sense of their professional identity. Groningen: PrintPartners Ipskamp B.V., Enschede, The Netherlands. 150 p.

Cruess, R. L., Cruess, S. R., Boudreau, J. D., Snell, L., \& Steinert, Y. (2015). A schematic representation of the professional identity formation and socialization of medical students and residents: A guide for medical educators. Academic Medicine, 90(6), 718-725.

Duobliené, L. (2017). The schizoid teacher situation: a danger for identity and a precondition for creativity [in Lithuanian]. Acta Paedagogica Vilnensia, 38, 11-24.

Eryilmaz, A. (2014). Perceived Personality Traits and Types of Teachers and Their Relationship to the Subjective Well-Being and Academic Achievements of Adolescents. Educational Sciences: Theory and Practice, 14(6), 2049-2062.

John, O. P., Naumann, L. P., \& Soto, C. J. (2008). Paradigm Shift to the Integrative Big-Five Trait Taxonomy: History, Measurement, and Conceptual Issues. In O. P. John, R. W. Robins, \& L. A. Pervin (Eds.) Handbook of personality: Theory and Research (pp. 114-158). New York, NY: Guilford Press.

Jokic, S., Pardanjac, M., \& Radosav, D. (2009). Teachers and use of information technology in education: Difficulties and opportunities. Proceedings of the 20th International DAAAM Symposium, 20(1), 1453-1454.

Kavanoz, S., \& Yüksel, H. G. (2017). Motivations and Concerns: Voices from Preservice Language Teachers. Australian Journal of Teacher Education, 42(8), 43-61.

Masschelein, J., \& Simons, M. (2015). Education in times of fast learning: the future of the school. Ethics and Education, 10(1), 84-95.

Morgan, A. (2017). Cultivating critical reflection: educators making sense and meaning of professional identity and relational dynamics in complex practice. Teaching Education, 28(1), 41-55.

Mount, M. K., Barrick, M. R., Scullen, S. M., \& Rounols, J. (2005). Higher - order dimensions of the big five personality traits and the big six vocational interest types. Personnel Psychology, 58, 447-478.

Paleksić, V., Narić, S., Vukotić, M., \& Stankovć, S. (2017). The Relationship between Personality Traits and Job Satisfaction of Teachers. Scripta Medica, 48(1), 11-16.

Rusbadrol, N. B., Mahmud, N., \& Arif, L. S. M. (2015). Association between Personality Traits and Job Performance among Secondary School Teachers. International Academic Research Journal of Social Science, 1(2), 1-6.

Sardabi, N., Biria, R., \& Golestan, A. A. (2018). Reshaping Teacher Professional Identity through Critical Pedagogy-Informed Teacher Education. International Journal of Instruction, 11(3), 617-634.

Schutz, P., Hong, J. Y., \& Francis, D. C. (2018). Research on Teacher Identity: Mapping Challenges and Innovations. Springer.

Thomas, M. A. M., \& Mockler, N. (2018). Alternative routes to teacher professional identity: Exploring the conflated sub-identities of Teach for America corps members. Education Policy Analysis Archives, 26(6), 1-25.

Wilson-Fleming, L., \& Wilson-Younge, D. (2012). Positive classroom environments = Positive academic results. Research paper. Alcorn State University. Retrieved from https:/files.eric.ed.gov/fulltext/ED536465.pdf 


\title{
NORMATIVE VALUES FOR THE TEST FOR CREATIVE THINKING-DRAWING PRODUCTION (TCT-DP), OVER THE SCHOOL YEARS IN PORTUGUESE PRIVATE SCHOOLS
}

\author{
Leonor Almeida ${ }^{1}$, Sara Ibérico Nogueira ${ }^{2}$, Tiago Souza Lima ${ }^{3}, \&$ Maria Manuel Sousa ${ }^{4}$ \\ ${ }^{I} I S G \mid$ Business \& Economics School/Associate Professor (Portugal) \\ ${ }^{2}$ Escola de Psicologia e Ciências da Vida, ULHT, Associate Professor (Portugal) \\ ${ }^{3}$ Universidade de Fortaleza, UNIFOR, Assistant Professor (Brazil) \\ ${ }^{4}$ Catholic University of Lisbon, Psychology Master (Portugal)
}

\begin{abstract}
The Test for Creative Thinking-Drawing Production (TCT-DP) has been widely used for the creative potential assessment. However, there are no tables of normative values for the different years of schooling and gender in the Portuguese private schools. This is our contribution in this work.

Take part in this study 294 individuals from Portugal, with age range from 15 to 22 years $(\mathrm{M}=17.7$, $\mathrm{SD}=1.64)$, most of them were women $(55 \%)$ and was enrolled in the $10^{\text {th }}(60.6 \%)$ and 12 th school year $(39.4 \%)$

The t-tests performed indicate that there are no statistically significant differences between the school years for the two factors of the TCT-DP and for a total factor. Regarding gender, statistically significant differences were observed only for the conventional factor, with women having a higher mean than men. The present study is the first phase of a larger research project that aims to characterize the levels of creativity and elaboration of normative tables of students of private schools.
\end{abstract}

Keywords: Creativity, TCT-DP, assessment, private schools.

\section{Introduction}

Considering the distinction between private and public education, we are currently developing some studies to characterize the levels of creativity in private education, given that we have already done it for students in public education (Ibérico Nogueira, Almeida, \& Lima, 2018). In particular, these are students of private education and, simultaneously, attending the professional branch at the 10th and 12th years of schooling.

Private education in Portugal (with the exception of higher education) has in quantitative terms and in frequency matters little expression. Portugal follows most European Union (Ministry of Education, 2015) countries.

We can characterize Portuguese educational establishments (compulsory education) as follows: a) Religious Schools where the schools held by religious congregations stand out. Their main objective is to educate their students based on humanistic and religious values. This type of institution gives priority to high-quality academic discipline and performance; b) Other Private Schools are distinguished from public schools in that they preserve excellent teaching, individual and individual attendance of the student, as well as great flexibility in the treatment of problem situations. Here too, great importance is given to the technological means used and the quality of teaching resources and facilities; c) Public schools, they emphasize the formation of the individual taking into account their participation in the social demands.

The sample collected for our study focuses on Private Schools and we intend to make a characterization of these students regarding their levels of creativity and to present its the normative values. It is also intended to carry out the comparative study of differences between gender and years of schooling in what concerns their creativity level. 


\section{Method}

\subsection{Participants}

Take part in this study 294 individuals from private schools, of the region of Lisbon and Porto with age range from 15 to 22 years $(\mathrm{M}=17.7, \mathrm{SD}=1.64)$, most of them were women $(55 \%)$ and was enrolled in the $10^{\text {th }}(60.6 \%)$ and 12 th school year $(39.4 \%)$.

\subsection{Instruments}

The TCT-DP was developed in 1985 by Urban and Jellen $(1986,1996)$ to assess the creative potential of individuals. It has a figurative pattern and its characteristics, evaluation criteria and its advantages are broadly presented by the authors and collaborators (Dollinger, Urban, \& James, 2004, Urban, 1991). Several studies of validation carried out in the Portuguese population reveal that the instrument has good psychometric qualities (e.g. Ibérico Nogueira, Almeida, \& Lima, 2017). It was also applied a sociodemographic questionnaire.

\subsection{Procedure}

The authors of this study contacted, via e-mail, the directors of several private schools in Lisboa and Porto to obtain permission to evaluate students in their institutional context. After permission was granted, we visited the schools and explained the study objectives to the teachers, psychologists and students, who were then asked to participate. All students and the charge of the student's education were informed regarding the instruments, the confidentiality of their answers and the permission to withdraw the study at any time. The students who agreed received the research protocol in the classroom setting.

\section{Results}

T-tests were performed to evaluate differences in averages for the factors of the TCT-DP according to gender and school year. In addition, for the construction of normative tables, descriptive statistics of mean and percentile were performed.

The results of t-tests performed indicate that there are no statistically significant differences between the school years for the two factors of the TCT-DP and for the total of TCT-DP. Regarding gender, statistically significant differences were observed only for the conventional factor $\mathrm{t}(246)=-2,33, p<0.05$, with women having a higher mean $(\mathrm{M}=11,7, \mathrm{SD}=3,10)$ than men $(\mathrm{M}=10,8$, $\mathrm{SD}=3,01)$.

Based on the results of the tests $t$, a table with the normative values (Table 1) was constructed for the total sample.

Table 1. Normative values.

\begin{tabular}{llll}
\hline & Conventional & Non-conventional & TCT - total \\
\hline Mean & 11.29 & 6.56 & 17.84 \\
Standard deviation & 3.09 & 4.43 & 5.97 \\
Minimum & 3.00 & 0.00 & 3.00 \\
Maximum & 22.00 & 26.00 & 38.00 \\
10 & 8.00 & 2.00 & 11.00 \\
20 & 9.00 & 3.00 & 12.00 \\
30 & 10.00 & 4.00 & 14.00 \\
40 & 10.00 & 5.00 & 16.00 \\
50 & 11.00 & 6.00 & 17.00 \\
60 & 12.00 & 7.00 & 19.00 \\
70 & 13.00 & 8.00 & 20.30 \\
80 & 14.00 & 10.00 & 23.00 \\
90 & 15.00 & 13.00 & 25.00 \\
\hline
\end{tabular}


In addition, a second normative table was constructed for the conventional factor, with percentile values separate for male and females, since these groups presented statistically different means in that factor (Table 2).

Table 2. Normative values for conventional factor.

\begin{tabular}{lll}
\hline & Male & Female \\
\hline Mean & 10.78 & 11.69 \\
Standard deviation & 3.01 & 3.10 \\
Minimum & 3.00 & 6.00 \\
Maximum & 18.00 & 22.00 \\
10 & 7.00 & 8.00 \\
20 & 8.00 & 9.00 \\
30 & 10.00 & 9.40 \\
40 & 10.00 & 10.00 \\
50 & 10.00 & 11.00 \\
60 & 12.00 & 12.00 \\
70 & 12.00 & 13.00 \\
80 & 13.00 & 15.00 \\
90 & 15.00 & 16.00 \\
\hline
\end{tabular}

\section{Discussion /conclusions}

In this study we developed a characterization of Private Schools students regarding their levels of creativity and we presented its the normative values. It is also carried out the comparative study of differences between gender and years of schooling in what concerns their creativity level. The results are consistent with those obtained by Ibérico Nogueira, Almeida and Lima (2018).

The present study is the first phase of a larger research project that aims to characterize the levels of creativity and the elaboration of normative tables for students of private schools. In the future, we will present their results as well as the comparisons between the averages obtained by public and private education.

\section{References}

Ibérico-Nogueira, S., Almeida, L., \& Lima, T. (2018, July). Normative values for the Test for Creative Thinking-Drawing Production (TCT-DP), over the school years in Portuguese context. 16th ICIE conference 2018 on Excellence, Innovation, \& Creativity in Basic-Higher Education \& Psychology - University Paris Descartes, July 3-6, 2018.

Ibérico Nogueira, S., Almeida, L., Lima, T. S. (2017). Factorial invariance across gender in the Portuguese version for the Test for Creative Thinking-Drawing Production. 15th ICIE International Conference on Excellence, Innovation, \& Creativity in Basic-Higher Education \& Psychology Latest Development in Research \& Practices Faculty of Psychology - University of Lisbon, Portugal, July 3-5, 2017.

Jellen, H., \& Urban, K. K. (1986). The TCT-DP (Test for Creative Thinking - Drawing Production): An instrument that can be applied to most age and ability groups. Creative Child and Adult Quarterly, 11, 138-155.

Ministério da Educação (2015). Ensino Particular - Ensino não oficial. Formas e Estatutos nos Estados Membros da União Europeia. Lisboa. ME, DEP-GEF.

Urban, K. K., \& Jellen, H. G. (1996). Manual of Test for Creative Thinking-Drawing Production (TCT-DP). Amsterdam: Harcourt Test Publishers. 


\title{
EMPATHY AND CLASSROOM CLIMATE AS PREDICTORS OF STUDENT'S WELL-BEING: AN INTERNATIONAL PILOT STUDY
}

\author{
Manja Veldin ${ }^{1}$, Ana Kozina ${ }^{1}$, Tina Vršnik Perše ${ }^{2}$, Maša Vidmar ${ }^{1}$, Ivana Jugović3 ${ }^{3}$, \\ \& Magnus Oskarsson ${ }^{4}$ \\ ${ }^{1}$ Educational Research Institute (Slovenia) \\ ${ }^{2}$ Faculty of Education at University of Maribor (Slovenia) \\ ${ }^{3}$ Institute for Social Research in Zagreb (Croatia) \\ ${ }^{4}$ Mid Sweden University (Sweden)
}

\begin{abstract}
One of the key ingredients of social, emotional and intercultural competencies, which contributes significantly to a positive and inclusive classroom climate is empathy (Zorza, 2015). Empathy enables us to understand others and feel connected with them (Eisenberg, et al., 1996). High ability of empathy, which includes cognitive (e.g. taking the perspective of another) and emotional components (e.g. personal distress), is associated with numerous positive effects on relationships and behaviour (e.g. prosocial behaviour), while the lack of it is associated with many negative effects (e.g. aggression) (Davis, 1980). Establishment of positive relationships with others and empathy are associated with psychological well-being of students (Shanafelt et al, 2005). The aim of the present study was to analyse the associations between empathy, perceived classroom climate and well-being among students of the 8th-grade. We will present the analysis from three EU countries ( $N$ : Slovenia: 107, Sweden: 86, and Croatia: 79) who participated in the pilot of the European Erasmus KA3+ Hand in Hand project. We analysed predictive power of empathy (IRI; Davis, 1980) and the perceived classroom climate (Positive class climate; Stöber, 2002) on the well-being (WEMWBS; Stewart-Brown et al., 2011). Results show, that in all countries, personal distress (one component of empathy) is an important predictor of the student's well-being. Results are discussed in light of guidelines for school practice.
\end{abstract}

Keywords: Empathy, classroom climate, well-being, students.

\section{Introduction}

Subjective well-being of students and its predictors have high importance in the promotion of adolescents positive functioning. One of them is empathy (Shanafelt et al., 2005) that enables us to understand others and feel connected with them (Eisenberg, et al., 1996). It represents one of the key ingredients of social, emotional and intercultural (SEI) competencies, which contribute significantly to a positive and inclusive classroom climate (Zorza, 2015). High ability of empathy, which includes cognitive (fantasy and perspective taking) and emotional components (emphatic concern and personal distress) (Davis, 1980), is associated with numerous positive effects on relationships and behaviour (e.g. prosocial behaviour; Decety et al., 2016), while the lack of it is associated with many negative effects (e.g. aggression; Jolliffe \& Farrington, 2006). It has been shown that empathy (and via its positive effect on the positive relationships with others), is connected to higher perceived psychological well-being of students (Shanafelt et al., 2005). Furthermore, the positive classroom climate, which involves positive relationships, classroom management, and structure, is also connected to well-being (Cocoradă \& Orzea, 2017). The aim of the present study was to analyse the associations between empathy, perceived classroom climate and well-being among students of the 8th-grade in three EU countries. 


\section{Method}

\subsection{Participants}

A convenience sample of $8^{\text {th }}$-grade (13 to 14-year-olds) students from three EU countries (N: Slovenia: 107; 47,7 \% males; Sweden: 86; 44,2 \% males; and Croatia: 79; 68,4 \% males) were invited to participate in the pilot of the Hand in Hand project, which aims to increase the social, emotional (e.g. self-awareness, self-management) and intercultural competencies of students and school staff.

\subsection{Instruments}

Interpersonal Reactivity Index (IRI; Davis, 1980) was used as a measure of empathy. It consists of four scales; two of them labelled as emotional empathy (empathic concern, personal distress) and the other two as cognitive empathy (perspective taking, fantasy). Each has 7 items on a 4-point Likert scale (1 - "Never", 4 - "Often"). Cronbach's alpha scores ranged from .65 to .74 (Slovenia) and .61 to .79 (Sweden) and from .66 to .76 (Croatia). Positive class climate (Positives Klassenklima-KLAKL; Stöber, 2002) was used to measure the perceived positive classroom climate. It includes 10 items on a 4-point Likert scale (1 - "Strongly disagree", 4 - "Strongly agree"). Cronbach's alpha scores ranged from .68 (Slovenia) and .76 (Sweden) to .78 (Croatia). The Warwick-Edinburgh Mental Well-being Scale (WEMWBS; Stewart-Brown et al., 2011) was used to assess the perceived well-being of students. It consists of 14 items on a 4-point Likert scale (1 - "Never", 4 - "Often"). Cronbach's alpha scores ranged from .78 (Slovenia) and .79 (Croatia) to .86 (Sweden).

\subsection{Procedure}

From December 2017 to March 2018, students completed a battery of questionnaires tapping SEI competencies and demographic variables either in the paper version (Slovenia and Croatia) or online (Sweden). For the purpose of this study, we only present data for the selected measures. Reliability tests, descriptive statistics, t-test and multiple linear regression for each country were calculated with IBM SPSS Statistics 21.

\section{Results}

In Table 1, we can see the means and standard deviations for the samples from each participating country. Both, Slovenian students as Croatian, report more frequently experiencing emphatic concern than Sweden students, $(t(186)=4,128, p=.000$ and $t(158)=-2,104, p=.037)$. On the other hand, the Croatian students report the most frequently experienced personal distress, significantly higher than in Sweden that has the lowest average at this scale, $t(157)=-2,347, p=.020$. There were no statistically significant differences between countries on other scales.

Table 1. Descriptive statistics of empathy, classroom climate and well-being of students across countries.

\begin{tabular}{lllcccc}
\hline & \multicolumn{2}{c}{ Slovenia } & \multicolumn{2}{c}{ Sweden } & \multicolumn{2}{c}{ Croatia } \\
& $M$ & $S D$ & $M$ & $S D$ & $M$ & \\
\hline Empathy & & & & & & \\
Fantasy & 2,523 & 0,650 & 2,483 & 0,617 & 2,570 & 0,627 \\
Empathic concern & 2,964 & 0,497 & 2,693 & 0,502 & 2,853 & 0,637 \\
$\quad$ Perspective taking & 2,613 & 0,499 & 2,594 & 0,637 & 2,613 & 0,532 \\
Personal distress & 2,297 & 0,529 & 2,159 & 0,618 & 2,357 & 0,561 \\
Positive class climate & 2,580 & 0,388 & 2,555 & 0,462 & 2,491 & 0,484 \\
Well-being & 3,063 & 0,398 & 3,100 & 0,534 & 3,184 & 0,438 \\
\hline
\end{tabular}

In Table 2, multiple linear regression for each country is presented, with all four subscales of empathy and classroom climate as predictors included. Personal distress, a subscale of empathy is a significant negative predictor in all participating countries and the only significant one in the Croatian sample. If we use personal distress as our only predictor of the selected outcome, it explains 20,6\% (Slovenia), 12,1 \% (Sweden) and 9,6 \% of the well-being's variance. In the Slovenian sample, emphatic concern is also an important positive predictor. In Sweden, that has 40,7 \% of the well-being variance explained, another empathy subscale (fantasy) is an important positive predictor, and as the only one from our samples, has a significant contribution of the positive classroom climate to the explained variance. 
Table 2. Four components of empathy and positive class climate as predictors of well-being across countries.

\begin{tabular}{|c|c|c|c|c|c|}
\hline & $B(S E)$ & $\bar{\beta}$ & $t$ & $\mathrm{R}^{2}$ & $\mathrm{R}^{2 *}$ \\
\hline \multicolumn{6}{|c|}{ Slovenia $(F(5,98)=7,293, p=.000)$} \\
\hline Constant & $3,210(, 357)$ & & $8,987 * * *$ & & \\
\hline Fantasy &,$- 008(, 062)$ & $-0,014$ & $-0,137$ & & \\
\hline Empathic concern & $210(, 091)$ & 0,265 & $2,309 *$ & & \\
\hline Perspective taking &,$- 023(, 090)$ & $-0,029$ & $-0,253$ & & \\
\hline Personal distress &,$- 381(, 70)$ & $-0,513$ & $-5,441 * * *$ & & \\
\hline Positive class climate & ,069 (,093) & 0,069 & 0,750 & ,271 & ,234 \\
\hline \multicolumn{6}{|c|}{ Sweden $(\mathrm{F}(5,69)=9,454 ; \mathrm{p}=.000)$} \\
\hline Constant & $2,106(, 436)$ & & $4,834 * * *$ & & \\
\hline Fantasy & ,362 (,101) & 0,400 & $3,567 * *$ & & \\
\hline Empathic concern & ,046 (,126) & $-0,041$ & $-0,361$ & & \\
\hline Perspective taking &,$- 066(, 099)$ & $-0,075$ & $-0,669$ & & \\
\hline Personal distress &,$- 366(, 101)$ & $-0,405$ & $-3,628 * *$ & & \\
\hline Positive class climate & ,459 (,127) & $\mathbf{0 , 3 7 9}$ & $3,610^{* *}$ & ,407 & ,364 \\
\hline \multicolumn{6}{|c|}{ Croatia $(F(5,72)=3,888 ; p=004)$} \\
\hline Constant & 2,701 (,410) & & $6,595 * * *$ & & \\
\hline Fantasy & $0,012(, 078$ & 0,017 & 0,154 & & \\
\hline Empathic concern & $0,088(, 086)$ & 0,127 & 1,026 & & \\
\hline Perspective taking & $0,159(, 092)$ & 0,192 & 1,729 & & \\
\hline Personal distress & $-0,277(, 096)$ & $-0,352$ & $-2,875^{* *}$ & & \\
\hline Positive class climate & $0,175(, 100)$ & 0,193 & 1,761 & ,213 & 158 \\
\hline
\end{tabular}

\section{Conclusions}

Our study shows that personal distress (one aspect of empathy), is an important predictor of well-being in all participating countries. This supports the development of an international programme, aiming at reducing its influences. Students that have troubles in regulating their emotions (and distinguishing between their own and feelings of others) and get overwhelmed when someone is in distress (the focus is on themselves and not on the other person) report lower well-being than their peers in all participating countries. One of the examples of intervention could be focused on self- and others awareness (e.g. how to distinguish between our own feelings and feeling of others) and on self-regulation (e.g. regulation of own emotions, thoughts, actions).

\section{References}

Cocoradă, E., \& Orzea, I. E. (2017). Relationships between wellbeing, resilience and school climate. Bulletin of the Transilvania University of Brasov. Series VII, Social Sciences, Law., 10(2), 191-200.

Davis, M. H. (1980). A multidimensional approach to individual differences in empathy. JSAS Catalog of Selected Documents in Psychology, 10, 1-19.

Decety J, Bartal IB-A, Uzefovsky F, \& Knafo-Noam A. (2016). Empathy as a driver of prosocial behaviour: highly conserved neurobehavioural mechanisms across species. Phil. Trans. R. Soc. B 371:20150077.

Eisenberg, N., Fabes, R. A., Murphy, B., Karbon, M., Smith, M., \& Maszk, P. (1996). The relations of children's dispositional empathy-related responding to their emotionality, regulation, and social functioning. Developmental psychology, 32(2), 195.

Jolliffe, D., \& Farrington, D. P. (2006). Examining the relationship between low empathy and bullying. Aggressive behavior, 32(6), 540-550.

Shanafelt, T. D., West, C., Zhao, X., Novotny, P., Kolars, J., Habermann, T., \& Sloan, J. (2005). Relationship between increased personal well-being and enhanced empathy among. Journal of general internal medicine, 20(7), 559-564.

Stewart-Brown, S. L., Platt, S., Tennant, A., Maheswaran, H., Parkinson, J., Weich, S., ... \& Clarke, A. (2011). The Warwick-Edinburgh Mental Well-being Scale (WEMWBS): a valid and reliable tool for measuring mental well-being in diverse populations and projects. Journal of Epidemiology and Community Health, 65(Suppl 2), A38-A39.

Stöber, J. (2002). Skalendokumentation „Persönliche Ziele von SchülerInnen“. Hallesche Berichte zur Pädagogischen Psychologie, 3, 40-41.

Zorza, J.P., Marino, J. \& Mesas, A.A. (2015). The influence of effortful control and empathy on perception of school climate. Eur J Psychol Educ 30:457-472 


\title{
EDU-CAR: QUALITATIVE ANALYSIS OF AN INTERVENTION FOR THE DEVELOPMENT OF SOCIO-EMOTIONAL AND CAREER COMPETENCES ${ }^{1}$
}

\author{
Mara Leal, \& Lucy Melo-Silva \\ Department of Psychology, Faculty of Philosophy, Sciences and Letters at Ribeirão Preto, \\ University of São Paulo (Brazil)
}

\begin{abstract}
This study aims to qualitatively analyze the communication strategies used by the psychologist and the participants in a career intervention that aimed at the development of socioemotional and career skills in high school students. A total of 44 students (55\% boys, ages: $M=15.16$, SD = 0.7) from two public high school classes in the interior of São Paulo, Brazil participated in the intervention. The program was structured in two modules, the first one for the work of the socioemotional competences and the second module for the work of the career competences. The program comprised a total of 12 sessions. The sessions were held weekly. The data were obtained through audio records that were transcribed and analyzed based on content analysis. Data were analyzed by intervention session and by classroom. The registration of 20 sessions constitutes the corpus of analysis of this study. Questions about the quality of the activities and about the quality of student participation, in their opinions, were evaluated. The analysis of the frequencies of the registers of the categories of interventions of the psychologist and of the reactions of the students allowed verifying the effectiveness of the intervention. The results show that the school was identified as a place of preparation for work and that the topics covered were considered important for life. Most of the students considered the quality of the activities developed in the intervention as good, and their participation as regular.
\end{abstract}

Keywords: Intervention, qualitative, socio-emotional skills, career development, high school.

${ }^{1}$ Grant \#2015/12677-0, São Paulo Research Foundation (FAPESP). 


\title{
CONTENT OF FATHER AND MOTHER STEREOTYPES IN JAPAN, COMPARED TO OVERALL GENDER STEREOTYPES
}

\author{
Mizuka Ohtaka \\ Department of Politics and Public Administration, Faculty of Law, Yamanashi Gakuin University (Japan)
}

\begin{abstract}
Suzuki (2017) reviewed the studies on gender inequality and concluded that gender stereotypes contribute to the persistence of gender discrimination in the workplace and at home. It has also been verified that the content of father (Troilo, 2013) and mother stereotypes (Ganong \& Coleman, 1995) differed from overall gender stereotypes in American society. This study investigated whether the content of father and mother stereotypes was dissimilar to that of overall gender stereotypes in Japan. That is, does the content of father (versus men) and mother (versus women) stereotypes differ from the typically held gender stereotypes? A survey was conducted among undergraduates $(N=266$; Men $=106$, Women $=160)$, with a mean age of 19.05 years $(S D=1.02$ years). The results indicated that the content of father stereotype was different from that of men and that of mother stereotype was different from that of women. This implies that the idea that 'fathers (rather than men) should work outside the home and mothers (rather than women) should keep the house', is held in Japanese society. In the future, it would be helpful to examine not only explicit stereotypes but also implicit stereotypes about fathers and mothers. Further, it would be useful to study stereotypes held by older and/or less educated adults.
\end{abstract}

Keywords: Stereotype, father, mother, gender.

\section{Introduction}

Gender stereotype is identified as one of the factors contributing to gender inequality in offices and families (Suzuki, 2017, for review). Troilo (2013) demonstrated that in the U.S.A., the content of the father stereotype was dissimilar to that for men (e.g., American fathers were thought to be Hardworking and Busy, when compared with American men). Similarly, Ganong and Coleman (1995) substantiated that the content of the mother stereotype differed from that for women (e.g., American mothers were believed to Act for the good of family and Favor her own children, when compared with American women). In Japan too, are parent stereotypes different from overall gender stereotypes? This study tries to verify whether in Japan, the content of father stereotype differs from that for men and whether the content of mother stereotype differs from that for women.

\section{Methods}

This study conducted a survey among 266 undergraduates. They were 18-22 years old, of both genders, unmarried, born and raised in Japan, and their native language was Japanese. They randomly received one of four self-report questionnaires on either fathers, mothers, men, or women, followed by the 85 descriptors (Table 1). These descriptors were generated from two pilot studies in a similar way to previous studies (Ganong \& Coleman, 1995; Troilo, 2013).

They were asked to list a percentage for each descriptor on a 11-point scale ranging from 0 to $100 \%$ (e.g., 'What percentage of Japanese fathers do you think have each of the following descriptors? For example, if you think that all Japanese fathers have the characteristic, you would choose $100 \%$. If you think that $20 \%$ of Japanese fathers have the characteristic, you would choose $20 \%$. Do not think of the fathers you know, but of fathers in general'). The 85 descriptors were randomly sorted to reduce order effects. 


\section{Results}

\subsection{Respondents}

Table 1. The Results of Analysis of Variance.

\begin{tabular}{|c|c|c|c|c|c|c|c|c|c|c|}
\hline Category & Descriptors & Fathers & Mothers & Men & Women & & & parent & sex & interaction \\
\hline & Strong & 63.24 & 49.86 & 68.28 & 31.45 & $F(3,258)=49.62$ & *** & ** & $* * *$ & $* * *$ \\
\hline Men $>$ & Powerful & 64.14 & 52.39 & 65.34 & 36.45 & $F(3,257)=32.26$ & $* * *$ & ** & *** & *** \\
\hline \multirow[t]{2}{*}{ Fathers } & Lazy & 54.29 & 47.89 & 62.20 & 50.00 & $F(3,257)=8.01$ & $* * *$ & $*$ & *** & \\
\hline & Selfish & 50.14 & 40.14 & 55.00 & 47.90 & $F(3,256)=7.52$ & $* * *$ & $* *$ & $* * *$ & \\
\hline & Physically strong & 61.86 & 48.03 & 68.47 & 36.07 & $F(3,257)=34.25$ & *** & & **** & *** \\
\hline Wen $=$ & Strong constitution & 62.06 & 50.70 & 64.92 & 40.48 & $F(3,256)=19.90$ & $* * *$ & & $* * *$ & ** \\
\hline & Work than children & 52.54 & 33.19 & 56.27 & 38.23 & $F(3,257)=22.17$ & $* * *$ & $t$ & $* * *$ & \\
\hline & Work & 85.51 & 64.20 & 79.65 & 58.39 & $F(3,253)=35.25$ & *** & ** & **** & \\
\hline & Hardworking & 76.96 & 65.29 & 70.18 & 60.97 & $F(3,252)=10.00$ & $* * *$ & $*$ & $* * *$ & \\
\hline & Breadwinner & 68.57 & 49.43 & 60.18 & 28.23 & $F(3,255)=40.57$ & $* * *$ & $* * *$ & $* * *$ & * \\
\hline Fathers & Big & 62.86 & 44.71 & 62.54 & 32.10 & $F(3,255)=40.31$ & *** & $* *$ & *** & ** \\
\hline & Masculine & 62.43 & 45.74 & 56.55 & 33.87 & $F(3,254)=24.74$ & *** & ** & *** & \\
\hline & Looking down on & 57.25 & 55.44 & 56.72 & 42.62 & $F(3,252)=7.70$ & $* * *$ & ** & $* *$ & $*$ \\
\hline & Well built & 56.86 & 45.00 & 55.42 & 32.26 & $F(3,257)=25.65$ & $* * *$ & $* *$ & $* * *$ & * \\
\hline & Busy & 74.43 & 75.44 & 66.03 & 63.55 & $F(3,254)=5.87$ & ** & *** & & \\
\hline & Very busy & 68.86 & 74.71 & 64.04 & 62.90 & $F(3,253)=5.25$ & ** & $* * *$ & & \\
\hline & Reliable & 66.00 & 72.21 & 55.61 & 54.52 & $F(3,253)=14.01$ & $* * *$ & $* * *$ & & \\
\hline & Dependable & 65.86 & 70.14 & 56.21 & 50.97 & $F(3,256)=13.91$ & $* * *$ & $* * *$ & & * \\
\hline & Reassuring & 64.86 & 68.57 & 54.92 & 53.33 & $F(3,255)=10.05$ & $* * *$ & $* * *$ & & \\
\hline & Trustworthy & 63.14 & 68.12 & 56.78 & 50.83 & $F(3,254)=10.55$ & $* * *$ & $* * *$ & & $*$ \\
\hline Fathers & Sociable & 60.56 & 66.38 & 57.93 & 56.13 & $F(3,257)=4.91$ & $* *$ & $* *$ & & $\dagger$ \\
\hline$\fallingdotseq$ & Robust & 60.00 & 65.94 & 55.09 & 50.81 & $F(3,255)=6.90$ & $* * *$ & $* * *$ & & * \\
\hline Mothers & Tough & 63.10 & 64.20 & 60.70 & 50.32 & $F(3,255)=5.81$ & ** & $* *$ & $\dagger$ & $*$ \\
\hline & Brave & 60.42 & 63.19 & 50.00 & 46.94 & $F(3,256)=10.38$ & $* * *$ & *** & & \\
\hline & Cool & 50.74 & 57.50 & 47.54 & 37.54 & $F(3,250)=10.64$ & $* * *$ & $* * *$ & & $* *$ \\
\hline & Courageous & 58.75 & 59.71 & 51.69 & 44.26 & $F(3,257)=8.98$ & $* * *$ & $* * *$ & & $\dagger$ \\
\hline & Polite & 63.24 & 63.57 & 54.07 & 61.11 & $F(3,259)=3.60$ & * & * & & \\
\hline & Sincere & 60.71 & 60.88 & 52.54 & 53.23 & $F(3,255)=4.20$ & ** & $* * *$ & & \\
\hline & Strict & 55.56 & 59.58 & 49.30 & 52.79 & $F(3,257)=3.71$ & $*$ & $* *$ & $\begin{array}{l}\dagger \\
\text { r }\end{array}$ & \\
\hline & Caring & 62.54 & 73.68 & 47.72 & 62.13 & $F(3,253)=20.10$ & $* * *$ & *** & **** & \\
\hline & Mature & 69.72 & 72.86 & 45.76 & 61.45 & $F(3,259)=24.87$ & $* * *$ & $* * *$ & $* * *$ & $* *$ \\
\hline & Thoughtful & 61.74 & 71.74 & 56.03 & 60.49 & $F(3,253)=9.79$ & $* * *$ & $* * *$ & $* *$ & \\
\hline Fathers & Amazing & 59.58 & 71.18 & 47.72 & 56.77 & $F(3,254)=13.76$ & $* * *$ & $* * *$ & $* * *$ & \\
\hline$<$ & Kind & 64.37 & 70.29 & 59.48 & 62.42 & $F(3,256)=4.24$ & ** & ** & $*$ & \\
\hline Mothers & Watch over & 64.79 & 68.55 & 50.17 & 58.52 & $F(3,256)=11.37$ & $* * *$ & $* * *$ & $*$ & \\
\hline & Understanding & 58.47 & 68.55 & 45.00 & 55.97 & $F(3,257)=16.60$ & $* * *$ & $* * *$ & $* * *$ & \\
\hline & Fine & 62.82 & 69.30 & 52.03 & 55.16 & $F(3,259)=10.69$ & $* * *$ & $* * *$ & $*$ & \\
\hline & Generous & 56.03 & 64.93 & 49.48 & 51.61 & $F(3,251)=9.03$ & $* * *$ & $* * *$ & * & \\
\hline & Childrearing & 40.70 & 79.57 & 36.32 & 71.94 & $F(3,255)=87.15$ & *** & * & *** & \\
\hline & Love family & 66.23 & 77.71 & 59.83 & 69.03 & $F(3,256)=11.66$ & $* * *$ & $* *$ & $* * *$ & \\
\hline & Love children & 63.57 & 77.71 & 57.07 & 65.48 & $F(3,256)=13.93$ & $* * *$ & *** & *** & \\
\hline & Take children's sides & 60.87 & 77.50 & 57.97 & 67.42 & $F(3,254)=12.21$ & $* * *$ & $* *$ & *** & \\
\hline & Cherish family & 65.86 & 76.06 & 56.10 & 66.45 & $F(3,258)=12.51$ & $* * *$ & *** & *** & \\
\hline & Family oriented & 51.29 & 75.36 & 47.93 & 63.06 & $F(3,255)=27.75$ & $* * *$ & $* *$ & $* * *$ & $\dagger$ \\
\hline Mothers & Warm & 59.72 & 72.90 & 52.76 & 62.26 & $F(3,256)=15.27$ & *** & *** & *** & \\
\hline & Capacious & 54.71 & 71.86 & 53.79 & 58.52 & $F(3,255)=13.04$ & $* * *$ & ** & $* * *$ & $* *$ \\
\hline & Like children & 60.28 & 70.58 & 49.32 & 63.39 & $F(3,257)=16.38$ & $* * *$ & $* * *$ & $* * *$ & \\
\hline & Friendly & 54.29 & 68.55 & 48.10 & 56.45 & $F(3,255)=14.61$ & $* * *$ & $* * *$ & $* * *$ & \\
\hline & Interfering & 45.14 & 68.29 & 31.86 & 52.42 & $F(3,257)=47.19$ & $* * *$ & *** & *** & \\
\hline & Dedicated & 54.51 & 66.14 & 50.34 & 60.82 & $F(3,256)=9.76$ & $* * *$ & * & *** & \\
\hline & Worrier & 52.36 & 65.88 & 47.89 & 58.23 & $F(3,255)=11.25$ & $* * *$ & $* *$ & $* * *$ & \\
\hline & Stabilize family & 60.00 & 63.86 & 52.07 & 62.90 & $F(3,255)=5.50$ & ** & * & $* *$ & \\
\hline & Do housework & 38.87 & 76.62 & 36.21 & 70.16 & $F(3,255)=69.43$ & *** & $\dagger$ & *** & \\
\hline & Busy with childrearing & 27.46 & 73.82 & 27.76 & 70.00 & $F(3,255)=107.46$ & $* * *$ & & $* * *$ & \\
\hline & Cooperative & 54.64 & 64.93 & 54.83 & 62.26 & $F(3,254)=6.18$ & $* * *$ & & $* * *$ & \\
\hline & Emotional & 49.71 & 64.43 & 47.54 & 62.74 & $F(3,253)=12.68$ & $* * *$ & & $* * *$ & \\
\hline & Cheerful & 53.52 & 61.88 & 56.44 & 61.29 & $F(3,257)=4.61$ & ** & & ** & \\
\hline & Preciuos & 34.20 & 58.12 & 35.09 & 54.84 & $F(3,253)=23.59$ & $* * *$ & & $* * *$ & \\
\hline & Pure & 41.55 & 52.43 & 36.21 & 51.13 & $F(3,257)=10.06$ & $* * *$ & & $* * *$ & \\
\hline & Soft & 37.36 & 61.88 & 37.37 & 66.72 & $F(3,255)=44.16$ & $* * *$ & & $* * *$ & \\
\hline$=$ & Gracious & 50.14 & 57.50 & 47.02 & 63.06 & $F(3,254)=10.57$ & $* * *$ & & $* * *$ & * \\
\hline & Calculating & 44.57 & 53.48 & 42.76 & 58.23 & $F(3,255)=9.78$ & $* * *$ & & $* * *$ & \\
\hline & Pretty & 32.82 & 54.00 & 35.52 & 57.54 & $F(3,256)=30.25$ & $* * *$ & & $* * *$ & \\
\hline & Sweet & 31.86 & 52.54 & 33.22 & 56.89 & $F(3,257)=26.12$ & $* * *$ & & $* * *$ & \\
\hline & Refined & 35.07 & 50.74 & 32.54 & 55.97 & $F(3,254)=25.44$ & $* * *$ & & $* * *$ & $\dagger$ \\
\hline & Tidy & 30.42 & 53.19 & 36.90 & 55.57 & $F(3,256)=24.56$ & $* * *$ & $\dagger$ & $* * *$ & \\
\hline & Elegant & 26.09 & 48.29 & 27.59 & 54.52 & $F(3,255)=35.19$ & $* * *$ & & $* * *$ & \\
\hline & Modest & 33.86 & 46.87 & 28.95 & 52.26 & $F(3,252)=20.58$ & $* * *$ & & $* * *$ & * \\
\hline & Feminine & 26.48 & 53.94 & 35.25 & 67.21 & $F(3,258)=54.22$ & *** & $* * *$ & $* * *$ & \\
\hline & Pretty hair & 30.14 & 49.00 & 35.93 & 63.55 & $F(3,258)=37.80$ & $* * *$ & $* * *$ & $* * *$ & $\dagger$ \\
\hline & Feel lonely & 48.33 & 51.74 & 50.69 & 62.58 & $F(3,257)=6.67$ & $* * *$ & ** & $* *$ & $\dagger$ \\
\hline Mothers & Blackhearted & 32.11 & 43.77 & 42.98 & 61.29 & $F(3,255)=23.62$ & $* * *$ & $* * *$ & $* * *$ & \\
\hline & Cute & 27.00 & 48.94 & 33.86 & 59.19 & $F(3,251)=32.79$ & $* * *$ & $* *$ & $* * *$ & \\
\hline & Brilliant & 26.34 & 46.97 & 32.41 & 57.90 & $F(3,253)=40.05$ & $* * *$ & $* * *$ & $* * *$ & \\
\hline & Beautiful & 23.53 & 49.13 & 30.68 & 56.39 & $F(3,253)=46.74$ & $* * *$ & $* *$ & $* * *$ & \\
\hline & Fresh & 37.68 & 52.90 & 46.90 & 52.90 & $F(3,254)=12.05$ & $* * *$ & $*$ & $* * *$ & $*$ \\
\hline Women & Coward & 32.32 & 46.67 & 47.07 & 59.02 & $F(3,253)=22.91$ & *** & $* * *$ & $* * *$ & \\
\hline$>$ Men & Mentally weak & 36.29 & 42.75 & 49.31 & 53.44 & $F(3,254)=9.51$ & $* * *$ & $* * *$ & $*$ & \\
\hline & Active & 50.74 & 58.70 & 57.89 & 55.32 & $F(3,252)=2.85$ & * & & & * \\
\hline & Moody & 46.44 & 49.85 & 53.73 & 53.55 & $F(3,257)=2.13$ & $\dagger$ & * & & \\
\hline & Quiet & 48.00 & 40.59 & 42.46 & 46.13 & $F(3,253)=2.44$ & $\dagger$ & & & $*$ \\
\hline Others & Immovable & 55.92 & 55.22 & 49.82 & 52.26 & $F(3,255)=1.65$ & & * & & \\
\hline & Calm & 47.50 & 42.75 & 42.37 & 46.13 & $F(3,258)=1.54$ & & & & $*$ \\
\hline & Audacious & 50.71 & 55.57 & 53.39 & 48.69 & $F(3,256)=1.58$ & & & & $*$ \\
\hline & Stubborn & 55.63 & 55.57 & 54.48 & 51.13 & $F(3,257)=0.81$ & & & & \\
\hline
\end{tabular}


The mean age of respondents was $19.05(S D=1.02)$ years old. The respondents comprised 106 men (39.85\%) and 160 women (60.15\%). 73 (33 men and 40 women) answered the questionnaires on fathers, 71 (25 men and 46 women) answered those on mothers, 59 (20 men and 39 women) answered those on men and 63 (28 men and 35 women) answered those on women.

\subsection{Analysis of variance}

The data were analysed using a two-way analysis of variance. The analysis design used two independent variables: whether the target was parents or not (a between factor), and men or women (a between factor), and the dependent variable was each descriptor. Table 1 shows the results of analysis of variance.

First, it was found that the content of stereotypes of fathers, mothers, men, and women differed from each other. The respondents believed that fathers rather than men, mothers, and women have the seven characteristics Work, Hardworking, Breadwinner, Big, Masculine, Looking down on, and Well built.

They thought that mothers rather than women, fathers and men have the 14 characteristics: Childrearing, Love family, Love children, Take children's sides, Cherish family, Family oriented, Warm, Capacious, Like children, Friendly, Interfering, Dedicated, Worrier, and Stabilise family and that mothers rather than fathers, women and men have the 9 characteristics Caring, Mature, Thoughtful, Amazing, Kind, Watch over, Understanding, Fine, and Generous.

They believed that men rather than fathers, women, and mothers have the four characteristics Strong, Powerful, Lazy and Selfish. They thought that women rather than mothers, men, and fathers have the eight characteristics Feminine, Pretty hair, Feel lonely, Black hearted, Cute, Brilliant, Beautiful, and Fresh and that women rather than men, mothers and fathers have the two characteristics Coward and Mentally weak.

Next, it was also indicated that the content of parent stereotypes was different from that of overall gender stereotypes. The respondents believed that fathers and mothers rather than men and women have the following 15 characteristics: Busy, Very busy, Reliable, Dependable, Reassuring, Trustworthy, Sociable, Robust, Tough, Brave, Cool, Courageous, Polite, Sincere, and Strict.

On the other hand, the respondents thought that men and fathers rather than women and mothers have the 3 characteristics Physically strong, Strong constitution, and Work than children and mothers and women rather than fathers and men have the following 16 characteristics Do housework, Busy with childrearing, Cooperative, Emotional, Cheerful, Precious, Pure, Soft, Gracious, Calculating, Pretty, Sweet, Refined, Tidy, Elegant, and Modest. This means that the content of parent stereotypes overlaps with that of overall gender stereotypes.

\section{Discussion}

These results showed that the content of father and mother stereotypes differed from that of overall gender stereotypes and suggest that Japanese have the idea that 'fathers (rather than men) should work outside the home, and mothers (rather than women) should keep the house'. However, it should be noted that the content that repeated in parent stereotypes and overall gender stereotypes implies that 'both men and fathers should work outside the home, and both mothers and women should keep the house' and this point needs further verification.

Furthermore, Park, Smith, and Correll (2010) investigated not explicit but implicit stereotypes and found that fathers were strongly associated with professional images and mothers were more strongly associated with childcare images. Therefore, it will deepen our understanding to compare implicit parent stereotypes to implicit overall gender stereotypes. In addition, although this study targeted only undergraduates, future research should target older and/or less educated adults in order to generalise the findings.

\section{Acknowledgements}

This work was supported by JSPS KAKENHI Grant Number JP18K18298.

\section{References}

Ganong, L. H. \& Coleman, M. (1995). The content of mother stereotypes. Sex Roles, 32, 495-512.

Park, B., Smith, J. A., \& Correll, J. (2010). The persistence of implicit behavioural associations for moms and dads. Journal of Experimental Social Psychology, 46, 809-815.

Suzuki, A. (2017). Mechanisms of persistence in gender inequality: Perspective of work and family. Japanese Psychological Review, 60, 62-80.

Troilo, J. (2013). The drinker and the nurturer: College students' perceptions of men and fathers. Journal of Applied Social Psychology, 43, 1089-1096. 


\title{
THE EFFECTS OF PSYCHOLOGICAL GAMES ON NONVERBAL COMMUNICATION SKILLS OF THE YOUTH
}

\author{
Koshi Makino \\ Department of Business Administration, Setsunan University (Japan)
}

\begin{abstract}
This study examined the effects of psychological games on nonverbal communication skills. Werewolf-Game was used as a psychological game. Werewolf game has about 10 participants and they were divided into two teams, werewolf team and citizen team. Werewolf tells a lie and attacks the citizens, citizens try to find the wolf by conversation and expel it. As for this game, members of werewolf team have to tell lies and others must find out them, so nonverbal communication skills also become very important.

Participants were 60 university students (36 males and 24 females, Average age is 20.62.). Half participants were divided into an experimental group, and the others were into the control group. The experimental group members played werewolf-games 6 times, and control group members did not. Their nonverbal communication skills (3 factors) were measured: nonverbal expression, nonverbal sensitivity, and nonverbal control. The questionnaire was carried out 3 times: 2 weeks before the games, 2 weeks after the games, and 4 weeks after.

According to ANOVA $(2 \times 3)$, interaction effect was significant. There was no significant difference in the means of all scales between experimental and control group before the games. Two weeks after the games, nonverbal expression and nonverbal control skills in experimental group became higher than before, and those two skills were higher than those of control group. In addition, those two skills were maintained high four weeks later. These results indicate the possibility that nonverbal communication skills are promoted by the training which used psychological games.
\end{abstract}

Keywords: Psychological games, nonverbal communication skills, communication skill training, Werewolf-Game.

\section{Introduction}

Communication skills are a part of social skills, which work more directly for interpersonal relationship. Makino (2010) have researched on communication skills of junior-school students and communication skills training for them in Japan. Makino (2012) examined the association between communication skills and friendship, their mental health. According to Makino (2012), as the person who had high communication skills for the same-sex, the friend relations were good and the mental health state was good. Communication skills are indispensable for daily life. On the other hand, we often use nonverbal communication in everyday life. According to Wada (1991), the nonverbal communication skills are classified in three: nonverbal expression, nonverbal sensitivity, and nonverbal control skill. This study aims at the improvement of the nonverbal communication skills using a psychological game as one of the communication skill trainings. That is because that psychological game needs the skill to read feelings from the expression of the others, or the skill to control one's expressions not to show one's real feelings. This study aims at the improvement of the nonverbal communication skills using a psychological game as one of the communication skill trainings.

\section{Objectives}

The purpose of this study was to examine the effects of psychological games on nonverbal communication skills of the youth. This study used Werewolf-Game as a psychological game. Werewolf game has about 10 participants and they were divided into 2 teams, werewolf team and citizen team. Werewolf tells a lie and attacks the citizens, citizens try to find the wolf by conversation and expel it. In this game, werewolf team members lie and try to deceive citizen members and citizen members have to find out their lies, so nonverbal communication of the participants is very important to win. This game was very popular even in Japan in 2013. This study examined the effects of psychological games on nonverbal communication skills. And if the games work to improve their nonverbal communication skills, this study tried to find which skill was trained by the games. This research will make a substantial contribution to communication studies if it becomes clear that some psychological games are useful for nonverbal communication skill trainings. 


\section{Methods}

\subsection{Participants}

Participants were 60 university students (who have experienced few werewolf-games before, 36 males and 24 females, Average age is 20.62.). Half participants were divided into an experimental group, and the others were into the control group. The experimental group members played werewolf-games 6 times, and control group members did not.

\subsection{Procedure}

First of all, participants completed a questionnaire before this research in the classrooms. The questionnaire was presented as a study about daily life among university students. They were told to answer the questions without discussing them with others. After the first investigation, they were divided into 2 groups. Half participants were divided into an experimental group, and the others were the control group. The experimental group members played werewolf-games 6 times for two weeks. On the other hand, control group members did not. Two weeks after the game, the participants of both groups answered the questionnaire again ( 2 weeks after the games). Furthermore, the participants of both groups answered the questionnaire once again (4 weeks after the games).

\subsection{Measures}

The questionnaire was made up of seven scales (and six scales were not analyzed in this study) plus demographic items. It took about 20 minutes to complete.

Nonverbal Communication skills scale. The nonverbal communication skills self-report scale was arranged from Wada (1991). It included 12 items that were rated on a seven-point scale (1=extremely poor, 7= extremely good). Examples of those items were "I can hold down my feelings to reflect on my face." and "I can read the feelings of the others from their expressions." and so on.

According to Wada (1991), the nonverbal communication skills scale was consisted of three factors: nonverbal expression, nonverbal sensitivity, and nonverbal control skill. This study uses these 3 factors as the nonverbal communication skills.

\section{Results}

\subsection{The comparison between experimental group and control group before the games}

The analysis design of this study is ANOVA (2(between: experimental, control) $\times 3$ (within: before, 2 weeks after the games, 4 weeks after the games). The dependent variables are 3 nonverbal communication skill factors.

Table 1 shows means of each skill before the games. As a result of ANOVA of the mean of each factor score between experimental group and control group before the psychological games, there was no significant difference between them. This result revealed that there was no difference in their nonverbal communication skills between experimental and control group before the games.

Table 1. Mean scores of each factor before, 2 weeks, and 4 weeks after the games in two groups

\begin{tabular}{l|ccc|ccc}
\hline $\begin{array}{l}\text { Non-verbal } \\
\text { Communication } \\
\text { Factors }\end{array}$ & \multicolumn{3}{|c|}{ Control group } & \multicolumn{3}{c}{ Experimental group } \\
\hline & Before & $\begin{array}{c}\text { 2 weeks } \\
\text { later }\end{array}$ & $\begin{array}{c}4 \text { weeks } \\
\text { later }\end{array}$ & Before & $\begin{array}{c}\text { 2 weeks } \\
\text { later }\end{array}$ & $\begin{array}{c}4 \text { weeks } \\
\text { later }\end{array}$ \\
1. Nonverbal expression & 4.47 & 4.53 & 4.57 & 4.51 & 4.92 & 4.76 \\
& $(0.98)$ & $(1.01)$ & $(0.92)$ & $(0.85)$ & $(0.97)$ & $(1.17)$ \\
2. Nonverbal sensitivity & 4.88 & 4.78 & 4.90 & 4.85 & 5.02 & 4.93 \\
& $(1.00)$ & $(0.88)$ & $(1.07)$ & $(0.84)$ & $(0.96)$ & $(1.13)$ \\
3. Nonverbal control & 3.95 & 3.98 & 4.21 & 4.25 & 4.75 & 4.61 \\
& $(1.29)$ & $(1.14)$ & $(1.03)$ & $(1.06)$ & $(1.09)$ & $(1.07)$ \\
\hline
\end{tabular}

\subsection{The comparison between experimental group and control group after the games}

Table 1 shows means of each skill 2 weeks after and 4 weeks after the games. As a result of ANOVA (2(between: experimental, control) $\times 3$ (within: before, 2 weeks after the games, 4 weeks after the games) of the mean of each factor score, there were significant differences in two factors: nonverbal expression skill and nonverbal control skill (see Figure 1 \& Figure 2). 2 weeks after the games, nonverbal expression and nonverbal control skills in experimental group became higher than before, and those two skills were higher than those of control group. In addition, those two skills were maintained high 4 weeks 
later, but they were not higher than those of before the games. There was no significant difference in nonverbal sensitivity skill. These results showed that the participants of experimental group learned how to show their feelings on their faces and how to control their feelings against their true feelings depending on the scene through the games compared with the participants of control group. And those high two skills were maintained four weeks later, which means this effect is not temporary. However, nonverbal sensitivity is considered very important for the were-wolf game, it was not raised up by the games.

Figure 1. Nonverbal expression skill before and after the games.

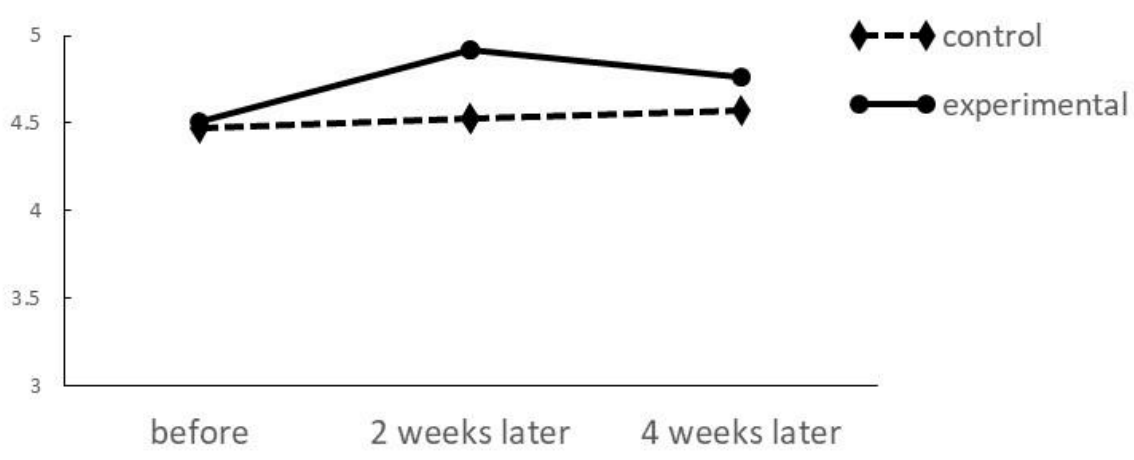

Figure 2. Nonverbal control skill before and after the games.

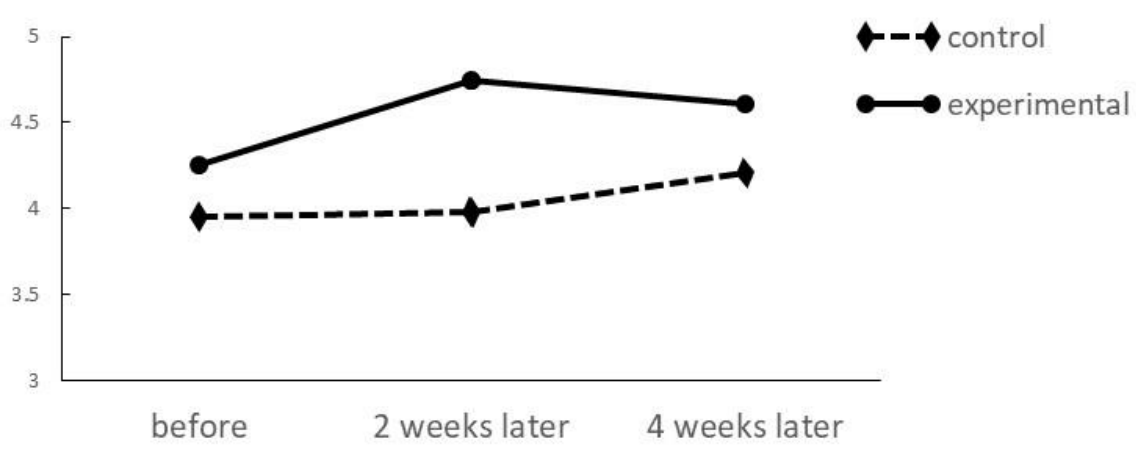

\section{Conclusions}

This study examined the effects of psychological games on nonverbal communication skills of the youth. The psychological games in this experiment raised two nonverbal communication skills of the participants in the experimental group and those two skills were higher than those of control group, but the psychological mechanism was not elucidated. The participants acquired skills how to show their feelings on their faces and how to control their feelings against their true feelings depending on the scene while enjoying the games. And those two skills were maintained high four weeks later. These results indicate the possibility that parts of nonverbal communication skills are promoted by the training using the psychological games.

\section{References}

Makino, K. (2010). A Development of the Communication Skills Training for Junior High School Students (3). - The Effects of Communication Skills Training on Junior High School Students in Japan. - Journal of Business Administration and Information, 18, 1-9.

Makino, K. (2012). The Communication Skills and Friendship in Adolescence - The Sex and Grade Differences of Communication Skills for Friends of the Same and Opposite Sex - Journal of Business Administration and Information, 20, 17-32.

WADA, M. (1991). A study of interpersonal competence: Construction of nonverbal skill scale and social skill scale The Japanese Journal of Experimental Social Psychology. 31, 49-59. 


\title{
CONTENT VALIDITY OF THE DESCRIPTIVE/INJUNCTIVE NORM PREFERENCE SCALE
}

\author{
Yoriko Sano $^{1}$ \& Norihiro Kuroishi ${ }^{2}$ \\ ${ }^{1}$ College of Interhuman Symbiotic Studies, Kanto Gakuin University (Japan) \\ ${ }^{2}$ Institute for Educational Research and Service, International Christian University (Japan)
}

\begin{abstract}
The purpose of this study was to examine a content validity of the descriptive/injunctive norm preference scale (DINPS). This scale measures a tendency which people prefer injunctive norms (i.e. the social rules we have to comply) or descriptive norms (i.e. the perceptions of actual behavior showed by almost all people). Three scales were adopted to consider a content validity of DINPS. First, the need for uniqueness scale (Snyder \& Fromkin, 1977, 1980) was used because this scale measured a stable individual deference which people want to be unique. Second, the F scale (Adorno et al., 1951) was used to assess that people agree or disagree with 9 subscales as "conventionalism", "authoritarian submission", "authoritarian aggression", "anti-intraception", "superstition and stereotypy", "power and toughness", "destructiveness and cynicism", "projectivity", and "sex". Finally, the individual and social orientedness scale made by Ito (1993) was used. This scale measured two aspects: 1) social orientedness concerned the orientation to others and the external (interpersonal) adjustment, 2) individual orientedness concerned the orientation to self and the internal adjustment. Forty hundred respondents compete these four scales by on line survey. According to correlational analyses, it was found that there was a content validity of DINPS modestly.
\end{abstract}

Keywords: Content validity, descriptive norm, injunctive norm, scale.

\section{Introduction}

Kuroishi \& Sano (2018) developed the scale which measured the preference for descriptive or injunctive norms. This scale, named DINPS (i.e. descriptive/injunctive preference norm scale), was consisted of three subscales such as "Apprehension of deviance from descriptive", "Regard for injunctive" and "Aversion to injunctive norms". "Apprehension of deviance from descriptive" meant that one's concern whether one deviated from descriptive norms or not ("I am worried that I am not doing the same thing as everyone else" etc.). "Regard for injunctive" represented one's value and respect for injunctive norms ("Rules are important for everyone to live comfortably" etc.). "Aversion to injunctive norms" showed the tendency which one hated injunctive norms ("I do not like customs and traditions" etc.).

The purpose of this study was to examine a content validity of DINPS. Three scales, the need for uniqueness scale (Snyder \& Fromkin, 1977, 1980), F scale (Adorno et al., 1951), and the individual and social orientedness scale (Ito, 1993), were adopted. As the need for uniqueness scale measures a stable individual deference which people want to be unique, the scale is considered as contrary concept with DINPS. The F scale measures the potentially antidemocratic personality, therefore, the F scale includes constructive concepts that one value following social rules and injunctive norms. The individual and social orientedness scale measure two aspects: 1) "Social orientedness" concerned the orientation to others and the external (interpersonal) adjustment, 2) "Individual orientedness" concerned the orientation to self and the internal adjustment. Especially, it is assumed that "Social orientedness" relates with DINPS, because "Social orientedness" represented that one's concerns about others around and external adjustments in a community or society one belongs to.

\section{Methods}

Web questionnaire panel survey was used to collect data. Four hundred Japanese (male 200, female 200, aged 20-59) participated in this study. Respondents completed four scales as bellow. 1) The DINPS 55 items originally made by Kuroishi \& Sano (2018), which was consisted of three subscales, "Apprehension of deviance from descriptive", "Regard for injunctive", and "Aversion to 
injunctive norms". 2) The need for uniqueness scale 32 items (Snyder \& Fromkin, 1977, 1980). 3) The F scale 29 items (Adorno et al., 1951) which was consist of nine subscales as "Conventionalism", "Authoritarian submission", "Authoritarian aggression", "Anti-intraception", "Superstition and stereotypy", "Power and toughness", "Destructiveness and cynicism", "Projectivity", and "Sex". 4) The individual and social orientedness scale 30 items made by Ito (1993) which measured two aspects a social orientedness (positive/negative) and an individual orientedness (positive/negative).

\section{Results and discussion} in Table1.

Almost all subscales were normal distributions. Descriptive statistics of four scales were shown

Table1. Descriptives of four scales.

\begin{tabular}{llcc}
\hline & \multicolumn{1}{c}{ Subscale } & $M$ & $S D$ \\
\hline \multirow{4}{*}{ DINPS } & Apprehension of deviance from descriptive (a) & 2.94 & .65 \\
& Regard for injunctive (b) & 3.42 & .55 \\
& Aversion to injunctive norms (c) & 2.93 & .56 \\
\hline Need for uniqueness scale (d) & & 2.89 & .34 \\
\hline \multirow{5}{*}{ F scale } & Conventionalism (e) & .47 & .78 \\
& Authoritarian submission (f) & .25 & .64 \\
& Authoritarian aggression (g) & .10 & .72 \\
& Anti-intraception (h) & .19 & .72 \\
& Superstition and stereotypy (i) & .23 & .68 \\
& Power and toughness (j) & -.09 & .65 \\
& Destructiveness and cynicism (k) & .08 & .93 \\
& Projectivity (l) & -.21 & .78 \\
& Sex (m) & -.05 & .85 \\
\hline \multirow{5}{*}{ Individual and social orientedness scale } & Social Orientedness (positive) (n) & 3.31 & .62 \\
& Social Orientedness (negative) (o) & 3.02 & .66 \\
& Individual Orientedness(positive) (p) & 3.20 & .55 \\
& Individual Orientedness(negative) (q) & 2.97 & .61 \\
\hline
\end{tabular}

Note: DINPS, the need for uniqueness scale and the individual and social proentedness scale were measured 5-point Likert scale (1-5). The F scale was used 7 point Likert scale (1-7) and transformed as follows: 1 into -3 , 2 into -2, 3 into -1, 4 into 0, 5 into 1, 6 into 2 , and 7 into 3.

Correlational analyses were conducted to test a content validity of DINPS shown in Table 2. As we expected, "Apprehension of deviance from descriptive" and "Regard for injunctive" were correlated negatively with "Need for uniqueness" ( $r=-.51, p<.001$ and $r=-.38, p<.001$, respectively), on the other hand, "Aversion to injunctive norms" were correlated positively with "Need for uniqueness" $(r=.36, p<.001)$.

Also, "Apprehension of deviance from descriptive" and "Regard for injunctive" were positively correlated with social orientedness scale. These results were consistent with an assumption. As "Social orientedness" measured one's concern for others/society and external adjustment, it was obvious that observing or concerning for descriptive norm and injunctive norm fitted conceptually with "Social orientedness".

Table 2. Inter-subscales correlation coefficients.

\begin{tabular}{|c|c|c|c|c|c|c|c|c|c|c|}
\hline & $\mathrm{d}$ & e & $\mathrm{f}$ & g & $\mathrm{h}$ & $\mathrm{i}$ & $\mathrm{j}$ & $\mathrm{k}$ & l & $\mathrm{m}$ \\
\hline \multicolumn{11}{|l|}{ DINPS } \\
\hline $\begin{array}{l}\text { a:Apprehension of } \\
\text { deviance from descriptive }\end{array}$ & $-.51^{* * *}$ & .10 & $.27^{* * * *}$ & $.21^{* *}$ & $.17^{* *}$ & $.19^{* * *}$ & $.23^{* * *}$ & $.27^{* * *}$ & $.19^{* * * *}$ & $.24^{* * *}$ \\
\hline b:Regard for injunctive & $-.38^{* * *}$ & $.42^{* * *}$ & $.39^{* * *}$ & $.25^{* * *}$ & $.22^{* * *}$ & $.34^{* * *}$ & .07 & $.15^{* *}$ & -.02 & $.13^{* *}$ \\
\hline $\begin{array}{l}\text { c:Aversion to injunctive } \\
\text { norms }\end{array}$ & $.36^{* * *}$ & -.07 & .04 & $.13^{* *}$ & $.11^{*}$ & $.21^{* * *}$ & $.24^{* * *}$ & $.11^{*}$ & $.31^{* * *}$ & $.18^{* * *}$ \\
\hline
\end{tabular}


Table 2. (Continued).

\begin{tabular}{|c|c|c|c|c|}
\hline & $\mathrm{n}$ & 0 & p & q \\
\hline DINPS & & & & \\
\hline $\begin{array}{l}\text { a:Apprehension of } \\
\text { deviance from descriptive }\end{array}$ & $.28^{* * *}$ & $.72^{* * *}$ & $-.38^{* * *}$ & -.05 \\
\hline b:Regard for injunctive & $.54^{* * *}$ & $.23^{* * *}$ & $.16^{* *}$ & -.00 \\
\hline $\begin{array}{l}\text { c:Aversion to injunctive } \\
\text { norms }\end{array}$ & -.08 & $.16^{* *}$ & .08 & $.53^{* * *}$ \\
\hline
\end{tabular}

The F scale was related with injunctive norm. Specifically, "Conventionalism" and "Authoritarian submission" of the F scale were positively correlated with "Regard for injunctive". In addition, "Aversion to injunctive norms" was positively correlated with "Projectivity", "Power and toughness" and "Superstition and stereotypy" ( $r=.31, p<.001, r=.24, p<.001, r=.21, p<.001$, respectively).

\section{Conclusion}

This study examined a content validity of the DINPS. As results showed, DINPS related adequately with three scales which had associated psychological concepts. This study conducted in only Japan. As descriptive/injunctive norm preference was universal psychological process, cross culture studies were needed in future.

\section{Acknowledgments}

This research was supported by "Society for Human and Environmental Studies" at Kanto Gakuin University.

\section{References}

Adorno, T. W., Frenkel-Brunswik, E., Levinson, D. J., \& Sanford, R. N. (1951). The Authoritarian Personality, New York.

Ito Minako. (1993). Construction of an individual and social orientedness scale and its reliability and validity. The Japanese Journal of Psychology, 64, 115-122.

Kuroishi, N., \& Sano, Y. (2018). Development of a descriptive/injunctive norm preference scale. Poster Presentation at International Psychological Applications Conference and Trends, Porto, Portugal.

Snyder, C. R., \& Fromkin, H. L. (1977). Abnormality as a positive characteristic: The development and validation of a scale mesureing need for uniqueness. Journal of Abnormal Psychology, 86, 515-527.

Snyder, C. R., \& Fromkin, H. L. (1980). Uniqueness: The human pursuit of difference. New York: Plenum Press. 


\title{
ROAD LESS TRAVELED: MOTIVATIONS AND PATHWAYS OF FILIPINO LESBIANS AND GAYS IDENTIFYING AS EX-GAYS
}

\author{
Nel Jayson Santos, Aaron Vichard Ang, Sigrid Joyce Dela Paz, \& Daniel Jan Duque \\ Department of Psychology, De La Salle University-Manila (Philippines)
}

\begin{abstract}
This study explores the motivations and the pathways of past self- identifying Filipino lesbians and gays. In this study, the researchers seek to (1) understand the motivations that influenced ex-gays in modifying their sexual orientation and (2) delve more into the different pathways (stages and/or steps) that are involved in the sexual orientation modification of ex-gays specifically in the Philippine context. A semi-structured interview was conducted with 10 self- identified Filipino ex-gays. Inductive thematic analysis was then used to analyze the data and to identify common themes. Four themes emerged as motivations of ex- gays respectively; Identity Dissonance, Spiritual Conviction, Cognitive Reconstruction, and Influential Role Models. Likewise, three themes materialized as common pathways taken by ex- gays, namely; Church Involvement, Accountability/Support Groups, and Adaptation of New Lifestyle. Moreover, the study clarifies the existing definition of being an ex-gay.
\end{abstract}

Keywords: Ex-gay, sexual orientation, and sexual orientation modification process.

\section{Introduction}

\subsection{Motivations of ex-gays}

Ex-gay refers to individuals that experienced some modification in their sexual orientation ranging from exclusive homosexuality to exclusive heterosexuality, or ones either curious, attempting, or claiming to have altered their sexual orientation (Weiss et al., 2010). Prior studies, identify religion as one prevalent motivation for sexual reorientation (Haldeman, 1994). It can be noted that in some cases the individual's spiritual identity proves to be more salient compared to their sexual identity leading them to prioritize faith over sexual orientation (Bartoli \& Gillemi, 2008). Although not all ex-gays in the previous studies reported change, most either (1) no longer interpreted their same-sex attractions requiring them to identify as gays/lesbians or (2) found themselves not entirely homosexual and/or predominantly straight. In addition, factors like strained family relationships, understanding the nature of one's sexual attractions, adoption of new of perspectives, and receiving supportive atmosphere with other ex-gays are also reported as motivations for sexual reorientation (Robinson \& Spivey, 2007).

\subsection{Pathways of ex-gays}

In accordance with Throckmorton (2002), sexual orientation modification process of ex-gays is generally associated with different religious organizations. One of the major Ex-gay Christian ministry in the United States and Canada is the Exodus International, that intends to help lesbian and gay (LG) individuals to lessen their same- sex attraction and eventually promote abstinence over homosexual desires (Burack, 2015). The community utilizes techniques that include strengthening the individual's level of religiosity, and heterosexual identity (Shidlo \& Schroeder, 2002). Likewise, Rosenbuth (2000), describes JONAH, a Jewish ex-gay ministry in Brooklyn, that utilizes Jewish principles aligned with the Torah to help LG individuals in dealing with their homosexual issues. Furthermore, Evergreen International, a Church of Jesus Christ of Latter Day Saints ministry in Utah, also utilizes the religion's teachings and doctrines to aid in same-sex attraction and homosexual behaviors (Robinson, 1998). In the Philippines, religious organizations that promote the ex-gay movement among Filipino Lesbian and Gays are also present (UNDP \& USAID, 2014). One of such organization is the Courage Philippines, an Apostolate of the Catholic that gives spiritual guidance and support to Filipino Lesbians and Gays experiencing same-sex attraction (Campbell, 2010). It centers on helping members to fully surrender themselves to God and cultivate a life of interior chastity (Courage Philippines, 2012). According to Robinson and Spivey (2007), most participants in the west disclosed developing negative and traumatic experiences after going through activities such as engaging in masculine-oriented hobbies, and putting blame on parenting shortcomings for their homosexuality. 


\subsection{Present study}

The literature conveys that the identified motivations and pathways of ex-gays are largely studied in the western context (Weiss et al., 2010). Although ex-gay ministries and their overall goals towards sexual reorientation were presented, specific processes and activities done is yet to be explored. Moreover, motivations of the ex-gay individuals are still to be expanded upon and affirmed with respect to a different cultural context. In the present study, motivation is defined as the factors behind an individual's decision to change. While pathways refer to the overall modification process including practice, activities, techniques, and/or steps taken. The term ex-gay will be limited to lesbian and gay individuals that are previously attracted to the members of the same sex and/or currently claim to have an attraction to the opposite sex or deny any attraction at all. In general, the study aims to answer the following questions:

1. What are the motivations that influenced Filipino ex-gays to modify their sexual orientation?

2. What are the pathways (practices, activities, and/ or steps) involved in the sexual orientation modification of Filipino ex-gays?

\section{Method}

A semi-structured interview was used to aid in determining the participant's motivation in associating themselves as ex-gays and the pathways (stages and or steps) of they came to a point of identifying themselves as former gays. Ten self-identified Filipino ex-gays (four former lesbians and six former gays) participated in the study. Each participant received a consent form via email containing the nature of the study which the researcher ensured was understood and duly signed. Participants were then audio recorded and interviewed for around 45 minutes to an hour, using English and Filipino. Afterwards, the data was transcribed and analyzed for patterns and themes using the Inductive Thematic Analysis approach related to the motivations and pathways of identifying as ex-gays. Throughout the paper pseudonyms were used to identify the participants.

\section{Results}

\subsection{Motivations of Filipino lesbian and gays in modifying their sexual orientation}

Identity Dissonance. Most of the participants manifested having identity dissonance as their initial motivation in modifying their sexual orientation (9 out of 10 participants). Identity Dissonance was described by the participants as having an internal disconnect which included re-emerging feelings of discomfort such as having doubts and guilt at certain points in their homosexual lifestyle. A participant from an ex-gay ministry mentioned about being unhappy when he was still living his homosexual lifestyle and having hesitations toward his personal character.

Spiritual Conviction. Spiritual conviction as a product of faith appear to be a central motivation of Filipino Lesbian and Gays in modifying their sexual orientation (10 out of 10 participants). Although, brought up religiously, not all reported to be religious prior to modifying their sexual orientation. Some are already disconnected with their religiosity in order to realize their lesbian/gay identity but reported being reignited into faith through spiritual encounters or callings that transpired at a particular point of their lives whether through a church service, church activity, and/or answered prayers. These factors led them to compel for change. A participant from a Christian ministry described that he was motivated by his faith and personal encounters with Jesus Christ through bible readings.

Cognitive Reconstruction. Cognitive Reconstruction involves forming new perspectives with regards to their sexual orientation and manifesting a deeper understanding of one's identity (9 out of 10 participants). It also includes the realization of the participants regarding the repetitive and tiring cycle of same-sex relationships and increased awareness of the health related diseases brought about by homosexual relationships. A respondent described that same-sex relationships does not usually last, and may often increase the risk of sexually transmitted diseases

Influential role models. Almost all of the participants mentioned about witnessing a role model that motivated and inspired them to modify their sexual orientation ( 9 out of 10 participants). The role model may also include a person having experienced the same struggle or situation as the participant. In which, these role models have succeeded and persevered in their own attempts to turn away from their homosexual lifestyle. A participant mentioned that how inspiring it is to see people successful in their own pursuits in modifying their lifestyles.

\subsection{Pathways involved in the sexual orientation modification of Filipino lesbians and gays}

Church Involvement. Church involvement appeared to be the common pathway involved in their sexual orientation modification (7 out of 10 participants). Church involvements referred to activities and practices that enriched their faiths. This includes attending church services and activities of the 
church such as bible studies \& retreats, fellowships, and outreach programs. Two participants stated that the different church activities that they had aided them in modifying their sexual orientation.

Accountability/Support Group. All participants reported accountable and supportive environments. Holding sharing meetings helped them to know about themselves. Also, having healthy platonic relationships with their support groups were the primary reasons why most of the participants came back and continued in their sexual modification efforts. A participant mentioned that having supportive environment with fellow community members helped them to know more about themselves.

Adaptation of New Lifestyle. All participants reported avoiding situations and practices that would have make them vulnerable to the gay lifestyle in their sexual reorientation process (10 out of 10 participants). This included ceasing use of dating apps, and limiting interactions with their gay peers. On the other hand, each participant also reported starting certain activities and practices that helped divert their attention from their homosexual lifestyle. This includes frequent church activities and having more personal time of reflection through Bible reading and praying. A participant reported spending more of his time with his family and avoiding places that would make him vulnerable to the homosexual lifestyle.

\section{Discussion}

The findings in the study affirmed faith, as the central driving force in an ex-gay's sexual orientation modification process. Although participants identified parenting and dysfunctional family dynamics as one contributing factor to their gay/lesbian identity, expression of any negativity towards their parents were not seen. Instead, most participants reported better family relationships as they chose to identify as ex-gays. Furthermore, participants in the study reported experiencing no physically or emotionally demanding activities. This may suggest that the approach of Philippine ex-gay communities is less aggressive than that of the ministries in the west, where negative and traumatic experiences from therapy were noted. Lastly, when asked about their current sexual orientation, participants admitted still having same sex attractions, although less powerful and intimate as before. Most of them were not familiar, and as well as hesitant towards the term "ex-gay". However, they preferred to be described as individuals who have turned away from the gay lifestyle striving to live as how God wanted them to be.

\section{References}

Bartoli, E., and Gillemi, A. R. (2008). Continuing to Depolarize the Debate on Sexual Orientation and Religion: Identity and the Therapeutic Process. Professional Psychology: Research and Practice, 39(2), 202-209.

Burack, C. (2015). From Heterosexuality to Holiness: Psychoanalysis and Ex-gay Ministries. Psychoanalysis, Culture \& Society, 20(3), 220-227. doi:10.1057/pcs.2015.25

Courage Philippines. (2012). Powerful Ex-gay Testimony: Danny Wallace. Retrieved from http://couragephilippines.blogspot.com/

D'Augelli, A. R. (1994). Identity development and sexual orientation: Toward a model of lesbian, gay, and bisexual development. In E. J. Trickett, R. J. Watts, \& D. Birman (Eds.), Human diversity: Perspectives on people in context (pp. 312-333). San Francisco: Jossey- Bass.

Haldeman, D. (1994). The Practice and Ethics of Sexual Orientation Conversion Therapy. Journal of Consulting and Clinical Psychology, 62,221-227.

Manalastas, E. J., Ojanen, T. T., Torre, B. A., Ratanashevorn, R., Hong, B. C. C., Kumaresan, V., \& Veeramuthu, V. (2017). Homonegativity in Southeast Asia: Attitudes Toward Lesbians and Gay Men in Indonesia, Malaysia, the Philippines, Singapore, Thailand, and Vietnam. Asia-Pacific Social Sciences Review, 17(1), 25-33.

Robinson, C. and Spivey, S. (2007). The Politics of Masculinity in the Ex-Gay Movement. Gender and Society, 21 (5), 650-675

Shidlo, A. \& Schroeder, M. (2002). Changing Sexual orientation: A Consumers' Report. Professional Psychology: Research and Practice, 33(3), 249-259. doi:10.1037/0735-7028.33.3.249

UNDP., and USAID. (2014). Being LGBT in Asia: The Philippines Country Report. Retrieved from https://www.usaid.gov/sites/default/files/documents/1861/2014\%20UNDPUSAID\%20Philippines\%20LGBT\%20Country\%20Report\%20-\%20FINAL.pdf

Weiss, E., Morehouse, J., Yeager, T., \& Berry, T. (2010). A Qualitative Study of Ex-gay and Ex-Ex-gay Experiences. Journal of Gay \& Lesbian Mental Health, 14(4), 291-319. doi:10.1080/19359705.2010.506412 


\title{
UNIVERSITY TEACHERS' PROFESSIONAL IDENTITY: A ROLE OF SELF-DETERMINATION THEORY
}

\author{
Kristina Kovalcikiene, Aurelija Stelmokiene, Loreta Gustainiene, \\ \& Giedre Geneviciute-Janone \\ Department of Psychology, Vytautas Magnus University (Lithuania)
}

\begin{abstract}
Based on scientific literature, professional identity development of teachers in higher education leads to many positive work outcomes and is one of the essential elements of psychological well-being. This study investigated the role of Self-Determination theory (SDT) in the role of university teachers' sense of professional identity. SDT explains how its components play in producing highly motivated individuals, leading to the development of one's sense of self. Thus, the aim of this study is to identify the relationship between university teachers' professional identity and SDT. Sample consisted of 257 university teachers from Lithuania. Professional Identity Questionnaire (Kovalcikiene, 2014) was used to measure university teachers' identification with three professional roles: researcher, teacher and practitioner (or service provider). The SDT components (autonomy, competence and relatedness) were assessed using Basic Psychological Need Satisfaction and Frustration Scale - Work Domain (Chen et al., 2015). The results revealed that all components of self-determination, i.e. autonomy, competence and relatedness were related ( $\mathrm{p}<.01$, in all cases) with university teachers' identification with all three professional roles (researcher, teacher and practitioner) as well as overall professional identity. The regression analysis revealed that only competence of university teachers significantly associated with their professional identity: researcher, teacher, practitioner and overall ( $\mathrm{p}<.001$, in all cases). Scientific contribution of this research implies empirical study of the role of SDT in university teachers' professional identity.
\end{abstract}

Keywords: Professional identity, self-determination theory, university teachers.

\section{Introduction}

Most studies in the field of teachers' professional identity have mainly focused on school teachers and far less attention is paid to teachers working at higher education institutions (Jermolajeva \& Bogdanova, 2017). Professional identity development of teachers in higher education ensures the quality of teaching, work efficiency, job satisfaction, motivation, occupational commitment (Canrinus et al., 2012; Scartezini \& Monereo, 2016) and is one of the essential elements of psychological well-being (Skinner et al., 2019).

Concept of university teachers' professional identity encompasses their personal identity, social identity and role identity in an intertwined way (Stets \& Burke, 2000), and is usually defined in terms of teachers' perceptions of themselves as teachers based on their interpretations of their continuing interaction with their context (Canrinus, 2011). Traditionally, university teachers play three main roles in the university context: researcher, teacher, and practitioner or service provider (e.g. psychologist). Each of these roles is associated with different contexts, responsibilities, values, and expectations, because each role leads them to interact with different others, e.g. students, university staff, labour market representatives, academics in their area of research, etc. (Scartezini \& Monereo, 2016).

In the scientific literature of professional identity development in higher education, there is a wide variety of theoretical frameworks, and unfortunately, there is a lack of consensus among researchers as to the most effective approach to professional identity development (Trede et al., 2012). In this study, widely applied Self-Determination theory (SDT) is examined. A fundamental principle of SDT is that internalization of behavior and integration to the self is supported by the fulfillment of three basic psychological needs: autonomy (behaving with a sense of volition, endorsement, willingness, and own choice), competence (mastering one's environment), and relatedness (feeling emotionally connected to others) (Ryan \& Deci, 2002). These needs must be ongoingly satisfied in order to maintain autonomous motivation, high-quality performance, and well-being (Deci, et al., 2017). Moreover, SDT explains how its components play in producing highly motivated individuals, leading to the development of one's sense 
of self (Mylrea et al., 2017). The conceptual model, which was offered by Mylrea et al. (2017), of integration of SDT components and professional identity is presented in Figure 1. Thus, this study aimed to identify the relationship between university teachers' professional identity (researcher, teacher, and practitioner) and SDT components (autonomy, competence, and relatedness).

Figure 1. Conceptualization of the role of SDT components in the development of professional identity ${ }^{\text {. }}$

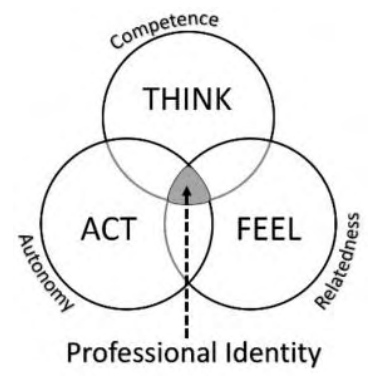

\section{Methods}

The sample consisted of 257 university teachers (34\% of males and 65\% of females) in Lithuania. The average age -46.4 years $(S D=10.23)$. The average of work experience at present university - 15.23 years $(S D=8.83)$. Participants filled up a self-administered on-line questionnaire. Response rate was 23.31 percent. Professional Identity Questionnaire (Kovalcikiene, 2014) was modified (short version) for university teachers to measure their identification with three professional roles: researcher (Cronbach $\alpha=.987$ ), teacher (.846) and practitioner (.979). The SDT components - need for autonomy (.798), competence (.847) and relatedness (.887) - were assessed using Basic Psychological Need Satisfaction and Frustration Scale - Work Domain (Chen et al., 2015).

\section{Results}

The results revealed that SDT components of autonomy, competence and relatedness is related ( $p<.01$, in all cases) with university teachers' identification with all three professional roles (researcher, teacher and practitioner) as well as overall professional identity (Table 1).

Table 1. Descriptive statistics and correlation matrix for research variables $(N=257)$.

\begin{tabular}{|c|c|c|c|c|c|c|c|c|c|c|c|c|}
\hline & Variable & Min. & Max. & Mean & SD & 1 & 2 & 3 & 4 & 5 & 6 & 7 \\
\hline \multirow{6}{*}{$\bar{a}$} & Autonomy & 1 & 7 & 5.60 & .92 & 1.00 & & & & & & \\
\hline & Competence & 1 & 7 & 5.95 & .80 & $.718 * *$ & 1.00 & & & & & \\
\hline & Relatedness & 1 & 7 & 5.38 & 1.15 & $.644^{* *}$ & $.519 * *$ & 1.00 & & & & \\
\hline & Researcher & 1 & 5 & 3.89 & .82 & $.297 * *$ & $.364 * *$ & $.176^{* *}$ & 1.00 & & & \\
\hline & Teacher & 1 & 5 & 4.12 & .74 & $.229 * *$ & $.304^{* *}$ & $.163 * *$ & $.436 * *$ & 1.00 & & \\
\hline & Practitioner & 1 & 5 & 4.01 & .72 & $.221 * *$ & $.317^{* * *}$ & $.178 * *$ & $.534 * *$ & $.740 * *$ & 1.00 & \\
\hline
\end{tabular}

Notes: $* *$ correlation is significant at the .01 level.

The regression analysis revealed (Table 2) that only need for competence of university teachers is associated with their professional identity ( $\mathrm{p}<.001$, in all cases). Mastering environment and being in "control" of work related activities are important for university teachers' professional identity.

Table 2. SDT components in prediction of university teachers' professional identity $(N=257)$.

\begin{tabular}{|c|c|c|c|c|c|c|}
\hline \multirow{2}{*}{ Variables } & \multicolumn{3}{|c|}{ Standardized coefficients $(\beta)$} & \multicolumn{3}{|c|}{ Model characteristics } \\
\hline & Autonomy & Competence & Relatedness & $\mathrm{R}^{2}$ & $\mathrm{~F}$ & Sig. \\
\hline Researcher & .107 & $.317 * *$ & -.057 & .137 & 13.405 & $<.001$ \\
\hline Teacher & .023 & $.289 * *$ & -.002 & .093 & 8.634 & $<.001$ \\
\hline Practitioner & -.029 & $.077 *$ & .049 & .101 & 9.462 & $<.001$ \\
\hline Overall PI & .053 & $.388 * *$ & -.020 & .157 & 15.724 & $<.001$ \\
\hline
\end{tabular}

Notes: *coefficients are significant at the .05 level; **coefficients are significant at the .01 level.

1 Source: Mylrea, M. F., Gupta, T. S., \& Glass, B. D. (2017). Developing Professional Identity in Undergraduate Pharmacy Students: A Role for Self-Determination Theory. Pharmacy, 5 (16), p. 6. 


\section{Discussion and conclusions}

The results revealed that the university teachers' professional identity is linked to the satisfaction of their basic psychological needs of autonomy, competence and relatedness. This confirms the conceptual model of professional identity and SDT proposed by Mylrea et al. (2017). This also confirms the fundamental principle of SDT that satisfaction of basic needs leads to the development of one's sense of self (Deci et al., 2017; Mylrea et al., 2017). The way teachers perceive themselves depends on what teachers think, feel and actually do, and vice versa. Therefore, professional development of university teachers should cover their competences, interconnection and autonomy needs. It is important, because research in organizational psychology usually has tended to take the perspectives of either the employees (i.e., well-being of university teachers) or the profits of organization (i.e., satisfied students or high university ratings). SDT provides the concepts that guide the creation of policies, practices, and environments that promote both well-being and high-quality performance (Deci et al., 2017). Moreover, the results highlighted the importance of need for competence. This confirms the results of Van Lankveld el al. (2017) systematic analysis on psychological processes underlying the development of teacher's professional identity in the university context. In their study, a sense of competence was found to be a key indicator of the development of university teacher identity. It means that university teachers must have a clear vision of their competences and be able to gear the development (if needed).

The added value or scientific contribution of this study is twofold: first, empirically confirmed conceptual interaction between SDT and professional identity contributes to the studies of evidence in this field; secondly, this model was tested in the sample of university teachers. Providing support in three basic areas (autonomy, competence and relatedness), and especially in the area of competence, may have the potential for university teachers to increase their sense of professional identity, as well as their motivation levels (in accordance with SDT).

\section{References}

Canrinus, E. T. (2011). Teachers' sense of their professional identity. Groningen: PrintPartners Ipskamp B.V., Enschede, The Netherlands. 150 p.

Canrinus, E. T., Helms-Lorenz, M., Beijaard, D., Buitink, J., \& Hofman, A. (2012). Self-efficacy, Job Satisfaction, Motivation and Commitment: Exploring the Relationships Between Indicators of Teachers' Professional Identity. European Journal of Psychology of Education, 27, 115-132.

Chen, B., Vansteenkiste, M., Beyers, W., Boone, L., Deci, E. L. et al. (2015). Basic psychological need satisfaction, need frustration, and need strength across four cultures. Motiv Emot, 39, 216-236.

Deci, E. L., Olafsen, A. H., \& Ryan, R. M. (2017). Self-Determination Theory in Work Organizations: The State of a Science. Annual Review of Organizational Psychology and Organizational Behavior, 4, 19-43.

Jermolajeva, J., \& Bogdanova, T. (2017). Professional Identity of Higher Education Teachers in Samples of Riga and Smolensk. Proceedings of the International Scientific Conference SOCIETY. INTEGRATION. EDUCATION, 197-207.

Kovalcikiene, K. (2014). The importance of psychological, social and academic environmental factors for postgraduate (doctoral) students' professional identity expression (Doctoral dissertation, in Lithuanian). Retrieved from https://vb.vdu.lt/object/elaba:2184689/2184689.pdf

Mylrea, M. F., Gupta, T. S., \& Glass, B. D. (2017). Developing Professional Identity in Undergraduate Pharmacy Students: A Role for Self-Determination Theory. Pharmacy, 5 (16), 1-9.

Ryan, R. M., \& Deci, E. L. (2002). An overview of self-determination theory: An organismic dialectical perspective. In E. L. Deci \& R. M. Ryan (Eds.), Handbook of self-determination research (pp. 3-33). Rochester, NY: University of Rochester Press.

Scartezini, R. A., \& Monereo, C. (2016). The development of university teachers' professional identity: a dialogical study. Research Papers in Education, 33 (1), 42-58.

Stets, J. E., \& Burke, P. J. (2000). Identity Theory and Social Identity Theory. Social Psychology Quarterly, 63 (3), 224-237.

Skinner, B., Leavey, G., \& Rothi, D. (2019). Managerialism and teacher professional identity: impact on well-being among teachers in the UK. Educational Review. Retrieved from https://doi.org/10.1080/00131911.2018.1556205.

Trede, F., Macklin, R., \& Bridges, D. (2012). Professional identity development: A review of the higher education literature. Stud. High. Educ., 37, 365-384.

Van Lankveld, T., Schoonenboom, J., Volman, M., Croiset, G., \& Beishuizen, J. (2017). Developing a teacher identity in the university context: a systematic review of the literature. Higher Education Research \& Development, 36 (2), 325-342. 


\title{
SELF-CONCEPT AND ANXIETY OF POTENTIAL SLOVAK LEADERS
}

\author{
Lenka Ďuricová, \& Beata Žitniaková Gurgová \\ Department of Psychology, Faculty of Education, Matej Bel University in Banska Bystrica (Slovakia)
}

\begin{abstract}
Purpose: The purpose of this paper is to examine self-concept and anxiety of the Slovak university students as future leaders. The positive self-concept and emotional stability are considered important characteristics of effective leaders.

Research method: Our research sample consisted of 199 students (mean age=20.04; SD=1.58;) of Matej Bel University (field of study: management and education). The available and intentional sampling was used. The research tools were: State-trait anxiety inventory (Slovak version: Müllner, Ruisel, \& Farkaš, 1980) and Piers-Harris self-concept scale for children and adolescent 2 (Czech version, 2015; translation to Slovak: Duricová \& Ladnová, 2018). Reliability (inner consistency) of measured variables was acceptable (Cronbach alpha from .558 to .902 for state and trait anxiety and all self-concept dimensions. The correlation research study was conducted.

Results: Our findings prove negative moderate or strong statistically significant correlations between all self-concept dimensions and state and trait anxiety (from -.262 to $-.567 ; \mathrm{p}=.001$ ). There has been proved strong significant relation between the total self-concept and state and trait anxiety (-.556; -.684; $\mathrm{p}=0.001)$. Research limitations: Data were self-reported. The sample size.

Value: This paper presents the contribution to the research of relevant personal factors of tomorrow's leaders in education and economics in Slovakia.

The paper was supported by The Slovak Research and Development Agency: APVV 17-0557 project.
\end{abstract}

Keywords: leadership, trait anxiety, state anxiety, self-concept.

\section{Introduction}

According to Bass and Bass (2009), the leadership literature has not shed light on a concerted definition or its constitution. Yukl (2009, p.13) defines leadership as "the process of influencing others to understand and agree about what needs to be done and how to do it, and the process of facilitating individual and collective efforts to accomplish shared objectives." Simplifying the definition, leadership is the ability to influence a group towards the achievement of goals. Leadership is a demanding, unrelenting job with enormous pressures and grave responsibilities. Kirkpatrick and Locke (1991) believes it takes a special kind of person to master the challenges of opportunity.

One of the earliest approaches to understanding leadership was the trait approach emphasizing on the personality of leaders. Contemporary research suggests the importance of five personality traits to determine leader's effectiveness and the leadership style (Judge \& Bono, 2000; Judge et al., 2002; Anderson, 2006; Hassan et al., 2016).

The study of leader traits has a long history. There is constantly reported self-confidence among leader's core traits associated with emotional stability. Every major review of the leadership literature lists self-confidence as an essential characteristic for effective leadership (Bass, 1990; Locke, 1991; Yukl, 2009; House \& Aditya, 1997). There are many reasons why a leader needs self-confidence (Kirkpatrick \& Locke, 1991; Northouse, 2016; Axelrod, 2017; Holsinger, 2018). A great deal of information must be gathered and processed. A constant series of problems must be solved and decisions made. A person riddled with self-doubt would never be able to take the necessary actions nor command the respect of others. Self-confidence plays an important role in decision-making and in gaining others' trust. They have a positive attitude about themselves and are able to press ahead with the belief that, if and when they make a wrong decision, any setback can be overcome. Self-confidence helps effective leaders remain even-tempered. Emotional stability is especially important when resolving interpersonal conflicts and when representing the organization. Leaders who derail are less able to handle pressure and more prone to moodiness, angry outbursts, and inconsistent behaviour, which undermines their interpersonal relationships with subordinates, peers, and superiors. On the contrary, the successful leaders are calm, confident, and predictable even during crisis. Emotional stability operates as relevant variable even in profile approach to 
effective leadership (Parr et al., 2016). Emotional stability is also in positive relationship to up-to-date network leadership performance (Grift, 2016).

The aim of our research study was to examine self-concept and anxiety of the Slovak university students as future leaders. They represent tomorrow's leaders in education and economics in Slovakia. Our objective was to verify the relationship between student's positive self-concept (perceived as the assumption of his future self-confidence) and his state and trait anxiety (the lack of emotional stability). Considering listed empirical research we state these hypothesis:

H1: There is statistically significant negative correlation between student's state anxiety and their total self-concept.

H2: There is statistically significant negative correlation between student's trait anxiety and their total self-concept.

\section{Methods}

The research was carried out in Matej Bel University in Banska Bystrica, Slovakia. Our research sample consisted of 199 university students (mean age $=20.04$; $\mathrm{SD}=1.58$ ). The available and intentional sampling was used (late adolescence and field of university study: management or education). The research was realized anonymously by the means of questionnaire set:

1. The Piers-Harris Children and Adolescent's Self-Concept Scale 2 (Czech version: Obereignerů et al., 2015; translation to Slovak: Duricová \& Ladnová, 2018). Except of total score (TOT), the questionnaire includes 6 subscales evaluating specific domains of self-concept $(\alpha=$ Cronbach alpha): behavioural adjustment (BEH; $\alpha=.647$ ), intellectual and school status (INT; $\alpha=0.558$ ), physical appearance (PHY; $\alpha=.696$ ), freedom from anxiety (FRE; $\alpha=.721$ ), popularity (POP; $\alpha=.626$ ), happiness and satisfaction (HAP; $\alpha=.762$ ).

2. State-trait anxiety inventory (Slovak version: Müllner, Ruisel, \& Farkaš, 1980), measures state anxiety (the current level of anxiety; $\alpha=.902$ ) and trait anxiety (anxiety as an individual disposition; $\alpha=$.899).

Obtained data were processed by the statistical program SPSS using the procedures of descriptive and inductive statistics.

\section{Results}

Since our variables didn't fulfil the condition of normal distribution, non-parametric procedure was used to analyse the data. Based on the results (Table 1), we can state that there are strong negative statistically significant relations between the total self-concept and state and trait anxiety. More detailed analysis brought negative moderate statistically significant correlations between all self-concept dimensions and state and trait anxiety. Especially strong is the negative correlation between trait anxiety and freedom from anxiety and happiness and satisfaction.

Table 1. Correlation analysis (Spearman) of student's anxiety and self-concept $(N=199)$.

\begin{tabular}{|c|c|c|c|c|c|c|c|c|c|}
\hline Variables & 1 & 2 & 3 & 4 & 5 & 6 & 7 & 8 & 9 \\
\hline M (mean) & 11.75 & 10.42 & 7.74 & 6.94 & 7.87 & 8.29 & 41.31 & 39.45 & 46.11 \\
\hline $\begin{array}{l}\text { SD } \\
\text { (stand.dev.) }\end{array}$ & 2.06 & 2.45 & 2.36 & 2.49 & 2.17 & 2.04 & 8.03 & 10.24 & 9.62 \\
\hline 1. BEH & & $.360^{* *}$ & .115 & $.191^{* *}$ & $.152^{*}$ & $.220^{* *}$ & $.501^{* *}$ & $-.320 \% *$ & $-.262^{* * *}$ \\
\hline CI $(95 \%)$ & & $.23-.49$ & $-.02-.25$ & $.07-.32$ & $.02-.03$ & $.08-.36$ & $.40-.60$ & $-.45-.18$ & $-.39-.13$ \\
\hline 2. INT & & & $.406^{* *}$ & $.185^{* *}$ & $.160^{*}$ & $.306^{* *}$ & $.587^{* *}$ & $-.337^{* *}$ & $-.322^{* * *}$ \\
\hline $\operatorname{CI\rho }(95 \%)$ & & & $.29-.52$ & $.05-.32$ & $.03-.29$ & $.18-.44$ & $.49-.67$ & $-.46-.20$ & $-.44-.18$ \\
\hline 3. PHY & & & & $.441^{* *}$ & $.466^{* *}$ & $.721^{* *}$ & $.708^{* *}$ & $-.321 * *$ & $-.475^{* *}$ \\
\hline CI $(95 \%)$ & & & & $.32-.55$ & $.35-.57$ & $.64-.79$ & $.63-.78$ & $-.45-.19$ & $-.58-.36$ \\
\hline 4. FRE & & & & & $.480^{* *}$ & $.511^{* *}$ & $.716^{* *}$ & $-.485^{* *}$ & $-.668^{* *}$ \\
\hline 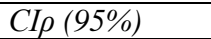 & & & & & $.35-.59$ & $.39-.62$ & $.64-.79$ & $-.59-.79$ & $-.75-58$ \\
\hline 5. POP & & & & & & $.383^{* *}$ & $.625^{* *}$ & $-.334^{* *}$ & $-.387^{* * *}$ \\
\hline CI $(95 \%)$ & & & & & & $.25-.51$ & $.52-.71$ & $-.46-.20$ & $-.50-.25$ \\
\hline 6. HAP & & & & & & & $.727^{* *}$ & $-.452^{* * *}$ & $-.567^{* *}$ \\
\hline 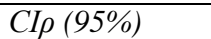 & & & & & & & $.64-.80$ & $-.57-.32$ & $-.67-.45$ \\
\hline 7. TOT & & & & & & & & $-.556^{* * *}$ & $-.684^{* *}$ \\
\hline CI $(95 \%)$ & & & & & & & & $-.65-.45$ & $-.75-.60$ \\
\hline 8. State anxiety & & & & & & & & & $.702^{* *}$ \\
\hline$C I \rho(95 \%)$ & & & & & & & & & $.62-.76$ \\
\hline 9. Trait anxiety & & & & & & & & & \\
\hline
\end{tabular}




\section{Conclusion}

Our results confirm strong negative correlation between student's self-concept and his/her level of anxiety. The stronger positive self-concept the lower the anxiety is. This finding corresponds with previous empirical research (Judge et al., 1998; Judge \& Bono, 2000; Judge et al., 2002). Trait anxiety has direct association to a leader health status (Lindorff, 1995). The most common drugs used by managers are for treating depression, insomnia and anxiety (St-Hilaire \& Gilbert, 2018). Mortensen (2014) also deals with disastrous outcomes of leader's chronic anxiety. In terms of prevention, it is then desirable to develop student's positive self-concept during the study.

By longitudinal study Benson (2018) confirmed that a leader's self-confidence can be developed in youth in a leadership training program and that a leader's effectiveness and leader's self-confidence will persist over time to become permanent. Hollenbeck and Hall (2004) found that education enhances an individual's level of self-efficacy by providing knowledge and skills. Here we can see an opportunity in developing new study programs for future teachers and economists as future leaders.

\section{References}

Anderson, J. A. (2006). Leadership, personality and effectiveness. The Journal of Socio-Economics, 35, $1078-1091$.

Axelrod, R. H. (2017). Leadership and Self-Confidence. In J. Marques, S. Dhiman (Eds.) Leadership Today: Practices for Personal and Professional Performance (297-313). Switzerland: Springer International Publishing.

Bass, B.M. (1990). Stogdill's handbook of leadership. New York: Free Press.

Bass, B. M., \& Bass, R. (2009). The Bass handbook of leadership: Theory, research, and managerial applications. New York,NY: Simon and Schuster.

Benson, L. (2018). Permanency of Leader Self-Confidence Development: A Longitudinal Comparative Analysis. PEOPLE: International Journal of Social Sciences, 4(2), 906-931.

Duricová, L. \& Ladnová, Z. (2018). Perceived parenting style and self-concept of Slovak pubescent youth. The New Educational Review 52 (2), 55-65.

Grift, M. (2016). The role of emotional stability, extraversion and the motivation to affiliate on network leadership performance. Master thesis: Utrecht University.

Hassan, H., Asad, S. \& Hoshino, Y. (2016). Determinants of leadership style in big five personality dimensions. Universal Journal of Management, 4 (4), 161-179.

Hollenbeck, G.P. \& Hall, D. (2004). Self-Confidence and Leader Performance. Organizational Dynamics, 33 (3). 254-269.

Holsinger, J.W. (2018). Traits, skills, and styles of leadership. In J.W Holsinger \& E.L. Carlton, Leadership for Public Health: Theory and Practice. Health Administration Press.

House, R.J., \& Aditya, R.N. (1997). The social scientific study of leadership: Quo vadis? Journal of Management, 23, 409-473.

Judge, T.A., Erez, A. \& Bono, J.E. (1998). The power of being positive: the relation between positive self-concept and job performance. Human Performance, 11 (2-3), 167-187.

Judge, T. A., \& Bono, J. E. (2000). Five-factor model of personality and transformational leadership. Journal of applied psychology, 85, 751-765.

Judge, T. A., Bono, J. E., Ilies, R., \& Gerhardt, M. W. (2002). Personality and leadership: a qualitative and quantitative review. Journal of applied psychology, 87, 765-780.

Kirkpatrick, S.A. \& Locke, E.A. (1991). Leadership: do traits matter? Academy of Management Executive, 5 (2), 48-60.

Locke, E.A. (1991). The essence of leadership: The four keys to leading successfully. New York: Lexington Books

Lindorff, M. (1995). Predictors of symptom increase among managers - the role of environmental, personal and situational characteristics. Stres Medicine, 11(1), $41-50$

Mortensen, R. (2014). Anxiety, work and coping. The Psychologist - Manager Journal, 17 (3), 178-181

Müllner, J., Ruisel, I. \& Farkaš, G. (1980). Dotazník na meranie úzkosti a úzkostlivosti. Bratislava: Psychodiagnostické a didaktické testy.

Northouse, P. G. 2016. Leadership: Theory and Practice, 7th ed. Los Angeles: Sage.

Parr, A. D., Lanza, S. T., \& Bernthal, P. (2016). Personality Profiles of Effective Leadership Performance in Assessment Centres. Human performance, 29(2), 143-157.

St-Hilaire, F. \& Gilbert, M.H. (2018) What the leaders need to know about managers' mental health? Organizational Dynamics, 2018, https://doi.org/10.1016/j.orgdyn.2018.11.002

Yukl, G. (2009). Leadership in Organizations. New Delhi: Dorling Kindersley. 


\title{
PERCEPTION OF (THE) PSYCHOLOGICAL MOTIVES OF TATTOOING OF ALL AGES
}

\author{
Anna Winiarek ${ }^{1}, \&$ Ludwika Wojciechowska ${ }^{2}$ \\ ${ }^{1}$ Polish Academy of Science, Institute of Psychology (Poland) \\ ${ }^{2}$ University of Zielona Góra, Faculty of Education, Psychology and Sociology (Poland)
}

\begin{abstract}
Reviewing the current psychological research, one can reach a conclusion that previously tattooing the body has been mainly seen through the perspective of clinical psychology - usually as body self-mutilation, proving a negative attitude to oneself, or as the occurrence of mental disorders. Tattooing yourself was also often associated with other risk-taking behaviors such as the use of psychoactive substances.

Currently, more and more people are tattooing themselves, more and more tattoo studios are being set up. Therefore, the idea was to look again at the motives of people's tattoos and how tattoos are currently perceived by people of all ages.

The study is question, which was carried out from October 2018 till April 2019, focuses on the perception of the motives of tattooing trough different age cohorts.

A total of 407 people from different development stage (pre-school period, early school-age period, adolescents, early adults, middle adults and late adults) took part in the study. The study was conducted using semi-structured interview.

The collected data were analyzed using the $x^{2}$ test, the $V$-Cramer test and with the Bonferroni method.

The results of the research have shown that people of different ages exhibit a different perspective of the motives for tattooing.

In addition, it turns out that the motives of tattooing are perceived not only as a negative, but also as a positive phenomenon, for example, indicating personal development and the pursuit of self-realization in accordance with assumption of Abraham Maslow's hierarchy of needs.

Detailed results will be presented during the conference.

The results obtained in the study will be used to create a standardized questionnaire to study the psychological motives of tattooing and another body modifications. The study is also the first stage of a broad research project on the mechanisms of body modifications among people of all ages.
\end{abstract}

Keywords: Development psychology, social psychology, personal growth, body modification, tattooing. 


\title{
A CASE STUDY ON THE PROCESS OF INTERPERSONAL RELATIONSHIP FORMATION BETWEEN MUSLIMS AND JAPANESE HOSTS
}

\author{
Sachiko Nakano \\ Yamaguchi University (Japan)
}

\begin{abstract}
This study aims to explore the process of interpersonal relationship formation between international Muslim students and their Japanese hosts in Japan. I conducted a semi-structured interview with eight Muslim international students in Japan, and analyzed the data by using the Trajectory Equifinality Model. Results showed that, step by step, the students underwent seven similar experiences in the interpersonal relationship formation process with Japanese hosts: (1) assumption of cultural differences, (2) consideration of coping policy, (3) contact with the Japanese, (4) perception of cultural differences, (5) occurrence of difficulties, (6) consideration of coping strategies, and (7) coping strategies. Difficulties based on cross-cultural conflict hindered the formation of relations with hosts. The results suggested, therefore, that the key to building relationships is learning how to cope with difficulties. In addition, the combination of cultural preservation and interpersonal relationship formation was divided into three types. The first was to adjust criteria for the observance of religious mandates, so as to also become acquainted with Japanese religious customs. The second was to limit relations with the Japanese, because religious discipline and compliance with mandates is top priority. The third option was not to give up on exchanges with the Japanese or on culture preservation, so as to be able to explain the norms of their own culture and to carry them out with understanding. This study revealed effective skills that make it possible to both preserve the mother culture and form relationships.
\end{abstract}

Keywords: Muslim students in Japan, interpersonal relationship, cross-cultural contact. 


\title{
STIGMATIZATION OF PEOPLE WITH ALCOHOL DEPENDENCE COMPARED TO PEOPLE WITH SCHIZOPHRENIA
}

\author{
Jana Serebriakova, \& Maria Hildegard Walter \\ Department of Psychology, University of Innsbruck (Austria)
}

\begin{abstract}
Previous stigma research has shown considerable differences between various disorders. However, most studies neglected the impact on stigmatization that behaviour, diagnosis and the label as a disease have separated from each other. To counteract this limitation we examined differences in public conceptions of the most stigmatized mental illnesses: alcohol dependence and schizophrenia.

We analysed responses to vignettes describing individuals meeting DSM-III-R criteria for alcohol dependence and schizophrenia, without mentioning the name of the disease. The purpose is to explore whether there are differences regarding 1) the stigma, containing personal attributes (stereotypes), emotional reactions (prejudices) and social distance (discrimination), 2) the impact of labelling the behaviour in the vignette as a disease, and 3) the impact of giving the right diagnosis.

We found differences of stigmatization between alcohol dependence and schizophrenia. Contrary to previous research, labelling the behaviour in the vignettes as a disease has a positive impact on stigmatization towards people with alcohol dependence and schizophrenia. One of the most notable differences between the two disorders is that, regarding alcohol dependence, giving the right diagnosis affects all components of stigma negatively, while giving the diagnosis schizophrenia shows less negative reactions.

Our findings illustrate the need for differentiation between mental disorders as well as between the different components of stigma. Moreover, labelling with a disease or diagnosis has a various influence on stigmatization between alcohol dependence and schizophrenia. The described differences of public conceptions have important implications for the planning of anti-stigma programs and may help developing more customized interventions.
\end{abstract}

Keywords: Stigmatization, alcohol dependence, addiction, schizophrenia.

\section{Introduction}

A considerable part of stigma research in psychiatry has dealt with mental disorder in general. However, previous research has shown considerable differences between various mental disorders (Aydin \& Fritsch, 2015). Therefore, a systematic comparison between lay beliefs, attitudes and reactions to those suffering from alcohol dependence and schizophrenia, will be provided. The public stigma has relevant consequences for the stigmatized (Anagnostopoulos \& Hantzi, 2011). Perceptions of public stigma can conduce to the experience of self-stigma, which influences seeking professional help (Schomerus, 2009). Furthermore, it has negative effects on quality of treatments, process of the disease, integration and quality of life for the stigmatized (Meise, Fleischhacker \& Schöny, 2002).

In psychiatric attitude research, the stigma of alcohol dependence received less attention than the stigma of other mental disorders (Schomerus et al., 2010). Previous studies showed that people with alcohol dependence are more stigmatized than people with schizophrenia (Pescosolido et al., 2010). As reported in previous studies, the public appears somewhat more likely to identify symptoms of schizophrenia as an indication of mental disorder than symptoms of alcohol dependence. Furthermore, alcohol dependence is also considered as an infringement of social norms (Schomerus et al., 2011). In a review of studies, Link et al. (1987) showed that although most studies found some effects of labelling mental illness, but these effects were either not significant across all the outcomes or didn't hint towards more severe labels leading to more severe rejection. 


\section{Objectives}

Alcohol dependence and schizophrenia appear to be the most stigmatized mental disorders and should be main targets of interventions aimed at reducing the stigma of mental illness (Pescosolido et al., 2010). A better differentiation of the stigma components of these two disorders may allow us to develop more customized interventions that may prove more successful than broad and unspecific approaches.

Therefore, in our study similarities and differences will be examined regarding 1) the stigma, containing personal attributes (stereotypes), emotional reactions (prejudices) and the desire for social distance (discrimination), 2) the impact of labelling the behaviour in the vignette as a disease, and 3) the impact of giving the right diagnosis.

\section{Methods}

\subsection{Design and sample}

The sample consisted of 637 German-speaking participants (70 per cent female). Participants were aged 16 to 71 years $(M=25.5, S D=7.9)$.

The presented study was conducted as an online survey targeting German-speaking participants between February and March 2017. We placed advertisements in the mailing list of the university and Facebook-posts. Participation was voluntary and included electronically obtained underwriting of their anonymity for all participants at the beginning of the survey. To avoid bias, we told the participants in the beginning that we consider the attitude, but did not mention that the survey is about attitude toward mental disorders. During the survey, participants obtained two vignettes containing a diagnostically unlabeled psychiatric case history and filled out questionnaires measured personal attributes, emotional reactions and social distance towards the persons described in the vignettes, two questions about the labelling of the problem in the vignettes and questionnaires on socio-demographic details.

\subsection{Instruments}

Vignette. The survey began with the presentation of a vignette containing a diagnostically unlabeled psychiatric case history. The case histories either represented a case of alcohol dependence or schizophrenia. Angermeyer and Matschinger (2003a) used DSM-III-R criteria for the construction of the vignettes, but the symptoms in the vignettes also fulfilled the current criteria of ICD-10 for the respective disorder. The samples were presented with both vignettes.

Personal attributes. A list of nine personal attributes was created by Angermeyer (2003b) to cover two important components of the stereotype of mental illness: dangerousness and dependency. Respondents were asked to indicate on a five-point Likert scale ranging from "definitely true" to "definitely not true" to what extent these attributes apply to the person described in the vignette.

Emotional reactions. A list of eleven five-point Likert-scaled items ( 1 = definitely the case, 5 = definitely not the case), representing three ways to respond to individuals with mental illness (fearful, adverse and prosocial emotions), was used to assess the respondents' emotional reactions to the person described in the vignette (Sulzenbacher, 2004).

Social distance. For the assessment of respondent's desire for social distance, we used a scale developed by Matschinger, Rost and Angermeyer (2000). It includes seven items representing the following social relationships: rent a room, commonplace of work, neighborhood, member of the same social circle, personal job brokering, marriage into one's family and child care. Answers, to what extent they would, in those situations, accept the person described in the vignette, were given on a five-point Likert scale offering choices from "most certainly" to "certainly not".

Labelling. The respondents were asked, if they would indicate the problem described in the vignette as a disease. If they answer in the affirmative, we used an open-ended question to indicate the diagnosis of the disease. Two main categories were distinguished: correct and incorrect diagnosis.

\section{Discussion}

Summarizing our findings, a number of differences between alcohol dependence and schizophrenia emerge. While the person in the alcohol dependence-vignette elicits a greater desire for social distance, more emotions that are adverse and less prosocial emotions, the person in the schizophrenia-vignette was frequently perceived as more dangerous and dependent as well as elicits more emotions that are fearful. The symptoms of both disorders in the vignette are identified as an indication of a disease by the majority of the respondents. Contrary to previous research (Angermeyer \& Matschinger, 2003a), labelling the behavior in the vignettes as a disease has a positive impact on stigmatization towards people with alcohol dependence and schizophrenia. People, who label the described problem in the 
vignette as a disease, perceive in both cases more prosocial emotions and perceive the person in the vignette as more dependent. In the alcohol dependence-vignette, they furthermore perceive less adverse emotions, than people, who did not label the problem in the vignette as a disease. It seems like the label has a protective mechanism toward stigmatization. One of the most notable differences between the two disorders is that, regarding alcohol dependence, giving the right diagnosis affects all components of stigma negatively, while giving the diagnosis schizophrenia shows fewer negative reactions. In the case of alcohol dependence, giving the individual in the vignette the right diagnosis has negative effects on the reactions towards them (greater desire for social distance, adverse emotions and perception as dangerous). By contrast, in the case of schizophrenia, giving the right diagnosis only has a negative effect on the perceived danger. The differences in the case of alcohol dependence could be resulted from the fact that alcohol dependence is seen as a disease, a weakness of will as well as an infringement of social norms.

Those described differences of public conceptions have important implications for the planning of anti-stigma programs, because they show that there are no homogenous and over all mental disorders generalizable anti-stigma strategies. Therefore, it should be the main target to develop more customized interventions, based on the differences across various mental disorders.

\section{Conclusions}

Our findings illustrate the need for differentiation between mental disorders as well as between the different components of stigma. Moreover, labelling with a disease or diagnosis has various influence on stigmatization between alcohol dependence and schizophrenia. The described differences of public conceptions have important implications for the planning of anti-stigma programs and may help developing more customized interventions.

\section{References}

Anagnostopoulos, F., \& Hantzi, A. (2011). Familiarity with and Social Distance from People with Mental Illness: Testing the Mediating Effects of Prejudiced Attitudes. Journal of Community \& Applied Social Psychology, 21(5), 451-460.

Angermeyer, M. C., \& Matschinger, H. (2003a). The stigma of mental illness: effects of labelling on public attitudes towards people with mental disorder. Acta Psychiatrica Scandinavia, 108(4), 304-309.

Angermeyer M. C., \& Matschinger H. (2003b). Public beliefs about schizophrenia and depression: similarities and differences. Social Psychiatry and Psychiatric Epidemiology, 38(9), 526-534.

Aydin, N., \& Fritsch, K. (2015). Stigma und Stigmatisierung von psychischen Krankheiten. [Stigma and stigmatization of mental disorders]. Psychotherapeut, 60(3), 245-257.

Link, B. G., Cullen, F. T., Frank, J., \& Wozniak, J. F. (1987). The social rejection of former mental patients. Understanding why labels matter. American Journal of Sociology, 92(6), 1461-1500.

Matschinger, H., Rost, J., \& Angermeyer, M. C. (2000). Probleme der Populationsheterogenität bei der Messung und Erklärung von Einstellungsdimensionen. Darstellung am Beispiel sozialer Distanz gegenüber psychisch Erkrankten. [Problems of the population-heterogeneity at the measurement and explanation of attitude-dimensions. Illustration using the example of social distance toward people with mental disorders]. In Reinecke, J., \& Tarnei, C. (Eds.), Angewandte Klassifikationsanalyse in den Sozialwissenschaften (pp. 159-177). Münster: Waxmann.

Meise, U., Fleischhacker, W.W., \& Schöny, W. (2002). Es ist leichter ein Atom zu zerstören, als ein Vorurteil. [It is easier to destroy an atom than a prejudice]. Neuropsychiatrie, 16(1-2),1-4.

Pescosolido, B. A., Martin, J. K., Long, J. S., Medina, T. R., Phelan, J. C., \& Link, B. G. (2010). “A Disease Like Any Other"? A Decade of Change in Public Reactions to Schizophrenia, Depression, and Alcohol Dependence. The American Journal of Psychiatry, 167(11), 1321-1330.

Schomerus, G. (2009). Steine auf dem Weg. Stigma und Hilfesuchverhalten. [Stones on the way. Stigma and seeking help behavior]. Psychiatrische Praxis, 36(2), 53-54.

Schomerus, G., Holzinger, A., Matschinger, H., Lucht, M., \& Angermeyer, M. C. (2010). Public attitudes towards alcohol dependence. Psychiatrische Praxis, 37(3), 111-118.

Schomerus, G., Lucht, M., Holzinger, A., Matschinger, H., Carta, M. G., \& Angermeyer, M. C. (2011). The Stigma of Alcohol Dependence Compared with Other Mental Disorders: A Review of Population Studies. Alcohol and Alcoholism, 46(2), 105-112.

Sulzenbacher, H. (2004). Die Relevanz des Begriffs „Schizophrenie“für die soziale Einstellung zu schizophren Erkrankten: Eine Untersuchung bei Studierenden [The relevance of the concept "Schizophrenia" for the social attitude toward schizophrenic people]. (Unpublished doctoral dissertation). Leopold-Franzens-Universität, Innsbruck, AUT. 


\title{
HETEROGENEITY OF THINKING: CASE OF RELIGION
}

\author{
Alisa Rekunova \\ Tallinn University/Institute of Natural Sciences and Health (Estonia)
}

\begin{abstract}
According to Lev Vygotsky's cultural-historical theory, thinking goes through four stages: syncretic concepts, everyday concepts, pseudoconcepts, and true (structural-systemic, by Aaro Toomela) concepts. Toomela suggested, that religious beliefs tend to form on the stage of everyday concepts. The first step of the work was to distinguish different stages of concepts in the human mind by examining the words the person uses during an interview. The second step was to examine whether there any contradictions between religious and scientific concepts. Through the framework of the theory of heterogeneity of thinking, it was analyzed if and how the person comes to terms with contradictory areas of life.
\end{abstract}

Keywords: Contradictions, cultural-historical psychology, heterogeneity of thinking, religion.

\section{Introduction}

According to the theory of word meaning structure developed by Lev Vygotsky (1934) and later modified by Aaro Toomela (2017), it can be sensory-based thought, everyday (or situation) thought, logical thought, and structural-systemic thought. However, people tend to switch between levels of thought due to heterogeneity of the mind and thinking.

Heterogeneity of the mind means that in different areas of thought the level of thinking can be developmentally different. Nevertheless, mainstream psychology usually does not account heterogeneity of the mind and works with massive amounts of statistical data excluding individual differences (Toomela 2007, 2008).

According to Toomela (2017), religious beliefs tend to form on the stage of everyday concepts when a child is not able to distinguish pseudoconcepts from logical or structural-systemic concepts.

Recent studies show controversial results. For example, some of them show that religiosity decreases with the level of education (Kanazawa, 2010; Gervais \& Norenzayan, 2012); the others show the opposite picture (Iannaccone, 1998).

Thus, the main question is: is it correct that conflicts are resolved easier by people who have higher education or there are some other characteristics that matte more? And if these characteristics exist, what are they?

\section{Methods}

To explore people's conflicts and resolving of these conflicts, it was decided to use qualitative research. 20 semi-structured interviews were held in Russian. Religious beliefs, attitude towards religion, level of education, and personal experience were examined.

There were 20 participants: 2 people without higher education, 7 people with higher education, and 11 scientists of different spheres.

\section{Results}

In the research, there were three different kinds of approaches to faith: 8 believers, 8 non-believers (atheists), and 4 undecided (we can call them agnostics).

Some of the respondents said that scientific or professional and religious spheres do not overlap, therefore, we can assume they do not see any conflict. Some of them, on contrary, hesitate to answer and we can conclude they see the conflicts but they do not want (or do not believe they are able to) to solve it. Finally, the third category of respondents say that religious beliefs and scientific concepts cannot coexist in the human mind. 


\section{Discussion and summary}

People who tend to think on everyday level of thought, usually don't see conflicts; people who think in logical concepts, see conflicts but prefer not to think about that by different reasons; and people who have access to structural-systemic concepts, see conflicts and solve them.

There are differences between people who have relatively easy access to logical thought compared to those who mostly tend to think in everyday concepts.

The level of thought is connected to the level of education, nevertheless, there are other factors that matter. Some of the respondents were brought up in religious families and later realized themselves as atheists or agnostics. However, the opposite way from an atheist or an agnostic to a believer is a very rare case.

\section{References}

Gervais, W. M., \& Norenzayan, A. (2012). Analytic thinking promotes religious disbelief. Science, 336, 493-496.

Iannaccone, L. R. (1998). Introduction to the economics of religion. Journal of Economic Literature, 36, 1465-1496.

Kanazawa, S. (2010). Why liberals and atheists are more intelligent. Social Psychology Quarterly, 73(1), 33-57.

Toomela, A. (2007). Culture of Science: Strange History of the Methodological Thinking in Psychology. Integr Psych Behav, 41, 6-20.

Toomela, A. (2008). Variables in Psychology: A Critique of Quantitative Psychology. Integr Psych Behav, 42, 245-265.

Toomela, A. (2017). Religion as a form of everyday conceptual thought: Evidence from orthodox icons. In: Brown, S. \& Tateo, L. (Ed.). The method of imagination. Charlotte, NC: Information Age Publishing, 191-224.

Vygotsky, L. S. (1934). Myshlenije i rech. Psikhologicheskije issledovanija. (Thinking and speech. Psychological investigations.). Moscow: Gosudarstvennoje Social'no-ekonomicheskoje Izdatel'stvo. 



\section{* VIRTUAL PRESENTATIONS *}

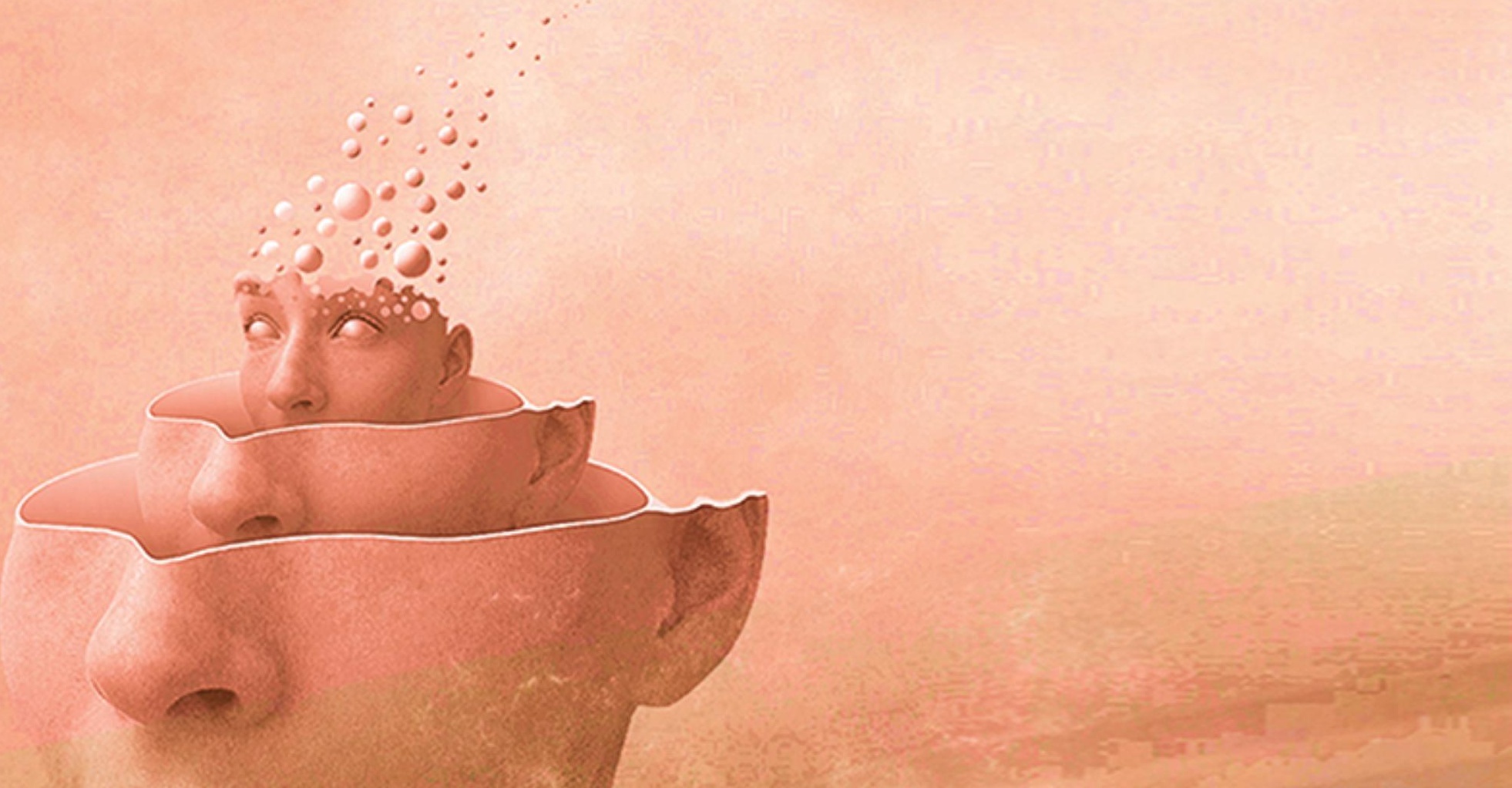





\title{
PARENTAL SUPPORT, MENTORING RELATIONSHIP AND 30-DAY PREVALENCE OF ALCOHOL USE AMONG HIGH SCHOOL BOYS AND GIRLS
}

\author{
Marianna Berinšterová, Monika Magdová, \& Miroslava Bozogáňová \\ Institute of Pedagogy and Psychology, Faculty of Humanities and Natural Sciences \\ University of Presov (Slovakia)
}

\begin{abstract}
Parental and nonparental significant adults are important developmental assets for adolescents. Previous studies confirm positive association of mentoring, parent-child relationship and substance use prevention. The aim of this paper is to explore a mediational effect of mentoring in relation to perceived paternal and maternal support and a 30-day prevalence of alcohol use among high school students in Slovakia.

Items from the ESPAD study were used for measuring the 30-day prevalence of alcohol use. The Resilience and Youth Development Module questionnaire was used and adapted for exploring the perceived support from the mother and the father. In addition, a single item measure for examining the existence of a mentoring relationship was used. 177 students (53.7\% girls; Mage=1.92; SD=1.69) participated in the research. Descriptive analysis and binary logistic regression were used for data analyses in SPSS 16. Analyses were conducted separately among boys and girls.

Out of the 177 students, 70 (39.5\%) reported the existence of a mentoring relationship and 113 students (63.8\%) reported alcohol use in the last 30 days. Results did not confirm significant mediational effect of a mentoring relationship on examined variables. Nevertheless, both the existence of a mentoring relationship and alcohol use in the last 30 days were more frequently found among boys who perceived a lower level of paternal and maternal support. Perceived paternal and maternal support were not significantly associated with the examined variables among girls.

Results point to different correlates of alcohol use depending on gender and also distinctions of significant parental and nonparental adult roles in the middle and late adolescents' development. Suggestions for counselling significant adults and adolescents are discussed.
\end{abstract}

Keywords: Significant adult, mentoring relationship, alcohol use, adolescents.

\section{Introduction}

A significant adult is an important protective factor against risky behaviour of adolescents (Galbo, 1986). Resilience theories claim that a supportive relationship from the adults in the child's environment serves as a developmental asset that leads to the satisfaction of the needs of autonomy, competence and relatedness (Constantine, Benard, Diaz, 1999).

Several authors suggest that gender-specific differences in parent-child relationships are less researched (Luk, Farhat, Iannotti, et al., 2010). Older research demonstrated that fathers provide more instrumental support and mothers affective support (Starrels, 1994). Gender correlates with parent-child relationships (Biblarz, Stacy, 2010). Positive association was also found between both mother-child and father-child communication and young people's life satisfaction (Levin, Currie, 2010). The attention of this field is justified in the context of substance use prevention because of confirmed gender-specific risk factors of substance use (Amaro, Blake, Schwartz, \& Flinchbaugh, 2001).

The adolescent's social world expands, including extrafamilial relationships (Arnett, 2004). A nonparental significant adult can act as a natural mentor (Rhodes \& Lowe, 2008). Positive consequences of mentoring were found in the areas of attitudes, health, relations - family bonding, school bonding, motivation, career outcomes, life skills, self-efficacy, school performance, and emotional regulation (Zand et al., 2009; Eby, Allen, Evans, Ng, \& DuBois, 2008; Jucovy, 2003).

Non-parental adults might have a functional role in which they provide support and guidance to others and serve as role models. Thus, individuals in different social roles, such as older friends, family members, but also romantic partners, might serve as a mentoring capacity to adolescents. 


\section{Objectives}

The aim of this paper is to explore a mediational effect of mentoring in relation to perceived paternal and maternal support and a 30-day prevalence of alcohol use among high school students in Slovakia. Previous research suggests gender-specific relationships between parental support and substance use of adolescents (Luk, Farhat, Iannotti, \& Simons-Morton, 2010).

\section{Methods}

\subsection{Research sample}

The sample consisted of 177 students (53.7\% girls; Mage=1.92; SD=1.69) from two high schools in Slovakia. The selection of schools was highly dependent on the willingness of school administrators to participate in the study. Data collection took place during school lessons, in the presence of an external administrator. Parental approval with school activities for the given school year has been extended to the participation in the research.

\subsection{Research questionnaire}

Data were collected by means of using the following items:

3.2.1. Single item measure of alcohol use in the last 30 days from the questionnaire of international study Espad (Hibell, Guttormsson, Ahlström, et al., 2011). Think back over the LAST 30 DAYS. On how many occasions (if any) did you drink alcohol? Responses were dichotomized: not at all (0), at least once.

\subsubsection{Single item measure for examining the existence of a mentoring relationship was} used, supplemented by a note about a mentoring relationship definition as follows. Mentoring relationship = this is a relationship between you and an adult who is not your parent or caretaker. He/she is someone you can go to if you need support, leadership and advice, someone who inspires you, encourages you to try your best in life.

Item was assessed on a two-point scale (yes - no).

3.2.3. RYDM questionnaire. Resilience and Youth Development Module questionnaire (Constantine, Benard, \& Diaz, 1999; Hanson \& Kim, 2007) was used and adapted for exploring the perceived support from the mother and the father. Parental support was understood as consisting of relationships perceived as caring, and include adults' high expectations (Constantine, Benard \& Diaz, 1999). Twenty-six items exploring parental support were assessed on a four-point Likert scale. Item examples include statements such as "My mother (father) really cares about me", "My mother (father) always wants me to do my best". The reliability (Cronbach $\alpha$ ) of the subscale in our sample was 0.955 .

\subsection{Statistical analysis}

Research data were processed by SPSS.16. Descriptive and regression analyses were used. For the needs of mediational analysis with dichotomic mediator and outcome variables, binary logistic regression was used. Sobel test (Herr, N. R. (n.d.)) determined value of Z-score. Analyses were conducted separately among boys and girls.

\section{Results}

\subsection{Descriptive analysis}

From the total sample of 177 respondents, 70 (39.5\%) reported the existence of a natural mentoring relationship. Out of 82 boys, 37 (45.1\%) reported the existence of a mentor. Out of 95 girls, 33 (34.7\%) reported the existence of a mentor. (31.6\%).

64 students (36.2\%) reported alcohol use in the last 30 days: 34 boys (41.5\%) and 30 girls 


\subsection{Mediational analysis in the sample of boys}

Two models for the sample of boys were conducted, examining maternal support (Figure 1) and paternal support (Figure 2) in relation to a 30-day prevalence of alcohol use with the mediational effect of mentoring. Boys with a higher level of perceived maternal and paternal support were more likely to report the existence of a mentoring relationship. Paternal support was also negatively associated with the 30-day prevalence of alcohol use. Sobel test for the Model $1(z=-0,764)$ and Model $2(z=-0,231)$ did not confirm significant mediational effects.

Figure 1. Mediational effect of a mentoring relationship in relation to maternal support and a 30-day prevalence of alcohol use among boys (Model 1).

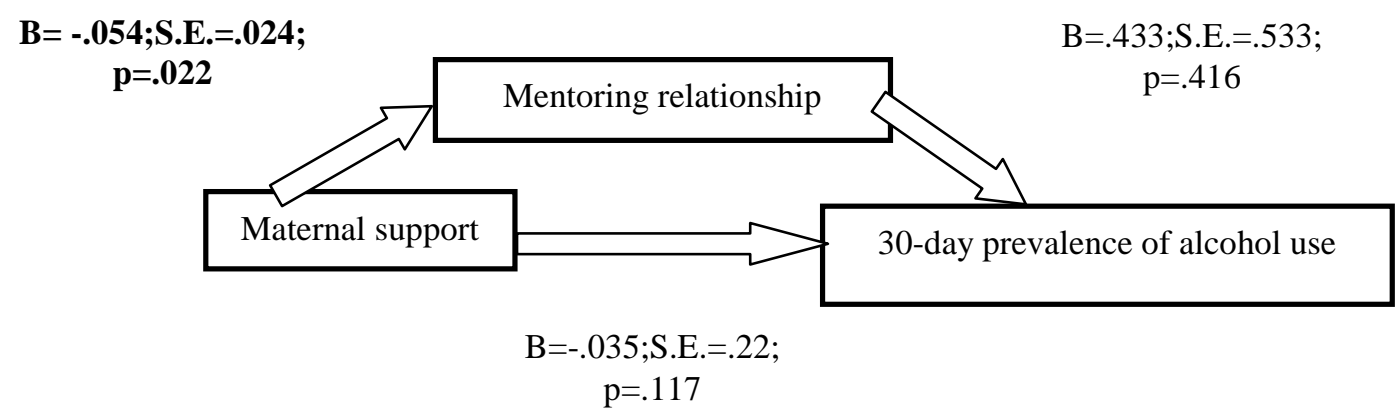

Figure 2. Mediational effect of a mentoring relationship in relation to paternal support and a 30-day prevalence of alcohol use among boys (Model 2.)

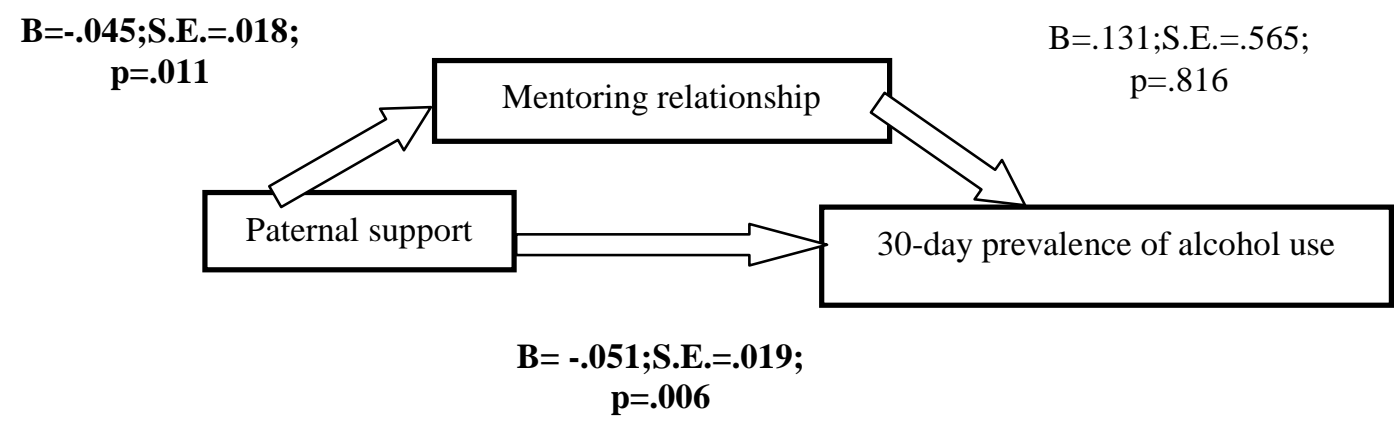

\subsection{Mediational analysis in the sample of girls}

Also, two models for the sample of girls were conducted examining maternal support (Figure 3) and paternal support (Figure 4) in relation to a 30-day prevalence of alcohol use with the mediational effect of mentoring. Associations between the variables were not significant. Sobel test for the Model 3 $(\mathrm{z}=-0,28)$ and Model $4(\mathrm{z}=-0,298)$ did not confirm significant mediational effects.

Figure 3. Mediational effect of a mentoring relationship in relation to paternal support and a 30-day prevalence of alcohol use among girls (Model 3).

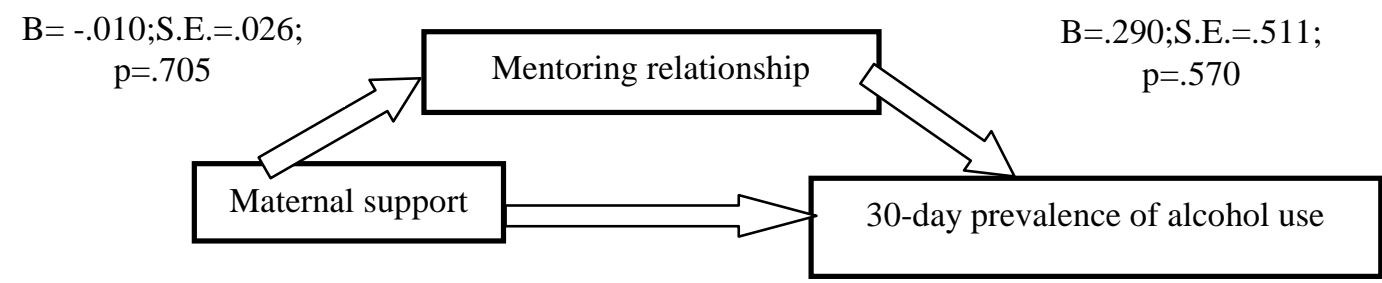

$$
\begin{gathered}
\mathrm{B}=.30 ; \text { S.E. }=.028 ; \\
\mathrm{p}=.289
\end{gathered}
$$


Figure 4. Mediational effect of a mentoring relationship in relation to paternal support and a 30-day prevalence of alcohol use among girls (Model 4).

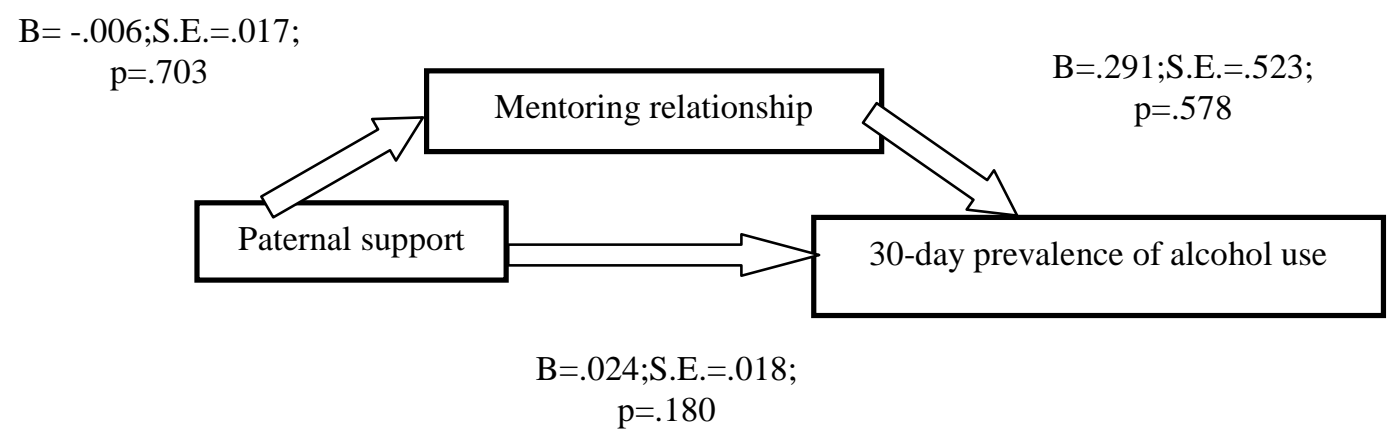

\section{Discussion and conclusion}

The aim of this paper was to explore a mediational effect of mentoring in relation to the perceived paternal and maternal support and a 30day prevalence of alcohol use among high school students in Slovakia. Although the Sobel test did not confirm a mediational effect, some significant associations were found, especially among boys:

- the association among the perceived maternal support and the existence of a mentor relationship, the perceived parental support and the existence of a mentoring relationship. Boys who perceived lower levels of paternal and maternal support were more likely to report a mentoring relationship. Among girls, this association was also negative but not significant

- The negative association between paternal support and the 30-day prevalence of alcohol use. Those boys who perceived lower levels paternal support were more likely to use alcohol in the last 30 days. This association was not significant for the maternal support. In contrast, previous research found that the fathers had less influence and were less involved in a relationship with the child than the mothers (Stoker \& Swadi, 1990, Williams \& Kelly, 2005).

No significant relationships among the variables was found among girls, in contrast with previous research which found that parental control and parental emotional support were more strongly related to substance use outcomes in girls than in boys, and also that girls were prone to supportive communication with their fathers (Choquet, Hassler, Morin, et al., 2008; Demidenko, Manion, Lee, 2015).

No significant association between a mentoring relationship and a 30-day prevalence of alcohol use was found, although relationships with a significant nonparental adult is considered to have multiple positive consequences. The explanation should be rooted in cultural and social norms. Alcohol use is considered a part of traditions and local social norms. It was found that descriptive norms are directly associated with alcohol use among students (Magdová, 2018).

\subsection{Limitations of the research}

Of course, this research has some limits, including the social desirability of answers, the sample size and also the limited amount of attention paid to the person of a mentor. Further research should focus on the socio-demographic, personality and social-psychology variables related to nonparental significant adults in the context of substance use.

The research suggests a protective role of parental support in the context of substance use. Resilience theory sees these developmental assets as a buffer against negative influences. The father-son relationship occupies a unique position. However, further research is needed to describe these genderspecific variations more closely. 


\section{References}

Amaro, H., Blake, S. M., Schwartz, P. M., \& Flinchbaugh, L. J. (2001). Developing theory-based substance abuse prevention programs for young adolescent girls. The Journal of Early Adolescence, 21(3), 256-293.

Arnett, J. J. (2004). A longer road to adulthood. Emerging adulthood, 3-25.

Biblarz, T. J., \& Stacey, J. (2010). How does the gender of parents matter? Journal of marriage and family, 72(1), 3-22.

Constantine, N., Benard, B., \& Diaz, M. (1999, June). Measuring protective factors and resilience traits in youth: The healthy kids resilience assessment. In: Seventh annual meeting of the Society for Prevention Research, New Orleans, LA.

Demidenko, N., Manion, I., \& Lee, C. M. (2015). Father-daughter attachment and communication in depressed and nondepressed adolescent girls. Journal of Child and Family Studies, 24(6), 1727-1734.

Eby, L. T., Allen, T. D., Evans, S. C., Ng, T., \& DuBois, D. L. (2008). Does mentoring matter? A multidisciplinary meta-analysis comparing mentored and non-mentored individuals. Journal of vocational behavior, 72(2), 254-26.

Galbo, J. J. (1986). Adolescents' perceptions of significant adults: Implications for the family, the school and youth serving agencies. Children and Youth Services Review, 8(1), 37-51.

Hanson, T. L., \& Kim, J. O. (2007). Measuring Resilience and Youth Development: The Psychometric Properties of the Healthy Kids Survey. Issues \& Answers. REL 2007-No. 34. Regional Educational Laboratory West.

Herr, N.R. (n.l.). Mediation with Dichotomous Outcomes. http://www.nrhpsych.com/mediation/logmed.html

Hibell, B., Guttormsson, U., Ahlström, S., Balakireva, O., Bjarnason, T., Kokkevi, A., \& Kraus, L. (2011). Substance use among students in 35 European countries. Stockholm: The Swedish Council for Information on Alcohol and Other Crugs (CAN).

Jucovy, L. (2003). Mentoring children of prisoners in Philadelphia. Public/Private Ventures. Retrieved November 12, 2017 from http://www.ppv.org/ ppv/publications/assets/21_publication.pdf.

Levin, K. A., \& Currie, C. (2010). Family structure, mother-child communication, father-child communication, and adolescent life satisfaction: A cross-sectional multilevel analysis. Health Education, 110(3), 152-168.

Luk, J. W., Farhat, T., Iannotti, R. J., \& Simons-Morton, B. G. (2010). Parent-child communication and substance use among adolescents: Do father and mother communication play a different role for sons and daughters? Addictive behaviors, 35(5), 426-431.

Magdová Brutovská, M. (2018). Uloga društvenih normi u strukturnom modelu koji objašnjava uporabu alkohola među studentima. Društvena istraživanja: časopis za opća društvena pitanja, 27(4), 605-628.

Rhodes, J., \& Lowe, S. R. (2008). Youth mentoring and resilience: Implications for practice. Child care in practice, $14(1), 9-17$.

Starrels, M. E. (1994). Gender differences in parent-child relations. Journal of family Issues, 15(1), 148-165.

Zand, D. H., Thomson, N., Cervantes, R., Espiritu, R., Klagholz, D., LaBlanc, L., \& Taylor, A. (2009). The mentor-youth alliance: The role of mentoring relationships in promoting youth competence. Journal of adolescence, 32(1), 1-17. 


\title{
THE ADDICTIVE PERSONALITY: MYTH OR CORNERSTONE OF PREVENTION AND TREATMENT?
}

\author{
Donna L. Roberts \\ PhD, Department of Social Sciences \& Economics, Embry-Riddle Aeronautical University (USA)
}

\begin{abstract}
The concept of an addictive personality offers an attractive and intuitive construct for defining the parameters of prevention, diagnosis and treatment of substance abuse and addictive disorders. However, despite the intellectual and practical appeal, it remains controversial, with researchers and practitioners heavily debating its fundamental validity. In a seminal research study, Alan R. Lang, in conjunction with the US National Academy of Science, found that while there was no single identifiable personality type that could be used to exclusively determine susceptibility to addiction or constitute an "addictive personality," there are, however, significant personality factors that tend to underlie addictive behavior. Specifically, these included, impulsivity, sensation seeking, nonconformity, social alienation, tolerance for deviance, and heightened stress coupled with lack of coping skills. Proponents of the addictive personality paradigm argue that these common characteristics represent critical information for intervention and treatment, as well as in the realm of development and prevention. Conversely, opponents contend that not only does empirical research not support the conclusion, but neither does the theoretical framework of addiction as disease. They contend that this view is deterministic and undermines the complex and multifaceted nature of addiction, thus limiting the approaches to treatment and potentially increasing the stigma associated with addictive disorders. Currently, most researchers and practitioners would argue against the notion of a deterministic addictive personality, but consider the potential of individual personality factors to influence behavior in both positive and negative ways and thus, incorporate relevant strategies into a comprehensive treatment plan.

As we now face a global epidemic of opioid abuse that surpasses previous waves of drug use in both magnitude and severity, perhaps it is time to reevaluate the role of personality as a contributor in both the development and rehabilitation of addiction and how such information, taken in context with other relevant factors, can best serve to combat the widespread epidemic.
\end{abstract}

Keywords: addiction, personality, addictive personality, substance abuse

\section{Introduction - personality, addiction and the addictive personality defined}

Psychologists have defined personality as "the set of psychological traits and mechanisms within the individual that are organized and relatively enduring and that influence his or her interactions with, and adaptations to, the intrapsychic, physical and social environments" (Larsen \& Buss, 2018, p. 4). As such, it represents a comprehensive and stable characterization of an individual.

According to the American Society of Addiction Medicine, addiction is "a primary, chronic disease of brain reward, motivation, memory and related circuitry. Dysfunction in these circuits leads to characteristic biological, psychological, social and spiritual manifestations. This is reflected in an individual pathologically pursuing reward and/or relief by substance use and other behaviors. Addiction is characterized by inability to consistently abstain, impairment in behavioral control, craving, diminished recognition of significant problems with one's behaviors and interpersonal relationships, and a dysfunctional emotional response" (ASAM, 2019, para. 1).

Thus, consideration of an addictive personality assumes that the tendency to adopt and maintain these addictive behaviors is woven into the very fabric of the individuals overarching patterns of thought, emotion and manner of behaving. This perspective posits that certain personality types are more predisposed - some even assert destined - to develop and maintain psychological and/or physical addiction. 


\section{The addictive personality - myth or reality?}

The designation of 'addictive personality' does not appear among the ten personality disorders of the current Diagnostic and Statistical Manual of Mental Disorders (DMS), Fifth Edition. Furthermore, the DSM-5 lists nine specific types of substance addictions - alcohol; caffeine; cannabis; hallucinogens; inhalants; opioids; sedatives, hypnotics, and anxiolytics; stimulants; and tobacco, with substance abuse and substance dependence measured on a continuum from mild to severe. These types are further described by eleven distinct criteria, none of which refer to personality characteristics (American Psychiatric Association, 2013).

While the terms 'addictive personality' and 'addictive personality disorder' are largely not officially recognized in academic and professional research and practice, they abound in the general dialogue, having their roots in the fundamental stereotype of addiction as a 'character disorder' and the underlying desire to be able to cleanly and clearly identify a targeted cause in order to modify its effect. These designations appear in venues as diverse as Healthguidance.org, HighBeam Research (operated by Cengage Learning), Psychology Today, the New York Times, Addiction.com, Medic8.com, Recovery.org and WebMD.com. While these sources are not generally considered scholarly research, they no doubt have influence upon the social consciousness as well as aspects of the recovery movement.

Researchers have long been interested in identifying individuals at risk for developing substance abuse disorders. With respect to distinguishing individual differences, the trait of extraversion was frequently targeted for examination. Specifically, early on, various researchers hypothesized that individuals respond differently to alcohol consumption based on their level of extraversion (Eysenck, 1957; McDougall, 1929; Sher \& Wood, 2005). While some correlations have been noted, the research findings have proven inconsistent and have not supported a general association between specific temperaments and addictive tendencies.

More recently, the concept became popularized in the social consciousness in the 1980s when Alan R. Lang conducted seminal research as a meta-analysis in an attempt to determine the nature of an addictive personality, if, in fact, one existed. His intention therein was stated as a practical and applied purpose - "If we can better identify the personality factors, they can help us devise better treatment and can open up new strategies to intervene and break the patterns of addiction" (Lang, 1983, p. 235). Lang's research, conducted for the United States National Academy of Sciences, concluded that, "While there is no single set of traits that is definitive of an 'addictive personality', several 'significant personality factors' exist, including: 1) impulsivity; 2) sensation-seeking; 3) non-conformity combined with weak commitment to socially valued goals for achievement; 4) social alienation and tolerance for deviance; and 5) heightened stress coupled with a lack coping skills" (Lang, 1983, p. 233). While not representative of a personality type per se, these conclusions did highlight aspects of trait and temperament that may play a significant role in the development of sustainment of addictive behaviors.

Proponents of the view that personality plays an influential role in addiction argue,

There is little question of whether personality is associated with problematic alcohol involvement (such as alcohol use disorders; AUDs); it clearly is. However, the question remains: how or why is personality related to risky drinking and AUDs? Although the identification of an 'addictive personality' (i.e., a specific configuration of personality characteristics linked to addictive behaviors) has largely been abandoned, numerous studies demonstrate links between specific personality traits and problematic alcohol involvement. More specifically, although traits related to impulsivity/disinhibition appear to demonstrate the most robust and consistent relation with alcohol involvement, all five-factor traits (i.e., openness to experience, conscientiousness, extraversion, agreeableness, neuroticism) have been shown to correspond, with varying degrees of consistency, to alcohol use and outcomes (Sher, Trull, Bartholow, and Vieth, 1999, Malouff, Thorsteinsson, Rooke, and Schutte, 2007, Trull and Sher, 1994). (Littlefield, Littlefield, \& Sher, 2010).

Despite the equivocal results, research continues to examine potential correlations between personality-like influences and the development of addiction. Building on the previous research, Whiteside and Lynam (2001) employed factor analyses to identify four distinct personality facets related with impulsive-like behavior: sensation seeking, lack of planning, lack of persistence, and urgency (acting rashly when distressed). Other researchers have explored associations between specific traits and their related behaviors (Cyders et al., 2007, Dick et al., 2010; Smith et al., 2007). Figure 1 represents the 
Psychobiological Model of Temperament and Character which depicts the relationship between personality and habit systems, an integral aspect of addictive tendencies (Cloninger, 1994). Still others have examined the relationship between motives related to addictive behaviors and personality traits (Grant, Stewart, O’Connor, Blackwell, \& Conrod, 2007). Despite these efforts, clear and consistent connections to broad personality traits and types remain elusive.

Figure 1. Psychobiological Model of Temperament and Character (Cloninger, 1994).

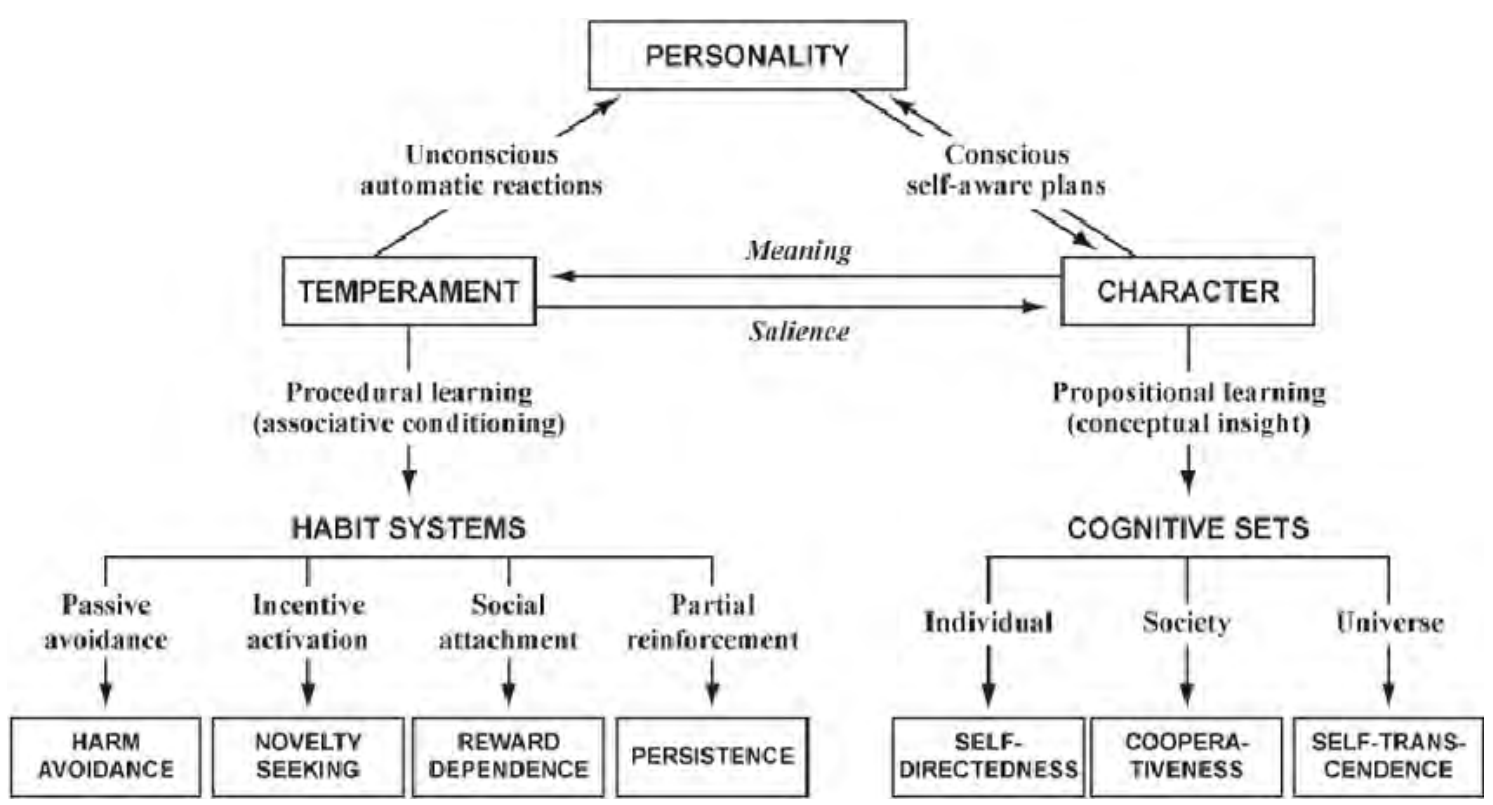

\section{Conclusion}

The fact that the empirical research does not conclusively support a clear and defined picture of an addictive personality, is not surprising, but nor does it negate the potential insights to be gained by considering personality as an important factor in both the development and recovery of substance abuse disorders. Empirical research that attempts to yield consistent predictive results from personality research often instead yields equivocal results, in part due to the difficulty in determining a comprehensive yet specific framework for defining personality.

There is significant debate about whether personality, as we currently define and measure it, even provides a valid and reliable explanation and/or predictor of behavior. Beyond that, there is significant disagreement about how to define personality in a measurable, specific, yet comprehensive way. Among themselves, personality psychologists do not agree various fundamental aspects of the construct - e.g., trait vs. type - and thus, do not embrace a single set of stable personality measures that consistently yield valid and reliable predictive results. Furthermore, there is sufficient evidence that factors other than personality profiles - e.g., situational aspect, abilities and motivation - also influence performance (George \& Jones 2011). Equivocal results have indeed been the status quo for decades of research linking personality to specific behavior.

Personality and addiction both represent complex constructs with numerous interacting variables. Despite efforts of researchers to refine and fine tune measurements, methodologies and procedures, results have provided few direct links between the variables that comprise these complex constructs. Human behavior in any context is always a complex and multifaceted combination of various internal and external variables working in concert to produce what might appear as a singular aspect of behavior. It is, as the Gestalists argue, more than the sum of its parts.

That said, because a concept (particularly a concept as intuitive and fundamental as personality) has not been perfectly honed, in an empirical sense, it does not mean that it has no clinical value. When we focus our microscope down to a more micro level, we see that addiction and attempts at recovery, represent individual stories of pain and healing, strength and weakness, failure and success. It is, after all, this level at which true change is made and at which the real purpose of psychology - understanding the human condition in order to make it better - exists. Therefore, any tool, any construct, any conceptualization that leads to growth and recovery of the human spirit is valid beyond its psychometric properties. 


\section{References}

American Psychiatric Association (APA). (2013). Diagnostic and statistical manual of mental disorders (5th ed.). Arlington, VA: Author.

American Society of Addiction Medicine (ASAM). (2019). Definition of addiction. Retrieved from: https://www.asam.org/resources/definition-of-addiction

Cloninger C. R. (1994) Temperament and personality. Current Opinions in Neurobiology, (4), 266-273

Cyders, M. A., Smith, G. T., Spillane, N. S., Fischer, S., Annus, A. M., \& Peterson, C. (2007). Integration of impulsivity and positive mood to predict risky behavior: Development and validation of a measure of positive urgency. Psychological Assessment, 19, 107- 118.

Dick, D. M., Smith, G., Olausson, P., Mitchell, S. H., Leeman, R. F., O’Malley, S. S., et al. (2010). Understanding the construct of impulsivity and its relationship to alcohol use disorders. Addiction Biology, 15, 217- 226.

Eysenck, H. J. (19957). Drugs and personality I. Theory and methodology. The British Journal of Psychiatry, 103:119-131.

George, J., \& Jones, G . (2011). Understanding and managing organizational behavior. (6 ${ }^{\text {th }}$ ed.). Upper Saddle River, NJ: Prentice Hall.

Grant, V. V., Stewart, S. H., O’Connor, R. M., Blackwell, E., \& Conrod, P. J. (2007). Psychometric evaluation of the five-factor Modified Drinking Motives Questionnaire-Revised in undergraduates. Addictive Behaviors, 32, 2611- 2632.

Lang, A. R. (1983). "Addictive Personality: A Viable Construct?". In Levison, Peter K., Gerstein, Dean R., Maloff, Deborah R. Commonalities in Substance Abuse and Habitual Behavior. New York, NY: Lexington Books. pp. 157-236.

Larsen, R. R., \& Buss, D. M. (2017). Personality psychology: Domains of knowledge about human nature, $6^{\text {th }}$ edition. New York, NY: McGraw-Hill.

Littlefield, A. K., Littlefield, A. K., \& Sher, K. J. (2010). The multiple, distinct ways that personality contributes to alcohol use disorders New York, NY: Wiley-Blackwell Publishing Ltd. doi:10.1111/j.1751-9004.2010.00296.x

Malouff, J., Thorsteinsson, E., Rooke, S., \& Schutte, N. (2007). Alcohol involvement and the five factor model of personality: A meta-analysis. Journal of Drug Education, 37, 277- 294.

McDougall W. (1929). The chemical theory of temperament applied to introversion and extroversion. The Journal of Abnormal and Social Psychology, (24), 293-309

Sher, K. J., Trull, T. J., Bartholow, B., \& Vieth, A. (1999). Personality and alcoholism: Issues, methods, and etiological processes. In H. Blane \& K. Leonard (Eds.), Psychological Theories of Drinking and Alcoholism, 2nd ed., pp. 55-105). New York, NY: Plenum.

Sher, K. J., Wood M. D. (2005). Subjective effects of alcohol II: Individual differences. In: Earleywine M, Editor. Mind altering drugs: Scientific evidence for subjective experience, pp. 86-134. New York, NY: Oxford. pp. 86-134.

Smith, G. T., Fischer, S., Cyders, M. A., Annus, A. M., Spillane, N. S., \& McCarthy, D. M. (2007). On the validity and utility of discriminating among impulsivity-like traits. Assessment, 14, $155-170$.

Trull, T. J., \& Sher, K. J. (1994). Relationship between the five-factor model of personality and Axis I disorders in a nonclinical sample. Journal of Abnormal Psychology, 103, 350- 360.

Whiteside, S. P., \& Lynam, D. R. (2001). The five factor model and impulsivity: Using a structural model of personality to understand impulsivity. Personality and Individual Differences, 30, 669-689. 


\title{
SPIRITUALITY, QUALITY OF LIFE, DEPRESSION AND DEMENTIA
}

\author{
Océane Agli \\ Department of Psychology, EA 2114, University of Tours (France)
}

\begin{abstract}
This study aimed to observe dementia's role in the relationship between spirituality, quality of life, and depression in aging. The sample included 61 participants between 65 and 98 years old, separated into two groups: participants diagnosed with dementia $(\mathrm{N}=31)$ and control participants $(\mathrm{N}=30)$. There was no significant difference in spirituality between demented and control participants; however, different patterns of correlation were observed between spirituality, depression, and quality of life in these groups. Although the level of spirituality did not differ despite dementia, this pathology would appear to play a role in the relationship between spirituality, quality of life, and depression.
\end{abstract}

Keywords: Dementia, depression, elderly, quality of life, spirituality.

\section{Introduction}

According to Rivier, Hongler, and Sutter (2008), spirituality is a human cognitive approach which seeks to give meaning to life, to set values, and sometimes to seek transcendence, resulting in a spiritual identity. This is part of human development, especially in adults and the elderly. For Dalby (2006), spirituality corresponds to a search for meaning and purpose at a time of life when earlier sources of meaning and purpose may be diminishing. Several studies have highlighted that positive effects of spirituality on mental health (Ellison \& Fan, 2008). In the elderly these benefits can also be observed, on quality of life and wellbeing (Koenig, 1994), stress (Levin, 1996) and depression (Koenig et al., 1992).

To evaluate spirituality, Peterman, Fitchett, Brady, Hernandez, and Cella (2002) developed a brief, reliable, valid measure of spiritual well-being, the Functional Assessment of Chronic Illness Therapy-Spiritual Well-being Scale (FACIT-Sp). A few years later, a short version was created (Canada, Murphy, Fitchett, \& Peterman, 2008): the FACIT-Sp12 item, based on three dimensions: mean, peace, and faith.

When dementia is diagnosed, patients often experience a situation of huge stress, which plunges them into a loss of control and questions their identity. Indeed, dementia is a degenerative neural syndrome characterized by the emergence of neuropsychological deficits, which impact the individual's autonomy. It combines a memory disorder with impairment in other cognitive functions (diagnostic criteria of Diagnostic and Statistical Manual of Mental Disorders-V, 2013). Mood and behavioral disorders (depression, anxiety, psychotic symptoms, agitation, and apathy) are also common. All these symptoms have an impact on the subject's capacity to reason and adapt and disrupt social and relational daily life (Auriacombe \& Orgogozo, 2004). Nevertheless, several studies have shown that dementia patients at a mild to moderate stage (with preserved language skills) are able to correctly estimate their quality of life (Brod, Steward, Sands, \& Walton, 1999).

A systematic review of how spirituality can help to cope with the early stages of Alzheimer's disease has been conducted (Beuscher \& Beck, 2008). Among the six studies reviewed, three emphasized the importance of maintaining contact with family, or even with the environment and nature (Phinney, 1998; Stuckey, Post, Ollerton, FallCreek, \& Whitehouse, 2002). This allows people to connect with their spiritual side, which seems extremely important to improve their quality of life and ability to cope with the disease. A more recent systematic review (Agli, Bailly, \& Ferrand, 2014) underlined the beneficial effects of spirituality among older people with dementia, especially regarding quality of life (Dalby, Sperlinger, \& Boddington, 2012; Jolley et al., 2010; MacKinlay \& Trevitt, 2010). In a longitudinal study, MacKinlay (2012) observed a reduction in levels of depression at a three-month follow-up in participants belonging to a pastoral group. In a study by Stuckey et al. (2002), the authors specified that participants did not focus on the future. Instead, they centered on the present hope, living day by day, to manage better and cope with their disease. 
However, methodological limitations reduce the scope of the results obtained by the studies cited previously: spirituality is often assimilated with religion, the selection criteria are not clearly explained, and the diagnosis of dementia is not always clearly established. On the other hand, if spirituality allows a better quality of life, we do not know if maintaining a good quality of life would allow spiritual development, since no measure of a direct link between spirituality and quality of life has been conducted. The objectives of this study were therefore: (a) to examine whether older people with dementia reported significantly different spirituality scores than the control participants, and (b) to examine the role of dementia in the relationship between spirituality, quality of life, and depression among older people.

\section{Method}

\subsection{Participants}

Sixty-one participants between 65 and 98 years old living in four nursing homes in the Centre region in France were recruited. Participants who had psychotic or addictive disorders (particularly alcoholism) were excluded. The common inclusion criterion was age 65 or older. The experimental design of this study involved dividing the participants into two groups: a test group that represented participants with dementia, and a control group for comparison. The test group $(\mathrm{N}=31)$ consisted of participants diagnosed with dementia (established prior to entering the home and reported in their medical file, or established since entering the institution, through neuropsychological assessments conducted during memory consultations, or using brain scanners). For the control group $(\mathrm{N}=30)$, participants had no dementia pathology. There were 47 women $(77.05 \%)$ and 14 men (22.95\%). The mean age was 87.23 years old (SD = 6.48; 65-98 years old).

\subsection{Procedure}

The study protocol was approved by the management of the nursing homes. Each participant was informed that his or her participation in the study was voluntary and anonymous. The families and/or guardians of the participants with dementia were also notified of the progress of the protocol. An information document and a written consent form were signed in duplicate. The protocols were collected by a psychologist, in a hetero-evaluation, in a quiet room. To protect the identity of the participants, data were collected using codes consisting of a combination of letters and numbers.

\subsection{Measures}

To evaluate spirituality, the FACIT-Sp12 (Canada et al., 2008) was used. This scale was recently validated with 11 items on French older people (Agli, Bailly, \& Ferrand, 2017) and can be used on people with cognitive impairment. Participants evaluated items on a 5-point Likert scale (from 0 "not at all" to 4 "very much"). The questionnaire provides four scores: one per dimension and one overall. A high overall score reflects a high level of spirituality. The internal consistency of these subscales is good (Peace $\alpha=.66$; Faith $\alpha=.77$ ), with the exception of the mean dimension, which is just below the threshold $(\alpha=.59)$. The overall scale has good internal consistency $(\alpha=.81)$.

The subjects' cognitive level was evaluated using the Mini Mental State Evaluation (Folstein, Folstein, \& McHugh, 1975). The threshold score for the early stage of dementia is set at 10. Below this score, cognitive impairment is considered to be too high and can create a bias of understanding instructions and/or language.

To assess quality of life, the French version (Schwebel et al., 2010) of the Dementia Quality of Life scale (DQoL) (Brod et al., 1999) was used. This scale, assessing quality of life for the elderly with dementia, is composed of 29 items divided into five dimensions: self-esteem, positive emotions and humor, negative emotions, the sense of belonging, and aesthetics. A thirtieth item evaluates the overall quality of life (DQoL30): “Overall, how would you rate your quality of life?". A high score reflects a good quality of life. The DQoL has been validated for patients with a minimum score of 12 on the MMSE (Mini-Mental State Examination). In this study, there was good internal consistency for the five subdimensions of the scale (Aesthetics $\alpha=.80$; Sense of belonging $\alpha=.72$; Self-esteem $\alpha=.70$; Positive emotions and humor $\alpha=.82$; Negative emotions $\alpha=.89$ ).

Finally, the level of depression was evaluated with the short version of the Geriatric Depression Scale (Yesavage et al., 1982) and validated in French by Bourque, Blanchard and Vézina (1990). A recent study validated its use with elderly people with cognitive impairment with a MMSE score of 10 (Conradsson et al., 2013). The Cronbach alpha for the current study sample was also good $(\alpha=.74)$. 


\subsection{Data analysis}

To see if spirituality, quality of life, and depression were differently expressed according to cognitive level (presence or absence of dementia), we performed a comparison of means. Data tended not to follow a normal distribution and we therefore conducted a non-parametric Mann-Whitney test on the two independent samples (dementia group and control group). In a second step, rank correlations (measured by the Spearman coefficient) between the dimensions of the DQoL/GDS and the factors of the FACIT were performed. Effect size can be defined as small $(0.3<\mathrm{r}<0.5)$, moderate $(0.5<\mathrm{r}<0.7)$, or large $(0.7<\mathrm{r})$. All analyzes were performed using Statistica V13.3 software.

\section{Results}

\subsection{Comparisons of means of participants with dementia versus controls}

Participants with dementia had a marginally higher depression score $(\mathrm{Z}=1.93, \mathrm{p}=.054)$ than control participants. In addition, it is important to note that participants with dementia reported a score above the threshold of $5(\mathrm{M}=6.45$; $\mathrm{SD}=3.25)$, indicating the presence of mild depression, whereas control participants report a score slightly below this threshold $(M=4.97$; $S D=3.15)$. Participants in the dementia group also reported significantly lower self-esteem scores than control participants $(Z=-2.15$, $\mathrm{p}=.031$ ). Finally, no significant effect was observed on the other dimensions of quality of life, particularly spirituality. In other words, participants with dementia had similar scores of quality of life (sense of aesthetics, sense of belonging, positive and negative emotions, and overall quality of life) and spirituality (mean, peace, faith and general spirituality) to control participants.

\subsection{Links between spirituality, quality of life and depression}

The correlation analyzes between FACIT, DQoL and GDS revealed different patterns of correlation among the demented and control participants. In particular, regarding correlations greater than 0.50 indicating a large effect size, we can observe more significant correlations among control participants than among older people with dementia. Thus, the mean factor was strongly associated with sense of aesthetics $(r=0.69)$, sense of belonging $(r=0.53)$, positive emotions and humor $(r=0.64)$ and the overall item of quality of life $(\mathrm{r}=0.52)$ in the control group. The peace factor was also strongly correlated with four dimensions of DQoL (sense of belonging, self-esteem, positive and negative emotions) in the same group. In contrast, in the dementia group, the mean factor was only strongly correlated with the overall item of quality of life $(r=0.50)$, whereas the peace factor was mainly associated with sense of aesthetics $(r=0.52)$, self-esteem $(r=0.53)$ and positive emotions/humor $(\mathrm{r}=0.59)$. No significant correlation was observed between the faith factor and quality of life in the control group, whereas for participants with dementia two moderate correlations were found with the aesthetic research $(r=0.44)$ and positive emotions/humor $(r=0.45)$.

Regarding the link between spirituality and depression, only the mean factor was negatively correlated (moderate effect size) with depression in the dementia group $(r=-0.36)$. On the other hand, in the control group, mean $(r=-0.64)$ and peace $(r=-0.56)$ factors were strongly correlated to depression, as well as the overall measure of spirituality $(r=-0.64)$. Finally, it should be noted that the factor faith was not significantly correlated with depression, for either group.

\section{Discussion}

The study objectives were: (a) to examine whether older people with dementia report significantly different spirituality scores from the control participants, and (b) to examine the role of dementia in the relationship between spirituality, quality of life, and depression among older people. First, the results show no significant difference between older people with dementia and control participants concerning spirituality. Indeed, the demented participants had similar scores to the control participants for each dimension (Mean, Peace, Faith) and also for the overall measure (total score FACIT). In other words, dementia does not interfere with the communication of spiritual needs (as long as the person's ability to understand and express oneself is preserved). These results confirm those of MacKinlay and Trevitt (2010). Thus, older people with dementia can rely on their spiritual resources in the same way as older people without cognitive impairment.

On the other hand, among the five dimensions of DQoL, and also the overall quality-of-life item, only the self-esteem dimension presented a significantly different mean from control participants. Thus, although older people with dementia are able to maintain a satisfactory quality of life (Byrne-Davis, Bennett, \& Wilcock, 2006), it appears that they have a more negative judgment of themselves. Because anosognosia occurs only in the advanced stages of dementia, ill people are long aware of their difficulties 
and their loss of capacity. It is possible that this perception is related to their decline in self-esteem and an increasing feeling of being worthless.

In a second analysis, correlations were performed to observe links between spirituality, quality of life, and depression. The results mainly show more significant and strong correlations among control participants. Indeed, in the dementia group, the mean dimension was associated only with the overall quality-of-life item. In other words, the more participants give meaning to their existence, the higher they rate their quality of life. The peace dimension was correlated with three other factors of the DQoL: sense of aesthetics, self-esteem, and positive emotions/humor. This corroborates Trevitt and MacKinlay's (2006) study, which emphasized the preserved abilities of insight and humor in dementia. Thus, the peace dimension correlates more with quality of life than with the mean dimension. The latter is a cognitive dimension more strongly associated with physical health, whereas the former is an emotional factor and is more related to mental health (Canada et al., 2008). This means that older people with dementia seem more concerned about their mental health in relation to their deficits. Finally, two significant moderate correlations were found between the faith dimension and quality of life (sense of aesthetics and positive emotions/humor). On the other hand, among the control participants no significant link was found between this more religious dimension and quality of life, as if realization of the disease revealed a desire to make a bond between religious beliefs and the search for beauty through the environment, which is in line with results in the literature (Phinney, 1998; Stuckey et al., 2002). It should be noted that items corresponding to the sense of aesthetics dimension of the DQoL mainly concern appreciation of nature (3 items out of 5).

Regarding the connection between spirituality and depression, more negative correlations were observed in control participants (Peace and Mean), whereas only the mean factor was correlated with depression in participants with dementia. Thus, for older people the stronger the sense of meaning they have in their lives, the less depressed they feel. It is possible that awareness of their pathology has created or reactivated this need for meaning in order to cope better with symptoms and changes in daily life (Auriacombe \& Orgogozo, 2004). That is to say that the faith dimension is not significantly correlated to depression in either group.

Nevertheless, each participant was asked what criteria led to the diagnosis of dementia. In a patient's medical file, it is not uncommon to see the words "neurodegenerative syndrome," but with no documents (results of medical examinations, neuropsychological tests, family testimonials, etc.) attesting to this conclusion. In addition, it is important to note the difficulty sometimes encountered in differentiating between the diagnosis of dementia and other pathologies. Furthermore, when a probable diagnosis is made, the choice of the MMSE-which remains universal - to determine the stage of progression of the disease (mild, moderate, or advanced stage) could be questioned. In fact, non-demented older people may fail this test, and older people with dementia may succeed. Dementia syndromes may differ according to the time of day (morning/evening, after family visit, before meal, etc.), the place (home, institution, hospital, etc.), the person (personality, mood, etc.), the interlocutors and how they are perceived, or the family. It should also be noted that the small sample in our study represents a limit because it is not necessarily representative of the population, and is insufficient for highly developed statistical analysis. It could thus be interesting to conduct a similar study, but this time analyzing by age group, considering the wide age range (from 65 to 98 years) in our study.

In conclusion, in this study different patterns of correlation are observed between demented and control older people concerning the relationship between spirituality, quality of life, and depression. It would be interesting to continue this research in terms of concrete applications. Thus, spirituality trajectories could be observed in the same way as changes in individual dementia syndromes and quality of life. Finally, if the spirituality concept persists in pathological aging, it would also be interesting to study what happens in advanced stages of neurodegenerative diseases, when language abilities are reduced and understanding is impaired.

\section{References}

Agli, O., Bailly, N., \& Ferrand, C. (2014). Spirituality/religion in the elderly with dementia: A systematic review. International Psychogeriatrics, 27(5), 715-725. doi:10.1017/ S1041610214001665

Agli, O., Bailly, N., \& Ferrand, C. (2017). Validation of the functional assessment of chronic illness therapy - spiritual well-being (FACIT-Sp12) on French old people. Journal of Religion and Health, 56(2), 464-476. doi:10.1007/s10943-016-0220-0

American Psychiatric Association. (2013). DSM-5: diagnostic and statistical manual of mental disorders (5th edition). Washington D.C: American Psychiatric Association

Auriacombe, S., \& Orgogozo, J. M. (2004). Syndrome démentiel. [Demential syndrome]. EMC-Neurologie, 1(1), 55-64. doi:10.1016/j.emcn.2003.10.003 
Beuscher, L., \& Beck, C. (2008). A literature review of spirituality in coping with early-stage Alzheimer's disease. Journal of Clinical Nursing, 17(5a), 88-97. doi:10.1111/j.13652702.2007.02126.x

Bourque, P., Blanchard, L., \& Vezina, J. (1990). Étude psychométrique de l’Échelle de dépression gériatrique. [Psychometric study of the Geriatric Depression Scale]. Revue Canadienne Du Vieillissement, 9(4), 348-355. doi:10.1017/S0714980800007467

Brod, M., Steward, S. L., Sands, L., \& Walton, P. (1999). Conceptualizations and measurement of quality of life in dementia: The dementia quality of life instrument (DQoL). The Gerontologist, 39(1), 25-35. doi:10.1093/geront/39.1.25

Byrne-Davis, L. M., Bennett, P. D., \& Wilcock, G. K. (2006). How are quality of life ratings made? Toward a model of quality of life in people with dementia. Quality of Life Research, 15(5), 855-865. doi:10.1007/s11136-005-5416-9

Canada, A. L., Murphy, P. E., Fitchett, G., Peterman, A., \& Schover, L. R. (2008). A 3-factor model for the FACIT-Sp. Psycho-Oncology, 17(9), 908-916. doi:10.1002/pon.v17:9

Conradsson, M., Rosendahl, R., Littbrand, H., Gustafson, Y., Olofsson, B., \& Lövheim, H. (2013). Usefulness of the geriatric depression scale 15-item version among very old people with and without cognitive impairment. Aging and Mental Health, 17(5), 638-645. doi:10.1080/13607863.2012.758231

Dalby, P. (2006). Is there a process of spiritual change or development associated with ageing? A critical review of research. Aging and Mental Health, 10(1), 4-12. doi:10.1080/ 13607860500307969

Dalby, P., Sperlinger, D. J., \& Boddington, S. (2012). The lived experience of spirituality and dementia in older people living with mild to moderate dementia. Dementia: the International Journal of Social Research and Practice, 11(1), 75-94. doi:10.1177/ 1471301211416608

Ellison,C.G.,\&Fan,D.(2008).Dailyspiritualexperiencesandpsychologicalwell-beingamong us adults. Social Indicators Research, 88(2), 247-271. doi:10.1007/s11205-007-9187-2

Folstein, M. F., Folstein, S. E., \& McHugh, P. R. (1975). Mini-Mental State: A practical method for grading the cognitive state of patients for the clinician. Journal of Psychiatric Research, 12(3), 189-198. doi:10.1016/0022-3956(75)90026-6

Jolley, D., Benbow, S. M., Grizzell, M., Willmott, S., Bawn, S., \& Kingston, P. (2010). Spirituality and faith in dementia. Dementia: The International Journal of Social Research and Practice, 9(3), 311-325. doi:10.1177/1471301210370645

Koenig, H. G., Cohen, H. J., Blazer, D. G., Pieper, C., Meador, K. G., Shelp, F., ... DiPasquale, B. (1992). Religious coping and depression among elderly, hospitalized medically ill men. American Journal of Psychiatry, 149(12), 1693-1700. doi:10.1176/ajp.149.12.1693

Levin, J. S. (1996). How religion influences morbidity and health: Reflections on natural history, salutogenesis and host resistance. Social Science Medicine, 43(5), 849-864. doi:10.1016/02779536(96)00150-5

MacKinlay, E. (2012). Resistance, resilience, and change: The person and dementia. Journal of Religion, Spirituality \& Aging, 24(1-2), 80-92. doi:10.1080/15528030.2012.633048

MacKinlay, E., \& Trevitt, C. (2010). Living in aged care: Using spiritual reminiscence to enhance meaning in life for those with dementia. International Journal of Mental Health Nursing, 19(6), 394-401. doi:10.1111/j.1447-0349.2010.00684.x

Peterman, A. H., Fitchett, G., Brady, M. J., Hernandez, L., \& Cella, D. (2002). Measuring spiritual well-being in people with cancer: The functional assessment of chronic illness therapy-Spiritual well-being scale (FACIT-Sp). Annals of Behavioral Medicine, 24(1), 49-58. doi:10.1207/S15324796ABM2401_06

Phinney, A. (1998). Living with dementia from the patient's perspective. Journal of Gerontological Nursing, 24(6), 8-15. doi:10.3928/0098-9134-19980601-06

Rivier, C., Hongler, T., \& Suter, C. (2008). La spiritualité en soins palliatifs. [Spirituality in palliative care]. Guide Des Soins Palliatifs Du Medecin Vaudois, 6(5), 1-26.

Schwebel, G., Dramé, M., Jolly, D., Boyer, F., Morrone, I., Di Pollina, L., ... Novella, J. L. (2010). Validation psychométrique du questionnaire dementia quality of life (DQoL) en langue française. [Psychometric validation of the dementia quality of life questionnaire in French language]. La Revue De Gériatrie, 35(2), 93-100.

Stuckey, J. C., Post, S. G., Ollerton, S., FallCreek, S. J., \& Whitehouse, P. J. (2002). Alzheimer's disease, religion and the ethics of respect for spirituality a community dialogue. Alzheimer's Care Quarterly, 3(3), 199-207.

Trevitt, C., \& MacKinlay, E. (2006). "I am just an ordinary person...”: Spiritual reminiscence in older people with memory loss. Journal of Religion, Spirituality \& Aging, 18(2-3), 79-91. doi:10.1300/J496v18n02_07

Yesavage, J. A., Brink, T. L., Rose, T. L., Lum, O., Huang, V., Adey, M., \& Leirer, V. O. (1982). Development and validation of a geriatric depression screening scale: A preliminary report. Journal of Psychiatric Research, 17(1), 37-49. doi:10.1016/0022-3956(82)90033-4 


\title{
BRIEF THERAPY APPROACHES IN THE TREATMENT OF SUBSTANCE ABUSE AND ADDICTION
}

\author{
Donna L. Roberts \\ PhD, Department of Social Sciences \& Economics, Embry-Riddle Aeronautical University (USA)
}

\begin{abstract}
The use of brief intervention and brief therapy techniques that are focused on short-term, problem-specific approaches, has become an increasingly important part of the continuum of care in the treatment of substance abuse problems and addictive disorders. With the health care system changing to a managed model of care and with changes in reimbursement policies for substance abuse treatment, these short, problem-specific approaches can be valuable in the treatment of addictive disorders. They provide the opportunity for clinicians to increase positive outcomes by using these modalities independently as stand-alone interventions and, more commonly, as additions to other forms of substance abuse and mental health treatment. Furthermore, they can be used in diverse venues, including opportunistic settings (e.g., primary care, home health care) and specialized substance abuse treatment settings (inpatient and outpatient).

Appropriate for a variety of substance abuse problems from at-risk use to dependence, brief interventions and brief therapies can help clients reduce or stop abuse, act as a first step in the treatment process to determine if clients can stop or reduce on their own, and/or act as a method to change specific behaviors before or during treatment. Currently, the psychotherapy of substance abuse utilizes a wide variety of treatment modalities based on diverse theoretical perspectives, including, cognitive-behavioral, strategic and interactional, humanistic and existential, psychodynamic, family systems and group therapy.
\end{abstract}

Keywords: Brief therapy, substance abuse, addiction therapy, addiction, substance abuse treatment.

\section{Introduction}

In the realm of psychological disorders and their treatment, addictions have long been considered serious, pervasive and characteristically challenging to overcome. The National Institute on Drug Abuse (NIDA) defines addiction as "a chronic, relapsing brain disease that is characterized by compulsive drug seeking and use, despite harmful consequences," considering it treatable and manageable, but not curable (NIDA, 2014). They further note that recovery is a long-term (often lifetime) and complex process. The pervasiveness of the disease and its subsequent domination of the addict, can typically render individuals non-functional in virtually every aspect of their lives. While fully immersive rehabilitation might be necessary in the detoxification phase, it is usually impractical for the long-term course of the disease and its cycles of relapse.

Brief, or solution-focused therapy is generally considered most effective for short-term, strategic intervention with a limited and pragmatic focus. While this seems in opposition to the broad and comprehensive nature of treatment necessary to address the complex array of symptoms and causes present in addiction, brief therapy techniques have come to serve an important role in the continuum of treatment. With the healthcare system embracing a managed model of care, and with fluctuations in reimbursement policies for substance abuse treatment, these short, problem-specific approaches can be valuable and practical in the treatment of addictive disorders. The flexibility of their approach allows them to be used independently as a primary means of intervention strategy, or in combination with a more comprehensive treatment regimen. Furthermore, they are adaptable to various clinical settings, both inpatient and outpatient.

Brief interventions and brief therapies can be employed at various stages and in various contexts of substance abuse. The variety and flexibility of the procedures and practices of brief therapy can serve in aspects of treatment from prevention to maintenance of sobriety to relapse counseling. Currently, the psychotherapy of substance abuse utilizes a wide variety of treatment modalities based on diverse theoretical perspectives, including, cognitive-behavioral, strategic and interactional, humanistic and existential, psychodynamic, family systems and group. Regardless of setting or therapeutic orientation, the basic goal of treatment is to reduce the risk of harm at all levels - addicts, their families, and the larger community - that typically results from chronic substance abuse. 


\section{Brief interventions and brief therapies}

Brief interventions and brief therapies represent differing strategies in the spectrum of treatment options and can be identified by their somewhat differing outcome goals. Brief interventions are "those practices that aim to investigate a potential problem and motivate an individual to begin to do something about his substance abuse, either by natural, client-directed means or by seeking additional substance abuse treatment." The distinction between these modalities is further clarified by considering the purpose and intended outcome. Specifically, "interventions are generally aimed at motivating a client to perform a particular action (e.g., to enter treatment, change a behavior, think differently about a situation), whereas therapies are used to address larger concerns (such as altering personality, maintaining abstinence, or addressing long-standing problems that exacerbate substance abuse)" (Barry, 1999, pp. 67-69).

Brief interventions can be conducted in a variety of substance abuse treatment settings and thus may be facilitate by different treatment staff or other health care professionals. The fundamental goal of any brief intervention is to reduce the risk of harm that could result from continued use of substances. The specific goals for treatment are based on an assessment of the individual, typical substance consumption patterns, consequences and problems resulting from the abuse, and the setting (or settings) of the brief intervention (Barry, 1999; Bien, 1993; McMurran, 1994; Rotgers, Keller \& Morgenstern, 1996). Typical distinctions between the goals of brief interventions as applied in different settings are outlined in Table 1.

Table 1. Goals of Brief Interventions According to Setting.

Goals of Brief Interventions According to Setting

Source: Center for Substance Abuse Treatment. Brief Interventions and Brief Therapies for Substance Abuse. (1999); Adapted from Bien et al., 1993 and Barry, 1999, p. 76

\begin{tabular}{|c|c|}
\hline Setting & Purpose/Goal \\
\hline Opportunistic Setting & $\begin{array}{l}\text { - Facilitate referrals for additional specialized treatment (e.g., a nurse } \\
\text { identifying substance-abusing clients through screening and advising } \\
\text { them to seek further assessment or treatment) } \\
\text { - Affect substance abuse directly by recommending a reduction in } \\
\text { hazardous or at-risk consumption patterns (e.g., a primary care } \\
\text { physician advising hazardous or at-risk drinkers to cut down, } \\
\text { National Alcohol Screening Day) or establishing a plan for } \\
\text { abstinence }\end{array}$ \\
\hline $\begin{array}{l}\text { Neutral Environments } \\
\text { (e.g., individuals } \\
\text { responding to media } \\
\text { advertisements) }\end{array}$ & $\begin{array}{l}\text { - Assess substance abuse behavior and give supportive advice about } \\
\text { harm reduction (e.g., a public health initiative to screen people in } \\
\text { shopping malls and provide feedback and advice) }\end{array}$ \\
\hline Health Care Setting & - $\quad$ Facilitate referrals for additional specialized treatment \\
\hline $\begin{array}{l}\text { Substance Abuse } \\
\text { Treatment Programs }\end{array}$ & $\begin{array}{l}\text { - Act as a temporary substitute for more extended treatment for persons } \\
\text { seeking assistance but waiting for services to become available } \\
\text { (e.g., an outpatient treatment center that offers potential clients } \\
\text { assessment and feedback while they are on a waiting list) } \\
\text { Act as a motivational prelude to engagement and participation in } \\
\text { more intensive treatment (e.g., an intervention to help a client commit } \\
\text { to inpatient treatment when the assessment deems it appropriate, but } \\
\text { the client believes outpatient treatment is adequate) } \\
\text { Facilitate behavior change related to substance abuse or associated } \\
\text { problems }\end{array}$ \\
\hline
\end{tabular}

Brief therapy is "a systematic, focused process that relies on assessment, client engagement, and rapid implementation of change strategies" (Barry, 1999, 2011, p. 150). Brief therapies differ from brief interventions in that their goal is a more in-depth examination of underling dysfunctional patterns in thought and behavior (McMurran, 1994; Rotgers, Keller \& Morgenstern, 1996). Specifically, "in addition to the goals of brief interventions, the goals of brief therapy in substance abuse treatment is remediation of some specified psychological, social, or family dysfunction as it pertains to substance abuse, focusing primarily on present concerns and stressors rather than on historical antecedents" (Barry 1999, 2011, 
p. 80). However, while its focus encompasses examination of more than the immediate circumstances of interventions, brief therapy "differs from longer term therapy in that it focuses more on the present, downplays psychic causality, emphasizes the effective use of therapeutic tools in a shorter time, and focuses on a specific behavioral change rather than large-scale or pervasive change" (Barry, 1999, 2011, p. 151).

In general, interventions focus on encouraging an addict (or recovering addict) to engage in a specific behavior (e.g., entering a treatment program or course of therapy, refrain from using in that moment, apply logic to think differently about a situation, change immediate circumstances). In contrast, therapies are part of a broader and more comprehensive package of behavior modification and address more established dysfunctions (e.g., a personality, maintaining abstinence and preventing relapse, or confronting long-standing issues that contribute to substance abuse) (Heather, 1994). The Center for Substance Abuse Treatment has identified the following key differences that help distinguish brief interventions from brief therapies:

- Length of the sessions (from 5 minutes for an intervention to more than six 1-hour therapy sessions)

- Extensiveness of assessment (which will be greater for therapies than for interventions)

- Setting (nontraditional treatment settings such as a social service or primary care setting, which will use interventions exclusively, versus traditional substance abuse treatment settings where therapy or counseling will be used in addition to interventions)

- Personnel delivering the treatment (brief interventions can be administered by a wide range of professionals, but therapy requires training in specific therapeutic modalities)

- Materials and media used (certain materials such as written booklets or computer programs may be used in the delivery of interventions but not therapies) (Barry, 1999, 2011, p. 68).

Although the fundamental purpose and goals, as well as the theoretical foundations, for brief interventions and brief therapies can differ significantly, in practice, these distinctions less obvious, and perhaps less critical. Both attempt to address the complex of behavior patterns that encompass substance abuse and its associated problems. Both may be necessary in providing adequate care and treatment. As such, they work together to address different needs along a continuum of patient behavior over time and treatment cycles.

\section{Approaches to brief therapy and their theoretical foundations}

There are a variety of different theoretical approaches and corresponding modalities of treatment available to the clinician for the treatment of substance abuse. Table 2 outlines the major issues involved in several different treatment approaches.

Table 2. Approaches to Brief Therapy.

\begin{tabular}{|c|l|}
\hline \multicolumn{2}{|c|}{$\begin{array}{c}\text { Approaches to Brief Therapy } \\
\text { Source: Center for Substance Abuse Treatment (2012). Brief Interventions and Brief Therapies for } \\
\text { Substance Abuse, pp. 42-43 }\end{array}$} \\
\hline Cognitive Therapy & $\begin{array}{l}\text { This therapy posits that substance abuse disorders reflect habitual, } \\
\text { automatic, negative thoughts and beliefs that must be identified and } \\
\text { modified to change erroneous ways of thinking and associated behaviors. } \\
\text { The desire to use substances is typically activated in specific, often } \\
\text { predictable high-risk situations, such as upon seeing drug paraphernalia } \\
\text { or experiencing boredom, depression, or anxiety. This approach helps } \\
\text { clients examine their negative thoughts and replace them with more } \\
\text { positive beliefs and actions. Many relapse prevention strategies use } \\
\text { cognitive processes to identify triggering events or emotional states that } \\
\text { reactivate substance use and replace these with more healthful responses. }\end{array}$ \\
\hline Behavioral Therapy & $\begin{array}{l}\text { Using this approach, which is based on learning theories, the therapist } \\
\text { teaches the client specific skills to improve identified deficiencies in } \\
\text { social functioning, self-control, or other behaviors that contribute to } \\
\text { substance use disorder. Some of the techniques that are used include } \\
\text { assertiveness training, social skills training, contingency management, } \\
\text { behavior contracting, community reinforcement and family training } \\
\text { (CRAFT), behavioral self-control training, coping skills, and stress } \\
\text { management. }\end{array}$ \\
\hline
\end{tabular}




\begin{tabular}{|c|c|}
\hline $\begin{array}{c}\text { Cognitive-Behavioral } \\
\text { Therapy }\end{array}$ & $\begin{array}{l}\text { This approach combines elements of cognitive and behavioral therapies, } \\
\text { but in most substance abuse treatment settings it is considered a separate } \\
\text { therapy. This approach focuses on learning and practicing a variety of } \\
\text { coping skills. The emphasis is placed on developing coping strategies, } \\
\text { especially early in the therapy. Cognitive-behavioral therapy is thought to } \\
\text { work by changing what the client does and thinks rather than just } \\
\text { focusing on changing how the client thinks. }\end{array}$ \\
\hline $\begin{array}{c}\text { Strategic/Interactional } \\
\text { Therapies }\end{array}$ & $\begin{array}{l}\text { These approaches seek to understand a client's viewpoint on a problem, } \\
\text { what meaning is attributed to events, and what ineffective interpersonal } \\
\text { interactions and coping strategies are being applied. By shifting the focus } \\
\text { to competencies, not weaknesses and pathology, the therapist helps } \\
\text { clients change their perception of the problem and apply existing personal } \\
\text { strengths to finding and applying a more effective solution. }\end{array}$ \\
\hline Solution-Focused Therapy & $\begin{array}{l}\text { Using this approach, the therapist helps a client with a substance abuse } \\
\text { disorder recognize the exceptions to use as a means to reinforce and } \\
\text { change behavior. Future behavior is based on finding solutions to } \\
\text { problem behaviors. Little or no time is spent talking about the problem; } \\
\text { rather, therapy is focused on solutions that have already worked for the } \\
\text { client in the past. }\end{array}$ \\
\hline $\begin{array}{l}\text { Humanistic/Existential } \\
\text { Therapy }\end{array}$ & $\begin{array}{l}\text { These therapies assume that the underlying cause of substance abuse } \\
\text { disorders is a lack of meaning in one's life, a fear of death, } \\
\text { disconnectedness from people, spiritual emptiness, or other } \\
\text { overwhelming anxieties. Through unconditional acceptance, clients are } \\
\text { encouraged to improve their self-respect, self-motivation, and growth. } \\
\text { The approach can be a catalyst for seeking alternatives to substances in } \\
\text { order to fill the emptiness experienced and expressed as substance abuse. }\end{array}$ \\
\hline Psychodynamic Therapy & $\begin{array}{l}\text { The psychodynamic therapist works with the assumption that a person's } \\
\text { problems with substances are rooted in unconscious and unresolved past } \\
\text { conflicts, especially in early family relationships. The goal is to help the } \\
\text { client gain insight into underlying causes of manifest problems, } \\
\text { understand what function substance abuse is serving, and strengthen } \\
\text { present defenses to work through the problem. A strong therapeutic } \\
\text { alliance with the therapist assists the client to make positive changes. }\end{array}$ \\
\hline Interpersonal Therapy & $\begin{array}{l}\text { This therapy, which combines elements of cognitive and psychodynamic } \\
\text { therapies, was originally developed to work with clients with depression } \\
\text { but has been used successfully with substance-abusing clients. It focuses } \\
\text { on reducing the client's dysfunctional symptoms and improving social } \\
\text { functioning by concentrating on a client's maladaptive patterns of } \\
\text { behavior. It is supportive in nature, providing encouragement, } \\
\text { reassurance, reduction of guilt, and help in modifying the client's } \\
\text { environment. }\end{array}$ \\
\hline Family Therapy & $\begin{array}{l}\text { While not a distinct "school" of therapy, family therapy is a modality that } \\
\text { either treats the client as part of a family system or considers the entire } \\
\text { family as "the client." It examines the family system and its hierarchy to } \\
\text { determine dysfunctional uses of power that lead to negative or } \\
\text { inappropriate alignments or poor communication patterns and that } \\
\text { contribute to substance use disorder by one or more family members. The } \\
\text { therapist helps family members discover how their own system operates, } \\
\text { improve communication and problem-solving skills, and increase the } \\
\text { exchange of positive reinforcement. }\end{array}$ \\
\hline Group Therapy & $\begin{array}{l}\text { This modality (also not a distinct theoretical school) uses many of the } \\
\text { techniques and theories described to accomplish specified goals. In some } \\
\text { group therapy, the group itself and the processes that emerge are central } \\
\text { to helping clients see themselves in the reactions of others, although the } \\
\text { content and focus of the groups vary widely. }\end{array}$ \\
\hline
\end{tabular}




\section{Conclusion}

Brief interventions and time-limited therapies represent an important component in the repertoire of substance abuse therapy options, either as the primary therapeutic intervention or, perhaps most effectively, in combination with other treatment modalities. Various treatment perspectives can be adapted to a time-limited model in order to address the most incapacitating dysfunctional behaviors in a straight-forward and goal-directed manner. Regardless of the specific theoretical approach, brief therapy intends to focus treatment time on real world issues in order to allow the patient to become more functional as soon as possible. Their incorporation into comprehensive treatment programs has proven particularly effective. In general, brief therapies serve as practical, accessible and efficient means of using resources to address the multitude of issues that affect and are affected by substance abuse.

\section{References}

Barry, K. L. (1999). Brief Interventions and Brief Therapies for Substance Abuse: (Treatment Improvement Protocol (TIP) Series, No. 34. Rockville, MD: Substance Abuse and Mental Health Services Administration's (SAMHSA) Center for Substance Abuse Treatment (CSAT).

Bien, T. H., Miller, W. R., \& Tonigan, J. S. (1993). Brief interventions for alcohol problems: A review. Addiction 88:315-336.

Center for Substance Abuse Treatment. Brief Interventions and Brief Therapies for Substance Abuse. (1999). Treatment Improvement Protocol (TIP) Series, No. 34.) Chapter 1-Introduction to Brief Interventions and Therapies. Rockville (MD): Substance Abuse and Mental Health Services Administration. Retrieved from: https://www.ncbi.nlm.nih.gov/books/NBK64950/

Center for Substance Abuse Treatment. (2012). Brief Interventions and Brief Therapies for Substance Abuse: Treatment Improvement Protocol (TIP) Series, No. 34. Rockville (MD): Substance Abuse and Mental Health Services Administration. Retrieved from: https://www.ncbi.nlm.nih.gov /books/NBK64947/pdf/Bookshelf_NBK64947.pdf

Heather, N. (1994). Brief interventions on the world map. Addiction 89(6):665-667.

McMurran, M. (1994). Psychology of addiction (Contemporary Psychology, Vol. 10). New York, NY: Taylor \& Francis.

NIDA. (2014, July 1). Drugs, Brains, and Behavior: The Science of Addiction. Retrieved from https://www.drugabuse.gov/publications/drugs-brains-behavior-science-addiction.

Rotgers, F., Keller, D.S., \& Morgenstern, J. (Eds.). (1996). Treating substance abuse: Theory and technique. New York, NY: Guilford Press. 


\title{
GENDER-BASED LINEAR CORRELATION OF EMOTIONAL QUOTIENT WITH STUDY HABITS AND ACADEMIC SUCCESS OF JUNIOR COLLEGE STUDENTS
}

\author{
Mary Ann I. Diaz ${ }^{1}$, Elenita M. Tiamzon ${ }^{2}$, M. ${ }^{a}$ Cristina J. Estioco ${ }^{3}$, \\ \& John Mark S. Borbon ${ }^{2}$ \\ ${ }^{I}$ Guidance and Counseling Office, World Citi Colleges (Philippines) \\ ${ }^{2}$ Research and Publication Office, World Citi Colleges (Philippines) \\ ${ }^{3}$ Office of the Assistant Vice President, World Citi Colleges (Philippines)
}

\begin{abstract}
The main aim of the study is to examine the emotional quotient, study habits, and academic success of the Junior College Students that are useful to the school administrators, guidance and counseling practitioners, faculty, researchers, and to the respondents themselves to have a better understanding and come up with an intervention program. The main source of data came primarily from the three hundred thirty-eight (338) Junior College students who were enrolled for the Second Semester, Academic Year 2017-2018 at World Citi Colleges, Quezon City. Two sets of standardized tests were utilized in the study; the Study Habits and Attitudes (SSHA) by Brown, W.F \& Holtzman which consist of 100 items and the Baron Emotional Quotient Inventory (BarOn EQ-i:S) by Reuven Bar-On. According to BarOn, emotional intelligence is a combination of emotional and social competencies that determine how effective a person relate with himself, others and cope with daily demands and pressures. Emotional intelligence is an important factor in determining a persons' ability to succeed in life and directly influences his over-all well - being. Emotional Intelligence is developed over time, changes throughout life, and can be improved through training and remedial programs. It includes intrapersonal, interpersonal, stress management, adaptability, and general mood, that when manage well, this will lead to effective performance. Findings from the study revealed that respondents have effective skills in interpersonal and adaptability scales; stress management and general mood for female only but with low emotional and social capacity on interpersonal and total EQ, and stress management and general mood. Female respondents displayed effective study habits as compared to male respondents. Respondents are average performers in terms of academics. Gender has a potential impact on emotional quotient, study habits and academic performance. Respondents who are emotionally intelligent are expected to display effective study habits and attitudes that lead to high academic performance. There exists linear correlation between gender and emotional quotient, gender and study habits and gender and academic performance. Based from the results an intervention program was created.
\end{abstract}

Keywords: Academic success, adaptability, emotional quotient, stress management, study habits and attitudes.

\section{Introduction}

In the educational setting, the work of school counselors entails a lot of responsibilities. School counseling is an emotionally demanding, dynamic and fast-paced profession that can be inspiring and rewarding as well as discouraging and taxing. Counselors do not only face a range of emotional issues to students and parents they serve, but also in the administrators and teachers with whom they collaborate and consult. Pellitteri et.al. (2006) stated that counselors are in the unique and challenging position of balancing the perspective, wants, and need of all these parties, essentially serving as the "emotional center" of the complex system of the school community. Therefore, counselors become increasingly pressed to manage not only the emotions of the people around them but their own emotions as well. And as the emotional center, it is essential that they also develop the foundational skills of emotional intelligence, not only sets a necessary example of leadership but support the counselors own well-being and personal adjustment to a challenging work environment particularly into serving the students. 
Interestingly, the researcher has been in the helping profession for many years and has obtained vital information that was believed contributory to the present study. She has witnessed how well the students performed in academics and how difficult these students maintained their grades as well. Random interviews were conducted to validate demographic profile of students as well as pressing issues that hinders them to achieved high academic performance. Some notable issues raised were about student motivation, failing grades, learning difficulties, peer pressure, study habits, overwhelming demand of academic activities, and family concerns like financial, separation of parents, and sibling rivalry. These growing challenges that students are confronted with, others have attempted give up and made no means to bounce back. Aquino (1980) validated these problems as he pointed out that most counseling problems in schools are related to educational achievement which is perennial and the most common concern for administrators, educators, and counselors.

With the initiatives of the different organizations to foster students' academic success and the in support to the endeavor of the institution to provide quality education to its clientele, the researcher aimed to determine the correlation of emotional quotient with study habits and academic success of the Junior College students with the hope that the result creates a positive impact on their academic performance.

\section{Methods}

\subsection{Research design}

The researchers used the quantitative descriptive correlational method of research in the study. This type of research method is mainly use to describe and correlates the influence of emotional intelligence and study habits of scholars towards academic success.

\subsection{Population and sampling}

The researchers considered the entire population of three hundred thirty-eight (338) Junior College students who belong to the different programs. Purposive Sampling was used. This sampling technique is a non- probability technique that involves the conscious selection by the researcher of certain people to include in a study. Participants are selected because they have particular characteristics that are of interest to the researcher.

\subsection{Respondents of the study}

The respondents involved in the study are the currently enrolled Junior College students of World Citi Colleges for the Second Semester, School Year 2017-2018 from 10 different programs.

\subsection{Research instruments}

The researchers utilized two standardized instruments: BarOn Emotional Quotient Inventory (BarOn EQ-EQ:iS) by Reuven Bar-On) and The Survey of Study Habits and Attitudes (SSHA).

\subsection{Data collection and analysis}

The administration of the two standardized tests was conducted in the classroom and in the guidance testing room of World Citi Colleges. An orientation on the day of the administration was conducted to assure that respondents understand the purpose of the tests, how it should be answered to acquire the desired data. The BarOn Emotional Quotient Inventory was initially administered. Materials were collected right after the respondents have completed the inventory. The second test was the Survey of Study Habits and Attitudes. The answer sheets and the test booklets were distributed to the respondents. Individual test of respondents were scored in a standardized manner as indicated in the manual of the tests being utilized. Results were tabulated, analyzed and interpreted. Analysis was done in the presence of the statistical treatment such as frequency, percentage, weighted mean, Pearson's Product Moment Correlation Coefficient and Linear Regression.

\section{Results and discussion}

\subsection{Emotional quotient as measured by BarOn EQ:i}

According to Emotional Quotient as measured by BarOn EQ:iS. The results show that male and female respondents garnered the same range of scores of 84 and below in intrapersonal, and total EQ. This was evident by the mean score of 83.8 for male and 84.0 for female respondents in intrapersonal and 81.1 for male and 53.1 for female in total EQ which both fall on the description of "for enrichment. The findings of the study contradict the study conducted by Kafetsios (2004) that emotional intelligence has a significant difference in gender groups. 


\subsection{The study habits of junior college students}

The result shows that majority of male and female of the respondents got a score ranging from 49 \& below which corresponds to "low" description or have a practice of an ineffective study skills in all seven scales. The result signifies that male and female showed study habits in common. Based from the student's responses, students revealed that they do not have efficient time management, lack planning and concentration in their studies, have poor reading skills, ineffective test-taking techniques and failure to inform teachers of their difficulties with school work and ask for their help.

\subsection{Academic success of respondents as measured by the general weighted average}

The results show that majority of the respondents both the male and female obtained a grade ranging from 2.26 to 2.99 and 2.25 to 1.75 . The data denotes that majority of the respondents were performing within the description of average and fair only. This means that while majority of the respondents scored low in study habits, they displayed moderate in academic performance.

\subsection{Correlation between gender and emotional quotient}

Table 1. Correlation between the Gender and Emotional Quotient of the Respondents.

\begin{tabular}{|c|c|c|c|c|}
\hline Sources & $\mathbf{r}$ & $\mathbf{r}^{2}$ & VI & Decision \\
\hline Gender/Emotional Quotient & 0.963 & 0.927 & Significant & Reject $\mathrm{H}_{0}$ \\
\hline
\end{tabular}

Table 1 presents the correlation of gender and emotional quotient of respondents. The coefficient of correlation that exists between the two variables is about 93\%. The correlation is considered very high as indicated by the $\mathrm{r}$ - value of 0.963 . The result signifies that there is a significant correlation between gender and emotional quotient of the respondents.

Table 2. Coefficients.

\begin{tabular}{|c|c|c|c|}
\hline \multirow{2}{*}{ MODEL } & \multicolumn{2}{|c|}{ Unstandardized Coefficients } & Standardized Coefficients \\
\cline { 2 - 4 } & B & Std. Error & Beta \\
\hline Constant & 1.089 & 9.038 & 0.555 \\
\hline Male & 0.553 & 0.128 & 0.519 \\
\hline Female & 0.437 & 0.108 & \\
\hline
\end{tabular}

In order to get the correlational analysis between the emotional quotient and the gender, the table shows the estimated total emotional quotient of student is 1.089. The regression coefficient or slope coefficient for the male students is 0.553 . The given predictor is 0.553 that for two respondents whose emotional quotient differed by 10 on the average can also be said that the total emotional quotient are differed by 5.53. For female is 1.089. The regression coefficient or slope coefficient for the female students is 0.437 . The given predictor is 0.437 , for two students whose emotional quotient differed by 10 , the average total emotional quotient are differed by 4.37 . The results above show that the dependency of the emotional quotient to the male junior college students is about $55 \%$ and that of the female junior college students is about $44 \%$.

\subsection{Correlation between the gender and study habits}

Table 3. Correlation between the Gender and Study habits of the Respondents.

\begin{tabular}{|c|c|c|c|c|}
\hline Sources & $\mathbf{R}$ & $\mathbf{r}^{2}$ & VI & Decision \\
\hline Gender/ Study habits & 0.926 & 0.857 & Significant & Reject $\mathrm{H}_{0}$ \\
\hline
\end{tabular}

Table 3 presents the correlation between gender and study habits of the respondents. The correlation is considered very high as indicated by the $r$ - value of 0.926 . The coefficient of correlation that exists between the two variables is about $86 \%$. The data implies that there is a significant correlation between the gender and study habits of respondents. This may be due to the fact the females are considerably having routine behaviors imbibed in them to feed their hunger and quench their thirst for knowledge and learning.

Table 4. Coefficients.

\begin{tabular}{|c|c|c|c|}
\hline \multirow{2}{*}{ MODEL } & \multicolumn{2}{|c|}{ Unstandardized Coefficients } & Standardized Coefficients \\
\cline { 2 - 4 } & B & Std. Error & Beta \\
\hline Constant & 0.373 & 3.770 & 0.472 \\
\hline Male & 0.544 & 0.165 & 0.787 \\
\hline Female & 0.530 & 0.096 & \\
\hline
\end{tabular}


In order to get the correlational analysis between the study habits and the gender, the table shows the estimated study habits for the respondents where the gender is male is 0.373 . The regression coefficient or slope coefficient for the male students is 0.544 . It can be said that for two students whose emotional quotient differed by 10 on the average can also be said that the total emotional quotient are differed by 5.44. The results above show that the dependency of the study habits to the male junior college students is about $54 \%$ and that of the female junior college students is about $53 \%$.

\subsection{Correlation between the gender and academic success}

Table 5. Correlation between the Gender and Academic Success of the Respondents.

\begin{tabular}{|c|c|c|c|c|}
\hline Sources & $\mathbf{R}$ & $\mathbf{r}^{2}$ & VI & Decision \\
\hline Gender/Academic Success & 0.987 & 0.975 & Significant & Reject $\mathrm{H}_{0}$ \\
\hline
\end{tabular}

Table 5 presents the correlation of gender and academic success of respondents. The correlation is considered very high as indicated by the $r$ - value of 0.987 . The coefficient of correlation that exists between the two variables is about $99 \%$. The result shows that there is a significant correlation between the gender and academic success of the respondents.

Table 6. Coefficients.

\begin{tabular}{|c|c|c|c|}
\hline \multirow{2}{*}{ MODEL } & \multicolumn{2}{|c|}{ Unstandardized Coefficients } & Standardized Coefficients \\
\cline { 2 - 4 } & $\mathrm{B}$ & Std. Error & Beta \\
\hline Constant & 0.076 & 0.150 & 0.628 \\
\hline Male & 0.576 & 0.080 & 0.431 \\
\hline Female & 0.398 & 0.081 & \\
\hline
\end{tabular}

In order to get the correlational analysis between the academic success and the gender, the table shows the estimated academic success for a student where the gender is male is 0.076 . The regression coefficient or slope coefficient for the male students is 0.576 . This indicates that the slope of the regression line is positive. It can be said that for two students whose emotional quotient differed by 10 on the average can also be said that the total emotional quotient are differed by 5.76. The results above show that the dependency of the study habits to the male junior college students is about $58 \%$ and that of the female junior college students is about $40 \%$.

\subsection{Correlation between emotional quotient, study habits and academic success of the junior college students}

Table 7. Correlation between the Emotional Quotient, Study Habits and Academic Success of the Junior College students.

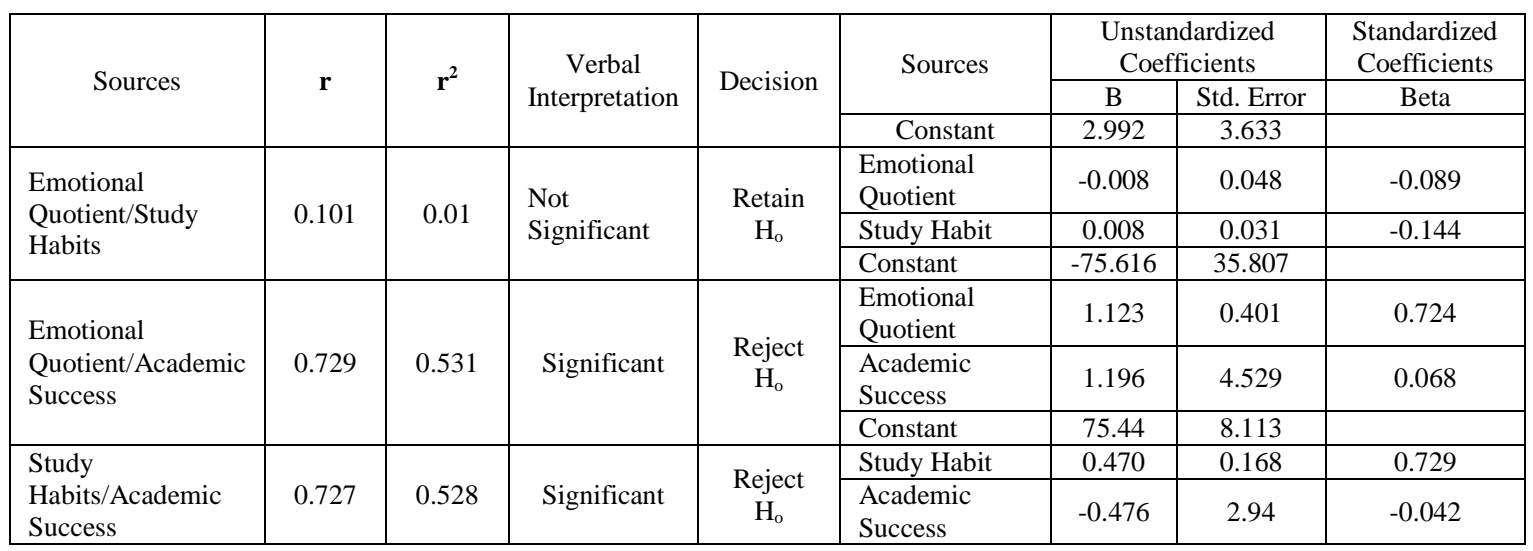

There exists no significant correlation between the emotional quotient and study habits. On the other hand, there exist significant correlation between emotional quotient and academic success, and between study habits and academic success. This is indicated by the $\mathrm{r}$ - value of $0.101,0.729$, and 0.727 . There exists a very low correlation between the emotional quotient and study habits and moderately high correlation on the emotional quotient and academic success and study habits and academic success.

In order to get the correlational analysis between the emotional quotient and the study habits, emotional quotient and academic success, and study habits and academic success the table shows the estimated academic success for a student is 2.992, estimated study habits for a student is -75.616 , and estimated emotional quotient for a student is 75.44. The results above show that the dependency of the 
emotional quotient and the study habit to the academic success of the students is about $0.8 \%$; the emotional quotient to the study habits of the students is about $72 \%$ and the academic success of the students to the study habits is about 7\%; the emotional quotient to the study habits of the student is about $73 \%$ and that of the academic success of the students to the study habits is about $-4 \%$.

\section{References}

Aquino, L. B. (2011). Study habits and attitudes of freshmen students: Implications or academic intervention programs.

Bar-On, R. (2002). Baron emotional quotient inventory: Short. Technical manual. Canada. Multihealth Systems, Inc.

Barry, J. (2006). The effect of socio-economic status on academic achievement. A research for Bachelor of Arts, Wichita State University.htth://www.soar.wichita.edu/dspace/bitstream/10057/616/1/t06115.pdf

Bahman, S. \& Maffini, H. (2008). Developing children's emotional intelligence. New York. Continuum International Publishing Group.

Fernandez-Berrocal, P., Cabello, R., Castillo, R., \& Extremera, N. (2012). Gender differences in emotional intelligence: the mediating effect of age. Behavioral Psychology, Vol. 20, N1, 2012, pp. 77-89

Brown, W. \& Holtzman, W. (1967). Survey of study habits and attitudes. Manual. New York. The Psychological Corporation.

Cerna, M., \& Pavliushehenko, K. (2015). Influence of study habits on academic performance of international college students in Shanghai. Higher Education Studies; Vol. 5, No. 4.

Child D (1981). Psychology and the teacher. London: Holt, Rinehart and Winston.

Choi, N. (2005). Self-efficacy and self-concept as predictors of college students' academic performance. Psychology in the Schools, 42(2), 197-205. doi:10.1002/pits.20048

Clark, C. S., Callister, R., \& Wallace, R. (2003). Undergraduate management skills courses and students emotional intelligence. Journal of Management Education, 27(1), 3-23.

Crede, M. \& Kuncel, N. (2008). Study Habits, Skills, and Attitudes: The Third Pillar Supporting Collegiate Academic Performance. University at Albany, SUNY, and University of Minnesota

Crow, D.L. \& Crow, A. (2007). Educational psychology. Delhi: Surject Publications

Eiland, T.T \& Walz, S. (2012). Student Attitudes towards Failure in College.

Fernandez-Berrocal, P., Cabello, R., Castillo, R., \& Extremera, N. (2012). Gender differences in emotional intelligence: the mediating effect of age. Behavioral Psychology, Vol. 20, N1, 2012, pp. 77-89

Freeman \& Mors (1993). The impact of study habits on the academic performance of students.

Freedman, J, Ghini, M. \& Jensen, A. (2005). Six seconds emotional intelligence assessment (SEI). San Francisco: Six Seconds Gardner, H. (1983). Frames of Mind. New York. Basic Books.

Goleman, D. (1995). Emotional intelligence: Why It can matter more than IQ? New York: Bantom Books.

Good, T.L.(1998). Pygmalion grows up: Studies in the expectation communication process. New York: Longman

Gordon, V.N. (2006). Career Advising: An academic advisor's guide. San Fancisco: Jossey-Bass.

Jennings, N.Lovett, S., Cuba, L, Single, J \& Lindkvist, H. (2014). What would make this year successful for you? How students define success in college. http:/www.aacu.org/publicationsresearch/periodicals/what-would-make-successful-year-you-how-students-define-success

Jorfi, H. \& Yaccob, H. (2012). Role of gender in emotional intelligence: Relationship among EI, communication effectiveness and job satisfaction (A case study of Iran). https://www.questia.com/read/1P3-2762924001/role-of-gender-in-emotional- intelligencerelationship

Kvapil, L. (2007). The impact of emotional intelligence on the academic performance of at-risk high school students. University of the Incarnate Word, 2007, 214; 3255951

Licuanan, P. (2013). Enhanced Policies and Guidelines on Student Affairs and Services. CHED Memorandum No. 9.

Mallery, B. (2017) What is emotional intelligence? 2 theories and measures. An initiative of positive psychology and positran. http://positivepsychology.org.uk/emotional-intelligence-mayer-saloveytheory/

Pellitteri, J., Stern, R., Shelton C. \& Muller-Ackerman, B. (2006). Emotionally intelligent school counseling. Mahwah, New Jersey: Lawrence Erlbaum Associates, Inc., Publishers. 


\title{
RESTING STATE EEG POWER ANALYSIS IN FILIPINO CHILDREN WITH DYSLEXIA
}

\author{
Katherine Ko', Roann Ramos ${ }^{2,3}$, \& Rosalito De Guzman ${ }^{4}$ \\ ${ }^{1}$ Department of Psychology, College of Science, University of Santo Tomas (Philippines) \\ ${ }^{2}$ RWTH Aachen University Hospital (Germany) \\ ${ }^{3}$ College of Education Graduate Studies, De La Salle University-Dasmarinas (Philippines) \\ ${ }^{4}$ The Graduate School, University of Santo Tomas (Philippines)
}

\begin{abstract}
Dyslexia is a neurocognitive disorder characterized by severe and persistent reading difficulties despite normal intellectual functioning and appropriate schooling. To better understand the neural underpinnings of dyslexia, this study investigated the neurophysiological differences between normal readers (NR group) and readers with dyslexia (DYS group) by analyzing their brain activity at eyes-closed resting state using mobile electroencephalography (mEEG). The results revealed that the DYS group exhibited an overall larger power activation in the theta and beta frequency bands, as well as a dominance of delta, theta, and beta frequencies across all scalp sites. Increased delta and theta activity was found in the left frontal region, whereas significantly stronger beta power was found in the right hemisphere. Moreover, weaker alpha activity was observed in the left frontal and right posterior regions. These findings provide evidence of an atypical and less integrated linguistic network in dyslexia.
\end{abstract}

Keywords: Dyslexia, reading, mobile electroencephalography, resting state.

\section{Introduction}

Dyslexia is a neurodevelopmental disorder characterized by severe and persistent reading deficits in both children and adults despite normal intellectual functioning and having been provided with educational opportunities (Pina Rodrigues et al., 2017). As a multifaceted and heterogeneous disorder that persists across the lifespan, it has been carefully and intensively studied by researchers who have attempted to determine its genetic, neurobiological, and cognitive components (Snowling, 2013).

One approach to understanding dyslexia is through analyzing resting state activity, an area of interest in cognitive neuroscience wherein intrinsic functional connectivity at rest permits the brain to allocate resources and prepare itself for changes stemming from the internal or external environment. This allows researchers to make predictions about the resting state network as a determining factor of underlying neural activity. Research in this area has provided valuable evidence on deviant network organization for neurological disorders and generated much understanding about the neural characteristics of healthy brain development (Alcauter et al., 2017; Gracia-Tabuenca, Moreno, Barrios, \& Alcauter, 2018). Brain activity can be obtained using mobile electroencephalography (mEEG), a neuroimaging tool that allows researchers to observe patterns of brain frequencies. Each frequency band has a purpose and an underlying function: A dominance of slow frequencies (i.e., delta and theta) when one is engaged in a cognitive task would suggest slow brain activity and possibly even cognitive dysfunction (Kamel \& Saeed Malik, 2015), whereas faster frequencies (i.e., beta and gamma) are dominant when the brain is actively processing information (Magazzini \& Singh, 2018). The alpha band, which is dominant at resting state, is associated with cortical and behavioral inhibition (Bastos et al., 2015, Marshall, O'Shea, Jensen, \& Bergmann, 2015). These frequencies are used to explain the differences in brain activation between normal controls and those with neurological deficits.

Results from resting state EEG studies on dyslexia and other learning disorders have typically reported greater delta and theta power as well as weaker alpha and beta power (Papagiannopoulou \& Lagopoulos, 2016; Roca-Stappung, Fernandez, Bosch-Bayard, Harmony, Ricardo-Garcell, 2017). Studies generally indicate remarkably elevated low frequency activity, particularly in the theta band, in the left hemisphere which reflects an atypical linguistic network, implicating the presence of brain abnormalities in children with dyslexia prior to reading acquisition (De Vos et al., 2017; Fraga González 
et al., 2016; Morillon, Liégeois-Chauvel, Arnal, Bénar, \& Giraud, 2012; Pagnotta et al., 2015; van der Mark et al., 2011). Babiloni et al. (2012) reported abnormal alpha rhythms, whereas a number of studies have observed abnormally stronger beta power in the right hemisphere, indicating task-related overexcitability (De Vos et al., 2017; Dimitriadis et al., 2016, 2018; Hoeft et al., 2011; Jiménez-Bravo et al., 2017; Lizarazu et al., 2015; Power, Colling, Mead, Barnes, \& Goswami, 2016; Simos et al., 2011).

\section{Method}

\subsection{Participants}

The participants were divided the Dyslexia (DYS) group $(n=5$; mean age $=9.61$; $\mathrm{DD}=1.7$ ) and the Normal Reader $(\mathrm{NR})$ group $(\mathrm{n}=4$ mean age $=9.61$; $\mathrm{SD}=1.00)$. For both groups, non-verbal IQ results obtained using the Raven's Colored Matrices (Raven, Raven, \& Court, 2003) were found to be within the normal range (at least at 75th percentile). The DYS group had been previously diagnosed with Specific Learning Disorder with an impairment in reading by a professional (medical doctor, clinical/educational psychologist, or special educator/reading specialist). The NR group, on the other hand, presented with no history of reading difficulties. All participants were male, right-handed, with normal vision (as previously assessed by their physicians), and free of any co-morbid conditions, such as attention deficit/hyperactivity disorder, autism spectrum disorders, and any speech/language and visual impairments. Furthermore, parental consent and child assent were obtained before data gathering.

\subsection{Data collection and analysis}

Brain signals were obtained by the Emotiv EPOC Neuroheadset (Emotiv Systems, Inc., 2013), a non-invasive, high-resolution, neuro-signal acquisition and processing wireless headset designed for contextualized research (see Figure 1). It has 14 channels (AF3, F7, F3, FC5, T7, P7, O1, O2, P8, T8, FC6, F4, F8, and AF42) distributed according to the internationally accepted 10-20 system of electrode placement and includes two references in the CMS/DRL noise cancellation configuration P3/P4 locations. Only 12 channels were included in the study (i.e., T7 and T8 were excluded).

Figure 1. The Emotiv EPOC Neuroheadset and its scalp locations.
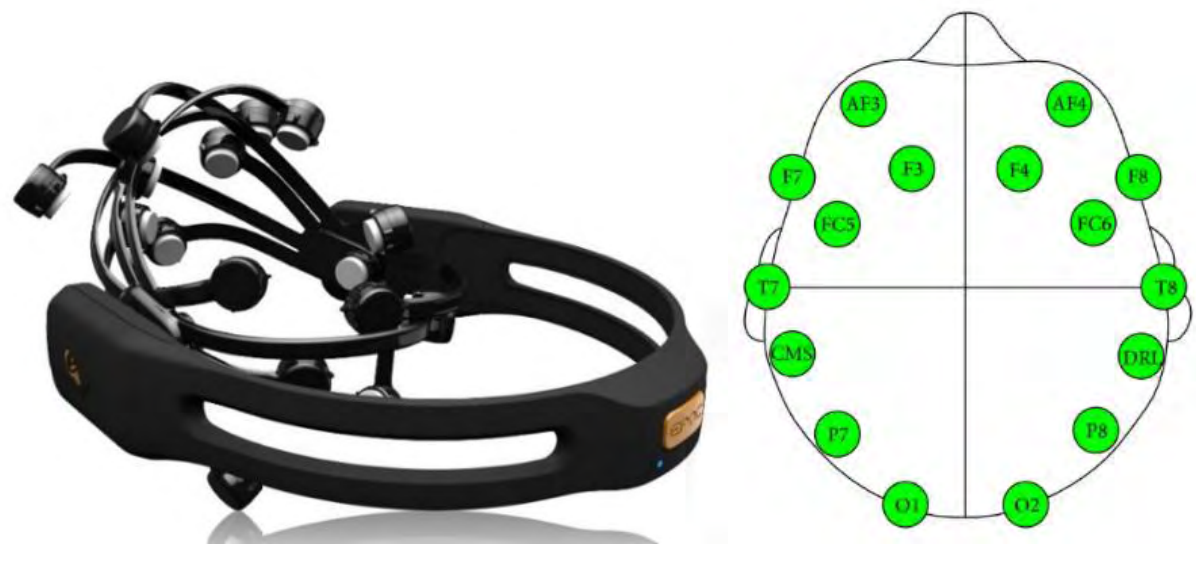

Data were transferred via Bluetooth to the computer and raw EEG data were acquired using the EmotivPRO software. Further signal processing was carried out using EEGLAB, an open source MATLAB toolbox for processing data from EEG. The EEG recordings were segmented into epochs to be extracted, visually inspected, and cleaned for artifacts. Absolute power analyses using fast Fourier transform (FFT) for delta (1-4 Hz), theta (4-8 Hz), alpha (8-12 Hz), and beta (12-25 Hz). Mann-Whitney U tests were performed using IBM SPSS Statistics version 25.0. The participants wore the EPOC headset throughout the experiment. Before putting on the headset, the 14 electrode recesses were fitted with a moist felt pad. The headset was then placed on the participant's head and subjected to software set-up. After verifying that the built-in battery was fully charged and the wireless signal reception was reported as good, the experiment began. Each participant wore the headset for five minutes. 


\section{Results}

Significant group differences were observed for the theta $(U=1, p=.03)$ and beta $(U=0$, $\mathrm{p}=.01$ ) frequency bands, wherein the DYS group exhibited overall stronger power for these bands. Tests comparing electrode sites indicate that the DYS group obtained significantly stronger theta power in the frontal and left parietal regions. Stronger beta power was mostly observed in the right frontal and left parietal region. On the other hand, the NR group demonstrated stronger alpha power values in the left frontal and right occipital regions. Significant inter- and intra-hemispheric differences were limited to the delta, theta, and alpha bands (see Figure 2). For the DYS group, delta power is significantly left-lateralized in the frontal region, whereas theta power is bilaterally distributed. Alpha and beta power are notably right-lateralized. The NR group, on the other hand, demonstrate a more stable resting network.

Figure 2. Power distributions according to frequency band for Resting State. Power (in dB) is represented by colors (dark red $=$ very high, orange $=$ high, yellow $=$ average, light blue $=$ low, dark blue $=$ very low $)$.

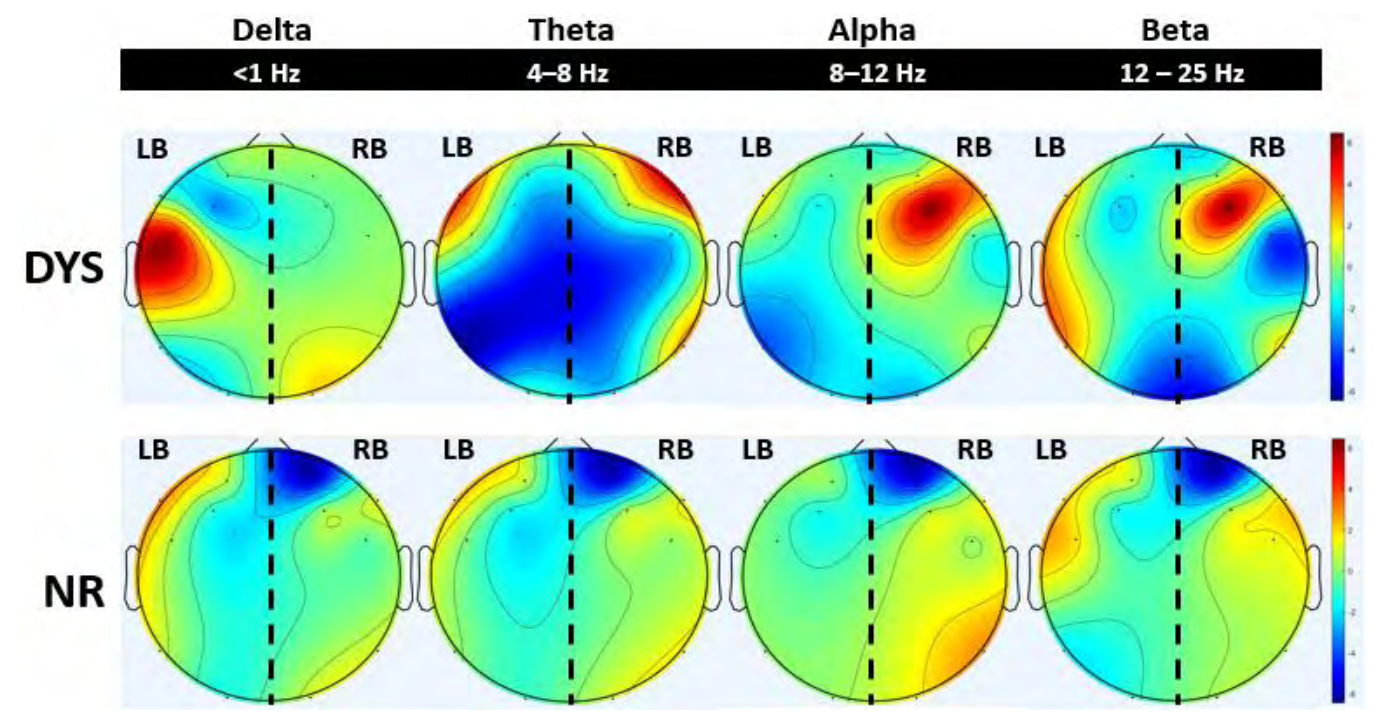

\section{Discussion}

The results revealed that the DYS group exhibited an overall larger power activation in the theta and beta frequency bands, as well as a dominance of delta, theta, and beta frequencies across all scalp sites. In the DYS group, increased delta and theta activity was found in the left frontal region. Abnormalities in the theta band (i.e., overactivation) at resting state have been implicated as a distinct neural signature in dyslexia, suggesting a less integrated network, as well as reduced communication in readers with dyslexia compared to controls. Thus, the observed increase in low frequency activity during eyes closed resting state in children with dyslexia is a strong indicator of the presence of an atypical network (De Vos et al., 2017; Fraga González et al., 2016; Pagnotta et al., 2015; Papagiannopoulou \& Lagopoulos, 2016). The frontal reading network involves the left inferior frontal gyrus which plays a key role in speech articulation. Left-hemispheric hypoactivation characterized by an abnormal modulation of delta and theta frequencies is reflective of altered connectivity patterns that have been found to have crucial consequences in processing speech input (van der Mark et al., 2011).

An attenuation of beta frequencies was observed in the left hemisphere as compared to the right hemisphere. The current findings agree with other studies that have reported abnormally stronger beta power in the right hemisphere (De Vos et al., 2017; Dimitriadis et al., 2013, 2016, 2018; Jiménez-Bravo et al., 2017; Lizarazu et al., 2015; Power et al., 2016). At rest, this right-lateralized overexcitability may be attributed to task-related overactivation in the right hemisphere (Hoeft et al., 2011; Simos et al., 2011). Moreover, weaker alpha activity was observed in the DYS group compared to the NR group, especially in the left frontal and right posterior regions. Comparable results were obtained by Babiloni et al. (2012) and Papagiannopoulou and Lagopoulos (2016). 


\section{Conclusion}

Analyzing eyes-closed resting state EEG rhythms is essential to better understand the role of abnormal cortical sources in brain-based deficits. The findings of this study confirmed a less integrated language network as evidenced by a dominance of theta activity in the left frontal region at resting state in children with dyslexia. Moreover, atypical alpha and beta activity were also observed. More studies are needed to further explore the neurophysiological characteristics of resting state activity in children with dyslexia.

\section{References}

Alcauter, S., García-Mondragón, L., Gracia-Tabuenca, Z., Moreno, M. B., Ortiz, J.J., \& Barrios, F. A. (2017). Resting state functional connectivity of the anterior striatum and prefrontal cortex predicts reading performance in school-age children. Brain and Language, 174, 94-102. doi:10.1016/j.bandl.2017.07.007

Babiloni, C., Stella, G., Buffo, P., Vecchio, F., Onorati, P., Muratori, C., ... Rossini, P.M. (2012). Cortical sources of resting state EEG rhythms are abnormal in dyslexic children. Clinical Neurophysiology, 123(12), 2384-2391. doi: 10.1016/j.clinph.2012.05.002

Bastos, A. M., Fries, P., Litvak, V., Moran, R., Friston, K.J., \& Bosman, C.A. (2015). A DCM study of spectral asymmetries in feedforward and feedback connections between visual areas V1 and V4 in the monkey. NeuroImage, 108, 460-475. doi: 10.1016/j.neuroimage.2014.12.081

De Vos, A., Vanvooren, S., Vanderauwera, J., Ghesquière, P., \& Wouters, J. (2017). Atypical neural synchronization to speech envelope modulations in dyslexia. Brain Language, 164, 106-117. doi: 10.1016/j.bandl.2016.10.002

Delorme, A., \& Makeig, S. (2004). EEGLAB: An open source toolbox for analysis of single-trial EEGdynamics including independent component analysis. Journal of Neuroscience Methods, 134, 9-21. doi: 10.1016/j.jneumeth.2003.10.009

Dimitriadis, S.I., Laskaris, N.A., Simos, P.G., Fletcher, J.M., \& Papanicolaou, A.C. (2016). Greater repertoire and temporal variability of cross-frequency coupling (CFC) modes in resting-state neuromagnetic recordings among children with reading difficulties. Frontiers in Human Neuroscience, 10(163). doi: 10.3389/fnhum.2016.00163

Dimitriadis, S.I., Simos, P.G., Fletcher, J.M., \& Papanicolaoue, A.C. (2018). Aberrant resting-state functional brain networks in dyslexia: Symbolic mutual information analysis of neuromagnetic signals. International Journal of Psychophysiology, 126, 20-29. doi:10.1016/j.ijpsycho.2018.02.008

Emotiv Systems, Inc. (2013). EmotivPRO Software. San Francisco, CA: Emotiv Systems, Inc.

Fraga González, G., Zarić, G., Tijms, J., Bonte, M., Blomert, L., \& van der Molen, M.W. (2014). Brain potential analysis of visual word recognition in dyslexics and typically reading children. Frontiers in Human Neuroscience, 8(474). doi: 10.3389/fnhum.2014.00474

Hoeft, F., McCandliss, B.D., Black, J.M., Gantman, A., Zakerani, N., Hulme, C., ... Gabrieli, J.D.E. (2011). Neural systems predicting long-term outcome in dyslexia. Proceedings of the National Academy of Sciences, 108(1), 361-366. doi: 10.1073/pnas.1008950108

Gracia-Tabuenca, Z., Moreno, M.B., Barrios, F.A., \& Alcauter, S. (2018). Hemispheric asymmetry andhomotopy of resting state functional connectivity correlate with visuospatial abilities in schoolage children. NeuroImage. doi: 10.1016/j.neuroimage.2018.03.051

Jiménez-Bravo, M., Marrero, V., \& Benítez-Burraco, A. (2017). An oscillopathic approach to developmental dyslexia: From genes to speech processing. Behavioural Brain Research, 329(1), 84-95. doi: 10.1016/j.bbr.2017.03.048

Kamel, N., \& Saeed Malik, A. (Eds.). (2015). EEG/ERP analysis: Methods and applications. Boca Raton, FL: CRC Press.

Lizarazu, M., Lallier, M., Molinaro, N., Bourguignon, M., Paz-Alonso, P. M., Lerma-Usabiaga, G., ...Carreiras, M. (2015). Developmental evaluation of atypical auditory sampling in dyslexia: Functional and structural evidence. Human Brain Mapping, 36, 4986-5002. doi:10.1002/hbm.22986.117

Magazzini, L., \& Singh, K. D. (2018). Spatial attention modulates visual gamma oscillations across the human ventral stream. NeuroImage, 166, 219-229. doi: 10.1016/j.neuroimage.2017.10.069 
Marshall, T.R., O'Shea, J., Jensen, O., \& Bergmann, T.O. (2015). Frontal eye fields control attentional modulation of alpha and gamma oscillations in contralateral occipitoparietal cortex. Journal of Neuroscience, 35(4), 1638-1647. doi: 10.1523/jneurosci.3116-14.2015

MathWorks, Inc. (2016). MATLAB and Statistics Toolbox Release 2012b. Natick, MA: The MathWorks, Inc.

Morillon, B., Liégeois-Chauvel, C., Arnal, L.H., Bénar, C.G., \& Giraud, A.L. (2012). Asymmetric function of theta and gamma activity in syllable processing: an intra-cortical study. Frontiers in Psychology, 3(248). doi: 10.3389/fpsyg.2012.00248

Pagnotta, M.F., Zouridakis, G., Lianyang Li, Lizarazu, M., Lallier, M., Molinaro, N., \& Carreiras, M. (2015). Low frequency overactivation in dyslexia: Evidence from resting state magnetoencephalography. $37^{\text {th }}$ Annual International Conference of the IEEE Engineering in Medicine and Biology Society (EMBC), 6959-6962. doi: 10.1109/embc.2015.7319993

Papagiannopoulou, E. A., \& Lagopoulos, J. (2016). Resting state EEG hemispheric power asymmetry in children with dyslexia. Frontiers in Pediatrics, 4(11). doi: 10.3389/fped.2016.00011

Pina Rodrigues, A., Rebola, J., Jorge, H., Ribeiro, M.J., Pereira, M., van Asselen, M., \& Castelo Branco, M. (2017). Visual perception and reading: New clues to patterns of dysfunction across multiple visual channels in developmental dyslexia. Investigative Ophthalmology \& Visual Science, 58, 309-317. doi: 10.1167/iovs.16-20095

Power, A.J., Colling, L.J., Mead, N., Barnes, L., \& Goswami, U. (2016). Neural encoding of the speech envelope by children with developmental dyslexia. Brain \& Language, 160, 1-10. doi: 10.1016/j.bandl.2016.06.006

Raven, J., Raven, J. C., \& Court, J. H. (2003). Manual for Raven's Progressive Matrices and Vocabulary Scales. San Antonio, TX: Harcourt Assessment.

Roca-Stappung, M., Fernandez, T., Bosch-Bayard, J., Harmony, T., \& Ricardo-Garcell, J. (2017). Electroencephalographic characterization of subgroups of children with learning disorders. PLoS ONE, 12(7), e0179556. doi: 10.1371/journal.pone.0179556

Simos, P.G., Rezaie, R., Fletcher, J.M., Juranek, J., Passaro, A.D., Li, Z., .. Papanicolaou, A.C. (2011). Functional disruption of the brain mechanism for reading: effects of comorbidity and task difficulty among children with developmental learning problems. Neuropsychology, 25(4), 520-534. doi: 10.1037/a0022550

Snowling, M. J. (2013). Early identification and interventions for dyslexia: A contemporary view. Journal of Research in Special Educational Needs, 13(1), 7-14. doi: 10.1111/j.14713802.2012.01262.x

van der Mark, S., Klaver, P., Bucher, K., Maurer, U., Schulz, E., Brem, S., ... Brandeis, D. (2011). The left occipitotemporal system in reading: Disruption of focal fMRI connectivity to left inferior frontal and inferior parietal language areas in children with dyslexia. NeuroImage, 54, 2426-2436. doi: 10.1016/j.neuroimage.2010.10.002 


\title{
POSITIVE SELF-ESTEEM, SATISFACTION AND THE UNPLUGGED PROGRAM AMONG SLOVAK SCHOOLCHILDREN
}

\author{
Olga Orosová, Maria Bacikova-Sleskova, \& Beata Gajdosova \\ Department of Educational Psychology and Health Psychology, Faculty of Arts, \\ Pavol Jozef Šafárik University in Košice (Slovakia)
}

\begin{abstract}
Background: The aims of this study were to explore the changes in schoolchildren's positive self-esteem, satisfaction with themselves and their surroundings following the Unplugged program as well as the indirect effect of Unplugged on satisfaction through self-esteem. Method: In the school year 2013/2014, the program Unplugged was implemented in Slovak primary schools. The study was carried out as a cluster randomized controlled trial with data collection conducted immediately before program implementation (T1), immediately after program implementation (T2), and at three months (T3) after program implementation. The program included 1195 participating schoolchildren ( $\mathrm{M}=11.52 ; 47.4 \%$ boys). The schools were randomly assigned to an experimental $(n=540)$ and control group $(n=655)$. The experimental group was exposed to the program Unplugged which consisted of 12 lessons (http://www.eudap.net/). The effect of participating in Unplugged and gender was explored through the changes in positive self-esteem and satisfaction with themselves and surroundings. This was followed across the three contact points at the baseline (T1) and two follow-up measures (T2, T3) with group (experimental/control) and gender as the between-subject factors. GLM Repeated Measures were used for the data analyses. The indirect effects of the group (experimental/control) on satisfaction through self-esteem were explored using the Hayes' PROCESS tool. In order to explore whether the indirect effect was moderated by gender, separate analyses were performed on each respective part of the data. Results: There was a significant main effect of time measures on the self-esteem expressed by the schoolchildren. The contrast analysis revealed that positive self-esteem was significantly higher at the second follow-up (T3) than at the first follow-up test (T2). There was a significant interaction effect between self-esteem and the group. This effect indicated that the level of self-esteem differed between the participants and non-participants of the Unplugged program. The contrast analysis revealed that positive self-esteem was significantly higher at the second follow-up (T3) than at the baseline test (T1) among the participants of the Unplugged program. The partial indirect effects of the group (experimental/control) on satisfaction through self-esteem was found at the second follow-up test (T3) among girls. Conclusion: The results have confirmed a short-term effect of the Unplugged program in increasing the positive self-esteem of schoolchildren, as well as an indirect effect of satisfaction in girls through positive self-esteem three months after program implementation.
\end{abstract}

Keywords: Unplugged program, self-esteem, satisfaction, schoolchildren.

\section{Introduction}

Self-esteem, satisfaction with life, satisfaction with themselves and well-being have created important personal components of health-related interventions. It has been found that interventions increasing self-esteem, life satisfaction and optimistic life orientation with the aim of reducing bullying among classmates were likely to not only benefit those who were victimized, but all students (Låftman \& Modin, 2017; Blood et al., 2011). Self-esteem development may be the target of interventions aiming to improve quality of life in addition to treating adolescents' psychopathology (Jozefiak et al., 2017). Moreover, well-being, self-control, and self-esteem have been identified as important factors in preventing problematic internet use (Mei, Yau, Chai et al., 2016).

Self-esteem mediates the association between quality of life and suicidal ideation (Fulginiti, \& Brekke, 2015) as well as between the perception of one's body image and psychological distress (Duchesne, Dion, Lalande et al., 2017). There was also a mediation effect of self-esteem found on well-being and social anxiety (Fernandes, 2018). Self-esteem has also been identified as an important component in the prevention of eating problems in adolescents (Barker\& Bornstein, 2010; Tirlea et al., 2016). 
Gender differences and personal characteristics have been found to be important factors in the effectiveness of school-based drug use prevention programs; factors that could cast light on the psychological mechanism of the program effect (Vigna-Taglianti et al., 2009). Research has confirmed that school-based prevention programs, which target multiple risk behaviours and help build self-esteem and life skills, are more likely to be effective in preventing drug use (Warren, 2016). However, there have been inconsistent results about the drug use prevention program effects among boys and girls (Vigna-Taglianti et al., 2009).

This study aimed to explore the effectiveness of the school-based curriculum „Unplugged“ among Slovak schoolchildren. This program to prevent substance use is based on the social influence approach and addresses social and personal skills, knowledge, and normative beliefs (Vigna-Taglianti et al., 2014). The data-based drug use prevention programs have been seldom accessible at Slovak elementary schools. Schoolchildren's positive self-esteem, satisfaction with themselves and with their surroundings and the moderation effect of gender were the indicators chosen in this study for examining the effectiveness of the Unplugged program.

\section{Objectives}

The objectives of this study were to explore the changes in schoolchildren's positive self-esteem, satisfaction with themselves and with their surroundings following the Unplugged program as well as the indirect effect of Unplugged on satisfaction through self-esteem.

\section{Methods}

\subsection{Sample and study design}

In the school year 2013/2014, the program Unplugged was implemented in Slovak primary schools. The study was carried out as a cluster randomized controlled trial with data collection conducted immediately before program implementation (T1), immediately after program implementation (T2) and at three months (T3) after program implementation. The program included 1195 participating schoolchildren ( $M=11.52 ; 47.4 \%$ boys). The schools were randomly assigned to an experimental $(n=540)$ and control group $(n=655)$. The experimental group was exposed to the program Unplugged which consisted of 12 lessons (http://www.eudap.net/). The effect of participating in Unplugged and gender was explored through the changes in positive self-esteem and satisfaction with themselves and surroundings. This was followed across the three contact points at the baseline (T1) and two follow-up measures (T2, T3) with group (experimental/control) and gender as the between-subject factors.

\subsection{Method}

The measures included in the present study were:

Self-Liking and Self-Competence Scale (SLCS-R)

Self-esteem was measured using the 16-item self-liking and competence scale-revised (SLCS-R) (Tafarodi\& Swann, 2001). Based on Principal Component analysis, a 7-item component of positive self-esteem (Crombach $\alpha=0.793$ ) was used (Berinsterova, 2015). The items were assessed on a 5-point Likert scale ( 1 - totally disagree, 5 - totally agree), where higher scores indicated a higher level of positive self-esteem.

Satisfaction with themselves and surroundings (ESPAD 2011)

Satisfaction was measured by three questions: "How satisfied are you usually with the family's economic situation? How satisfied are you usually with your health? How satisfied are you usually with yourself?" The responses ranged from not at all satisfied (1) to very satisfied (5), where higher scores indicated more satisfying/positive relationships with themselves and their surrounding (Cronbach alpha=0.765).

\subsection{Statistical analyses}

GLM Repeated Measures were used for the data analyses. The indirect effects of the group (experimental/control) on satisfaction through self-esteem were explored using the Hayes' PROCESS tool (with satisfaction at the baseline measure as the covariate variable). In order to explore whether the indirect effect was moderated by gender, separate analyses were performed on each respective part of the data.

\section{Results}

There was a significant main effect of time measures on the self-esteem expressed by schoolchildren (Table 1,2). The contrast analysis (Table 3) revealed that positive self-esteem was significantly higher at the second follow-up (T3) than at the first follow-up test (T2). 
There was a significant interaction effect between self-esteem and the group (Table 2). This effect indicated that the level of self-esteem differed between the participants and non-participants of the Unplugged program. The contrast analysis revealed that positive self-esteem was significantly higher at the second follow-up (T3) than at the baseline test (T1) among the participants of the Unplugged program (Figure 1).

Table 1. Descriptive characteristics of the sample.

\begin{tabular}{|c|c|c|c|c|c|c|c|c|c|c|c|c|c|}
\hline & & \multicolumn{2}{|c|}{$\mathrm{S}^{\mathrm{T} 1}$} & \multicolumn{2}{|c|}{$\mathrm{SE}^{\mathrm{T} 1}$} & \multicolumn{2}{|c|}{$\mathrm{S}^{\mathrm{T} 2}$} & \multicolumn{2}{|c|}{$\mathrm{SE}^{\mathrm{T} 2}$} & \multicolumn{2}{|c|}{$\mathrm{S}^{\mathrm{T} 3}$} & \multicolumn{2}{|c|}{$\mathrm{SE}^{\mathrm{T} 3}$} \\
\hline Group & Gender & Mean & SD & Mean & SD & Mean & SD & Mean & SD & Mean & SD & Mean & SD \\
\hline \multirow{3}{*}{ CG } & Boys & 12.78 & 2.61 & 26.90 & 6.56 & 12.64 & 2.78 & 23.79 & 8.35 & 12.58 & 2.41 & 25.56 & 6.79 \\
\hline & Girls & 12.93 & 2.14 & 27.45 & 5.36 & 12.63 & 2.28 & 27.27 & 6.38 & 12.57 & 2.51 & 27.02 & 6.15 \\
\hline & Total & 12.87 & 2.35 & 27.22 & 5.89 & 12.64 & 2.50 & 25.79 & 7.47 & 12.58 & 2.46 & 26.40 & 6.46 \\
\hline \multirow{3}{*}{ EG } & Boys & 12.73 & 2.00 & 27.34 & 5.96 & 12.66 & 2.09 & 27.07 & 5.60 & 12.31 & 2.24 & 27.48 & 5.22 \\
\hline & Girls & 12.71 & 2.44 & 27.57 & 5.20 & 12.67 & 2.35 & 27.43 & 5.86 & 12.83 & 1.93 & 28.67 & 6.42 \\
\hline & Total & 12.72 & 2.26 & 27.48 & 5.49 & 12.67 & 2.24 & 27.29 & 5.74 & 12.60 & 2.08 & 28.21 & 6.00 \\
\hline \multirow{3}{*}{ Total } & Boys & 12.76 & 2.35 & 27.09 & 6.30 & 12.65 & 2.49 & 25.17 & 7.48 & 12.46 & 2.34 & 26.37 & 6.24 \\
\hline & Girls & 12.84 & 2.28 & 27.50 & 5.28 & 12.65 & 2.31 & 27.35 & 6.13 & 12.68 & 2.28 & 27.78 & 6.31 \\
\hline & Total & 12.80 & 2.31 & 27.33 & 5.72 & 12.65 & 2.39 & 26.45 & 6.80 & 12.59 & 2.30 & 27.20 & 6.31 \\
\hline
\end{tabular}

Notes: CG = control group, EG = experimental group (the Unplugged program), T1 = baseline measure, T2 = the first follow-up measure immediately after program implementation, $\mathrm{T} 3=$ the second follow-up measure three months after program implementation, $\mathrm{S}=$ Satisfaction with themselves and surroundings, SE = Positive self-esteem

Table 2. The repeated-measures effects of time on the Positive Self-esteem and the Satisfaction with themselves and surroundings.

\begin{tabular}{|l|c|c|l|c|c|}
\hline Positive self-esteem (SE) & F & p-value & $\begin{array}{l}\text { Satisfaction with themselves } \\
\text { and surroundings (S) }\end{array}$ & F & p-value \\
\hline SE & 4.27 & 0.014 & S & 2.36 & 0.095 \\
\hline SE x Group & 3.09 & 0.046 & S x Group & 0.35 & 0.708 \\
\hline SE x Gender & 2.57 & 0.077 & S x Gender & 0.84 & 0.434 \\
\hline SE x Group x Gender & 2.83 & 0.060 & S x Group x Gender & 1.55 & 0.213 \\
\hline
\end{tabular}

Table 3. The time contrasts of the Positive Self-esteem and the Satisfaction with themselves and surroundings.

\begin{tabular}{|c|c|c|c|c|c|c|c|}
\hline Positive self-esteem (SE) & SE & $\mathrm{F}$ & p-value & $\begin{array}{l}\text { Satisfaction with } \\
\text { themselves and } \\
\text { surroundings (S) }\end{array}$ & S & $\mathrm{F}$ & p-value \\
\hline \multirow{2}{*}{ SE } & T1 vs T2 & 0.51 & 0.697 & \multirow{2}{*}{ 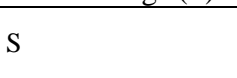 } & T1 vs T2 & 4.29 & 0.039 \\
\hline & T2 vs T3 & 5.08 & 0.025 & & T2 vs T3 & 0.68 & 0.412 \\
\hline \multirow{2}{*}{ SE x Group } & T1 vs T2 & 5.02 & 0.026 & \multirow{2}{*}{ S x Group } & T1 vs T2 & 0.37 & 0.544 \\
\hline & T2 vs T3 & 0.01 & 0.930 & & T2 vs T3 & 0.03 & 0.871 \\
\hline \multirow{2}{*}{ SE x Gender } & T1 vs T2 & 1.96 & 0.162 & \multirow{2}{*}{ S x Gender } & T1 vs T2 & 0.80 & 0.372 \\
\hline & T2 vs T3 & 0.73 & 0.394 & & T2 vs T3 & 1.68 & 0.195 \\
\hline \multirow{2}{*}{ SE x Group x Gender } & T1 vs T2 & 0.01 & 0.966 & \multirow{2}{*}{ S x Group x Gender } & T1 vs T2 & 2.73 & 0.099 \\
\hline & $\mathrm{T} 2$ vs $\mathrm{T} 3$ & 4.10 & 0.043 & & $\mathrm{~T} 2$ vs $\mathrm{T} 3$ & 1.60 & 0.206 \\
\hline
\end{tabular}

The partial indirect effects of the group (experimental/control) on satisfaction with themselves and surroundings through positive self-esteem was found at the second follow-up test (T3), three months after the Unplugged program implementation only among girls (Figure 2).

Figure 1. Positive self-esteem x group interaction.

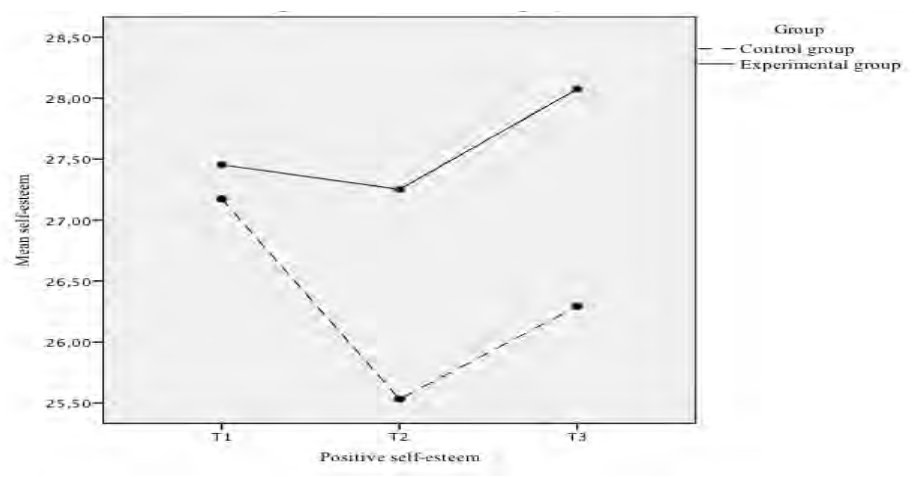


Figure 2. Diagram of the indirect effect of group on the satisfaction with themselves and surroundings through positive self-esteem among girls.

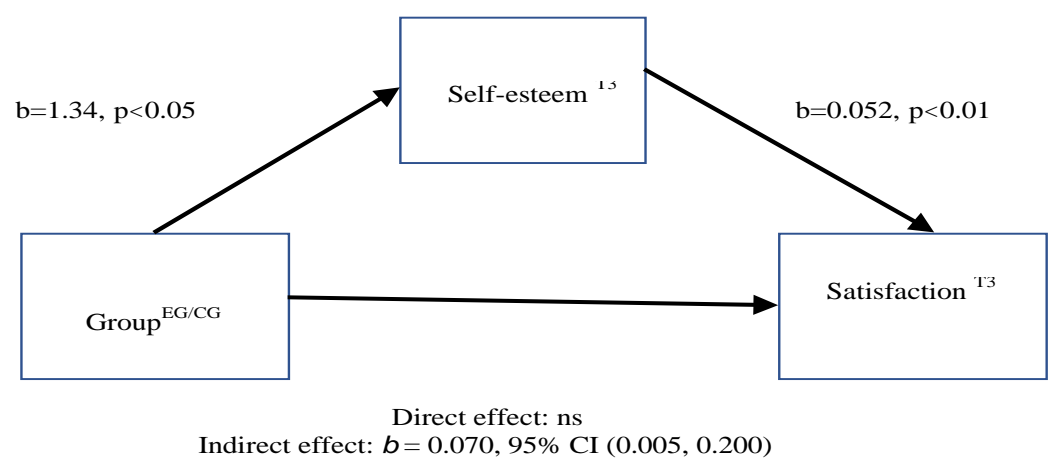

implementation,

\section{Discussion and conclusions}

The results of this study have confirmed the partial indirect effect of the Unplugged program on satisfaction with themselves and surroundings through positive self-esteem that was found at the second follow-up test (three months after the Unplugged program implementation) only among girls. In line with these findings, a previous study confirmed the effectiveness of the Unplugged program through the development of a positive relationship with the teacher, empathy, decrease of conflicts, increased self-awareness and self-esteem and better school results (Carpi et al., 2015). The results of this study are also consistent with the statement that the Unplugged program, as a comprehensive social influence school curricula against substance misuse, may perform differently among girls and boys due to developmental and personality factors (Vigna-Taglianti, et al., 2009). Similarly, the effect of group-based, low-dose intervention on improving self-esteem was also found among girls from diverse cultural backgrounds (Tirlea et al., 2016). The mediation role of self-esteem between the Unplugged program participation and lifetime prevalence of cigarette smoking, a short-term indirect mediation effect of positive self-esteem in the program Unplugged, has also been confirmed by our previous analyses (Berinšterová et al., 2015).

On the other hand, while it was found that the Unplugged program was effective in reducing smoking, episodes of drunkenness and the use of cannabis in the short term, this association was confined to boys, with age and self-esteem as possible explanations of this difference (Vigna-Taglianti, et al., 2014).

This study found a significant main effect of time measures on the self-esteem expressed by schoolchildren and the levels of self-esteem differed between the participants and non-participants of the Unplugged program. The results confirmed the effectiveness of the Unplugged program via positive self-esteem three months after program implementation. However, the main effect of time measures on self-esteem did not differ between boys and girls. The results of this study supported a gender specific indirect effect of the program although a direct effect of the program for both genders.

In conclusion, the Unplugged program evaluation suggests that a comprehensive social-influence approach in the school setting could be effective in the direct and indirect improvement of personal skills and supports the need for a gender specific program effect evaluation. The level of psychological education of teachers responsible for the Unplugged program implementation, which is strongly based on interactive group work and quality of program implementation, will be able to deliver the program effect. The future of the Unplugged program implementation, as well as examining program effectiveness should reflect ,a need to adapt substance-use prevention programs to gender, peer gender, and the gender balance of schools..." (Grard, Kunst, Kuipers et all., 2018, p. 8).

\section{Acknowledgements}

This work was supported by Research and Development support Agency under the contract No. APVV-0253-11 and APVV-15-0662. 


\section{References}

Barker, E. T., \& Bornstein, M. H. (2010). Global self-esteem, appearance satisfaction, and self-reported dieting in early adolescence. The journal of early adolescence, 30(2), 205-224.

Berinsterova, M. (2015). The effectiveness of the school program of universal prevention "Unplugged". Dissertation thesis. Kosice: Pavol Jozef Šafárik University in Košice.

Berinšterová, M., Orosová, O., \& Miovský, M. (2015). Short term effectiveness of the Unplugged program in Slovakia: the Mediational role of self-esteem. European Journal of Public Health, 25(suppl_3).

Blood, G. W., Blood, I. M., Tramontana, G. M., Sylvia, A. J., Boyle, M. P., \& Motzko, G. R. (2011). Self-reported experience of bullying of students who stutter: Relations with life satisfaction, life orientation, and self-esteem. Perceptual and motor skills, 113(2), 353-364.

Carpi, A., Ferrari, M. B., Poggi, L., Chiesa, S., Costa, A., De, A. L., Rebolini, G., \& Gabutti, G. (2015). Health promotion in Local Health Unit 4 Chiavarese--Liguria Region, Italy." Unplugged" project: needs, methodology and implementation. Minerva pediatrica, 67(6), 495-503.

Duchesne, A. P., Dion, J., Lalande, D., Bégin, C., Émond, C., Lalande, G., \& McDuff, P. (2017). Body dissatisfaction and psychological distress in adolescents: Is self-esteem a mediator? Journal of health psychology, 22(12), 1563-1569.

Fernandes, H. M. (2018). Physical Activity and Mental Health in Adolescents: The mediating effect of Self-Esteem and Body Satisfaction. Revista de psicologia del deporte, 27(1), 67-75.

Fulginiti, A., \& Brekke, J. S. (2015). Escape from discrepancy: Self-esteem and quality of life as predictors of current suicidal ideation among individuals with schizophrenia. Community mental health journal, 51(6), 654-662.

Grard, A., Kunst, A., Kuipers, M., Richter, M., Rimpela, A., Federico, B., \& Lorant, V. (2018). Same-sex friendship, school gender composition, and substance use: a social network study of 50 European schools. Substance use \& misuse, 53(6), 998-1007.

Hibell, B., Guttormsson, U., Ahlström, S., Balakireva, O., Bjarnason, T., Kokkevi, A., \& Kraus, L. (2012). The 2011 ESPAD report: substance use among students in 36 European countries. Swedish Council for Information on Alcohol and Other Drugs. Stockholm. European Monitoring Centre for Drugs and Drug Addiction. Council of Europe. Cooperation Group to Combat Drug Abuse and Illicit Trafficking in Drugs (Pompidou Group).

Jozefiak, T., Kayed, N. S., Ranøyen, I., Greger, H. K., Wallander, J. L., \& Wichstrøm, L. (2017). Quality of life among adolescents living in residential youth care: do domain-specific self-esteem and psychopathology contribute? Quality of Life Research, 26(10), 2619-2631.

Låftman, S., \& Modin, B. (2017). Peer victimization among classmates-Associations with students' internalizing problems, self-esteem, and life satisfaction. International journal of environmental research and public health, 14(10), 1218.

Mei, S., Yau, Y. H., Chai, J., Guo, J., \& Potenza, M. N. (2016). Problematic Internet use, well-being, self-esteem and self-control: Data from a high-school survey in China. Addictive behaviors, 61, 74-79.

Tafarodi, R. W., \& Swann Jr, W. B. (2001). Two-dimensional self-esteem: Theory and measurement. Personality and individual Differences, 31(5), 653-673.

Tirlea, L., Truby, H., \& Haines, T. P. (2016). Pragmatic, randomized controlled trials of the Girls on the Go! Program to improve self-esteem in girls. American journal of health promotion, 30(4), 231-241.

Vigna-Taglianti, F. D., Galanti, M. R., Burkhart, G., Caria, M. P., Vadrucci, S., \& Faggiano, F. (2014). "Unplugged," a European school-based program for substance use prevention among adolescents: Overview of results from the EU-Dap trial. New directions for youth development, 2014(141), 67-82.

Vigna-Taglianti, F., Vadrucci, S., Faggiano, F., Burkhart, G., Siliquini, R., Galanti, M. R., \& EU-Dap Study Group. (2009). Is universal prevention against youths' substance misuse really universal? Gender-specific effects in the EU-Dap school-based prevention trial. Journal of Epidemiology \& Community Health, 63(9), 722-728.

Warren, F. (2016). 'What Works' in Drug Education and Prevention? 7 December 2016. [Social Research series] 


\title{
PSYCHOLOGICAL LITERACY OF TEACHERS IN THE ROLE OF YOUTH MENTORS
}

\author{
Marianna Berinšterová, Katarína Fuchsová, \& Monika Magdová \\ Institute of Pedagogy and Psychology, Faculty of Humanities and Natural Sciences \\ University of Presov (Slovakia)
}

\begin{abstract}
The concept of psychological literacy is important in education, but its use is lacking in Slovakia as well as mentoring relationships in school environment. Psychological literacy is defined as the ability to understand basic psychological principles and the ability to use these principles in practice. Mentoring of youth is understood as the existence of a nonparental significant adult who provides social support. Knowledge and skills needed for this role as part of psychological literacy should be included in the curriculum of education students.

The aim of the contribution was to explore the understanding of psychological literacy of teachers in Slovakia in terms of knowledge, competencies and attitudes, specifically for the role of the natural mentor of pupils.

Three focus groups were conducted with 18, 20 and 24 participants (82\% of women; Mage=41,4; 68\% of teachers have 15-20 years of practice) and two moderators in three regions of Slovakia. Participants were primary and secondary school teachers, selected based on willingness to participate in the research.

Six theoretical areas of psychological literacy were identified: (1) respecting psychological patterns in pedagogical practice; (2) counselling interview (3) competencies for cooperation in the school context; (4) commitment to the teaching profession; (5) life skills (critical thinking; self-regulation; social competence); (6) ability to handle stressful situations. These six theoretical areas will be the base for the Q-methodology creation and further for the preparation of theoretical model of psychological literacy of teachers.

Research will also contribute to the innovation of the psychological training of future teachers at universities in Slovakia. The understanding of the concept of mentoring in the school context and the role of a teacher as a mentor is discussed.
\end{abstract}

Keywords: Psychological literacy, teachers, education, mentoring.

\section{Introduction and objectives}

An interest in exploring psychological literacy has increased in recent years. The concept of psychological literacy is defined as "the general capacity to adaptively and intentionally apply psychology to meet personal, professional and societal needs" (Cranney, Botwood, Morris, 2012) and challenges (Roberts, Heritage, Gasson, 2015). More detailed conceptualisation McGovern et al. (2010) offers following elements:

(1) Understanding the basic concepts and principles of psychology;

(2) Thinking critically;

(3) Having problem-solving skills;

(4) Understanding scientific research practices;

(5) Communicating well in different contexts;

(5) Applying psychological principles to personal, social, or organizational problems;

(5) Acting ethically;

(6) Having cultural competence and respecting diversity;

(7) Having self and other awareness and understanding.

Research of psychological literacy is most often related to the study of psychological issues. British Psychological Society (BPS) states the following demands on the study of psychology: " $a$ coherent set of knowledge, skills and values that underpin students' psychological literacy and which enable them to apply psychology to real life contexts. (p.26). 
Psychological literacy of teachers is a specific topic in this case, given the multiple impact on cognitive, emotional and social development of children and adolescents. A teacher is not only a person who communicates information in a professional way. A teacher is a significant nonparental adult for many children and youth (Galbo, 1986; Galbo, Demetrulias, 1996). Supportive relationship with a nonparental significant adult is an important developmental asset. The needs of autonomy, competence and relatedness can be fulfilled in this relationship. In Slovakia, formal mentoring programs are rarely implemented and research of this area is minimally conducted.

However, the concept of psychological literacy of teachers is rarely mentioned and examined. In Slovakia, there is a need for innovations in the curricula of educational study programs. Increasing the capacity to be a natural mentor requires respect for functional and interactive psychological literacy (Sokolová, Lemešová, Jursová, Zacharová, 2014). Based on this knowledge, the aim of the research was formulated: to identify components that can form the concept of psychological literacy of teachers. The statements of teachers expressed in focus groups were used for the categorization of these components.

\section{Methods}

\subsection{Research sample}

The sample selection criterion was the geographical location due to the expectation of greater diversity of the absolved study preparation of teachers. For the needs of this research, 3 schools were selected, in pursuance of their willingness to participate in the research. In each school single focus group of teachers who educate pupils in the age of $11-15$ years (2 schools) and 15-19 years (1 school) was created.

Although the typical rule of focus groups is that participant don't know each other, our focus groups were created from colleagues who work together. Some research-based support for this approach can be found (Beckman, Haunschild, \& Phillips, 2004). Group dynamics of well-known participants and shared educational situation could contribute the creativity and expressing opinions, the essential parts of focus group research methods.

\subsection{The procedure of focus groups}

Research method of focus groups was conducted in June-July 2018 as a part of regular teambuilding activities for teachers at the end of the school year. Recommended methodological procedure was used (Švaříček, Šed'ová 2007). The same 2 persons facilitate the discussion in the all three groups. The process had the following phases:

(1) Opening phase. In this phase, the aim was to introduce the topic of the focus groups, research project, the way of data collection and analysis, the persons of moderators of the discussion (researchers) and explain the etical aspects of this research.

(2) Motivational phase. This phase consists of presentation of research results, that claim the multiple positive correlates of natural mentoring relationship of adolescents, while special attention was paid to the teacher in the role of natural mentor. In the end of the motivational part, psychological preparation of teachers was mentioned appealing to their own study experiences and profession needs in terms of the topic of focus groups.

(3) Discussion phase. Three questions were presented to teachers. Questions focused on knowledge, competencies and attitudes of teachers in the role of natural mentors.

- What kind of knowledge a teacher needs in the role of natural mentor?

- Imagine yourself in the role of natural mentor of pupils. Which competencies would you need to improve?

- $\quad$ Try to identify attitudes, that could be helpful for teachers in the role of mentor. Discussion was recorded with the permission of the participants.

(4) Ending phase. In this phase participants were invited to give the feedback to the course of focus group, facilitator approach, the topic and questions. The element of nonformal learning was added in the form of appreciation of the activity of the participants.

\section{Results}

Records of focus groups were transcribed and assessed by 3 researchers individually. Relevant issues in transcripts were labelled. Coding of the answers brings categories that are described below. Number and percentage of occurrence of these answers are listed. 
Table 1. Categories of answers on question 1: "What kind of knowledge a teacher needs in the role of natural mentor?".

\begin{tabular}{clc|c} 
CATEGORIES OF ANSWERS & N & $\%$ \\
1. & How to motivate pupils & 18 & 16.66 \\
\hline 2. & Problem behaviour of children and adolescent & 16 & 14.81 \\
\hline 3. & Behaviour disorders & 12 & 11.11 \\
\hline 4. & Learning disabilities & 12 & 11.11 \\
\hline 5. & How to handle aggression in the school context & 11 & 10.19 \\
\hline 6. & Personality typologies & 9 & 8.33 \\
\hline 7. & Stress situations and coping & 7 & 6.48 \\
\hline 8. & Effective communication & 7 & 6.48 \\
\hline 9. & Learning styles & 6 & 5.55 \\
\hline 10. Developmental specifics of pupils & 4 & 3.70 \\
\hline 11. Emotional intelligence & 4 & 3.70 \\
\hline 12. Gifted children & 2 & 1.85
\end{tabular}

Answers for the first question were coded into 12 categories. Final number of answers was 108. The most occurred categories were "how to motivate children", "problem behaviour of children and adolescents" "behaviour disorders" and "learning disabilities".

Table 2. Categories of answers on question 2: "Imagine yourself in the role of natural mentor of pupils. Which competencies would you need to improve?".

\begin{tabular}{lc|c} 
CATEGORIES OF ANSWERS & N & $\mathbf{\%}$ \\
\hline 13. Ability to talk with pupils about their personal problem & 30 & 72.58 \\
\hline 14. Solving conflicts with pupils & 22 & 48.39 \\
\hline 15. Cooperation with pupils & 18 & 45.16 \\
\hline 16. Relaxation methods & 14 & 38.7 \\
\hline 17. Cooperation with parents & 14 & 38.7 \\
\hline 18. Coping with own negative emotions & 14 & 38.7 \\
\hline 19. Motivational interview & 12 & 30.65 \\
\hline 20. Self-control & 8 & 16.13 \\
\hline 21. Asking the right questions & 8 & 24.19 \\
\hline 22. Understanding of nonverbal communication & 9 & 15.51 \\
\hline 23. Cooperation with colleagues & 7 & 11.29 \\
\hline 24. Management of classes with diverse learning and behaviour & & \\
\hline disorders & 6 & 9.67 \\
\hline 25. Critical thinking & 3 & 4.83 \\
\hline 26. Ability to develop creativity of pupils & 3 & 4.83
\end{tabular}

Number of categories found in the answers to the question 2 is 14 . The total number of answers was 159. Teachers most frequently stated that they would need to improve the ability to talk with pupils about their personal problems, the competence to solve conflicts with pupils, and the cooperation with them. 
Table 3. Categories of answers on question 3: "Try to identify attitudes, that could be helpful for teachers in the role of mentor".

\begin{tabular}{lc|c} 
& $\mathbf{N}$ & $\mathbf{\%}$ \\
\hline 27. Interest in the interaction with pupils not just during classes & 53 & 88.33 \\
\hline 28. Interest in learning outcomes of children & 31 & 51.66 \\
\hline 29. Openness toward parents of pupils & 25 & 41.66 \\
\hline 30. Openness to consultation with experts (psychologists, supervisors) & 4 & 6.66 \\
\hline 31. Willingness to learn new things & 3 & 4.54 \\
\hline & &
\end{tabular}

The most frequented answer to the question concerning the attitude helpful for the teacher in the role of mentor was "interest in the interaction with pupils not just during classes". Overall, 5 categories were created from all 116 answers.

\subsection{Summary of categories and model proposal}

In the research, 31 categories of answers were identified. By summarising these categories and studying other researches (Canrinus, Helms-Lorenz, Beijaard, Buitink, Hofman,2012; Cranney, Morris, Botwood, 2015; Mangrulkar, Whitman, Posner, 2001; McGovern, Corey, Cranney, et al., 2010; Sharp, 2014; Rhodes, DuBois, 2008) in the area of mentoring and psychological literacy, components of psychological literacy of teachers in the role of mentor are proposed. These components are considered:

(1) respecting psychological patterns in pedagogical practice and knowledge from psychology

(2) counselling interview

(3) life skills - the ability of a person to behave adaptively in their interaction with the environment (critical thinking; self-regulation; cooperation in the school context; the ability to handle stressful situations)

(4) commitment to the teaching profession;

\section{Discussion and conclusion}

The aim of the contribution was to explore the understanding of psychological literacy of teachers in the role of natural mentors by the focus groups of 62 teachers. 31 categories of psychological literacy in terms of knowledge, competencies and attitudes were found and summarised to 4 components of specific model of psychological literacy. Research results point to the following:

The need of specific understanding of psychological literacy for the teaching profession. Conceptualisation of psychological literacy often highlights the reflection of psychological knowledge and its critical assessment (Coulson, Homewood, 2016). On the other hand, a significant part of the found categories was related to counselling interview, cooperation and own life skills (Mangrulkar, Whitman, Posner, 2001), and forming of professional relationships in the school context. Although relationship is professional, it is still an interpersonal relationship.

Perception of the aspects of natural mentoring relationships. Many teachers perceived themselves as educators rather than natural mentors. The research of mentoring in Slovakia is rare. There is a need for the deeper research of this area and dissemination of the results among teachers and youth workers.

One of the limits of the research was social desirability, especially in focus groups consisting of members that know each other. Furthermore, subjective views of researchers would impact the formation of categories of answers. Another research is needed to confirm the proposed components of psychological literacy of teachers.

The results of this contribution will be used for creation of Q methodology and another research of psychological literacy of teachers, and for innovation of study of education in Slovakia. According to Dunn, Cautin and Gurung (2011), the curricular change is needed in "education of educators", with balance between graduate and undergraduate needs. 


\section{References}

Beckman, C. M., Haunschild, P. R., \& Phillips, D. J. (2004). Friends or strangers? Firm-specific uncertainty, market uncertainty, and network partner selection. Organization science, 15(3), 259-275.

British Psychological Society (BPS) (2012b). The future of A-level psychology. Leicester: BPS. Retrieved 16.1.2019, from: http://www.bps.org.uk/system/files/

Canrinus, E. T., Helms-Lorenz, M., Beijaard, D., Buitink, J., \& Hofman, A. (2012). Self-efficacy, job satisfaction, motivation and commitment: exploring the relationships between indicators of teachers' professional identity. European journal of psychology of education, 27(1), 115-132.

Coulson, D., \& Homewood, J. (2016). Developing psychological literacy: is there a role for reflective practice? Journal of University Teaching \& Learning Practice, 13(2), 5.

Dunn, D. S., Cautin, R. L., \& Gurung, R. A. R. (2011). Curriculum matters: Structure, content, and psychological literacy. In J. Cranney \& D. S. Dunn (Eds.), The psychologically literate citizen: Foundations and global perspectives (pp. 15-26). New York, NY, US: Oxford University Press. Retrieved 25.2.2019 form http://dx.doi.org/10.1093/acprof:oso/9780199794942.003.0017

Cranney J, Botwood L, Morris S. (2012). National Standards for Psychological Literacy and GlobalCitizenship: Outcomes of Undergraduate Psychology. Office for Learning \& Teaching, Government of Australia. Sydney, NSW.

Cranney, J., Morris, S., \& Botwood, L. (2015). Psychological literacy in undergraduate psychology education. In Dunn, D.S. (Ed.). The Oxford handbook of undergraduate psychology education.

Galbo, J. J. (1986). Adolescents' perceptions of significant adults: Implications for the family, the school and youth serving agencies. Children and Youth Services Review, 8(1), 37-51.

Galbo, J. J., \& Demetrulias, D. M. (1996). Recollections of nonparental significant adults during childhood and adolescence. Youth \& Society, 27(4), 403-420.

McGovern TV, Corey L, Cranney J, et al. (2010). Psychologically literate citizens. In: Halpern, D.F., (ed). Undergraduate Education in Psychology: A Blueprint for the Future of the Discipline. Washington, DC: American Psychological Association, 9-27.

Rhodes,J. E., \& DuBois, D. L. (2008).Mentoring relationships and programs for youth. Current Directions in Psychological Science, 17, 254-258.

Roberts, L. D., Heritage, B., \& Gasson, N. (2015). The measurement of psychological literacy: a first approximation. Frontiers in psychology, 6, 105.

Sharp, P. (2014). Nurturing Emotional Literacy: A Practical for Teachers, Parents and those in the Caring Professions. London: Routledge.

Švaříček, R. Šed’ová K. a kol. (2007). Kvalitatívní výskum v pedagogických vědách. Praha: Portál, $384 \mathrm{~s}$. ISBN 978-80-7367-313-0. 


\title{
PARENTAL STRATEGIES AND ADOLESCENTS' PROBLEM BEHAVIOUR IN SCHOOL ENVIRONMENT: THE MEDIATION EFFECT OF RULES INTERNALIZATION
}

\author{
Ondrej Kalina, \& Maria Bacikova \\ Department of Educational Psychology \& Health Psychology, Faculty of Arts, \\ PJ Safarik University in Kosice (Slovakia)
}

\begin{abstract}
Research regarding parent-child processes has demonstrated that rules set up by parents towards their children plays protective role in problem behaviour in school environment. However, recent studies have revealed inconsistent associations among parental strategies which parents used to set up the rules and adolescents' problem behaviour. This study explores two different parenting strategies of rules setting to test the hypothesis that an autonomy-supportive strategy would relate negatively, whereas controlling strategy would relate positively, to adolescents problem behaviour. Moreover, mediation analyse was explored to test whether associations among parenting strategies and adolescents' problem behaviour could be explained by level of internalization of parental rules.

A cross-sectional representative dataset from elementary schools was used $(\mathrm{N}=581, \mathrm{M}=13.5$ years, $\mathrm{SD}$ $=0.59,51.7 \%$ of boys). Problem behaviour of adolescent were measured by 21 items scale (e.g. "Did you take something what did not belongs to you?"). Two types of parental strategies were explored: (1) autonomy - supportive strategy was measured by 7 items scale (e.g. "My father/mother allows me to make decision by my own"); (2) controlling strategy was measured by 8 items scale (e.g."My father/mother reminds me my previous mistakes whet criticizing me"). Internalization of parenting rules was assessed by 22 items questionnaire which consist from four separate scales (1) identification; (2) introjection; (3) external regulation and (4) rebellion. Linear regression models and mediation analyses were used for data analysis in SPSS 21.

As it was predicated the parenting strategies were significantly associated with level of problem behaviour of adolescents. Higher autonomy-supportive strategy by mother and father was related with less problem behaviour ( $\mathrm{B}=-0.124^{*}$ in mothers; $\mathrm{B}=-0.147^{*}$ in fathers). On the other hand, higher controlling strategy by mother and father was related to higher rates of problem behaviour $(\mathrm{B}=0.172 *$ in mothers, $\mathrm{B}=0.201^{*}$ in fathers). The association among autonomy - supportive strategy and problem behaviour was partially mediated by identification $\left(-1.461^{* * *}\right)$ and by introjection $\left(-0.0721^{*}\right)$. The association among controlling strategy and problem behaviour was partially mediated by rebellion $\left(0.0953^{* * *}\right)$.

The results of this study point to importance of parental strategies in adolescence period as autonomy and controlling ways of setting rules may have opposite effect on problem behaviour. Moreover, both strategies impact the processes of rules internalization (identification, introjection and rebellion) which seems to be very important in this context.
\end{abstract}

Keywords: Internalization, problem behaviour, parental strategies, psychological control.

\section{Introduction}

Problem behaviour of Slovak school aged children has increased in recent years. The international study on teaching and learning by OECD (Jensen et al. 2012) has given alarming results in terms of school pupils' behavior. Teachers in participating countries of the study has reported late arrivals (39.4\%; in Slovakia: 13\%) and absence of students (45.8\%; in Slovakia: 39.8\%). Given the absence and late arrival of pupils, the situation of Slovak teachers compared to the international average appears to be more flattering. However, other aspects of the discipline are to the detriment of Slovak school pupils. Compared to the international average, Slovak school teachers are more often confronted with distracting pupils during their lessons, their lies and cheating, vulgarism or the destruction of school furniture. 
There is no doubt about the increase in non-discipline in schools, but it should be borne in mind that the issue of discipline goes beyond the institution of the school, because the level of discipline reflects the social situation. Discipline at school is to some extent a mirrored reflection of discipline in society and family in particular. School and society in this sense represent continuous vessels (Bendl, 2001). But what is the society that produces an increasing number of problem behavior pupils other than "previous" society? On such question, there can be multiple answers, and one of them is the nature of the relationship, which is no longer based on the asymmetry of the "superior and subordinate" relationship compared to traditional society and family as well. The relationship between a child and adults (parents) is based on partnership in a democratic structure and the child's obedience is then more difficult to claim.

The most frequents parental strategy is rules implementation in various context of child behaviour. Several different personality theories converge to suggestion that internalization of parental rules is an important determinant of the likelihood that parental rules will result in desired outcomes (Deci \& Ryan, 2000; Grusec \& Goodnow, 1994; Kochanska, 2002). Internalization is generally described as individual, active process through which external regulations is transformed into personally held values and through which they may integrate regulations into the self (Deci, Eghrari, Patrick, \& Leone, 1994). The Self-determination theory distinct three levels of internalized form of behavioural: (1) external regulation, behaviour is characterized only to comply with an external demands in order to get a reward or to avoid a punishment therefore individuals may perceive pressure and control to comply with an externally imposed rules (e.g. children may follow their parents' guidelines regarding problem behaviour to avoid parental punishment); (2) introjected regulation, in often characterized as motivation from an internalized pressuring voice as the source of motivation for behaviour is guilt, shame or worry (Assor, Vansteenkiste, \& Kaplan, 2009). For instance, adolescents may follow parental guidelines for problem behaviour domains to avoid feeling guilty about their behaviour; (3) identified regulation, in which person has personally identified with the importance of a behaviour and accepted it as a regulation of her own. E.g. adolescents follow parental rules regarding problem behaviour because they understand why their parents ask them to do so and because they view their behaviour as conducive to their self-endorsed goals.

Self-determination theory also propose that a lack of internalization and the behavioural problems associated with such a lack may increase controlling and decrease autonomy-supportive parenting (Grolnick, 2003). Parents usually prohibit their child from numerous types of problems behaviours by threatening to punish the child or by withdrawing privileges. Moreover, parents also use emotional type of pressure as they appeal to the child's feelings of guilt and shame or by limiting their love and acceptance when the child does not follow the rules. These types of covert control are consistent with the concept of psychological control (Assor, Roth, \& Deci, 2004; Soenens, Vansteenkiste, Luyten, Duriez, \& Goossens, 2005). In contrast to controlling styles of prohibition, parents may use an autonomysupportive style of prohibition, as they take the adolescent's frame of reference and provide a relevant and clear rationale for prohibition while also minimizing pressure (Deci et al., 1994).

We expected that adolescents in this study would be more likely to internalize their parents' rules regarding problem behaviour when they perceive their parents using an autonomy-supportive style. In other words, an autonomy-supportive style is facilitative of the process of internalization and thus would be associated with identified regulation. In contrast, we expect that adolescents in this study would be less likely to internalize parental rules when parents will use a controlling style. A controlling style of prohibition would thus foster more controlled and less internalized reasons for adopting parents' guidelines regarding problem behaviour.

Therefore the aim of this study is to explore the association among two types of parental strategies and problem behaviour of adolescent in schools. We also expect that rules internalization will mediate this relationship.

\section{Methods}

\subsection{Sample}

Participants were recruited from the national project VEGA focused on parental processes in context of health risky behaviour of adolescents. This analysis is based on data from 581 (51.1\% males; mean age $=13.5$; $\mathrm{SD}=0.65$ ) students from 12 basic school from Slovakia.

\subsection{Measures}

Problem behaviour of adolescent as outcome variable was measured by 21 items scale (e.g. "Did you take something what did not belongs to you?") as each respondent indicate the answer on first 18 items on five point scale and last three items on four point scale. The score ranges from 21 to 106 point as 
the higher score indicates higher level of problem behaviour. Cronbach's alpha for problem behaviour was 0.840 .

Two types of parental strategies were explored:

(1) autonomy-supportive strategy was measured by 7 items scale (e.g. "My father/mother allows me to make decision by my own") using the Autonomy Support Scale of the Perceptions of Parents Scale (Grolnick, Ryan, \& Deci, 1991). The score ranges from 7 to 35 points as the higher score indicates higher level of autonomy-supportive strategy. The sum scores were computed separately for mother and father. Cronbach's alpha for autonomy-supportive strategy was 0.527 .

(2) controlling strategy was measured by 8 items scale (e.g."My father/mother reminds me my previous mistakes whet criticizing me") using Psychological Control Scale-Youth Self-Report (Barber, 1996). The score ranges from 8 to 40 points as the higher score indicates higher level of controlling strategy. The sum scores were computed separately for mother and father. Cronbach's alpha for controlling strategy was 0.747 .

Internalization of parenting rules was assessed by Internalization of parental rules in the moral domain (Soenens, Vansteenkiste, 2009) 22 questionnaire which consist from four separate scales for (1) identification; (2) introjection; (3) external regulation and (4) rebellion. The sum scores were computed separately for mother and father. Cronbach's alphas for these scales ranges from 0.728 to 0.866 .

\subsection{Statistical analyses}

Firstly, we selected only those respondents which completely answered the questionnaire regarding problem behaviour and other explored variables. After that using regression models we explored whether types of parental strategies were associated with problem behaviour of their children and similarly we explored whether type of internalization of rules will mediate this relationship.

\section{Results}

As it was predicated the parenting strategies were significantly associated with level of problem behaviour of adolescents. Higher autonomy-supportive strategy by mother and father was related with less problem behaviour ( $\mathrm{B}=-0.124^{*}$ in mothers; $\mathrm{B}=-0.147^{*}$ in fathers). On the other hand, higher controlling strategy by mother and father was related to higher rates of problem behaviour $(\mathrm{B}=0.172 *$ in mothers, $\mathrm{B}=0.201^{*}$ in fathers). The association among autonomy - supportive strategy and problem behaviour was partially mediated by identification $\left(-1.461^{* * *}\right)$ and by introjection $\left(-0.0721^{*}\right)$. The association among controlling strategy and problem behaviour was partially mediated by rebellion $\left(0.0953^{* * *}\right)$.

\section{Discussion and conclusion}

The results of this study point to importance of parental strategies in adolescence period as autonomy and controlling ways of setting rules may have opposite effect on problem behaviour. Moreover, both strategies impact the processes of rules internalization (identification, introjection and rebellion) which seems to be very important in this context.

\section{Acknowledgements}

This work was supported by the Slovak Research and Development Agency under the contract No. APVV-15-0662 and0253-11 and the Slovak Academy of Sciences under Contract No. VEGA 1/0623/17.

\section{References}

Assor A, Roth G, Deci EL. (2004). The emotional costs of parents' conditional regard: A selfdetermination theory analysis. Journal of Personality, 72, 47-88.

Assor A, Vansteenkiste M, Kaplan A. (2009). Approach and avoidance introjected motivations: Approach is better than avoidance, but identified motivation is better than both. Journal of Educational Psychology, 101, 482-497.

Bendl, S. (2005). Ukaznena trida. Prague: Triton. 
Deci, E. L., \& Ryan, R. M. (2000). The "what" and "why" of goal pursuits: Human needs and the selfdetermination of behavior. Psychological Inquiry, 11 ,227-268.

Deci EL, Eghrari H, Patrick BC, Leone DR. (1994). Facilitating internalization: The self-determination theory perspective. Journal of Personality, 62, 119-142.

Grolnick WS. (2003). The psychology of parental control: How well-meant parenting backfires. Mahwah, NJ: Erlbaum.

Grolnick WS, Ryan RM, Deci E. (1991). Inner resources for school achievement: Motivational mediators of children's perceptions of their parents. Journal of Educational Psychology, 83, 508-517.

Grusec JE, Goodnow JJ. (1994). Impact of parental discipline methods on the child's internalization of values: A reconceptualization of current points of view. Developmental Psychology, 30, 4-19.

Jensen B, et al. (2012). The Experience of New Teachers: Results from TALIS 2008, OECD Publishing. http://dx.doi.org/10.1787/9789264120952-en

Kochanska, G. (2002). Committed compliance, moral self, and internalization: A mediational model. Developmental Psychology, 38, 339-351.

Barber, BK. (1996). Parental psychological control: Revisiting a neglected construct. Child Development, 67, 3296-3319.

Soenens B, Vansteenkiste, M, Luyten P, Duriez B, Goossens, L. (2005). Maladaptive perfectionistic self-representations: The mediational link between psychological control and adjustment. Personality and Individual Differences, 38, 487-498. 


\title{
DYNAMICS OF SOCIO-CULTURAL AND LINGUISTIC IDENTITY IN THE PROCESS OF SOCIALIZATION IN A MULTICULTURAL SOCIETY
}

\author{
Tatiana Martsinkovskaya ${ }^{1}$, Vasilisa Orestova ${ }^{2}$ Ekaterina Kiseleva $^{2}$, Maria Soboleva ${ }^{2}$, \\ \& Evgenia Kriger ${ }^{2}$ \\ ${ }^{1}$ Institute of psychology, Russian State university for the humanities; Psychological Institute RAE (Russia) \\ ${ }^{2}$ Russian State university for the humanities (Russia)
}

\begin{abstract}
The relevance of the problem of socialization in multicultural space is extremely high. Its significance is connected with the processes of globalization and increasing migration. In this situation, the sociocultural and, especially, linguistic identity can be analyzed as a bonding and bridging social capital that helps or complicates socialization depending on the specifics of the social situation.

These assumptions were checked in the empirical research of the socio-cultural and linguistic identity of the three groups of ethnic Germans: living in Russia, in the CIS and those, who moved to Germany.

The obtained data showed the bilingualism of the majority of respondents. At the same time, respondents from Russia and Germany consider both Russian and German languages as native, while respondents from the CIS no longer regard German as their mother language. For them ethnic, not linguistic identity is the most essential for socialization in a multicultural society.

For Germans living in Russia, on the contrary, both languages help in communication and in professional activity. For the Germans who now live in Germany, the Russian language helps in understanding their sociocultural specifics. The future is associated with the German language.

Our respondents answer that the main group of socialization for them is the family. This was especially marked in the answers of respondents living in Russia and Germany. So we can state that in a complex multicultural environment the family for them is not only a group of socialization, but also an object of identity, partly playing the role of the psychological defense and support in a changing world.

The obtained material also showed the tendency towards individualization. For the respondents from Russia and Germany the main identity groups, besides family, are professional groups and groups with common interests.

Thus, it can be concluded that linguistic identity plays the role of both - bonding and bridging social capital. In positive socialization, sociocultural identity plays the role of a bridging rather than a connecting capital. Mixed linguistic identity is predominantly a positive moment, increasing socialization in a multicultural environment.
\end{abstract}

Keywords: Socialization, transitivity, identity, multicultural world.

\section{Introduction}

Relevance and significance of socialization in the modern changing and multicultural space is connected both with social transitivity and expansion of interpersonal and intergroup communication. (Bauman, 2008, Belinskaya, 2005, Belinskaya, Dubovskaya, 2009). Globalization and mass migrations of people spread out socialization borders and require knowledge of culture, the language of the new social environment and the positive attitude towards norms and standards of native and foreign culture. (Habermas, 2003).

The fluidity and variability of values and norms is, in many ways, the cause of the growing anxiety and tension of people who find it increasingly difficult to adapt to the ever-changing "rules of the game". Therefore, we can talk about the principle continuity of the process of socialization. The cardinal and contradictory nature of the changes has led to the uncertainty in the social expectations of people and ambivalence in their attitude to value orientations and norms. This resulted in negative changes in the content of the social identity of the older generation, and, as a consequence, the complexity in transmission of norms and values to the younger generation (Andreeva, 2012, Martsinkovskaya, 2015).

The expansion of migration processes and the associated large-scale transformations of the social and cultural context raise the problem of socialization not only for children, but also for adults. Therefore, the concept of resocialization is currently being modernized. Language socialization is the focus of many 
investigations, but the transmission of norms and standards in different social groups is analyzed very rarely, though in big and small cities the process of such transmission is quite different.

It is no coincidence that in recent years the question of constructing a linguistic identity, primarily the linguistic identity of small nations, has become one of the priority activities of the European community. No less attention is paid to the question of combining of the state and minority languages in the content of their linguistic identity (Andreeva, 2012, Martsinkovskaya, 2013). In conditions of a transitive society, inculturation, acceptance and appropriation of culture are one of the important factors determining the success of socialization in new conditions (Martsinkovskaya, 2015). Therefore, it is culture that can become the basis for the formation of the socio-cultural identity of people in a new situation. Unlike culture, the role of language in the process of formation of a sociocultural identity is ambiguous (Dontsov, Stefanenko, Utalieva, 1997). Apparently, we can talk about the complex relationship of language, culture and identity (sociocultural, ethnic, personal, group, etc.).

It seems that for the analysis of sociocultural and, especially, linguistic identity it may be productive to use concepts of bonding and bridging social capital (Portes, 1998, Putnam, 2002, Bourdieu, 2002, Coleman, 2001), which helps or complicates socialization depending on the specifics of the social situation.

\section{The aim of the study}

These assumptions were checked in the empirical research of the socio-cultural and linguistic identity of the three groups of ethnic Germans: living in Russia, in the CIS and those, who moved to Germany.

The purpose of this study was to examine the role of native/native languages and cultures in the process of socialization in multicultural space.

We assumed that in different social-cultural conditions the role of language in the process of socialization will change significantly. The main parameter from our point of view is not the knowledge of the language, but the emotional attitude to it and its connection with the general culture (national and world).

\section{Participants and methods.}

Research was conducted on base:

- Association "Russian Germans", 125 respondents aged from 20 till 30 years;

- Association "Russian Germans" in CIS, 97 respondents aged from 21 till 30 years;

years;

- Association "Russian Germans" in Frankfurt and Berlin, 83 respondents aged from 19 till 30 the study.

The study was conducted in 2016-2017. All the participants gave their consent to participate in

\section{Methods}

Technique "Socialization". Martsinkovskaya, Khuzeeva (Identity and socialization, 2015), which estimate the level of socialization and emotional comfort;

The questionnaire "Attitude to language". Martsinkovskaya, Khuzeeva; (Identity and socialization, 2015);

The questionnaire "Structure of identity". Martsinkovskaya, Khuzeeva, (Identity and socialization, 2015).

\section{Results}

The obtained data showed the bilingualism of the majority of respondents. Majority of respondents answer, that native are both languages - Russian and German. They also stress their positive attitude to both languages. Respondents from Russia and Germany consider both Russian and German languages as native, while respondents from the CIS no longer regard German as their mother language. They live in countries, were new, native for these countries language is a mother language (mainly Kazakh language). That's why even Russian language is not the main for them. For them ethnic, not linguistic identity is the most essential for socialization in a multicultural society. So different social situation lead to different attitude not for language itself, but for its role in future life (figure 1). 
Figure 1. Do you think that your future is connected with native language and culture.

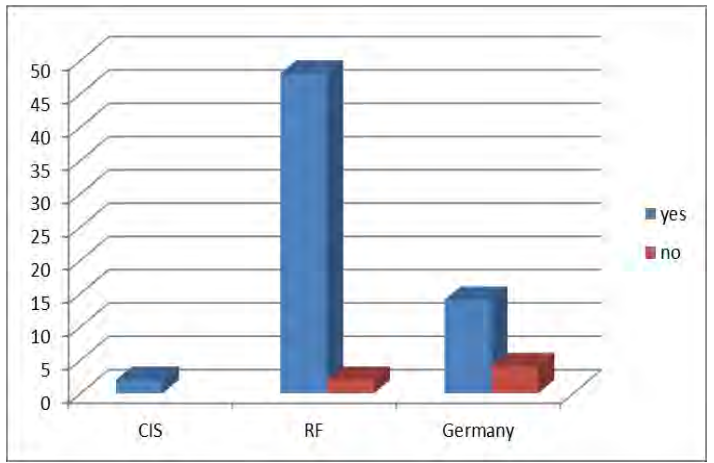

The obtained data also show that for Germans living in Russia and in Germany, languages help to realize their national and socio-cultural identity. For respondents from CIS their national identity is not connected with languages. But Russian and German helps them to communicate with elder relatives. In Russia and Germany both languages help in communication and in professional activity. Although for the Germans who now live in Germany, the Russian language helps in communications, the professional future is associated mainly with the German language (figure 2).

Figure 2. What for do you need your native language.

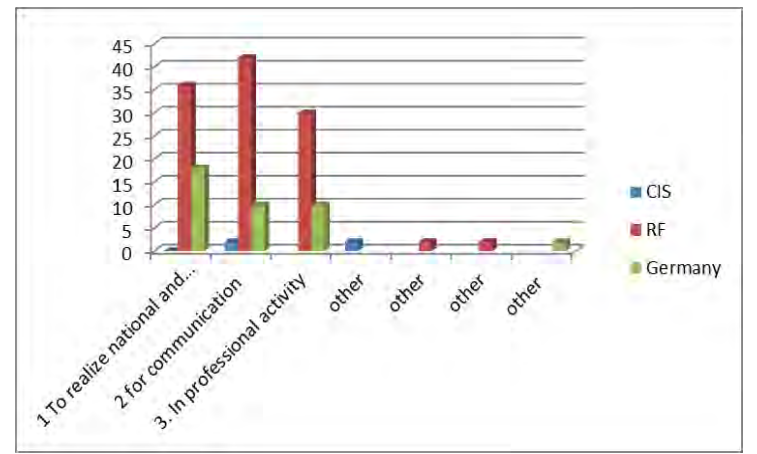

The analysis of the process of socialization showed that for the majority of our respondents from all countries the main group of socialization is the family. As it can be expected, the family is also the leading identity group (figure 3).

Figure 3. The structure of social-cultural identity.

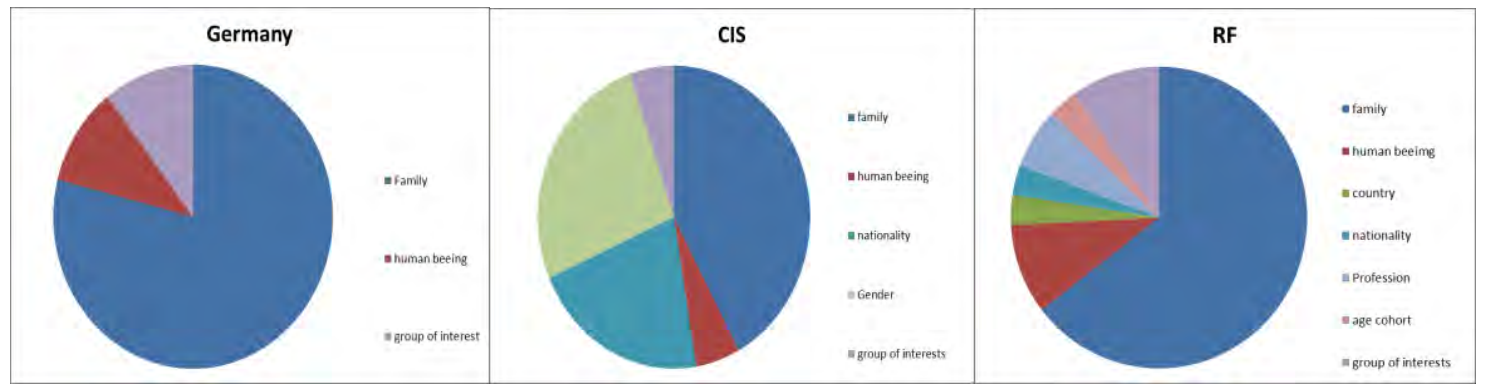

The role of the family was especially marked in the answers of respondents living in Russia and Germany. So we can state that in a complex multicultural environment the family for them is not only a group of socialization, but also an object of identity, partly playing the role of the psychological defense and support in a changing world. For the respondents from CIS the important identity group is nationality, which corresponds with their linguistic identity.

The obtained material also showed the tendency towards individualization. For the respondents from Russia and Germany the main identity groups, besides family, are professional groups and groups with common interests.

It corresponds with the data, obtained in answers to the questions about structure of identity. Personal identity is very important for all respondents, especially from Russia and Germany (figure 4). 
Perhaps, the collectivism in mentality of main CIS countries decreases this tendency in answers of respondents from CIS. A little bit lower data in Germany may be connected with adaptation in new social environment.

Figure 4. Personal identity.

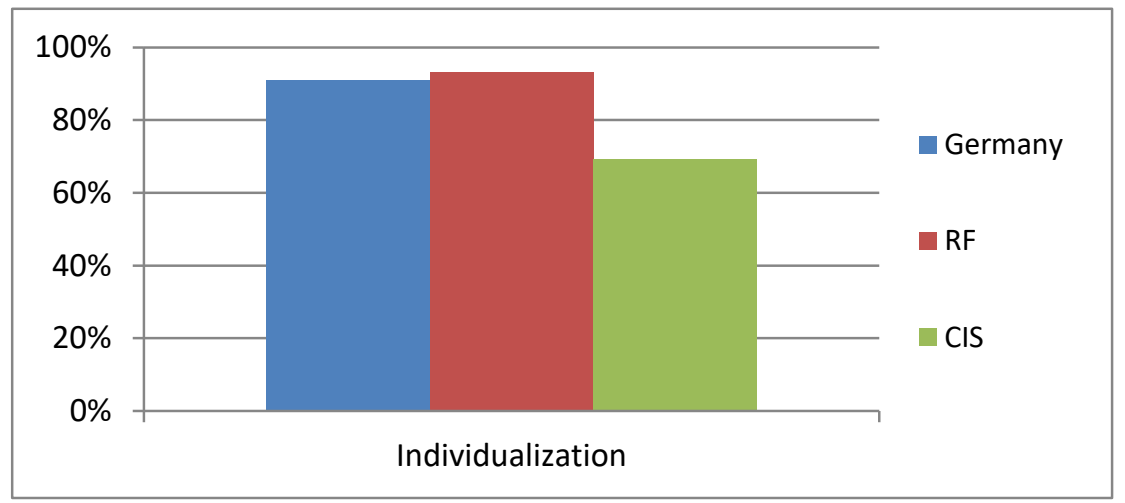

This material is proved in comparative analysis of contents of whole identity (Figure 5).

Figure 5. Content of identity in different groups.

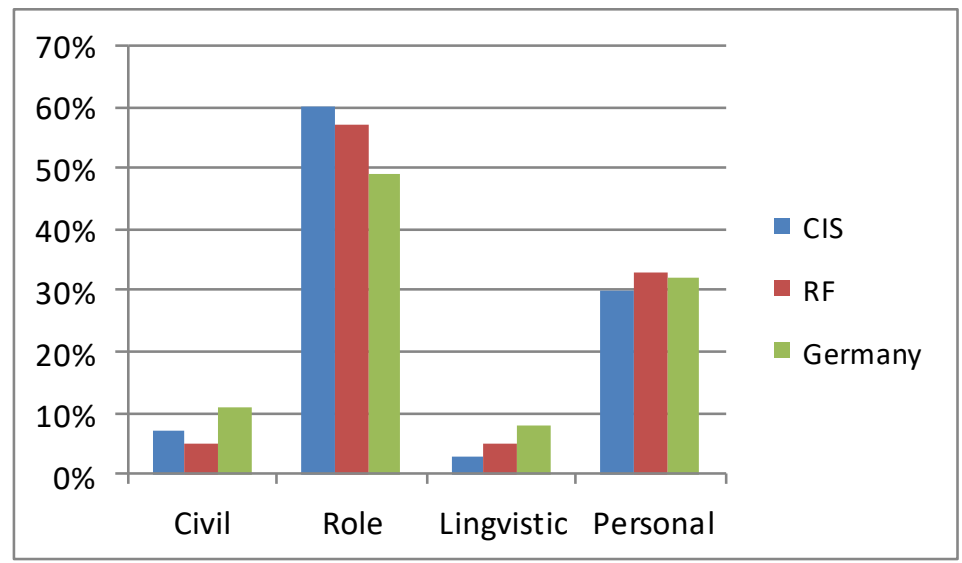

As we can see the linguistic and civil identity don't mean too much for respondents from all countries. But it is important to mark that not personal, as they say, but role identity plays the leading role, especially in CIS.

\section{Discussion}

The analysis of the role of socio-cultural and linguistic identity shows that they can be viewed and function both as bonding and bridging social capital. In the context of a mono cultural environment, it is the sociocultural identity, that is, the identification of oneself with a given culture, with a specific social environment, that helps to establish trustworthy contacts. And we can see that in some aspects, such variant is arising in CIS, where "Russian Germans" completely identified themselves with main culture and social environment. That's why we can say that their identity implies the mutual acceptance of a person and a group. Such mutual acceptance is also connected with the development of positive social emotions to norms and standards of behavior, ways of organizing interaction, constructing new contacts. Thus, in this situation, the sociocultural identity functions as a bonding social capital, especially when joint activities are needed for achieving an important goal or for overcoming obstacles.

In a situation of transitivity, which occur during formation CIS after SSSR it's the social-cultural identity which performs as bonding social capital, that helped "Russian Germans" to maintain their national integrity and, for elder generation, to maintain the continuity of individual stages of life. That's why it is national (ethnic) identity is more important for them in comparison with linguistic.

However, with significant transformations, this form of social capital can become a source of social isolation, isolation of "mother" culture from other social and cultural groups. It can also perform as either stigmatized or aggressive positions towards others. Happily, there is no stigmatized or aggressive 
phenomenology in answers of all our respondents. But may be the leading position of family and role identity is the socialization phenomenology is the mark of negative consequences of such connection of the social-cultural identity with the bonding social capital.

In this respect, linguistic identity is much more flexible. It presupposes, first of all, the possibility of adequate communication between members of one group, linguistic or social. The presence of semantic dominants and slang words, adverbs which also present in the language and entering into the linguistic identity, increases the confidence of contacts between members of the group and optimizes their interaction. That's why the position of "Russian Germans" in Russia and Germany, for whom the linguistic identity is very important, seems more productive.

It is due to the fact, that the functioning of identity in the context of bridging social capital becomes more optimal for intra-group and inter-group communications and interactions in the modern world. Constant changes in group and social structures presuppose the flexibility of both sociocultural and linguistic identity, especially when people change the place of residence and / or constant contacts with people speaking another language. The mainly positive socialization of majority of people, who moved from Russia to Germany, may be connected with the fact that socialization in the framework of bridging social capital is not connected with refuse or forgotten of the native language or culture, as it is implicit in the classical concepts of identity. It often was state before that there are only two variants of socialization in multicultural society - identification with one group, culture, language or marginalization of a person.

Our material show that the study of sociocultural and linguistic identity within the framework of bridging social capital can explain the positive dynamic of socialization in multicultural society (Germany, Moscow) because our respondents have a good knowledge of new cultural standards and language, and the ability to flexibly use the available knowledge in constructing new contacts and cooperation with people belonging to a different culture.

\section{Conclusions}

Thus, it can be concluded that linguistic identity plays the role of both - bonding and bridging social capital in different social circumstances.

In positive socialization, sociocultural identity plays the role of a bridging rather than a connecting capital.

Mixed linguistic identity is predominantly a positive moment, increasing socialization in a multicultural environment.

Ethnic and linguistic aspects of culture can act as intolerant parameters that obstruct the development of sociocultural identity in the multicultural space.

\section{References}

Andreeva, G.M. (2012). Presentation of identity in the context of the interaction. Psychological studies. Vol. 5, 26. 1. URL: http://psystudy.ru

Bauman, Z. (2008). Liquid modernity. Saint-Peterburg: Piter.

Belinskaya, E.P. (2005). People in a changing world: socio-psychological perspective. M.: Prometheus.

Belinskaya, E.P., Dubovskaya E.M. (2009). Variability and constancy as factors of socialization. Psychological studies. V,ol. 5, 7. 5. URL: http://psystudy.ru

Bourdieu, P. (2002). Forms of capital. Economic sociology. Vol. 3, 5, 60-74

Dontsov, A, Stefanenko T, Utalieva Z. (1997) Language as a factor of ethnic identity. Questions of psychology. 4.75-86.

Coleman, G. (2001). The social and human capital. Social science and modernity. 3. 121-139

Martsinkovskaya, T. (Eds.). (2015) Identity and socialization in contemporary world M.: MPGU.

Martsinkovskaya, T. (2013). Social space: theoretical and empirical analysis. Psychological studies. Vol. 6. 30. 12. URL: http://psystudy.ru

Martsinkovskaya ,T. (2015). The problem of socialization in the historical-genetic paradigm. M.: Smisl.

Habermas, Yu. (2003). The philosophical discourse of modernity. M.: Whole world.

Harre, R. (1995) Metaphysics and methodology: some recommendations for psycho-social studies. Social psychology: self-reflection of marginality. M.: UNION RAN.

Portes, A. (1998) Social capital: Its origins and applications in modern sociology. Annual Review of Sociology. Vol. 24: 1-24

Putnam, R. D. (2002) Democracies in Flux: The Evolution of Social Capital in Contemporary Society. New York: Oxford University Press 


\title{
VIOLENCE AGAINST TEACHERS IN THE SCHOOL ENVIRONMENT: AN EXPLORATIVE STUDY
}

\author{
Isabella Corradini, \& Assunta Marano \\ Themis Research Centre (Italy)
}

\begin{abstract}
Violence in school against teachers is a current problem characterized by complex dynamics (Espelage et al., 2013; Mc Mahon et al., 2014), and can take many forms, from verbal assaults to psychological and physical attacks.

Our study has explored the teachers' perception and their experiences with respect to this phenomenon. The tool used for the study is composed by a questionnaire (QUIVAT, Questionnaire investigating Violence Against Teacher, Corradini and Marano, 2018) supplemented by focus group discussions to better analyze teachers' safety in the school environment, the characteristics of their specific workplace, their proposals about prevention measures.

Four primary and four upper secondary schools in a major European city (included 54 teachers: 24 of them from primary and 30 from secondary schools) were involved in the study.

We conducted data collection and integrated analysis (ATLAS.ti; IBM SPSS) by reducing the data into themes through a process of coding and condensing the codes in seven categories. The outcomes of this exploratory study show that $63.4 \%$ of teachers, at all school levels, has experienced some forms of school-related violence, and the verbal type is the one they are most exposed to. Regression analysis indicates that the causes of the problem are mainly attributed to situational and social/environmental factors. We discuss these results considering also the self-reported health implications by those teachers exposed to the violence and the importance of prevention measures.
\end{abstract}

Keywords: Violence, school, stress, victimization, prevention.

\section{Introduction}

School violence is a current and relevant topic, even if in the last years research has strongly focused on bullying at school among students. Violence against teachers is characterized by varied dynamics involving all school personnel (Espelage et al., 2013; Mc Mahon et al., 2014) and including bullying, intimidation, verbal assault, property damage, etc. (Espelage and Horne, 2008). Hence, violence against teachers can take many forms of aggression, from relational to physical ones (Crick, 1996). One of the most relevant theory used to analyse violence in schools is the social-ecological theory (Bronfenbrenner, $1977 ; 1979 ; 1994)$, that looks at behavior within the system of the interactions between a child, immediate environment (family, school, and peers) and larger social environment (community, society, culture), as well as interactions among different levels of the environment.

According to the literature concerning the impacts of violence on teachers who are victims of school violence (e.g. Daniels, Bradley, \& Hays, 2007; Dzuka and Dalbert, 2007) the consequences include individual and psychological aspects, such as depression, anxiety and somatic symptoms; teachers' performance and their life satisfaction can also be affected.

Given that school violence is a public health problem (Centers for Disease Control and Prevention, 2016), studies are also focusing on risks factors for assaults against teachers (Gerberich et al., 2014). Moreover, considering the complexity of the issue, prevention should be based on a broad framework (Espelage et al., 2013) referring to the social-ecological theory, able to include activities assessment and teachers' training. 


\section{Design and methods}

This pilot study aims to analyze teachers' perception and their experiences on the phenomenon of the violence against them in the school environment. The study has been conducted on 54 teachers ( 24 from primary and 30 from upper secondary schools in Rome, Italy).

Each participant completed the QUIVAT, Questionnaire investigating Violence Against Teacher (Corradini and Marano, 2018), a self-report measures of the scholastic relationship, composed by 24 multiple-choice questions. We developed this instrument on the basis of the social-ecological theory (Bronfenbrenner, 1977), as a theoretical and empirical derived questionnaire from a previous work conducted with teachers to select the most significant items.

After the questionnaire administration, part of the sample participated in focus group discussions to better analyze teachers' safety in the school environment, the characteristics of their specific workplace, and their proposals about prevention measures.

For the analysis the sample was stratified with respect to the following variables: age (Percentage/Range 25-34: 20\%; 35-44: 32\%; 45-54: 36\%; 55-64: 12\%); teaching seniority (Percentage/Range 1-10: 23\%; 11-21: 35\%; 21-30: 23\%; more than 30: 21\%) and gender $(\mathrm{M}=68 \% ; \mathrm{M}=32 \%)$. Moreover, the sample was balanced in terms of employment status and level of education.

\section{Analysis and results}

Analysis were conducted using quantitative statistical method to identify violence factors against teachers and their possible correlations and consequences.

Descriptive analysis (frequencies, means, and standard deviation) was conducted for demographic and teacher's career variables. Comparisons between continuous distributions were carried out by means of the analysis of variance (ANOVA). The Pearson correlation coefficient and stepwise regression analysis was also used. All these statistical analyses were performed using the SPSS software package (IBM SPSS statistical software version 23), Chicago, Illinois, USA. We conducted data collection and integrated analysis (ATLAS.ti 7 Scientific Software) reducing the data into themes through a process of coding and condensing the codes in seven categories as following:

1. Awareness of the type of violence;

2. Reporting violence facts to the authority;

3. Main causes determining violence;

4. Protective and risk factors for teachers;

5. Consequences;

6. Measures to prevent and contrast the phenomenon;

7. School policies.

We now describe the main outcomes of our study.

Code 1. Awareness of the type of violence

Referring to the QUIVAT questions, teachers' responses are polarized only on two typologies: physical and verbal assaults. In our questionnaire the term "physical assault" refers to both intentional actions with physical contact and actions not directed toward teachers, such as the launch of objects in the classroom that however physically hits them. With the term "verbal assault" we refer to a hostile communication, e.g. insulting, shouting, intimidating. In our study, 63.4\% of teachers has experienced some form of school-related violence: more than one third declares that the verbal assault is the one they are most exposed to, while $2 \%$ has suffered from physical assaults. Moreover, $18 \%$ complains a disrespectful communication, not in person but on the Internet, at the limit of defamation: they are mocked and offended by students or their parents in several online chats.

Code 2. Reporting violence facts to the authority

Different answers are codified in this category. 39\% of teachers declares they activated student disciplinary proceedings; $2 \%$ advised police and judicial systems; $48 \%$ contacted students' parents. Among intervention strategies, especially when the perpetrator is a student, teachers can decide to involve student's parents also to prevent future violence (Anderman et al., 2018).

Code 3. Main causes determining violence

The respondents report multiple causes of violence in schools. In particular, a $42 \%$ of them stresses the role of school situational factors (e.g. the level of school organization, lack of clarity in parent engagement, lack of managerial support), and a 56\% social/environmental factors (e.g. economic system, political system, mass media). Teachers are aware that an integration of these factors is needed to explain the phenomenon of violence. 
Code 4. Protective and risk factors for teachers

Given that risk factors can be also considered as protective factors depending on the mechanisms activated by the interaction between individuals and environment (Rutter, 1979), factors in this category are codified together. Class cohesion and student participation are considered the most relevant protecting or risk factors (contextual factors) by 38\% of teachers; teachers' competences (cultural factors) are considered important by $21 \%$ of the sample, and personality (individual factors) by $12 \%$.

Code 5. Consequences

Violence can have an impact both on organizational aspects and on teachers' health.

Many teachers ( $>=30 \%$ ) declare some consequences related to organizational and educational aspects, such as "Lost days of work", "Lost instructional time" and "Student disciplinary proceedings involving school, police, judicial systems, social services, and parents".

Moreover, 36\% of teachers who experienced some form of school-related violence reports emotional distress and the difficulty of finding strategies to manage it.

Code 6. Measures to prevent and contrast the phenomenon

In this category teacher answers are polarized on two typologies: effective classroom management practices (45\%) and promoting academic engagement (34\%). An important aspect concerns teachers' preparation for managing school violence. In fact, $51 \%$ of the sample complains the lack of specific training to manage the problem. Teacher preparation should be an important area to develop for teachers' professional training, given that they lack of sufficient expertise and skill to manage the problem (Espelage et al., 2013).

Code 7. School policies

For $58 \%$ of the teachers, school violence prevention programs are fundamental to manage the problem. Moreover, even though training is considered fundamental by teachers for their preparedness, it has to be part of a systemic approach developed by school institutions.

Figure 1 shows the seven categories represented semantically in network views, pointing out the interrelations and the main outcomes between data transcriptions.

Finally, several stepwise multiple regression analyses were carried out. The results $(\mathrm{p}<.05)$ show an influence of situational and social/environmental factors on teachers' perception.

Figure 1. Atlas.ti 7: A network presentation of the seven categories.

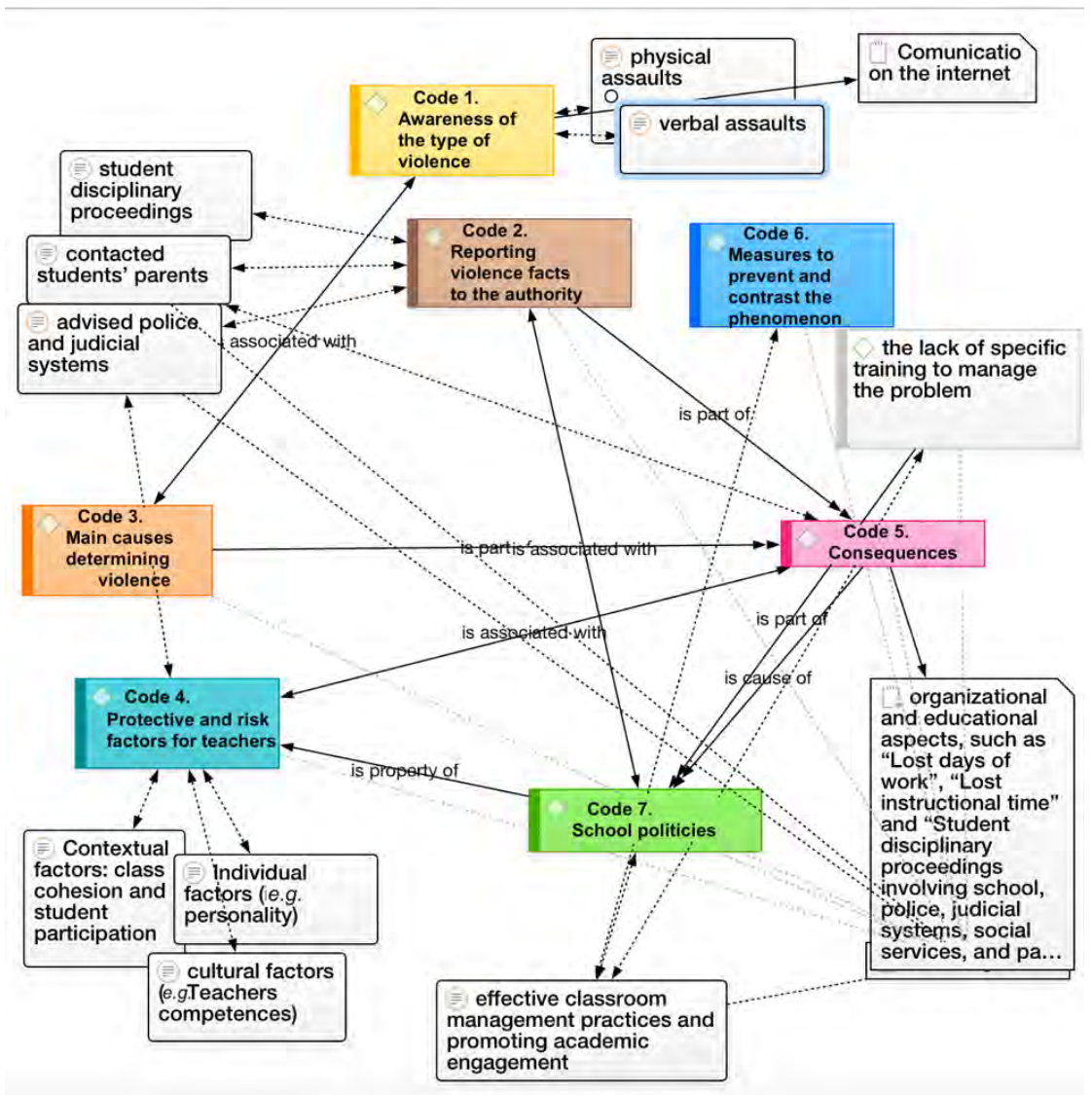




\section{Conclusion and future work}

Given the nature of our research design and its results, it seems reasonable to conclude that school management and socio-ecological school environment have significant predictive value for teachers' violence perception.

The outcomes show that situational and social/environmental factors are particularly important to teachers to understand the phenomenon of violence against them, thus favouring the idea that adoption of appropriate measures can be effective to tackle the problem. For this purpose, a systemic approach including a special training for teachers has to be developed by school institutions. These findings have to be interpreted in the light of the limitation represented by a small size of our sample. Nevertheless, the results are in accordance with a recent metanalysis (Longobardi et al. 2018) and literature review (Espelage et al., 2013, Gerberich et al., 2014; Mc Mahon et al., 2014). Finally, we think that teachers' involvement, given their awareness about the specific workplace context, is fundamental for the effectiveness of prevention programs (Corradini, Marano, Paolinelli, 2017).

We are planning to work with a larger sample and an extended questionnaire to investigate additional factors affecting violence against teachers in the school environment.

\section{References}

Anderman, E.M., Espelage, D.L., Reddy, L.A., McMahon S.D., Martinez, A., Lane, K.L., Reynolds, C., Paul, N. (2018). Teachers' reactions to experiences of violence: an attributional analysis, Social Psychology of Education, 21, issue 3, 621-653.

Bronfenbrenner, U. (1977). Toward an experimental ecology of human development, American Psychologist, 32, 513-531.

Bronfenbrenner, U. (1979). The ecology of human development: Experiments in nature and design. Cambridge, MA, Harvard: University Press.

Bronfenbrenner, U. (1994). Ecological models of human development. In T. Husen \& T. N. Postlethwaite (Eds.), International encyclopedia of education (2nd ed., Vol. 3, 1643-1647). Oxford, UK: Pergamon Press.

Centers for Disease Control and Prevention. (2016). Understanding school violence, [https://www.cdc.gov/violenceprevention/pdf/School_Violence_Fact_Sheet-a.pdf].

Corradini, I., Marano, A. (2018). QUIVAT, Questionnaire investigating Violence Against Teacher, Themis Research Centre, Internal Report.

Corradini, I., Marano, A., Paolinelli F. (2017). On burnout perception among secondary school teachers: a pilot study, in Proceedings of International Psychological Applications Conference and Trends (InPACT) 2017.

Crick, N. R. (1996). The role of overt aggression, relational aggression, and prosocial behavior in the prediction of children's future social adjustment. Child Development, 67(5), 2317-2327.

Daniels, J. A., Bradley, M. C., \& Hays, M. (2007). The impact of school violence on school personnel: Implications for psychologists, Professional Psychology: Research and Practice, 38, 652-659.

Dzuka, J., \& Dalbert, C. (2007). Student violence against teachers: Teachers' well-being and the belief in a just world, European Psychologist, 12, 253-260.

Espelage, D.L., \& Horne, A. (2008). School violence and bullying prevention: From research based explanations to empirically based solutions. In S. Brown \& R. Lent (Eds.), Handbook of counseling psychology (4th ed., pp. 588 -598). Hoboken, NJ: Wiley.

Espelage, D., Anderman, E.M., Brown, V.E., Jones, A., Lane, K.L., McMahon, S.D., Reynolds, C.R. (2013). Understanding and preventing violence directed against teachers: Recommendations for a national research, practice, and policy agenda, American Psychologist, 68(2), 75-87.

Gerberich, S. G., Nachreiner, N. M., Ryan, A. D., Church, T. R., McGovern, P. M., Geisser, M. S., Mongin, S.J., Watt, G.D., Feda, D.M., Stage, S.K., Pinder, E.D. (2014). Case-control study of student-perpetrated physical violence against educators, Annals of Epidemiology, Elsevier, 24, 325-332.

Longobardi, C., Badenes-Ribera, L., Fabris, M.A., Martinez, A., McMahon S.D., (2018), Prevalence of student violence against teachers: A meta-analysis, Psychology of Violence. Advance online publication.

McMahon, S.D., Martinez, A., Espelage, D., Rose, C., Reddy, L.A., Lane, K., Brown, V. (2014). Violence directed against teachers: Results from a national survey. Psychology in the Schools, 51(7), 753-766

Rutter, M. (1979). Protective factors in children's responses to stress and disadvantage. In M. W. Kent \& J. E. Rolf, Primary prevention of psychopathology, Vol. 3: Social competence in children (pp. 49-74). Hanover, NH: University Press of New England. 


\title{
ATTITUDES OF CITIZENS TO THE POLICY OF HOMELESSNESS IN ST. PETERSBURG
}

\author{
Andrey Zaytsev, \& Olga Medyanik \\ Department of Political Psychology, St. Petersburg state University (Russia)
}

\begin{abstract}
The problem of homelessness in Saint-Petersburg is very acute at the moment. According to statistics, there are estimated 12000 homeless people in the streets, with that number growing every year. This problem relates to studies in social and political psychology. A psychological research was conducted, aimed at researching socio- psychological characteristics and features of homelessness so that a typology of homeless people can be created in order to socialize them. Preparatory stage of this study was a through observation of homeless people, including an in-depth interview for a better study. At this point, following types of homeless people were proposed: hustlers, vagrants, people ready for change, people not ready for change, escapists using drugs and alcohol, and reasons for losing home were identified as well. Taking results of preparatory stage into account, the design of main study was made, including a scale multi-factor questionnaire. 60 homeless people in Saint- Petersburg were surveyed. They were asked to evaluate the scale of agreement with statements divided into blocks. Blocks were formed from different spheres in order to capture all the psychological reasons for becoming homeless. Among others, there was family block to evaluate the family relationships, block of dependency on alcohol or drugs, block of socialization to evaluate readiness to embrace change, block of mental disorders.

Exploratory factor analysis of questionnaire data united the signs of factors, which we gave following names: factor of readiness/not readiness to accept change, factor of family conflict in childhood, factor of divorce/leaving/isolation from family, factor of forgetfulness or capacity to leave relationships, adventurer factor, vagrancy factor, alcoholism due to post-traumatic disorder. Psychological features of homelessness in the following study are seen in the boundaries of psychological science as evading risk, danger, threat, responsibility, overcoming fear, as a way to escape reality creating personal defense mechanism against internal and external threats and fears. The questionnaire for homelessness helps identify and predict psychological and sociological features which can leave the person on the streets and thrown out of society, which can help create patterns for socializing homeless people and preventing homelessness in Saint-Petersburg.
\end{abstract}

Keywords: Homeless people types, psychological reasons for homelessness, vagrancy, problem of socializing homeless people.

\section{Introduction}

The aim of the study was a development in a typology of homeless citizens based on their socio-political and socio-psychological reasons for disorders in socialization of personality. Objectives of the study: to identify the psychological factors and causes that lead Russian citizens to homelessness; to assess the willingness of homeless people to change their lives; to obtain a deeper psychological portrait of the homeless and to develop a socio-psychological typology of the homeless.

The object of the study was homeless citizens living in St. Petersburg and other regions regardless of age, gender, experience and history of homelessness, social status and subjective income.

The subject was the study of psychological characteristics that affect the formation of homelessness.

The main hypothesis was the assumption that homeless citizens may have different typological psychological characteristics depending on the causes of socialization of personality disorders.

The study period was August-November 2018.

The choice of the place for research was "Maltese help service" as well as urban "night stay social homes" and other places of stay for the homeless. 
The design of the questionnaire included statements that took into account the following characteristics and factors of the homeless person:

- the factor of satisfaction with the quality of life and confidence in the future;

- the factor of personality formation in childhood under the influence of negative cases of interaction with family and friends;

- the factor of the individual's propensity to dromomania;

- the factor of willingness of the individual to change.

There were denoted the psychological characteristics of the behavior of homeless citizens, for various reasons, who were homeless and without documents:

* factor of willingness of the subject to changes in his life. This possibility indicates the willingness or unwillingness of the subject to change in life, the willingness to take responsibility; childhood

* the factor of conflict and destructive relations of the respondent with his / her family in

* the factor of the respondent's willingness for isolation from the family in childhood.

* the factor of psychological mobility, the ability to forget/get out of a relationship or some living space, as well as the respondent's propensity to adventurism.

* the factor of the respondent's propensity to dromomania, namely the inability and unwillingness of a person to move away from the "dependent" lifestyle;

* the factor of avoiding the "hard" reality with the help of alcohol or drugs.

The procedure of final statistical processing and interpretation of the received information was done.

The results of the study made it possible to draw significant conclusions and highlight the typology of homeless citizens

\section{Homeless questionnaire} Zaitsev).

According to the results of the research obtained by this questionnaire (O. V. Medyanik, A. D.

\subsection{Social disadaptation}

The survey participants showed an average degree of agreement that they understand people who run away from home $(\mathrm{M}=3.24, \sigma=2.5)$, about half of the respondents faced a similar situation when there was a good reason to run away from home. Also, almost half of the respondents expressed understanding of people who leave the house and often change their habitat $(M=3.33, \sigma=2.5)$. One of these reasons may be to draw attention to yourself during a long absence in the house $(M=3.05, \sigma=2.55)$. Man, running away from home, forced a family to worry about him, thereby attracting attention to himself, which he lacked in the family.

The subjects responded to the remaining statements of this unit with a low degree of agreement. "I believe that real life is only on the street" ( $M=1.38, \sigma=1.19)$. It can be concluded that, despite the current situation of the respondents, they do not agree with this statement, we can even say that the homeless are not satisfied with their current situation. For them, their living on the street is not real life. This is some form of escape from reality, a protective reaction from a homeless existence.

The analysis of the average values of the first block showed that the homeless faced to some extent a situation when they had to leave the house, for example, to attract the missing attention to themselves or as a result of avoiding adverse factors that can negatively affect the emerging psyche. Homeless people also understand that they are in a difficult situation, but they try to abstract from reality.

\subsection{Mental illness}

In this block, subjects expressed low or below-average agreement on all statements. This indicates an adequate perception of the homeless by ordinary citizens. If the subjects answered the statements "I am often told that I am abnormal" ( $\mathrm{M}=1.79, \sigma=1.52)$, "People say your head's not right" $(\mathrm{M}=1.79, \sigma=1.44)$,"People are avoiding me, because they think that I am not quite adequate person" $(\mathrm{M}=1.98, \sigma=1.81)$ with a high degree of agreement it could be said that ordinary citizens have a negative attitude to the homeless, as well as the homeless treat themselves to people who are not all right with the head. However, this trend is not observed.

The analysis of the average values of the second block shows that neither the homeless nor ordinary people perceive the homeless as unhealthy and inadequate people. This block is aimed at identifying the attitude of citizens to the homeless and the final values can be argued that ordinary citizens do not consider homeless abnormal and mentally ill people. 


\subsection{Alcoholism and drug addiction}

The homeless interviewed responded with a fairly high level of agreement with the statement "I am a non-drinking person" $(\mathrm{M}=4.31, \sigma=2.23)$, as well as with a low level of agreement with the statement " In alcohol I seek my salvation" $(\mathrm{M}=1.55, \sigma=1.44)$. It can be concluded that the majority of the homeless interviewed remain or have already become non-drinking people. Low level of consent is also present in the following statements:" I understand people who cannot live without alcohol "(M=2.65, $\sigma=2.22)$," Only with the help of alcohol or drugs people can accept the heavy reality " $(\mathrm{M}=1.72, \sigma=1.78)$. From the above results on this block, we can distinguish the following points: among the homeless more than half are non-drinking people, while almost all respondents agree that alcohol or drugs will not help life to get better, but aggravate their situation.

\subsection{Family relation}

In this block, the respondents showed an average result for the first two statements "I thought there are terrible relationships in my family" $(M=3.02, \sigma=2.26)$, "I preferred to communicate more with my peers than with my family" $(M=3.20, \sigma=2.24)$. Such results may indicate that about half of the respondents had unstable or simply bad relations within the family, so it was easier for respondents to keep in touch on the street with those who understand them than to tolerate bad relations at home. This can be a serious psychological reason that led a person to the street. As a result of traumatic events occurring at home, people simply ran away from the influence of harmful factors on the street.

\subsection{Prospects of socialization}

As can be seen from table 1, the majority of respondents are non-drinking people $(\mathrm{M}=4.31 \sigma=2.23)$, but somehow less than half consume alcohol.

Also, based on the results of comparing the averages, we can say that almost all respondents are ready to do anything to change their lives for the better $(M=5.66, \sigma=2.10)$. These indicators show that the homeless have not lost hope of returning to a normal life, and it is worth noting that the majority of the homeless agree to get a job if they are offered $(M=4.35, \sigma=2.18)$. It is also worth noting that despite the current state, almost all homeless people believe in tomorrow $(\mathrm{M}=5.50, \sigma=2.37)$ and they are ready to start a new life if they are helped $(\mathrm{M}=5.72, \sigma=1.98)$. Also, most of the homeless want and are willing to work $(\mathrm{M}=5.67, \sigma=2.14)$.

According to the results of this block, the majority of homeless people understand the difficult situation they are in and, nevertheless, almost all of them are ready and want to change their lives for the better, which in the future can significantly help in the process of socialization of homeless people.

Summing up the data of descriptive statistics, we can say that even though the homeless are deprived of shelter, support, financial component, they are not deprived of faith in the future and hope for a better life. We can also say that a large number of homeless people had difficult relationships within the family, which is why the person left the house, running away from the negative influence of relatives, perhaps avoiding moral or physical violence. As a result, the man had to run away from home and spend time on the street. This is one of the essential reasons why a person has come to this way of life. The unbearable desire to leave the house and go to another reality in which there is no moral and physical violence is the main reason why the homeless were on the street.

The homeless themselves understand that this way of life does not suit them, but they believe that tomorrow everything can become better. They are ready to take up work, to build their lives in a new way. However, it is worth noting, judging by the results of the questionnaire, the homeless are not able or simply do not want to take responsibility for themselves and are not able to independently correct their current situation. There are paternalistic attitudes towards others, which should help them or give attention and care, which they were deprived, perhaps, in childhood. They have not fully passed the children's socialization, forming the skills of living together in a family with relatives. They expect that help will come to them from the state or from people who care about them.

Almost all homeless people are ready and willing to work, but without help they do not want and are not able to find a job. According to the survey, the homeless do not consider themselves to be sick and abnormal people who should stay away, which is also a positive aspect in the further socialization of the homeless. The homeless also believe that ordinary citizens do not consider them sick or mentally unhealthy people and "healthy" society does not treat them with prejudice or hostility.

Let us also refer to the results of table 2, which describes the reasons why a person was homeless, the distribution by sex, as well as by territorial affiliation.

\subsection{Causes of homelessness}

As a result of the study, men were almost twice as many as women. At the same time on 3 accounts for 2 local homeless. The most common reason why people become homeless is "fraud". Then there are "family reasons", and close the three "judicial reasons". Such factors as: "the problem with 
employment", "lack of support from the state", "the presence of addictions" and "problems with documents" distributed between a roughly equal number of votes, about 5 for each category. At the end of the list is the category "Debts".

\section{Factor analysis of the questionnaire}

The first factor by weight was called the factor of willingness \unwillingness to change, which combined the characteristics of a person's habituation to his way of life and not seeing the need to change the way of life that a person leads now, namely - the person does not hesitate to borrow money from others (0.291), and also does not want or cannot take responsibility (0.660). At the same time, the homeless person understands that he is avoided because he is considered not quite adequate and normal person (0.798)

We also received a number of categories that can be called "inverse" to the factor. Namely, the homeless are ready to do much to return to normal life $(-0.645)$, they believe in tomorrow $(-0.667)$. If they are helped from outside, they are ready to change (-0.549), and respondents have a desire and willingness to work (-0.595).

The second weight factor was named the factor of conflict with relatives in childhood, incorporates the belief that the runaway of the respondents from the house will make them worried (0.331), while homeless seemed to be in their families was a terrible relationship (0.628). They are also sure that their parents were cold to them $(0.712)$, they also think that in childhood they were treated rudely (0.7), and in childhood they were subjected to physical or psychological violence (0.688). Their parents often quarreled (0.830), they also felt that as a child no one loved them (0.799) and they did not feel really needed $(0.634)$. It is also worth noting that the people raised them drank a lot $(0.398)$. The inclusion of the issue of alcohol consumption seems to be due to correlation.

The third factor by weight is also related to the family sphere, but it is more indicative of how much the respondent is prone to break/care/isolation from the family. This factor included a number of beliefs, such as understanding of people who run away from home $(-0.634)$, which leave the house without any reason $(-0.608)$, who leaves the house because of the strong anxiety $(-0.660)$, as well as people who cannot live without alcohol (-0.546). Also, everyone was worried about the respondents when they ran away from home (-0295). The homeless interviewed also noted that they needed constant care and supervision $(-0.431)$, as well as respondents preferred to communicate with peers than with family $(-0.416)$

Next factor is the factor of psychological destructive mobility, the ability to forget/get out of a relationship or some living space, which can be called "street adventurism". A number of homeless respondents are sure that real life is only on the street (0.643). Respondents easily part with relatives (0.407). At the same time, respondents often change their habitat (0.582), and the people around them are convinced of the abnormality of the respondent (0.669) and that they have problems with the head (0.465). Also, respondents tend to avoid problems with alcohol (0.657).

The fifth factor combined the features of the factors, which are called as the factor of dromomania. These included the following features of the factor: some respondents like to roam the streets for no reason (0.612), they also feel that the walls are pressing on them $(0.571)$, some of them do not like to think about tomorrow (0.540). Some responders have limitations in mobility $(0,410)$. The respondents also noted that most of the money goes to alcohol (0.628), while they are ready to undergo treatment for addictions (0.582) and work if they receive such an offer $(0.533)$.

The last factor reveals alcohol addiction as a result of children's reaction to psychological traumas and is named by us as a factor of alcoholization as a result of post-traumatic stress disorder. A number of respondents who grew up without parents $(0.467)$ believes that children can run away from home if they are already independent $(0,427)$. Also, some respondents admit that they see their salvation in alcohol (0.571) and this is the only way to come to terms with the hard reality (0.592). At the same time, they do not believe that they will ever be able to stop drinking (0.357) and overcome their addiction on their own (0.724).

The factor analysis allowed us to determine a number of significant psychological reasons why a person was on the street. These reasons include: conflicts with family, as a result of which a person simply could not be in a traumatic situation and left home; alcoholism and drug addiction, as a result of which spheres of human life one after another are destroyed and in the result a person is on the street without everything; children's psychological trauma, which caused a significant devastating effect on the psyche of the child, as a result of which the person is not able to continue to maintain a normal lifestyle.

As a result, the above reasons can develop a person dromomania, it leads to the fact that a person will not be able to take responsibility for his/ her life. There is a great chance for starting alcohol or drug addiction as a way to "escape" from reality. A person develops adventurism, a tendency to a dependent lifestyle, resentment and distrust of the rest of the world.

Exploratory factor analysis of the questionnaire data combined the features of the factors that we gave the following names: the factor of willingness/unwillingness to change, the factor of conflict with 
family in childhood, the factor of breaking/leaving/ isolation with the family, the factor of the ability to forget or get out of a relationship, the factor of adventurism, the factor of dromomania, the factor of alcoholism as a consequence of post-traumatic stress disorder. These factors made it possible to distinguish the typology of the homeless.

\section{Summary}

The results of our study can be used in social policy to work with homeless citizens. Firstly, we have established objective reasons why a person was on the street. The most common reasons were: "fraud", "problems with relatives" and "judicial reasons", "alcoholism". Male homeless were almost twice as many as female, and two-thirds of the homeless were "local" who were born in St. Petersburg.

Secondly, we are able to identify the psychological characteristics of homelessness, i.e. the psychological reasons why a person became homeless. Among such causes can be distinguished a runaway from home as a consequence of the traumatic psyche of the situation. The potential homeless in the family had a bad relationship, where the child witnessed beatings, quarrels, alcohol or drug using. Also, a person could become homeless if he was subjected to mental or physical violence in childhood. For the reasons listed above, the person was not able to be in the house and preferred to spend time on the street, where he/she got used to interact with their peers and where there was a high risk to fall into the wrong company. Also, many homeless people have signs of alcohol and drug addiction, which developed as a result of "escaping from reality". However, there is a positive change: the homeless, who have this addiction, were ready to undergo treatment for alcoholism and drug addiction.

Among the homeless were the people with dromomania. The percentage of such people is very small, but it cannot be excluded. People with dromomania do not think their lives are bad, and they are not willing to work or change their lives in any way. As a rule, these are people with a very long experience of homelessness. At the same time, the vast majority of homeless people understand that their life situation can be better and they are not satisfied with the current state. Almost all homeless people are ready and willing to work, they also believe in tomorrow and do not lose optimism. The majority of homeless people answered the question about their dreams and desires that they want to have their own roof over their heads.

As a result, we can also make a small conclusion that the homeless are not able to change their lives on their own. In their failures homeless blame the state, once close people, the system, etc., but in rare cases themselves. They are afraid or unwilling to take responsibility for their lives. In fact, they let it take its course on the principle of "come what may." Most of the homeless can be helped to socialize, as they are marked by a high degree of willingness to change for the better, and most importantly, they have hope for the best, but it is necessary to overcome a number of complex psychological barriers.

The observation process shown that the homeless are extremely distrustful. Most of them repeatedly betrayed and deceived close and unfamiliar people. Many were left without a home because of black realtors and deception from close relatives. Once on the street froma high degree of credulity and naivety, they were disappointed in people and in life, and they may first need psychological help and support from society and the state.

The second point is, as described above, the inability to take responsibility. For a long time living on the principle of "be that it will be" a person loses ability to make serious decisions, to make any efforts aimed at change. In this case, you need to provide a person with support in the face of the same people as he or competent professionals who will be able to gradually teach a person to independence and step by step independently move to the intended goals.

In order to help the homeless return to normal life, it is necessary to build a complex and gradual system that the homeless will have to go through. In order to reduce the number of homeless people in St. Petersburg in the future, it is necessary to focus on the maintenance of friendly relations in the family, the creation of special rehabilitation centers where the homeless could receive medical, psychological and legal assistance, the creation of hostels for the homeless and also need to find an opportunity to provide them with jobs, including them in the society with help of these measures.

\section{References}

Alekseeva. A.S.; 2003. Homeless as an Object Social Discreditation. Ecsocman. 05.11.2003

Kochetkova. T.N.; 2012. The Role of Society in Forming the Self-Relationship of the Personality of a Homeless Person. Cyberleninka. 16.07.2013

Osinskiy. I. I.; Habaeva. I. M.; Badlaeva. I. B.; 2003. Homeless - Social Bottom of Society. Ecsocman. 23.03.2003

Volkov. V.V.; 2010. Homelessness in Modern Russia: The Problem of Typology of Homeless. Cyberleninka. 12.02.2010 


\title{
ORGANIZATIONAL SUPPORT AND WORK ENGAGEMENT OF DEAF AND HARD OF HEARING EMPLOYEES: DIVERSITY MANAGEMENT CHALLENGES
}

\author{
Biljana Maleš Mutić, Svetlana Čizmić, Milica Vukelić, \& Ivana B. Petrović \\ Department of Psychology, Faculty of Philosophy, University of Belgrade (Serbia)
}

\begin{abstract}
The management of workforce diversity has been attracting attention among researchers and practitioners in the field of work and organizational psychology over the past ten years. Nevertheless, little is known whether existing concepts, theoretical models and assessment approaches could explain to the utmost work behaviour of minority employees. Work engagement is a positive, affective-motivational state of work-related well-being characterized by vigor, dedication and absorption. As it is deeply embedded in Job Demands-Resources Model (JD-R), it is usually an outcome of job resources such as positive work climate and organizational support. Such supportive climate could be crucial in integrating and supporting diverse workers. It could be particularly challenging to shape the proper form of organizational support for employees that use different channels of communication.

The current study explored the relationship between Work Engagement and Perceived Organizational Support among 101 hard of hearing and deaf persons working at different organizations in Serbia. These concepts were measured with Utrecht Work Engagement Scale (UWES) and Survey of Perceived Organizational Support (SPOS) in paper and pencil form, supported by video translation in Serbian sign language.

Perceived organizational support significantly positively correlated with work engagement in the entire sample of hard of hearing and deaf employees $(r=0.35, p<.001)$. Nevertheless, when subsamples were divided by the degree of hearing impairment $(56-70 \mathrm{~dB}$; $71-90 \mathrm{~dB}$; more than $91 \mathrm{~dB})$, the correlation persisted only among employees with the hearing impairment between 71-90dB $(r=0.64, p<.001)$. The correlation was insignificant in groups of employees with the hearing impairment between 56-70dB and with the hearing impairment of more than $91 \mathrm{~dB}$. The significant differences in levels of work engagement were found only between the employees with the hearing impairment between 56-70dB $(M=37.55$, $S D=7.06)$ and employees with the hearing impairment of more than $91 \mathrm{~dB}(M=43.53, S D=6.45)$; $F(2,84)=5.189, p<0.01$.

The results were discussed in the context of complex interplay between level of impairment, work engagement and properly shaped organizational support. The research is solid standpoint for deeper and further research of these concepts within sensitive working populations and provides research input for diversity management.
\end{abstract}

Keywords: Hard of hearing and deaf employees, work engagement, perceived organizational support, diversity management, Serbia.

\section{Introduction}

The management of workforce diversity has been attracting attention among researchers and practitioners in the field of work and organizational psychology over the past ten years (Otaye-Ebede, 2018). In addition to ensuring the presence of minorities in organizations through affirmative actions, it is also of the utmost importance to find means for taking into account their specificities so that their potentials are fully utilized and their well-being supported. There are some studies that show that people with impairment have an equally good or even better performance at work than those without impairment (e.g. Schur, 2002).

Little is known whether existing concepts, theoretical models and assessment approaches could fully explain work behaviour of minority employees. Work engagement is a positive, affective-motivational state of work-related well-being characterized by vigor, dedication and absorption (Bakker, Schaufeli, Leiter \& Taris, 2008). Employees could be engaged when they find their work 
meaningful, when they have feeling that they do a valuable work, and if and when they feel they are able to transform and redirect their work endeavors to effective activities. Engaged employees are, at the same time, cognitively and emotionally connected with their work.

The concept of work engagement is deeply embedded in Job Demands-Resources Model (JD-R) (Bakker \& Demerouti, 2016), and it is usually conceptualized as an outcome of job resources such as positive work climate and organizational support. According to Tims and colleagues (2012), increasing job resources could produce higher levels of work engagement and, thus, equip employees with mechanisms to deal with the negative aspects of job requirements. Social and organizational support is certainly valuable work resource that employees can have in dealing with challenging work demands (Bakker, Demerouti \& Xanthopoulou, 2012). Perceived Organizational Support (POS) has been operationalized as a general belief about the extent to which the organization appreciates contribution of employees and takes care of their well-being (Eisenberger, Huntington, Hutcison, \& Sowa, 1986). Some previous research has shown that there is a positive correlation between perceived organizational support and work engagement and job satisfaction (Caesens \& Stinglhamber, 2014; Rodwell \& Munro, 2013).

Supportive work climate could be crucial in integrating diverse workers. It is particularly challenging to shape a proper form of organizational support for employees that use different communication modalities. A workplace could be seen as a specifically complex environment for employees with sensory (e.g. hearing) impairment, and it is not surprising that many of them have difficulties finding long-term employment. According to some research, employers often emphasize the communication barrier (the important mediator of employers' support), as the most challenging aspect in integration of employees with sensory (e.g. hearing) impairment (Foster, 1992). The quality of the relationship between an employee with sensory impairment and an employer is perceived as a critical factor for maintaining employment, as well as their organizational integration (Allen, Rawlings \& Schildroth, 1989). Employees with sensory impairment have tendency to withdraw from the work environment that is highly challenging for them (Scherich, 1996), e.g. a team work that implies simultaneous communication. Thus, the aim of the current study was to explore the relationship between perceived organizational support and work engagement among hard of hearing and deaf persons working at different organizations in Serbia.

\section{Method}

\subsection{Procedure and sample}

The sample comprised of 101 hard of hearing and deaf employees (49.5\% were women, 91\% with secondary education) from various publicly and privately owned organizations, from Serbia. Participation in this research was anonymous, voluntary and not compensated. There were $8.9 \%$ of employees with hearing impairment between 20 and $40 \mathrm{~dB}$; 5\% with hearing impairment between 41 and $55 \mathrm{~dB} ; 19.8 \%$ with hearing impairment between $56-70 \mathrm{~dB} ; 32.7 \%$ employees with hearing impairment between 71-90 dB, and 33.7\% with hearing impairment of more than $91 \mathrm{~dB}$.

\subsection{Instruments}

Work Engagement was measured by the Serbian short version of the Utrecht Work Engagement Scale, UWES-S (Petrović, Vukelić \& Čizmić, 2017) that consists of nine items followed by a seven-point scale (from $0=$ Never, to $6=$ Always), Cronbach's alpha coefficient was 0.90 . The perceived organizational support was measured using the Serbian version of the 8-item form of the Survey of Perceived Organizational Support, SPOS (Eisenberger et al., 1986), previously used in research on a large sample of employees from Serbia (Vukelić, Čizmić, Petrović, Tenjović \& Giorgi, 2015). Each SPOS item was followed by a 7-point Likert scale, Cronbach's alpha coefficient was 0.87. Both scales were translated into Serbian sign language and presented in form of a video. After seeing each video-statement (fully translated with Likert scale anchors), employees were entering their responses in the printed paper form of the questionnaire. Questionnaire was followed by sociodemographic inventory. Cronbach's alpha coefficient of the Serbian sign language version of UWES-9 was 0.81, while Cronbach's alpha for the Serbian sign language version of SPOS-8 was 0.83 . 


\section{Results}

The results have shown that perceived organizational support significantly positively correlated with work engagement in the entire sample of hard of hearing and deaf working persons $(\mathrm{r}=0.35, \mathrm{p}<.001)$. Nevertheless, when subsamples were divided by the degree of hearing impairment (56-70 dB; 71-90 dB; more than $91 \mathrm{~dB}$ ), the correlation persisted only among employees with the hearing impairment between 71-90 dB ( $\mathrm{r}=0.64, \mathrm{p}<.001)$. The most affirmatively evaluated statements of SPOS within this group were: the organization takes pride in my accomplishments at work (with 64\% answers moderately to strongly agree) and the organization would take into the account and respect my complaints (also 64\% answers moderately to strongly agree). The correlation was insignificant in the group of employees with the hearing impairment between 56-70 dB and with the hearing impairment of more than $91 \mathrm{~dB}$. The significant differences in the level of work engagement were found only between the employees with the hearing impairment between 56-70 dB and employees with the hearing impairment of more than $91 \mathrm{~dB}$ (Table 1); F(2,84) = 5.189, $\mathrm{p}<.01, \eta 2=0.11$.

Table 1. Means and standard deviations of work engagement for groups of employees with different levels of hearing impairment.

\begin{tabular}{ccc}
\hline Level of hearing impairment $(\mathrm{dB})$ & $M$ & $S D$ \\
\hline $56-70$ & 4.17 & .78 \\
$71-90$ & 4.42 & .82 \\
91and more & 4.84 & .72 \\
\hline
\end{tabular}

\section{Conclusion}

Presented research has raised several important questions. The first one is the nature and intensity of relations between organizational support and work engagement within different segments of employees with hearing impairment. It is also necessary to understand all social, work and emotional aspects that could affect work engagement and productivity of employees with hearing impairment. Future research would benefit from mixed-method approach as qualitative exploration could give deeper insight into diverse workforce perception of organizational social circumstances. The results can be fully understood only with taking into the account complex interplay between level of impairment, work engagement and organizational support. The second one is the necessity of translating and/or adjusting the assessment approach for employees with sensory impairment both carefully considering both the questions/statements and the answers/rating scales. In order to be able to shape interventions that would cover diverse workforce, it is necessary for employers to establish well-designed and meaningful assessments (Otaye-Ebede, 2018). The research is solid standpoint for deeper and further research of these concepts within sensitive working populations and provides research input for diversity management. The most important practical implication of the presented research is the cautionary advice for organizational consultants to carefully check assessment instruments aimed for exploring and developing organizational interventions that should cover diverse workforce.

\section{References}

Allen, T. E., Rawlings, B. W. \& Schildroth, A.N. (1989). Deaf students and the school-to-work transition. Baltimore, USA: Paul H. Brooks Publishing.

Bakker, A. B., \& Demerouti, E. (2016). Job demands-resources theory: taking stock and looking forward. Journal of Occupational Health Psychology, 22(3), 273-285. doi: 10.1037/ocp0000056

Bakker, A. B., Demerouti, E., \& Xanthopoulou, D. (2012). How do engaged employees stay engaged. Ciencia \& Trabajo, 14(1), 15-21.

Bakker, A. B., Schaufeli, W. B., Leiter, M. P., \& Taris, T. W. (2008). Work engagement: An emerging concept in occupational health psychology. Work \& stress, 22(3), 187-200. doi: 10.1080/02678370802393649

Caesens, G., \& Stinglhamber, F. (2014). The relationship between perceived organizational support and work engagement: The role of self-efficacy and its outcomes. Revue Européenne de Psychologie Appliquée/European Review of Applied Psychology, 64(5), 259-267. doi: 10.1016/j.erap.2014.08.002 
Eisenberger, R., Huntington, R., Hutchison, S., \& Sowa, D. (1986). Perceived organizational support. Journal of Applied Psychology, 71(3), 500-507. doi: 0021-9010/86

Foster, S. B. (1987). Employment experiences of deaf college graduates: An interview study. Journal of Rehabilitation of the Deaf, 21(1), 1-15.

Otaye-Ebede, L. (2018). Employees' perception of diversity management practices: scale development and validation. European Journal of Work and Organizational Psychology, 27(4), 462-476. doi: 10.1080/1359432X.2018.1477130

Petrović, I. B., Vukelić, M., \& Čizmić, S. (2017). Work engagement in Serbia: Psychometric properties of the Serbian version of the Utrecht Work Engagement Scale (UWES). Frontiers in Psychology, 8, 1799. doi: 10.3389/fpsyg.2017.01799

Rodwell, J., \& Munro, L. (2013). Well-being, satisfaction and commitment: the substitutable nature of resources for maternity hospital nurses. Journal of advanced nursing, 69(10), 2218-2228. doi: 10.1111/jan.12096

Scherich, D. L. (1996). Job accommodations of persons who are deaf or hard of hearing: Current practices and recommendations. Journal of Rehabilitation, 62(2), 27-35.

Schur, L. (2002). The Difference a Job Makes: The Effects of Employment Among People with Disabilities. Journal of Economic, 36(2), 339-347. doi: 10.1080/00213624.2002.11506476

Tims, M., Bakker, A. B. \& Derks, D. (2012). The development and validation of the Job Crafting Scale. Journal of Vocational Behavior, 80(1), 173-186. doi: 10.1016/j.jvb.2011.05.009

Vukelić, M., Čizmić, S., Petrović, I. B., Tenjović, L., \& Giorgi, G. (2015). Psychometric properties of the Serbian version of the negative acts questionnaire: Revised. Psihologija, 48(1), 19-33. doi: 10.2298/PSI1501019V 


\title{
ALCOHOL USE IN ASSOCIATION WITH UNORGANIZED SOCIAL ACTIVITIES IN FREE TIME AND ACCESSIBILITY OF ALCOHOL AMONG EARLY ADOLESCENTS
}

\author{
Lenka Abrinkova ${ }^{1}$, Olga Orosová ${ }^{2}$, Maria Bacikova-Sleskova ${ }^{2}$, \& Marcela Stefaňáková ${ }^{\mathbf{1}}$ \\ ${ }^{1}$ Department of Psychology, PJ Safarik University in Kosice (Slovakia) \\ ${ }^{2}$ Department of Educational Psychology and Psychology of Health, PJ Safarik \\ University in Kosice (Slovakia)
}

\begin{abstract}
Objectives: This study aimed to explore the role of unorganized social activities in free time and the accessibility of alcohol in explaining alcohol use in the past 30 days among early adolescents.

Methods: A representative sample of 653 (52.9\% girls) early adolescents (mean age $=11.72$ at T1; mean age $=13.09$ at T5) was collected within a five-wave longitudinal design with a baseline (T1), 3-month follow up (T2), 6-month follow-up (T3), 12-month follow-up (T4) and 15-month follow-up (T5) measurement. Alcohol use in the past 30 days and accessibility of alcohol was measured by the questionnaire of the international study ESPAD. Student's free time activities questionnaire was also used from ESPAD to represent unorganized social activities. Respondent's alcohol use (dichotomized: 0-not used, 1-used) served as the outcome variable and accessibility of alcohol and unorganized social activities served as the independent variables. Binary logistic regressions were used to analyse the data at every measurement period. The role of gender as a potential moderator of alcohol use was further addressed. Alcohol use at T1 was used as a control variable.

Findings: In each of the 5 measurement points, both accessibility of alcohol and participation in unorganized social activities increased the probability of alcohol use while controlling for alcohol use at T1. Gender differences in alcohol use were found at T1 with boys reporting higher alcohol use than girls. Moreover, a moderation effect of gender and accessibility of alcohol on alcohol use was confirmed at T1 showing that the probability of alcohol use was the highest for the girls with a high accessibility to alcohol.

Conclusions: This study contributes to the current knowledge regarding predictors of alcohol use among early adolescents.
\end{abstract}

Keywords: Alcohol drinking, unorganized social activities, alcohol accessibility.

\section{Introduction}

It has been widely claimed that organized leisure activities such as art, hobbies or youth organizations contribute to the healthy development of adolescents and reduce the risk of delinquency (Munger, 2015). On the other hand, peer oriented unorganized activities such as hanging out with friends and partying tend to provide opportunities for alcohol abuse and anti-social behaviour (Persson et al., 2007). Environmental approaches which prevent drinking among young people have mainly focused on modifying the context in which drinking occurs. Yet, despite great efforts, alcohol remains available to young people who access it from a variety of retail and social sources (Flewling et al, 2013). The accessibility of alcohol is perceived as prominent among the factors associated with a higher level of alcohol consumption (Popova, 2009). However, few studies have addressed the potential of unorganized social activities and accessibility of alcohol in the developmental period of early adolescents where peer-oriented activities play a key role in adolescent's life (Brauer et al., 2015) and alcohol is more available for them (Flewling et al, 2013). Thus, we aimed to explore these variables over a longer time period. 


\section{Design}

A longitudinal design was used.

\section{Objectives}

The objective of this study was to examine the role of unorganized social activities in free time and accessibility of alcohol in explaining alcohol use in the past 30 days among early adolescents.

\section{Methods}

\subsection{Sample and procedure}

The data were collected in five waves of the longitudinal study with a baseline in September 2013 (T1), a 3 month follow up (T2), 6 month follow up (T3), 12 month follow up (T4) and 15 month follow up (T5) in 31 randomly selected Slovak primary schools. The sample consisted of 653 (52.9\% girls) early adolescents (mean age $=11.72$ at T1; mean age $=13.09$ at T5).

\subsection{Measures}

The early adolescents were asked to fill in a paper version of the questionnaire regarding their alcohol use in the past 30 days, accessibility of alcohol and participation in unorganized social activities in free time. Participation in the study was anonymous.

- Alcohol use in the past 30 days was explored by the question: "On how many occasions (if any) have you had any alcoholic beverage to drink during the last 30 days?" The possible answers were: $0,1-2,3-5,6-9,10-19,20-39$, 40 or more. We dichotomized the variable into "not used alcohol $=0$ " versus "used alcohol $=1$ ".

- The accessibility of alcohol was identified by the following question: „If you wanted, would it be difficult or easy for you to obtain alcohol?", with response options: impossible (1) to very easy (5)

- Participation in unorganized social activities were assessed by a single item measuring how often you go out in the evening (to a disco, cafe, party, etc.) with possible answers from never (1) to almost every day (5)

\subsection{Statistical analyses}

Five separate binary logistic regression models were used for every measurement period to assess the association between alcohol use in the past 30 days and accessibility of alcohol and participation in unorganized social activities. Alcohol use in the past 30 days served as the dependent variable while accessibility of alcohol and participation in unorganized social activities served as independent variables. A T-test was performed to access the gender differences in all independent variables. In order to assess the potentially different effects in gender, all interactions with gender were tested. Alcohol use at T1 was used as a control variable.

\subsection{Results}

The results showed that about $9.3 \%$ of early adolescents $(12.7 \%$ boys, $6.4 \%$ girls $)$ in $\mathrm{T} 1$ had had experience with alcohol use in the past 30 days while in T5 the number of early adolescents with experience of alcohol use in the past 30 days had risen to $21.3 \%$ (25.1\% boys, $18.3 \%$ girls). Gender differences in alcohol use were found at $\mathrm{T} 1$ with boys reporting higher alcohol use than girls.

The descriptive analysis and gender differences in all explored variables in every measurement period are presented in Table 1. Generally, participation in unorganized social activities rises among boys and girls within 18 months with significant gender differences in T2. The rising tendencies of scores within 18 months were also identified in accessibility of alcohol in both boys and girls with significant gender differences in T3. 
Table 1. The descriptive characteristics in the measured variables according to gender.

\begin{tabular}{|l|l|l|l|l|l|l|}
\hline & \multicolumn{2}{|c|}{ Girls } & \multicolumn{2}{c|}{ Boys } & \multirow{2}{*}{ Sig. } \\
\hline & Mean & SD & Mean & SD & t & Sig. \\
\hline T1 Unorganized social activities & 1.67 & 1.19 & 1.63 & 1.06 & -0.47 & 0.63 \\
\hline T2 Unorganized social activities & 1.56 & 1.05 & 1.78 & 1.18 & 2.18 & $<.05$ \\
\hline T3 Unorganized social activities & 1.70 & 1.09 & 1.86 & 1.19 & 1.15 & 0.12 \\
\hline T4 Unorganized social activities & 1.95 & 1.23 & 2.01 & 1.28 & 0.47 & 0.63 \\
\hline T5 Unorganized social activities & 2.02 & 1.33 & 2.00 & 1.30 & 0.13 & 0.89 \\
\hline T1 Accessibility of alcohol & 1.75 & 1.20 & 1.81 & 1.29 & 0.55 & 0.57 \\
\hline T2 Accessibility of alcohol & 1.95 & 1.32 & 2.14 & 1.42 & 1.40 & 0.16 \\
\hline T3 Accessibility of alcohol & 2.14 & 1.42 & 2.50 & 1.58 & 2.39 & $<.05$ \\
\hline T4 Accessibility of alcohol & 2.53 & 1.51 & 2.72 & 1.61 & 1.11 & 0.26 \\
\hline T5 Accessibility of alcohol & 2.76 & 1.54 & 2.97 & 1.60 & 1.19 & 0.23 \\
\hline
\end{tabular}

Accessibility of alcohol and participation in unorganized social activities were significantly associated with the experience of alcohol use in the past 30 days in all 5 measurement periods. Early adolescents with higher levels of accessibility of alcohol and higher participation in unorganized social activities tend to drink more.

Table 2. Regression models for alcohol use in five measurement periods among early adolescents.

\begin{tabular}{|c|c|c|c|c|c|c|}
\hline \multicolumn{7}{|c|}{ Model T1 $\left(R^{2}=0,12\right)$} \\
\hline \multirow{2}{*}{ Predictors } & \multirow{2}{*}{ B } & \multirow{2}{*}{ S.E } & \multirow{2}{*}{$\operatorname{Exp}(B)$} & \multicolumn{2}{|c|}{ 95\% C. I for $\operatorname{Exp}(B)$} & \multirow{2}{*}{ Sig } \\
\hline & & & & Lower & Upper & \\
\hline Gender* & -2.40 & 0.77 & 0.90 & 0.02 & 0.41 & $<.05$ \\
\hline Unorganized social activities & 0.36 & 0.12 & 1.43 & 1.12 & 1.83 & $<.001$ \\
\hline Accessibility of alcohol & 0.39 & 0.13 & 1.47 & 1.13 & 1.92 & $<.001$ \\
\hline Interaction Gender X Accessibility of alcohol & 0.62 & 0.22 & 1.88 & 1.20 & 2.95 & $<.001$ \\
\hline \multicolumn{7}{|c|}{ Model T2 $\left(R^{2}=0,11\right)$} \\
\hline Alcohol use in T1 & 0.53 & 0.50 & 1.70 & 0.62 & 4.60 & 0.29 \\
\hline Gender* & 0.44 & 0.36 & 1.56 & 0.76 & 3.18 & 0.22 \\
\hline Unorganized social activities & 0.54 & 0.12 & 1.71 & 1.35 & 2.17 & $<.001$ \\
\hline Accessibility of alcohol & 0.39 & 0.12 & 1.48 & 1.16 & 1.89 & $<.001$ \\
\hline \multicolumn{7}{|c|}{ Model T3 $\left(R^{2}=0,16\right)$} \\
\hline Alcohol use in T1 & 0.93 & 0.44 & 2.54 & 1.07 & 6.03 & $<.05$ \\
\hline Gender* & 0.03 & 0.31 & 1.03 & 0.56 & 1.92 & 0.90 \\
\hline Unorganized social activities & 0.32 & 0.12 & 1.37 & 1.07 & 1.75 & $<.05$ \\
\hline Accessibility of alcohol & 0.60 & 0.11 & 1.81 & 1.46 & 2.25 & $<.001$ \\
\hline \multicolumn{7}{|c|}{ Model T4 $\left(R^{2}=0,26\right)$} \\
\hline Alcohol use in T1 & -0.10 & 0.52 & 0.90 & 0.32 & 2.53 & 0.84 \\
\hline Gender* & -0.20 & 0.36 & 0.81 & 0.40 & 1.65 & 0.56 \\
\hline Unorganized social activities & 0.59 & 0.13 & 1.80 & 1.37 & 2.36 & $<.001$ \\
\hline Accessibility of alcohol & 0.92 & 0.15 & 2.52 & 1.86 & 3.44 & $<.001$ \\
\hline \multicolumn{7}{|c|}{ Model T5 $\left(R^{2}=0,20\right)$} \\
\hline Alcohol use in T1 & 0.40 & 0.45 & 1.49 & 0.61 & 3.60 & 0.37 \\
\hline Gender* & -0.32 & 0.31 & 0.72 & 0.39 & 1.33 & 0.30 \\
\hline Unorganized social activities & 0.25 & 0.11 & 1.29 & 1.03 & 1.60 & $<.05$ \\
\hline Accessibility of alcohol & 0.72 & 0.12 & 2.07 & 1.62 & 2.65 & $<.001$ \\
\hline
\end{tabular}

Note: * boys as a reference group

Regarding the moderation effect, the relationship between alcohol use and accessibility of alcohol varies depending on gender in T1. Girls with a high accessibility of alcohol show a higher probability of alcohol use compared to boys with a high accessibility of alcohol. Thus, it seems that gender moderates the relationship between accessibility of alcohol and alcohol use in the past 30 days. The visualisation can be seen in Figure 1. The moderation effects in other measurement periods were tested but insignificant and are thus not displayed. 
Figure 1. Interaction effect of gender and accessibility of alcohol on alcohol use in T1.

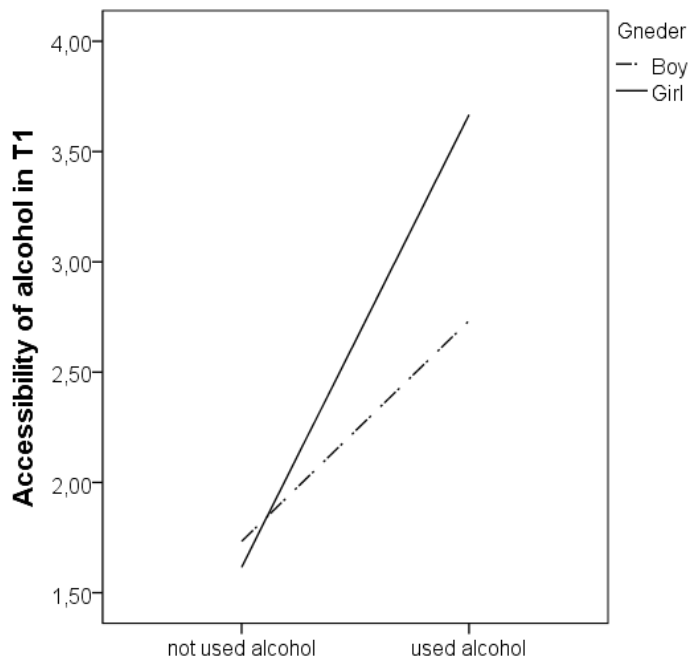

\section{Discussion and conclusion}

The findings of this study show that accessibility of alcohol and participation in unorganized social activities play an important role in alcohol use over a longer time period in early adolescence. Moreover, a significant and unique moderator effect of gender on the relationship between accessibility of alcohol and alcohol use was also found.

In our study, the participation in unorganized social activities was directly associated with alcohol use. We observed that with rising age, there is an increase in participation in unorganized social activities for boys and girls. This can be associated with the developmental period characterized by the higher need for the presence of peers, a lack of structure and the absence of adult supervision making risk behaviour both easier and more rewarding (Osgood et al., 2005). These situations are most prevalent in public locations e-g. bars or discos (Forsyth \& Barnard, 2000). With regard to drinking location and leisure time activities, it has been shown that drinking alcohol at home is associated with lower alcohol consumption than drinking outside the home (Clapp et al., 2006) and the likelihood of alcohol use increased in the presence of peers (Grune et al. ,2017).

In our study, it was found that a higher level of accessibility of alcohol was associated with a higher level of alcohol use. Despite centralised controls of alcohol availability (taxes), promotion regulation, controlled opening hours and age restriction (Hay et. al., 2009), early adolescent can access alcohol either in the home environment (Komro et al., 2007) or during unorganized social activities with peers. Furthermore, girls with higher accessibility of alcohol are more likely to use alcohol when compared to boys. Thus, it seems that girls are more prone to consume alcohol when it is accessible for them.

Moreover, the results show that with the increasing age, there is an increase of explained variance in every regression model suggesting that unorganized social activities and accessibility of alcohol play a larger role in explaining alcohol use with increasing age. Thus the general importance of any activity, class or youth groups (scouts or youth clubs), sports, dance activities (yoga, aerobics, dance class) to provide an organized and safe way of spending free time for early adolescents must be stressed.

The current results contribute to the previous findings mainly with respect to the moderating role of gender and might be beneficial in preventing alcohol use among boys and girl in early adolescents.

\section{Acknowledgements}

This work was supported by Research and Development support Agency under the contract No. APVV 0253-11 and APVV-15-0662. 


\section{References}

Brauer, J. R., De Coster, S. (2015). Social relationships and delinquency: Revisiting parent and peer influence during adolescence. Youth \& Society, 47(3), 374-394. doi: 10.1177/0044118X12467655

Clapp, J. D., Reed, M. B., Holmes, M. R., Lange, J. E., \& Voas, R. B. (2006). Drunk in public, drunk in private: The relationship between college students, drinking environments and alcohol consumption. American Journal of Drug and Alcohol Abuse, 32, 275-285. doi:10.1080/00952990500481205

Flewelling R. L., Grube, J. W., Paschall, M. J., Biglan, A., Kraft, A., Black, C., Hanley, S.M., Ringwalt, C., Wiesen, C., \&, Ruscoe, J. (2013). Reducing youth access to alcohol: findings from a community-based randomized trial. American journal of community psychology, 51(1-2), 264-77. doi: 10.1007/s10464-012-9529-3.

Forsyth, A., Barnard, M. (2000). Preferred drinking locations of Scottish adolescents. Health \& Place, 6, 105-115. doi:10.1016/S1353-8292(00)00002-2

Grüne, B., Piontek, D., Sleczka, P., Kraus, L., \& Pogarell, O. (2017). Drinking location and drinking culture and their association with alcohol use among girls and boys in Europe. Journal of studies on alcohol and drugs, 78(4), 549-557. doi: 10.15288/jsad.2017.78.549.

Komro, K.A., Maldonado-Molina, M.M., Tobler, A.L., Bonds, J.R., \& Muller K.E. (2007). Effects of home access and availability of alcohol onyoung adolescents' alcohol use. Addiction, 102, 1597-1608. doi:10.1111/j.1360-0443.2007.01941.x

Munger, R. L. (2005). Changing Children's Behavior by Changing the People, Places and Activities in Their Lives. Boys Town Press.

Osgood, D. W., Anderson, A. L. and Shaffer, J. N. (2005). Unstructured Leisure in the After - School Hours, in J. L. Mahoney, W. L. Reed and J. S. Eccles (eds) Organized Activities as Contexts of Development:Extracurricular Activities, After-School and Community Programs, pp.45-64. London: Lawrence Erlbaum Associates Journal Articles.

Persson, A., Kerr, M. and Stattin, H. (2007). Staying in or Moving Away from Structured Activities: Explanations Involving Parents and Peers, Developmental Psychology 43 (1): 197-207. doi: 10.1037/0012-1649.43.1.197

Popova S, Giesbrecht N, Bekmuradov D, Patra J. (2009). Hours and Days of Sale and Density of Alcohol Outlets: Impacts on Alcohol Consumption and Damage: A Systematic Review. Alcohol and Alcoholism 44: 500-516. doi: 10.1093/alcalc/agp054 


\title{
INCREASING CAPACITY IN WORK WITH WAR VICTIMS AND MIGRANTS: PRAGMATIC EMPOWERMENT TRAINING (PET)
}

\author{
Charles David Tauber ${ }^{1}$, \& Sandra Marić ${ }^{2}$ \\ ${ }^{1}$ M.D., CEO, Coalition for Work with Psychotrauma and Peace (Croatia and Netherlands) \\ ${ }^{2}$ MA, Deputy CEO, Coalition for Work with Psychotrauma and Peace (Croatia and Netherlands)
}

\begin{abstract}
According to a variety of publications and in our experience, there is a very large deficit in the number of people available to work with the psychological traumas of war and migration and other disasters, primarily those created by humans. While symptoms of such traumas may be relieved by drugs to some degree, drug treatment does not solve the basic issues. The consequences are a lack of functionality of the victims of trauma at various levels, as well as increased physical illness. Further, the training and employment of professionals is costly and time-consuming. In work by the first author since 1988 with asylum seekers and refugees in The Netherlands and since 1995 in the Western Balkans, we have found that "barefoot therapists", aka "peer supporters" from the beneficiary communities can be trained using participatory methods in about 150 hours to provide at very least first-line assistance using methods based on Carl Rogers' participatory education model. This also has the advantage of greater acceptance by the beneficiaries, especially in situations in which psychological treatment is stigmatized.
\end{abstract}

Keywords: Psychological trauma treatment, war trauma, peer support, asylum seekers, integration.

\section{Introduction}

There is a large amount of psychological traumatization among war victims and migrants as well as among members of minority groups in many parts of the world, not the least of them Europe and North America. In a series of articles in The Lancet in 2007, the standpoints of which were reiterated in October $2018^{1}$, it was said that more than $90 \%$ of the people needing psychological assistance weren't getting it. This, of course, is a much more severe situation in war-torn regions and among marginalized groups. The psychological issues cause a wide variety of additional consequences, such as a lack of functionality within families and societies, physical ailments, difficulty in integration into receiving societies, economic difficulties, and domestic and civil violence. It also is obvious that there are insufficient resources to train a sufficient number of psychologists and psychiatrists to deal with these numbers of people in adequate ways, particularly in environments where resources already are scarce. A further issue is that receiving psychological assistance is highly stigmatized in many cultures, especially in those groups most in need of assistance. Still another issue is the lack of understanding of the specific cultural, religious, and experiential factors by practitioners outside of the beneficiary groups.

The method now known as Pragmatic Empowerment Training (PET) has been developed through the experience of the first author in work with asylum seekers and refugees, first with various groups in The Netherlands starting in 1988, and later with the victims of war in the Western Balkans since 1995 as head of the Coalition for Work with Psychotrauma and Peace (CWWPP), and currently again with the victims of the migrant crisis in Europe and elsewhere. The aim of the method is to train people without previous education who are members of the beneficiary groups to assist one another in the first instance, referring to professionals only where absolutely necessary. The education and psychological supervision is given without charge online and onsite. The educational and therapeutic philosophy is that of Carl Rogers' participatory education.

\footnotetext{
${ }^{1}$ Lancet Global Mental Health Group, (2007). Scaling up services for mental disorders-a call for action. Lancet 2007; 370, $1241-52$.
} 


\section{The Issues}

\subsection{Recognition}

One of the primary issues is the lack of recognition by governments, inter-governmental agencies, large non-governmental organizations, and donors that psychological assistance and relief is essential for the recovery of people after war, torture, and other mistreatment. Frequently, it is seen by those in leadership as "soft" and often has been characterized as "irrelevant" in comparison to such factors as politics and economics. Psychological trauma has not been considered to be a primary factor in integration into society during and after conflict and in work with vulnerable groups, such as migrants.

Where such aid is given at all, it given for very short periods of time, frequently for only one or a very few sessions. Also, drugs rather than talk therapy almost always are used. The recent findings from research show us that level of use anti-depressives for 2017 is double in war-affected Vukovar-Sirmium County in comparison with the rest of the Croatia ${ }^{2}$. Further, Croatian police in 2007 reported that about a third of drivers in the region were using some sort of psychoactive drug. This applies even in situations that are somewhat more stable, such as recovery, integration, and development. Additionally, with very few exceptions, work on psychological trauma is not considered to be part of peacebuilding and reconciliation processes.

The problem of dealing with traumatic reactions is that, in most societies, it is overly medicalized. We strongly believe that it should be more socially and culturally sensitive. The term "psychosocial" attempts to express the recognition that there always is a close, ongoing circular interaction between an individual's psychological state and his or her social environment ${ }^{3}$. Such an approach is directly intertwined with peacebuilding work. In a broader concept of healing in which the individual is not treated as separate from his or her social context, the shift from "victim" to "survivor" is as much a question of social justice as a question of any personal process undertaken by the individual.

Summerfield argues that: "If symbolic acts are not linked with the delivery of truth, justice, and social change, they run the danger of being seen as a strategy to "buy off" the survivors. Social reform is the best medicine; for victims of war and atrocity this means public recognition and justice. Health and illness have social and political roots: post-traumatic reactions are not just a private problem, with the duty on the individual to recover, but an indictment of the socio-political forces that produced them." 4

A common definition of a traumatic situation is one that threatens the physical or psychological status of a person or entity or those of someone or something related in some way to the person or entity. Thus, a simple example would be an auto accident or a near miss to a person or to someone close. It might even be something similar with the same type of car or in a street that the person uses frequently. It also could be the loss of jobs in the same sector. Also, it might be something that happened in the past to people of the same ethnic group or the same religion or the same gender. Thus, we all constantly are being exposed to traumatic situations.

The first framework for traumatic incidents was developed in the 1970s because of the long-term psychological effects of the Vietnam War on soldiers in the USA. In the mid 1980s, the International Society for Traumatic Stress Studies was established as one of the first formal initiatives to recognize trauma as a distinct multidisciplinary field of study that explores how trauma affects not only individuals but also communities. Wars, natural disasters, climate change, forced migration, and their impact on individuals and communities has recently become an integral area of study. ${ }^{5}$

\subsection{Consequences of traumatization}

We have seen that the psychological consequences of such traumatization can be varied and can differ significantly between individuals, cultures, and situations. Commonly, they include depression, anger, anxiety, loss of direction, dissociation, and relational issues. There also is reactive psychosis. We must note that we call these "reactions" rather than "disorders", as we feel that people not reacting to such circumstances would be more ill than those having them. We also see that, while there are patterns to such reactions, the specific reactions are highly individual.

Somatic reactions are frequently present and are underestimated, in our view. These can affect every system in the body. Most frequently, we have seen circulatory system reactions, digestive system

\footnotetext{
2 Draganić, P., Škribulja, M., Oštarčević, S. (2019). Potrošnja lijekova u Hrvatskoj 2013. - 2017. Agencija za lijekove i medicinske proizvode- Halmed pg. 145. Retrieved 3, 15, 2019, from :http://www.halmed.hr/fdsak3jnFsk1Kfa/publikacije/Potrosnja-lijekova-uHrvatskoj-2013-2017.pdf\#page33

${ }^{3}$ Agger, I. (2001). Psychosocial assistance during ethnopolitical warfare in the former Yugoslavia. In D. Chriot \& M. Seligman (Eds.), Ethno-political warfare: Causes, consequences and a possible solution (pp. 305-318). Washington DC: American Psychological Association.

${ }^{4}$ Summerfield, D. (2000). War and Mental Health: a Brief Overview. British Medical Journal 321: 232-235.

${ }^{5}$ Psychologists for Social Responsibility, (2002). Personal and Community Reconstruction, Resilience and Empowerment in Times of Ethnopolitical Conflict. Washington, D.C.
} 
reactions, and immune system reactions, as well as endocrine system reactions, including sexual ones. In one study carried out by the Croatian Ministry of Defenders, it was found that the life span of war veterans was considerably decreased ${ }^{6}$. We thus feel that physicians also need to be involved in work with people reacting to trauma.

The societal consequences of trauma also seem to be mostly unrecognized. We have seen strong ecoomic consequences of trauma in the dysfunctionality at various levels mentioned above. Thus, there is a lack of concentration among workers, problems in planning, problems in execution of work, etc. Furthermore, psychological trauma leads to violence. This is true within families, that is, domestic violence, as well as within the community. We believe that unresolved psychological traumatization is a major unrecognized cause of violence and terrorism.

\subsection{Levels of traumatization and need for work}

Traumatic situations occur at a variety of levels. At an individual level, this can be intrapersonal, that is, a shift in the situation within the person, or it can be one or more reactions to an external situation. There also can be a traumatic situation at the family level. Further, traumatic situations can occur at the group level. A group here indicates people who have something in common. Additionally, trauma can occur at regional and national levels. An example of traumatic events at the global level would be climate change. Thus, it is seen that traumatic situations not only occur individually but also collectively and must be dealt with accordingly at the micro or macro levels. This has strong implications for peacebuilding.

Vamik Volkan commented that trauma in individual victims may cause new social and political processes at a broader social level and may result in altered behavior being transmitted from one generation to another. Neglecting the effects of trauma in one generation may lead to future generations carrying the suffering of previous ones, which Volkan terms transgenerational transmission. ${ }^{7}$ This type of transgenerational transmission can go on for hundreds, and some researchers say thousands of years. Recent studies have indicated that there may be a genetic component to it. It is extremely important in peacebuilding, as it may lead to future conflicts, psychological and physical suffering, and impaired functioning at the group and individual levels. Thus, it is important to work with the original trauma as early as possible.

\subsection{Capacity vs. elitism: Who works with the beneficiaries?}

We have stated earlier in this paper that there is a very great lack of capacity to deal with the psychological issues and reactions of people in violent and post-violent situations, as well as with vulnerable groups. This gap between need and availability of treatment is especially wide in low-income countries and middle-income countries ${ }^{8}$. In many places, this has been restricted to licensed psychologists and psychiatrists and, very occasionally, social workers. Yet, at least in some respects, this is not efficient, effective, or practical. We argue for the creation of a new group of people, that is, those without previous education in these fields, whom we call "barefoot therapists" or "peer supporters" who, in our experience, can be trained in about 120 hours and then supervised on an ongoing basis.

In terms of effectiveness, peers know the issues of one another far better than professionals. Also, peers can relate to one another more easily than people at a "higher" level. Furthermore, there are more peers than professionals available within the community. Thus, we would argue that, if peers can be adequately trained and supervised and know the limits to which they can work properly, they are a better resource than "professionals". Our experience is that they can become even more professional than the professionals in quite a number of instances. One problem in this is that they are not recognized officially and that the quality of their education and supervision is not controlled.

This issue of capacity will not be able to be solved by training more professionals. That is too costly in terms of time and resources. Peer support has a long tradition in informal services and may complement mental health care promoting recovery orientation and destigmatization. Non-specialist health professionals, lay workers, affected individuals, and caregivers with training and appropriate supervision by mental health specialists are able to detect, diagnose, treat, and support individuals with mental disorders and reduce caregiver burden. ${ }^{9}$ This also provides a sustainable pool of people within vulnerable communities on a long-term basis through community-based programs and task-shifting approaches.

\footnotetext{
${ }^{6}$ Ministarstvo branitelja, (2012). Praćenje pobola i smrtnosti hrvatskih branitelja iz Domovinskog rata I članova njihovih obitelji.

${ }^{7}$ Volkan, V. (1997). Bloodlines: From Ethnic Pride to Ethnic Terrorism. New York: Farrar, Straus and Giroux.

${ }^{8}$ Kohn, R., et al. (2004). The treatment gap in mental health care. Bulletin of the World Health Organization, 82: 858-866.

${ }^{9}$ Kakuma, R., Minas, H., van Ginneken N., Dal Poz M.R., et all. (2011). Human resources for mental health care: current situati on and strategies for action. Lancet, Nov 5; 378(9803):1654-63.
} 


\section{Pragmatic Empowerment Training (PET)}

The PET program was first developed in response to the requests of volunteers working with asylum seekers and refugees in The Netherlands in the late 1980s and early 1990s. When staff at asylum seeker centers and non-governmental organizations there, as well as social workers, nurses, and some physicians heard about what we were doing, they also requested it. We also have used PET with war victims in various locations in Croatia, Bosnia, and Serbia since the mid-1990s.

PET is based on Carl Rogers' concepts of participatory education. Rogers' Person-Centered Approach implies that, in a positive and safe climate of empathy and trust, a person is able to build personal power, self-acceptance, self-awareness, and self-trust, and that the person can be trusted to move toward psychological health without this being imposed by another person from the outside. ${ }^{10}$ For the most part, the participants determine topics that they wish to learn. They use their own experience in the learning process. We establish a climate that is pervaded by three conditions, that is, congruence, empathy, and unconditional positive regard. Thus, there is more discussion and practice than lecturing. The role of the trainer is changed to that of facilitator. Because people are working with one another, the cultural barriers are considerably lower than there would be in conventional education. We believe in the tendency of the group as a whole, and every individual in it, to find a way into deepened understanding of person-centered theory and practice. In other words, we are committed to the consistency between the theory of the approach and our community learning process.

In general, small groups meet for about an hour and a half to two hours per week. We carry out groups onsite and online. The online groups have a number of advantages. First, they save travel costs and time. Time schedules thus become more flexible. Further, online work allows people from different places to come together and share knowledge and experience. We do not set limits to the number of sessions.

Additionally, the nature of the discussions allows for supervision as well as direct education. Thus, participants can gain insight into their practical cases and issues from the experience of others. The program is adapted to the needs and desires of the participants in each specific group. In general, it includes a general introduction to work with people, self-care, inter-personal and group communication, an introduction to psychology and counseling, this concentrating on the specific situations of the participants, non-violent conflict transformation, civil society, human rights, and integration of vulnerable groups into society. In general, no charge is made for the program. We issue certificates of participation and completion, these stating what the program has included and the number of hours of participation.

\section{Results}

It is very difficult to quantify the results of PET. Thus, we only can use anecdotal evidence to justify the technique. What people who have been through the program tell us is that it provides them with the tools to provide care that otherwise would not be provided to people who otherwise would not seek it in places in which such care otherwise would not be available. They also tell us that they prefer to work with their peers rather than with the so-called professionals. In this sense, it opens up a variety of additional opportunities for work with potential beneficiaries.

We have used PET in variety of situations with a large number of beneficiary groups. We have found that calling the groups "educational" rather than "therapy" groups frequently, but not always, overcomes stigmatization. Our clients will refuse therapy, and they complain about their existential problems. We frequently can hear, "if I have a job, or papers, or a house I wouldn't have a problem". Another issue here is taking the work out of the pathological sphere, that is, speaking about "natural" "post-traumatic stress reactions" rather than about post-traumatic stress "disorder" or "syndrome". This is critical in recruiting and working with beneficiaries, in our experience.

One situation in which PET has been useful is with volunteers and staff members working in difficult situations with vulnerable people such as asylum seekers and refugees. These situations are present in the West and in violent and post-violent situations. Unfortunately, most organizations that use volunteers and even those who employ humanitarian staff, that is, inter-governmental and large non-governmental organizations, do not provide psychological training or psychological supervision. Thus, there are high levels of burnout and damage to beneficiaries.

Another situation in which we have used PET is with groups of the victims of war, both civilians and (former) military, in villages. Further, we have worked with specific demographic groups, such as women, youth, former soldiers, etc. We see PET as being useful in situations where the provision of

${ }^{10}$ Rogers, R. C., Lyon, H. C., Tausch, R. (2013). On Becoming an Effective Teacher-Person-centered Teaching, Psychology, Philosophy, and Dialogues with Carl R. Rogers and Harold Lyon. London: Routledge. 
psychological assistance is difficult because of a lack of trained personnel and where resources are extremely limited. This applies to disaster situations, including wars and natural disasters. We also see PET being used with vulnerable groups, particularly those that are suspicious of "establishment" experts. Examples are minority groups, groups of migrants in the process of integration, etc.

\section{Conclusions}

Post conflict environments have long-term negative psychological impacts. Attention must be devoted to the work on psychological trauma and its accompanying physical and societal consequences. Practitioners working in post-conflict regions also are repeatedly exposed to severe trauma and therefore have become susceptible to its long-term consequences on health and emotional wellbeing. They need to be familiar with the basic concepts of trauma. Neglecting the effects of trauma in one generation may lead to future generations carrying the suffering of previous ones, as it may lead to future conflicts, psychological and physical suffering, and impaired functioning at the group and individual levels. We also strongly believe that the healing of the wounds of trauma is a pre-requisite for social integration, and is a condition for peace. Psychological trauma has not been considered to be a primary factor in integration into society during and after conflict and in work with vulnerable groups.

The gap between need and availability of treatment is especially wide in low-income countries and middle-income countries. In many places, this has been restricted to licensed psychologists and psychiatrists and, very occasionally, social workers. Yet, at least in some respects, this is not efficient, effective, or practical. In brief, currently, there is a insufficient number of professionals to deal with the situation, nor can a sufficient number be trained within the foreseeable future. In less developed societies, there are strong stigma against mental health. We thus see the best solution in well-trained and well-supervised "barefoot therapists", who also may be called "peer supporters".

PET is a valuable technique that we see as deserving more attention and recognition, particularly in front line low resource situations and particularly in situations in which mental health care is highly stigmatized. PET is based on Carl Rogers' concepts of Person-Centered Approach that implies that person can be trusted to move toward psychological health without the another being imposed from the outside. Its financial costs and the amount of initial training required are relatively low. It may be carried out online, allowing the efficiency to be even greater. We are in the process of translating the content into self-paced online format.

We welcome inquiries and collaboration.

\section{References}

Lancet Global Mental Health Group, (2007). Scaling up services for mental disorders-a call for action. Lancet 2007; 370, 1241-52.

Draganić, P., Škribulja, M., Oštarčević, S. (2019). Potrošnja lijekova u Hrvatskoj 2013. - 2017. Agencija za lijekove i medicinske proizvode- Halmed pg. 145. Retrieved 3, 15, 2019, from: http://www.halmed.hr/fdsak3jnFsk1Kfa/publikacije/Potrosnja-lijekova-u-Hrvatskoj-20132017.pdf\#page33

Agger, I. (2001). Psychosocial assistance during ethnopolitical warfare in the former Yugoslavia. In D. Chriot \& M. Seligman (Eds.), Ethno-political warfare: Causes, consequences and a possible solution (pp. 305-318). Washington DC: American Psychological Association.

Summerfield, D. (2000). War and Mental Health: a Brief Overview. British Medical Journal 321: 232-235.

Psychologists for Social Responsibility, (2002). Personal and Community Reconstruction, Resilience and Empowerment in Times of Ethnopolitical Conflict. Washington, D.C.

Ministarstvo branitelja, (2012). Praćenje pobola i smrtnosti hrvatskih branitelja iz Domovinskog rata I članova njihovih obitelji.

Volkan, V. (1997). Bloodlines: From Ethnic Pride to Ethnic Terrorism. New York: Farrar, Straus and Giroux.

Kohn, R., et al. (2004). The treatment gap in mental health care. Bulletin of the World Health Organization, 82: 858-866.

Kakuma, R., Minas, H., van Ginneken N., Dal Poz M.R., et all. (2011). Human resources for mental health care: current situation and strategies for action. Lancet, Nov 5; 378(9803):1654-63.

Rogers, R. C., Lyon, H. C., Tausch, R. (2013). On Becoming an Effective Teacher-Person-centered Teaching, Psychology, Philosophy, and Dialogues with Carl R. Rogers and Harold Lyon. London: Routledge. 


\title{
MIGRATION FACTORS RELATED TO EMIGRATION INTENTIONS AMONG UNIVERSITY STUDENTS IN SLOVAKIA
}

\author{
Bohuš Hajduch $^{1}$, Olga Orosová $^{\mathbf{2}}, \boldsymbol{\&}$ Marcela Štefaňáková ${ }^{1}$ \\ ${ }^{1}$ Department of Psychology, Faculty of Arts, P.J. Safarik University in Košice (Slovakia) \\ ${ }^{2}$ Department of Educational Psychology and Psychology of Health, Faculty of Arts, P.J. Safarik \\ University in Košice (Slovakia)
}

\begin{abstract}
Background: In Slovakia, there has been an increase in the number of students who aim to move abroad. We hypothesized that experience with living abroad might increase the likelihood of emigration intentions because people (in this study, university students) with migration experience might feel more confident after carrying out and experiencing their emigration intentions.

Objectives: The main objective of this study was to explore whether factors such as emigration self-efficacy (ESE), evaluation of migration experience (EME) and the frequency of migration experience (FME) are related to the emigration intentions (EI) of young people (under 30 years old) in Slovakia. The moderation effect of gender in the relationship between the independent variables and dependent variable (EI) was also tested.

Methods: The sample consisted of 474 university students from Slovakia $(M=22.4, S D=2.13)$ from which 76.8\% were women, all participating in the Student Life Cohort Study (SLiCE 2016) which mostly focuses on the emigration/migration intentions and risk behaviour of university students. The data for this sample were collected via an online survey. Standard multiple regression was used and the analysis was adjusted for gender.

Findings: Our model explained $26.1 \%$ of the variance in EI. ESE was found to make the largest unique contribution to explaining emigration intentions $(\beta=0.373$, p $\alpha<0.001)$ followed by EME $(\beta=0.138$, $p \alpha<0.05)$ and FME $(\beta=0.121, p \alpha<0.05)$. Therefore, higher emigration self-efficacy, more positive migration experience and more frequent migration experience all have a positive unique effect on EI. Gender was not significant so we couldn't confirm the moderation effect of gender in the relationship between the independent variables and the dependent variable (EI).

Conclusion: We found that emigration self-efficacy, evaluation of migration experience and the frequency of migration experience were related to emigration intentions among young people in Slovakia. These results contribute to a better understanding of the role of these factors in explaining emigration behaviour among young people in Slovakia.
\end{abstract}

Keywords: Emigration intentions, emigration self-efficacy, migration experience, university students.

\section{Introduction}

Migration has been widely studied in recent decades although it has still not been fully explored and understood. The most important reason could be the relatively high complexity of migration: structural factors interact with individual characteristics, social embedding, life course events and psychological characteristics. Migration is also not stable. On the contrary, it is a process over time (Kley, 2009). With the development of information technology, transport and international connections, the world is getting more connected than ever before. This globalization naturally means a rise in migratory behaviour. In Slovakia, there has been an increase in the number of students who aim to move abroad. The Statistical Office of the Slovak Republic indicates that students with secondary education (53.68\%), followed by university students (21.55\%) are the most numerous group of Slovak emigrants in terms of education (Káčerová, Horváthová, 2014). Bahna (2009) found that young people from Slovakia aged 18-24 had expressed their willingness to leave permanently (36\%) or only for a certain time (53.3\%). Men generally showed increased willingness to travel abroad. According to the economic activity of the respondents, the overwhelming majority of students (up to 92.9\%) expressed their willingness to leave for work abroad, while $47.4 \%$ of the students expressed their willingness to leave permanently. Also, a more 
frequent and more positive experience with living abroad increased the emigration intentions of students. We can see the importance of studying migration behaviour, especially among the young generation (students) which is confronted with the possibilities of voluntary migration already in high school. According to Kley (2009), completing school as a life-course event is the most important predictor of considering leaving the home city. This could be explained by people's need to plan their future and forthcoming situations and therefore, consider the possibility of leaving the home city.

While examining migration behaviour, it often comes up that actual migration may be experienced differently than originally expected. Changes in migration intentions occur. This could be explained by the fact that pre-migration intentions are not always in accordance with the real course of migration because many obstacles and new opportunities may occur during the migration process. We could perceive migrants as open and self-reliant individuals. The influence of personality characteristics in the process of migration was confirmed by Canache et al. (2013) who found openness to experience as well as extraversion to be positively related to emigration intentions. Gajdošová (2017) found that a higher self-efficacy and a need for change were significant for both genders in predicting emigration plans of Slovak university students. Also, people without migration experience have been found to be less willing to migrate again, compared to those with migration experience (Kupiszewski, 1996).

\section{Objective}

The main objective of this study was to explore whether factors such as emigration self-efficacy, evaluation of migration experience and the frequency of migration experience significantly contribute to the explanation of emigration intentions among young people (under 30 years old) in Slovakia. Based on the theoretical background, we hypothesized that higher emigration self-efficacy, more positive migration experience and more frequent migration experience would all have a positive and unique effect on emigration intentions among university students in Slovakia. We also tested the moderation effect of gender in the relationship between independent variables and dependent variable (emigration intentions).

\section{Research sample and procedure}

The research sample consisted of 474 university students from Slovakia $(M=22.4, S D=2.13)$ from which 76.8\% were women, who participated in the Student Life Cohort Study (SLiCE 2016) which focuses on risk behaviour and emigration/migration intentions of university students. The data for this sample were collected via an online survey. For the purposes of our study, standard multiple regression was used and the analysis was adjusted for gender.

\section{Measures}

Emigration intentions and the factors related to emigration intentions in our research sample were measured by the following variables:

- Emigration intentions were measured by a 5-item scale (Chan-Hoong \& Soon, 2011) which required respondents to rate the frequency with which they thought about working or living in another country for the following reasons: (1) overseas education, (2) better job prospects, (3) setting up a business, (4) to work and live in another country for an extended period of time, and (5) emigrating to another country to live there permanently. Each of these five items were scored on a 5-item Likert scale from 1 - never to 5 - all the time. Cronbach's alpha was 0.86. A higher overall score denotes a greater desire to migrate.

- Emigration self-efficacy was measured by three items: "If I wanted, I could easily leave abroad", "I am convinced that I would manage the process of leaving abroad" and "I am convinced that I would manage my stay abroad." Respondents rated their answers on 5-point Likert type scale, ranged from 1 - completely disagree to 5 - completely agree. A higher overall score indicates higher self-efficacy.

- Emigration experience, perceived as one's previous experience with living abroad, was measured with these two questions:

○ The first question was regarding the frequency of living abroad: "How many times have you left your country to live abroad?" rated from 1 - I have never been abroad, 2 - I have never lived abroad, 3 - once, 4 - twice, 5 - three times or more.

- The second question: "How would you now assess your experience with living abroad?" regards the positive, neutral or negative experience with emigration, rated from 1 - very bad, to 5 - very good. 


\section{Results}

The results show certain significant gender differences among young people in Slovakia ( $<30 \mathrm{yr})$. Males scored higher in self-efficacy and higher in frequency of their migration experience, compared to females. Further details can be found in Table 1.

Table 1. The descriptive characteristics in the measured variables according to gender in our research sample.

\begin{tabular}{|c|c|c|c|c|}
\hline Variable & Men & Women & $\begin{array}{c}\text { Theoretical } \\
\text { range }\end{array}$ & $\begin{array}{l}\text { T-test } \\
\text { value }\end{array}$ \\
\hline $\begin{array}{l}\text { Emigration } \\
\text { intentions }\end{array}$ & $\begin{array}{c}M=13.52 \\
(\mathrm{SD}=4.92)\end{array}$ & $\begin{array}{l}M=12.87 \\
(S D=5.2)\end{array}$ & $5-25$ & -1.16 \\
\hline $\begin{array}{l}\text { Emigration } \\
\text { self-efficacy }\end{array}$ & $\begin{array}{c}M=12.49 \\
(\mathrm{SD}=2.24)\end{array}$ & $\begin{array}{c}M=11.41 \\
(S D=2.68)\end{array}$ & $3-15$ & $-3.83 * * *$ \\
\hline $\begin{array}{l}\text { Evaluation of } \\
\text { migration } \\
\text { experience }\end{array}$ & $\begin{array}{c}\mathrm{M}=3.31 \\
(\mathrm{SD}=2.22)\end{array}$ & $\begin{array}{c}\mathrm{M}=3.39 \\
(\mathrm{SD}=2.28)\end{array}$ & $1-5$ & 0.317 \\
\hline $\begin{array}{c}\text { Frequency of } \\
\text { migration } \\
\text { experience }\end{array}$ & $\begin{array}{c}M=2.1 \\
(\mathrm{SD}=1.15)\end{array}$ & $\begin{array}{c}\mathrm{M}=1.8 \\
(\mathrm{SD}=1.1)\end{array}$ & $1-5$ & $-2.082 *$ \\
\hline
\end{tabular}

Next, our model explained $26.1 \%$ of the variance in emigration intentions. The factor emigration self-efficacy made the largest unique contribution in explaining emigration intentions $(\beta=0.373$, $p \alpha<0.001)$ followed by the factor evaluation of migration experience $(\beta=0.138, p \alpha<0.05)$ and the factor frequency of migration experience $(\beta=0.121, p \alpha<0.05)$. These findings indicate that a higher emigration self-efficacy, more positive migration experience and more frequent migration experience, all have a positive and unique effect on emigration intentions among university students in Slovakia.

Gender was not significant, so we couldn't confirm the moderation effect of gender in the relationship between the independent variables and the dependent variable (emigration intentions). More details are presented in Table 2.

Table 2. The results of linear regression analysis for each independent variable in relation to emigration intentions of young people in Slovakia (adjusted for gender).

\begin{tabular}{|c|c|c|c|}
\hline Variable & $\boldsymbol{\beta}$ & $\mathbf{t}$ & $\mathbf{p}$ \\
\hline $\begin{array}{c}\text { Emigration } \\
\text { self-efficacy }\end{array}$ & 0.373 & 8.53 & $<0.001$ \\
\hline $\begin{array}{c}\text { Evaluation of } \\
\text { migration } \\
\text { Experience }\end{array}$ & 0.138 & 2.48 & 0.014 \\
\hline $\begin{array}{c}\text { Frequency of } \\
\text { migration } \\
\text { Experience }\end{array}$ & 0.121 & 2.22 & 0.027 \\
\hline
\end{tabular}

\section{Discussion}

Many theories of migration point to the economic and demographic factors in attempting to answer what is behind people's intentions to migrate to another country (e.g. Massey et al., 1998). This question arises because migration to another country is often seen as a significant loss of highly talented 
and skilled people, who migrate in search for better career opportunities, better working conditions and a higher salary (Giannoccolo, 2009). Similar research has been conducted in Slovakia and found that job opportunities, the possibility of improving language skills and opportunity to travel and new experiences are the most important reasons for university students who are considering leaving (Benka \& Orosová, 2017). These factors alone, however, do not explain why some people who live in a very similar environmental and socioeconomic conditions, want to migrate to another country whereas others do not.

In order to understand the phenomenon of migration, we examined various psychological factors and their relation to emigration intentions. We confirmed that university students with a higher emigration self-efficacy, more positive migration experience and more frequent migration experience have greater intentions to leave Slovakia. This is in line with Kley (2009) who found that migration experience could be influenced by a bad migration experience, but also by other factors such as the level of income, breaking up with a partner or finding another one. On the other hand, Speare et al. (1975) found that migration experience did not affect considering but only planning migration. It was interpreted as the effect of a migrant's learned skills in dealing with uncertainty and the necessity of making new friends and adapting to their new environment. The main reason could be the fact that people with migration experience are more confident and self-efficient because they have already experienced migration, and therefore, are more prepared and more likely to migrate again. We can agree that migration is an experience full of uncertainty and novelty, mostly because migrants are exploring new locations and cultures, meeting new people and building new relationships. It is important to highlight that this alone is perceived as a positive phenomenon, but only in the case of people's future return as a well-educated workforce. This case certainly applies to Slovakia as like other European countries, Slovakia is also facing globalization and exodus of young and well-educated people to countries with better career opportunities and more developed economies. Therefore, Slovakia, as well as other countries, try to prevent this loss of well-educated students, which could be perceived as a negative phenomenon in the case they choose not to return to their home country.

The biggest limitation of this study concerns the measurement of the factors evaluating migration experience and frequency of migration experience, which we assessed by only single item measures. In the future, it would be helpful to direct our attention to a more complex examination of the relationship between migration experience and emigration intentions and include additional psychological factors important for students' efforts to emigrate. In addition, this study only focused on Slovak university students, so there is potential to study these psychological factors internationally, to provide a deeper understanding of migration behaviour outside of Slovakia. Based on our own research (Hajduch, Orosová, Kulanová, Hal, Lukács, 2018) we have already found that students in Belgium and Slovakia who want to stay in their country of origin (stayers) are more satisfied in life than leavers. Also, the highest percentage of undecided students from the explored countries was found in Belgium (56,5\%). These results could be an indication of another important factors related to emigration intentions not only in Slovakia, but also internationally.

\section{Conclusion}

In this study, we have addressed psychological factors such as emigration self-efficacy, evaluation of migration experience and the frequency of migration experience as important factors related to emigration intentions among university students in Slovakia. We have found that students who want to leave their home country report a higher level of emigration self-efficacy, more positive migration experience and also more frequent migration experience. Although many studies point to mostly economic reasons for migration, they cannot provide the full answer to why some individuals in similar economic conditions choose to stay and some choose to leave their home country. Therefore, we believe that exploring migration from an eclectic perspective may be beneficial for a better understanding of migration behaviour among the young generation of students in Slovakia and outside Slovakia as well.

\section{Acknowledgements}

This work was supported by Research and Development support Agency under the contract No. APVV-0253-11, APVV-15-0662 and Scientific Grant Agency VEGA 1/0713/15. 


\section{References}

Bahna, M. 2009. Pracovná migrácia zo Slovenska po vstupe do EÚ v kontexte krajín EÚ [Labor migration from Slovakia after joining $E U$ in the context of $E U$ countries]. Available at: http://www.sociologia.sav.sk/cms/uploaded/1216_attach_Pracovna_migracia_zo_Slovenska_v_ko ntexte_EU_10.pdf

Benka, J. (2017). Psychological contexts of students' migration from the perspective of the Self-determination theory. In: Orosová, O., Gajdošová, B. (Eds.), Emigration intentions and risk behaviour among university students, p. 147-166

Canache, D., M. Hayes, J. J. Mondak, S. C. Wals. (2013). Openess, extraversion and the intention to emigrate. Journal of Research in Personality 47, 351-355

Chan Hoong, L., \& Soon, D. A. (2011). Study of Emigration Attitudes of Young Singaporeans. Institute of Policy Studies, IPS Working Papers, (19). Retrieved from http://kyspp.nus.edu.sg/ips/wpcontent/uploads/sites/2/2013/06/wp19_ v2.pdf.

Gajdošová, B. (2017). Intrapersonal and interpersonal factors favouring and hindering plans for long-term migration abroad of university students after finishing university. In: Orosová, O., Gajdošová, B. (Eds.), Emigration intentions and risk behaviour among university students, p. 186-203

Giannoccolo, P, (2009). The Brain Drain: A Survey of the Literature. Università degli Studi di Milano-Bicocca, Department of Statistics, Working Paper No. 2006-03-02. Available at SSRN: https://ssrn.com/abstract=1374329

Hajduch, B., Orosová, O., Kulanová, M., Hal, G. V., Lukács, A. (2018). Life satisfaction and emigration plans of students from four EU countries. European Journal of Public Health, Volume 28, Issue suppl_4, 1 November 2018, cky218.049, available at: https://doi.org/10.1093/eurpub/cky218.049

Káčerová, M., Horváthová, M. (2014). Zahraničná migrácia Slovenska - demografické a priestorové aspekty. Slovak Statistics and Demography, 2, 33-51.

Kley, S. (2009). Explaining migration as a process of cumulative causation in the life course. In: Migremus Arbeitspapiere Nr. 2/2009

Kupiszewski, M. (1996). The future of East-West migration in Europe. In F. W. Carter, P. Jordan, \& V. Rey (Eds.), Central Europe after the fall of the Iron Curtain: Geopolitical perspectives, spatial patterns and trends (pp. 247-268). Frankfurt am Main, Germany: Peter Lang, Europaischer Verlag der Wissenschaften.

Massey, D. S., Arango, J., Hugo, G., Kouaouchi, A., Pellegrino, A., \& Taylor, J. E. (1998). Worlds in motion: Understanding international migration at the end of the millennium. Oxford, England: Clarendon Press.

Speare, A., Goldstein, S. and Frey, W. H. (1975). Residential Mobility, Migration, and Metropolitan Change. Cambridge: Ballinger Publishing. 


\title{
WORKPLACE RELATIONSHIPS AS A SYMPTOM OF ORGANIZATIONAL CULTURE AND A LEVER FOR CULTURE TRANSFORMATION
}

\author{
Olga Tararukhina \\ Ph.D., Saint Petersburg State University (Russia)
}

\begin{abstract}
This theoretical paper proposes an original approach to transforming organizational culture through the lever of workplace relationships. It explores the concept of organizational culture at five levels, from the most visible to the most deep and fundamental, the complexity of workplace relationships, their kinds and types. It further articulates how workplace relationships appear as a symptom of organizational culture, and how culture, both societal and organizational, influences interpersonal relationships at work, as well as lives in those relationships, and how a company's culture can be influenced and transformed through workplace relationships.
\end{abstract}

Keywords: Organizational culture, workplace relationship, professional relationship.

\section{Introduction}

Comparative meta-analysis of theoretical and empirical studies of workplace relationships in four societal cultures - in United States, China, Russia, and Sweden - revealed significant differences not only in how relationships work, but also in the place they occupy in the culture: value, purpose, key characteristics of workplace professional relationships, even time perspective, are very different (Tararukhina, 2016). It confirmed that the ways people hold and develop relationships, are reflective of societal culture. Culture and relationships are intertwined: relationships reflect culture, culture sustains and evolves through relationships. By nature of what culture is, organizational culture can also be reflected through workplace relationships: through processes of internal adaptation (Schein, 2004), employee behavior in the workplace (Cooke, 2015).

In the last decade, the subject of organizational culture has been getting more attention in popular business publications and magazines, as well as academic research, aiming to harness the power of culture in helping the company achieve significant business results, or in getting in the way of company's abilities to deliver. Yet, one of the carriers of culture, and levers of its transformation, workplace relationships, is typically left out of equation, giving the floor to the level of teams and groups. This paper proposes an original theoretical approach, that is based on empirical and theoretical research, as well as practical consulting work, to leveraging workplace relationships for culture transformation.

\section{State of the art}

Currently, the phrase "organizational culture" seems to be used so loosely that it may mean run the gamut between leadership effectiveness, employee engagement, reputation, climate, communication. Under these circumstances, it is only natural that when it comes to culture change, it would defer to the tools from traditional change management (primarily training and communication) or organizational design, at best.

Literature review shows an abundant amount of research and theories about human activity in the workplace, including fields like trust or motivation, and rather broad areas of leadership, negotiations, communication, conflict resolution, influence. These approaches are aiming to approach professional relationships through the perspective of the individual's skills, abilities, and personality and behavior; or through group dynamics and team effectiveness approaches, none of which were not intended to address the dyadic level of dynamics in organization.

With a few exceptions (such as Schutz, 1966, 2004; Fiske, 1992; Schein, 2004), most of those approaches fail to get to the core of what a relationship is in the workplace: what it consists of, and what is this intangible element that seems to make or break the effectiveness of task execution: some things get longer to execute and take more resources than others. However, when it comes to culture transformation, even these researchers seem to focus on the organizational and individual level, as opposed to dyadic level of culture. 


\subsection{Organizational culture transformation}

Existing approaches to culture transformation follow the way their authors understand the culture itself. Many propose change management approaches to the process that evolves and transforms, but doesn't change "like a used car" (Katzenbach, 2012). Some would say that it is semantics, but transformation suggests transforming at the core, while change suggests replacement of an element.

Organizational culture constantly evolves, while also remains whole and complete in each moment. That is achieved through culture also being a system of a complex set of elements at different levels. Even when some elements of culture could be perceived as healthy or dysfunctional, these elements are a part of one system, they are in relationship with one another: they reinforce, balance, support, hinder etc. Any one element (e.g. behavior X) is deeply interconnected and may even be interdependent with others (value $\mathrm{X}$ and behavior $\mathrm{Y}$ ). Eliminating one is not only impossible (it will be re-installed by the rest of the system), it is also impractical: even when seen as dysfunctional, it is a source of past success, stability, and meaning. Therefore, there is no culture that one can prescribe as good for every company out there; culture is organization-specific, nor can it be simply replaced by another, there needs to be a thoughtful approach that appreciates and leverages the evolutionary nature of culture, that helps it evolve to the desired state without making the "old" one wrong or bad.

\subsection{Organizational culture and workplace relationships}

There have been several substantial approaches proposed to articulate the way organizational culture determines and rules relationships. Schutz (1966, 2004) identified interpersonal needs (inclusion, control, and openness) that are defined behaviorally in action and feeling, as well as directionally: self-other, other-self, and self-self. According to Schutz (2004), these dimensions at all levels determine what culture could deliver the best performance when individuals have their interpersonal needs met (based on the needs). This approach refers to one-to-many dynamics when it comes to the organizational culture work. However, interpersonal needs appear to hold important in the one-to-one dynamic as well.

Fiske (1992, 2019) proposed that people in all cultures use these models to organize much of their everyday social cognition according to four kinds of relationships:

- market pricing: "relationships are oriented to socially meaningful ratios or rates such as prices, wages, interest, rents, tithes, or cost-benefit analyses" (Fiske, 2019),

- authority ranking: "people have asymmetric positions in a linear hierarchy in which subordinates defer, respect, and (perhaps) obey, while superiors take precedence and take pastoral responsibility for subordinates" (Fiske, 2019),

- communal sharing: "a relationship in which people treat some dyad or group as equivalent and undifferentiated with respect to the social domain in question" (Fiske, 2019), and

- equality matching: "people keep track of the balance or difference among participants and know what would be required to restore balance" (Fiske, 2019).

These four kinds can describe all relationships within certain situations: the same pair of relationship partners can engage in all four of those in the same day, depending on the situation they are in and the subject they are interacting about.

Schein (2004) proposed that individuals create assumptions through answering questions about the proper ways to relate to each other in order to make the group safe, comfortable, and productive:

- about identity and role in the group (Who am I supposed to be in this group and what will be my role?);

- $\quad$ about power and influence in the group (Will my needs for influence and control be met?);

- about needs and goals (Will the group's goals allow me to meet my own needs?);

- about acceptance and intimacy in the group (Will I be accepted, respected, and loved in this group? How close will our relationship be?).

This approach allows to see how individual can adapt to, and be included into the group, a relationship between one-to-many. However, this approach arguably does not translate very well to the the dyadic level, one-to-one interpersonal relationship, where both individuals create dynamics from the beginning, there is nothing to be included in, both relationship partners by nature of what is an interpersonal relationship, create one "from scratch". The social needs articulated by Schein (2004), appear relevant to the one-to-one relationship as well.

\section{New perspectives / contributions}

From the brief overview above, it becomes clear that the theory of organizational culture needs clarification in terms of its determining professional relationships and being reflected through them, which would also allow for the development of a culture transformation approach that is grounded in social processes of culture rather than change management. Research on workplace relationships (or professional relationships) needs to address the phenomenon of the relationship itself at the dyadic level, as opposed to indirect description and studies of what influences them: there needs to be more clarity as to what a professional relationship consists of, how it develops, what are its kinds etc. 
This paper presents an attempt to meet all three requests.

\subsection{Original theory of organizational culture}

Schein (2004) proposed a model of organizational culture that consists of three levels: artifacts, espoused behaviors and values, and underlying assumptions. The proposed theory suggests that there is a need for more specificity, for discernment of layers of Schein's level 2, "espoused behaviors and values". Those are distinctly different layers of culture, because: 1) they have different functions; 2) they are experienced differently, and 3) they have a different impact. We can observe a behavior, such as a smiling (or not) coworker coming up to us in the hallway; we know what the norms and rules are in each kind of meeting, what is expected of each role in each meeting; and after we got used to the culture, we almost never question why we do what we do, what's important about doing it the "way we do it around here".

By making this clarification, the proposed model of organizational culture has 5 layers, that like layers of an onion, reveal culture from its most visible elements (language, space, symbols) to the most latent ones (basic assumptions):

Figure 1. "Onion" model of the five layers of organizational culture.

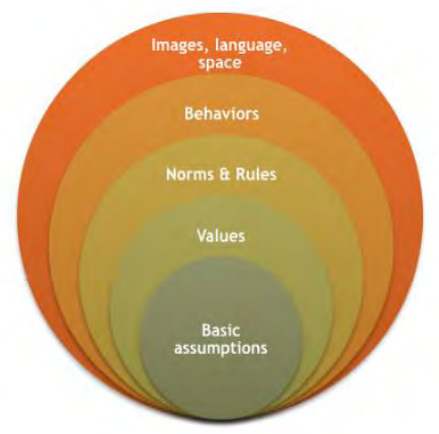

In this model, the layers represent:

- Images / language / space: observable, tangible materials and objects;

- Behaviors: observable actions people take in interactions and in doing their work;

- Norms: shared understanding of the distinction between what's right and wrong, what's acceptable and what's not; Rules: dos and don'ts as well as knowing or the ability to forecast their consequences;

- Values: what is held important when members of the organization take action;

- Basic assumptions: beliefs that we assume to be true and do not question or typically even discuss.

Following Schein's definition, culture in this model is also defined as "the shared way things are done around here" that develops through socialization, trial and error on the way to success. Its key functions are to make things predictable, familiar; to explain what things mean; to determine what to pay attention to and what to react to, and how to act/react; to keep the organization's memory about what leads to success, stability and meaning (Schein, 2004).

It is also important to underscore what does not appear in the description above. Since culture evolves through socialization, through learning from other people about culture of a specific organization (e.g. incumbents enculturate newcomers), and as a result of this learning process, culture not only lives in group meetings, it gets transmitted and transformed through the vehicle of interpersonal workplace relationships: they are an important carrier of organizational culture.

\subsection{Original theory of workplace relationships}

What is unique about workplace relationships, is that they are both personal and professional, they develop in the context of organizational and societal culture, they are influenced by the roles relationship partners play (e.g., boss and employee), there is an implied and explicit hierarchy that may be different from the hierarchy in the society where the relationship takes place. To add another dimension to an already complex system, consider this: one can't end a professional relationship, unless they change the company; and coworkers change roles: today's boss can be a peer tomorrow. Add to the picture politics and potential incivility, as well as emergent introduction of robots, and we get the contemporary system of professional relationships.At work, employees have several types of relationships, main four include: with the immediate manager, with peers we have immediate work with in common, other leasers in the organization, and other peers who we don't share immediate work. Professional relationships that are connected through hierarchy or through work in common are stronger than those that are only connected through belonging to the same organization. 
Presented here original approach to the concept of workplace relationships (Tararukhina, 2016), is focused on the relationship itself, its quality, not the relationship partners as individuals or their behaviors. First, according to our theory of workplace relationships (Tararukhina, Gurieva, 2015; Tararukhina, 2016), they consist of:

- Clarity: understanding roles of relationship partners, as well as each other's' identities and motivation;

- Significance: attributing value, importance to the other person (both as a person and as a coworker);

- Trust: ability to confide in the other, be vulnerable to their actions, take the risk on them;

- Closeness: intensity, flow of exchange of thoughts and feelings with each other; experience of being connected.

Second, there are four kinds of relationships (Tararukhina, 2016): the degree to which relationship is focused on meeting the yearnings of each relationship partner, determines where and how the satisfaction with the relationship is tilted between them in a difficult situation, when there is tension between relationship partners. The kinds of relationships are:

- Imposing and Pleasing: one of the partners insists that their yearnings (Wright and Wright, 2012) be met at the expense of the other; one of the partners insists that the yearnings of another be met at the expense of their own;

- Resonance: both partners successfully meet the yearnings of each other and treat them as equally important to both of them;

- Dissonance: both partners retreat from meeting their own and the other's yearnings.

All four kinds of relationships exist in all organizations, so to understand culture through relationships, we need to understand not only the prevailing kinds of relationships, but also how each of these relationships reflects the layers of culture, what is more common for a specific organization, and what is this organization's unique "flavor" of each kind of relationship. These kinds of workplace relationships show up in all the layers of culture, from language to basic assumptions, and can reflect culture as its symptoms. They are also symptoms of societal cultures (Tararukhina, 2016, 2019a, b, c).

To identify that, the following questions need to be answered:

- What are the language, behaviors, norms/rules, and values of Imposing and Pleasing in this organization?

- What are the language, behaviors, norms/rules, and values of Resonance in this organization?

- What are the language, behaviors, norms/rules, and values of Dissonance in this organization?

\subsection{Original approach to transforming organizational culture through workplace relationships}

Culture transformation typically is thought of in conjunction with a big shift in business strategy, or market, or political environment. There is also a natural transformation that occurs in organizational cultures, as the company grows, matures, and evolves. It takes place at all levels - organizational, team level, and at the dyadic level (in interpersonal relationships). At organizational level, culture integrates (honoring consistency of the sum of parts, "overarching culture") and differentiates (honoring diversity of subcultures). At the team/group level, the focus is on increasing group performance through productivity and culture. At the dyadic level, it appears as influencing culture by choices about what dots to connect, what questions to ask and focus on, what to highlight as important, what to reinforce. These micro-moves can create significant shifts over time. It happens both formally and informally; sources of power help the influence, as well as closeness of the relationship, through intersubjectivity (Buber, 1992).

When new employees join the group, the organization has to enculturate them. It is also on the new members/employees to seek to learn new culture. One of the most important things newcomers learn is the rules of engagement in their new organization. Professional relationships are actually one of the most tangible ways to experience and understand what organizational culture is like, as well as go to the meetings. When culture is transforming, relationship partners can lean on their relationships quality (determined by trust, closeness, significance, clarity) to navigate from the old towards the new culture:

- At the Behaviors layer: relationships moderate observable actions;

- At the Norms and Rules layer: relationships are a locus of meaning making;

- At the Values layer: relationships provide relative importance of actions and results, from the perspective of the new culture, help outline, underscore the importance and meaning of the new culture.

\section{Practical implications}

This approach provides a more tangible and grounded way to achieve successful culture transformation in organizations than change management and communication alone, because it leverages the element of culture that connects the individuals at the most human level, their yearnings. This approach is agnostic of industry and kind of company, which makes it very flexible for organizational leaders to 
understand and execute. It can help organizational leaders inform their leadership styles, increase their versatility and relational skills, and help others do the same.

\section{Originality and value}

This approach and theory, contrary to most known to us, concerns with the quality of professional relationships, their content, kinds, and development, and how they reflect organizational culture; though that, this approach proposes an original perspective on culture transformation that has the potential to be more powerful and sustainable than behavior training alone.

\section{Questions for future research}

Going forward, one avenue of research could be to identify whether there is a correlation between the kinds of business strategies and kinds of workplace relationships, due to the significant relationship between organizational culture and business strategy.

\section{References}

Boisnier A., Chatman j. (2002). The role of subcultures in agile organizations. Retrieved from http://faculty.haas.berkeley.edu/chatman/papers/20_CulturesSubculturesDynamic.pdf

Buber M. (1992). On intersubjectivity and cultural creativity. Chicago and London: The University of Chicago Press.

Cooke R. (2015, October). How Culture Really Works. Paper presented at the 1st Ultimate culture conference. Chicago, IL.

Fiske A.P. (1992). The four elementary forms of sociality: Framework for a unifying theory of social relations. Psychological Review, 1992, Vol. 99, N 4, 689-723.

Fiske A.P. (2019). Human Sociality. Retrieved from http://www.sscnet.ucla.edu/anthro/faculty/fiske/relmodov.htm

Gibb J. (1991). Trust. A new vision of human relationships for business, education, family, and personal living. Newcastle Publishing, CA.

Katzenbach J. (2012). Culture change that sticks. Harvard Business Review. July-August 2012. Retrieved from https://hbr.org/2012/07/cultural-change-that-sticks

Kluckhohn F.L., Strodtbeck F.R. (1961). Variations in value orientations. Row, Peterson and Company.

Lewin K. (1997). Resolving social conflicts. Field theory in social science. (Reprinted ed.) American Psychological Association.

Myasishchev V.N. (1995). Psihologiya otnoshenij. [Psychology of relationships]. Moskva-Voronezh: NPO MODEHK.

Obozov N.N. (1979). Mezhlichnostnoe obshchenie. [Interpersonal interaction]. Leningrad: Izdatel'stvo LGU.

Quinn R.E. (1996). Deep change: Discovering the leader within. John Wiley \& Sons.

Schein E. (2004). Organizational culture and leadership. (3rd ed.) San Francisco, CA: John Wiley \& Sons.

Schutz W. (1966). The interpersonal underworld. Science \& Behavior Books, Inc.

Schutz W. (2004). The human element. Business Consultants, Inc.

Tararukhina O. (2016). Cross-cultural variations in satisfaction with interpersonal relationships in the workplace. Doctoral dissertation, Saint Petersburg State University, Saint Petersburg, Russia. Retrieved from http://www.dslib.net/soc-psixologia/kross-kulturnye-osobennostiudovletvorjonnosti-mezhlichnostnymi-otnoshenijami-v.html

Tararukhina O.V., Gurieva S.D. (2015). Social'no-psihologicheskaya model' razvitija mezhlichnostnyh otnoshenij v organizacii [Social-psychological model of development of interpersonal relationships in the workplace]. // Sovremennye issledovaniya social'nyh problem. 2015, 1 (21), 26-40.

Tararukhina O. (2019, March) a. Specifics of Workplace Relationships in Russia. Paper presented at the third biennial International Convention of Psychological Science (ICPS). Paris, France.

Tararukhina O. (2019, March) b. American Business Culture through the Lens of Workplace Relationships. Paper presented at the third biennial International Convention of Psychological Science (ICPS). Paris, France.

Tararukhina O. (2019, April) c. Workplace Relationships as Symptoms of the US Business Culture. Paper presented at the annual meeting of Midwestern Pryschological Association. Chicago, IL.

Worley C., Williams T., Lawler E. (2014) The agility factor: Building adaptable organizations for superior performance. San Francisco, CA: Jossey-Bass.

Wright, R., Wright J. (2012). Transformed! The science of spectacular living. Nashville: Turner Publishing. 


\title{
CYBERBULLYING VS. TRADITIONAL BULLYING - DO VICTIMS REACT DIFFERENTLY?
}

\author{
Meyran Boniel-Nissim \\ School of Social Sciences and Humanities, Kinneret Academic College, (Israel)
}

\begin{abstract}
Bullying is a deliberate use of force or coercion to abuse or intimidate others. Cyberbullying is bullying that takes place in the virtual space over digital devices like cell phones, computers, and tablets. In view of the differences between physical and virtual spaces, it can be presumed that differences would be found between the bullying victims' coping strategies. This study hypothesized that children who are victims of traditional bullying tend to report it at school, whereas cyberbullying victims use the characteristics of the virtual space (anonymity, invisibility, lack of eye contact) to retaliate against their aggressors. The sample included $1,2953^{\text {rd }}$ - to $9^{\text {th }}$-graders, of which 955 responded that they had been victims of at least one type of bullying (either traditional or cyber). The study found that victims of traditional bullying tended to act to break out of the situation more than cyberbullying victims did. Moreover, it was found that victims of traditional bullying tended to report it at school, and cyberbullying victims tended to report it to parents. However, the hypothesis that victims of cyberbullying would use cyber characteristics to retaliate was not supported. Additionally, a link was found between the victims' age and their use of anti-bullying strategies: The younger they were, victims of traditional bullying tended to react.
\end{abstract}

Keywords: Cyberbullying, traditional bullying, victims, school, action strategies.

\section{Coping strategies of victims of traditional bullying and cyberbullying}

The literature links bullying behaviors and victimization behaviors, so that the victim may at times exhibit bullying behavior and vice versa (Machmutow, Perren, Sticca, \& Alsaker, 2012; Völlink, Bolman, Dehue, \& Jacobs, 2013). Consequently, another action strategy available to the victim is causing harm, whether to the bully or to others. A study conducted on children and adolescents (aged 8-18) found a significant relationship between victims' online behavior and bullies' online behavior (Boniel-Nissim \& Dolev-Cohen, 2012). Furthermore, evidence indicates that cyberbullying victims, mainly boys, perform antisocial behaviors as observers of cyberbullying (Cao \& Lin, 2015). Victims who choose action strategies of repaying in the same coin, called in the literature bully-victim, tend to express emotions (for instance anger or nervousness) and to respond more aggressively to stressful situations, and usually do not opt for solution-oriented response strategies (Völlink et al., 2013). Conversely, response strategies characterized by assertiveness and seeking support were found to moderate the relationship between cyberbullying victims and depression symptoms. Namely, the more victims were active and positive about changing their situation, the lower levels of depression they exhibited (Machmutow et al., 2012).

In view of the paucity of research literature on action strategies, and the greater understanding that there is a difference between bullying phenomena in various spaces, which might require different response strategies, the aim of this study was to examine children's response strategies to traditional bullying and cyberbullying. Based on the research literature, it can be assumed that a significant difference would be found between the uses of action strategies in the various spaces. It is expected that the supervision in the education system would encourage the use of seeking help and problem-solving with the help of the school staff when the bullying occurs on school grounds. Additionally, the face-to-face encounter with the bully, which increases the likelihood of physical harm, would increase the use of avoidance strategies. On the other hand, unsupervised cyberspace, which is characterized by disinhibition, would increase the use of retaliation through the virtual space.

Hence, this study hypothesized that a significant difference would be found between strategies employed by victims of cyberbullying as opposed to victims of traditional bullying.

1. Victims of traditional bullying tend to report to the education system more than victims of cyberbullying do.

2. Victims of traditional bullying tend to use avoidance more than victims of cyberbullying do.

3. Victims of cyberbullying tend to use bullying behavior towards the bully, via the internet, compared to victims of traditional bullying. 


\section{Methods}

The data were gathered online through 'Small-talk' panel during February-March 2015. 'Small-talk' panel is a children's internet panel, operated by a research institute, which enables examining opinions and conducting surveys among young children (ages 8-14; elementary and middle school). The panel includes about 1,500 Hebrew-speaking members, whose parents signed the legal regulations and agreed to let their children answer questionnaires, which they receive through an online link. The panel is run under strict Esomar guidelines.

A notification to the parents about the survey was published on the panel with a link to the questionnaire. After agreeing to participate, any panel member (child) could access the link and answer the questionnaire. The child could leave the questionnaire at any stage. The children were given the option to be helped by a parent if the questions were unclear. The questionnaire opened with a question about age and school grade in order to facilitate a sample limited to the required age. The quota sampling method was used; namely, when it reached the target number of respondents that answered fully and properly, the questionnaire was removed. The required quota was 1350 children. Fifty-five children partially completed the questionnaire and therefore were removed from the total sample.

\section{Sample}

The sample included 1,295 respondents aged 9-14 (3rd to 9th grade) who answered the questionnaire fully. Of them, 955 reported that they had been subjected to some kind of bullying (traditional or cyber). Nine hundred and thirteen children were affected in the physical space (95.6\%), 605 were affected in cyberspace (63.3\%), and 548 (57.4\%) reported having been victimized in both. Most children were in elementary school (3rd grade $-9 \%$, 4th grade $-27 \%$, 5th grade $-26 \%$, 6th grade $-18 \%$, 7 th grade $-8 \%$, 8th grade $-7 \%$, 9 th grade $-5 \%)$. Forty-one percent were boys.

\section{Measures}

Personal details: age, grade, gender

Traditional bullying (Lev-Wiesel, Sarid, \& Sternberg, 2013): twenty six items concerning social rejection by other children at school. Each item is phrased as a statement that expresses a form of bullying/social rejection, answered on a Likert scale of 1 (never) to 5 (every day or almost every day). The questionnaire addresses five kinds of bullying: Rejection (for example, "Friends ignored me"); physical harm (for example, "Friends threw things at me"); insults (for example, "Friends called me names"); accusations (for example, "Friends blamed me for bad things that had happened"); and manipulation (for example, "Friends threatened me"). The questionnaire was examined by means of Cronbach's alpha internal consistency coefficient, and high reliability was found $(\alpha=0.96)$.

Cyberbullying (Menesini, Nocentini, \& Calussi, 2011): A list of 16 situations of cyberbullying, answered on a Likert scale of 1 (never) to 5 (every day or almost every day). Cyberbullying situations relate to four types: Public humiliation (for example, "Someone changed my picture in a negative way and published it"); cruelty (for example, "Someone cursed me online"); deception (for example, "Someone deceived me online"); and harassment (for example, "I received unwelcome sexual messages from someone I know"). The instructions made it clear that the virtual space included the internet, social networks and smartphone apps. The questionnaire had high reliability ( $\alpha=0.92)$.

Action strategies (Rolider, Lapidot-Lefler, \& Levy, 2000): The questionnaire raises action possibilities when the child experiences bullying. The items include reporting action (seeking help from a parent/friend/school staff); offensive actions based on disinhibition and retaliation ("harm the bully", "harm other children", "use the internet to retaliate"); avoidance actions ("stay at home", "do nothing", distance myself physically or disconnect from the bully"); and direct communication with the bully ("ask the bully to stop"). The questionnaire includes 13 items on a yes/no scale and was presented separately for traditional bullying and for cyberbullying.

\section{Findings}

Traditional bullying (at school): Sixty-nine percent of the respondents reported some form of bullying over the last year. The percentage of bullying at school was significantly higher among $3^{\text {rd }}-4^{\text {th }}$ grade children than among $5^{\text {th }}-6^{\text {th }}$ grade and $7^{\text {th }}-9^{\text {th }}$ grade children $\left(\chi^{2}=35.23, \mathrm{p}<.001\right)$. Eighty-three percent of the $3^{\text {rd }}-4^{\text {th }}$ grade children reported having experienced bullying and harassment at school, 69\% in $5^{\text {th }}-6^{\text {th }}$ grade, and $62 \%$ in $7^{\text {th }}-9^{\text {th }}$ grade. No significant difference was found in bullying frequency between boys (70\%) and girls (68\%).

Cyberbullying: Forty-eight percent of the children reported occurrences of cyberbullying. A significant difference was found between boys (40\%) and girls (52\%) (Chisq. $=17.87, \mathrm{p}<.001$ ). No 
significant differences were found between the age groups victimized by cyberbullying, which ranged between $45 \%$ and $48 \%$.

Action strategies of victims of traditional bullying: of the respondents who experienced traditional harassment $(\mathrm{N}=913), 6 \%$ stated that they would do nothing. Hence, $94 \%$ used at least one strategy to cope with bullying. The frequency of the various strategies, from the most frequent to the least frequent, was: seeking help from someone at school (59\%), distancing oneself from the bully (55\%), telling the bully to stop (51\%), seeking help from a family member (46\%), seeking help from a fellow student (24\%), retaliating against the bully (23\%), going to the police (4\%), staying at home (4\%), harming the bully via social networks (2\%), and harming other children (2\%). In general, it seems that reporting actions are more frequent in cases of bullying at school, as are avoidance actions (distancing oneself from the bully).

Action strategies of victims of cyberbullying: of the victims of cyberbullying ( $\mathrm{N}=605), 10 \%$ stated that they would take no action. On the other hand, 90\% employ various strategies (at least one) against their situation. The frequency of the various strategies, from the most frequent to the least frequent, was: seeking help from family (44\%), telling the bully to stop (36\%), disconnecting or blocking the bully (33\%), seeking help from school staff (26\%), hurting the bully (15\%), seeking help from a fellow student (14\%), going to the police (9\%), harming the bully via cyberspace (6\%), staying at home (2\%), harming other children (1\%), and damaging other children's property (1\%). Contrary to the hypothesis, it seems that the strategy of retaliating via cyberspace was reported at a very low frequency.

Action strategies of victims of traditional bullying and cyberbullying by gender: we found a significant difference between boys and girls regarding retaliation against and harming the bully. A higher percentage of boys who were victims only of traditional bullying retaliated than girls did (25\% vs. $7 \%$ ). Girls who were victimized by both traditional bullying and cyberbullying responded more than boys did by distancing themselves from the bully (19\% vs. $11 \%)$. Girls sought help from someone at school and from fellow students more than boys did.

When examining the differences between boys and girls in the physical space, it is obvious that the strategy of retaliating was more frequent among boys (37\%) than among girls (10\%). Conversely, distancing oneself from the bully was more characteristic of girls (62\%) than of boys (48\%). Reaching out for help from a family member was more common among girls (50\%) as opposed to boys (42\%), as was seeking help from school staff $-64 \%$ for girls and $53 \%$ for boys.

The differences between boys and girls maintain a similar trend when examining cyberbullying victims (see Table 2). The strategy of retaliating was more frequent among boys (26\%) than among girls (7\%). Seeking help from a family member was more frequent among girls (48\%) than among boys (37\%), as was seeking help from school staff (31\% and 19\%, respectively). On the other hand, the strategy of disconnecting from the bully by means of blocking him/her online was equally frequent among boys and girls (33\%).

Action strategies of victims of traditional bullying and cyberbullying by age: when the harm is in physical space, a strategy of retaliating and hurting the bully was more common among $3^{\text {rd }}-4^{\text {th }}$ grades and $7^{\text {th }}-9^{\text {th }}$ grades. In all three age groups, responses were less when the bullying only took place in cyberspace. It was also found that seeking help from school staff was frequent in instances of traditional bullying, and decreased the older the victims were. Seeking help from family members also decreased the older the victims of traditional bullying were. Conversely, the use of this strategy increased when they were victims of cyberbullying.

When examining the differences between victims of traditional bully by age groups, it is obvious that the strategy of retaliating was more frequent among $7^{\text {th }}-9^{\text {th }}$ grades $(30 \%)$ than among $3^{\text {rd }}-4^{\text {th }}(19 \%)$ and $5^{\text {th }}-6^{\text {th }}$ grades $(18 \%)$. Seeking help from school staff decreased significantly with age: in $3^{\text {rd }}-4^{\text {th }}$ grades $70 \%$ sought help at school, whereas in $5^{\text {th }}-6^{\text {th }}$ grades $58 \%$ and in $7^{\text {th }}-9^{\text {th }}$ grades $52 \%$ did so.

When the harm was inflicted in cyberspace, the strategy of retaliating against the offender also increased with age: $18 \%$ in $7^{\text {th }}-9^{\text {th }}$ grades versus $14 \%$ in 5 th-6th grades and $9 \%$ in $3^{\text {rd }}-4^{\text {th }}$ grades. The strategy of doing nothing was more frequent in lower grades: $15 \%$ in $3^{\text {rd }}-4^{\text {th }}$ grades, $11 \%$ in $5^{\text {th }}-6^{\text {th }}$ grades, and only $8 \%$ in $7^{\text {th }}-9^{\text {th }}$ grade. It should be noted that the opposite happened when the bullying took place in physical space.

Comparison between action strategies of traditional bullying and cyberbullying victims: the use of action strategies by victims of cyberbullying was less than the use of action strategies by victims of traditional bullying. The findings indicated a statistically significant difference in most response strategies to traditional bullying versus cyberbullying. In general, the percentage of respondents that employed an action strategy was higher in instances of traditional bullying. The strategy that was more frequent among cyberbullying victims was to retaliate through cyberspace. Additional strategies that were significantly higher in cyberspace victims were "does nothing" and "does something else" (i.e., an action that was not specified in the questionnaire). There were only two action strategies for which no significant difference was found between traditional bullying and cyberspace victims - staying at home and seeking help from the family. 


\section{Discussion and conclusion}

Victims of cyberbullying are less inclined to act than victims of traditional bullying are. These findings are supported by previous research that shows that cyberbullying victims tend not to report these transgressions (Agatston, Kowalski, \& Limber, 2012; Parris et al., 2012). The reasons for not reporting are varied and include, among others, fear of losing access to technology (Cassidy, Jackson, \& Brown, 2009); fear that adults would not really be able to help, the bully's anonymity that makes concrete reporting difficult, mistrust of educators to understand or take the problem seriously, fear of being blamed for their aggressive reaction toward the bully, embarrassment, and fear of being labelled a victim (Agatston et al., 2012; Cassidy et al., 2009; Smith, 2012; Smith \& Slonje, 2010).

Avoidance strategies are used at a different frequency when the bullying occurs in the physical space in contrast to the virtual space. Six present of the victims of traditional bullying (at school) stated that they would not do anything against the bully versus $10 \%$ of cyberbullying victims. Since the literature has indicated that the impacts of cyberbullying on victims is similar to those of traditional bullying - and include depression, low self-esteem, anxiety, suicidal thoughts, and psychosomatic symptoms such as headaches and sleeping problems (Menesini \& Nocentini, 2012; Olweus, 2012; Smith, 2012) - it can be assumed that the high incidence of "do nothing" does not stem from a feeling that cyberbullying is less severe. It could be that children are more threatened by taking action online, or lack the appropriate tools to defend themselves. Moreover, it would seem that they act in other ways that were not mentioned in the questionnaire, because a considerable percentage replied that they "do something else".

The hypothesis that victims of traditional bullying are more likely to seek help at school was supported. It should be noted that although seeking help from school staff is the most frequent choice of traditional bullying victims, it is the fourth most frequent choice, and at a significantly lower percentage, of cyberbullying victims. Perhaps, cyberbullying victims do not experience school as the place for aid and assistance, because the bullying did not take place on school grounds. Schools do conduct interventions on the issue of cyberbullying, but it seems that unlike traditional bullying, children do not perceive cyberbullying as the school's responsibility.

However, whereas seeking help from parents is the most common choice of cyberbullying victims, this strategy is in fourth place when it comes to traditional bullying victims. It is possible that this mirror image is due to the data on seeking help at school. Namely, the natural turning for help to the school staff when bullying occurs on school grounds reduces the need to tell parents. Correspondingly, in instances of cyberbullying, the lessened seeking of help at school increases the need to tell parents and ask for their help. The ranking of this strategy is different for the physical and virtual spaces, but the frequency of seeking help from parents by victims of traditional bullying and cyberbullying is not significantly different.

As expected, victims of traditional bullying respond primarily by seeking help at school (59\%) and by physically distancing themselves from the bully (55\%). Concerning victims of cyberbullying, it seems that the option to strike back online (in view of the internet's disinhibition features) was not corroborated in this study, showing low frequency (6\%) relative to other possible action strategies. However, it should be noted that comparing the response strategies of victims of traditional and cyberbullying revealed that victims of cyberbullying tens to retaliate against the bully online at a significantly higher frequency than victims of traditional bullying do.

In cases of cyberbullying, it seems that disconnecting or blocking the bully is the most common strategy (33\%). This finding is supported by a study that found that in cases of cyberbullying, those involved are afraid to be perceived as 'informers', and also feel that there is not much that can be done about it, so they prefer avoidance strategies (such as deleting messages) over using other response strategies (Parris et al., 2012).

In this study, the frequency of sharing and seeking help from one's peer group was low (14\%), nevertheless research has shown that the victim's best chance to receive effective help is from friends who come to his/her assistance (Li, 2010). Furthermore, strategies of seeking help following victimization in the physical space are more frequent the younger the victim is. The findings show that $70 \%$ of $3^{\text {rd }}-4^{\text {th }}$ grade children seek help from school staff as opposed to $52 \%$ of $7^{\text {th }}-9^{\text {th }}$ graders, and $52 \%$ of $3^{\text {rd }}-4^{\text {th }}$ grade children go to someone in their family for help as opposed to $41 \%$ of the older children ( $7^{\text {th }}-9^{\text {th }}$ grades). Additionally, girls were found to use more action strategies than boys do, except for actions that involve retaliation against the bully, in which boys showed higher frequency. On the other hand, unlike victims of traditional bullying, no differences were found between the various age groups of cyberbullying victims. But, the gender distinction was maintained here as well: girls tend to use more action strategies than boys do, except for actions that involve retaliation against the bully, in which boys showed higher frequency. It could be that the reason for this is that bullying among girls is usually concerned with damaging one's reputation rather than using physical force, and as such fits the definition of cyberbullying (Smith, 2015). Moreover, most incidences of cyberbullying occur on social networks, which girls use more (Whittaker \& Kowalski, 2015). 


\section{References}

Agatston, P., Kowalski, R., \& Limber, S. (2012). Youth views on cyberbullying. In J.W. Patchin \& S. Hinduja (Eds.), Cyberbullying Prevention and Response: Expert Perspectives (pp. 57-71). New York: Routledge.

Boniel-Nissim, M., \& Dolev-Cohen, M. (2012). Children writing on walls: The Facebook culture. Study and Research of Teacher Training, 13, 207-231. (Hebrew)

Cao, B., \& Lin, W. Y. (2015). How do victims react to cyberbullying on social networking sites? The influence of previous cyberbullying victimization experiences. Computers in Human Behavior, 52, 458-465. doi:10.1016/j.chb.2015.06.009

Cassidy, W., Jackson, M., \& Brown, K. N. (2009). Sticks and stones can break my bones, but how can pixels hurt me? Students' experiences with cyber-bullying. School Psychology International, 30, 383-402. doi:10.1177/0143034309106948

Kowalski, R. M., Morgan, C. A., \& Limber, S. P. (2012). Traditional bullying as a potential warning sign of cyberbullying. School Psychology International, 33, 505-519. doi:10.1177/0143034312445244

Lev-Wiesel, R., Sarid, M., \& Sternberg, R. (2013). Measuring social peer rejection during childhood: Development and validation. Journal of Aggression, Maltreatment \& Trauma, 22(5), 482-492.

Li, Q. (2010). Cyberbullying in high schools: A study of students' behaviors and beliefs about this new phenomenon. Journal of Aggression, Maltreatment \& Trauma, 19, 372-392. doi:10.1080/10926771003788979

Machmutow, K., Perren, S., Sticca, F., \& Alsaker, F. D. (2012). Peer victimization and depressive symptoms: Can specific coping strategies buffer the negative impact of cyber victimization? Emotional and Behavioural Difficulties, 17, 403-420. doi: 10.1080/13632752.2012.704310

Menesini, E., Nocentini, A., \& Calussi, P. (2011). The measurement of cyberbullying: Dimensional structure and relative item severity and discrimination. Cyberpsychology, Behavior, and Social Networking, 14(5), 267-274.

Menesini, E., \& Nocentini, A. (2012). Peer education intervention: Face-to-face versus online. In A. Costabile \& B. A. Spears (Eds.), The Impact of Technology on Relationships in Educational Settings (pp. 139-150). New York, NY: Routledge.

Niemelä, S., Brunstein-Klomek, A., Sillanmäki, L., Helenius, H., Piha, J., Kumpulainen, K., \& Sourander, A. (2011). Childhood bullying behaviors at age eight and substance use at age 18 among males. A nationwide prospective study. Addictive Behaviors, 36, 256-260. doi:10.1016/j.addbeh.2010.10.012

Olweus, D. (2012). Cyberbullying: An overrated phenomenon? European Journal of Developmental Psychology, 9, 520-538. doi: 10.1080/17405629.2012.682358

Parris, L., Varjas, K., Meyers, J., \& Cutts, H. (2012). High school students' perceptions of coping with cyberbullying. Youth \& Society, 44, 284-306. doi:10.1177/0044118X11398881

Rolider, A., Lapidot-Lefler, N., \& Levy, R. (2000). The phenomenon of bullying in schools in Israel. Emek Yizrael Academy. (Hebrew)

Smith, P. K. (2012). Cyberbullying and cyber aggression. In S. R. Jimerson, A. B. Nickerson, M. J. Mayer, \& M. J. Furlong (Eds.), Handbook of school violence and school safety: International research and practice (pp. 93-103). New York: Routledge/Taylor \& Francis Group.

Smith, P. K. (2015). The nature of cyberbullying and what we can do about it. Journal of Research in Special Educational Needs, 15, 176-184. doi:10.1111/1471-3802.1211

Smith, P. K., \& Slonje, R. (2010). Cyberbullying: The nature and extent of a new kind of bullying, in and out of school. In S. Jimerson, S. Swearer, \& D. Espelage (Eds.), Handbook of bullying in schools (pp. 249-262). New York: Routledge.

Völlink, T., Bolman, C. A., Dehue, F., \& Jacobs, N. C. (2013). Coping with cyberbullying: Differences between victims, bully-victims and children not involved in bullying. Journal of Community \& Applied Social Psychology, 23, 7-24. doi.10.1002/casp.2142

Whittaker, E., \& Kowalski, R. M. (2015). Cyberbullying via social media. Journal of School Violence, 14, 11-29. doi: 10.1080/15388220.2014.949377 


\title{
EFFECTIVE MEASURE OF ORGANIZATIONAL CULTURE
}

\author{
Olga Tararukhina \\ Ph.D., Saint Petersburg State University (Russia)
}

\begin{abstract}
This theoretical paper presents a theory of organizational culture and a framework approach to measuring it through two aspects that connect the "hard" business results with "soft" culture and people experiences: through the strength of organizational culture on one hand, and on the other hand, through the impact culture has on the business of the organization. Ensuing practical approach includes ways of effective study of organizational culture, meaningful diagnosis that is based on the function and impact of culture that delivers business insight for leaders to exercise effective management of what can be done about culture, given the diagnostic results. It further articulates ways to ensure alignment of culture with the business strategy in order to help deliver sustainable and holistic results.
\end{abstract}

Keywords: Organizational culture, culture measure, strategy, alignment, framework.

\section{Introduction}

The subject of organizational culture has been receiving an increasing number of publications, studies, by both scholars and practitioners. Every big enough management and organizational consulting company seems to offer an assessment of organizational culture and its change. This interest may be due to the increasing evidence of how organizational culture can be the single most important factor of the company's business success and growth, or stagnation and failure.

As with most things in business, simple and quantifiable measurement of progress is typically preferred to assess, track, understand, and evaluate whether change efforts in fact bring desired results. This leads to many kinds of simplifications of the subject or organizational culture, how it evolves, what can be done with it and about it, and what cannot (Schein, 2015). Unfortunately, the nuances and complexity of culture cannot be omitted when working with organizational culture without losing its impact. Therefore, a reliable and meaningful organizational culture assessment is required to do diagnosis, to plan for appropriate interventions and to measure progress towards the desired culture that supports organizational success.

This paper presents an original approach to measuring organizational culture: measuring the impact culture has on the business, in a way that provides actionable insights for business leaders, who can apply their learnings to make meaningful and thoughtful transformation in their organizations, that are not within the typical change management toolbox, which arguably is more effective than most culture assessments known to us.

This approach is developed based on our theoretical and empirical research, as well as practitioner consultant work.

\section{State of the art}

There is a significant number of ways the organizational culture is defined and approached; there is no one universal or typical way of understanding and describing what it is: when scholars or practitioners of organizational culture use the word "culture", most commonly they are likely to imply things that differ from what their clients in business may imply. That difference extends beyond the concept itself, to the simplicity vs. complexity of their approach to culture assessment and change, the depth of analysis and how actionable the insights would be. 


\subsection{Organizational culture}

Perspectives on what is organizational culture include: employee morale; employee value proposition; organizational health; shared purpose; reputation; how work gets done; written and unwritten rules of how to work together; core values; behaviors and mindsets; mental models and assumptions... Most of these definitions as well as others are not inherently wrong, but they less informed, less complete than needed to help create meaningful impact on the business. Focusing on one or two elements of culture, or just an aspect of the larger concept that culture is, may be enough to convince a busy business client, but not enough to create approaches to sustainable work with organizational culture. Furthermore, this abundance of definitions may be adding the perception of abstraction and vagueness to something that already is a seemingly intangible concept for many business leaders.

One of the most helpful, coherent, substantial, thorough theories or organizational culture and methods to working with it, was developed by E. Schein. He articulated (Schein, 2004) three levels of organizational culture as well as that organizational culture as a process of the group solving problems and answering questions of external adaptation and those of internal integration. He also postulated that culture keeps the organization's memory about what leads to success (Schein, 2004).

\subsection{Organizational culture measure}

Most practitioner efforts (with only a few examples know to us) intending to help companies improve their business results, typically focus on measuring such elements of internal organizational life as: employee engagement, job satisfaction, climate of the work environment, or leadership effectiveness. Each of these approaches is too reductionist and superficial for adequately assess and offer grounds to affect organizational culture.

Another problem with most of these approaches is their reliance exclusively or primarily on self-reporting employee survey data. Surveys are known to be susceptible to a possible variation of interpretations even when taken in the same organization (e.g. when asked to which degree respondents agree with a certain statement that can have multiple interpretations from the respondent's perspective). Employees may be looking to portray the organization in the best way possible to help their leader look good. Employees tend to get used to ("trained on") a survey that is repeated over time, as to what is the "right way" to answer the questions. Lastly, results may be difficult to make meaning of without adding anecdotal, qualitative color.

Another school of thought in measuring culture takes the approach of archetyping, which appears to be helpful at the first glance, when it helps business leaders understand and grasp the results. However, when the organization begins to work on culture and potentially takes the assessment again, archetype may become a label with a negative effect on understanding of the true intent of the insights and efforts.

This group of approaches to assessing organizational culture also seems to prescribe what works best for all organizations or specific industry, making an assumption about a certain "ideal" without discerning between different identities, purposed, missions, and strategies that differ significantly among the companies, and may condition their cultures.

\section{New perspectives / contributions}

After a wide variety of approaches briefly described above have been deployed with different degrees of immediate and long-term, sustainable success, there should probably come a time where there is a need for a more unifying theory of organizational culture, that would include a measure that provides an effective and meaningful insight.

\subsection{Original theory of organizational culture}

As described in the article "Workplace Relationships as a Symptom on Organizational Culture and a Lever for Culture Transformation" (Tararukhina, 2019) made a clarification to earlier model of organizational culture (Schein, 2004). The proposed model of organizational culture has 5 layers, that like layers of an onion, reveal culture from its most visible elements (language, space, symbols) to the most latent ones (basic assumptions). In this model, the layers represent:

-Images / language / space: observable, tangible materials and objects;

-Behaviors: observable actions people take in interactions and in doing their work;

-Norms: shared understanding of the distinction between what's right and wrong, what's acceptable and what's not; Rules: understanding of dos and don'ts as well as knowing or the ability to forecast their consequences;

-Values: what is held important when members of the organization take action;

-Basic assumptions: beliefs that we assume to be true and do not question or typically even discuss. 
Following Schein's definition, culture in this model is also defined as "the shared way things are done around here" that develops through socialization, trial and error on the way to success. Its key functions are to make things predictable, familiar; to explain what things mean; to determine what to pay attention to and what to react to, and how to act/react; to keep the organization's memory about what leads to success, stability and meaning (Schein, 2004).

It is also important to underscore what does not appear in the description above. Since culture evolves through socialization, through learning from other people about culture of a specific organization (e.g. incumbents enculturate newcomers), and as a result of this learning process, culture not only lives in group meetings, it gets transmitted and transformed through the vehicle of interpersonal workplace relationships: they are an important carrier of organizational culture.

\subsection{Original culture measure framework}

If culture is "how we do things around here", business strategy is "how we will move forward to achieve our goals" (Rumelt, 2011). Because of the impact culture can have on the execution of strategy (Schein, 2004, 2015; Cooke, 2015; Katzenbach, 2012; Work Effects; Quinn, 1996), for an organization to be successful with its strategy, it not only needs to be the right strategy for this market at this time, but its culture also needs to be in its service. To be effective, organizational culture needs to be: consistent with the organization's purpose and identity, help execute business strategy, and explains how to belong here. Culture that leads organization to be successful needs to meet several criteria at the same time, it would be a mistake to think that when the business strategy shifts, organizational culture needs no attention, it would just catch up, and perform just as well.

Given that: a) culture of a specific organization is not good and not bad, b) given the criteria of an effective culture outlined above, and c) given the importance of its relationship with strategy; given that, the most meaningful way to assess what may need shifting in the culture, is through exactly those impacts: 1) whether culture is supporting the strategy, or is in the way; 2) how consistent (tight) is the culture. Consistency here means consistency of behaviors, norms, and values - how loosely or tightly they are observed and repeated, how consistent is its meaning throughout the organization. Therefore, the proposed original culture measure framework represents four scenarios. Each scenario depends on high/low consistency of the culture, and the kind of its relationship to strategy (whether it hinders or helps:

Figure 1. Approach to measuring organizational culture.

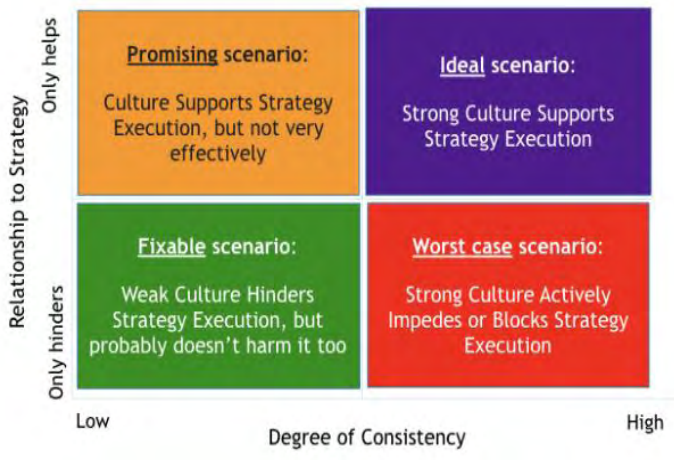

Consistency is created through social processes, such as connectedness between members, consistency with company's identity and purpose, history that employees have together, clarity of the original values (and whether they are still important). In highly consistent cultures people would have similar interpretations of the same behavior, have a shared understanding and interpretation of the rules and norms, and would hold the same meaning for those cultural norms, holding the same things as important (e.g., being on time, or quality over quantity).

There is an ideal scenario in this framework presented in Fig.1. The difference between an ideal scenario in the proposed original framework and the archetype-based culture models is that this ideal scenario would only be ideal for this specific company, no other, because it takes into consideration only this company and its strategy, without benchmarking to other organizations and industries. Each company's uniqueness is therefore respected. Ideal scenario takes place when a strong culture supports strategy execution; promising scenario is observed when culture supports strategy execution, but not very effectively because culture is relatively loose; fixable scenario is a result of a weak culture hindering strategy execution (which may not harm the strategy too much); and worst case scenario that takes place when a strongly consistent culture actively impedes or blocks strategy execution. 
What's tangible, meaningful, and actionable in this approach to culture measurement, is that as opposed to the typical abstract, isolated phenomenon, culture appears as the driver of business results, which it is. This way, it becomes clear how and why somebody asking a question in a meeting or how effective are the meetings (not the amount of ping pong tables or free breakfasts) is helping the company advance in the market place.

Probably not surprisingly, suggested methods to identify consistency of culture and its relationship to strategy for this framework are largely qualitative: interviews, observations. Organizational network analysis can answer questions about variations in behavior, norms, and values, the way they show up in interpersonal situations (Cross, Parker, 2004; Connected Commons). Stories solidify and reinforce those meanings and rules made in the culture, they keep the organizational narrative going, sustain the culture; would be analyzed using content analysis. Finally, other frameworks can be used to help the organization identify, where they are culturally on a certain continuum for each of the elements that matter to the organization. Those frameworks can be either prescribed after having been tested in various industries and kinds of companies (Work Effects: 10 defined dimensions), or can be determined by a group of employees of different levels, representing the organization in its diversity (can be any number of dimensions, as long as they are meaningful for the organization).

\subsection{Culture transformation approach based on the results of original culture measure framework}

Assuming the company would want to move to the ideal scenario from any other place the company finds its culture to be in the framework, the journey will start with that aspect of the scenario that would be easier to make shifts in: either the relationship to strategy, or the culture consistency. The suggested way out of the worst case scenario would not be directly to the ideal scenario, because when the culture consistency is strongly against the strategy, that is the scenario in which culture truly would "eat strategy for breakfast".

To move from the promising scenario to the ideal scenario, company needs to focus on increasing consistency of culture by increasing amount of interactions between employees, both formally and informally. Especially leaders, who would need to connect the dots for the employees more often, ask questions that draw attention to norms and rules, highlight values, reinforce behaviors.

To move from the fixable scenario to the ideal scenario, the organization would need to first move to the promising scenario by focusing on reinforcing what is supporting the strategy.

To move from the worst case scenario: To move to the ideal scenario, first move to the fixable scenario, for the culture needs to be "loosened up" in a way that the unquestionable norms and rules could be questioned, to make them ready to be shifted (in the direction of supporting the strategy), in as much as it may appear uncomfortable. Options may include bringing in new people; start doing things in a new and different way; question why we have been doing things the way we have. Then, after the culture would have shifted to support the strategy, next steps are to work on solidifying cultural elements into more consistency and strongly held norms, rules, and values. But only after the right norms, rules, and values were identified and clarified.

Lastly, it is important to remember that even in the ideal scenario culture needs ongoing attention and effort. Leaders can't assume that their work is done here, they need to keep cultivating the culture, for it in turn to sustain and moderate the organization.

\subsection{Alignment of organizational culture to business strategy}

As it was demonstrated above, it is extremely important for culture to be aligned to the business strategy, for the organization to be able deliver sustainable and holistic results. In order to successfully align culture to strategy, it is important to keep in mind that culture needs to follow and support the business strategy, not the other way around. It is helpful to lean on workplace relationships between individuals, whether with peers or with leaders (Tararukhina, 2016), which are the biggest leverage to help redirect/align culture to business strategy; especially relationships employees have with their leaders. From that perspective, leadership behaviors influencing culture are key: modeling, connecting the dots, reinforcing, asking questions that support shifting values, norms, and behaviors towards the desired culture, help employees see how what they do impacts strategy execution and organizational goals. It is also helpful to maintain the organizational inquiry and conversation about why the organization goes about working the way it does: is it in service of the strategy, or is it hindering the strategy.

The alignment, however, is not an event. Just like culture transformation cannot be an event of change (Katzenbach, 2012; Worley, Williams, Lawler, 2014), alignment needs to be an evolutionary process. For both alignment considerations above to work at their best, for culture transformation to happen in the most effective and efficient way, as well as for the diagnostic methods suggested above, it is advisable to strive for psychological safety (Edmondson, 2019). 


\section{Practical implications}

Described original approach and framework provide meaningful and actionable insights that help organizational leaders make better decisions about their companies' internal processes, allows them to have a clearer perspective into why certain things are more challenging to introduce in the organization than others, and a more tangible and most likely less expensive controls over the next steps that would drive business and financial results. Therefore, this framework produces action steps that are more tangible for business leaders to take, and are more connected to their daily work and business results that they are ultimately accountable for, without losing integrity of the complexity of culture.

\section{Originality and value}

This approach and theory, contrary to most known to us, raises questions about the diversity of points of view on organizational culture and possible time to converge into a more consistent approach, attempts to bring the definition of culture into one; specifies and completes the theory of what organizational culture consists of; provides a framework of culture measure that is objective, that produces actionable insights, in a way that respects each company's uniqueness, agnostic of industries, and provides a roadmap of progressively shifting the culture in an evolutionary way.

\section{Questions for future research}

Going forward, what appears interesting for theoretical and empirical research would be, for example, how culture diagnostics through this approach can help identify and prove that some business strategies are poor, and should be abandoned as inconsistent with the company's identity, because pursuing them would have rather negative consequences, at least lost time and resources.

\section{References}

Connected Commons. (2019). Retrieved April 3, 2019 from https://connectedcommons.com

Cooke R. (2015, October). How Culture Really Works. Paper presented the 1st Ultimate culture conference. Chicago, IL.

Cross R., Parker A. (2004). The hidden power of social networks. Boston, Mass.: Harvard Business School Press.

Edmondson A.C. (2019). The fearless organization. John Wiley \& Sons.

Human Synergistics. (2019). Retrieved April 3, 2019 from https://www.humansynergistics.com

Katzenbach J. (2012). Culture change that sticks. Harvard Business Review. July-August 2012. Retrieved from https://hbr.org/2012/07/cultural-change-that-sticks

Organizational Culture Assessment Instrument Online. (2019). Retrieved April 3, 2019 from https://www.ocai-online.com

Quinn R.E. (1996). Deep change: Discovering the leader within. John Wiley \& Sons.

Rumelt R.P. (2011). Good strategy / bad strategy. The difference and why it matters. NY, NY: Crown Business.

Schein E. (2004), Organizational culture and leadership. (3rd ed.) San Francisco, CA: John Wiley \& Sons.

Schein E. (2015, October). When and How to Think about Culture. Paper presented at the 1st Ultimate culture conference. Chicago, IL.

Tararukhina O. (2016). Cross-cultural variations in satisfaction with interpersonal relationships in the workplace. Doctoral dissertation, Saint Petersburg State University, Saint Petersburg, Russia. Retrieved from http://www.dslib.net/soc-psixologia/kross-kulturnye-osobennostiudovletvorjonnosti-mezhlichnostnymi-otnoshenijami-v.html

Tararukhina O. (2019, May). Workplace Relationships as a Symptom of Organizational Culture and a Leverfor Culture Transformation. Paper presented at the InPACT 2019 conference. Zagreb, Croatia.

Work Effects. (2019). Retrieved April 3, 2019 from http://work-effects.com

Worley C., Williams T., Lawler E. (2014) The agility factor: Building adaptable organizations for superior performance. San Francisco, CA: Jossey-Bass. 


\title{
THE MEANING-BASED APPROACH TO ASSESSING THE IMAGES OF YOUNG AND ELDERLY WOMEN AND MEN
}

\author{
Shulamith Kreitler \\ School of Psychological Sciences, Tel-Aviv University (Israel)
}

\begin{abstract}
The study was inspired by the widely documented evidence about socio-cultural changes in the status and behavior of women in recent decades. The objective was to compare the images that younger and older women have of younger and older women and to compare these images with the images they have of younger and older men. It was expected that the differences would be larger in regard to the images of women than of men. The participants were 80 younger (25-40 years old) and 80 older women (50-65 years old). The method consisted in administering a meaning-based questionnaire of 43 items which provided scores for four clusters referring to perceptual-sensory, actional-dynamic, experiential-cognitive and contextual-situational aspects. The assessed images were of young women, older women, young man and older men. Mean comparisons between and within groups showed highly significant differences in the images of women and men, in most clusters, more in regard to women than to men. The findings indicate major differences in conceptions relating to gender and age. The meaning-based assessment tool proved to be sufficiently sensitive to differences and sufficiently comprehensive to provide an overall perspective.
\end{abstract}

Keywords: Gender, age, meaning, conception, women.

\section{Introduction}

In recent years big changes have taken place in the status and functioning of women, mainly in Western society. These changes have come about through the fight of women, legal regulations concerning equality of rights, changes in social structures and life style and the increased participation of women in education, economy and politics. In most Western societies' women have come a long way towards equality in employment, education, management and politics (Eagly, Diekman, Johannessen-Schmidt, \& Koenig, 2004; McLean \& Ramsey, 2002). The internet and media have also played their role in regard to women's freedom (Butkowski, Dixon \& Week, 2019). As a result, women have become more active in different public positions, express more openly their views, expect to be treated in a more egalitarian manner and fight more openly for their rights and the manner in which they are treated (Cotter, Hermsen, \& Vanneman, 2011). Parallel changes have come about in fashion styles, involvement in sport and sexual freedom. It was expected that these socio-cultural changes would be reflected in the conceptions of women about 'being a woman'.

\section{Objectives}

The study focused on comparing the conception of women of two age groups about a young and an elderly woman. The two age groups were young women, growing up in the already changed world of the last two decades, and older women of former generations. The hypotheses were that there would be significant differences in the conceptions of younger women and those of older women, particularly in regard to the image of a young woman. Further, it was expected that younger women would evidence larger differences than older women in their conceptions of young and old women. More specifically, major differences in the images of younger and older women were expected in regard to the activities of women and their physical appearance. The rationale was that these domains represent those on which the majority of the feminist and equal right movements focused (Thoma, Hediger, Sundaram et al., 2012). For control purposes also the images of younger and older men were studied. It was expected that younger and older women would have more similar conceptions of younger and older men and would have fewer differences between the images of younger and older men. The rationale was that the socio-cultural differences in the last few generations seem to have affected the life of men to a lesser degree than the life of women. 


\section{Method}

\subsection{Design}

The basic design of the study consisted in between-group and within-group comparisons. The groups were younger and older women, while the compared constructs were young woman and elderly woman, young man and older man.

\subsection{Participants}

The participants were 160 women including 80 younger women ( 25 to 40 years old) and 80 older women (50 to 65 years old). In each sample over $70 \%$ were married and engaged in some kind of occupation. The women were recruited from offices in a university and a hospital as well as from internet groups.

\subsection{Tools}

The major tool of the study was the meaning-based questionnaire of concepts, which is grounded theoretically in the cognitive theory of meaning (Kreitler, 2014; Kreitler \& Kreitler, 1990). Accordingly, it includes items that refer to the basic content categories of meaning (called meaning dimensions) which are used by individuals to express or communicate meanings of any kind of stimuli. The meaning categories have been identified on the basis of a rich body of data that included meaning responses of several thousands of subjects in different countries and cultures, differing in age, education, profession and gender, who were asked to communicate the meaning of a variety of referents differing in abstraction, and content spheres (e.g. human, animal, plant, nature, or artificial/synthetic). Examples of the meaning categories are the action of the referent, its sensory features, time and space qualities, emotional aspects, development and possessions. Meaning categories of this kind represent basic cognitive processes applied in any kind of cognitive operation (e.g., problem solving, decision making, cognitive aspects of personality tendencies or traits) as well as communications of meanings of all kinds (Kreitler, 2013; Kreitler, 2014). The 22 meaning dimensions were used for generating items that would represent various aspects of the studied images. Similarly constructed questionnaires were used in other domains (e.g., Kreitler, 2018). After being administered to pretest samples that enabled removing unclear items or those producing responses with no variability, the questionnaire included 43 items. The subjects were requested to check in regard to each item how characteristic it was for the studied construct. There were four response options ranging from "Highly characteristic" to "not at all characteristic", scored as 4 to 1 , respectively. The Cronbach alpha reliability was .76. For purposes of data elaboration the items were grouped into the four following clusters, on the basis of their content, as in other similarly constructed questionnaires: items focused on sensory-perceptual aspects (10 items), actional-dynamic aspects (12 items), experiential-cognitive aspects (11 items), and contextual-situational aspects (10 items). [see Appendix for the questionnaire and scoring). Four questionnaires were prepared with the same format referring to the image of a young woman (see Appendix), to the image of an elderly woman, to the image of a young man and to the image of an elderly man.

\subsection{Procedure.}

Every subject got the four questionnaires, in random order. The questionnaires were administered unanimously in a sequence. The following demographic information was requested: year of birth, marital status, years of education, and occupation.

\section{Results}

Table 1 presents the results concerning the four examined images, i.e., of younger woman, elderly woman, young man, older man. The findings consist of means and mean comparisons of the responses in the four clusters of items, i.e. perceptual-sensory, actional dynamic, experiential-cognitive and contextual-situational. Considering each image separately shows that for young women, the highest clusters in regard to young woman are perceptual-sensory and contextual aspects, for older women and young man actional-dynamic and experiential-cognitive aspects, and for older man actional-dynamic and contextual-situational aspects. For older women, the highest scores in regard to young woman are perceptual-sensory and contextual aspects, for older women actional-dynamic and contextual aspects, for young man experiential-cognitive and contextual aspects, and for older man perceptual-sensory and contextual aspects. This summary indicates a lot of variability across groups and constructs of images.

The same conclusion is supported by the results of mean comparisons in terms of t-tests. The within-group comparisons indicate that in the sample of young women, the images of young and elderly women differ significantly in three clusters except the actional-dynamic. The same holds for the images of young man and old man, because the difference in the actional-dynamic cluster is significant only on 
the $\mathrm{p}<.05$ level which does not reach the Bonferroni criterion. In the sample of older women, the images of young and elderly women differ significantly only in the perceptual-sensory and experiential-cognitive clusters, and the images of young man and old man differ significantly in the experiential-cognitive and contextual clusters, whereas the differences in the other clusters are either nonsignificant or do not pass the Bonferroni criterion.

Comparing the images of women and of men in the two samples shows that in all clusters the differences are significant.

Table 1. Comparing the responses of younger and older women in regard to the images of a young and an older woman and of young and older man.

\begin{tabular}{|c|c|c|}
\hline Young women & Older women & \\
\hline Image of young woman & Image of young woman & $\begin{array}{c}\text { t-test bet. } \\
\text { Groups }\end{array}$ \\
\hline $\begin{array}{lll}\mathrm{a} & 29.3 & {[3.4]} \\
\end{array}$ & $\begin{array}{lll}\mathrm{a} & 25.4 & \text { [3.9] } \\
\end{array}$ & $6.742 * * *$ \\
\hline $25.6 \quad[2.8]$ & $\begin{array}{lll}\mathrm{b} & 20.2 & {[2.0]} \\
\end{array}$ & $14.037 * * *$ \\
\hline $22.8 \quad[3.4]$ & $\begin{array}{lll}\text { c } & 21.3 \quad \text { [3.3] } \\
\end{array}$ & $2.832 * *$ \\
\hline $28.5 \quad[4.1]$ & $\begin{array}{lll}d & 26.8 & \text { [4.3] } \\
\end{array}$ & $2.559 * *$ \\
\hline Image of elderly woman & Image of elderly woman & \\
\hline $\begin{array}{lll}\mathrm{a} & 21.6 & {[4.2]} \\
\end{array}$ & $\begin{array}{lll}\mathrm{a} & 19.3 & {[4.2]} \\
\end{array}$ & $3.463 * *$ \\
\hline $\begin{array}{lll}\mathrm{b} & 24.9 & {[4.5]} \\
\end{array}$ & b $22.3 \quad[4.5]$ & $3.795 * *$ \\
\hline $28.5[4.5]$ & $\begin{array}{lll}\text { C } & 22.4 & {[4.0]} \\
\end{array}$ & $9.062^{* * *}$ \\
\hline $\begin{array}{lll}\mathrm{d} & 19.6 & {[4.8]} \\
\end{array}$ & $\begin{array}{lll}d & 27.2 & {[4.5]} \\
\end{array}$ & $10.332^{* * *}$ \\
\hline $\begin{array}{l}\text { t-test within group } \\
\text { (young woman and } \\
\text { elderly woman) }\end{array}$ & $\begin{array}{c}\text { t-test within group } \\
\text { (young woman and elderly } \\
\text { woman) }\end{array}$ & \\
\hline a-a $14.798 * * *$ & a-a $10.815^{* * *}$ & \\
\hline b-b $1.181 \mathrm{~ns}$ & b-b 1.998* & \\
\hline $\begin{array}{ll}\mathrm{C}-\mathrm{c} & 9.039 * * * \\
\end{array}$ & c-c $3.149 * *$ & \\
\hline d-d $12.610^{* * *}$ & d-d $0.575 \mathrm{~ns}$ & \\
\hline Image of young man & Image of young man & \\
\hline $\begin{array}{lll}\mathrm{a} & 27.3 & {[4.5]} \\
\end{array}$ & $\begin{array}{lll}\mathrm{a} & 29.6 & {[5.4]} \\
\end{array}$ & $2.927 * *$ \\
\hline $\begin{array}{lll}\mathrm{b} & 32.4 & {[5.1]} \\
\end{array}$ & $\begin{array}{llll}b & 31.4 & {[5.4]} \\
\end{array}$ & $1.204 \mathrm{~ns}$ \\
\hline $\begin{array}{lll}\text { c } & 30.7 & {[4.7]} \\
\end{array}$ & $\begin{array}{lll}\text { C } & 32.2 & {[4.9]} \\
\end{array}$ & $1.976^{*}$ \\
\hline $\begin{array}{lll}\mathrm{d} & 31.4 & {[4.0]} \\
\end{array}$ & $\begin{array}{lll}d & 34.1 & \text { [3.5] } \\
\end{array}$ & $4.544^{* *}$ \\
\hline Image of older man & Image of older man & \\
\hline $\begin{array}{lll}\mathrm{a} & 29.5[4.8] \\
\end{array}$ & $\begin{array}{lll}\mathrm{a} & 31.4 & {[4.0]} \\
\end{array}$ & $2.720 * *$ \\
\hline b $\quad 30.8$ [4.5] & b 29.5 [4.9] & $1.748^{*}$ \\
\hline $\begin{array}{lll}\text { C } & 28.2 & {[4.9]} \\
\end{array}$ & C 27.8 [4.9] & $0.516 \mathrm{~ns}$ \\
\hline $\begin{array}{lll}\mathrm{d} & 33.6 & {[5.0]} \\
\end{array}$ & d 31.3 [4.7] & $2.998 * *$ \\
\hline $\begin{array}{c}\text { t-test within group } \\
\text { (young man and older } \\
\text { man) }\end{array}$ & $\begin{array}{c}\text { t-test within group } \\
\text { (young man and older } \\
\text { man) }\end{array}$ & \\
\hline $2.991 * *$ & $0.295 \mathrm{~ns}$ & \\
\hline $2.104 *$ & $2.331 *$ & \\
\hline $3.293 * *$ & $5.679 * * *$ & \\
\hline $\begin{array}{ll}\text { d-d } & 3.073^{* *} \\
\end{array}$ & d-d $4.274 * * *$ & \\
\hline $\begin{array}{c}\text { t-test within group } \\
\text { (young woman and young } \\
\text { man) }\end{array}$ & $\begin{array}{c}\text { t-test within group } \\
\text { (elderly woman and older } \\
\text { man) }\end{array}$ & \\
\hline $3.172 * *$ & $18.660 * * *$ & \\
\hline b-b $10.454 * * *$ & $11.024 * * *$ & \\
\hline $\begin{array}{ll}\text { C-C } & 9.870 * * * \\
\end{array}$ & $7.636 * * *$ & \\
\hline d-d $4.528^{* *}$ & $7.636 * * *$ & \\
\hline
\end{tabular}

Note. $a=$ =Perceptual-sensory aspects; $b=$ actional-dynamic aspects; $c=$ experiential-cognitive aspects; $d=$ contextual-situational aspects . ${ }^{a}$ In each column the first number is the mean, the second, in brackets is the standard deviation. 


\section{Discussion}

The results support the major hypothesis of the study that younger and older women would differ in the manner in which they conceptualize and present the images of young and elderly women. The two samples differed significantly in regard to all clusters concerning the images of young and elderly women. As expected, the differences in the conceptions of younger women tended to be larger than in older women (significant changes in three vs two clusters). Again, as expected, there are major differences in both groups in the perceptual-sensory items that refer to external appearance, but contrary to expectation not in regard to activity. However, the two samples differed also in their images of young and old men yet to a lesser degree than in regard to the images of women. The differences in regard to both male images were significant only in regard to the perceptual-sensory and contextual clusters. Hence there is some indication for support of the hypothesis in regard to the images of men.

\section{Conclusions}

The findings imply that both gender and age are important parameters that contribute to shaping the images of women and men. Younger women's conceptions differed somewhat more than those of older women. The fact that the differences in the images are so salient indicates that when dealing with them it is advisable to consider the images of women and men, old and young, as they are without generalizing. Another important conclusion is that the images of young and old women and men are reflected in all four clusters of features that encompass the physical appearance (perceptual-sensory cluster), action and behavior (actional-dynamic cluster), inner world (experiential-cognitive cluster) and external-situational characteristics (contextual -situational cluster). Hence, the meaning-based tool proved to be adequate for assessing the images of figures differing in gender and age in being both sensitive to differences as well as sufficiently comprehensive to reflect overall characteristics.

\section{References}

Butkowski, C. P., Dixon, T. L., \& Weeks, K. (2019). Body surveillance on instagram: Examining the role of selfie feedback investment in young adults women's body image concerns. Sex Role, Jan. 5, no pp.

Cotter, D., Hermsen, J. M., \& Vanneman, R. (2011). The end of the gender revolution? Gender role attitudes from 1977 to 2008. American Journal of Sociology, 117 (1), 259-289.

Eagly, A. H., Diekman, A. B., Johannesen-Schmidt, M.C., \& Koenig, A. M. (2004). Gender gaps in sociopolitical attitudes: A social psychological analysis. Journal of Personality and Social Psychology, 87(6), 796-816.

Kreitler, S. (2013). The construction of meaning. In D. Cervone, M. Fajkowska, M. W. Eysenck \& T. Maruszewski (Eds.). Personality Dynamics: Embodiment, Meaning Construction, and the Social World. Clinton Corners, NY: Eliot Werner Publications.

Kreitler, S. (2014). Meaning and its manifestations: The Meaning System. In S. Kreitler \& T. Urbanek (Eds.) Conceptions of meaning (pp. 3-32). Hauppauge, NY: Nova Publishers.

Kreitler, S. (2018). Meaningfulness of life and existential distress. Advances in Clinical and Experimental Psychology, 1(1), 1-10. DOI: 10.29011/ ACEP-101. 100001.

Kreitler, S., \& Kreitler, H. (1990). Cognitive foundations of personality traits. New York: Plenum.

McLean, S. A. \& Ramsey, J. (2002). Human rights, reproductive freedom, medicine and the law. Medical Law International, 5(4), 239-258.

Thoma, M. E., Hediger, M. L., Sundaram, R....Louis, G. M. B. (2012). Comparing apples and pears: Women's perceptions of their body size and shape. Journal of Women's Health, 21(10), 1074-1081. 
Appendix

\section{The Image of a Young Woman}

The following items describe the features that may characterize a normative young woman at present, in the current period. Please check in regard to each item to what extent you think it characterizes a young woman. Note: The four response alternatives are: Highly characteristic, Characteristic to a medium extent, Characteristics to a small extent, Not at all characteristic

1. Being active, doing things, performing things

2. Getting help, getting things from others, having others arrange things for her

3. Feeling that she belongs to something or someone

4. Developing, being in a state of development, feeling that she develops, that her life develops

5. Having a good ability to think, to understand, to analyze, to solve problems; having a good memory

6 . Having a body with good proportions and dimensions

7. Dealing with things of which there are many, whose quantity is large

8. Caring about places in which she stays - country, location, home

9. Concerned about being esteemed, about whether others think well of her; whether others value her and her work

10. Being pressured by time; stressed by lack of time; being often late

11. Owning many things, having possessions, feeling that many things belong to her

12. Feeling that she has a task or purpose in life, that her work has a goal

13. Being aware of the causes for her behavior and for the things that she does

14. Being aware of the things that are affected by her, of the things and the people with whom she is in contact

15. Being concerned about having always the right kind of bodily weight

16. Having many emotional experiences, reacting emotionally to people and things, interested in learning new emotions, being able to feel deeply about things

17. Being concerned about having results to what she does or says; being aware of the results of her actions

18. Being in contact with people, having people around her most of the time

19. Being aware of belonging to a certain nation, to a certain community, to a certain group, gender, organization, team, feeling that she is part of a family

20. Having a variegated life, living a life of different kinds

21. Behaving in an aggressive manner; trying to control others and situations

22. Being aware of how things happen, of the manner in which life occurs

23. Being aware of different materials fulfilling a certain role in her life, e.g., water, air, metals and crystals, cotton and wool

24. Having a life with a certain structure, a life that is a continuum of events interrelated in some form; having an organized life

25. Having changes in her life

26. Wanting many things

27. Evoking in other people emotions like love or longing, sometimes even fear or anger, being loved

28. Having certain opinions and beliefs, believing in certain things

29. Being a source of inspiration for others, evoking in them thinking or understanding

30. Looking well, being physically beautiful, handsome

31. Being healthy, being in a good physical state

32. Being interested in learning to know many tastes, having food that tastes well, being able to taste things with an interesting taste

33. Having interesting and exciting physical sensations

34. Being able to listen a lot to music that she likes, enjoying music

35. Feeling pain in different body parts

36. Liking to be exposed in her environment to colors and forms that she likes

37. Concerned about being rich, having a lot of money

38. Leading a stable life, based on routine

39. Having a profession, a career; being achievement-oriented

40. Being concerned about having a skin and hair in good colors

41. Being in good control of her body

42. Contributing to others' welfare and well-being

43. Manipulative; managing things in ways that benefit her 


\title{
THE ROLE OF TRAINING OR HINTS RELATING TO "THINKING IN OPPOSITES” IN INSIGHT PROBLEM SOLVING
}

\author{
Erika Branchini ${ }^{1}$, Roberto Burro ${ }^{1}$, Elena Capitani ${ }^{2}$, Ugo Savardi $^{1}$, \& Ivana Bianchi $^{3}$ \\ ${ }^{I}$ Department of Human Sciences, University of Verona (Italy) \\ ${ }^{2}$ Department of Education, Cultural Heritage and Tourism, University of Macerata (Italy) \\ ${ }^{3}$ Department of Humanities, Section of Philosophy and Human Sciences, University of Macerata (Italy)
}

\begin{abstract}
In recent years, there has been a growing desire to remove the mystery from insight, the underlying processes of which are not fully understood (Gilhooly et al., 2015). The present research focuses on the hypothesis that "thinking in opposites" might facilitate the process of representational change required for solving visuo-spatial insight problems (Bianchi et al., 2019; Branchini et al., 2015, 2016). There is evidence in Cognitive Science that opposites (or contrasts) are a central cognitive structure in space perception (e.g. Bianchi et al., 2017) and language (e.g. Jones et al., 2012) and they are fundamental to inductive (Gale and Ball, 2012) and deductive reasoning (Augustinova, 2008). They are also involved in creative thinking (Rothenberg, 2001).

In particular, the study reports the results of research carried out with the aim of investigating whether prompting problem solvers to use a strategy based on the manipulation of opposites (i.e. inside-outside, large-small) would improve their performance (Bianchi et al., 2019). Two hundred and forty undergraduate students were asked to analyze the spatial features inherent to six problems in terms of opposites before embarking on their search for a solution. Two hint and two training conditions were studied.

The results show that success rates increased when the participants were explicitly trained to use opposites.

In relation to the current debate on the factors which facilitate insight problem solving (e.g. Ahmed and Patrick, 2006; Cunningham and MacGregor, 2008; Patrick et al., 2015), our results add evidence that a prompt "to think in opposites" is a facilitating factor when it is given explicitly (i.e. as part of training). The findings are also discussed in relation to a debate on the same subject regarding the differences between the role of Type 1 processes (which are automatic, unconscious and associative) and that of Type 2 processes (which are controlled, conscious and analytical) (e. g. Weisberg, 2015, 2018). In particular, we provide suggestions concerning the nature of the role of opposites in the generation of potential solutions (Type 1 processes) and in the evaluation of these various potential alternatives with a view to homing in on a single solution (Type 2 processes).
\end{abstract}

Keywords: Insight problem solving, opposites, representational change, training versus hint, Type 1 versus Type 2 processes.

\section{Introduction}

Some problems appear trivial at a first glance, yet in reality finding their solution turns out to be very difficult and time-consuming. Despite the initial impression, there may not be an obvious solution to the problem and the application of strategies which are familiar to the problem solver (i.e. which have been used in the past to solve similar problems) are not necessarily successful. When the solution is finally found, it often appears suddenly and unexpectedly and is frequently accompanied by an "aha" experience. This is why they are known as "insight" problems (Öllinger and Knoblich, 2009). A representational change is needed in order to solve this type of problem (Ohlsson, 2011).

The way in which people tackle these problems is not fully understood and there has been a growing desire to remove the mystery surrounding them by means of theory driven experiments (Gilhooly et al., 2015). A question which remains open concerns whether there are any factors which may facilitate insight, either on an explicit or implicit level. This issue is related to the more general debate on the role of automatic and unconscious (Type 1) processes versus controlled and conscious (Type 2) 
processes in insight problem solving (Weisberg, 2015; 2018). While hints represent implicit suggestions or prompts (e.g. Bröderbauer et al., 2013 and on the efficacy of hints see for instance Bröderbauer et al., 2013), training programs explicitly teach problem solvers a strategy or procedure that can be recalled later on to solve problems with a similar structure (Patrick and Ahmed, 2014; on the efficacy of training procedures, see for instance Patrick et al., 2015). The study discussed in this paper (and presented in detail in Bianchi et al., 2019) delves deeper into this issue while specifically testing the role of "thinking in opposites" as a facilitating factor. Some studies have put forward the idea that the process of representational change required to solve visuo-spatial insight problems can be facilitated by "thinking in opposites" (Bianchi et al., 2019; Branchini et al., 2015; Branchini et al., 2016). However, what these studies did not clarify was whether a prompt to use opposites works better when proposed as an explicit strategy and systematically applied or as a hint acting at a less systematically-driven level.

\section{Design}

Four conditions were tested. These resulted from a $2 \times 2$ factorial experimental design with one factor being the Type of Facilitation (two levels: Hint and Training) and another factor the Identification of Opposite properties (two levels: Overt Listing and Covert Listing).

In the Training conditions, before the six visuo-spatial insight problems were presented to the participants, they were given a brief training session in which an experimenter used three example problems to demonstrate how a three step strategy based on the manipulation of spatial opposites might help them to solve the problems. The first step consisted of identifying all the spatial properties inherent to the problem (both in the written text and in the figure); the second step involved identifying the corresponding opposite for each property found in step one. In the third step they were asked to assess whether using these opposite properties might help them to find the solution. In one variant of the Training condition, the participants were asked to write down a list of the opposites identified in the second step (Overt Listing Training condition, OLT); in another condition, this was not required (Covert Listing Training condition, CLT).

In the Hint conditions, before the six problems were presented, the participants were told that transforming the spatial properties of the problem into their opposites would help them to find the solution. To this end, they were requested to identify all of the spatial properties inherent to the problem (both in the written text and in the figure) and determine their corresponding opposites before embarking on their search for the solution. There were two Hint conditions in this case too. In one condition (the Overt Listing Hint condition, OLH), the participants were asked to write down the list of opposites they had identified and in another condition (the Covert Listing Hint condition, $\mathrm{OCH}$ ), the participants were not asked to make an explicit list.

For demonstration purposes, three example problems were used both in the training and in the hint conditions. In all of the conditions, the participants were in small groups of three members. Previous studies had in fact proved the benefits of "contrast thinking" which arises when people work in groups rather than individually (Augustinova, 2008; Branchini et al., 2016).

\section{Objectives}

The aim of the study was to explore how different levels of awareness and systematicity pertaining to the use of opposites might affect visuo-spatial insight problem solving. The two types of facilitation tested (i.e. Hint versus Training) presupposed two different levels of awareness in the application of a strategy based on opposites. The two conditions relating to the identification of the opposites inherent to the problem (i.e. Overt Listing versus Covert Listing) stimulated different levels of systematicity when the participants manipulated the structure of the problem.

\section{Method}

Two hundred and forty undergraduate students at the University of Verona, Italy took part in the study (111 males, 129 females; $M_{\text {age }}=21.83$ years, $S D=7.26$ years) in small groups of three members. Sixty participants (i.e. 20 groups) were randomly assigned to each of the four conditions studied: the Overt Listing Training condition (OLT), the Covert Listing Training condition (CLT), the Overt Listing Hint condition (OLH) and the Covert Listing Hint condition (CLH).

Six target problems were used with the order of presentation randomized between groups: the "pigs in a pen" problem (Schooler et al., 1993); the "triangle" problem (De Bono, 1969); the "deer" problem (origin unknown); the "eight-coin" problem (Ormerod et al., 2002); the "five-square" problem 
(Katona, 1940) and the "circumference" problem (Köhler, 1969). Three example problems were used in the instruction booklets in all of the training and hint conditions. These were the "parallelogram" problem and the "altar window" problem (both devised by Wertheimer, 1919/1945) and the "nine dot" problem (devised by Maier, 1930). The instructions were printed on sheets of paper given to the participants. These were read out by the experimenter who then answered any questions as necessary. The experimenter then handed out a booklet containing the six problems to be solved. Since a group problem solving task was foreseen, only one booklet per group was provided and the participants were repeatedly encouraged to talk aloud while trying to find the solution together. They were given seven and a half minutes for each problem.

\section{Discussion}

Two main aspects emerged from the results of the study (for further details and analysis, see Bianchi et al 2019; all data were analyzed using GLMM - Borenstein, Hedges, Higgins, \& Rothstein, 2009).

One aspect refers to success rates. The participants in the Training conditions solved a greater number of problems than the participants in the Hint conditions (main effect of Type of Facilitation: $\chi 2_{(1, \mathrm{~N}=80)}=6.530, p=.011$, z-ratio $\left.=-3.993, d=-0.446\right)$. This suggests that explicitly teaching a strategy based on the manipulation of opposite features works better that simply giving a hint to think in terms of opposites.

The second aspect refers to the effect of overtly listing the opposites identified in the problem structure in the preliminary phase. Overt listing improved the participants' ability to solve the problems (i.e. they solved a greater number of problems) in the Hint condition but not in the Training condition (significant interaction between the Type of Facilitation and Identification of Opposites: $\chi 2_{(1, \mathrm{~N}=80)}=4.453, p=.035$; post hoc OLH vs OLH: $S T=0.986, S E=0.354, z$-ratio $=2.781, p=0.03$, $d=0.311)$.

These results suggest that a certain degree of systematicity in the application of the prompt to think in opposites is needed. In the training condition, this systematicity seemed to have been sufficiently guaranteed by the three steps that the participants were instructed to follow (see the Design section). Conversely, in the hint condition, asking participants to write down the lists of opposites identified in the preliminary phase helped them to remain anchored to the manipulation of these characteristics when searching for the solution and this led to a better success rate. This anchoring effect emerged both from an analysis of the sketches done by the participants when they were searching for the solution and from the verbal dialogues between the members of each group. These analyses were possible since the sketches were collected after each session and video recordings were made during the sessions.

\section{Conclusion}

The findings reported in this article support previous evidence regarding the facilitating effect of "thinking in opposites" in visuo-spatial insight problem solving (Bianchi et al., 2019; Branchini et al., 2016; Branchini et al., 2015a, 2015b; Branchini et al., 2009). In particular they indicate that this strategy works more effectively when systematically applied, that is, when contextualized in an explicit procedure (in this case involving three steps) or when hinted at after an overt identification of the opposites to be focused on.

Various studies have shown that the restructuring of the problem which is a necessary part of the process is facilitated when problem solvers are given training during which it is revealed that some of the tacit assumptions they are making work as real constraints and prevent them from seeing the solution (Ahmed and Patrick, 2006; Cunningham and MacGregor, 2008; Patrick et al., 2015). Adopting a strategy of thinking in terms of opposites helps to overcome these constraints by suggesting the "obvious" direction which the transformation of the structure of the problem should take. We say "obvious" since intuitively the concept of opposites is very readily evoked by people (even at a very early age), despite the fact that invariably they are unable to give an explicit definition of the concept (Bianchi et al., 2011; Casasola, 2008; Casasola et al., 2003; Savardi and Bianchi, 2008). The "intuitivity" of the concept of opposites connects Opposites to Type 1 processes. Conversely, when Opposites provide the framework for a systematic revision of alternative possible manipulations, they seem to work more in terms of Type 2 (i.e. conscious) processes.

Evidence that opposites support visuo-spatial insight problem solving (Bianchi et al., 2019; Branchini et al., 2016; Branchini et al., 2015a, 2015b; Branchini et al., 2009) is in line with other studies in the field of Cognitive Science which have demonstrated the role of opposites in several human cognitive abilities. These range from perception (Bianchi et al., 2017) to the organization of conceptual 
spaces (Jones et al., 2012), to inductive (Gale and Ball, 2012) and deductive reasoning (Agustinova, 2008) and also to creative thinking (Rothenberg, 2001).

Two limitations of the present state of art of research on the benefits of thinking in terms of opposites in problem solving concern, firstly, the generalizability of these conclusions beyond visuo-spatial problem solving and, secondly, its generalizability beyond the group condition. Why is "thinking in opposites" associated with better performance when problem solvers are in small groups rather than working individually? One hypothesis is that when problem solvers work in groups, this facilitates "U-turns" in thinking processes and disconfirming strategies since it is easier to disconfirm the suggestions of others than one's own ideas (on confirmation biases see for instance Kuhn and Udell, 2007; Mercier and Sperber, 2011). In any case, further studies are needed to better understand whether there are any weaknesses in the "thinking in opposites" strategy when used by individuals in problem solving.

\section{References}

Ahmed, A., \& Patrick, J. (2006). Making implicit assumptions explicit in verbal insight problem solving. In Cognitive Science Society (Ed.), Proceeding of 28th Annual Conference of the Cognitive Science Society in Cooperation with 5th International Conference of Cognitive Science Society (pp. 986-991). Red Hook, NY: Curran Associates, Inc.

Augustinova, M. (2008). Falsification cueing in collective reasoning: Example of Wason selection task. European Journal of Social Psychology, 38 (5), 770-785.

Bianchi, I., Branchini, E., Burro, R., Capitani, E., \& Savardi, U. (2019). Overtly prompting people to "think in opposites" supports insight problem solving. Thinking \& Reasoning. Retrieved from: https://doi.org/10.1080/13546783.2018.1553738

Bianchi, I., Paradis, C., Burro, R., van de Weijer, J., Nyström, M., \& Savardi, U. (2017). Identification of opposites and intermediates by eye and by hand. Acta Psychologica, 180, 175-189.

Bianchi, I., Savardi, U., \& Kubovy, M. (2011). Dimensions and their poles: A metric and topological theory of opposites. Language and Cognitive Processes, 26 (8), 1232-1265.

Borenstein, M., Hedges, L. V., Higgins, J. P. T., \& Rothstein, H. R. (2009). Introduction to meta-analysis. London: Wiley.

Branchini, E., Bianchi, I., Burro, R., Capitani, E., \& Savardi, U. (2016). Can contraries prompt intuition in insight problem solving? Frontiers in Psychology, 7:1962.

Branchini, E., Burro, R., Bianchi, I., \& Savardi, U. (2015). Contraries as an effective strategy in geometrical problem solving. Thinking \& Reasoning, 21 (4), 397-430.

Branchini, E., Burro, R., \& Savardi, U. (2009). Contraries in productive thinking. In U. Savardi (Ed.), The Perception and Cognition of Contraries (pp. 203-224). Milan: McGraw-Hill.

Branchini, E., Savardi, U., \& Bianchi, I. (2015). Productive thinking: the role of perception and perceiving opposition. Gestalt Theory, 37, 7-24.

Bröderbauer, S., Huemer, M., \& Riffert, F. (2013). On the effectiveness of incidental hints in problem solving revisiting Norman Maier and Karl Duncker. Gestalt Theory, 35, 349-364.

Casasola, M. (2008). The development of infants' spatial categories. Current Directions in Psychological Science, 17, 21-25.

Casasola, M., Cohen, L. B., \& Chiarello, E. (2003). Six-month-old infants' categorization of containment spatial relations. Child Development, 74, 679-693.

Cunningham, J. B., \& MacGregor, J. N. (2008). Training insightful problem solving: Effects of realistic and puzzle-like contexts. Creativity Research Journal, 20, 291-296.

De Bono, E. (1969). The mechanism of mind. New York, NY: Penguin.

Gale, M., \& Ball, L. J. (2012). Contrast class cues and performance facilitation in a hypothesis testing task: Evidence for an iterative counterfactual model. Memory \& Cognition, 40, 408-419.

Gilhooly, K., Ball, L., \& Macchi, L. (2015). Insight and creative thinking processes: Routine and special. Thinking \& Reasoning, 21 (1), 1-4.

Jones, S., Murphy, M. L., Paradis, C., \& Willners, C. (2012). Antonyms in English: Construals, constructions, and canonicity. Studies in English language. Cambridge: Cambridge University Press.

Katona, G. (1940). Organizing and memorizing: Studies in the psychology of learning and teaching. New York, NY: Columbia University.

Köhler, W. (1969). The task of Gestalt psychology. Princeton, NJ: Princeton University Press. 
Kuhn, D., \& Udell, W. (2007). Coordinating own and other perspectives in argument. Thinking and Reasoning, 13, 90-104.

Maier, N. R. F. (1930). Reasoning in humans. On direction. Journal of Comparative Psychology, 10 (2), 115-143.

Mercier, H., \& Sperber, D. (2011). Why do humans reasons? Arguments for an argumentative theory. Behavioral and Brain Sciences, 34, 57-111.

Ohlsson, S. (2011). Deep learning: How the mind overrides experience. Cambridge: Cambridge University Press.

Öllinger, M., \& Knoblich, G. (2009). Psychological research on insight problem solving. In H. Atmanspacher \& H. Primas (Eds.), Recasting reality (pp. 275-300). Berlin, Germany: Springer.

Ormerod, T. C., MacGregor, J. N., \& Chronicle, E. P. (2002). Dynamics and constraints in insight problem solving. Journal of Experimental Psychology: Learning, Memory \& Cognition, 28, 791-799.

Patrick, J., \& Ahmed, A. (2014). Facilitating representation change in insight problems through training. Journal of Experimental Psychology: Learning, Memory \& Cognition, 40, 532-543.

Patrick, J., Ahmed, A., Smy, V., Seeby, H., \& Sambrooks, K. (2015). A cognitive procedure for representation change in verbal insight problems. Journal of Experimental Psychology: Learning, Memory \& Cognition, 41, 746-759.

Rothenberg, A. (2001). Bipolar illness, creativity, and treatment. Psychiatric Quarterly, 72 (2), 131-147.

Schooler, J. W., Ohlsson, S., \& Brooks, K. (1993). Thoughts beyond words: When language overshadows insight. Journal of Experimental Psychology: General, 122 (2), 166-183.

Weisberg, R. W. (2015). Toward an integrated theory of insight in problem solving. Thinking \& Reasoning, 21, 5-39.

Weisberg, R. W. (2018). Problem solving. In L. J. Ball \& V. A. Thompson (Eds.), The Routledge International Handbook of Thinking and Reasoning (pp. 1239-1274). New York, NY: Routledge.

Wertheimer, M. (1945). Productive thinking. New York: Harper. First appearance Über Schulzbprozesse productiven Denken (Original work published in 1919). 


\title{
KNOWLEDGE OF PROSPECTIVELY RELEVANT INFORMATION IMPROVES SEARCH PERFORMANCE
}

\author{
Margit Höfler $^{1,2}$, Vanessa Kuwal ${ }^{1}$, Sebastian A. Bauch ${ }^{1}$, \& Anja Ischebeck ${ }^{1}$ \\ ${ }^{1}$ Institute of Psychology, University of Graz (Austria) \\ ${ }^{2}$ Department for Clinical Neurosciences and Preventive Medicine, Center for Dementia Studies, Danube, \\ University Krems (Austria)
}

\begin{abstract}
When searching for a target among distractors, we are able to focus our attention to those properties of the target or parts of the display relevant for the current search while ignoring irrelevant properties. However, it is unclear whether we can make use of irrelevant information when we are aware that these properties will become relevant in future searches. The aim of the current experiment was therefore to investigate whether and how the pre-knowledge about which parts of the display will become relevant in future searches affects the current and future searches in the same display. To this end, we had 20 participants perform two blocks of searches while their eye movements were recorded. During the first block, participants searched in 10 different search displays for different target letters that were superimposed on drawings of objects. Each search display was repeated 10 times, thus resulting in 100 searches per block. Half of the participants were instructed to ignore the objects accompanying the letters; the other half of the participants were told that, in a second block of searches, the objects would have to be searched for. Hence, in the latter case, participants were aware that the objects would become relevant in a further search task. The results showed that, in the first block (letter search), participants performed the search task equally fast, regardless of their pre-knowledge about the objects' prospective relevance in the second block. In the second block (object search), search performance was better for those participants who knew about the objects' relevance in advance compared to participants who had been told to ignore the objects. This suggests that participants are able to use relevant information from a previous search for future searches, without compromising search performance in the first search.
\end{abstract}

Keywords: Visual search, eye tracking, item relevance.

\section{Introduction}

When searching for a cup of strawberry yogurt of a familiar brand in the supermarket, you will perhaps restrict your attention to properties of the target objects that will help you find these objects quickly, for example, a brand's typical logo or color. At the same time you may ignore other properties of the target that are irrelevant to the search, for example, the precise shape of the cup. However, it is unclear whether we really succeed in using irrelevant information from previous searches in a later search environment, where this information eventually turns relevant. In order to preserve our limited working memory resources (Cowan, 2001), it would be efficient to process only such properties of targets that are relevant for the search task. There is evidence that memory is better for properties of targets objects that are relevant for the actual (visual search) task than for properties of target objects that are irrelevant (e.g., Williams, Henderson, \& Zacks, 2005). However, some irrelevant information might nevertheless be incidentally processed when a scene is inspected regardless of whether it will be of use in the future or not. For example, using a real-world setting in which participants were free to move around the environment while their eye movements were being recorded, Tatler and Tatler (2013) investigated memory for different objects by comparing the effect of three different task instructions. Participants were either asked to memorize as much as possible about all objects (undirected memory task) or only certain objects (directed memory task) in the environment, or received no memory instruction at all (free-viewing task). During a subsequent memory test, memory for four object properties (identity, color, location, and the relative distance to other objects) was tested. The results showed that memory performance was better in the directed and undirected memory task than in the free viewing task, with no differences between both (directed and undirected) memory tasks. However, object memory was above chance also in the free-viewing task. This indicated incidental memory for objects even if the task did not require 
memorization (see also Williams et al., 2005). This suggests that, although participants were able to restrict their attentional and memory resources to objects and properties that were essential for a certain task, task-irrelevant information also left some traces in memory.

It is unclear, however, whether task-irrelevant information is stored during visual serial search when participants do not expect a further search in the same environment. Võ and Wolfe (2012) had participants search repeatedly through different scenes for different target objects. Before this main search task, however, they were asked to pre-search the scenes for letters superimposed on some of the objects. When participants searched for target letters they also viewed the (irrelevant) objects located under those letters. Võ and Wolfe were interested in whether the irrelevant information about object identity during the pre-search would enhance search performance in the subsequent object search task. However, performance in the subsequent object search task did not change due to the pre-search (but see Hollingworth, 2012). The authors concluded that looking for objects (i.e. searching for specific objects) is different from looking at objects. Critically, however, participants in Võ and Wolfe were not aware that the objects behind those letters might become relevant in a future search. It is therefore possible that a more explicit instruction to memorize the objects might have enhanced search performance in the main search. In the present study, we investigated whether the knowledge about a future search modulated the processing of irrelevant item identity information in an earlier search. We assumed that memory for irrelevant item identity information would depend on the instruction. We either instructed participants that they could use the information in a subsequent search or not. To this end, we modified the repeated-search paradigm of Võ and Wolfe (2012). Participants had to complete two blocks of searches in which they searched displays repeatedly that consisted of ten objects superimposed by letters. All participants searched for letters superimposed on objects in the first block and for the objects in the second block. Half of the participants was explicitly told that they would have to search the display for the objects in the second block (object-relevant condition), whereas the other half was instructed to ignore the objects (object-irrelevant condition). If participants could use the irrelevant information during the first block as instructed, search performance in the second block should be faster in the object-relevant as compared to the object-irrelevant group.

\section{Methods}

\subsection{Design}

To test the effect of instruction on memory in repeated visual search we applied a 2 (search block: block 1, block 2) × 2 (instruction type: object-relevant, object-irrelevant) design. During each block, they were presented with 10 different search displays that they had to search consecutively for 10 times each. In each search display, 10 items were presented. Items consisted of the picture of an object with a superimposed letter, such that object and letter were clearly discernible. All participants searched the displays for different target letters in the first block and for different target objects in the second block. Half of the participants were instructed that the objects behind the letters would become relevant during a further block (object-relevant condition) whereas the other half was instructed to ignore the objects (object-irrelevant condition). In each block, we measured manual response times and number of fixations needed to find the letters and objects.

\subsection{Participants}

20 participants (13 female, 7 male; average age: 24.0 years, range: 20 - 30 years, $S D=3.4$ ) took part in the experiment. All participants reported normal or corrected to normal vision. The experiment was approved by the local ethics committee. Written informed consent was obtained from all participants.

\subsection{Stimuli}

100 different objects (individual size: $\max 1.8^{\circ}$ ) were chosen from the Microsoft® Clipart database and covered ten different categories (kitchen, bathroom, nature, animals, fairy-tale/fantasy characters, office, means of transportation, musical instruments, fruits, and sports equipment). In each of the ten displays, only items from the same category were presented. Stimuli were presented using Experiment Builder (SR Research, Canada) on a 21" CRT Monitor with a resolution of $1152 \times 864$ pixel and a refresh rate of $60 \mathrm{~Hz}$. Stimuli were presented randomly within an invisible $6 \times 6$ grid and could deviate from the center of each grid cell $\pm 0.16^{\circ}$. Each object was superimposed by a red letter in Arial font (bold). The letters were $0.45^{\circ}$ (20px) high and presented in red color (RGB: 255, 0, 0). They were randomly chosen from all capital letters of the Latin alphabet excluding the letter "I". We created the stimulus materials such that the letters did not occlude the objects completely and that the letters themselves were discriminable from the respective object (e.g., we did not use objects that were mainly of

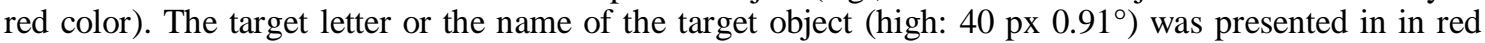
(RGB: 255, 0 ,0) Arial font (bold). 


\subsection{Apparatus}

We used an EyeLink 1000 (SR Research, Canada) to track the eye movements of the participants. We tracked from one eye with an average spatial resolution better than $0.4^{\circ}$. Manual responses were measured using a Gamepad (Microsoft ${ }^{\circledR}$ Sidewinder). The distance from the participants’ eyes to the monitor was approximately $83 \mathrm{~cm}$.

\subsection{Procedure}

Participants sat in a dimly lit, sound proof cabin. They were instructed that they would have to search several search displays for different target letters. Participants were instructed to search for the letters in Block 1 and either ignore the objects behind the letters (objects-irrelevant condition) or to process these objects as they would become relevant in a later block of searches (objects-relevant condition).

Each block started with a 9-point calibration of the eye tracker. In both blocks, each trial started with the presentation of a fixation disc (which also served as a drift correction for the eye tracker) at the center of the screen. Immediately afterwards, the target letter (in the first block) or the target object (in the second block) was presented for 1,000 msec (see Figure 1). The target object was written in German. Then the search display was presented. The task of the participants was to search for the respective target in the search display and to press the trigger on the game pad with the right index finger when the target was found. The target was always present. Participants were told to search as fast and as accurately as possible. With the button press, a new trial started with the presentation of a new target. In the first block, participants searched the same display for ten different target letters before a new display was presented. In the second block, we presented search displays with the objects only and participants were asked to search for a target object. As the overlying letters were omitted in the displays, participants could not rely on possibly learned association between the letters and objects when they searched for the letters in the first search block. Within the 10 searches in the same display, the same target (letter or object) was never searched twice. The order of the presentation of the displays was randomized as well as the order of the targets. There was a short pause between the first and the second block. The participants completed 10 practice trials with a different set of items than in the actual experiment. The experiment lasted about 45 minutes.

Figure 1. Sequence of events in Block 1 and 2. Each of the ten different displays was presented ten times consecutively. In Block 1, participants had to search for different target letters in displays with ten objects with the target letters superimposed on them. Half of the participants were instructed to ignore the objects behind the letters, the other half to process them. In Block 2, participants searched for different target objects (letters removed).

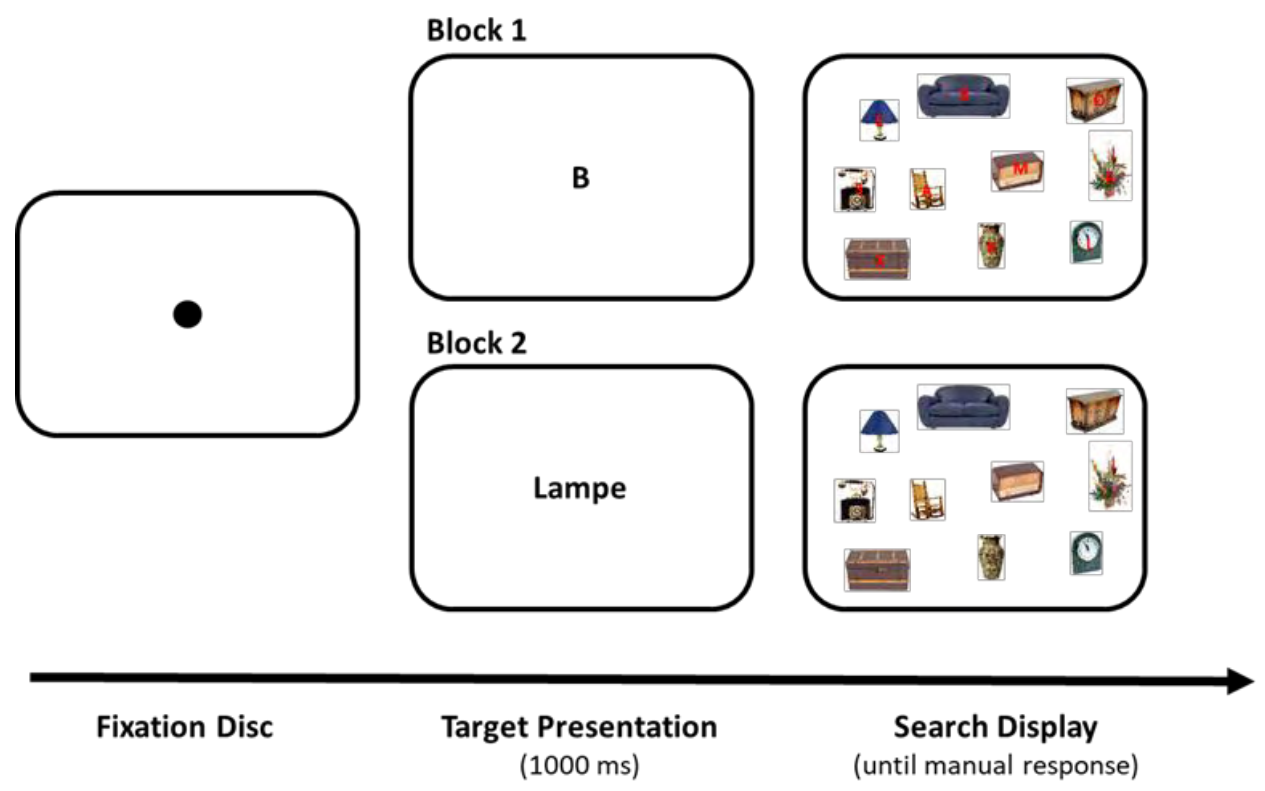




\section{Results and discussion}

In total, 4,000 trials were available for analysis (20 participants x 200 trials). For each fixation, we computed the Euclidean distances relative to all items in the display and the item with the smallest distance was then determined as the item fixated. We excluded all trials in which participants did not fixate the respective target item during the trial. This was the case for 504 trials (12.6 \%) of the trials.

We computed the mean response time, averaged across individual means separately for each block and instruction group (see Figure 2). In Block 1, participants needed on average 2,050 ms $(S D=225)$ to find the target letter in the object-relevant condition and $1,939 \mathrm{~ms}(S D=283)$ in the object-irrelevant condition. In Block 2, participants needed 1,056 ms $(S D=233)$ if they had been instructed to process the objects in Block 1 and $1,190 \mathrm{~ms}(S D=225)$ if they had been instructed to ignore the objects. A $2 \times 2$ ANOVA with block as within and instruction (object relevant vs. object irrelevant) as between-factor revealed a significant main effect of block, $F(1,18)=294.96, p<.001, \eta_{p}{ }^{2}=0.93$, but no main effect of instruction, $F<1$. However, the interaction was significant, $F(1,18)=5.03, p=.038$, $\eta_{p}{ }^{2}=0.22$. In particular, the difference in search performance between Block 1 and Block 2 was reliably larger for the object-relevant group (994 ms) compared to the object-irrelevant group (749 ms), $t(18)=2.43, p=.038$. This suggests that participants who were instructed to process the objects during Block 1 benefited in Block 2 from this information more than participants who were instructed to ignore the objects.

To investigate whether this benefit in the second block is also reflected in the oculomotor system, we conducted a further ANOVA with the number of fixations until the first target fixation as dependent variable. Participants inspected in the first block $9.3(S D=0.77)$ items in the object-relevant condition and $8.7(S D=1.17)$ item in the object-irrelevant condition. In the second block they fixated 6.3 items both in the object-relevant and irrelevant condition ( $S D=0.8$ and 1.3 , respectively). We found a significant main effect of block with fewer fixations in the second block than in the first block, $F(1,18)=99.80, p<.001, \eta_{p}{ }^{2}=0.85$. The main effect of instruction as well as the interaction was not significant, both $F<1$. This suggests that number or fixated items during search is less sensitive for measuring the effects of task instruction. Further research is necessary to investigate how participants benefit from pre-knowledge about prospective item relevance.

Figure 2. Mean response time separately for each block and instruction condition.

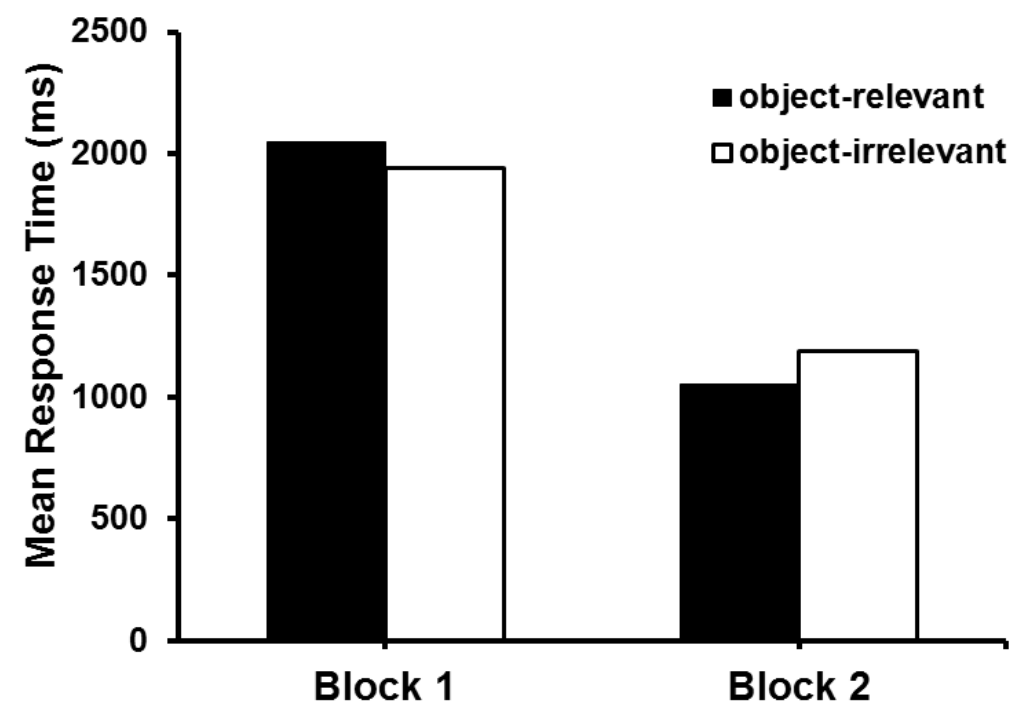

\section{Conclusion}

In the present paper we investigated whether knowledge about the future relevance of currently irrelevant target properties modulated search times in current as well as in future searches. We found that participants that were explicitly informed about the future relevance of the currently irrelevant target property (i.e. the object identity) showed a greater difference in search times between search block one and two. Our findings show, at least when analyzing the manual responses, that participants could benefit from irrelevant information during visual search when they were informed that this information would become relevant in a later search. This indicates that traces of irrelevant information can be kept in memory during visual search. 


\section{Acknowledgements}

This work was supported by a grant from the Austrian Science Fund (FWF): P 28546 to M.H. We are grateful to Jasmin Jennetten for her support during the preparation of the manuscript.

\section{References}

Hollingworth, A. (2012). Task Specificity and the Influence of Memory on Visual Search: Comment on Höfler, M., Gilchrist, I.D., \& Körner, C. (2015). Guidance toward and away from distractors in repeated visual search. Journal of Vision, 15(5), 1-14. doi:10.1167/15.5.12

Oberauer, K. \& Eichinger, S. (2013). Visual working memory declines when more features must be remembered for each object. Memory \& Cognition, 41, 1212 - 1227.

Tatler, B. \& Tatler, S. L. (2013). The influence of instructions on object memory in a real-world setting. Journal of Vision, 13(2):5. doi: 10.1167/13.2.5.

Võ, M. L.-H., \& Wolfe, J. M. (2012). When does repeated search in scenes involve memory? Looking at versus looking for objects in scenes. Journal of Experimental Psychology: Human Perception and Performance, 38(1), 23-41.

Williams, C.C., Henderson, J.M., \& Zacks, R. T. (2005). Incidental visual memory for targets and distractors in visual search. Perception \& Psychophysics, 67(5) 816-827. https://doi.org/10.3758/BF03193535 
*WORKSHOPS *

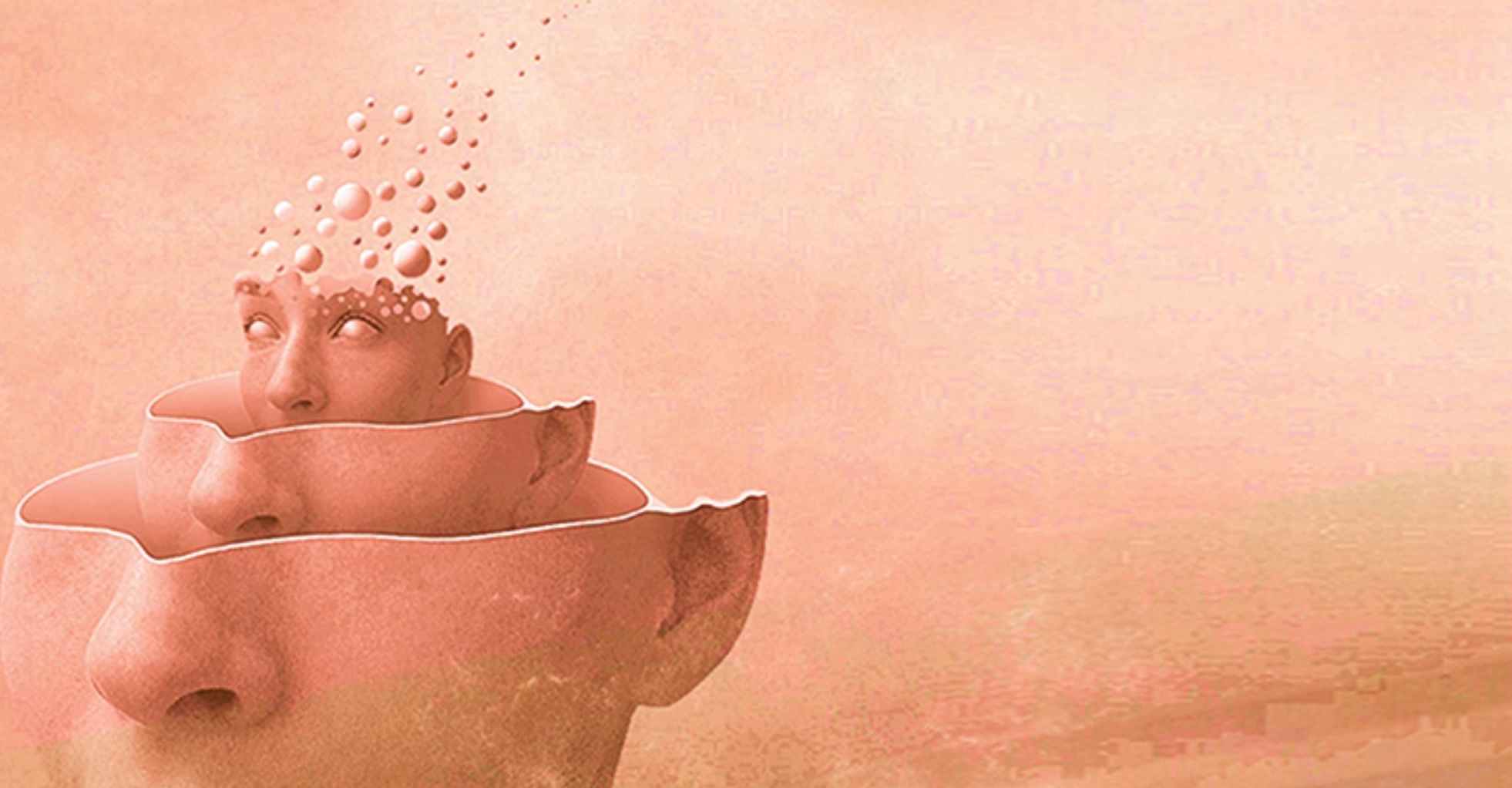





\title{
ADOLESCENT FRONTAL LOBE BRAIN DEVELOPMENT: EFFECTS OF SOCIAL AND ECONOMIC DEPRIVATION AND IMPLICATIONS FOR INTELLECTUAL ASSESSMENT, ACADEMIC REMEDIATION, AND COURT CASE DISPOSITION
}

\author{
Michael Lindsey \\ JD, PhD, Adjunct Professor Psychology Department, Southern Methodist University, President-Nestor \\ Consultants, Inc., Dallas, Texas (USA)
}

\begin{abstract}
Neuroscience has documented the substantive growth of frontal lobe gray matter during the adolescent years, similar to the brain growth spurt in early childhood - both precursors of preparation for quantitative and qualitative adaptive learning. Several United States Supreme Court decisions (Roper v. Simmons; Graham v. Florida; JDB v. North Carolina; Miller v. Alabama) have affirmed the historical chronological age of 'majority' being 18 years old, is inconsistent with what it means to be an adult. Mature cognitive processing is more appropriately characterized by the "Jean Piagetian" formal operations stage, i.e., abstract thinking, logical thinking, decision-making, and long-term planning. Formal operations is now acknowledged to be achieved during a young adult's mid-20's years of age.

"Adult" (mature) cognitive functioning is a combination of adequate brain tissue (gray matter), and learning (white matter). White matter is accumulated as a person grows, develops adaptive schema, has experiential practice, is taught, learns from modeling, and observations. We can thus infer that the more diverse are these ways of 'becoming an adult,' the more competent the young adult will be.

Current educational and IQ normative data we have on adolescents is from majority (i.e., non-ethnic majority) youth. Not yet answered is what are the norms for ethnic minority young adults (mid -20's), who have social, economic, academic, and/or experiential deprivation?

If such life experiences result in less white matter, and less complex white matter - are consequently, the normative data on "deprived" ethnic minority youth significantly different from majority youth? If yes, the implications are enormous for such issues as: (1) educational remediation; (2) IQ testing, (3) juvenile court case disposition, (4) the youth's lifelong success, (5) citizenship behaviors, (6) self, and (7) familial sufficiency.

This workshop will explore these complex issues, and make recommendations for the following next steps: empirical data collection, 'intervention’ program changes, and policy reforms.
\end{abstract}

Keywords: Brain, adolescent, IQ, judges. 


\title{
COMPREHENSIVE TREATMENT FOR TIC DISORDERS
}

\author{
Omar Rahman, \& Adam Lewin \\ University of South Florida (USA)
}

\begin{abstract}
Purpose: This workshop will help mental health professionals increase knowledge of tic disorders.

Background: Tic disorders, including Tourette's, affect around 1 in 100 children, with many continuing to have tics in adulthood. Impairment can be in the areas of emotional difficulties, social problems, family problems, school/work, physical problems, sleep issues, etc. Often, the nature of tics is unpredictable and uncontrollable, which leads to an increased sense of frustration.

Tic disorders are often comorbid with other conditions, such as OCD, ADHD, depression, and anxiety. In addition, many individuals with Tourette's have difficulty with emotional control, outbursts, and rage. Often, these are misunderstood by others and addressed in unhelpful ways.

Knowledge about the nature of tics, common issues, and treatment strategies, is typically low, even in the mental health field. Additionally, tics can sometimes be difficult to identify, or may be confused with other movements or sounds. Attendees of this workshop will better be able to identify tics, common problems, and successful treatment strategies.

Key points: We will discuss:

-Differential identification of tics (vs. compulsions, movements, stereotypies)

-Comprehensive Behavioral Interventions for Tics (CBIT), including Habit Reversal Training

-Pharmacological treatment

-Managing typical comorbid conditions and reducing dysfunction

-Ongoing research

Procedure:

-Information will be presented by an expert in a discussion format, with interaction and questions from attendees. Short videos will be used for demonstrating common tics and interventions. There will be interactive demonstration of some of the major techniques.

Description of the participants: Participants will be mental health professionals or others who wish to develop knowledge in identification and treatment of tic disorders. Maximum number will be 40 .
\end{abstract}

Keywords: Tics, Tourette's, CBIT, HRT. 


\section{AUTHOR INDEX}

\begin{tabular}{|c|c|c|c|}
\hline Abrinkova, L. & 384 & Combalbert, N. & $64,69,191$ \\
\hline Adamczyk, K. & 6 & Cormenzana, S. & 198 \\
\hline Afiatin, $\mathrm{T}$. & 119 & Corradini, I. & 371 \\
\hline Agli, O. & 332 & Corral, S. & 198 \\
\hline Almeida, L. & 282,288 & Dalpé, J. & 270,276 \\
\hline Alvarez, I. & 198 & Daukilas, S. & 285 \\
\hline Ang, A. & 304 & De Guzman, R. & 347 \\
\hline Anisimova, $\mathrm{T}$. & 207 & Deperrois, R. & 64 \\
\hline Aslan, A. & 92 & Deyneka, O. & 124,173 \\
\hline Atıc1, M. & 143 & Diaz, M. & 342 \\
\hline Attabib, N. & 54 & Dominiak, V. & 155 \\
\hline Aykaç, B. & 92 & Dougez, C. & 191 \\
\hline Bacikova, M. & 362 & Dudchenko, Z. & 155 \\
\hline Bacikova-Sleskova, M. & 352,384 & Dufresne-Tassé, C. & 106 \\
\hline Bakuleva, K. & 207 & Duque, D. & 304 \\
\hline Barel, E. & 232 & Ďuricová, L. & 310 \\
\hline Bauch, S. & 424 & Easvaradoss, V. & 227 \\
\hline Bekiroğlu, B. & 177 & El-Hage, W. & 69 \\
\hline Ben-Yehudah, G. & 279 & Émond, A. & 101 \\
\hline Berinšterová, M. & 323,357 & Erdem, A. & 91,145 \\
\hline Bertron-Gatier, L. & 261 & Eremina, D. & 14 \\
\hline Best, L. & 48,54 & Estioco, M. & 342 \\
\hline Bianchi, I. & 419 & Ferreira, M. & 237 \\
\hline Boniel-Nissim, M. & 404 & Folomeeva, $\mathrm{T}$. & 195 \\
\hline Borbon, J. & 342 & Freeze, $\mathrm{T}$. & 48 \\
\hline Bordarie, J. & 159 & Fuchsová, K. & 357 \\
\hline Both, L. & 33 & Gadd, K. & 212 \\
\hline Bozdağ, F. & 92 & Gajdosova, B. & 352 \\
\hline Bozogáňová, M. & 323 & Gál, Z. & 78,86 \\
\hline Branchini, E. & 419 & Gangloff, B. & 150 \\
\hline Bulgan, G. & 144 & Gaudet, D. & 48,54 \\
\hline Burro, R. & 419 & Gençöz, F. & 28 \\
\hline Caetano, T. & 43,258 & Geneviciute-Janone, G. & 307 \\
\hline Caño, A. & 267 & Gonen, M. & 91 \\
\hline Capitani, E. & 419 & Goupil, G. & 270,276 \\
\hline Carp, E. & 74 & Gouveia, K. & 237 \\
\hline Celejewska, A. & 6 & Gurgová, B. & 310 \\
\hline Chitashvili, M. & 247 & Gurieva, S. & 124 \\
\hline Ciric, T. & 181 & Gustainiene, L. & 307 \\
\hline Čizmić, S. & 380 & Hajduch, B. & 394 \\
\hline Coban, A. & 145 & Havighurst, S. & 91 \\
\hline
\end{tabular}


Herrero, M.

Höfler, M.

Iakovleva, M.

Ikui, Y.

İnceler, Y.

Ischebeck, A.

Iyi, T.

Jain, S.

Jankovic, I.

Joseph, E.

Jugović, I.

Jussila, A.

Kalina, O.

Kaliska, L.

Kasik, L.

Kasperski, R.

Katagiri, K.

Kılıç, N.

Kiseleva, E.

Ko, K.

Kõiv, K.

Köroğlu, D.

Kourkoutas, E.

Kovalcikiene, K.

Kozina, A.

Kreitler, S.

Kriger, E.

Kruglova, N.

Kuncewicz, D.

Kuncewicz, D.

Kuroishi, N.

Kuru, H.

Kütük, M.

Kuwal, V.

Landry, F.

Leal, $M$.

Lehtikunnas, L.

Lewin, A.

Liduma, A.

Lima, $\mathrm{T}$.

Lindsey, M.

Loginova, I.

Lopes, J.

Lubinskaya, E.

Machavariani, D.
198

424

10

255

28

424

145

227

222

227

291

134

362

129

78,86

279

23

177

366

347

124, 134

28

59

285, 307

291

414

366

10

264

264

273, 301

202

143

424

270, 276

294

212

53, 432

124

282, 288

431

18

43,258

10

247
Magdová, M.

323, 357

Makino, K.

298

Malleh, N.

150

Marano, A.

371

Marić, S.

389

Martínez-Pampliega, A.

198

Martsinkovskaya, T.

366

Matthys, C.

263

McPhee, R.

48

Medyanik, O.

169,375

Melo-Silva, L.

294

Msall, K.

111

Mutić, B.

380

Nakano, S.

314

Nguy, V.

Nikiforov, G.

39

155

Nikolaeva, O.

10

Nogueira, S.

282, 288

Ohtaka, M.

295

Or, M.

59

Orestova, V.

366

Orosová, O.

352, 384, 394

Oskarsson, $\mathrm{M}$.

291

Osowiecka, M.

6

Ouhmad, N.

69

Özdemir, H.

Papadaki, A.

164, 177, 186

Paquette, R.

Pavlich, C.

59

270, 276

Paz, S.

6

304

Peitel, T.

134

Perše, $\mathrm{T}$.

291

Petriaeva, O.

18

Petrovic, D.

96, 114

Petrović, I.

380

Popescu, A.

74

Poraj-Weder, M.

263

Prabhakaran, T.

227

Proctor, C.

48

Quintanar, L.

83

Rahman, O.

53, 432

Ramadas, E.

Ramdhani, N.

43, 258

119

347

134 
Razzak, R.

Reis, J.

Rekunova, A.

Roberts, D.

Rodionova, E.

Rodríguez-Naranjo, C.

Rone, S.

Rubin, O.

Russell, R.

Sakmar-Balkan, E.

Saltukoğlu, G.

Samuylova, I.

Sano, Y.

Santos, N.

Sanz-Vázquez, M.

Savardi, U.

Segrin, C.

Semchuk, J.

Serebriakova, J.

Shalev, O.

Shchelkova, O.

Soboleva, M.

Sokhadze, O.

Sollarova, E.

Solovieva, Y.

Soro, J.

Sousa, M.

Štefaňáková, M

Stelmokiene, A.

Stojiljkovic, S.

Sushentsov, E.

328,337

155

267

124

38

48

202

164,186

207, 217

273, 301

304

198

419

6

39

315

59

3,10

366

247

129

83

237

288

384,394

307

181,222

3
Taillandier-Schmitt, A.

191

Tararukhina, O.

399, 409

Tatar, A.

164, 177, 186

Tauber, C.

389

Tiamzon, E.

342

Todorovic, J.

181,222

Topcu, M.

28

Toyama, M.

138

Trepanowski, R.

6

Trifu, S.

74

Tzischinsky, O.

232

Usmanova, E.

3

Valiev, A.

3

Vaz-Cerniglia, C. 261

Veldin, M.

291

Vidmar, M.

291

Vinokurov, F.

195

Volkova, O.

18

Vracar, S.

114

Vukelić, M.

380

Walter, M.

315

Wąsowicz, G.

263

Wiles, E.

119

Winiarek, A.

313

Wojciechowska, L.

313

Wong, W.

39

Yamazaki, Y.

138

Zaytsev, A.

375

Zuzeviciute, V.

124 
